
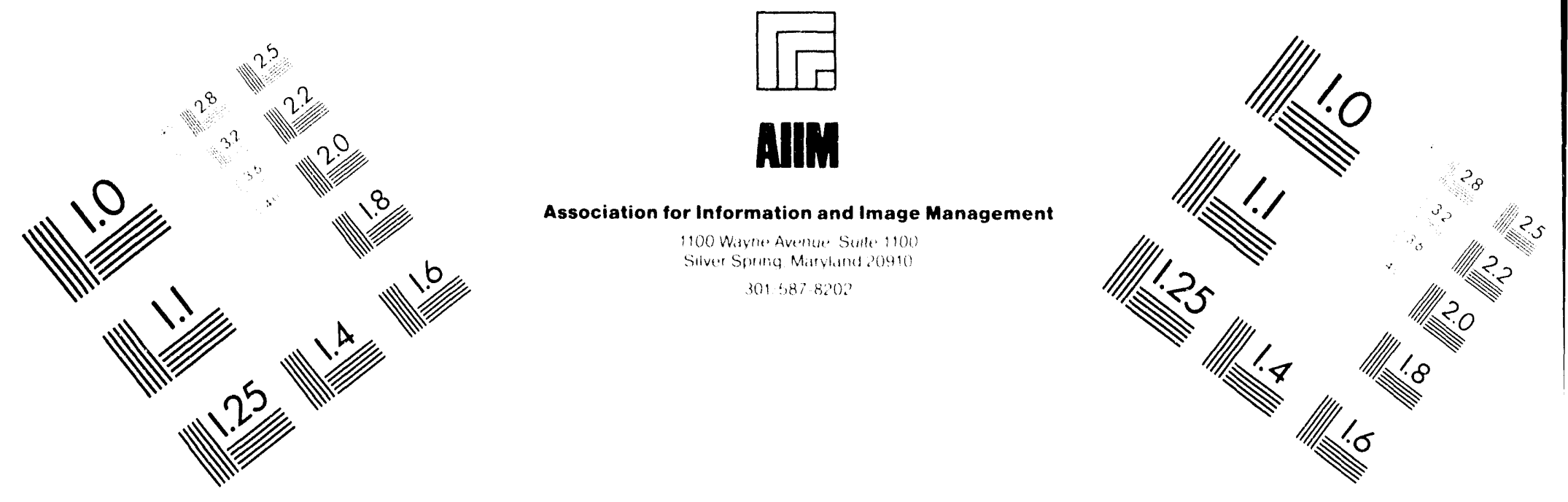

\title{
Centimeter
}

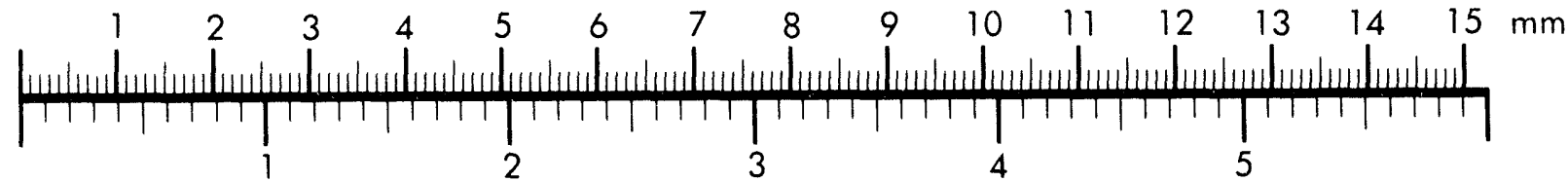

Inches
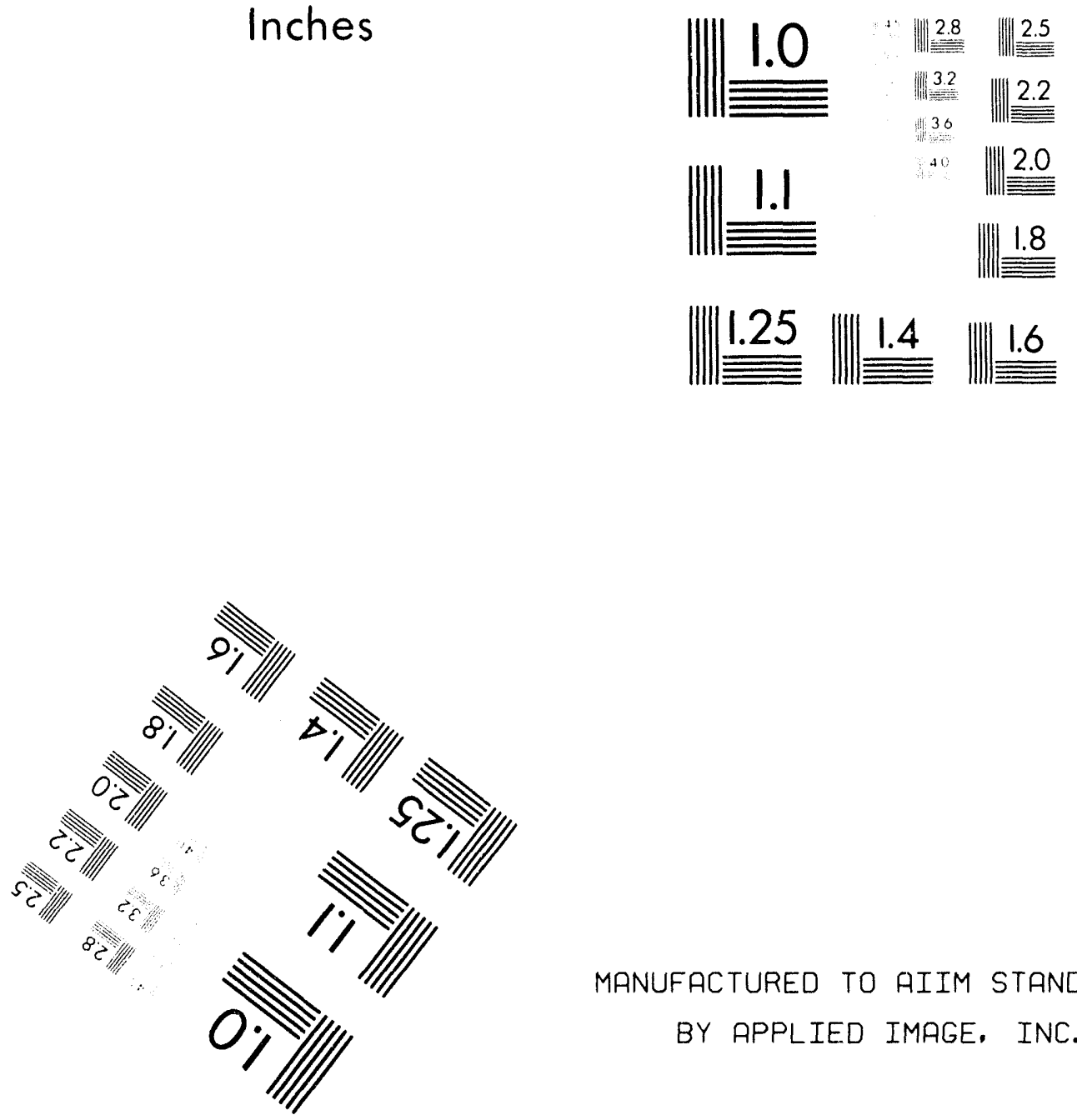

MANUFACTURED TO AIIM STANDARDS

BY APPLIED IMAGE, INC.

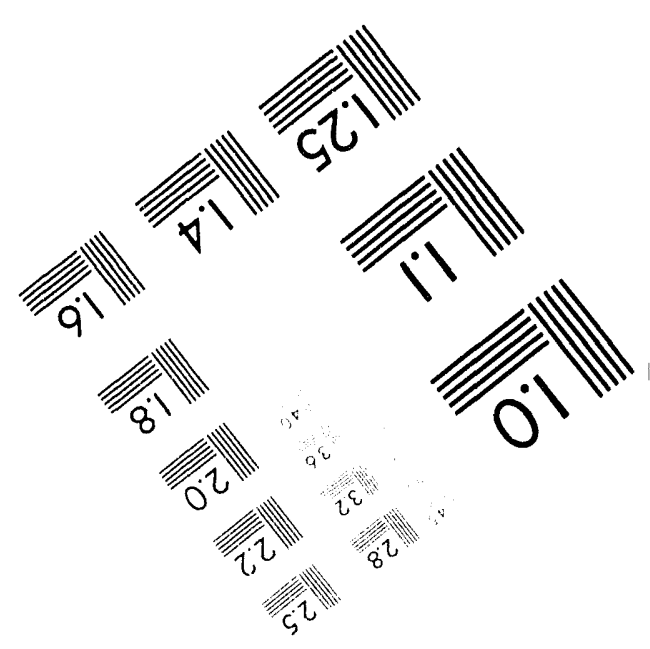



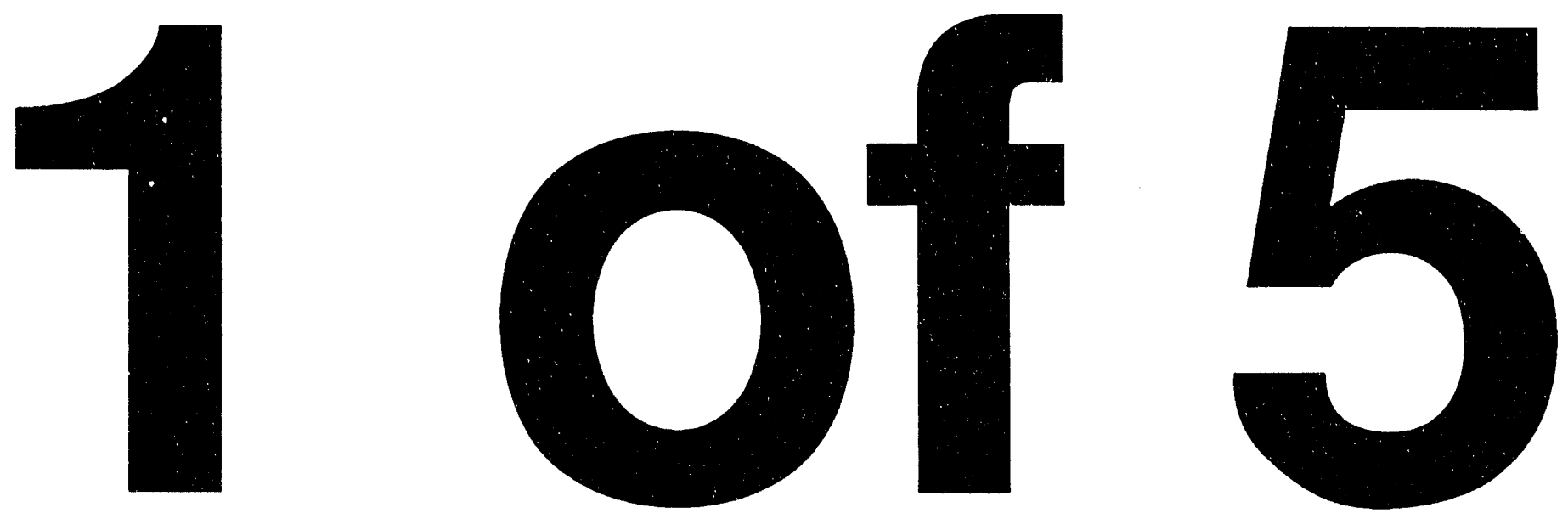
DOE/RL-93-56-4

\section{Quarterly Report of RCRA Groundwater Monitoring Data for Period October 1, 1993 through December 31, 1993}

Geohydrologic Engineering Function

Westinghouse Haniord Company

Environmental Division

Date Published

April 1994

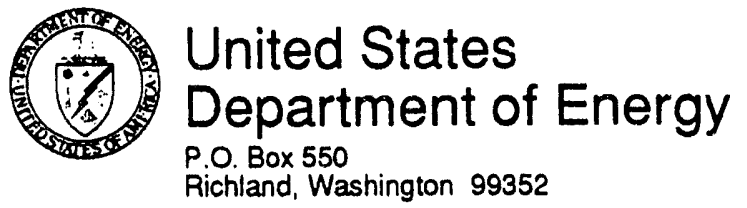

Approved for Public Release 


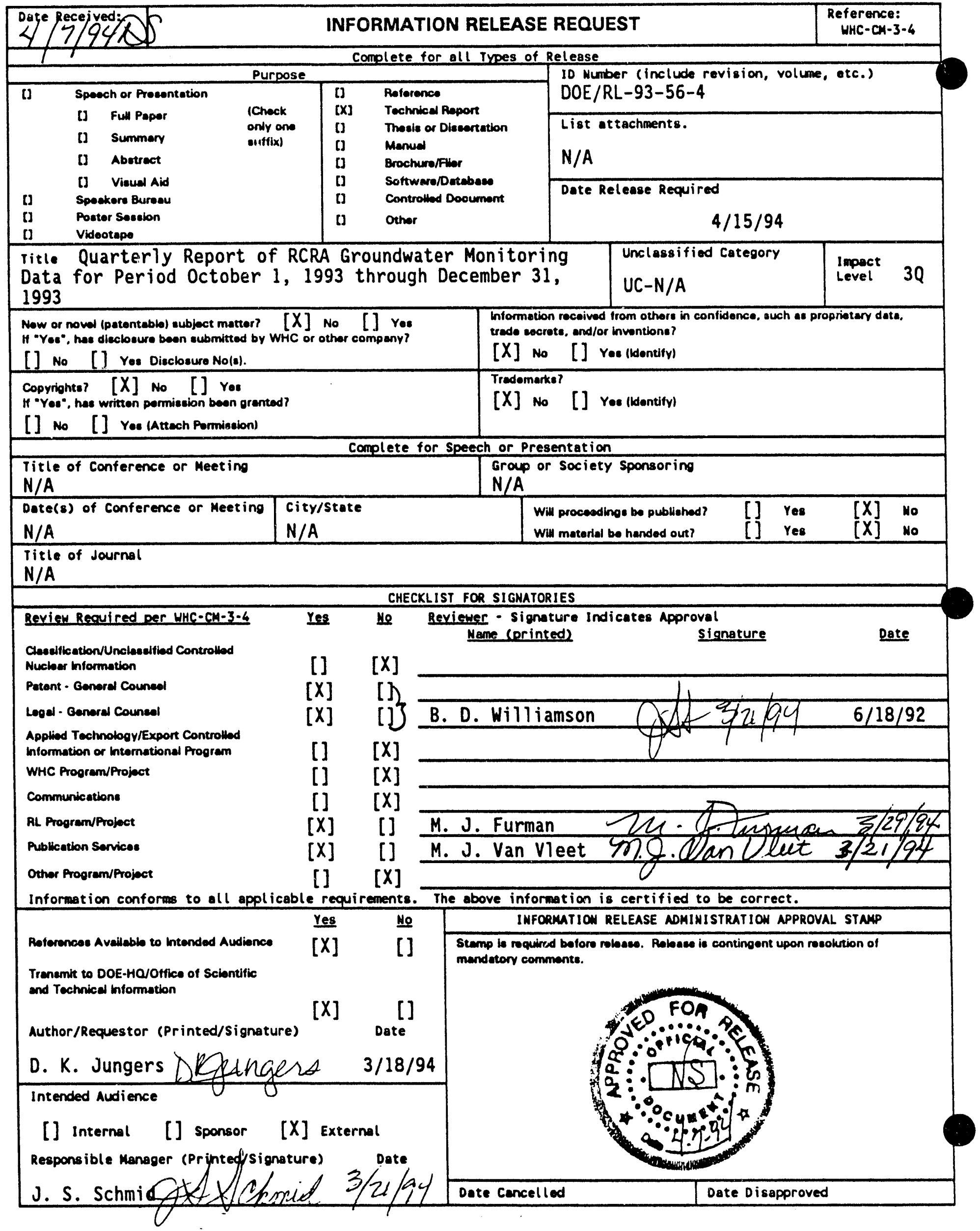


DOE/RL-93-56-4

\section{CONTENTS}

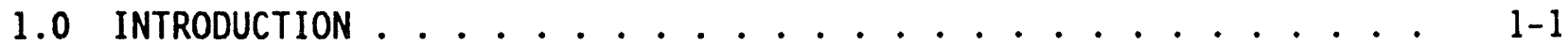

1.1 LABORATORY AND SAMPLING STATUS . . . . . . . . . . . . . 1-1

1.2 QUALITY CONTROL PROGRAM . . . . . . . . . . . . . . . 1-1

1.2.1 Introduction ................. . 1-1

1.2.2 External Quality Control Program .......... . 1-5

1.2.3 Internal Quality Control Program . . . . . . . . . . . 1-6

1.2.4 Nonconformance/Incident Reports . . . . . . . . . . 1-11

1.2.5 Quality Assurance Occurrences . . . . . . . . . . 1-12

1.3 QUALITY CONTROL DEFINITIONS . . . . . . . . . . . . . . . 1-14

1.4 DATA TABLES . . . . . . . . . . . . . . . . . . . . . . . 1-16

1.4.1 Water Level Measurement Report . . . . . . . . . . . 1-16

1.4.2 Constituent List and Summary of Results ....... . 1-16

1.4.3 Constituents with at Least One Detected Value . . . . 1-17

1.4.4 Contamination Indicator Parameters . . . . . . . . 1-18

1.4.5 Codes and Abbreviations ............. . 1-18

1.5 DATA EVALUATION ................. . . . . . . . . . . 1

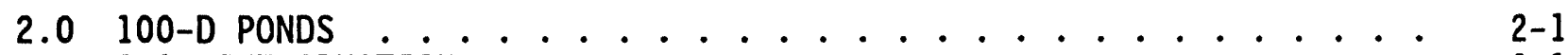

2.1 INTRODUCTION . . . . . . . . . . . . . . . . . 2-1

2.2 WATER LEVEL MEASUREMENTS . . . . . . . . . . . . . . . 2-1

2.3 WATER CHEMISTRY DATA ................... . . 2-1

$3.0183-H$ SOLAR EVAPORATION BASINS . . . . . . . . . . . . . . 3-1

3.1 INTRODUCTION . . . . . . . . . . . . . . . . . 3-1

3.2 WATER LEVEL MEASUREMENTS . . . . . . . . . . . . . . . 3-1

3.3 WATER CHEMISTRY DATA ................ . . . 3-2

$4.0100-N$ RCRA SITES . . . . . . . . . . . . . . . . . . . 4-1

4.1 INTRODUCTION ....................... 4-1

4.2 WATER LEVEL MEASUREMENTS .................. . . 4-1

4.3 WATER CHEMISTRY DATA . . . . . . . . . . . . . . . . . 4-1

4.3.1 1301-N Liquid Waste Disposal Facility . . . . . . . . 4-2

4.3.2 1324-N Surface Impoundment and
1324-NA Percolation Pond . . . . . . . . . . . . 4-2

4.3.3 1325-N Liquid Waste Disposal Facility . . . . . . . . 4-2

4.3.4 100-NR-2 Operable Unit.............. 4-2

5.0 GROUT TREATMENT FACILITY . . . . . . . . . . . . . . . 5-1

5.1 INTRODUCTION . . . . . . . . . . . . . . . . 5-1

5.2 WATER LEVEL MEASUREMENTS .................. . . 5-1

5.3 WATER CHEMISTRY DATA . . . . . . . . . . . . . . 5-2

6.0 216-B-3 POND SYSTEM .................... . . . 6-1

6.1 INTRODUCTION ..................... . . . . . . . .

6.2 WATER LEVEL DATA ..................... . . . 6-1

6.3 WATER CHEMISTRY DATA ................... . . . . . .

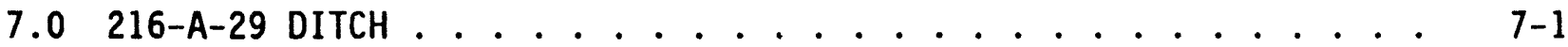

7.1 INTRODUCTION .................... . . . . . . 7

7.2 WATER LEVEL MEASUREMENTS . . . . . . . . . . . . . . . . . . $7-1$

7.3 WATER CHEMISTRY DATA . . . . . . . . . . . . . . . . . . $7-2$ 


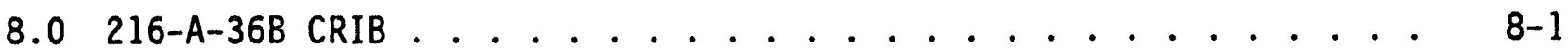

8.1 INTRODUCTION ....................... 8-1

8.2 WATER LEVEL MEASUREMENTS . . . . . . . . . . . . . . 8-1

8.3 WATER CHEMISTRY DATA ................... . . 8-2

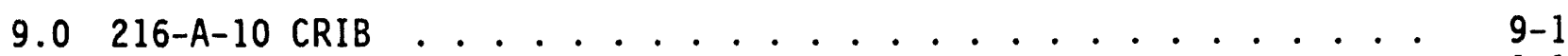

9.1 INTRODUCTION ...................... . . . 9-1

9.2 WATER LEVEL MEASUREMENTS .................... . . . . . $9-1$

9.3 WATER CHEMISTRY DATA . . . . . . . . . . . . . . . . 9-2

10.0 216-B-63 TRENCH ....................... . . . 10-1

10.1 INTRODUCTION .................... . . . . 10-1

10.2 WATER LEVEL MEASUREMENTS ................. . . . . 10-1

10.3 WATER CHEMISTRY DATA . . . . . . . . . . . . . 10-2

$11.0216-5-10$ POND AND DITCH ................... . . . . . . . . . .

11.1 INTRODUCTION ..................... . . . . 11-1

11.2 WATER LEVEL MEASUREMENTS . . . . . . . . . . . . . . 11-2

11.3 WATER CHEMISTRY DATA . . . . . . . . . . . . . 11-2

$12.0216-U-12$ CRIB . . . . . . . . . . . . . . . . . . . 12-1

12.1 INTRODUCTION ..................... . . . . . . . . . . . . . . .

12.2 WATER LEVEL MEASUREMENTS . . . . . . . . . . . . . . 12-2

12.3 WATER CHEMISTRY DATA . . . . . . . . . . . . . 12-2

13.0 LIQUID EFFLUENT RETENTION FACILITY . . . . . . . . . . . . . 13-1

13.1 INTRODUCTION . . . . . . . . . . . . . . . . . 13-1

13.2 WATER LEVEL MEASUREMENTS . . . . . . . . . . . . . . . . 13-1

13.3 WATER CHEMISTRY DATA .................... $13-1$

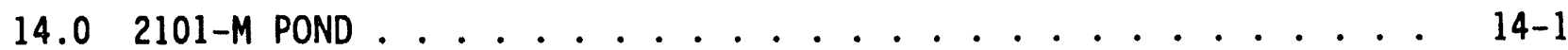

14.1 INTRODUCTION ......................... . . . . . . . . . . . .

14.2 WATER LEVEL DATA ..................... . . . 14-1

14.3 WATER CHEMISTRY DATA ................. 14-1

15.0 LOW-LEVEL BURIAL GROUNDS ................... . . 15-1

15.1 INTRODUCTION .................... . . . . . . . . . . .

15.2 WATER LEVEL MEASUREMENTS . . . . . . . . . . . . . . . 15-1

15.3 WATER CHEMISTRY DATA . . . . . . . . . . . . . 15-1

16.0 SINGLE-SHELL TANKS . . . . . . . . . . . . . . . . 16-1

16.1 INTRODUCTION ................... 16-1

16.2 WATER LEVEL MEASUREMENTS . . . . . . . . . . . . 16-2

16.3 WATER CHEMISTRY DATA . . . . . . . . . . . . . . . . 16-3

16.3.1 Waste Management Area A-AX . . . . . . . . . 16-4

16.3.2 Waste Management Area B-BX-BY . . . . . . . . . . . 16-4

16.3.3 Waste Management Area $C$. . . . . . . . . . . . . 16-4

16.3.4 Waste Management Area S-SX ... . . . . . . . . 16-4

16.3.5 Waste Management Area T... . . . . . . . . . 16-4

16.3.6 Waste Management Area TX-TY . . . . . . . . . 16-5

16.3.7 Waste Management Area U . . . . . . . . . . 16-6 
DOE/RL-93-56-4

CONTENTS (cont.)

17.0300 AREA PROCESS TRENCHES . . . . . . . . . . . . . 17-1

17.1 INTRODUCTION . ................ . . . . . . .

17.2 WATER LEVEL MEASUREMENTS . . . . . . . . . . . . 17-1

17.3 WATER CHEMISTRY DATA .............. 17-1

18.0 NONRADIOACTIVE DANGEROUS WASTE LANDFILL . . . . . . . . . 18-1

18.1 INTRODUCTION .................. 18-1

18.2 WATER LEVEL DATA . . . . . . . . . . . . . . 18-1

18.3 WATER CHEMISTRY DATA ................ 18-1

19.0 REFERENCES ................... . . 19-1 


\section{LIST OF FIGURES}

1-1 Locations of the RCRA Groundwater Monitoring Projects and Landmarks on the Hanford Site . . . . . . . . . . . . . 1-2

2-1 Monitoring Well Location Map for the 100-D Ponds . . . . . . . 2-2

3-1 Monitoring Well Location Map for the 183-H Solar Evaporation Basins Network ..................... 3-3

4-1 Monitoring Well Locations for 1301-N, 1324-N/NA, and 1325-N Facilities................... . 4-3

5-1 Monitoring Well Locations for the Grout Treatment Facility . . . 5-3

6-1 216-B-3 Pond Groundwater Monitoring Network . . . . . . . . . . . 6-3

7-1 Monitoring Well Locations for the 216-A-29 Ditch . . . . . . . 7-3

8-1 Monitoring Well Locations for the 216-A-36B Crib . . . . . . . 8-3

9-1 Monitoring Well Locations for the 216-A-10 Crib. . . . . . . . 9-3

10-1 Well Location Map for the 216-3-63 Trench. . . . . . . . . . 10-3

11-1 Monitoring Well Location for the 216-S-10 Facility . . . . . . 11-3

12-1 Well Location Map for 216-U-12 Crib............... 12-3

13-1 Monitoring Well Locations for the Liquid Effluent Retention Facility .................. 13-3

14-1 Monitoring Well Locations for the 2101-M Pond . . . . . . . . . 14-3

15-1 Monitoring Well Locations for Low-Level Waste Management Area 1... . . . . . . . . . . . . 15-3

15-2 Monitoring Well Locations for Low-Level Waste Management Area 2... . . . . . . . . . . . 15-4

15-3 Monitoring Well Locations for Low-Level Waste Management Area 3... . . . . . . . . . . . 15-5

15-4 Monitoring Well Locations for Low-Level Waste Management Area 4. . . . . . . . . . . . . . 15-6

15-5 Monitoring We11 Locations for Low-Leve1 Waste Management Area 5 . . . . . . . . . . . . . . . . .

16-1 Single-She11 Tank Waste Management Areas in the 200 East Area . . . . . . . . . . . . . . . . . 16-7 


\section{LIST OF FIGURES (cont.)}

16-2 Single-She11 Tank Waste Management Areas in the 200 West Area . . . . . . . . . . . . . . 16-8

16-3 Monitoring Well Locations for the Single-She1l Tank Waste Management Areas in the 200 East Area ... . . . . . . . 16-9

16-4 Monitoring Well Locations for the Single-She11 Tank Waste Management Areas in the 200 West Area .......... 16-10

17-1 Monitoring We11 Locations for the 300 Area Process Trenches . . 17-2

18-1 Monitoring We11 Locations for the Nonradioactive Dangerous Waste Landfill and Solid Waste Landfill .......... 18-2 


\section{LIST OF TABLES}

1-1 Completeness ...................... 1-4

1-2 Field Duplicates and Field Blanks Evaluation Results, Fourth Quarter 1993

1-3 Breakdown of Field Duplicates Exceeding Quality Control Limits ................. . . 1-7

1-4 Breakdown of Field Blanks Exceeding Quality Control Limits . . . 1-8

1-5 Frequency of Quality Control Samples, Fourth Quarter $1993 \ldots$. . . 1-8

1-6 Radiochemistry Quarterly Report Summary . . . . . . . . . . 1-9

1-7 Summary of EPA Water Pollution, Water Supply, and Interlaboratory Comparison Study ............. 1-11

1-8 Analysis Method Code Definitions .............. 1-19

1-9 Drinking Water Standards . . . . . . . . . . . . 1-22

2-1 Monitoring Well Purpose and Sampling Schedule in the 100-D Ponds Network ............... 2-. 2-3

2-2 RCRA Water Level Measurement Report 100-D Ponds, Fourth Quarter $1993 \ldots . . . . . . . . . .2-4$

2-3 Constituent List and Summary of Results for the 100-D Pond Data for Reporting Period October 1 through December 31, 1993 . . 2-7

2-4 Constituents with at Least One Detected Value for the 100-D Pond Data for Reporting Period October 1 through December 31, 1993 . . 2-9

2-5 Contamination Indicator Parameters for the 100-D Pond Data for Reporting Period October 1 through December 31, 1993...... 2-12

3-1 Monitoring We1l Position and Sampling Schedule for the 183-H Solar Evaporation Basins Network . . . . . . . . . . 3-4

3-2 RCRA Water Level Measurement Report 183-H Solar Evaporation Basins, Fourth Quarter 1993 ... . . . . . 3-6

3-3 Constituent List and Summary of Results for 183-H Solar Evaporation Basins Data for Reporting Period October 1 through December 31, 1993 .............. 3-10

3-4 Constituents with at Least One Detected Value for the 183-H Solar Evaporation Basins Data for Reporting Period October 1 through December 31, 1993 


\section{LIST OF TABLES (cont.)}

3-5 Contamination Indicator Parameters for the 183-H Solar

Evaporation Basins Data for Reporting Period October 1

through December 31, 1993 ................. 3-19

4-1 Monitoring Well Purpose and Sampling Schedule in

the 100-N Area..................... . . . 4-4

4-2 RCRA Water Level Measurement Report 100-N Area,

Fourth Quarter 1993 ................... 4-6

4-3 Constituent List and Summary of Results for 100-N RCRA Sites

Data for Reporting Period October 1 through December 31, 1993 . . 4-12

4-4 Constituents with at Least One Detected Value for the

100-N RCRA Sites Data for Reporting Period October 1

through December 31, 1993 .................. 4-15

4-5 Contamination Indicator Parameters for the 100-N RCRA Sites

Data for Reporting Period October 1 through December 31, 1993 . . 4-26

4-6 Drinking Water Standards Exceeded in 100-N Area RCRA Sites . . . 4 4-28

5-1 Monitoring Well Purpose and Sampling Schedule for the Grout

Treatment Facility Network . . . . . . . . . . . . . . 5-4

5-2 RCRA Water Level Measurement Report Grout Treatment

Facility, Fourth Quarter 1993 ............... 5-5

5-3 Constituent List and Summary of Results for the Grout Treatment

Facility Data for Reporting Period October 1 through

December 31, 1993 .................. . . 5-7

5-4 Constituents with at Least One Detected Value for the Grout

Treatment Facility Data for Reporting Period October 1

through December 31, 1993 ................. 5-10

5-5 Contamination Indicator Parameters for the Grout Treatment

Facility Data for Reporting Period October 1

through December 31,1993 ................ . 5-16

5-6 Summary Table of Flagged Data for Reporting Period October 1 through December 31, 1993 ................. 5-17

6-1 Monitoring Well Purpose and Sampling Schedule for the 216-B-3 Pond System Network . . . . . . . . . . . . 6-4

6-2 RCRA Water Level Measurement Report 216-B-3 Pond System, Fourth Quarter 1993 . . . . . . . . . . . . . 6-5

6-3 Constituent List and Summary of Results for the 216-B-3 Pond Data for Reporting Period October 1 through December 31, 1993 . . . . . 


\section{LIST OF TABLES (cont.)}

6-4 Constituents with at Least One Detected Value for the 216-B-3 Pond Data for Reporting Period October 1

through December 31, 1993 .. . . . . . . . . . . . . 6-12

6-5 Contamination Indicator Parameters for the 216-B-3 Pond Data

for Reporting Period October 1 through December 31, 1993 . . . . 6-21

7-1 Monitoring Well Purpose and Sampling Schedule for the 216-A-29 Ditch . . . . . . . . . . . . . . . . . . . 7-4

7-2 RCRA Water Level Measurement Report 216-A-29 Ditch, Fourth Quarter 1993 ................... . 7-5

7-3 Constituent List and Summary of Results for the 216-A-29 Ditch Data for Reporting Period October 1 through December 31, 1993 . . 7-8

7-4 Constituents with at Least One Detected Value for the 216-A-29 Ditch Data for Reporting Period October 1 through December 31, 1993 ................. 7-13

7-5 Contamination Indicator Parameters for the 216-A-29 Ditch Data for Reporting Period October 1 through

8-1 Monitoring Well Purpose and Sampling Schedule for the 216-A-36B Crib Network

8-2 RCRA Water Level Measurement Report 216-A-36B Crib, Fourth Quarter 1993

8-3 Constituent List and Summary of Results for the 216-A-36B Crib Data for Reporting Period October 1 through December 31, 1993

8-4 Constituents with at Least One Detected Value for the 216-A-36B Crib Data for Reporting Period October 1 through December 31, 1993

8-5 Contamination Indicator Parameters for the 216-A-36B Crib Data for Reporting Period October 1 through December $31,1993$.

9-1 Monitoring Well Purpose and Sampling Schedule for the 216-A-10 Crib Network

9-2 RCRA Water Level Measurement Report 216-A-10 Crib, Fourth Quarter 1993

9-3 Constituent List and Summary of Results for the 216-A-10 Crib Data for Reporting Period October 1 through December 31, 1993 


\section{LIST OF TABLES (cont.)}

9-4 Constituents with at Least One Detected Value for the 216-A-10 Crib Data for Reporting Period October 1

through December 31, 1993................. . 9-10

9-5 Contamination Indicator Parameters for the 216-A-10 Crib Data for Reporting Period October 1 through December 31, 1993 . . . . . . 9-14

10-1 Monitoring Well Purpose and Sampling Schedule for the 216-B-63 Trench Network................ . . 10-4

10-2 RCRA Water Level Measurement Report 216-B-63 Trench, Fourth Quarter 1993 ................. 10-5

10-3 Constituent List and Summary of Results for the 216-B-63 Trench Data for Reporting Period October 1 through December 31, 1993 ................. . 10-7

10-4 Constituents with at Least One Detected Value for the 216-B-63 Trench Data for Reporting Period October 1 through December 31, 1993 ................. 10-10

10-5 Contamination Indicator Parameters for the 216-B-63 Trench Data for Reporting Period October 1 through December 31, 1993 .................... 10-16

10-6 Water Quality Standards Exceeded at the 216-B-63 Trench . . . . 10-18

11-1 Monitoring Well Purpose and Sampling Schedule for the 216-S-10 Facility................... . . . 11-4

11-2 RCRA Water Level Measurement Report 216-S-10 Facility, Fourth Quarter 1993

11-3 Constituent List and Summary of Results for the 216-S-10 Facility Data for Reporting Period October 1 through December 31, 1993 ............... . 11-6

11-4 Constituents with at Least One Detected Value for the 216-S-10 Facility Data for Reporting Period Octcber 1

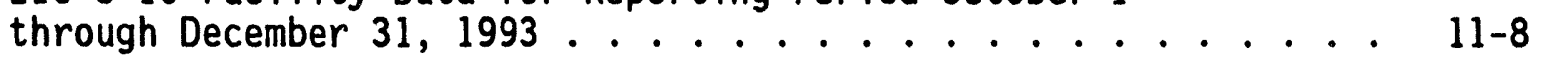

11-5 Contamination Indicator Parameters for the 216-S-10 Facility Data for Reporting Period October 1 through December 31, 1993 . . 11-9

12-1 Monitoring We11 Purpose and Sampling Schedule for the 216-U-12 Crib.................... . . 12-4

12-2 RCRA Water Level Measurement Report for the 216-U-12 Crib, Fourth Quarter 1993 


\section{LIST OF TABLES (cont.)}

12-3 Constituent List and Summary of Results for the 216-U-12 Crib

Data for Reporting Period October 1 through December 31, 1993 . . 12-6

12-4 Constituents with at Least One Detected Value for the

216-U-12 Crib Data for Reporting Period October 1 through

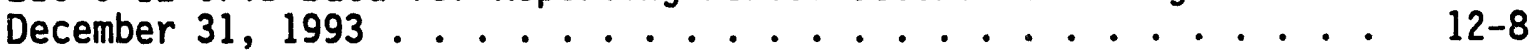

12-5 Contamination Indicator Parameters for the 216-U-12 Crib Data for Reporting Period October 1 through December 31, 1993 . . . 12-9

13-1 Monitoring Well Purpose and Sampling Schedule for the Liquid Effluent Retention Facility Network. . . . . . . . . . 13-4

13-2 RCRA Water Level Measurement Report for the LERF, Fourth Quarter ..................... 13-5

13-3 Constituent List and Summary of Results for the Liquid Effluent Retention Facility Data for Reporting Period October 1 through December 31, 1993 ............... 13-6

13-4 Constituents with at Least One Detected Value for the Liquid Effluent Retention Facility Data for Reporting Period October 1 through December 31, 1993................. 13-9

13-5 Contamination Indicator Parameters for the Liquid Effluent Retention Facility Data for Reporting Period October 1 through December 31, 1993 .............. 13-12

14-1 Monitoring Well Purpose and Sampling Schedule for the 2101-M Pond...................... 14-4

14-2 RCRA Water Level Measurement Report for the 2101-M Pond, Fourth Quarter 1993

14-3 Constituent List and Summary of Results for the 2101-M Pond Data for Reporting Period October 1 through December 31, 1993 . . 14-6

14-4 Constituents with at Least One Detected Value for the 2101-M Pond Data for Reporting Period October 1 through December 31, 1993 .................. 14-8

14-5 Contamination Indicator Parameters for the 2101-M Pond Data for Reporting Period October 1 through December 31, 1993 . . . . 14-9

15-1 Low-Leve? Burial Grounds . . . . . . . . . . . . . . . 15-8

15-2 Monitoring Well Purpose and Sampling Schedule for Low-Level Waste Management Area 1. . . . . . . . . . 15-9

15-3 Monitoring Well Purpose and Sampling Schedule for Low-Level Waste Management Area 2................. 15-10 


\section{LIST OF TABLES (cont.)}

15-4 Monitoring Well Purpose and Sampling Schedule for Low-Level

Waste Management Area 3 .. . . . . . . . . . . 15-11

15-5 Monitoring We11 Purpose and Sampling Schedule for Low-Leve1

Waste Management Area 4 .............. . 15-12

15-6 Honitoring We11 Purpose and Sampling Schedule for Low-Leve1

Waste Management Area 5 .............. . 15-13

15-7 RCRA Water Level Measurement Report for the LLWMA-1,

Fourth Quarter 1993 ................. 15-14

15-8 RCRA Water Level Measurement Report for the LLWMA-2,

Fourth Quarter 1993 ............... 15-17

15-9 RCRA Water Level Measurement Report for the LLWMA-3,

Fourth Quarter 1993 ............... 15-19

15-10 RCRA Water Leve1 Measurement Report for the LLWMA-4, Fourth Quarter 1993 ................ . 15-22

15-11 RCRA Water Level Measurement Report for the LLWMA-5, Fourth Quarter 1993 ................. 15-24

15-12 Constituent List and Summary of Results for the Low-Level

Waste Burial Grounds WMA 1 Data for Reporting Period

October 1 through December 31,1993 ............ 15-25

15-13 Constituents with at Least One Detected Value for the

Low-Level Waste Burial Grounds WMA 1 Data for Reporting

Period October 1 through December 31, 1993 . . . . . . . . 15-28

15-14 Contamination Indicator Parameters for the Low-Level Waste Burial Grounds WMA 1 Data for Reporting Period October 1 through December 31, 1993 ........... 15-39

15-15 Constituent List and Summary of Results for the Low-Leve1

Waste Burial Grounds WMA 2 Data for Reporting Period

October 1 through December 31,1993 ............ 15-40

15-16 Constituents with at Least One Detected Value for the Low-Level Waste Burial Grounds WMA 2 Data for Reporting

Period October 1 through December 31, 1993 ......... . 15-43

15-17 Contamination Indicator Parameters for the Low-Level Waste Burial Grounds WMA 2 Data for Reporting Period October 1 through December 31,1993 ............ 15-51

15-18 Constituent List and Summary of Results for the Low-Level Waste Burial Grounds WMA 3 Data for Reporting

Period October 1 through December 31, 1993 


\section{LIST OF TABLIES (cont.)}

15-19 Constituents with at Least One Detected Value for the Low-Level Waste Burial Grounds WMA 3 Data for Reporting Period October 1 through December 31, 1993 . . . . . . . . . 15-56

15-20 Contamination Indicator Parameters for the Low-Level Waste Burial Grounds WMA 3 Data for Reporting Period October 1 through December 31, 1993

15-21 Constituent List and Summary of Results for the Low-Leve1

Waste Burial Grounds WMA 4 Data for Reporting Period October 1 through December 31, 1993

15-22 Constituents with at Least One Detected Value for the Low-Level Waste Burial Grounds WMA 4 Data for Reporting Period October 1 through December 31, 1993 . . . . . . . . 15-73

15-23 Contamination Indicator Parameters for the Low-Level Waste Burial Grounds WMA 4 Data for Reporting Period October 1 through December 31, 1993 ............ 15-83

15-24 Constituent List and Summary of Results for the Low-Level Waste Burial Grounds WMA 5 Data for Reporting Period October 1 through December 31,1993 ........... 15-85

15-25 Constituents with at Least One Detected Value for the Low-Level Waste Burial Grounds WMA 5 Data for Reporting Period October 1 through December 31, 1993 . . . . . . . . 15-88

15-26 Contamination Indicator Parameters for the Low-Level Waste Burial Grounds WMA 3 Data for Reporting Period October 1 through December 31, 1993 ............... 15-92

15-27 Water Quality Standards Exceeded at Low-Level Waste Management Area 1. . . . . . . . . . . . . . . 15-93

15-28 Water Quality Standards Exceeded at Low-Leve1 Waste Management Area 2................... 15-93

15-29 Water Quality Standards Exceeded at Low-Level Waste Management Area 3. . . . . . . . . . . . . . 15-94

15-30 Water Quality Standards Exceeded at Low-Level Waste Management Area 4.................... 15-95

15-31 Water Quality Standards Exceeded at Low-Level Waste Management Area 5................... 15-96

16-1 Groundwater Monitoring Wells for the Single-Shell Tanks . . . 16-11

16-2 RCRA Water Level Measurement Report for the Single-Shell Tanks, Fourth Quarter 1993 
LIST OF TABLES (cont.)

16-3 Constituent List and Summary of Results for the Single-Shell Tank Waste Management Area A-AX Data for Reporting Period October 1 through December 31,1993 ............ 16-20

16-4 Constituents with at Least One Detected Value for the Single-She 11 Tank Waste Management Area A-AX Data for Reporting Period October 1 through December 31, 1993.

16-5 Contamination Indicator Parameters for the Single-Shell Tank

Waste Management Area A-AX Data for Reporting Period

October 1 through December 31, 1993

16-6 Constituent List and Summary of Results for the Single-Shell

Tank Waste Management Area B-BY-BX Data for Reporting Period October 1 through December 31, 1993 ............ 16-23

16-7 Constituents with at Least One Detected Value for the Single-Shell Tank Waste Management Area B-BY-BX Data for Reporting Period October 1 through December 31, 1993... . . 16-25

16-8 Contamination Indicator Parameters for the Single-Shell Tank Waste Management Area B-BY-BX Data for Reporting Period October 1 through December 31, 1993 ............ 16-27

16-9 Constituent List and Summary of Results for the Single-Shell Tank Waste Management Area C Data for Reporting Period October 1 through December 31, 1993 . . . . . . . . . 16-28

16-10 Constituents with at Least One Detected Value for the Single-She11 Tank Waste Management Area C Data for Reporting Period October I through December 31, 1993

16-11 Contamination Indicator Parameters for the Single-Shell Tank Waste Management Area C Data for Reporting Period October 1 through December 31,1993 ............... 16-30

16-12 Constituent List and Summary of Results for the Single-She 11 Tank Waste Management Area S-SX Data for Reporting Period October 1 through December 31, 1993 ............ 16-31

16-13 Constituents with at Least One Detected Value for the Single-She11 Tank Waste Management Area S-SX Data for Reporting Period October 1 through December 31, 1993 . . . . . 16-32

16-14 Contamination Indicator Parameters for the Single-She11 Tank Waste Management Area S-SX Data for Reporting Period October 1 through December 31, 1993. . . . . . . . . . 16-33

16-15 Constituent List and Summary of Results for the Single-She11 Tank Waste Management Area T Data for Reporting Period October 1 through December 31, 1993 


\section{LIST OF TABLES (cont.)}

16-16 Constituents with at Least One Detected Value for the

Single-She11 Tank Waste Management Area T Data for Reporting Period October 1 through December 31, 1993 . . . . . . . . 16-36

16-17 Contamination Indicator Parameters for the Single-Shell Tank Waste Management Area T Data for Reporting Period October 1 through December 31,1993 . . . . . . . . . . . . . 16-38

16-18 Constituent List and Summary of Results for the Single-Shell Tank Waste Management Area TX-TY Data for Reporting Period October 1 through December 31, 1993 ........... 16-39

16-19 Constituents with at Least One Detected Value for the Single-She11 Tank Waste Management Area TX-TY Data for Reporting Period October 1 through December 31, 1993 . . . . . 16-41

16-20 Contamination Indicator Parameters for the Single-Shel1 Tank Waste Management Area TX-TY Data for Reporting Period October 1 through December 1993 .............. 16-43

16-21 Constituent List and Summary of Results for the Single-Shel1 Tank Waste Management Area U Data for Reporting Period October 1 through December 31, 1993.............. 16-44

16-22 Constituents with at Least One Detected Value for the Single-She11 Tank Waste Management Area U Data for Reporting Period October 1 through December 31, 1993 .......... 16-45

16-23 Contamination Indicator Parameters for the Single-Shell Tank Waste Management Area U Data for Reporting Period October 1 through December 31,1993 .............. 16-46

17-1 Monitoring Well Purpose and Sampling Schedule for the 300 Area Process Trenches Network . . . . . . . . . . 17-3

17-2 RCRA Water Level Measurement Report for the 300 Area Process Trenches, Fourth Quarter 1993 . . . . . . . . . . 17-4

17-3 Constituent List and Summary of Results for the 300 Area Process Trenches Data for Reporting Period October 1 through December 31, 1993 . . . . . . . . . . . . . . . 17-9

17-4 Constituents with at Least One Detected Value for the 300 Area Process Trenches Data for Reporting Period October 1 through December 31, 1993

17-5 Contamination Indicator Parameters for the 300 Area Process Trenches Data for Reporting Period October 1 through December 31, 1993 ................. 17-15 


\section{DOE/RL-93-56-4}

\section{LIST OF TABLES (cont.)}

18-1 Monitoring We11 Purpose and Sampling Schedule for the Nonradioactive Dangerous Waste Landfill Network . . . . . . . 18-3

18-2 RCRA Water Level Measurement Report for the NRDWL, Fourth Quarter $1993 \ldots$. . . . . . . . . . . . 18-4

18-3 Constituent List and Summary of Results for the NRDWL Data for Reporting Period October 1 through December 31, 1993 ............... 18-7

18-4 Constituents with at Least One Detected Value for the NRDWL Data for Reporting Period October 1 through December 31, 1993 ............. . 18-8

18-5 Contamination Indicator Parameters for the NRDWL Data for Reporting Period October 1 through December 31, 1993 .. . . . 18-10 


\section{LIST OF TERMS}

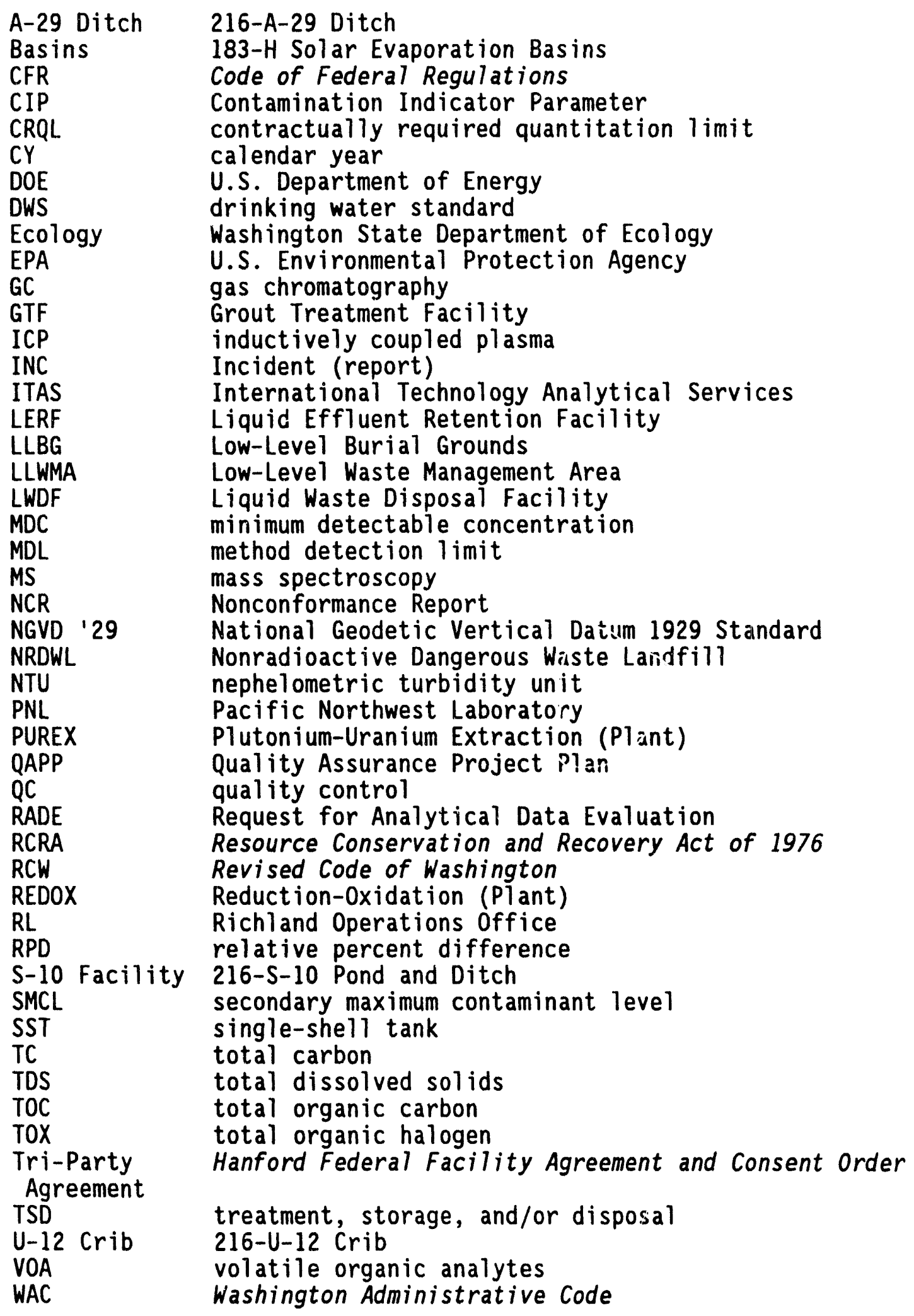


DOE/RL-93-56-4

LIST OF TERMS (cont.)

WHC

WMA

WP

WS

Westinghouse Hanford Company

waste management area

Water Pollution

Water Supply 


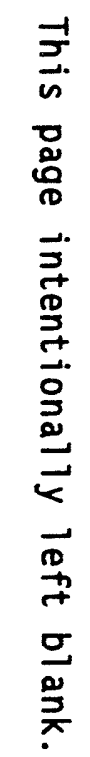

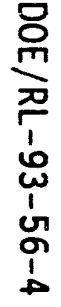

$\bullet$ 
$D O E / R L-93-56-4$

CONTENTS

1.0 INTRODUCTION . . . . . . . . . . . . . . . . . . . . 1-1

1.1 LABORATORY AND SAMPLING STATUS ............... . . 1-1

1.2 QUALITY CONTROL PROGRAM . . . . . . . . . . . . . . . . 1-1

1.2.1 Introduction . . . . . . . . . . . . . . . . . . . 1-1

1.2.2 External Quality Control Program . . . . . . . . . . 1-5

1.2.3 Internal Quality Control Program . . . . . . . . . . 1-6

1.2.4 Nonconformance/Incident Reports . . . . . . . . . . 1-11

1.2.5 Quality Assurance Occurrences . . . . . . . . . . . 1-12

1.3 QUALITY CONTROL DEFINITIONS . . . . . . . . . . . . . . 1-14

1.4 DATA TABLES . . . . . . . . . . . . . . . . . . . . . . . . 1-16

1.4.1 Water Level Measurement Report . . ........... 1-16

1.4.2 Constituent List and Summary of Results ...... . . . 1-16

1.4.3 Constituents with at Least One Detected Value . . . . . 1-17

1.4.4 Contamination Indicator Parameters . . . . . . . . . . . 1-18

1.4.5 Codes and Abbreviations . . . . . . . . . . . . . . . . 1-18

1.5 DATA EVALUATION ....................... . . . . . . . . . 1 


\section{LIST OF FIGURES}

1-1 Locations of the RCRA Groundwater Monitoring Projects and Landmarks on the Hanford Site ............. 1-2

\section{LIST OF TABLES}

1-1 Completeness ...................... . . 1-4

1-2 Field Duplicates and Field Blanks Evaluation Results, Fourth Quarter $1993 \ldots . . . . . . . . .1-7$

1-3 Breakdown of Field Duplicates Exceeding Quality Control Limits............... . . . 1-7

1-4 Breakdown of Field Blanks Exceeding Quality Control Limits . . . 1-8

1-5 Frequency of Quality Control Samples, Fourth Quarter 1993 . . . 1-8

1-6 Radiochemistry Quarterly Report Summary . . . . . . . . . . 1-9

1-7 Summary of EPA Water Pollution, Water Supply, and Interlaboratory Comparison Study............ 1-11

1-8 Analysis Method Code Definitions .... . . . . . . . . 1-19

1-9 Drinking Water Standards ................ 1-22 
$\mathrm{DOE} / \mathrm{RL}-93-56-4$

QUARTERLY REPORT OF RCRA GROUNDWATER MONITORING DATA FOR

PERIOD OCTOBER 1, 1993 THROUGH DECEMBER 31, 1993

\subsection{INTRODUCTION}

Hanford Site interim-status groundwater monitoring projects are conducted as either background, indicator parameter evaluation, or groundwater quality assessment monitoring programs as defined in the Resource Conservation and Recovery Act of 1976 (RCRA); and Interim Status Standards for Owners and Operators of Hazardous Waste Treatment, Storage, and Disposal Facilities, as amended (40 Code of Federal Regulations [CFR] 265). Compliance with the 40 CFR 265 regulations is required by the Washington Administrative Code (WAC) 173-303. This report contains data from Hanford Site groundwater monitoring projects. The location of each facility is shown in Figure 1-1.

Westinghouse Hanford Company (WHC) manages the RCRA groundwater monitoring projects for federal facilities on the Hanford Site. Project management, specifying data needs, performing quality control (QC) oversight, managing data, and preparing project sampling schedules are all parts of this responsibility. Pacific Northwest Laboratory (PNL) administers the contract for analytical services and provides groundwater sampling services to WHC for the RCRA groundwater monitoring program.

This quarterly report contains data received between November 20 and February 25, 1994, which are the cutoff dates for this reporting period. This report may contain not only data from the October through December quarter but al so data from earlier sampling events that were not previously reported.

\subsection{LABORATORY AND SAMPLING STATUS}

DataChem Laboratories of Salt Lake City, Utah, performs the hazardous chemicals analyses for the Hanford Site. Analyses for coliform bacteria are performed by Columbia/Biomedical Laboratories and for dioxin by TMS Analytical Services, Inc.; both are under subcontract to DataChem Laboratories. International Technology Analytical Services (ITAS) Richland, Washington, performs the radiochemical analyses.

\subsection{QUALITY CONTROL PROGRAM}

M. H. Edrington/J. C. Johnston/T. X. Washington Westinghouse Hanford Company

\subsubsection{Introduction}

The QC Program is based on guidance from the U.S. Environmental Protection Agency (EPA), the RCRA Groundwater Monitoring Technical Enforcement Guidance Document (EPA 1986a), and Chapter One: Quality Control, from Test Methods for Evaluating Solid Waste (EPA 1986b). 


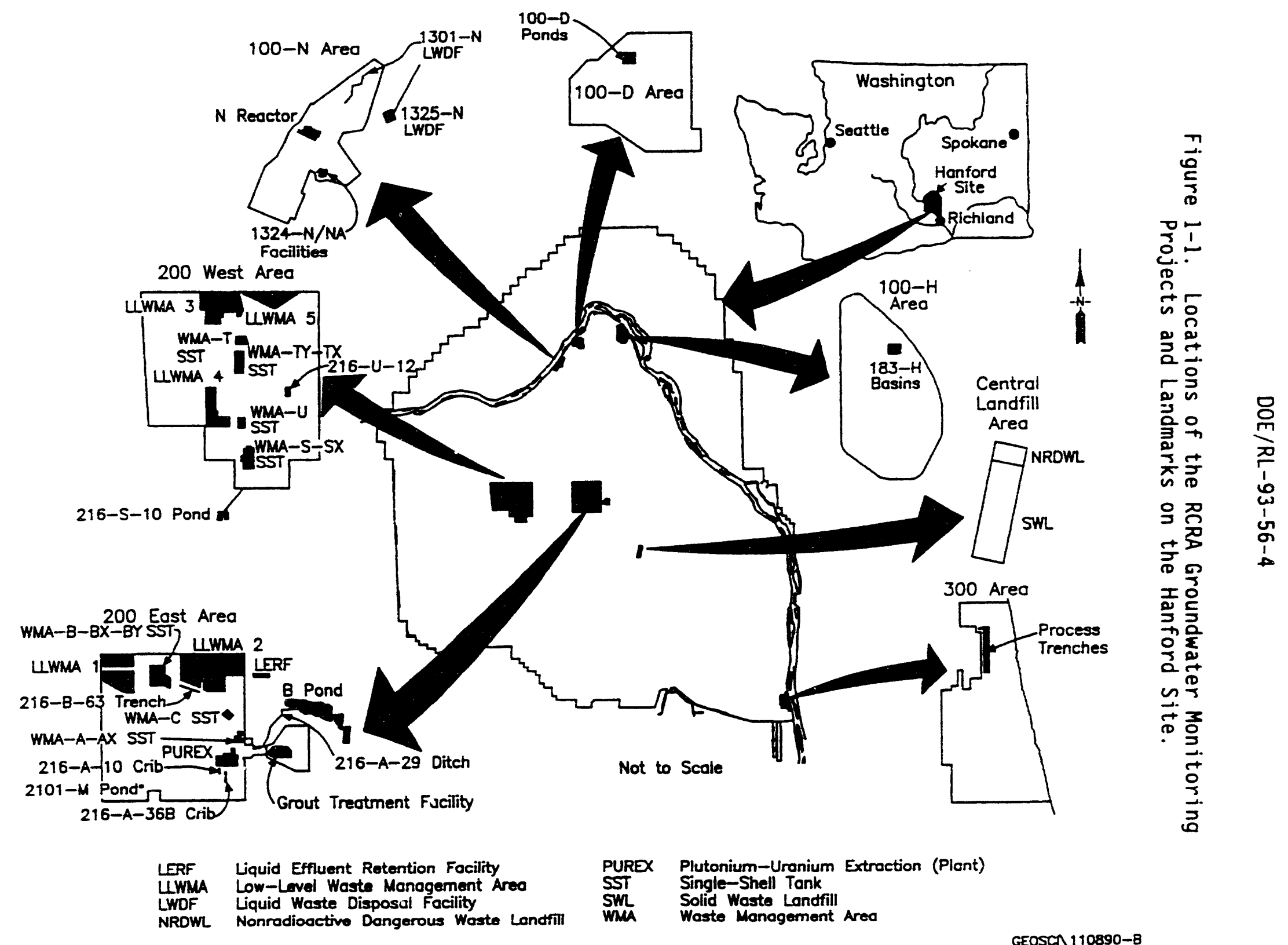

GEOSCA $110890-8$ 
1.2.1.1 Data Quality Objectives. The QC Program uses the five measures of data quality: precision, accuracy, representativeness, completeness, and comparability, along with applicable program-specific quality parameters to evaluate the quality of the data and the analytical laboratories analyzing the samples. Target values for precision and accuracy are specified in Quality Assurance Project Plan (QAPP) for RCRA Groundwater Monitoring Activities (WHC 1992).

1. Precision is evaluated using data results from laboratory duplicates, matrix spike duplicates (see Section 1.2.3), field duplicates, and blind samples (see Section 1.2.2).

2. Accuracy is evaluated using data results from laboratory matrix spikes; laboratory control samples; EPA Water Pollution (WP), Water Supply (WS), and Interlaboratory Performance Evaluation Programs (see Section 1.2.3); and by blind samples (see Section 1.2.2).

3. Representativeness expresses the degree to which RCRA facility groundwater monitoring data represent the real composition of the groundwater in the aquifer. Goals for data representativeness for groundwater monitoring programs are addressed qualitatively by the specification of well construction, sampling locations, sampling intervals, and sampling and analyses techniques in the groundwater monitoring plan for each RCRA facility.

4. Completeness is defined as the percentage of measurements made that are judged to be valid. Completeness is determined by the number of data unflagged during validation divided by the total number of data evaluated multiplied by 100 . The calculated percentages used in reporting completeness are conservative figures and are based on the data flags $P, F, D, Q$, and/or $H$. Table 1-1 illustrates project completeness for the data presented in this report.

5. Comparability is used to ensure that samples analyzed by different laboratories or by the same laboratory over different time periods are comparable. For the October through December quarter of 1993, only the primary hazardous chemistry and radiochemistry laboratories were requested to analyze samples and submit data. Samples were analyzed in accordance with Test Methods for Evaluating Solid Waste (EPA 1986b) and other applicable approved methods. Comparability of field measurements is determined by following approved sampling procedures that ensure consistency among sampling events. 
Table 1-1. Completeness.

\begin{tabular}{|c|c|c|c|}
\hline Project & $\begin{array}{c}\text { Total number } \\
\text { of evaluated } \\
\text { data }\end{array}$ & $\begin{array}{c}\text { Number of } \\
\text { unflagged } \\
\text { results }\end{array}$ & $\begin{array}{c}\text { Percent } \\
\text { complete } \\
\text { (unflagged) }\end{array}$ \\
\hline $100-D$ & 348 & 345 & 99 \\
\hline $100-N$ & 1,590 & 1,421 & 89 \\
\hline $183-\mathrm{H}$ & 943 & $87 !$ & 92 \\
\hline 2101-M & 216 & 215 & 100 \\
\hline $216-A-10$ & 1,069 & 1,005 & 94 \\
\hline $216-A-29$ & 2,780 & 2,624 & 94 \\
\hline $216-A-36 B$ & 1,158 & 1,127 & 97 \\
\hline $216-B-3$ & 1,575 & 1,406 & 89 \\
\hline $216-B-63$ & 1,303 & 1,170 & 90 \\
\hline $216-5-10$ & 355 & 345 & 97 \\
\hline $216-U-12$ & 262 & 258 & 98 \\
\hline 300 APT & 320 & 316 & 99 \\
\hline Grout & 1,054 & 1,006 & 95 \\
\hline LERF & 333 & 318 & 95 \\
\hline LLBG WMA-1 & 1,899 & 1,824 & 96 \\
\hline LLBG WMA-2 & 1,684 & 1,505 & 89 \\
\hline LLBG WMA-3 & 2,678 & 2,513 & 94 \\
\hline LLBG WMA-4 & 2,319 & 2,022 & 87 \\
\hline LLBG WMA-5 & 500 & 463 & 93 \\
\hline NRDWL & 293 & 243 & 83 \\
\hline SST $A / A X$ & 16 & 16 & 100 \\
\hline SST $B / B X / B Y$ & 172 & 160 & 93 \\
\hline SST C & 2 & 2 & 100 \\
\hline SST S/SX & 48 & 48 & 100 \\
\hline SST T & 219 & 191 & 87 \\
\hline SST TX/TY & 337 & 305 & 91 \\
\hline SST U & 124 & 116 & 94 \\
\hline
\end{tabular}

APT $=$ area process trench .

LERF = Liquid Effluent Retention Facility.

LLBG = Low-Level Burial Grounds.

NRDWL = Nonradioactive Dangerous Waste Landfill.

SST $=$ single-shell tank .

WMA = waste management area . 
1.2.1.2 Program-Specific Validation Flags. Program-specific validation flags are assigned during the process of evaluating RCRA groundwater data. These flags include (1) $\mathrm{H}$ flags for exceeding holding times; (2) Q flags for field blank and duplicate data outside of the evaluation criteria; (3) F flags for data under the Request for Analytical Data Evaluation (RADE) process;

(4) D flags for data associated with a Nonconformance Report (NCR)/Incident (INC) report; and (5) P flags for data identified as a "potential problem."

1. Holding time exceedances have been flagged in the quarterly reports with an $H$ flag. This flag means that the samples were analyzed beyond the method-required holding times as stated in the contract. The $\mathrm{H}$-flagged data may be used qualitatively, but no regulatory decisions should be made based on a single flagged analytical result.

2. Field blanks that indicate contaminations, and field duplicate samples that show greater than $25 \%$ relative percent difference (RPD), are identified in the quarterly project data tables with a $Q$ flag. This flag means that the field $Q C$ data associated with the sample data were outside limits established in the QAPP (WHC 1992). The Q-flagged data can be used qualitatively, but no regulatory decisions should be made based on a single-flagged data point.

3. The RADE system is used to track and evaluate data that appear to be inconsistent with the current pattern or with historical trends (see Section 1.5).

4. NCR and INC reports are received from the laboratory and are used to document occurrences that may affect data quality. Data points affected by these reports are flagged with a 'D.' The $D$-flagged data may be used qualitatively, but no regulatory decision should be made based on a single-flagged data point (see Section 1.2.4).

5. Data points related to isolated occurrences determined by the specific project scientist are flagged with a ' $P$, ' indicating a potential problem with the data. Explanations for use of the flag will be discussed in the specific project chapter.

\subsubsection{External Quality Control Program}

The external $Q C$ program uses three kinds of $Q C$ samples to evaluate quality in the field and laboratory. These are field duplicates, field blanks, and blind samples. (See page 1-14 for definitions of the above QC samples.)

The analytical results of QC samples are judged to be acceptable if the following evaluation criteria are met.

- Field duplicates--Results of field duplicate pairs must have precision as measured by RPD within $\pm 25 \%$. 
- Blanks--Four kinds of blanks are used to check for contamination resulting from field activities and/or bottle preparation. These are full trip blanks, daily trip blanks, field transfer blanks, and bottle blanks.

Except for common laboratory contaminants, results above the 1 imit of two times the method detection limit (MDL) are identified as suspected contamination. For common laboratory contaminants, such as acetone, methylene chloride, 2-butanone, toluene, and phthalate esters, sample results less than five times the MDL are qualified as nondetects.

- Blind samples--Results must fall within 2.0 standard deviations from the mean recovery of the known concentration of samples submitted to the laboratory.

Table 1-2 provides a summary of the analysis results of QC samples available for the October through December quarter of 1993.

Samples associated with duplicate or blank analytes that exceeded the QC limits are discussed below and are flagged with a ' $Q$ ' in the data tables.

Precision based on field duplicate analysis was determined to be $97.2 \%$ acceptable. Table 1-3 shows a breakdown of the 71 duplicate results that fell outside the QC limits.

of the 4,268 blanks analyzed during the October through December quarter of 1993, only 67 exceeded the QC limit for 98.4\% acceptability. Table 1-4 shows a breakdown of the constituents detected in the blanks.

Volatile organics by gas chromatography/mass spectroscopy (GC/MS) accounted for 26 of the 77 blanks exceeding QC limits for the quarter. The following constituents were found to be present in the field blanks: 1,1,1-trichloroethane (11), methylene chloride (8), carbon disulfide (6), and carbon tetrachloride (1). For $19(73 \%)$ of these field blanks in which the above-mentioned constituents exceeded QC limits, the laboratory's internal QC process identified these as samples contaminated in the laboratory. The laboratory assigned and reported these values with B qualifiers. No field duplicate results were found to exceed $Q C$ limits for volatile organics by GC/MS.

Table 1-5 shows the required and actual sampling frequencies for QC samples. Sampling frequencies were met for field transfer blanks, daily trip blanks, and full trip blanks. Because of sampler error, three duplicate samples were not collected.

\subsubsection{Internal Quality Control Program}

The internal $Q C$ program uses four types of $Q C$ data to establish and monitor performance in the laboratory. These data are laboratory blanks, matrix spikes, matrix duplicates, and EPA studies (WP and WS). 
Table 1-2. Field Duplicates and Field Blanks Evaluation Results, Fourth Quarter 1993.

\begin{tabular}{|l|c|c|c|}
\hline & $\begin{array}{c}\text { Number of } \\
\text { analyses }\end{array}$ & $\begin{array}{c}\text { Number of results } \\
\text { outside of criteria }\end{array}$ & $\begin{array}{c}\text { Acceptable } \\
(\%)\end{array}$ \\
\hline Duplicates & 2,542 & 71 & 97.2 \\
\hline Blanks & 4,268 & 67 & 98.4 \\
\hline
\end{tabular}

Table 1-3. Breakdown of Field Duplicates Exceeding Quality Control Limits.

\begin{tabular}{|c|c|c|c|c|}
\hline $\begin{array}{l}\text { Method } \\
\text { analysis } \\
\text { code }\end{array}$ & Method description & $\begin{array}{l}\text { Number of } \\
\text { flagged } \\
\text { results }\end{array}$ & $\begin{array}{c}\text { Total } \\
\text { number of } \\
\text { analyses }\end{array}$ & $\begin{array}{l}\text { Percent of } \\
\text { total out } \\
\text { of limits }\end{array}$ \\
\hline 17 & $\begin{array}{l}\text { Pesticides and } \\
\text { polychlorinated biphenyls }\end{array}$ & 4 & 230 & 1.7 \\
\hline 25 & $\begin{array}{l}\text { Volatile organics } \\
\text { (gas chromatography) }\end{array}$ & 4 & 136 & 2.9 \\
\hline 30 & Phenols & 1 & 192 & .52 \\
\hline 34 & $\begin{array}{l}\text { Inductively coupled } \\
\text { plasma metals }\end{array}$ & 26 & 920 & 2.8 \\
\hline $40,43,48$ & $\begin{array}{l}\text { Graphite furnace atomic } \\
\text { absorption metals }\end{array}$ & 8 & 132 & 6.1 \\
\hline 54,129 & Ammonium & 1 & 13 & 7.7 \\
\hline 122 & Total organic carbon & 2 & 24 & 8.3 \\
\hline 124 & Anions & 6 & 182 & 3.3 \\
\hline 135 & Gross alpha & 6 & 7 & 85.7 \\
\hline 136 & Gross beta & 4 & 7 & 57.1 \\
\hline 137 & Radium & 3 & 6 & 50.0 \\
\hline 140 & $\begin{array}{l}\text { Cesium-137, cobalt- } 60 \text {, } \\
\text { ruthen ium-106 }\end{array}$ & 4 & 12 & 33.3 \\
\hline 148 & Uranium-235 & 2 & 3 & 66.7 \\
\hline
\end{tabular}


Table 1-4. Breakdown of Field Blanks Exceeding Quality Control Limits.

\begin{tabular}{|c|l|c|c|c|}
\hline $\begin{array}{c}\text { Method } \\
\text { analysis } \\
\text { code }\end{array}$ & \multicolumn{1}{|c|}{ Method description } & $\begin{array}{c}\text { Number of } \\
\text { flagged } \\
\text { results }\end{array}$ & $\begin{array}{c}\text { Total } \\
\text { number of } \\
\text { analyses }\end{array}$ & $\begin{array}{c}\text { Percent } \\
\text { of total } \\
\text { out of } \\
\text { limits }\end{array}$ \\
\hline 16 & $\begin{array}{l}\text { Volatile organics } \\
\text { (gas chromatography/ } \\
\text { mass spectroscopy) }\end{array}$ & 26 & 2,530 & 1.0 \\
\hline 19 & Semivolatile organics & 1 & 167 & 0.6 \\
\hline 25 & $\begin{array}{l}\text { Volatile organics } \\
\text { (gas chromatography) }\end{array}$ & 2 & 85 & 2.4 \\
\hline 34 & $\begin{array}{l}\text { Induct ively coupled plasma } \\
\text { metals }\end{array}$ & 18 & 665 & 2.7 \\
\hline 54,129 & Ammonium & 3 & 7 & 42.9 \\
\hline 62 & Perchlorate & 1 & 1 & 100 \\
\hline 67 & Total organic halogen & 1 & 45 & 2.2 \\
\hline 122 & Total organic carbon & 1 & 62 & 1.6 \\
\hline 124 & Anions & 5 & 133 & 3.8 \\
\hline 126 & Turbidity & 1 & 3 & 88.9 \\
\hline 127 & Total carbon & 33.3 \\
\hline
\end{tabular}

Table 1-5. Frequency of Quality Control Samples, Fourth Quarter 1993.

\begin{tabular}{|l|c|c|c|c|}
\hline \multicolumn{1}{|c|}{$\begin{array}{c}\text { Quality control } \\
\text { samples }\end{array}$} & $\begin{array}{c}\text { Required } \\
\text { frequencies }\end{array}$ & $\begin{array}{c}\text { Number of } \\
\text { wells } \\
\text { sampled }\end{array}$ & $\begin{array}{c}\text { Number of } \\
\text { samples } \\
\text { taken }\end{array}$ & $\begin{array}{c}\text { Actual } \\
\text { frequency } \\
(\%)\end{array}$ \\
\hline Duplicates & $5 \%$ & 269 & 13 & 4.8 \\
\hline Daily trip blank & $\begin{array}{c}\text { One each } \\
\text { sampling day }\end{array}$ & 50 & 50 & 100 \\
\hline Full trip blank & $5 \%$ & 269 & 15 & 5.6 \\
\hline Field transfer blank & $5 \%$ & 269 & 17 & 6.3 \\
\hline Bottle blanks & $\begin{array}{c}\text { Per lot of } \\
\text { bottles }\end{array}$ & N/A & 0 & N/A \\
\hline
\end{tabular}

$N / A=$ not applicable. 
Every quarter the contracted laboratories each supply their own $Q C$ report, which includes data quality information on matrix spikes, matrix duplicates, and blanks in the form of precision and accuracy. The contracted laboratories also supply a report of their results on EPA's WP, WS, and radiochemical intercomparison studies on a quarterly basis. The results of EPA studies independently verify the continuation of laboratory performance and are expressed as the percentage of EPA-accepted results. Each half year the contracted laboratories al so supply an $\mathrm{MDL} / \mathrm{minimum}$ detectable concentration (MDC) report. The MDLs and MDCs are required to be below the contractually required quantitation limit (CRQL). The CRQL is not associated with a quantitation limit as the name suggests. It is intended to be the lowest analyte concentration in a given matrix that a laboratory can be expected to detect consistently. The CRQL is agreed on under the contractual statement of work. A laboratory NCR is issued when the MDL is greater than the CRQL. WHC reviews each of these reports and summarizes the results in Table 1-6.

Table 1-6. Radiochemistry Quarterly Report Summary.

\begin{tabular}{|c|c|c|c|}
\hline $\begin{array}{c}\text { Fourth } \\
\text { quarter } \\
1993\end{array}$ & $\begin{array}{c}\text { Duplicates } \\
\text { (precision, in } \\
\% \text { acceptable) }\end{array}$ & $\begin{array}{c}\text { Spikes } \\
\text { (accuracy, in } \\
\text { acceptable) }\end{array}$ & (in \% acceptable) \\
\hline $\begin{array}{c}\text { October } \\
\text { through } \\
\text { December }\end{array}$ & 98.8 & 98.6 & 99.6 \\
\hline
\end{tabular}

Precision and accuracy results from the radiochemistry laboratory, summarized in Table 1-6, indicate the performance of all customers submitting water matrix samples. WHC samples represent only a part of the performance summary. These figures represent samples analyzed during the period of october through December.

Radiochemistry precision and accuracy figures are gleaned from the radiochemistry laboratory's quarterly report, with percent acceptatility calculated from duplicates and spikes, respectively. These results, along with radiochemistry blank data, are summarized in Table 1-6.

For the October through December quarter of 1993, the radiochemistry laboratory analyzed 170 duplicates, of which 168 were within control 1 imits, for an acceptability of $98.8 \%$. The duplicates, which exceeded the $3 \sigma$ control limits, were reported by the laboratory to have a large amount of sediment. The laboratory offers nonhomogeneity of the samples as a possible explanation for the duplicates falling out of precision.

The radiochemistry laboratory analyzed 219 spikes, of which 216 fell within control limits, for an acceptability of $98.6 \%$. The laboratory reports that two of the samples were lost due to technician error, involving heating the samples to dryness. Another sample was reported to have been lost because of contamination. 
Of 275 blanks analyzed for the October through December quarter of 1993, 274 fell within control limits, for an acceptability of $99.6 \%$. The radiochemistry laboratory reports that contamination was the explanation for the single blank out of control limits. The laboratory reviewed the associated data and either accepted it or reanalyzed the samples based on this review.

A disproportionate and unacceptable number of total organic carbon (TOC) and total carbon (TC) results are still being reported by the hazardous chemistry laboratory with a B qualifier assigned. Investigation into the $B$ qualifier issue has been initiated by the QC task team through the contract administrator. The contract administrator is preparing to meet with laboratory management and personnel in an effort to resolve this problem.

The contract administrator brought this issue before the hazardous chemistry laboratory's management and received response from them with a lengthy explanation of the $B$ qualifier issue regarding TOC. The laboratory reviewed all results for laboratory blanks processed with RCRA groundwater TOC samples. Upon completion of this review, the laboratory concluded that there are no serious problems with these laboratory blank results. The laboratory also maintains that contamination is not a problem and that their system is in control at or above the CRQL level for TOC. The QC team has requested from the contract administrator a more complete clarification from the laboratory for the large percentage of B-qualified results for TOC and other analyses such as barium, calcium, iron, and sodium. The $Q C$ team has also requested to be informed of the criterion used by the laboratory to assign a B qualifier.

Because nearly $40 \%$ of the field blanks exceeding $Q C$ limits were volatile organic analytes (VOA) analyzed by GC/MS, the internal QC section focuses its evaluation on the laboratory results for this method. Review of the data provided for laboratory blanks, indicates that no VOAs exceeded laboratory control limits for blanks. The data also present spike recoveries that range from 93.6 to $104 \%$, all well within acceptable control limits. The RPD for matrix spike duplicates ranged from 4.62 to 5.41 , also well with in acceptable QC limits. Although the laboratory's QC samples are within control limits, much of the VOA field data were reported with $B$ qualifiers, and a large portion of the $Q$ flags for field blanks were assigned because of VOA results. An investigation is warranted to uncover the meaning of or the reason for this apparent discrepancy in data quality.

EPA WP samples were analyzed by the hazardous chemistry laboratory during December 1993. There were 132 blind samples sent for inductively coupled plasma (ICP) metals, anions, ammonium ion, $\mathrm{pH}$, specific conductance, total dissolved solids, alkalinity, pesticides, cyanide, volatile organics, polychlorinated biphenyls, TOC, and chemical oxygen demand. All results, except one, were in acceptable ranges. The unacceptable result was for sulfate. The laboratory contractor was notified of this deficiency. This results in 131 out of 132 acceptable results, or $99 \%$ acceptability (Table 1-7). 
Table 1-7. Summary of EPA Water Pollution, Water Supply, and Interlaboratory Comparison Study (in percent acceptable).

\begin{tabular}{|c|c|c|c|}
\hline $\begin{array}{c}\text { Fourth quarter } \\
1993\end{array}$ & $\begin{array}{c}\text { EPA water } \\
\text { pollution }\end{array}$ & $\begin{array}{c}\text { EPA water } \\
\text { supply }\end{array}$ & $\begin{array}{c}\text { EPA interlab } \\
\text { comparison }\end{array}$ \\
\hline $\begin{array}{c}\text { October through } \\
\text { December }\end{array}$ & $99 \%$ & $\begin{array}{c}\text { Samples not sent } \\
\text { for this quarter }\end{array}$ & $91.7 \%$ \\
\hline
\end{tabular}

$E P A=$ U.S. Environmental Protection Agency.

Hazardous chemistry contracted laboratory.

Radiochemistry contracted laboratory.

EPA intercomparison samples were analyzed by the radiochemistry laboratory during December 1993 through February 1994. Twenty-four samples were analyzed for gross alpha, gross beta, radium-225,228, strontium-89,90, tritium, uranium, cobalt-60, cesium-134,137, ruthenium-106, barium-133, and zinc-65. Two sample results, radium-228 and cesium-134, were outside of the $\pm 25 \%$ bias. The laboratory contractor was notified of these deficiencies. This results in 22 out of 24 acceptable results, or $91.7 \%$ acceptability (see Table 1-7).

\subsubsection{Nonconformance/Incident Reports}

NCR and INC reports are methods of documentation by which contract laboratories can inform laboratory contractors and their customers of any problems encountered with the analysis, data, and/or data deliverable. This method of documentation identifies occurrences, deficiencies, and/or issues that can potentially have an adverse effect on the data integrity. These may include, but are not limited to, the following:

- Lost sample

- Broken bottle

- Instrument malfunction

- Calibration standard out of acceptable range

- Laboratory control standard out of acceptable range

- Matrix spike recovery out of acceptable range

- Procedural noncompliance

- Chain of custody discrepancies

- Shipping temperature out of acceptable range

- Misreported data.

During this reporting period, 29 NCR/INC reports were transmitted by the hazardous chemistry laboratory and 2 reports were transmitted by the radiochemistry laboratory. The following is a breakdown of the INC reports by the area of analysis (number of reports received and number of samples affected by those reports). The hazardous chemistry laboratory reported the following: herbicides ( 3 reports, 11 samples); phenols ( 9 reports, 12 samples); total organic halogen (TOX) (2 reports, 20 samples); anions ( 3 reports, 18 samples); volatiles analyzed by GC (2 reports, 10 samples); volatiles analyzed by GC/MS ( 3 reports, 5 samples); pesticides ( 2 reports, 
25 samples); TOC ( 2 reports, 3 samples); cyanides (1 report, 5 samples); and ammonium ion (2 reports, 34 samples). The aforementioned reports affected less than $1 \%$ of the respective data.

The radiochemistry laboratory transmitted two reports during the reporting period. The first report documented a calculation error; the samples were recalculated and re-reported with the correct values. The second report documented an alpha/peak software problem. The laboratory reevaluated all potentially affected data and determined that the software problem did not affect any WHC data.

\subsubsection{Quality Assurance Occurrences}

Quality assurance occurrences are situations that potentially affect the quality of the data. These situations are discussed through summaries of inspections of laboratory services and/or system and performance audits/ surveillances. Section 1.2.5.1 discusses occurrences during the current quarter, while Section 1.2.5.2 updates past occurrences.

Inspection of laboratory services and/or system and performance audits/surveillances for the RCRA groundwater monitoring program are performed throughout the year by the laboratory contractor, WHC, and/or the U.S. Department of Energy (DOE) on various aspects of this program. Quality assurance requirements and data quality objectives are defined in the Quality Assurance Project Plan for RCRA Groundwater Monitoring Activities (WHC 1992). Results of these oversight activities are documented with the audit/ surveillance and/or inspecting organization(s).

1.2.5.1 0ccurrences for Current Quarter. During the reporting period the hazardous chemistry laboratory responded to the lingering findings and observations from the previous two inspection of services. The contract administrator has since determined the responses to be insufficient and has requested adequate responses by January 31, 1994. The result of the laboratory responses will be documented in the next quarterly report.

1.2.5.2 Status of Previous Occurrences. An inspection of services of the hazardous chemistry laboratory was conducted August 4-6, 1993. The inspection was in response to increasing concern for the quality of ICP metals data and as a followup on the previous inspection of TOX. A summary of the inspection findings and observations is provided below.

The inspection team found evidence supporting eight findings and three observations; they are as follows:

Finding \#1: Re-profiling of ICP instrumentation was found to have occurred without the required recalibration.

Finding \#2: For TOX analyses, instances were found when the $B$ column was greater than $10 \%$ of the total (of the $A+B$ columns) without the required reanalysis being performed. 
Finding \#3: Documentation of training for a TOX analyst was such that it could not be determined that required training per Rev. I of IW-BA-9020 was accomplished before the analysis of samples by the analyst.

Finding \#4: Data were misreported for TOX analyses.

Finding \#5: The February 1993 MDL study included misreported data.

Finding \#6: ICP data generated since implementation of the February 1993 MDL study values have been misreported.

Finding \#7: The hazardous chemistry laboratory and noncompliances with procedure QC-DC-006, Rev. 3, governing NCRs.

Finding \#8: Type II water being used did not comply with the preparation requirements of American Society for Testing and Materials procedure 01193.

Observation \#1: Blank subtraction was not performed when average blank values were less than the MDL per IW-BA-9020, Rev. 1.

Observation \#2: The hazardous chemistry laboratory procedure was not being followed for glassware cleaning.

Observation \#3: Calibration standards were being used past the expiration dates.

The above findings and observations were forwarded to the hazardolls chemistry laboratory and require a written response to address each issue.

Many TOX duplicate results for the second and third quarters of calendar year (CY) 1992 exceeded the RPD. These exceedances corresponded to laboratory blanks containing from 10 to $60 \mathrm{ppb}$ of TOX. These incidents led to an inspection of the TOX procedure, analysis, and records at the hazardous chemistry laboratory during November 1992. An inspection report was issued by the laboratory contractor in December 1992 with three findings and three observations. The findings were (1) lack of appropriate qualifiers and comment codes in reported data, (2) inconsistencies in subtraction of background for TOX analysis, and (3) lack of submission of internal laboratory QC blind samples. The observations were (1) procedural noncompliances for TOX analytical testing, (2) use of a nonstandard equation for calculating RPD, and (3) lack of a system for ensuring that analysts are provided laboratory QC data summaries. It was later discovered that only half of the TOX data were being reported electronically because of miscommunication between the hazardous chemistry laboratory and the laboratory contractor. All affected ToX data from this inspection have been flagged with a ' $P$ ' for potential problem. In future quarterly reports, TOX issues will be updated through the most recent ToX inspection (see Section 1.2.5.1). 


\subsection{QUALITY CONTROL DEFINITIONS}

Accuracy. The closeness of agreement between an observed value and a true value. Accuracy is assessed by means of reference samples and percent recoveries.

Blind sample. A sample that contains a concentration of analyte that is known to the supplier but unknown to the analyzing laboratory. The analyzing laboratory is informed that the sample is a QC sample and not a field sample. The blind, double blind, and matrix matched double blind samples are used to assess accuracy and monitor the performance of the analytical laboratory(ies) with prepared or purchased materials from EPA QC samples/concentrates or primary materials.

Bottle blank. A sample that contains only Type II reagent water. The bottle blank contains one sample for each bottle size, with at least enough bottles to include all constituents analyzed by a specific project, except radionuclides. Bottle blanks shall be submitted to the primary laboratory per lot of bottles. Bottle blanks are filled in the analytical laboratory under the sample preparation procedures. Bottle blanks do not go into the field.

Contractually required quantitation limit. A value intended to be the lowest analyte concentration in a given matrix that the laboratory can be expected to achieve consistently; agreed upon under the contract statement of work.

Daily trip blank. A sample that contains only Type II reagent water. The daily trip blank is used to check for sample contamination by volatile organic compounds arising from conditions encountered during the collection of samples. The daily trip blank is not opened in the field. One daily trip blank is collected for each day that sampling occurs.

Double blind sample. A sample that contains a concentration of analyte that is known to the supplier but is unknown to the analyzing laboratory. The analyzing laboratory is not informed that the sample is a QC sample. All attempts are made to make this sample appear like a field sample. For example, the double blind sample should be submitted to the laboratory within the same time period and with a sample identification number similar to that of the field samples. The double blind sample does not include matrix matching.

External quality control sample. Any QC sample prepared without the knowledge of the analytical laboratory.

Field duplicate sample. A sample used to determine repeatability of an analytical measurement on identical samples collected as close as possible to the same time at the same location. These samples are stored in separate containers and are analyzed independently by the same laboratory. 
$\mathrm{DOE} / \mathrm{RL}-93-56-4$

Field transfer blank. A sample that contains only Type II reagent water. The field transfer blank is used to check for sample contamination by volatile organic compounds arising from conditions encountered during the collection of samples. The field transfer blank is taken during the collection of samples. The field transfer blank is filled at the sampling site by pouring Type II reagent water from a cleaned container into a volatile organic analysis vial. At least 1 field transfer blank is collected for each 20 samples, or 1 per sampling batch.

Full trip blank. A sample that contains only Type II reagent water and preservative, as required. A full trip blank is used to check for contamination in sample bottles and sample preparation. The full trip blank is analyzed for all constituents of interest on all types of sample bottles used during that sampling period. The frequency of collection for a full trip blank is 1 per 20 samples, or 1 per sampling batch. A full trip blank is filled in the analytical laboratory under the sample preparation procedures. The full trip blank is not opened in the field.

Internal quality control sample. Any QC sample prepared by the analytical laboratory and used to establish and monitor the quality of the analytical laboratory.

Limit of detection. The lowest concentration level that is statistically different from a blank. This is calculated by the average blank signal plus three standard deviations for the blank analyses.

Matrix matched double blind sample. A matrix matched double blind sample contains a concentration of analyte that is known to the supplier but unknown to the analyzing laboratory. The sample matrix has been altered to closely match that of the field samples.

Method detection limit. The minimum concentration of a substance that can be measured and reported with $99 \%$ confidence that the analyte concentration is greater than zero and is determined from analysis of a sample in a given matrix type containing the analyte.

Minimum detectable concentration. Required level of analytical detection for radiochemical samples.

Precision. The agreement among a set of individual measurements of the same property, usually under prescribed similar conditions. Precision is calculated by using RPD of the duplicate/replicate analyses. These samples should contain concentrations of analyte above the MDL and may involve the use of matrix spikes.

Reliable detection level. A detection limit set at two times the concentration of the MDL, so the risk of both false positives and false negatives falls below $1 \%$.

Iype II reagent water. Distilled or deionized water that is free of contaminants that may interfere with the analytical test in question. 


\subsection{DATA TABLES}

\section{J. A. Serkowski \\ Westinghouse Hanford Company}

Four different formats are used to display water level measurements and groundwater sample analytical results. This section describes the meaning of each table column heading, defines the abbreviations used, explains the data qualifiers and flags, and lists analysis method codes and relevant regulatory standards.

\subsubsection{Water Level Measurement Report}

The Water Level Measurement Report has four columns as follows:

- Well--Well in which measurement was made.

- Date--Date of measurement.

- Depth to water--Depth, in feet, from well casing reference point to top of water in well.

- Water level--Elevation, in feet above mean sea level, of water level computed by subtracting depth to water from casing reference elevation.

Wells are grouped according to the zone that they monitor beginning with the top of the unconfined aquifer and continuing downward to the confined aquifer.

Only measurements made during the reporting quarter are included in the tables. In addition to measurements made during sampling, which are marked with an asterisk $\left({ }^{*}\right)$, measurements are routinely performed at other times to permit collection of data for an entire network within a 1- or 2-day time period.

Data that are judged to be suspect by the project scientist are flagged with ' + ' in the table. Before data are designated as suspect, checks are conducted to determine if a transcription error occurred between the field sheet and the electronic database.

\subsubsection{Coristituent List and Summary of Results}

The Constituent List and Summary of Results table (Summary table) is the first of three tables that present the results of groundwater sample analyses. The Summary table displays statistics based on the complete data set for the reporting period. The complete data set consists of all analysis results requested by the project associated with samples collected before the end of the current reporting quarter, which arrived at WHC before the cutoff date for the current quarterly report (see Section 1.1 for the current cutoff date), and which have not been reported in a previous quarterly. Therefore, data 
collected during previous quarters may appear in this document if the laboratory did not report the data until the current reporting period.

The fields in the Summary table are as follows:

- Constituent name--Name of the analyzed constituent being summarized; the Short name is an abbreviation used in the Constituents with at Least One Detected Value table (Section 1.4.3); the (Method), when present, is a code (Section 1.4.5) used to distinguish between different analytical methods for the same constituent.

- Units--Units in which the MDL and drinking water standards (DWS) are reported in this table.

- Laboratory MDL--The MDL computed by the laboratory for a constituent. This field is blank for radionuclides where the MDL is defined as the value of the total error reported by the laboratory for each result.

- DWS limit/agency--The DWS used for comparison to the reported results; see Section 1.4 .5 for Agency definitions and a complete listing of standards used.

- Number of samples--Total is the number of sample results from all wells in the project contained in the complete data set for that constituent; $>M D L$ is the number of results that exceed the MDL; $>$ DWS is the number of results that exceed the 1isted DWS.

Summarized constituents are grouped as Contamination Indicator Parameters (CIP), Drinking Water Parameters, Groundwater Quality Parameters, and SiteSpecific and other Constituents. Within groups, rows are ordered alphabetically by the full constituent name.

If a constituent has an MDL that exceeds the DWS, exceedance of the DWS cannot be determined, so an asterisk $\left(^{*}\right)$ is placed in the $\geq$ DWS field.

\subsubsection{Constituents with at Least One Detected Value}

The Constituents with at Least One Detected Value table (Detected table) is a subset of the complete data set. Only constituents that were detected above the MDL in at least one sample collected for the project during the current reporting period are reported in the Detected table.

The first three column headings are as follows:

- Well name--Well from which sample was collected.

- Collection date--Date sample was collected.

- Sample number--Unique number assigned to a well sample. 
The remaining columns contain analytical results and qualifiers for the constituent. The structure of the column header is as follows.

Constituent short name

Method code/reporting units

MDL/DWS (suffix).

The Summary table (Section 1.4.2) shows the relationship between the full constituent name and the short name. The analytical method code is defined in Table 1-8. Abbreviations used for reporting units are listed in Section 1.4.5. On the third line, a period appears in place of the MDL or DWS if a value does not exist. The one-letter suffix that may be added to the DWS identifies the regulation associated with the standard and is described in Section 1.4.5.

Analysis results are displayed with two digits to the right of the decimal point. This is a consequence of the generating software and does not reflect the accuracy of the results. The laboratories normally report the result with three significant digits. Radionuclide values may be negative because the laboratory subtracts a background reading from the measured result. The letter, or group of letters, that may follow the result are data qualifiers and flags. A complete explanation of what each flag means is provided in Section 1.4.5.

\subsubsection{Contamination Indicator Parameters}

The CIP table has a format similar to the Detected table (Section 1.4.3) but includes only data for $\mathrm{pH}$, specific conductance, TOC, and TOX. All results for these parameters, whether detected or not, are listed in the CIP table. The analysis method code is not part of the column header in this report.

\subsubsection{Codes and Abbreviations}

Abbreviations--The abbreviations used in the data tables are as follows:

COL coliform colonies per 100 milliliters

DWS drinking water standard

$\mathrm{ft} \quad$ feet

MDL method detection limit

ms1 mean sea level

NTU nephelometric turbidity unit

$\mathrm{pC} i / \mathrm{L} \quad$ picocuries per liter

ppb parts per billion

ppm parts per million

$\mu$ mho micromhos per centimeter. 
Table 1-8. Analysis Method Code Definitions.

\begin{tabular}{|c|c|}
\hline Method code & Method name \\
\hline 16 & $5 H-8468240^{\circ}$ \\
\hline 17 & $\mathrm{SH}-8468080^{\circ}$ \\
\hline 19 & SW-846 $8270^{\circ}$ \\
\hline 25 & SH-846 8010/8020" \\
\hline 29 & SW-846 $8140^{\circ}$ \\
\hline 30 & SH-846 8040 \\
\hline 34 & SW-846 6010 \\
\hline 36 & ASTM $D-1385^{\circ}$ \\
\hline 40 & SW-846 $7421^{\circ}$ \\
\hline 41 & SW-846 $7470^{\circ}$ \\
\hline 42 & SH-846 $7841^{\circ}$ \\
\hline 43 & SW-846 $7060^{\circ}$ \\
\hline 48 & SW-846 $7740^{\circ}$ \\
\hline 49 & $\mathrm{SH}-8468150^{\circ}$ \\
\hline 51 & SW-846 8280 \\
\hline 52 & ASTM D-1067-A \\
\hline 54 & ASTM D-1426-D \\
\hline 56 & SW-846 9010" \\
\hline 62 & In-house ion chromatography \\
\hline 63 & SW-846 $9030^{\circ}$ \\
\hline 65 & Standard Methods $" 209 \mathrm{~B}^{\mathrm{c}}$ \\
\hline 67 & SW-8469020 \\
\hline 69 & SW-846 9131" \\
\hline 73 & ASTM $D-1125-A$ \\
\hline 93 & Field probe, PH \\
\hline 94 & Field probe, conductivity \\
\hline 122 & SW-8469060 \\
\hline 124 & ASTM D-4327-88 \\
\hline 125 & ASTM D-1293 \\
\hline 126 & Standard Methods $\# 214 A^{C}$ \\
\hline 127 & ASTM $D-2579-A$ \\
\hline 129 & ASTM D-1426-C \\
\hline 130 & EPA Method 300. $0^{\circ}$ \\
\hline 135 & SH-846 9310, Alpha" \\
\hline 136 & SH-846 9310, Beta" \\
\hline 137 & SH-846 9315, Radium \\
\hline 139 & ITAS I-129 Low level \\
\hline 140 & ITAS Ganme scan \\
\hline 141 & ITAS Sr-90 \\
\hline 142 & ITAS H-3 \\
\hline 143 & ITAS TC-99 \\
\hline 144 & SW-846 9132" \\
\hline 145 & ITAS Gross U \\
\hline 146 & ITAS Isotopic PU \\
\hline 147 & ITAS Am-24i \\
\hline 148 & ITAS I sotopic U \\
\hline 168 & USEPA HACH $\mathrm{COO}^{\circ}$ \\
\hline 357 & EPA $600,310.2$ \\
\hline 358 & SH-846 $9012^{\circ}$ \\
\hline
\end{tabular}


Agencies and suffixes--Agency codes and DWS suffixes identify the regulatory origin of the standard of the DWS. Agency codes are used in the Summary table, and DWS suffixes are used in the Detected and CIP tables. The codes are defined as follows:

Agency Suffix Requlatory basis

EPA None Maximum contaminant levels in 40 CFR 141, "National Primary Drinking Water Regulations."

EPAS S Secondary maximum contaminant leve1s in 40 CFR 143, "National Secondary Drinking Water Regulations."

EPAI $\quad i \quad$ EPA interim primary drinking water standards in 40 CFR 265, Appendix III.

Data qualifiers and flags--Data qualifiers and flags used in the Detected and CIP tables are assigned by the laboratory and by the WHC QC team, respectively. Qualifiers reflect conditions occurring in the laboratory relating to the analytical procedure. Flags serve a wider function of alerting the data user to the limitations of the reported value. Qualifiers and flags can be appended to each other to form a string of letters when several factors apply to a result. The qualifiers and flags used are as follows:

Laboratory qualifiers:

B - Blank associated with analyte is contaminated

D - Analyzed sample is diluted

E - Concentration is out of instrument calibration range

$\mathrm{J}$ - Concentration is estimated

$L$ - Concentration is below the CRQL but above the MDL

$U$ - Concentration is below the indicated value.

Data flags:

D - Result associated with a documented laboratory nonconformance

$F$ - Suspect data currently under review

$H$ - Laboratory holding time exceeded

G - Reviewed data that are considered valid

P - Potential problem; see text associated with table

$Q$ - Result associated with suspect QC data

$R$ - Reviewed data that have been rejected

$Y$ - Reviewed data that continues to be suspect

+ - Suspect water level data

* - CRQL is greater than DWS, so exceedance of DWS is undetermined.

More complete descriptions of some of these qualifiers and flags are presented in Section 1.2, Quality Control Program. 
Analysis method codes--Analys is method codes are used as an abbreviation for the laboratory method used to perform an analysis. A complete listing of the analysis method codes used in the Summary and Above CRQL tables is shown in Table 1-8.

DWSS--DWSs are used in all of the chemistry tables to provide a standard with which to compare sample results. The DWSs are based on standards found in 40 CFR 265, 40 CFR 141, and 40 CFR 143. Where there is overlap in constituents addressed by these regulations, 40 CFR 265 takes precedence over 40 CFR 141, which, in turn, takes precedence over 40 CFR 143. A complete listing of the DWSs used in the Summary, Above CRQL, and CIP tables is shown in Table 1-9.

\subsection{DATA EVALUATION}

Data evaluation is a process through which suspect data are identified and/or investigated. At present, the data evaluation process consists of evaluation of data called out in RADEs, submitted by data users, and the statistical evaluation of CIP data. The statistical evaluations of CIP data are presented within the chapters for individual sites.

Thirty-five RADEs were submitted during the fourth quarter of 1993 in time for inclusion in this report. Of the submitted RADEs, 10 are for radionuclides, 8 are for field parameters ( $\mathrm{pH}$ and specific conductance), 7 are for ICP metals, 4 are for anions, 3 are for coliform, 2 are for organics, 2 are for turbidity, and 1 each for TOC and ammonium ion. One RADE, for an unfiltered bailed sample that apparently contained a significant quantity of solid material, is not included.

F flags are used to indicate that an RADE has been submitted. After evaluation the $F$ flag may be changed to ' $G$,' indicating acceptable data; ' $Y$, ' indicating suspect data; or ' $R$,' indicating unusable data. 
Table 1-9. Drinking Water Standards. (sheet 1 of 2)

\begin{tabular}{|c|c|c|c|c|}
\hline Const ituent name & short name & DwS & Units & Agency \\
\hline 1,1,1-Trichloroethane & $1,1,1-T$ & 200 & ppb & EPA \\
\hline 1,1-Dichloroethene & DICETHY & 7 & ppob & EPA \\
\hline 1,2-Dibromo-3-chloropropane & DIBRCHL & 0.2 & ppob & EPA \\
\hline 1,2-D ibromoethane & DIBRETH & 0.05 & ppb & EPA \\
\hline 1,2-Dichlorobenzene & 12-dben & 600 & ppob & EPA \\
\hline 1,2-Dichloroethane & $1,2-01 C$ & 5 & ppb & EPA \\
\hline 1,2-D ichloropropane & DICPANE & 5 & ppob & EPA \\
\hline 1,4-Dichlorobenzene & 14-ctben & $\pi$ & ppb & EPA \\
\hline $2,4,5-T P$ & $2,4,5 \mathrm{TP}$ & 50 & ppo & EPA \\
\hline $2,4-D$ & $2,4-0$ & 100 & ppb & EPAI \\
\hline Arsenic & ARSENIC & 50 & ppb & EPA \\
\hline Barium & BARIUN & 1,000 & ppb & EPAI \\
\hline Benzene & BENZENE & 5 & ppo & EPA \\
\hline Cadnium & CADMIUA & 10 & ppb & EPAI \\
\hline Carbon tetrachloride & TETRANE & 5 & ppb & EPA \\
\hline Cesium- 137 & CS-137 & 200 & $\mathrm{PCi} / \mathrm{L}$ & EPA \\
\hline Chlordane & CHLOANE & 2 & ppb & EPA \\
\hline Chloride & CHLORID & 250,000 & ppb & EPAS \\
\hline Chlorobenzene & CHLROB & 100 & ppb & EPA \\
\hline Chromium & CHROMUM & 50 & ppb & EPAI \\
\hline cis-1,2-D ichl oroethylene & CIS12DE & 70 & ppb & EPA \\
\hline Cobalt -60 & co- 60 & 100 & $\mathrm{pCi} / \mathrm{L}$ & EPA \\
\hline Coliforms & COLIFORM & 1 & $\mathrm{COL}$ & EPAI \\
\hline Copper & COPPER & 1,000 & ppb & EPAS \\
\hline Endrin & ENDRIN & 0.2 & ppb & EPA \\
\hline Ethylbenzene & ETHBENZ & 700 & ppb & EPA \\
\hline Flworide & FLUORID & 1,400 & ppb & EPAI \\
\hline gamma-BHC (L indane) & g-BHC & 4 & ppb & EPAI \\
\hline Gross alpha & ALPHA & 15 & $\mathrm{PCi} / \mathrm{L}$ & EPA \\
\hline Heptachlor & HEPILOR & 0.4 & ppb & EPA \\
\hline Heptachlor epoxide & HEPTIDE & 0.2 & ppo & EPA \\
\hline lodine-129 & $1-129$ & 1 & $\mathrm{PCi} / \mathrm{L}$ & EPA \\
\hline Iron & IRON & 300 & ppb & EPAS \\
\hline Lead & LEAD & 50 & ppb & EPAI \\
\hline Manganese & MANGESE & 50 & ppb & EPAS \\
\hline Mercury & MERCURY & 2 & $p p b$ & EPA \\
\hline
\end{tabular}


Table 1-9. Drinking Water Standards. (sheet 2 of 2)

\begin{tabular}{|c|c|c|c|c|}
\hline Constituent name & Short name & DWS & Units & Agency" \\
\hline Methoxychlor & METHLOR & 100 & ppb & EPAI \\
\hline Nitrate & NITRATE & 45,000 & ppb & EPA \\
\hline Nitrite & NITRITE & 1,000 & ppb & EFA \\
\hline Pentachlorophenol & PENTCHP & 1 & ppb & EPA \\
\hline pH & PH & $6.5-8.5$ & PH & EPAS \\
\hline Radiun & RADIUN & 5 & $\mathrm{pCi} / \mathrm{L}$ & EPAI \\
\hline Ruthenium- 106 & RU- 106 & 30 & $\mathrm{pCi} / \mathrm{L}$ & EPA \\
\hline Selenium & SELENUM & 10 & ppb & EPAI \\
\hline silver & SILVER & 50 & ppb & EPAI \\
\hline strontium-90 & SR-90 & 8 & $\mathrm{pCi} / \mathrm{L}$ & EPA \\
\hline styrene & STYRENE & 100 & ppb & EPA \\
\hline sulfate & SULFATE & 250,000 & ppo & EPAS \\
\hline Technet ium- 99 & TC- 99 & 900 & $\mathrm{pCi} / \mathrm{L}$ & EPA \\
\hline Tetrachloroethene & PERCENE & 5 & ppb & EPA \\
\hline Toluene & TOLUENE & 1,000 & ppob & EPA \\
\hline Total dissolved solids & TOS & 500 & ppm & EPAS \\
\hline Toxaphene & TOXAENE & 5 & ppb & EPAI \\
\hline trans-1,2-Dichloroethylene & TRANDCE & 100 & ppb & EPA \\
\hline Trichloroethene & TRICENE & 5 & ppb & EPA \\
\hline Tritium & TRITIUA & 20,000 & $\mathrm{pCi} / \mathrm{L}$ & EPA \\
\hline Vinyl chloride & VINYIDE & 2 & ppb & EPA \\
\hline Xylenes (total) & XYLENE & 10,000 & ppb & EPA \\
\hline Zinc & ZINC & 5,000 & ppb & EPAS \\
\hline
\end{tabular}

COL $=$ coliform colonies per 100 milliliters.

$\mathrm{pCi} / \mathrm{L}=$ picocuries per liter.

ppb = parts per billion.

see section 1.4 .5 for ogency listing. 
$D O E / R L-93-56-4$

This page intentionally left blank. 
DOE/RL-93-56-4

\section{CONTENTS}

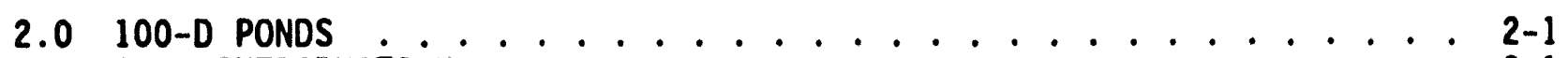

2.1 INTRODUCTION ...................... 2-1

2.2 WATER LEVEL MEASUREMENTS . . . . . . . . . . . 2-1

2.3 WATER CHEMISTRY DATA ............... 2- 


\section{LIST OF FIGURES}

2-1 Monitoring Well Location Map for the 100-D Ponds........ 2-2

\section{LIST OF TABLES}

2-1 Monitoring Well Purpose and Sampling Schedule in the 100-D Ponds Network ................ . 2-3

2-2 RCRA Water Level Measurement Report for the 100-D Ponds, Fourth Quarter 1993 . . . . . . . . . . . . . . . 2-4

2-3 Constituent List and Summary of Results for the 100-D Pond Data for Reporting Period October 1 through December 31, $1993 \ldots$. . . 2-7

2-4 Constituents with at Least One Detected Value for the 100-D Pond Data for Reporting Period October 1 through December 31, 1993 . . . 2-9

2-5 Contamination Indicator Parameters for the 100-D Pond Data for Reporting Period October 1 through December 31, $1993 \ldots . .2-12$ 


\subsection{0-D PONDS \\ M. J. Hartman \\ Westinghouse Hanford Company}

\subsection{INTRODUCTION}

The 100-D Ponds system is an interim-status RCRA disposal unit located in the 100-D Area of the Hanford Site (Figure 2-1). Four wells make up the 100-D Ponds sampling network (Table 2-1). The 100-D Ponds are monitored under an indicator evaluation program ( 40 CFR 265).

The 100-D Ponds unit was constructed in 1977 for disposal of nonradioactive effluents derived from 100-D Area operating facilities. The 100-D Ponds are located in the former 188-D ash disposal basin and consist of a settling pond and a percolation pond, separated by a dike.

Effluent to the 100-D Ponds originated from two sources: a filter plant and engineering testing laboratories. Some past discharges may have included dangerous waste (Hartman 1991a). The 183-D Filter Plant still discharges low volumes of nondangerous effluent to the 100-D Ponds (WHC 1990a).

\subsection{MATER LEVEL MEASUREMENTS}

Water levels are measured monthly in wells in and near the 100-D Area. Water level data are 1 isted in Table 2-2. The December measurement in well D5-13 and the November measurement in well 699-92-49 were outside the expected range and are suspected errors.

\subsection{MATER CHEMISTRY DATA}

The 100-D Ponds wells were all sampled as scheduled last quarter. Data are listed in Tables 2-3 through 2-5. Data flags are discussed in Section 1.4. The "D" flags on sulfate data indicate the samples were diluted for analysis.

Drinking water standards were exceeded for chromium and iron in unfiltered samples from wells $08-4$ and D8-6.

Specific conductance, $\mathrm{pH}$, and TOC at wells D8-4 and D8-6 did not exceed critical mean values. Well D8-5 is not used for detection purposes per agreement with the Washington State Department of Ecology (Ecology). 
Figure 2-1. Monitoring Well Location Map for the 100-D Ponds.

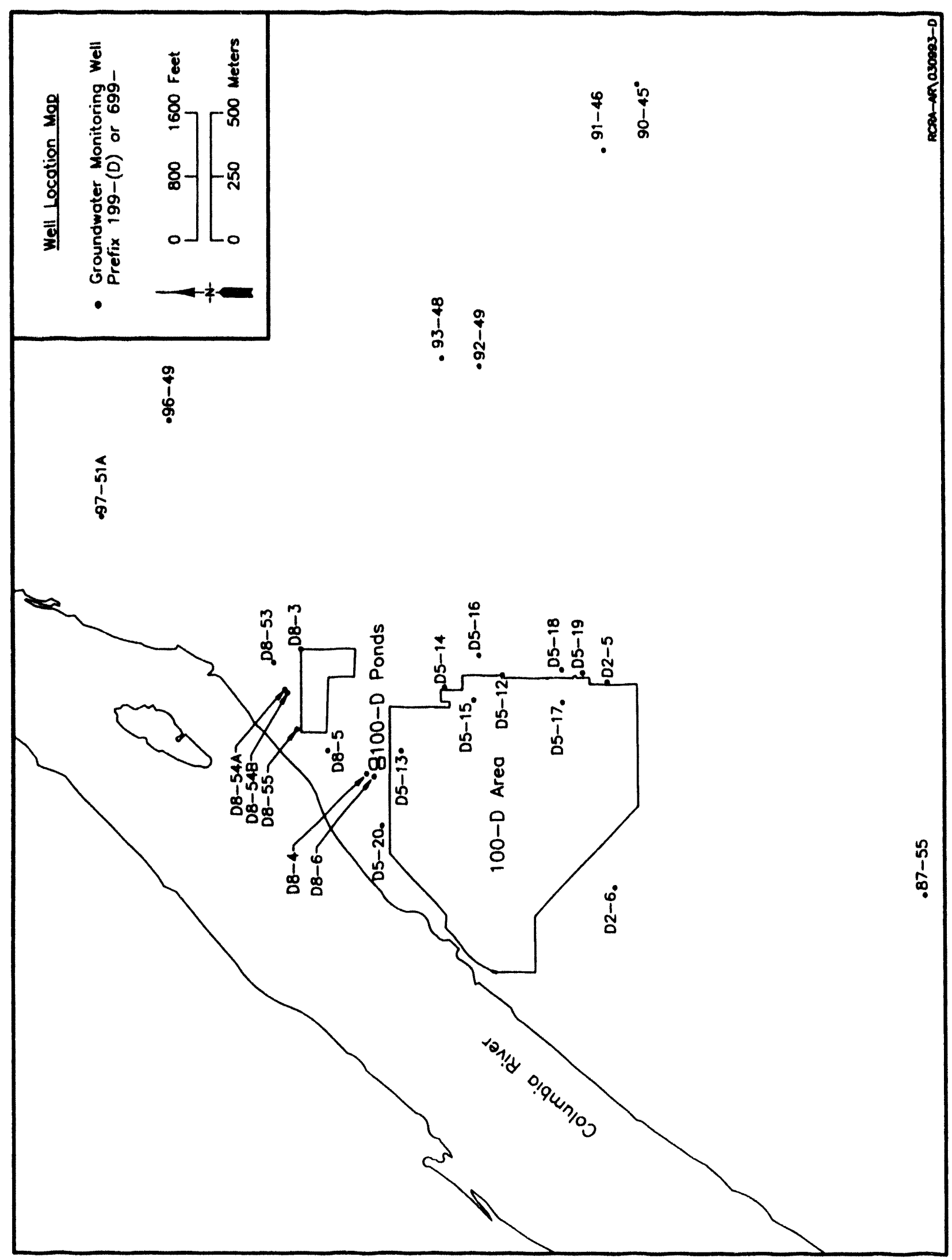


Table 2-1. Monitoring Well Purpose and Sampling Schedule in the 100-0 Ponds Network.

\begin{tabular}{|c|c|c|c|}
\hline $\begin{array}{c}\text { Well no. } \\
(199-)\end{array}$ & $\begin{array}{c}\text { Relative } \\
\text { position }\end{array}$ & $\begin{array}{c}\text { Sample } \\
\text { frequency }\end{array}$ & $\begin{array}{c}\text { Sample date, } \\
\text { 4th Qtr 1993 }\end{array}$ \\
\hline D5-13 & Upgradient & Semiannualiy & $12 / 7 / 93$ \\
\hline D8-4 & Downgradient & Semiannually & $12 / 8 / 93$ \\
\hline D8-5“ & Downgradient & Semiannually & $12 / 7 / 93$ \\
\hline D8-6 & Downgradient & Semiannually & $12 / 7 / 93$ \\
\hline
\end{tabular}

Note: The hydrogeologic unit monitored is the uppermost portion of the unconfined aquifer, which comprises unconsolidated sand and gravels of the Ringold Formation.

Well D8-5 will not be used for detection purposes, per agreement with the Washington State Department of Ecology. 
Table 2-2. RCRA Water Level Measurement Report for the 100-D Ponds, Fourth Quarter 1993. (sheet 1 of 3 )

\begin{tabular}{|c|c|c|c|}
\hline Well & Date & $\begin{array}{l}\text { Depth to } \\
\text { water (ft) }\end{array}$ & $\begin{array}{l}\text { Water level } \\
\text { elevation } \\
\text { above msl }(\mathrm{ft})\end{array}$ \\
\hline \multicolumn{4}{|c|}{ Wells Monitoring the Top of the Unconfined Aquifer } \\
\hline $199-D 2-5$ & $\begin{array}{l}10 / 05 / 93 \\
11 / 16 / 93 \\
12 / 20 / 93\end{array}$ & $\begin{array}{l}75.25 \\
75.41 \\
75.46\end{array}$ & $\begin{array}{l}385.05 \\
384.89 \\
384.84\end{array}$ \\
\hline $199-02-6$ & $\begin{array}{l}10 / 05 / 93 \\
11 / 16 / 93 \\
12 / 20 / 93\end{array}$ & $\begin{array}{l}85.49 \\
85.71 \\
85.51\end{array}$ & $\begin{array}{l}383.79 \\
383.57 \\
383.77\end{array}$ \\
\hline 199-D5-12 & $\begin{array}{l}10 / 05 / 93 \\
11 / 16 / 93 \\
12 / 20 / 93\end{array}$ & $\begin{array}{l}85.44 \\
85.61 \\
85.66\end{array}$ & $\begin{array}{l}384.19 \\
384.02 \\
383.97\end{array}$ \\
\hline $199-05-13$ & $\begin{array}{l}10 / 05 / 93 \\
11 / 16 / 93 \\
12 / 20 / 93\end{array}$ & $\begin{array}{l}87.47 \\
88.18 \\
85.68\end{array}$ & $\begin{array}{c}384.02 \\
383.31 \\
385.81+\end{array}$ \\
\hline $199-05-14$ & $\begin{array}{l}10 / 05 / 93 \\
11 / 16 / 93 \\
12 / 20 / 93\end{array}$ & $\begin{array}{l}87.77 \\
88.06 \\
88.03\end{array}$ & $\begin{array}{l}383.92 \\
383.63 \\
383.66\end{array}$ \\
\hline 199-D5-15 & $\begin{array}{l}10 / 05 / 93 \\
11 / 16 / 93 \\
12 / 20 / 93\end{array}$ & $\begin{array}{l}87.34 \\
87.58 \\
87.58\end{array}$ & $\begin{array}{l}384.19 \\
383.95 \\
383.95\end{array}$ \\
\hline $199-D 5-16$ & $\begin{array}{l}10 / 05 / 93 \\
11 / 16 / 93 \\
12 / 20 / 93\end{array}$ & $\begin{array}{l}89.05 \\
89.22 \\
89.25\end{array}$ & $\begin{array}{l}383.84 \\
383.67 \\
383.64\end{array}$ \\
\hline $199-05-17$ & $\begin{array}{l}10 / 05 / 93 \\
11 / 16 / 93 \\
12 / 20 / 93\end{array}$ & $\begin{array}{l}84.77 \\
84.92 \\
85.00\end{array}$ & $\begin{array}{l}384.72 \\
384.57 \\
384.49\end{array}$ \\
\hline $199-05-18$ & $\begin{array}{l}10 / 05 / 93 \\
11 / 16 / 93 \\
12 / 20 / 93\end{array}$ & $\begin{array}{l}82.27 \\
82.41 \\
82.49\end{array}$ & $\begin{array}{l}384.41 \\
384.27 \\
384.19\end{array}$ \\
\hline $199-05-19$ & $\begin{array}{l}10 / 05 / 93 \\
11 / 16 / 93 \\
12 / 20 / 93\end{array}$ & $\begin{array}{l}80.04 \\
80.13 \\
80.25\end{array}$ & $\begin{array}{l}384.76 \\
384.67 \\
384.55\end{array}$ \\
\hline $199-05-20$ & $\begin{array}{l}10 / 05 / 93 \\
11 / 16 / 93 \\
12 / 20 / 93\end{array}$ & $\begin{array}{l}86.07 \\
85.94 \\
85.25\end{array}$ & $\begin{array}{l}382.03 \\
382.16 \\
382.85\end{array}$ \\
\hline
\end{tabular}


Table 2-2. RCRA Water Level Measurement Report for the 100-D Ponds, Fourth Quarter 1993. (sheet 2 of 3)

\begin{tabular}{|c|c|c|c|}
\hline Well & Date & $\begin{array}{r}\text { Depth to } \\
\text { water (ft) }\end{array}$ & $\begin{array}{l}\text { Water level } \\
\text { elevation } \\
\text { above } \mathrm{ms} 1(\mathrm{ft})\end{array}$ \\
\hline \multicolumn{4}{|c|}{ Wells Monitoring the Top of the Unconfined Aquifer } \\
\hline 199-D8-3 & $\begin{array}{l}10 / 05 / 93 \\
11 / 16 / 93 \\
12 / 20 / 93\end{array}$ & $\begin{array}{l}69.09 \\
68.57 \\
68.92\end{array}$ & $\begin{array}{l}379.90 \\
380.42 \\
380.07\end{array}$ \\
\hline $199-08-4$ & $\begin{array}{l}10 / 05 / 93 \\
11 / 16 / 93 \\
12 / 20 / 93\end{array}$ & $\begin{array}{l}85.44 \\
85.50 \\
85.26\end{array}$ & $\begin{array}{l}383.29 \\
383.23 \\
383.47\end{array}$ \\
\hline $199-D 8-5$ & $\begin{array}{l}10 / 05 / 93 \\
11 / 16 / 93 \\
12 / 20 / 93\end{array}$ & $\begin{array}{l}71.17 \\
71.04 \\
70.42\end{array}$ & $\begin{array}{l}381.32 \\
381.45 \\
382.07\end{array}$ \\
\hline $199-08-53$ & $\begin{array}{l}10 / 05 / 93 \\
11 / 16 / 93 \\
12 / 20 / 93\end{array}$ & $\begin{array}{l}56.32 \\
55.57 \\
54.97\end{array}$ & $\begin{array}{l}379.71 \\
380.46 \\
381.06\end{array}$ \\
\hline $199-D 8-54 A$ & $\begin{array}{l}10 / 05 / 93 \\
11 / 16 / 93 \\
12 / 20 / 93\end{array}$ & $\begin{array}{l}63.01 \\
62.29 \\
61.69\end{array}$ & $\begin{array}{l}379.77 \\
380.49 \\
381.09\end{array}$ \\
\hline $199-D 8-54 B$ & $\begin{array}{l}10 / 05 / 93 \\
11 / 16 / 93 \\
12 / 20 / 93\end{array}$ & $\begin{array}{l}62.15 \\
61.51 \\
60.98\end{array}$ & $\begin{array}{l}380.36 \\
381.00 \\
381.53\end{array}$ \\
\hline $199-08-55$ & $\begin{array}{l}10 / 05 / 93 \\
11 / 16 / 93 \\
12 / 20 / 93\end{array}$ & $\begin{array}{l}59.58 \\
58.87 \\
58.09\end{array}$ & $\begin{array}{l}379.77 \\
380.48 \\
381.26\end{array}$ \\
\hline $199-08-6$ & $\begin{array}{l}10 / 05 / 93 \\
11 / 16 / 93 \\
12 / 20 / 93\end{array}$ & $\begin{array}{l}93.28 \\
93.77 \\
93.02\end{array}$ & $\begin{array}{l}383.22 \\
382.73 \\
383.48\end{array}$ \\
\hline $699-87-55$ & $\begin{array}{l}10 / 05 / 93 \\
11 / 16 / 93 \\
12 / 20 / 93\end{array}$ & $\begin{array}{l}72.93 \\
73.11 \\
73.05\end{array}$ & $\begin{array}{l}385.70 \\
385.52 \\
385.58\end{array}$ \\
\hline $699-90-45$ & $\begin{array}{l}10 / 05 / 93 \\
11 / 16 / 93 \\
12 / 20 / 93\end{array}$ & $\begin{array}{l}37.67 \\
37.73 \\
37.74\end{array}$ & $\begin{array}{l}383.87 \\
383.81 \\
383.80\end{array}$ \\
\hline $699-91-46$ & $\begin{array}{l}10 / 05 / 93 \\
11 / 16 / 93 \\
12 / 20 / 93\end{array}$ & $\begin{array}{l}33.31 \\
33.34 \\
33.36\end{array}$ & $\begin{array}{l}383.75 \\
383.72 \\
383.70\end{array}$ \\
\hline
\end{tabular}


Table 2-2. RCRA Water Level Measurement Report for the 100-D Ponds, Fourth Quarter 1993. (sheet 3 of 3)

\begin{tabular}{cccc}
\hline Well & Date & $\begin{array}{c}\text { Depth to } \\
\text { water (ft) }\end{array}$ & $\begin{array}{c}\text { Water level } \\
\text { elevation } \\
\text { above ms }(\mathrm{ft})\end{array}$ \\
\hline Wells Monitoring the Top of the Unconfined Aquifer \\
\hline $699-92-49$ & $10 / 05 / 93$ & 49.06 & 382.82 \\
& $11 / 16 / 93$ & 51.56 & $380.32+$ \\
$699-93-48$ & $12 / 20 / 93$ & 48.95 & 382.93 \\
& $10 / 05 / 93$ & 55.18 & 382.61 \\
$699-96-49$ & $11 / 16 / 93$ & 55.29 & 382.50 \\
& $12 / 20 / 93$ & 55.33 & 382.46 \\
& $10 / 05 / 93$ & 38.01 & 381.22 \\
$699-97-51 A$ & $11 / 16 / 93$ & 38.13 & 381.10 \\
& $12 / 20 / 93$ & 38.13 & 381.10 \\
& $10 / 05 / 93$ & 21.68 & 380.59 \\
& $11 / 16 / 93$ & 21.65 & 380.62 \\
& $12 / 20 / 93$ & 21.57 & 380.70 \\
\hline
\end{tabular}

Notes: 1. Water level elevations are calculated by subtracting the measured depth to water from the surveyed elevation for the well.

2. Depth-to-water values are transcribed from field records.

3. Elevations marked with $a$ ' $^{\prime}$ ' are outside of the expected range and are suspected of error.

4. To convert feet to meters multiply by 0.3048 . 
Table 2-3. Constituent List and Summary of Results for the 100-D Pond Data for Reporting Period 0ctober 1 through December 31, 1993. (sheet 1 of 2)

CONTAMINATION INOICATOR PARAMETERS

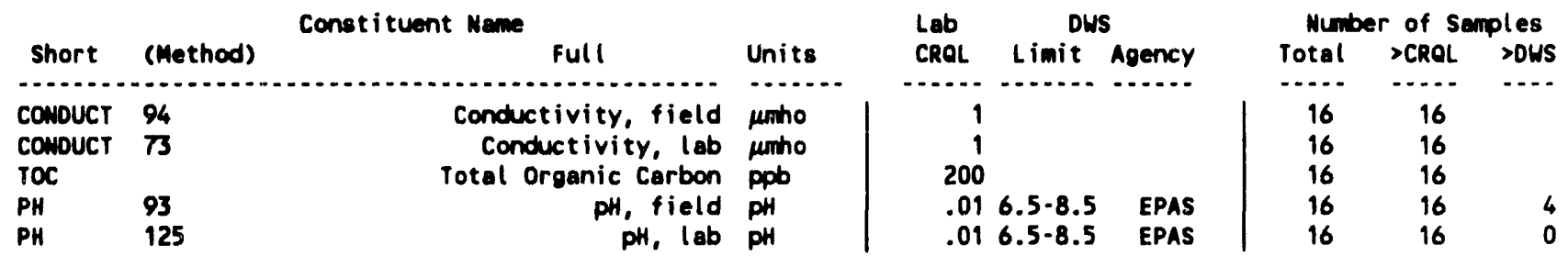

DRINKING WATER PARAMETERS

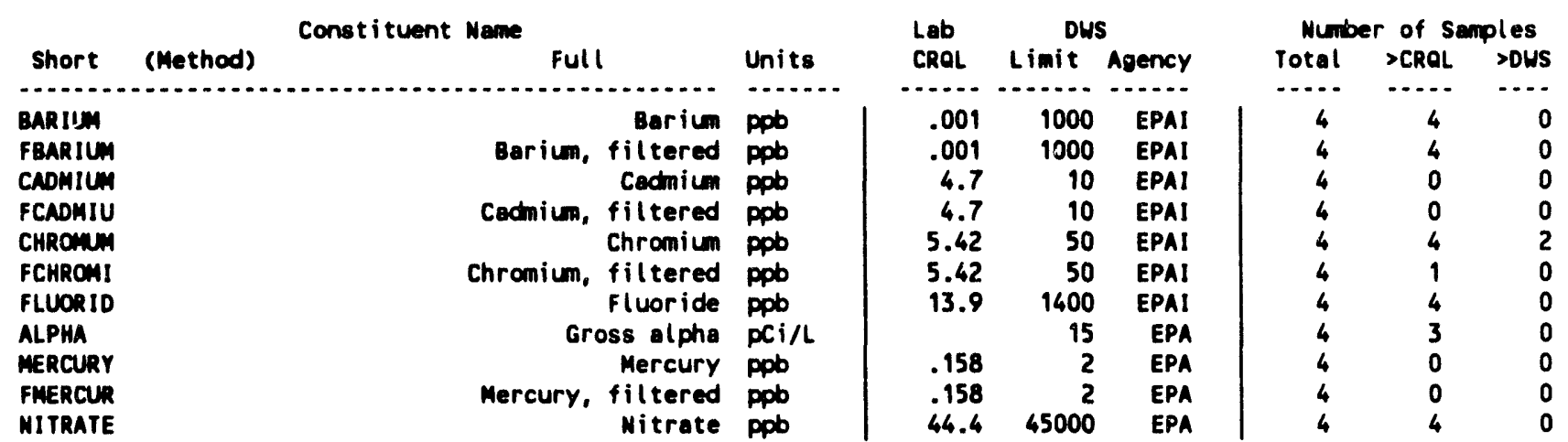

GROUNOWATER QUALITY PARAMETERS

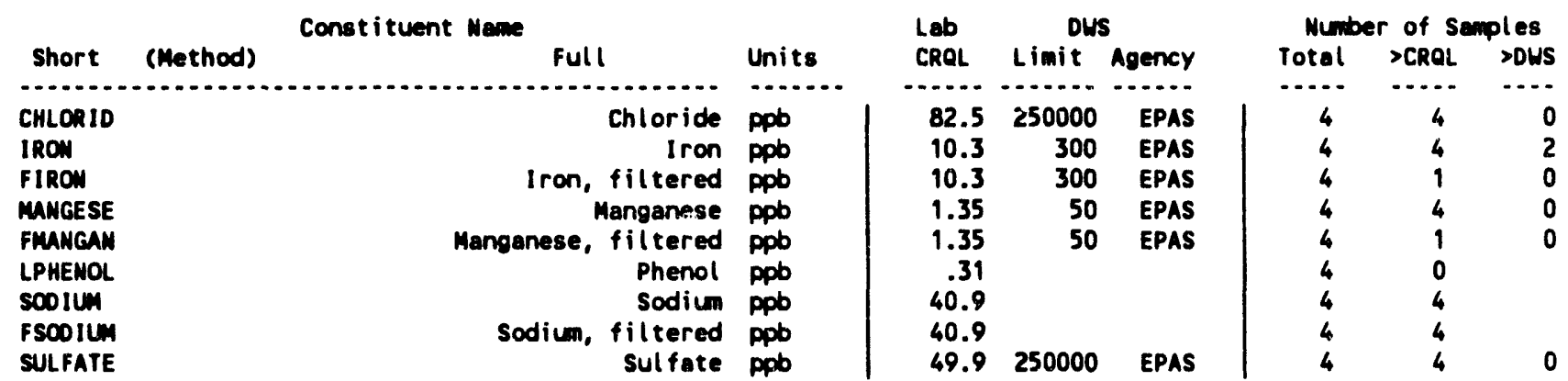

SITE SPECIFIC AND OTHER CONSTITUENTS

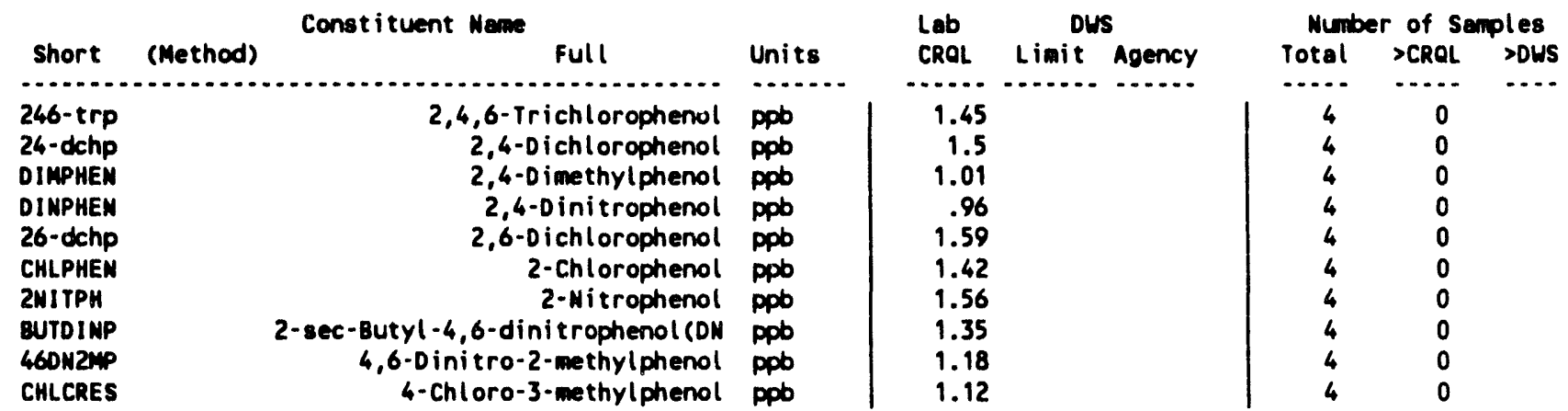


Table 2-3. Constituent List and Summary of Results for the 100-D Pond Data for Reporting Period October 1 through December 31, 1993. (sheet 2 of 2)

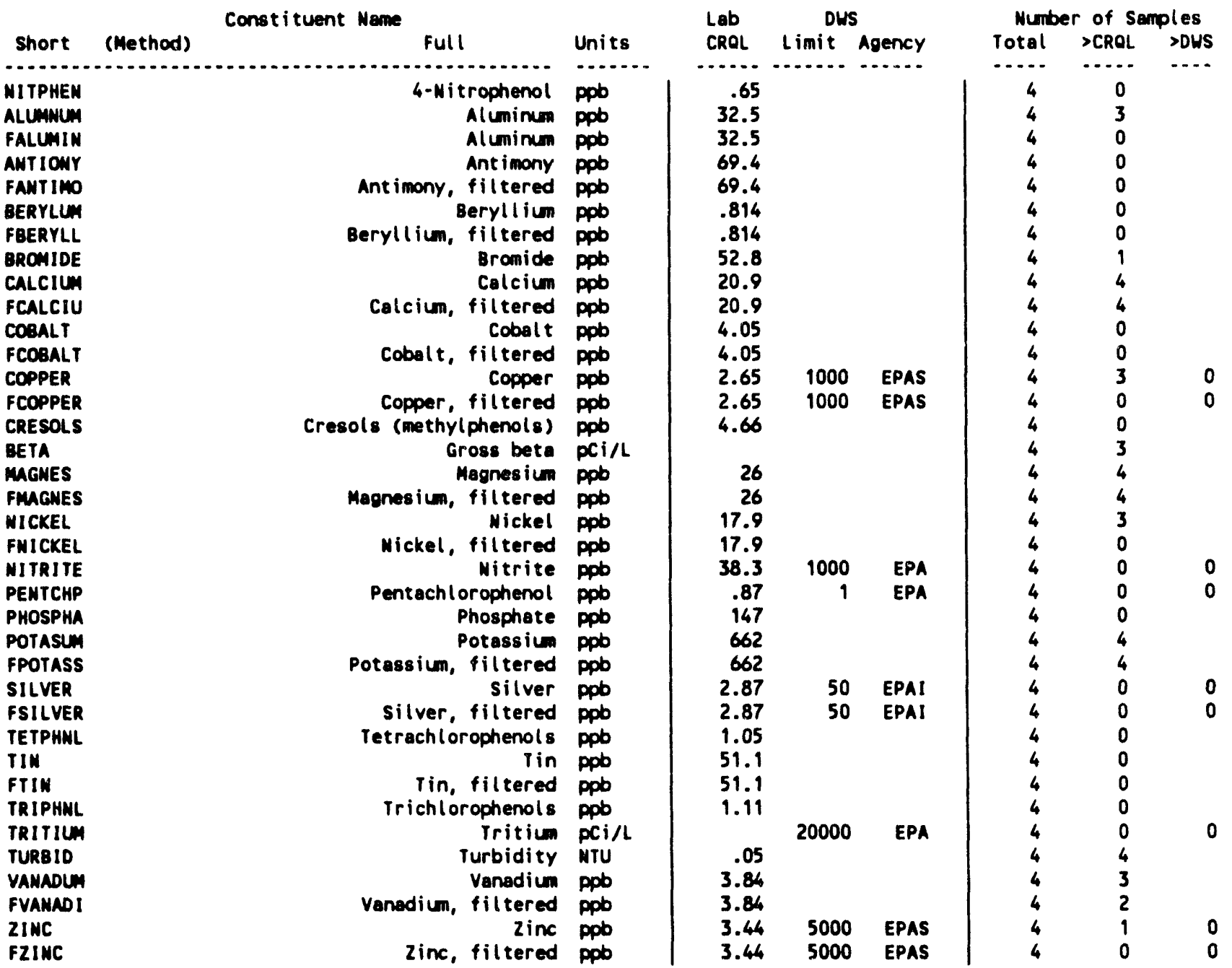

For explanstion of this table, see Section 1.4 of report. 
Table 2-4. Constituents with at Least One Detected Value for the 100-D Pond Data for Reporting Period October 1 through December 31, 1993. (sheet 1 of 3)

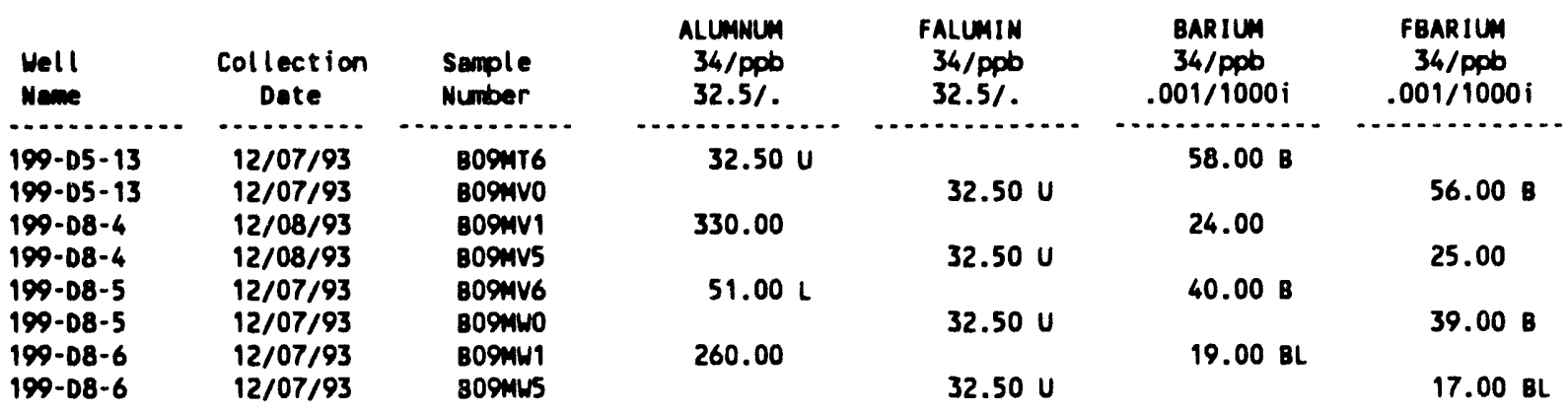

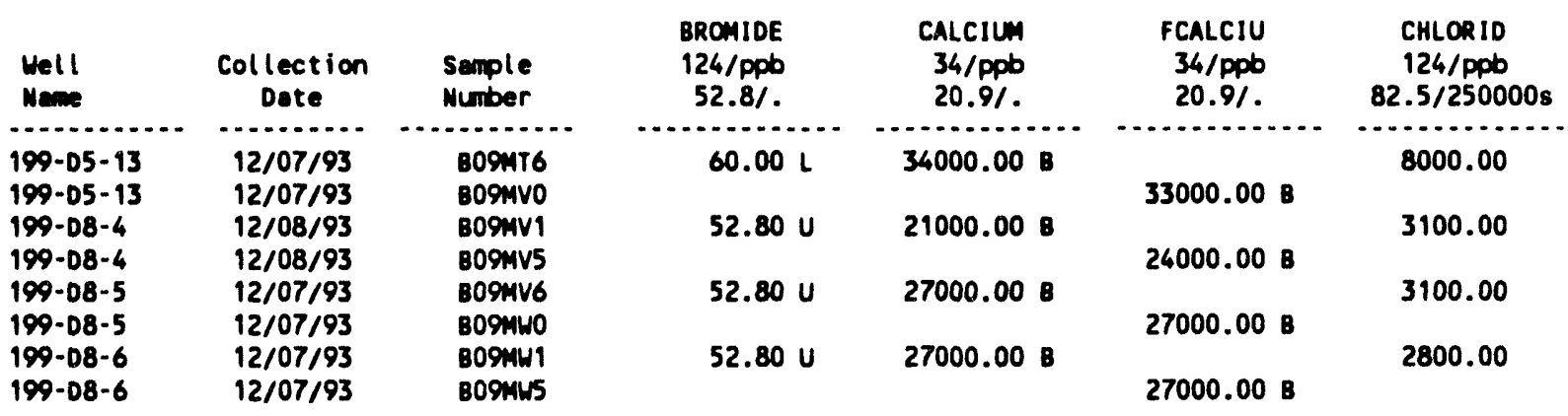

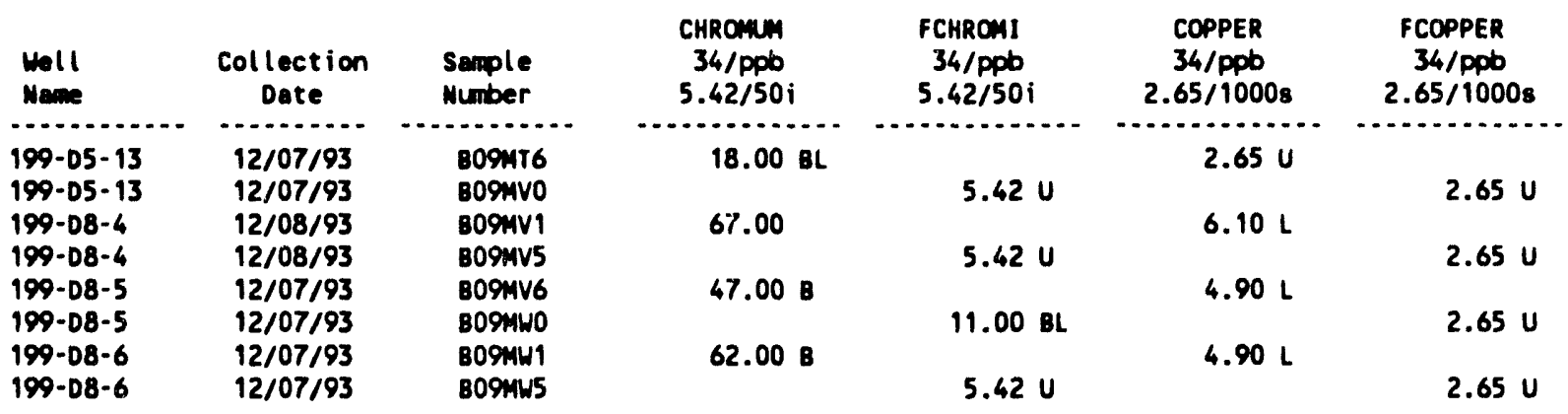

\begin{tabular}{|c|c|c|c|c|c|c|}
\hline $\begin{array}{l}\text { Well } \\
\text { Nesce }\end{array}$ & $\begin{array}{c}\text { Collection } \\
\text { Date }\end{array}$ & $\begin{array}{l}\text { Sample } \\
\text { Number }\end{array}$ & $\begin{array}{l}\text { FLUORID } \\
124 / \mathrm{ppD} \\
13.9 / 1400 \mathrm{i}\end{array}$ & $\begin{array}{c}\text { ALPHA } \\
135 / \mathrm{pCi} / \mathrm{L} \\
. / 15\end{array}$ & $\begin{array}{c}\text { BETA } \\
136 / \mathrm{PCi} / \mathrm{L} \\
.1 .\end{array}$ & $\begin{array}{c}\text { IROW } \\
34 / \mathrm{ppb} \\
10.3 / 300 \mathrm{~s}\end{array}$ \\
\hline $\begin{array}{l}199-05-13 \\
199-08-4 \\
199-08-5 \\
199-08-6\end{array}$ & $\begin{array}{l}12 / 07 / 93 \\
12 / 08 / 93 \\
12 / 07 / 93 \\
12 / 07 / 93\end{array}$ & $\begin{array}{l}\text { BO9MT6 } \\
\text { B09MV1 } \\
\text { B09MV6 } \\
\text { B09MU1 }\end{array}$ & $\begin{array}{l}300.00 \\
200.00 \\
300.00 \\
200.00\end{array}$ & $\begin{array}{l}2.07 \\
.72 U \\
1.03 \\
1.14\end{array}$ & $\begin{array}{l}3.75 \\
3.72 \\
5.94 \\
.85 \mathrm{U}\end{array}$ & $\begin{array}{l}77.00 \mathrm{~B} \\
630.00 \\
190.00 \mathrm{~B} \\
450.00 \mathrm{~B}\end{array}$ \\
\hline
\end{tabular}


Table 2-4. Constituents with at Least One Detected Value for the 100-D Pond Data for Reporting Period October 1 through December 31,1993 . (sheet 2 of 3 )

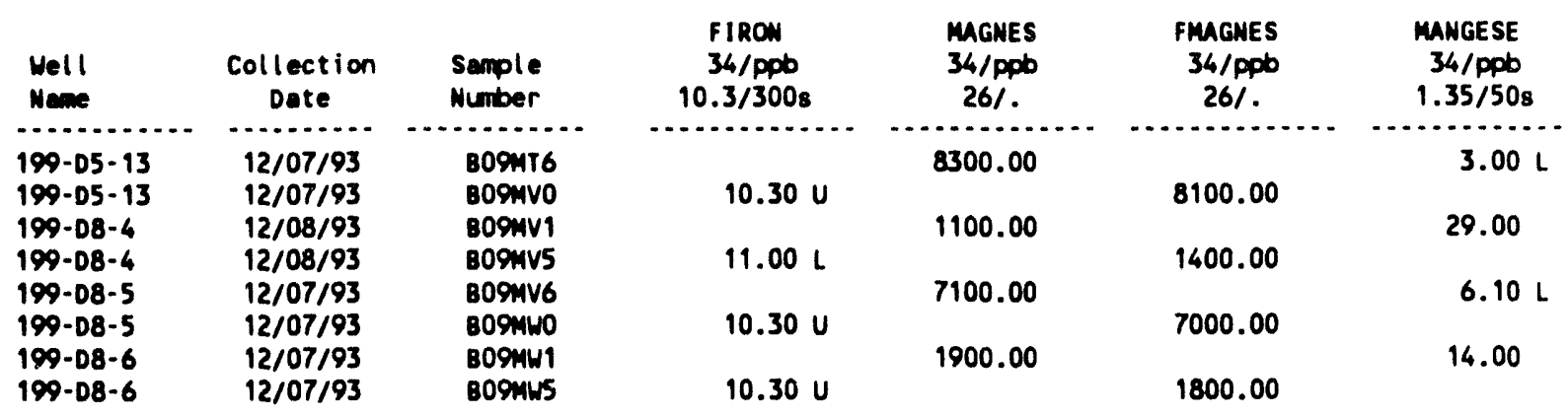

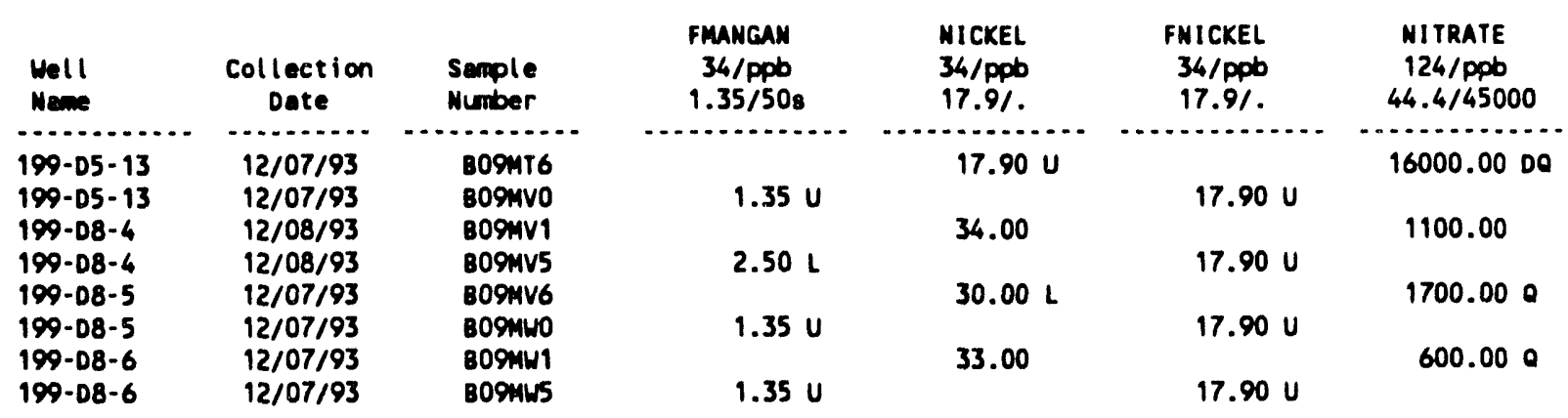

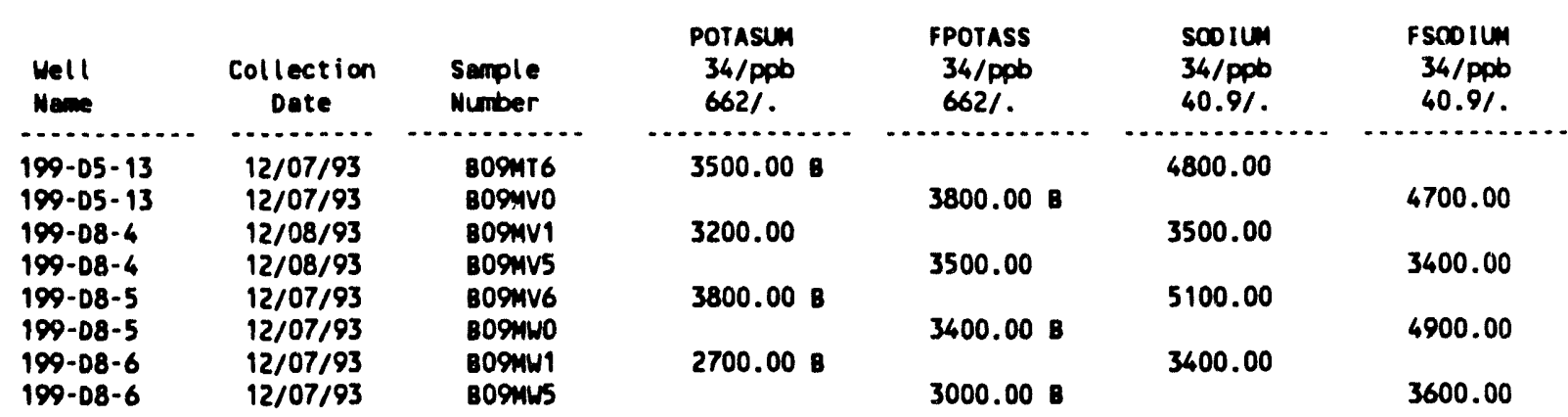

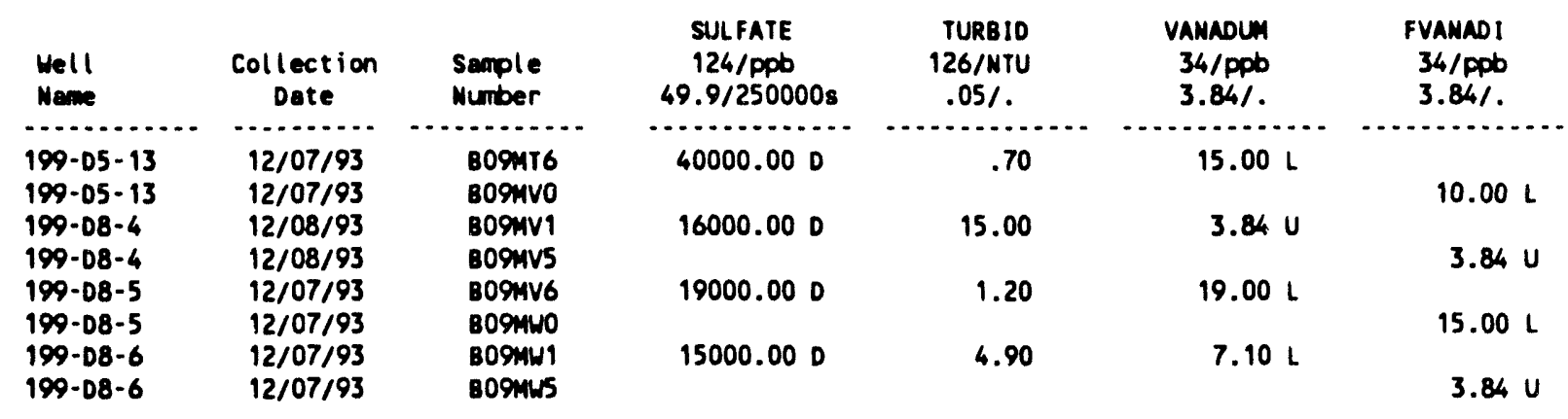


Table 2-4. Constituents with at Least One Detected Value for the 100-D Pond Data for Reporting Period October 1 through December 31 , 1993. (sheet 3 of 3 )

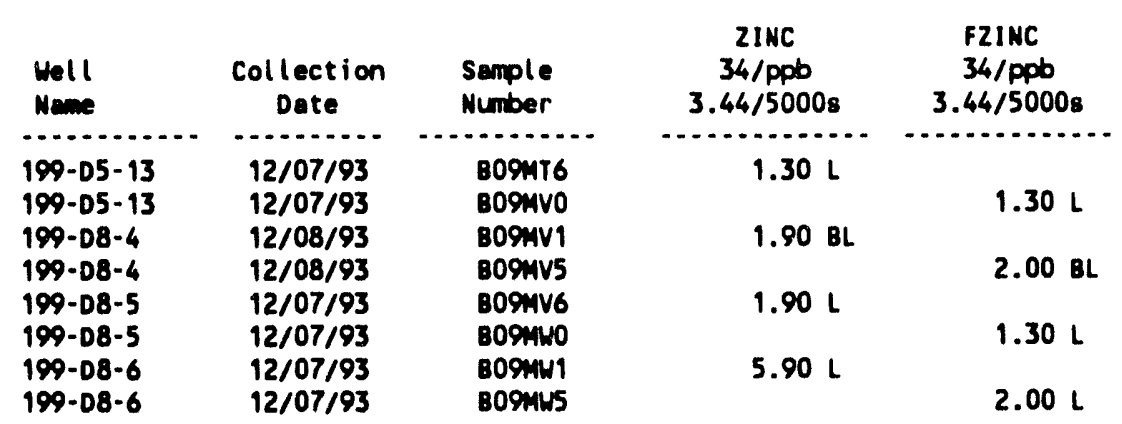

For explenation of this table, see Section 1.4 of report. 
Table 2-5. Contamination Indicator Parameters for the 100-D Pond Data for Reporting Period October 1 through December 31, 1993.

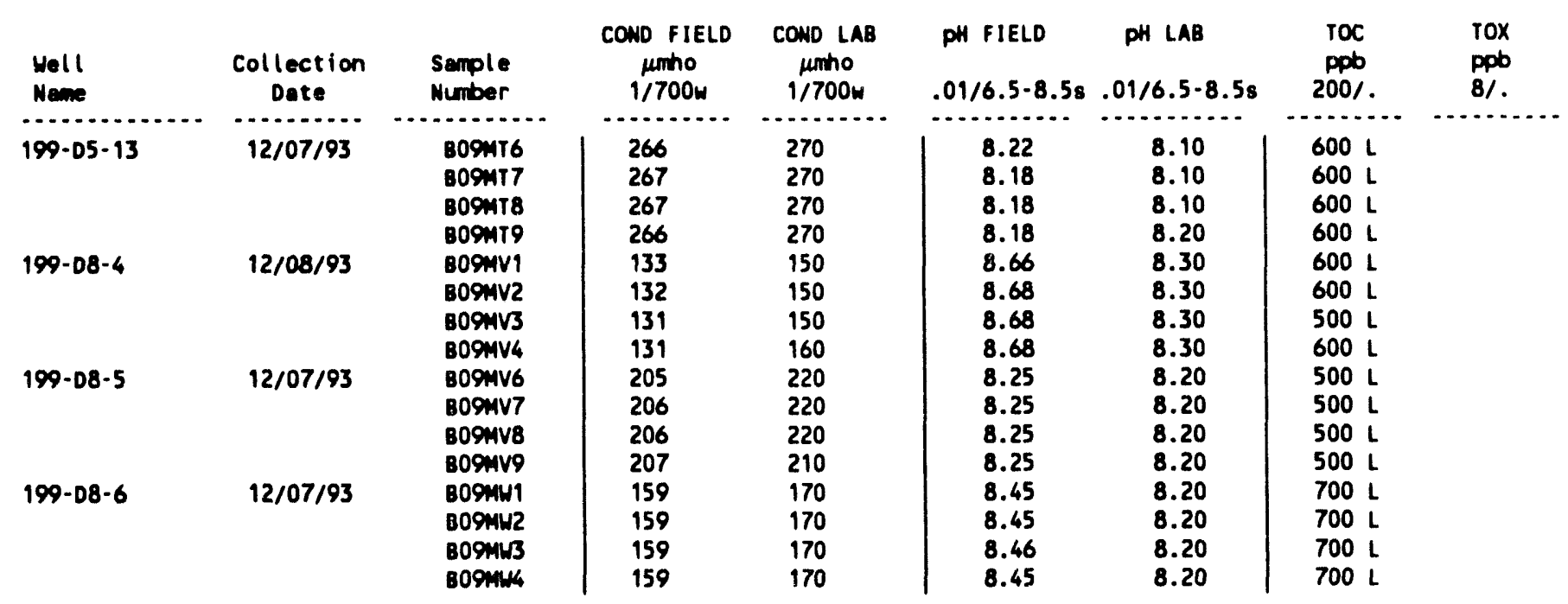

For explanation of this table, see Section 1.4 of report. 


\section{DOE/RL-93-56-4}

\section{CONTENTS}

$3.0183-H$ SOLAR EVAPORATION BASINS . . . . . . . . . . . . 3-1

3.1 INTRODUCTION . ..................... 3-1

3.2 WATER LEVEL MEASUREMENTS . . . . . . . . . . . . 3-1

3.3 WATER CHEMISTRY DATA . . . . . . . . . . . 3-2 


\section{LIST OF FIGURES}

3-1 Monitoring Well Location Map for the 183-H Solar Evaporation

Basins Network . . . . . . . . . . . . . . . . . 3-3

\section{LIST OF TABLES}

3-1 Monitoring Well Position and Sampling Schedule for the 183-H Solar Evaporation Basins Network........... 3-4

3-2 RCRA Water Level Measurement Report for the 183-H Solar Evaporation Basins, Fourth Quarter $1993 \ldots$. . . . . . . .6

3-3 Constituent List and Summary of Results for 183-H Solar Evaporation Basins Data for Reporting Period October 1 through December 31, 1993 ................ . . 3-10

3-4 Constituents with at Least One Detected Value for the 183-H Solar Evaporation Basins Data for Reporting Period October 1 through December $31,1993 \ldots$. . . . . . . . . . . . . . .

3-5 Contamination Indicator Parameters for the 183-H Solar Evaporation Basins Data for Reporting Period October 1 through

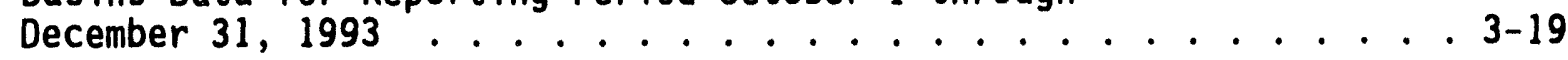


DOE/RL-93-56-4

\title{
3.0 183-H SOLAR EVAPORATION BASINS
}

\author{
R. E. Peterson \\ Westinghouse Hanford Company
}

\subsection{INTRODUCTION}

The 183-H Solar Evaporation Basins (Basins) are a RCRA-regulated treatment, storage, and/or disposal (TSD) facility located along the Columbia River in the 100 Areas of the Hanford Site. Originally the Basins were part of the water treatment facility that prepared coolant for the 105-H Reactor. Subsequent to that mission, between 1973 and 1985, the Basins were used for storage and volume reduction (by solar evaporation) of liquid waste from nuclear fuel processing activities conducted in the 300 Area. The predominant waste consisted of nitric acid solutions that had been neutralized with sodium hydroxide before discharge into the Basins. The solutions contained various metals and radioactive constituents. Hazardous waste has been removed from the facility and the basin walls have been partially decontaminated. A soil investigation has been completed. An application for permanent closure is under review by Ecology. Groundwater monitoring is being conducted as an assessment level program under 40 CFR 265 interim status standards.

When TSD operations began in 1973, only two groundwater wells existed in the 100-H Area, neither of which monitored conditions near the Basins. During the operational life of the Basins, limited groundwater monitoring occurred. In 1974, a single downgradient well was installed (199-H4-3). In 1983, two additional downgradient wells and one upgradient well were installed. Eighteen additional monitoring wells, which were constructed to meet RCRA standards, were installed in 1986 and 1987 after the facility ceased operation. These wells were installed in response to a Consent Agreement and Compliance Order entered into by Ecology, EPA, and DOE (Ecology and EPA 1986).

Twenty-three wells were in use during 1993 for obtaining RCRA groundwater samples. One additional well (199-H4-15C) was used exclusively for water level measurements. A location map for the 100-H Area is shown in Figure 3-1. The purpose of each RCRA well, its sampling frequency, and the dates sampled during the quarter, are summarized in Table 3-1. Nitrate, sodium, gross alpha, and gross beta are used to monitor the extent of the plume attributable to the Basins' waste. Wells 199-H4-3, 199-H4-4, 199-H4-9, and 199-H4-12A are assumed to be within the plume.

\subsection{WATER LEVEL MEASUREMENTS}

During this quarter, water levels were measured in the monitoring well network at monthly intervals. Water levels also were recorded at the time of sample collection for water quality analyses. Water level measurements obtained during the fourth quarter of 1993 are presented in Table 3-2. The water level in well 199-H4-15CQ, a piezometer that monitors the bottom of the uppermost aquifer system, is now at or slightly above the top-of-casing reference point. Levels in this piezometer began a steady rise during 1992 , reflecting a possible connection between this piezometer and 199-H4-15CP, a deeper piezometer that monitors the uppermost confined aquifer in basalt. 


\subsection{WATER CHEMISTRY DATA}

Fourth quarter sampling was completed in the majority of the Basins' network wells during October 1993. Three wells were also sampled monthly to provide enhanced monitoring of the plume during cleanup and closure activities. A summary of fourth quarter 1993 results is presented in Table 3-3. Analytical results for constituents with at least one value above the MDL are presented in Tables 3-4 and 3-5. Blank entries in these tables indicate either the constituent was not included in the analysis ilst or the results are not yet available. If analytical results for previous sampling episodes were received during the current quarter, they are included in the tables.

For constituents relevant to assessing the 183-H plume, EPA interim DWSs (40 CFR 265, Appendix III) are exceeded for chromium, gross alpha, technetium, and nitrate (see Table 3-3). Gross alpha activity is correlated with uranium in basin waste solutions. Gross beta activity, which is also elevated, is correlated with technetium. Iron and manganese also exceed DWSs; however, these constituents are not related to basin waste solutions.

The pulse in 183-H indicator constituents and other water quality parameters that were reported in the previous second and third quarterly reports for 1993 (DOE-RL 1993b, 1994b) appears to cont inue into the fourth quarter, although levels have declined somewhat. The pulse is apparent in nitrate, sodium, sulfate, chromium, gross alpha, and gross beta concentrations in wells 199-H4-3 and 199-H4-4, which intercept the plume. These wells have al so shown increased turbidity levels during 1993. Other wells outside of the 183-H plume also exhibit the pulse.

The quarterly assessment of the groundwater plume has not been influenced significantly by $Q C$ problems, although some results for barium, calcium, chloride, fluoride, gross beta, and zinc have been flagged with a ' $Q$ ' as the result of blank or duplicate samples being out of acceptable limits. One value for nitrite in an upgradient well appears anomalous and is flagged for additional review. TOX results continue to experience QC problems. 
Figure 3-1. Monitoring Well Location Map for the 183-H Solar Evaporation Basins Network.

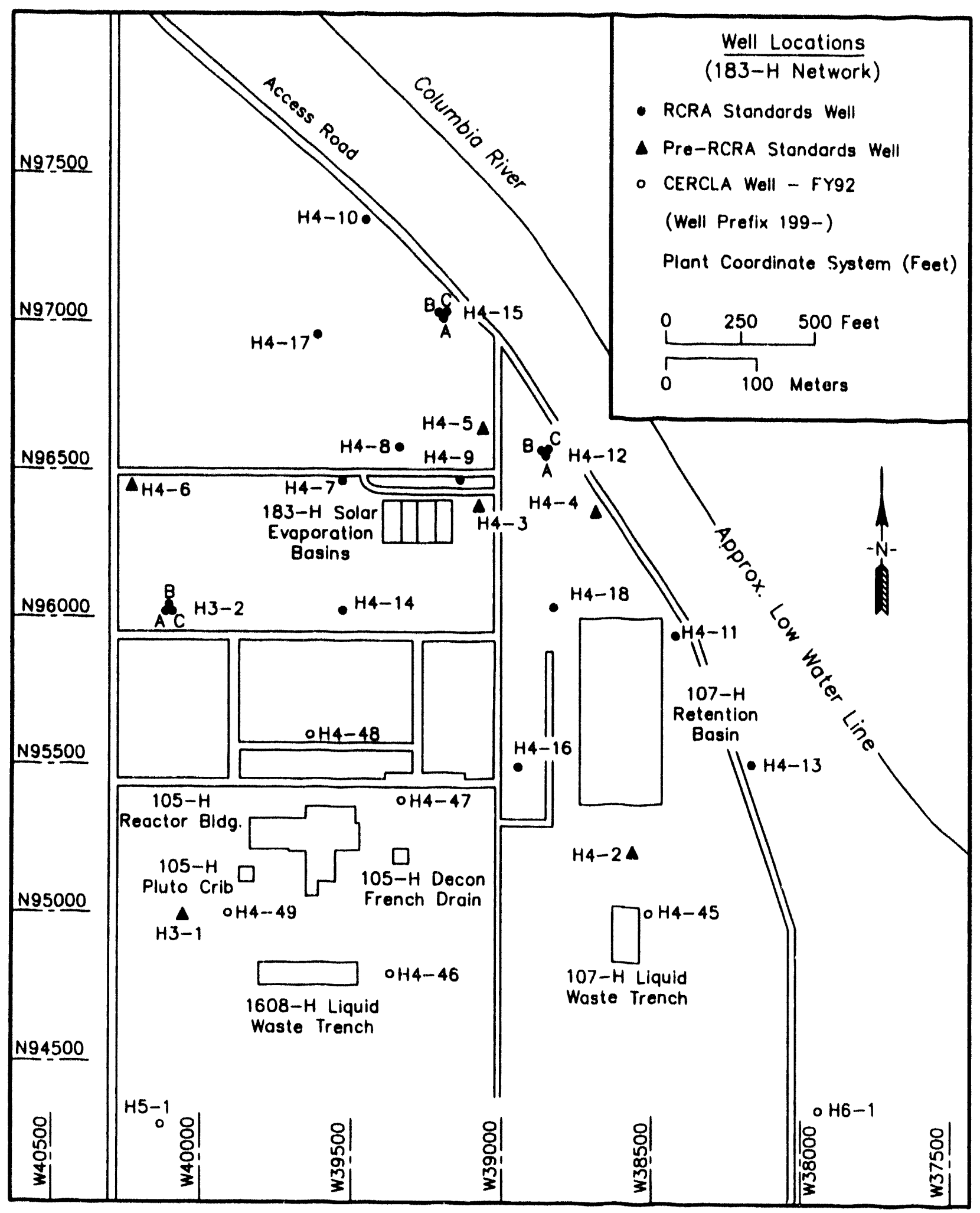

RCRA-AR\122993J 
Table 3-1. Monitoring Well Position and Sampling Schedule for the 183-H Solar Evaporation Basins Network. (sheet 1 of 2)

\begin{tabular}{|c|c|c|c|c|}
\hline $\begin{array}{l}\text { Well no. } \\
(199-)\end{array}$ & $\begin{array}{l}\text { Relative } \\
\text { position }\end{array}$ & Hydrogeologic unit & $\begin{array}{c}\text { Sample } \\
\text { frequency }\end{array}$ & $\begin{array}{l}\text { Sample date, } \\
\text { 4th Qtr } 1993\end{array}$ \\
\hline $\mathrm{H3}-1$ & Upgradient & Hanford: Water Table & Annually & Not sampled \\
\hline$H 3-2 A$ & Upgradient & Hanford: Water Table & Quarterly & $10 / 06 / 93$ \\
\hline$H 3-2 B$ & Upgradient & Hanford: Water Table & Annually & Not sampled \\
\hline $\mathrm{H} 3-2 \mathrm{C}$ & Upgradient & Ringold: Mid-Depth & Quarterly & $10 / 06 / 93$ \\
\hline $\mathrm{H} 4-10$ & Upgradient & Hanford: Water Table & Annually & Not sampled \\
\hline $\mathrm{H} 4-11$ & Downgradient & Hanford: Water Table & Quarterly & $10 / 05 / 93$ \\
\hline$H 4-12 A$ & Downgradient & Hanford: Water Table & Quarterly & $10 / 06 / 93$ \\
\hline$H 4-12 B$ & Downgradient & Hanford: Water Table & Annually & Not sampled \\
\hline $\mathrm{H} 4-12 \mathrm{C}$ & Downgradient & Ringold: Mid-Depth & Quarterly & $10 / 05 / 93$ \\
\hline $\mathrm{H} 4-13$ & Downgradient & Hanford: Water Table & Annually & Not sampled \\
\hline H4-14 & Upgradient & Hanford: Water Table & Quarterly & $10 / 05 / 93$ \\
\hline $\mathrm{H} 4-15 \mathrm{~A}$ & Downgradient & Hanford: Water Table & Quarterly & $10 / 05 / 93$ \\
\hline $\mathrm{H} 4-15 \mathrm{~B}$ & Downgradient & Hanford: Water Table & Annually & Not sampled \\
\hline $\mathrm{H} 4-15 \mathrm{Cq}$ & Downgradient & Ringold: Deep & Monthly & Piezometer \\
\hline $\mathrm{H} 4-15 \mathrm{Cr}$ & Downgradient & Ringold: Mid-Depth & Monthly & Piezometer \\
\hline $\mathrm{H} 4-15 \mathrm{Cs}$ & Downgradient & Ringold: Mid-Depth & Monthly & Piezometer \\
\hline H4-16 & Downgradient & Hanford: Water Table & Annually & Not sampled \\
\hline $\mathrm{H} 4-17$ & Upgradient & Hanford: Water Table & Annually & $10 / 06 / 93$ \\
\hline $\mathrm{H} 4-18$ & Downgradient & Hanford: Water Table & Quarterly & $10 / 06 / 93$ \\
\hline H4-3 & Downgradient & Hanford: Water Table & Monthly & $\begin{array}{l}10 / 06 / 93 \\
11 / 02 / 93 \\
12 / 08 / 93\end{array}$ \\
\hline$H 4-4$ & Downgradient & Hanford: Water Table & Monthly & $\begin{array}{l}10 / 06 / 93 \\
11 / 16 / 93 \\
12 / 08 / 93\end{array}$ \\
\hline $\mathrm{H} 4-5$ & Downgradient & Hanford: Water Table & Quarterly & $10 / 06 / 93$ \\
\hline $\mathrm{H} 4-6$ & Upgradient & Hanford: Water Table & Quarterly & $10 / 06 / 93$ \\
\hline H4-7 & Upgradient & Hanford: Water Table & Quarterly & $10 / 06 / 93$ \\
\hline
\end{tabular}


Table 3-1. Monitoring Well Position and Sampling Schedule for the 183-H Solar Evaporation Basins Network. (sheet 2 of 2)

\begin{tabular}{|c|c|c|c|c|}
\hline $\begin{array}{c}\text { Well no. } \\
(199-)\end{array}$ & $\begin{array}{c}\text { Relative } \\
\text { position }\end{array}$ & Hydrogeologic unit & $\begin{array}{c}\text { Sample } \\
\text { frequency }\end{array}$ & $\begin{array}{c}\text { Sample date, } \\
\text { 4th Qtr 1993 }\end{array}$ \\
\hline H4-8 & Upgradient & Hanford: Water Table & Quarterly & $10 / 08 / 93$ \\
\hline H4-9 & Downgradient & Hanford: Water Table & Monthly & $\begin{array}{l}10 / 06 / 93 \\
11 / 02 / 93 \\
12 / 06 / 93\end{array}$ \\
\hline
\end{tabular}

- Hydrogeologic units include the sandy gravels of the Hanford formation and silty sands of the Ringold Formation. Water levels are measured monthly in all wells.

Water level measurement only. 
Table 3-2. RCRA Water Level Measurement Report for the 183-H Solar Evaporation Basins, Fourth Quarter 1993. (sheet 1 of 4)

\begin{tabular}{|c|c|c|c|}
\hline Well & Date & $\begin{array}{l}\text { Depth to } \\
\text { water (ft) }\end{array}$ & $\begin{array}{l}\text { Water level } \\
\text { elevation } \\
\text { above msl ( } f t)\end{array}$ \\
\hline \multicolumn{4}{|c|}{ Wells Monitoring the Top of the $U$} \\
\hline $199-\mathrm{H} 3-1$ & $\begin{array}{l}10 / 05 / 93 \\
11 / 16 / 93 \\
12 / 20 / 93\end{array}$ & $\begin{array}{l}45.86 \\
46.07 \\
46.12\end{array}$ & $\begin{array}{l}375.62 \\
375.41 \\
375.36\end{array}$ \\
\hline $199-H 3-2 A$ & $\begin{array}{l}10 / 05 / 93 \\
11 / 16 / 93 \\
12 / 20 / 93\end{array}$ & $\begin{array}{l}42.73 \\
42.87 \\
42.89\end{array}$ & $\begin{array}{l}375.10 \\
374.96 \\
374.94\end{array}$ \\
\hline 199-H3-2B & $\begin{array}{l}10 / 05 / 93 \\
11 / 16 / 93 \\
12 / 20 / 93\end{array}$ & $\begin{array}{l}43.32 \\
43.48 \\
43.48\end{array}$ & $\begin{array}{l}375.10 \\
374.94 \\
374.94\end{array}$ \\
\hline $199-\mathrm{H} 4-10$ & $\begin{array}{l}10 / 05 / 93 \\
11 / 16 / 93 \\
12 / 20 / 93\end{array}$ & $\begin{array}{l}32.10 \\
31.13 \\
30.29\end{array}$ & $\begin{array}{l}372.34 \\
373.31 \\
374.15\end{array}$ \\
\hline $199-\mathrm{H} 4-11$ & $\begin{array}{l}10 / 05 / 93 \\
10 / 05 / 93 \\
11 / 16 / 93 \\
12 / 20 / 93\end{array}$ & $\begin{array}{l}45.64 \\
45.57 \\
44.43 \\
44.16\end{array}$ & $\begin{array}{l}371.20^{\star} \\
371.27 \\
372.41 \\
372.68\end{array}$ \\
\hline $199-H 4-12 A$ & $\begin{array}{l}10 / 05 / 93 \\
11 / 16 / 93 \\
12 / 20 / 93\end{array}$ & $\begin{array}{l}41.45 \\
40.41 \\
40.36\end{array}$ & $\begin{array}{l}372.05 \\
373.09 \\
373.14\end{array}$ \\
\hline 199-H4-12B & $\begin{array}{l}10 / 05 / 93 \\
11 / 16 / 93 \\
12 / 20 / 93\end{array}$ & $\begin{array}{l}41.46 \\
40.43 \\
40.38\end{array}$ & $\begin{array}{l}372.06 \\
373.09 \\
373.14\end{array}$ \\
\hline $199-\mathrm{H} 4-13$ & $\begin{array}{l}10 / 05 / 93 \\
11 / 16 / 93 \\
12 / 20 / 93\end{array}$ & $\begin{array}{l}47.29 \\
46.11 \\
45.99\end{array}$ & $\begin{array}{l}370.91 \\
372.09 \\
372.21\end{array}$ \\
\hline $199-\mathrm{H} 4-14$ & $\begin{array}{l}10 / 05 / 93 \\
10 / 05 / 93 \\
11 / 16 / 93 \\
12 / 20 / 93\end{array}$ & $\begin{array}{l}45.90 \\
45.87 \\
45.96 \\
45.95\end{array}$ & $\begin{array}{l}374.69 \star \\
374.72 \\
374.63 \\
374.64\end{array}$ \\
\hline
\end{tabular}


Table 3-2. RCRA Water Level Measurement Report for the 183-H Solar Evaporation Basins, Fourth Quarter 1993. (sheet 2 of 4)

\begin{tabular}{|c|c|c|c|}
\hline Well & Date & $\begin{array}{l}\text { Depth to } \\
\text { water }(f t)\end{array}$ & $\begin{array}{l}\text { Water level } \\
\text { elevation } \\
\text { above msl (ft) }\end{array}$ \\
\hline \multicolumn{4}{|c|}{ Wells Monitoring the Top of the } \\
\hline $199-H 4-15 A$ & $\begin{array}{l}10 / 05 / 93 \\
10 / 05 / 93 \\
11 / 16 / 93 \\
12 / 20 / 93\end{array}$ & $\begin{array}{l}34.98 \\
34.94 \\
33.91 \\
33.91\end{array}$ & $\begin{array}{l}372.23 \star \\
372.27 \\
373.30 \\
373.30\end{array}$ \\
\hline $199-H 4-15 B$ & $\begin{array}{l}10 / 05 / 93 \\
11 / 16 / 93 \\
12 / 20 / 93\end{array}$ & $\begin{array}{l}34.65 \\
33.64 \\
33.62\end{array}$ & $\begin{array}{l}372.27 \\
373.28 \\
373.30\end{array}$ \\
\hline $199-H 4-16$ & $\begin{array}{l}10 / 05 / 93 \\
11 / 16 / 93 \\
12 / 20 / 93\end{array}$ & $\begin{array}{l}50.15 \\
50.16 \\
50.16\end{array}$ & $\begin{array}{l}374.08 \\
374.07 \\
374.07\end{array}$ \\
\hline $199-H 4-17$ & $\begin{array}{l}10 / 05 / 93 \\
10 / 06 / 93 \\
11 / 16 / 93 \\
12 / 20 / 93\end{array}$ & $\begin{array}{l}45.79 \\
45.80 \\
45.41 \\
45.37\end{array}$ & $\begin{array}{l}373.30 \\
373.29 \star \\
373.68 \\
373.72\end{array}$ \\
\hline 199-H4-18 & $\begin{array}{l}10 / 05 / 93 \\
10 / 06 / 93 \\
11 / 16 / 93 \\
12 / 20 / 93\end{array}$ & $\begin{array}{l}48.57 \\
48.57 \\
48.25 \\
48.13\end{array}$ & $\begin{array}{l}373.25 \\
373.25^{\star} \\
373.57 \\
373.69\end{array}$ \\
\hline 199-H4-3 & $\begin{array}{l}10 / 05 / 93 \\
10 / 06 / 93 \\
11 / 02 / 93 \\
11 / 16 / 93 \\
12 / 20 / 93\end{array}$ & $\begin{array}{l}47.23 \\
47.27 \\
47.05 \\
46.86 \\
46.72\end{array}$ & $\begin{array}{l}373.06 \\
373.02^{\star} \\
373.24^{\star} \\
373.43 \\
373.57\end{array}$ \\
\hline $199-\mathrm{H} 4-4$ & $\begin{array}{l}10 / 05 / 93 \\
10 / 06 / 93 \\
11 / 16 / 93 \\
11 / 16 / 93 \\
12 / 20 / 93\end{array}$ & $\begin{array}{l}41.95 \\
42.00 \\
40.88 \\
40.82 \\
40.75\end{array}$ & $\begin{array}{l}371.75 \\
371.70^{\star} \\
372.82 \\
372.88^{\star} \\
372.95\end{array}$ \\
\hline $199-\mathrm{H} 4-5$ & $\begin{array}{l}10 / 05 / 93 \\
10 / 06 / 93 \\
11 / 16 / 93 \\
12 / 20 / 93\end{array}$ & $\begin{array}{l}43.93 \\
43.97 \\
43.06 \\
42.98\end{array}$ & $\begin{array}{l}372.28 \\
372.24^{\star} \\
373.15 \\
373.23\end{array}$ \\
\hline
\end{tabular}


Table 3-2. RCRA Water Level Measurement Report for the 183-H Solar Evaporation Basins, Fourth Quarter 1993. (sheet 3 of 4)

\begin{tabular}{|c|c|c|c|}
\hline Well & Date & $\begin{array}{l}\text { Depth to } \\
\text { water (ft) }\end{array}$ & $\begin{array}{l}\text { Water level } \\
\text { elevation } \\
\text { above msl (ft) }\end{array}$ \\
\hline \multicolumn{4}{|c|}{ Wells Monitoring the Top of the } \\
\hline $199-\mathrm{H} 4-6$ & $\begin{array}{l}10 / 05 / 93 \\
10 / 06 / 93 \\
11 / 16 / 93 \\
12 / 20 / 93\end{array}$ & $\begin{array}{l}44.53 \\
44.50 \\
44.63 \\
44.65\end{array}$ & $\begin{array}{l}375.05 \\
375.08^{\star} \\
374.95 \\
374.93\end{array}$ \\
\hline $199-\mathrm{H} 4-7$ & $\begin{array}{l}10 / 05 / 93 \\
10 / 06 / 93 \\
11 / 16 / 93 \\
12 / 20 / 93\end{array}$ & $\begin{array}{l}46.53 \\
45.56 \\
46.45 \\
46.43\end{array}$ & $\begin{array}{l}374.06 \\
375.03^{\star}+ \\
374.14 \\
374.16\end{array}$ \\
\hline 199-H4-8 & $\begin{array}{l}10 / 05 / 93 \\
10 / 06 / 93 \\
11 / 16 / 93 \\
12 / 20 / 93\end{array}$ & $\begin{array}{l}46.52 \\
46.55 \\
46.22 \\
46.19\end{array}$ & $\begin{array}{l}373.48 \\
373.45 \star \\
373.78 \\
373.81\end{array}$ \\
\hline $199-\mathrm{H} 4-9$ & $\begin{array}{l}10 / 05 / 93 \\
11 / 02 / 93 \\
11 / 16 / 93 \\
12 / 20 / 93\end{array}$ & $\begin{array}{l}45.19 \\
44.73 \\
44.63 \\
44.54\end{array}$ & $\begin{array}{l}372.89 \\
373.35 * \\
373.45 \\
373.54\end{array}$ \\
\hline
\end{tabular}

Wells Monitoring Upper Levels in the Unconfined Aquifer (Ringold Formation)

\begin{tabular}{llll}
\hline $199-H 3-2 C$ & $10 / 05 / 93$ & 43.37 & 374.85 \\
& $11 / 16 / 93$ & 43.43 & 374.79 \\
$12 / 20 / 93$ & 43.41 & 374.81 \\
$199-H 4-12 C$ & $10 / 05 / 93$ & 41.74 & $371.78 *$ \\
& $10 / 05 / 93$ & 41.72 & 371.80 \\
& $11 / 16 / 93$ & 40.83 & 372.69 \\
& $12 / 20 / 93$ & 40.69 & 372.83 \\
\hline
\end{tabular}

Well Monitoring Mid-Level in the Unconfined Aquifer (Ringold Formation)

\begin{tabular}{llll}
\hline $199-H 4-15 C R$ & $10 / 05 / 93$ & 33.76 & 373.61 \\
& $11 / 16 / 93$ & 33.85 & 373.52 \\
& $12 / 20 / 93$ & 33.88 & 373.49
\end{tabular}


Table 3-2. RCRA Water Level Measurement Report for the 183-H Solar Evaporation Basins, Fourth Quarter 1993. (sheet 4 of 4)

\begin{tabular}{|c|c|c|c|}
\hline Well & Date & $\begin{array}{l}\text { Depth to } \\
\text { water }(f t)\end{array}$ & $\begin{array}{l}\text { Water level } \\
\text { elevation } \\
\text { above msl (ft) }\end{array}$ \\
\hline \multicolumn{4}{|c|}{$\begin{array}{l}\text { Well Monitoring Mid-Level in the Unconfined Aquifer } \\
\text { (Ringold Formation) }\end{array}$} \\
\hline $199-\mathrm{H} 4-15 \mathrm{CS}$ & $\begin{array}{l}10 / 05 / 93 \\
11 / 16 / 93 \\
12 / 20 / 93\end{array}$ & $\begin{array}{l}35.33 \\
34.34 \\
34.27\end{array}$ & $\begin{array}{l}372.11 \\
373.10 \\
373.17\end{array}$ \\
\hline
\end{tabular}

Well Monitoring Bottom of Uppermost Aquifer System (Ringold Formation)

\begin{tabular}{llll}
\hline $199-H 4-15 C Q$ & $11 / 16 / 93$ & -0.01 & 407.28 \\
\hline
\end{tabular}

Notes: 1. Water level elevations are calculated by subtracting the measured depth to water from the surveyed elevation for the well.

2. Depth-to-water values are transcribed from field records.

3. Elevations marked with an $1 * 1$ were measured at the time of sampling.

4. Elevations marked with a ' + ' are outside of the expected range and are suspected of error.

5. To convert feet to meters multiply by 0.3048 .

6. Negative depth-to-water value indicates flowing conditions and approximate water level elevation. 
Table 3-3. Constituent List and Summary of Results for 183-H Solar Evaporation Basins Data for Reporting Period October 1 through December 31, 1993.

(sheet 1 of 2)

CONTAMINATION INDICATOR PARAMETERS

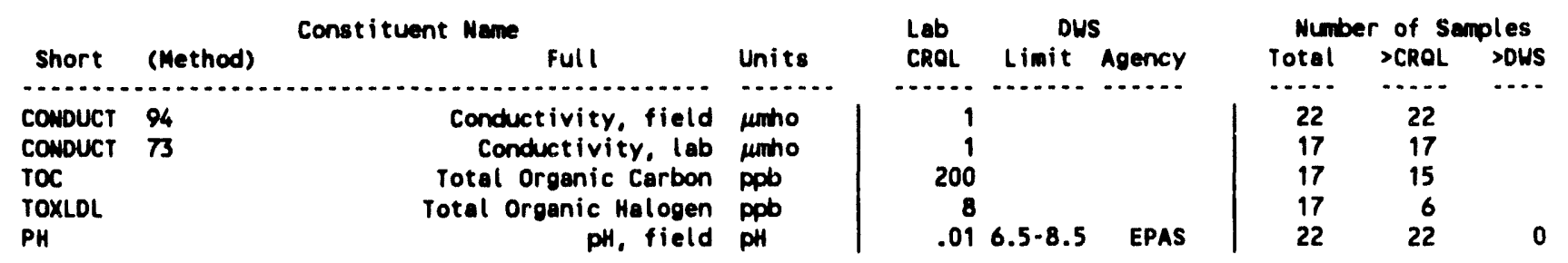

DRINKING WATER PARAMETERS

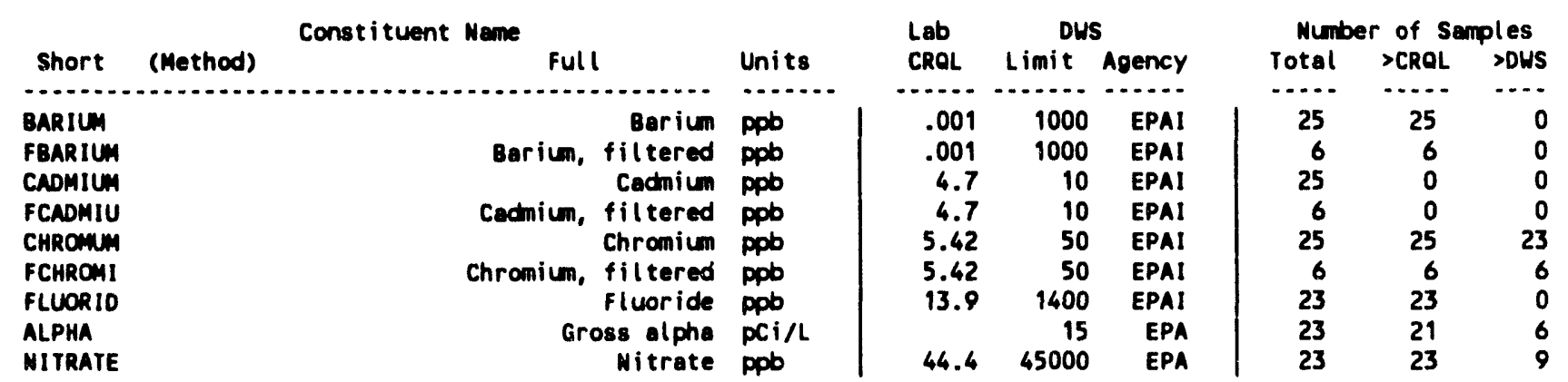

GROUNDWATER QUALITY PARAMETERS

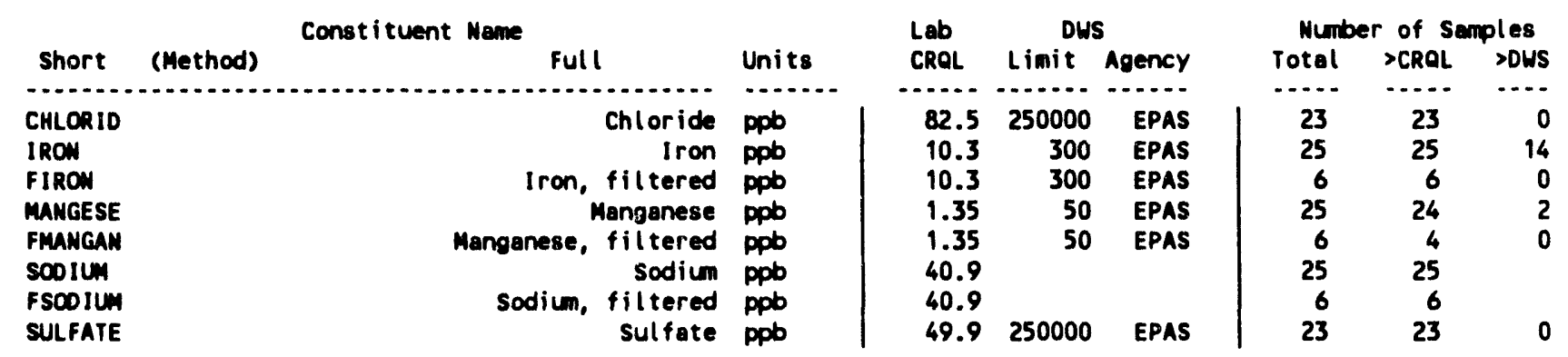

SITE SPECIFIC AND OTHER CONSTITUENTS

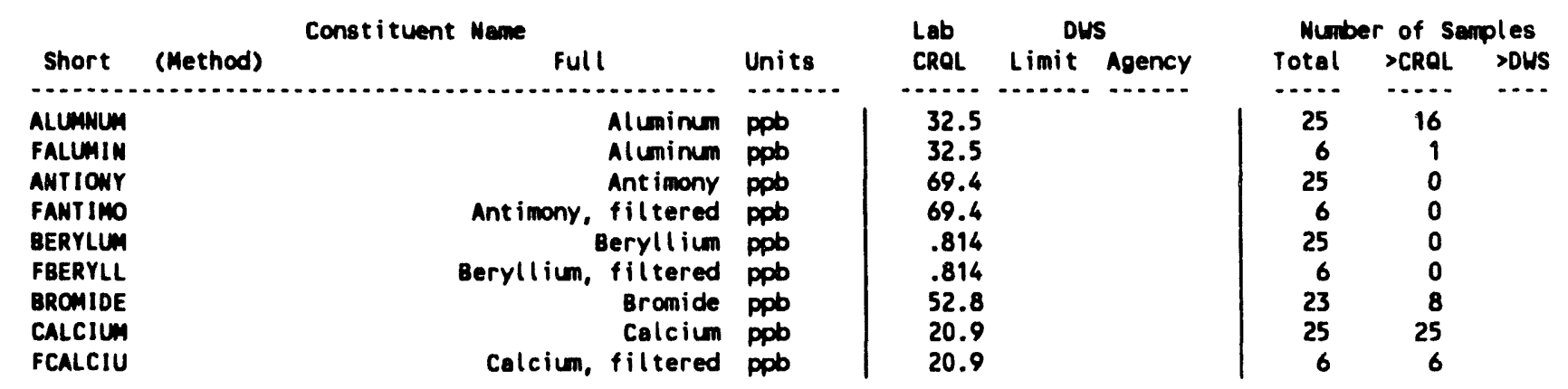


Table 3-3. Constituent List and Summary of Results for 183-H Solar Evaporation Basins Data for Reporting Period October 1 through December 31, 1993.

(sheet 2 of 2)

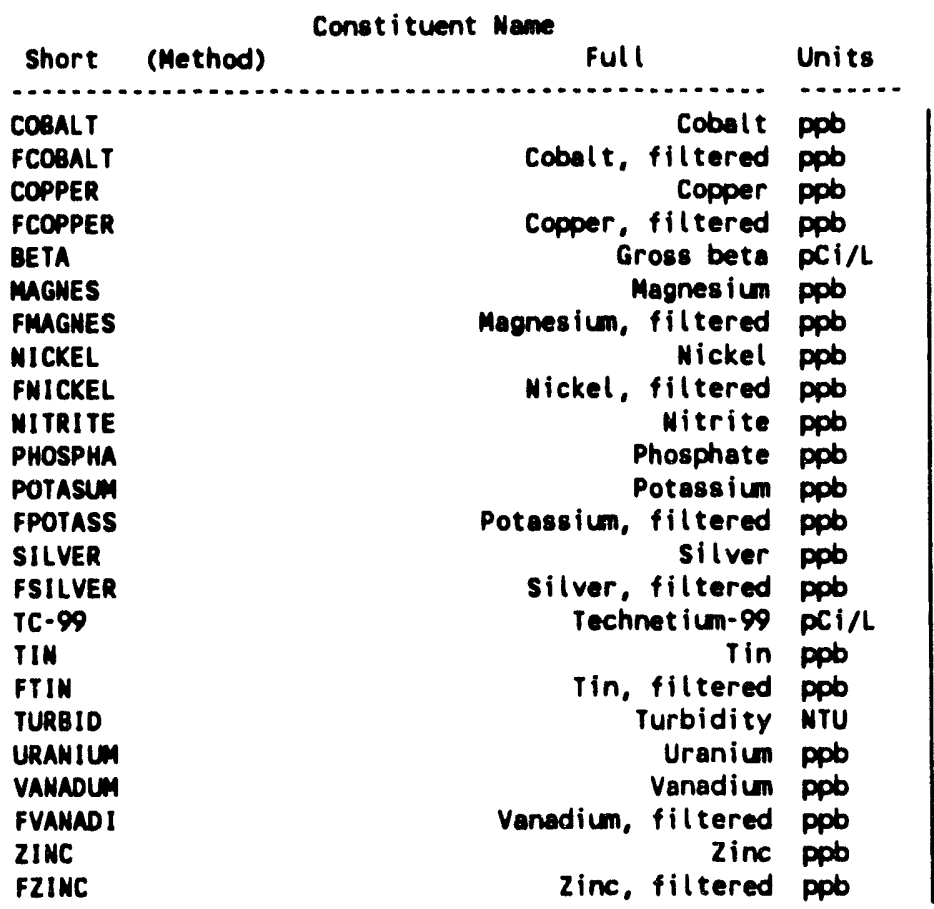

\begin{tabular}{|c|c|c|}
\hline Lab & OWS & \\
\hline CROL & Limit & Agency \\
\hline 4.05 & & \\
\hline 4.05 & & \\
\hline 2.65 & 1000 & $\begin{array}{l}\text { EPAS } \\
\text { FPAS }\end{array}$ \\
\hline 2.65 & 1000 & EPAS \\
\hline 26 & & \\
\hline 26 & & \\
\hline 17.9 & & \\
\hline 17.9 & & \\
\hline 38.3 & 1000 & EPA \\
\hline 662 & & \\
\hline 662 & & \\
\hline 2.87 & 50 & EPAI \\
\hline 2.87 & $\begin{array}{r}50 \\
900\end{array}$ & $\begin{array}{r}\text { EPAI } \\
\text { EPA }\end{array}$ \\
\hline 51.1 & & \\
\hline 51.1 & & \\
\hline .05 & & \\
\hline 3.84 & & \\
\hline $\begin{array}{l}3.84 \\
3.44\end{array}$ & 5000 & EPAS \\
\hline 3.44 & 5000 & EPAS \\
\hline
\end{tabular}

$\begin{array}{rrr}\begin{array}{c}\text { Mumber of Semples } \\ \text { Total }\end{array} & >\text { CRQL } & >\text { > W } \\ \cdots . . & \cdots \ldots & \cdots \\ 25 & 0 & \\ 6 & 0 & \\ 25 & 16 & 0 \\ 6 & 2 & 0 \\ 23 & 23 & \\ 25 & 25 & \\ 6 & 6 & \\ 25 & 10 & \\ 6 & 0 & \\ 23 & 1 & 0 \\ 23 & 0 & \\ 25 & 25 & \\ 6 & 6 & \\ 25 & 11 & 0 \\ 6 & 0 & 0 \\ 17 & 7 & 1 \\ 25 & 0 & \\ 6 & 0 & \\ 17 & 17 & \\ 17 & 17 & \\ 25 & 19 & \\ 6 & 1 & \\ 25 & 20 & 0 \\ 6 & 2 & 0\end{array}$

For explanation of this table, see Section 1.4 of report. 
Table 3-4. Constituents with at Least One Detected Value for the 183-H Solar Evaporation Basins Data for Reporting Period October 1 through December 31, 1993. (sheet 1 of 7)

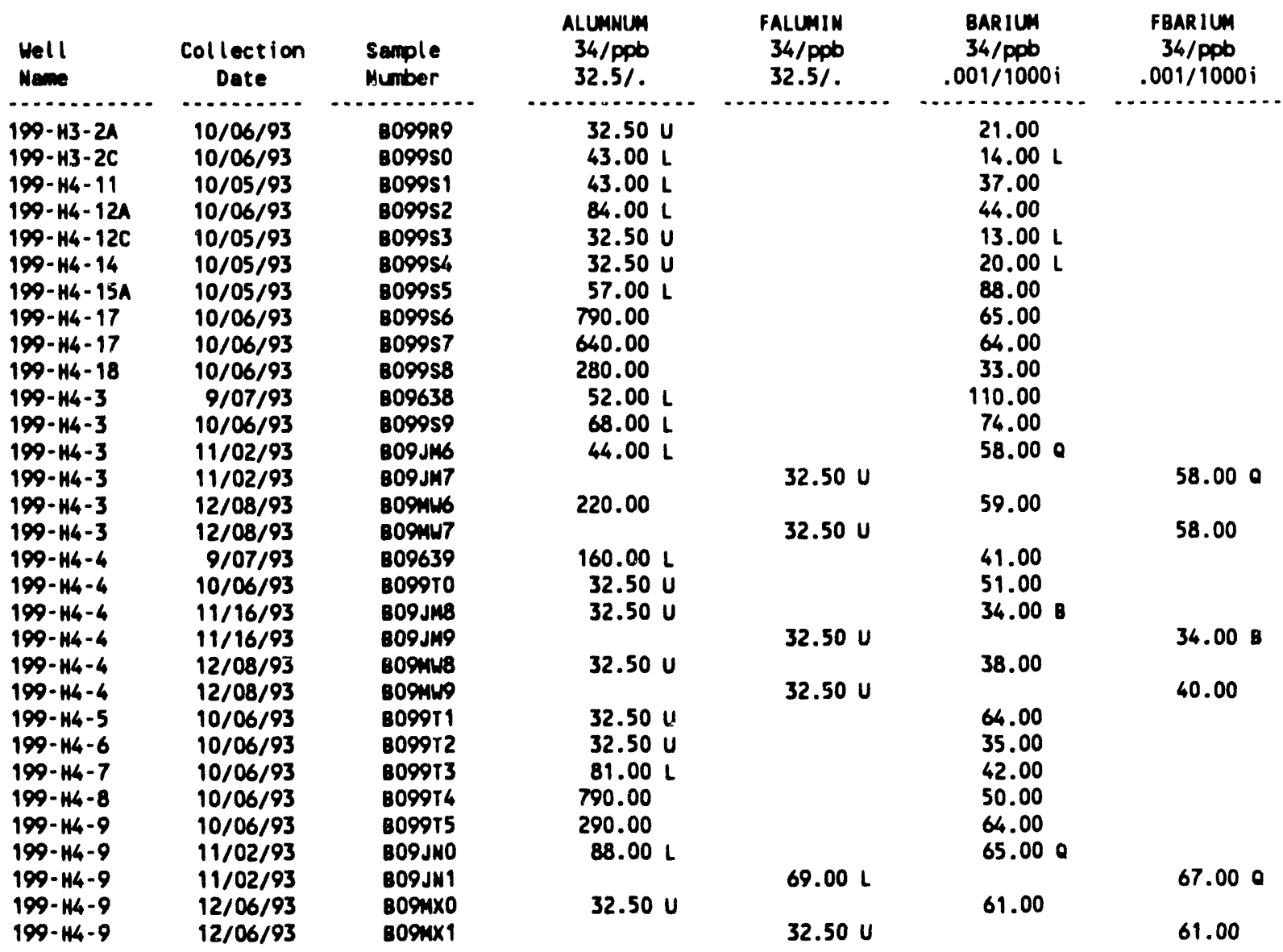

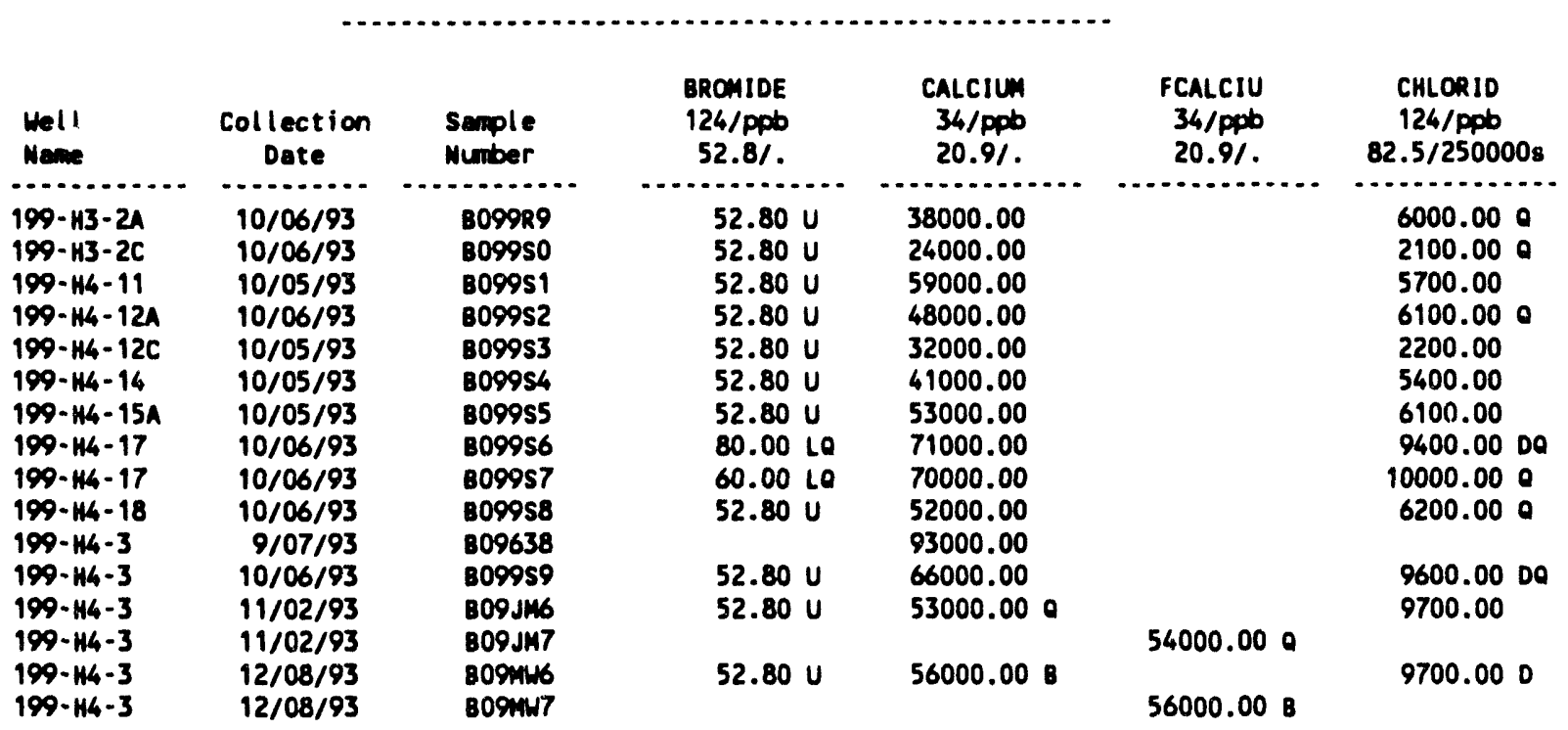


Table 3-4. Constituents with at Least One Detected Value for the 183-H Solar Evaporation Basins Data for Reporting Period October 1

through December 31, 1993. (sheet 2 of 7)

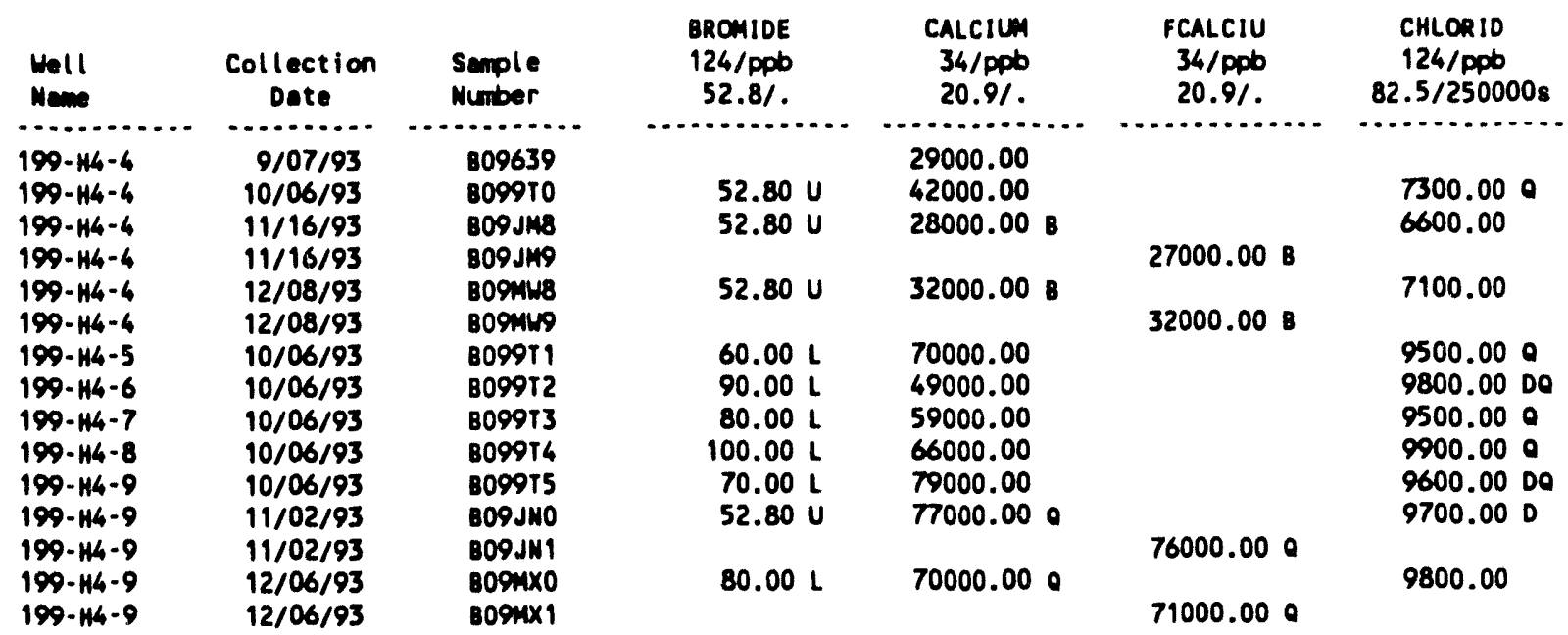

\begin{tabular}{|c|c|c|c|c|c|c|}
\hline $\begin{array}{l}\text { Hell } \\
\text { Neme }\end{array}$ & $\begin{array}{l}\text { Collection } \\
\text { Date }\end{array}$ & $\begin{array}{l}\text { Semple } \\
\text { Number }\end{array}$ & $\begin{array}{c}\text { CHROMum } \\
34 / \mathrm{ppb} \\
5.42 / 50 \mathrm{i}\end{array}$ & $\begin{array}{l}\text { FCHROOII } \\
34 / \mathrm{ppb} \\
5.42 / 50 \mathrm{i}\end{array}$ & $\begin{array}{c}\text { COPPER } \\
34 / \text { Ppb } \\
2.65 / 10008\end{array}$ & $\begin{array}{c}\text { FCOPPER } \\
34 / \mathrm{ppb} \\
2.65 / 1000 \mathrm{~s}\end{array}$ \\
\hline $\begin{array}{l}199-H 3-2 A \\
199-H 3-2 C \\
199-H 4-11 \\
199-114-12 A \\
199-144-12 C \\
199-H 4-14 \\
199-H 4-15 A \\
199-H 4-17 \\
199-H 4-17 \\
199-144-18 \\
199-H 4-3 \\
199-H 4-3 \\
199-H 4-3\end{array}$ & $\begin{array}{c}10 / 06 / 93 \\
10 / 06 / 93 \\
10 / 05 / 93 \\
10 / 06 / 93 \\
10 / 05 / 93 \\
10 / 05 / 93 \\
10 / 05 / 93 \\
10 / 06 / 93 \\
10 / 06 / 93 \\
10 / 06 / 93 \\
9 / 07 / 93 \\
10 / 06 / 93 \\
11 / 02 / 93\end{array}$ & $\begin{array}{l}8099 R 9 \\
809950 \\
809951 \\
800952 \\
809953 \\
809954 \\
B 09955 \\
B 09956 \\
809957 \\
809958 \\
809638 \\
\text { B09959 } \\
\text { B09 Jn6 }\end{array}$ & $\begin{array}{l}42.00 \\
23.00 \\
74.00 \\
54.00 \\
310.00 \\
160.00 \\
130.00 \\
440.00 \\
490.00 \\
270.00 \\
230.00 \\
190.00 \\
160.00\end{array}$ & & $\begin{array}{r}3.20 \mathrm{~L} \\
2.65 \mathrm{U} \\
2.65 \mathrm{U} \\
2.65 \mathrm{U} \\
3.10 \mathrm{~L} \\
3.60 \mathrm{~L} \\
2.65 \mathrm{U} \\
9.70 \mathrm{~L} \\
10.00 \mathrm{~L} \\
7.20 \mathrm{~L} \\
2.65 \mathrm{U} \\
18.00 \mathrm{~L} \\
7.00 \mathrm{~L}\end{array}$ & \\
\hline $199-H 4-3$ & $\begin{array}{l}11 / 02 / 93 \\
12 / 08 / 93\end{array}$ & $\begin{array}{l}\text { B09.JM7 } \\
\text { B094n6 }\end{array}$ & & 130.00 & & $11.00 \mathrm{~L}$ \\
\hline $\begin{array}{l}199-144-3 \\
199-14-3\end{array}$ & $\begin{array}{l}12 / 08 / 93 \\
12 / 08 / 93\end{array}$ & $\begin{array}{l}\text { BOshnt } \\
\text { Bosmin }\end{array}$ & 230.00 & 120.00 & $13.00 \mathrm{~L}$ & $5.40 \mathrm{~L}$ \\
\hline $\begin{array}{l}199-144-4 \\
199-144-4\end{array}$ & $\begin{array}{r}9 / 07 / 93 \\
10 / 06 / 93 \\
11 / 16 / 03\end{array}$ & $\begin{array}{l}809639 \\
\text { BO9910 } \\
\text { B09J48 }\end{array}$ & $\begin{array}{l}130.00 \\
120.00 \\
120.00\end{array}$ & & $\begin{array}{l}2.65 \mathrm{U} \\
4.00 \mathrm{~L} \\
4.70 \mathrm{~L}\end{array}$ & \\
\hline $\begin{array}{l}199-144-4 \\
199-144-4 \\
199-144-4\end{array}$ & $\begin{array}{l}11 / 16 / 93 \\
11 / 16 / 93 \\
12 / 08 / 93\end{array}$ & $\begin{array}{l}\text { BO9J JM9 } \\
\text { BO9MUB }\end{array}$ & 120.00 & 100.00 & $2.65 \mathrm{U}$ & $2.65 \mathrm{U}$ \\
\hline $\begin{array}{l}199-14-4 \\
199-44\end{array}$ & $12 / 08 / 93$ & Bosing & & 87.00 & & $2.65 \mathrm{U}$ \\
\hline $\begin{array}{l}199-H 4-5 \\
199-44-6\end{array}$ & $\begin{array}{l}10 / 06 / 93 \\
10 / 06 / 93\end{array}$ & $\begin{array}{l}\text { B09911 } \\
\text { B09912 }\end{array}$ & $\begin{array}{l}120.00 \\
190.00\end{array}$ & & $\begin{array}{l}2.80 \mathrm{~L} \\
5.20 \mathrm{~L}\end{array}$ & \\
\hline $\begin{array}{l}199-H 4-7 \\
199-H 4-8\end{array}$ & $\begin{array}{l}10 / 06 / 93 \\
10 / 06 / 93\end{array}$ & $\begin{array}{l}809913 \\
\text { B09914 }\end{array}$ & $\begin{array}{l}150.00 \\
380.00\end{array}$ & & $\begin{array}{l}2.65 \mathrm{U} \\
8.90 \mathrm{~L}\end{array}$ & \\
\hline $199-44-9$ & $10 / 06 / 93$ & BO9915 & $\begin{array}{l}380.00 \\
430.00\end{array}$ & & $8.40 \mathrm{~L}$ & \\
\hline $199-144-9$ & $11 / 02 / 93$ & B09JHO & 120.00 & & $3.50 \mathrm{~L}$ & \\
\hline $\begin{array}{l}199-H 4-9 \\
199-H 4-9\end{array}$ & $\begin{array}{l}11 / 02 / 93 \\
12 / 06 / 93\end{array}$ & $\begin{array}{l}\text { B09 JN1 } \\
\text { 8094X0 }\end{array}$ & 99.00 & 89.00 & $2.65 \mathrm{U}$ & $2.65 \mathrm{U}$ \\
\hline $199-114-9$ & $12 / 06 / 93$ & Bo991X1 & & 92.00 & & $2.65 \mathrm{U}$ \\
\hline
\end{tabular}


Table 3-4. Constituents with at Least One Detected Value for the 183-H Solar Evaporation Basins Data for Reporting Period October 1

through December 31, 1993. (sheet 3 of 7)

\begin{tabular}{|c|c|c|c|c|c|c|}
\hline $\begin{array}{l}\text { Well } \\
\text { Mame }\end{array}$ & $\begin{array}{c}\text { Collection } \\
\text { Date }\end{array}$ & $\begin{array}{l}\text { Semple } \\
\text { Number }\end{array}$ & $\begin{array}{c}\text { FLUORID } \\
124 / \mathrm{ppb} \\
13.9 / 1400 \mathrm{i}\end{array}$ & $\begin{array}{c}\text { ALPHA } \\
135 / \mathrm{pCi} / \mathrm{L} \\
. / 15\end{array}$ & $\begin{array}{c}\text { BETA } \\
136 / \mathrm{pCI} / \mathrm{L} \\
. / .\end{array}$ & $\begin{array}{c}\text { IROW } \\
34 / \mathrm{ppb} \\
10.3 / 3008\end{array}$ \\
\hline $\begin{array}{l}199-H 3-2 A \\
199-H 3-2 C \\
199-H 4-11 \\
199-H 4-12 A \\
199-H 4-12 C \\
199-H 4-14 \\
199-H 4-15 A \\
199-H 4-17 \\
199-H 4-17 \\
199-H 4-18 \\
199-H 4-3 \\
199-H 4-3 \\
199-H 4-3 \\
199-H 4-3 \\
199-H 4-4 \\
199-H 4-4 \\
199-H 4-4 \\
199-H 4-4 \\
199-H 4-5 \\
199-H 4-6 \\
199-H 4-7 \\
199-H 4-8 \\
199-H 4-9 \\
199-H 4-9 \\
199-H 4-9\end{array}$ & $\begin{array}{l}10 / 06 / 93 \\
10 / 06 / 93 \\
10 / 05 / 93 \\
10 / 06 / 93 \\
10 / 05 / 93 \\
10 / 05 / 93 \\
10 / 05 / 93 \\
10 / 06 / 93 \\
10 / 06 / 93 \\
10 / 06 / 93 \\
9 / 07 / 93 \\
10 / 06 / 93 \\
11 / 02 / 93 \\
12 / 08 / 93 \\
9 / 07 / 93 \\
10 / 06 / 93 \\
11 / 16 / 93 \\
12 / 08 / 93 \\
10 / 06 / 93 \\
10 / 06 / 93 \\
10 / 06 / 93 \\
10 / 06 / 93 \\
10 / 06 / 93 \\
11 / 02 / 93 \\
12 / 06 / 93\end{array}$ & 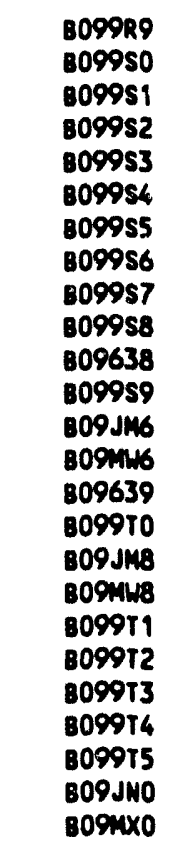 & $\begin{array}{l}400.00 \\
500.00 \\
200.00 \\
400.00 \\
200.00 \\
200.00 \\
200.00 \\
600.00 \\
300.00 \\
300.00 \\
\\
1300.00 \\
1100.00 \\
1200.00 \\
\\
800.00 \\
400.00 \\
600.00 \\
700.00 \\
500.00 \\
400.00 \\
300.00 \\
600.00 \\
500.00 \\
500.00\end{array}$ & $\begin{array}{c}1.09 \\
.73 u \\
3.23 \\
8.38 \\
2.35 \\
2.07 \\
1.85 \\
3.26 \\
4.17 \\
5.10 \\
\\
201.00 \\
64.80 \\
128.00 \\
\\
31.10 \\
32.20 \\
30.10 \\
2.87 \\
1.85 \\
1.35 u \\
5.29 \\
\because .10 \\
8.94 \\
8.19\end{array}$ & $\begin{array}{c}3.92 \\
4.11 \\
42.30 \\
25.20 \\
12.10 \\
4.81 \\
5.28 \\
10.800 \\
8.40 \\
13.20 \\
\\
352.00 \\
384.00 \\
392.00 \\
\\
199.00 \\
106.00 \\
156.00 \\
6.08 \\
5.31 \\
5.02 \\
6.30 \\
40.90 \\
249.00 \\
51.70\end{array}$ & $\begin{array}{c}89.00 \\
81.00 \\
120.00 \\
180.00 \\
110.00 \\
110.00 \\
160.00 \\
2400.00 \\
2500.00 \\
1000.00 \\
2800.00 \\
880.00 \\
620.00 \\
1600.00 \\
1600.00 \\
170.00 \\
300.00 \\
720.00 \\
470.00 \\
670.00 \\
370.00 \\
2400.00 \\
1800.00 \\
230.00 \\
63.00\end{array}$ \\
\hline $\begin{array}{l}\text { Hell } \\
\text { Nene }\end{array}$ & $\begin{array}{c}\text { Collection } \\
\text { Date }\end{array}$ & $\begin{array}{l}\text { Semple } \\
\text { Number }\end{array}$ & $\begin{array}{c}\text { FIROW } \\
\text { 34/ppb } \\
10.3 / 300 \mathrm{~s}\end{array}$ & $\begin{array}{c}\text { MAGNES } \\
34 / \text { Ppb } \\
26 \% .\end{array}$ & $\begin{array}{c}\text { FMAGNES } \\
34 / \text { ppb } \\
26 / .\end{array}$ & $\begin{array}{l}\text { MANGESE } \\
34 / \mathrm{ppb} \\
1.35 / 50 \mathrm{~s}\end{array}$ \\
\hline $\begin{array}{l}199-H 3-2 A \\
199-H 3-2 C \\
199-H 4-11 \\
199-H 4-12 A \\
199-H 4-12 C \\
199-H 4-14 \\
199-H 4-15 A \\
199-H 4-17 \\
199-H 4-17 \\
199-H 4-18 \\
199-H 4-3 \\
199-H 4-3 \\
199-H 4-3\end{array}$ & $\begin{array}{l}10 / 06 / 93 \\
10 / 06 / 93 \\
10 / 05 / 93 \\
10 / 06 / 93 \\
10 / 05 / 93 \\
10 / 05 / 93 \\
10 / 05 / 93 \\
10 / 06 / 93 \\
10 / 06 / 93 \\
10 / 06 / 93 \\
9 / 07 / 93 \\
10 / 06 / 93 \\
11 / 02 / 93\end{array}$ & $\begin{array}{l}\text { B099R9 } \\
\text { B099SO } \\
\text { B099S1 } \\
\text { B099S2 } \\
\text { B099S3 } \\
\text { B099S4 } \\
\text { B099S5 } \\
\text { B099S6 } \\
\text { B099S7 } \\
\text { B099S8 } \\
\text { B09638 } \\
\text { B099S9 } \\
\text { B09JM6 }\end{array}$ & $\ldots$ & $\begin{array}{r}8100.00 \\
8000.00 \\
8200.00 \\
7200.00 \\
11000.00 \\
7600.00 \\
10000.00 \\
13000.00 \\
13000.00 \\
8700.00 \\
17000.00 \\
11000.00 \\
9400.00\end{array}$ & $\cdots$ & $\begin{array}{r}2.20 \mathrm{~L} \\
2.60 \mathrm{~L} \\
2.60 \mathrm{~L} \\
5.20 \mathrm{~L} \\
2.20 \mathrm{~L} \\
2.60 \mathrm{~L} \\
1.80 \mathrm{~L} \\
50.00 \\
50.00 \\
21.00 \\
100.00 \\
18.00 \\
26.00\end{array}$ \\
\hline $\begin{array}{l}199-114-3 \\
199-144-3 \\
199-114-3\end{array}$ & $\begin{array}{l}11 / 02 / 93 \\
12 / 08 / 93 \\
12 / 08 / 93\end{array}$ & $\begin{array}{l}\text { B09JM7 } \\
\text { B09MW6 } \\
\text { B09M47 }\end{array}$ & $\begin{array}{l}28.00 \\
14.00 \mathrm{~L}\end{array}$ & 10000.00 & $\begin{array}{r}9800.00 \\
10000.00\end{array}$ & 30.00 \\
\hline $\begin{array}{l}199-\mathrm{H4}-4 \\
199-\mathrm{H4}-4 \\
199-\mathrm{H4}-4 \\
199-\mathrm{H4}-4 \\
199-\mathrm{H4}-4 \\
199-\mathrm{H4}-4\end{array}$ & $\begin{array}{r}9 / 07 / 93 \\
10 / 06 / 93 \\
11 / 16 / 93 \\
11 / 16 / 93 \\
12 / 08 / 93 \\
12 / 08 / 93\end{array}$ & $\begin{array}{l}\text { B09639 } \\
\text { B09910 } \\
\text { B09JM8 } \\
\text { B09JM9 } \\
\text { B09MU8 } \\
\text { B09MU9 }\end{array}$ & $\begin{array}{l}210.00 \mathrm{~B} \\
21.00\end{array}$ & $\begin{array}{l}4900.00 \\
6500.00 \\
4700.00 \\
5100.00\end{array}$ & $\begin{array}{l}4400.00 \\
5200.00\end{array}$ & $\begin{array}{l}27.00 \\
3.10 \mathrm{~L} \\
4.10 \mathrm{~L} \\
5.60 \mathrm{~L}\end{array}$ \\
\hline
\end{tabular}


Table 3-4. Constituents with at Least One Detected Value for the 183-H Solar Evaporation Basins Data for Reporting Period October 1

through December 31, 1993. (sheet 4 of 7)

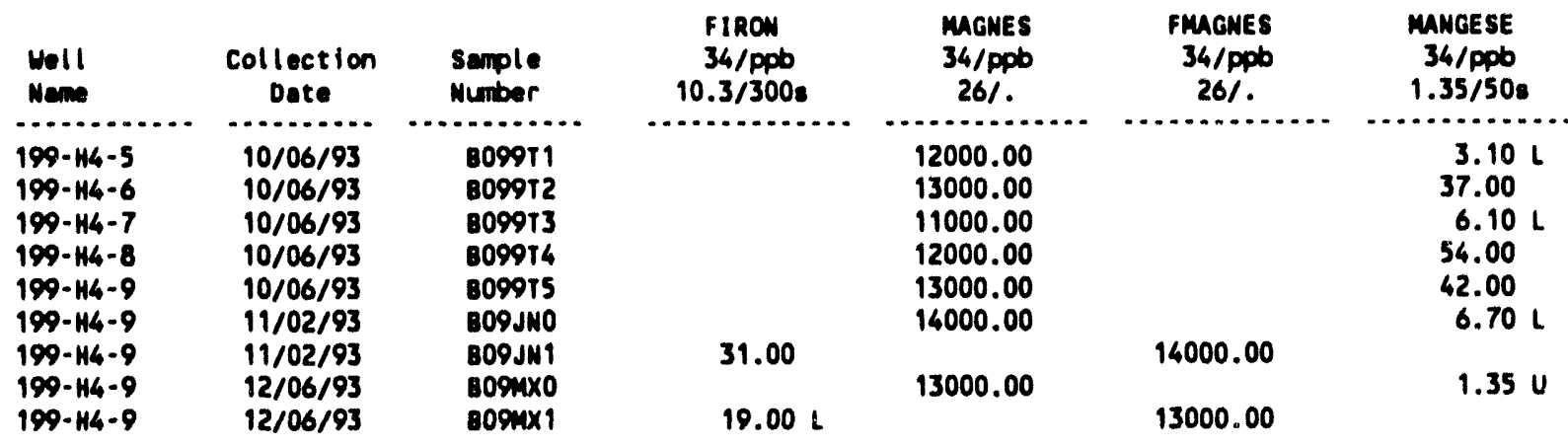

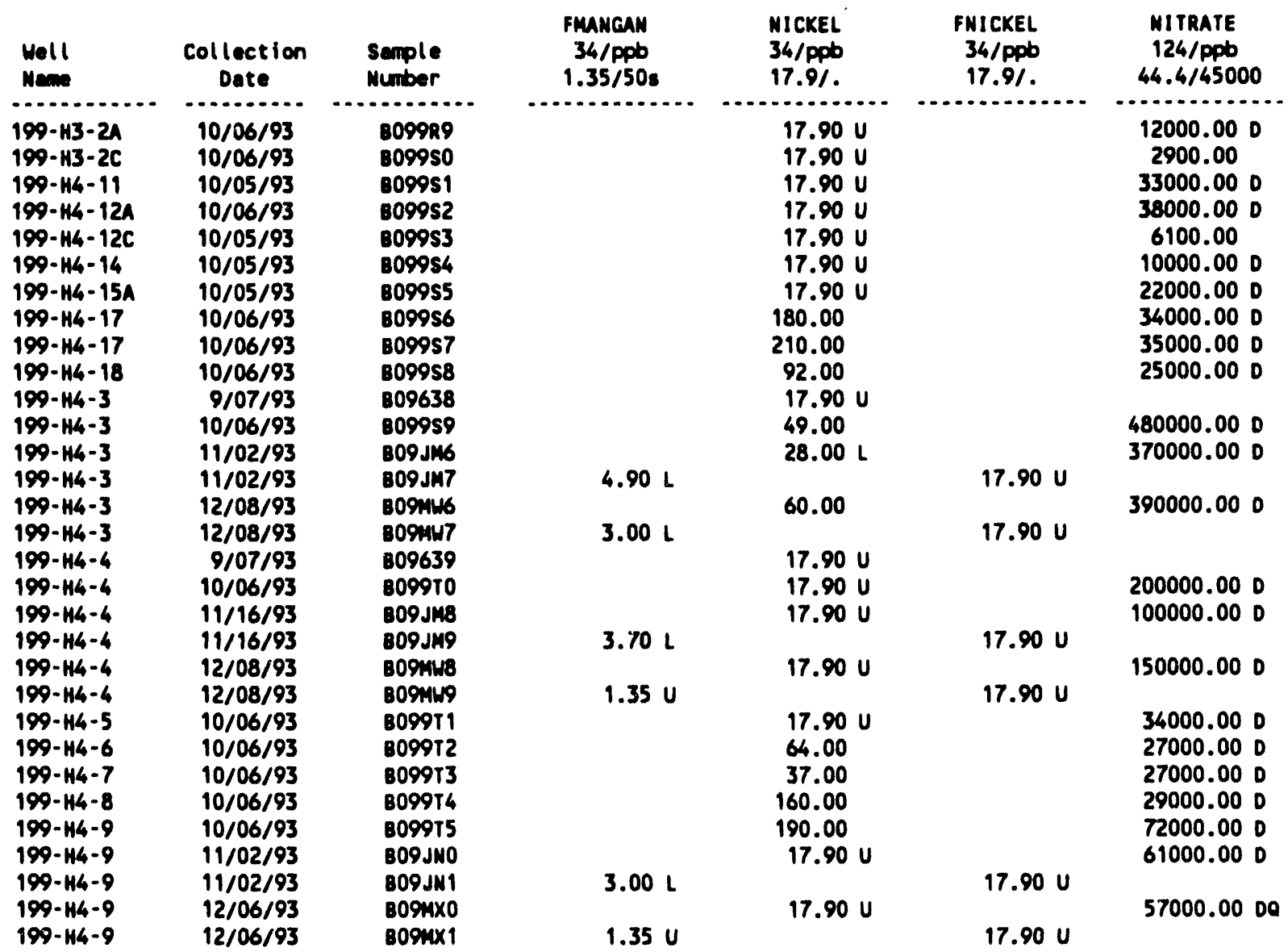


Table 3-4. Constituents with at Least One Detected Value for the 183-H Solar Evaporation Basins Data for Reporting Period October 1 through December 31, 1993. (sheet 5 of 7)

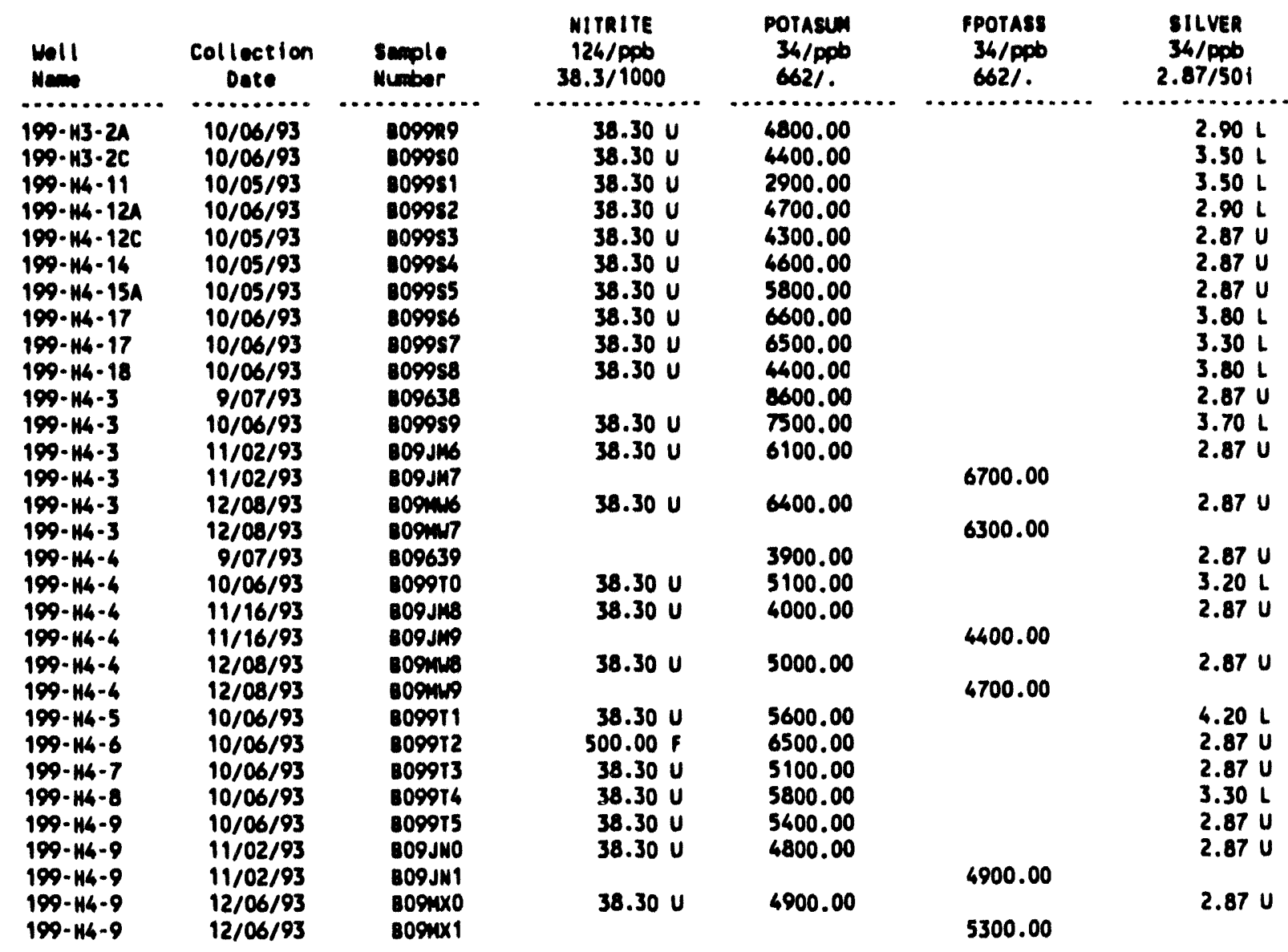

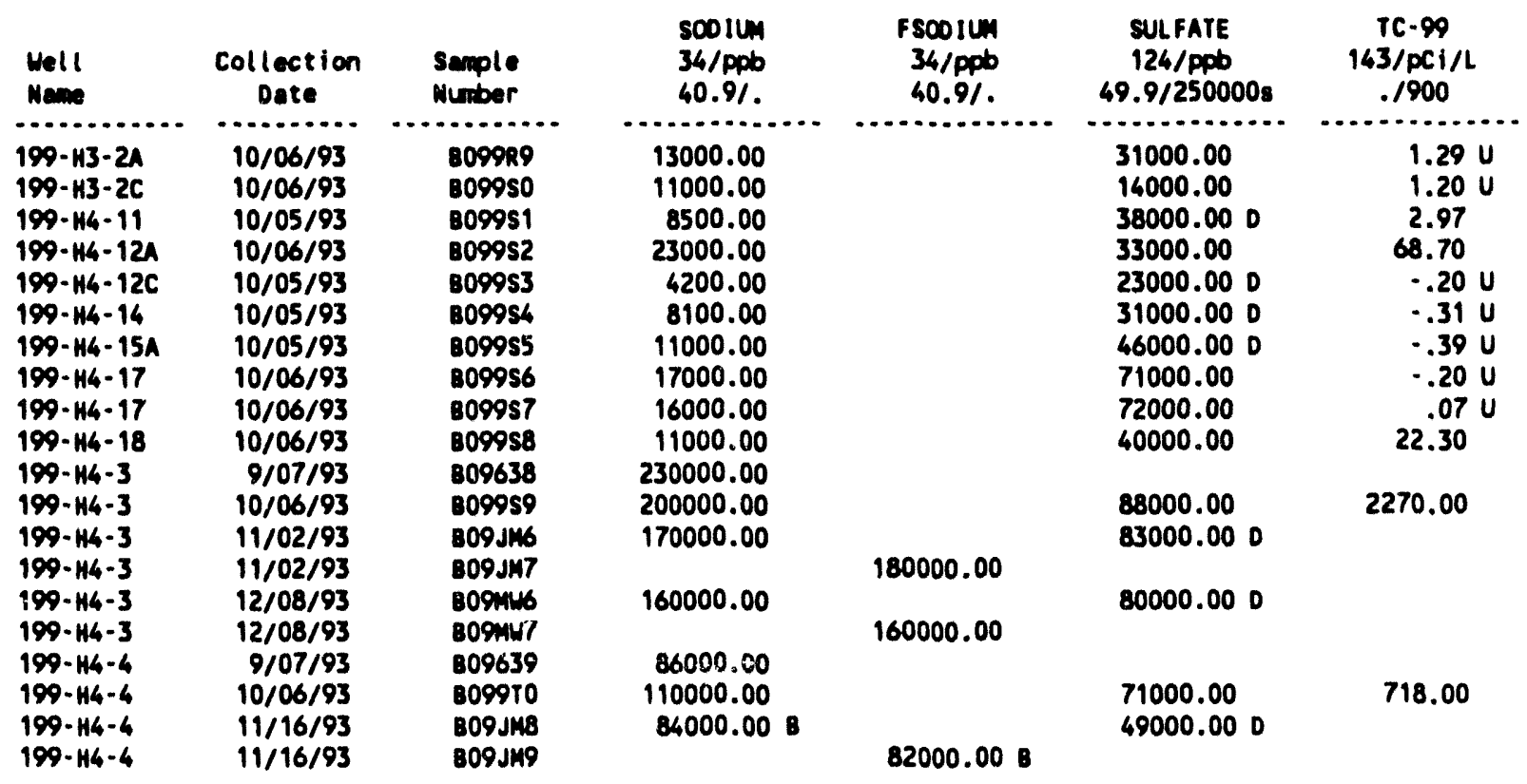


Table 3-4. Constituents with at Least One Detected Value for the 183-H Solar Evaporation Basins Data for Reporting Perlod October 1

through December 31, 1993. (sheet 6 of 7)

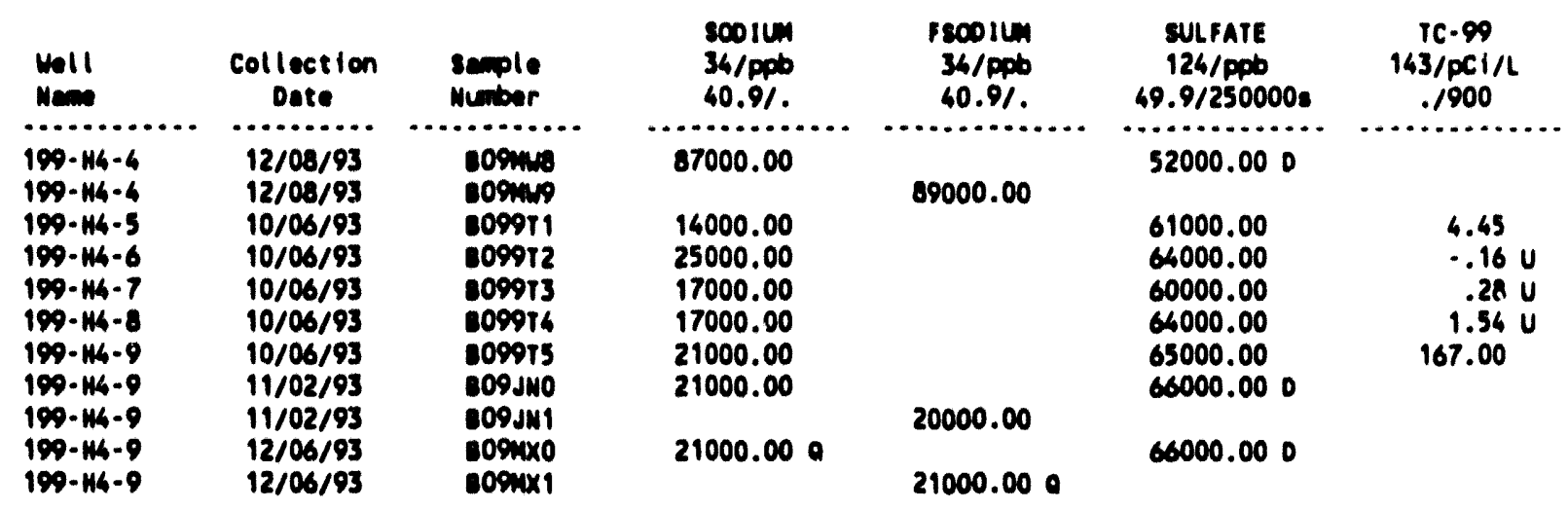

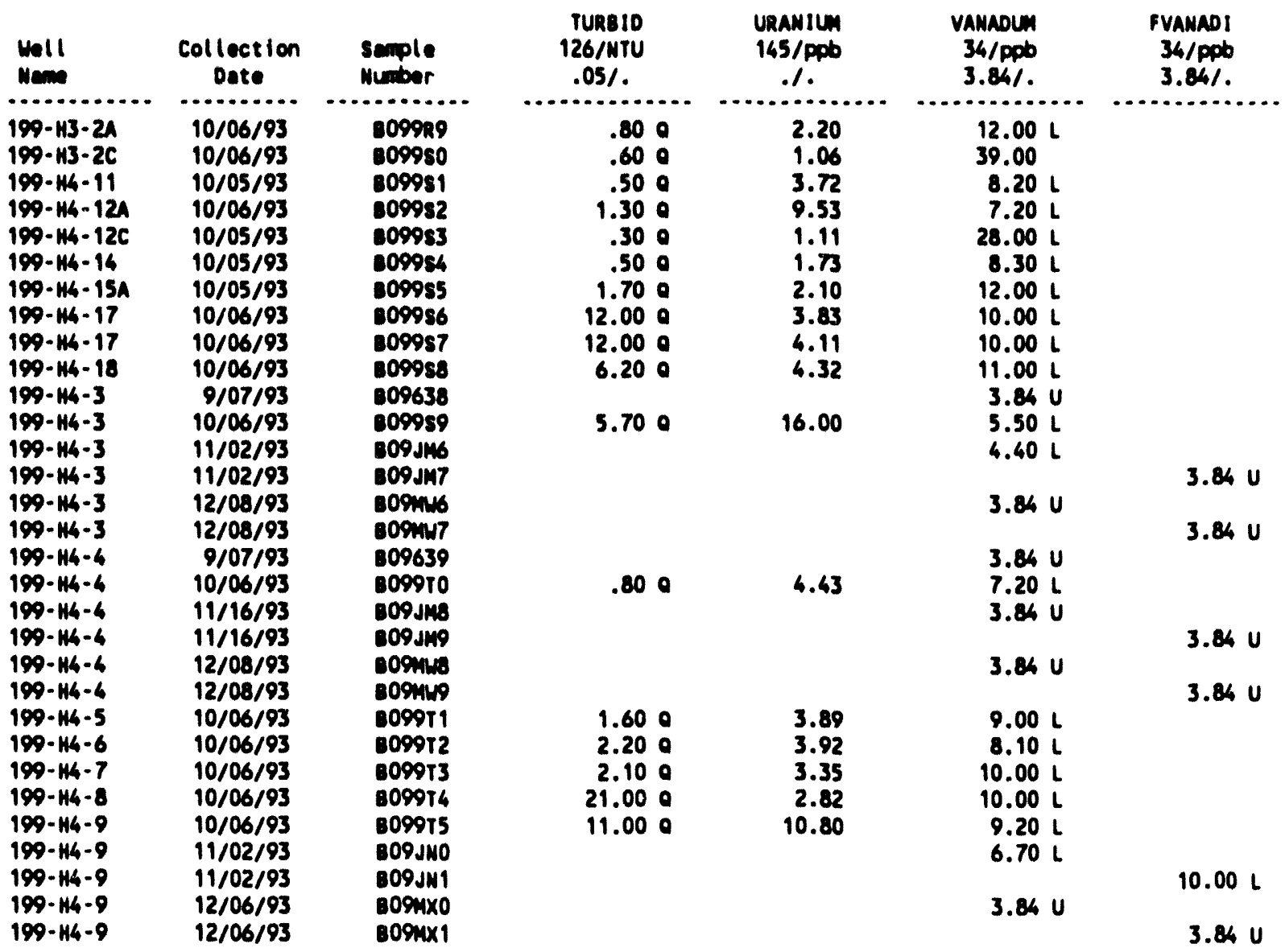


Table 3-4. Constituents with at Least One Detected Value for the 183-H Solar Evaporation Basins Data for Reporting Period October 1

through December 31, 1993. (sheet 7 of 7)

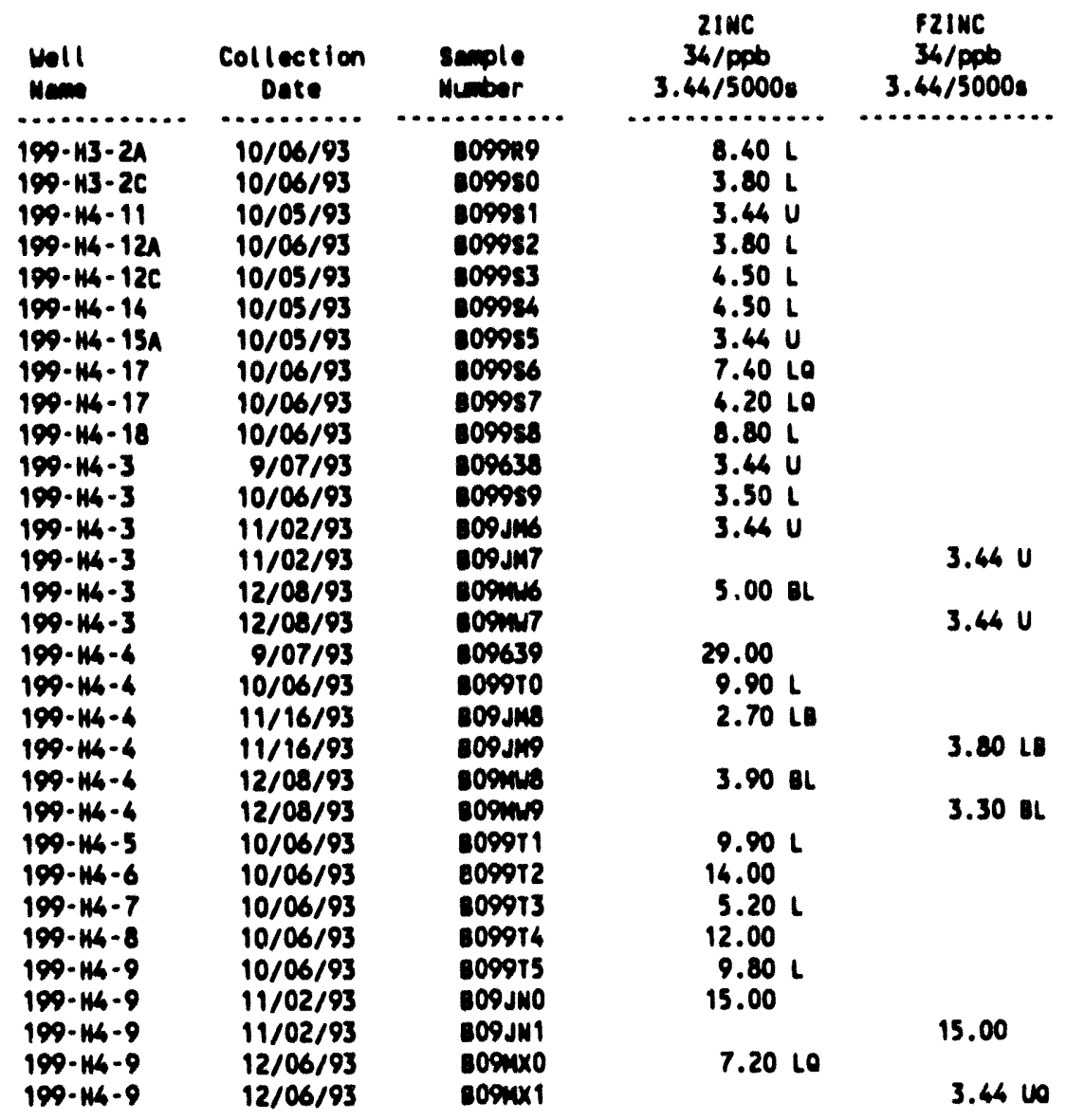

For explenation of this table, see section 1.4 of report. 
Table 3-5. Contamination Indicator Parameters for the 183-H Solar Evaporation Basins Data for Reporting Period October 1 through December 31, 1993.

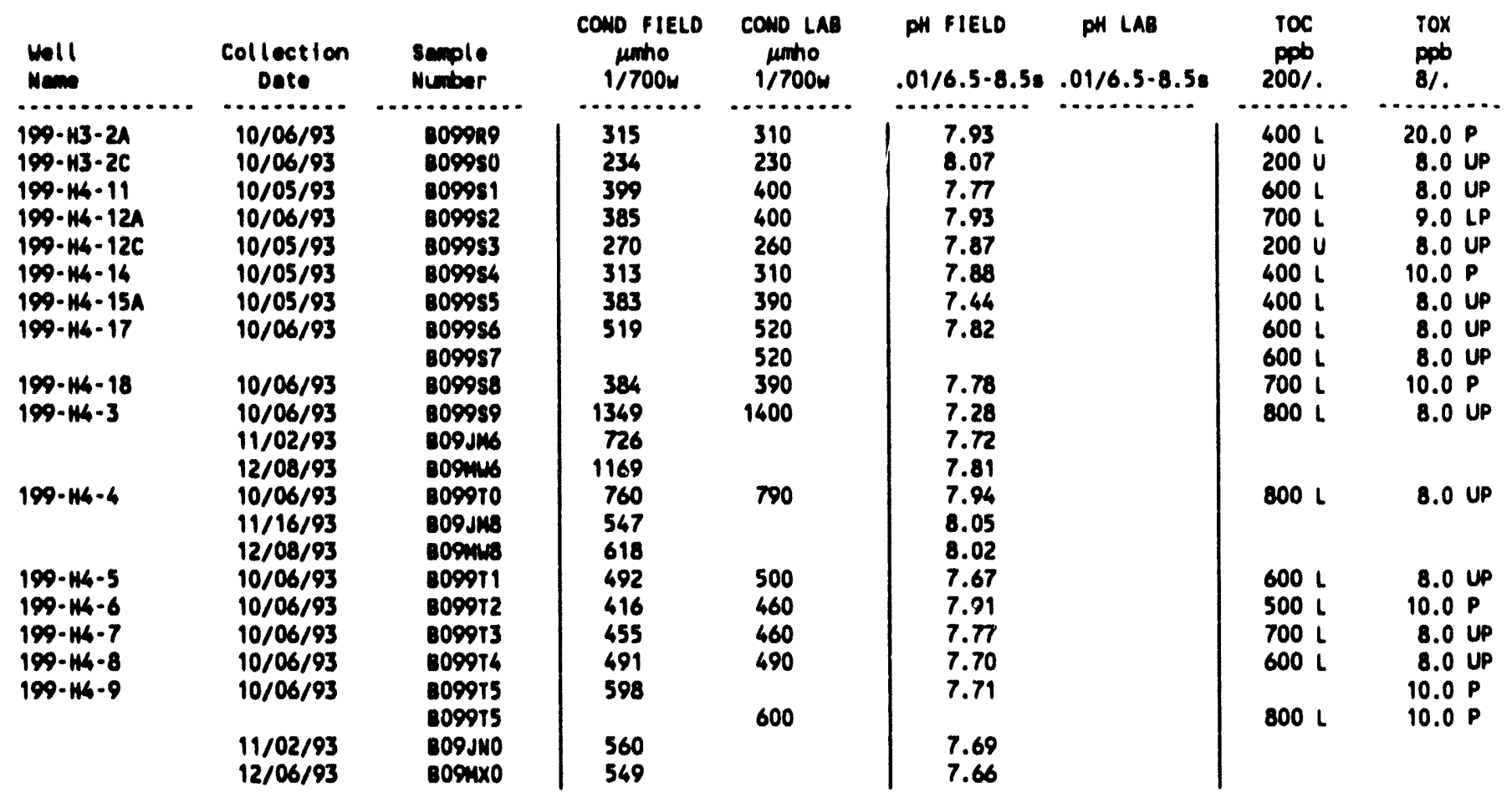

For explenation of this teble, see section 1.4 of report. 
DOE/RL-93-56-4

This page intentionally left blank. 
DOE/RL-93-56-4

\section{CONTENTS}

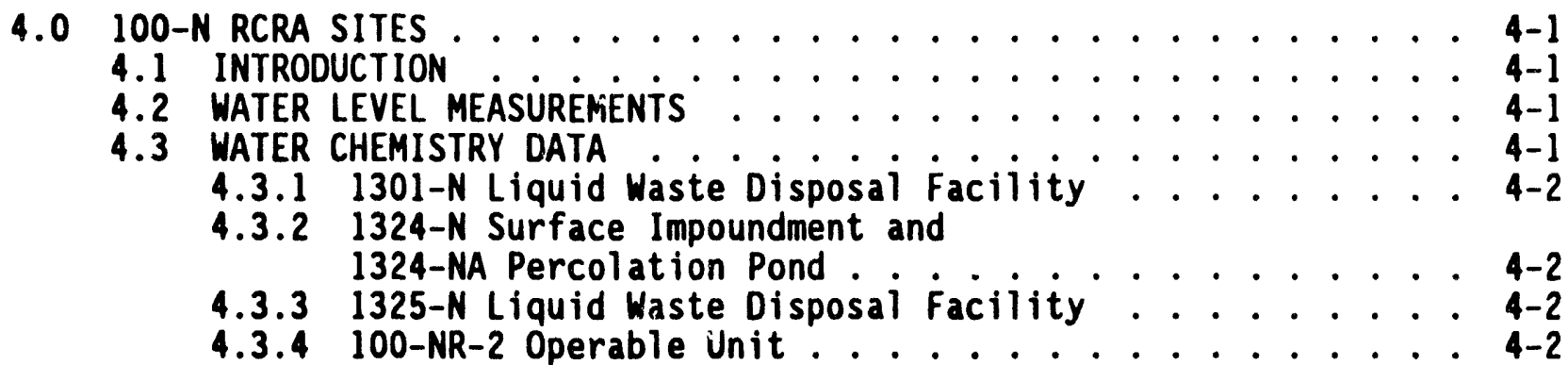


DOE/RL-93-56-4

\section{LIST OF FIGURES}

4-1 Monitoring Well Locations for 1301-N, 1324-N/NA, and $1325-N$ Facilities ............... . . . . . . . .

\section{LIST OF TABLES}

4-1 Monitoring Well Purpose and Sampling Schedule in the 100-N Area . . 4-4

4-2 RCRA Water Level Measurement Report for the 100-N Area, Fourth Quarter $1993 \ldots$. . . . . . . . . . . 4-6

4-3 Constituent List and Summary of Results for 100-N RCRA Sites Data for Reporting Period October 1 through December 31, 1993 . . . 4-12

4-4 Constituents with at Least One Detected Value for the 100-N RCRA Sites Data for Reporting Period October 1 through December 31,1993 ............... . . 4-15

4-5 Contamination Indicator Parameters for the 100-N RCRA Sites Data for Reporting Period October 1 through December 31, 1993 . . . 4-26

4-6 Drinking Water Standards Exceeded in 100-N Area RCRA Sites . . . 4-28 


\title{
4.0 100-N RCRA SITES
}

\author{
M. J. Hartman \\ Westinghouse Hanford Company
}

\subsection{INTRODUCTION}

Four interim-status RCRA disposal units are located in the 100-N Area of the Hanford Site: (1) the 1301-N Liquid Waste Disposal Facility (LWDF), (2) the 1324-N Surface Impoundment, (3) the 1324-NA Percolation Pond, and (4) the 1325-N LWDF (Figure 4-1). The 1324-N and 1324-NA units are monitored as a single site. RCRA monitoring began in late 1987. The 1324-N/NA site has been monitored under a groundwater quality assessment program since 1989 (40 CFR 265.93). The 1325-N site is monitored under an indicator evaluation program (40 CFR 265.92). Background concentrations are being reestablished for the 1301-N LWDF.

The 1301-N and 1325-N units were used for disposal of liquid effluent from the $N$ Reactor and associated facilities. Primary waste constituents were radionuclides, including strontium-90, tritium, and cobalt-60. Minor amounts of hazardous waste also were present in the waste streams (e.g., hydrazine). The 1324-N/NA units received corrosive waste. The 100-N RCRA units are currently not in use.

Data from the 100-NR-2 groundwater operable unit monitoring program are included in the RCRA quarterly report. Sampling is coordinated between RCRA and 100-NR-2 to avoid redundancy. Sampling, analysis, data validation, and verification are done according to the same protocols as RCRA.

Well locations in the 100-N Area are illustrated in Figure 4-1. Many of these wells are monitored monthly for water levels. Fewer wells are used for groundwater sampling. Sampling networks are listed in Table 4-1.

\subsection{HATER LEVEL MEASUREMENTS}

Water levels are measured monthly in most of the 100-N Area wells. Data are listed in Table 4-2. Measurements made during sampling in wells $\mathrm{N}-2$ and $\mathrm{N}-3$ are outside the expected range and are suspected errors. The December measurement in we $11 \mathrm{~N}-76$ showed a decrease from November; nearby wells increased. The measurement of $\mathrm{N}-76$ is a suspected error.

\subsection{WATER CHEMISTRY DATA}

This section includes a summary of sampling and analysis activities in the 100-N Area. Groundwater chemistry data are listed in Tables 4-3 through 4-5. The tables include data from samples collected in previous quarters that were not presented in the last quarterly report. Data flags are discussed in Sections 1.2 and 1.4. All D flags in this quarter's data indicate that samples were diluted for analysis. DWSs were exceecied for the constituents listed in Table 4-6. 


\subsubsection{1-N Liquid Waste Disposal Facility}

All of the wells in the 1301-N network were scheduled for sampling during the past quarter. Wells N-57 and N-67 were sampled with a bailer because of low water levels. The bailed wells were not purged and no filtered samples were collected.

Wells N-57 and N-67 had excessively high turbidity ( 820 and 990 nephelometric turbidity units [NTU], respectively). Other constituents in these wells (e.g., metals and radionuclides) were also elevated, and the samples are believed to be nonrepresentative of groundwater quality. Two of four values for laboratory $\mathrm{pH}$ in well $\mathrm{N}-14$ were anomalously low. Strontium-90 in well $\mathrm{N}-3$ was much higher than historical data and did not agree with gross beta activity. Data evaluations have been requested and the results are flagged with an ' $F$.'

\subsubsection{4-N Surface Impoundment and 1324-NA Percolation Pond}

The assessment monitoring program at the 1324-N/NA site has been revised (Hartman 1993). The wells in the revised network were sampled as scheduled during the last quarter.

\subsubsection{5-N Liquid Waste Disposal Facility}

Most of the 1325-N monitoring wells are sampled semiannually. Only the newest we11, $N-81$, was sampled during the last quarter.

\subsubsection{0-NR-2 Operable Unit}

The 100-NR-2 wells are sampled semiannually. The only wells in the 100-NR-2 network that were sampled during the quarter were wells that are also in RCRA networks. Those data are discussed above. 


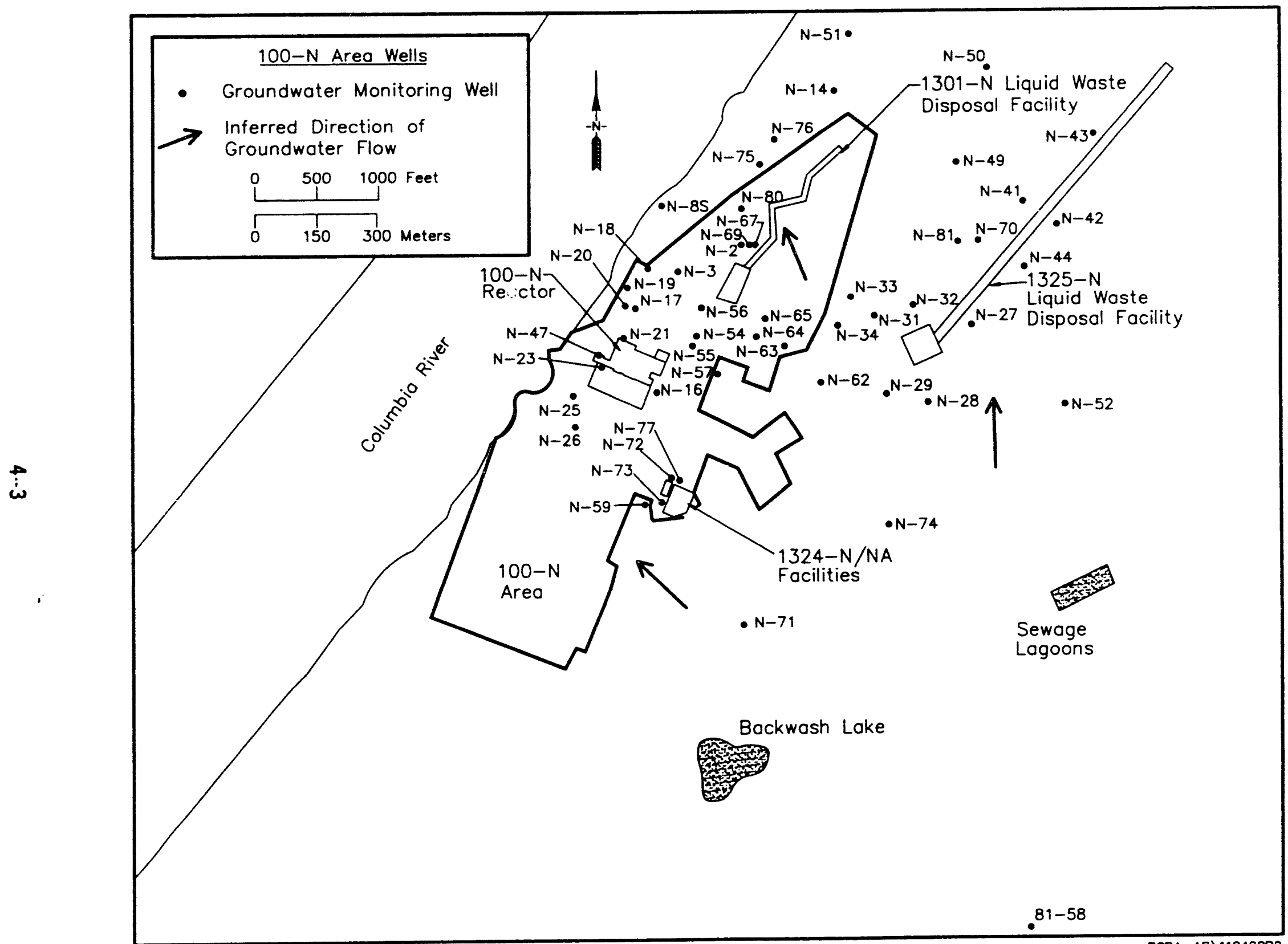

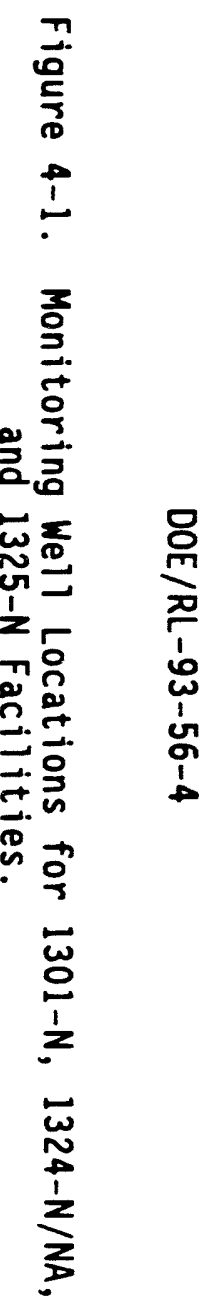


Table 4-1. Monitoring Well Purpose and Sampling Schedule in the 100-N Area. (sheet 1 of 2)

\begin{tabular}{|c|c|c|c|c|}
\hline $\begin{array}{l}\text { Well no. } \\
(199-)\end{array}$ & RCRA site & $\begin{array}{l}\text { Relative } \\
\text { position }\end{array}$ & $\begin{array}{c}\text { Sample } \\
\text { frequency }\end{array}$ & $\begin{array}{l}\text { Sample date, } \\
\text { 4th Qtr } 1993\end{array}$ \\
\hline$N-2$ & $1301-N$ & Downgradient & Quarterly & $11 / 9 / 93$ \\
\hline$N-3$ & $1301-N, N R-2$ & Downgradient & Quarterly & $11 / 9 / 93$ \\
\hline$N-14$ & $1301-N$ & Downgradient & Quarterly & $11 / 16 / 93$ \\
\hline$N-16$ & NR-2 & Downgradient & Semiannually & Not $s^{-1}$-duled \\
\hline$N-17$ & NR-2 & Downgradient & Semiannually & Not scheduled \\
\hline$N-19$ & NR-2 & Downgradient & Semiannually & Not scheduled \\
\hline$N-20$ & NR-2 & Downgradient & Semiannually & Not scheduled \\
\hline$N-21$ & NR-2 & Downgradient & Semiannually & Not scheduled \\
\hline$N-25$ & NR-2 & Downgradient & Semiannually & Not scheduled \\
\hline$N-26$ & $N R-2$ & Downgradient & Semiannually & Not scheduled \\
\hline $\mathrm{N}-27$ & $1325-N$ & Downgradient & Semiannually & Not scheduled \\
\hline$N-29$ & $1325-N$ & Downgradient & Semiannually & Not scheduled \\
\hline$N-32$ & $1325-N, N R-2$ & Downgradient & Semiannually & Not scheduled \\
\hline$N-34$ & $1301-N, N R-2$ & Upgradient & Quarterly & $11 / 11 / 93$ \\
\hline$N-41$ & $1325-N$ & Downgradient & Semiannually & Not scheduled \\
\hline$N-43$ & $1325-N$ & Downgradient & Semiannually & Not scheduled \\
\hline$N-49$ & NR-2 & Downgradient & Semiannually & Not scheduled \\
\hline$N-50$ & NR-2 & Downgradient & Semiannually & Not scheduled \\
\hline$N-51$ & NR-2 & Downgradient & Semiannually & Not scheduled \\
\hline$N-54$ & NR-2 & Downgradient & Semiannually & Not scheduled \\
\hline$N-57$ & $1301-N$ & Upgradient & Quarterly & $11 / 12 / 93^{\circ}$ \\
\hline$N-59$ & $1324-N / N A$ & Downgradient & Quarterly & $10 / 4 / 93$ \\
\hline$N-64$ & NR-2 & Downgradient & Semiannually & Not scheduled \\
\hline$N-67$ & $1301-N, N R-2$ & Downgradient & Quarterly & $11 / 16 / 93^{\circ}$ \\
\hline$N-69$ & $1301-N$ & $\begin{array}{c}\text { Downgradient; } \\
\text { bottom of } \\
\text { aquifer }\end{array}$ & Quarterly & $11 / 9 / 93$ \\
\hline$N-70$ & $1325-N, N R-2$ & $\begin{array}{c}\text { Downgradient; } \\
\text { bottom of } \\
\text { aquifer }\end{array}$ & Quarterly & Not scheduled \\
\hline
\end{tabular}



Table 4-2. RCRA Water Level Measurement Report for the 100-N Area, Fourth Quarter 1993. (sheet 1 of 6)

\begin{tabular}{|c|c|c|c|}
\hline Well & Date & $\begin{array}{l}\text { Depth to } \\
\text { water (ft) }\end{array}$ & $\begin{array}{l}\text { Water level } \\
\text { elevation } \\
\text { above msl (ft) }\end{array}$ \\
\hline \multicolumn{4}{|c|}{ Wells Monitoring the Top of the Unconfined Aquifer } \\
\hline $199-N-14$ & $\begin{array}{l}10 / 06 / 93 \\
11 / 16 / 93 \\
11 / 17 / 93 \\
12 / 21 / 93\end{array}$ & $\begin{array}{l}70.59 \\
70.21 \\
70.18 \\
69.58\end{array}$ & $\begin{array}{l}383.09 \\
383.47^{\star} \\
383.50 \\
384.10\end{array}$ \\
\hline $199-N-16$ & $\begin{array}{l}10 / 06 / 93 \\
11 / 18 / 93 \\
12 / 21 / 93\end{array}$ & $\begin{array}{l}71.57 \\
71.63 \\
71.28\end{array}$ & $\begin{array}{l}385.67 \\
385.61 \\
385.96\end{array}$ \\
\hline $199-N-17$ & $\begin{array}{l}10 / 06 / 93 \\
11 / 18 / 93 \\
12 / 21 / 93\end{array}$ & $\begin{array}{l}77.98 \\
77.34 \\
76.68\end{array}$ & $\begin{array}{l}383.73 \\
384.37 \\
385.03\end{array}$ \\
\hline $199-N-18$ & $11 / 18 / 93$ & 74.94 & 384.11 \\
\hline $199-N-19$ & $\begin{array}{l}10 / 06 / 93 \\
11 / 18 / 93 \\
12 / 21 / 93\end{array}$ & $\begin{array}{l}71.24 \\
70.39 \\
69.80\end{array}$ & $\begin{array}{l}383.20 \\
384.05 \\
384.64\end{array}$ \\
\hline $199-N-2$ & $\begin{array}{l}10 / 06 / 93 \\
11 / 09 / 93 \\
11 / 17 / 93 \\
12 / 21 / 93\end{array}$ & $\begin{array}{l}75.64 \\
71.93 \\
75.52 \\
75.13\end{array}$ & $\begin{array}{l}384.12 \\
387.83^{\star}+ \\
384.24 \\
384.63\end{array}$ \\
\hline $199-N-20$ & $\begin{array}{l}10 / 06 / 93 \\
11 / 18 / 93 \\
12 / 21 / 93\end{array}$ & $\begin{array}{l}72.76 \\
71.93 \\
71.40\end{array}$ & $\begin{array}{l}383.59 \\
384.42 \\
384.95\end{array}$ \\
\hline $199-N-21$ & $\begin{array}{l}10 / 06 / 93 \\
11 / 18 / 93 \\
12 / 21 / 93\end{array}$ & $\begin{array}{l}73.82 \\
72.83 \\
72.56\end{array}$ & $\begin{array}{l}383.65 \\
384.64 \\
384.91\end{array}$ \\
\hline $199-N-23$ & $\begin{array}{l}10 / 06 / 93 \\
11 / 18 / 93 \\
12 / 21 / 93\end{array}$ & $\begin{array}{l}73.62 \\
72.77 \\
72.05\end{array}$ & $\begin{array}{l}383.18 \\
384.03 \\
384.75\end{array}$ \\
\hline $199-N-25$ & $\begin{array}{l}10 / 06 / 93 \\
11 / 18 / 93 \\
12 / 21 / 93\end{array}$ & $\begin{array}{l}43.87 \\
42.82 \\
41.85\end{array}$ & $\begin{array}{l}382.38 \\
383.43 \\
384.40\end{array}$ \\
\hline
\end{tabular}


Table 4-2. RCRA Water Level Measurement Report for the 100-N Area, Fourth Quarter 1993. (sheet 2 of 6)

\begin{tabular}{|c|c|c|c|}
\hline Well & Date & $\begin{array}{l}\text { Depth to } \\
\text { water (ft) }\end{array}$ & $\begin{array}{l}\text { Water level } \\
\text { elevation } \\
\text { above msl }(\mathrm{ft})\end{array}$ \\
\hline \multicolumn{4}{|c|}{ Wells Monitoring the Top of the Unconfined Aquifer } \\
\hline $199-N-26$ & $\begin{array}{l}10 / 06 / 93 \\
11 / 18 / 93 \\
12 / 21 / 93\end{array}$ & $\begin{array}{l}73.56 \\
72.52 \\
71.69\end{array}$ & $\begin{array}{l}382.70 \\
383.74 \\
384.57\end{array}$ \\
\hline $199-N-27$ & $\begin{array}{l}10 / 06 / 93 \\
11 / 18 / 93 \\
12 / 21 / 93\end{array}$ & $\begin{array}{l}62.10 \\
62.39 \\
62.41\end{array}$ & $\begin{array}{l}387.50 \\
387.21 \\
387.19\end{array}$ \\
\hline $199-N-28$ & $\begin{array}{l}10 / 06 / 93 \\
11 / 18 / 93 \\
12 / 21 / 93\end{array}$ & $\begin{array}{l}76.53 \\
76.76 \\
76.84\end{array}$ & $\begin{array}{l}388.21 \\
387.98 \\
387.90\end{array}$ \\
\hline $199-N-29$ & $\begin{array}{l}10 / 06 / 93 \\
11 / 18 / 93 \\
12 / 21 / 93\end{array}$ & $\begin{array}{l}77.54 \\
77.81 \\
77.80\end{array}$ & $\begin{array}{l}388.21 \\
387.94 \\
387.95\end{array}$ \\
\hline $199-N-3$ & $\begin{array}{l}10 / 06 / 93 \\
11 / 09 / 93 \\
11 / 17 / 93 \\
12 / 21 / 93\end{array}$ & $\begin{array}{l}76.00 \\
71.56 \\
75.31 \\
75.69\end{array}$ & $\begin{array}{l}383.39 \\
387.83^{\star}+ \\
384.08 \\
383.70\end{array}$ \\
\hline $199-N-31$ & $\begin{array}{l}11 / 18 / 93 \\
12 / 21 / 93\end{array}$ & $\begin{array}{l}76.61 \\
76.58\end{array}$ & $\begin{array}{l}386.02 \\
386.05\end{array}$ \\
\hline $199-N-32$ & $\begin{array}{l}10 / 06 / 93 \\
11 / 18 / 93 \\
12 / 21 / 93\end{array}$ & $\begin{array}{l}75.56 \\
75.87 \\
75.87\end{array}$ & $\begin{array}{l}387.03 \\
386.72 \\
386.72\end{array}$ \\
\hline $199-N-33$ & $\begin{array}{l}10 / 06 / 93 \\
11 / 18 / 93 \\
12 / 21 / 93\end{array}$ & $\begin{array}{l}73.79 \\
75.47 \\
75.60\end{array}$ & $\begin{array}{l}386.58 \\
384.90 \\
384.77\end{array}$ \\
\hline $199-N-34$ & $\begin{array}{l}10 / 06 / 93 \\
11 / 11 / 93 \\
11 / 18 / 93 \\
12 / 21 / 93\end{array}$ & $\begin{array}{l}72.94 \\
73.18 \\
73.25 \\
73.29\end{array}$ & $\begin{array}{l}387.21 \\
386.97 \star \\
386.90 \\
386.86\end{array}$ \\
\hline $199-N-41$ & $\begin{array}{l}10 / 06 / 93 \\
11 / 18 / 93 \\
12 / 21 / 93\end{array}$ & $\begin{array}{l}73.73 \\
73.77 \\
73.49\end{array}$ & $\begin{array}{l}384.38 \\
384.34 \\
384.62\end{array}$ \\
\hline
\end{tabular}


Table 4-2. RCRA Water Level Measurement Report for the 100-N Area, Fourth Quarter 1993. (sheet 3 of 6)

\begin{tabular}{|c|c|c|c|}
\hline Well & Date & $\begin{array}{l}\text { Depth to } \\
\text { water (ft) }\end{array}$ & $\begin{array}{l}\text { Water level } \\
\text { elevation } \\
\text { above msl (ft) }\end{array}$ \\
\hline \multicolumn{4}{|c|}{ Wells Monitoring the Top of the Unconfined Aquifer } \\
\hline $199-N-42$ & $\begin{array}{l}10 / 06 / 93 \\
11 / 18 / 93 \\
12 / 21 / 93\end{array}$ & $\begin{array}{l}70.68 \\
70.71 \\
70.77\end{array}$ & $\begin{array}{l}384.96 \\
384.93 \\
384.87\end{array}$ \\
\hline $199-N-49$ & $11 / 18 / 93$ & 66.89 & 384.37 \\
\hline $199-N-50$ & $\begin{array}{l}11 / 18 / 93 \\
12 / 21 / 93\end{array}$ & $\begin{array}{l}80.45 \\
79.93\end{array}$ & $\begin{array}{l}383.45 \\
383.97\end{array}$ \\
\hline $199-N-51$ & $\begin{array}{l}10 / 06 / 93 \\
11 / 18 / 93 \\
12 / 21 / 93\end{array}$ & $\begin{array}{l}80.17 \\
78.96 \\
78.95\end{array}$ & $\begin{array}{l}382.55 \\
383.76 \\
383.77\end{array}$ \\
\hline $199-N-52$ & $\begin{array}{l}10 / 06 / 93 \\
11 / 18 / 93 \\
12 / 21 / 93\end{array}$ & $\begin{array}{l}75.86 \\
76.06 \\
76.16\end{array}$ & $\begin{array}{l}388.34 \\
388.14 \\
388.04\end{array}$ \\
\hline $199-N-54$ & $\begin{array}{l}10 / 06 / 93 \\
11 / 18 / 93 \\
12 / 21 / 93\end{array}$ & $\begin{array}{l}72.78 \\
72.82 \\
72.49\end{array}$ & $\begin{array}{l}385.22 \\
385.18 \\
385.51\end{array}$ \\
\hline $199-N-55$ & $\begin{array}{l}10 / 06 / 93 \\
11 / 18 / 93 \\
12 / 21 / 93\end{array}$ & $\begin{array}{l}73.45 \\
73.20 \\
72.81\end{array}$ & $\begin{array}{l}384.90 \\
385.15 \\
385.54\end{array}$ \\
\hline $199-N-56$ & $\begin{array}{l}10 / 06 / 93 \\
11 / 18 / 93 \\
12 / 21 / 93\end{array}$ & $\begin{array}{l}73.89 \\
73.86 \\
73.48\end{array}$ & $\begin{array}{l}384.70 \\
384.73 \\
385.11\end{array}$ \\
\hline $199-N-57$ & $\begin{array}{l}10 / 06 / 93 \\
11 / 12 / 93 \\
11 / 18 / 93 \\
12 / 21 / 93\end{array}$ & $\begin{array}{l}71.26 \\
71.41 \\
71.60 \\
71.50\end{array}$ & $\begin{array}{l}387.00 \\
386.85^{\star} \\
386.66 \\
386.76\end{array}$ \\
\hline $199-N-59$ & $\begin{array}{l}10 / 04 / 93 \\
11 / 18 / 93 \\
12 / 22 / 93\end{array}$ & $\begin{array}{l}72.68 \\
72.81 \\
74.99\end{array}$ & $\begin{array}{l}387.39 \star \\
387.26 \\
385.08+\end{array}$ \\
\hline
\end{tabular}


Table 4-2. RCRA Water Level Measurement for the 100-N Area, Fourth Quarter 1993. (sheet 4 of 6 )

\begin{tabular}{|c|c|c|c|}
\hline Well & Date & $\begin{array}{l}\text { Depth to } \\
\text { water (ft) }\end{array}$ & $\begin{array}{l}\text { Water level } \\
\text { elevation } \\
\text { above msl ( } f t)\end{array}$ \\
\hline \multicolumn{4}{|c|}{ Wells Monitoring the Top of the Unconfined Aquifer } \\
\hline $199-N-62$ & $\begin{array}{l}10 / 06 / 93 \\
11 / 18 / 93 \\
12 / 21 / 93\end{array}$ & $\begin{array}{l}75.84 \\
76.10 \\
76.21\end{array}$ & $\begin{array}{l}388.28 \\
388.02 \\
387.91\end{array}$ \\
\hline $199-N-63$ & $\begin{array}{l}10 / 06 / 93 \\
11 / 18 / 93 \\
12 / 21 / 93\end{array}$ & $\begin{array}{l}79.28 \\
79.64 \\
79.66\end{array}$ & $\begin{array}{l}387.95 \\
387.59 \\
387.57\end{array}$ \\
\hline $199-N-64$ & $\begin{array}{l}10 / 06 / 93 \\
11 / 17 / 93 \\
12 / 21 / 93\end{array}$ & $\begin{array}{l}67.68 \\
68.03 \\
68.02\end{array}$ & $\begin{array}{l}387.47 \\
387.12 \\
387.13\end{array}$ \\
\hline $199-N-65$ & $\begin{array}{l}10 / 06 / 93 \\
11 / 17 / 93 \\
12 / 21 / 93\end{array}$ & $\begin{array}{l}70.06 \\
70.35 \\
70.34\end{array}$ & $\begin{array}{l}386.92 \\
386.63 \\
386.64\end{array}$ \\
\hline $199-N-67$ & $\begin{array}{l}10 / 06 / 93 \\
11 / 16 / 93 \\
11 / 17 / 93 \\
12 / 21 / 93\end{array}$ & $\begin{array}{l}74.19 \\
74.41 \\
74.24 \\
73.96\end{array}$ & $\begin{array}{l}384.78 \\
384.56^{\star} \\
384.73 \\
385.01\end{array}$ \\
\hline $199-N-71$ & $\begin{array}{l}10 / 06 / 93 \\
11 / 12 / 93 \\
11 / 18 / 93 \\
12 / 22 / 93\end{array}$ & $\begin{array}{l}73.45 \\
73.70 \\
73.72 \\
73.73\end{array}$ & $\begin{array}{l}389.57 \\
389.32^{\star} \\
389.30 \\
389.29\end{array}$ \\
\hline $199-N-72$ & $\begin{array}{l}10 / 06 / 93 \\
11 / 12 / 93 \\
11 / 18 / 93 \\
12 / 21 / 93\end{array}$ & $\begin{array}{l}70.44 \\
70.84 \\
70.78 \\
70.60\end{array}$ & $\begin{array}{l}388.54 \\
388.14^{\star} \\
388.20 \\
388.38\end{array}$ \\
\hline $199-N-73$ & $\begin{array}{l}10 / 07 / 93 \\
11 / 12 / 93 \\
11 / 18 / 93 \\
12 / 22 / 93\end{array}$ & $\begin{array}{l}75.41 \\
75.57 \\
75.72 \\
75.64\end{array}$ & $\begin{array}{l}387.85 \\
387.69 \star \\
387.54 \\
387.62\end{array}$ \\
\hline $199-N-74$ & $\begin{array}{l}10 / 06 / 93 \\
11 / 18 / 93 \\
12 / 21 / 93\end{array}$ & $\begin{array}{l}68.27 \\
68.47 \\
68.55\end{array}$ & $\begin{array}{l}389.37 \\
389.17 \\
389.09\end{array}$ \\
\hline
\end{tabular}


Table 4-2. RCRA Water Level Measurement Report for the 100-N Area, Fourth Quarter 1993. (sheet 5 of 6 )

Well Date $\begin{gathered}\text { Depth to } \\ \text { water (ft) }\end{gathered} \begin{gathered}\text { Water level } \\ \text { elevaiton } \\ \text { above msl (ft) }\end{gathered}$

Wells Monitoring the Top of the Unconfined Aquifer

\begin{tabular}{llll}
\hline $199-N-75$ & $10 / 06 / 93$ & 74.07 & 382.71 \\
& $11 / 11 / 93$ & 71.91 & $384.87 \star$ \\
& $11 / 18 / 93$ & 73.39 & 383.39 \\
$12 / 21 / 93$ & 72.17 & 384.61 \\
$199-N-76$ & $10 / 06 / 93$ & 69.52 & 382.60 \\
& $11 / 11 / 93$ & 67.95 & $384.17 \star$ \\
& $11 / 18 / 93$ & 68.70 & 383.42 \\
$199-N-8 S$ & $12 / 21 / 93$ & 68.94 & $383.18+$ \\
& $10 / 06 / 93$ & 23.89 & 381.23 \\
& $11 / 17 / 93$ & 22.99 & 382.13 \\
$699-81-58$ & $12 / 21 / 93$ & 21.48 & 383.64 \\
& $10 / 06 / 93$ & 46.80 & 392.75 \\
& $11 / 18 / 93$ & 46.89 & 392.66 \\
& $12 / 21 / 93$ & 46.93 & 392.62 \\
\hline
\end{tabular}

Wells Monitoring the Bottom of the Unconfined Aquifer

\begin{tabular}{llll}
\hline $199-N-69$ & $10 / 06 / 93$ & 74.75 & 384.61 \\
& $11 / 09 / 93$ & 74.87 & $384.49 *$ \\
$11 / 17 / 93$ & 74.77 & 384.59 \\
$12 / 21 / 93$ & 74.45 & 384.91 \\
$199-N-70$ & $10 / 06 / 93$ & 68.94 & 385.76 \\
& $11 / 18 / 93$ & 69.23 & 385.47 \\
$199-N-77$ & $12 / 21 / 93$ & 69.14 & 385.56 \\
& $10 / 06 / 93$ & 71.08 & 388.36 \\
& $11 / 12 / 93$ & 70.24 & $389.20^{\star}+$ \\
& $11 / 18 / 93$ & 71.42 & 388.02 \\
& $12 / 21 / 93$ & 71.25 & 388.19
\end{tabular}


Table 4-2. RCRA Water Level Measurement for the 100-N Area, Fourth Quarter 1993. (sheet 6 of 6 )

Notes: 1. Water level elevations are calculated by subtracting the measured depth to water from the surveyed elevation for the well.

2. Depth-to-water values are transcribed from field records.

3. Elevations marked with an ' $*$ ' were measured at the time of sampling.

4. Elevations marked with a ' + ' are outside of the expected range and are suspected of error.

5. To convert feet to meters multiply by 0.3048 . 

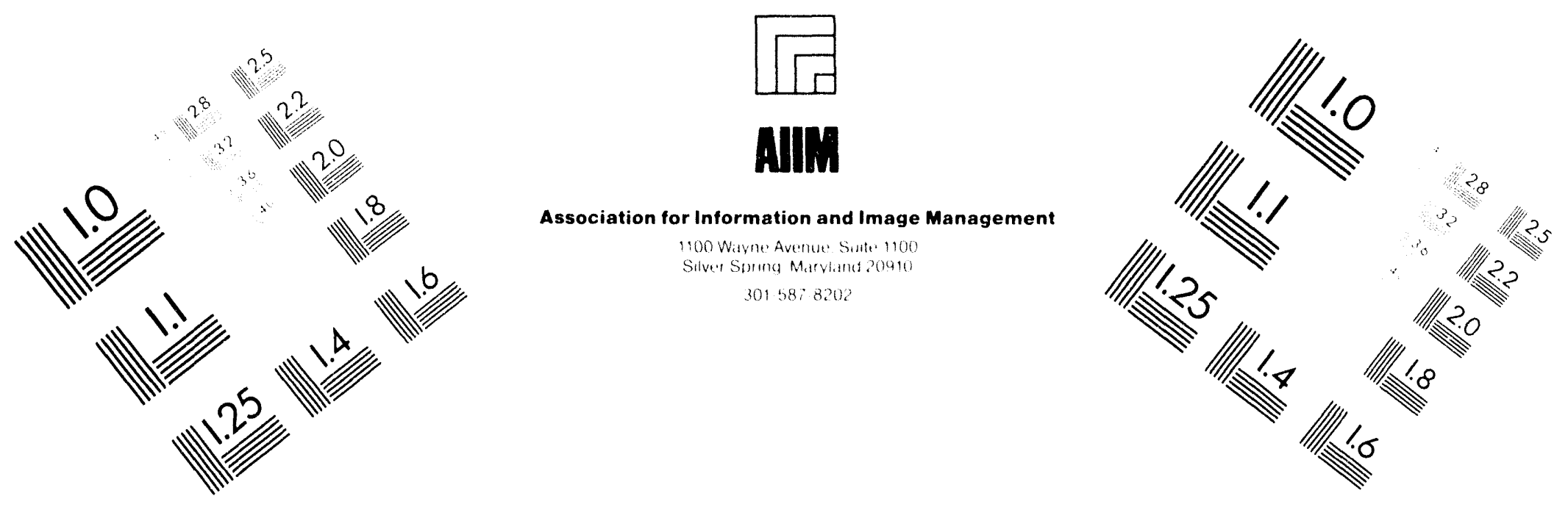

\section{Centimeter}

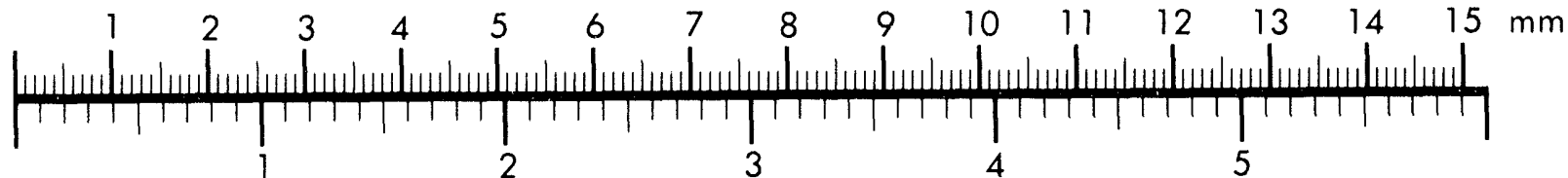

Inches
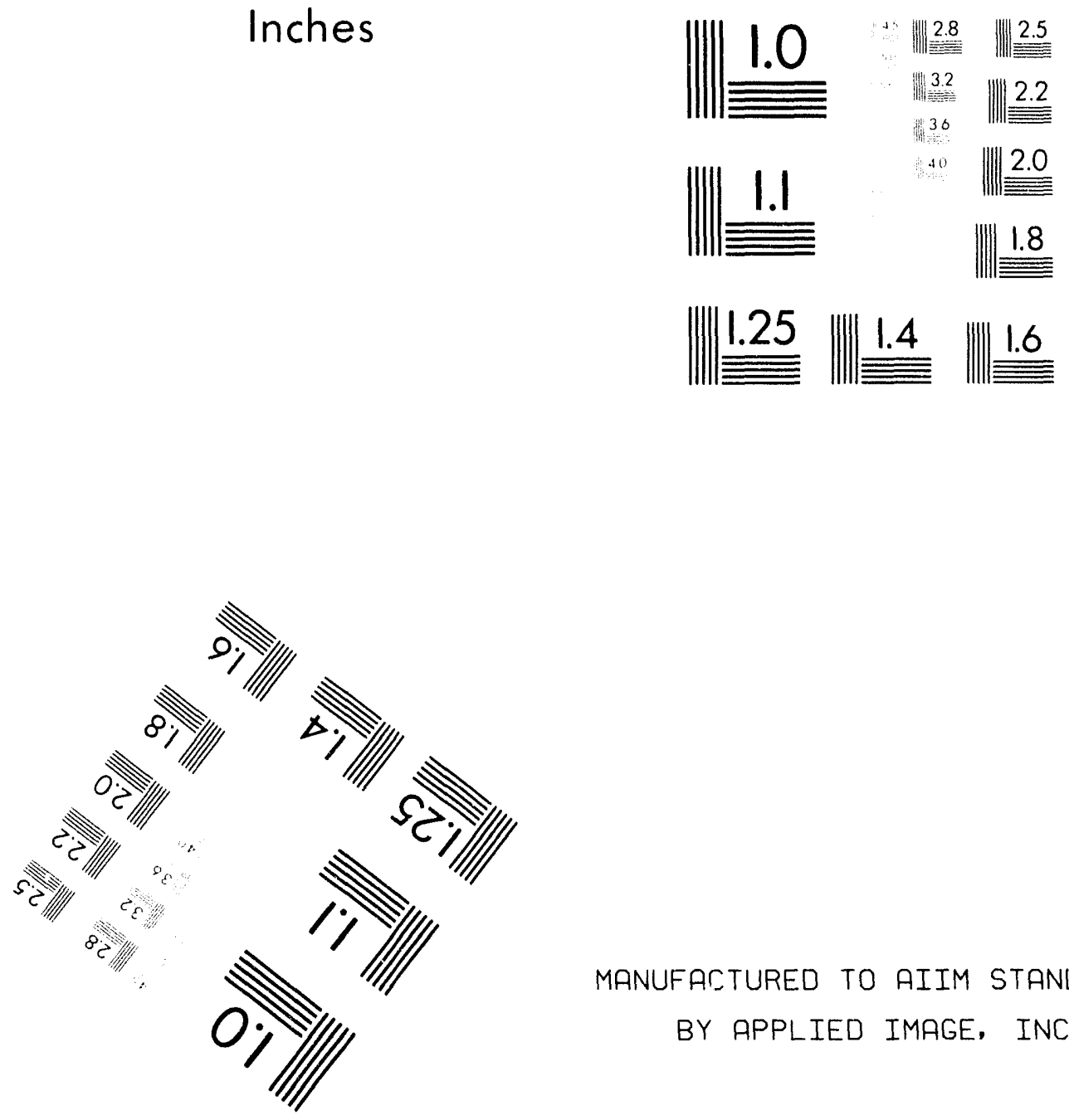

MANUFACTURED TO AIIM STANDARDS

BY APPLIED IMAGE, INC.

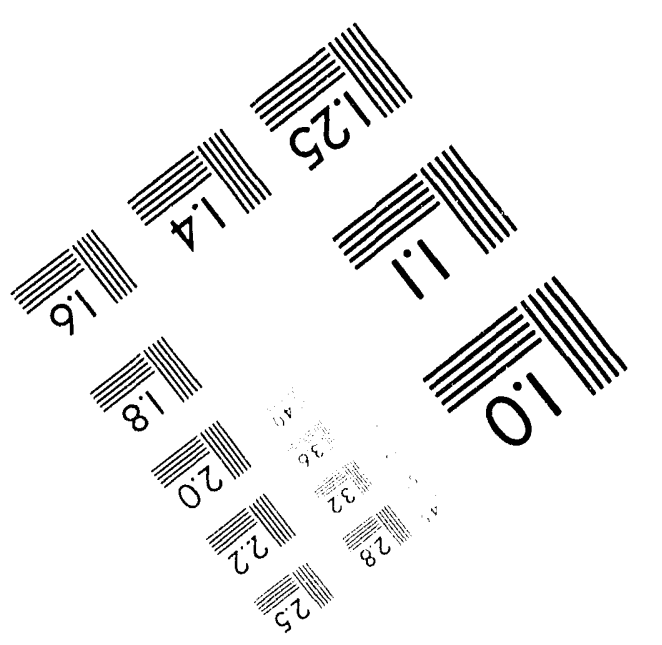



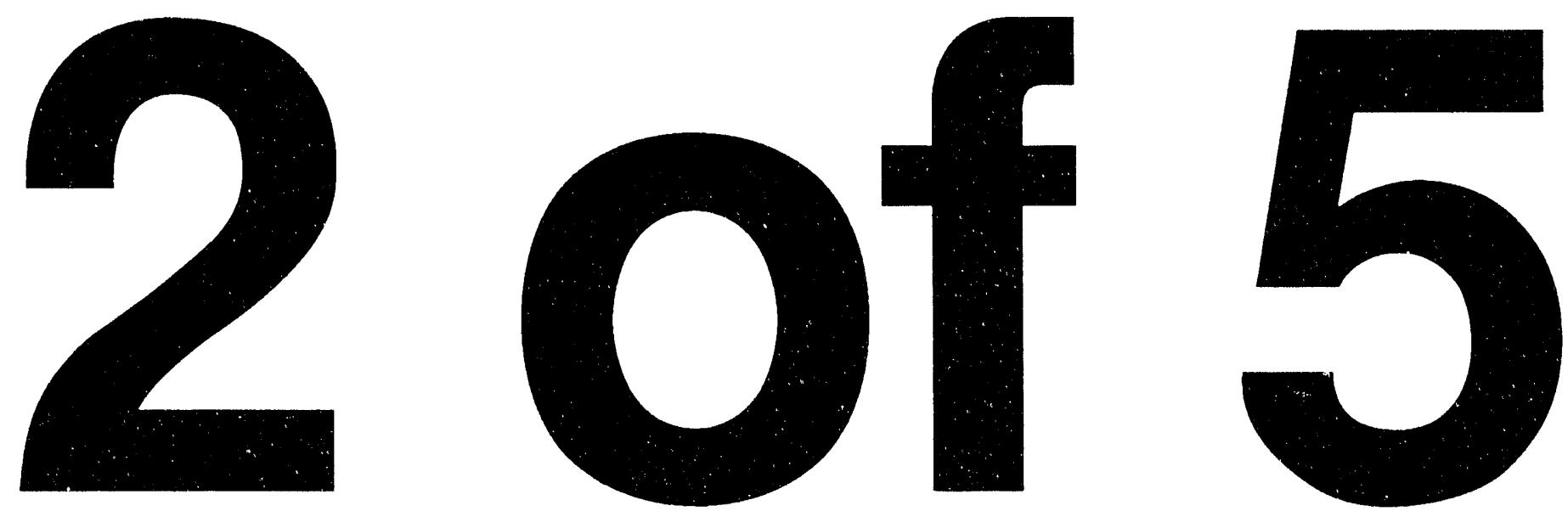
Table 4-3. Constituent List and Summary of Results for 100-N RCRA Sites Data for Reporting Period October 1 through December 31, 1993. (sheet 1 of 3)

CONTAMIHATION INDICATOR PARAMETERS

\begin{tabular}{|c|c|c|c|c|c|c|c|c|c|}
\hline \multirow[b]{2}{*}{ Short } & \multirow[b]{2}{*}{ (Method) } & \multirow{2}{*}{ Constituent Nawe Full } & \multirow[b]{2}{*}{ Units } & \multirow{2}{*}{$\begin{array}{l}\text { Lab } \\
\text { CROL }\end{array}$} & \multicolumn{2}{|c|}{ DuS } & \multicolumn{3}{|c|}{ Number of Samples } \\
\hline & & & & & Limit & Agency & Total & $>\mathrm{CRQL}$ & $>$ DWS \\
\hline & 0 & colota & (n) & $\cdots$ & & & 5 & 55 & \\
\hline CONOUCT & 94 & Conductivity, field & $\mu$ mino & 1 & & & 55 & 55 & \\
\hline CONDUCT & 73 & Conductivity, lab & $\boldsymbol{\mu}$ mino & 1 & & & 69 & 69 & \\
\hline TOC & & Total Orgenic Carbon & ppb & 200 & & & 64 & 57 & \\
\hline TOXLDL & & Total Organic Halogen & ppb & 8 & & & 57 & 12 & \\
\hline PH & 93 & PH, field & PH & .01 & $6.5-8.5$ & EPAS & 55 & 55 & 0 \\
\hline PH & 125 & pH, lab & pH & .01 & $6.5-8.5$ & EPAS & 60 & 60 & 2 \\
\hline
\end{tabular}

DRINKING WATER PARAMETERS

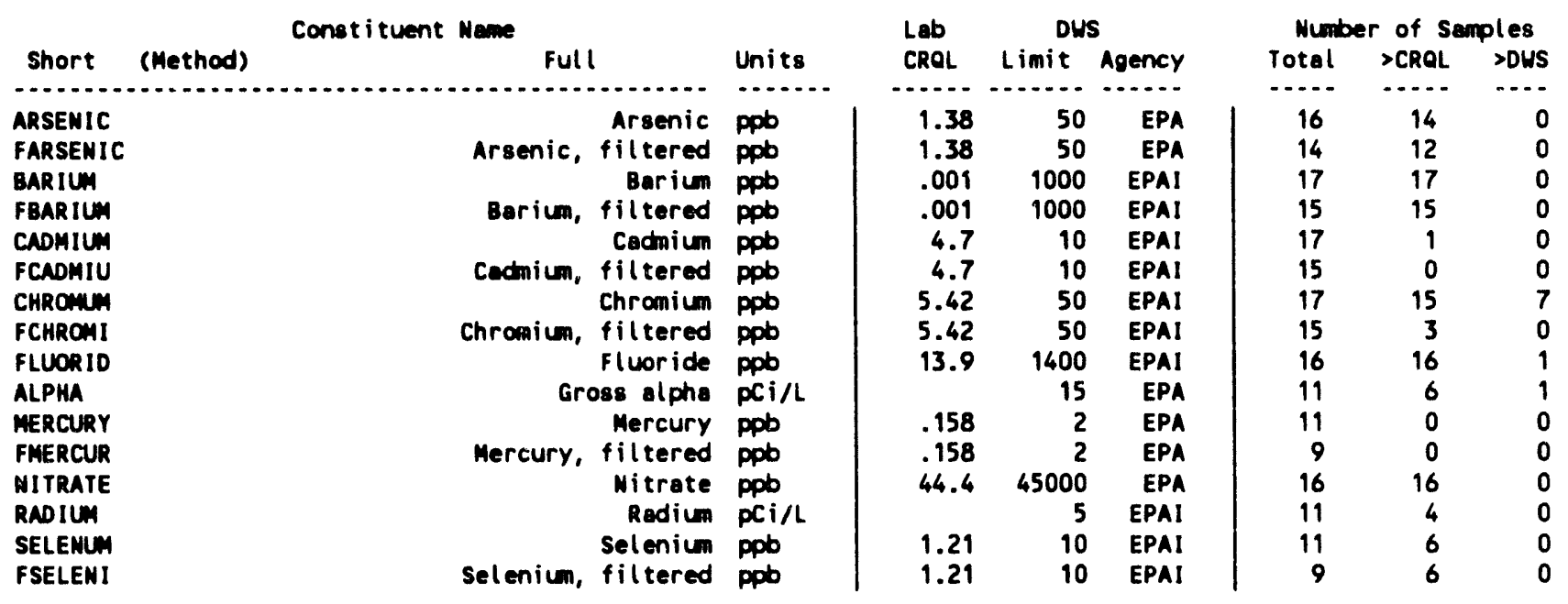

GROUMDWATER QUALITY PARAMETERS

\begin{tabular}{|c|c|c|c|c|c|c|c|c|c|}
\hline \multirow[b]{2}{*}{ Short } & \multirow[b]{2}{*}{ (Method) } & \multirow{2}{*}{ Constituent Name Full } & \multirow[b]{2}{*}{ Units } & \multirow{2}{*}{$\begin{array}{l}\text { Lab } \\
\text { CROL }\end{array}$} & \multicolumn{2}{|c|}{ DWS } & \multicolumn{3}{|c|}{ Number of Semples } \\
\hline & & & & & Limit & Agency & Total & $>$ CRQL & $>$ DWS \\
\hline CHLORIO & & Chloride & ppb & 82.5 & 250000 & EPAS & 16 & 16 & 0 \\
\hline IRON & & Iron & ppb & 10.3 & 300 & EPAS & 17 & 17 & 10 \\
\hline FIRON & & Iron, filtered & ppb & 10.3 & 300 & EPAS & 15 & 13 & 0 \\
\hline MANGESE & & Menganese & ppob & 1.35 & 50 & EPAS & 17 & 17 & 5 \\
\hline FMANGAN & & Manganese, filtered & ppb & 1.35 & 50 & EPAS & 15 & 9 & 0 \\
\hline LPHENOL & & Phenol & ppob & .31 & & & 11 & 0 & \\
\hline soolum & & Sodium & ppob & 40.9 & & & 17 & 17 & \\
\hline FSOOIUN & & Sodiun, filtered & ppb & 40.9 & & & 15 & 15 & \\
\hline SULFATE & & Sulfate & ppo & 49.9 & 250000 & EPAS & 16 & 16 & 2 \\
\hline
\end{tabular}

SITE SPECIFIC AND OTHER CONSTITUENTS

\begin{tabular}{|c|c|c|c|c|c|c|c|c|c|}
\hline \multirow[b]{2}{*}{ short } & \multicolumn{2}{|r|}{ Constituent Name } & \multirow[b]{2}{*}{ Units } & \multirow{2}{*}{$\begin{array}{l}\text { Lab } \\
\text { CROL }\end{array}$} & \multicolumn{2}{|c|}{ DWS } & \multicolumn{3}{|c|}{ Number of Samples } \\
\hline & (Method) & Full & & & Limit & Agency & Total & $>C R Q L$ & $>$ DWS \\
\hline $\begin{array}{l}1,1,1-T \\
1,1,2-T \\
1,1-D I C \\
1,2-D I C\end{array}$ & & $\begin{array}{r}\text { 1,1,1-irichloroethane } \\
1,1,2-\text { Trichloroethane } \\
\text { 1,1-D ichloroethane } \\
\text { 1,2-Dichloroethane }\end{array}$ & $\begin{array}{l}\text { ppb } \\
\text { ppb } \\
\text { ppb } \\
\text { ppb }\end{array}$ & $\begin{array}{l}.072 \\
.043 \\
.337 \\
.139\end{array}$ & 200 & EPA & $\begin{array}{l}5 \\
5 \\
5 \\
5\end{array}$ & $\begin{array}{l}0 \\
0 \\
0 \\
0\end{array}$ & 0 \\
\hline
\end{tabular}


Table 4-3. Constituent List and Summary of Results for 100-N RCRA Sites Data for Reporting Period October 1 through December 31, 1993. (sheet 2 of 3)

\begin{tabular}{|c|c|c|c|c|c|c|c|c|}
\hline & Const ituent Mane & & Lab & DWS & & Numt & of $\mathrm{Sa}$ & oles \\
\hline Short & (Method) & Units & CRQL & Limit & Agency & Total & $>C R Q L$ & $>$ DWS \\
\hline$\ldots \ldots$ & 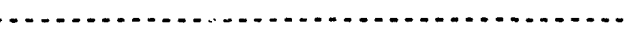 & $\ldots \ldots$ & $\cdots \cdots$ & $\ldots \ldots$ & $\ldots \ldots$ & -.... & $-\cdots$ & $\cdots$ \\
\hline $14-d$ ben & 1,4-Dichlorobenzene & ppb & .107 & $\sqrt{3}$ & EPA & 5 & 0 & 0 \\
\hline 246-trp & 2,4,6-Trichl orophenol & ppb & 1.45 & & & 11 & 0 & \\
\hline $24-d c h p$ & 2,4-Dichlorophenol & ppb & 1.5 & & & 11 & 0 & \\
\hline DIMPWEM & 2,4-0 imethylphenol & ppob & 1.01 & & & 11 & 0 & \\
\hline DINPHEN & 2,4-D initrophenol & ppob & .96 & & & 11 & 0 & \\
\hline 26-dehp & 2,6-0 ishlorophenol & pob & 1.59 & & & 11 & 0 & \\
\hline CHLPHEN & 2-Chlorophenol & ppob & 1.42 & & & 11 & 0 & \\
\hline 2NITPH & 2-Nitrophenol & ppo & 1.56 & & & 11 & 0 & \\
\hline BUTDINP & 2-sec-Butyl-4,6-dinitrophenol (DN & ppo & 1.35 & & & 11 & 0 & \\
\hline 460N2MP & 4,6-0 initro-2-methylphenol & ppob & 1.18 & & & 11 & 0 & \\
\hline CHLCRES & 4-Chloro-3-nethylphenol & ppo & 1.12 & & & 11 & 0 & \\
\hline NITPHEN & 4-Nitrophenol & ppo & .65 & & & 11 & 0 & \\
\hline ALKALIN & Alkal inity & Ppm & 1.96 & & & 5 & 5 & \\
\hline ALLANUM & Aluminum & ppob & 32.5 & & & 17 & 9 & \\
\hline FALUMIN & Aluminum & ppb & 32.5 & & & 15 & 0 & \\
\hline ANTIONY & Ant imony & ppb & 69.4 & & & 17 & 1 & \\
\hline FANTIMO & Ant imony, filtered & ppo & 69.4 & & & 15 & 1 & \\
\hline $58-125$ & Ant imony-125 & $\mathrm{pCi} / \mathrm{L}$ & & & & 11 & 4 & \\
\hline BENZENE & Benzene & ppb & .105 & 5 & EPA & 5 & 0 & 0 \\
\hline BERYLUM & Beryllium & ppb & .814 & & & 17 & 0 & \\
\hline FBERYLL & Beryllium, filtered & ppob & .814 & & & 15 & 0 & \\
\hline BRONIDE & Bromide & pop & 52.8 & & & 16 & 2 & \\
\hline CALCIUN & Calciun & ppb & 20.9 & & & 17 & 17 & \\
\hline FCALCIU & Colcium, filtered & ppo & 20.9 & & & 15 & 15 & \\
\hline TETRANE & Carbon tetrachloride & ppb & .121 & 5 & EPA & 5 & 0 & 0 \\
\hline CS-137 & Cesium- 137 & $\mathrm{pCi} / \mathrm{L}$ & & 200 & EPA & 11 & 1 & 0 \\
\hline CHLFORM & Chloroform & ppb & .043 & & & 5 & 5 & \\
\hline COBALT & Cobalt & ppb & 4.05 & & & 17 & 2 & \\
\hline FCOBALT & Cobalt, filtered & ppo & 4.05 & & & 15 & 0 & \\
\hline$c 0-60$ & Cobelt $t-60$ & $\mathrm{pCi} / \mathrm{L}$ & & 100 & EPA & 11 & 3 & 0 \\
\hline COL I FORM & Col iforms & $\mathrm{col}$ & 1 & 1 & EPAI & 8 & 0 & 0 \\
\hline COPPER & Copper & ppb & 2.65 & 1000 & EPAS & 17 & 12 & 0 \\
\hline FCOPPER & Copper, filtered & ppob & 2.65 & 1000 & EPAS & 15 & 6 & 0 \\
\hline CRESOLS & Cresols (methylphenols) & ppo & 4.66 & & & 11 & 0 & \\
\hline ETKBENZ & Ethylbenzene & ppob & .046 & 700 & EPA & 5 & 0 & 0 \\
\hline BETA & Gross beta & $\mathrm{pCi} / \mathrm{L}$ & & & & 11 & 11 & \\
\hline LHYDRAZ & Hydrazine & ppo & 1.89 & & & 11 & 0 & \\
\hline LEAD & Lead & ppb & .508 & 50 & EPAI & 16 & 13 & 1 \\
\hline FLEAD & Lead, fil tered & ppob & .508 & 50 & EPAI & 14 & 6 & 0 \\
\hline MAGNS & Magnes ium & ppob & 26 & & & 17 & 17 & \\
\hline FMAGNES & Magnesium, fillered & ppb & 26 & & & 15 & 15 & \\
\hline METHYCH & Methylene chloride & ppb & .056 & & & 5 & 0 & \\
\hline NICKEL & Nickel & ppb & 17.9 & & & 17 & 7 & \\
\hline FNICKEL & Nickel, filtered & ppb & 17.9 & & & 15 & 0 & \\
\hline NITRITE & Nitrice & ppo & 38.3 & 1000 & EPA & 16 & 3 & 0 \\
\hline PENTCHP & Pentechlorophenol & ppb & .87 & 1 & EPA & 11 & 0 & 0 \\
\hline PHOSPHA & Phosphate & ppo & 147 & & & 16 & 0 & \\
\hline POTASUM & Potassium & ppb & 662 & & & 17 & 17 & \\
\hline FPOTASS & Potassium, filtered & ppb & 662 & & & 15 & 15 & \\
\hline$R U-106$ & Ruthenivm-106 & $\mathrm{PCi} / \mathrm{L}$ & & 30 & EPA & 11 & 0 & 1 * \\
\hline SILVER & silver & ppob & 2.87 & 50 & EPAI & 17 & 7 & 0 \\
\hline FSILVER & silver, filtered & ppob & 2.87 & 50 & EPAI & 15 & 7 & 0 \\
\hline$S R-90$ & stront ium- 90 & $\mathrm{PCi} / \mathrm{L}$ & & 8 & EPA & 11 & 10 & 10 \\
\hline PERCENE & Tetrechloroethene & ppo & .049 & 5 & EPA & 5 & 0 & 0 \\
\hline TETPHNL & Tetrachlorophenols & ppo & 1.05 & & & 11 & 0 & \\
\hline TIN & Tin & ppb & 51.1 & & & 17 & 1 & \\
\hline FTIN & Tin, filtered & ppo & 51.1 & & & 15 & 0 & \\
\hline TOLUENE & Tol vene & ppo & .056 & 1000 & EPA & 5 & 1 & 0 \\
\hline TC & Total Carbon & ppb & 200 & & & 5 & 5 & \\
\hline
\end{tabular}


Table 4-3. Constituent List and Summary of Results for 100-N RCRA Sites Data for Reporting Period October 1 through December 31, 1993. (sheet 3 of 3)

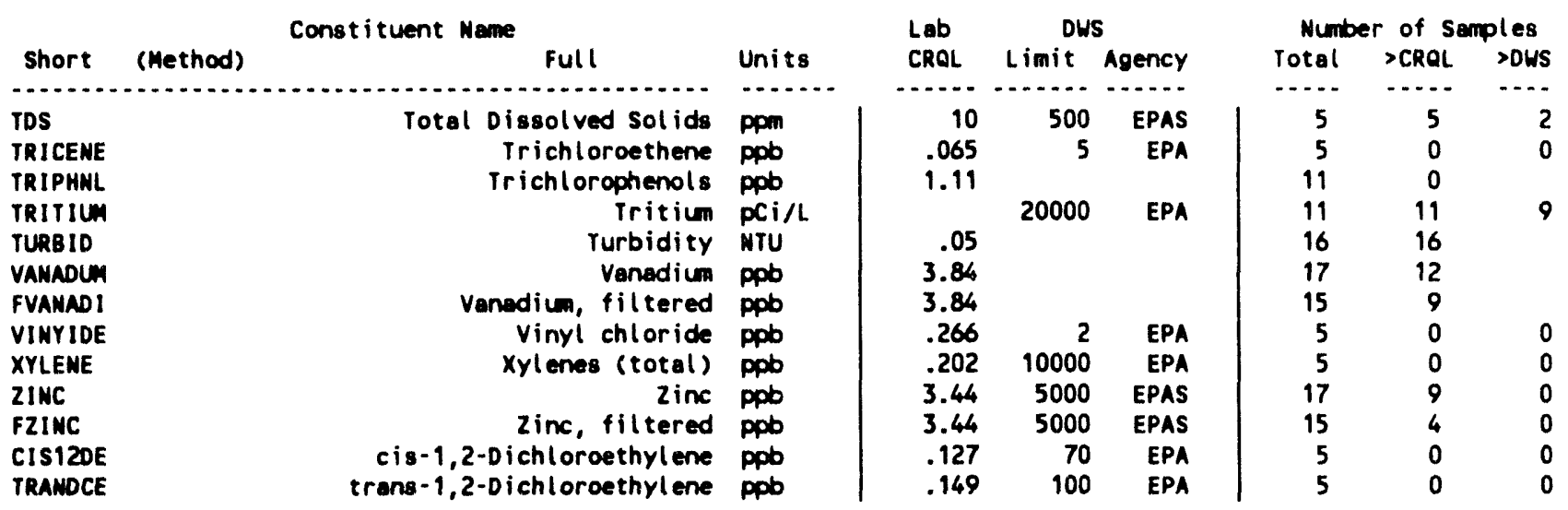

For explanation of this table, see section 1.4 of report. 
Table 4-4. Constituents with at Least One Detected Value for the 100-N RCRA Sites Data for Reporting Period October 1 through December 31, 1993. (sheet 1 of 11)

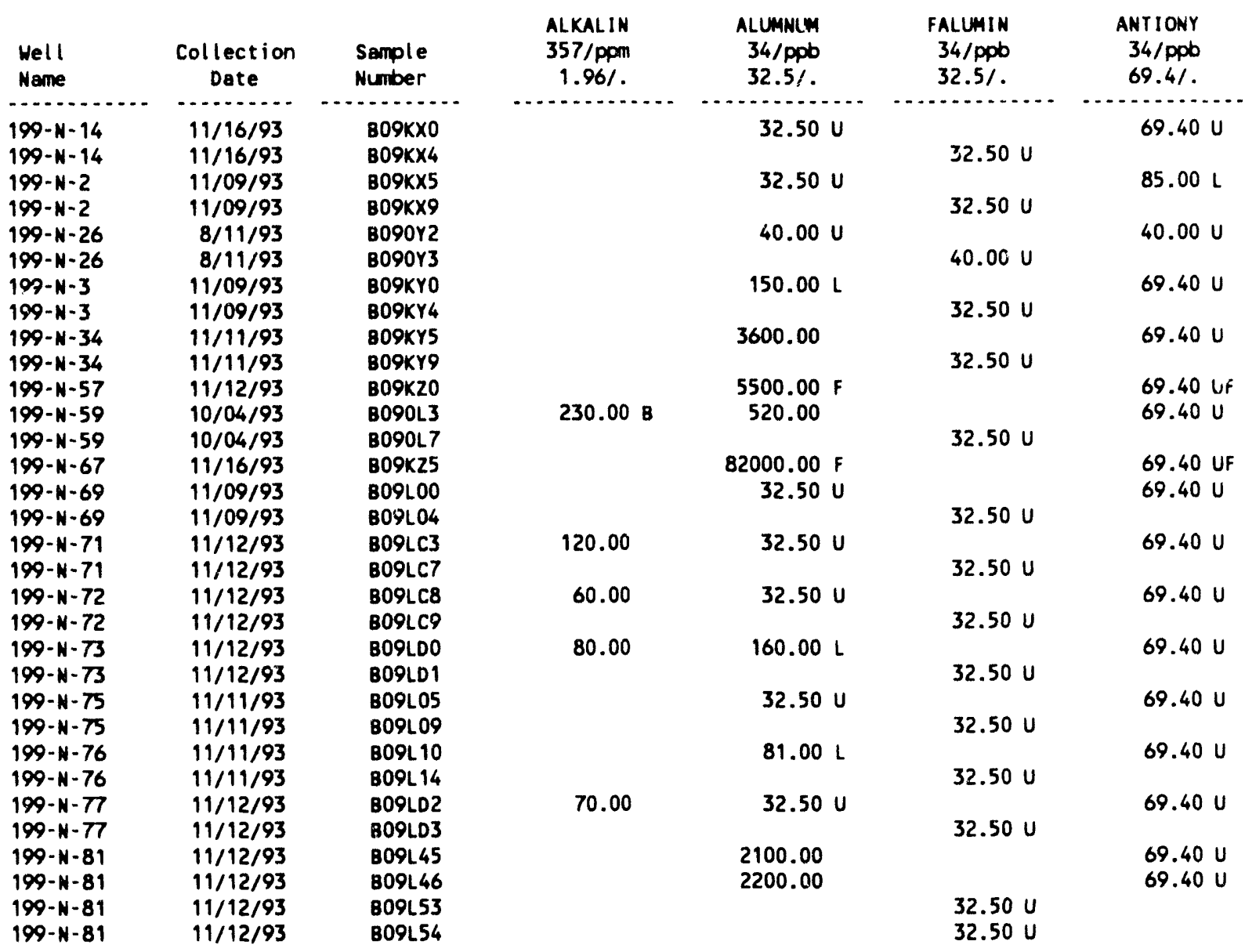

\begin{tabular}{|c|c|c|c|c|c|c|}
\hline $\begin{array}{l}\text { Well } \\
\text { Name }\end{array}$ & $\begin{array}{c}\text { Collection } \\
\text { Date }\end{array}$ & $\begin{array}{l}\text { Sample } \\
\text { Number }\end{array}$ & $\begin{array}{l}\text { FANT IMO } \\
34 / \mathrm{ppb} \\
69.4 / .\end{array}$ & $\begin{array}{c}\text { SB-125 } \\
140 / \mathrm{pCi} / \mathrm{L} \\
. /\end{array}$ & $\begin{array}{r}\text { ARSENIC } \\
43 / \mathrm{ppb} \\
1.38 / 50\end{array}$ & $\begin{array}{l}\text { FARSENIC } \\
43 / \mathrm{ppb} \\
1.38 / 50\end{array}$ \\
\hline - n. & ............... & 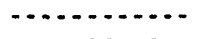 & $\cdots \cdots$ & . & & \\
\hline $199-N-14$ & $11 / 16 / 93$ & B09KX0 & & $13.70 \mathrm{U}$ & $2.40 \mathrm{~L}$ & \\
\hline $199-N-14$ & $11 / 16 / 93$ & B09KX4 & $69.40 \mathrm{U}$ & & & $1.38 \mathrm{U}$ \\
\hline $199-N-2$ & $11 / 09 / 93$ & B09KX5 & & $-3.93 U$ & $2.40 \mathrm{~L}$ & \\
\hline $\begin{array}{l}199-N-2 \\
199-N-26\end{array}$ & $\begin{array}{r}11 / 09 / 93 \\
8 / 11 / 93\end{array}$ & $\begin{array}{l}\text { 809KX9 } \\
\text { B090Y3 }\end{array}$ & $\begin{array}{l}69.40 \mathrm{U} \\
40.00 \mathrm{U}\end{array}$ & & & $2.00 \mathrm{~L}$ \\
\hline $199-N-3$ & $11 / 09 / 93$ & BO9KYO & & $.63 \mathrm{U}$ & $2.00 \mathrm{~L}$ & \\
\hline $199-N-3$ & $11 / 09 / 93$ & B09KY4 & $69.40 \mathrm{U}$ & & & $2.00 \mathrm{~L}$ \\
\hline $199-N-34$ & $11 / 11 / 93$ & B09KYS & & $7.33 u$ & $3.80 \mathrm{~L}$ & \\
\hline $199-N-34$ & $11 / 11 / 93$ & 809kY9 & $69.40 \mathrm{U}$ & & & $2.60 \mathrm{~L}$ \\
\hline $199-N-57$ & $11 / 12 / 93$ & 809K20 & & -17.40 UF & $20.00 \mathrm{~F}$ & \\
\hline $199-N-59$ & $10 / 04 / 93$ & $13090 \mathrm{L3}$ & & & 6.10 & \\
\hline $199-N-59$ & $10 / 04 / 93$ & B090L7 & $69.40 \mathrm{U}$ & & & 11.00 \\
\hline $199-N-67$ & $11 / 16 / 93$ & 809K25 & & $19.40 \mathrm{~F}$ & $6.50 \mathrm{~F}$ & \\
\hline $199-N-69$ & $11 / 09 / 93$ & BOOLOO & & $-5.52 U$ & $2.00 \mathrm{~L}$ & \\
\hline $199-N-69$ & $11 / 09 / 93$ & BO9LOC & $69.40 \mathrm{U}$ & & & $2.00 \mathrm{~L}$ \\
\hline
\end{tabular}


Table 4-4. Constituents with at Least One Detected Value for the 100-N RCRA Sites Data for Reporting Period October 1 through December 31, 1993. (sheet 2 of 11 )

\begin{tabular}{|c|c|c|c|c|c|c|}
\hline $\begin{array}{l}\text { Well } \\
\text { Name }\end{array}$ & $\begin{array}{c}\text { Collection } \\
\text { Date }\end{array}$ & $\begin{array}{l}\text { Semple } \\
\text { Number }\end{array}$ & $\begin{array}{l}\text { FANTIMO } \\
34 / \text { ppD } \\
69.4 / .\end{array}$ & $\begin{array}{c}\text { SB-125 } \\
140 / \text { PC } / / L \\
. /\end{array}$ & $\begin{array}{r}\text { ARSENIC } \\
43 / \mathrm{ppb} \\
1.38 / 50\end{array}$ & $\begin{array}{c}\text { FARSENIC } \\
43 / \mathrm{ppb} \\
1.38 / 50\end{array}$ \\
\hline $199-N-71$ & $11 / 12 / 93$ & B09LC3 & & & 11.00 & \\
\hline $199-N-71$ & $11 / 12 / 93$ & BO9LC7 & $69.40 \mathrm{U}$ & & & 10.00 \\
\hline $199-N-72$ & $11 / 12 / 93$ & B09LC8 & & & $4.10 \mathrm{~L}$ & \\
\hline $199-N-72$ & $11 / 12 / 93$ & B09LC9 & $69.40 \mathrm{U}$ & & & $4.00 \mathrm{~L}$ \\
\hline $199-N-73$ & $11 / 12 / 93$ & BO9LDO & & & $2.60 \mathrm{~L}$ & \\
\hline $199-N-73$ & $11 / 12 / 93$ & BOQLDI & $110.00 \mathrm{~L}$ & & & $2.80 \mathrm{~L}$ \\
\hline $199-N-75$ & $11 / 11 / 93$ & B09L05 & & $-1.47 U$ & $1.38 \mathrm{U}$ & \\
\hline $199-N-75$ & $11 / 11 / 93$ & BOSL09 & $69.40 \mathrm{U}$ & & & $1.38 \mathrm{U}$ \\
\hline $199-N-76$ & $11 / 11 / 93$ & $809 L 10$ & & 16.20 & $1.38 \mathrm{U}$ & \\
\hline $199-N-76$ & $11 / 11 / 93$ & $809 L 14$ & $69.40 \mathrm{U}$ & & & $2.00 \mathrm{~L}$ \\
\hline $199-N-77$ & $11 / 12 / 93$ & B09LD2 & & & $4.30 \mathrm{~L}$ & \\
\hline $199-N-77$ & $11 / 12 / 93$ & B09LD3 & $69.40 \mathrm{U}$ & & & $3.80 \mathrm{~L}$ \\
\hline $\begin{array}{l}199-N-81 \\
199-N-81\end{array}$ & $\begin{array}{l}11 / 12 / 93 \\
11 / 12 / 93\end{array}$ & $\begin{array}{l}B 09 L 45 \\
B 09 L 46\end{array}$ & & $\begin{array}{l}19.60 \\
17.30\end{array}$ & $\begin{array}{l}4.30 \mathrm{~L} \\
4.90 \mathrm{~L}\end{array}$ & \\
\hline $\begin{array}{l}199-N-81 \\
199-N-81\end{array}$ & $\begin{array}{l}11 / 12 / 93 \\
11 / 12 / 93\end{array}$ & $\begin{array}{l}\text { BO9L53 } \\
\text { B09L54 }\end{array}$ & $\begin{array}{l}69.40 U \\
69.40 U\end{array}$ & & & $\begin{array}{l}3.40 \mathrm{LO} \\
2.30 \mathrm{LO}\end{array}$ \\
\hline
\end{tabular}

\begin{tabular}{|c|c|c|c|c|c|c|c|}
\hline $\begin{array}{l}\text { Well } \\
\text { Nene }\end{array}$ & $\begin{array}{c}\text { Collection } \\
\text { Date }\end{array}$ & $\begin{array}{l}\text { Sample } \\
\text { Number }\end{array}$ & $\begin{array}{c}\text { BAR IUM } \\
34 / \text { ppb } \\
.001 / 1000 \mathrm{i}\end{array}$ & $\begin{array}{c}\text { FBARILM } \\
34 / \text { ppb } \\
.001 / 1000 i\end{array}$ & $\begin{array}{c}\text { BROMIDE } \\
124 / p p b \\
52.8 /\end{array}$ & $\begin{array}{l}\text { CADMIUM } \\
34 / p p b \\
4.7 / 10 i\end{array}$ & \\
\hline$\cdots+\cdots+\cdots$ & $\ldots$ & $\cdots$ & . & - & $\ldots \ldots$ & $\ldots \ldots$ & - \\
\hline $199-N-14$ & $11 / 16 / 93$ & BO9KX0 & $14.00 \mathrm{LB}$ & & $52.80 \mathrm{U}$ & 4.70 & $u$ \\
\hline $199-N-14$ & $11 / 16 / 93$ & $809 \times \times 4$ & & $14.00 \mathrm{LB}$ & & & \\
\hline $199-N-2$ & $11 / 09 / 93$ & $809 \times \times 5$ & 23.00 & & $52.80 \mathrm{U}$ & 4.70 & $U$ \\
\hline $199-N-2$ & $11 / 09 / 93$ & B09KX9 & & 23.00 & & & \\
\hline $199-N-26$ & $8 / 11 / 93$ & B090Y2 & 45.00 & & & 2.00 & U \\
\hline $197-N-26$ & $8 / 11 / 93$ & B090Y 3 & & 39.00 & & & \\
\hline $199-N-3$ & $11 / 09 / 93$ & BO9kYO & 110.00 & & $100.00 \mathrm{~L}$ & 4.70 & $u$ \\
\hline $199-N-3$ & $11 / 09 / 93$ & B09KY 4 & & 110.00 & & & \\
\hline $199-N-34$ & $11 / 11 / 93$ & B09KY 5 & $79.00 \mathrm{BO}$ & & $52.80 \mathrm{U}$ & 4.70 & $U$ \\
\hline $199-N-34$ & $11 / 11 / 93$ & B09KY9 & & $27.00 \mathrm{BO}$ & & & \\
\hline $199-N-57$ & $11 / 12 / 93$ & 809K20 & $260.00 \mathrm{~F}$ & & $60.00 \mathrm{LF}$ & 4.70 & UF \\
\hline $199-N-59$ & $10 / 04 / 93$ & B090L3 & 20.00 & & $52.80 \mathrm{U}$ & 4.70 & $\mathrm{U}$ \\
\hline $199-N-59$ & $10 / 04 / 93$ & 809017 & & $14.00 \mathrm{~L}$ & & & \\
\hline $199-N-67$ & $11 / 16 / 93$ & B09K25 & $990.00 \mathrm{BF}$ & & 52.80 UF & 9.70 & LF \\
\hline $199-N-69$ & $11 / 09 / 93$ & BOSLOO & $17.00 \mathrm{~L}$ & & $52.80 \mathrm{U}$ & 4.70 & $U$ \\
\hline $199-N-69$ & $11 / 09 / 93$ & 809104 & & $14.00 \mathrm{~L}$ & & & \\
\hline $199-N-71$ & $11 / 12 / 93$ & B09LC3 & $16.00 \mathrm{~L}$ & & $52.80 \mathrm{U}$ & 4.70 & $U$ \\
\hline $199-N-71$ & $11 / 12 / 93$ & B09LC7 & & $16.00 \mathrm{~L}$ & & & \\
\hline $199 \cdot N-72$ & $11 / 12 / 93$ & BO9LC8 & $16.00 \mathrm{~L}$ & & $52.80 \mathrm{U}$ & 4.70 & $U$ \\
\hline $199-N-72$ & $11 / 12 / 93$ & BO9LC9 & & $17.00 \mathrm{~L}$ & & & \\
\hline $199-N-73$ & $11 / 12 / 93$ & BO9LDO & 51.00 & & $52.80 \mathrm{U}$ & 4.70 & $U$ \\
\hline $199 \cdot N-73$ & $11 / 12 / 93$ & B09LD1 & & 48.00 & & & \\
\hline $199 \cdot N-75$ & $11 / 11 / 93$ & BOSLOS & $27.00 \mathrm{BO}$ & & $52.80 \mathrm{U}$ & 4.70 & U \\
\hline $199 \cdot N-75$ & $11 / 11 / 93$ & BOSLOQ & & 27.0080 & & & \\
\hline $199-N-76$ & $11 / 11 / 93$ & B09L10 & $19.00 \mathrm{LBO}$ & & $52.80 \mathrm{U}$ & 4.70 & $U$ \\
\hline $199-N-76$ & $11 / 11 / 93$ & BO9L 14 & & $16.00<80$ & & & \\
\hline $199-N-77$ & $11 / 12 / 93$ & B09LD2 & $16.00 \mathrm{~L}$ & & $52.80 u$ & 4.70 & U \\
\hline $199-$ - 77 & $11 / 12 / 93$ & BO9LD3 & & $17.00 \mathrm{~L}$ & & & \\
\hline $199-N-81$ & $11 / 12 / 93$ & 809145 & 49.00 & & $52.80 \mathrm{U}$ & 4.70 & U \\
\hline $199-N-81$ & $11 / 12 / 93$ & 809146 & 49.00 & & $52.80 \mathrm{U}$ & 4.70 & $U$ \\
\hline $199-N-81$ & $11 / 12 / 93$ & $B 09 L 53$ & & 31.00 & & & \\
\hline $199-N-81$ & $11 / 12 / 93$ & B09L54 & & 32.00 & & & \\
\hline
\end{tabular}


Table 4-4. Constituents with at Least One Detected Value for the 100-N RCRA Sites Data for Reporting Period October I through December 31, 1993. (sheet 3 of 11 )

\begin{tabular}{|c|c|c|c|c|c|c|}
\hline $\begin{array}{l}\text { Well } \\
\text { Nane }\end{array}$ & $\begin{array}{c}\text { Collection } \\
\text { Date }\end{array}$ & $\begin{array}{l}\text { Semple } \\
\text { Number }\end{array}$ & $\begin{array}{r}\text { FCADMIU } \\
34 / \text { ppb } \\
4.7 / 10 \mathrm{i}\end{array}$ & $\begin{array}{c}\text { CALCIUM } \\
34 / \mathrm{ppb} \\
20.9 /\end{array}$ & $\begin{array}{l}\text { FCALCIU } \\
34 / \mathrm{ppb} \\
20.9 \%\end{array}$ & $\begin{array}{c}\text { CS }-137 \\
140 / P C i / L \\
. / 200\end{array}$ \\
\hline $199-N-14$ & $11 / 16 / 93$ & $809 \mathrm{~K} \times 0$ & & $26000.00 \mathrm{~B}$ & & $2.52 \mathrm{U}$ \\
\hline $199-N-14$ & $11 / 16 / 93$ & $809 \mathrm{~K} \times 4$ & $4.70 \mathrm{U}$ & & $26000.00 \mathrm{~B}$ & \\
\hline $199-N-2$ & $11 / 09 / 93$ & 809kX5 & & 33000.00 & & $.89 \mathrm{U}$ \\
\hline $199-N-2$ & $11 / 09 / 93$ & $809 \mathrm{k} \times 9$ & $4.70 \mathrm{U}$ & & 33000.00 & \\
\hline $199-N-26$ & $8 / 11 / 93$ & 8090Y2 & & 44000.00 & & \\
\hline $199-N-26$ & $8 / 11 / 93$ & $8090 Y 3$ & $2.00 \mathrm{U}$ & & 41000.00 & \\
\hline $199-N-3$ & $11 / 09 / 93$ & BO9KYO & & 120000.00 & & $1.39 \mathrm{U}$ \\
\hline $199-N-3$ & $11 / 09 / 93$ & B09KY4 & $4.70 \mathrm{U}$ & & 120000.00 & \\
\hline $199-N-34$ & $11 / 11 / 93$ & B09KY5 & & $48000.00 \mathrm{BQ}$ & & $4.59 \mathrm{U}$ \\
\hline $199-N-34$ & $11 / 11 / 93$ & 809KY9 & $4.70 \mathrm{U}$ & & $43000.00 \mathrm{BO}$ & \\
\hline $199-N-57$ & $11 / 12 / 93$ & B09KZO & & $91000.00 \mathrm{~F}$ & & -.52 UF \\
\hline $199-N-59$ & $10 / 04 / 93$ & B090L3 & & 18000.00 & & \\
\hline $199-N-59$ & $10 / 04 / 93$ & B090L7 & $4.70 \mathrm{U}$ & & 16000.00 & \\
\hline $199-N-67$ & $11 / 16 / 93$ & B09K25 & & $78000.00 \mathrm{BF}$ & & -1.08 UF \\
\hline $199-N-69$ & $11 / 09 / 93$ & BOSLOO & & 24000.00 & & $3.11 \mathrm{U}$ \\
\hline $199-N-69$ & $11 / 09 / 93$ & B09LO4 & $4.70 \mathrm{U}$ & & 17000.00 & \\
\hline $199-N-71$ & $11 / 12 / 93$ & BO9LC3 & & 16000.00 & & \\
\hline $199-N-71$ & $11 / 12 / 93$ & B09LC7 & $4.70 \mathrm{U}$ & & 15000.00 & \\
\hline $199-N-72$ & $11 / 12 / 93$ & BO9LC8 & & 26000.00 & & \\
\hline $199-N-72$ & $11 / 12 / 93$ & BO9LC9 & $4.70 \mathrm{U}$ & & 26000.00 & \\
\hline $199-N-73$ & $11 / 12 / 93$ & BOSLDO & & 21000.00 & & \\
\hline $199-N-73$ & $11 / 12 / 93$ & BO9LD1 & $4.70 \mathrm{U}$ & & 21000.00 & \\
\hline $\begin{array}{l}199-N-75 \\
199-N-75\end{array}$ & $\begin{array}{l}11 / 11 / 93 \\
11 / 11 / 93\end{array}$ & $\begin{array}{l}\text { BOPLOS } \\
\text { BOSLOS }\end{array}$ & $4.70 \mathrm{U}$ & $27000.00 \mathrm{BO}$ & $27000.00 \mathrm{BO}$ & $-.40 \mathrm{U}$ \\
\hline $199-N-76$ & $11 / 11 / 93$ & BO9L 10 & & 28000.00 BO & & $4.42 \mathrm{U}$ \\
\hline $199-N-76$ & $11 / 11 / 93$ & B09L 14 & $4.70 \mathrm{U}$ & & $28000.00 \mathrm{BO}$ & \\
\hline $199-N-77$ & $11 / 12 / 93$ & 809LD2 & & 26000.00 & & \\
\hline $199-N-77$ & $11 / 12 / 93$ & B09LD3 & $4.70 \mathrm{U}$ & & 25000.00 & \\
\hline $\begin{array}{l}199-N-81 \\
199-N-81\end{array}$ & $\begin{array}{l}11 / 12 / 93 \\
11 / 12 / 93\end{array}$ & $\begin{array}{l}B 09 L 45 \\
B 09 L 46\end{array}$ & & $\begin{array}{l}37000.00 \\
38000.00\end{array}$ & & $\begin{array}{c}7.430 \\
-2.51 \text { uQ }\end{array}$ \\
\hline $\begin{array}{l}199-N-81 \\
199-N-81\end{array}$ & $\begin{array}{l}11 / 12 / 93 \\
11 / 12 / 93\end{array}$ & $\begin{array}{l}809153 \\
809 L 54\end{array}$ & $\begin{array}{l}4.70 \mathrm{U} \\
4.70 \mathrm{U}\end{array}$ & & $\begin{array}{l}36000.00 \\
36000.00\end{array}$ & \\
\hline
\end{tabular}

\begin{tabular}{|c|c|c|c|c|c|c|}
\hline $\begin{array}{l}\text { Well } \\
\text { Name }\end{array}$ & $\begin{array}{c}\text { Collection } \\
\text { Date }\end{array}$ & $\begin{array}{l}\text { Sample } \\
\text { Number }\end{array}$ & $\begin{array}{c}\text { CHLARID } \\
124 / \mathrm{ppb} \\
82.5 / 250000 \mathrm{~s}\end{array}$ & $\begin{array}{l}\text { CHLFORM } \\
25 / \mathrm{ppb} \\
.043 / .\end{array}$ & $\begin{array}{c}\text { CHROMUM } \\
34 / \text { PPD } \\
5.42 / 50 \mathrm{i}\end{array}$ & $\begin{array}{l}\text { FCHROMI } \\
34 / \mathrm{ppb} \\
5.42 / 50 \mathrm{i}\end{array}$ \\
\hline $199-N-14$ & $11 / 16 / 93$ & BOQKXO & 1700.00 & & $5.42 \mathrm{U}$ & \\
\hline $199-N-14$ & $11 / 16 / 93$ & B09KX4 & & & & $5.42 \mathrm{U}$ \\
\hline $199-N-2$ & $11 / 09 / 93$ & $809 \mathrm{k} \times 5$ & 1500.00 & & $7.00 \mathrm{~L}$ & \\
\hline $199-N-2$ & $11 / 09 / 93$ & B09KX9 & & & & $5.42 \mathrm{U}$ \\
\hline $199-N-26$ & $8 / 11 / 93$ & B090Y2 & & & 45.00 & \\
\hline $199-N-26$ & $8 / 11 / 93$ & B090Y3 & & & & $6.00 \mathrm{U}$ \\
\hline $199-N-3$ & $11 / 09 / 93$ & B09KYO & $17000.00 \mathrm{D}$ & & 26.00 & \\
\hline $199-N-3$ & $11 / 09 / 93$ & B09KY4 & & & & $9.90 \mathrm{~L}$ \\
\hline $199-N-34$ & $11 / 11 / 93$ & B09KY5 & $3600.00 \mathrm{~B}$ & & 63.00 & \\
\hline $199-N-34$ & $11 / 11 / 93$ & B09KY9 & & & & $7.20 \mathrm{~L}$ \\
\hline $199-N-57$ & $11 / 12 / 93$ & 809K20 & $19000.00 \mathrm{DF}$ & & $110.00 \mathrm{~F}$ & \\
\hline $199-N-59$ & $10 / 04 / 93$ & B090L3 & 5100.00 & 1.70 & 360.00 & \\
\hline $199-N-59$ & $10 / 04 / 93$ & B090L7 & & & & $7.60 \mathrm{~L}$ \\
\hline
\end{tabular}


Table 4-4. Constituents with at Least One Detected Value for the 100-N RCRA Sites Data for Reporting Period October 1 through December 31, 1993. (sheet 4 of 11 )

\begin{tabular}{|c|c|c|c|c|c|c|}
\hline $\begin{array}{l}\text { Well } \\
\text { Name }\end{array}$ & $\begin{array}{l}\text { Collection } \\
\text { Date }\end{array}$ & $\begin{array}{l}\text { Sample } \\
\text { Number }\end{array}$ & $\begin{array}{c}\text { CHLOR 1D } \\
124 / \text { ppb } \\
82.5 / 250000 \text { s }\end{array}$ & $\begin{array}{l}\text { CHLFORM } \\
25 / \mathrm{ppb} \\
.043 \% .\end{array}$ & $\begin{array}{c}\text { CHROMUM } \\
34 / \mathrm{ppb} \\
5.42 / 50 \mathrm{i}\end{array}$ & $\begin{array}{l}\text { FCHROMI } \\
34 / \mathrm{ppt} \\
5.42 / 50 \mathrm{i}\end{array}$ \\
\hline $199-N-67$ & $11 / 16 / 93$ & B09K25 & $1900.00 \mathrm{~F}$ & & $1100.00 \mathrm{~F}$ & \\
\hline $\begin{array}{l}199-N-69 \\
199-N-69\end{array}$ & $\begin{array}{l}11 / 09 / 93 \\
11 / 09 / 93\end{array}$ & BO9L04 & & & & $5.42 \mathrm{U}$ \\
\hline $199-N-71$ & $11 / 12 / 93$ & B09LC3 & 3200.00 & 6.20 & 56.00 & \\
\hline $199-N-71$ & $11 / 12 / 93$ & B09LC7 & & & & $5.42 \mathrm{U}$ \\
\hline $199-N-72$ & $11 / 12 / 93$ & B09LC8 & $9500.00 \mathrm{D}$ & $62.00 \mathrm{D}$ & $9.50 \mathrm{~L}$ & \\
\hline $199-N-72$ & $11 / 12 / 93$ & BOALC9 & & & & $5.42 \mathrm{U}$ \\
\hline $199 \cdot N-73$ & $11 / 12 / 93$ & BOQLDO & 5300.00 & 10.00 & 29.00 & \\
\hline $199-N-73$ & $11 / 12 / 93$ & B09LD1 & & & & $5.42 \mathrm{U}$ \\
\hline $199-N-75$ & $11 / 11 / 93$ & BO9L05 & $1400.00 \mathrm{~B}$ & & $9.50 \mathrm{~L}$ & \\
\hline $199-N-75$ & $11 / 11 / 93$ & BO9LO9 & & & & $5.42 \mathrm{U}$ \\
\hline $199-N-76$ & $11 / 11 / 93$ & B09L 10 & $1900.00 \mathrm{~B}$ & & 28.00 & \\
\hline $199-N-76$ & $11 / 11 / 93$ & B09L 14 & & & & $5.42 \mathrm{U}$ \\
\hline $199-N-77$ & $11 / 12 / 93$ & B09LD2 & $11000.00 \mathrm{D}$ & 83.000 & $5.42 \mathrm{U}$ & \\
\hline $199-N-77$ & $11 / 12 / 93$ & B09LD3 & & & & $5.42 \mathrm{U}$ \\
\hline $199-N-81$ & $11 / 12 / 93$ & $B 09 L 45$ & 3700.00 & & 120.00 & \\
\hline $199-N-81$ & $11 / 12 / 93$ & B09L46 & 3700.00 & & 150.00 & \\
\hline $199-N-81$ & $11 / 12 / 93$ & $B 09153$ & & & & 5.42 \\
\hline $199-N-81$ & $11 / 12 / 93$ & B09L54 & & & & 5.42 \\
\hline
\end{tabular}

\begin{tabular}{|c|c|c|c|c|c|c|c|c|c|}
\hline $\begin{array}{l}\text { Well } \\
\text { Name }\end{array}$ & $\begin{array}{c}\text { Collection } \\
\text { Date }\end{array}$ & $\begin{array}{l}\text { Sample } \\
\text { Number }\end{array}$ & $\begin{array}{l}\text { COBALT } \\
34 / \mathrm{ppb} \\
4.05 /\end{array}$ & & $\begin{array}{c}\text { FCOBALT } \\
34 / \text { Ppb } \\
4.05 / .\end{array}$ & & $\begin{array}{c}\text { co-60 } \\
140 / \mathrm{pCi} / \mathrm{L} \\
. / 100\end{array}$ & $\begin{array}{c}\text { COPPER } \\
34 / P P b \\
2.65 / 1000\end{array}$ & \\
\hline$\cdots$ & $\cdots$ & $\ldots \ldots$ & $\ldots$ & $\cdots$ & & $\cdots$ & $\cdots \cdots$ & - & - \\
\hline $\begin{array}{l}199-N-14 \\
199-N-14\end{array}$ & $\begin{array}{l}11 / 16 / 93 \\
11 / 16 / 93\end{array}$ & $\begin{array}{l}809 \times \times 0 \\
809 \times \times 4\end{array}$ & 4.05 & $U$ & 4.05 & & $-3.82 U$ & 2.651 & \\
\hline $\begin{array}{l}199-N-2 \\
199-N-2\end{array}$ & $\begin{array}{l}11 / 09 / 93 \\
11 / 09 / 93\end{array}$ & $\begin{array}{l}809 K \times 5 \\
809 K \times 9\end{array}$ & 4.05 & U & 4.05 & $U$ & $1.63 \mathrm{U}$ & 3.601 & $\mathrm{~L}$ \\
\hline $\begin{array}{l}199-N-26 \\
199-N-26\end{array}$ & $\begin{array}{l}8 / 11 / 93 \\
8 / 11 / 93\end{array}$ & $\begin{array}{l}\text { B090Y2 } \\
\text { 8090Y3 }\end{array}$ & 5.00 & $U$ & 5.00 & $U$ & & $6.00 \mathrm{~L}$ & $\mathrm{U}$ \\
\hline $\begin{array}{l}199-N-3 \\
199-N-3\end{array}$ & $\begin{array}{l}11 / 09 / 93 \\
11 / 09 / 93\end{array}$ & $\begin{array}{l}\text { BO9KYO } \\
\text { B09KY4 }\end{array}$ & 4.05 & $U$ & 4.05 & $U$ & $5.28 \mathrm{U}$ & 3.601 & $L$ \\
\hline $\begin{array}{l}199-N-34 \\
199-N-34\end{array}$ & $\begin{array}{l}11 / 11 / 93 \\
11 / 11 / 93\end{array}$ & $\begin{array}{l}\text { B09KY5 } \\
\text { 809KY9 }\end{array}$ & 4.05 & va & 4.05 & UQ & $-2.91 U$ & 25.00 & \\
\hline $\begin{array}{l}199-N-57 \\
199-N-59\end{array}$ & $\begin{array}{l}11 / 12 / 93 \\
10 / 04 / 93\end{array}$ & $\begin{array}{l}\text { B09K20 } \\
\text { B090L } 3\end{array}$ & $\begin{array}{l}4.05 \\
4.05\end{array}$ & $\begin{array}{l}\text { UF } \\
\text { U }\end{array}$ & & & 1.03 UF & $\begin{array}{r}88.00 \\
9.20\end{array}$ & $\begin{array}{l}B F \\
L\end{array}$ \\
\hline $199-N-59$ & $10 / 04 / 93$ & B090L7 & & & 4.05 & $U$ & & & \\
\hline $\begin{array}{l}199-N-67 \\
199-N-69\end{array}$ & $\begin{array}{l}11 / 16 / 93 \\
11 / 09 / 93\end{array}$ & $\begin{array}{l}\text { BO9K25 } \\
\text { B09L00 }\end{array}$ & $\begin{array}{r}62.00 \\
5.90\end{array}$ & $\mathbf{F}$ & & & $\begin{array}{c}22.60 \mathrm{~F} \\
9.26\end{array}$ & $\begin{array}{r}330.00 \\
6.00\end{array}$ & F \\
\hline $199 \cdot N-69$ & $11 / 09 / 93$ & BO9LOL & & & 4.05 & $U$ & & & \\
\hline $\begin{array}{l}199-N-71 \\
199-N-71\end{array}$ & $\begin{array}{l}11 / 12 / 93 \\
11 / 12 / 93\end{array}$ & $\begin{array}{l}\text { B09LC3 } \\
\text { B09LC7 }\end{array}$ & 4.05 & $U$ & 4.05 & $\mathrm{U}$ & & 8.001 & L8 \\
\hline $\begin{array}{l}199-N-72 \\
199-N-72\end{array}$ & $\begin{array}{l}11 / 12 / 93 \\
11 / 12 / 93\end{array}$ & $\begin{array}{l}\text { BO9LC8 } \\
\text { BO9LC9 }\end{array}$ & 4.05 & U & 4.05 & $U$ & & 4.601 & LB \\
\hline $\begin{array}{l}199-N-73 \\
199-N-73\end{array}$ & $\begin{array}{l}11 / 12 / 93 \\
11 / 12 / 93\end{array}$ & $\begin{array}{l}\text { BO9LDO } \\
\text { BO9LD }\end{array}$ & 4.05 & u & 4.05 & $U$ & & 4.601 & LB \\
\hline $\begin{array}{l}199-N-75 \\
199-N-75\end{array}$ & $\begin{array}{l}11 / 11 / 93 \\
11 / 11 / 93\end{array}$ & $\begin{array}{l}\text { B09L05 } \\
\text { B09L09 }\end{array}$ & 4.05 & ve & 4.05 & Un & $-.82 \mathrm{U}$ & 2.651 & $u$ \\
\hline $\begin{array}{l}199-N-76 \\
199-N-76\end{array}$ & $\begin{array}{l}11 / 11 / 93 \\
11 / 11 / 93\end{array}$ & $\begin{array}{l}\text { BO9L } 10 \\
\text { BO9L } 14\end{array}$ & 4.05 & vo & 4.05 & vo & $-1.22 \mathrm{U}$ & 2.65 & $\mathbf{U}$ \\
\hline $\begin{array}{l}199-N-77 \\
199-N-77\end{array}$ & $\begin{array}{l}11 / 12 / 93 \\
11 / 12 / 93\end{array}$ & $\begin{array}{l}\text { B09LD2 } \\
\text { B09LD3 }\end{array}$ & 4.05 & $U$ & 4.05 & U & & 2.651 & $\mathbf{U}$ \\
\hline
\end{tabular}


Table 4-4. Constituents with at Least One Detected Value for the 100-N RCRA Sites Data for Reporting Period October 1 through December 31, 1993.

(sheet 5 of 11 )

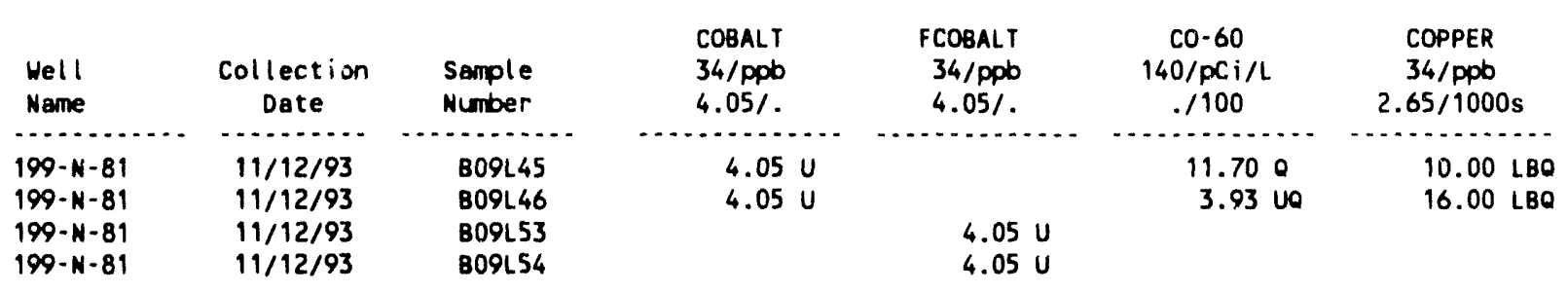

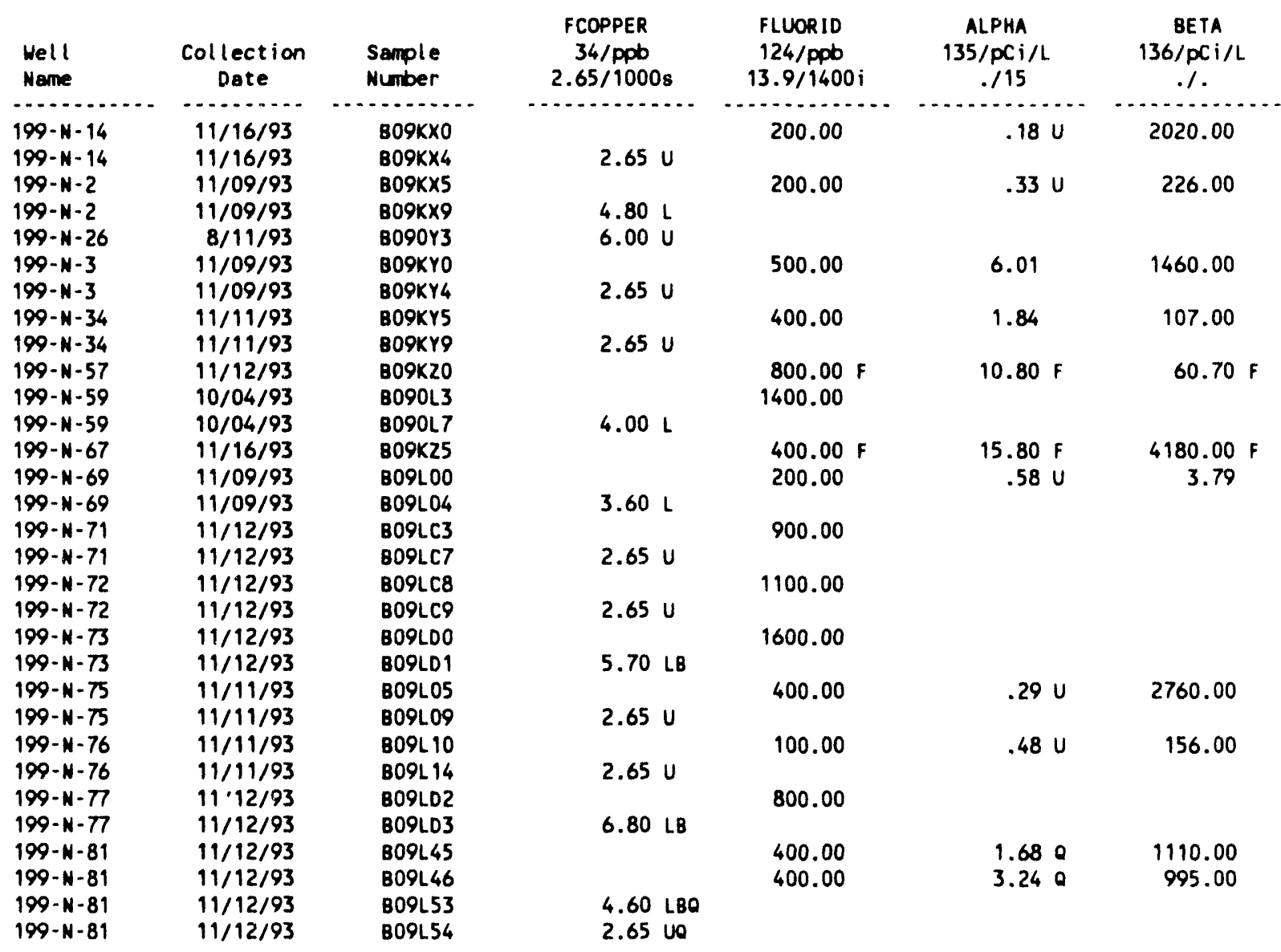

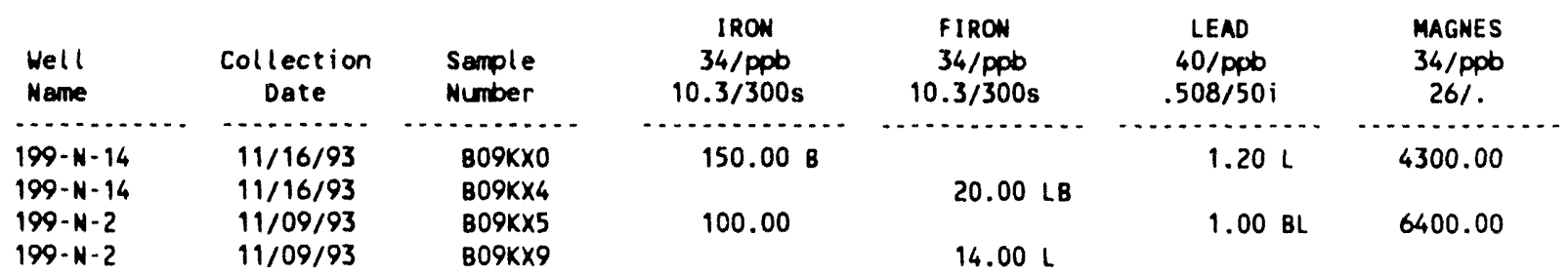


Table 4-4. Constituents with at Least One Detected Value for the 100-N RCRA Sites Data for Reporting Period October 1 through December 31, 1993. (sheet 6 of 11 )

\begin{tabular}{|c|c|c|c|c|c|c|}
\hline $\begin{array}{l}\text { Well } \\
\text { Name }\end{array}$ & $\begin{array}{c}\text { Collection } \\
\text { Date }\end{array}$ & $\begin{array}{l}\text { Semple } \\
\text { Number }\end{array}$ & $\begin{array}{c}\text { IRON } \\
34 / \mathrm{ppb} \\
10.3 / 300 \mathrm{~s}\end{array}$ & $\begin{array}{c}\text { FIRON } \\
34 / \mathrm{ppb} \\
10.3 / 300 \mathrm{~s}\end{array}$ & $\begin{array}{l}\text { LEAD } \\
40 / \mathrm{ppb} \\
.508 / 50 \mathrm{i}\end{array}$ & $\begin{array}{c}\text { MAGNES } \\
34 / \text { PPD } \\
26 \%\end{array}$ \\
\hline $\begin{array}{l}199-N-26 \\
199-N-26\end{array}$ & $\begin{array}{l}8 / 11 / 93 \\
8 / 11 / 93\end{array}$ & $\begin{array}{l}\text { B090Y2 } \\
\text { B090Y3 }\end{array}$ & 590.000 & 10.00 vo & & 8800.00 \\
\hline $\begin{array}{l}199-N-3 \\
199-N-3\end{array}$ & $\begin{array}{l}11 / 09 / 93 \\
11 / 09 / 93\end{array}$ & $\begin{array}{l}\text { B09KYO } \\
\text { B09KY4 }\end{array}$ & 1300.00 & 26.00 & $3.10 \mathrm{BL}$ & 21000.00 \\
\hline $\begin{array}{l}199-N-34 \\
199-N-34\end{array}$ & $\begin{array}{l}11 / 11 / 93 \\
11 / 11 / 93\end{array}$ & $\begin{array}{l}\text { B09KY5 } \\
\text { B09KY9 }\end{array}$ & $7700.00 \mathrm{~B}$ & $22.00 \mathrm{~B}$ & $3.00 \mathrm{~L}$ & 9800.00 \\
\hline $\begin{array}{l}199-N-57 \\
199-N-59\end{array}$ & $\begin{array}{l}11 / 12 / 93 \\
10 / 04 / 93\end{array}$ & $\begin{array}{l}\text { B09K20 } \\
\text { B090L3 }\end{array}$ & $\begin{array}{c}54000.00 \text { BOF } \\
2800.00\end{array}$ & & $\begin{array}{r}39.00 \mathrm{~F} \\
1.40 \mathrm{BL}\end{array}$ & $\begin{array}{r}20000.00 F \\
3400.00\end{array}$ \\
\hline $199-N-59$ & $10 / 04 / 93$ & 809017 & & 44.00 & & \\
\hline $\begin{array}{l}199-N-67 \\
199-N-69\end{array}$ & $\begin{array}{l}11 / 16 / 93 \\
11 / 09 / 93\end{array}$ & $\begin{array}{l}\text { 809K25 } \\
809 L 00\end{array}$ & $\begin{array}{c}210000.00 \mathrm{BF} \\
120.00\end{array}$ & & $\begin{array}{r}280.00 \mathrm{~F} \\
3.50 \mathrm{BL}\end{array}$ & $\begin{array}{r}38000.00 \\
4800.00\end{array}$ \\
\hline $199-N-69$ & $11 / 09 / 93$ & B09LOL & & 22.00 & & \\
\hline $\begin{array}{l}199-N-71 \\
199-N-71\end{array}$ & $\begin{array}{l}11 / 12 / 93 \\
11 / 12 / 93\end{array}$ & $\begin{array}{l}\text { Bo9lC3 } \\
\text { B09LC7 }\end{array}$ & $300.00 \mathrm{BQ}$ & $29.00 \mathrm{BQ}$ & $.51 \mathrm{U}$ & 4500.00 \\
\hline $\begin{array}{l}199-N-72 \\
199-N-72\end{array}$ & $\begin{array}{l}11 / 12 / 93 \\
11 / 12 / 93\end{array}$ & $\begin{array}{l}\text { BO9LC8 } \\
\text { BO9LC9 }\end{array}$ & 91.0080 & 17.00 LBQ & $1.30 \mathrm{~L}$ & 5400.00 \\
\hline $\begin{array}{l}199-N-73 \\
199-N-73\end{array}$ & $\begin{array}{l}11 / 12 / 93 \\
11 / 12 / 93\end{array}$ & $\begin{array}{l}\text { BOSLDO } \\
\text { B09LD1 }\end{array}$ & $450.00 \mathrm{BO}$ & $27.00 \mathrm{BO}$ & $.51 \mathrm{U}$ & 4300.00 \\
\hline $\begin{array}{l}199-N-75 \\
199 \cdot N-75\end{array}$ & $\begin{array}{l}11 / 11 / 93 \\
11 / 11 / 93\end{array}$ & $\begin{array}{l}\text { BOSLOS } \\
\text { B09L09 }\end{array}$ & $35.00 \mathrm{~B}$ & $10.30 \mathrm{U}$ & $.51 \mathrm{U}$ & 4400.00 \\
\hline $\begin{array}{l}199-N-76 \\
199-N-76\end{array}$ & $\begin{array}{l}11 / 11 / 93 \\
11 / 11 / 93\end{array}$ & $\begin{array}{l}\text { B09L10 } \\
\text { BOSL } 14\end{array}$ & $340.00 \mathrm{~B}$ & $12.00 \mathrm{LB}$ & $.90 \mathrm{~L}$ & 4600.00 \\
\hline $\begin{array}{l}199-\mathrm{N}-77 \\
199-\mathrm{N}-77\end{array}$ & $\begin{array}{l}11 / 12 / 93 \\
11 / 12 / 93\end{array}$ & $\begin{array}{l}\text { BO9LD2 } \\
\text { B09LD3 }\end{array}$ & $52.00 \mathrm{BO}$ & $13.00 \mathrm{LBO}$ & $1.10 \mathrm{~L}$ & 5700.00 \\
\hline $\begin{array}{l}199-N-81 \\
199-N-81\end{array}$ & $\begin{array}{l}11 / 12 / 93 \\
11 / 12 / 93\end{array}$ & $\begin{array}{l}B 09 L 45 \\
809 L 46\end{array}$ & $\begin{array}{l}3600.00 \mathrm{BQ} \\
3800.00 \mathrm{BQ}\end{array}$ & & $\begin{array}{l}1.10 \mathrm{LQ} \\
1.80 \mathrm{LQ}\end{array}$ & $\begin{array}{l}7400.00 \\
7600.00\end{array}$ \\
\hline $\begin{array}{l}199-N-81 \\
199-N-81\end{array}$ & $\begin{array}{l}11 / 12 / 93 \\
11 / 12 / 93\end{array}$ & $\begin{array}{l}\text { B09L53 } \\
\text { B09L54 }\end{array}$ & & $\begin{array}{l}18.00 \text { LBO } \\
14.00 \text { LBO }\end{array}$ & & \\
\hline
\end{tabular}

\begin{tabular}{|c|c|c|c|c|c|c|}
\hline $\begin{array}{l}\text { Well } \\
\text { Mame }\end{array}$ & $\begin{array}{c}\text { Collection } \\
\text { Date }\end{array}$ & $\begin{array}{l}\text { Sample } \\
\text { Number }\end{array}$ & $\begin{array}{c}\text { FMAGNES } \\
34 / \text { ppb } \\
26 / .\end{array}$ & $\begin{array}{c}\text { MANGESE } \\
34 / \mathrm{ppb} \\
1.35 / 50 \mathrm{~s}\end{array}$ & $\begin{array}{c}\text { FMANGAN } \\
34 / \mathrm{ppb} \\
1.35 / 50 \mathrm{~s}\end{array}$ & $\begin{array}{l}\text { NICKEL } \\
34 / \text { ppb } \\
17.9 / .\end{array}$ \\
\hline (n)........... & (1) & and & & 1 & & \\
\hline $199-N-14$ & $11 / 16 / 93$ & $809 \mathrm{~K} \times 0$ & & $4.10 \mathrm{~L}$ & & $17.90 \mathrm{U}$ \\
\hline $\begin{array}{l}199-N-14 \\
199-N-2\end{array}$ & $\begin{array}{l}11 / 16 / 93 \\
11 / 09 / 93\end{array}$ & $\begin{array}{l}\text { 809KX4 } \\
\text { 809KX5 }\end{array}$ & 4200.00 & $3.20 \mathrm{~L}$ & $1.35 \mathrm{U}$ & $17.90 \mathrm{U}$ \\
\hline $199-N-2$ & $11 / 09 / 93$ & $809 \mathrm{~K} \times 9$ & 6500.00 & & $1.35 \mathrm{U}$ & \\
\hline $199-N-26$ & $8 / 11 / 93$ & B090Y2 & & 18.00 & & 35.00 \\
\hline $\begin{array}{l}199-N-26 \\
199-N-3\end{array}$ & $\begin{array}{r}8 / 11 / 93 \\
11 / 09 / 93\end{array}$ & $\begin{array}{l}\text { B090Y3 } \\
\text { B09KYO }\end{array}$ & 8100.00 & 44.00 & $3.00 \mathrm{U}$ & 17.90 \\
\hline $199-N-3$ & $11 / 09 / 93$ & $809 K Y 4$ & 21000.00 & & $1.50 \mathrm{~L}$ & (1) \\
\hline $199-N-34$ & $11 / 11 / 93$ & B09KY5 & & 350.00 & & 58.00 \\
\hline $199-N-34$ & $11 / 11 / 93$ & B09KY9 & 7900.00 & & $2.50 \mathrm{~L}$ & \\
\hline $199-N-57$ & $11 / 12 / 93$ & B09K20 & & $540.00 \mathrm{~F}$ & & $62.00 \mathrm{~F}$ \\
\hline $199 \cdot N-59$ & $10 / 04 / 93$ & $8090 \mathrm{~L} 3$ & & 44.00 & & 180.00 \\
\hline $199 \cdot N-59$ & $10 / 04 / 93$ & B090L7 & 3100.00 & & $4.30 \mathrm{~L}$ & \\
\hline $199-N-67$ & $11 / 16 / 93$ & B09K25 & & $2700.00 \mathrm{~F}$ & & $680.00 \mathrm{~F}$ \\
\hline $199-N-69$ & $11 / 09 / 93$ & BOSL00 & & $3.30 \mathrm{~L}$ & & $17.90 \mathrm{U}$ \\
\hline $199-N-69$ & $11 / 09 / 93$ & BO9LO4 & 3400.00 & & $1.35 \mathrm{U}$ & \\
\hline $199-N-71$ & $11 / 12 / 93$ & B09LC3 & & $7.70 \mathrm{~L}$ & & $17.90 \mathrm{U}$ \\
\hline $199-N-71$ & $11 / 12 / 93$ & B09LC7 & 4400.00 & & $2.40 \mathrm{~L}$ & \\
\hline $199-N-72$ & $11 / 12 / 93$ & B09LC8 & & $3.40 \mathrm{~L}$ & & $17.90 \mathrm{U}$ \\
\hline
\end{tabular}


Table 4-4. Constituents with at Least One Detected Value for the 100-N RCRA Sites Data for Reporting Period October 1 through December 31, 1993.

(sheet 7 of 11 )

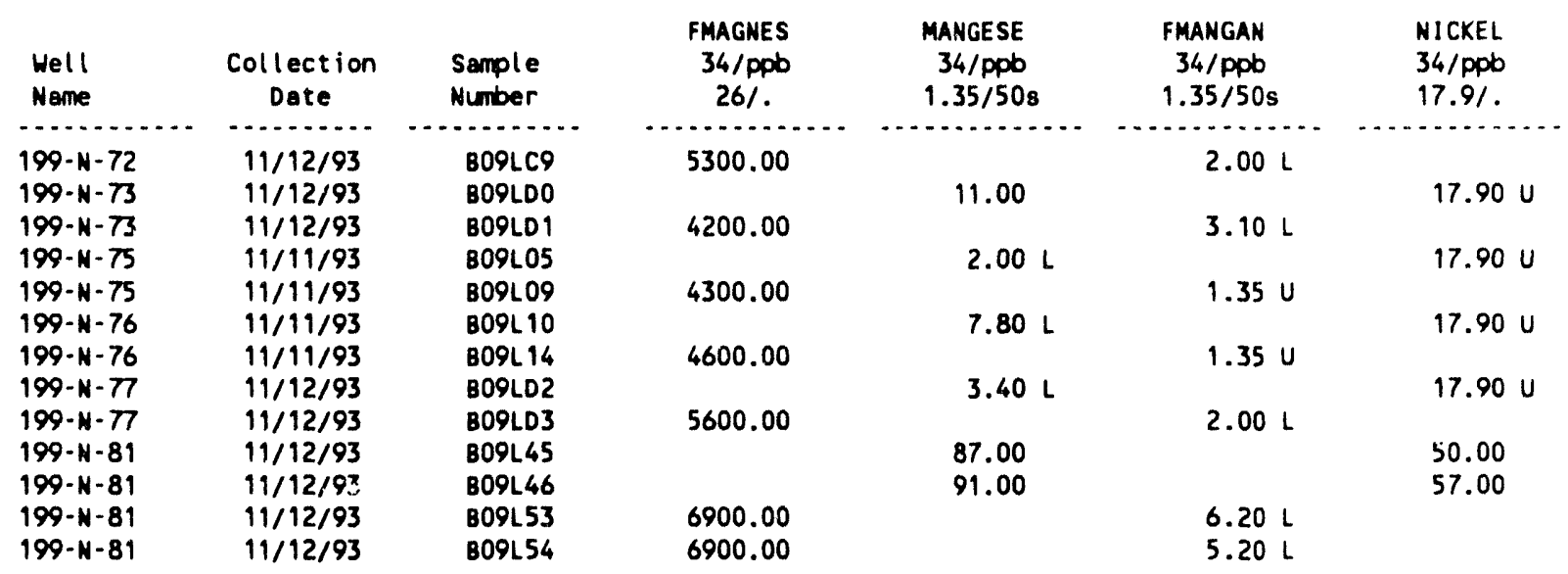

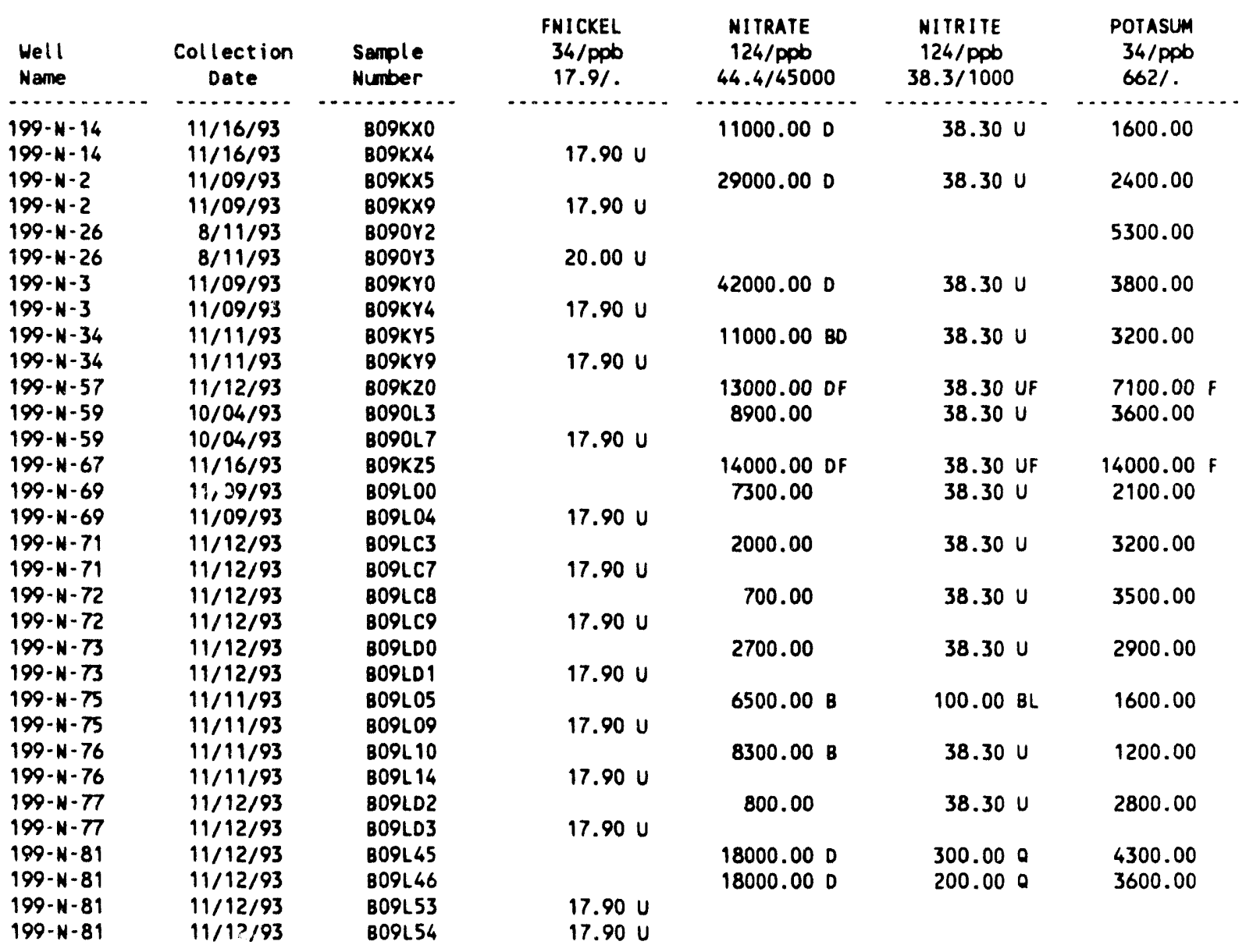


Table 4-4. Constituents with at Least One Detected Value for the 100-N RCRA Sites Data for Reporting Period October 1 through December 31, 1993.

(sheet 8 of 11 )

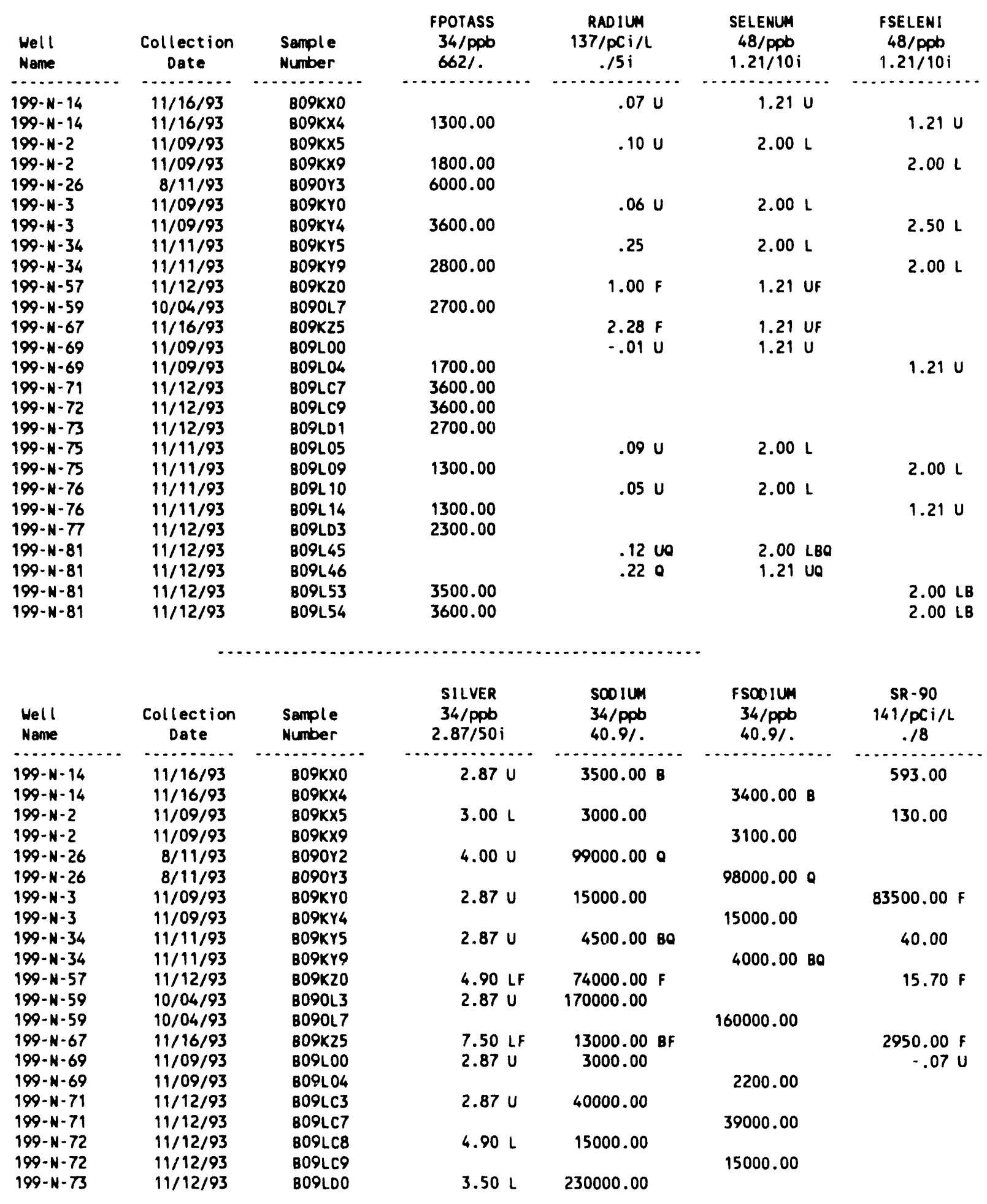


Table 4-4. Constituents with at Least One Detected Value for the 100-N RCRA Sites Data for Reporting Period October 1 through December 31, 1993.

(sheet 9 of 11 )

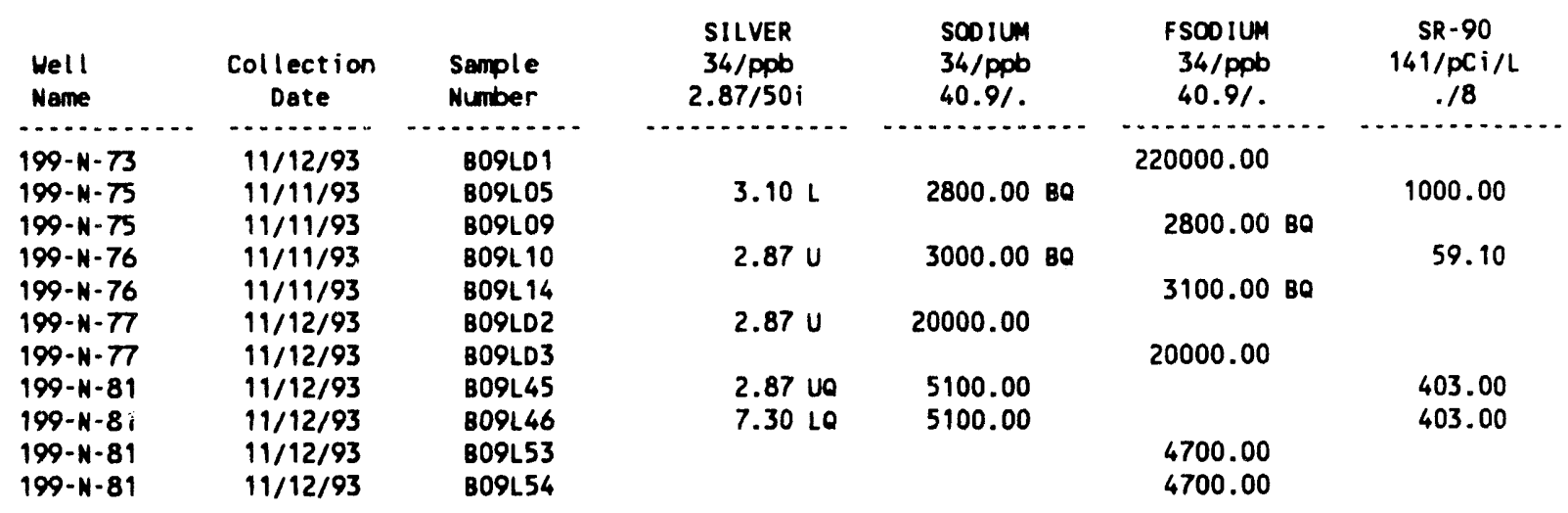

\begin{tabular}{|c|c|c|c|c|c|c|}
\hline $\begin{array}{l}\text { Heil } \\
\text { Name }\end{array}$ & $\begin{array}{c}\text { Collection } \\
\text { Date }\end{array}$ & $\begin{array}{l}\text { Sample } \\
\text { Number }\end{array}$ & $\begin{array}{c}\text { SULFATE } \\
124 / \mathrm{ppb} \\
49.9 / 250000 \mathrm{~s}\end{array}$ & $\begin{array}{l}\text { TIN } \\
34 / \text { ppb } \\
51.1 / .\end{array}$ & $\begin{array}{c}\text { FTIN } \\
34 / \mathrm{ppb} \\
51.1 / .\end{array}$ & $\begin{array}{l}\text { TOLUENE } \\
25 / \text { ppb } \\
.056 / 1000\end{array}$ \\
\hline $199-N-14$ & $11 / 16 / 93$ & B09KXO & $15000.00 \mathrm{D}$ & $51.10 \mathrm{U}$ & $\cdots$ & \\
\hline $199-N-14$ & $11 / 16 / 93$ & B09KX4 & & & $51.10 \mathrm{U}$ & \\
\hline $199-N-2$ & $11 / 09 / 93$ & $809 \times \times 5$ & 14000.000 & $51.10 \mathrm{U}$ & & \\
\hline $199-N-2$ & $11 / 09 / 93$ & B09KXS & & & $51.10 \mathrm{U}$ & \\
\hline $199-N-26$ & $8 / 11 / 93$ & B090Y2 & & $20.00 U$ & & \\
\hline $199-N-26$ & $8 / 11 / 93$ & B090Y3 & & & $20.00 \mathrm{U}$ & \\
\hline $199-N-3$ & $11 / 09 / 93$ & BO9KYO & $150000.00 \mathrm{D}$ & $51.10 \mathrm{U}$ & & \\
\hline $199-N-3$ & $11 / 09 / 93$ & BO9KY4 & & & $51.10 \mathrm{U}$ & \\
\hline $\begin{array}{l}199-N-34 \\
199-N-34\end{array}$ & $\begin{array}{l}11 / 11 / 93 \\
11 / 11 / 93\end{array}$ & $\begin{array}{l}\text { B09KY5 } \\
\text { B09KY9 }\end{array}$ & $58000.00 \mathrm{D}$ & $51.10 \mathrm{va}$ & 51.10 Ua & \\
\hline $199-N-57$ & $11 / 12 / 93$ & BO9K20 & 270000.00 DF & 51.10 UF & & \\
\hline $199-N-59$ & $10 / 04 / 93$ & B090L3 & 190000.000 & $51.10 \mathrm{U}$ & & $.06 \mathrm{U}$ \\
\hline $199-N-59$ & $10 / 04 / 93$ & B090L7 & & & $51.10 \mathrm{U}$ & \\
\hline $199-N-67$ & $11 / 16 / 93$ & $809 \times 25$ & $14000.00 \mathrm{DF}$ & $55.00 \mathrm{LF}$ & & \\
\hline $\begin{array}{l}199-N-69 \\
199-N-69\end{array}$ & $\begin{array}{l}11 / 09 / 93 \\
11 / 09 / 93\end{array}$ & $\begin{array}{l}\text { BO9LOO } \\
\text { BO9LO4 }\end{array}$ & 12000.000 & $51.10 \mathrm{U}$ & 5110 & \\
\hline $199-N-71$ & $11 / 12 / 93$ & BO9LC3 & 24000.000 & $51.10 \mathrm{U}$ & & $.06 \mathrm{BL}$ \\
\hline $199-N-71$ & $11 / 12 / 93$ & BO9LC7 & & & $51.10 \mathrm{U}$ & \\
\hline $\begin{array}{l}199-N-72 \\
199-N-72\end{array}$ & $\begin{array}{l}11 / 12 / 93 \\
11 / 12 / 93\end{array}$ & $\begin{array}{l}\text { BOSLC8 } \\
\text { BO9LC9 }\end{array}$ & $37000.00 \mathrm{D}$ & $51.10 \mathrm{U}$ & $51.10 \mathrm{U}$ & $.06 \mathrm{U}$ \\
\hline $\begin{array}{l}199-H-73 \\
199-H-73\end{array}$ & $\begin{array}{l}11 / 12 / 93 \\
11 / 12 / 93\end{array}$ & $\begin{array}{l}\text { BOSLDO } \\
\text { BOSLD1 }\end{array}$ & $410000.00 \mathrm{D}$ & $51.10 \mathrm{U}$ & $51.10 \mathrm{U}$ & $.06 \mathrm{U}$ \\
\hline $199-N-75$ & $11 / 11 / 93$ & BO9L05 & 11000.000 & $51.10 \mathrm{va}$ & & \\
\hline $199-N-75$ & $11 / 11 / 93$ & B09L09 & & & 51.10 vo & \\
\hline $\begin{array}{l}199-N-76 \\
199-N-76\end{array}$ & $\begin{array}{l}11 / 11 / 93 \\
11 / 11 / 93\end{array}$ & $\begin{array}{l}\text { BO9L } 10 \\
\text { B09L } 14\end{array}$ & 11000.000 & $51.10 \mathrm{ve}$ & 51.10 ve & \\
\hline $\begin{array}{l}199-N-77 \\
199-N-77\end{array}$ & $\begin{array}{l}11 / 12 / 93 \\
11 / 12 / 93\end{array}$ & $\begin{array}{l}\text { BO9LD2 } \\
\text { B09LD } 3\end{array}$ & 31000.000 & $51.10 \mathrm{U}$ & $51.10 \mathrm{U}$ & $.06 v$ \\
\hline $\begin{array}{l}199-H-81 \\
199-H-81\end{array}$ & $11 / 12 / 93$ & $809 L 45$ & 35000.000 & $51.10 \mathrm{U}$ & & \\
\hline $\begin{array}{l}199-N-81 \\
199-N-81\end{array}$ & $11 / 12 / 93$ & $\begin{array}{l}\text { B09L46 } \\
\text { B09L53 }\end{array}$ & & 31.100 & $51.10 \mathrm{U}$ & \\
\hline $199-N-81$ & $11 / 12 / 93$ & B09L54 & & & $51.10 \mathrm{U}$ & \\
\hline
\end{tabular}


Table 4-4. Constituents with at Least One Detected Value for the 100-N RCRA Sites Data for Reporting Period October 1 through December 31, 1993.

(sheet 10 of 11 )

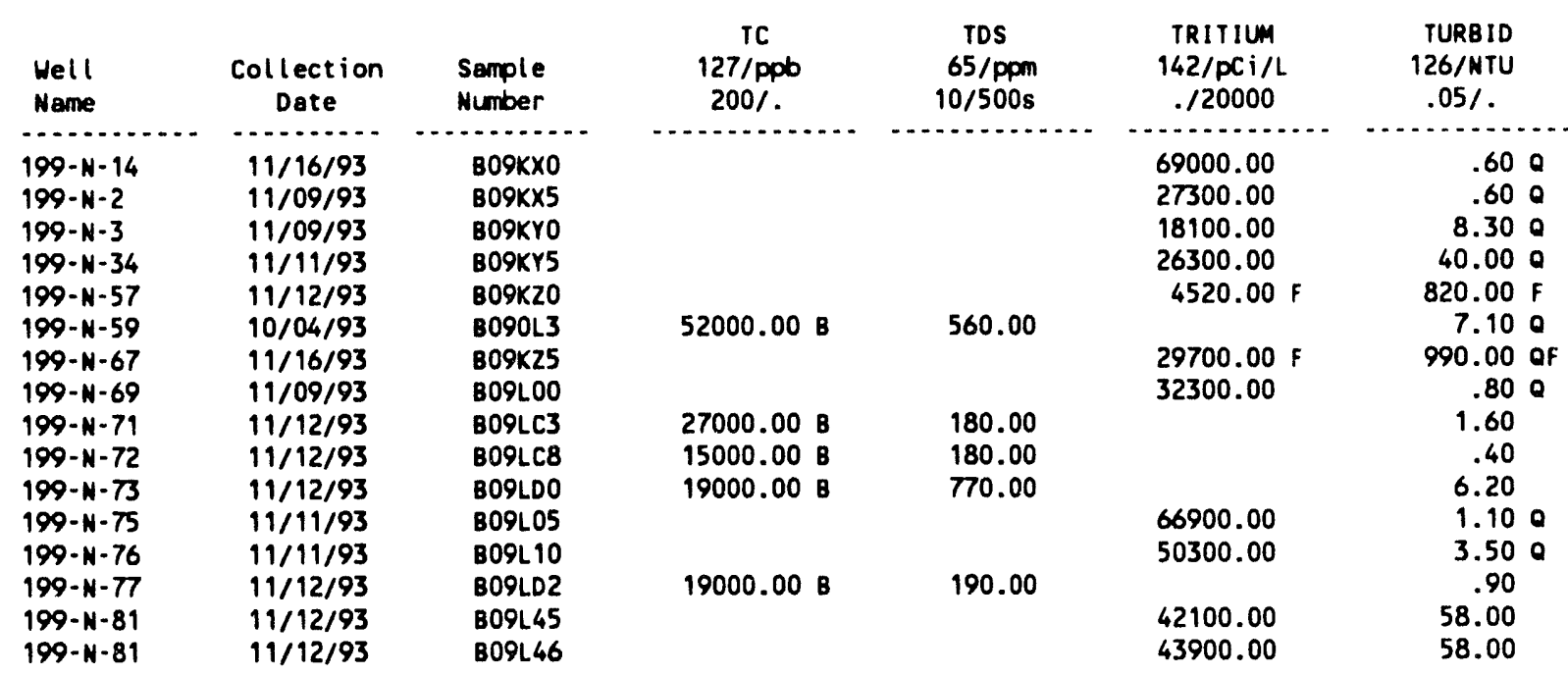

\begin{tabular}{|c|c|c|c|c|c|c|}
\hline $\begin{array}{l}\text { Well } \\
\text { Name }\end{array}$ & $\begin{array}{l}\text { Collection } \\
\text { Dete }\end{array}$ & $\begin{array}{l}\text { Sample } \\
\text { Number }\end{array}$ & $\begin{array}{c}\text { VANADUM } \\
34 / \text { PpD } \\
3.84 / .\end{array}$ & $\begin{array}{l}\text { FVANADI } \\
34 / \mathrm{ppb} \\
3.84 / .\end{array}$ & $\begin{array}{c}\text { 2INC } \\
34 / \mathrm{ppb} \\
3.44 / 5000 \mathrm{~s}\end{array}$ & $\begin{array}{c}\text { FZ1NC } \\
34 / \mathrm{ppb} \\
3.44 / 5000 \mathrm{~s}\end{array}$ \\
\hline $0_{-4}$ & ; & poovyo & 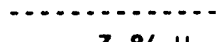 & & . & \\
\hline $\begin{array}{l}199-N-14 \\
199-N-14\end{array}$ & $11 / 16 / 93$ & $\begin{array}{l}\text { B09KX0 } \\
\text { B09KX4 }\end{array}$ & $3.84 \mathrm{U}$ & & $3.44 \mathrm{U}$ & \\
\hline $\begin{array}{l}199-N-14 \\
199-N-2\end{array}$ & $\begin{array}{l}11 / 16 / 93 \\
11 / 09 / 93\end{array}$ & $\begin{array}{l}\text { 809KX4 } \\
809 \mathrm{~K} \times 5\end{array}$ & $4.50 \mathrm{~L}$ & $7.70 \mathrm{~L}$ & $3.44 \mathrm{U}$ & $2.80 \mathrm{LB}$ \\
\hline $199-N-2$ & $11 / 09 / 93$ & B09KX9 & 桨 & $3.84 \mathrm{U}$ & 0.0 & $3.44 \mathrm{U}$ \\
\hline $199-N-26$ & $8 / 11 / 93$ & B090Y2 & $9.00 \mathrm{U}$ & & 5.00 vo & \\
\hline $199-N-26$ & $8 / 11 / 93$ & B090Y3 & & $9.00 \mathrm{U}$ & & 5.00 va \\
\hline $199-N-3$ & $11 / 09 / 93$ & B09KYO & $4.60 \mathrm{~L}$ & & 27.00 & \\
\hline $199-N-3$ & $11 / 09 / 93$ & B09KY4 & & $6.90 \mathrm{~L}$ & & 18.00 \\
\hline $199-N-34$ & $11 / 11 / 93$ & B09KY5 & $20.00 \mathrm{~L}$ & & 140.00 & \\
\hline $199-N-34$ & $11 / 11 / 93$ & B09KY9 & & $3.84 \mathrm{U}$ & & 52.00 \\
\hline $199-N-57$ & $11 / 12 / 93$ & 809K20 & $100.00 \mathrm{~F}$ & & $2100.00 \mathrm{~F}$ & \\
\hline $199-N-59$ & $10 / 04 / 93$ & $8090 \mathrm{~L} 3$ & $16.00 \mathrm{~L}$ & & 21.00 & \\
\hline $199-N-59$ & $10 / 04 / 93$ & B090L7 & & $13.00 \mathrm{~L}$ & & $7.70 \mathrm{~L}$ \\
\hline $\begin{array}{l}199-N-67 \\
199-N-69\end{array}$ & $\begin{array}{l}11 / 16 / 93 \\
11 / 09 / 93\end{array}$ & $\begin{array}{l}\text { B09K25 } \\
\text { BO9L00 }\end{array}$ & $\begin{array}{r}270.00 \mathrm{~F} \\
3.84 \mathrm{U}\end{array}$ & & $\begin{array}{c}3000.00 \mathrm{BF} \\
3.44 \mathrm{U}\end{array}$ & \\
\hline $199-N-69$ & $11 / 09 / 93$ & BO9L 04 & & $4.50 \mathrm{~L}$ & & $3.44 \mathrm{U}$ \\
\hline $199-N-71$ & $11 / 12 / 93$ & B09LC3 & 34.00 & & $3.44 \mathrm{U}$ & \\
\hline $199-N-71$ & $11 / 12 / 93$ & B09LC7 & & 33.00 & & $3.44 \mathrm{U}$ \\
\hline $199-N-72$ & $11 / 12 / 93$ & BO9LC8 & $12.00 \mathrm{~L}$ & & $3.44 \mathrm{U}$ & \\
\hline $199-N-72$ & $11 / 12 / 93$ & B09LC9 & & $16.00 \mathrm{~L}$ & & $3.44 \mathrm{U}$ \\
\hline $199-N-73$ & $11 / 12 / 93$ & BO9LDO & $19.00 \mathrm{~L}$ & & $3.44 \mathrm{U}$ & \\
\hline $199-N-73$ & $11 / 12 / 93$ & B09LD1 & & $14.00 \mathrm{~L}$ & & $3.44 \mathrm{U}$ \\
\hline $199-N-75$ & $11 / 11 / 93$ & B09L05 & $3.84 \mathrm{U}$ & & 14.00 & \\
\hline $199-N-75$ & $11 / 111 / 93$ & B09L09 & & $3.84 \mathrm{U}$ & & 13.00 \\
\hline $199-N-76$ & $11 / 11 / 93$ & B09L 10 & $3.84 \mathrm{U}$ & & 22.00 & \\
\hline $199-N-16$ & $11 / 11793$ & 809L 14 & & $3.84 U$ & & 3.440 \\
\hline $\begin{array}{l}199-N-77 \\
199-N-77\end{array}$ & $\begin{array}{l}11 / 12 / 95 \\
11 / 12 / 93\end{array}$ & $\begin{array}{l}\text { BO9LD2 } \\
\text { BOSLD }\end{array}$ & $12.00 \mathrm{~L}$ & & $3.44 \mathrm{U}$ & \\
\hline $12 Y-4=11$ & 17 & BUYLUD & & $12.00 \mathrm{~L}$ & & 3.440 \\
\hline
\end{tabular}


Table 4-4. Constituents with at Least One Detected Value for the 100-N RCRA Sites Data for Reporting Period October 1 hrough December 31, 1993. (sheet 11 of 11 )

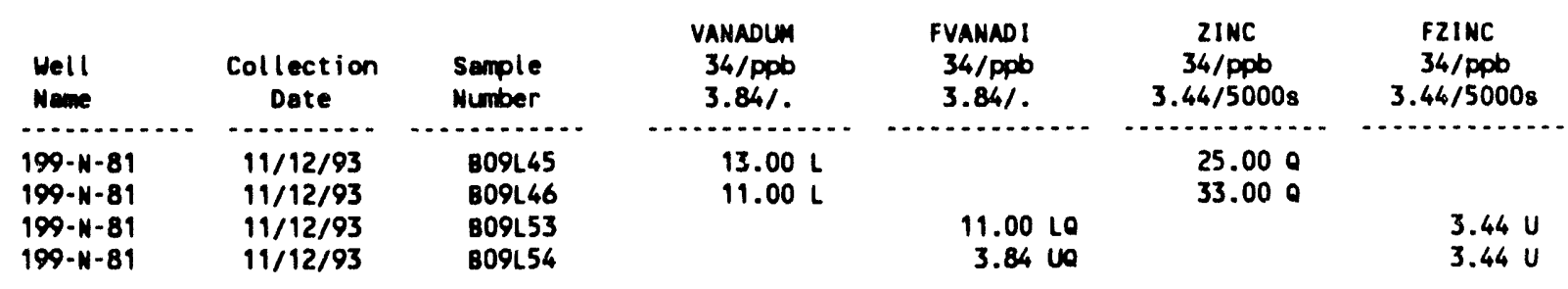

For explanation of this table, see Section 1.4 of report. 
Table 4-5. Contamination Indicator Parameters for the 100-N RCRA Sites Data for Reporting Period October 1 through December 31, 1993. (sheet 1 of 2)

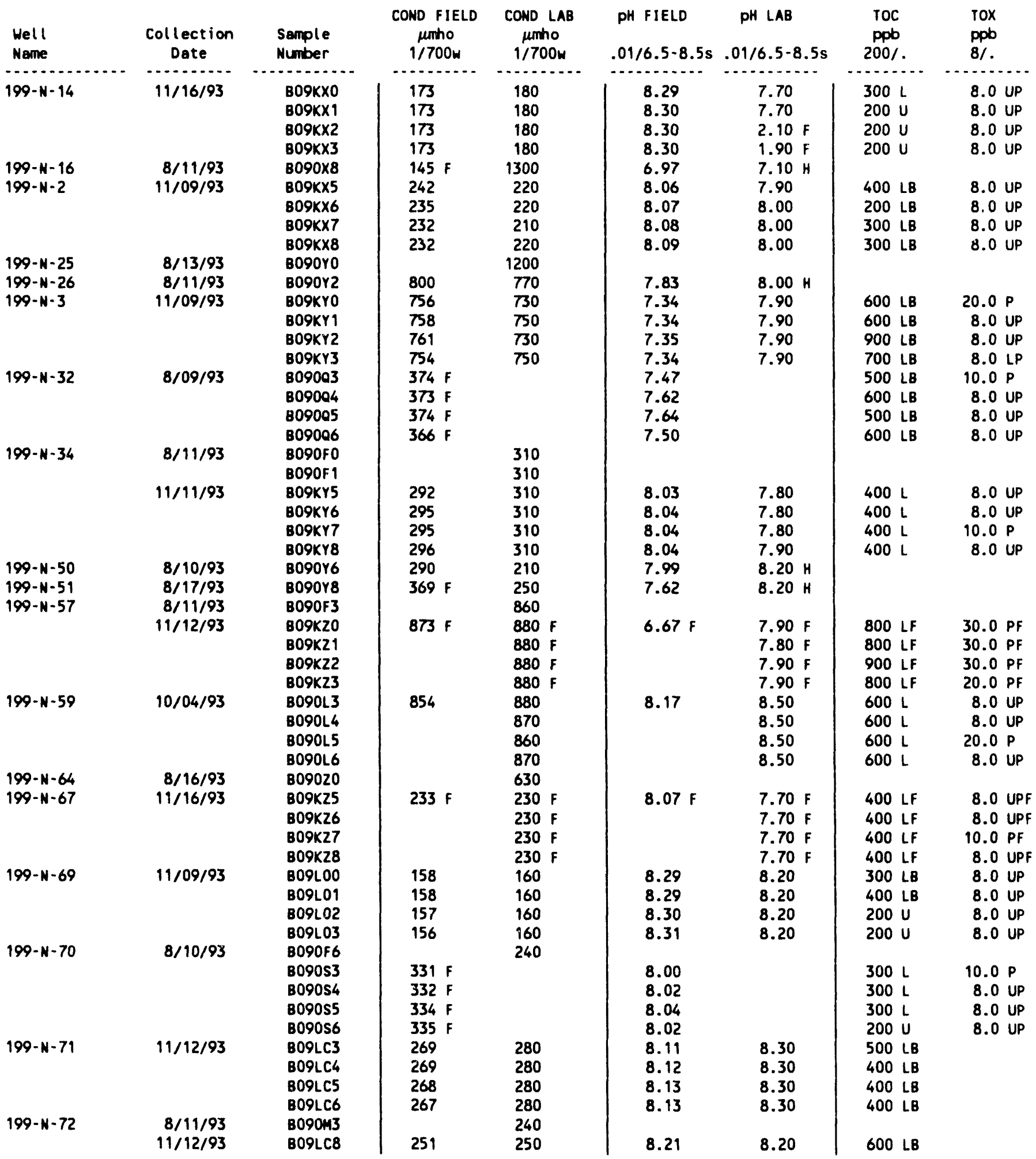


Table 4-5. Contamination Indicator Parameters for the 100-N RCRA Sites Data for Reporting Period October 1 through December 31, 1993. (sheet 2 of 2)

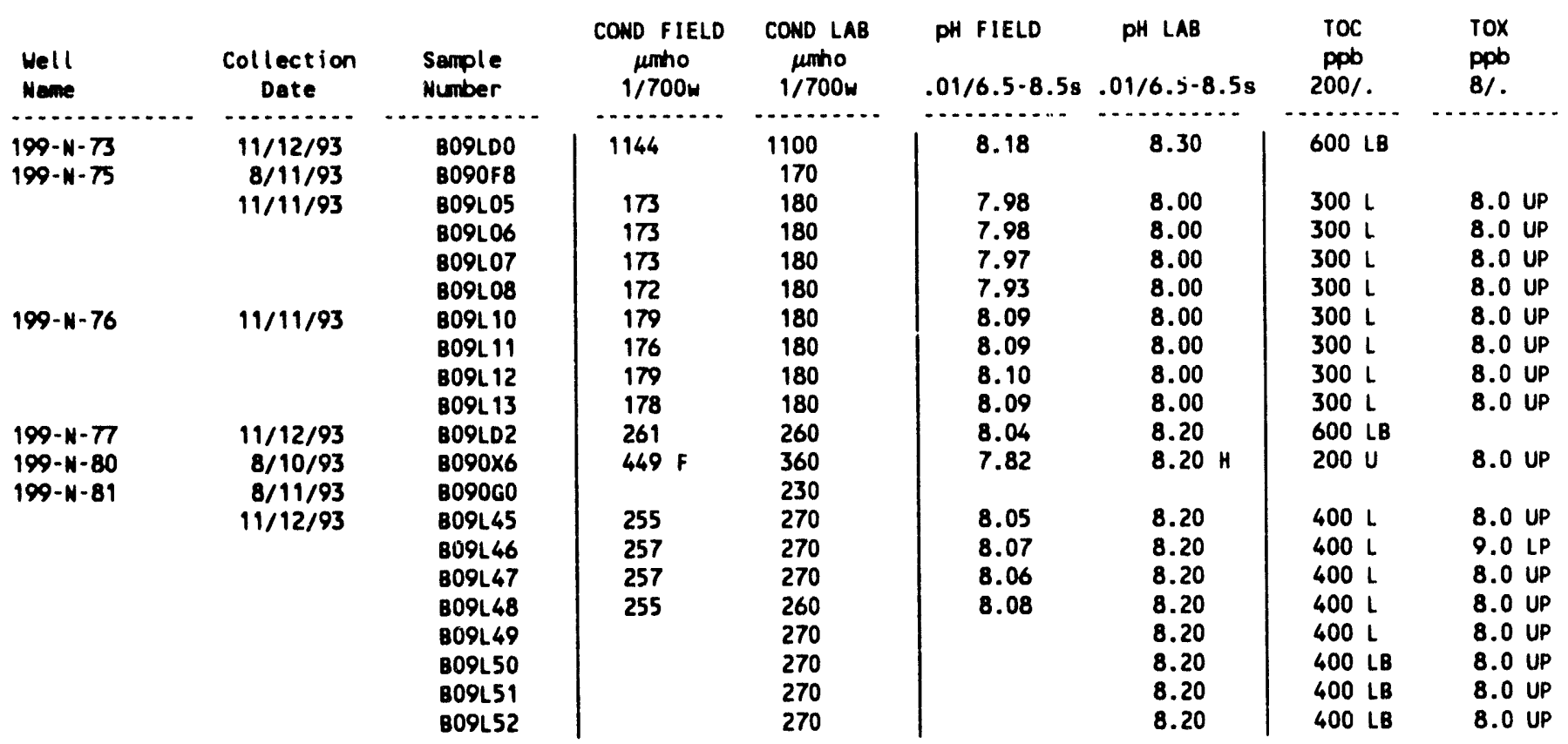


DOE /RL-93-56-4

CONTENTS

5.0 GROUT TREATMENT FACILITY . . . . . . . . . . . . . . 5-1

5.1 INTRODUCTION . . . . . . . . . . . . . . . . 5-1

5.2 WATER LEVEL MEASUREMENTS . . . . . . . . . . . . 5-1

5.3 WATER CHEMISTRY DATA . . . . . . . . . . . 5-2 
$\mathrm{DOE} / \mathrm{RL}-93-56-4$

\section{LIST OF FIGURES}

5-1 Monitoring Well Locations for the Grout Treatment Facility . . . . . 5-3

\section{LIST OF TABLES}

5-1 Monitoring Well Purpose and Sampling Schedule for the Grout Treatment Facility Network . . . . . . . . . . . . . . . . . 5-4

5-2 RCRA Water Level Measurement Report for the Grout Treatment Facility, Fourth Quarter 1993 ............... . 5-5

5-3 Constituent List and Summary of Results for the Grout Treatment Facility Data for Reporting Period October 1 through December 31, 1993

5-4 Constituents with at Least One Detected Value for the Grout Treatment Facility Data for Reporting Period October 1 through December 31, 1993

5-5 Contamination Indicator Parameters for the Grout Treatment Facility Data for Reporting Period October 1 through December 31, 1993 . . . 5-16

5-6 Summary Table of Flagged Data for Reporting Period October 1 through December 31,1993 ................ . . 5-17 
$D O E / R L-93-56-4$

\subsection{GROUT TREATMENT FACILITY}

L. C. Swanson

Westinghouse Hanford Company

\subsection{INTRODUCTION}

The Grout Treatment Facility (GTF) was a treatment and disposal facility regulated under the RCRA and "Dangerous Waste Regulations" (WAC 173-303). Figure 5-1 shows the location of this facility. The GTF was placed into cold standhv after the conclusion of recent Hanford Federal Facility Agreement and Consent Order (Tri-Party Agreement) renegotiations between Ecology, EPA, and the U.S. Department of Energy (DOE) (Ecology et al. 1992). These three parties agreed to eliminate the $M-01$ series of milestones that directed GTF operations, and to establish a new series of milestones (M-60) redirecting disposal of the mixed hazardous and low-level radioactive waste by means of a vitrification process. In short, this redirection eliminates grouting as a disposal method for this waste, and puts the GTF groundwater monitoring program on hold. A finalized change request to the Tri-Party Agreement was approved in January 1994.

In response to the status change, the groundwater monitoring and sampling programs were discontinued in November 1993, pending a renewed mission. Outstanding laboratory analys is results for groundwater samples collected before November 1993 will be reported in RCRA quarterly reports. Once all sample results are reported, the GTF section will be removed from the quarterly and annual reports. Some of the GTF monitoring wells may still be sampled in support of other 200 East Area groundwater activities (e.g., the 216-A-29 Ditch, tracking of 200 East Area groundwater plumes, and 200 East Area water table maps).

Grout treatment would have involved a process of blending a slurry of selected mixed hazardous and radioactive liquid waste with grout-forming solids and chemical additives. This slurry would then be poured into subterranean vaults for hardening and long-term storage.

The GTF RCRA groundwater monitoring network consisted of nine downgradient wells and four upgradient wells, as listed in Table 5-1. Well locations are depicted in Figure 5-1. The RCRA detection-level monitoring program followed 40 CFR 265 and the Interim-Status Ground Water Monitoring Plan for the Grout Treatment Facility (Teel et al. 1989). Table 5-1 1ists the final sampling network and the monitoring status for each of the wells.

\subsection{WATER LEVEL MEASUREMENTS}

During this quarter, water levels were measured either monthly or quarterly in the 13 network wells and several other monitoring wells located in the area of the GTF. Water level measurements also were recorded when the network wells were sampled. 
$\mathrm{DOE} / \mathrm{RL}-93-56-4$

Some data are flagged with a ' + ,' because of inconsistency with past water level measurements. We11 299-E25-33 needs to be resurveyed to account for the $4.6-\mathrm{m}(15-\mathrm{ft})$ stickup that resulted from recent wellhead modifications during grout vault construction work. Water levels for all of the network wells are listed in Table 5-2.

\subsection{WATER CHEMISTRY DATA}

Groundwater sample results are reported for 10 wells this quarter. Other wells were not sampled because the groundwater monitoring program was terminated as described above. Table 5-1 lists each GTF well and the sampling frequency and dates for this quarter. Table 5-3 is a summary listing of the analysis results for all of the wells. Table 5-4 contains the analysis results for any constituents that had at least one detected value. Table 5-5 lists the analysis results for the four indicator parameters: specific conductance, field $\mathrm{pH}, \mathrm{TOC}$, and TOX.

Almost all of the constituent concentrations for this quarter are below the DWSs. Those that did exceed the DWSs followed historical trends, such as unfiltered chromium, unfiltered iron, and tritium; however, all of the filtered species for these constituents did fall below the DWS. A turbidity reading of 6.7 NTUs was higher than normal at well 299-E25-31, and probably reflects wellhead remediation work performed at this well the previous quarter. Turbidity was also high at well 299-E25-45 at 18 NTUs. The reason for this increase and the high coliform at well 299-E25-44 (5 most probable number [MPN]) is not known.

Constituents that exceeded the MDLs also followed historical trends. The indicator parameters fell below (and above for $\mathrm{pH}$ ) the critical mean for all reported wells, except for specific conductance in well 299-E25-33. The higher value of $320 \mu \mathrm{mho} / \mathrm{cm}$ is consistent with past readings at this well.

Several flags were attached to particular laboratory data, including arsenic, lead, nitrate, selenium, $\mathrm{PH}$, TOC, and TOX. Flagged data are listed in Table 5-6 for each applicable well and constituent, except for TOX, because all of the TOX data are flagged. The TOX flag (a $P$ flag) indicates a potential problem with the quality of the laboratory data. The TOX issues are still being addressed. Each of the flags is defined in the footnotes of Table 5-6. 
Figure 5-1. Monitoring Well Locations for the Grout Treatment Facility.

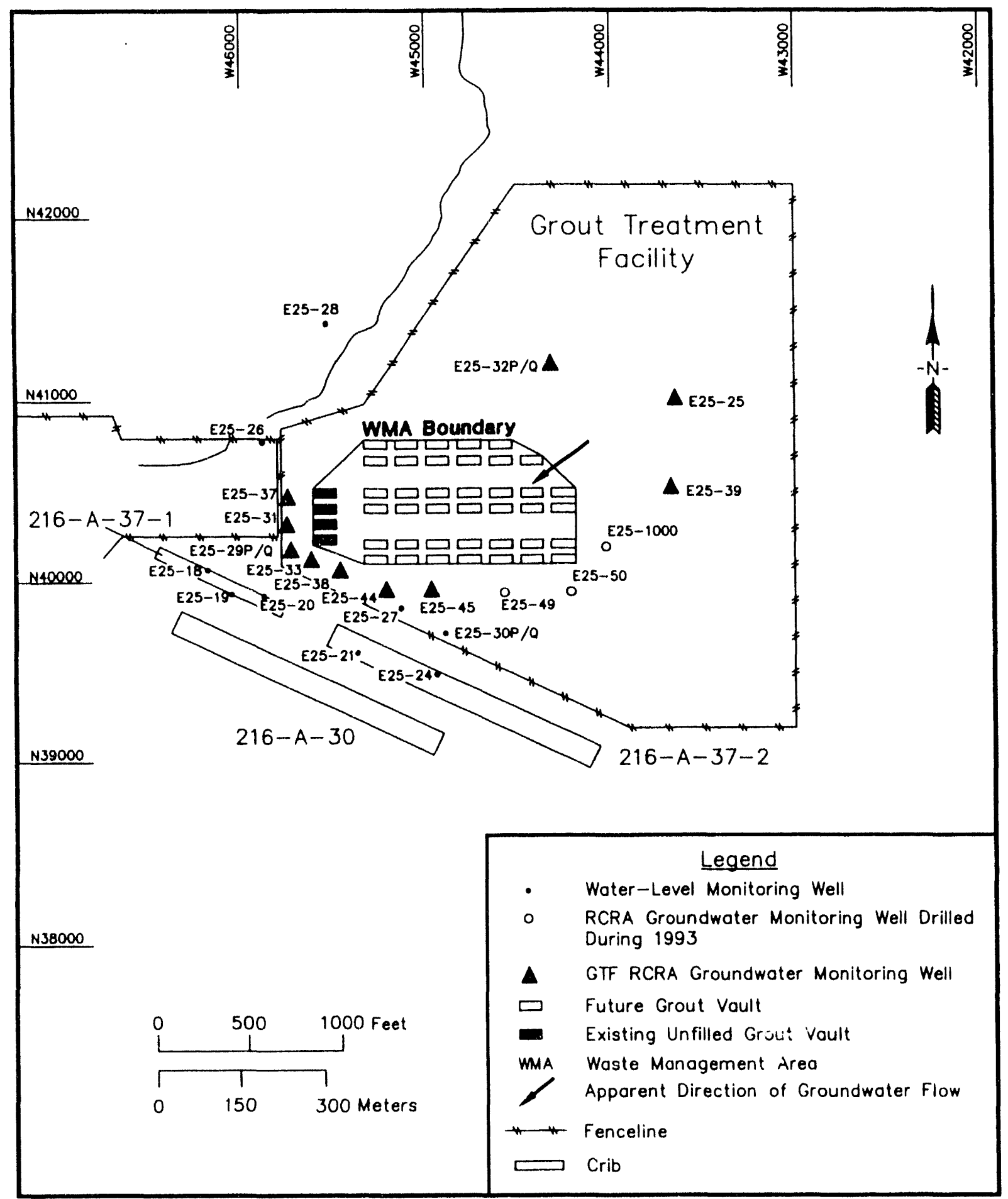


Table 5-1. Monitoring Well Purpose and Sampling Schedule for the Grout Treatment Facility Network.

\begin{tabular}{|c|c|l|c|c|}
\hline $\begin{array}{c}\text { Well no. } \\
(299-)\end{array}$ & $\begin{array}{c}\text { Relative } \\
\text { position }\end{array}$ & Hydrogeologic unit & $\begin{array}{c}\text { Sample } \\
\text { frequency }\end{array}$ & $\begin{array}{c}\text { Sample date, } \\
\text { 4th Qtr 1993 }\end{array}$ \\
\hline E25-25 & Upgradient & Hanford: Water Table & Semiannually & $10 / 15 / 93$ \\
\hline E25-32P & Upgradient & Hanford: Water Table & Quarterly & $10 / 13 / 93$ \\
\hline E25-39 & Upgradient & Hanford: Water Table & Semiannually & $10 / 14 / 93$ \\
\hline E25-29P & Downgradient & Hanford: Water Table & Semiannually & $10 / 13 / 93$ \\
\hline E25-31 & Downgradient & Hanford: Water Table & Quarterly & $10 / 13 / 93$ \\
\hline E25-33 & Downgradient & Hanford: Water Table & Semiannually & $10 / 20,93$ \\
\hline E25-37 & Downgradient & Hanford: Water Table & Semiannually & $10 / 19 / 93$ \\
\hline E25-38 & Downgradient & Hanford: Water Table & Semiannually & $10 / 20 / 93$ \\
\hline E25-44 & Downgradient & Hanford: Water Table & Quarterly & $10 / 14 / 93$ \\
\hline E25-45 & Downgradient & Hanford: Water Table & Quarterly & $10 / 14 / 93$ \\
\hline
\end{tabular}

${ }^{a}$ Hydrogeologic units include the sandy gravels of the Hanford formation. 
Table 5-2. RCRA Water Level Measurement Report for the Grout Treatment Facility, Fourth Quarter 1993. (sheet 1 of 2)

\begin{tabular}{lccc}
\hline We11 & Date & $\begin{array}{c}\text { Depth to } \\
\text { water }(\mathrm{ft})\end{array}$ & $\begin{array}{c}\text { Water leve1 } \\
\text { elevation } \\
\text { above ms }\end{array}$ \\
\hline $299-\mathrm{ft})$
\end{tabular}


Table 5-2. RCRA Water Level Measurement Report for the Grout Treatment Facility, Fourth Quarter 1993. (sheet 2 of 2)

Notes: 1. Water level elevations are calculated by subtracting the measured depth to water from the surveyed elevation for the well.

2. Depth-to-water values are transcribed from field records.

3. Elevations marked with an $|*|$ were measured at the time of sampling.

4. Elevations marked with a ' + ' are outside of the expected range and are suspected of error.

5. To convert feet to meters multiply by 0.3048 . 
Table 5-3. Constituent List and Summary of Results for the Grout Treatment Facility Data for Reporting Period October 1 through December 31, 1993.

(sheet 1 of 3 )

CONTAMINATION INOICATOR PARAMETERS

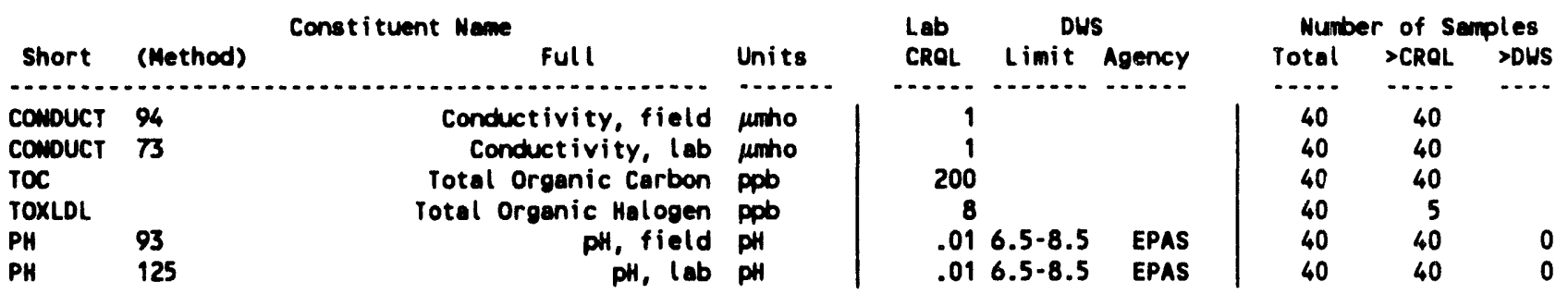

DRINKING WATER PARAMETERS

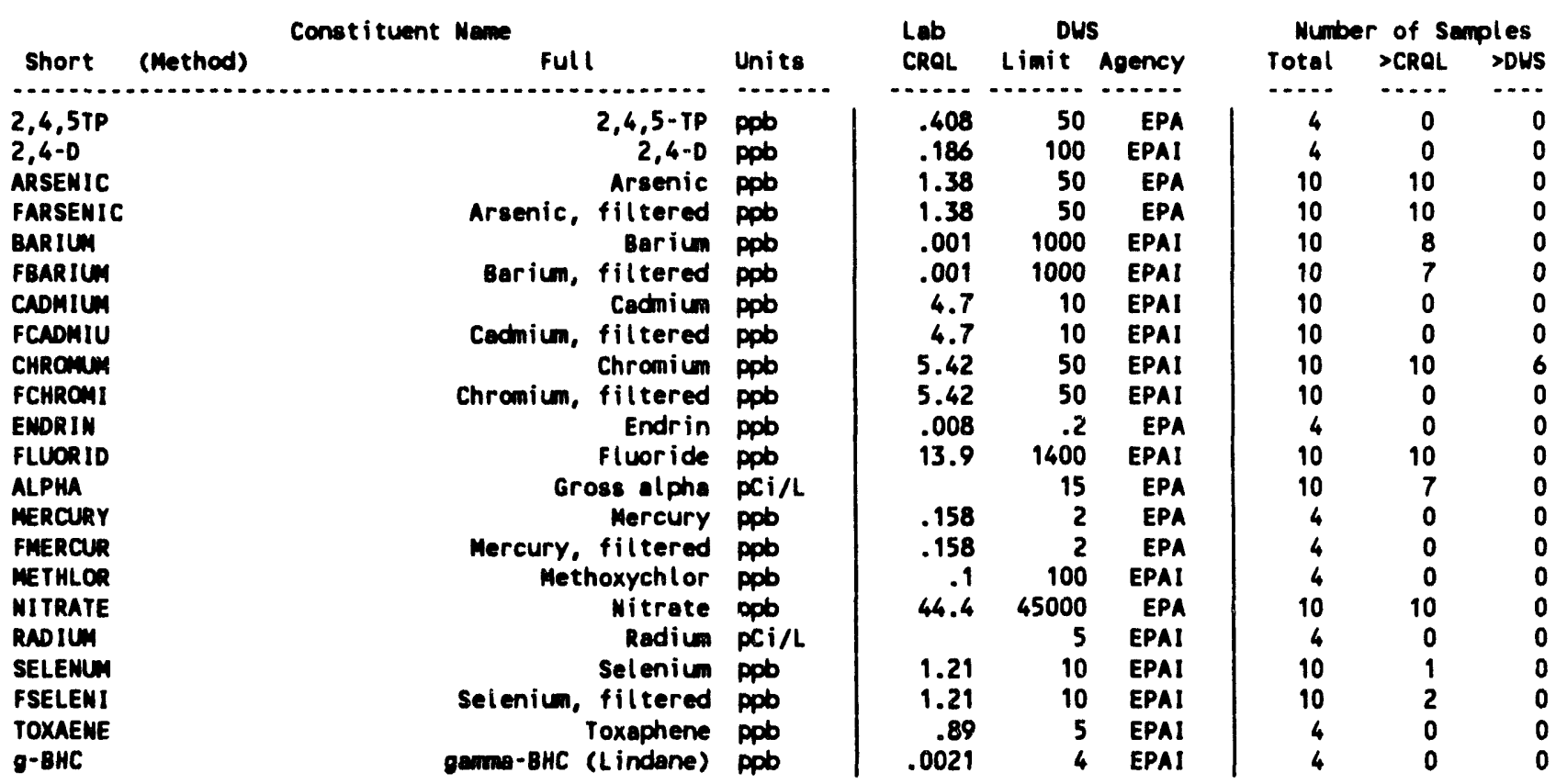

GROUNDWATER QUALITY PARAMETERS

\begin{tabular}{|c|c|c|c|c|c|c|c|c|c|}
\hline & & Const ituent Mase & & Lab & DWS & & Num & $r$ of $s$ & ples \\
\hline Short & (Method) & Full & Units & CROL & Limit & Agency & Totel & $>$ CROL & $>$ DWS \\
\hline CHLORID & & Chloride & ppob & 82.5 & 250000 & EPAS & 10 & 10 & 0 \\
\hline IROW & & Iron & ppb & 10.3 & 300 & EPAS & 10 & 10 & 7 \\
\hline FIRON & & Iron, filtered & ppo & 10.3 & 300 & EPAS & 10 & 8 & 0 \\
\hline MANGESE & & Manganese & ppb & 1.35 & 50 & EPAS & 10 & 8 & 0 \\
\hline FMANGAN & & Manganese, filtered & ppob & 1.35 & 50 & EPAS & 10 & 6 & 0 \\
\hline LPHENOL & & Phenol & ppob & .31 & & & 10 & 1 & \\
\hline soolun & & Sodiun & ppb & 40.9 & & & 10 & 10 & \\
\hline Fsoolun & & Sodium, filtered & ppb & 40.9 & & & 10 & 10 & \\
\hline SULFATE & & Sulfate & ppob & 49.9 & 250000 & EPAS & 10 & 10 & 0 \\
\hline
\end{tabular}


Table 5-3. Constituent List and Summary of Results for the Grout Treatment Facility Data for Reporting Period October I through December 31, 1993.

(sheet 2 of 3 )

SITE SPECIFIC AND OTHER CONSTITUENTS

\begin{tabular}{|c|c|c|c|c|c|c|c|c|c|}
\hline & & Const ituent Neme & & Lab & DUS & & Nunt & $r$ of $\mathrm{Se}$ & ples \\
\hline Short & (Method) & Full & Units & CROL & Limit & Agency & Total & $>$ CROL & $>$ DWS \\
\hline $2,4,5-T$ & & $2,4,5-T$ & ppob & .384 & , & $-8-0.0$ & 4 & 0 & $\cdots$ \\
\hline $246-\operatorname{trp}$ & & 2,4,6-Trichlorophenol & ppb & 1.45 & & & 10 & 0 & \\
\hline 24-dchp & & 2,4-Dichlorophenol & ppob & 1.5 & & & 10 & 0 & \\
\hline DIMPHEN & & 2,4-D imethylphenol & ppob & 1.01 & & & 10 & 0 & \\
\hline DINPHEN & & 2,4-0 initrophenol & ppob & .96 & & & 10 & 0 & \\
\hline 26-dchp & & 2,6-D ichlorophenol & ppb & 1.59 & & & 10 & 0 & \\
\hline CHLPHEN & & 2-Chlorophenol & ppb & 1.42 & & & 10 & 0 & \\
\hline 2NITPH & & 2-Witrophenol & ppob & 1.56 & & & 10 & 0 & \\
\hline BUTDINP & 30 & 2-sec-Butyl-4,6-dini trophenol (DN & ppob & 1.35 & & & 10 & 0 & \\
\hline BUTOINP & 49 & 2-sec-Butyl-4,6-dini trophenol (DN & ppb & .269 & & & 4 & 0 & \\
\hline & & $4.41-000$ & ppb & .0005 & & & 4 & 0 & \\
\hline DDE & & $4.4^{\prime}-D D E$ & ppb & .0009 & & & 4 & 0 & \\
\hline DOT & & $4,4 \cdot-D D T$ & ppb & .011 & & & 4 & 0 & \\
\hline 46DN2UP & & 4,6-Dinitro-2-nethylphenol & ppob & 1.18 & & & 10 & $\mathbf{0}$ & \\
\hline CHLCRES & & 4-Chloro-3-methylphenol & ppb & 1.12 & & & 10 & 0 & \\
\hline MITPHEN & & 4-Nitrophenol & ppb & .65 & & & 10 & 0 & \\
\hline ALDRIN & & Aldrin & ppb & .05 & & & 4 & 0 & \\
\hline -8-BHC & & Alpha-BHC & ppb & .012 & & & 4 & 0 & \\
\hline ALLAMUNA & & Aluminum & ppb & 32.5 & & & 10 & 7 & \\
\hline FALUMIN & & Aluminum & ppb & 32.5 & & & 10 & 1 & \\
\hline Amion IU & & Mmonium ion & ppb & 38.5 & & & 2 & 2 & \\
\hline ANTIONY & & Ant imony & ppb & 69.4 & & & 10 & 0 & \\
\hline FANTIMO & & Ant imony, filtered & ppb & 69.4 & & & 10 & 0 & \\
\hline BERYLUM & & Beryllium & ppo & .814 & & & 10 & 1 & \\
\hline FBERYLL & & Beryllium, filtered & ppb & .814 & & & 10 & 1 & \\
\hline b-BHC & & Beta-BHC & ppo & .0026 & & & 4 & 0 & \\
\hline BROMIDE & & Bromide & ppb & 52.8 & & & 10 & 0 & \\
\hline CALCIUM & & Calcium & ppb & 20.9 & & & 10 & 10 & \\
\hline FCALCIU & & Calciun, filtered & ppob & 20.9 & & & 10 & 10 & \\
\hline CHLOANE & & Chlordane & ppb & .0057 & 2 & EPA & 4 & 0 & 0 \\
\hline COBALT & & Cobalt & ppb & 4.05 & & & 10 & 2 & \\
\hline FCOBALT & & Cobolt, filtered & ppb & 4.05 & & & 10 & 3 & \\
\hline COL I FORM & & Coliforms & COL & & 1 & EPAI & 4 & 1 & 1 \\
\hline COPPER & & Copper & ppb & 2.65 & 1000 & EPAS & 10 & 4 & 0 \\
\hline FCOPPER & & Copper, filtered & ppo & 2.65 & 1000 & EPAS & 10 & 0 & 0 \\
\hline CRESOLS & & Cresols (methylphenols) & ppb & 4.66 & & & 10 & 0 & \\
\hline d-BHC & & Delta-BHC & ppb & .0014 & & & 4 & 0 & \\
\hline DIELRIN & & Dieldrin & ppob & .019 & & & 4 & 0 & \\
\hline ENDO1 & & Endosulfan $I$ & ppb & .0029 & & & 4 & 0 & \\
\hline ENDO2 & & Endosulfan II & ppob & .004 & & & 4 & 0 & \\
\hline ENDSFAN & & Endosulfan sulfate & ppb & .0072 & & & 4 & 0 & \\
\hline ENDRALD & & Endrin Aldehyde & ppob & .011 & & & 4 & 0 & \\
\hline BETA & & Gross bete & $\mathrm{pCi} / \mathrm{L}$ & & & & 10 & 10 & \\
\hline MEPTLOR & & Heptachlor & ppb & .0019 & .4 & EPA & 4 & 0 & 0 \\
\hline HEPTIDE & & Heptachlor epoxide & ppb & .0008 & .2 & EPA & 4 & 0 & 0 \\
\hline LHYDRAZ & & Hydrazine & ppob & 1.89 & & & 2 & 0 & \\
\hline LEAD & & Lead & ppo & .508 & 50 & EPAI & 4 & 3 & 0 \\
\hline FLEAD & & Lead, filtered & ppb & .508 & 50 & EPAI & 4 & 2 & 0 \\
\hline MGGNES & & Magnes ium & ppb & 26 & & & 10 & 10 & \\
\hline FMAGNES & & Magnesium, filtered & ppb & 26 & & & 10 & 10 & \\
\hline NICKEL & & Nickel & ppo & 17.9 & & & 10 & 6 & \\
\hline FNICKEL & & Nickel, filtered & ppb & 17.9 & & & 10 & 1 & \\
\hline MITRITE & & Nitrite & ppb & 38.3 & 1000 & EPA & 10 & 0 & 0 \\
\hline
\end{tabular}


Table 5-3. Constituent List and Summary of Results for the Grout Treatment Facility Data for Reporting Period October 1 through December 31, 1993. (sheet 3 of 3 )

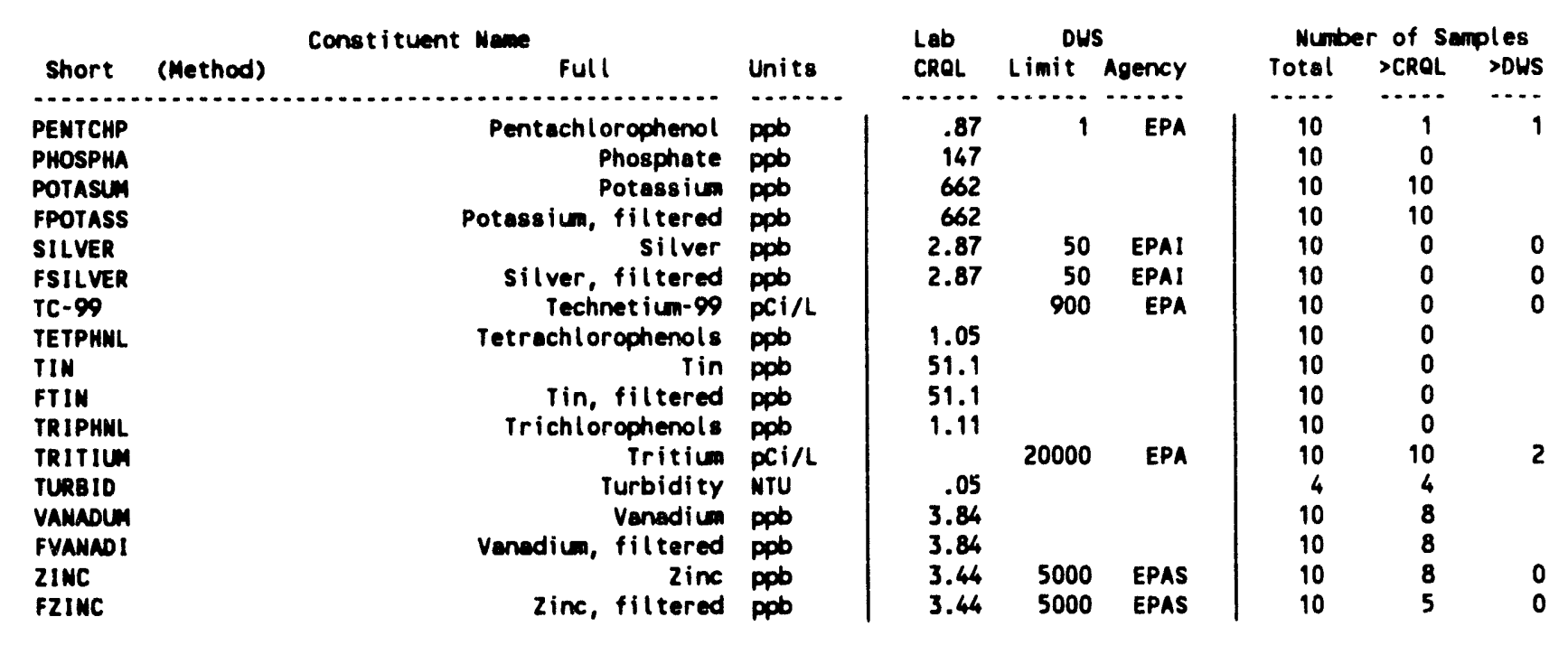

For explanation of this table, see Section 1.4 of report. 
Table 5-4. Constituents with at Least One Detected Value for the Grout Treatment Facility Data for Reporting Period October 1

through December 31, 1993. (sheet 1 of 6)

\begin{tabular}{|c|c|c|c|c|c|c|}
\hline $\begin{array}{l}\text { Well } \\
\text { Nene }\end{array}$ & $\begin{array}{l}\text { Collection } \\
\text { Date }\end{array}$ & $\begin{array}{l}\text { Semple } \\
\text { Number }\end{array}$ & $\begin{array}{l}\text { ALUMNUN } \\
34 / \text { PpD } \\
32.5 \%\end{array}$ & $\begin{array}{l}\text { FALUMIN } \\
34 / \mathrm{ppb} \\
32.5 /\end{array}$ & $\begin{array}{c}\text { AMHONIU } \\
129 / \mathrm{ppb} \\
38.51 .\end{array}$ & $\begin{array}{r}\text { ARSENIC } \\
43 / \mathrm{ppb} \\
1.38 / 50\end{array}$ \\
\hline $\begin{array}{l}299-E 25-25 \\
299-E 25-25 \\
299-E 25-29 P \\
299-E 25-29 P \\
299-E 25-31 \\
299-E 25-31 \\
299-E 25-32 P \\
299-E 25-32 P \\
299-E 25-33 \\
299-E 25-33 \\
299-E 25-37 \\
299-E 25-37 \\
299-E 25-38 \\
299-E 25-38 \\
299-E 25-39 \\
299-E 25-39 \\
299-E 25-44 \\
299-E 25-44 \\
299-E 25-45 \\
299-E 25-45\end{array}$ & $\begin{array}{l}10 / 15 / 93 \\
10 / 15 / 93 \\
10 / 13 / 93 \\
10 / 13 / 93 \\
10 / 13 / 93 \\
10 / 13 / 93 \\
10 / 13 / 93 \\
10 / 13 / 93 \\
10 / 20 / 93 \\
10 / 20 / 93 \\
10 / 19 / 93 \\
10 / 19 / 93 \\
10 / 20 / 93 \\
10 / 20 / 93 \\
10 / 14 / 93 \\
10 / 14 / 93 \\
10 / 14 / 93 \\
10 / 14 / 93 \\
10 / 14 / 93 \\
10 / 14 / 93\end{array}$ & $\begin{array}{l}\text { B09CN7 } \\
\text { B09CP1 } \\
\text { B09CP2 } \\
\text { B09CP6 } \\
\text { B09BJ8 } \\
\text { B09BJ9 } \\
\text { B09BKD } \\
\text { B09BK1 } \\
\text { 809C02 } \\
\text { B09CQ6 } \\
\text { B09CQ7 } \\
\text { B09CR1 } \\
\text { B09CR2 } \\
\text { B09CR6 } \\
\text { B09CR7 } \\
\text { B09CS1 } \\
\text { B09CT5 } \\
\text { B09CT9 } \\
\text { B09CV0 } \\
\text { B09CV4 }\end{array}$ & $\begin{array}{l}32.50 \mathrm{U} \\
32.50 \mathrm{U} \\
100.00 \mathrm{~L} \\
32.50 \mathrm{U} \\
43.00 \mathrm{~L} \\
320.00 \\
130.00 \mathrm{~L} \\
160.00 \mathrm{~L} \\
37.00 \mathrm{~L} \\
430.00\end{array}$ & $\begin{array}{l}32.50 U \\
32.50 \mathrm{U} \\
32.50 \mathrm{U} \\
32.50 \mathrm{U} \\
32.50 \mathrm{U} \\
32.50 \mathrm{U} \\
35.00 \mathrm{~L} \\
32.50 \mathrm{U} \\
32.50 \mathrm{U} \\
32.50 \mathrm{U}\end{array}$ & $\begin{array}{l}60.00 \mathrm{LQ} \\
60.00 \mathrm{LQ}\end{array}$ & $\begin{array}{c}9.10 \\
3.60 \mathrm{~L} \\
14.00 \\
5.70 \\
7.40 \\
3.40 \mathrm{~L} \\
15.00 \\
15.00\end{array}$ \\
\hline $\begin{array}{l}\text { Well } \\
\text { Neme }\end{array}$ & $\begin{array}{c}\text { Collection } \\
\text { Date }\end{array}$ & $\begin{array}{l}\text { Semple } \\
\text { Number }\end{array}$ & $\begin{array}{l}\text { FARSENIC } \\
43 / \mathrm{ppb} \\
1.38 / 50\end{array}$ & $\begin{array}{c}\text { BARIUM } \\
\text { 34/Ppb } \\
.001 / 1000 i\end{array}$ & $\begin{array}{l}\text { FBARIUM } \\
\text { 34/ppb } \\
.001 / 1000 i\end{array}$ & $\begin{array}{c}\text { BERYLUM } \\
34 / \text { ppb } \\
.814 / .\end{array}$ \\
\hline $\begin{array}{l}299-E 25-25 \\
299-E 25-25 \\
299-E 25-29 P \\
299-E 25-29 P \\
299-E 25-31 \\
299-E 25-31 \\
299-E 25-32 P \\
299-E 25-32 P \\
299-E 25-33 \\
299-E 25-33 \\
299-E 25-37 \\
299-E 25-37 \\
299-E 25-38 \\
299-E 25-38 \\
299-E 25-39 \\
299-E 25-39 \\
299-E 25-44 \\
299-E 25-44 \\
299-E 25-45 \\
299-E 25-45\end{array}$ & $\begin{array}{l}10 / 15 / 93 \\
10 / 15 / 93 \\
10 / 13 / 93 \\
10 / 13 / 93 \\
10 / 13 / 93 \\
10 / 13 / 93 \\
10 / 13 / 93 \\
10 / 13 / 93 \\
10 / 20 / 93 \\
10 / 20 / 93 \\
10 / 19 / 93 \\
10 / 19 / 93 \\
10 / 20 / 93 \\
10 / 20 / 93 \\
10 / 14 / 93 \\
10 / 14 / 93 \\
10 / 14 / 93 \\
10 / 14 / 93 \\
10 / 14 / 93 \\
10 / 14 / 93\end{array}$ & $\begin{array}{l}\text { B09CN7 } \\
\text { B09CP1 } \\
\text { B09CP2 } \\
\text { B09CP6 } \\
\text { B09BJ8 } \\
\text { B098J9 } \\
\text { B09BKO } \\
\text { B09BK1 } \\
\text { B09C02 } \\
\text { B09C06 } \\
\text { 809C07 } \\
\text { B09CR1 } \\
\text { B09CR2 } \\
\text { B09CR6 } \\
\text { B09CR7 } \\
\text { B09CS1 } \\
\text { B09C15 } \\
\text { B09CT9 } \\
\text { B09CV0 } \\
\text { B09CV4 }\end{array}$ & $\begin{array}{c}2.50 \mathrm{BL} \\
13.00 \\
9.30 \\
3.10 \mathrm{~L} \\
13.00 \\
5.30 \\
8.90 \\
3.70 \mathrm{~L} \\
8.20 \\
5.90\end{array}$ & $\begin{array}{l}.00 \mathrm{U} \\
21.00 \\
27.00 \\
14.00 \mathrm{~L} \\
39.00 \\
16.00 \mathrm{~L} \\
23.00 \\
.00 \mathrm{U} \\
24.00 \\
20.00\end{array}$ & $\begin{array}{c}.00 \mathrm{U} \\
20.00 \\
25.00 \\
13.00 \mathrm{~L} \\
41.00 \\
12.00 \mathrm{~L} \\
22.00 \\
.00 \mathrm{U} \\
23.00 \\
.00 \mathrm{U}\end{array}$ & $\begin{array}{l}.81 U \\
.81 U \\
.81 U \\
.81 U \\
.81 U \\
1.00 \mathrm{U} \\
.81 U \\
.81 U \\
.81 U \\
.81 U\end{array}$ \\
\hline
\end{tabular}


Table 5-4. Constituents with at Least One Detected Value for the Grout Treatment Facility Data for Reporting Period October 1

through December 31, 1993. (sheet 2 of 6)

\begin{tabular}{|c|c|c|c|c|c|c|}
\hline $\begin{array}{l}\text { Well } \\
\text { Neme }\end{array}$ & $\begin{array}{c}\text { Collection } \\
\text { Date }\end{array}$ & $\begin{array}{l}\text { Semple } \\
\text { Nunber }\end{array}$ & $\begin{array}{c}\text { FBERYLL } \\
34 / \mathrm{ppb} \\
.814 / .\end{array}$ & $\begin{array}{l}\text { CALCIUN } \\
34 / \text { pob } \\
20.91\end{array}$ & $\begin{array}{l}\text { FCALCIU } \\
34 / \mathrm{ppb} \\
20.91 .\end{array}$ & $\begin{array}{c}\text { CHLORID } \\
124 / \text { ppb } \\
82.5 / 2500008\end{array}$ \\
\hline $\begin{array}{l}299-E 25-25 \\
299-E 25-25 \\
299-E 25-29 P \\
299-E 25-29 P \\
299-E 25-31 \\
299-E 25-31 \\
299-E 25-32 P \\
299-E 25-32 P \\
299-E 25-33 \\
299-E 25-33 \\
299-E 25-37 \\
299-E 25-37 \\
299-E 25-38 \\
299-E 25-38 \\
299-E 25-39 \\
299-E 25-39 \\
299-E 25-44 \\
299-E 25-44 \\
299-E 25-45 \\
299-E 25-45\end{array}$ & $\begin{array}{l}10 / 15 / 93 \\
10 / 15 / 93 \\
10 / 13 / 93 \\
10 / 13 / 93 \\
10 / 13 / 93 \\
10 / 13 / 93 \\
10 / 13 / 93 \\
10 / 13 / 93 \\
10 / 20 / 93 \\
10 / 20 / 93 \\
10 / 19 / 93 \\
10 / 19 / 93 \\
10 / 20 / 93 \\
10 / 20 / 93 \\
10 / 14 / 93 \\
10 / 14 / 93 \\
10 / 14 / 93 \\
10 / 14 / 93 \\
10 / 14 / 93 \\
10 / 14 / 93\end{array}$ & $\begin{array}{l}\text { 809CN7 } \\
\text { B09CP1 } \\
\text { B09CP2 } \\
\text { B09CP6 } \\
\text { B098J8 } \\
\text { B098J9 } \\
\text { B09BK0 } \\
\text { B098K1 } \\
\text { 809CO2 } \\
\text { B09CO6 } \\
\text { B09CA7 } \\
\text { B09CR1 } \\
\text { B09CR2 } \\
\text { B09CR6 } \\
\text { B09CR7 } \\
\text { B09CS1 } \\
\text { B09CTS } \\
\text { B09CT9 } \\
\text { B09CV0 } \\
\text { B09CV4 }\end{array}$ & $\begin{array}{l}.81 U \\
.81 U \\
.81 U \\
.81 U \\
.81 U \\
1.80 L \\
.81 U \\
.81 U \\
.81 U \\
.81 U\end{array}$ & $\begin{array}{l}22000.00 \\
26000.00 \\
24000.00 \\
23000.00 \\
30000.00 \\
23000.00 \\
25000.00 \\
21000.00 \\
20000.00\end{array}$ & $\begin{array}{l}22000.00 \\
26000.00 \\
24000.00 \\
23000.00 \\
30000.00 \\
23000.00 \\
26000.00 \\
21000.00 \\
20000.00 \\
19000.00\end{array}$ & $\begin{array}{l}1500.00 \\
2400.00 \\
1800.00 \\
1500.00 \\
3200.00 \\
1500.00 \\
2500.00 \\
1700.00 \\
3000.00\end{array}$ \\
\hline
\end{tabular}

\begin{tabular}{|c|c|c|c|c|c|c|}
\hline $\begin{array}{l}\text { Hell } \\
\text { Name }\end{array}$ & $\begin{array}{l}\text { Collection } \\
\text { Date }\end{array}$ & $\begin{array}{l}\text { Semple } \\
\text { Number }\end{array}$ & $\begin{array}{l}\text { CHROMUM } \\
34 / p p b \\
5.42 / 50 i\end{array}$ & $\begin{array}{c}\text { FCHRONI } \\
34 / \text { ppb } \\
5.42 / 50 i\end{array}$ & $\begin{array}{l}\text { COBALT } \\
34 / \text { ppb } \\
4.05 \%\end{array}$ & $\begin{array}{c}\text { FCOBALT } \\
34 / p p b \\
4.05 \%\end{array}$ \\
\hline $\begin{array}{l}299-E 25-25 \\
299-E 25-25\end{array}$ & $\begin{array}{l}10 / 15 / 93 \\
10 / 15 / 93\end{array}$ & $\begin{array}{l}\text { BO9CN7 } \\
\text { BO9CP1 }\end{array}$ & 41.00 & $5.42 \mathrm{U}$ & $4.05 \mathrm{U}$ & $4.05 \mathrm{U}$ \\
\hline 299-E25-29P & $10 / 13 / 93$ & BO9CP2 & 130.00 & & $13.00 \mathrm{~L}$ & \\
\hline $\begin{array}{l}\text { 299-E25-29P } \\
299-E 25-31\end{array}$ & $10 / 13 / 93$ & $\begin{array}{l}\text { 809CP6 } \\
8098.18\end{array}$ & 95.00 & $5.42 \mathrm{U}$ & 4.050 & $6.10 \mathrm{~L}$ \\
\hline 299-E25-31 & $10 / 13 / 93$ & в09BJ9 & 7.00 & $5.42 U$ & 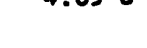 & $5.60 \mathrm{~L}$ \\
\hline 299-E25-32P & $10 / 13 / 93$ & 8098BKO & 58.00 & & $14.00 \mathrm{~L}$ & \\
\hline 299-E25-32P & $10 / 13 / 93$ & B09BK1 & & $5.42 \mathrm{U}$ & & $6.10 \mathrm{~L}$ \\
\hline 299-E25-33 & $10 / 20 / 93$ & 809c02 & 33.00 & & $4.05 \mathrm{U}$ & \\
\hline $\begin{array}{l}299-\text { E25-33 } \\
299-\text { E25-37 }\end{array}$ & $\begin{array}{l}10 / 20 / 93 \\
10 / 19 / 93\end{array}$ & $\begin{array}{l}\text { 809C06 } \\
\text { 809C07 }\end{array}$ & 270.00 & $5.42 \mathrm{U}$ & $4.05 \mathrm{U}$ & $4.05 U$ \\
\hline $299-E 25-37$ & $10 / 19 / 93$ & BO9CRI & & $5.42 \mathrm{U}$ & & $4.05 \mathrm{U}$ \\
\hline 299-E25-38 & $10 / 20 / 93$ & B09CR2 & 160.00 & & $4.05 \mathrm{U}$ & \\
\hline 299-E25-38 & $10 / 20 / 93$ & B09CR6 & & $5.42 \mathrm{U}$ & & $4.05 \mathrm{U}$ \\
\hline 299-E25-39 & $10 / 14 / 93$ & BO9CR7 & 210.00 & & $4.05 \mathrm{U}$ & \\
\hline $\begin{array}{l}299-E 25-39 \\
299-E 25-44\end{array}$ & $\begin{array}{l}10 / 14 / 93 \\
10 / 14 / 93\end{array}$ & $\begin{array}{l}\text { 809CS1 } \\
\text { B09CT5 }\end{array}$ & & $5.42 \mathrm{U}$ & $4.05 U$ & $4.05 \mathrm{U}$ \\
\hline $\begin{array}{l}299--E 25-44 \\
299-E 25-44\end{array}$ & $\begin{array}{l}10 / 14 / 93 \\
10 / 16 / 93\end{array}$ & $\begin{array}{l}\text { B09CT5 } \\
\text { B09CT9 }\end{array}$ & 34.00 & $5.42 \mathrm{U}$ & 4.050 & $4.05 \mathrm{U}$ \\
\hline 299-E25- 45 & $10 / 14 / 93$ & 809cVo & 33.00 & & $4.05 \mathrm{U}$ & \\
\hline & $10 / 14 / 93$ & $809 \mathrm{CV} 4$ & & $5.42 \mathrm{U}$ & & $4.05 \mathrm{U}$ \\
\hline
\end{tabular}


Table 5-4. Constituents with at Least One Detected Value for the Grout Treatment Facility Data for Reporting Period October 1

through December 31, 1993. (sheet 3 of 6)

\begin{tabular}{|c|c|c|c|c|c|c|}
\hline $\begin{array}{l}\text { Mell } \\
\text { Mane }\end{array}$ & $\begin{array}{c}\text { Collection } \\
\text { Date }\end{array}$ & $\begin{array}{l}\text { Sample } \\
\text { Number }\end{array}$ & $\begin{array}{l}\text { COL IFORM } \\
144 / \mathrm{COL} \\
1 / 1 \mathrm{i}\end{array}$ & $\begin{array}{c}\text { COPPER } \\
34 / p p b \\
2.65 / 10008\end{array}$ & $\begin{array}{l}\text { FCOPPER } \\
34 / \mathrm{ppb} \\
2.65 / 1000 \mathrm{~s}\end{array}$ & $\begin{array}{l}\text { FLUDRID } \\
124 / \text { ppb } \\
13.9 / 1400 i\end{array}$ \\
\hline $\begin{array}{l}299-E 25-25 \\
299-E 25-25 \\
299-E 25-29 P \\
299-E 25-29 P \\
299-E 25-31 \\
299-E 25-31 \\
299-E 25-32 P \\
299-E 25-32 P \\
299-E 25-33 \\
299-E 25-33 \\
299-E 25-37 \\
299-E 25-37 \\
299-E 25-38 \\
299-E 25-38 \\
299-E 25-39 \\
299-E 25-39 \\
299-E 25-44 \\
299-E 25-44 \\
299-E 25-45 \\
299-E 25-65\end{array}$ & $\begin{array}{l}10 / 15 / 93 \\
10 / 15 / 93 \\
10 / 13 / 93 \\
10 / 13 / 93 \\
10 / 13 / 93 \\
10 / 13 / 93 \\
10 / 13 / 93 \\
10 / 13 / 93 \\
10 / 20 / 93 \\
10 / 20 / 93 \\
10 / 19 / 93 \\
10 / 19 / 93 \\
10 / 20 / 93 \\
10 / 20 / 93 \\
10 / 14 / 93 \\
10 / 14 / 93 \\
10 / 14 / 93 \\
10 / 14 / 93 \\
10 / 14 / 93 \\
10 / 14 / 93\end{array}$ & $\begin{array}{l}\text { B09CN7 } \\
\text { 809CP1 } \\
\text { B09CP2 } \\
\text { B09CP6 } \\
\text { B09BJ8 } \\
\text { B09BJ9 } \\
\text { B09BKO } \\
\text { 809BK1 } \\
\text { B09CA2 } \\
\text { B09CQ6 } \\
\text { B09CA7 } \\
\text { B09CR1 } \\
\text { B09CR2 } \\
\text { B09CR6 } \\
\text { B09CR7 } \\
\text { B09CS1 } \\
\text { B09CT5 } \\
\text { B09CT9 } \\
\text { B09CVO } \\
\text { B09CV4 }\end{array}$ & $\begin{array}{l}1.00 \mathrm{U} \\
1.00 \mathrm{U}\end{array}$ & $\begin{array}{l}2.65 \mathrm{U} \\
2.65 \mathrm{U} \\
4.00 \mathrm{~L} \\
3.00 \mathrm{~L} \\
2.65 \mathrm{U} \\
7.60 \mathrm{~L} \\
11.00 \mathrm{~L} \\
2.65 \mathrm{U} \\
2.65 \mathrm{U}\end{array}$ & $\begin{array}{l}2.65 U \\
2.65 U \\
2.65 U \\
2.65 U \\
2.65 U \\
2.65 U \\
2.65 U \\
2.65 U \\
2.65 U \\
2.65 U\end{array}$ & $\begin{array}{r}200.00 \\
700.00 \\
200.00 \\
200.00 \\
1000.00 \\
300.00 \\
700.00 \\
200.00 \\
600.00 \\
700.00\end{array}$ \\
\hline
\end{tabular}

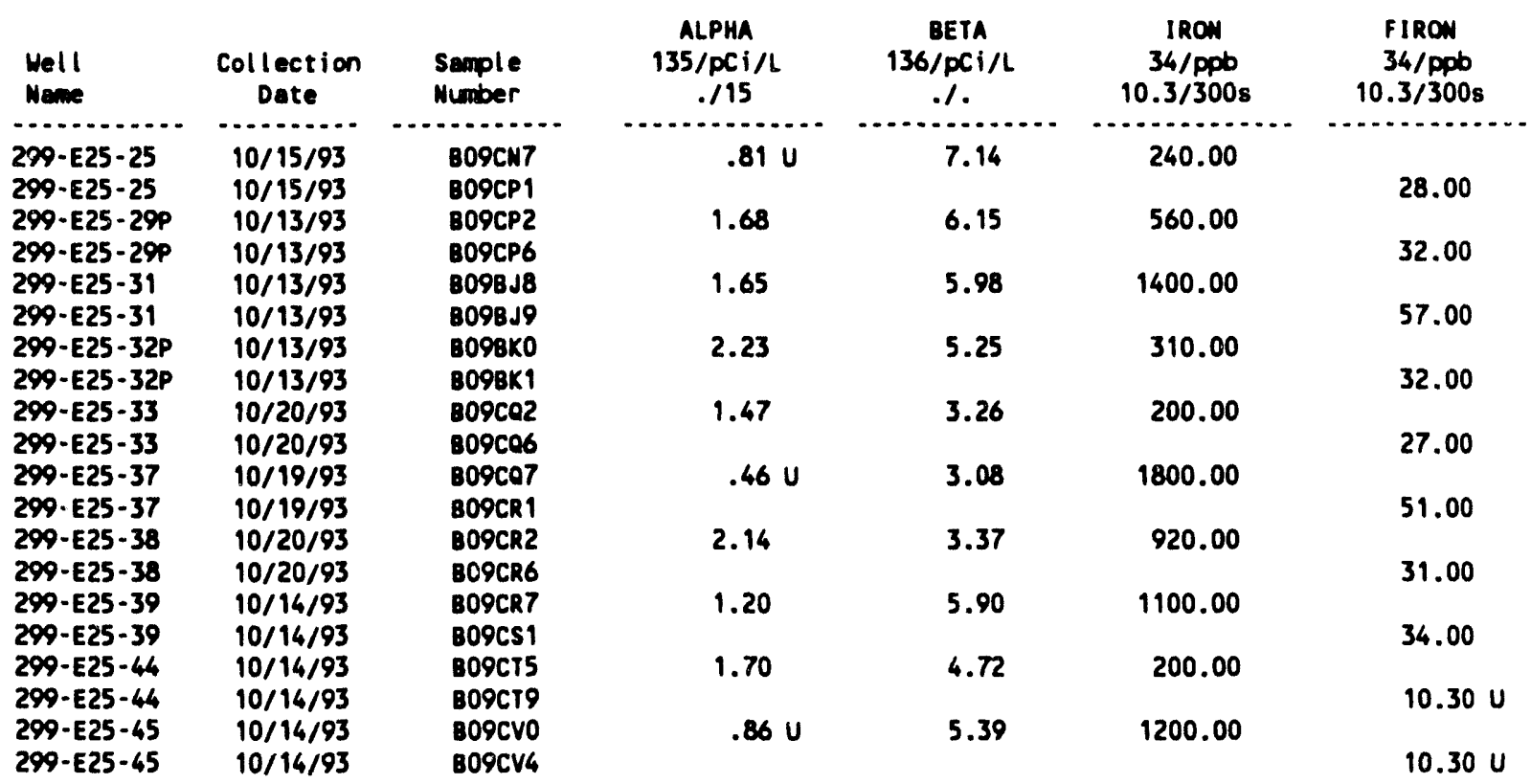


Table 5-4. Constituents with at Least One Detected Value for the Grout Treatment Facility Data for Reporting Period October 1

through December 31,1993 . (sheet 4 of 6)

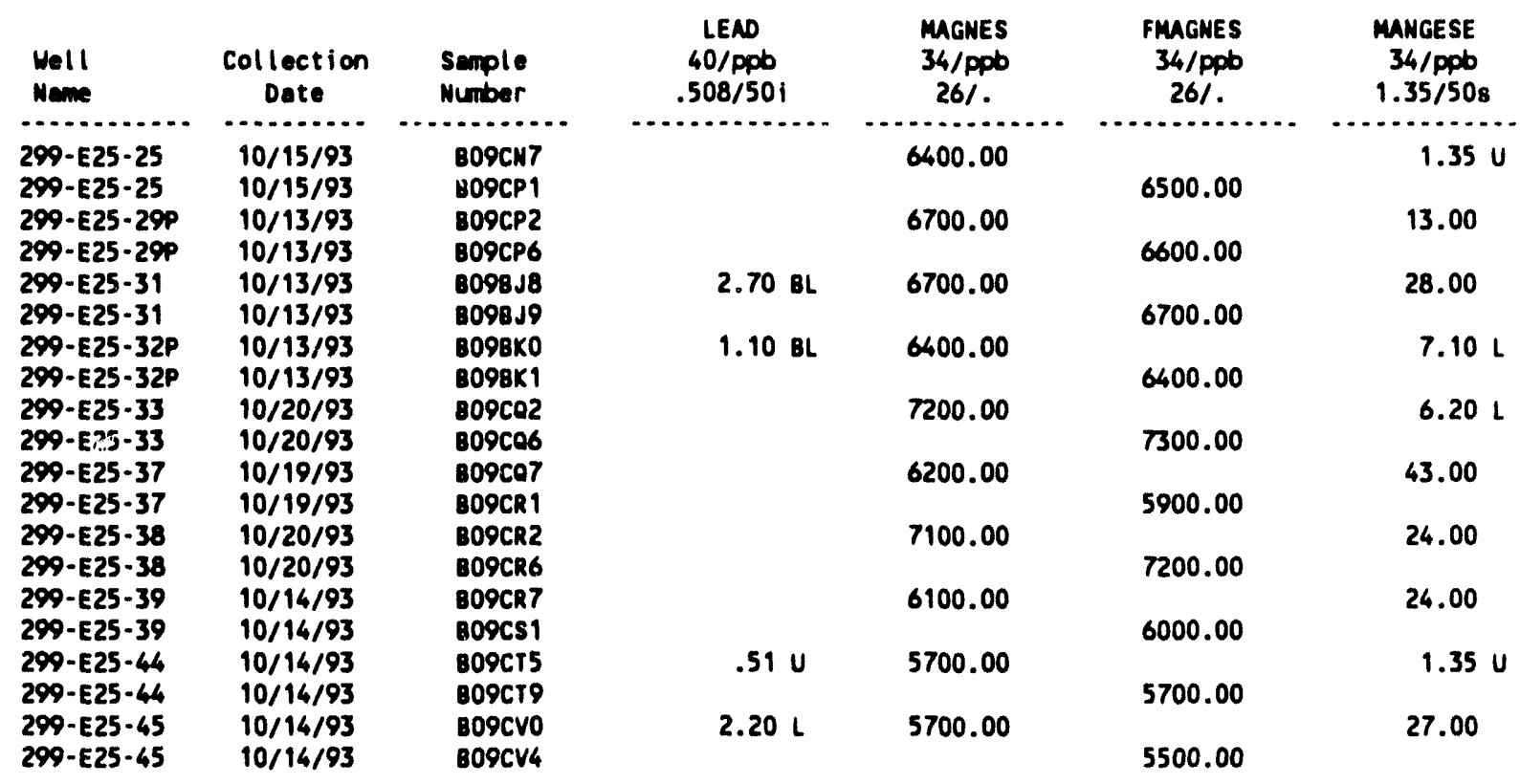

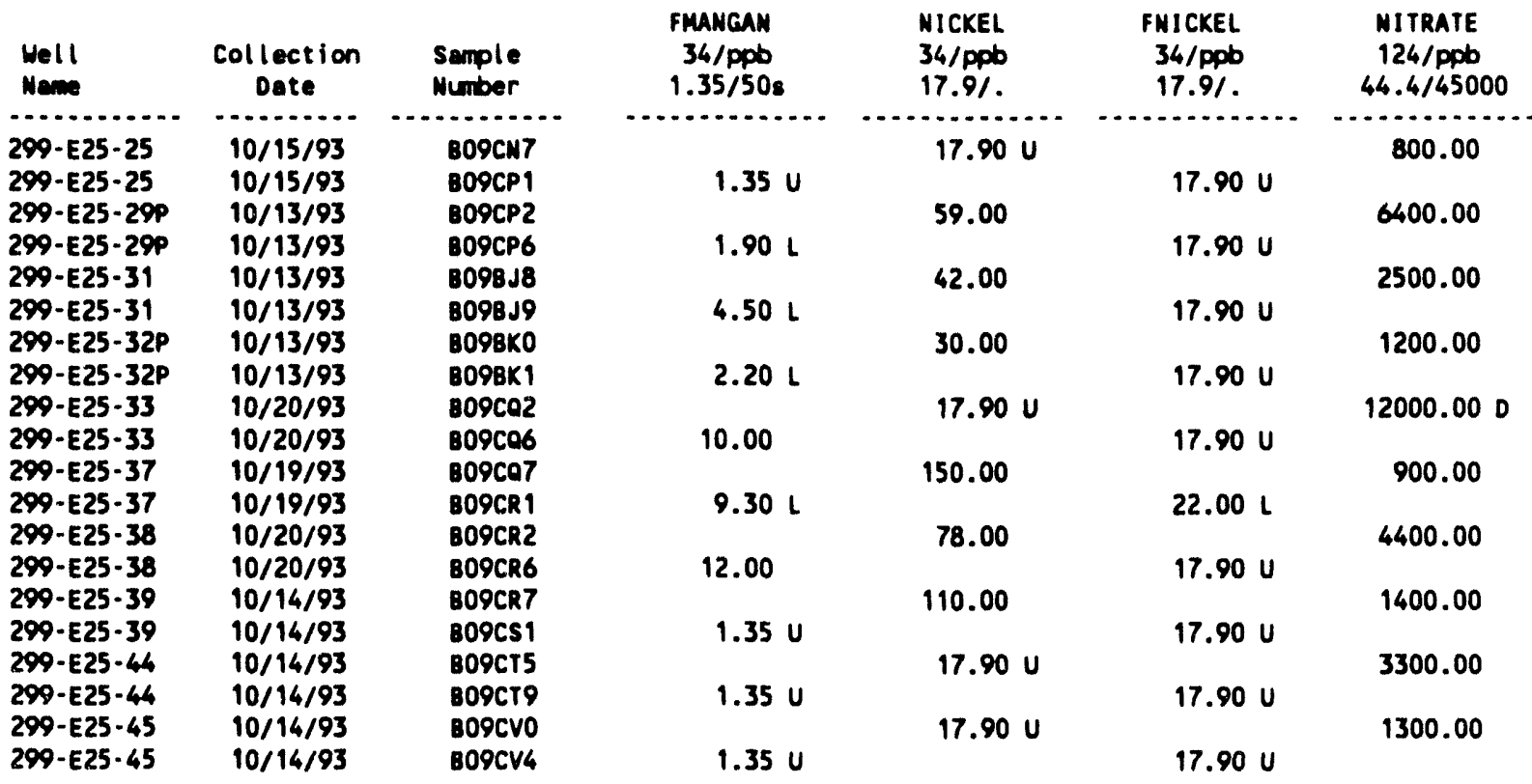


Table 5-4. Constituents with at Least One Detected Value for the Grout Treatment Facility Data for Reporting Perlod October 1

through December 31, 1993. (sheet 5 of 6)

\begin{tabular}{|c|c|c|c|c|c|c|}
\hline $\begin{array}{l}\text { Well } \\
\text { Neme }\end{array}$ & $\begin{array}{c}\text { Collection } \\
\text { Dete }\end{array}$ & $\begin{array}{l}\text { semple } \\
\text { Number }\end{array}$ & $\begin{array}{c}\text { PENTCHP } \\
30 / \text { PPb } \\
.87 / 1\end{array}$ & $\begin{array}{l}\text { LPHENOL } \\
\text { 30/PPD } \\
.31 \%\end{array}$ & $\begin{array}{l}\text { POTASUW } \\
34 / \text { PYb } \\
6621 .\end{array}$ & $\begin{array}{l}\text { FPOTASS } \\
34 / \text { Ppb } \\
662 \%\end{array}$ \\
\hline $\begin{array}{l}299-E 25-25 \\
299-E 25-25 \\
299-E 25-29 P \\
299-E 25-29 P \\
299-E 25-31 \\
299-E 25-31 \\
299-E 25-32 P \\
299-E 25-32 P \\
299-E 25-33 \\
299-E 25-33 \\
299-E 25-37 \\
299-E 25-37 \\
299-E 25-38 \\
299-E 25-38 \\
299-E 25-39 \\
299-E 25-39 \\
299-E 25-44 \\
299-E 25-44 \\
299-E 25-45 \\
299-E 25-45\end{array}$ & $\begin{array}{l}10 / 15 / 93 \\
10 / 15 / 93 \\
10 / 13 / 93 \\
10 / 13 / 93 \\
10 / 13 / 93 \\
10 / 13 / 93 \\
10 / 13 / 93 \\
10 / 13 / 93 \\
10 / 20 / 93 \\
10 / 20 / 93 \\
10 / 19 / 93 \\
10 / 19 / 93 \\
10 / 20 / 93 \\
10 / 20 / 93 \\
10 / 14 / 93 \\
10 / 14 / 93 \\
10 / 14 / 93 \\
10 / 14 / 93 \\
10 / 14 / 93 \\
10 / 14 / 93\end{array}$ & $\begin{array}{l}\text { B09CN7 } \\
\text { B09CP1 } \\
\text { 809CP2 } \\
\text { B09CP6 } \\
\text { B09BJ8 } \\
\text { B098J9 } \\
\text { 809BK0 } \\
\text { B09BK1 } \\
\text { B09CA2 } \\
\text { B09CA6 } \\
\text { B09CA7 } \\
\text { B09CR1 } \\
\text { B09CR2 } \\
\text { B09CR6 } \\
\text { B09CR7 } \\
\text { B09CS1 } \\
\text { B09C15 } \\
\text { B09CTP } \\
\text { B09CVO } \\
\text { B09CV4 }\end{array}$ & $\begin{array}{l}1.60 \mathrm{~L} \\
.87 U \\
.87 U \\
.87 U \\
.87 U \\
.87 U \\
.87 U \\
.87 U \\
.87 U \\
.87 U\end{array}$ & $\begin{array}{l}1.90 \mathrm{~L} \\
.31 U \\
.31 U \\
.31 U \\
.31 U \\
.31 U \\
.31 U \\
.31 U \\
.31 U \\
.31 U\end{array}$ & $\begin{array}{l}3700.00 \\
4400.00 \\
4300.00 \\
3500.00 \\
4700.00 \\
4600.00 \\
4600.00 \\
3900.00 \\
4300.00 \\
4700.00\end{array}$ & $\begin{array}{l}3100.00 \\
4600.00 \\
4700.00 \\
4000.00 \\
4200.00 \\
4500.00\end{array}$ \\
\hline $\begin{array}{l}\text { Well } \\
\text { Nene }\end{array}$ & $\begin{array}{c}\text { Collection } \\
\text { Date }\end{array}$ & $\begin{array}{l}\text { Somple } \\
\text { Number }\end{array}$ & $\begin{array}{l}\text { SELENUW } \\
48 / \text { ppb } \\
1.21 / 101\end{array}$ & $\begin{array}{l}\text { FSELENI } \\
48 / \mathrm{ppb} \\
1.21 / 10 \mathrm{i}\end{array}$ & $\begin{array}{l}\text { soolly } \\
34 / p p b \\
40.9 /\end{array}$ & $\begin{array}{l}\text { Fsoolum } \\
34 / \mathrm{ppb} \\
40.9 \%\end{array}$ \\
\hline $\begin{array}{l}299-E 25-25 \\
299-E 25-25 \\
299-E 25-29 P \\
299-E 25-29 P \\
299-E 25-31 \\
299-E 25-31 \\
299-E 25-32 P \\
299-E 25-32 P \\
299-E 25-33 \\
299-E 25-33 \\
299-E 25-37 \\
299-E 25-37 \\
299-E 25-38 \\
299-E 25-38 \\
299-E 25-39 \\
299-E 25-39 \\
299-E 25-44 \\
299-E 25-44 \\
299-E 25-45 \\
299-E 25-45\end{array}$ & $\begin{array}{l}10 / 15 / 93 \\
10 / 15 / 93 \\
10 / 13 / 93 \\
10 / 13 / 93 \\
10 / 13 / 93 \\
10 / 13 / 93 \\
10 / 13 / 93 \\
10 / 13 / 93 \\
10 / 20 / 93 \\
10 / 20 / 93 \\
10 / 19 / 93 \\
10 / 19 / 93 \\
10 / 20 / 93 \\
10 / 20 / 93 \\
10 / 14 / 93 \\
10 / 14 / 93 \\
10 / 14 / 93 \\
10 / 14 / 93 \\
10 / 14 / 93 \\
10 / 14 / 93\end{array}$ & 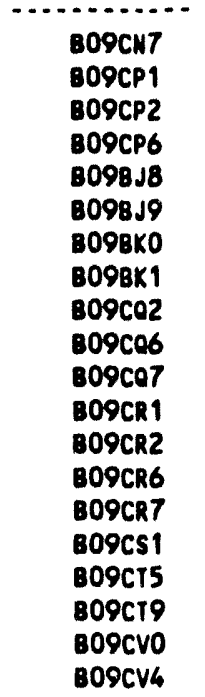 & $\begin{array}{l}1.21 \mathrm{U} \\
1.21 \mathrm{U} \\
1.21 \mathrm{U} \\
1.21 \mathrm{U} \\
1.21 \mathrm{U} \\
2.00 \mathrm{LB} \\
1.21 \mathrm{U} \\
1.21 \mathrm{U} \\
1.21 \mathrm{U} \\
1.21 \mathrm{U}\end{array}$ & $\begin{array}{l}1.21 \mathrm{U} \\
1.21 \mathrm{U} \\
1.21 \mathrm{U} \\
1.21 \mathrm{U} \\
2.00 \mathrm{~L} \\
2.00 \mathrm{LB} \\
1.21 \mathrm{U} \\
1.21 \mathrm{U} \\
1.21 \mathrm{U} \\
1.21 \mathrm{U}\end{array}$ & $\begin{array}{r}5100.00 \\
19000.00 \\
15000.00 \\
5700.00 \\
25000.00 \\
7300.00 \\
10000.00 \\
6000.00 \\
15000.00 \\
16000.00\end{array}$ & $\begin{array}{r}5100.00 \\
19000.00 \\
15000.00 \\
5700.00 \\
25000.00 \\
7000.00 \\
10000.00 \\
5900.00 \\
15000.00 \\
16000.00\end{array}$ \\
\hline
\end{tabular}


Table 5-4. Constituents with at Least One Detected Value for the Grout Treatment Facility Data for Reporting Period October 1

through December 31, 1993. (sheet 6 of 6)

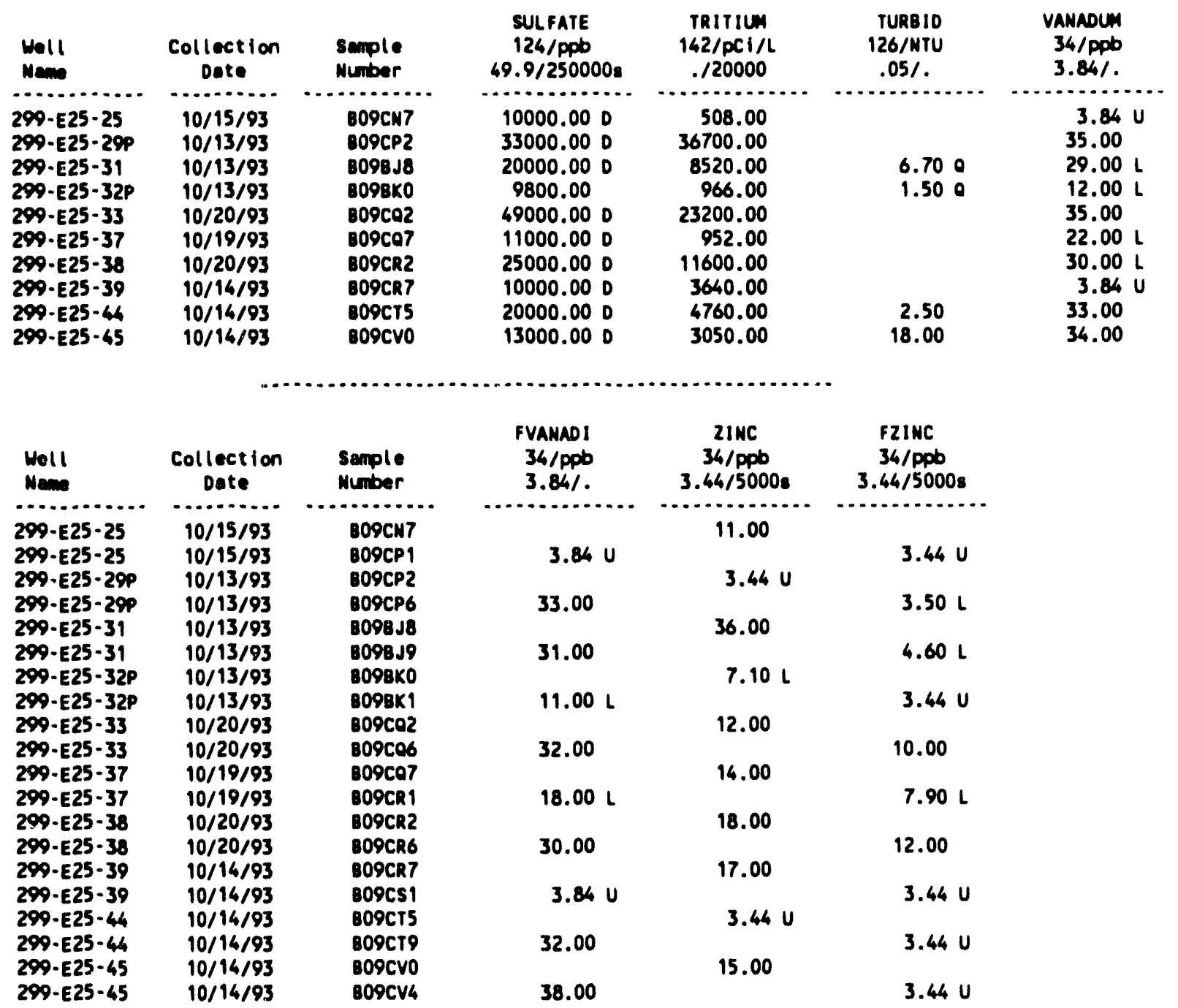

For explention of this table, see Section 1.4 of report. 
Table 5-5. Contamination Indicator Parameters for the Grout Treatment Facility Data for Reporting Period October 1 through December 31, 1993.

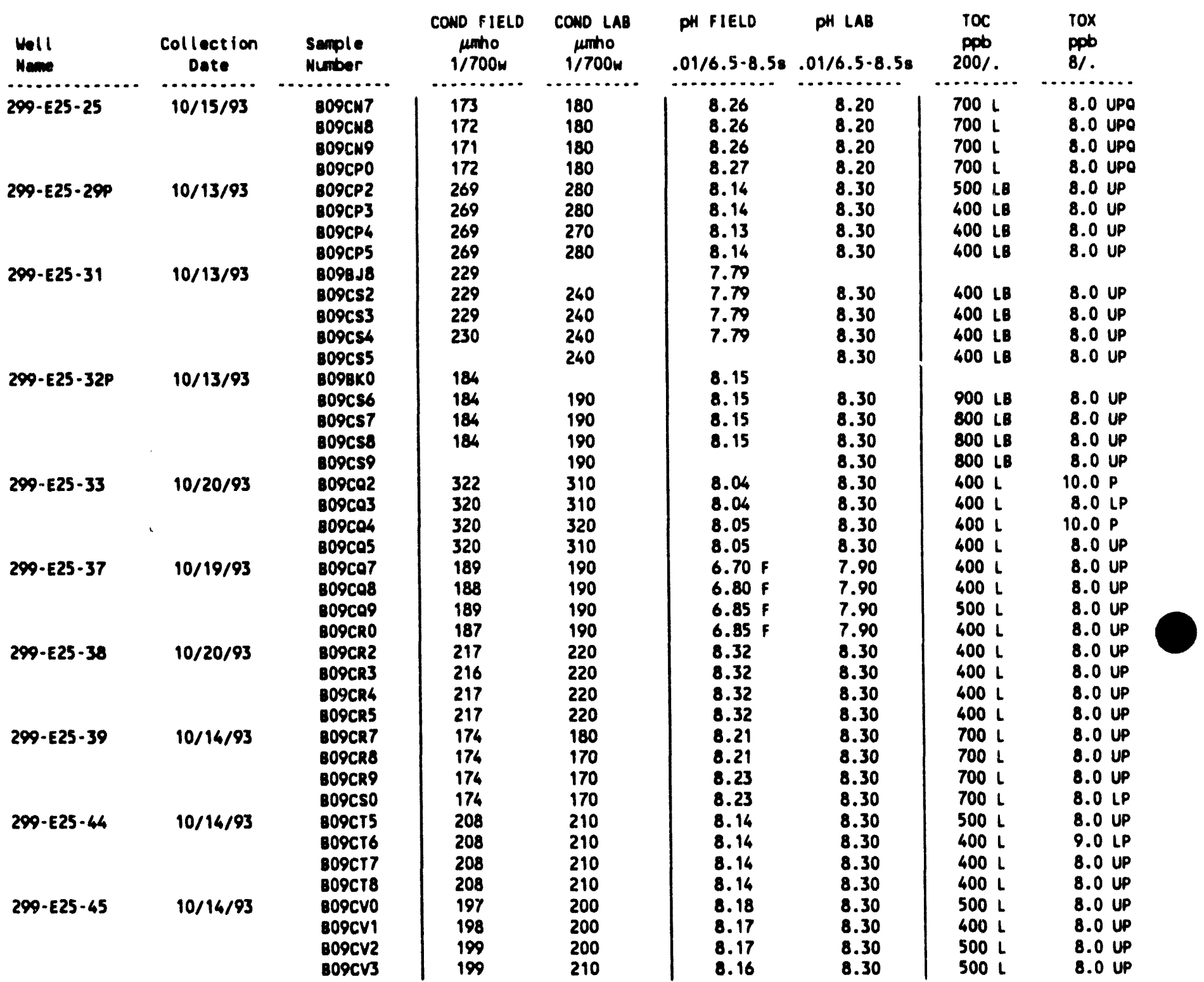

For explanation of this teble, see Section 1.4 of report. 
Table 5-6. Summary Table of Flagged Data for Reporting Period October 1 through December 31, 1993.

\begin{tabular}{|c|c|c|c|c|c|c|}
\hline $\mathrm{Well} 7^{\circ}$ & $E 25-25$ & $E 25-29 P$ & E25-31 & $E 25-32 P$ & E25-33 & E25-37 \\
\hline Constituent & $\begin{array}{c}\text { TOX } \\
\text { arsenic } \\
\end{array}$ & TOC & $\begin{array}{l}\text { TOC } \\
\text { lead }\end{array}$ & $\begin{array}{l}\text { TOC } \\
\text { lead }\end{array}$ & Nitrate & $\begin{array}{l}\text { Field pH } \\
\text { selenium }\end{array}$ \\
\hline $\mathrm{Flag}$ & $\begin{array}{c}Q \\
B Q\end{array}$ & B & $\begin{array}{l}B \\
B\end{array}$ & $\begin{array}{l}\text { B } \\
\text { B }\end{array}$ & D & $\begin{array}{l}F \\
B\end{array}$ \\
\hline Sample date & $10 / 15 / 93$ & $10 / 13 / 93$ & $10 / 13 / 93$ & $10 / 13 / 93$ & $10 / 20 / 93$ & $10 / 19 / 93$ \\
\hline
\end{tabular}

Well names are all preceded by 299 -.

${ }_{B}=$ blank contamination.

$D=$ sample diluted.

$F=$ formally under investigation.

$Q=$ associated with out-of-limits analysis.

TOC = total organic carbon.

TOX = total organic halogen. 


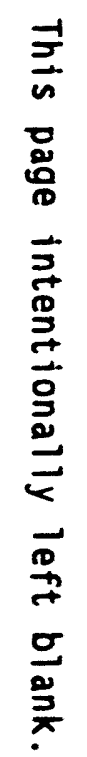

8
0
00
10
0
1
0
0
1
1

0 
DOE/RL-93-56-4

\section{CONTENTS}

$6.0216-B-3$ POND SYSTEM . . . . . . . . . . . . . . . 6-1

6.1 INTRODUCTION ................... . . . . . . .

6.2 WATER LEVEL DATA . . . . . . . . . . . . . . . . . 6-1

6.3 WATER CHEMISTRY DATA ............... . . 6-1 
DOE/RL-93-56-4

LIST OF FIGURES

6-1 216-B-3 Pond Groundwater Monitoring Network . . . . . . . . . 6-3

\section{LIST OF TABLES}

6-1 Monitoring Well Purpose and Sampling Schedule

for the 216-B-3 Pond System Network . . . . . . . . . . . . . 6-4

6-2 RCRA Water Level Measurement Report for the 216-B-3 Pond System, Fourth Quarter 1993 . . . . . . . . . . . . . . 6-5

6-3 Constituent List and Summary of Results for the 216-B-3 Pond Data for Reporting Period October 1 through December 31, 1993 . . . . 6-9

6-4 Constituents with at Least One Detected Value for the 216-B-3 Pond Data for Reporting Period October 1 through December 31, 1993 . . . 6-12

6-5 Contamination Indicator Parameters for the 216-B-3 Pond Data for Reporting Period October 1 through December 31, $1993 \ldots$..... 6-21 


\title{
6.0 216-B-3 POND SYSTEM
}

\author{
D. B. Barnett \\ Westinghouse Hanford Company
}

\subsection{INTRODUCTION}

The 216-B-3 Pond System is located east of the 200 East Area and consists of a main pond, three interconnected lobes for wastewater disposal, and several ditches leading to the ponds (Figures $1-1$ and $6-1$ ). These surface impoundments cover approximately 41.3 hectares (102 acres). Table 6-1 1ists the groundwater wells and their monitoring status.

The 216-B-3 Pond System groundwater monitoring well locations are shown in Figure 6-1. Detection monitoring began at the 216-B-3 Pond System in November 1988 and continued through June 1990, when assessment monitoring was scheduled to begin because of elevated levels of TOX in two downgradient monitoring wells (699-43-41E and 699-43-4lF). Assessment monitoring actually began in June 1991 when groundwater sampling on the Hanford Site resumed, following a hiatus, as described in the Groundwater Quality Assessment Plan for the 216-8-3 Pond System (Harris 1990). Thus far, no specific constituents have been linked to the elevated TOX results. It is anticipated that the groundwater monitoring program will return to detection level monitoring in CY 1994.

\subsection{WATER LEVEL DATA}

Water levels were measured at least three times in all wells during the October through December 1993 period, as shown in Table 6-2. Results reported for wells 699-40-40B (measured October 7, 1993) and 699-42-37 (measured October 8, 1993) are outside of the expected range and may be in error.

\subsection{MATER CHEMISTRY DATA}

A11 wells scheduled for the October through December 1993 sampling were sampled during October, except upgradient we 11 299-E18-1, which was sampled in December. Results for the period that are unavailable as of the writing of this report will be reported in future quarterly reports.

The list of constituents for which data were received and summary of results available are given in Table 6-3. The results of the available analyses, for constituents with at least one value above the MDL, are reported in Table 6-4. Results of analyses for CIPs are reported in Table 6-5. Explanations of data flags and qualifiers are given in Section 1.4 . 
Constituents exceeding DWS during the October through December 1993 period include chromium in unfiltered samples from wells 299-E32-4, 699-40-36, 699-40-39, 699-40-40A, 699-40-40B, 699-41-40, 699-42-39A, 699-42-39B, 699-43-4lE, 699-43-41G, 699-43-42J, 699-44-42, and 699-44-43B (2 samples); iron in unfiltered samples from wells 299-E32-4, 699-40-36, 699-40-39, 699-40-40A, 699-40-40B, 699-41-40, 699-42-37, 699-42-39A, 699-42-39B, 699-42-40A, 699-43-41E, 699-43-41G, 699-43-42J, and 699-44-43B; manganese in unfiltered samples from wells 699-40-36, 699-40-39, 699-40-40A, 699-40-40B, 699-41-35, 699-41-40, 699-42-37, 699-42-39B, 699-42-40A, and 699-43-41G; and tritium from wells 699-41-40, 699-41-42, 699-42-39A, 699-42-39B, 699-42-42B, 699-43-40, 699-43-41E, 699-43-41F, 699-43-41G, and 699-44-39B. Results in Table 6-4 show an unusually large number of $Q$ flags for results of ammonium and turbidity. The reasons for this flag are discussed in Section 1.2.

Results for several constituents from well 699-40-39 exceed or fall below historical ranges for these constituents. The constituents include several metals and tritium. It is possible that high turbidity ( 340 NTUs) in the sample is responsible for these departures. The affected constituents are flagged in Table 6-4, and an RADE has been submitted to investigate the reason for the anomalies.

An RADE has also been submitted for the result for field $\mathrm{pH}$ from well 699-41-42. This result exceeds the upper limit of DWS for $\mathrm{pH}$, and is the highest result received for this well thus far. 


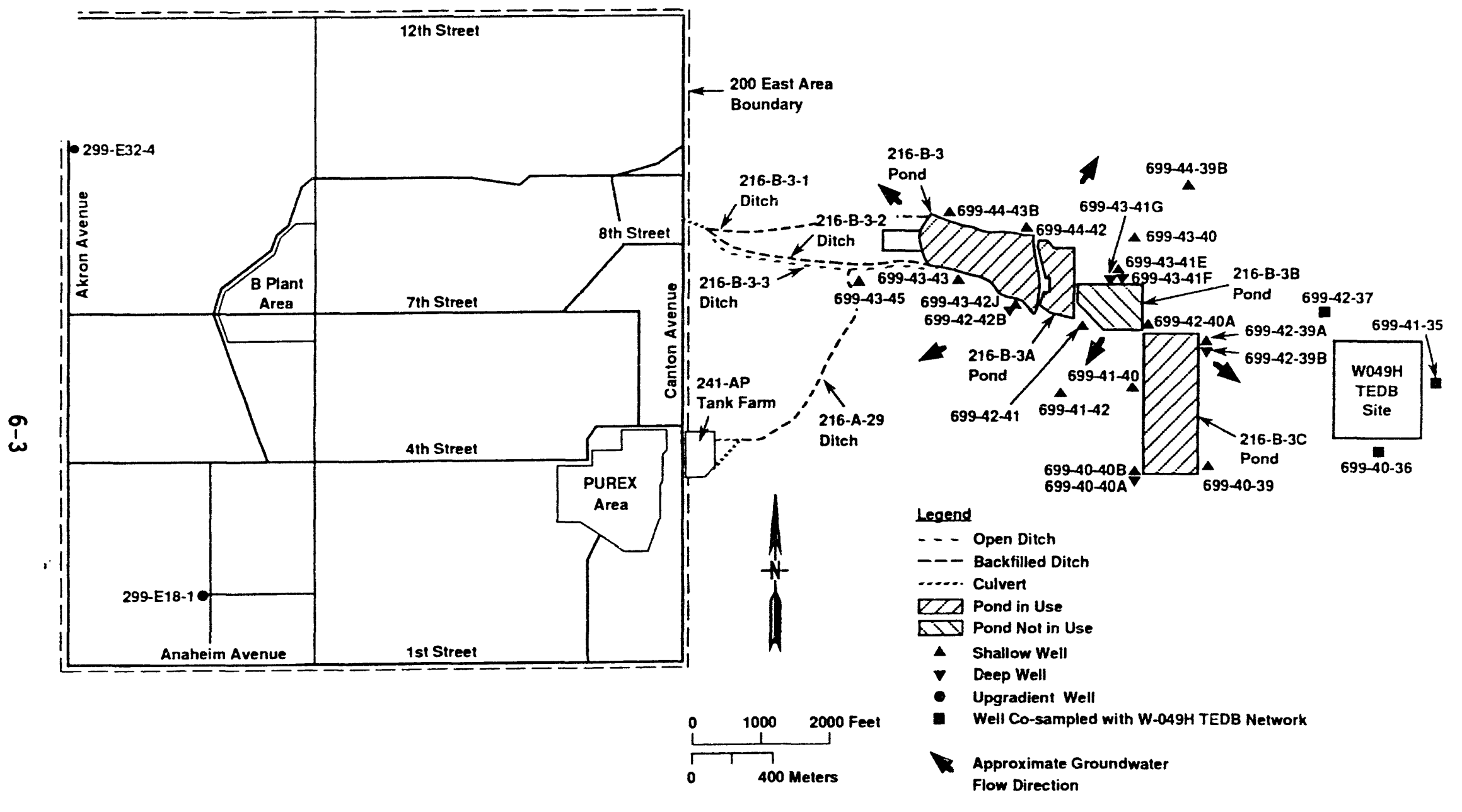

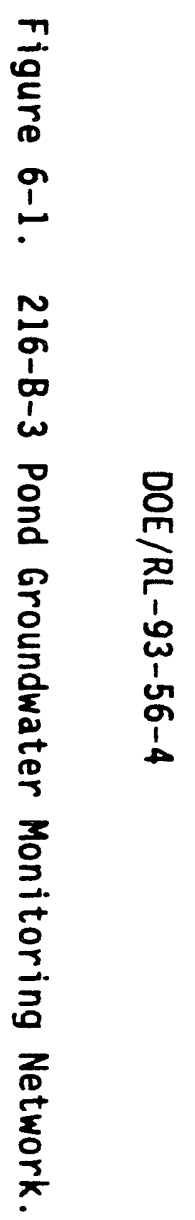

H9312019.1 
Table 6-1. Monitoring Well Purpose and Sampling Schedule for the 216-B-3 Pond System Network.

\begin{tabular}{|c|c|c|c|c|}
\hline Well no. & $\begin{array}{l}\text { Relative } \\
\text { position }\end{array}$ & Hydrogeologic & $\begin{array}{c}\text { Sample } \\
\text { frequency }\end{array}$ & $\begin{array}{l}\text { Sample date, } \\
\text { 4th Qtr } 1993\end{array}$ \\
\hline $299-\mathrm{E} 18-1^{\circ}$ & Upgradient & Hanford: Water Table & Quarterly & $12 / 15 / 93$ \\
\hline $299-E 32-4^{6}$ & Upgradient & Hanford: Water Table & Quarterly & $10 / 15 / 93$ \\
\hline $699-40-36^{c}$ & Downgradient & Ringold: Top of Confined & Quarterly & $10 / 08 / 93$ \\
\hline $699-40-39$ & Downgradient & Ringold: Upper Semiconfined & Quarterly & $10 / 19 / 93$ \\
\hline $699-40-40 A$ & Downgradient & Ringold: Lower Semiconfined & Quarterly & $10 / 07 / 93$ \\
\hline $699-40-40 B$ & Downgradient & Ringold: Upper Semiconfined & Quarterly & $10 / 07 / 93$ \\
\hline $699-41-35^{c}$ & Downgradient & Ringold: Top of Confined & Quarterly & $10 / 08 / 93$ \\
\hline $699-4$ & Down & Ringold: Upper Semiconfined & Quarterly & $1 / 93$ \\
\hline $699-41-42$ & Downgradient & Ringold: Top of Confined & Quarterly & $10 / 11 / 93$ \\
\hline $699-42-37^{c}$ & Downgradient & Ringold: Top of Confined & Quarterly & $10 / 08 / 93$ \\
\hline $699-42-39 A$ & Downgr: & Ringold: Upper Semiconfined & Quarterly & $10 / 07 / 93$ \\
\hline $699-42-39 B$ & Downgradient & Ringold: Lower Semiconfined & Quarteriy & $10 / 07 / 93$ \\
\hline $699-42-40 \mathrm{~A}$ & Downgradient & Ringold: Upper Semiconfined & Quarterly & $10 / 11 / 93$ \\
\hline $699-42-41$ & Downgradient & Ringold: Water Table & Quarterly & $10 / 11 / 93$ \\
\hline $699-42-42 B$ & Downgradient & Ringold: Lower Unconfined & Quarterly & $10 / 13 / 93$ \\
\hline $699-43-40$ & Downgradient & Ringold: Water Table & Quarterly & $10 / 11 / 93$ \\
\hline $699-43-41 E$ & Downgradient & Ringold: Upper Semiconfined & Quarterly & $10 / 11 / 93$ \\
\hline $699-43-41 F$ & Downgradient & Ringold: Lower Semiconfined & Quarterly & $10 / 11 / 93$ \\
\hline $699-43-41 G$ & Downgradient & 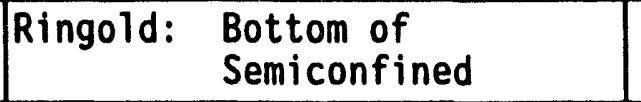 & Quarterly & \\
\hline $699-43-42 \mathrm{~J}$ & Downgradient & Ringold: Water Table & Quarterly & $10 / 13 / 93$ \\
\hline $699-43-43^{d}$ & Downgradient & Hanford/Ringold: Water Table & Quarterly & $10 / 13 / 93$ \\
\hline $699-43-45^{d}$ & Downgradient & Hanford/Ringold: Water Table & Quarterly & $10 / 13 / 93$ \\
\hline $699-44-39 B$ & Downgradient & Ringold: Water Table & Quarterly & $10 / 11 / 93$ \\
\hline $699-44-42$ & Downgradient & Ringold: Water Table & Quarterly & $10 / 13 / 93$ \\
\hline $699-44-43 B$ & Downgradient & Ringold: Water Table & Quarterly & $10 / 12 / 93$ \\
\hline
\end{tabular}

Well shared with the 2101-M Pond network.

Well shared with the Low-Level Waste Burial Grounds network.

CWells shared with the W-O49H Treated Effluent Disposal Basin network.

Wells shared with the 216-A-29 Ditch network. 
Table 6-2. RCRA Water Level Measurement Report for the 216-B-3 Pond System, Fourth Quarter 1993. (sheet 1 of 4)

Well Date $\quad \begin{gathered}\text { Depth to } \\ \text { water }(\mathrm{ft})\end{gathered} \begin{gathered}\text { Water level } \\ \text { elevation } \\ \text { above msl (ft) }\end{gathered}$

Wells Monitoring the Unconfined Aquifer at the Water Table

\begin{tabular}{|c|c|c|c|}
\hline 299-E18-1 & $\begin{array}{l}10 / 12 / 93 \\
11 / 03 / 93 \\
12 / 13 / 93\end{array}$ & $\begin{array}{l}318.37 \\
318.25 \\
318.35\end{array}$ & $\begin{array}{l}401.87 \\
401.99 \\
401.89\end{array}$ \\
\hline 299-E32-4 & $\begin{array}{l}10 / 14 / 93 \\
10 / 15 / 93 \\
11 / 11 / 93 \\
12 / 16 / 93\end{array}$ & $\begin{array}{l}284.52 \\
284.34 \\
284.52 \\
284.69\end{array}$ & $\begin{array}{l}401.36 \\
401.54 * \\
401.36 \\
401.19\end{array}$ \\
\hline $699-41-42$ & $\begin{array}{l}10 / 04 / 93 \\
10 / 11 / 93 \\
11 / 15 / 93 \\
12 / 13 / 93\end{array}$ & $\begin{array}{l}233.44 \\
232.79 \\
233.19 \\
232.72\end{array}$ & $\begin{array}{l}410.47 \\
411.12^{\star} \\
410.72 \\
411.19\end{array}$ \\
\hline $699-42-40 A$ & $\begin{array}{l}10 / 04 / 93 \\
10 / 11 / 93 \\
11 / 15 / 93 \\
12 / 13 / 93\end{array}$ & $\begin{array}{l}125.58 \\
125.46 \\
124.90 \\
123.77\end{array}$ & $\begin{array}{l}419.95 \\
420.07 * \\
420.63 \\
421.76\end{array}$ \\
\hline $699-42-41$ & $\begin{array}{l}10 / 04 / 93 \\
10 / 11 / 93 \\
11 / 15 / 93 \\
12 / 13 / 93\end{array}$ & $\begin{array}{l}147.42 \\
147.29 \\
147.12 \\
146.54\end{array}$ & $\begin{array}{l}419.88 \\
420.01 * \\
420.18 \\
420.76\end{array}$ \\
\hline $699-43-40$ & $\begin{array}{l}10 / 04 / 93 \\
10 / 11 / 93 \\
11 / 15 / 93 \\
12 / 13 / 93\end{array}$ & $\begin{array}{l}123.61 \\
123.99 \\
123.87 \\
123.73\end{array}$ & $\begin{array}{l}418.59 \\
418.21 * \\
418.33 \\
418.47\end{array}$ \\
\hline $699-43-42 \mathrm{~J}$ & $\begin{array}{l}10 / 04 / 93 \\
10 / 13 / 93 \\
11 / 15 / 93 \\
12 / 13 / 93\end{array}$ & $\begin{array}{l}163.39 \\
163.25 \\
163.33 \\
162.87\end{array}$ & $\begin{array}{l}418.29 \\
418.43^{\star} \\
418.35 \\
418.81\end{array}$ \\
\hline $699-43-43$ & $\begin{array}{l}10 / 04 / 93 \\
10 / 13 / 93 \\
11 / 15 / 93 \\
12 / 13 / 93\end{array}$ & $\begin{array}{l}164.47 \\
164.42 \\
164.63 \\
164.40\end{array}$ & $\begin{array}{l}414.90 \\
414.95 \\
414.74 \\
414.97\end{array}$ \\
\hline
\end{tabular}


Table 6-2. RCRA Water Level Measurement Report for the 216-B-3 Pond System, Fourth Quarter 1993. (sheet 2 of 4)

\begin{tabular}{|c|c|c|c|}
\hline Well & Date & $\begin{array}{l}\text { Depth to } \\
\text { water (ft) }\end{array}$ & $\begin{array}{l}\text { Water level } \\
\text { elevation } \\
\text { above msl (ft) }\end{array}$ \\
\hline \multicolumn{4}{|c|}{ Wells Monitoring } \\
\hline $699-43-45$ & $\begin{array}{l}10 / 04 / 93 \\
10 / 13 / 93 \\
11 / 15 / 93 \\
12 / 13 / 93\end{array}$ & $\begin{array}{l}194.76 \\
194.76 \\
194.84 \\
194.78\end{array}$ & $\begin{array}{l}402.92 \\
402.92^{\star} \\
402.84 \\
402.90\end{array}$ \\
\hline $699-44-39 B$ & $\begin{array}{l}10 / 04 / 93 \\
10 / 11 / 93 \\
11 / 15 / 93 \\
12 / 13 / 93\end{array}$ & $\begin{array}{l}95.88 \\
96.00 \\
96.18 \\
95.34\end{array}$ & $\begin{array}{l}417.52 \\
417.40^{\star} \\
417.22 \\
418.06\end{array}$ \\
\hline $699-44-42$ & $\begin{array}{l}10 / 04 / 93 \\
10 / 13 / 93 \\
11 / 15 / 93 \\
12 / 13 / 93\end{array}$ & $\begin{array}{l}158.75 \\
158.65 \\
158.84 \\
158.55\end{array}$ & $\begin{array}{l}420.47 \\
420.57^{\star} \\
420.38 \\
420.67\end{array}$ \\
\hline $699-44-43 B$ & $\begin{array}{l}10 / 04 / 93 \\
10 / 12 / 93 \\
11 / 15 / 93 \\
12 / 13 / 93\end{array}$ & $\begin{array}{l}164.59 \\
164.60 \\
164.65 \\
164.77\end{array}$ & $\begin{array}{l}415.53 \\
415.52^{\star} \\
415.47 \\
415.35\end{array}$ \\
\hline
\end{tabular}

Wells Monitoring Confined or Semi-Confined Aquifer

\begin{tabular}{llll}
\hline $699-40-36$ & $10 / 04 / 93$ & 118.23 & 410.69 \\
& $10 / 08 / 93$ & 118.37 & $410.55^{\star}$ \\
$11 / 15 / 93$ & 118.25 & 410.67 \\
$12 / 13 / 93$ & 118.12 & 410.80 \\
$699-40-39$ & $10 / 04 / 93$ & 129.99 & 411.85 \\
& $10 / 19 / 93$ & 130.15 & $411.69 \star$ \\
& $11 / 15 / 93$ & 129.95 & 411.89 \\
$699-40-40 A$ & $12 / 13 / 93$ & 129.71 & 412.13 \\
& $10 / 04 / 93$ & 130.65 & 410.56 \\
& $10 / 07 / 93$ & 130.75 & $410.46 \star$ \\
& $11 / 15 / 93$ & 130.53 & 410.68 \\
& $12 / 13 / 93$ & 130.16 & 411.05
\end{tabular}


Table 6-2. RCRA Water Level Measurement Report for the 216-B-3 Pond System, Fourth Quarter 1993. (sheet 3 of 4)

\begin{tabular}{|c|c|c|c|}
\hline Well & Date & $\begin{array}{l}\text { Depth to } \\
\text { water (ft) }\end{array}$ & $\begin{array}{l}\text { Water level } \\
\text { elevation } \\
\text { above msl (ft) }\end{array}$ \\
\hline \multicolumn{4}{|c|}{ Wells Monitoring Confined o } \\
\hline $699-40-40 B$ & $\begin{array}{l}10 / 04 / 93 \\
10 / 07 / 93 \\
11 / 15 / 93 \\
12 / 13 / 93\end{array}$ & $\begin{array}{l}131.32 \\
130.85 \\
131.17 \\
130.81\end{array}$ & $\begin{array}{l}410.86 \\
411.33^{\star}+ \\
411.01 \\
411.37\end{array}$ \\
\hline $699-41-35$ & $\begin{array}{l}10 / 04 / 93 \\
10 / 08 / 93 \\
11 / 15 / 93 \\
12 / 13 / 93\end{array}$ & $\begin{array}{l}108.63 \\
108.86 \\
108.67 \\
108.63\end{array}$ & $\begin{array}{l}411.75 \\
411.52 \star \\
411.71 \\
411.75\end{array}$ \\
\hline $699-41-40$ & $\begin{array}{l}10 / 04 / 93 \\
10 / 11 / 93 \\
11 / 15 / 93 \\
12 / 13 / 93\end{array}$ & $\begin{array}{l}131.67 \\
131.64 \\
131.42 \\
131.00\end{array}$ & $\begin{array}{l}414.27 \\
414.30^{\star} \\
414.52 \\
414.94\end{array}$ \\
\hline $699-42-37$ & $\begin{array}{l}10 / 04 / 93 \\
10 / 08 / 93 \\
11 / 15 / 93 \\
12 / 13 / 93\end{array}$ & $\begin{array}{l}105.12 \\
105.92 \\
105.12 \\
105.09\end{array}$ & $\begin{array}{l}414.30 \\
413.50^{\star}+ \\
414.30 \\
414.33\end{array}$ \\
\hline $699-42-39 A$ & $\begin{array}{l}10 / 04 / 93 \\
10 / 07 / 93 \\
11 / 15 / 93 \\
12 / 13 / 93\end{array}$ & $\begin{array}{l}140.73 \\
140.17 \\
140.43 \\
139.77\end{array}$ & $\begin{array}{l}417.41 \\
417.97 \star \\
417.71 \\
418.37\end{array}$ \\
\hline $699-42-39 B$ & $\begin{array}{l}10 / 04 / 93 \\
10 / 07 / 93 \\
11 / 15 / 93 \\
12 / 13 / 93\end{array}$ & $\begin{array}{l}141.12 \\
140.20 \\
140.82 \\
140.27\end{array}$ & $\begin{array}{l}417.20 \\
418.12^{\star} \\
417.50 \\
418.05\end{array}$ \\
\hline $699-43-41 E$ & $\begin{array}{l}10 / 04 / 93 \\
10 / 11 / 93 \\
11 / 15 / 93\end{array}$ & $\begin{array}{l}131.02 \\
131.10 \\
130.85\end{array}$ & $\begin{array}{l}419.84 \\
419.76^{\star} \\
420.01\end{array}$ \\
\hline $699-43-41 F$ & $\begin{array}{l}10 / 04 / 93 \\
10 / 11 / 93 \\
11 / 15 / 93\end{array}$ & $\begin{array}{l}131.09 \\
131.09 \\
130.94\end{array}$ & $\begin{array}{l}419.92 \\
419.92^{\star} \\
420.07\end{array}$ \\
\hline $699-43-41 G$ & $\begin{array}{l}10 / 04 / 93 \\
10 / 11 / 93 \\
11 / 15 / 93\end{array}$ & $\begin{array}{l}136.25 \\
136.12 \\
136.27\end{array}$ & $\begin{array}{l}415.09 \\
415.22^{\star} \\
415.07\end{array}$ \\
\hline
\end{tabular}


Table 6-2. RCRA Water Level Measurement Report for the 216-B-3 Pond System, Fourth Quarter 1993. (sheet 4 of 4)

\begin{tabular}{llll}
\hline Wel1 & Date & $\begin{array}{c}\text { Depth to } \\
\text { water }(\mathrm{ft})\end{array}$ & $\begin{array}{c}\text { Water level } \\
\text { elevation } \\
\text { above ms }(\mathrm{ft})\end{array}$ \\
\hline Well Monitoring the Unconfined Aquifer Below the Water Table \\
\hline $699-42-42 B$ & $10 / 04 / 93$ & 166.61 & 416.62 \\
& $10 / 13 / 93$ & 166.17 & $417.06 *$ \\
$11 / 15 / 93$ & 166.49 & 416.74 \\
$12 / 13 / 93$ & 166.05 & 417.18 \\
\hline
\end{tabular}

Notes: 1. Water level elevations are calculated by subtracting the measured depth to water from the surveyed elevation for the well.

2. Depth-to-water values are transcribed from field records.

3. Elevations marked with an $1 * 1$ were measured at the time of sampling.

4. Elevations marked with a ' + ' are outside of the expected range and are suspected of error.

5. To convert feet to meters multiply by 0.3048 . 
Table 6-3. Constituent List and Summary of Results for the 216-B-3 Pond Data for Reporting Period October 1 through December 31, 1993. (sheet 1 of 3)

CONTAMIMATION IMDICATOR PARAMETERS

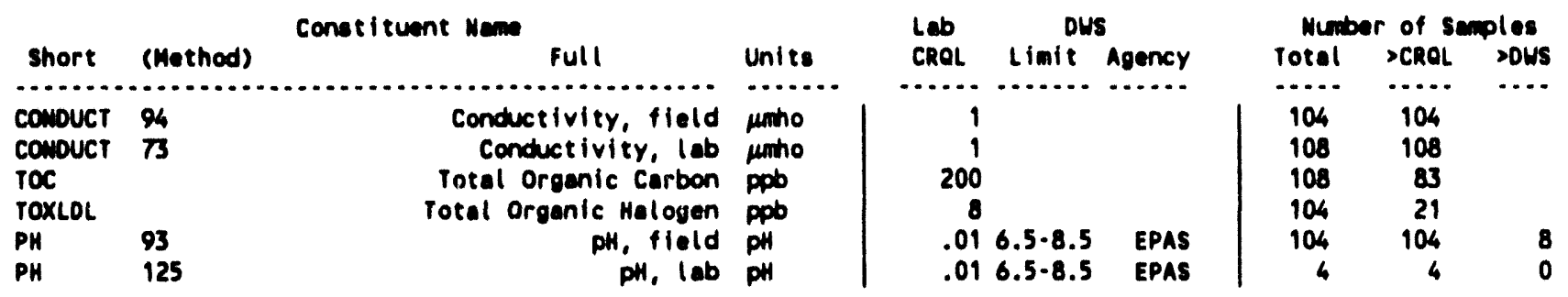

DRINKING MATER PARAMETERS

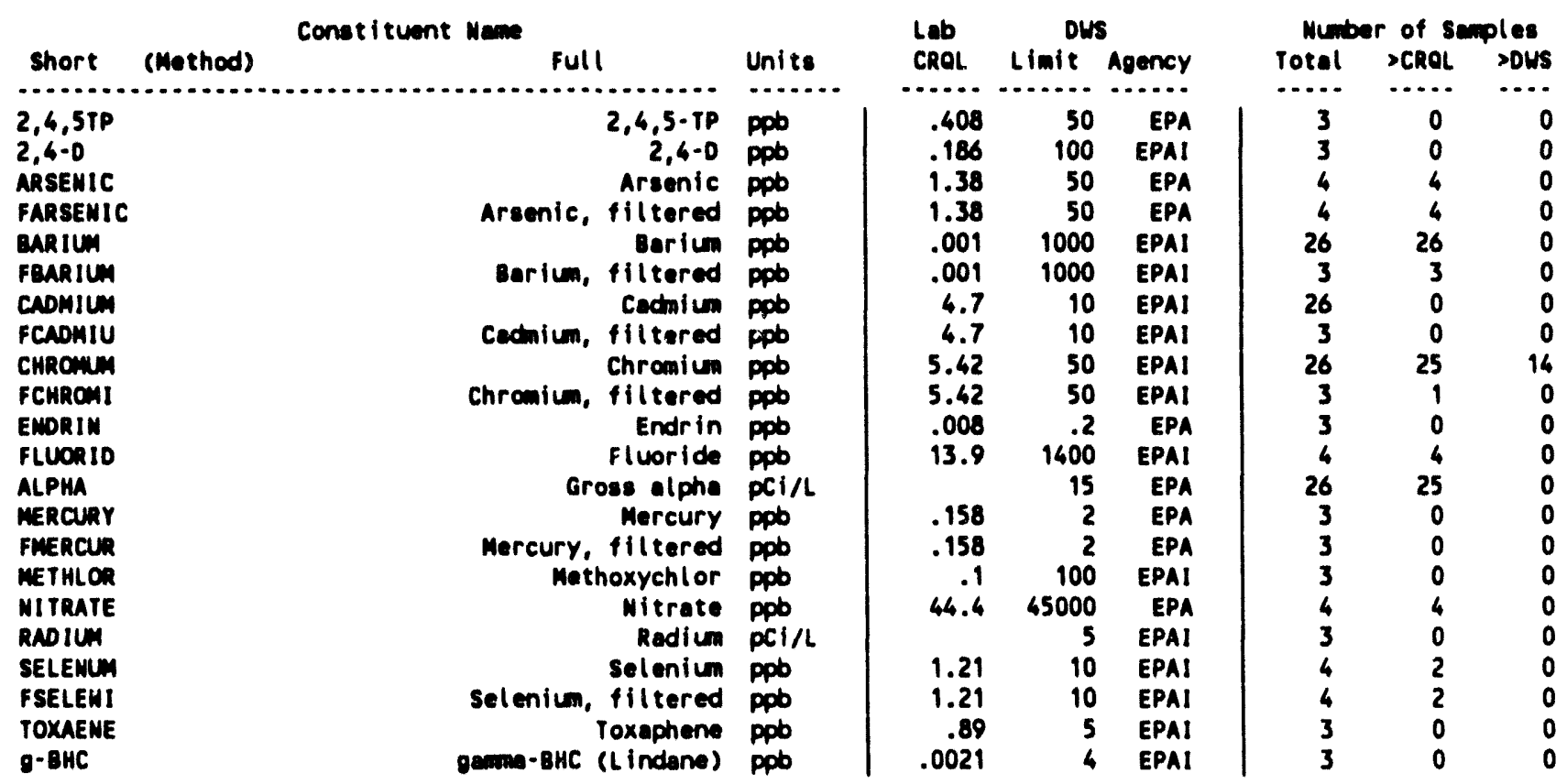

GROUNDWATER QUALITY PARAMETERS

\begin{tabular}{|c|c|c|c|c|c|c|c|c|c|}
\hline \multirow[b]{2}{*}{ Short } & \multicolumn{2}{|r|}{ Constituent Mame } & \multirow[b]{2}{*}{ Units } & \multirow{2}{*}{$\begin{array}{l}\text { Lab } \\
\text { CROL }\end{array}$} & \multicolumn{2}{|c|}{ Dus } & \multicolumn{3}{|c|}{ Number of smiples } \\
\hline & (Method) & Full & & & Limit & Agency & Total & $>$ CROL & $>$ DWS \\
\hline & 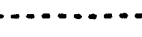 & $\cdots$ & $\cdots \cdots$ & $\cdots \ldots$ & ........ & ….. & $\cdots \cdot$ & $\ldots$. & $\cdots$ \\
\hline CHLORID & & Chloride & ppb & 82.5 & 250000 & EPAS & 4 & 4 & 0 \\
\hline IRON & & Iron & ppb & 10.3 & 300 & EPAS & 26 & 26 & 15 \\
\hline FIRON & & Iron, filltered & ppb & 10.3 & 300 & EPAS & 3 & 3 & 0 \\
\hline MANGESE & & Manganese & ppb & 1.35 & 50 & EPAS & 26 & 26 & 10 \\
\hline FMANGAN & & Manganese, filtered & ppb & 1.35 & 50 & EPAS & 3 & 2 & 0 \\
\hline LPHEMOL & & Phenol & ppb & .31 & & & 4 & 0 & \\
\hline Soolun & & Sodiun & ppob & 40.9 & & & 26 & 26 & \\
\hline Fsoolum & & Sodiun, filtered & ppb & 40.9 & & & 3 & 3 & \\
\hline SULFATE & & sulfate & ppb & 49.9 & 250000 & EPAS & 4 & 4 & 0 \\
\hline
\end{tabular}


Table 6-3. Constituent List and Summary of Results for the 216-B-3 Pond Data for Reporting Period October 1 through December 31, 1993. (sheet 2 of 3)

SITE SPECIFIC AMD OTHER CONSTITUENTS

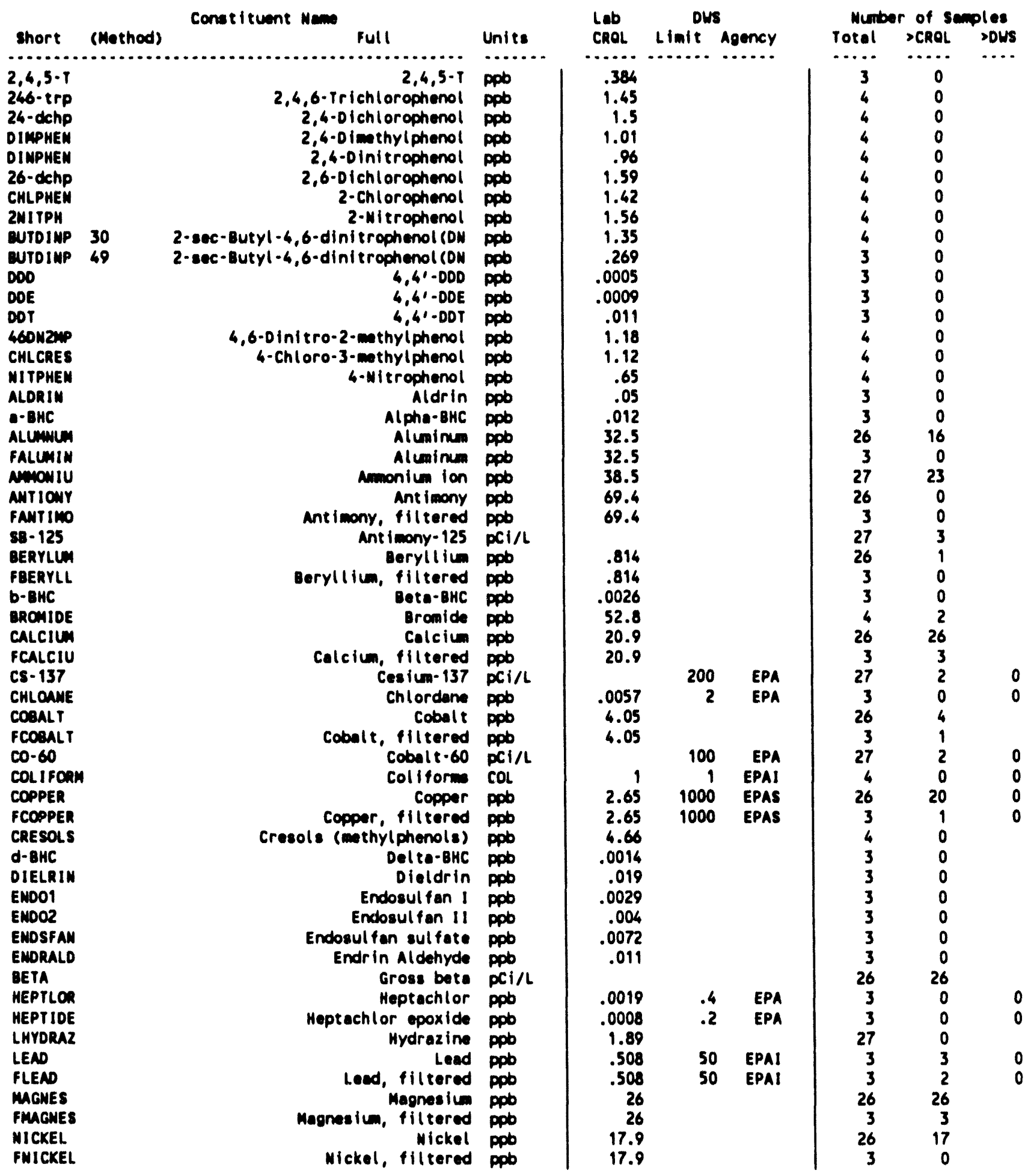


Table 6-3. Constituent List and Summary of Results for the 216-B-3 Pond Data for Reporting Period October 1 through December 31, 1993. (sheet 3 of 3)

\begin{tabular}{|c|c|c|c|c|c|c|c|c|c|}
\hline & & Constituent Mem & & Lab & DWS & & Num & $\mathbf{r}$ of 8 & ples \\
\hline short & (Method) & Full & Units & CRQL & Limit & Agency & Total & $>$ CROL & \\
\hline NITRITE & & Nitrite & $\infty$ & 38.3 & 1000 & EPA & 4 & 0 & 0 \\
\hline PENTCHP & & Pentachlorophenol & ppo & .87 & 1 & EPA & 4 & 0 & 0 \\
\hline PNOSPHA & & Phosphate & ppob & 147 & & & 4 & 0 & \\
\hline POTAEY & & Potassium & ppob & 662 & & & 26 & 26 & \\
\hline Fpotass & & Potessiun, filtered & ppb & 662 & & & 3 & 3 & \\
\hline$R U-106$ & & Ruthenium-106 & $P C_{1} / L$ & & 30 & EPA & 27 & 1 & 4* \\
\hline silven & & silver & ppo & 2.87 & so & EPAI & 26 & 0 & 0 \\
\hline FSILVER & & silver, filtered & ppob & 2.87 & 50 & EPAI & 3 & 0 & 0 \\
\hline TETPMNL & & Tetrachlorophenols & ppob & 1.05 & & & 4 & 0 & \\
\hline TIN & & Tin & ppob & 51.1 & & & 26 & 2 & \\
\hline FTIN & & rin, flltered & ppob & 51.1 & & & 3 & 0 & \\
\hline TDS & & Total Diseolved Sollide & ppm & 10 & 500 & EPAS & 27 & 27 & 0 \\
\hline TRIPHNL & & Trichlorophenols & ppb & 1.11 & & & 4 & 0 & \\
\hline TRtriun & & iritiun & $\mathrm{pCi} / \mathrm{L}$ & & 20000 & EPA & 26 & 20 & 10 \\
\hline Tundio & & Turbidity & NTU & .05 & & & 27 & 27 & \\
\hline unaniua & & Uraniun & ppo & & & & 2 & 2 & \\
\hline VAMADON & & Vanadium & ppob & 3.84 & & & 26 & 23 & \\
\hline FVAMADI & & Venadiun, filtered & ppob & 3.84 & & & 3 & 2 & \\
\hline ZINC & & Zine & ppob & 3.46 & $\begin{array}{l}5000 \\
5000\end{array}$ & EPAS & 26 & 19 & 0 \\
\hline FZINC & & E. filltered & ppb & 3.46 & 5000 & EPAS & 3 & 1 & $\mathbf{0}$ \\
\hline
\end{tabular}

For explenation of this teble, see Section 1.4 of report. 
Table 6-4. Constituents with at Least One Detected Value for the 216-B-3 Pond Data for Reporting Period October 1 through December 31, 1993. (sheet 1 of 9)

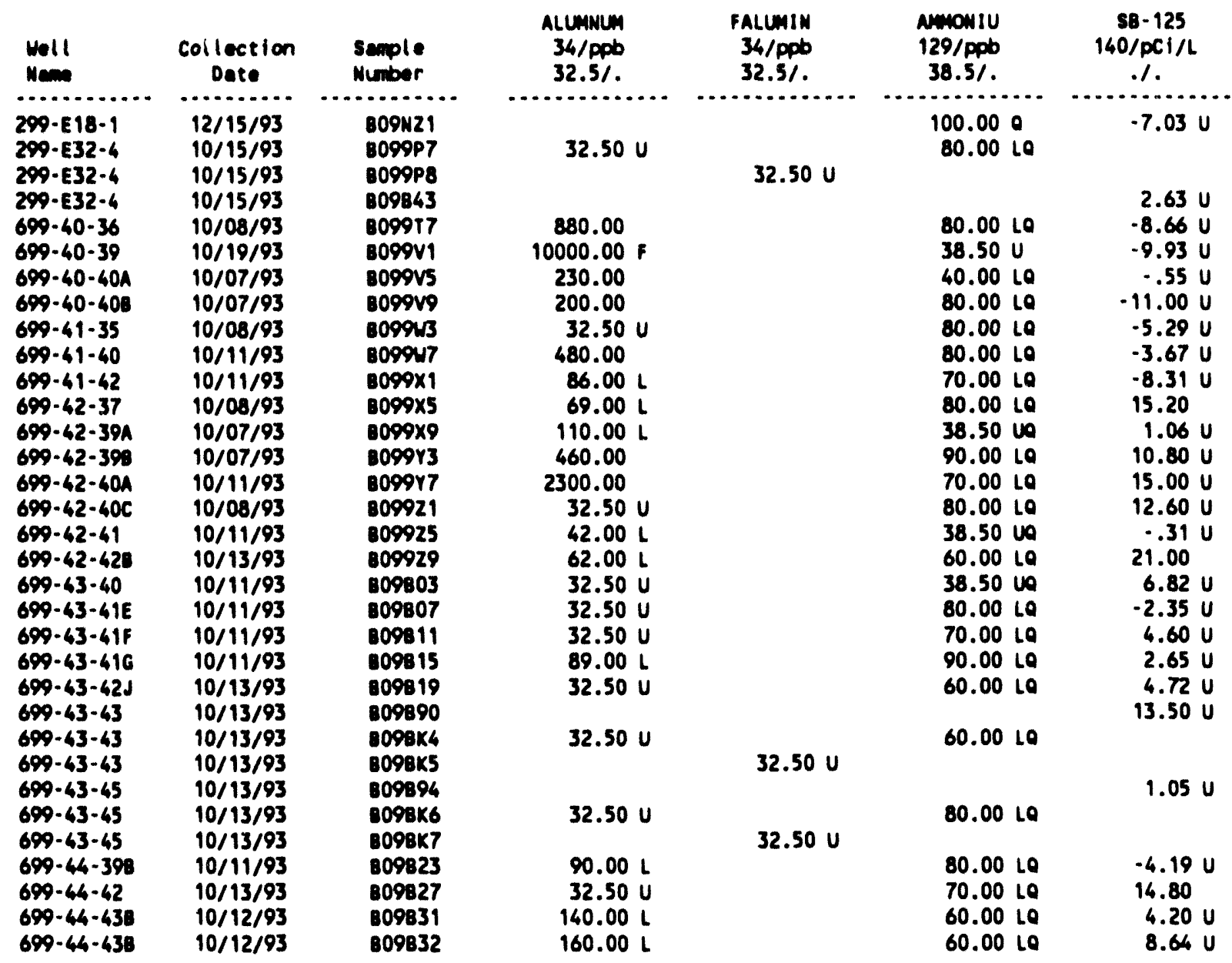

\begin{tabular}{|c|c|c|c|c|c|c|}
\hline $\begin{array}{l}\text { Mell } \\
\text { Menow }\end{array}$ & $\begin{array}{c}\text { Collection } \\
\text { Date }\end{array}$ & $\begin{array}{l}\text { Semple } \\
\text { Nutber }\end{array}$ & $\begin{array}{r}\text { ARSENIC } \\
43 / \mathrm{ppb} \\
1.38 / 50\end{array}$ & $\begin{array}{l}\text { FARSENIC } \\
43 / \mathrm{ppb} \\
1.38 / 50\end{array}$ & $\begin{array}{c}\text { BARIUA } \\
34 / \text { ppb } \\
.001 / 1000 \mathrm{i}\end{array}$ & $\begin{array}{l}\text { FBARIUN } \\
34 / \text { ppb } \\
.001 / 10001\end{array}$ \\
\hline $\begin{array}{l}299-E 18-1 \\
299-E 18-1 \\
299-E 32-4 \\
299-E 32-4 \\
699-40-36 \\
699-40-39 \\
699-40-40 A \\
699-40-408 \\
699-41-35 \\
699-41-40 \\
699-41-42 \\
699-42-37 \\
699-42-39 A \\
699-42-399\end{array}$ & $\begin{array}{l}12 / 15 / 93 \\
12 / 15 / 93 \\
10 / 15 / 93 \\
10 / 15 / 93 \\
10 / 08 / 93 \\
10 / 19 / 93 \\
10 / 07 / 93 \\
10 / 07 / 93 \\
10 / 08 / 93 \\
10 / 11 / 93 \\
10 / 11 / 93 \\
10 / 08 / 93 \\
10 / 07 / 93 \\
10 / 07 / 93\end{array}$ & 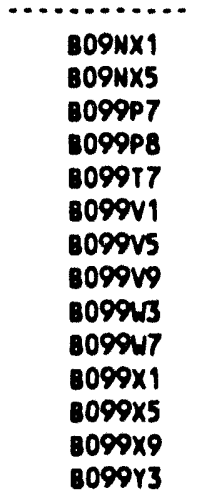 & $2.20 \mathrm{~L}$ & $\begin{array}{l}2.00 \mathrm{~L} \\
5.50 \mathrm{~B}\end{array}$ & $\begin{array}{c}74.00 \\
69.00 \\
190.00 \mathrm{~F} \\
96.00 \\
89.00 \\
130.00 \\
67.00 \\
31.00 \\
58.00 \\
55.00 \\
76.00\end{array}$ & 74.00 \\
\hline
\end{tabular}


Table 6-4. Constituents with at Least One Detected Value for the 216-B-3 Pond Data for Reporting Period October 1 through December 31, 1993.

(sheet 2 of 9 )

\begin{tabular}{|c|c|c|c|c|c|c|}
\hline Well & $\begin{array}{c}\text { Collection } \\
\text { Dete }\end{array}$ & $\begin{array}{l}\text { Semple } \\
\text { Number }\end{array}$ & $\begin{array}{l}\text { ARSENIC } \\
43 / \text { PPD } \\
1.38 / 50\end{array}$ & $\begin{array}{l}\text { FARSENIC } \\
43 / \mathrm{ppb} \\
1.38 / 50\end{array}$ & $\begin{array}{l}\text { BARIUA } \\
34 / \text { ppb } \\
.001 / 10001\end{array}$ & $\begin{array}{l}\text { FBARIUM } \\
34 / \mathrm{ppb} \\
.001 / 1000 \mathrm{i}\end{array}$ \\
\hline $\begin{array}{l}699-42-40 \mathrm{~A} \\
699-42-40 \mathrm{C} \\
699-42-41 \\
699-42-428 \\
699-43-40 \\
699-43-41 E \\
699-43-41 F \\
699-43-61 \mathrm{G} \\
699-43-42 \mathrm{~J} \\
699-43-43\end{array}$ & $\begin{array}{l}10 / 11 / 93 \\
10 / 08 / 93 \\
10 / 11 / 93 \\
10 / 13 / 93 \\
10 / 11 / 93 \\
10 / 11 / 93 \\
10 / 11193 \\
10 / 11 / 93 \\
10 / 13 / 93 \\
10 / 13 / 93\end{array}$ & $\begin{array}{l}809917 \\
809921 \\
809925 \\
809929 \\
809803 \\
809807 \\
809811 \\
809815 \\
809819 \\
8098 K 4\end{array}$ & 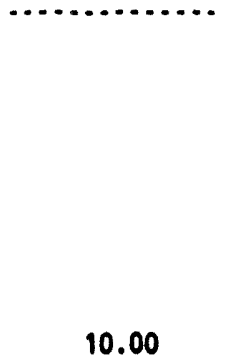 & $\cdots$ & $\begin{array}{r}33.00 \\
120.00 \\
7.30 \mathrm{~L} \\
25.00 \\
49.00 \\
36.00 \\
35.00 \\
51.00 \\
10.00 \mathrm{~L} \\
13.00 \mathrm{~L}\end{array}$ & \\
\hline $\begin{array}{l}699-43-43 \\
699-43-45 \\
699-43-45 \\
699-44-398 \\
699-44-42 \\
699-44-438 \\
699-44-438\end{array}$ & $\begin{array}{l}10 / 13 / 93 \\
10 / 13 / 93 \\
10 / 13 / 93 \\
10 / 11 / 93 \\
10 / 13 / 93 \\
10 / 12 / 93 \\
10 / 12 / 93\end{array}$ & $\begin{array}{l}8098 K 5 \\
8098 K 6 \\
8098 K 7 \\
809823 \\
809827 \\
809831 \\
809832\end{array}$ & & $\begin{array}{r}9.60 \\
12.00\end{array}$ & $\begin{array}{l}34.00 \\
20.00 \mathrm{~L} \\
34.00 \\
48.00 \\
49.00\end{array}$ & $\begin{array}{l}12.00 \mathrm{~L} \\
34.00\end{array}$ \\
\hline
\end{tabular}

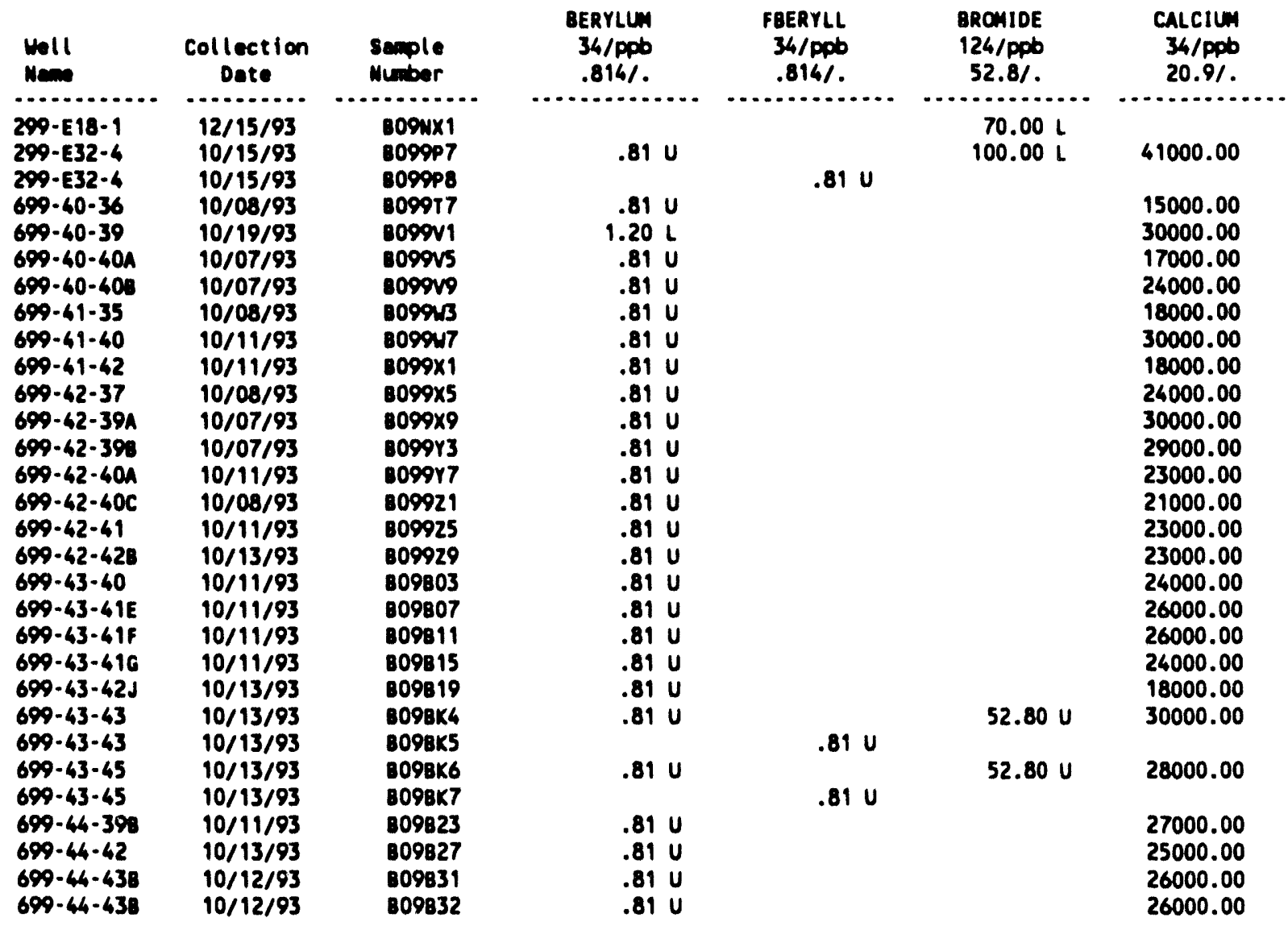


Table 6-4. Constituents with at Least One Detected Value for the 216-B-3 Pond Data for Reporting Period October 1 through December 31, 1993.

(sheet 3 of 9 )

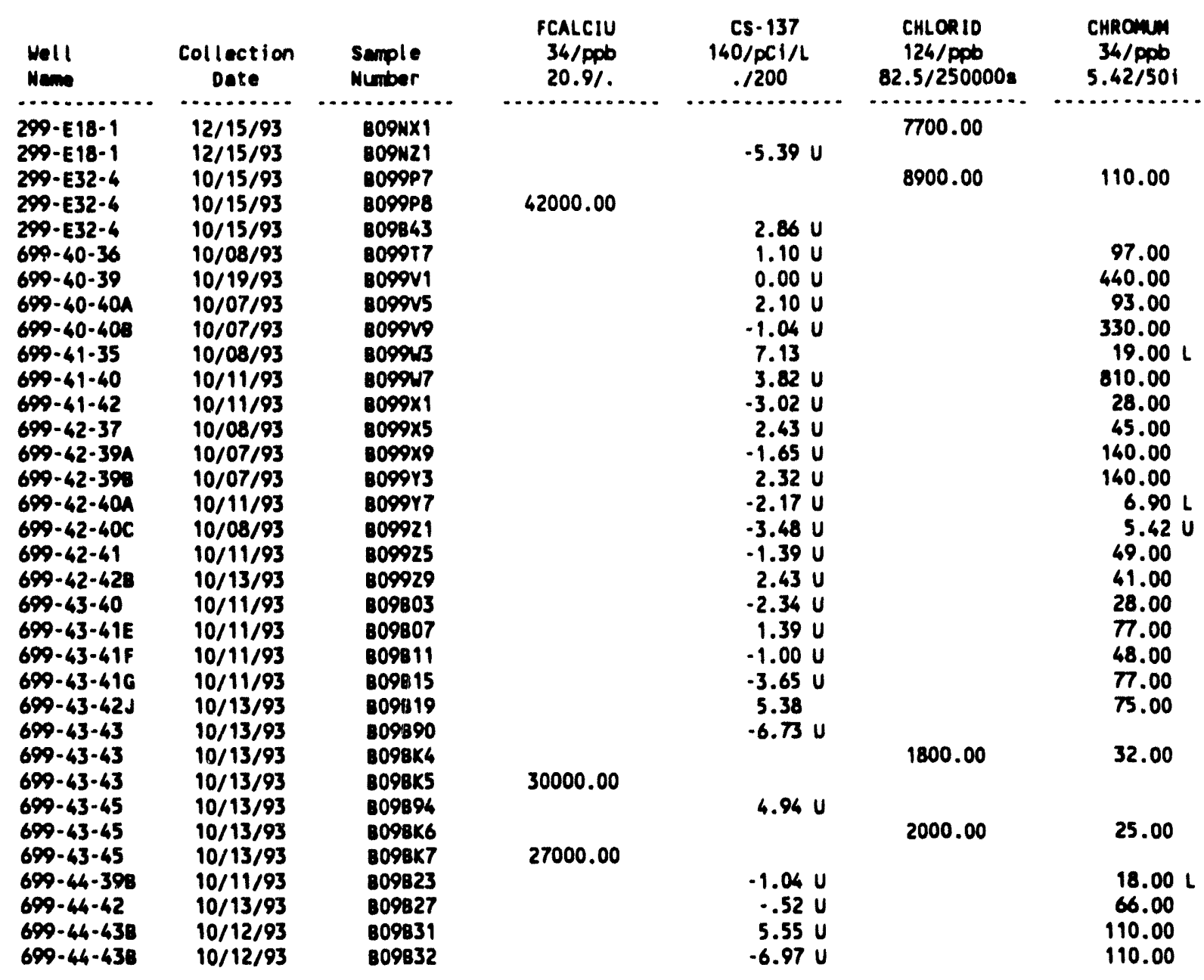

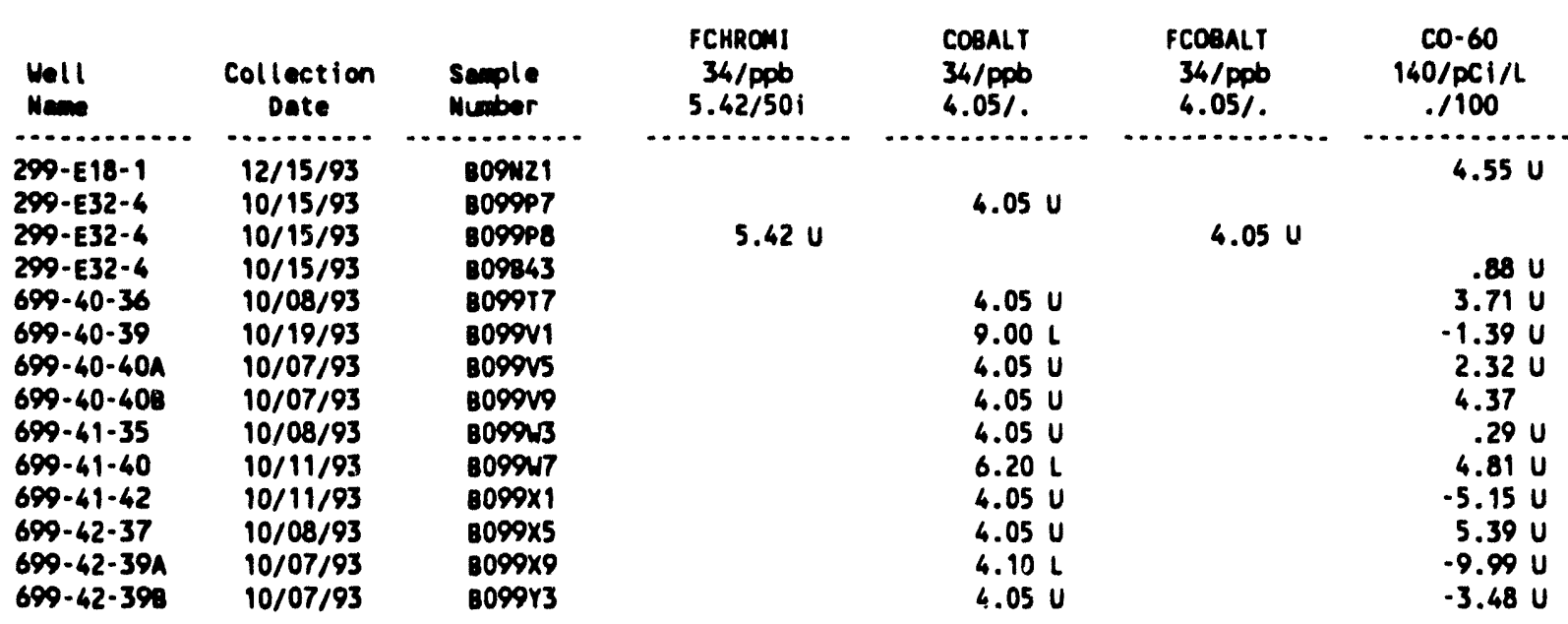


Table 6-4. Constituents with at Least One Detected Value for the 216-B-3 Pond Data for Reporting Period October 1 through December 31, 1993.

(sheet 4 of 9 )

\begin{tabular}{|c|c|c|}
\hline $\begin{array}{l}\text { Hell } \\
\text { Heane }\end{array}$ & $\begin{array}{c}\text { Collection } \\
\text { Date }\end{array}$ & $\begin{array}{l}\text { Sample } \\
\text { Number }\end{array}$ \\
\hline $\begin{array}{l}699-42-40 A \\
699-42-40 C \\
699-42-41 \\
699-42-42 B \\
699-43-40 \\
699-43-41 E \\
699-43-41 F \\
699-43-416 \\
699-43-42 J \\
699-43-43 \\
699-43-43 \\
699-43-43 \\
699-43-45 \\
699-43-45 \\
699-43-45 \\
699-44-398 \\
699-44-42 \\
699-44-43 B \\
699-44-43 B\end{array}$ & $\begin{array}{l}10 / 11 / 93 \\
10 / 08 / 93 \\
10 / 11 / 93 \\
10 / 13 / 93 \\
10 / 11 / 93 \\
10 / 11 / 93 \\
10 / 11 / 93 \\
10 / 111 / 93 \\
10 / 13 / 93 \\
10 / 13 / 93 \\
10 / 13 / 93 \\
10 / 13 / 93 \\
10 / 13 / 93 \\
10 / 13 / 93 \\
10 / 13 / 93 \\
10 / 11 / 93 \\
10 / 13 / 93 \\
10 / 12 / 93 \\
10 / 12 / 93\end{array}$ & $\begin{array}{l}\text { B099Y7 } \\
\text { B09921 } \\
809925 \\
809929 \\
809803 \\
809807 \\
809811 \\
809815 \\
809819 \\
809890 \\
8098 K 6 \\
8098 K 5 \\
809894 \\
8098 K 6 \\
809 B K 7 \\
B 09 B 23 \\
B 09827 \\
809 B 31 \\
B 09832\end{array}$ \\
\hline
\end{tabular}

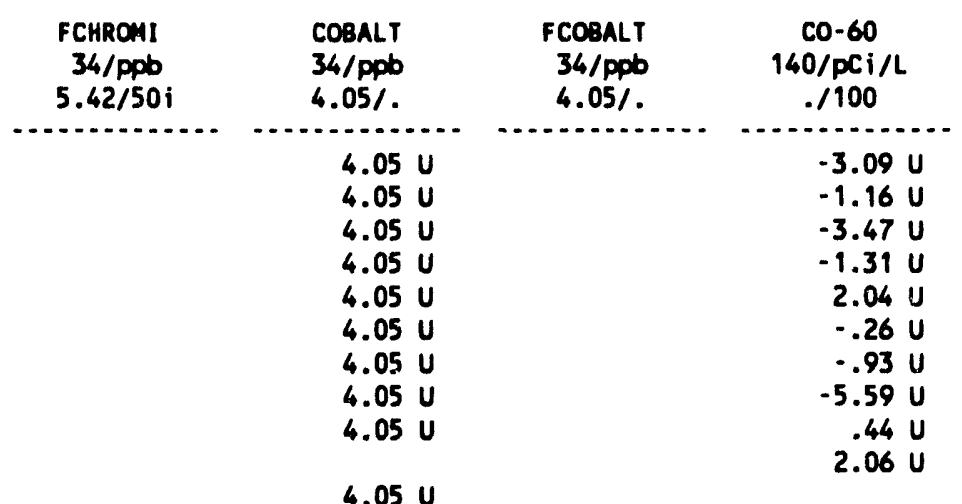

$5.42 \mathrm{U} \quad 6.10 \mathrm{~L}$

$\begin{array}{lll}5.50 \mathrm{~L} & 4.05 \mathrm{U} & 4.05 U\end{array}$
$-6.12 U$
$4.05 \mathrm{U}$
$8.10 \mathrm{~L}$
$4.05 \mathrm{U}$
$4.05 \mathrm{U}$

$$
\begin{array}{r}
7.14 \\
-5.73 U \\
-1.60 U \\
.52 U
\end{array}
$$

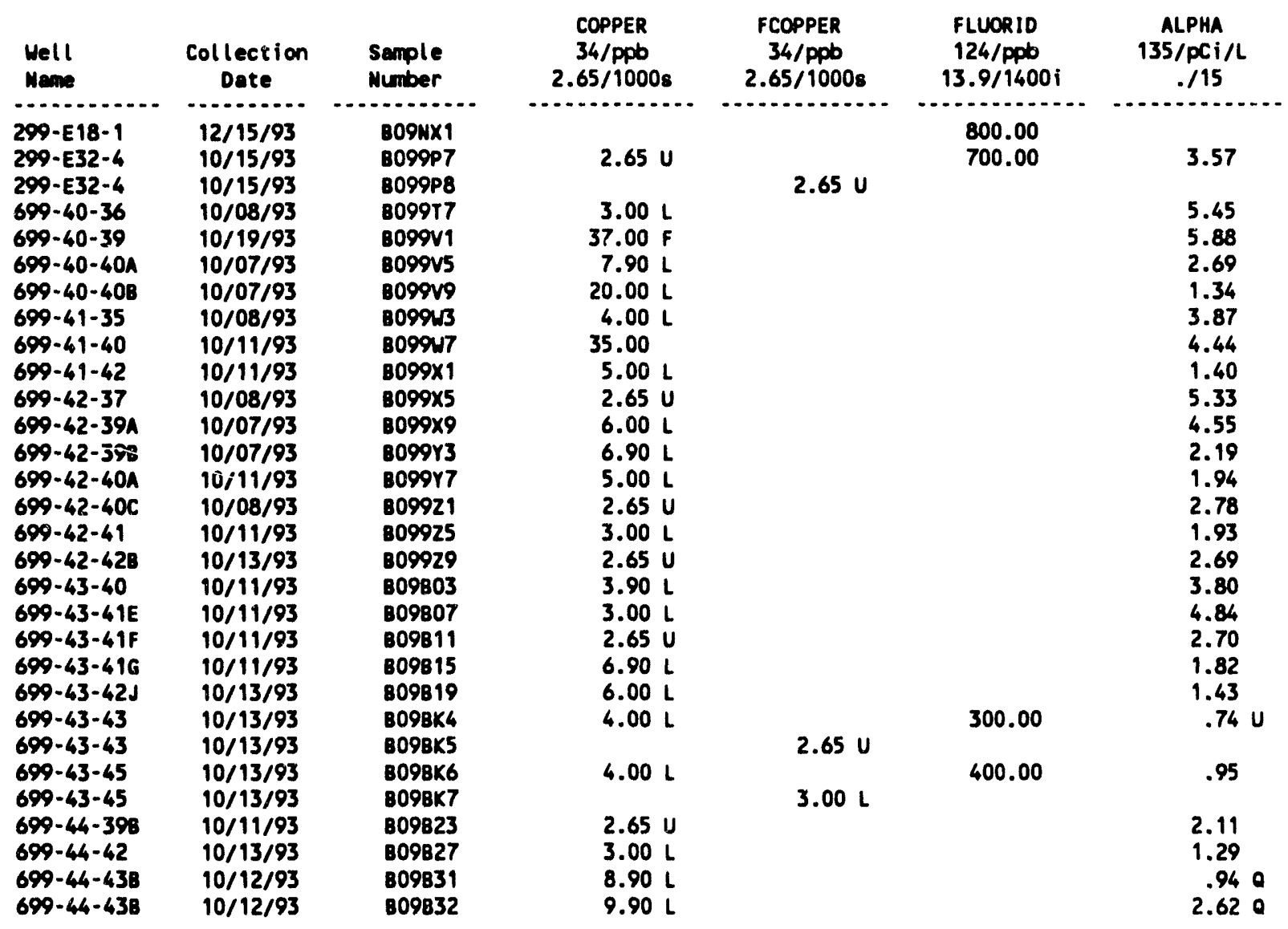


Table 6-4. Constituents with at Least One Detected Value for the 216-B-3 Pond Data for Reporting Period October 1 through December 31, 1993.

(sheet 5 of 9 )

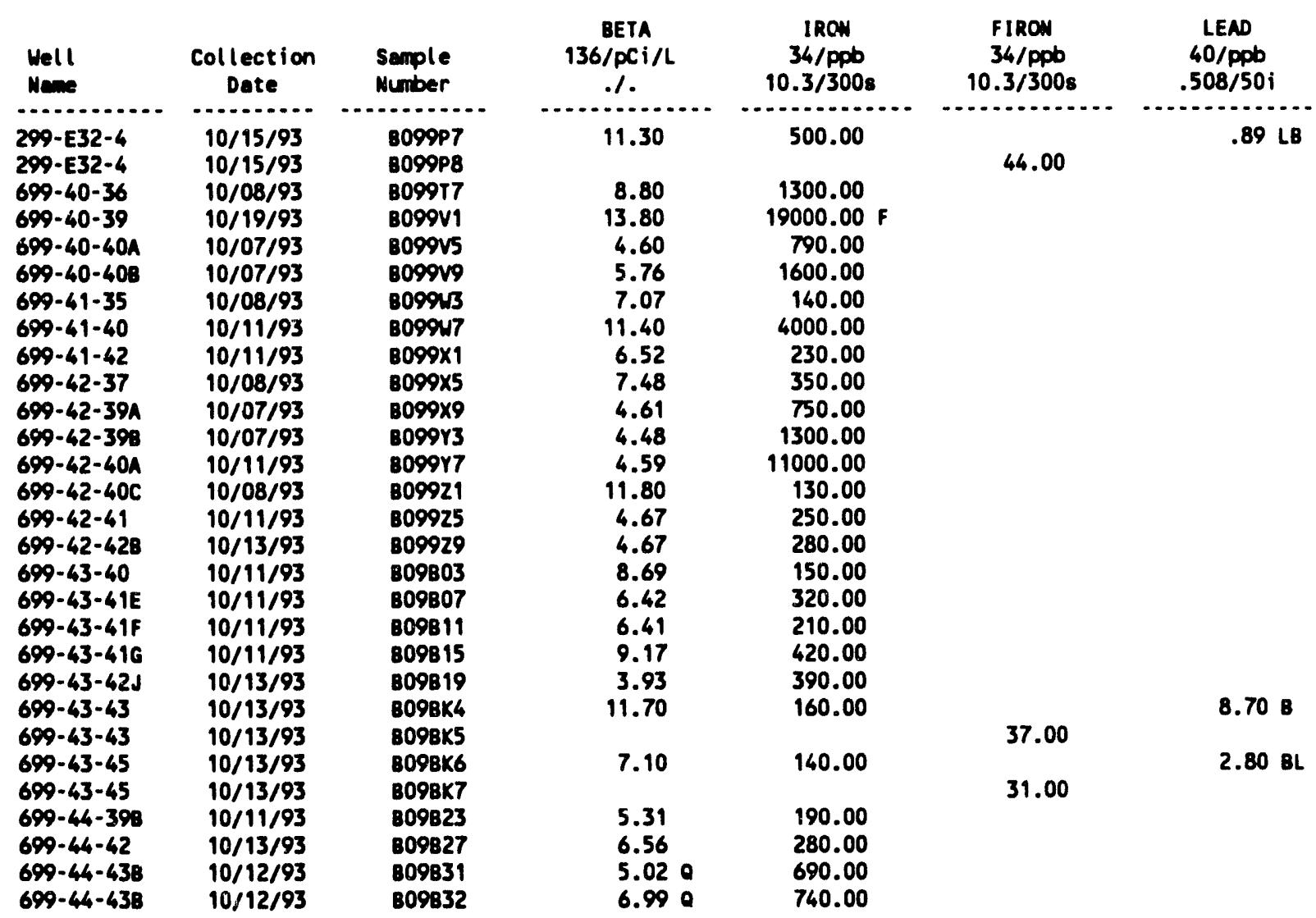

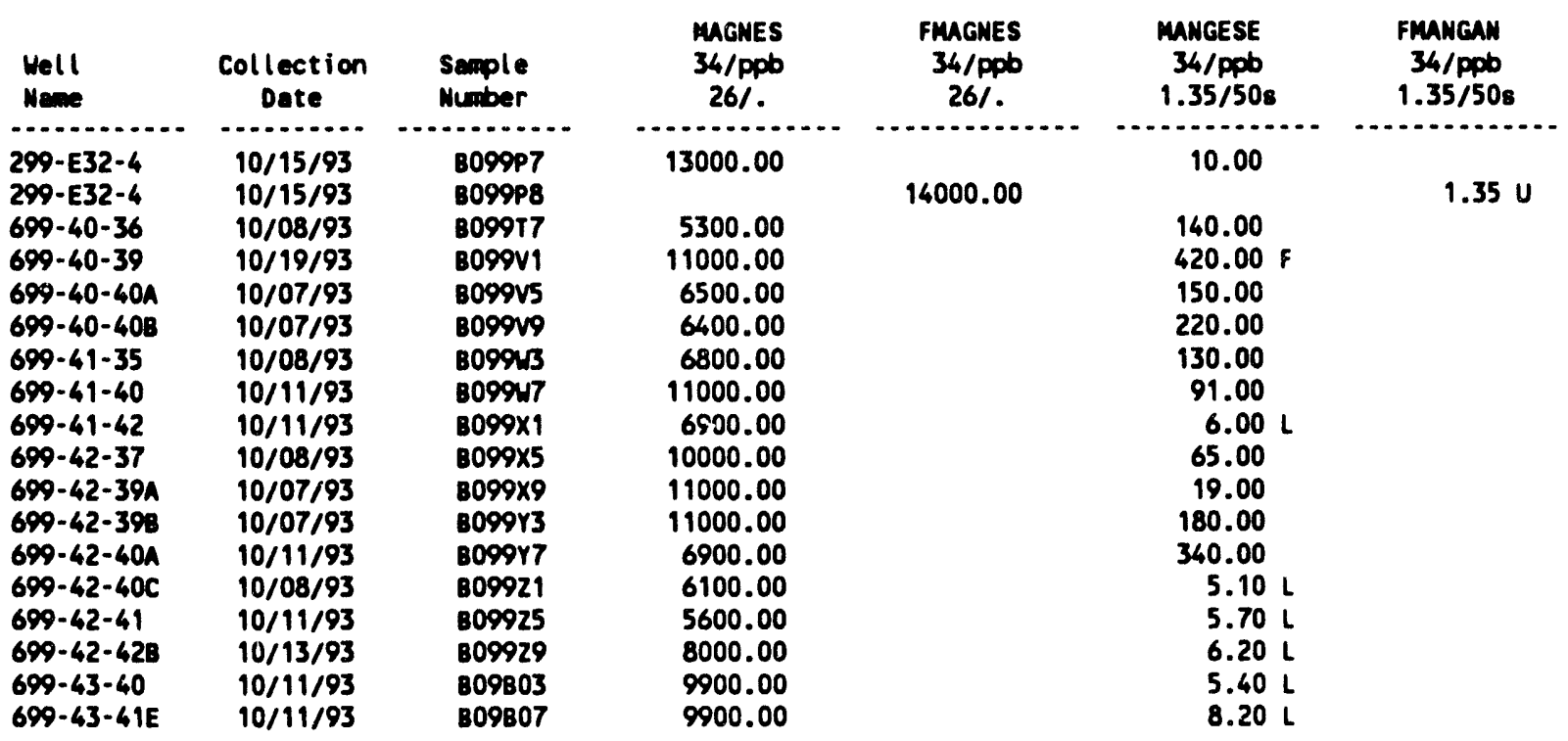


Table 6-4. Constituents with at Least One Detected Value for the 216-B-3 Pond Data for Reporting Period October 1 through December 31, 1993.

(sheet 6 of 9)

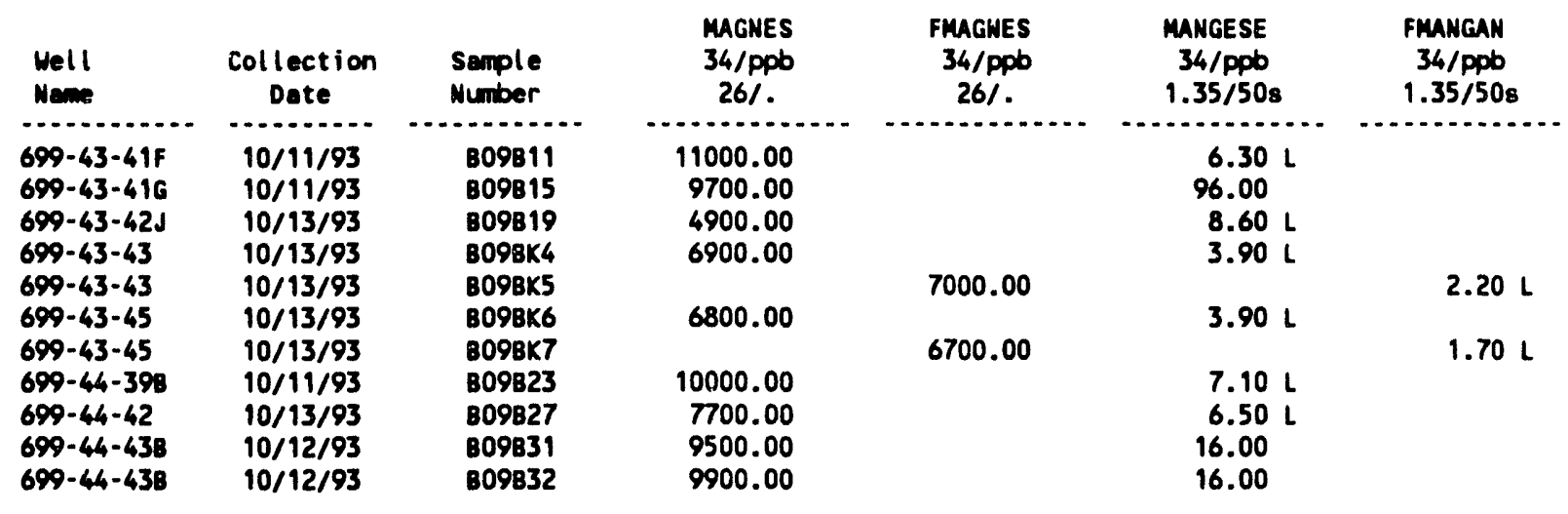

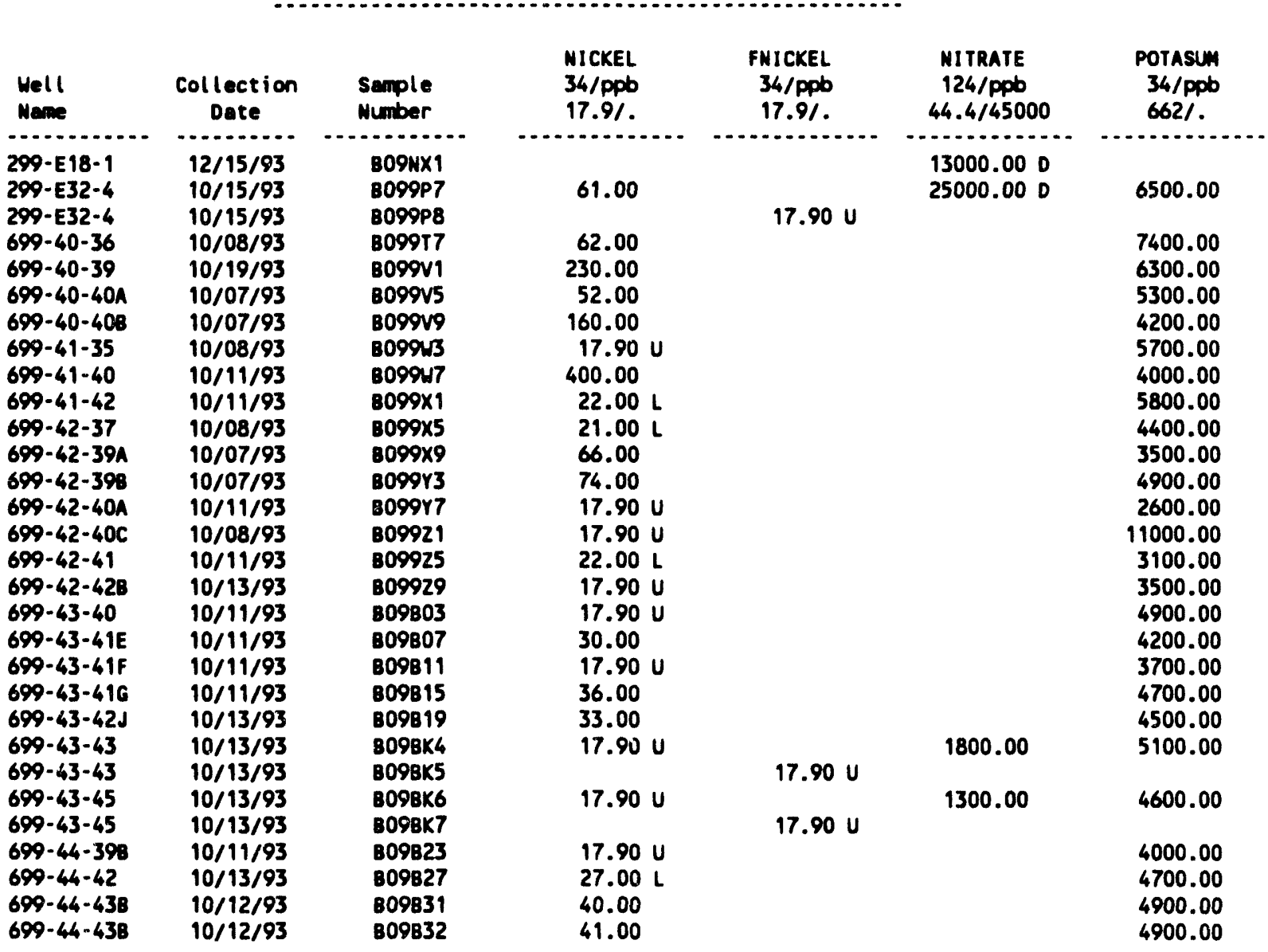


Table 6-4. Constituents with at Least One Detected Value for the 216-B-3 Pond Data for Reporting Period October 1 through December 31, 1993.

(sheet 7 of 9 )

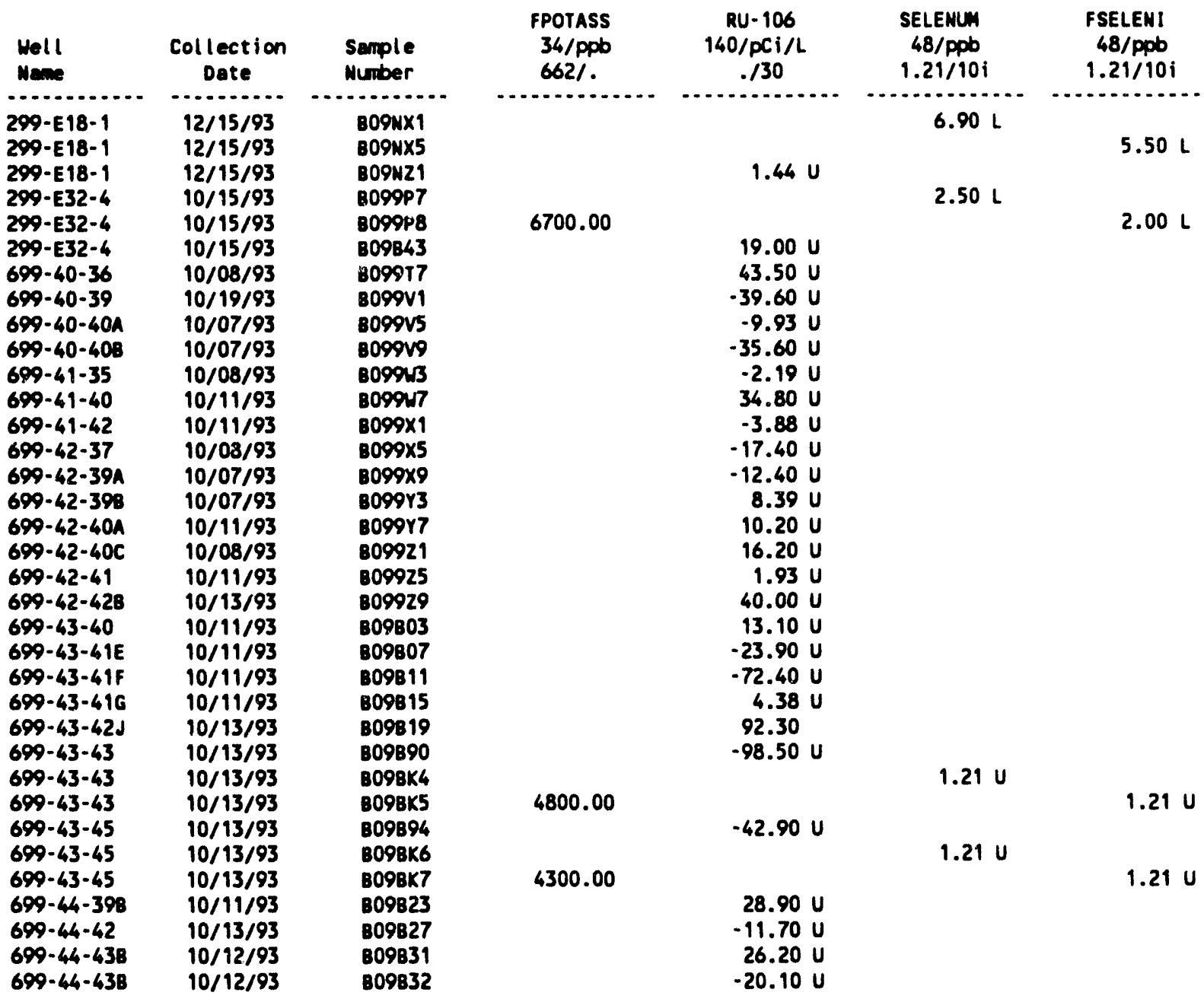

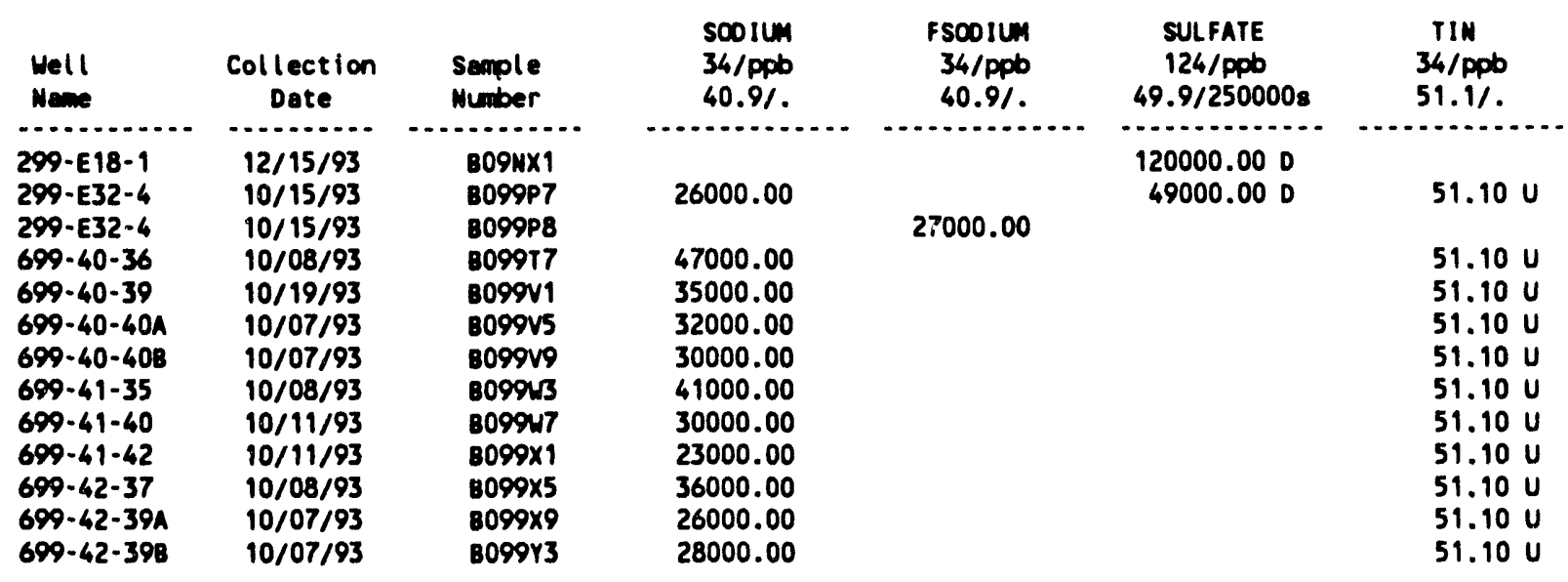


Table 6-4. Constituents with at Least One Detected Value for the 216-B-3 Pond Data for Reporting Period October 1 through December 31, 1993.

(sheet 8 of 9 )

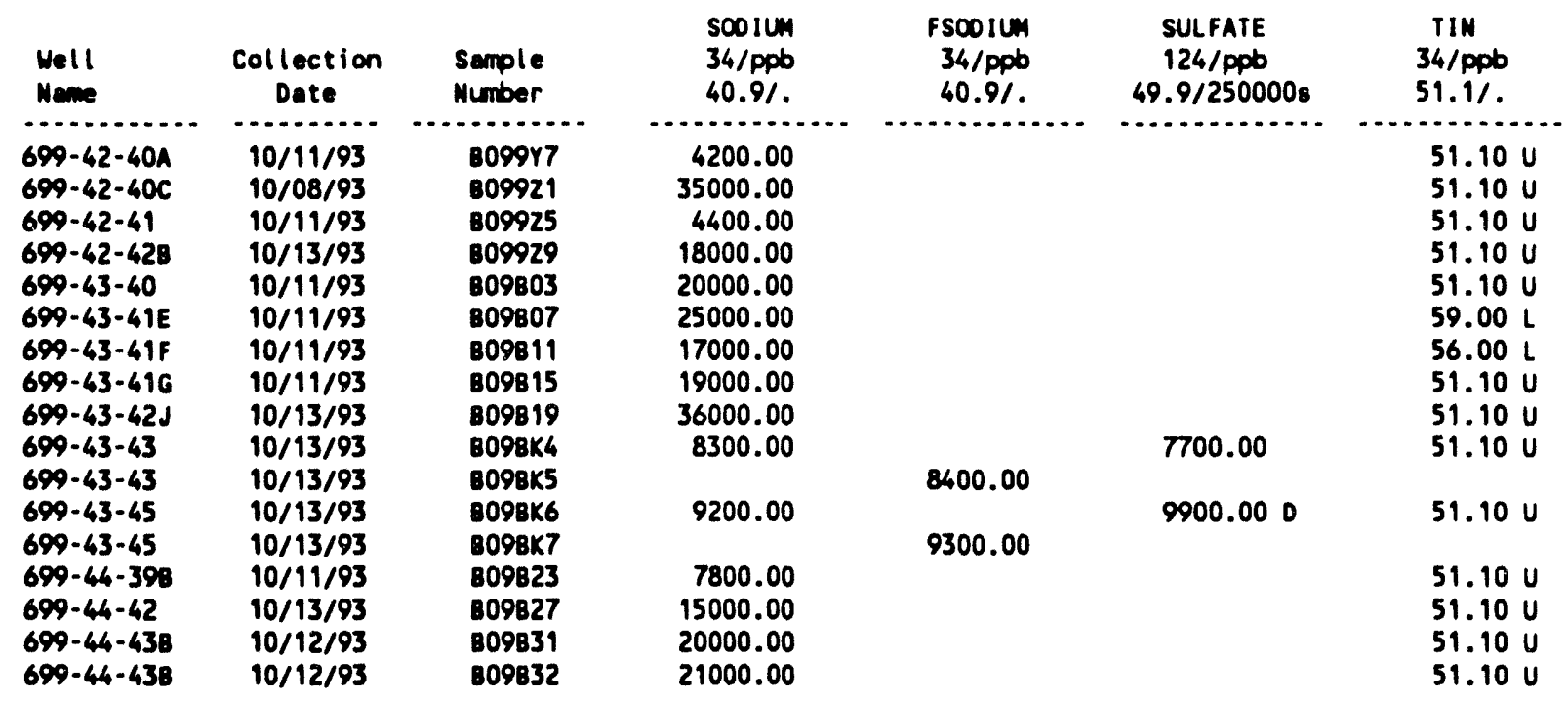

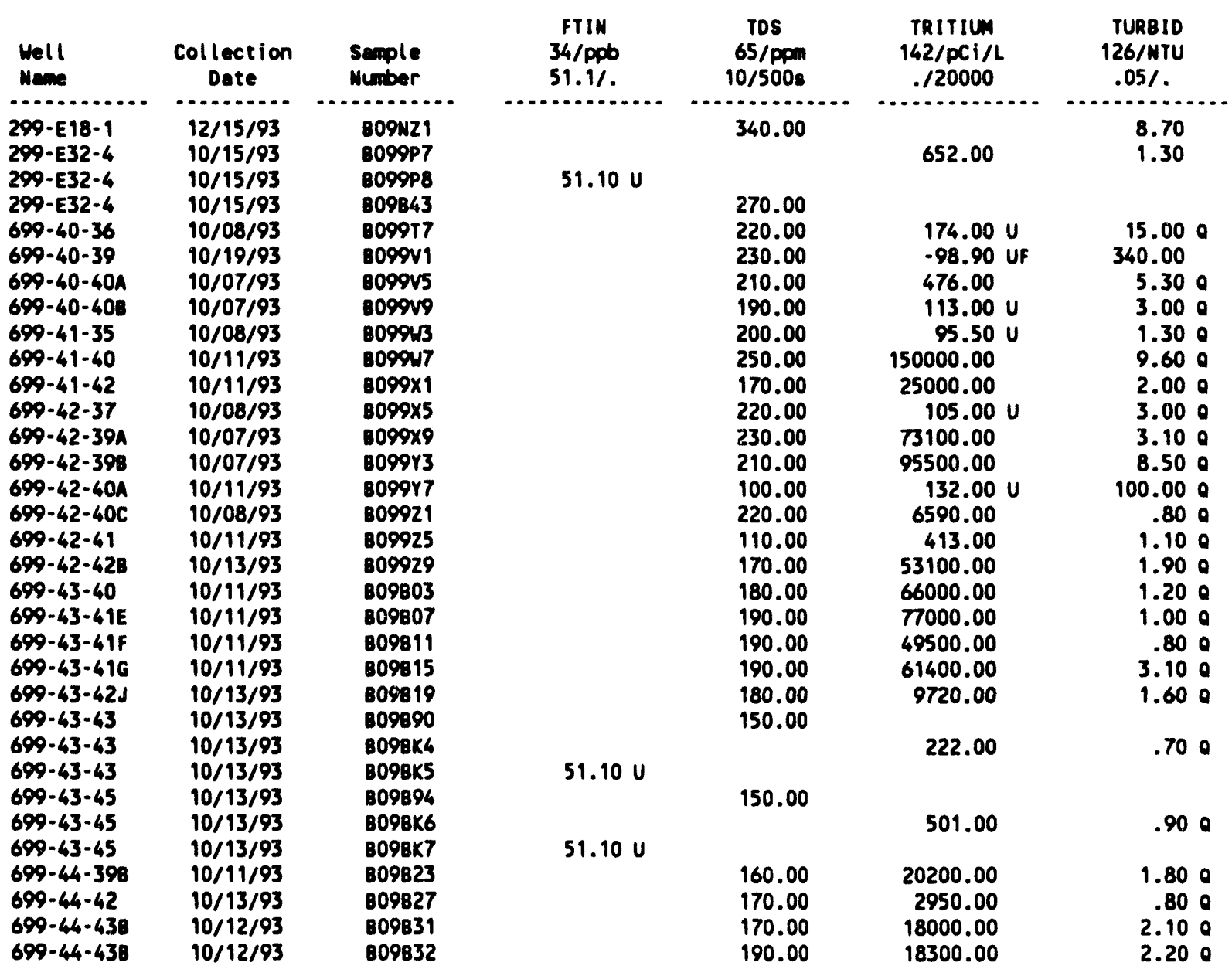


Table 6-4. Constituents with at Least One Detected Value for the 216-B-3 Pond Data for Reporting Period October 1 through December 31, 1993. (sheet 9 of 9 )

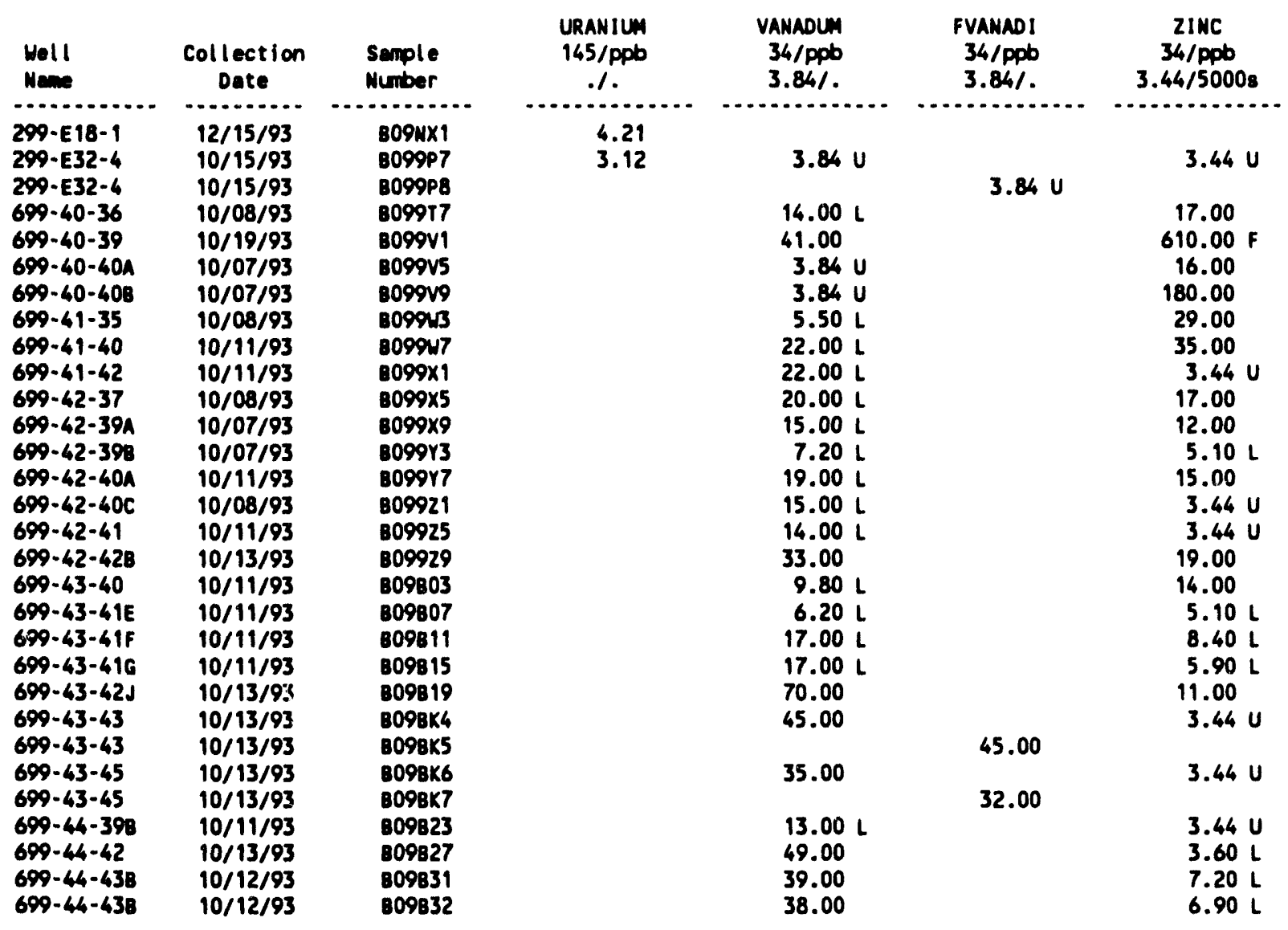

\begin{tabular}{|c|c|c|c|}
\hline $\begin{array}{l}\text { Nell } \\
\text { Nane }\end{array}$ & $\begin{array}{c}\text { Collection } \\
\text { Date }\end{array}$ & $\begin{array}{l}\text { Sample } \\
\text { Number }\end{array}$ & $\begin{array}{c}\text { F21NC } \\
34 / \mathrm{ppb} \\
3.44 / 5000 \mathrm{~s}\end{array}$ \\
\hline $\begin{array}{l}299-E 32-4 \\
699-43-43 \\
699-43-45\end{array}$ & $\begin{array}{l}10 / 15 / 93 \\
10 / 13 / 93 \\
10 / 13 / 93\end{array}$ & $\begin{array}{l}\text { 8099P8 } \\
\text { 8098K5 } \\
\text { 8098K7 }\end{array}$ & $\begin{array}{l}3.44 \mathrm{U} \\
3.44 \mathrm{U} \\
3.70 \mathrm{~L}\end{array}$ \\
\hline
\end{tabular}

For explanstion of this table, see Section 1.4 of report. 
Table 6-5. Contamination Indicator Parameters for the 216-8-3 Pond Data for Reporting Period úctober 1 through December 31, 1993. (sheet 1 of 2)

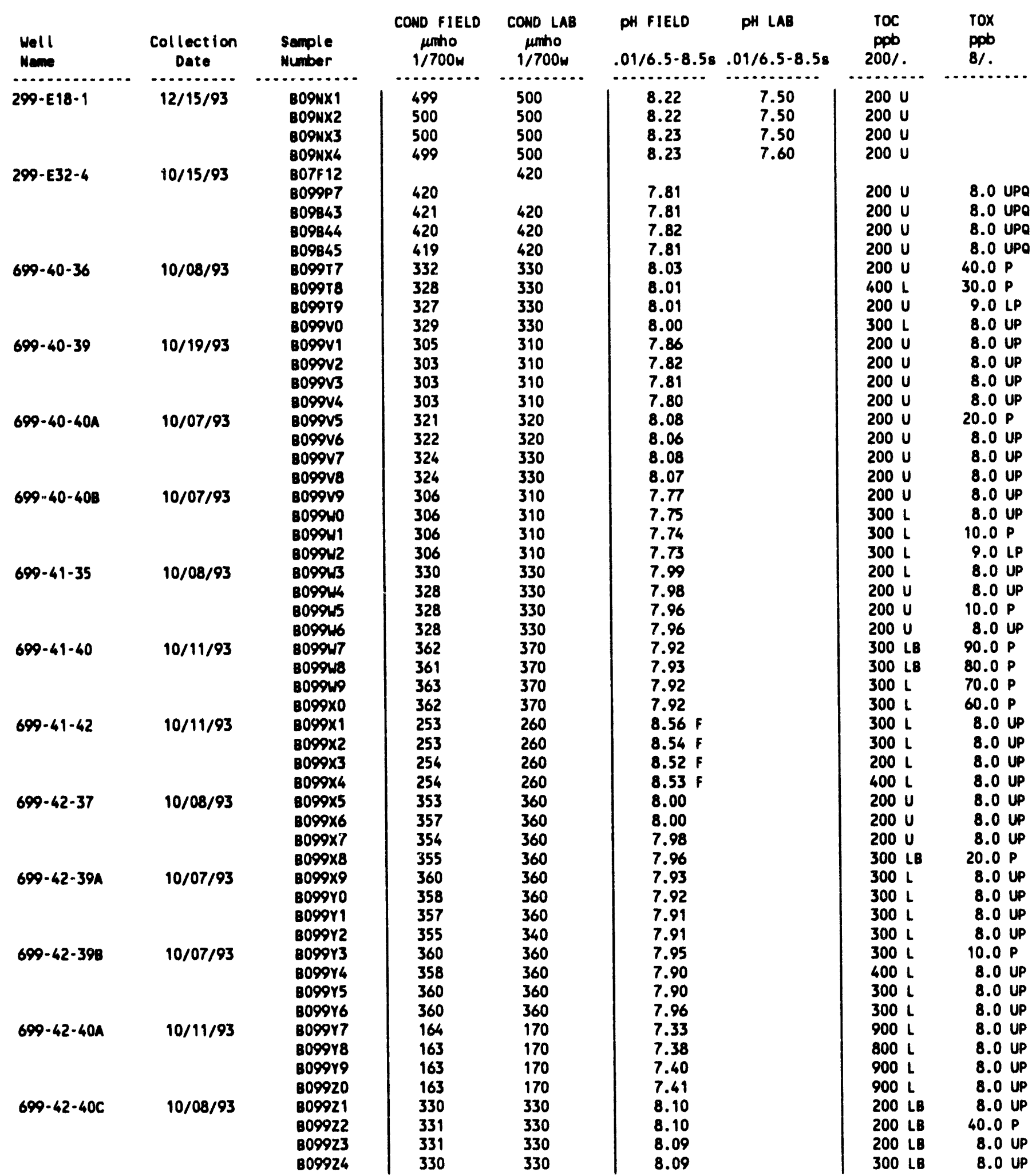


Table 6-5. Contamination Indicator Parameters for the 216-B-3 Pond Data for Reporting Period October 1 through December 31, 1993. (sheet 2 of 2)

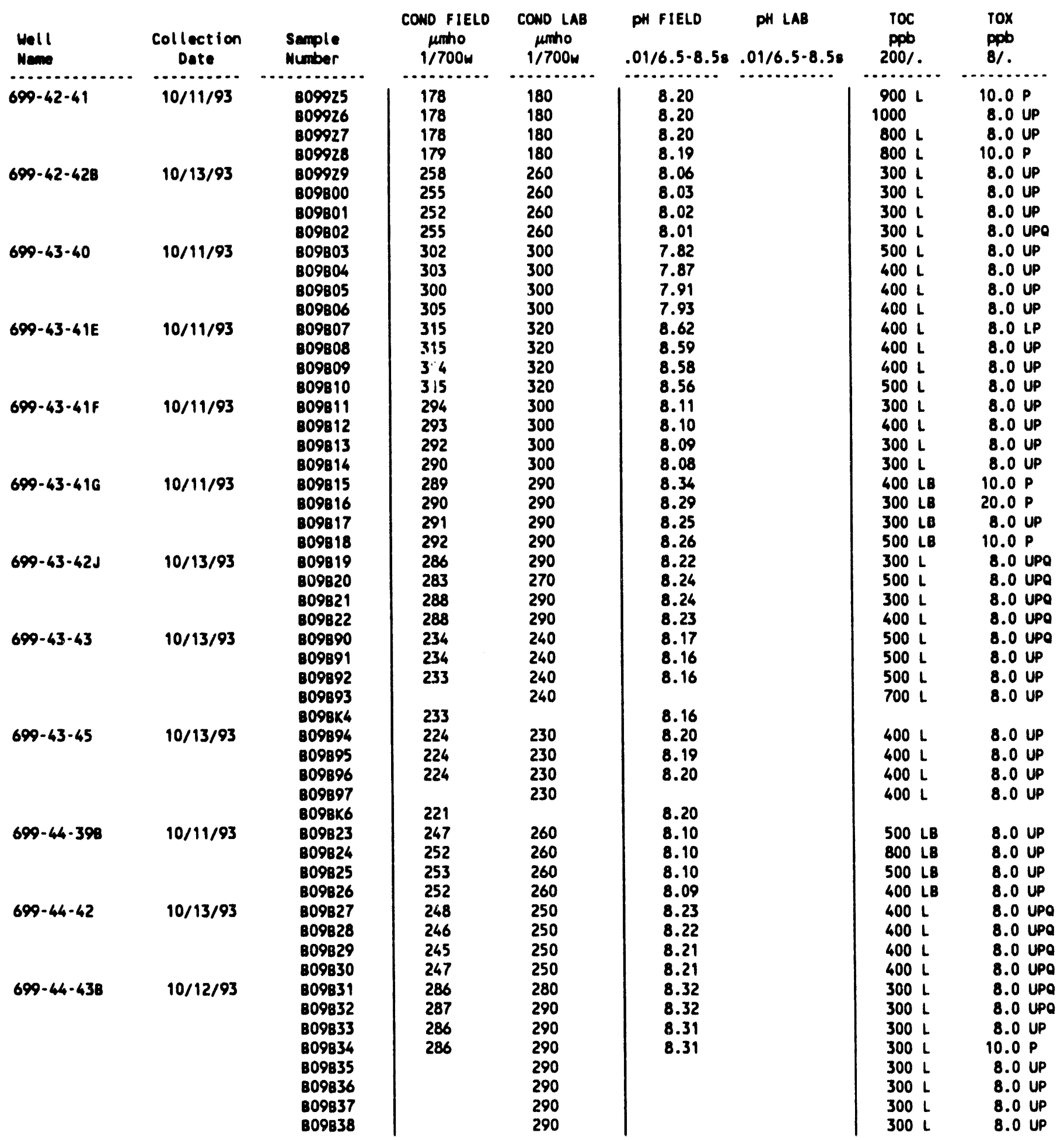

For explenation of this table, see Section 1.4 of report. 
DOE/RL-93-56-4

\section{CONTENTS}

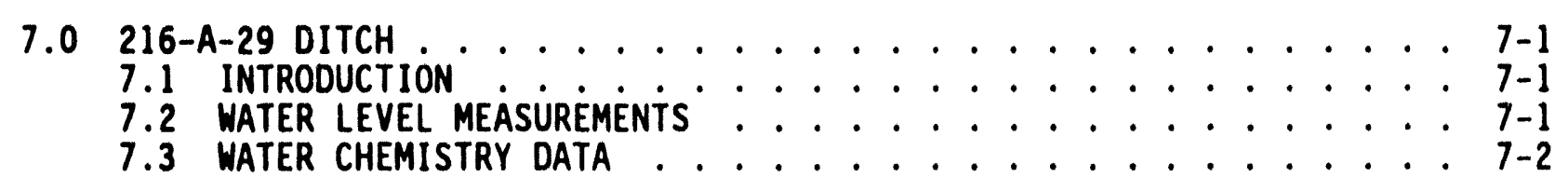


DOE/RL-93-56-4

\section{LIST OF FIGURES}

7-1 Monitoring We11 Locations for the 216-A-29 Ditch......... . 7-3

\section{LIST OF TABLES}

7-1 Monitoring Well Purpose and Sampling Schedule for the 216-A-29 Ditch . . . . . . . . . . . . . . . . . . . . . . 7-4

7-2 RCRA Water Level Measurement Report for the 216-A-29 Ditch, Fourth Quarter 1993 . . . . . . . . . . . . . . . . 7-5

7-3 Constituent List and Summary of Results for the 216-A-29 Ditch Data for Reporting Period October 1 through December 31, 1993 . . . 7-8

7-4 Constituents with at Least One Detected Value for the 216-A-29 Ditch Data for Reporting Period October 1 through December 31, 1993 .................... 7-13

7-5 Contamination Indicator Parameters for the 216-A-29 Ditch Data for Reporting Period October 1 through December 31, 1993 . . . . . . 7-27 


\section{$7.0 \quad 216-A-29$ DITCH}

6. L. Kasza

Westinghouse Hanford Company

\subsection{INTRODUCTION}

The 216-A-29 Ditch (A-29 Ditch) is located east of the 200 East Area in the central portion of the Hanford Site. From 1955 to July 1991, this excavated, unlined ditch received the effluent from the Plutonium-Uranium Extraction (PUREX) Plant Chemical Sewer Line and conducted it to the 216-B-3-3 Ditch and the B Pond System. After July 15, 1991, the flow to the A-29 Ditch was rerouted to the PUREX Cooling Water Line, and the site of the ditch was backfilled, graded, and stabilized as part of the Tri-Party Agreement (Ecology et al. 1992), Milestone M-17-10. Because the A-29 Ditch will not receive additional hazardous substances, the DOE proposed that the ditch be permanently closed under RCRA interim status (WAC 173-303-400; 40 CFR 265 Subpart G; DOE 1987).

Groundwater beneath the A-29 Ditch has been monitored by a RCRA groundwater monitoring network since 1988 because of earlier discharges of caustic and potentially hazardous chemicals to the ditch. Currently the A-29 Ditch is in a groundwater quality assessment monitoring program because of elevated specific conductance levels in one monitoring well (299-E25-35) (Chou et al. 1990). The groundwater monitoring activities for the A-29 Ditch also are directed by the Groundwater Monitoring Plan for the 216-A-29 Ditch (Kasza and Goodwin 1991).

The Ri:RA groundwater monitoring network consists of 3 upgradient and 10 downgradient wells. Nine additional wells in the surrounding area are used to provide supplementary data for the groundwater quality assessment investigation. Figure 7-1 shows the locations of the wells used to monitor groundwater quality for the A-29 Ditch. Table 7-1 presents pertinent data about these wells.

\subsection{WATER LEVEL MEASUREMENTS}

Water levels are measured monthly in the 13 wells comprising the A-29 Ditch monitoring network, and are measured quarterly in the 9 additional wells that provide supplemental analytical data for the groundwater quality assessment investigation. Water level measurements are also recorded whenever the wells are sampled.

Water level measurements obtained during this quarter are presented in Table 7-2. Several inconsistencies were noted when recent data were compared to the previously measured water levels. Water levels reported for well 299-E25-35 on October 20, 1993, well 299-E17-15 for October 15, 1993, and well 299-E22-36 for both the October 15 and December 7, 1993 measurements fell outside of the expected ranges. These data are flagged in Table 7-2. 


\subsection{MATER CHEMISTRY DATA}

Groundwater beneath the A-29 Ditch is sampled quarterly in accordance with the groundwater quality assessment plan (Chou et a1. 1990). The constituent 1 ist and summary of results are provided in Table 7-3. Reported analytical data are included in Tables 7-4 and 7-5. Table 7-4 provides a list of the wells and analytical results for chemical constituents with at least one value above MDL. In many cases, the detected values are from the wells that are monitored to provide comparative regional data for the groundwater quality assessment investigation. Results of analyses for CIPs are reported in Table 7-5.

Constituents reported in concentrations that exceed the DWS for the A-29 Ditch detection network wells include unfiltered chromium in wells 299-E25-32P, 299-E25-34, 299-E25-35, 299-E25-42, 299-E25-43 (two samples), 299-E25-48, 299-E26-12, and 299-E26-13; unfiltered iron in wells 299-E25-32P, 299-E25-34, 299-E25-35, 299-E25-42, 299-E25-43 (two samples), 299-E25-47, 299-E25-48, and 299-E26-12; and tritium in wells 299-E25-35 and 299-E25-42.

Constituents reported in concentrations that exceed the DWS at the wells that provide regional data for the A-29 Ditch groundwater quality assessment investigation include unfiltered chromium in we11 299-E17-15 (both times sampled), 299-E17-20, 299-E25-31 and 299-E25-36; unfiltered iron in wells 299-E17-15, 299-E17-20, 299-E25-11, 299-E25-18, 299-E25-19, 299-E25-31, and 299-E25-36; nitrate in wells 299-E17-15 (both times), 299-E17-20, 299-E25-19 and 299-E25-20; tritium in wells 299-E17-15 (both times), 299-E1720, 299-E25-11, 299-E25-18, 299-E25-19, and 299-E25-20.

Field-measured $\mathrm{PH}$ at well 299-E25-34 fell outside the acceptable range of the DWS at the October 12, 1993 sample collection. Review of the field record indicates no data transcription errors. The recorded value is outside the historic trend for the well, and measurement error is suspect. An RADE has been submitted to determine the validity of the measurement.

Data are flagged in Tables 7-4 and 7-5 according to the explanation found in Section 1.4. The $D$ flags for nitrate and sulfate indicate that the samples were diluted for analysis.

All analytical data for the fourth quarter 1993 sampling of the A-29 groundwater monitoring network were available and included in this report. 


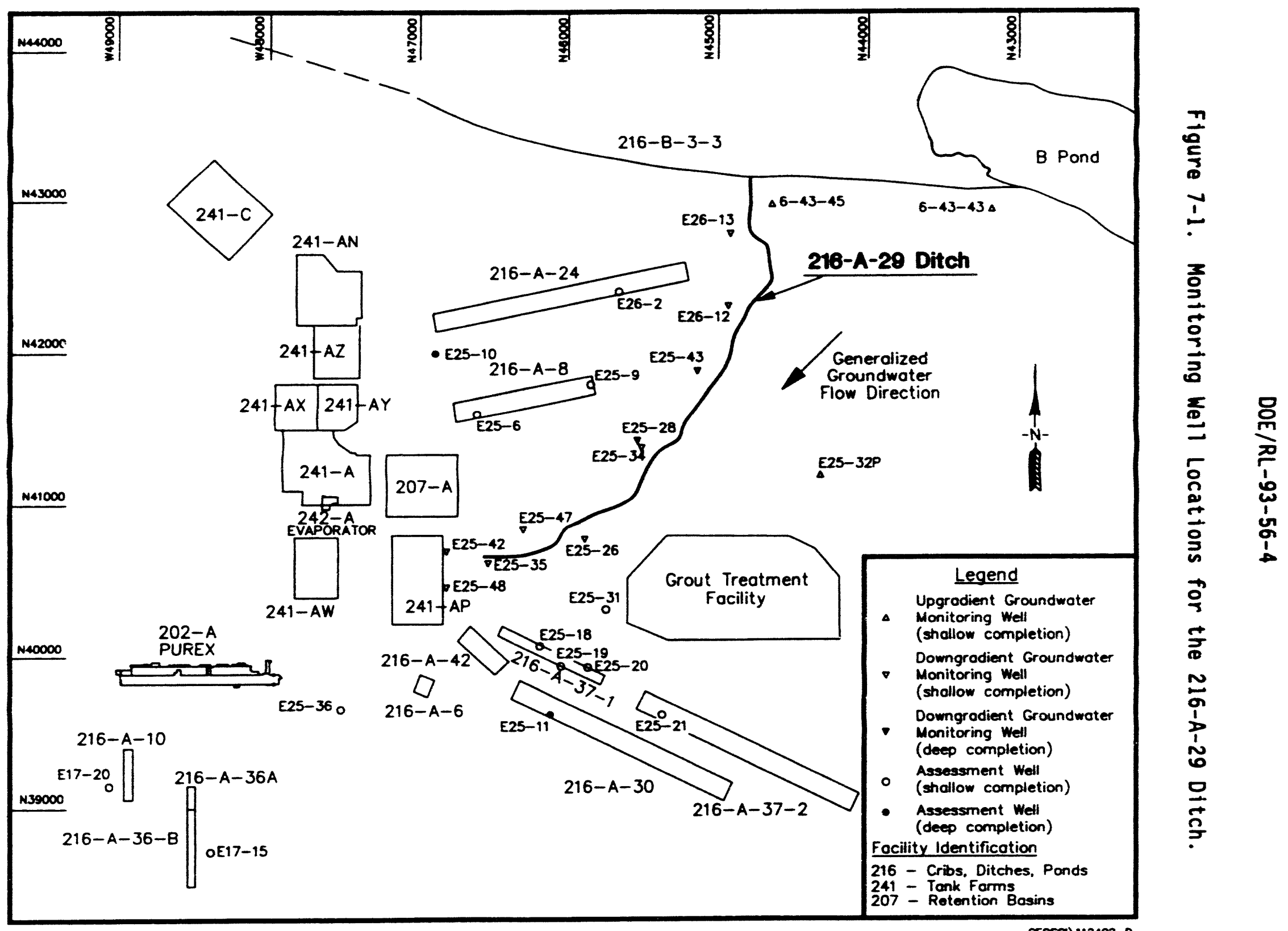


Table 7-1. Monitoring Well Purpose and Sampling Schedule for the 216-A-29 Ditch.

\begin{tabular}{|c|c|c|c|c|}
\hline Well no. & $\begin{array}{l}\text { Relative } \\
\text { position }\end{array}$ & Hydrogeologic unit & $\begin{array}{c}\text { Sample } \\
\text { frequency }\end{array}$ & $\begin{array}{l}\text { Sample date, } \\
\text { 4th Qtr } 1993\end{array}$ \\
\hline $699-43-43^{\circ}$ & Upgradient & Hanford: Water Table & Quarterly & $10 / 13 / 93$ \\
\hline $699-43-45^{\circ}$ & Upgradient & Hanford: Water Table & Quarterly & $10 / 13 / 93$ \\
\hline $299-E 25-32 p^{6}$ & Upgradient & Hanford: Water Table & Quarterly & $10 / 13 / 93$ \\
\hline 299-E25-26 & Downgradient & Hanford: Mid-Depth & Quarterly & Note \\
\hline $299-E 25-28$ & Downgradient & Ringold: Deep & Quarterly & $10 / 29 / 93$ \\
\hline $299-E 25-34$ & Downgradient & Hanford: Water Table & Quarterly & $10 / 12 / 93$ \\
\hline $299-E 25-35$ & Downgradient & Hanford: Water Table & Quarterly & $10 / 20 / 93$ \\
\hline 299-E25-42 & Downgradient & Hanford: Water Table & Quarterly & $10 / 12 / 93$ \\
\hline $299-E 25-43$ & Downgradient & Hanford: Water Table & Quarterly & $10 / 20 / 93$ \\
\hline 299-E25-47 & Dow: & Hanford: Water Table & Quarterly & 10 \\
\hline $299-E 25-48$ & Downgradient & Hanford: Water Table & Quarterly & $10 / 15 / 93$ \\
\hline 299-E26-12 & Downgradient & Hanford: Water Table & Quarterly & $10 / 12 / 93$ \\
\hline $299-E 26-13$ & Downgradient & Hanford: Water Table & Quarterly & $10 / 12 / 93$ \\
\hline $299-E 17-15^{c}$ & As ses sment & Ringold: Water Table & Quarterly & $\begin{array}{l}10 / 11 / 93 \\
12 / 08 / 93\end{array}$ \\
\hline $299-E 17-20^{d}$ & As ses sment & Ringold: Water Table & Quarterly & $10 / 15 / 93$ \\
\hline $299-E 25-11$ & As ses sment & $\begin{array}{l}\text { Undifferentiated } \\
\text { sediments: Mid-Depth }\end{array}$ & Quarterly & $10 / 21 / 93$ \\
\hline $299-E 25-18$ & As ses sment & $\begin{array}{l}\text { Undifferentiated } \\
\text { sediments: Mid-Depth }\end{array}$ & Quarterly & $10 / 10 / 93$ \\
\hline $299-E 25-19$ & Asses sment & $\begin{array}{l}\text { Undifferentiated } \\
\text { sediments: Mid-Depth }\end{array}$ & Quarterly & $11 / 18 / 93$ \\
\hline $299-E 25-20$ & Asses sment & $\begin{array}{l}\text { Undifferentiated } \\
\text { sediments: Mid-Depth }\end{array}$ & Quarterly & $10 / 13 / 93$ \\
\hline 299-E25-21 & As se s sment & Hanford: Water Table & Quarterly & $10 / 21 / 93$ \\
\hline $299-E 25-31$ & Asses sment & Hanford: Water Table & Quarterly & $10 / 13 / 93$ \\
\hline $299-E 25-36^{d}$ & As ses sment & Ringold: Water Table & Quarterly & $10 / 15 / 93$ \\
\hline
\end{tabular}

"Wells shared with the 216-B-3 Pond monitoring network.

Wells shared with the Grout Treatment Facility monitoring network.

'Wells shared with the 216-A-36B monitoring network.

Wells shared with the 216-A-10 monitoring network.

eThis well was not sampled because of pump problems; pump repaired and well sampled in the following quarter. 
Table 7-2. RCRA Water Level Measurement Report for the 216-A-29 Ditch, Fourth Quarter 1993. (sheet 1 of 3)

Well Date $\begin{gathered}\text { Depth to } \\ \text { water (ft) }\end{gathered} \begin{gathered}\text { Water level } \\ \text { elevation } \\ \text { above msl (ft) }\end{gathered}$

Wells in the Detection Monitoring System

\begin{tabular}{|c|c|c|c|}
\hline 299-E25-26 & $\begin{array}{l}10 / 04 / 93 \\
11 / 15 / 93 \\
12 / 13 / 93\end{array}$ & $\begin{array}{l}266.24 \\
266.57 \\
266.40\end{array}$ & $\begin{array}{l}402.28 \\
401.95 \\
402.12\end{array}$ \\
\hline 299-E25-28 & $\begin{array}{l}10 / 04 / 93 \\
11 / 15 / 93 \\
12 / 13 / 93\end{array}$ & $\begin{array}{l}260.27 \\
260.26 \\
260.30\end{array}$ & $\begin{array}{l}402.17 \\
402.18 \\
402.14\end{array}$ \\
\hline 299-E25-32P & $\begin{array}{l}10 / 04 / 93 \\
10 / 13 / 93 \\
11 / 15 / 93 \\
12 / 13 / 93\end{array}$ & $\begin{array}{l}267.95 \\
268.02 \\
268.04 \\
267.98\end{array}$ & $\begin{array}{l}402.09 \\
402.02^{\star} \\
402.00 \\
402.06\end{array}$ \\
\hline 299-E25-34 & $\begin{array}{l}10 / 04 / 93 \\
10 / 12 / 93 \\
11 / 15 / 93 \\
12 / 13 / 93\end{array}$ & $\begin{array}{l}260.78 \\
260.73 \\
260.82 \\
260.83\end{array}$ & $\begin{array}{l}402.09 \\
402.14^{\star} \\
402.05 \\
402.04\end{array}$ \\
\hline 299-E25-35 & $\begin{array}{l}10 / 04 / 93 \\
10 / 20 / 93 \\
11 / 15 / 93 \\
12 / 13 / 93\end{array}$ & $\begin{array}{l}272.46 \\
275.11 \\
272.48 \\
273.02\end{array}$ & $\begin{array}{l}401.93 \\
399.28^{\star}+ \\
401.91 \\
401.37\end{array}$ \\
\hline 299-E25-42 & $\begin{array}{l}10 / 04 / 93 \\
10 / 12 / 93 \\
11 / 15 / 93 \\
12 / 13 / 93\end{array}$ & $\begin{array}{l}281.24 \\
281.35 \\
281.24 \\
281.47\end{array}$ & $\begin{array}{l}402.05 \\
401.94^{\star} \\
402.05 \\
401.82\end{array}$ \\
\hline 299-E25-43 & $\begin{array}{l}10 / 04 / 93 \\
10 / 20 / 93 \\
11 / 15 / 93 \\
12 / 13 / 93\end{array}$ & $\begin{array}{l}247.50 \\
247.48 \\
247.60 \\
247.85\end{array}$ & $\begin{array}{l}402.39 \\
402.41^{\star} \\
402.29 \\
402.04\end{array}$ \\
\hline 299-E25-47 & $\begin{array}{l}10 / 04 / 93 \\
10 / 14 / 93 \\
11 / 15 / 93 \\
12 / 13 / 93\end{array}$ & $\begin{array}{l}271.58 \\
271.57 \\
271.67 \\
271.66\end{array}$ & $\begin{array}{l}402.19 \\
402.20^{\star} \\
402.10 \\
402.11\end{array}$ \\
\hline
\end{tabular}


Table 7-2. RCRA Water Level Measurement Report for the 216-A-29 Ditch, Fourth Quarter 1993. (sheet 2 of 3)

\begin{tabular}{|c|c|c|c|}
\hline Well & Date & $\begin{array}{l}\text { Depth to } \\
\text { water }(\mathrm{ft})\end{array}$ & $\begin{array}{c}\text { Water level } \\
\text { elevation } \\
\text { above msl (ft) }\end{array}$ \\
\hline \multicolumn{4}{|c|}{ Wells in the Detection Monitoring System } \\
\hline $299-E 25-48$ & $\begin{array}{l}10 / 04 / 93 \\
10 / 15 / 93 \\
11 / 15 / 93 \\
12 / 13 / 93\end{array}$ & $\begin{array}{l}280.18 \\
280.16 \\
280.10 \\
280.25\end{array}$ & $\begin{array}{l}402.13 \\
402.15^{\star} \\
402.21 \\
402.06\end{array}$ \\
\hline $299-E 26-12$ & $\begin{array}{l}10 / 04 / 93 \\
10 / 12 / 93 \\
11 / 15 / 93 \\
12 / 13 / 93\end{array}$ & $\begin{array}{l}228.13 \\
228.19 \\
228.25 \\
228.28\end{array}$ & $\begin{array}{l}402.61 \\
402.55^{\star} \\
402.49 \\
402.46\end{array}$ \\
\hline $299-E 26-13$ & $\begin{array}{l}10 / 04 / 93 \\
10 / 12 / 93 \\
11 / 15 / 93 \\
12 / 13 / 93\end{array}$ & $\begin{array}{l}202.52 \\
202.51 \\
202.60 \\
202.59\end{array}$ & $\begin{array}{l}402.50 \\
402.51^{*} \\
402.42 \\
402.43\end{array}$ \\
\hline $699-43-43$ & $\begin{array}{l}10 / 04 / 93 \\
10 / 13 / 93 \\
11 / 15 / 93 \\
12 / 13 / 93\end{array}$ & $\begin{array}{l}164.47 \\
164.42 \\
164.63 \\
164.40\end{array}$ & $\begin{array}{l}414.90 \\
414.95^{\star} \\
414.74 \\
414.97\end{array}$ \\
\hline $699-43-45$ & $\begin{array}{l}10 / 04 / 93 \\
10 / 13 / 93 \\
11 / 15 / 93 \\
12 / 13 / 93\end{array}$ & $\begin{array}{l}194.76 \\
194.76 \\
194.84 \\
194.78\end{array}$ & $\begin{array}{l}402.92 \\
402.92^{\star} \\
402.84 \\
402.90\end{array}$ \\
\hline
\end{tabular}

Supplemental Wells for Assessment Monitoring

\begin{tabular}{llll}
\hline $299-E 17-15$ & $12 / 06 / 93$ & 321.16 & 400.62 \\
$299-E 17-20$ & $10 / 15 / 93$ & 321.66 & $397.57^{\star}+$ \\
& $12 / 07 / 93$ & 317.65 & 401.58 \\
$299-E 25-11$ & $10 / 21 / 93$ & 279.46 & $401.82^{\star}$ \\
& $12 / 08 / 93$ & 279.35 & 401.93 \\
$299-E 25-18$ & $10 / 15 / 93$ & 277.50 & $401.55^{\star}$ \\
& $12 / 08 / 93$ & 277.10 & 401.95 \\
$299-E 25-19$ & $12 / 08 / 93$ & 275.29 & 401.91
\end{tabular}


Table 7-2. RCRA Water Level Measurement Report for the 216-A-29 Ditch, Fourth Quarter 1993. (sheet 3 of 3)

\begin{tabular}{lccc}
\hline We11 & Date & $\begin{array}{c}\text { Depth to } \\
\text { water }(\mathrm{ft})\end{array}$ & $\begin{array}{c}\text { Water level } \\
\text { elevation } \\
\text { above ms }\end{array}$ \\
& Supplemental Wells for Assessment Monitoring \\
\hline $299-E 25-20$ & $10 / 13 / 93$ & 274.89 & $401.41^{\star}$ \\
$299-E 25-21$ & $12 / 08 / 93$ & 274.43 & 401.87 \\
& $10 / 21 / 93$ & 275.51 & $401.76^{\star}$ \\
$299-E 25-31$ & $12 / 02 / 93$ & 275.56 & 401.71 \\
$299-E 25-36$ & $10 / 13 / 93$ & 272.46 & $402.18^{\star}$ \\
& $10 / 15 / 93$ & 306.72 & $400.67^{\star}+$ \\
$299-E 25-6$ & $12 / 07 / 93$ & 305.30 & $402.09+$ \\
$299-E 25-9$ & $12 / 08 / 93$ & 258.99 & 399.32 \\
$299-E 26-2$ & $12 / 08 / 93$ & 255.02 & 399.84 \\
\hline
\end{tabular}

Notes: 1. Water level elevations are calculated by subtracting the measured depth to water from the surveyed elevation for the well.

2. Depth-to-water values are transcribed from field records.

3. Elevations marked with an ' $*$ ' were measured at the time of sampling.

4. Elevations marked with a ' + ' are outside of the expected range and are suspected of error.

5. To convert feet to meters multiply by 0.3048 . 
Table 7-3. Constituent List and Summary of Results for the 216-A-29 Ditch Data for Reporting Period October 1 through December 31, 1993.

(sheet 1 of 5)

CONTAMIMATION INDICATOR PARAMETERS

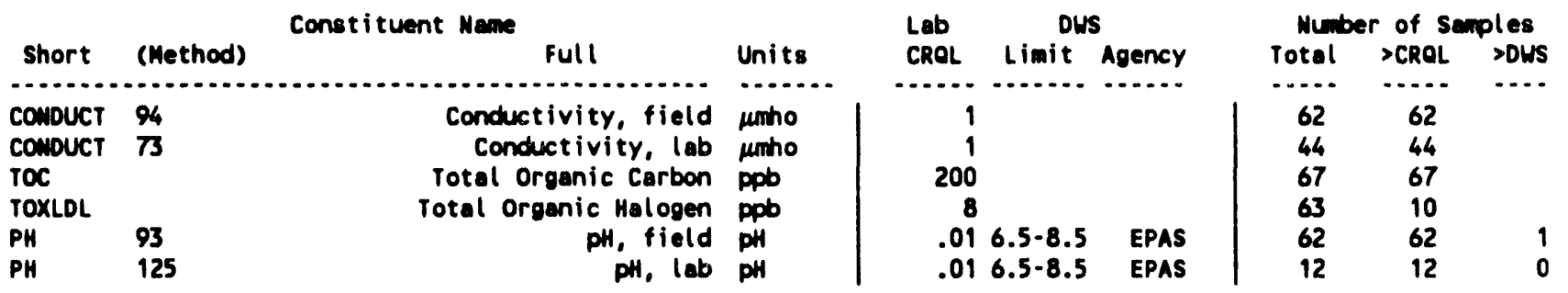

DRINKING MATER PARAMETERS

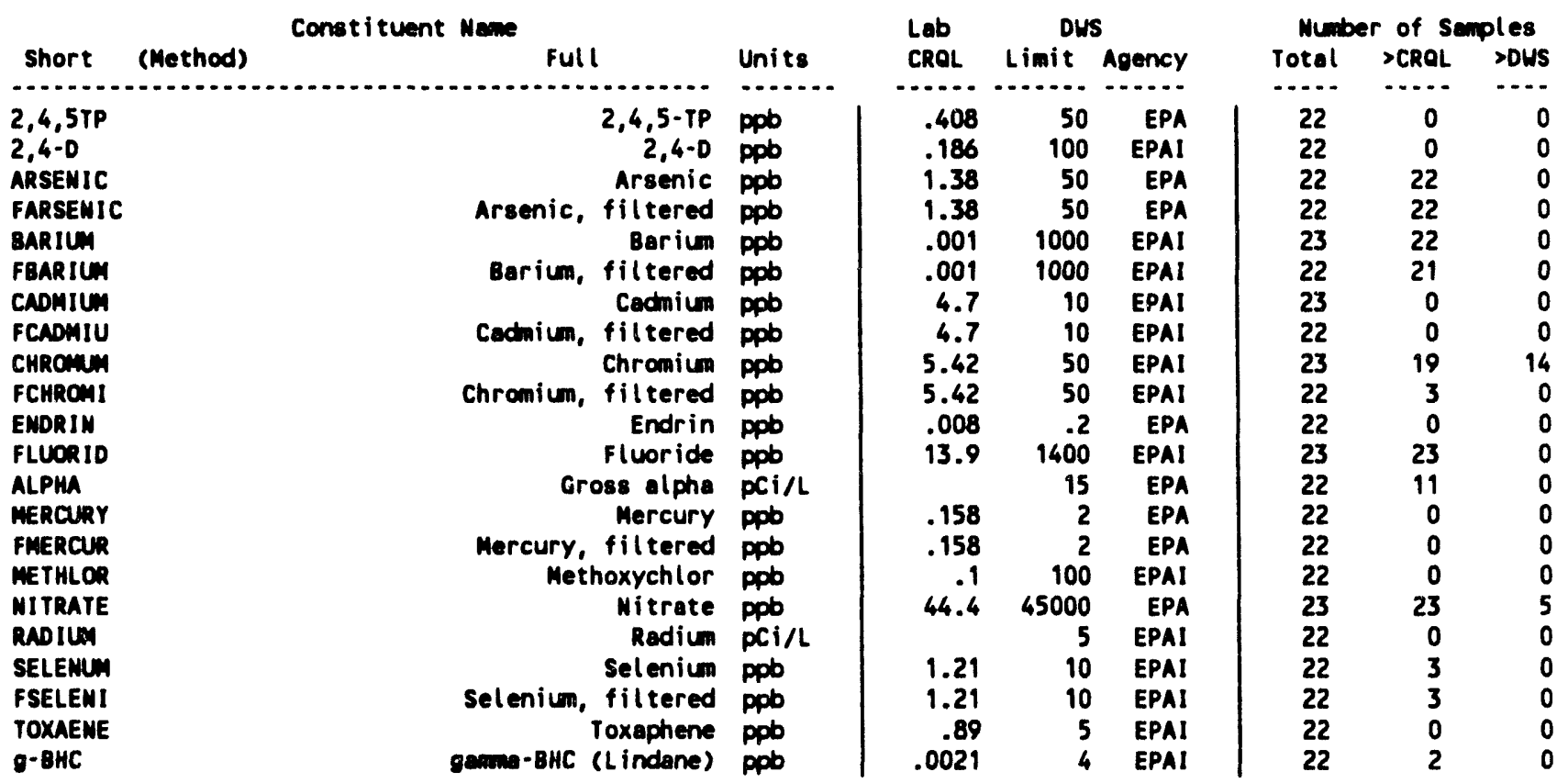

GROUMDHATER QUALITY PARAMETERS

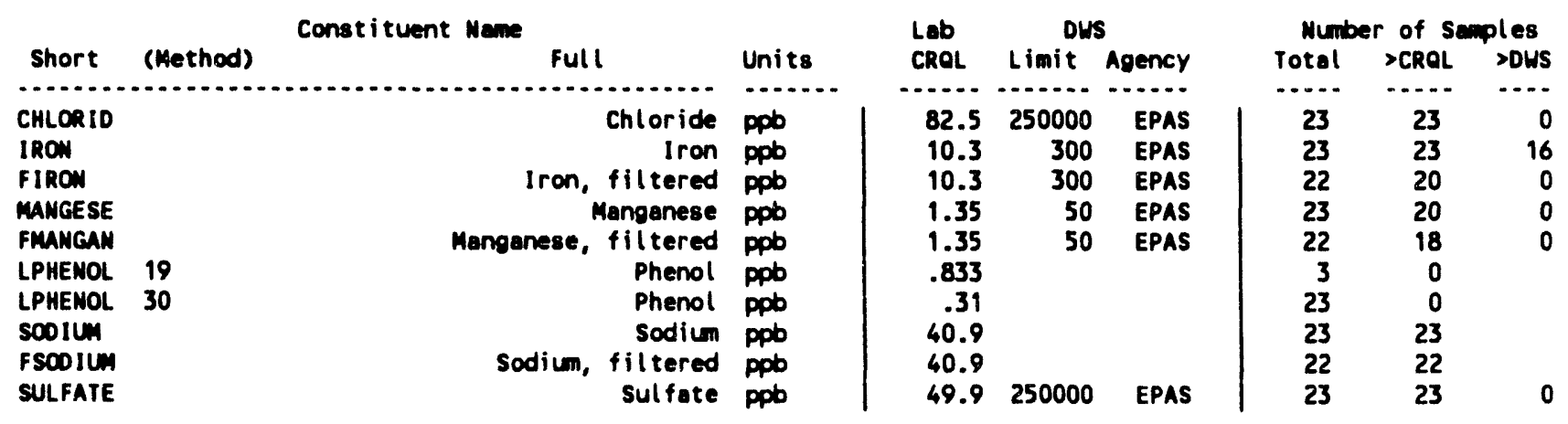


Table 7-3. Constituent List and Summary of Results for the 216-A-29 Ditch Data for Reporting Period October 1 through December 31, 1993.

(sheet 2 of 5 )

SITE SPECIFIC ANO OTHER CONSTITUENTS

\begin{tabular}{|c|c|c|c|c|c|c|c|c|c|}
\hline Short & (Method) & Constituent Mame & Units & $L_{c B b}$ & $\begin{array}{l}\text { Dus: } \\
\text { Limit }\end{array}$ & Agency & $\begin{array}{l}\text { Num } \\
\text { Total }\end{array}$ & $\begin{array}{l}r \text { of Se } \\
>\text { CRQL }\end{array}$ & Ples \\
\hline & & rots & (2) & $\cdots \cdots$ & & & $\ldots .$. & $\cdots \cdots$ & - \\
\hline $1,1,1-1$ & & 1,1,1-Trichloroethane & ppob & .64 & 200 & EPA & 2 & 0 & 0 \\
\hline $1,1,2-1$ & & 1,1,2-irichloroethane & ppb & .25 & & & 2 & 0 & \\
\hline $1,1-01 C$ & & 1,1-Dichlor oethane & ppob & .61 & & & 2 & 0 & \\
\hline TETRCHB & & $1,2,4,5$-Tetrachlorobenzene & ppob & 4.39 & & & 1 & 0 & \\
\hline TRICMLB & & 1,2,4-Trichlorobenzene & ppo & 4.14 & & & 1 & 0 & \\
\hline 12-cben & & 1,2-Dichlorobenzene & ppb & 3.77 & 600 & EPA & 1 & 0 & c \\
\hline $1,2-01 C$ & & 1.2-Dichloroethane & ppb & .45 & 5 & EPA & 2 & 0 & C \\
\hline 12-OCE & & 1,2-Dichloroethene & ppb & 1.2 & & & 2 & 0 & \\
\hline 13-dben & & 1,3-Dichlorobenzene & ppb & 7.85 & & & 1 & 0 & \\
\hline $\begin{array}{l}14-\text { dben } \\
14-\text { dben }\end{array}$ & $\begin{array}{l}16 \\
19\end{array}$ & $\begin{array}{l}\text { 1,4-Dichlorobenzene } \\
\text { 1,4-Dichlorobenzene }\end{array}$ & $\begin{array}{l}\text { Ppb } \\
\text { ppb }\end{array}$ & 4.63 & $\frac{75}{75}$ & $\begin{array}{l}\text { EPA } \\
\text { EPA }\end{array}$ & $\begin{array}{l}2 \\
1\end{array}$ & $\begin{array}{l}0 \\
0\end{array}$ & c \\
\hline MAPMOUI & & 1,4-Maph toquinone & ppo & 10 & & & 1 & 0 & \\
\hline IEUTYM & & 1-Butanol & Ppon & 13.1 & & & 2 & 0 & \\
\hline 1-naphe & & 1-Naphthylanine & ppb & 25.3 & & & 1 & 0 & \\
\hline TETRCHP & & 2,3,4,6-Tet rachlorophenol & ppb & 4.77 & & & 1 & 0 & \\
\hline $2,4,5-T$ & & $2,4,5-1$ & ppob & .384 & & & 22 & 0 & \\
\hline $\begin{array}{l}245-\operatorname{trp} \\
246-\operatorname{trp}\end{array}$ & 19 & $\begin{array}{l}\text { 2,4,5-Trichlorophenol } \\
\text { 2,4,6-Trichlorophenol }\end{array}$ & $\begin{array}{l}\text { ppb } \\
\text { ppb }\end{array}$ & $\begin{array}{l}4.01 \\
2.54\end{array}$ & & & $\begin{array}{l}1 \\
1\end{array}$ & $\begin{array}{l}0 \\
0\end{array}$ & \\
\hline $246-\operatorname{trp}$ & 30 & 2,4,6-Trichlorophenol & ppob & 1.45 & & & 23 & 0 & \\
\hline 24 -dchp & 19 & 2,4-Dichlorophenol & ppb & 2.8 & & & 3 & 0 & \\
\hline 24-dchp & 30 & 2,4-D ichlorophenol & ppb & 1.5 & & & 23 & 0 & \\
\hline OIMPNEN & 19 & 2,4-0 imethylphenol & ppob & 5.88 & & & 1 & 0 & \\
\hline DIMPHEN & 30 & 2,4-0 imethylphenol & ppb & 1.01 & & & 23 & 1 & \\
\hline DIMPHEN & 19 & 2,4-D initrophenol & ppb & 4.46 & & & 1 & 0 & \\
\hline DINPHEN & 30 & 2,4-Dinitrophenol & ppob & .96 & & & 23 & 0 & \\
\hline 24-dint & & 2,4-D initrotoluene & pppb & 2.59 & & & 1 & 0 & \\
\hline 26-dchp & $\begin{array}{l}19 \\
30\end{array}$ & 2,6-D ichlorophenol & ppb & $\begin{array}{l}3.47 \\
1.50\end{array}$ & & & $\begin{array}{r}1 \\
23\end{array}$ & & \\
\hline $\begin{array}{l}\text { 26-dchp } \\
\text { 26-dint }\end{array}$ & 30 & $\begin{array}{l}\text { 2,6-D ichlorophenol } \\
\text { 2,6-0 ini trotoluene }\end{array}$ & $\begin{array}{l}\text { Ppb } \\
\text { ppb }\end{array}$ & $\begin{array}{l}1.59 \\
2.85\end{array}$ & & & $\begin{array}{r}23 \\
1\end{array}$ & $\begin{array}{l}0 \\
0\end{array}$ & \\
\hline ACEFENE & & 2-Acetylaminofluorene & ppo & 2.83 & & & 1 & 0 & \\
\hline CHLMAPH & & 2-Chloronaphthalene & ppb & 3.02 & & & 1 & 0 & \\
\hline CHLPHEN & 19 & 2-Chlorophenol & ppb & 1.91 & & & 1 & 0 & \\
\hline CHLPHEN & 30 & 2-Chlorophenol & ppob & 1.42 & & & 23 & $\mathbf{0}$ & \\
\hline ZMEMAPH & & 2-Methylnaph thalene & ppob & 2.76 & & & 1 & 0 & \\
\hline ZNETHPH & & 2-Methylphenol & ppob & 1.8 & & & 3 & 0 & \\
\hline 2-napha & & 2-Naphthylemine & ppb & 15.6 & & & 1 & 0 & \\
\hline ONITAMI & & 2-Nitroaniline & ppb & 7.82 & & & 1 & 0 & \\
\hline 2NITPH & 19 & 2-Nitrophenol & ppb & 3.96 & & & 3 & 0 & \\
\hline 2NITPH & 30 & 2-Witrophenol & ppb & 1.56 & & & 23 & 0 & \\
\hline PICOLIN & & 2-Picoline & ppb & 5.83 & & & 1 & 0 & \\
\hline BUTDIMP & 30 & 2-sec-Butyl-4,6-dinitrophenol (DN & ppb & 1.35 & & & 23 & 0 & \\
\hline EUTDINP & 49 & 2-sec-Butyl-4,6-dini trophenol (ON & ppob & .269 & & & 22 & 0 & \\
\hline DICHBEN & & 3,3'-Dichlorobenz idine & ppob & 3.88 & & & 1 & 0 & \\
\hline DIMEYLB & & 3,3'-D ime thylbenzidine & ppob & 10.4 & & & 1 & 0 & \\
\hline METCKAN & & 3-Methylcholanthrene & ppb & 2.63 & & & 1 & 0 & \\
\hline MNITANI & & 3-Nitroeniline & ppob & 9.52 & & & 1 & 0 & \\
\hline & & $4,4 \cdot-0 D D$ & ppob & .0005 & & & 22 & 0 & \\
\hline DDE & & $4,4 \cdot-D D E$ & ppob & .0009 & & & 22 & 0 & \\
\hline DOT & & $4,4^{\prime}-0 D T$ & ppb & .011 & & & 22 & 0 & \\
\hline 460N2MP & 19 & 4,6-Dinitro-2-methylphenol & ppb & 5.54 & & & 1 & 0 & \\
\hline 460N2MP & 30 & 4,6-D initro-2-methylphenol & ppo & 1.18 & & & 23 & 0 & \\
\hline $\begin{array}{l}\text { AMIMOYL } \\
\text { BROPHEN }\end{array}$ & & $\begin{array}{r}\text { 4-Aminobiphenyl } \\
\text { 4-Bromophenylphenyl ether }\end{array}$ & $\begin{array}{l}\text { ppb } \\
\text { ppb }\end{array}$ & $\begin{array}{l}3.83 \\
2.34\end{array}$ & & & $\begin{array}{l}1 \\
1\end{array}$ & $\begin{array}{l}0 \\
0\end{array}$ & \\
\hline & & & & & & & & & \\
\hline
\end{tabular}


Table 7-3. Constituent List and Summary of Results for the 216-A-29 Ditch Data for Reporting Period October 1 through December 31, 1993.

(sheet 3 of 5 )

\begin{tabular}{|c|c|c|c|c|c|c|c|c|c|}
\hline \multirow{2}{*}{ short } & \multirow[b]{2}{*}{ (Method) } & \multirow{2}{*}{$\begin{array}{l}\text { Constituent Neme Full } \\
\text { Fun }\end{array}$} & \multirow[b]{2}{*}{ Units } & \multicolumn{3}{|c|}{ Dus } & \multicolumn{3}{|c|}{ Number of Samples } \\
\hline & & & & CROL & Limit & Agency & Total & $>$ CROL & $>$ DUS \\
\hline CHLCRES & 19 & 4-Chloro-3-wethylphenol & ppob & 7.41 & & & 1 & 0 & $\ldots$ \\
\hline CHLCRES & 30 & 4-Chloro-3-methylphenol & ppb & 1.12 & & & 23 & 0 & \\
\hline CHLANIL & & 4-Chloroenil ine & ppob & 13.2 & & & 1 & 0 & \\
\hline ४СРPETH & & 4-Chlorophenylphenyl ether & ppb & 3.04 & & & 1 & 0 & \\
\hline MIBK & & 4-Methyl-2-pentanone & ppob & .85 & & & 2 & 0 & \\
\hline WMETHPH & & 4-Methylphenol & ppb & 3.54 & & & 3 & 0 & \\
\hline NITRAMI & & 4-Nitroanil ine & ppb & 21.4 & & & 1 & 0 & \\
\hline MITPHEN & 19 & 4-Nitrophenol & ppb & 1.52 & & & 1 & 0 & \\
\hline MITPHEN & 30 & 4-Nitrophenol & ppb & .65 & & & 23 & 0 & \\
\hline 4NITRUI & & 4-Nitroquinol ine-1-oxide & ppob & 6 & & & 1 & 0 & \\
\hline MITRTOL & & 5-Mitro-0-toluidine & ppob & 5.25 & & & 1 & 0 & \\
\hline DIMaENZ & & 7, 12-D iwethylbenz [a] anthracene & Ppb & 1.82 & & & 1 & 0 & \\
\hline ACEMAPH & & Acenaphthene & ppb & 2.88 & & & 1 & 0 & \\
\hline ACEMATL & & Acenaphthylene & ppb & 3.96 & & & 1 & 0 & \\
\hline ACETONE & & Acetone & ppb & 13.4 & & & 2 & 0 & \\
\hline ACETOPH & & Acetophenone & ppob & 2.38 & & & 1 & 0 & \\
\hline ALORIN & & Aldrin & ppb & .05 & & & 22 & 0 & \\
\hline DIMPHM & & Alpha, al pho-D i methyl phenethyl ani & ppb & 37.8 & & & 1 & 0 & \\
\hline - BHC & & Alpha-Bhr & ppb & .012 & & & 22 & 1 & \\
\hline ALLANINA & & Aluminum & ppo & 32.5 & & & 23 & 12 & \\
\hline FALUAIN & & Aluminum & ppb & 32.5 & & & 22 & 1 & \\
\hline Amionit & & Amonium ion & ppb & 38.5 & & & 23 & 21 & \\
\hline AMILINE & & Aniline & ppb & 3.53 & & & 1 & 0 & \\
\hline AMTHRA & & Anthracene & ppb & 2.95 & & & 1 & 0 & \\
\hline ANTIONY & & Ant imony & ppob & 69.4 & & & 23 & 1 & \\
\hline FANTIMO & & Antimony, filtered & ppb & 69.4 & & & 22 & 0 & \\
\hline $58-125$ & & Ant imony- 125 & $\mathrm{pCi} / \mathrm{L}$ & & & & 5 & 0 & \\
\hline ARAMITE & & Aranite & ppob & 8.6 & & & 1 & 0 & \\
\hline BEMZEME & & Benzene & ppob & .65 & 5 & EPA & 2 & 0 & 0 \\
\hline BEMZAAN & & Benzo (a) anthracene & ppob & 2.34 & & & 1 & 0 & \\
\hline BENZOPY & & Benzo(a)pyrene & ppob & 1.7 & & & 1 & 0 & \\
\hline BENZBFL & & Benzo(b)f lworanthene & ppb & 4.41 & & & 1 & 0 & \\
\hline BGHIPER & & Benzo(ghi)perylene & ppob & 3.65 & & & 1 & 0 & \\
\hline BMZKFLU & & Senzo(k)f lworanthene & ppob & 2.21 & & & 1 & 0 & \\
\hline BENZOTH & & Benzothiazole & ppb & 2.55 & & & 3 & 0 & \\
\hline BEMZALC & & Benzyl alcohol & ppob & 5.16 & & & 1 & 0 & \\
\hline BERYLLA & & Baryllium & ppob & .814 & & & 23 & 4 & \\
\hline FBERYLL & & Berylliun, filtered & ppob & .814 & & & 22 & 1 & \\
\hline b-BHC & & Beta-BHC & ppo & .0026 & & & 22 & 1 & \\
\hline BIS2CMM & & Bis(2-Choroethoxy) methane & ppb & 7.13 & & & 1 & 0 & \\
\hline BISZCHE & & Bis(2-chloroethyl) ether & ppb & 2.9 & & & 1 & 0 & \\
\hline BISLETH & & Bis(2-chloroisopropyl) ether & ppb & 3.35 & & & 1 & 0 & \\
\hline BISZEPH & & Bis(2-ethylhexyl) phthalate & ppb & 4.07 & & & 3 & 0 & \\
\hline BROMIDE & & Bromide & ppb & 52.8 & & & 23 & 0 & \\
\hline BUTBENP & & Butylbenzylphthalate & ppob & 5.94 & & & 1 & 0 & \\
\hline CALCIUN & & Calcium & ppb & 20.9 & & & 23 & 23 & \\
\hline FCALCIU & & Colcium, filtered & ppb & 20.9 & & & 22 & 22 & \\
\hline CARBIOE & & Carbon disulfide & ppb & .95 & & & 2 & 0 & \\
\hline TETRAME & & Carbon tetrachloride & ppb & .87 & 5 & EPA & 2 & 0 & 0 \\
\hline cs-137 & & Cesium-137 & $\mathrm{PCi} / \mathrm{L}$ & & 200 & EPA & 5 & 0 & 0 \\
\hline CHLOAME & & Chlordane & ppob & .0057 & 2 & EPA & 22 & 0 & 0 \\
\hline CHLLATE & & Chlorobenzil ate & ppob & 8.69 & & & 1 & 0 & \\
\hline CHLFORM & & Chloroform & ppb & .4 & & & 2 & 0 & \\
\hline CHRYSEN & & Chrysene & ppb & 1.7 & & & 1 & 0 & \\
\hline COAALT & & Cobalt & ppob & 4.05 & & & 23 & 2 & \\
\hline FCOBALT & & Cobolt, filtered & ppb & 4.05 & & & 22 & 4 & \\
\hline$c 0-60$ & & Cobalt -60 & $\mathrm{PCi} / \mathrm{L}$ & & 100 & EPA & 5 & 0 & 0 \\
\hline COLIFORM & & Coliforme & COL & & 1 & EPAI & 22 & 1 & 0 \\
\hline COPPER & & Copper & ppb & 2.65 & 1000 & EPAS & 23 & 14 & 0 \\
\hline
\end{tabular}


Table 7-3. Constituent List and Summary of Results for the 216-A-29 Ditch Data for Reporting Period October 1 through December 31, 1993.

(sheet 4 of 5 )

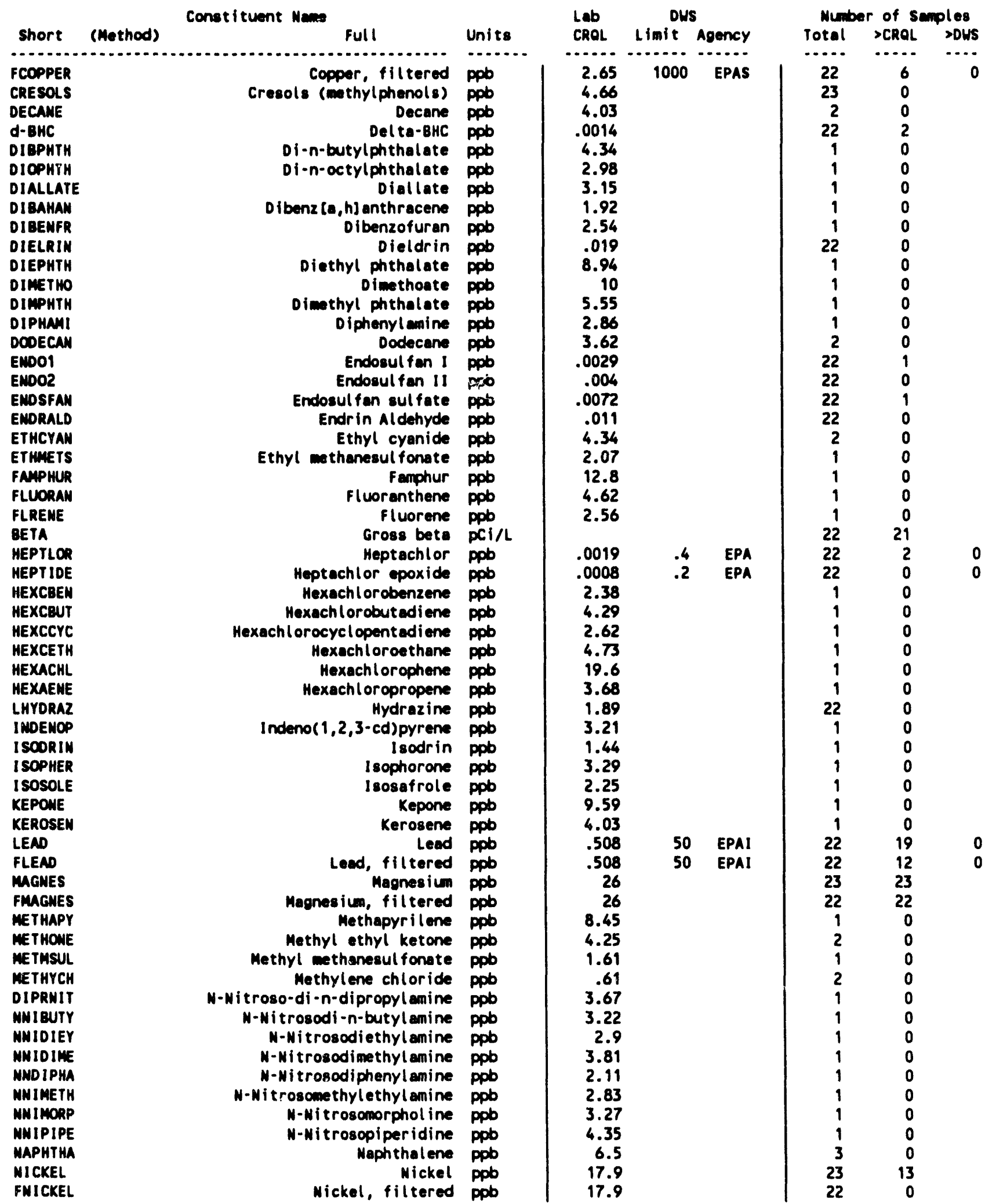


Table 7-3. Constituent List and Summary of Results for the 216-A-29 Ditch Data for Reporting Period October 1 through December 31, 1993.

(sheet 5 of 5 )

\begin{tabular}{|c|c|c|c|c|c|c|c|c|c|}
\hline & & Const itwent Meme & & Lab & Dus & & Numb & $r$ of $\mathbf{S e}$ & ples \\
\hline Short & (Method) & Full & Units & CROL & Limit & Agency & Total & $>$ CRQL & >DWS \\
\hline NITRITE & & Mitrite & (n) & 10. & - non & (n) & $\cdots$ & $\cdots$ & $\cdots$ \\
\hline NITBENZ & & Mitrite & Ppp & 38.3 & 1000 & EPA & 23 & 0 & 0 \\
\hline NITRPYR & & $\begin{array}{l}\text { Nitrobenzene } \\
\text { Nitrosopyrrol idine }\end{array}$ & $\begin{array}{l}\text { ppb } \\
\text { ppob }\end{array}$ & $\begin{array}{l}3.16 \\
2.78\end{array}$ & & & 1 & $\begin{array}{l}0 \\
0\end{array}$ & \\
\hline TRIPHOS & & $0,0,0$-Triethyl phosphorothioate & ppb & 5.19 & & & 1 & 0 & \\
\hline DIPMOS & & 0,0-diethyl0-2-pyraz inylphosphor & ppb & 3.04 & & & 1 & 0 & \\
\hline PARATHI & & Parathion & ppob & 3.9 & & & 1 & 0 & \\
\hline PENTCMB & & Pentachl orobenzene & ppob & 3.99 & & & 1 & 0 & \\
\hline PENTCAN & & Pentachloronitrobenzene (PCNB) & pppb & 2.49 & & & 1 & 0 & \\
\hline PENTCHP & 19 & Pentachlor ophenol & ppob & 8.07 & 1 & EPA & 3 & 0 & 3* \\
\hline PENTCHP & 30 & Pentachlorophenol & ppob & .87 & 1 & EPA & 23 & 0 & 0 \\
\hline PHENT IN & & Phenacet in & ppob & 4.78 & & & 1 & 0 & \\
\hline PHENANT & & Phenanthrene & ppb & 5.27 & & & 1 & 0 & \\
\hline PHOSPMA & & Phosphate & pppb & 147 & & & 23 & 0 & \\
\hline POTASUM & & Potassium & Ppp & 662 & & & 23 & 23 & \\
\hline FPOTASS & & Potassiun, filtered & pppb & 662 & & & 22 & 22 & \\
\hline PRONIDE & & Pronemide & pppb & 3.42 & & & 1 & 0 & \\
\hline PYRENE & & Pyrene & pppb & 3.69 & & & 1 & 0 & \\
\hline PYRIOIN & & Pyridine & ppb & 4.57 & & & 1 & 0 & \\
\hline RU-106 & & Rutheniun-106 & $\mathrm{PCi} / \mathrm{L}$ & & 30 & EPA & 5 & 0 & 0 \\
\hline SAFROL & & Sefrol & ppob & 3.22 & & & 1 & 0 & \\
\hline SILVER & & silver & ppb & 2.87 & 50 & EPAI & 23 & 0 & 0 \\
\hline FSILVER & & silver, filtered & ppb & 2.87 & 50 & EPAI & 22 & 0 & 0 \\
\hline TC- 99 & & Technet ium-99 & $\mathrm{PCI} / \mathrm{L}$ & & 900 & EPA & 2 & 0 & 0 \\
\hline PERCENE & & Tetrachloroethene & ppb & 1.1 & 5 & EPA & 2 & 0 & 0 \\
\hline TETPHNL & & Tetrachlorophenols & ppb & 1.05 & & & 23 & 0 & \\
\hline TETRADE & & Tetradecane & ppob & 2.43 & & & 2 & 0 & \\
\hline PYROPHOS & & Tetraethyldi thi opyrophosphate & ppo & 2.81 & & & 1 & 0 & \\
\hline TAF & & Tetrahydrofuran & ppob & 3.67 & & & 2 & 0 & \\
\hline TIN & & $\operatorname{Tin}$ & ppb & 51.1 & & & 23 & 1 & \\
\hline FTIN & & Tin, filtered & ppob & 51.1 & & & 22 & 0 & \\
\hline TOLUENE & & Tolvene & ppb & .73 & 1000 & EPA & 2 & 0 & 0 \\
\hline $\begin{array}{l}\text { TOS } \\
\text { TRIBUTPH }\end{array}$ & & $\begin{array}{l}\text { Total Dissolved Solids } \\
\text { Tributyl Phosphate }\end{array}$ & $\begin{array}{l}\text { ppm } \\
\text { pobb }\end{array}$ & $\begin{array}{r}10 \\
4.42\end{array}$ & $\mathbf{5 0 0}$ & EPAS & $\begin{array}{l}2 \\
3\end{array}$ & 2 & 0 \\
\hline TRICENE & & Trichloroethene & ppob & .7 & 5 & EPA & 2 & 0 & 0 \\
\hline TRIPHNL & & Trichlorophenols & ppo & 1.11 & & & 23 & 0 & \\
\hline TOICHPH & & Tris-2-chloroethyl phosphate & ppb & 2.88 & & & 3 & 0 & \\
\hline TRITIUN & & Tritiun & $\mathrm{pCi} / \mathrm{L}$ & & 20000 & EPA & 23 & 23 & 9 \\
\hline TURB ID & & Turbidity & NTU & .05 & & & 22 & 22 & \\
\hline$U-234$ & & Uranium-234 & $\mathrm{PCi} / \mathrm{L}$ & & & & 2 & 2 & \\
\hline$U-235$ & & Uranium-235 & $\mathrm{PCi} / \mathrm{L}$ & & & & 2 & 1 & \\
\hline U-238 & & Uranium-238 & $\mathrm{PCi}$ & & & & 2 & 2 & \\
\hline VANADUN & & $\begin{array}{l}\text { Venadium } \\
\text { Vanedium, filtered }\end{array}$ & ppb & 3.84 & & & 23 & 18 & \\
\hline FVANADI & & $\begin{array}{l}\text { Vanadium, filtered } \\
\text { Vinyl chloride }\end{array}$ & ppb & $\begin{array}{r}3.84 \\
.78\end{array}$ & & & $\begin{array}{r}22 \\
2\end{array}$ & $\begin{array}{r}17 \\
0\end{array}$ & \\
\hline $\begin{array}{l}\text { VINYIDE } \\
\text { XYLENE }\end{array}$ & & $\begin{array}{l}\text { Vinyl chloride } \\
\text { Xylenes (total) }\end{array}$ & $\begin{array}{l}\text { Ppb } \\
\text { ppb }\end{array}$ & 1.7 & 10000 & $\begin{array}{l}\text { EPA } \\
\text { EPA }\end{array}$ & 2 & 0 & $\begin{array}{l}0 \\
0\end{array}$ \\
\hline 2INC & & 2 inc & ppb & 3.44 & 5000 & EPAS & 23 & 15 & 0 \\
\hline FZINC & & zinc, filtered & ppb & 3.44 & 5000 & EPAS & 22 & 12 & 0 \\
\hline MCRESOL & & m-Cresol & ppb & 1.46 & & & 3 & 0 & \\
\hline DINBEHZ & & m-dinitrobenzene & ppb & 9.35 & & & 1 & 0 & \\
\hline OTOLHYD & & o-Toluidine & ppb & 2.27 & & & 1 & 0 & \\
\hline DIMENMS & & p-D ine thy laninoszobenzene & ppb & 3.36 & & & 1 & 0 & \\
\hline PHENOIA & & P-Phenylenedienine & ppo & 3.09 & & & 1 & 0 & \\
\hline SYMTRIN & & sym-Trinitrobenzene & ppb & 3.74 & & & 1 & 0 & \\
\hline
\end{tabular}

For explenation of this table, see section 1.4 of report. 
Table 7-4. Constituents with at Least One Detected Value for the 216-A-29 Ditch Data for Reporting Period October 1 through December 31, 1993. (sheet 1 of 14)

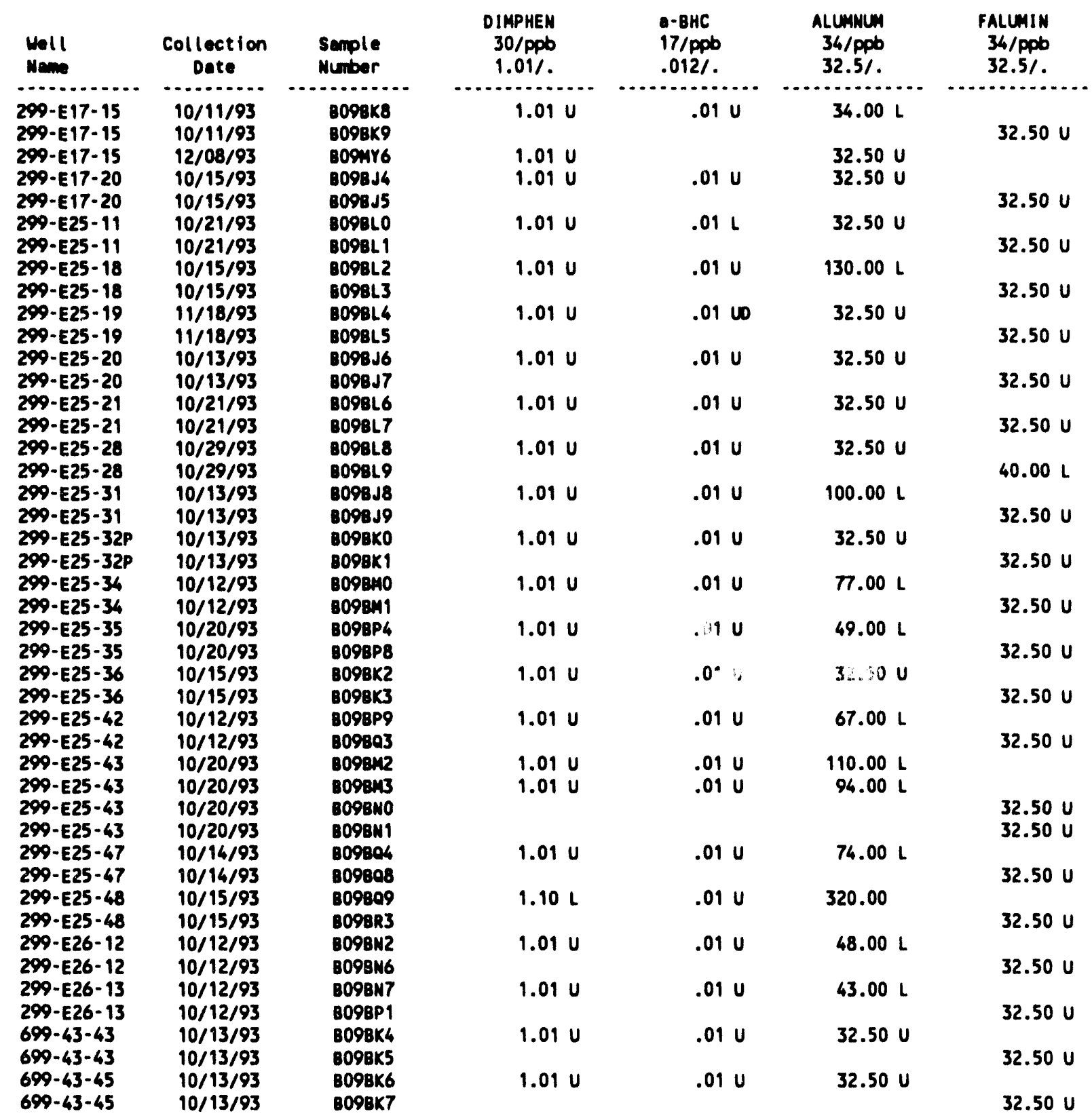

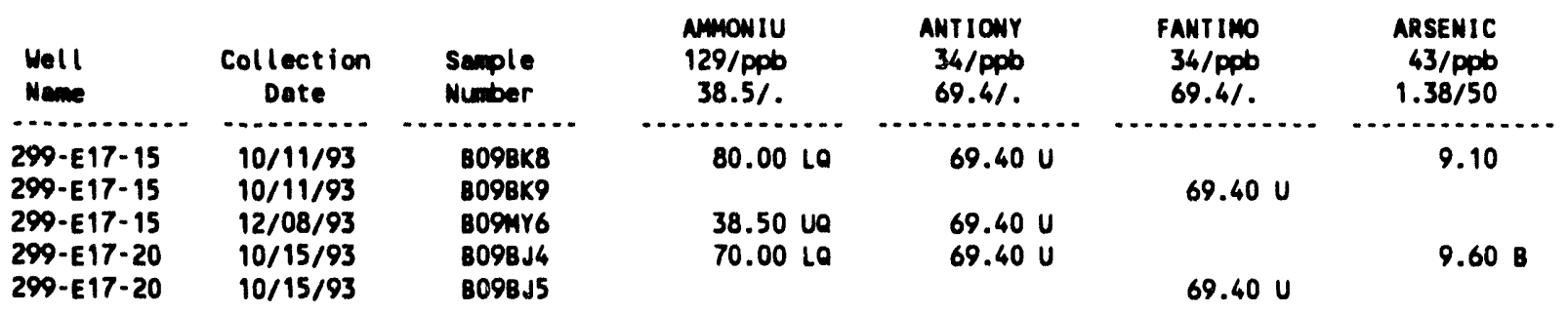


Table 7-4. Constituents with at Least One Detected Value for the 216-A-29 Ditch Data for Reporting Period October 1 through December 31, 1993. (sheet 2 of 14)

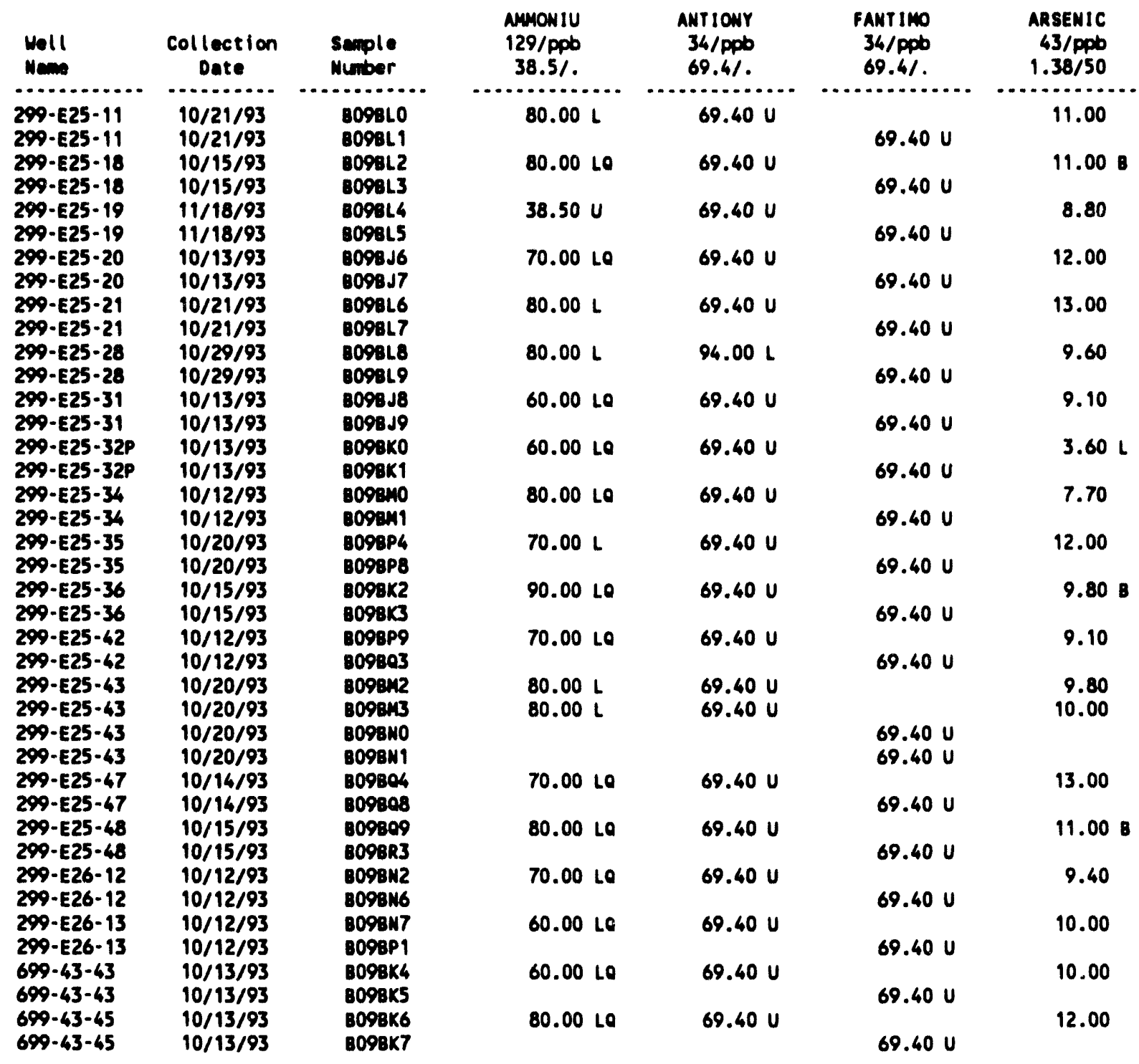

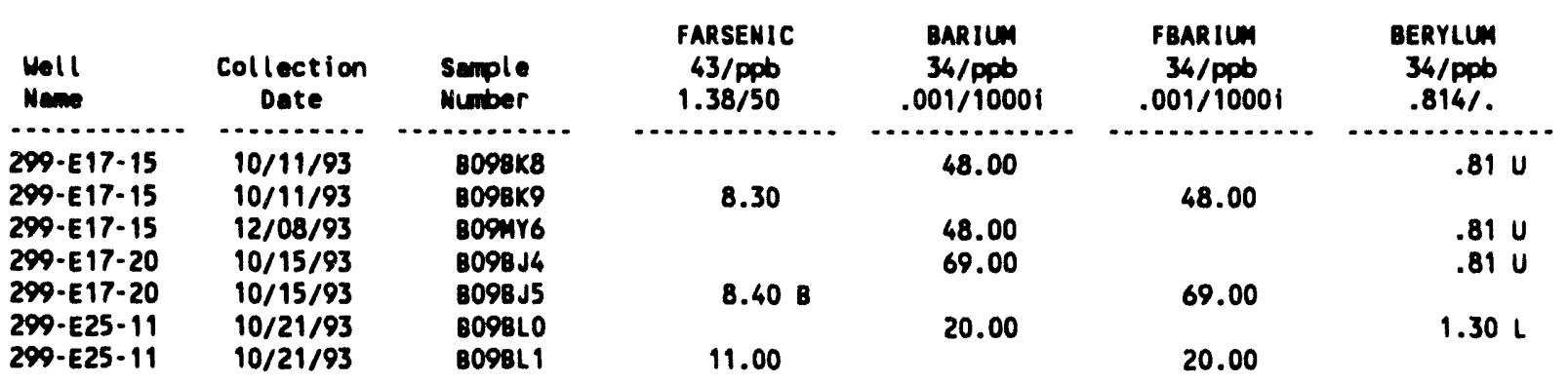


Table 7-4. Constituents with at Least One Detected Value for the 216-A-29 Ditch Data for Reporting Period October 1 through December 31, 1993. (sheet 3 of 14)

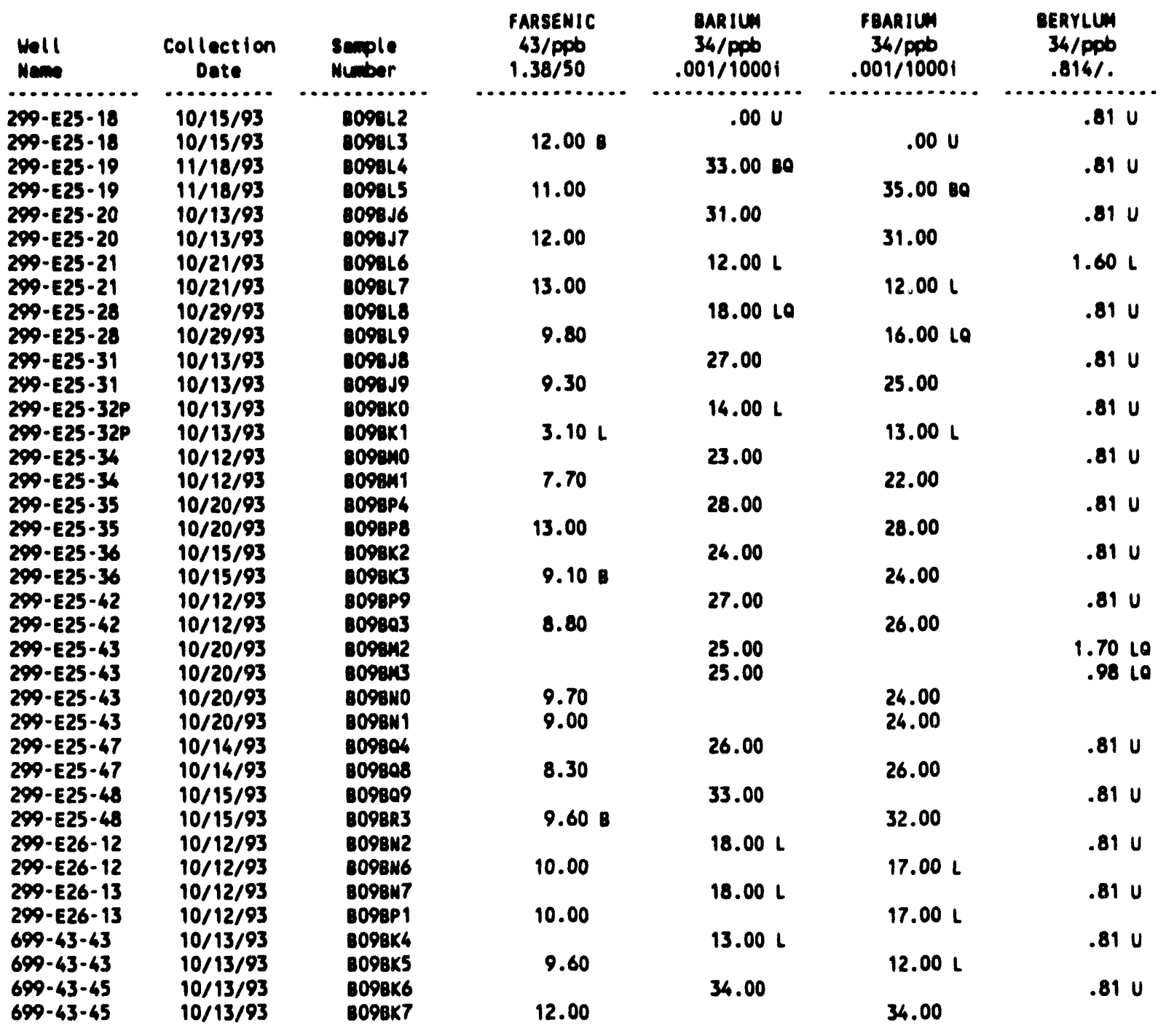

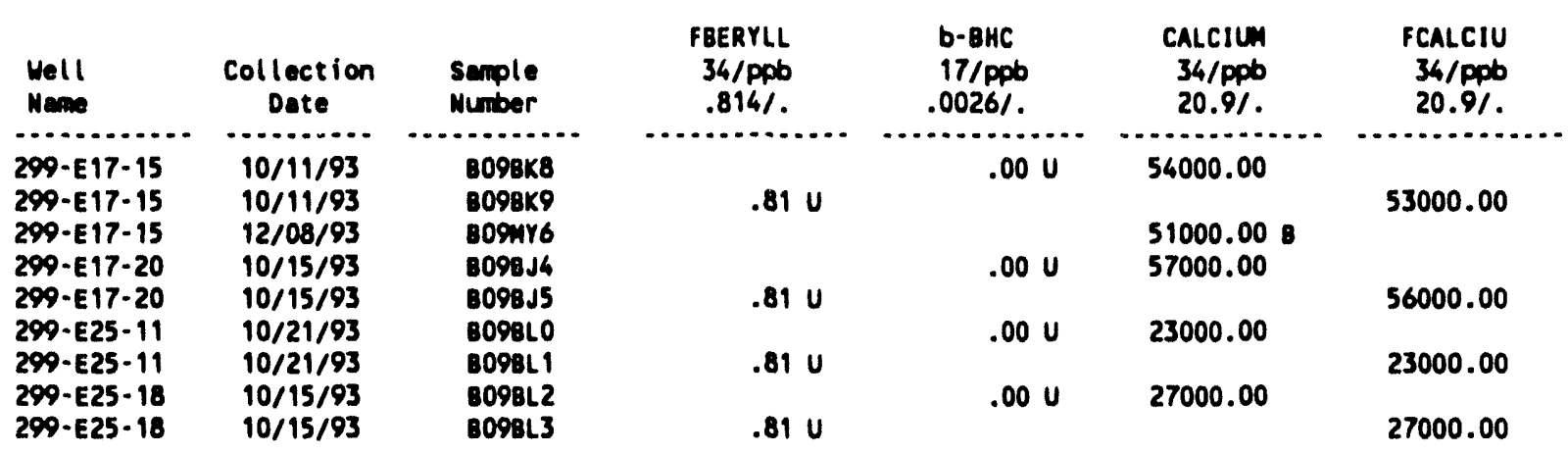


Table 7-4. Constituents with at Least One Detected Value for the 216-A-29 Ditch Data for Reporting Period October 1 through December 31, 1993. (sheet 4 of 14)

\begin{tabular}{|c|c|c|c|c|c|c|}
\hline Well & $\begin{array}{c}\text { Collection } \\
\text { Date }\end{array}$ & $\begin{array}{l}\text { semple } \\
\text { Murber }\end{array}$ & $\begin{array}{c}\text { FEearLL } \\
36 / \text { pob } \\
.814 / .\end{array}$ & $\begin{array}{l}\text { b-вHC } \\
17 / p \text { po } \\
.0026 / \text {. }\end{array}$ & $\begin{array}{l}\text { CALCIUA } \\
34 / p p b \\
20.9 / \text {. }\end{array}$ & $\begin{array}{l}\text { FCALCIU } \\
34 / p p b \\
20.9 /\end{array}$ \\
\hline 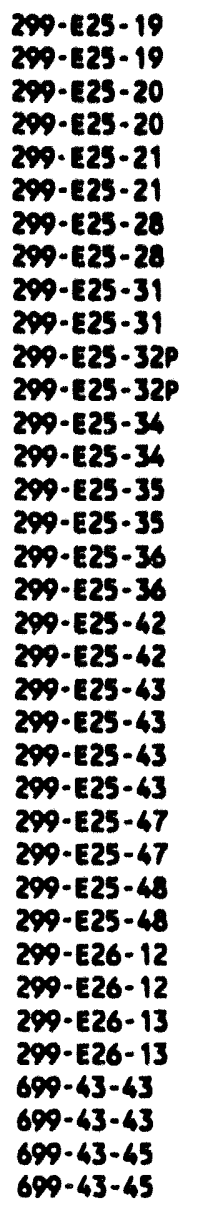 & $\begin{array}{l}11 / 18 / 93 \\
11 / 18 / 93 \\
10 / 13 / 93 \\
10 / 13 / 93 \\
10 / 21 / 93 \\
10 / 21 / 93 \\
10 / 29 / 93 \\
10 / 29 / 93 \\
10 / 13 / 93 \\
10 / 13 / 93 \\
10 / 13 / 93 \\
10 / 13 / 93 \\
10 / 12 / 93 \\
10 / 12 / 93 \\
10 / 20 / 93 \\
10 / 20 / 93 \\
10 / 15 / 93 \\
10 / 15 / 93 \\
10 / 12 / 93 \\
10 / 12 / 93 \\
10 / 20 / 93 \\
10 / 20 / 93 \\
10 / 20 / 93 \\
10 / 20 / 93 \\
10 / 16 / 93 \\
10 / 16 / 93 \\
10 / 15 / 93 \\
10 / 15 / 93 \\
10 / 12 / 93 \\
10 / 12 / 93 \\
10 / 12 / 93 \\
10 / 12 / 93 \\
10 / 13 / 93 \\
10 / 13 / 93 \\
10 / 13 / 93 \\
10 / 13 / 93\end{array}$ & 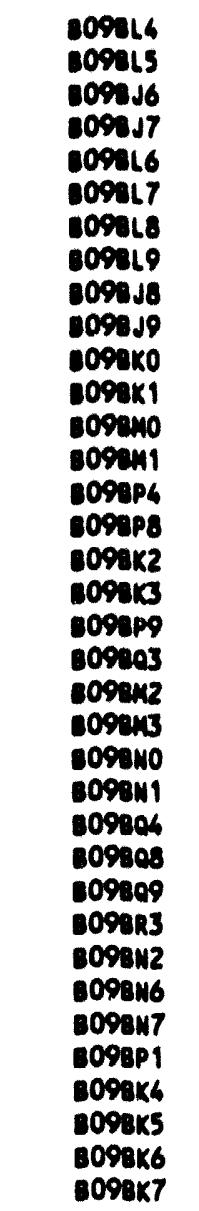 & $\begin{array}{l}.81 U \\
.81 U \\
.81 U \\
.81 U \\
.81 U \\
.81 U \\
.81 U \\
.81 U \\
.81 U \\
.81 U \\
2.10 L 0 \\
.81 U \\
.81 U \\
.81 U \\
.81 U \\
.81 U \\
.81 U \\
.81 U\end{array}$ & 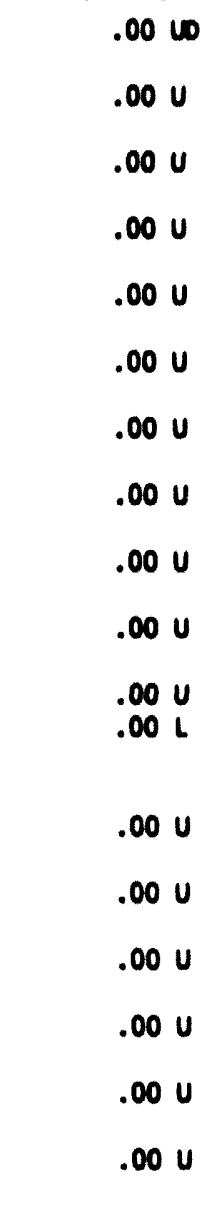 & $\begin{array}{l}29000.00 \\
36000.00 \\
18000.00 \\
23000.00 \\
24000.00 \\
23000.00 \\
26000.00 \\
32000.00 \\
30000.00 \\
31000.00 \\
28000.00 \\
28000.00 \\
24000.00 \\
25000.00 \\
32000.00 \\
31000.00 \\
30000.00 \\
28000.00\end{array}$ & $\begin{array}{l}29000.00 \\
36000.00 \\
18000.00 \\
23000.00 \\
24000.00 \\
23000.00 \\
26000.00 \\
32000.00 \\
30000.00 \\
32000.00 \\
28000.00 \\
28000.00 \\
24000.00 \\
25000.00 \\
32000.00 \\
30000.00 \\
30000.00 \\
227000.00\end{array}$ \\
\hline
\end{tabular}

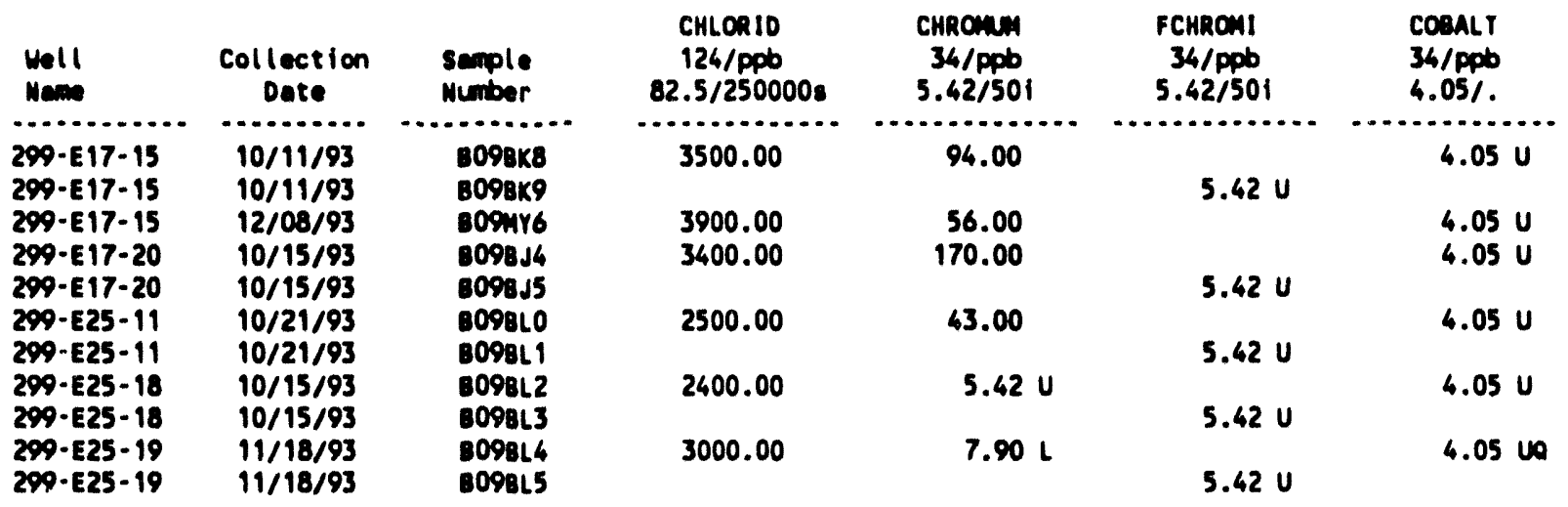


Table 7-4. Constituents with at Least One Detected Value for the 216-A-29 Ditch Data for Reporting Perlod October 1 through December 31, 1993. (sheet 5 of 14)

\begin{tabular}{|c|c|c|c|c|c|c|}
\hline $\begin{array}{l}\text { Well } \\
\text { Meane }\end{array}$ & $\begin{array}{c}\text { Collection } \\
\text { Dete }\end{array}$ & $\begin{array}{l}\text { Semple } \\
\text { Mutber }\end{array}$ & $\begin{array}{c}\text { CHLORIO } \\
124 / \mathrm{ppD} \\
82.5 / 250000 \mathrm{~s}\end{array}$ & $\begin{array}{c}\text { CHRomin } \\
34 / \mathrm{ppb} \\
5.42 / 501\end{array}$ & $\begin{array}{l}\text { FCHROA1 } \\
34 / p p b \\
5.42 / 501\end{array}$ & $\begin{array}{l}\text { cosalt } \\
34 / p \% b \\
4.05 / .\end{array}$ \\
\hline$\varphi \cdot E 25 \cdot 20$ & $10 / 13 / 93$ & cossus & 3100.00 & $5.42 \mathrm{U}$ & & $12.00 \mathrm{~L}$ \\
\hline $299-825-20$ & $10 / 13 / 93$ & cossu & 10000 & & $5.62 \mathrm{U}$ & \\
\hline $\begin{array}{l}299-E 25-21 \\
299-\varepsilon 25-21\end{array}$ & $\begin{array}{l}10 / 21 / 93 \\
10 / 21 / 93\end{array}$ & $\begin{array}{l}009266 \\
\operatorname{cosect7}\end{array}$ & 2500.00 & $5.42 \mathrm{U}$ & & $4.05 \mathrm{U}$ \\
\hline $299-\varepsilon 25-28$ & $10 / 29 / 93$ & coselo & 2300.00 & $5.42 \mathrm{U}$ & 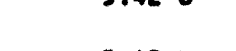 & $4.05 u$ \\
\hline $\begin{array}{l}299-E 25 \cdot 28 \\
299-E 25 \cdot 31\end{array}$ & $10 / 29 / 93$ & 109019 & & & $5.42 \mathrm{U}$ & \\
\hline $290-E 25-31$ & $\begin{array}{l}10 / 13 / 93 \\
10 / 13 / 93\end{array}$ & $\begin{array}{l}\cos 318 \\
\operatorname{cosevj9}\end{array}$ & 1800.00 & 95.00 & $5.42 \mathrm{U}$ & $4.05 \mathrm{U}$ \\
\hline $\begin{array}{l}299-\varepsilon 25-32 P \\
299-E 25-32 P\end{array}$ & $\begin{array}{l}10 / 13 / 93 \\
10 / 13 / 93\end{array}$ & $\begin{array}{l}\text { posaro } \\
\text { noserax } 1\end{array}$ & 1500.00 & 58.00 & & $14.00 \mathrm{~L}$ \\
\hline $\begin{array}{l}29-\varepsilon 25-34 \\
299-\varepsilon 25-34\end{array}$ & $\begin{array}{l}10 / 12 / 93 \\
10 / 12 / 93\end{array}$ & $\begin{array}{l}\text { 109eno } \\
\text { сояем1 }\end{array}$ & 2000.00 & 90.00 & $5.42 \mathrm{U}$ & $4.05 \mathrm{U}$ \\
\hline $\begin{array}{l}299-E 25 \cdot 35 \\
299-E 25 \cdot 35\end{array}$ & $\begin{array}{l}10 / 20 / 93 \\
10 / 20 / 93\end{array}$ & $\begin{array}{l}\text { noseph } \\
\text { noseps }\end{array}$ & 3400.00 & 81.00 & & $4.05 \mathrm{U}$ \\
\hline $\begin{array}{l}299-E 25-36 \\
299-E 25-36\end{array}$ & & $\begin{array}{l}\text { loperk2 } \\
\text { nopak3 }\end{array}$ & $\$ 100.00$ & 67.00 & $5.42 \mathrm{U}$ & $4.05 \mathrm{U}$ \\
\hline $\begin{array}{l}299-\varepsilon 25-42 \\
299-\varepsilon 25-42\end{array}$ & $\begin{array}{l}10 / 12 / 93 \\
10 / 12 / 93\end{array}$ & $\begin{array}{l}\text { losapg } \\
\text { sogeas }\end{array}$ & 4100.00 & 89.00 & $5.50 \mathrm{~L}$ & $4.05 \mathrm{U}$ \\
\hline $\begin{array}{l}299-525-43 \\
299-E 25-43\end{array}$ & $\begin{array}{l}10 / 20 / 93 \\
10 / 20 / 93\end{array}$ & $\begin{array}{l}\text { cosent2 } \\
\text { cosant3 }\end{array}$ & $\begin{array}{l}2200.00 \\
2200.00\end{array}$ & $\begin{array}{l}86.00 \\
83.00\end{array}$ & & $\begin{array}{l}4.05 U \\
4.05 U\end{array}$ \\
\hline $\begin{array}{l}299-E 25-43 \\
299-E 25-43\end{array}$ & $\begin{array}{l}10 / 20 / 93 \\
10 / 20 / 93\end{array}$ & $\begin{array}{l}\text { loseno } \\
\text { nosen1 }\end{array}$ & & & $\begin{array}{l}5.42 \mathrm{U} \\
5.42 \mathrm{U}\end{array}$ & \\
\hline $\begin{array}{l}290 \cdot E 25 \cdot-67 \\
299-E 25 \cdot-67\end{array}$ & $\begin{array}{l}10 / 16 / 93 \\
10 / 16 / 93\end{array}$ & $\begin{array}{l}\text { Bosed4 } \\
\text { coseas }\end{array}$ & 2700.00 & 36.00 & $5.42 \mathrm{U}$ & $.05 \mathrm{U}$ \\
\hline $\begin{array}{l}299-E 25-48 \\
299-E 25-40\end{array}$ & $\begin{array}{l}10 / 15 / 93 \\
10 / 15 / 93\end{array}$ & $\begin{array}{l}809909 \\
\text { co9sR3 }\end{array}$ & 3300.00 & 63.00 & $5.42 \mathrm{U}$ & $.05 \mathrm{U}$ \\
\hline $\begin{array}{l}299-\varepsilon 26-12 \\
299-\varepsilon 26-12\end{array}$ & $\begin{array}{l}10 / 12 / 93 \\
10 / 12 / 93\end{array}$ & $\begin{array}{l}\text { 8093N2 } \\
\text { LO9an6 }\end{array}$ & 1700.00 & 85.00 & & $4.05 \mathrm{U}$ \\
\hline $\begin{array}{l}\text { P. E26-13 } \\
29-E 26-13\end{array}$ & $\begin{array}{l}10 / 12 / 93 \\
10 / 12 / 93\end{array}$ & $\begin{array}{l}\text { logen } 7 \\
\text { cosep1 }\end{array}$ & 1800.00 & 66.00 & $5.42 \mathrm{U}$ & $4.05 U$ \\
\hline $\begin{array}{l}699-43-43 \\
699-43-63\end{array}$ & $\begin{array}{l}10 / 13 / 93 \\
10 / 13 / 93\end{array}$ & $\begin{array}{l}\text { no9ek6 } \\
\text { so9kK5 }\end{array}$ & 1800.00 & 32.00 & $5.42 \mathrm{U}$ & $4.05 \mathrm{U}$ \\
\hline $\begin{array}{l}9-43 \cdot 45 \\
9-63-45\end{array}$ & $\begin{array}{l}10 / 13 / 93 \\
10 / 13 / 93\end{array}$ & $\begin{array}{l}\text { 109ax6 } \\
\text { co9ax7 }\end{array}$ & 2000.00 & 25.00 & $5.50 \mathrm{~L}$ & $4.05 \mathrm{U}$ \\
\hline
\end{tabular}

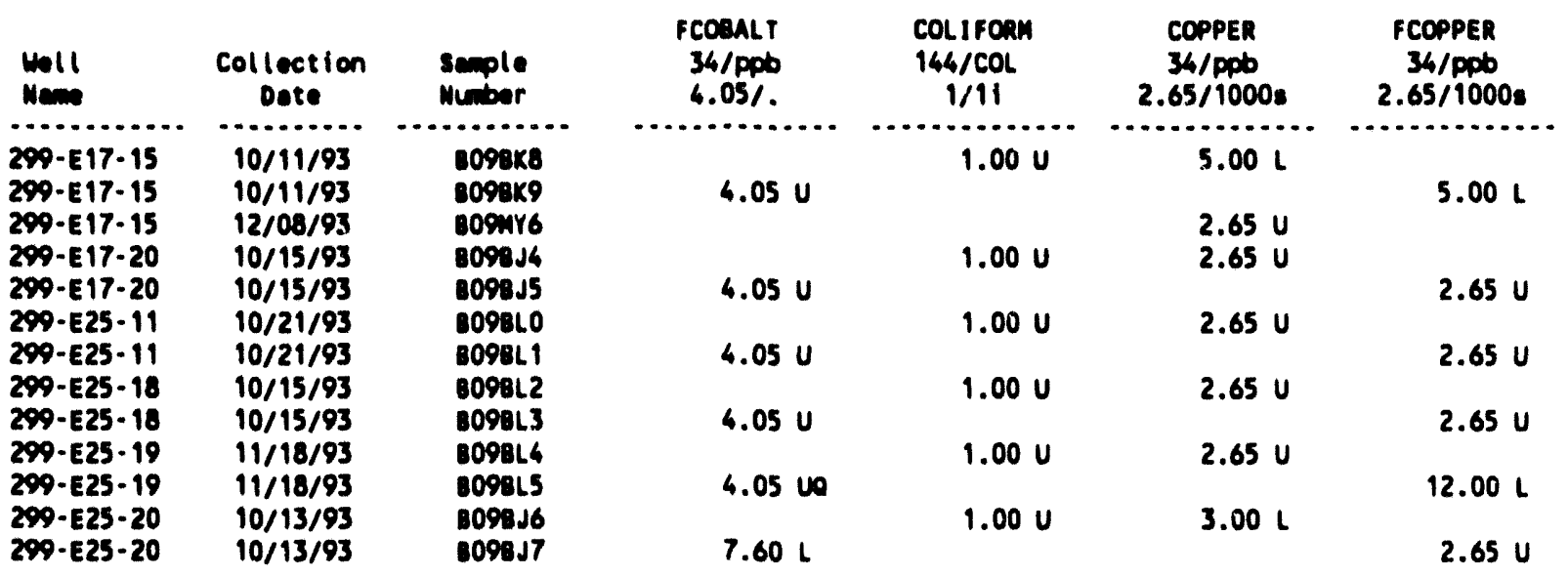


Table 7-4. Constituents with at Least One Detected Value for the 216-A-29 Ditch Data for Reporting Period October 1 through December 31, 1993. (sheet 6 of 14)

\begin{tabular}{|c|c|c|c|c|c|c|}
\hline $\begin{array}{l}\text { Mell } \\
\text { Nemen }\end{array}$ & $\begin{array}{c}\text { Collection } \\
\text { Dete }\end{array}$ & $\begin{array}{l}\text { Semple } \\
\text { Number }\end{array}$ & $\begin{array}{l}\text { FCOBALT } \\
34 / \text { ppo } \\
4.05 /\end{array}$ & $\begin{array}{l}\text { COL IFOAM } \\
144 / \mathrm{COL} \\
1 / 11\end{array}$ & $\begin{array}{c}\text { COPPER } \\
34 / \text { ppb } \\
2.65 / 1000\end{array}$ & $\begin{array}{c}\text { FCOPPER } \\
34 / \text { Ppb } \\
2.65 / 1000 \mathrm{~s}\end{array}$ \\
\hline 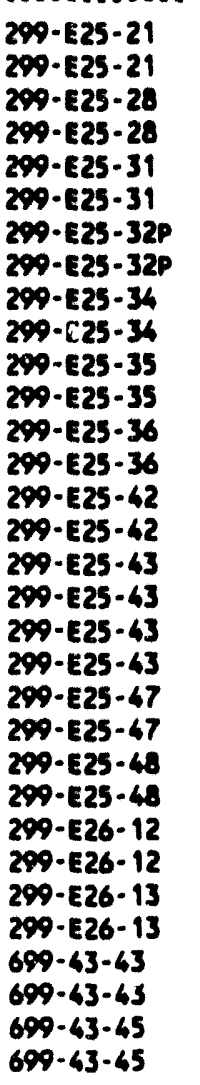 & $\begin{array}{l}10 / 21 / 93 \\
10 / 21 / 93 \\
10 / 29 / 93 \\
10 / 29 / 93 \\
10 / 13 / 93 \\
10 / 13 / 93 \\
10 / 13 / 93 \\
10 / 13 / 93 \\
10 / 12 / 93 \\
10 / 12 / 93 \\
10 / 20 / 93 \\
10 / 20 / 93 \\
10 / 15 / 93 \\
10 / 15 / 93 \\
10 / 12 / 93 \\
10 / 12 / 93 \\
10 / 20 / 93 \\
10 / 20 / 93 \\
10 / 20 / 93 \\
10 / 20 / 93 \\
10 / 14 / 93 \\
10 / 16 / 93 \\
10 / 15 / 93 \\
10 / 15 / 93 \\
10 / 12 / 93 \\
10 / 12 / 93 \\
10 / 12 / 93 \\
10 / 12 / 93 \\
10 / 13 / 93 \\
10 / 13 / 93 \\
10 / 13 / 93 \\
10 / 13 / 93\end{array}$ & 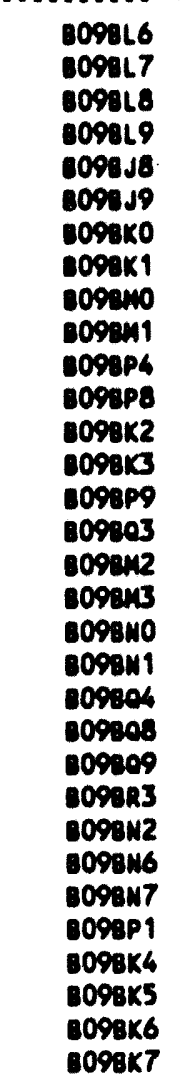 & $\begin{array}{l}4.05 U \\
4.05 U \\
5.60 \mathrm{l} \\
6.10 \mathrm{l} \\
4.05 U \\
4.05 U \\
4.05 U \\
4.05 U \\
4.05 U \\
4.05 U \\
4.05 U \\
4.05 U \\
4.05 U \\
4.05 U \\
6.10 \mathrm{4} \\
4.05 U\end{array}$ & $\begin{array}{l}1.00 \mathrm{U} \\
1.00 \mathrm{U} \\
1.00 \mathrm{U} \\
1.00 \mathrm{U} \\
1.00 \mathrm{U} \\
1.00 \mathrm{U} \\
1.00 \mathrm{U} \\
1.00 \mathrm{UH} \\
1.00 \mathrm{U} \\
1.00 \\
1.00 \mathrm{U} \\
1.00 \mathrm{U} \\
1.00 \mathrm{U} \\
1.00 \mathrm{U} \\
1.00 \mathrm{U} \\
1.00 \mathrm{U}\end{array}$ & $\begin{array}{l}2.65 \mathrm{U} \\
3.50 \mathrm{~L} \\
4.00 \mathrm{~L} \\
3.00 \mathrm{~L} \\
4.90 \mathrm{~L} \\
5.90 \mathrm{~L} \\
2.65 \mathrm{~V} \\
5.90 \mathrm{~L} \\
7.20 \mathrm{~L} \\
7.20 \mathrm{~L} \\
2.65 \mathrm{~V} \\
2.65 \mathrm{~V} \\
4.00 \mathrm{~L} \\
6.90 \mathrm{~L} \\
4.00 \mathrm{~L} \\
4.00 \mathrm{~L}\end{array}$ & $\begin{array}{l}2.65 U \\
4.701 \\
2.65 U \\
2.65 U \\
2.65 U \\
2.65 U \\
2.65 U \\
2.65 U\end{array}$ \\
\hline
\end{tabular}

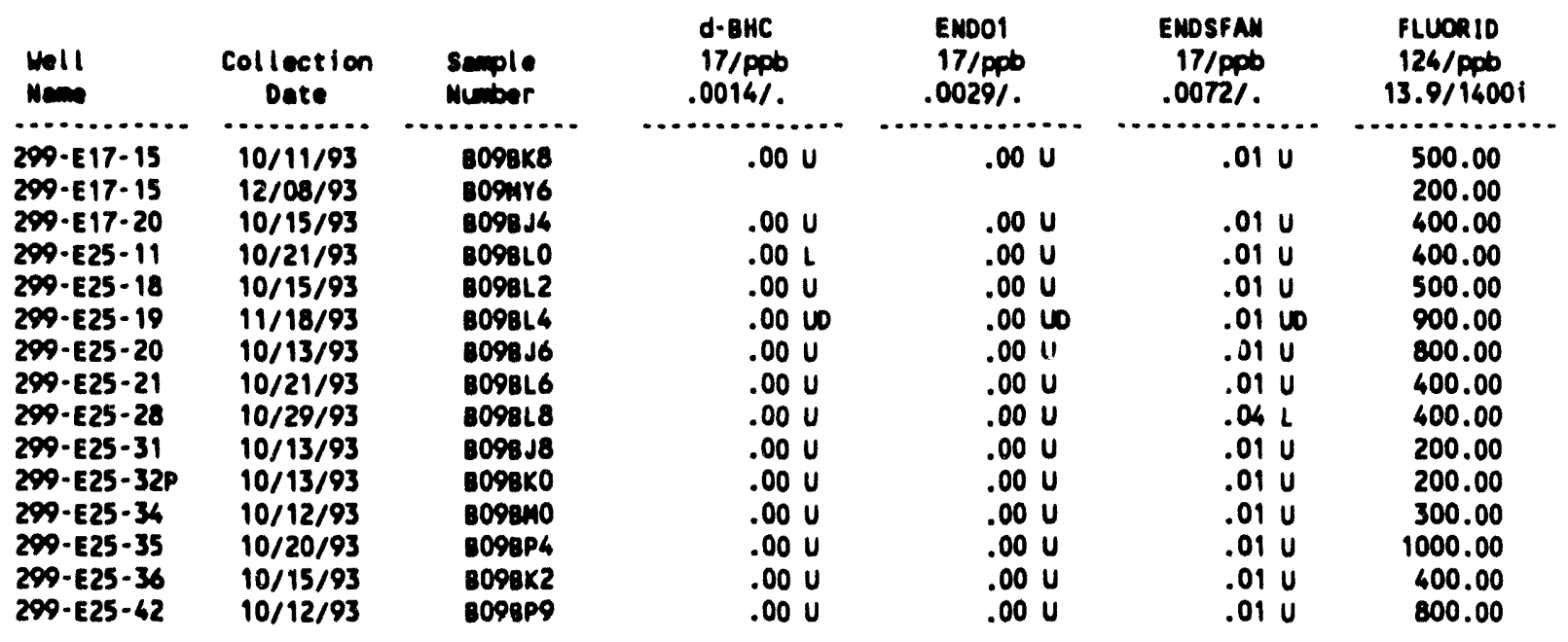


Table 7-4. Constituents with at Least One Detected Value for the 216-A-29 Ditch Data for Reporting Period October 1 through December 31, 1993. (sheet 7 of 14)

\begin{tabular}{|c|c|c|c|c|c|c|}
\hline $\begin{array}{l}\text { Well } \\
\text { Name }\end{array}$ & $\begin{array}{c}\text { Collection } \\
\text { Date }\end{array}$ & $\begin{array}{l}\text { Sample } \\
\text { Number }\end{array}$ & $\begin{array}{l}\text { d-BHC } \\
17 / \text { ppb } \\
.0014 / .\end{array}$ & $\begin{array}{l}\text { ENDO1 } \\
17 / \text { ppb } \\
.0029 / .\end{array}$ & $\begin{array}{c}\text { ENDSFAN } \\
17 / \text { ppb } \\
.0072 / .\end{array}$ & $\begin{array}{l}\text { FLUORID } \\
124 / \mathrm{ppb} \\
13.9 / 1400 \mathrm{i}\end{array}$ \\
\hline $\begin{array}{l}299-E 25-43 \\
299-E 25-43 \\
299-E 25-47 \\
299-E 25-48 \\
299-E 26-12 \\
299-E 26-13 \\
699-43-43 \\
699-43-45\end{array}$ & $\begin{array}{l}10 / 20 / 93 \\
10 / 20 / 93 \\
10 / 14 / 93 \\
10 / 15 / 93 \\
10 / 12 / 93 \\
10 / 12 / 93 \\
10 / 13 / 93 \\
10 / 13 / 93\end{array}$ & $\begin{array}{l}\text { B09BN2 } \\
\text { B098N3 } \\
\text { B09804 } \\
\text { 809Ba9 } \\
\text { 8098N2 } \\
\text { B09BN7 } \\
\text { B09BK4 } \\
\text { B09BK6 }\end{array}$ & $\begin{array}{l}.00 \mathrm{UQ} \\
.00 \mathrm{LQ} \\
.00 \mathrm{U} \\
.00 \mathrm{U} \\
.00 \mathrm{U} \\
.00 \mathrm{U} \\
.00 \mathrm{U} \\
.00 \mathrm{U}\end{array}$ & $\begin{array}{l}.00 \mathrm{U} \\
.00 \mathrm{LQ} \\
.00 \mathrm{U} \\
.00 \mathrm{U} \\
.00 \mathrm{U} \\
.00 \mathrm{U} \\
.00 \mathrm{U} \\
.00 \mathrm{U}\end{array}$ & $\begin{array}{l}.01 \mathrm{U} \\
.01 \mathrm{U} \\
.01 \mathrm{U} \\
.01 \mathrm{U} \\
.01 \mathrm{U} \\
.01 \mathrm{U} \\
.01 \mathrm{U} \\
.01 \mathrm{U}\end{array}$ & $\begin{array}{l}700.00 \\
700.00 \\
700.00 \\
400.00 \\
300.00 \\
400.00 \\
300.00 \\
400.00\end{array}$ \\
\hline
\end{tabular}

\begin{tabular}{|c|c|c|c|c|c|c|}
\hline $\begin{array}{l}\text { Hell } \\
\text { Nane }\end{array}$ & $\begin{array}{c}\text { Collection } \\
\text { Dete }\end{array}$ & $\begin{array}{l}\text { Semple } \\
\text { Number }\end{array}$ & $\begin{array}{c}\text { ALPHA } \\
135 / \mathrm{PCi} / \mathrm{L} \\
. / 15\end{array}$ & $\begin{array}{c}\text { BETA } \\
\text { 136/pCi/L } \\
. / .\end{array}$ & $\begin{array}{c}\text { HEPTLOR } \\
17 / \mathrm{ppb} \\
.0019 / .4\end{array}$ & $\begin{array}{c}\text { IRON } \\
\text { 34/ppb } \\
10.3 / 3008\end{array}$ \\
\hline $\begin{array}{l}299-E 17-15 \\
299-E 17-15 \\
299-E 17-20 \\
299-E 25-11 \\
299-E 25-18 \\
299-E 25-19 \\
299-E 25-20 \\
299-E 25-21 \\
299-E 25-28 \\
299-E 25-31 \\
299-E 25-32 P \\
299-E 25-34 \\
299-E 25-35 \\
299-E 25-36 \\
299-E 25-42 \\
299-E 25-43 \\
299-E 25-43 \\
299-E 25-47 \\
299-E 25-48 \\
299-E 26-12 \\
299-E 26-13 \\
699-43-43 \\
699-43-45\end{array}$ & $\begin{array}{l}10 / 11 / 93 \\
12 / 08 / 93 \\
10 / 15 / 93 \\
10 / 21 / 93 \\
10 / 15 / 93 \\
11 / 18 / 93 \\
10 / 13 / 93 \\
10 / 21 / 93 \\
10 / 29 / 93 \\
10 / 13 / 93 \\
10 / 13 / 93 \\
10 / 12 / 93 \\
10 / 20 / 93 \\
10 / 15 / 93 \\
10 / 12 / 93 \\
10 / 20 / 93 \\
10 / 20 / 93 \\
10 / 14 / 93 \\
10 / 15 / 93 \\
10 / 12 / 93 \\
10 / 12 / 93 \\
10 / 13 / 93 \\
10 / 13 / 93\end{array}$ & 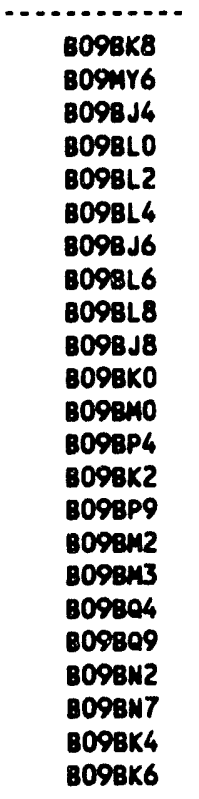 & $\begin{array}{l}4.44 \\
\\
5.26 \\
.37 \mathrm{U} \\
1.18 \mathrm{U} \\
.23 \mathrm{U} \\
.88 \mathrm{U} \\
.65 \mathrm{U} \\
. .03 \mathrm{U} \\
1.65 \\
2.23 \\
1.22 \\
1.13 \\
.79 \mathrm{U} \\
4.29 \\
1.50 \mathrm{U} \\
.98 \mathrm{U} \\
1.02 \mathrm{U} \\
2.02 \\
.93 \mathrm{U} \\
.92 \mathrm{U} \\
.74 \mathrm{U} \\
.95\end{array}$ & $\begin{array}{r}92.00 \\
33.80 \\
6.19 \\
6.87 \\
10.20 \\
10.50 \\
3.11 \\
1.07 u \\
5.98 \\
5.25 \\
3.50 \\
2.43 \\
8.39 \\
8.33 \\
3.600 \\
2.510 \\
9.14 \\
7.84 \\
5.25 \\
6.41 \\
11.70 \\
7.10\end{array}$ & $\begin{array}{l}.00 \mathrm{U} \\
.00 \mathrm{U} \\
.01 \mathrm{~L} \\
.00 \mathrm{U} \\
.00 \mathrm{U} \\
.00 \mathrm{U} \\
.00 \mathrm{U} \\
.00 \mathrm{U} \\
.00 \mathrm{U} \\
.00 \mathrm{U} \\
.00 \mathrm{U} \\
.00 \mathrm{U} \\
.00 \mathrm{U} \\
.00 \mathrm{U} \\
.00 \mathrm{U} \\
.01 \mathrm{~L} \\
.00 \mathrm{U} \\
.00 \mathrm{U} \\
.00 \mathrm{U} \\
.00 \mathrm{U} \\
.00 \mathrm{U} \\
.00 \mathrm{U}\end{array}$ & $\begin{array}{r}430.00 \\
270.00 \\
830.00 \\
440.00 \\
370.00 \\
310.00 \\
140.00 \\
220.00 \\
24.00 \\
1400.00 \\
310.00 \\
640.00 \\
530.00 \\
350.00 \\
480.00 \\
620.00 \\
550.00 \\
320.00 \\
1000.00 \\
390.00 \\
300.00 \\
160.00 \\
140.00\end{array}$ \\
\hline $\begin{array}{l}\text { Well } \\
\text { Mame }\end{array}$ & $\begin{array}{c}\text { Collection } \\
\text { Date }\end{array}$ & $\begin{array}{l}\text { Semple } \\
\text { Number }\end{array}$ & $\begin{array}{c}\text { FIROM } \\
\text { 34/ppb } \\
10.3 / 300 \mathrm{~s}\end{array}$ & $\begin{array}{l}\text { LEND } \\
\text { 40/ppb } \\
.508 / 50 i\end{array}$ & $\begin{array}{c}\text { MAGNES } \\
34 / \text { ppb } \\
26 / .\end{array}$ & $\begin{array}{c}\text { FMMGMES } \\
34 / P p b \\
26 / .\end{array}$ \\
\hline $\begin{array}{l}299-E 17-15 \\
299-E 17-15 \\
299-E 17-15 \\
299-E 17-20 \\
299-E 17-20 \\
299-E 25-11 \\
299-E 25-11 \\
299-E 25-18 \\
299-E 25-18\end{array}$ & $\begin{array}{l}10 / 11 / 93 \\
10 / 11 / 93 \\
12 / 08 / 93 \\
10 / 15 / 93 \\
10 / 15 / 93 \\
10 / 21 / 93 \\
10 / 21 / 93 \\
10 / 15 / 93 \\
10 / 15 / 93\end{array}$ & $\begin{array}{l}\text { B09BK8 } \\
\text { 8098KS } \\
\text { B09MY6 } \\
\text { 809BJL } \\
\text { B09BJ5 } \\
\text { B09BLO } \\
\text { BO9BL1 } \\
\text { B09BL2 } \\
\text { BO9BL3 }\end{array}$ & $\begin{array}{l}27.00 \\
36.00 \\
10.30 \mathrm{U}\end{array}$ & $\begin{array}{c}3.20 \mathrm{LB} \\
.51 \mathrm{U} \\
.64 \mathrm{LB}\end{array}$ & $\begin{array}{l}13000.00 \\
13000.00 \\
17000.00 \\
5700.00 \\
7500.00\end{array}$ & $\begin{array}{r}16000.00 \\
5600.00 \\
7600.00\end{array}$ \\
\hline
\end{tabular}


Table 7-4. Constituents with at Least One Detected Value for the 216-A-29 Ditch Data for Reporting Period October 1 through December 31, 1993. (sheet 8 of 14)



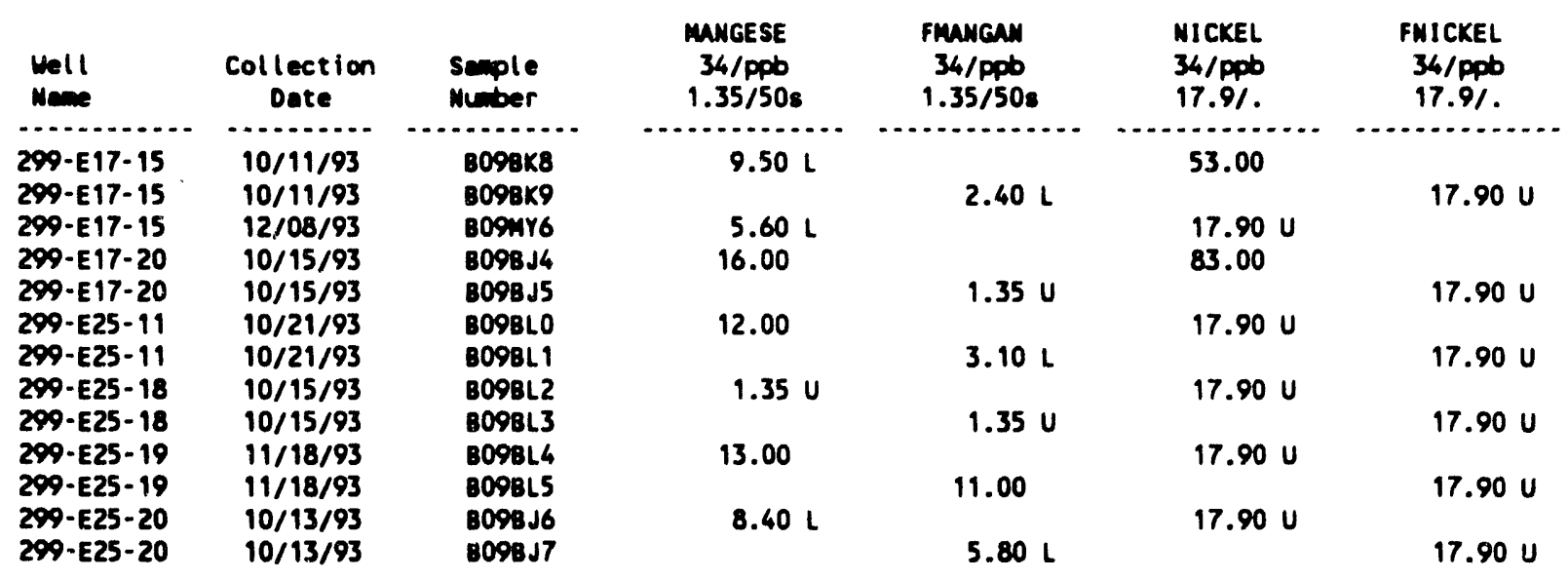


Table 7-4. Constituents with at Least One Detected Value for the 216-A-29 Ditch Data for Reporting Period October 1 through December 31, 1993. (sheet 9 of 14)

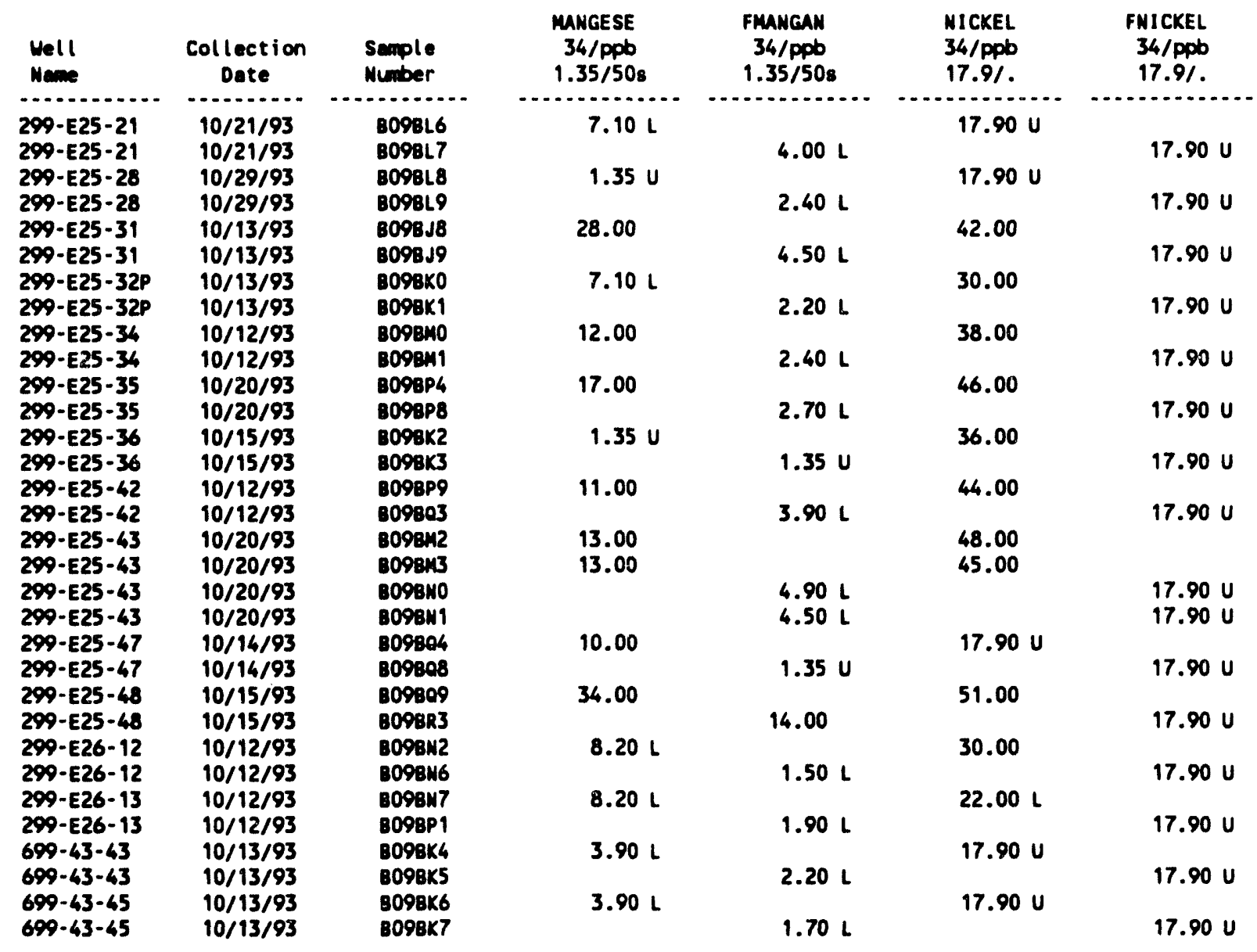

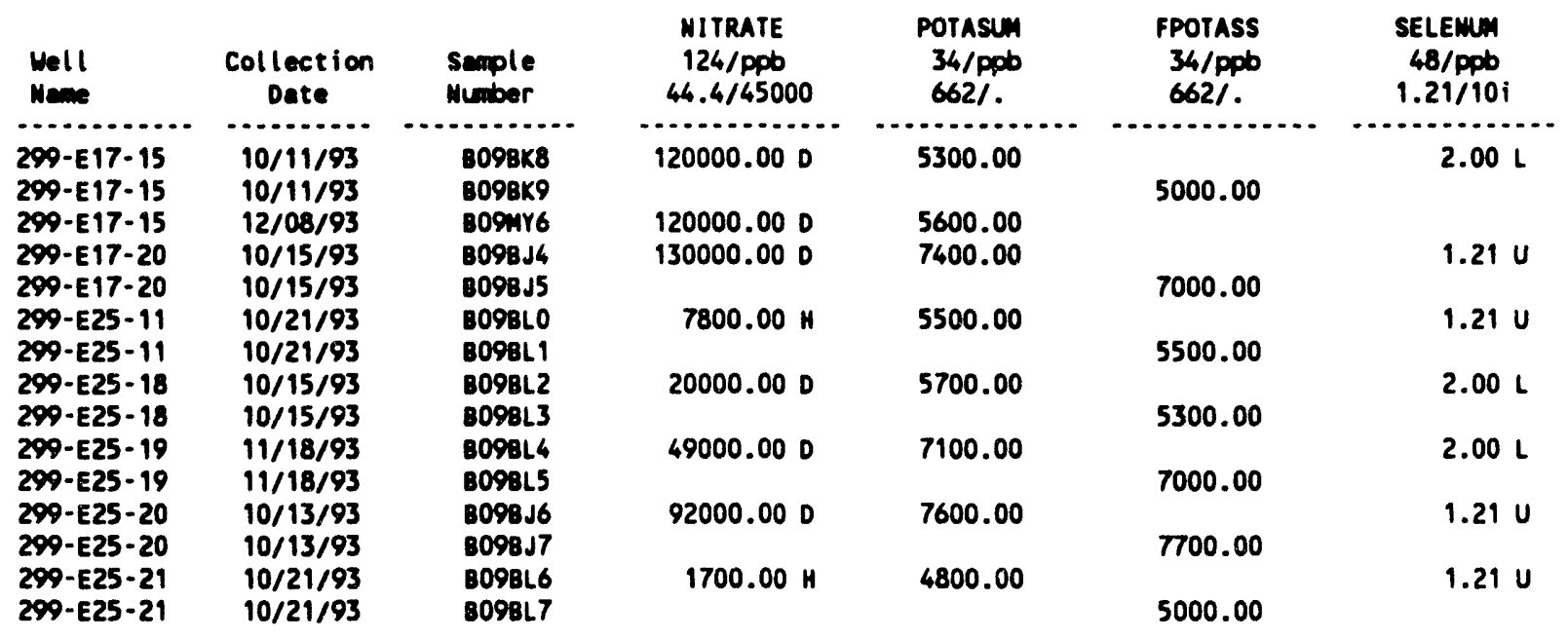


Table 7-4. Constituents with at Least One Detected Value for the 216-A-29 Ditch Data for Reporting Period October 1 through December 31, 1993. (sheet 10 of 14)

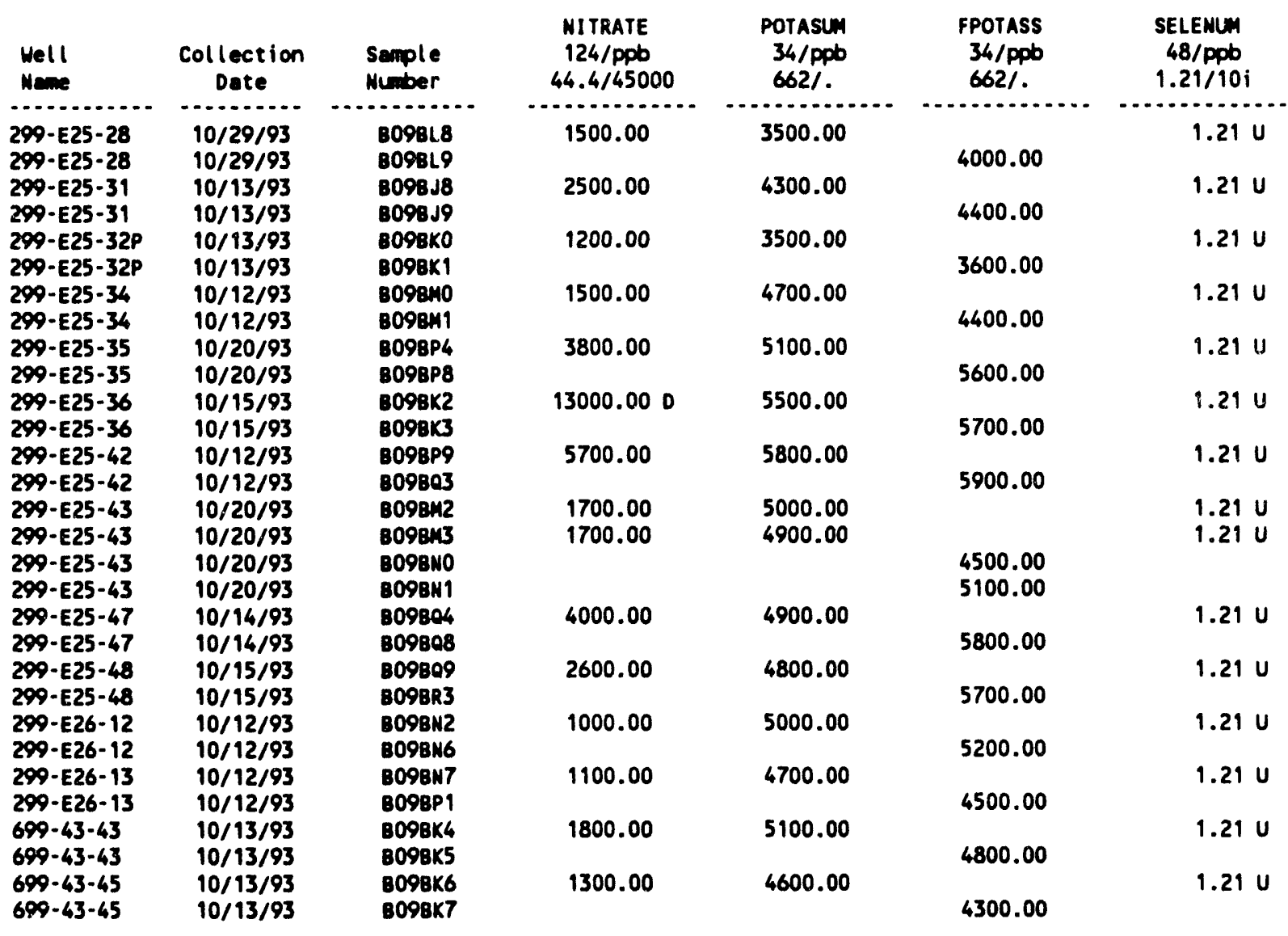

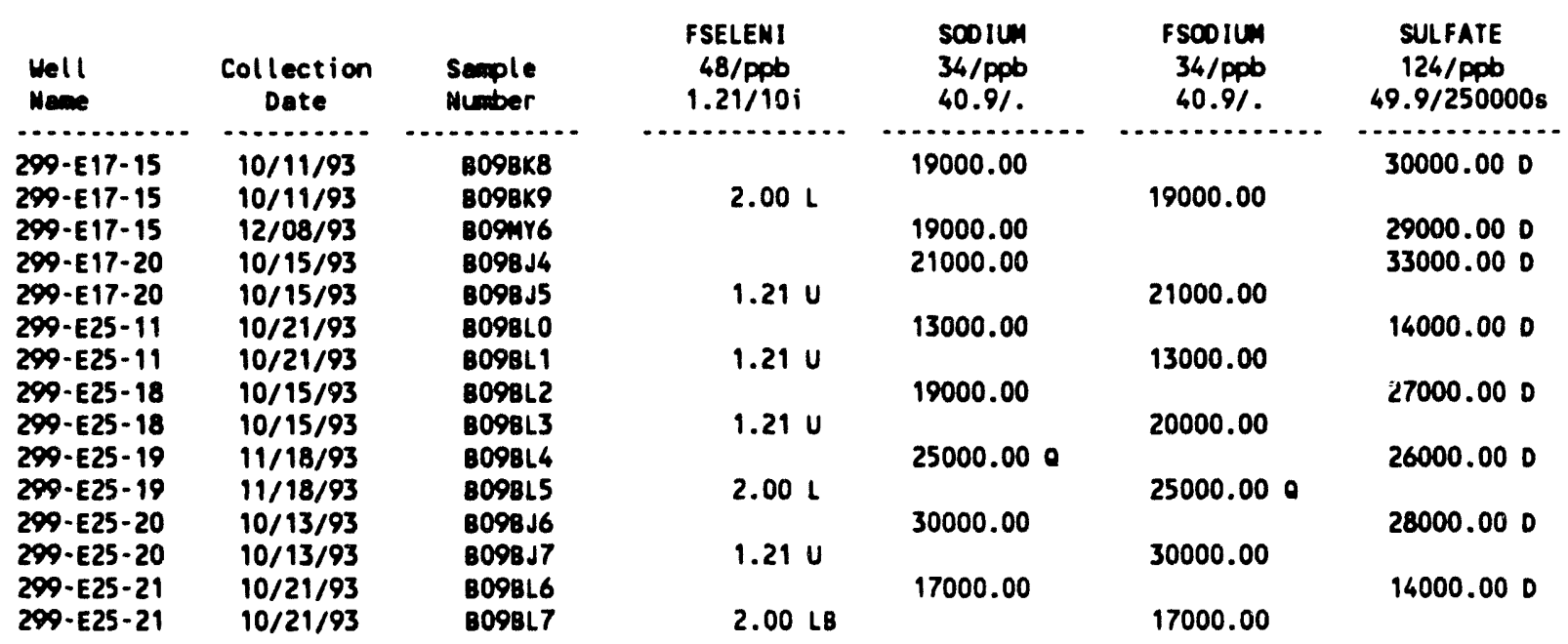


Table 7-4. Constituents with at Least One Detected Value for the 216-A-29 Ditch Data for Reporting Period October 1 through December 31, 1993. (sheet 11 of 14)

\begin{tabular}{|c|c|c|c|c|c|c|}
\hline $\begin{array}{l}\text { Well } \\
\text { Neme }\end{array}$ & $\begin{array}{c}\text { Collection } \\
\text { Date }\end{array}$ & $\begin{array}{l}\text { Semple } \\
\text { Number }\end{array}$ & $\begin{array}{c}\text { FSELENI } \\
48 / \mathrm{ppb} \\
1.21 / 10 \mathrm{i}\end{array}$ & $\begin{array}{l}\text { scolum } \\
34 / \mathrm{ppb} \\
40.9 / .\end{array}$ & $\begin{array}{c}\text { Fsoolun } \\
\text { 34/ppb } \\
40.91 .\end{array}$ & $\begin{array}{c}\text { SULFATE } \\
124 / \mathrm{ppb} \\
49.9 / 250000 \mathrm{~s}\end{array}$ \\
\hline $\begin{array}{l}299-E 25-28 \\
299-E 25-28 \\
299-E 25-31 \\
299-E 25-31 \\
299-E 25-32 P \\
299-E 25-32 P \\
299-E 25-34 \\
299-E 25-34 \\
299-E 25-35 \\
299-E 25-35 \\
299-E 25-36 \\
299-E 25-36 \\
299-E 25-42 \\
299-E 25-42 \\
299-E 25-43 \\
299-E 25-43 \\
299-E 25-43 \\
299-E 25-43 \\
299-E 25-47 \\
299-E 25-47 \\
299-E 25-48 \\
299-E 25-48 \\
299-E 26-12 \\
299-E 26-12 \\
299-E 26-13 \\
299-E 26-13 \\
699-43-43 \\
699-43-43 \\
699-43-45 \\
699-43-45\end{array}$ & $\begin{array}{l}10 / 29 / 93 \\
10 / 29 / 93 \\
10 / 13 / 93 \\
10 / 13 / 93 \\
10 / 13 / 93 \\
10 / 13 / 93 \\
10 / 12 / 93 \\
10 / 12 / 93 \\
10 / 20 / 93 \\
10 / 20 / 93 \\
10 / 15 / 93 \\
10 / 15 / 93 \\
10 / 12 / 93 \\
10 / 12 / 93 \\
10 / 20 / 93 \\
10 / 20 / 93 \\
10 / 20 / 93 \\
10 / 20 / 93 \\
10 / 14 / 93 \\
10 / 14 / 93 \\
10 / 15 / 93 \\
10 / 15 / 93 \\
10 / 12 / 93 \\
10 / 12 / 93 \\
10 / 12 / 93 \\
10 / 12 / 93 \\
10 / 13 / 93 \\
10 / 13 / 93 \\
10 / 13 / 93 \\
10 / 13 / 93\end{array}$ & 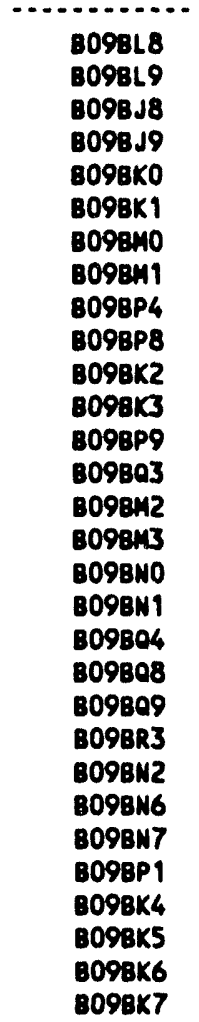 & $\begin{array}{l}1.21 U \\
1.21 U \\
1.21 U \\
1.21 U \\
1.21 U \\
1.21 U \\
1.21 U \\
1.21 U \\
1.21 U \\
1.21 U \\
1.21 U \\
1.21 U \\
1.21 U \\
1.21 U \\
1.21 U\end{array}$ & $\begin{array}{l}11000.00 \\
15000.00 \\
5700.00 \\
9500.00 \\
27000.00 \\
18000.00 \\
25000.00 \\
10000.00 \\
10000.00 \\
21000.00 \\
14000.00 \\
8700.00 \\
9200.00 \\
8300.00\end{array}$ & $\begin{array}{r}11000.00 \\
15000.00 \\
5700.00 \\
9600.00 \\
27000.00 \\
19000.00 \\
26000.00\end{array}$ & $\begin{array}{l}10000.00 \mathrm{D} \\
20000.00 \mathrm{D} \\
9800.00 \\
11000.00 \mathrm{D} \\
56000.00 \mathrm{D} \\
32000.00 \mathrm{D} \\
44000.00 \mathrm{D} \\
11000.00 \mathrm{D} \\
11000.00 \mathrm{D} \\
24000.00 \mathrm{D} \\
24000.00 \mathrm{D} \\
8200.00 \\
9300.00 \mathrm{D} \\
7700.00 \\
9900.00 \mathrm{D}\end{array}$ \\
\hline
\end{tabular}

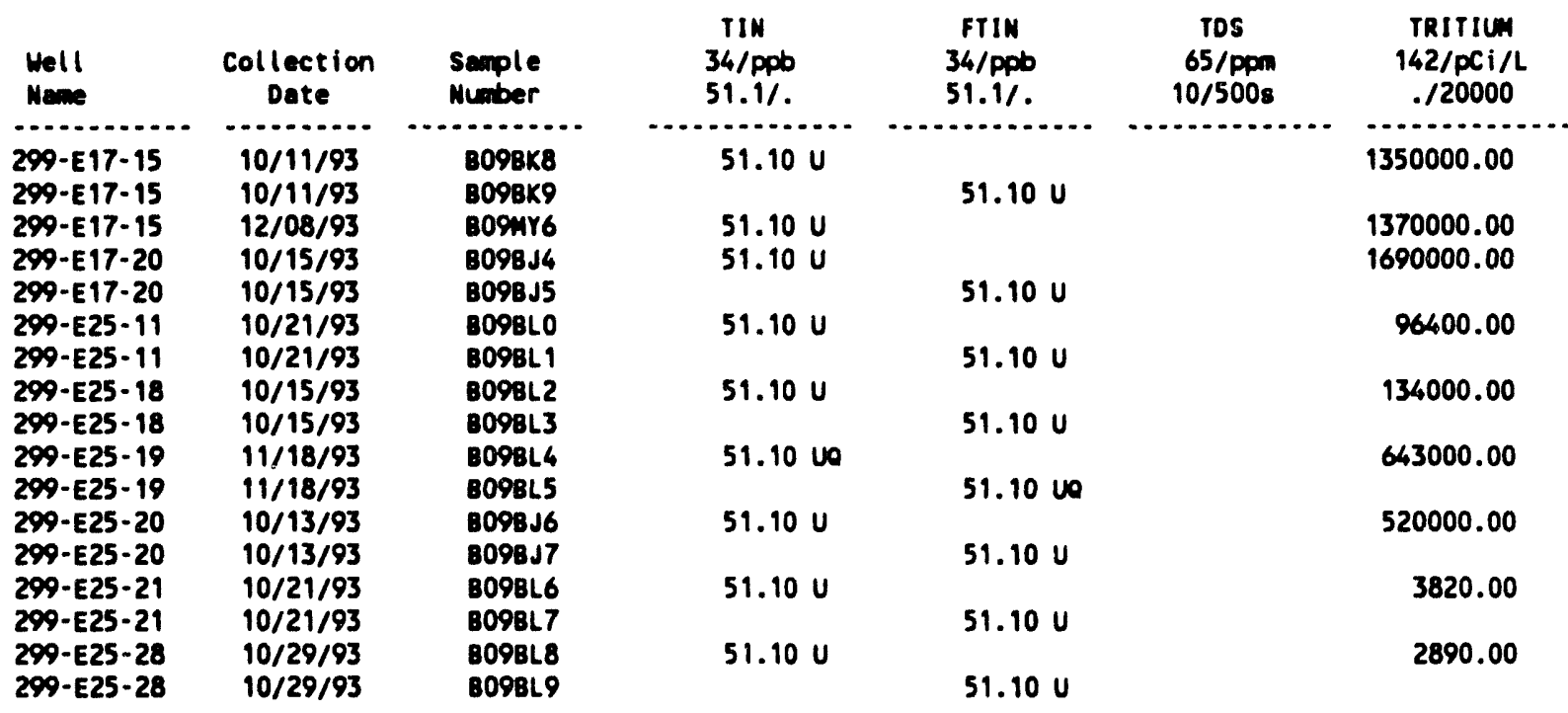


Table 7-4. Constituents with at Least One Detected Value for the 216-A-29 Ditch Data for Reporting Period October 1 through December 31, 1993. (sheet 12 of 14)

\begin{tabular}{|c|c|c|c|c|c|c|}
\hline $\begin{array}{l}\text { Mell } \\
\text { Name }\end{array}$ & $\begin{array}{c}\text { Collection } \\
\text { Date }\end{array}$ & $\begin{array}{l}\text { Semple } \\
\text { Number }\end{array}$ & $\begin{array}{l}T I N \\
34 / p p b \\
51.1 / .\end{array}$ & $\begin{array}{l}\text { FTIN } \\
34 / \text { ppb } \\
51.1 / .\end{array}$ & $\begin{array}{c}\text { TDS } \\
65 / \mathrm{ppm} \\
10 / 500 \mathrm{~s}\end{array}$ & $\begin{array}{c}\text { TRITIU } \\
\text { 142/PCi/L } \\
. / 20000\end{array}$ \\
\hline $\begin{array}{l}299-E 25-31 \\
299-E 25-31 \\
299-E 25-32 P \\
299-E 25-32 P \\
299-E 25-34 \\
299-E 25-34 \\
299-E 25-35 \\
299-E 25-35 \\
299-E 25-36 \\
299-E 25-36 \\
299-E 25-42 \\
299-E 25-42 \\
299-E 25-43 \\
299-E 25-43 \\
299-E 25-43 \\
299-E 25-43 \\
299-E 25-47 \\
299-E 25-47 \\
299-E 25-48 \\
299-E 25-48 \\
299-E 26-12 \\
299-E 26-12 \\
299-E 26-13 \\
299-E 26-13 \\
699-43-43 \\
699-43-43 \\
699-43-43 \\
699-43-45 \\
699-43-45 \\
699-43-45\end{array}$ & $\begin{array}{l}10 / 13 / 93 \\
10 / 13 / 93 \\
10 / 13 / 93 \\
10 / 13 / 93 \\
10 / 12 / 93 \\
10 / 12 / 93 \\
10 / 20 / 93 \\
10 / 20 / 93 \\
10 / 15 / 93 \\
10 / 15 / 93 \\
10 / 12 / 93 \\
10 / 12 / 93 \\
10 / 20 / 93 \\
10 / 20 / 93 \\
10 / 20 / 93 \\
10 / 20 / 93 \\
10 / 14 / 93 \\
10 / 14 / 93 \\
10 / 15 / 93 \\
10 / 15 / 93 \\
10 / 12 / 93 \\
10 / 12 / 93 \\
10 / 12 / 93 \\
10 / 12 / 93 \\
10 / 13 / 93 \\
10 / 13 / 93 \\
10 / 13 / 93 \\
10 / 13 / 93 \\
10 / 13 / 93 \\
10 / 13 / 93\end{array}$ & 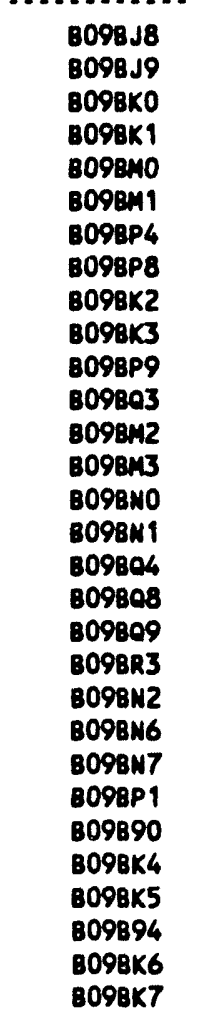 & $\begin{array}{l}51.10 \mathrm{U} \\
51.10 \mathrm{U} \\
51.10 \mathrm{U} \\
51.10 \mathrm{U} \\
51.10 \mathrm{U} \\
51.10 \mathrm{U} \\
51.10 \mathrm{U} \\
51.10 \mathrm{U} \\
51.10 \mathrm{U} \\
51.10 \mathrm{U} \\
56.00 \mathrm{~L} \\
51.10 \mathrm{U} \\
51.10 \mathrm{U} \\
51.10 \mathrm{U}\end{array}$ & $\begin{array}{l}51.10 \mathrm{U} \\
51.10 \mathrm{U} \\
51.10 \mathrm{U} \\
51.10 \mathrm{U} \\
51.10 \mathrm{U} \\
51.10 \mathrm{U} \\
51.10 \mathrm{U} \\
51.10 \mathrm{U} \\
51.10 \mathrm{U} \\
51.10 \mathrm{U} \\
51.10 \mathrm{U} \\
51.10 \mathrm{U} \\
51.10 \mathrm{U} \\
51.10 \mathrm{U}\end{array}$ & 150.00 & $\begin{array}{r}8520.00 \\
966.00 \\
1770.00 \\
29700.00 \\
7670.00 \\
25000.00 \\
1350.00 \\
1580.00 \\
14300.00 \\
8580.00 \\
511.00 \\
344.00\end{array}$ \\
\hline
\end{tabular}

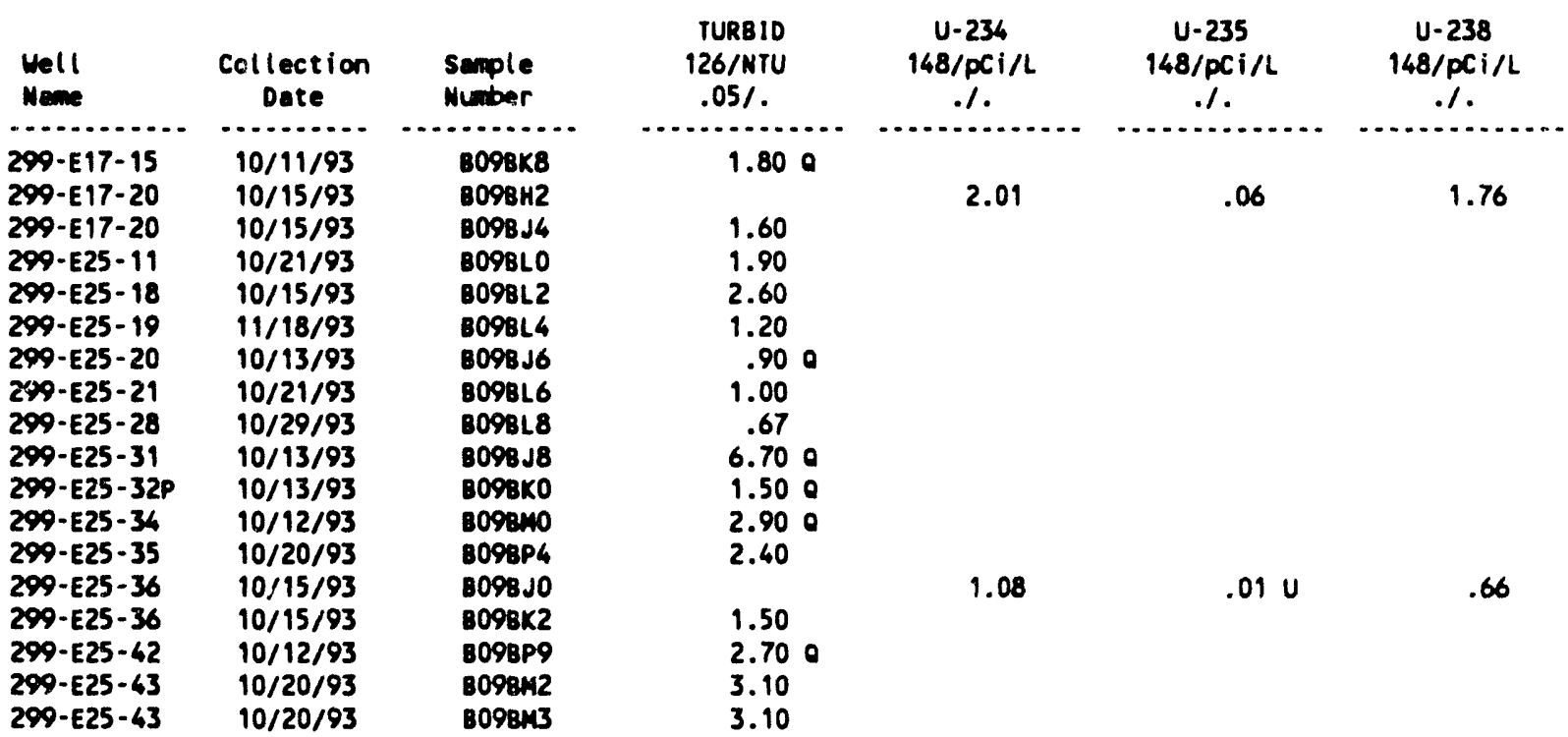


Table 7-4. Constituents with at Least One Detected Value for the 216-A-29 Ditch Data for Reporting Period October 1 through December 31, 1993. (sheet 13 of 14)

\begin{tabular}{|c|c|c|c|c|c|c|}
\hline $\begin{array}{l}\text { Well } \\
\text { Nene }\end{array}$ & $\begin{array}{c}\text { Collection } \\
\text { Dete }\end{array}$ & $\begin{array}{l}\text { Semple } \\
\text { Number }\end{array}$ & $\begin{array}{c}\text { TURBID } \\
\text { 126/NTU } \\
.05 / .\end{array}$ & $\begin{array}{c}U-234 \\
148 / \mathrm{pCi} / \mathrm{L} \\
. /\end{array}$ & $\begin{array}{c}\mathrm{U}-235 \\
148 / \mathrm{pCi} / \mathrm{L} \\
.1\end{array}$ & $\begin{array}{c}\text { U-238 } \\
148 / \mathrm{pCi} / \mathrm{L} \\
. /\end{array}$ \\
\hline $\begin{array}{l}299-E 25-47 \\
299-E 25-48 \\
299-E 26-12 \\
299-E 26-13 \\
699-43-43 \\
699-43-45\end{array}$ & $\begin{array}{l}10 / 14 / 93 \\
10 / 15 / 93 \\
10 / 12 / 93 \\
10 / 12 / 93 \\
10 / 13 / 93 \\
10 / 13 / 93\end{array}$ & $\begin{array}{l}\text { B09804 } \\
\text { 809809 } \\
\text { B098N2 } \\
\text { B098N7 } \\
\text { B098K4 } \\
\text { B098K6 }\end{array}$ & $\begin{array}{r}4.40 \\
12.00 \\
1.200 \\
1.600 \\
.700 \\
.900\end{array}$ & & & \\
\hline
\end{tabular}

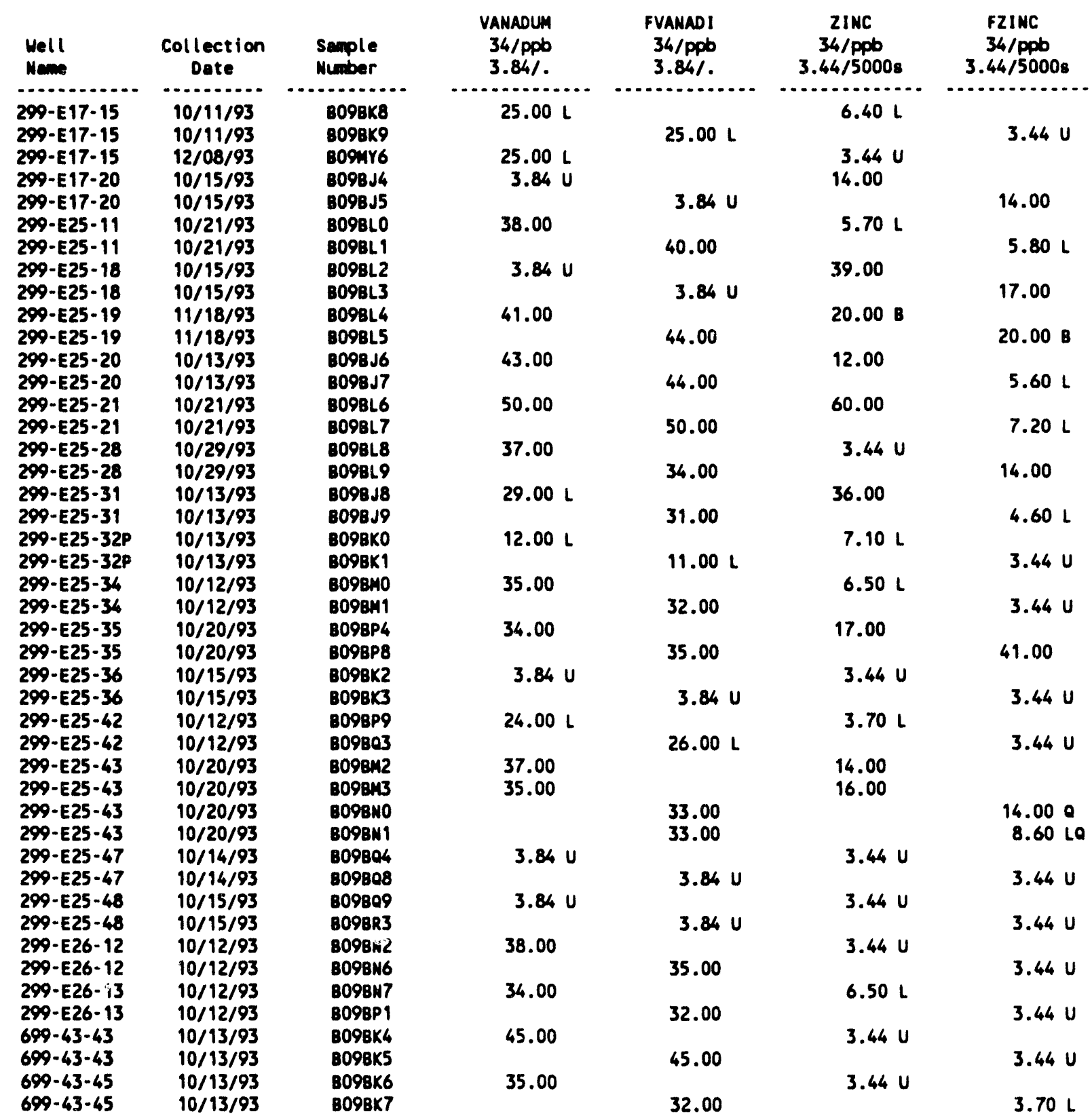


Table 7-4. Constituents with at Least One Detected Value for the 216-A-29 Ditch Data for Reporting Period October 1 through December 31, 1993. (sheet 14 of 14)

\begin{tabular}{|c|c|c|c|}
\hline $\begin{array}{l}\text { Heil } \\
\text { Neme }\end{array}$ & $\begin{array}{c}\text { Collection } \\
\text { Date }\end{array}$ & $\begin{array}{l}\text { Sample } \\
\text { Number }\end{array}$ & $\begin{array}{l}\text { g-8HC } \\
17 / p p b \\
.0021 / 4 i\end{array}$ \\
\hline $\begin{array}{l}299-E 17-15 \\
299-E 17-20 \\
299-E 25-11 \\
299-E 25-18 \\
299-E 25-19 \\
299-E 25-20 \\
299-E 25-21 \\
299-E 25-28 \\
299-E 25-31 \\
299-E 25-32 P \\
299-E 25-34 \\
299-E 25-35 \\
299-E 25-36 \\
299-E 25-42 \\
299-E 25-43 \\
299-E 25-43 \\
299-E 25-47 \\
299-E 25-48 \\
299-E 26-12 \\
299-E 26-13 \\
699-43-43 \\
699-43-45\end{array}$ & $\begin{array}{l}10 / 11 / 93 \\
10 / 15 / 93 \\
10 / 21 / 93 \\
10 / 15 / 93 \\
11 / 18 / 93 \\
10 / 13 / 93 \\
10 / 21 / 93 \\
10 / 29 / 93 \\
10 / 13 / 93 \\
10 / 13 / 93 \\
10 / 12 / 93 \\
10 / 20 / 93 \\
10 / 15 / 93 \\
10 / 12 / 93 \\
10 / 20 / 93 \\
10 / 20 / 93 \\
10 / 14 / 97 \\
10 / 15 / 9 \\
10 / 12 / 93 \\
10 / 12 / 93 \\
10 / 13 / 93 \\
10 / 13 / 93\end{array}$ & 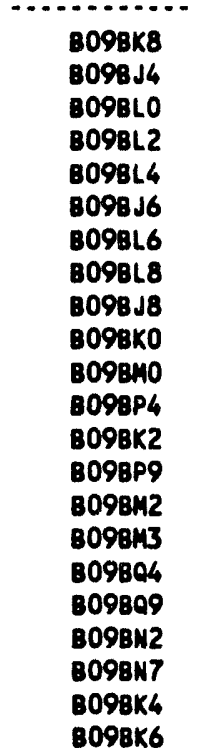 & $\begin{array}{l}.00 \mathrm{U} \\
.00 \mathrm{U} \\
.01 \mathrm{~L} \\
.00 \mathrm{U} \\
.00 \mathrm{U} \\
.00 \mathrm{U} \\
.00 \mathrm{U} \\
.00 \mathrm{U} \\
.00 \mathrm{U} \\
.00 \mathrm{U} \\
.00 \mathrm{U} \\
.00 \mathrm{U} \\
.00 \mathrm{U} \\
.00 \mathrm{U} \\
.00 \mathrm{U} \\
.01 \mathrm{LQ} \\
.00 \mathrm{U} \\
.00 \mathrm{U} \\
.00 \mathrm{U} \\
.00 \mathrm{U} \\
.00 \mathrm{U} \\
.00 \mathrm{U}\end{array}$ \\
\hline
\end{tabular}

For explenation of this table, see Section 1.4 of report. 
Table 7-5. Contamination Indicator Parameters for the 216-A-29 Ditch Data for Reporting Period October 1 through December 31, 1993. (sheet 1 of 2)

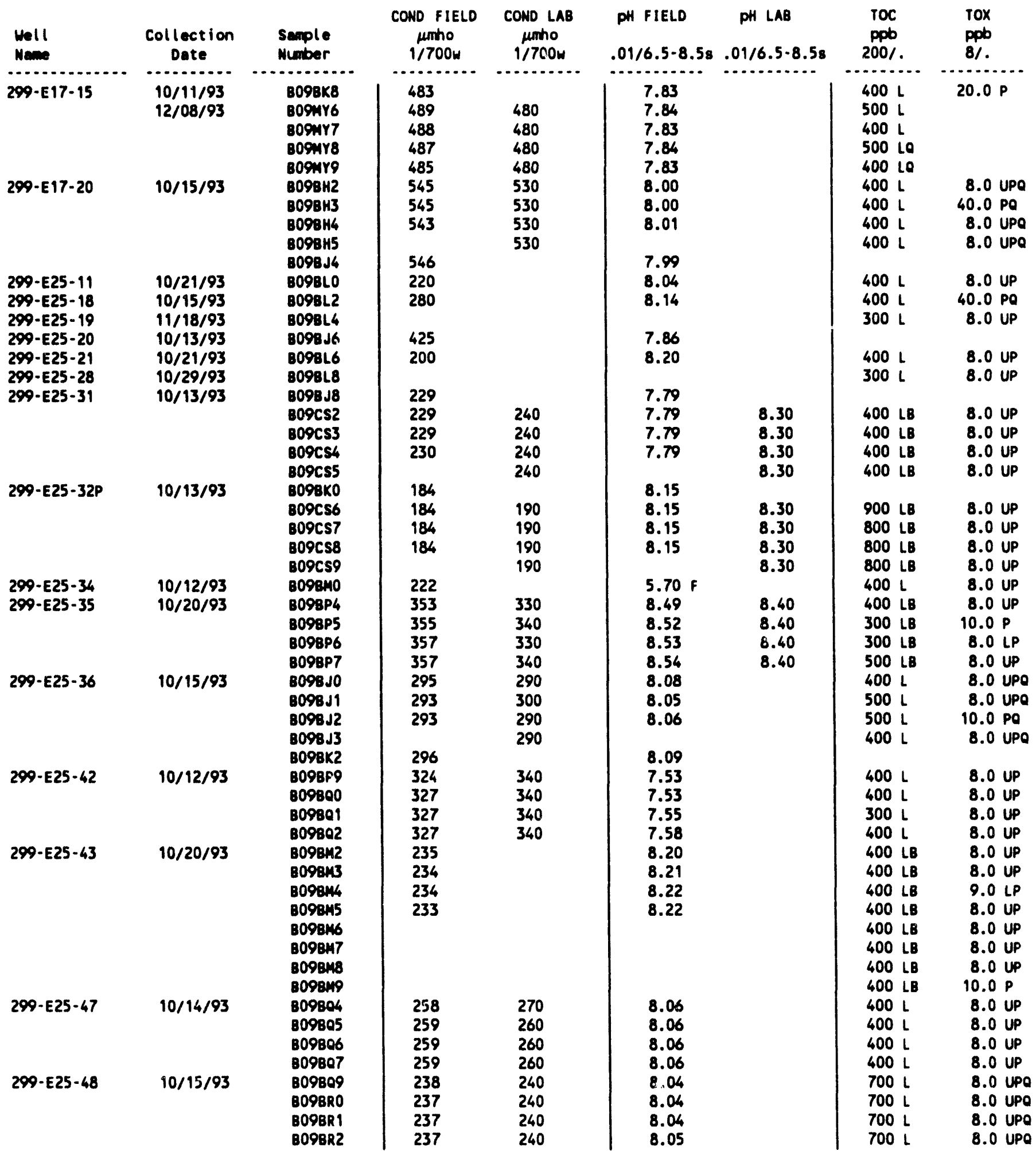


Table 7-5. Contamination Indicator Parameters for the 216-A-29 Ditch Data for Reporting Period October 1 through December 31, 1993. (sheet 2 of 2)

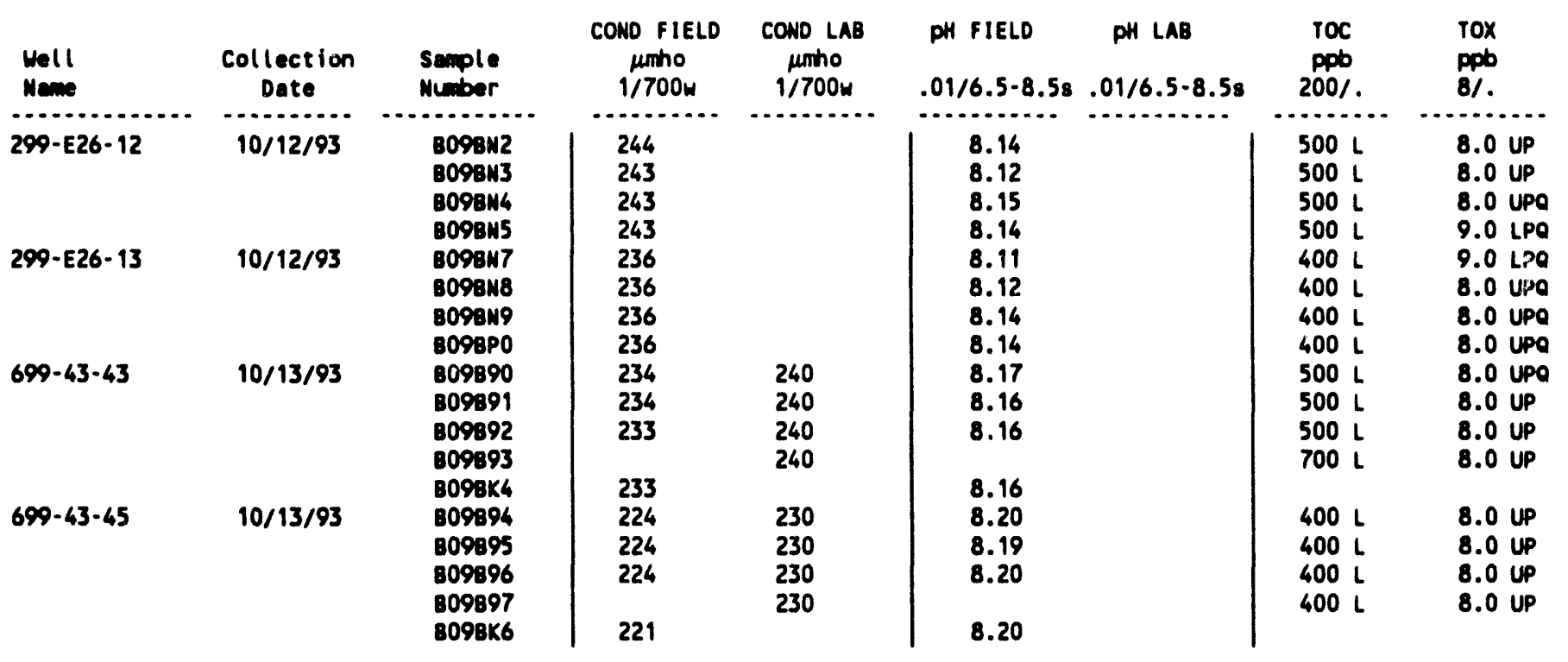

For explanation of this table, see Section 1.4 of report. 
DOE/RL-93-56-4

\section{CONTENTS}

$8.0216-A-36 B$ CRIB $\ldots \ldots \ldots . \ldots . \ldots . \ldots . \ldots 8-1$

8.1 INTRODUCTION ............... 8-1

8.2 WATER LEVEL MEASUREMENTS ............... 8-1

8.3 WATER CHEMISTRY DATA .............. 8-2 
DOE /RL-93-56-4

\section{LIST OF FIGURES}

8-1 Monitoring Well Locations for the 216-A-36B Crib . . . . . . . . 8-3

\section{LIST OF TABLES}

8-1 Monitoring Well Purpose and Sampling Schedule for the 216-A-36B Crib Network.................. . 8-4

8-2 RCRA Water Level Measurement Report for the 216-A-36B Crib,

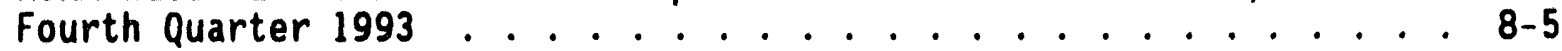

8-3 Constituent List and Summary of Results for the 216-A-36B Crib Data for Reporting Period October 1 through December 31, 1993 . . 8-6

8-4 Constituents with at Least One Detected Value for the 216-A-36B Crib Data for Reporting Period October 1 through December 31, 1993 ....................... 8-11

8-5 Contamination Indicator Parameters for the 216-A-36B Crib Data for Reporting Period October 1 through December 31, 1993 . . . 8-14 


\title{
8.0 216-A-36B CRIB
}

\author{
G. L. Kasza \\ Westinghouse Hanford Company
}

\subsection{INTRODUCTION}

The 216-A-36B Crib is a retired LWDF for the PUREX Plant. The crib is located in the 200 East Area approximately $365 \mathrm{~m}(1,200 \mathrm{ft})$ south of the PUREX Plant (Figures $1-1$ and $8-1$ ).

In accordance with the Tri-Party Agreement (Ecology et a1. 1992), Milestone M-20-34, RCRA Closure/Post Closure plans are to be submitted to Ecology and the EPA in March 1996. An interim-status RCRA groundwater monitoring network has been in place since May 1988.

The 216-A-36B Crib is the southernmost $152-m(500-\mathrm{ft})$ section of a crib originally known as the 216-A-36 Crib. Ammonia scrubber distillate from the PUREX Plant was disposed to the 216-A-36B Crib to percolate through the soll column. The original crib received liquid effluent from September 1965 to March 1966. Because a substantial inventory of radionuclides was disposed of to the crib, and assumed to have infiltrated sediments near the inlet of the crib, a grout curtain was emplaced to separate the crib into two sections: $A$ and B. Discharge to the 216-A-36B Crib resumed in March 1966 and continued until 1972 when the crib was temporarily removed from service. The 216-A-36B Crib was placed back in service in November 1982 and operated until it was retired from use in October 1987.

Ammonia scrubber distillate pumped into the 216-A-36B Crib consisted of a condensate from decladding operations in which zirconium cladding was removed from irradiated fuel by boiling in a solution of ammonium fluoride and ammonium nitrate. Some of the waste stream constituents included radionuclides (tritium, strontium-90, cesium-137, ruthenium-106, cobalt-60, and uranium) and hazardous substances (ammonium, fluoride, and nitrate).

The RCRA groundwater detection monitoring well network consists of six downgradient wells and one upgradient well, as listed in Table 8-1. All wells in the network meet RCRA construction standards except wells 299-E17-5 and 299-E17-9. These two older wells are constructed with perforated carbon steel casing. Well locations are shown in Figure 8-1. Background monitoring was performed for seven quarters. Indicator parameter critical means were calculated using four quarters of data from September 1988 to June 1989. Well 299-E17-15 is shared with the 216-A-29 Ditch facility groundwater monitoring network.

\subsection{WATER LEVEL MEASUREMENTS}

Water level measurements are made on a quarterly basis and during semiannual RCRA sample collection. Quarterly water level measurements collected d" "ing the October through December 1993 period are reported in 
Table 8-2. As noted in Table 8-2, water level measurements taken at the time of sampling were not avallable for inclusion in this report; they will be included in a future report.

\subsection{MATER CHEMISTRY DATA}

Wells in the 216-A-36B Crib groundwater monitoring network were sampled during this quarter as part of a semiannual sampling cycle. Well 299-E17-15 is shared $w$ th the 216-A-29 Ditch network and was sampled twice during the report pericd: (1) on October 11, 1993 for the groundwater quality assessment program (quarterly sampling schedule) for the 216-A-29 Ditch and (2) on December 8, 1993 during the semiannual sampling for the 216-A-36B Crib.

Table 8-3 gives the constituent 1 ist and a summary of the results. Table 8-4 provides the data for constituents with at least one detected value; Table 8-5 provides the results of indicator parameter analyses. All $D$ flags for nitrate and sulfate indicate that the samples were diluted for analysis. No indicator parameter replicate averages exceeded the calculated critical means values for the $216-A-36 B$ groundwater monitoring network.

The DWSs for unfiltered chromium were exceeded in samples from wells 299-E17-15 (two sampling events), 299-E17-16, and 299-E17-17 during the report period. The standards for unfiltered iron were exceeded in wells 299-E17-9, 299-E17-15, and 299-E17-17. The nitrate standard was exceeded in samples from wells 299-E17-9, 299-E17-14, 299-E17-15 (two samples), and 299-E17-16. Tritium levels exceeded the DWSs in all reported samples from the 216-A-36B groundwater monitoring network.

Most of the TOX along with several tritium and semivolatile organic analytical results were unavailable for inclusion in this report and will be presented in a future quarterly report.

An RADE was prepared to investigate the source of the erroneous specific conductance value reported for sample B09MZ7 from well 299-E17-17. 

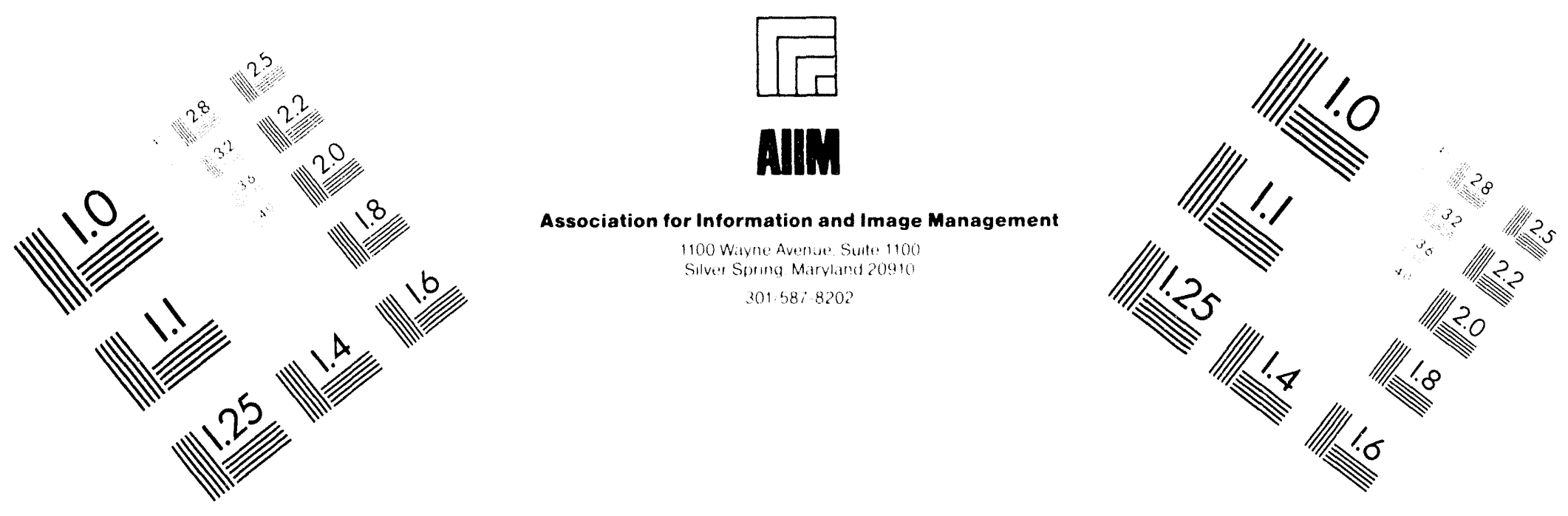

\section{Centimeter}

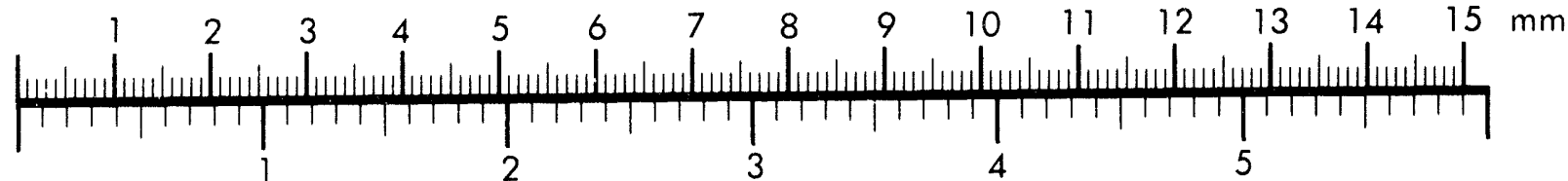

Inches
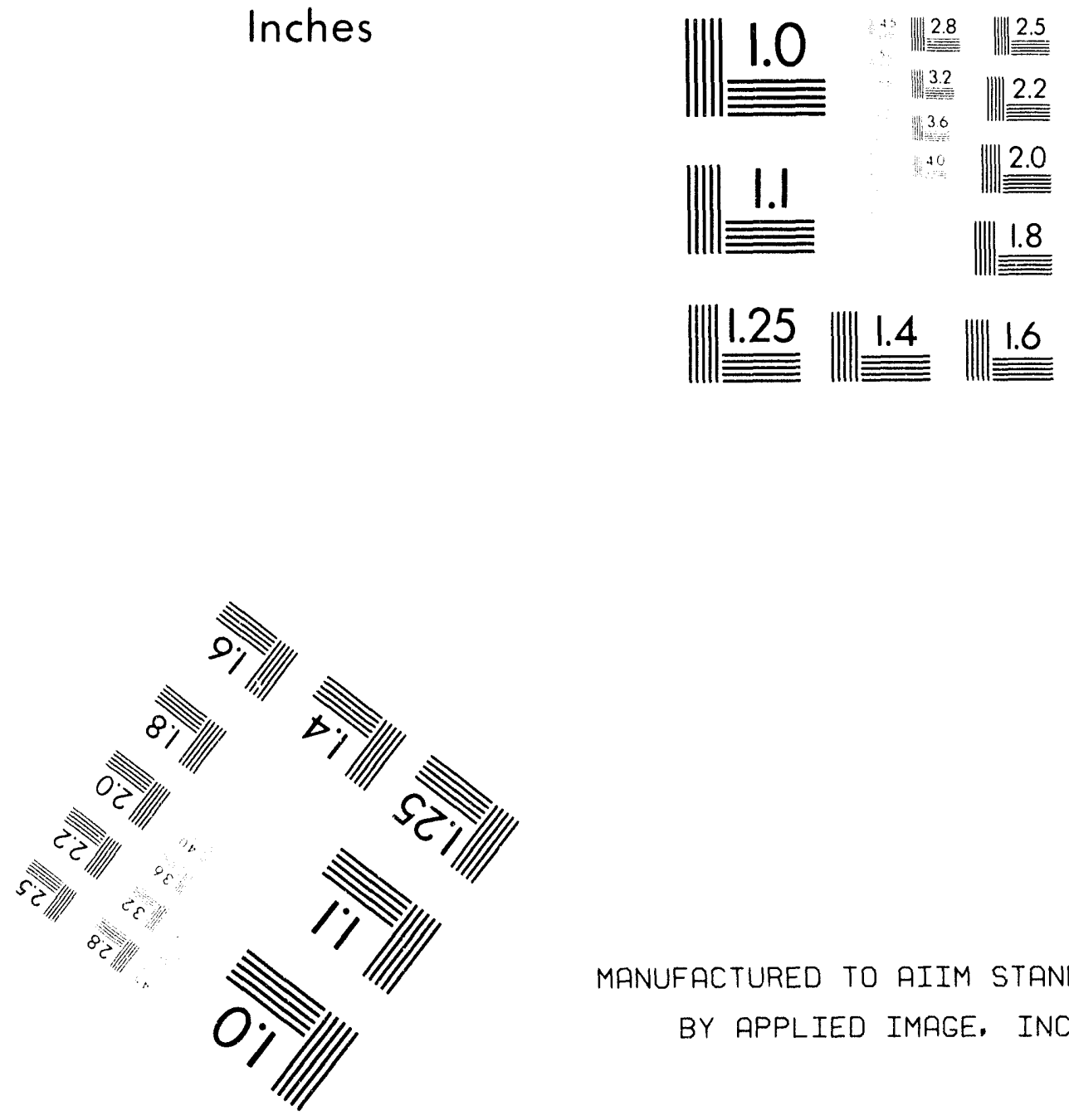

MANUFACTURED TO AIIM STANDARDS

BY APPLIED IMAGE. INC.

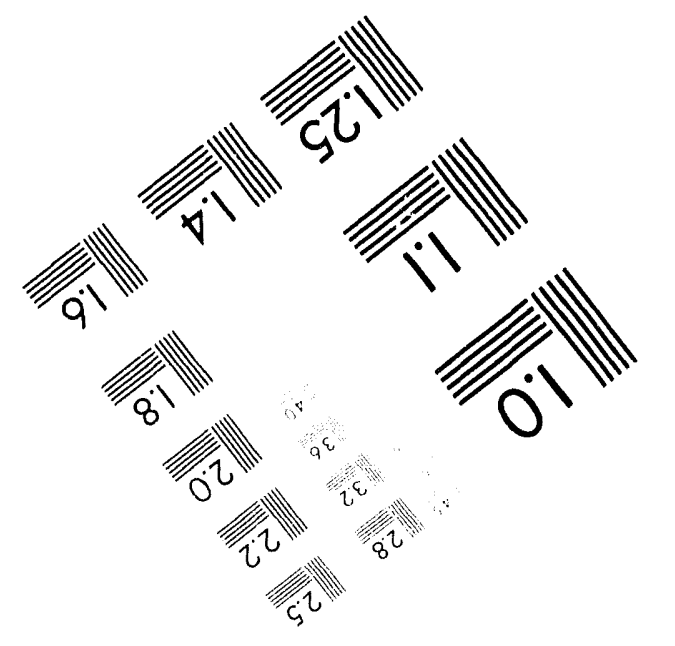



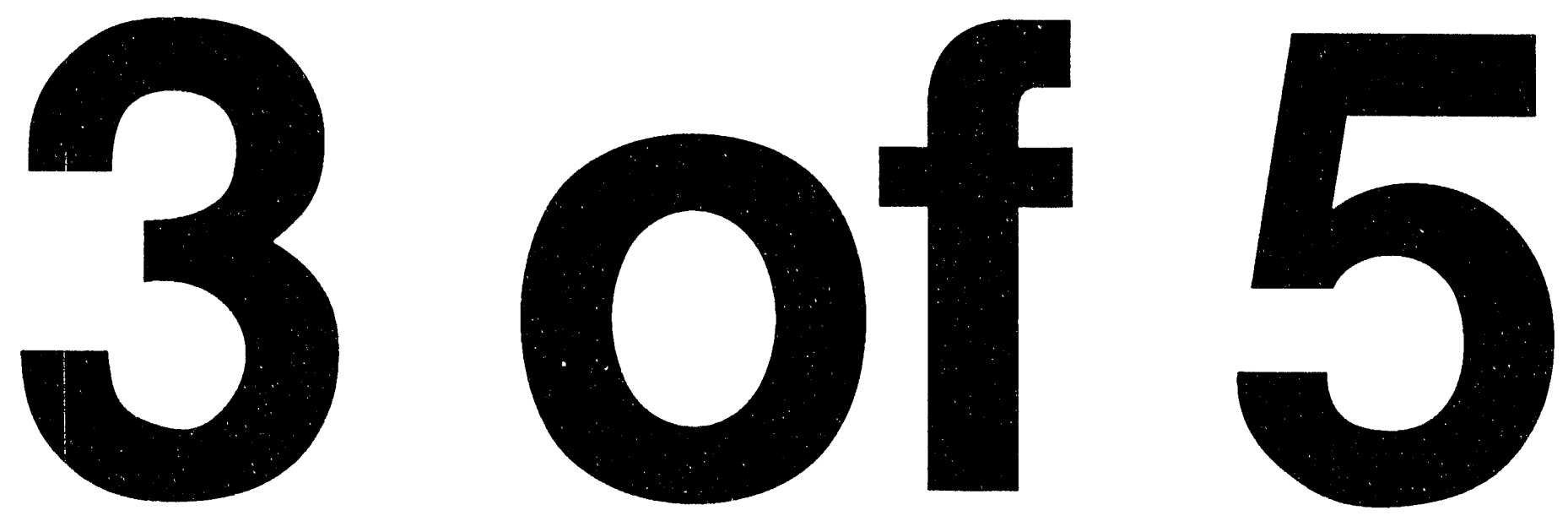


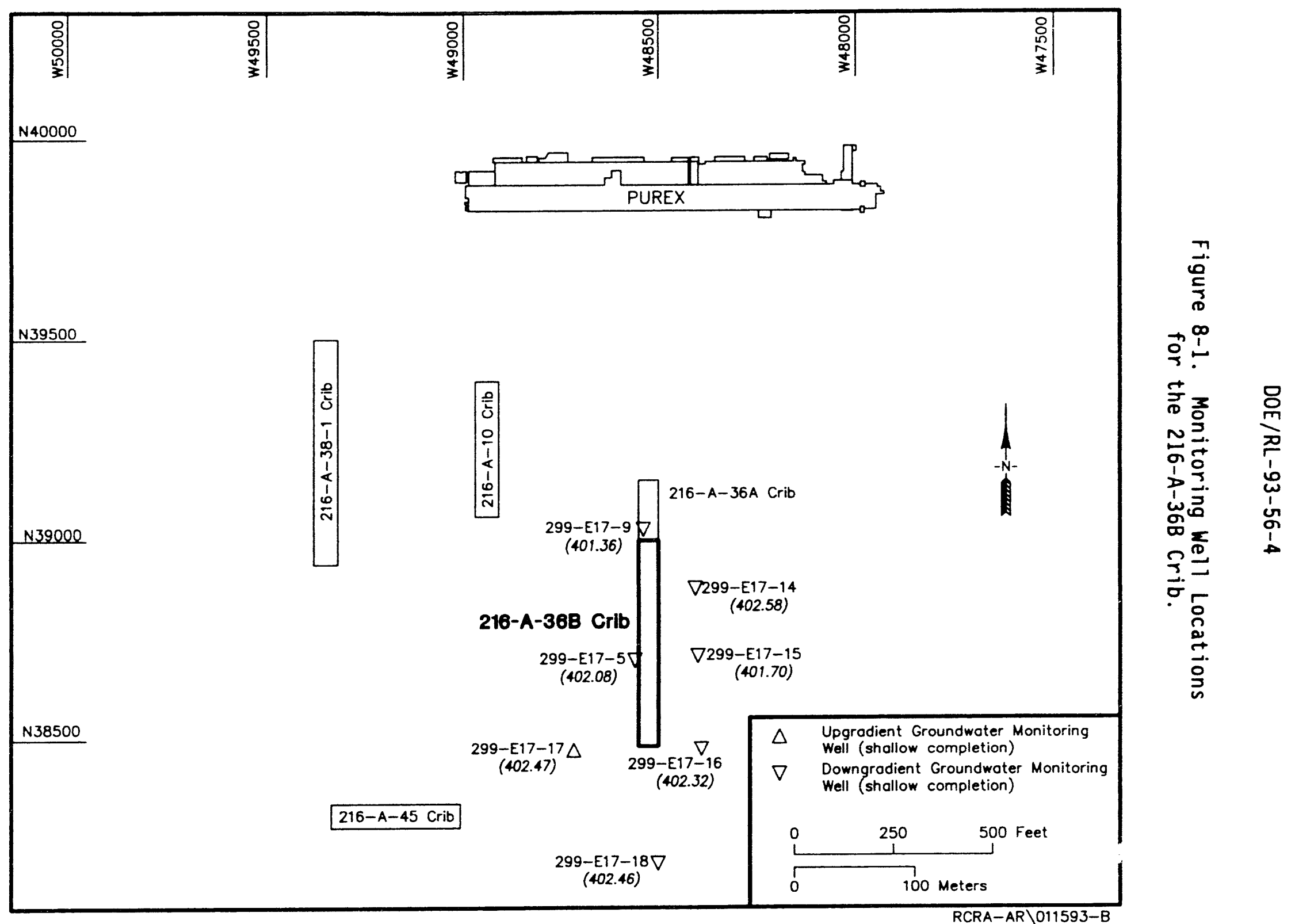


Table 8-1. Monitoring Well Purpose and Sampling Schedule for the 216-A-36B Crib Network.

\begin{tabular}{|c|c|l|l|c|}
\hline $\begin{array}{c}\text { Well no. } \\
(299-)\end{array}$ & $\begin{array}{c}\text { Relative } \\
\text { position }\end{array}$ & \multicolumn{1}{|c|}{ Hydrogeologic unit } & $\begin{array}{c}\text { Sample } \\
\text { frequency }\end{array}$ & $\begin{array}{c}\text { Sample date, } \\
\text { 4th Qtr 1993 }\end{array}$ \\
\hline E17-17 & Upgradient & Ringold: Water Table & Semiannually & $12 / 08 / 93$ \\
\hline E17-5 & Downgradient & Ringold: Water Table & Semiannually & $12 / 13 / 93$ \\
\hline E17-9 & Downgradient & Ringold: Water Table & Semiannually & $12 / 08 / 93$ \\
\hline E17-14 & Downgradient & Ringold: Water Table & Semiannually & $12 / 08 / 93$ \\
\hline E17-15 & Downgradient & Ringold: Water Table & Semiannually & $10 / 11 / 93$ \\
& & & & $12 / 08 / 93$ \\
\hline E17-16 & Downgradient & Ringold: Water Table & Semiannually & $12 / 03 / 93$ \\
\hline E17-18 & Downgradient & Ringold: Water Table & Semiannually & $12 / 13 / 93$ \\
\hline
\end{tabular}

"Well shared with the 216-A-29 Ditch network. 
Table 8-2. RCRA Water Level Measurement Report for the 216-A-36B Crib, Fourth Quarter 1993.

\begin{tabular}{lccc}
\hline Wel1 & Date & $\begin{array}{c}\text { Wepth to } \\
\text { water (ft) }\end{array}$ & $\begin{array}{c}\text { Water fevel } \\
\text { elevation } \\
\text { above ms ( } \mathrm{ft})\end{array}$ \\
\hline $299-\mathrm{f} 17-14$ & $12 / 06 / 93$ & 320.74 & 401.44 \\
$299-E 17-15$ & $12 / 06 / 93$ & 321.16 & 400.62 \\
$299-E 17-16$ & $12 / 06 / 93$ & 319.36 & 401.22 \\
$299-E 17-17$ & $12 / 06 / 93$ & 318.58 & 401.34 \\
$299-E 17-18$ & $12 / 06 / 93$ & 319.29 & 401.36 \\
$299-E 17-5$ & $12 / 06 / 93$ & 317.74 & 400.95 \\
$299-E 17-9$ & $12 / 06 / 93$ & 316.44 & 401.20 \\
\hline
\end{tabular}

Notes: 1. Water level measurements taken at time of sampling are not available for inclusion in this table.

2. Water level elevations are calculated by subtracting the measured depth to water from the surveyed elevation for the well.

3. Depth-to-water values are transcribed from field records.

4. To convert feet to meters multiply by 0.3048 .

5. Water level measurements taken at the time of sample collection were not available for inclusion in this report. 
Table 8-3. Constituent List and Summary of Results for the 216-A-36B Crib Data for Reporting Period October 1 through December 31, 1993. (sheet 1 of 5)

CONTAMINATION INDICATOR RARAMETERS

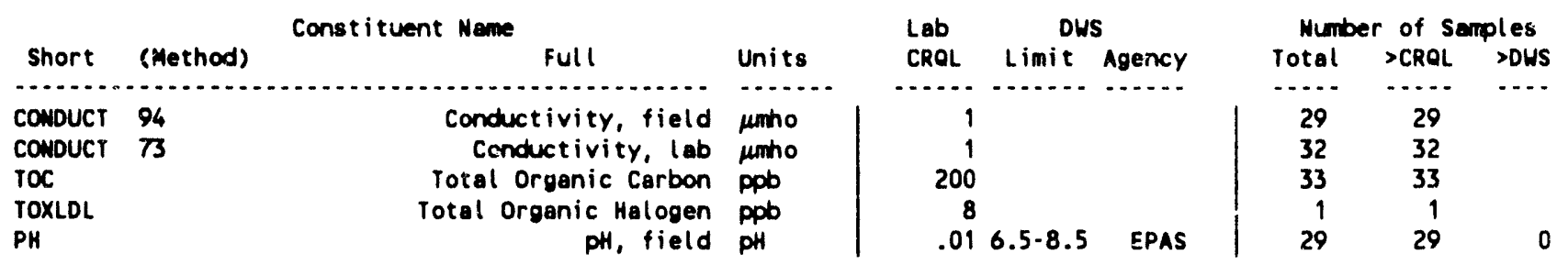

DRINKING WATER PARAMETERS

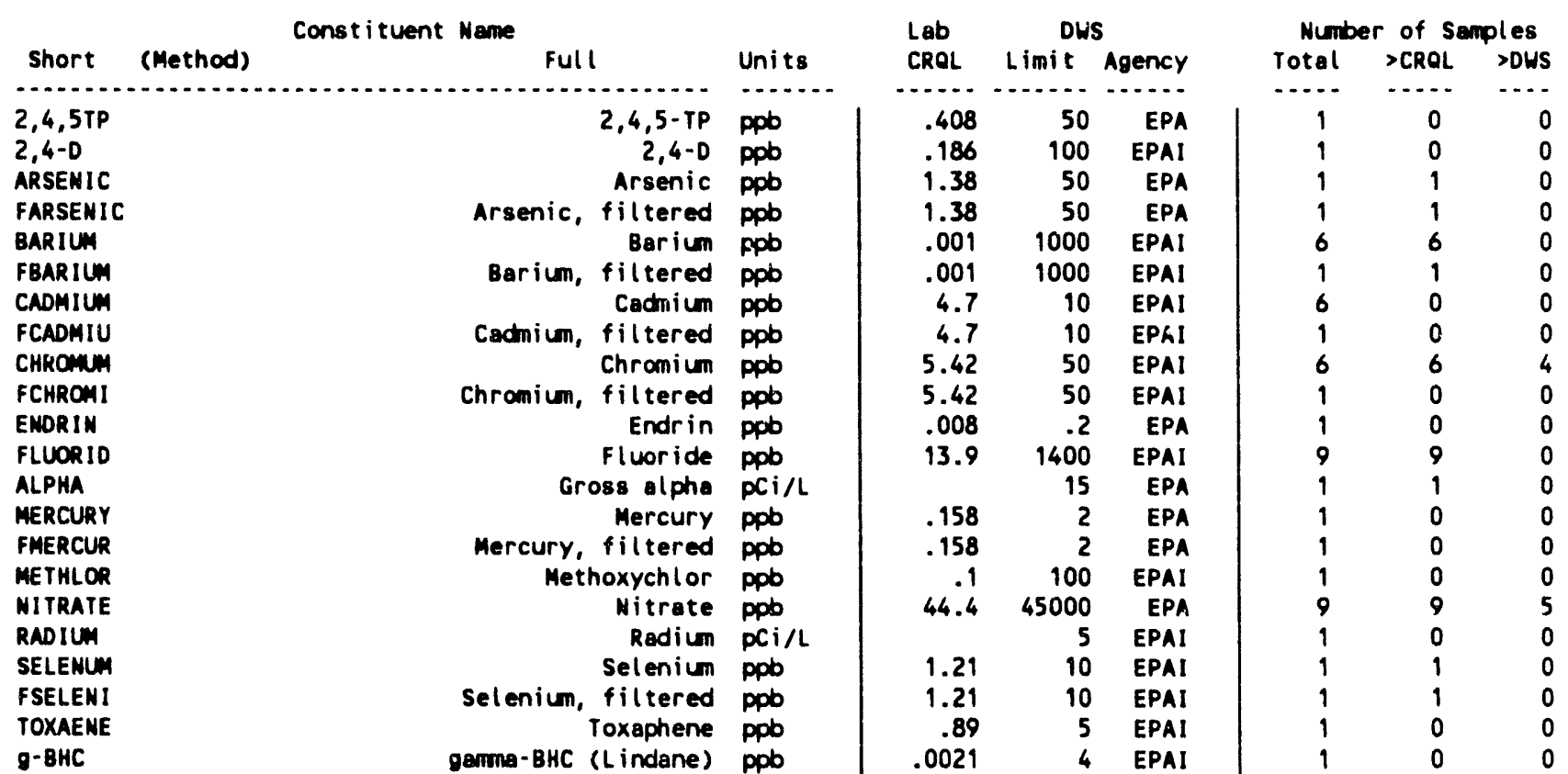

GROUNDWATER QUALITY PARAMETERS

\begin{tabular}{|c|c|c|c|c|c|c|c|c|c|}
\hline \multicolumn{4}{|c|}{ Constituent Name } & \multicolumn{3}{|c|}{ DUS } & \multicolumn{3}{|c|}{ Number of Semples } \\
\hline Short & (Method) & Full & Units & CROL & Limit & Agency & Total & $>$ CRQL & $>$ OWS \\
\hline$\ldots \ldots$ & 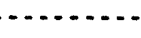 & 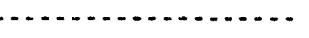 & $\ldots \ldots$ & $\cdots \cdots$ & $\cdots \cdots$ & $\ldots \ldots$ & $\ldots \ldots$ & $\ldots$. & $\ldots$ \\
\hline CHLOR ID & & Chloride & ppb & 82.5 & 250000 & EPAS & 9 & 9 & 0 \\
\hline IRON & & Iron & ppb & 10.3 & 300 & EPAS & 6 & 6 & 3 \\
\hline FIRON & & Iron, filtered & ppb & 10.3 & 300 & EPAS & 1 & 1 & 0 \\
\hline MANGESE & & Manganese & ppb & 1.35 & 50 & EPAS & 6 & 6 & 0 \\
\hline FMANGAN & & Menganese, filtered & ppb & 1.35 & 50 & EPAS & 1 & 1 & 0 \\
\hline LPHENOL & 19 & Phenol & opb & .833 & & & 5 & 0 & \\
\hline LPHENOL & 30 & Phenol & ppb & .31 & & & 9 & 0 & \\
\hline SODIun & & Sodium & ppo & 40.9 & & & 6 & 6 & \\
\hline FSODIUN & & Sodium, filtered & ppb & 40.9 & & & 1 & 1 & \\
\hline SULFATE & & Sulfate & ppb & 49.9 & 250000 & EPAS & 9 & 9 & 0 \\
\hline
\end{tabular}


Table 8-3. Constituent List and Summary of Results for the 216-A-36B Crib Data for Reporting Period October 1 through December 31, 1993. (sheet 2 of 5)

SITE SPECIFIC AND OTHER CONSTITUENTS

\begin{tabular}{|c|c|c|c|c|c|c|c|c|c|}
\hline \multirow[b]{2}{*}{ Short } & \multirow[b]{2}{*}{ (Method) } & \multirow[t]{2}{*}{ Const ituent Mame } & \multirow[b]{2}{*}{ Units } & \multicolumn{3}{|l|}{ Lab } & \multicolumn{3}{|c|}{ Number of Semples } \\
\hline & & & & CRQL & Limit & Agency & Total & $>$ CRQL & $>$ DWS \\
\hline & & (n) & $\cdots \cdot$ & (........ & $\cdots$ & $\cdots$ & $\cdots$ & $\cdots$ & $\cdots$ \\
\hline $\begin{array}{l}\text { TETRCHB } \\
\text { TRICHLB }\end{array}$ & & 1,2,4,5-Tetrachl or obenzene & ppb & 4.39 & & & $\begin{array}{l}5 \\
5\end{array}$ & 0 & \\
\hline 12-dben & & $\begin{array}{r}\text { 1,2,4-Trichlorobenzene } \\
\text { 1,2-0 ichl orobenzene }\end{array}$ & $\begin{array}{l}\text { ppo } \\
\text { ppob }\end{array}$ & 3.7 & 600 & EPA & $\begin{array}{l}3 \\
5\end{array}$ & $\begin{array}{l}0 \\
0\end{array}$ & 0 \\
\hline 13-dben & & 1,3-0 ichlorobenzene & ppob & 7.85 & & & 5 & 0 & \\
\hline 14-dben & & 1,4-0 ichlorabenzene & ppob & 4.64 & $\pi$ & EPA & 5 & 0 & 0 \\
\hline MAPHQUI & & 1,4-Naphtoquinore & ppo & 10 & & & 5 & 0 & \\
\hline 1-napha & & 1-Naphthylemine & ppb & 25.3 & & & 5 & 0 & \\
\hline $\begin{array}{l}\text { TETRCHP } \\
2,4,5-T\end{array}$ & & 2,3,4,6-Tetrachlorophenol & ppb & 4.77 & & & 5 & 0 & \\
\hline $\begin{array}{l}2,4,5-T \\
245-\text { trp }\end{array}$ & & $2,4,5-T$ & ppb & .384 & & & 1 & 0 & \\
\hline $\begin{array}{l}245-\operatorname{trp} \\
246-t r p\end{array}$ & & 2,4,5-Trichlorophenol & ppob & 4.01 & & & 5 & 0 & \\
\hline $\begin{array}{l}246-\operatorname{trp} \\
246-\operatorname{trp}\end{array}$ & 19 & 2,4,6-Irichlorophenol & ppb & 2.54 & & & $\begin{array}{l}5 \\
9\end{array}$ & 0 & \\
\hline $\begin{array}{l}246-\operatorname{trp} \\
24-d c h p\end{array}$ & $\begin{array}{l}30 \\
19\end{array}$ & $\begin{array}{r}\text { 2,4,6-Trichlorophenol } \\
\text { 2,4-D ichlorophenol }\end{array}$ & $\begin{array}{l}\text { ppb } \\
\text { ppb }\end{array}$ & $\begin{array}{r}1.45 \\
2.8\end{array}$ & & & $\begin{array}{l}9 \\
5\end{array}$ & $\begin{array}{l}0 \\
0\end{array}$ & \\
\hline 24-dchp & 30 & 2,4-Dichlorophenol & ppb & 1.5 & & & 9 & 0 & \\
\hline DIMPHEN & 19 & 2,4-D ime thyl phenol & ppb & 5.88 & & & 5 & 0 & \\
\hline DIMPHEN & 30 & 2,4-D ime thylphenol & ppb & 1.01 & & & 9 & 0 & \\
\hline DINPHEN & 19 & 2,4-D initrophenol & ppb & 4.46 & & & 5 & 0 & \\
\hline DINPHEN & 30 & 2,4-D initrophenol & ppb & .96 & & & 9 & 0 & \\
\hline 24-dint & & 2,4-D initrotoluene & ppb & 2.59 & & & 5 & 0 & \\
\hline 26-dchp & $\begin{array}{l}19 \\
30\end{array}$ & 2,6-Dichlorophenol & ppb & 3.47 & & & 5 & 0 & \\
\hline $\begin{array}{l}\text { 26-dchp } \\
26 \text {-dint }\end{array}$ & 30 & 2,6-Dichlorophenol & ppb & 1.59 & & & 9 & 0 & \\
\hline $\begin{array}{l}\text { 26-dint } \\
\text { ACEFENE }\end{array}$ & & $\begin{array}{l}\text { 2,6-0 ini trotoluene } \\
\text { 2-Actyleminof luorene }\end{array}$ & ppb & 2.85 & & & 5 & 0 & \\
\hline $\begin{array}{l}\text { ACEFENE } \\
\text { CHLWAPH }\end{array}$ & & $\begin{array}{l}\text { 2-Acetylaminof luorene } \\
\text { 2-Chloronaph thal ene }\end{array}$ & ppb & 2.83 & & & 5 & 0 & \\
\hline $\begin{array}{l}\text { CHLNAPH } \\
\text { CHLPHEN }\end{array}$ & & 2-Chloronaph thalene & ppb & 3.02 & & & 5 & 0 & \\
\hline CHLPHEN & & 2-Chlorophenol & ppb & 1.91 & & & 5 & 0 & \\
\hline CHLPHEN & 30 & 2-Chlorophenol & ppob & 1.42 & & & 9 & 0 & \\
\hline 2MENAPH & & 2-Methylnaphthalene & ppb & 2.76 & & & 5 & 0 & \\
\hline ZMETHPH & & 2-Methylphenol & ppb & 1.8 & & & 5 & 0 & \\
\hline 2-naphn & & 2-Naphthylamine & ppb & 15.6 & & & 5 & 0 & \\
\hline ONITANI & & 2-Nitroaniline & ppb & 7.82 & & & 5 & 0 & \\
\hline 2NITPH & 19 & 2-Nitrophenol & ppob & 3.96 & & & 5 & 0 & \\
\hline 2NITPH & 30 & 2-Nitrophenol & ppo & 1.56 & & & 9 & 0 & \\
\hline PICOLIN & & 2-Picoline & ppb & 5.83 & & & 5 & 0 & \\
\hline BUTDINP & 30 & 2-sec-Butyl-4,6-dinitrophenol (ON & ppo & 1.35 & & & 9 & 0 & \\
\hline BUTDINP & 49 & 2-8ec-Butyl-4,6-dinitrophenol (DN & ppob & .269 & & & 1 & 0 & \\
\hline DICHBEN & & $3,3^{i}-$ Dichlorobenz idine & ppo & 3.88 & & & 5 & 0 & \\
\hline DIMEYLB & & 3,3'-D imethylbenzidine & ppb & 10.4 & & & 5 & 0 & \\
\hline METCHAN & & 3-Methylcholenthrene & ppb & 2.63 & & & 5 & 0 & \\
\hline MNITANI & & 3-Nitroaniline & ppb & 9.52 & & & 5 & 0 & \\
\hline DDD & & $4,4^{\prime}-D D D$ & ppb & .0005 & & & 1 & 0 & \\
\hline DDE & & $4,4^{\prime}-D D E$ & ppo & .0009 & & & 1 & 0 & \\
\hline DOT & & $4,4^{\prime}-$ DDT & ppb & .011 & & & 1 & 0 & \\
\hline 460N2MP & 19 & 4,6-0 initro-2-methyiphenol & ppb & 5.54 & & & 5 & 0 & \\
\hline 460N2MP & 30 & 4,6-D initro-2-methylphenol & ppb & 1.18 & & & 9 & 0 & \\
\hline NMINOYL & & 4-Aminobiphenyl & ppb & 3.83 & & & 5 & 0 & \\
\hline BROPHEN & & 4-8romophenylphenyl ether & ppb & 2.34 & & & 5 & 0 & \\
\hline CHLCRES & 19 & 4-Chloro-3-methylphenol & ppb & 7.41 & & & 5 & 0 & \\
\hline CHLCRES & 30 & 4-Chloro-3-methylphenol & ppb & 1.12 & & & 9 & 0 & \\
\hline CHLANIL & & 4-Cnloroaniline & ppb & 13.2 & & & 5 & 0 & \\
\hline 4CPPETH & & 4-Chlorophenylphenyl ether & ppb & 3.04 & & & 5 & 0 & \\
\hline LMETHPH & & 4-Methylphenol & ppb & 3.54 & & & 5 & 0 & \\
\hline MITRANI & & 4-Mitroaniline & ppb & 21.4 & & & 5 & 0 & \\
\hline NITPHEN & 19 & 4-Nitrophenol & ppb & 1.52 & & & 5 & 0 & \\
\hline NITPHEN & 30 & 4-Nitrophenol & ppb & .65 & & & 9 & 0 & \\
\hline 4NITQUI & & 4-Nitroquinoline-1-oxide & ppb & 6 & & & 5 & 0 & \\
\hline NITRTOL & & 5-Nitro-o-toluidine & ppb & $5 .-5$ & & & 5 & 0 & \\
\hline
\end{tabular}


Table 8-3. Constituent List and Summary of Results for the 216-A-36B Crib Data for Reporting Period October 1 through December 31, 1993. (sheet 3 of 5)




Table 8-3. Constituent List and Summary of Results for the 216-A-36B Crib Data for Reporting Period October 1 through December 31, 1993. (sheet 4 of 5)

\begin{tabular}{|c|c|c|c|c|c|c|c|c|c|}
\hline \multirow[b]{2}{*}{ Short } & \multirow{2}{*}{\multicolumn{2}{|c|}{ 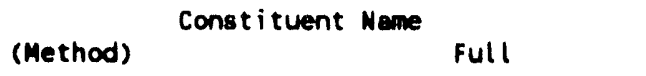 }} & \multirow[b]{2}{*}{ Units } & \multirow{2}{*}{$\begin{array}{l}\text { Lab } \\
\text { CROL }\end{array}$} & \multicolumn{2}{|c|}{ DUS } & \multicolumn{3}{|c|}{ Number of Samples } \\
\hline & & & & & Limit & Agency & Total & $>$ CRQL & $>$ DWS \\
\hline & & $\ldots$ & $\ldots \ldots$ & 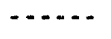 & $\cdots+\cdots$ & $\cdots+$ & $\ldots$. & $\ldots \ldots$ & $\cdots$ \\
\hline ENDSFAN & & Endosulfan sulfate & ppb & .0072 & & & 1 & 0 & \\
\hline ENDRALD & & Endr in Aldehyde & ppb & .011 & & & 1 & 0 & \\
\hline ETHMETS & & Ethyl methenesul fonate & ppob & 2.07 & & & 5 & 0 & \\
\hline FAMPHUR & & Fanphur & ppb & 12.8 & & & 5 & 0 & \\
\hline FLUORAN & & Fluoranthene & ppb & 4.62 & & & 5 & 0 & \\
\hline FLRENE & & Fluorene & ppb & 2.56 & & & 5 & 0 & \\
\hline BETA & & Gross beta & $\mathrm{pCi} / \mathrm{L}$ & & & & 1 & 1 & \\
\hline HEPTLOR & & Heptachlor & ppo & .0019 & .4 & EPA & 1 & 0 & 0 \\
\hline HEPTIDE & & Heptachlor epoxide & ppb & .0008 & .2 & EPA & 1 & 0 & 0 \\
\hline HEXCBEN & & Hexachlorobenzene & ppb & 2.38 & & & 5 & 0 & \\
\hline HEXCBUT & & Hexachlorobutadiene & ppb & 4.29 & & & 5 & 0 & \\
\hline HEXCCYC & & Hexach lorocyclopent ad i ene & ppo & 2.62 & & & 5 & 0 & \\
\hline HEXCETH & & Hexachloroethane & ppb & 4.73 & & & 5 & 0 & \\
\hline HEXACHL & & Hexachlorophene & ppb & 19.6 & & & 5 & 0 & \\
\hline HEXAENE & & Hexach loropropene & ppb & 3.68 & & & 5 & 0 & \\
\hline LHYDRAZ & & Hydrazine & ppb & 1.89 & & & 1 & 0 & \\
\hline INDENOP & & Indeno $(1,2,3-c d)$ pyrene & ppb & 3.21 & & & 5 & 0 & \\
\hline ISOORIN & & Isodrin & ppb & 1.44 & & & 5 & 0 & \\
\hline ISOPHER & & Isophorone & ppb & 3.29 & & & 5 & 0 & \\
\hline ISOSOLE & & Isosafrole & ppb & 2.25 & & & 5 & 0 & \\
\hline KEPONE & & Kepone & ppb & 9.59 & & & 5 & 0 & \\
\hline KEROSEN & & Kerosene & ppb & 4.03 & & & 5 & 0 & \\
\hline LEAD & & Lead & ppo & .508 & 50 & EPAI & 1 & 1 & 0 \\
\hline FLEAD & & Lead, filtered & ppb & .508 & 50 & EPAI & 1 & 1 & 0 \\
\hline MAGNES & & Magnesium & ppb & 26 & & & 6 & 6 & \\
\hline FMAGNES & & Magnesium, filtered & ppb & 26 & & & 1 & 1 & \\
\hline METHAPY & & Methapyrilene & ppb & 8.45 & & & 5 & 0 & \\
\hline METMSUL. & & Methyl methanesul fonate & ppb & 1.61 & & & 5 & 0 & \\
\hline DIPRNIT & & N-Nitroso-di-n-dipropylamine & ppb & 3.67 & & & 5 & 0 & \\
\hline NNIBUTY & & N-Nitrosodi-n-butylemine & ppb & 3.22 & & & 5 & 0 & \\
\hline NNIDIEY & & N-Nitrosodiethylamine & ppb & 2.9 & & & 5 & 0 & \\
\hline NN IDIME & & $N-N i$ trosodimethylemine & ppb & 3.81 & & & 5 & 0 & \\
\hline NND IPHA & & N-Nitrosodiphenylamine & ppb & 2.11 & & & 5 & 0 & \\
\hline NNIMETH & & $N$-Nitrosomethylethylamine & ppb & 2.83 & & & 5 & 0 & \\
\hline MN IMORP & & N-Nitrosomorphol ine & ppo & 3.27 & & & 5 & 0 & \\
\hline NNIPIPE & & N-Nitrosopiperidine & ppo & 4.35 & & & 5 & 0 & \\
\hline NAPHTHA & & Naphthalene & ppb & 6.5 & & & 5 & 0 & \\
\hline NICKEL & & Nickel & ppb & 17.9 & & & 6 & 2 & \\
\hline FNICKEL & & Nickel, filtered & ppb & 17.9 & & & 1 & 0 & \\
\hline NITRITE & & Nitrite & ppb & 38.3 & 1000 & EPA & 9 & 0 & 0 \\
\hline NITBENZ & & Nitrobenzene & ppb & 3.16 & & & 5 & 0 & \\
\hline NITRPYR & & Nitrosopyrrolidine & ppo & 2.78 & & & 5 & 0 & \\
\hline TRIPHOS & & $0,0,0$-Triethyl phosphorothioate & ppb & 5.19 & & & 5 & 0 & \\
\hline DIPHOS & & 0,0-diethyl0-2-pyraz inylphosphor & ppb & 3.04 & & & 5 & 0 & \\
\hline PARATHI & & Parathion & ppb & 3.9 & & & 5 & 0 & \\
\hline PENTCHB & & Pentachlor obenzene & ppb & 3.99 & & & 5 & 0 & \\
\hline PENTCHN & & Pentachloronitrobenzene (PCNB) & ppb & 2.49 & & & 5 & 0 & \\
\hline PENTCHP & 19 & Pentach lorophenol & ppb & 8.07 & 1 & EPA & 5 & 0 & $5 *$ \\
\hline PENTCHP & 30 & Pent ach l or ophenol & ppb & .87 & 1 & EPA & 9 & 0 & 0 \\
\hline PHENTIN & & Phenacet in & ppb & 4.78 & & & 5 & 0 & \\
\hline PHENANT & & Phenanthrene & ppb & 5.27 & & & 5 & 0 & \\
\hline РHOSPHA & & Phosphate & ppb & 147 & & & 9 & 0 & \\
\hline POTASUM & & Potassium & ppb & 662 & & & 6 & 6 & \\
\hline FPOTASS & & Potassium, filtered & ppb & 662 & & & 1 & 1 & \\
\hline PRONIDE & & Pronamide & ppob & 3.42 & & & 5 & 0 & \\
\hline PYRENE & & Pyrene & ppb & 3.69 & & & 5 & 0 & \\
\hline PYRIDIN & & Pyridine & ppb & 4.57 & & & 5 & 0 & \\
\hline
\end{tabular}


Table 8-3. Constituent List and Summary of Results for the 216-A-36B Crib Data for Reporting Period October 1 through December 31, 1993. (sheet 5 of 5)

\begin{tabular}{|c|c|c|c|c|c|c|c|c|c|}
\hline \multicolumn{4}{|c|}{ Constituent Name } & \multicolumn{3}{|c|}{ DWS } & \multicolumn{3}{|c|}{ Number of Semples } \\
\hline Short & (Method) & Full & Units & CRQL & Limit & Agency & Total & $>C R Q L$ & $>$ DUS \\
\hline 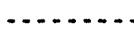 & $\cdots$ & - & $\ldots \ldots$ & $\ldots . .$. & $\ldots \ldots$ & $\ldots . .$. & $\ldots$ & $\ldots$. & $\ldots$ \\
\hline RU- 106 & & Ruthenium- 106 & $\mathrm{pCi} / \mathrm{L}$ & & 30 & EPA & 8 & 0 & $1 \star$ \\
\hline SAFROL & & Safrol & ppb & 3.22 & & & 5 & 0 & \\
\hline SILVER & & silver & ppb & 2.87 & 50 & EPAI & 6 & 0 & 0 \\
\hline FSILVER & & silver, filtered & ppb & 2.87 & 50 & EPAI & 1 & 0 & 0 \\
\hline TETPHNL & & Tetrachlorophenols & ppb & 1.05 & & & 9 & 0 & \\
\hline PYROPHOS & & Tetraethyldi thiopyrophosphate & ppb & 2.81 & & & 5 & 0 & \\
\hline TIN & & Tin & ppb & 51.1 & & & 6 & 0 & \\
\hline FTIN & & Tin, filtered & ppb & 51.1 & & & 1 & 0 & \\
\hline TRIBUTPH & & Tributyl Phosphate & ppb & 4.42 & & & 5 & 0 & \\
\hline TRIPHNL & & Trichlorophenols & ppb & 1.11 & & & 9 & 0 & \\
\hline TOICHPH & & Tris-2-chloroethyl phosphate & ppo & 2.88 & & & 5 & 0 & \\
\hline TRITIUA & & Tritiun & $\mathrm{pCi} / \mathrm{L}$ & & 20000 & EPA & 6 & 6 & 6 \\
\hline TURB 10 & & Turbidity & NTU & .05 & & & 1 & 1 & \\
\hline VANADUM & & Vanadium & ppb & 3.84 & & & 6 & 6 & \\
\hline FVANADI & & Vanadium, filtered & pob & 3.84 & & & 1 & 1 & \\
\hline ZINC & & zinc & ppb & 3.44 & 5000 & EPAS & 6 & 3 & 0 \\
\hline FZINC & & zinc, filtered & ppo & 3.44 & 5000 & EPAS & 1 & 0 & 0 \\
\hline MCRESOL & & $m$-Cresol & ppb & 1.44 & & & 5 & 0 & \\
\hline DINBENZ & & m-dinitrobenzene & pob & 9.35 & & & 5 & 0 & \\
\hline OTOLHYO & & o-Toluidine & pob & 2.27 & & & 5 & 0 & \\
\hline DIMEAMB & & p-D imethyleminoazobenzene & ppb & 3.36 & & & 5 & 0 & \\
\hline PHENDIA & & p-Phenylenediamine & ppb & 3.09 & & & 5 & 0 & \\
\hline SYMTRIN & & sym-Trinitrobenzene & ppb & 3.74 & & & 5 & 0 & \\
\hline
\end{tabular}

For explanation of this table, see Section 1.4 of report. 
Table 8-4. Constituents with at Least One Detected Value for the 216-A-36B Crib Data for Reporting Period October 1 through December 31, 1993. (sheet 1 of 3)

\begin{tabular}{|c|c|c|c|c|c|c|}
\hline $\begin{array}{l}\text { Well } \\
\text { Nane }\end{array}$ & $\begin{array}{c}\text { Collection } \\
\text { Date }\end{array}$ & $\begin{array}{l}\text { Sample } \\
\text { Number }\end{array}$ & $\begin{array}{l}\text { ALUMNUN } \\
34 / \mathrm{ppb} \\
32.51\end{array}$ & $\begin{array}{l}\text { FALUAIN } \\
34 / \text { ppb } \\
32.5 / .\end{array}$ & $\begin{array}{c}\text { AMMONIU } \\
129 / \mathrm{ppb} \\
38.5 /\end{array}$ & $\begin{array}{r}\text { ARSENIC } \\
43 / \text { ppo } \\
1.38 / 50\end{array}$ \\
\hline$\ldots \ldots$ & $\ldots \ldots$ & $\ldots \ldots$ & 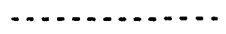 & 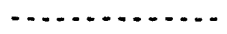 & $\ldots . . . .$. & $\ldots \ldots$ \\
\hline $\begin{array}{l}299-E 17-14 \\
299-E 17-15\end{array}$ & $\begin{array}{l}12 / 08 / 93 \\
10 / 11 / 93\end{array}$ & $\begin{array}{l}\text { BO9MY2 } \\
\text { BO9BK8 }\end{array}$ & $\begin{array}{l}32.50 \mathrm{U} \\
34.00 \mathrm{~L}\end{array}$ & & $\begin{array}{l}50.00<0 \\
80.00<a\end{array}$ & 9.10 \\
\hline 299-E 17-15 & $10 / 11 / 93$ & В098K9 & & $32.50 \mathrm{U}$ & & \\
\hline 299-E17-15 & $12 / 08 / 93$ & BO9MY & $32.50 \mathrm{U}$ & & 38.50 va & \\
\hline 299-E17-16 & $12 / 08 / 93$ & BOSMZO & $32.50 \mathrm{U}$ & & 38.50 va & \\
\hline $299-$ E $17-17$ & $12 / 08 / 93$ & BO9Mz4 & $32.50 \mathrm{U}$ & & 38.50 va & \\
\hline 299-E $17-18$ & $12 / 13 / 93$ & BO9Mz8 & & & 38.50 va & \\
\hline $299-E 17-5$ & $12 / 13 / 93$ & BO9N02 & & & 38.50 ua & \\
\hline 299-E17-5 & $12 / 13 / 93$ & B09N03 & & & 70.00 LO & \\
\hline 299-E17-9 & $12 / 08 / 93$ & B09N10 & 310.00 & & $60.00 \mathrm{LO}$ & \\
\hline
\end{tabular}

\begin{tabular}{|c|c|c|c|c|c|c|}
\hline $\begin{array}{l}\text { Hell } \\
\text { Nane }\end{array}$ & $\begin{array}{c}\text { Collection } \\
\text { Date }\end{array}$ & $\begin{array}{l}\text { Semple } \\
\text { Number }\end{array}$ & $\begin{array}{c}\text { FARSEN IC } \\
43 / \mathrm{ppb} \\
1.38 / 50\end{array}$ & $\begin{array}{c}\text { BAR IUM } \\
\text { 34/ppb } \\
.001 / 1000 \mathrm{i}\end{array}$ & $\begin{array}{l}\text { FBARIUM } \\
34 / \text { ppb } \\
.001 / 1000 i\end{array}$ & $\begin{array}{c}\text { CALCILH } \\
34 / \mathrm{ppb} \\
20.9 / .\end{array}$ \\
\hline 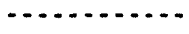 & $\cdots$ & $\ldots \ldots \ldots$ & , & - & $\cdots$ & $\cdots$ \\
\hline $\begin{array}{l}299-E 17-14 \\
299-E 17-15\end{array}$ & $\begin{array}{l}12 / 08 / 93 \\
10 / 11 / 93\end{array}$ & $\begin{array}{l}\text { B094Y2 } \\
\text { B098K8 }\end{array}$ & & $\begin{array}{l}49.00 \\
48.00\end{array}$ & & $\begin{array}{l}47000.00 \\
54000.00\end{array}$ \\
\hline 299-E17-15 & $10 / 11 / 93$ & B098K9 & 8.30 & & 48.00 & \\
\hline $\begin{array}{l}299-\text { E17- } 15 \\
299-\text { E17- } 16\end{array}$ & $\begin{array}{l}12 / 08 / 93 \\
12 / 08 / 93\end{array}$ & $\begin{array}{l}\text { BO9MY6 } \\
\text { BO9MzO }\end{array}$ & & $\begin{array}{l}48.00 \\
35.00\end{array}$ & & $\begin{array}{l}51000.00 \\
34000.00\end{array}$ \\
\hline 299-E17-17 & $12 / 08 / 93$ & Bo9m24 & & 40.00 & & 30000.00 \\
\hline 299-E17-9 & $12 / 08 / 93$ & $809 \times 10$ & & 75.00 & & 52000.008 \\
\hline
\end{tabular}

\begin{tabular}{|c|c|c|c|c|c|c|}
\hline $\begin{array}{l}\text { Hell } \\
\text { Nene }\end{array}$ & $\begin{array}{c}\text { Collection } \\
\text { Date }\end{array}$ & $\begin{array}{l}\text { Semple } \\
\text { Number }\end{array}$ & $\begin{array}{l}\text { FCALCIU } \\
34 / \text { ppo } \\
20.91 .\end{array}$ & $\begin{array}{c}\text { CHLORID } \\
124 / p p b \\
82.5 / 2500008\end{array}$ & $\begin{array}{l}\text { CHROMU } \\
\text { 34/pob } \\
5.42 / 50 \mathrm{i}\end{array}$ & $\begin{array}{l}\text { FCHROAI } \\
34 / \mathrm{ppb} \\
5.42 / 50 \mathrm{i}\end{array}$ \\
\hline$\ldots \ldots$ & $\ldots \ldots$ & $\ldots \ldots \ldots$ & $\ldots \ldots \ldots$ & $\ldots \ldots$ & 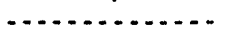 & $\ldots . . . .$. \\
\hline $\begin{array}{l}299-E 17-14 \\
299-E 17-15\end{array}$ & $\begin{array}{l}12 / 08 / 93 \\
10 / 11 / 93\end{array}$ & $\begin{array}{l}\text { Bo94Y2 } \\
\text { 809BK8 }\end{array}$ & & $\begin{array}{l}3800.00 \\
3500.00\end{array}$ & $\begin{array}{l}40.00 \\
94.00\end{array}$ & \\
\hline $299-E 17-15$ & $10 / 11 / 93$ & В09BKO & 53000.00 & & & $5.42 \mathrm{U}$ \\
\hline $\begin{array}{l}299-\text { E } 17-15 \\
299-\text { E } 17-16\end{array}$ & $\begin{array}{l}12 / 08 / 93 \\
12 / 08 / 93\end{array}$ & $\begin{array}{l}\text { BOMNY6 } \\
\text { BO9MZO }\end{array}$ & & $\begin{array}{l}3900.00 \\
4200.00\end{array}$ & $\begin{array}{l}56.00 \\
58.00\end{array}$ & \\
\hline 299-E17-17 & $12 / 08 / 93$ & BO9M24 & & 3500.00 & 150.00 & \\
\hline $299-E 17-18$ & $12 / 13 / 93$ & BOSMZ8 & & 4900.00 & & \\
\hline $\begin{array}{l}299-\text { E } 17-5 \\
299-\text { E } 17-5\end{array}$ & $\begin{array}{l}12 / 13 / 93 \\
12 / 13 / 93\end{array}$ & $\begin{array}{l}\text { BO9N02 } \\
\text { BO9N03 }\end{array}$ & & $\begin{array}{l}3300.00 \\
3400.00\end{array}$ & & \\
\hline 299-E17-9 & $12 / 08 / 93$ & BO9N10 & & 4600.00 & $8.50 \mathrm{~L}$ & \\
\hline
\end{tabular}

\begin{tabular}{|c|c|c|c|c|c|c|}
\hline $\begin{array}{l}\text { Hell } \\
\text { Nene }\end{array}$ & $\begin{array}{c}\text { Collection } \\
\text { Date }\end{array}$ & $\begin{array}{l}\text { Semple } \\
\text { Number }\end{array}$ & $\begin{array}{c}c 0-60 \\
140 / \mathrm{pCi} / \mathrm{L} \\
. / 100\end{array}$ & $\begin{array}{c}\text { COPPER } \\
34 / \mathrm{ppb} \\
2.65 / 10008\end{array}$ & $\begin{array}{l}\text { FCOPPER } \\
34 / \text { ppb } \\
2.65 / 1000 \mathrm{~s}\end{array}$ & $\begin{array}{l}\text { FLUOR ID } \\
124 / \mathrm{ppb} \\
13.9 / 1400 \mathrm{i}\end{array}$ \\
\hline - & $\ldots \ldots \ldots$ & $\cdots+\cdots$ & - & $\ldots \ldots$ & $\ldots \ldots \ldots$ & - -5 \\
\hline $\begin{array}{l}299-\text { E } 17-14 \\
299-\text { E } 17-15\end{array}$ & $\begin{array}{l}12 / 08 / 93 \\
10 / 11 / 93\end{array}$ & $\begin{array}{l}\text { BO9MYZ } \\
\text { B09BK8 }\end{array}$ & 5.29 & $\begin{array}{l}2.65 \mathrm{U} \\
5.00 \mathrm{~L}\end{array}$ & & $\begin{array}{l}500.00 \\
500.00\end{array}$ \\
\hline $\begin{array}{l}299-E 17-15 \\
299-E 17-15\end{array}$ & $\begin{array}{l}10 / 11 / 93 \\
12 / 08 / 93\end{array}$ & $\begin{array}{l}\text { В09BK9 } \\
\text { В09MY } 6\end{array}$ & $3.24 \mathrm{U}$ & $2.65 \mathrm{U}$ & $5.00 \mathrm{~L}$ & 200.00 \\
\hline $\begin{array}{l}299-\mathrm{E} 17-16 \\
299-\mathrm{E} 17-17\end{array}$ & $\begin{array}{l}12 / 08 / 93 \\
12 / 08 / 93\end{array}$ & $\begin{array}{l}\text { 809120 } \\
809124\end{array}$ & 24.00 & $2.65 \mathrm{U}$ & & 200.00 \\
\hline $\begin{array}{l}299-E 17-18 \\
299-E 17-5\end{array}$ & $\begin{array}{l}12 / 13 / 93 \\
12 / 13 / 93\end{array}$ & BO9M28 & $\begin{array}{l}1.22 \mathrm{U} \\
6.47 \mathrm{a}\end{array}$ & & & $\begin{array}{l}400.00 \\
900.000\end{array}$ \\
\hline $\begin{array}{l}\text { 299-E17-5 } \\
299-\text { E17-9 }\end{array}$ & $\begin{array}{l}12 / 13 / 93 \\
12 / 08 / 93\end{array}$ & $\begin{array}{l}\text { 809N03 } \\
\text { B09N10 }\end{array}$ & $\begin{array}{l}2.85 \mathrm{uO} \\
-.41 \mathrm{U}\end{array}$ & $2.65 \mathrm{U}$ & & $\begin{array}{l}400.000 \\
700.00\end{array}$ \\
\hline
\end{tabular}


Table 8-4. Constituents with at Least One Detected Value for the 216-A-36B Crib Data for Reporting Period October 1 through December 31, 1993. (sheet 2 of 3)

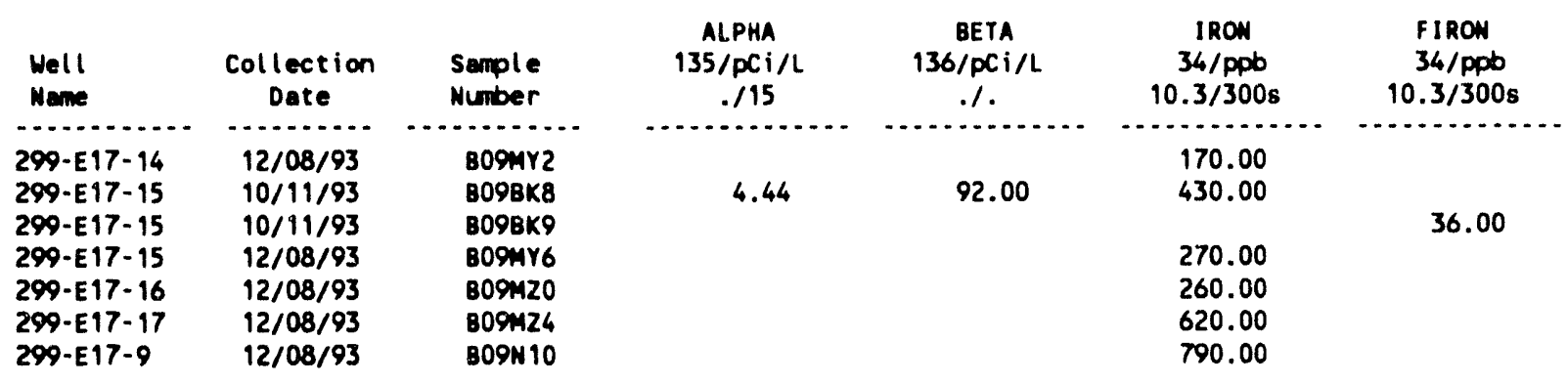

\begin{tabular}{|c|c|c|c|c|c|c|}
\hline $\begin{array}{l}\text { Well } \\
\text { Mane }\end{array}$ & $\begin{array}{c}\text { Collection } \\
\text { Date }\end{array}$ & $\begin{array}{l}\text { Semple } \\
\text { Number }\end{array}$ & $\begin{array}{l}\text { LEAD } \\
40 / \mathrm{ppb} \\
.508 / 50 \mathrm{i}\end{array}$ & $\begin{array}{c}\text { MAGNES } \\
34 / \text { ppb } \\
26 /\end{array}$ & $\begin{array}{c}\text { FMAGNES } \\
34 / \mathrm{ppb} \\
26 / .\end{array}$ & $\begin{array}{c}\text { MANGESE } \\
34 / \mathrm{ppb} \\
1.35 / 50 \mathrm{~s}\end{array}$ \\
\hline$\ldots \ldots \ldots$ & $\cdots+\cdots$ & $\cdots \cdots$ & - & 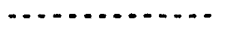 & $\ldots$ & $\ldots$ \\
\hline $\begin{array}{l}299-E 17-14 \\
299-E 17-15\end{array}$ & $\begin{array}{l}12 / 08 / 93 \\
10 / 11 / 93\end{array}$ & $\begin{array}{l}\text { B09MY2 } \\
\text { В09BK8 }\end{array}$ & $4.80 \mathrm{~L}$ & $\begin{array}{l}15000.00 \\
13000.00\end{array}$ & & $\begin{array}{l}4.10 \mathrm{~L} \\
9.50 \mathrm{~L}\end{array}$ \\
\hline 299-E17-15 & $10 / 11 / 93$ & B098K9 & & & 13000.00 & \\
\hline $299-E 17-15$ & $12 / 08 / 93$ & BO9MYG & & 13000.00 & & $5.60 \mathrm{~L}$ \\
\hline $299-E 17-16$ & $12 / 08 / 93$ & BO9M20 & & 9800.00 & & $4.60 \mathrm{~L}$ \\
\hline $299-E 17-9$ & $12 / 08 / 93$ & $\begin{array}{l}809124 \\
\text { B09N10 }\end{array}$ & & $\begin{array}{r}8900.00 \\
15000.00\end{array}$ & & $\begin{array}{l}15.00 \\
18.00\end{array}$ \\
\hline
\end{tabular}

\begin{tabular}{|c|c|c|c|c|c|c|}
\hline $\begin{array}{l}\text { Hell } \\
\text { Neme }\end{array}$ & $\begin{array}{c}\text { Collection } \\
\text { Date }\end{array}$ & $\begin{array}{l}\text { Semple } \\
\text { Number }\end{array}$ & $\begin{array}{c}\text { FMANGAN } \\
\text { 34/pob } \\
1.35 / 508\end{array}$ & $\begin{array}{l}\text { NICKEL } \\
34 / \text { pob } \\
17.9 /\end{array}$ & $\begin{array}{c}\text { FNICKEL } \\
34 / \mathrm{ppb} \\
17.91 .\end{array}$ & $\begin{array}{l}\text { NITRATE } \\
124 / \text { ppb } \\
44.4 \cdot 45000\end{array}$ \\
\hline$\cdots+\ldots$ & $\ldots \ldots$ & $\ldots \ldots \ldots$ & , n & $\ldots$ & $\ldots \ldots \ldots$ & $\ldots \ldots$ \\
\hline $\begin{array}{l}299-E 17-14 \\
299-E 17-15\end{array}$ & $\begin{array}{l}12 / 08 / 93 \\
10 / 11 / 93\end{array}$ & $\begin{array}{l}\text { B09MY2 } \\
\text { B098K8 }\end{array}$ & & $\begin{array}{l}17.90 \mathrm{U} \\
53.00\end{array}$ & & $\begin{array}{l}110000.00 \mathrm{D} \\
120000.00 \mathrm{D}\end{array}$ \\
\hline $299-E 17-15$ & $10 / 11 / 93$ & B098KO & $2.40 \mathrm{~L}$ & & $17.90 \mathrm{U}$ & \\
\hline 299-E17- 15 & $12 / 08 / 93$ & BO9MY6 & & $17.90 \mathrm{U}$ & & $120000.00 \mathrm{D}$ \\
\hline $\begin{array}{l}299-E 17-16 \\
299-E 17-17\end{array}$ & $\begin{array}{l}12 / 08 / 93 \\
12 / 08 / 93\end{array}$ & $\begin{array}{l}809 M 20 \\
809124\end{array}$ & & $\begin{array}{l}17.90 \mathrm{U} \\
57.00\end{array}$ & & $\begin{array}{l}55000.00 \\
38000.00\end{array}$ \\
\hline 299-E17-18 & $12 / 13 / 93$ & BO9M28 & & & & 33000.0080 \\
\hline 299-E17-5 & $12 / 13 / 93$ & BO9NO2 & & & & $26000.00 \mathrm{BD}$ \\
\hline 299-E17-5 & $12 / 13 / 93$ & B09N03 & & & & $27000.00 \mathrm{BO}$ \\
\hline 299-E17-9 & $12 / 08 / 93$ & BO9N10 & & $17.90 \mathrm{U}$ & & $120000.00 \mathrm{D}$ \\
\hline
\end{tabular}

\begin{tabular}{|c|c|c|c|c|c|c|}
\hline $\begin{array}{l}\text { Well } \\
\text { Neme }\end{array}$ & $\begin{array}{c}\text { Collection } \\
\text { Date }\end{array}$ & $\begin{array}{l}\text { Semple } \\
\text { Number }\end{array}$ & $\begin{array}{l}\text { POTASUM } \\
34 / \mathrm{ppb} \\
662 /\end{array}$ & $\begin{array}{l}\text { FPOTASS } \\
34 / \mathrm{ppb} \\
662 /\end{array}$ & $\begin{array}{l}\text { SELENUH } \\
48 / \mathrm{ppb} \\
1.21 / 10 \mathrm{i}\end{array}$ & $\begin{array}{c}\text { FSELENI } \\
48 / \mathrm{ppb} \\
1.21 / 10 \mathrm{i}\end{array}$ \\
\hline 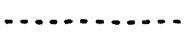 & $\ldots \ldots \ldots$ & $\ldots \ldots \ldots$ & $\ldots$ & $\ldots \ldots \ldots$ & - & $\ldots \ldots \ldots$ \\
\hline 299-E17-14 & $12 / 08 / 93$ & BO9MY2 & 5300.00 & & & \\
\hline 299-E17-15 & $10 / 11 / 93$ & В09BK8 & 5300.00 & & $2.00 \mathrm{~L}$ & \\
\hline 299-E17-15 & $10 / 11 / 93$ & B098K9 & & 5000.00 & & $2.00 \mathrm{~L}$ \\
\hline 299-E17-15 & $12 / 08 / 93$ & B09MY6 & 5600.00 & & & \\
\hline $\begin{array}{l}29 y-E 17-10 \\
200-517-17\end{array}$ & $\begin{array}{l}12 / 08 / 93 \\
12 / 08 / 03\end{array}$ & B09120 & 4700.00 & & & \\
\hline 299-E17-9 & $12 / 08 / 93$ & BO9N10 & 6700.00 & & & \\
\hline
\end{tabular}


Table 8-4. Constituents with at Least One Detected Value for the 216-A-36B Crib Data for Reporting Period October 1 through December 31, 1993. (sheet 3 of 3)

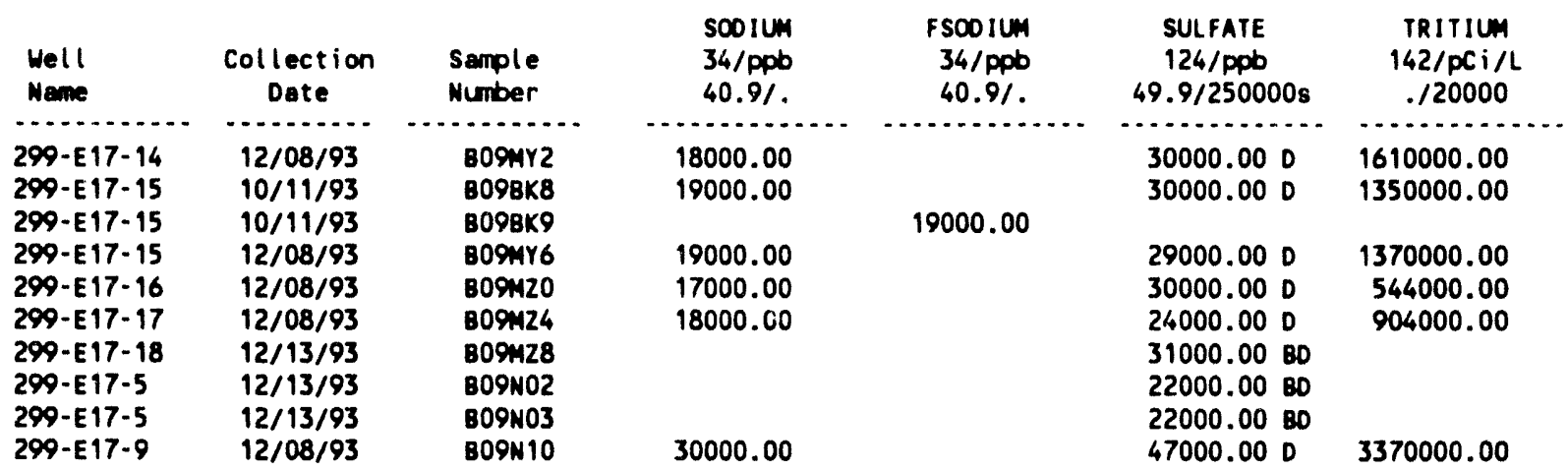

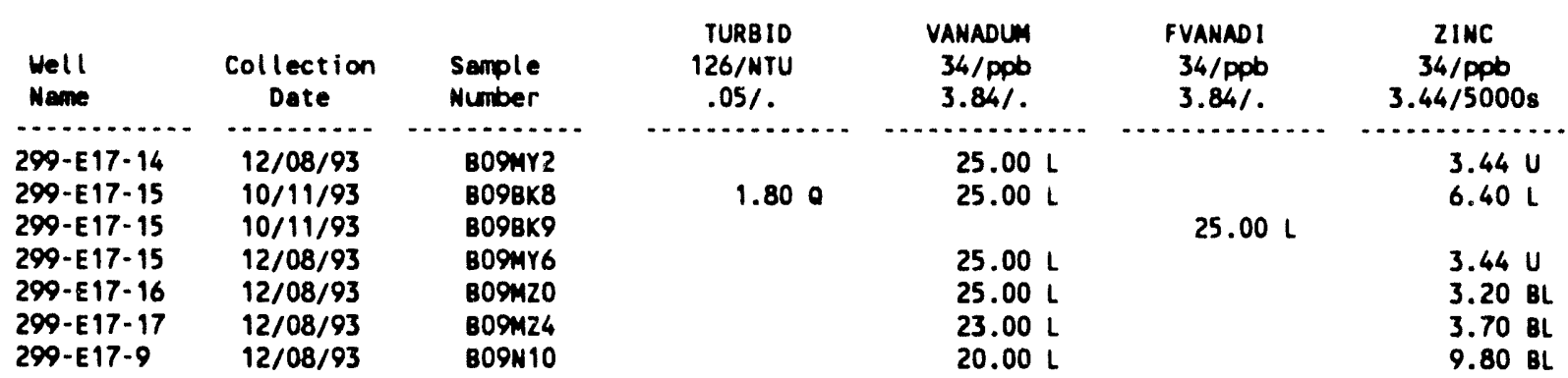

\begin{tabular}{|c|c|c|c|}
\hline $\begin{array}{l}\text { Hell } \\
\text { Name }\end{array}$ & $\begin{array}{c}\text { Collection } \\
\text { Date }\end{array}$ & $\begin{array}{l}\text { Semple } \\
\text { Number }\end{array}$ & $\begin{array}{c}\text { F2INC } \\
34 / \mathrm{ppb} \\
3.44 / 5000 \mathrm{~s}\end{array}$ \\
\hline$E 17-15$ & $10 / 11 / 93$ & B098KO & $3.44 \mathrm{U}$ \\
\hline
\end{tabular}

For explanation of this table, see Section 1.4 of report. 
Table 8-5. Contamination Indicator Parameters for the 216-A-36B Crib Data for Reporting Period October 1 through December 31, 1993.

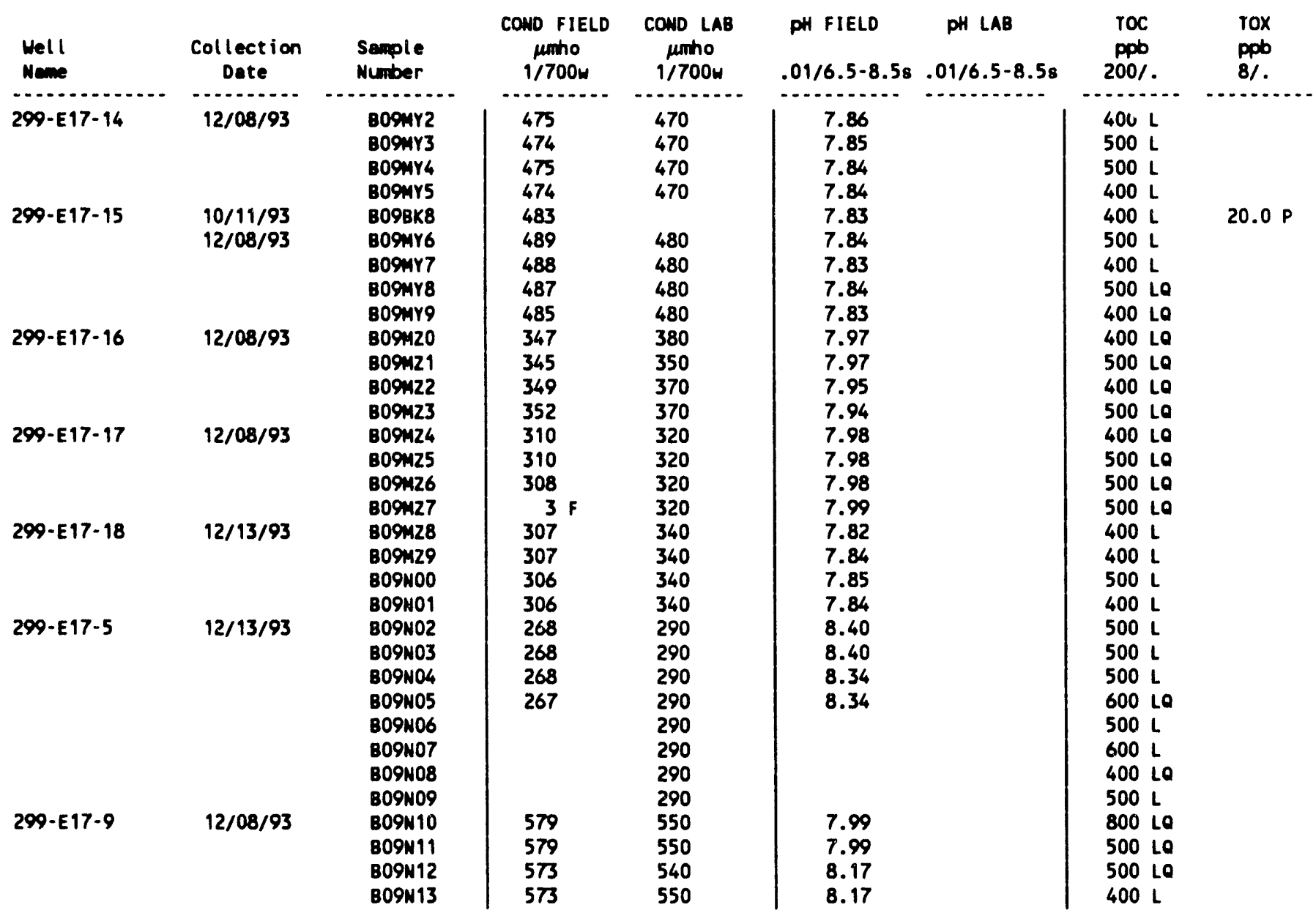

For explanotion of this table, see Section 1.4 of report. 
DOE/RL-93-56-4

\section{CONTENTS}

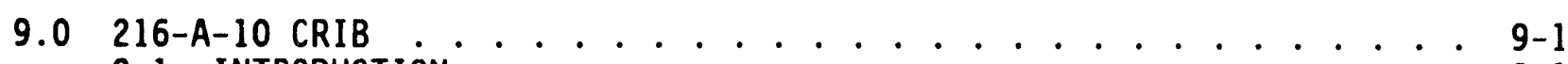

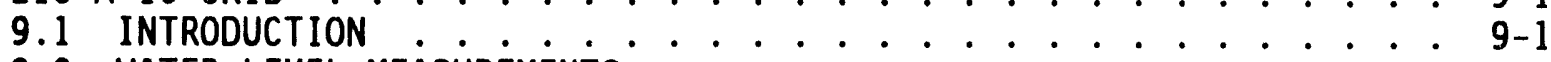

9.2 WATER LEVEL MEASUREMENTS . . . . . . . . . . . . . . . . . . . $9-1$

9.3 WATER CHEMISTRY DATA . . . . . . . . . . . . . . . . . . . . . 9-2 
DOE/RL-93-56-4

\section{LIST OF FIGURES}

9-1 Monitoring Well Locations for the 216-A-10 Crib. . . . . . . . . 9-3

\section{LIST OF TABLES}

9-1 Monitoring Well Purpose and Sampling Schedule for the 216-A-10 Crib Network .................. . 9-4

9-2 RCRA Water Level Measurement Report 216-A-10 Crib, Fourth Quarter 1993 . . . . . . . . . . . . . . 9-5

9-3 Constituent List and Summary of Results for the 216-A-10 Crib Data for Reporting Period October 1 through December 31, $1993 \ldots$. . . . 9-6

9-4 Constituents with at Least One Detected Value for the 216-A-10 Crib Data for Reporting Period October 1 through December 31, 1993 . . . 9-10

9-5 Contamination Indicator Parameters for the 216-A-10 Crib Data for Reporting Period October 1 through December 31, $1993 \ldots$. . . . 9-14 


\title{
$9.0216-A-10$ CRIB
}

\author{
G. L. Kasza \\ Westinghouse Hanford Company
}

\subsection{INTRODUCTION}

The 216-A-10 Crib is a retired LWDF for the PUREX Plant. The 216-A-10 Crib is located in the 200 East Area approximately $122 \mathrm{~m}(400 \mathrm{ft})$ south of the PUREX Plant (Figure 1-1).

In accordance with the Tri-Party Agreement (Ecology et al. 1992), Milestone M-20-33, RCRA Closure/Post-Closure plans are to be submitted to Ecology and the EPA in March 1996. An interim-status RCRA grolindwater monitoring network has been in place since November 1988 .

Several waste streams, collectively referred to as the Process Distillate Discharge, were disposed of in the crib to percolate through the soil column. Liquid waste was first disposed of for a 4-month period during the PUREX startup in 1956. In 1961, the 216-A-10 Crib replaced the 216-A-5 Crib and received PUREX effluent continuously until 1973. Periodic discharges were received in 1977, 1978, and 1981. From 1982 to 1987, effluent discharges resumed on a continuous basis. Discharge between 1981 and 1986 averaged $1 \times 10^{8} \mathrm{~L}\left(2.6 \times 10^{6} \mathrm{gal}\right)$ each year. In 1987, the 216-A-10 Crib was retired and replaced by the $216-\mathrm{A}-45 \mathrm{Crib}$.

The waste stream was characteristically acidic and contained concentrated salts. Some of the waste stream constituents included al iphatic hydrocarbon compounds, organic complexants, and radionuclides (plutonium, uranium, strontium-90, cobalt -60 , cesium-134 and -137 , ruthenium-103 and -106 , and tritium).

The RCRA groundwater detection monitoring system consists of six downgradient and two upgradient wells, as 1isted in Table 9-1. Of the downgradient wells, 299-E17-1 and 299-E24-2 are predated RCRA well construction standards and consequently are not used for statistical comparison purposes. Well locations are shown in Figure 9-1. Background monitoring was completed in the fourth quarter of 1989 following the first four quarters of groundwater sampling.

\subsection{WATER LEVEL MEASUREMENTS}

Water level measurements are made on a quarterly basis and during semiannual RCRA sample collection. Water level data collected during the fourth quarter of 1993 are reported in Table 9-2. Water levels recorded for wells 299-E17-20 (on October 15, 1993), 299-E24-18 (on December 7, 1993), 299-E24-2 (on October 20, 1993), and 299-E25-36 (on both October 15 and December 7, 1993) are outside of expected ranges and are suspected of error. 


\subsection{WATER CHEMISTRY DATA}

Wells in the 216-A-10 Crib groundwater monitoring network were sampled during the report period for the semiannual evaluation. Wells 299-E17-20 and 299-E25-36 also provide regional data for the 216-A-29 assessment monitoring program.

The list of analytes reported for the 216-A-10 network is provided in Table 9-3. Constituents with at least one detected value are provided in Table 9-4. The D flags for nitrate and sulfate indicate that the samples were diluted for analysis. Table 9-5 lists the results of the CIP analyses. No CIP replicate averages exceeded the calculated critical means.

DWSs for unfiltered chromium were exceeded in samples from wells 299-E17-19, 299-E17-20, 299-E24-16, 299-E24-17, 299-E24-18, and 299-E25-36. The DWS for unfiltered iron was exceeded in wells 299-E17-19, 299-E17-20, 299-E24-16, 299-E24-17, 299-E24-18, and 299-E25-36. Nitrate standards were exceeded in wells 299-E17-1, 299-E17-19, 299-E17-20, 299-E24-16 (two samples), and 299-E24-17. The standard for 106-ruthenium was exceeded in wells 299-E24-16 and 299-E24-18. Tritium standards were exceeded in all wells of the 216-A-10 monitoring network except well 299-E25-36.

RADEs have been prepared to investigate the large variation from historical concentrations for manganese and nitrite samples collected at well 299-E17-19 during this quarter.

Analytical data for isotopic uranium were unavailable for inclusion in this report and will be presented in a future quarterly report. 


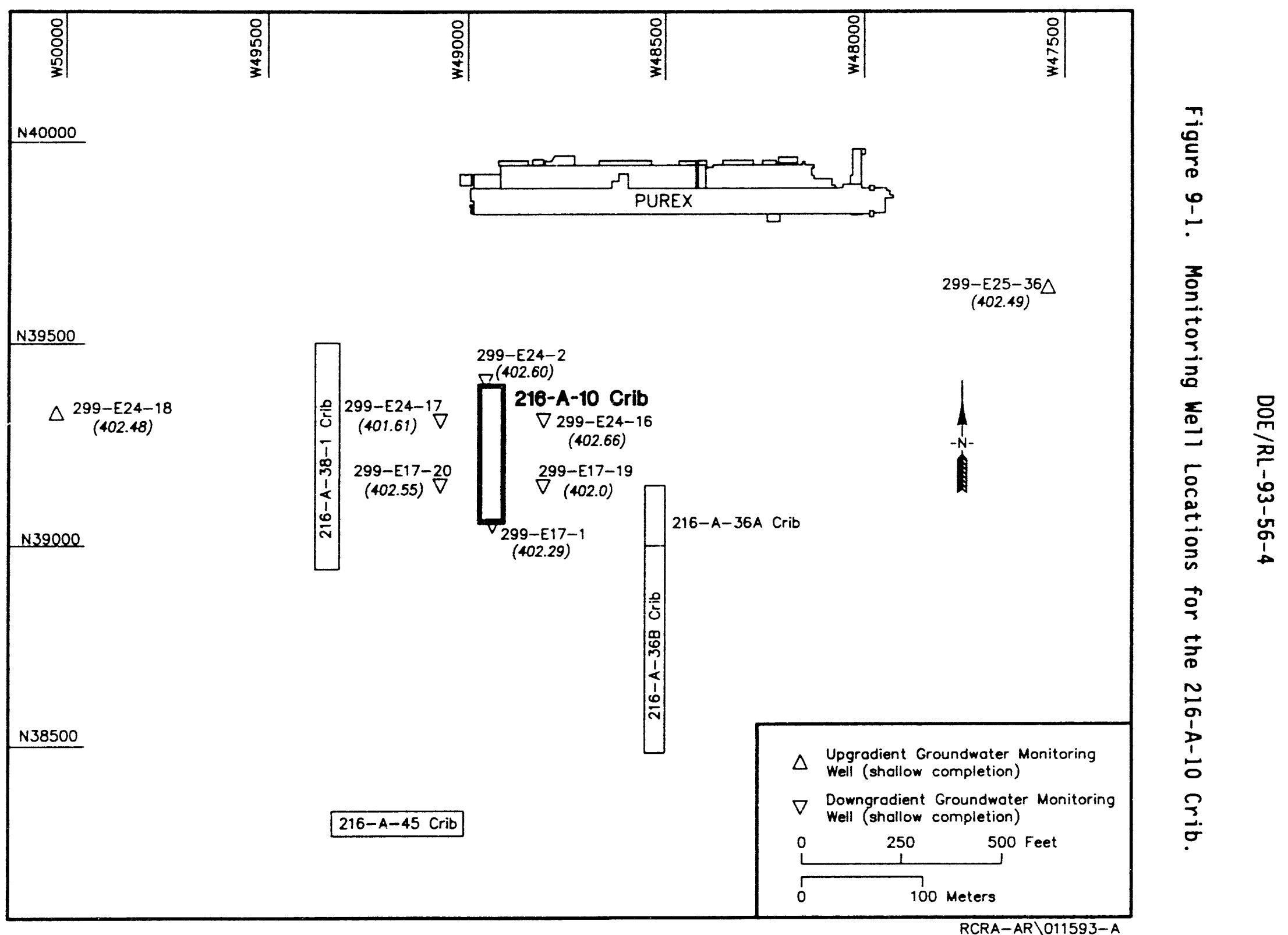


Table 9-1. Monitoring Well Purpose and Sampling Schedule for the 216-A-10 Crib Network.

\begin{tabular}{|c|c|c|c|c|}
\hline $\begin{array}{c}\text { Well no. } \\
(299-)\end{array}$ & $\begin{array}{c}\text { Relative } \\
\text { position }\end{array}$ & Hydrogeologic unit & $\begin{array}{c}\text { Sample } \\
\text { frequency }\end{array}$ & $\begin{array}{c}\text { Sample date, } \\
\text { 4th Qtr 1993 }\end{array}$ \\
\hline E24-18 & Upgradient & Ringold: Water Table & Semiannually & $10 / 21 / 93$ \\
\hline E25-36 & Upgradient & Ringold: Water Table & Semiannually & $10 / 15 / 93$ \\
\hline E17-1 & Downgradient & Ringold: Water Table & Semiannually & $10 / 12 / 93$ \\
\hline E17-19 & Downgradient & Ringold: Water Table & Semiannually & $10 / 20 / 93$ \\
\hline E17-20 & Downgradient & Ringold: Water Table & Semiannually & $10 / 15 / 93$ \\
\hline E24-2 & Downgradient & Ringold: Water Table & Semiannually & $10 / 20 / 93$ \\
\hline E24-16 & Downgradient & Ringold: Water Table & Semiannually & $10 / 11 / 93$ \\
\hline E24-17 & Downgradient & Ringold: Water Table & Semiannually & $10 / 11 / 93$ \\
\hline
\end{tabular}


Table 9-2. RCRA Water Level Measurement Report for the 216-A-10 Crib, Fourth Quarter 1993.

\begin{tabular}{|c|c|c|c|}
\hline Well & Date & $\begin{array}{l}\text { Depth to } \\
\text { water (ft) }\end{array}$ & $\begin{array}{l}\text { Water level } \\
\text { elevation } \\
\text { above msl (ft) }\end{array}$ \\
\hline 299-E17-1 & $\begin{array}{l}10 / 12 / 93 \\
12 / 07 / 93\end{array}$ & $\begin{array}{l}318.28 \\
317.60\end{array}$ & $\begin{array}{l}400.89 \star \\
401.57\end{array}$ \\
\hline 299-E17-19 & $\begin{array}{l}10 / 20 / 93 \\
12 / 07 / 93\end{array}$ & $\begin{array}{l}318.39 \\
318.68\end{array}$ & $\begin{array}{l}400.94^{\star} \\
400.65\end{array}$ \\
\hline $299-E 17-20$ & $\begin{array}{l}10 / 15 / 93 \\
12 / 07 / 93\end{array}$ & $\begin{array}{l}321.66 \\
317.65\end{array}$ & $\begin{array}{l}397.57^{\star}+ \\
401.58\end{array}$ \\
\hline 299-E24-16 & $10 / 11 / 93$ & 316.84 & $401.43^{\star}$ \\
\hline 299-E24-17 & $10 / 11 / 93$ & 318.05 & $400.64^{\star}$ \\
\hline 299-E24-18 & $\begin{array}{l}10 / 21 / 93 \\
12 / 07 / 93\end{array}$ & $\begin{array}{l}317.73 \\
316.96\end{array}$ & $\begin{array}{l}401.55^{\star} \\
402.32 t\end{array}$ \\
\hline 299-E24-2 & $\begin{array}{l}10 / 20 / 93 \\
12 / 07 / 93\end{array}$ & $\begin{array}{l}316.76 \\
315.51\end{array}$ & $\begin{array}{l}400.71^{\star}+ \\
401.96\end{array}$ \\
\hline 299-E25-36 & $\begin{array}{l}10 / 15 / 93 \\
12 / 07 / 93\end{array}$ & $\begin{array}{l}306.72 \\
305.30\end{array}$ & $\begin{array}{l}400.67^{\star}+ \\
402.09+\end{array}$ \\
\hline
\end{tabular}

Notes: 1. Water level elevations are calculated by subtracting the measured depth to water from the surveyed elevation for the well.

2. Depth-to-water values are transcribed from field records.

3. Elevations marked with an ' $*$ ' were measured at the time of sampling.

4. Elevations marked with a ' + ' are outside of the expected range and are suspected of error.

5. To convert feet to meters multiply by 0.3048 . 
Table 9-3. Constituent List and Summary of Results for the 216-A-10 Crib Data for Reporting Period October 1 through December 31, 1993. (sheet 1 of 4)

CONTAMINATION INDICATOR PARAMETERS

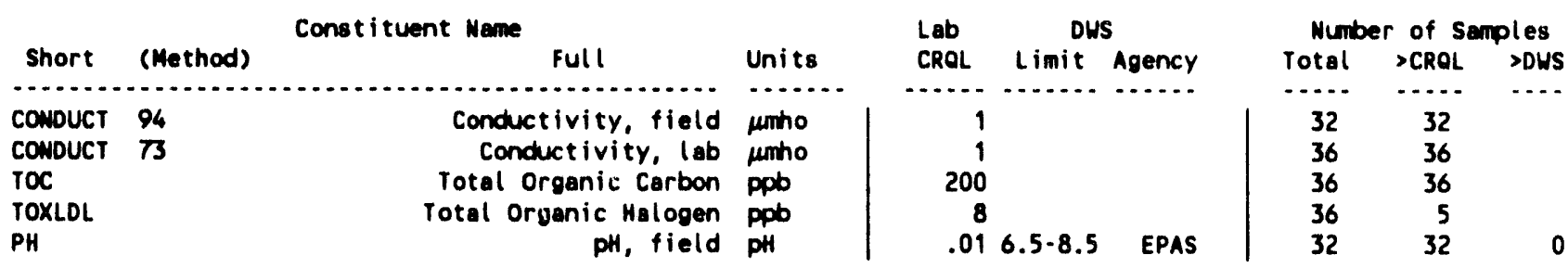

DRINKING WATER PARAMETERS

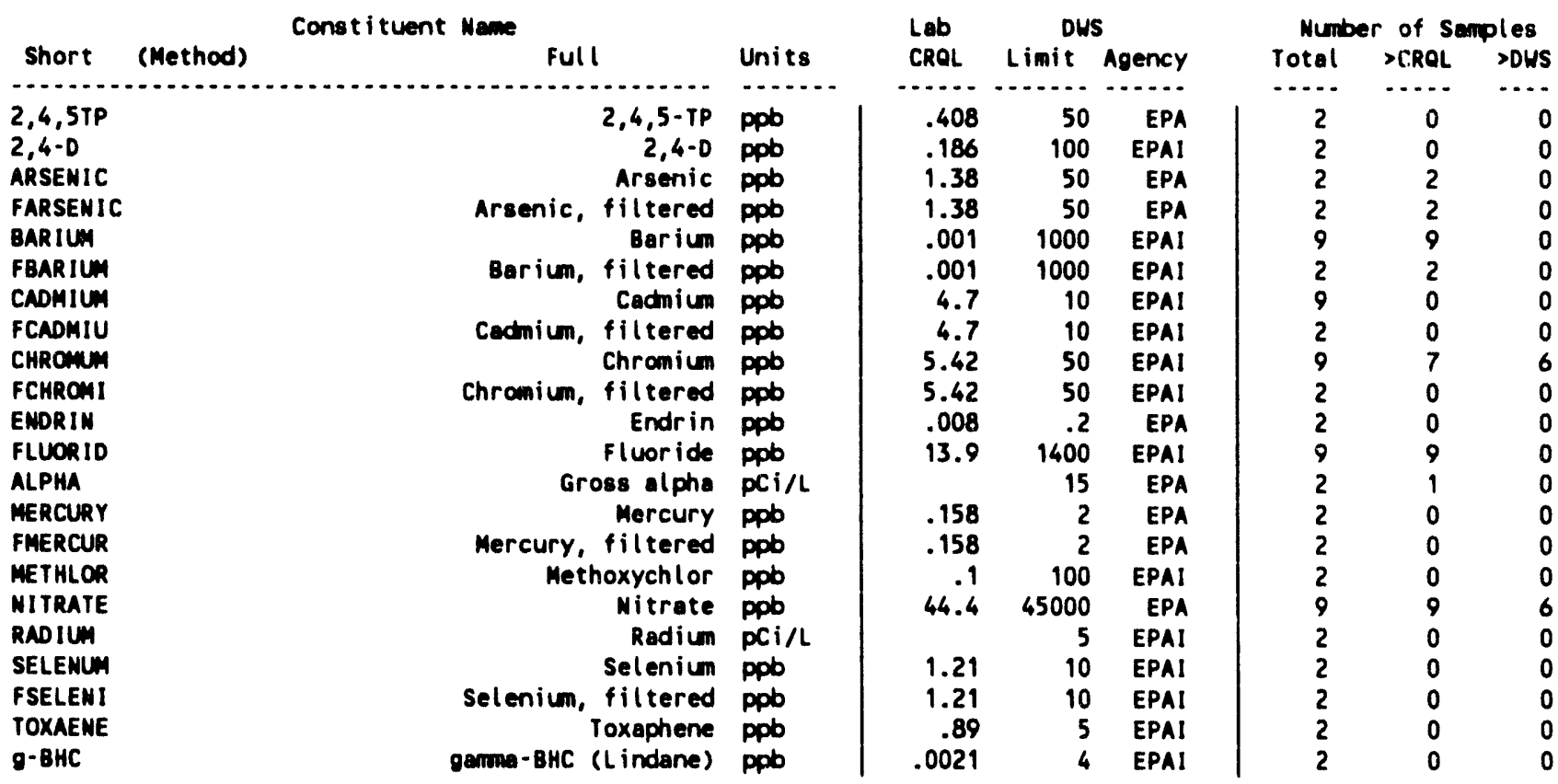

GROUNDWATER QUALITY PARAMETERS

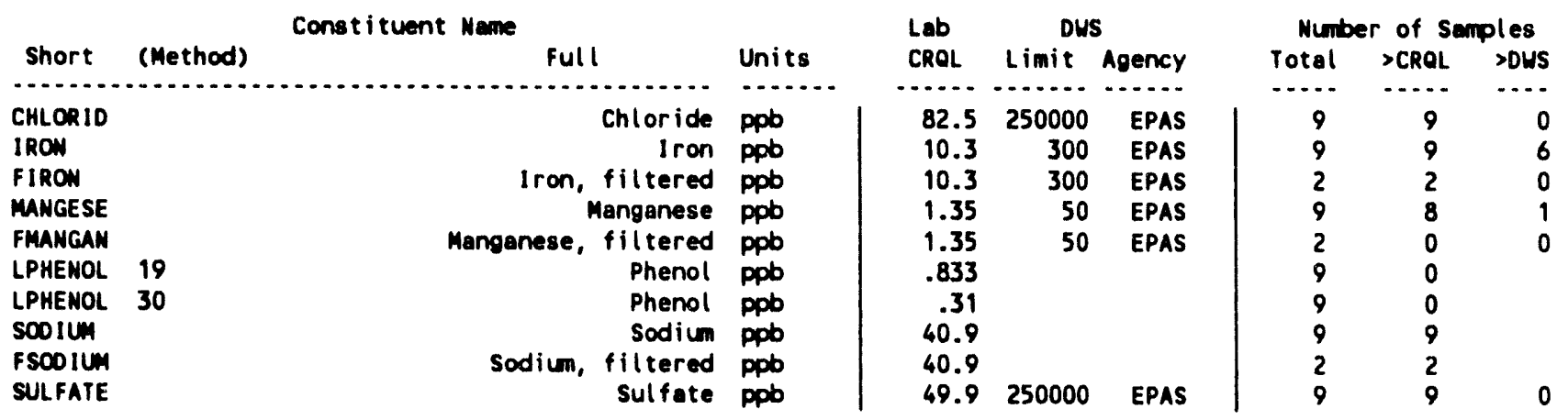


Table 9-3. Constituent List and Summary of Results for the 216-A-10 Crib Data for Reporting Period October 1 through December 31, 1993 . (sheet 2 of 4)

SITE SPECIFIC AMD OTHER CONSTITUENTS

\begin{tabular}{|c|c|c|c|c|c|c|c|c|c|}
\hline \multirow[b]{2}{*}{ Short } & \multirow{3}{*}{ (let } & \multirow[t]{2}{*}{ Const ituent Neme } & \multirow[b]{2}{*}{ Unit8 } & \multirow{2}{*}{$\begin{array}{l}\text { Lab } \\
\text { CRaL }\end{array}$} & \multicolumn{2}{|l|}{ DUS } & \multicolumn{3}{|c|}{ Number of Semples } \\
\hline & & & & & Limit & Agency & Total & $>$ CRQL & $>$ DWS \\
\hline$\ldots \ldots$ & & 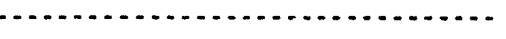 & 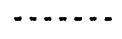 & $\cdots \cdots$ & $\cdots+\cdots$ & $\ldots \ldots$ & $\cdots \cdots$ & $\cdots \cdots$ & $\cdots$ \\
\hline $1,1,1-T$ & & 1,1,1-Trichloroethane & ppo & .64 & 200 & EPA & 9 & 1 & 0 \\
\hline $1,1,2-1$ & & 1,1,2-Trichloroethane & ppb & .25 & & & 9 & 0 & \\
\hline $1,1-01 C$ & & 1,1-Dichloroethane & ppb & .61 & & & 9 & 0 & \\
\hline $1,2-D I C$ & & 1.2-Dichloroethane & ppb & .45 & 5 & EPA & 9 & 0 & 0 \\
\hline 12-DCE & & 1,2-Dichloroethene & ppb & 1.2 & & & 9 & 0 & \\
\hline 14-dben & & 1,4-Dichlorobenzene & ppb & .63 & 75 & EPA & 9 & 0 & 0 \\
\hline 18UTYN & & 1-Butanol & ppm & 13.1 & & & 9 & 0 & \\
\hline $\begin{array}{l}2,4,5-1 \\
246-t 50\end{array}$ & & 2,4,5-T & ppb & .384 & & & 2 & 0 & \\
\hline $240-$ trp & 19 & $\begin{array}{r}\text { 2,4,6-Trichlorophenol } \\
\text { 2,4-0ichlorophenol }\end{array}$ & ppb & $\begin{array}{r}1.45 \\
2.8\end{array}$ & & & 9 & 0 & \\
\hline $24-d c h p$ & 30 & 2,4-D ichlorophenol & ppb & 1.5 & & & $\begin{array}{l}9 \\
9\end{array}$ & 0 & \\
\hline DIMPHEN & & 2,4-D inethylphenol & ppb & 1.01 & & & 9 & 0 & \\
\hline DINPHEN & & 2,4-0 initrophenol & ppb & .96 & & & 9 & 0 & \\
\hline 26-dchp & & 2,6-Dichlorophenol & ppb & 1.59 & & & 9 & 0 & \\
\hline CHLPHEN & & 2-Chlorophenol & ppo & 1.42 & & & 9 & 0 & \\
\hline ZMETHPH & & 2-Methylphenol & ppb & 1.8 & & & 9 & 0 & \\
\hline 2NITPH & 19 & 2-Nitrophenol & ppb & 3.96 & & & 9 & 0 & \\
\hline 2NITPH & 30 & 2-Nitrophenol & ppb & 1.56 & & & 9 & 0 & \\
\hline BUTOINP & 30 & 2-sec-Butyl-4,6-dinitrophenol (DN & ppb & 1.35 & & & 9 & 0 & \\
\hline BUTOINP & 49 & 2-sec-Butyl-4,6-dinitrophenol CDN & ppb & .269 & & & 2 & 0 & \\
\hline DDO & & $4,4^{\prime}-000$ & ppb & .0005 & & & 2 & 0 & \\
\hline DDE & & $4,4^{\prime}-$ DOE & ppb & .0009 & & & 2 & 0 & \\
\hline DOT & & $4.41-D D T$ & ppb & .011 & & & 2 & 0 & \\
\hline 460N2NP & & 4,6-0 initro-2-methyiphenol & ppb & 1.18 & & & 9 & 0 & \\
\hline CHLCRES & & 4-Chl oro-3-methylphenol & ppb & 1.12 & & & 9 & 0 & \\
\hline MIBK & & 4-Methyl-2-pentanone & ppb & .85 & & & 9 & 0 & \\
\hline WMETHPH & & 4-Methylphenol & ppb & 3.54 & & & 9 & 0 & \\
\hline NITPHEN & & 4-Nitrophenol & ppob & .65 & & & 9 & 0 & \\
\hline ACETONE & & Acetone & ppo & 13.4 & & & 9 & 0 & \\
\hline ALDRIN & & Aldrin & ppo & .05 & & & 2 & 0 & \\
\hline --ВКс & & Alphe-BHC & ppb & .012 & & & 2 & 0 & \\
\hline ALUNNUA & & Aluminum & ppb & 32.5 & & & 9 & 2 & \\
\hline FALUAIN & & Aluminum & ppb & 32.5 & & & 2 & 0 & \\
\hline AmMONIL & & Ammonium ion & ppo & 38.5 & & & 2 & 2 & \\
\hline ANTIONY & & Ant imony & $p p b$ & 69.4 & & & 9 & 0 & \\
\hline FANTIMO & & Ant imony, filtered & pob & 69.4 & & & 2 & 0 & \\
\hline $58-125$ & & Ant imony-125 & $\mathrm{PCi} / \mathrm{L}$ & & & & 9 & 0 & \\
\hline BENZENE & & Benzene & ppb & .65 & 5 & EPA & 9 & 0 & 0 \\
\hline BENZOTH & & Benzothiazole & ppo & 2.55 & & & 9 & 0 & \\
\hline BERYLUM & & Beryllium & $p p b$ & .814 & & & 9 & 2 & \\
\hline FBERYLL & & Beryllium, filtered & ppb & .814 & & & 2 & 0 & \\
\hline b-BHC & & Beta-BHC & ppb & .0026 & & & 2 & 0 & \\
\hline B1S2EPH & & Bis(2-ethylhexyl) phthalate & ppb & 4.07 & & & 9 & 0 & \\
\hline BROMIDE & & Bromide & ppb & 52.8 & & & 9 & 0 & \\
\hline calciun & & Calciun & ppb & 20.9 & & & 9 & 9 & \\
\hline FCALCIU & & Calcium, filtered & ppb & 20.9 & & & 2 & 2 & \\
\hline CARBIDE & & Carbon disulfide & ppb & .95 & & & 9 & 0 & \\
\hline TETRANE & & Carbon tetrachloride & ppb & .87 & 5 & EPA & 9 & 0 & 0 \\
\hline $\operatorname{cs}-137$ & & Cesium-137 & $\mathrm{pCi} / \mathrm{L}$ & & 200 & EPA & 9 & 0 & 0 \\
\hline CHLOANE & & Chlordane & ppo & .0057 & 2 & EPA & 2 & 0 & 0 \\
\hline CHLFORM & & Chloroform & ppb & .4 & & & 9 & 0 & \\
\hline COBALT & & Cobalt & ppb & 4.05 & & & 9 & 0 & \\
\hline FCOBALT & & Cobalt, filtered & ppb & 4.05 & & & 2 & 0 & \\
\hline
\end{tabular}


Table 9-3. Constituent List and Summary of Results for the 216-A-10 Crib Data for Reporting Period October 1 through December 31, 1993. (sheet 3 of 4)

\begin{tabular}{|c|c|c|c|c|c|c|c|c|c|}
\hline \multirow[b]{2}{*}{ Short } & \multirow{3}{*}{$\begin{array}{l}\text { (Method) } \\
\text { (M........... }\end{array}$} & \multirow[t]{2}{*}{ Const ituent Name } & \multirow[b]{2}{*}{ Units } & \multirow{2}{*}{$\begin{array}{l}\text { Lab } \\
\text { CROL }\end{array}$} & \multicolumn{2}{|c|}{ DUS } & \multicolumn{3}{|c|}{ Number of Samples } \\
\hline & & & & & Limit & Agency & Total & $>$ CRQL & $>$ DWS \\
\hline 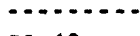 & & $\cdots \cdots$ & $\cdots \ldots$ & $\cdots \cdots$ & $\cdots-\cdots$ & $\cdots \cdots$ & $\cdots-\cdot$ & $\cdots \cdots$ & $\cdots \cdots$ \\
\hline$c 0-60$ & & Cobalt -60 & $p C i / L$ & & 100 & EPA & 9 & 0 & 0 \\
\hline COL I FORM & & Coliforms & $\mathrm{COL}$ & 1 & 1 & EPAI & 2 & 0 & 0 \\
\hline COPPER & & Copper & ppb & 2.65 & 1000 & EPAS & 9 & 5 & 0 \\
\hline FCOPPER & & Copper, filtered & ppb & 2.65 & 1000 & EPAS & 2 & 0 & 0 \\
\hline CRESOLS & & Cresols (methylphenols) & ppb & 4.66 & & & 9 & 0 & \\
\hline DECANE & & Decane & ppb & 4.03 & & & 9 & 0 & \\
\hline d-BHC & & Del ta-BHC & ppb & .0014 & & & 2 & 0 & \\
\hline DIELRIN & & Dieldrin & ppb & .019 & & & 2 & 0 & \\
\hline DODECAN & & Dodecane & ppob & 3.62 & & & 9 & 0 & \\
\hline ENDO1 & & Endosulfan 1 & ppb & .0029 & & & 2 & 0 & \\
\hline ENDO2 & & Endosulfan II & ppb & .004 & & & 2 & 0 & \\
\hline ENDSFAN & & Endosulfon sulfate & ppb & .0072 & & & 2 & 0 & \\
\hline ENDRALD & & Endrin Aldehyde & ppb & .011 & & & 2 & 0 & \\
\hline ETHCYAN & & Ethyl cyanide & ppb & 4.34 & & & 9 & 0 & \\
\hline BETA & & Gross beta & $\mathrm{pCi} / \mathrm{L}$ & & & & 2 & 2 & \\
\hline MEPTLOR & & Heptachlor & ppb & .0019 & .4 & EPA & 2 & 0 & 0 \\
\hline HEPTIDE & & Heptachlor epoxide & ppb & .0008 & .2 & EPA & 2 & 0 & 0 \\
\hline L HYDRAZ & & Hydrazine & ppb & 1.89 & & & 2 & 0 & \\
\hline LEAD & & Lead & ppb & .508 & 50 & EPAI & 2 & 2 & 0 \\
\hline FLEAD & & Lead, filtered & ppb & .508 & 50 & EPAI & 2 & 2 & 0 \\
\hline MAGNES & & Magnesium & ppb & 26 & & & 9 & 9 & \\
\hline FMANES & & Magnesiun, filtered & ppb & 26 & & & 2 & 2 & \\
\hline METHONE & & Methyl ethyl ketone & popb & 4.25 & & & 9 & 0 & \\
\hline METHYCH & & Methylene chloride & ppb & .61 & & & 9 & 0 & \\
\hline NAPHTHA & & Naphthal ene & ppb & 6.5 & & & 9 & 0 & \\
\hline NICKEL & & Nickel & ppb & 17.9 & & & 9 & 7 & \\
\hline FNICKEL & & Nickel, filtered & ppb & 17.9 & & & 2 & 0 & \\
\hline MITRITE & & Nitrite & ppb & 38.3 & 1000 & EPA & 9 & 1 & 1 \\
\hline PENTCHP & 19 & Pentechlorophenol & ppo & 8.07 & 1 & EPA & 9 & 0 & 9* \\
\hline PENTCHP & 30 & Pent ach lorophenol & ppb & .87 & 1 & EPA & 9 & 0 & 0 \\
\hline PHOSPHA & & Phosphate & ppb & 147 & & & 9 & 0 & \\
\hline POTASUA & & Potassium & ppb & 662 & & & 9 & 9 & \\
\hline FPOTASS & & Potassium, filtered & ppb & 662 & & & 2 & 2 & \\
\hline$R U-106$ & & Ruthenium-106 & $\mathrm{pCi} / \mathrm{L}$ & & 30 & EPA & 9 & 2 & 2 \\
\hline SILVER & & silver & ppb & 2.87 & 50 & EPAI & 9 & 0 & 0 \\
\hline FSILVER & & silver, filtered & ppb & 2.87 & 50 & EPAI & 2 & 0 & 0 \\
\hline PERCENE & & Tetrachloroethene & ppb & 1.1 & 5 & EPA & 9 & 0 & 0 \\
\hline TETPHNL & & Tetrachlorophenols & ppb & 1.05 & & & 9 & 0 & \\
\hline TETRADE & & Tetradecane & ppb & 2.43 & & & 9 & 0 & \\
\hline TAF & & Tetrahydrofuran & ppb & 3.67 & & & 9 & 0 & \\
\hline TIN & & Tin & ppb & 51.1 & & & 9 & 0 & \\
\hline FTIM & & Tin, filtered & ppb & 51.1 & & & 2 & 0 & \\
\hline TOLUENE & & Tolvene & ppb & .73 & 1000 & EPA & 9 & 0 & 0 \\
\hline TRIBUTPH & & Tributyl Phosphate & ppb & 4.42 & & & 9 & 0 & \\
\hline TRICENE & & Trichloroethene & ppb & .77 & 5 & EPA & 9 & 0 & 0 \\
\hline TRIPHNL & & Trichlorophenols & ppb & 1.11 & & & 9 & 0 & \\
\hline TOICHPH & & Tris-2-chlorcethyl phosphate & ppb & 2.88 & & & 9 & 0 & \\
\hline TRITIUN & & Tritium & $\mathrm{PCi} / \mathrm{L}$ & & 20000 & EPA & 9 & 9 & 8 \\
\hline TURBID & & Turbidity & NTU & .05 & & & 2 & 2 & \\
\hline$U-234$ & & Uranium-234 & $\mathrm{pCi} / \mathrm{L}$ & & & & 9 & 9 & \\
\hline$U-235$ & & Uranium-235 & $\mathrm{PCi} / \mathrm{L}$ & & & & 9 & 6 & \\
\hline$U-238$ & & Uranium-238 & $\mathrm{pCi} / \mathrm{L}$ & & & & 9 & 9 & \\
\hline VANADUM & & Vanadium & ppb & 3.84 & & & 9 & 7 & \\
\hline FVANAD I & & Vanediun, filtered & ppb & 3.84 & & & 2 & 0 & \\
\hline VINYIOE & & Vinyl chloride & ppb & .78 & 2 & EPA & 9 & 0 & 0 \\
\hline XYLENE & & Xylenes (total) & ppb & 1.7 & 10000 & EPA & 9 & 0 & 0 \\
\hline
\end{tabular}


Table 9-3. Constituent List and Summary of Results for the 216-A-10 Crib Data for Reporting Period October 1 through December 31, 1993. (sheet 4 of 4)

\begin{tabular}{|c|c|c|c|c|c|c|c|c|c|}
\hline \multirow[b]{2}{*}{ Short } & \multirow[b]{2}{*}{ (Method) } & \multirow[t]{2}{*}{ Constituent Neme } & \multirow[b]{2}{*}{ Un } & \multirow{2}{*}{$\begin{array}{l}\text { Lab } \\
\text { CRAL }\end{array}$} & \multicolumn{2}{|c|}{ DWS } & \multicolumn{3}{|c|}{ Number of Semples } \\
\hline & & & & & Limit & Agency & Total & $>C R Q L$ & $>$ OWS \\
\hline $\begin{array}{l}\text { ZINC } \\
\text { FZINC } \\
\text { MCRESOL }\end{array}$ & & $\begin{array}{r}\text { zinc } \\
\text { zinc, filtered } \\
m \text {-Cresol }\end{array}$ & $\begin{array}{l}\text { ppb } \\
\text { ppb } \\
\text { ppb }\end{array}$ & $\begin{array}{l}3.44 \\
3.44 \\
1.44\end{array}$ & $\begin{array}{l}5000 \\
5000\end{array}$ & $\begin{array}{l}\text { EPAS } \\
\text { EPAS }\end{array}$ & $\begin{array}{l}9 \\
2 \\
9\end{array}$ & $\begin{array}{l}8 \\
1 \\
0\end{array}$ & $\begin{array}{l}0 \\
0\end{array}$ \\
\hline
\end{tabular}

For explanation of this table, see section 1.4 of report. 
Table 9-4. Constituents with at Least One Detected Value for the 216-A-10 Crib Data for Reporting Period October 1 through December 31, 1993. (sheet 1 of 4)

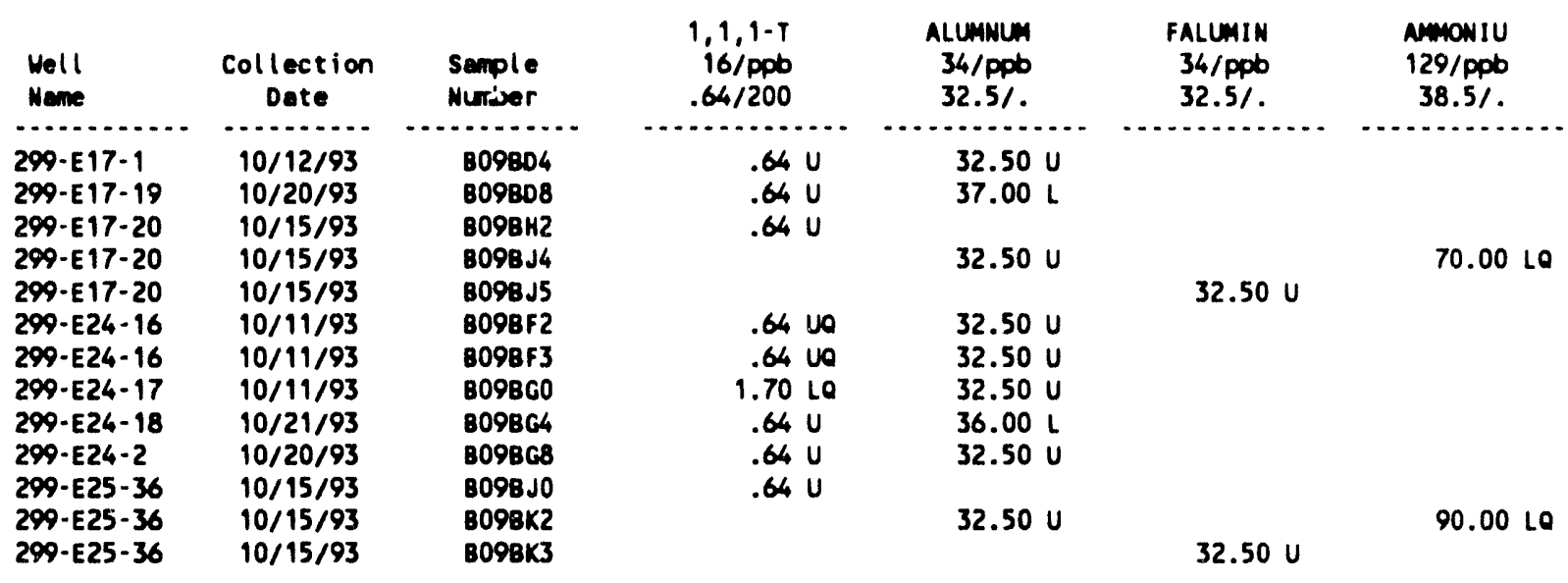

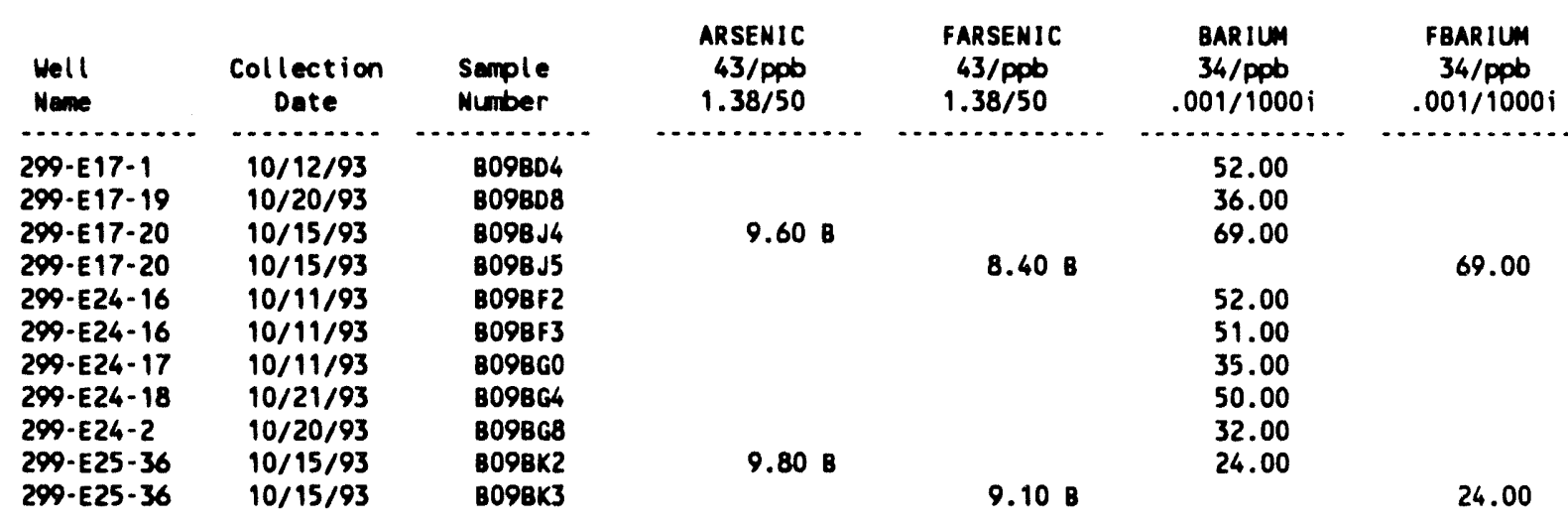

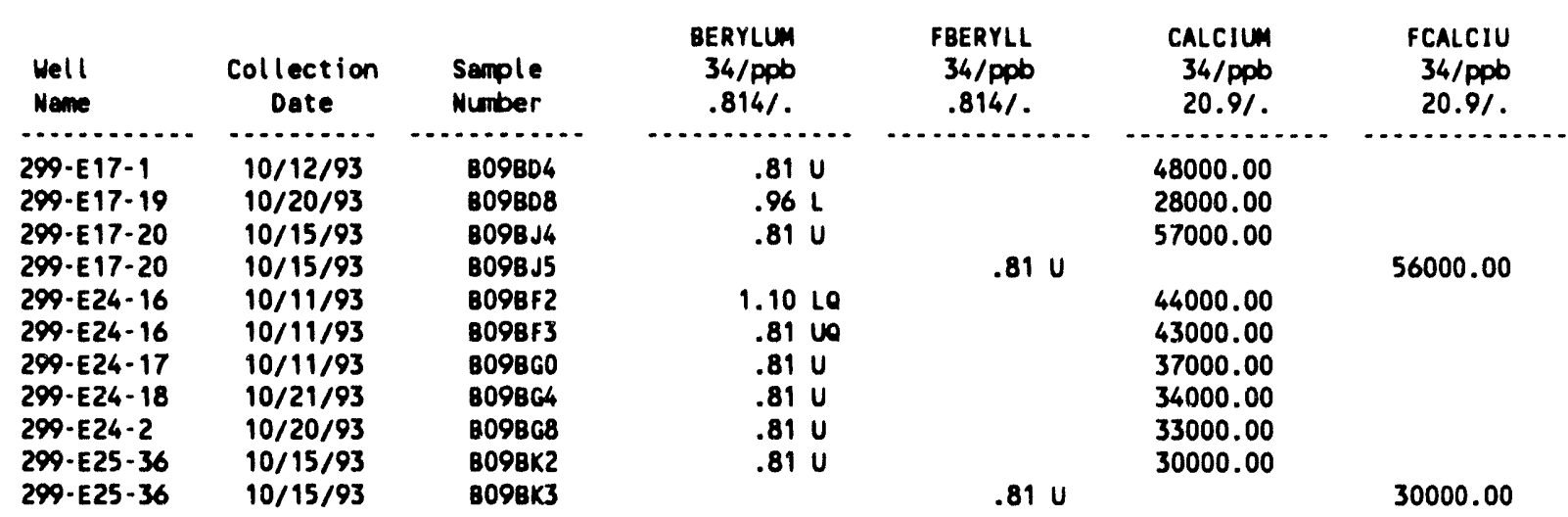


Table 9-4. Constituents with at Least One Detected Value for the 216-A-10 Crib Data for Reporting Period October 1 through December 31, 1993. (sheet 2 of 4)

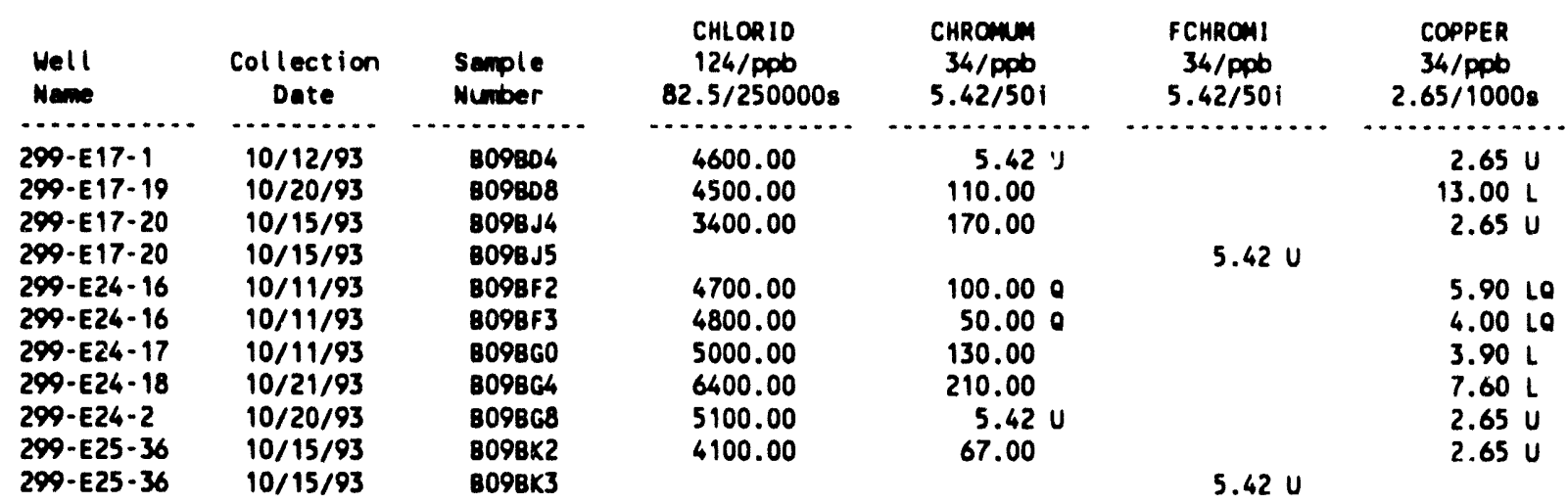

\begin{tabular}{|c|c|c|c|c|c|c|}
\hline $\begin{array}{l}\text { Nell } \\
\text { Newe }\end{array}$ & $\begin{array}{c}\text { Collection } \\
\text { Date }\end{array}$ & $\begin{array}{l}\text { Sample } \\
\text { Number }\end{array}$ & $\begin{array}{c}\text { FCOPPER } \\
34 / \mathrm{ppb} \\
2.65 / 10008\end{array}$ & $\begin{array}{l}\text { FLUORID } \\
124 / p p b \\
13.9 / 1400 i\end{array}$ & $\begin{array}{c}\text { ALPHA } \\
135 / P C i / L \\
. / 15\end{array}$ & $\begin{array}{c}\text { BETA } \\
136 / \mathrm{DC} / \mathrm{L} \\
.1\end{array}$ \\
\hline $\begin{array}{l}299-E 17-1 \\
299-E 17-19 \\
299-E 17-20 \\
299-E 17-20 \\
299-E 24-16 \\
299-E 24-16 \\
299-E 24-17 \\
299-E 24-18 \\
299-E 24-2 \\
299-E 25-36 \\
299-E 25-36\end{array}$ & $\begin{array}{l}10 / 12 / 93 \\
10 / 20 / 93 \\
10 / 15 / 93 \\
10 / 15 / 93 \\
10 / 11 / 93 \\
10 / 11 / 93 \\
10 / 11 / 93 \\
10 / 21 / 93 \\
10 / 20 / 93 \\
10 / 15 / 93 \\
10 / 15 / 93\end{array}$ & $\begin{array}{l}\text { 809BD4 } \\
\text { B09BD8 } \\
\text { B09BJ4 } \\
\text { B09BJ5 } \\
8098 F 2 \\
809 B F 3 \\
\text { B09BG0 } \\
\text { B09BG4 } \\
\text { B09BG8 } \\
\text { B09BK2 } \\
\text { B09BK3 }\end{array}$ & $\begin{array}{l}2.65 U \\
2.65 U\end{array}$ & $\begin{array}{l}600.00 \\
800.00 \\
400.00 \\
500.00 \\
400.00 \\
500.00 \\
800.00 \\
900.00 \\
400.00\end{array}$ & $.79 \mathrm{U}$ & 33.80 \\
\hline
\end{tabular}

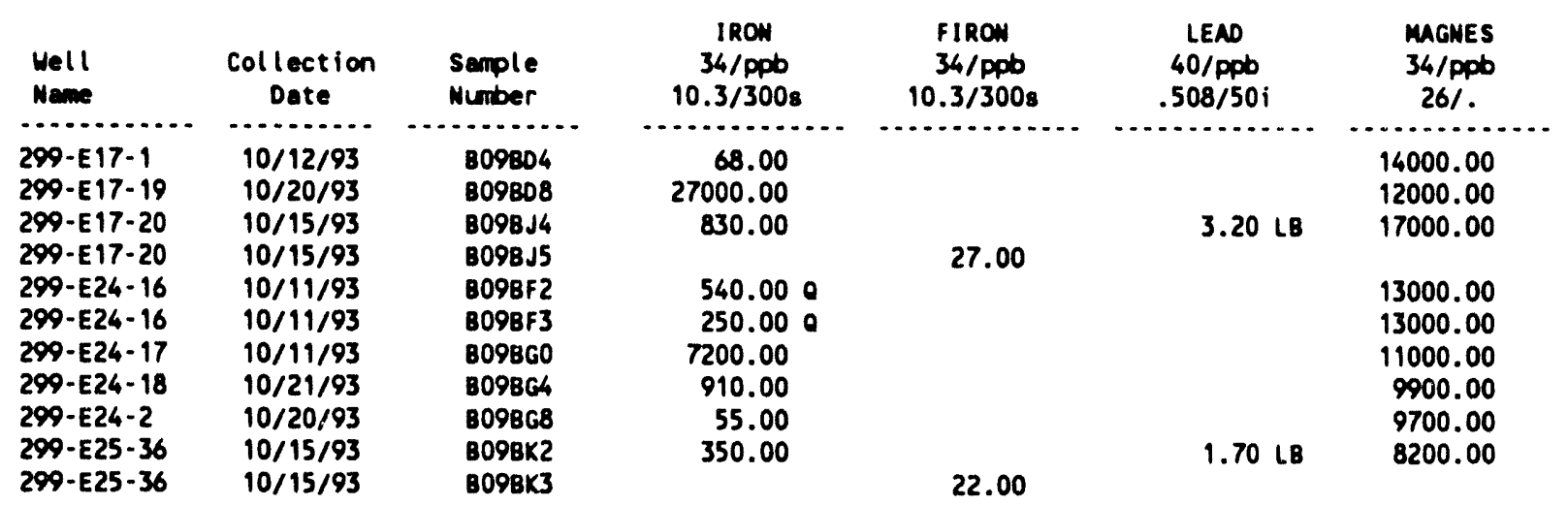


Table 9-4. Constituents with at Least One Detected Value for the 216-A-10 Crib Data for Reporting Period October 1 through December 31, 1993. (sheet 3 of 4)

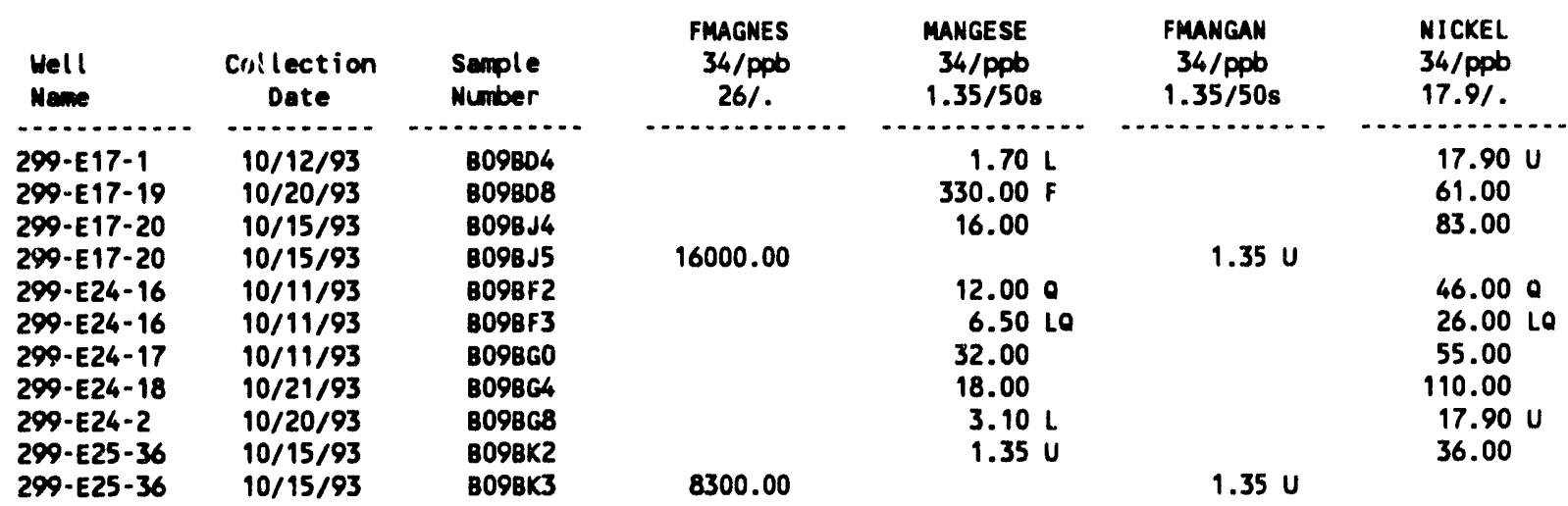

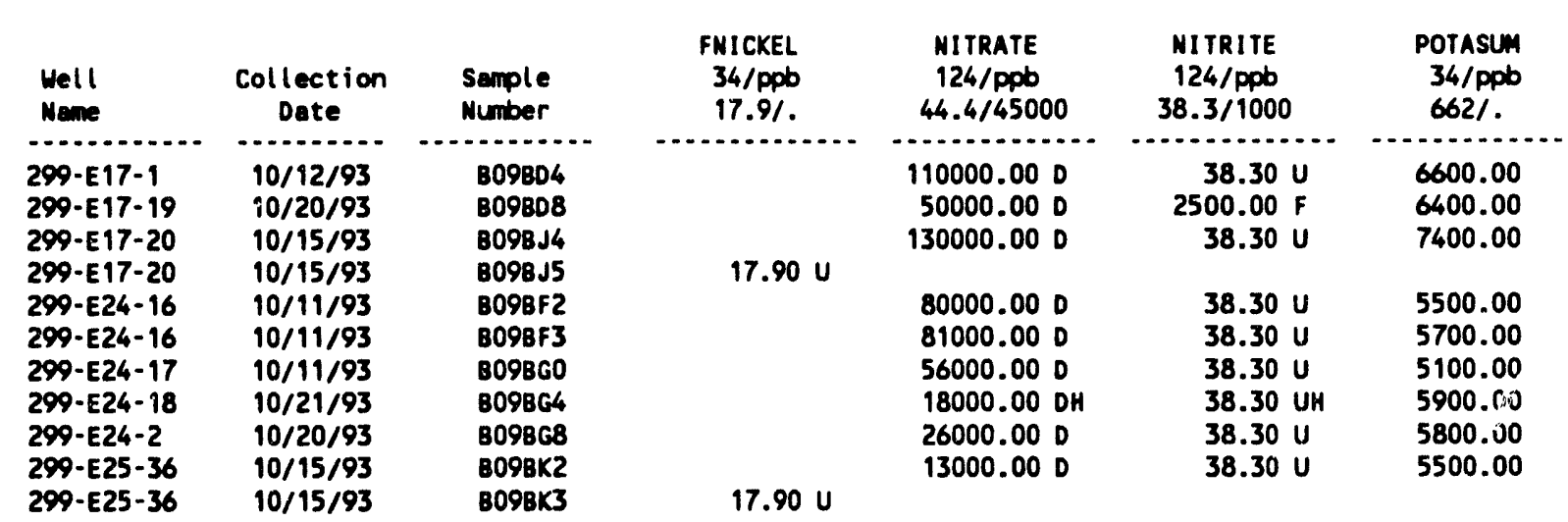

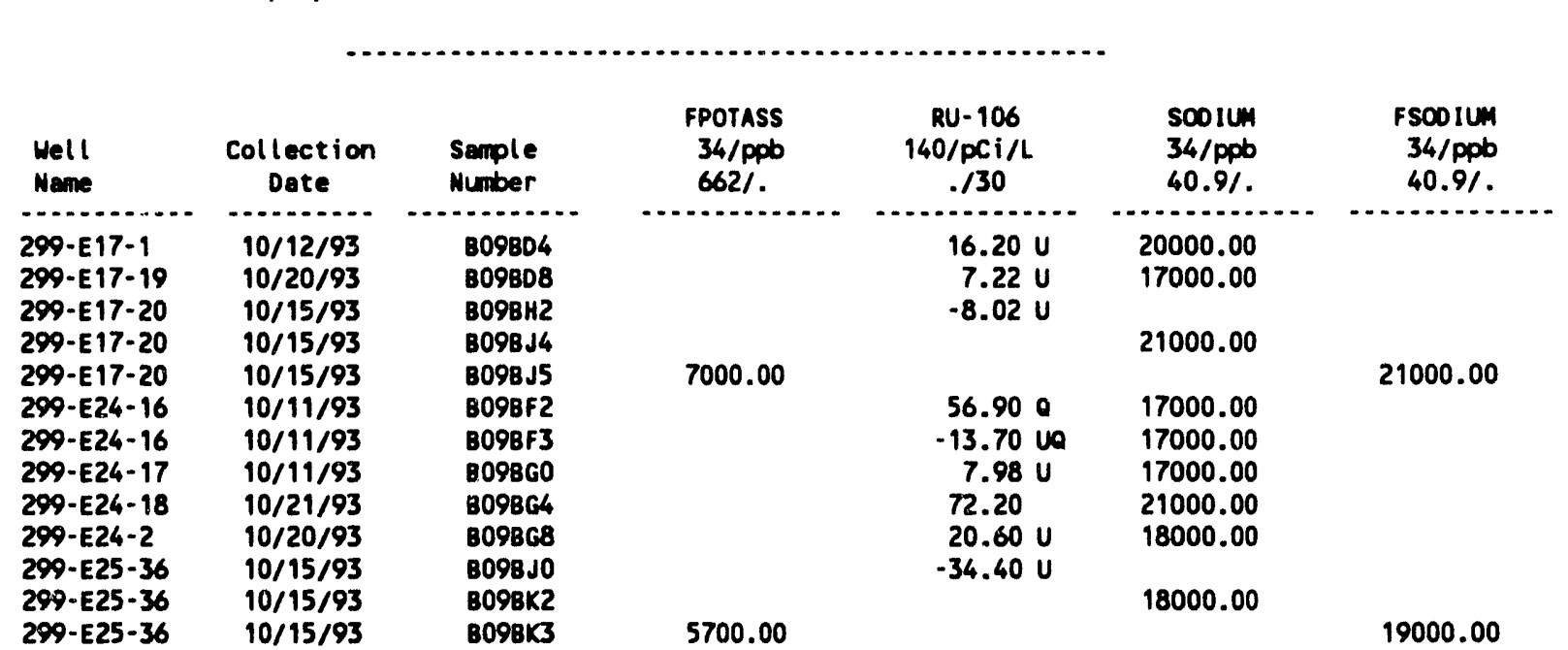


Table 9-4. Constituents with at Least One Detected Value for the 216-A-10 Crib Data for Reporting Period October 1 through December 31, 1993. (sheet 4 of 4)

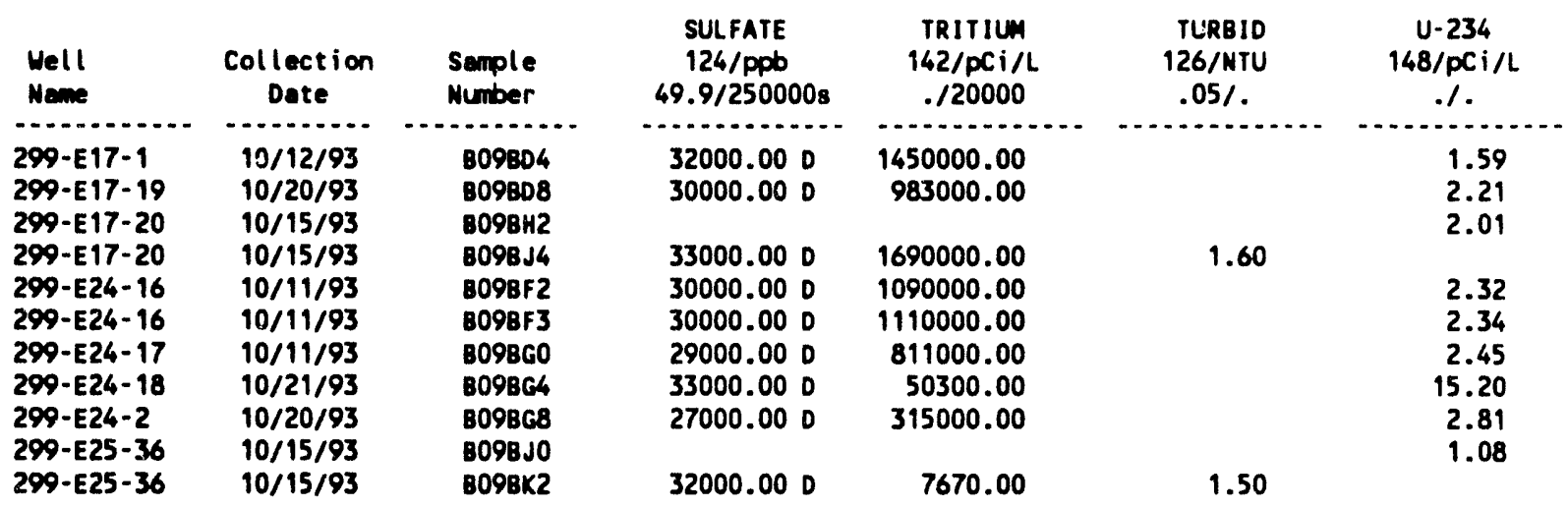

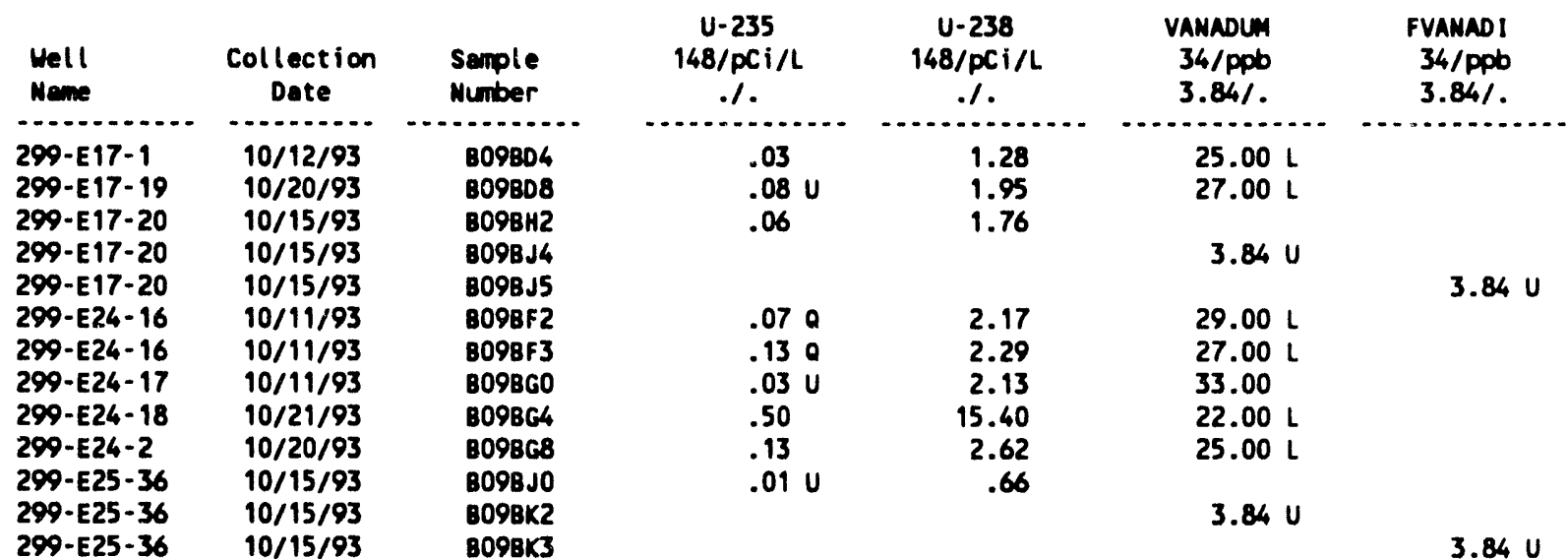

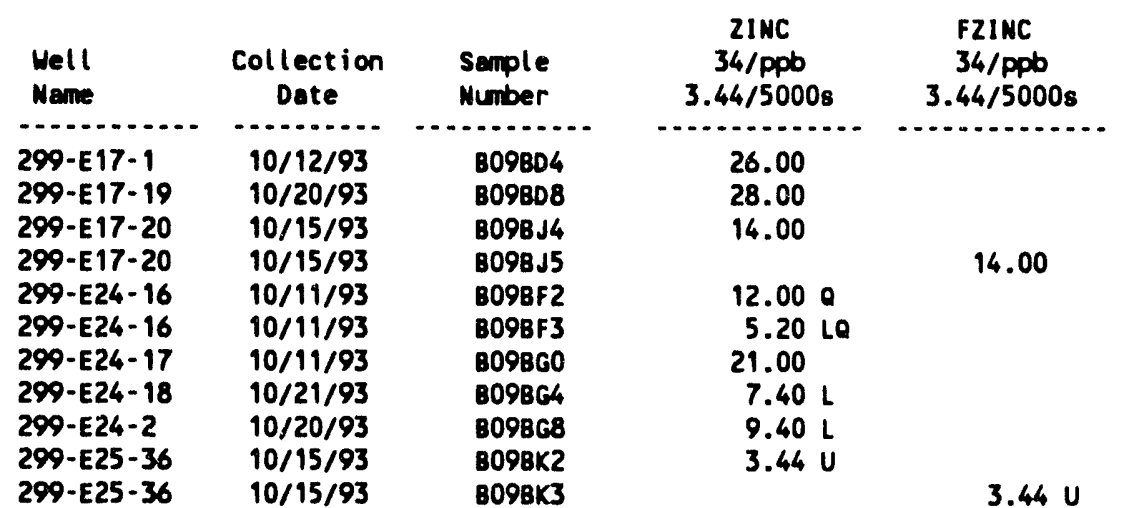

For explenation of this table, see section 1.4 of report. 
Table 9-5. Contamination Indicator Parameters for the 216-A-10 Crib Data for Reporting Period October 1 through December 31, 1993.

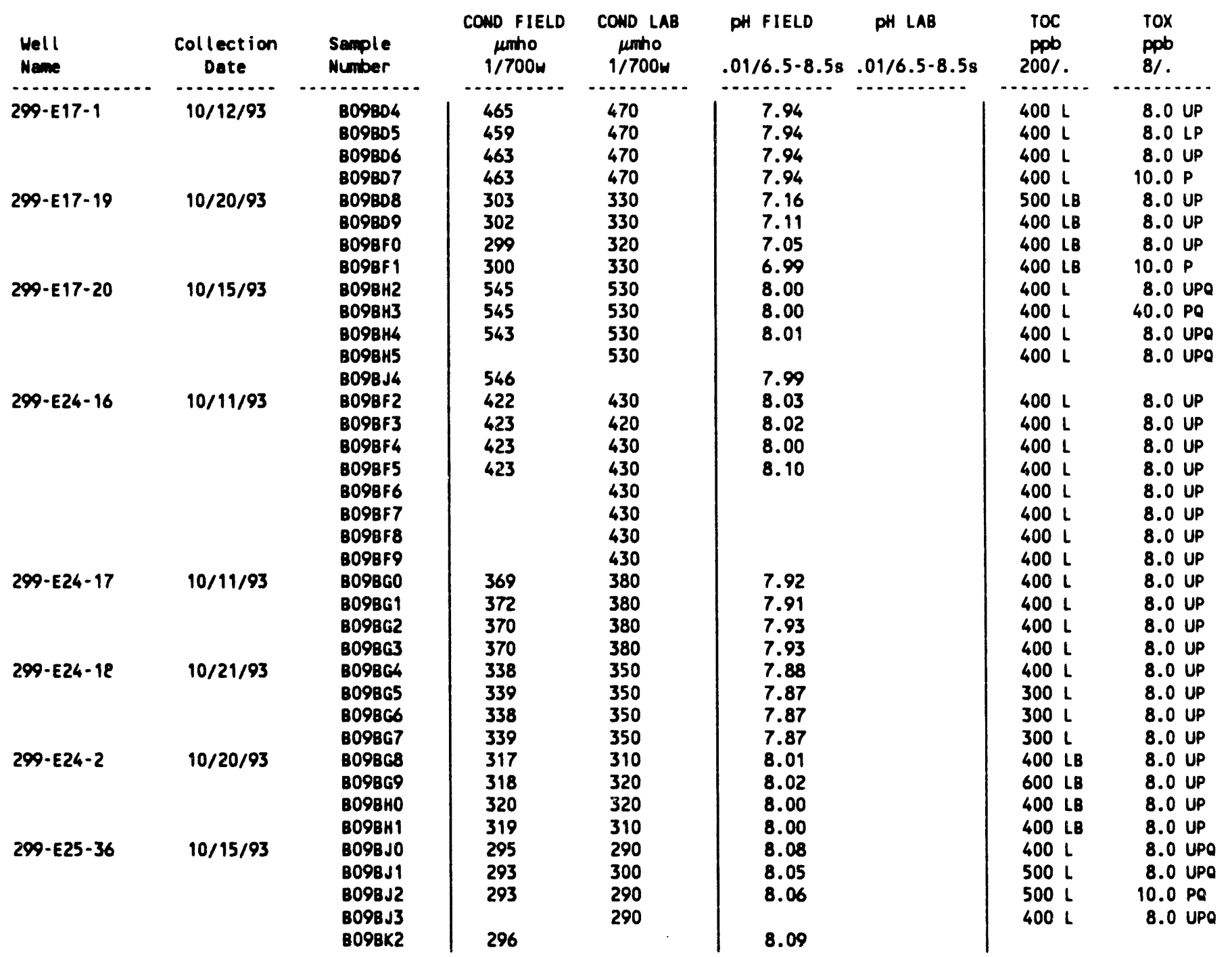

For explenation of this table, see Section 1.4 of report. 
$\mathrm{DOE} / \mathrm{RL}-93-56-4$

\section{CONTENTS}

10.0 216-B-63 TRENCH ...................... . . . 10-1

10.1 INTRODUCTION . . . . . . . . . . . . . . . . . . . . . . . . 10-1

10.2 WATER LEVEL MEASUREMENTS . . . . . . . . . . . . . . . 10-1

10.3 WATER CHEMISTRY DATA . . . . . . . . . . . . . . . 10-2 
DOE/RL-93-56-4

LIST OF FIGURES

10-1 Well Location Map for the 216-B-63 Trench. . . . . . . . . . . 10-3

\section{LIST OF TABLES}

10-1 Monitoring Well Purpose and Sampling Schedule for the 216-B-63 Trench Network................ . . 10-4

10-2 RCRA Water Level Measurement Report for the 216-B-63 Trench, Fourth Quarter 1993 . . . . . . . . . . . . . . . . 10-5

10-3 Constituent List and Summary of Results for the 216-B-63 Trench Data for Reporting Period October 1 through December 31, 1993. . 10-7

10-4 Constituents with at Least One Detected Value for the 216-B-63 Trench Data for Reporting Period October 1 through December $31,1993 .$. ............... 10-10

10-5 Contamination Indicator Parameters for the 216-B-63 Trench Data for Reporting Period October 1 through December 31, 1993 . . . . 10-16

10-6 Water Quality Standards Exceeded at the 216-B-63 Trench... . . 10-18 
$\mathrm{DOE} / \mathrm{RL}-93-56-4$

\subsection{6-B-63 TRENCH}

M. D. Sweeney

Westinghouse Hanford Company

\subsection{INTRODUCTION}

A RCRA detection-level groundwater monitoring network is being established for the 216-B-63 Trench. The 216-B-63 Trench received wastewater containing hazardous waste and radioactive materials from the $B$ Plant, located in the 200 East Area (Figures 1-1 and 10-1). Liquid effluent discharge to the 216-B-63 Trench ceased in February 1992. However, the trench is being retained to receive $B$ Plant discharges in the event of an emergency.

The 216-B-63 is an open, unlined, earthen trench that was in service from March 1970 until February 1992. The trench received $4.0 \times 10^{5}$ to $1.5 \times 10^{6} \mathrm{~L} /$ day $(100,000$ to $400,000 \mathrm{gal} / \mathrm{day})$ of liquid effluent from the B Plant chemical sewer. The effluent was a mixture of steam condensate and raw water. Documented hazardous discharges occurred from 1970 to October 1985, and consisted of aqueous sulfuric acid and sodium hydroxide solutions exceeding the $\mathrm{pH}$ limits of 2.0 and 12.5 , respectively. Radioactive soils were dredged from the trench in August 1970. In 1985, physical controls, radiation monitors, and operating procedures were modified to avoid inadvertent discharge of chemicals or radioactive substances to the wastewater stream.

Four quarters of groundwater background sampling have been completed for all of the wells in the network. Background levels have been established for the CIP in accordance with the requirements outlined in 40 CFR 265 InterimStatus Indicator-Evaluation Groundwater Monitoring Plan for the 216-B-63 Trench (PNL 1989). The nine wells within the network are now on a semiannual sampling program. Based on the present groundwater flow direction, seven of the wells are downgradient and five are upgradient of the trench (see Figure 10-1). The purpose of the wells, their sampling schedule, and the dates sampled are summarized in Table 10-1. As indicated in Table 10-1, some wells in the 216-B-63 network are shared with the Low-Level Burial Grounds (LLBG) and single-shell tanks (SST) groundwater monitoring networks.

\subsection{HATER LEVEL MEASUREMENTS}

Water level measurements obtained during the October through December 1993 period are presented in Table 10-2. Wells 299-E33-36, 299-E34-8, 299-E34-10, and 299-E33-37 were resurveyed in May 1993 to verify these elevations. Water in well 299-E33-36 remains lower than in nearby wells in the network and well 299-E33-33 has an outlier that may be caused by an error during sampling. Reasons for these apparent discrepancies are currently under investigation. 


\subsection{WATER CHEMISTRY DATA}

Groundwater samples were collected from the network in November 1993. The constituent list and summary of results are provided in Table 10-3. Constituents with at least one value above the MDL for all available data are presented in Table 10-4. Table 10-5 lists the results of the CIP; some results are shared with the LLBG. Laboratory $\mathrm{pH}$ values are no longer measured.

Constituents found in concentrations above DWS in samples from the October through December 1993 sampling period include chromium and iron. Table 10-6 lists the wells that have results above the DWS for these constituents. An RADE has been submitted for these results. Results for filtered samples for both iron and chromium continue to be well below DWS.

No CIP replicate averages exceeded the calculated critical means except for the $\mathrm{pH}$ noted below. One $\mathrm{pH}$ result generated in the field at the time of sampling exceeded the critical range in well 299-E27-8. This well was resampled on December 12, 1993, and the result indicated that the original measurement was in error.

Non-DWS constituents with laboratory qualifiers and QC flags indicating data problems include filtered and unfiltered barium, filtered and unfiltered calcium, chloride, filtered and unfiltered iron, filtered and unfiltered manganese, nitrate, filtered and unfiltered sodium, turbidity, and filtered and unfiltered zinc. The $D$ flags for nitrate indicated that the samples were diluted for analysis. 


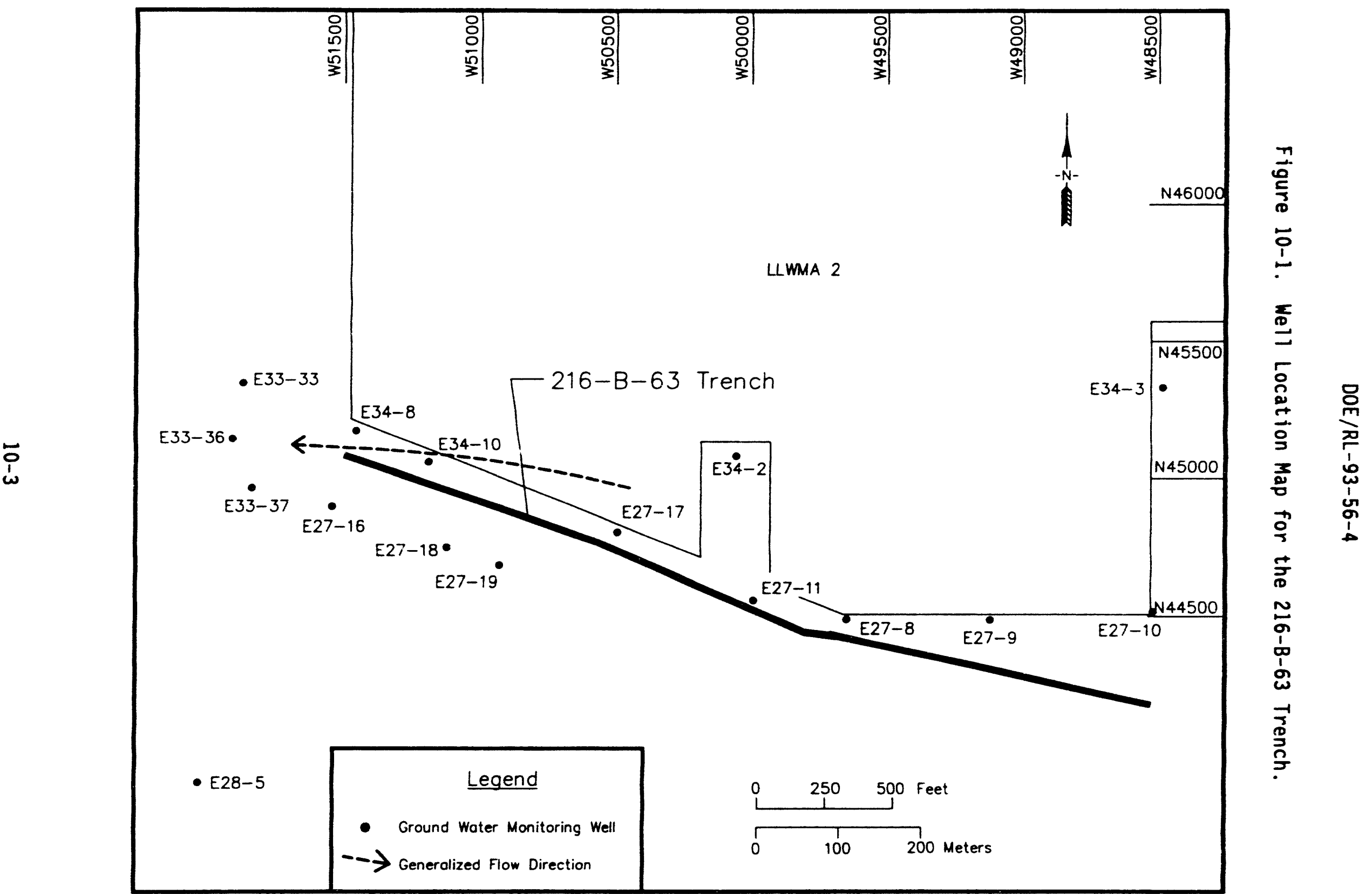

RCRA-AR $\backslash 121092 C 3$ 
Table 10-1. Monitoring Well Purpose and Sampling Schedule for the 216-B-63 Trench Network.

\begin{tabular}{|c|c|c|c|c|}
\hline $\begin{array}{c}\text { Well no. } \\
(299-)\end{array}$ & $\begin{array}{l}\text { Relative } \\
\text { position }\end{array}$ & Hydrogeolog & $\begin{array}{c}\text { Sample } \\
\text { frequency }\end{array}$ & $\begin{array}{l}\text { Sample date, } \\
\text { 4th Qtr } 1993\end{array}$ \\
\hline$E 27-8^{\circ}$ & Upgradient & Hanford: Water Table & Semiannually & $11 / 10 / 93$ \\
\hline$E 27-9^{\circ}$ & Upgradient & Hanford: Water Table & Semiannually & $11 / 10 / 93$ \\
\hline$E 27-11^{\bullet}$ & Upgradient & Hanford: Water Table & Semiannually & $11 / 11 / 93$ \\
\hline E27-16 & Downgradient & Hanford: Water Table & ally & $11 / 04 / 93$ \\
\hline$E 27-17^{\circ}$ & Upgradient & Hanford: Water Table & Semiannually & $11 / 11 / 93$ \\
\hline$E 27-18$ & Downgradient & Hanford: Water Table & Semiannually & $11 / 04 / 93$ \\
\hline E27-19 & Downgradient & Hanford: Water Table & Semiannually & $11 / 04 / 93$ \\
\hline$E 33-33^{b}$ & Downgradient & Hanford: Water Table & Semiannually & $11 / 05 / 93$ \\
\hline$E 33-36^{b}$ & Downgradient & Hanford: Water Table & Semiannually & $11 / 04 / 93$ \\
\hline$E 33-37$ & Downgradient & Hanford: Water Table & Semiannually & $11 / 04 / 93$ \\
\hline E34-8 & Downgradient & Hanford: Water Table & Semiannually & $11 / 05 / 93$ \\
\hline$E 34-10^{\circ}$ & Upgradient & Hanford: Water Table & Semiannually & $11 / 15 / 93$ \\
\hline
\end{tabular}

Well shared with the Low-Level Burial Grounds facility network.

Well shared with the single-shell tanks facility network. 


$$
\mathrm{DOE} / \mathrm{RL}-93-56-4
$$

Table 10-2. RCRA Water Level Measurement Report for the 216-B-63 Trench, Fourth Quarter 1993. (sheet 1 of 2)

\begin{tabular}{|c|c|c|c|}
\hline Well & Date & $\begin{array}{l}\text { Depth to } \\
\text { water }(f t)\end{array}$ & $\begin{array}{l}\text { Water level } \\
\text { elevation } \\
\text { above msl (ft) }\end{array}$ \\
\hline \multicolumn{4}{|c|}{ Wells Monitoring the Top o } \\
\hline $299-E 27-11$ & $\begin{array}{l}11 / 11 / 93 \\
12 / 10 / 93\end{array}$ & $\begin{array}{l}241.17 \\
241.79\end{array}$ & $\begin{array}{l}402.12^{\star} \\
401.50\end{array}$ \\
\hline 299-E27-16 & $11 / 04 / 93$ & 250.67 & $401.46^{\star}$ \\
\hline 299-E27-17 & $\begin{array}{l}11 / 11 / 93 \\
12 / 10 / 93\end{array}$ & $\begin{array}{l}232.29 \\
232.86\end{array}$ & $\begin{array}{l}402.45^{\star} \\
401.88\end{array}$ \\
\hline 299-E27-18 & $11 / 04 / 93$ & 248.91 & $401.24^{\star}$ \\
\hline 299-E27-19 & $11 / 04 / 93$ & 249.20 & $401.68^{*}$ \\
\hline 299-E27-8 & $\begin{array}{l}11 / 10 / 93 \\
12 / 10 / 93\end{array}$ & $\begin{array}{l}236.17 \\
236.19\end{array}$ & $\begin{array}{l}401.66^{\star} \\
401.64\end{array}$ \\
\hline 299-E27-9 & $\begin{array}{l}11 / 10 / 93 \\
12 / 10 / 93\end{array}$ & $\begin{array}{l}227.38 \\
227.47\end{array}$ & $\begin{array}{l}401.83^{\star} \\
401.74\end{array}$ \\
\hline 299-E33-33 & $\begin{array}{l}10 / 27 / 93 \\
11 / 05 / 93 \\
11 / 24 / 93 \\
12 / 15 / 93\end{array}$ & $\begin{array}{l}238.48 \\
237.39 \\
238.73 \\
238.45\end{array}$ & $\begin{array}{l}401.91 \\
403.00^{\star}+ \\
401.66 \\
401.94\end{array}$ \\
\hline 299-E33-36 & $\begin{array}{l}11 / 04 / 93 \\
12 / 08 / 93\end{array}$ & $\begin{array}{l}246.35 \\
246.13\end{array}$ & $\begin{array}{l}400.51^{\star} \\
400.73\end{array}$ \\
\hline 299-E33-37 & $12 / 08 / 93$ & 251.17 & 402.05 \\
\hline 299-E34-10 & $\begin{array}{l}11 / 15 / 93 \\
12 / 10 / 93\end{array}$ & $\begin{array}{l}237.21 \\
237.96\end{array}$ & $\begin{array}{l}402.5^{\star} \\
401.80\end{array}$ \\
\hline 299-E34-8 & $\begin{array}{l}11 / 05 / 93 \\
12 / 08 / 93\end{array}$ & $\begin{array}{l}239.69 \\
239.25\end{array}$ & $\begin{array}{l}401.05^{\star} \\
401.49\end{array}$ \\
\hline
\end{tabular}




\section{$D O E / R L-93-56-4$}

Table 10-2. RCRA Water Level Measurement Report for the 216-B-63 Trench, Fourth Quarter 1993. (sheet 2 of 2)

Notes: 1. Water level elevations are calculated by subtracting the measured depth to water from the surveyed elevation for the well.

2. Depth-to-water values are transcribed from field records.

3. Elevations marked with an ' $*$ ' were measured at the time of sampling.

4. Elevations marked with a ' + ' are outside of the expected range and are suspected of error.

5. To convert feet to meters multiply by 0.3048 . 
Table 10-3. Constituent List and Summary of Results for the 216-B-63 Trench Data for Reporting Period October 1 through December 31, 1993. (sheet 1 of 3)

CONTAMINATION INDICATOR PARAMETERS

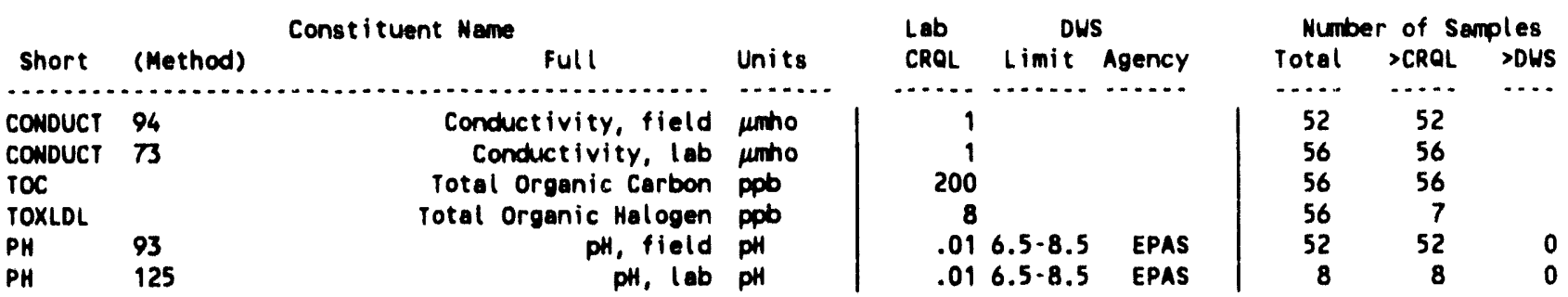

DRINKING WATER PARAMETERS

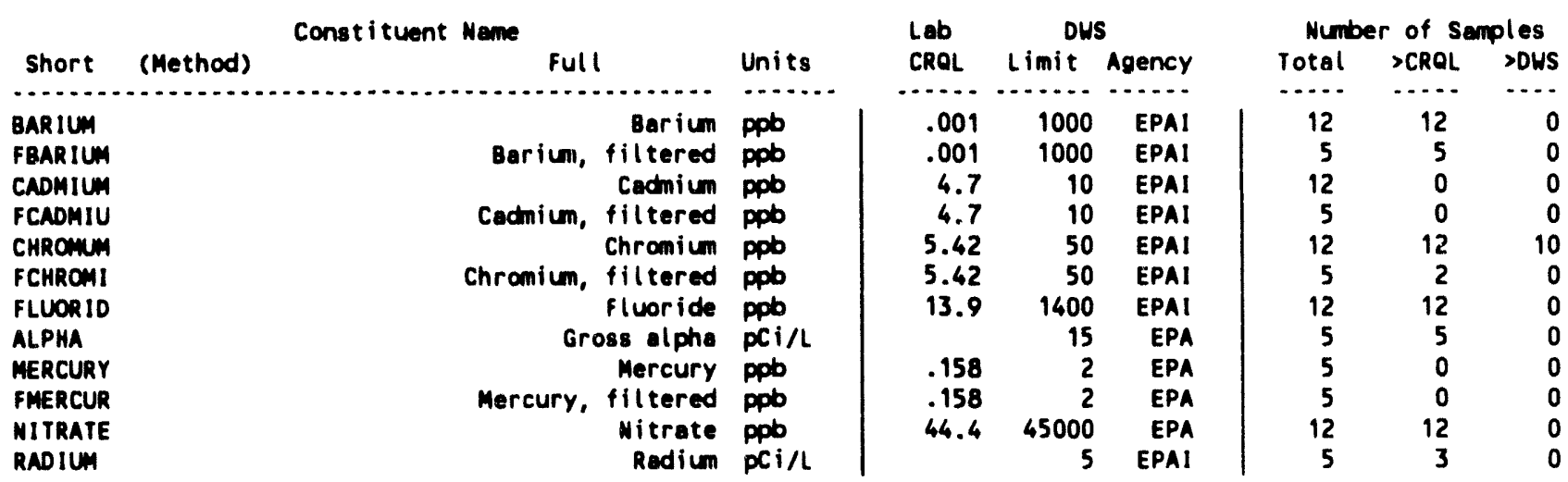

GROUNDWATER QUALITY PARAMETERS

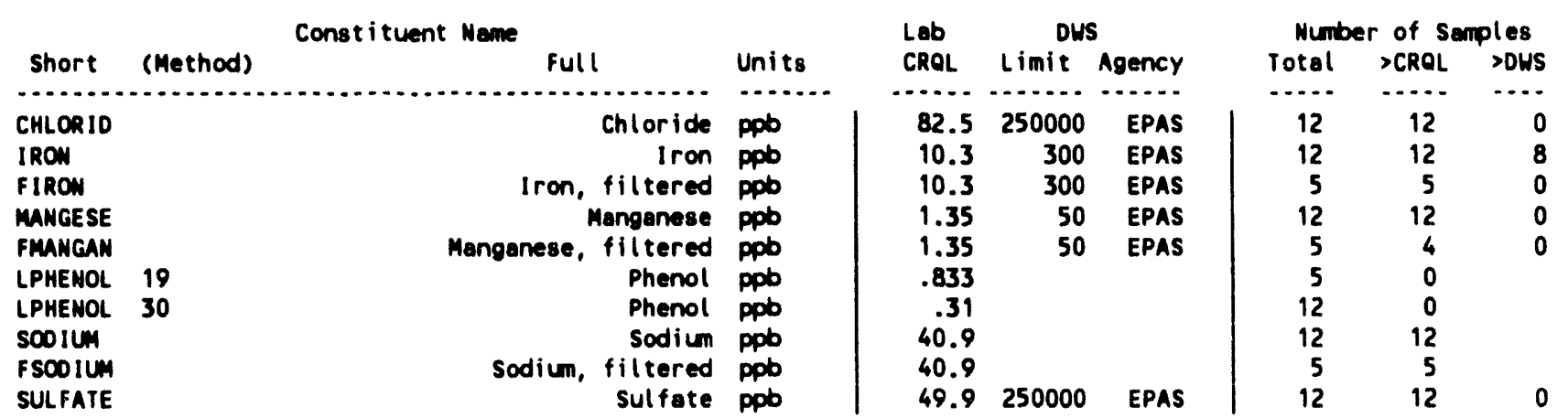


Table 10-3. Constituent List and Summary of Results for the 216-B-63 Trench Data for Reporting Period October 1 through December 31, 1993. (sheet 2 of 3)

SITE SPECIFIC AND OTHER CONSTITUENTS

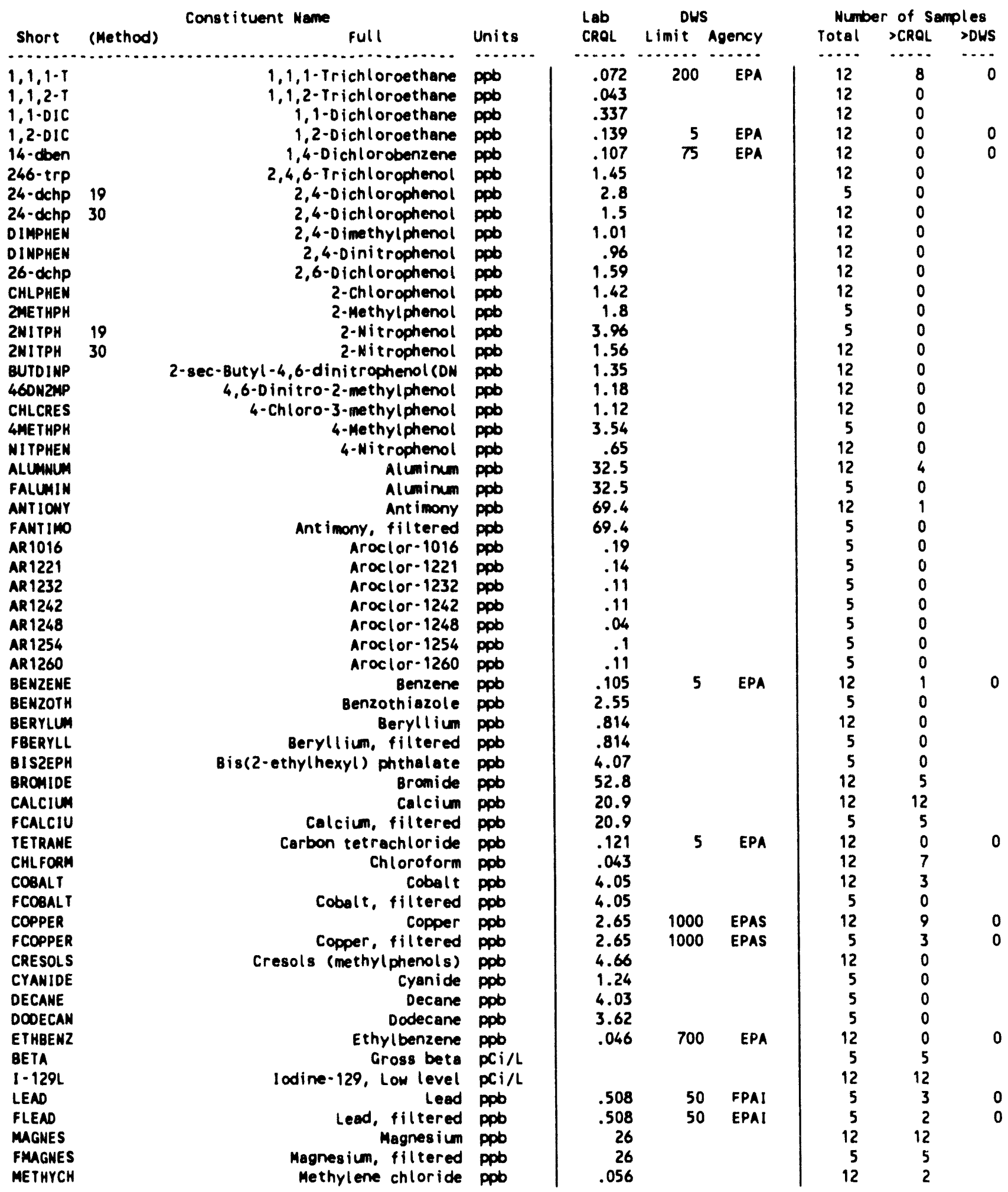


Table 10-3. Constituent List and Summary of Results for the 216-B-63 Trench Data for Reporting Period October 1 through December 31, 1993. (sheet 3 of 3)

\begin{tabular}{|c|c|c|c|c|c|c|c|c|c|}
\hline \multirow[b]{2}{*}{ Short } & \multirow[b]{2}{*}{ (Method) } & \multirow{2}{*}{$\begin{array}{l}\text { Constituent Nane Full } \\
\text { fone }\end{array}$} & \multirow[b]{2}{*}{ Units } & \multirow{2}{*}{$\begin{array}{l}\text { Lab } \\
\text { CROL }\end{array}$} & \multicolumn{2}{|c|}{ DWS } & \multicolumn{3}{|c|}{ Number of Samples } \\
\hline & & & & & Limit & Agency & Total & $>$ CRQL & $>$ DUS \\
\hline NAPHTHA & & Maphthal ene & pob & 6.5 & *.... & $\cdots . .$. & $\begin{array}{c}\cdots \\
5\end{array}$ & $\begin{array}{c}\cdots \\
0\end{array}$ & .... \\
\hline NICKEL & & $\begin{array}{l}\text { Nickel } \\
\text { Nichter }\end{array}$ & ppob & 17.9 & & & 12 & 10 & \\
\hline FNICKEL & & Nickel, filtered & ppb & 17.9 & & & 5 & 0 & \\
\hline NITRITE & & Nitrite & ppb & 38.3 & 1000 & EPA & 12 & 0 & 0 \\
\hline PENTCHP & 19 & Pentachl or ophenol & ppo & 8.07 & 1 & EPA & 5 & 0 & 5* \\
\hline PENTCHP & 30 & Pentachlorophenol & ppo & .87 & 1 & EPA & 12 & 0 & 0 \\
\hline PHOSPHA & & Phosphate & ppb & 147 & & & 12 & 0 & \\
\hline POTASUM & & Potassium & ppob & 662 & & & 12 & 12 & \\
\hline FPOTASS & & Potassium, filtered & ppb & 662 & & & 5 & 5 & \\
\hline SILVER & & silver & ppob & 2.87 & 50 & EPAI & 12 & 0 & 0 \\
\hline FSILVER & & silver, filtered & ppb & 2.87 & 50 & EPAI & 5 & 1 & 0 \\
\hline PERCENE & & Tetrachloroethene & ppb & .049 & 5 & EPA & 12 & 0 & 0 \\
\hline TETPHNL & & Tetrachlorophenols & ppb & 1.05 & & & 12 & 0 & \\
\hline TETRADE & & Tetradecane & ppb & 2.43 & & & 5 & 0 & \\
\hline TIA & & Tin & ppb & 51.1 & & & 12 & 0 & \\
\hline FTIN & & Tin, filtered & ppb & 51.1 & & & 5 & 0 & \\
\hline TOLUENE & & Tolvene & ppb & .056 & 1000 & EPA & 12 & 1 & 0 \\
\hline TRIBUTPH & & Tributyl Phosphate & ppb & 4.42 & & & 5 & 0 & \\
\hline TRICENE & & Trichloroethene & ppb & .065 & 5 & EPA & 12 & 0 & 0 \\
\hline TRIPHNL & & Trichlorophenols & ppb & 1.11 & & & 12 & 0 & \\
\hline TDICHPH & & Tris-2-chloroethyl phosphate & ppob & 2.88 & & & 5 & 0 & \\
\hline TRITIUM & & Tritium & $\mathrm{pCi} / \mathrm{L}$ & & 20000 & EPA & 12 & 12 & 0 \\
\hline TURBID & & Turbidity & NTU & .05 & & & 5 & 5 & \\
\hline URANIUM & & Uranium & ppo & & & & 5 & 5 & \\
\hline$u \cdot 234$ & & Urenium-234 & $\mathrm{pCi} / \mathrm{L}$ & & & & 12 & 12 & \\
\hline U-235 & & Uranium-235 & $\mathrm{pCi} / \mathrm{L}$ & & & & 12 & 9 & \\
\hline $0-238$ & & Uranium-238 & $\mathrm{pCi} / \mathrm{L}$ & & & & 12 & 12 & \\
\hline VAMADUM & & Vanedium & ppob & 3.84 & & & 12 & 12 & \\
\hline FVANADI & & Vanadium, filtered & ppb & 3.84 & & & 5 & 5 & \\
\hline VINYIDE & & Vinyl chloride & ppob & .266 & 2 & EPA & 12 & 0 & 0 \\
\hline XYLENE & & Xylenes (total) & ppb & .202 & 10000 & EPA & 12 & 0 & 0 \\
\hline ZINC & & Zinc & ppb & 3.44 & 5000 & EPAS & 12 & 7 & 0 \\
\hline FZINC & & zinc, filtered & ppb & 3.44 & 5000 & EPAS & 5 & 1 & 0 \\
\hline CISI2DE & & cis-1,2-Dichloroethylene & ppo & .127 & 70 & EPA & 12 & 0 & 0 \\
\hline MCRESOL & & $m$-Cresol & ppob & 1.44 & & & 5 & 0 & \\
\hline TRAMDCE & & trans-1,2-Dichloroethylene & ppo & .149 & 100 & EPA & 12 & 0 & 0 \\
\hline
\end{tabular}

For explanation of this table, see section 1.4 of report. 
Table 10-4. Constituents with at Least One Detected Value for the 216-B-63 Trench Data for Reporting Period October 1 through December 31, 1993. (sheet 1 of 6 )

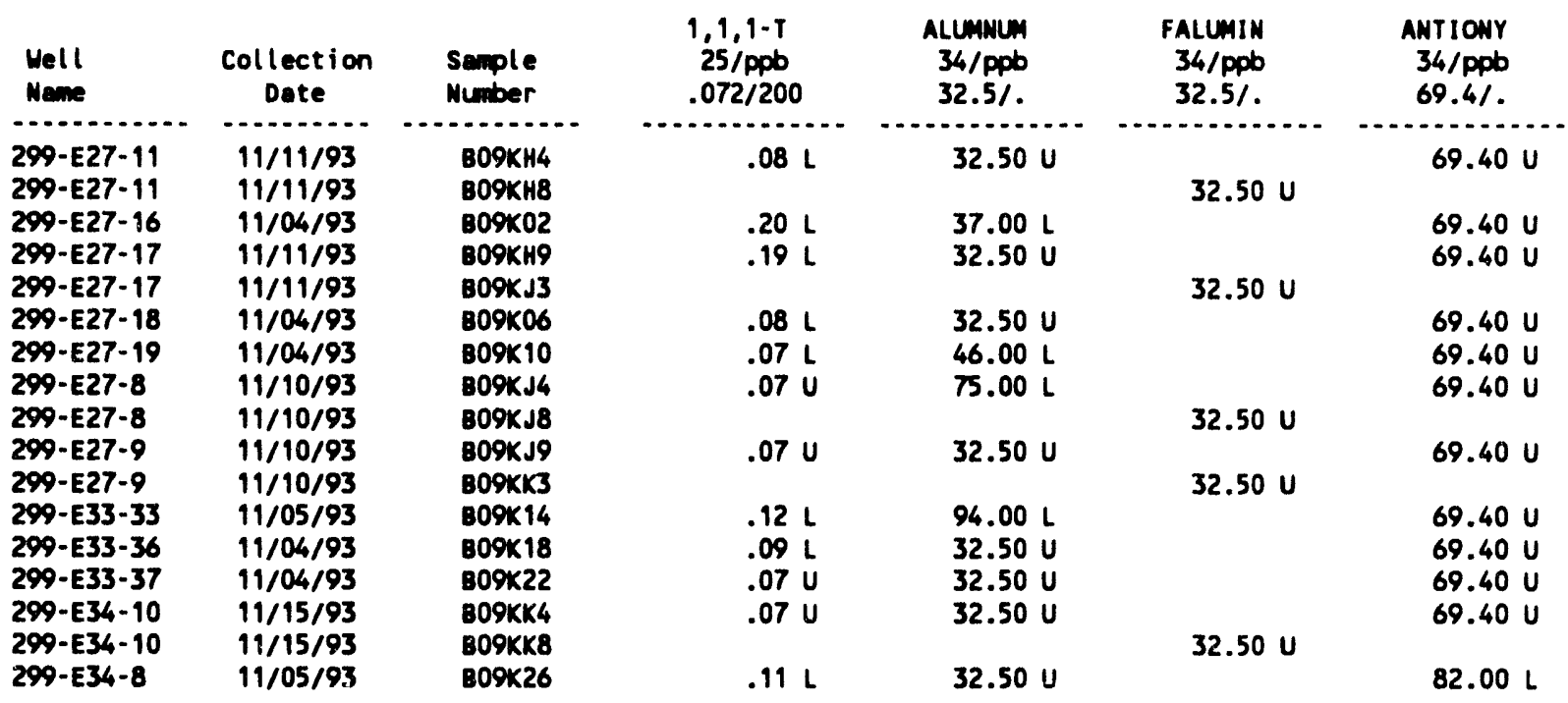

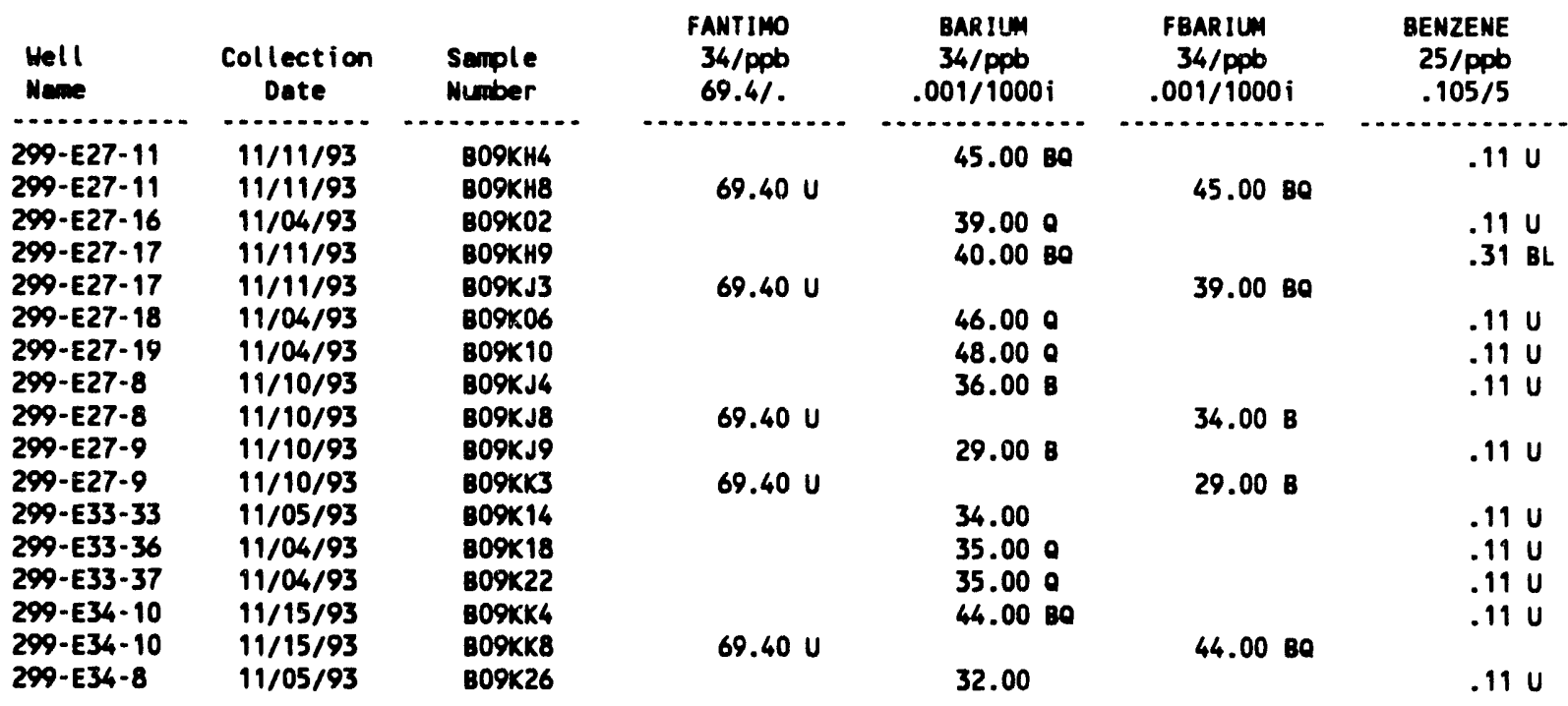

\begin{tabular}{|c|c|c|c|c|c|c|}
\hline $\begin{array}{l}\text { Hell } \\
\text { Name }\end{array}$ & $\begin{array}{c}\text { Collection } \\
\text { Date }\end{array}$ & $\begin{array}{l}\text { Semple } \\
\text { Number }\end{array}$ & $\begin{array}{c}\text { BRONIDE } \\
124 / \mathrm{ppb} \\
52.8 \%\end{array}$ & $\begin{array}{l}\text { CALCIUM } \\
34 / \mathrm{ppb} \\
20.9 \%\end{array}$ & $\begin{array}{c}\text { FCALCIU } \\
34 / \mathrm{ppb} \\
20.91\end{array}$ & $\begin{array}{c}\text { CHLORID } \\
124 / \text { ppb } \\
82.5 / 250000 \mathrm{~s}\end{array}$ \\
\hline $\begin{array}{l}299-E 27-11 \\
299-E 27-11\end{array}$ & $11 / 11 / 93$ & $\begin{array}{l}\text { 809KH4 } \\
\text { 809KH8 }\end{array}$ & $80.00 \mathrm{~L}$ & $43000.00 \mathrm{BO}$ & - & $9500.00 \mathrm{BD}$ \\
\hline $\begin{array}{l}299-\text { E27-16 } \\
299-\text { E27-17 }\end{array}$ & $\begin{array}{l}11 / 04 / 93 \\
11 / 11 / 93\end{array}$ & $\begin{array}{l}\text { B09K02 } \\
\text { B09KH9 }\end{array}$ & $\begin{array}{l}52.80 \mathrm{U} \\
70.00 \mathrm{~L}\end{array}$ & $\begin{array}{l}32000.000 \\
43000.00 \mathrm{B0}\end{array}$ & & $\begin{array}{l}5500.00 \\
9400.00 \mathrm{BD}\end{array}$ \\
\hline $\begin{array}{l}299-E 27-17 \\
299-E 27-18 \\
299-E 27-19\end{array}$ & $\begin{array}{l}11 / 11 / 93 \\
11 / 04 / 93 \\
11 / 04 / 93\end{array}$ & $\begin{array}{l}809 K J 3 \\
\text { B09K06 } \\
\text { B09K10 }\end{array}$ & $\begin{array}{r}52.80 \mathrm{U} \\
100.00 \mathrm{~L}\end{array}$ & $\begin{array}{l}35000.00 a \\
38000.00\end{array}$ & 43000.0080 & $\begin{array}{l}6500.00 \\
8600.00\end{array}$ \\
\hline
\end{tabular}


Table 10-4. Constituents with at Least One Detected Value for the 216-B-63 Trench Data for Reporting Period October 1

through December 31, 1993. (sheet 2 of 6)

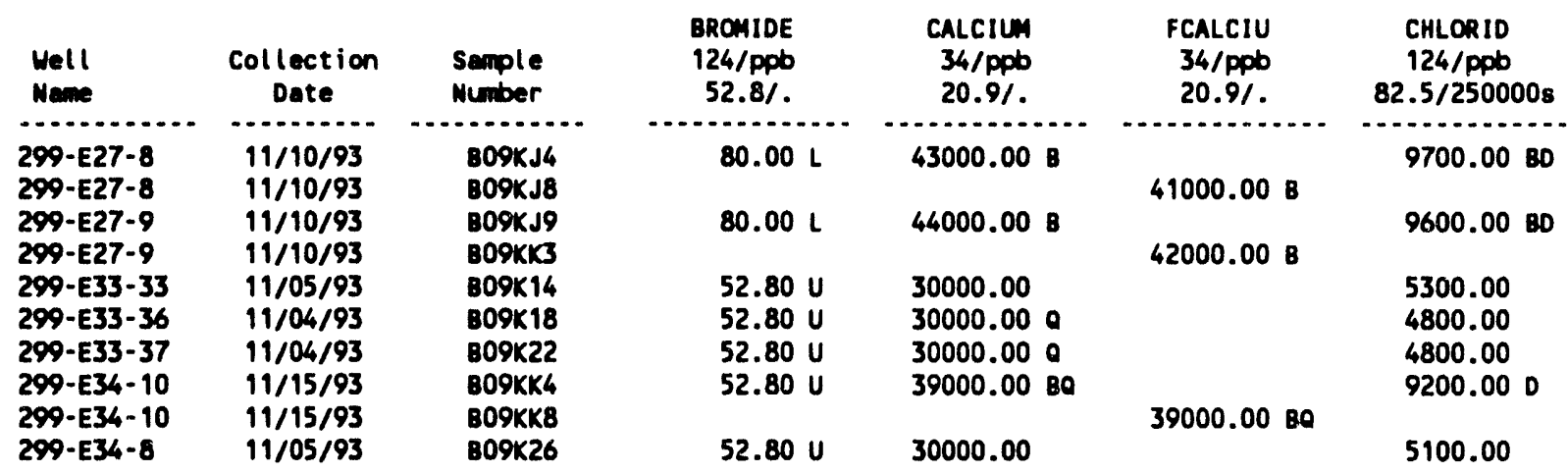

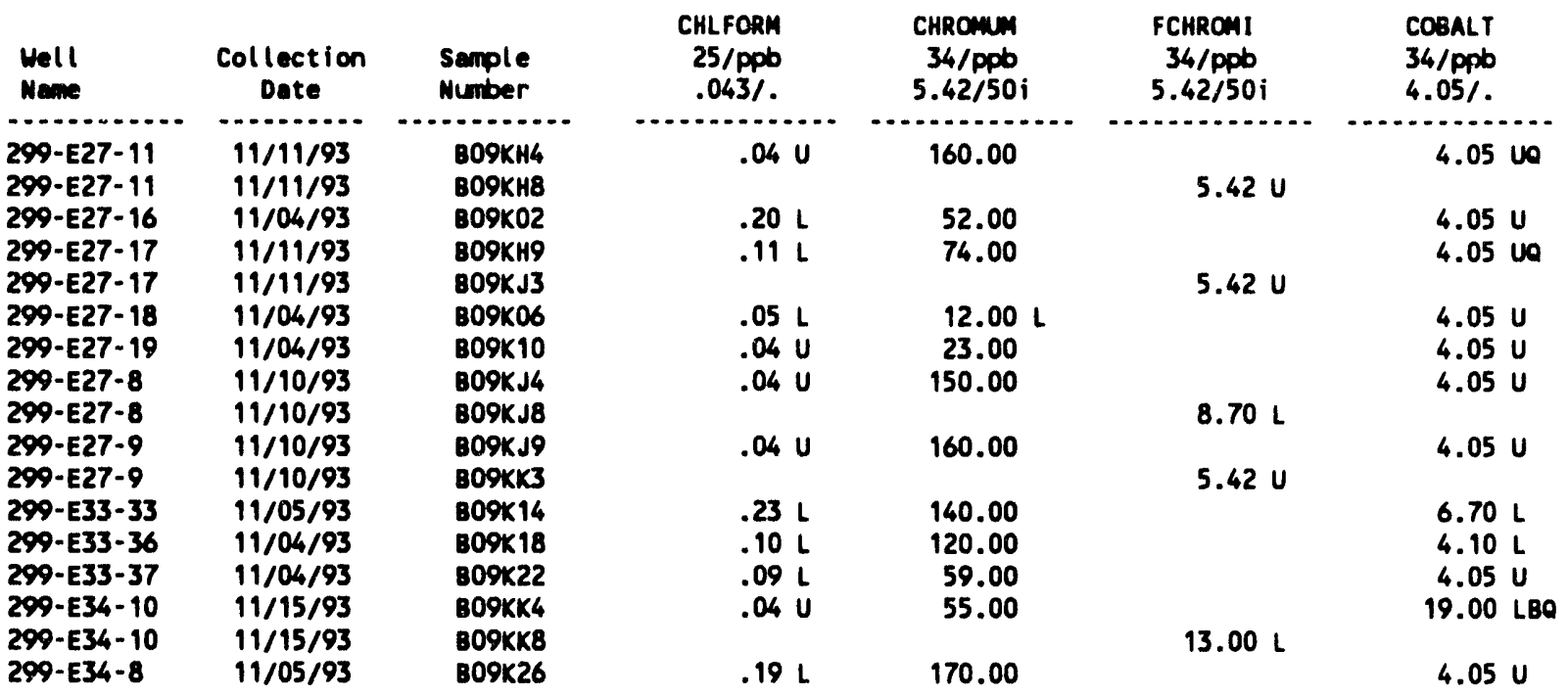

\begin{tabular}{|c|c|c|c|c|c|c|}
\hline $\begin{array}{l}\text { Well } \\
\text { Nene }\end{array}$ & $\begin{array}{c}\text { Collection } \\
\text { Date }\end{array}$ & $\begin{array}{l}\text { Semple } \\
\text { Number }\end{array}$ & $\begin{array}{c}\text { FCOBALT } \\
34 / \text { ppb } \\
4.05 \%\end{array}$ & $\begin{array}{c}\text { COPPER } \\
34 / \text { ppb } \\
2.65 / 1000 \mathrm{~s}\end{array}$ & $\begin{array}{c}\text { FCOPPER } \\
34 / \text { ppb } \\
2.65 / 1000 \mathrm{~s}\end{array}$ & $\begin{array}{c}\text { FLUORID } \\
124 / \text { ppb } \\
13.9 / 1400 \mathrm{i}\end{array}$ \\
\hline $\begin{array}{l}299-E 27-11 \\
299-E 27-11 \\
299-E 27-16 \\
299-E 27-17 \\
299-E 27-17 \\
299-E 27-18 \\
299-E 27-19 \\
299-E 27-8 \\
299-E 27-8 \\
299-E 27-9 \\
299-E 27-9 \\
299-E 33-33 \\
299-E 33-36 \\
299-E 33-37 \\
299-E 34-10 \\
299-E 34-10 \\
299-E 34-8\end{array}$ & $\begin{array}{l}11 / 11 / 93 \\
11 / 11 / 93 \\
11 / 04 / 93 \\
11 / 11 / 93 \\
11 / 11 / 93 \\
11 / 04 / 93 \\
11 / 104 / 93 \\
11 / 10 / 93 \\
11 / 10 / 93 \\
11 / 10 / 93 \\
11 / 10 / 93 \\
11 / 05 / 93 \\
11 / 04 / 93 \\
11 / 04 / 93 \\
11 / 15 / 93 \\
11 / 15 / 93 \\
11 / 05 / 93\end{array}$ & 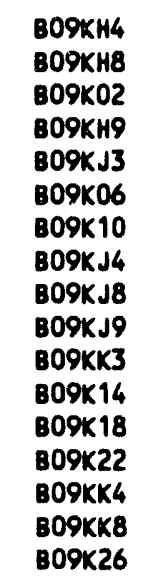 & $\begin{array}{l}4.05 \mathrm{U} \\
4.05 \mathrm{U}\end{array}$ & $\begin{array}{r}16.00 \mathrm{~L} \\
9.40 \mathrm{~L} \\
13.00 \mathrm{~L} \\
2.65 \mathrm{U} \\
2.65 \mathrm{U} \\
8.20 \mathrm{~L} \\
2.65 \mathrm{U} \\
14.00 \mathrm{~L} \\
7.00 \mathrm{~L} \\
4.70 \mathrm{~L} \\
7.50 \mathrm{~L} \\
16.00 \mathrm{~L}\end{array}$ & $\begin{array}{l}5.20 \mathrm{~L} \\
2.65 \mathrm{U}\end{array}$ & $\begin{array}{l}500.00 \\
300.00 \\
400.00 \\
500.00 \\
800.00 \\
400.00 \\
400.00 \\
600.00 \\
600.00 \\
600.00 \\
700.00 \\
600.00\end{array}$ \\
\hline
\end{tabular}


Table 10-4. Constituents with at Least One Detected Value for the 216-B-63 Trench Data for Reporting Period October 1 through December 31, 1993. (sheet 3 of 6)

\begin{tabular}{|c|c|c|}
\hline $\begin{array}{l}\text { Well } \\
\text { Name }\end{array}$ & $\begin{array}{c}\text { Collection } \\
\text { Date }\end{array}$ & $\begin{array}{l}\text { Senple } \\
\text { Number }\end{array}$ \\
\hline $\begin{array}{l}299-E 27-11 \\
299-E 27-11 \\
299-E 27-16 \\
299-E 27-17 \\
299-E 27-17 \\
299-E 27-18 \\
299-E 27-19 \\
299-E 27-8 \\
299-E 27-8 \\
299-E 27-9 \\
299-E 27-9 \\
299-E 33-33 \\
299-E 33-36 \\
299-E 33-37 \\
299-E 34-10 \\
299-E 34-10 \\
299-E 34-8\end{array}$ & $\begin{array}{l}11 / 11 / 93 \\
11 / 11 / 93 \\
11 / 04 / 93 \\
11 / 11 / 93 \\
11 / 11 / 93 \\
11 / 04 / 93 \\
11 / 04 / 93 \\
11 / 10 / 93 \\
11 / 10 / 93 \\
11 / 10 / 93 \\
11 / 10 / 93 \\
11 / 05 / 93 \\
11 / 04 / 93 \\
11 / 04 / 93 \\
11 / 15 / 93 \\
11 / 15 / 93 \\
11 / 05 / 93\end{array}$ & $\begin{array}{l}809 K 30 \\
809 K H 4 \\
809 K 02 \\
809 K 34 \\
809 K H 9 \\
809 K 06 \\
B 09 K 10 \\
B 09 K 38 \\
809 K J 4 \\
809 K 42 \\
B 09 K J 9 \\
B 09 K 14 \\
B 09 K 18 \\
B 09 K 22 \\
B 09 K 50 \\
B 09 K K 4 \\
B 09 K 26\end{array}$ \\
\hline
\end{tabular}

$\begin{array}{cc}\begin{array}{c}\text { ALPHA } \\ 135 / \text { PCi } / L \\ . / 15\end{array} & \begin{array}{c}\text { BETA } \\ 136 / \text { PCi } / L \\ . / .\end{array} \\ 3.35 & 7.49 \\ 1.57 & 7.22 \\ 2.37 & \\ 1.68 & 7.99 \\ & 9.38 \\ 1.14 & \\ & \end{array}$

\begin{tabular}{|c|c|}
\hline $\begin{array}{c}1-129 \\
139 / \mathrm{pCi} / \mathrm{L} \\
.1\end{array}$ & $\begin{array}{c}\text { IRON } \\
34 / \text { ppo } \\
10.3 / 300 \mathrm{~s}\end{array}$ \\
\hline 2.14 & \\
\hline $\begin{array}{l}2.39 \\
3.58\end{array}$ & $\begin{array}{l}690.00 \mathrm{~B} \\
200.00\end{array}$ \\
\hline $\begin{array}{l}5.27 \\
4.16 \\
3.27\end{array}$ & $\begin{array}{c}330.00 \mathrm{~B} \\
77.00 \\
140.00\end{array}$ \\
\hline 3.03 & $830.00 \mathrm{~B}$ \\
\hline $\begin{array}{l}3.11 \\
5.53 \\
6.64 \\
2.02\end{array}$ & $\begin{array}{l}660.00 \mathrm{~B} \\
630.00 \\
510.00 \\
250.00\end{array}$ \\
\hline 1.58 & $\begin{array}{l}310.00 \\
720.00\end{array}$ \\
\hline
\end{tabular}

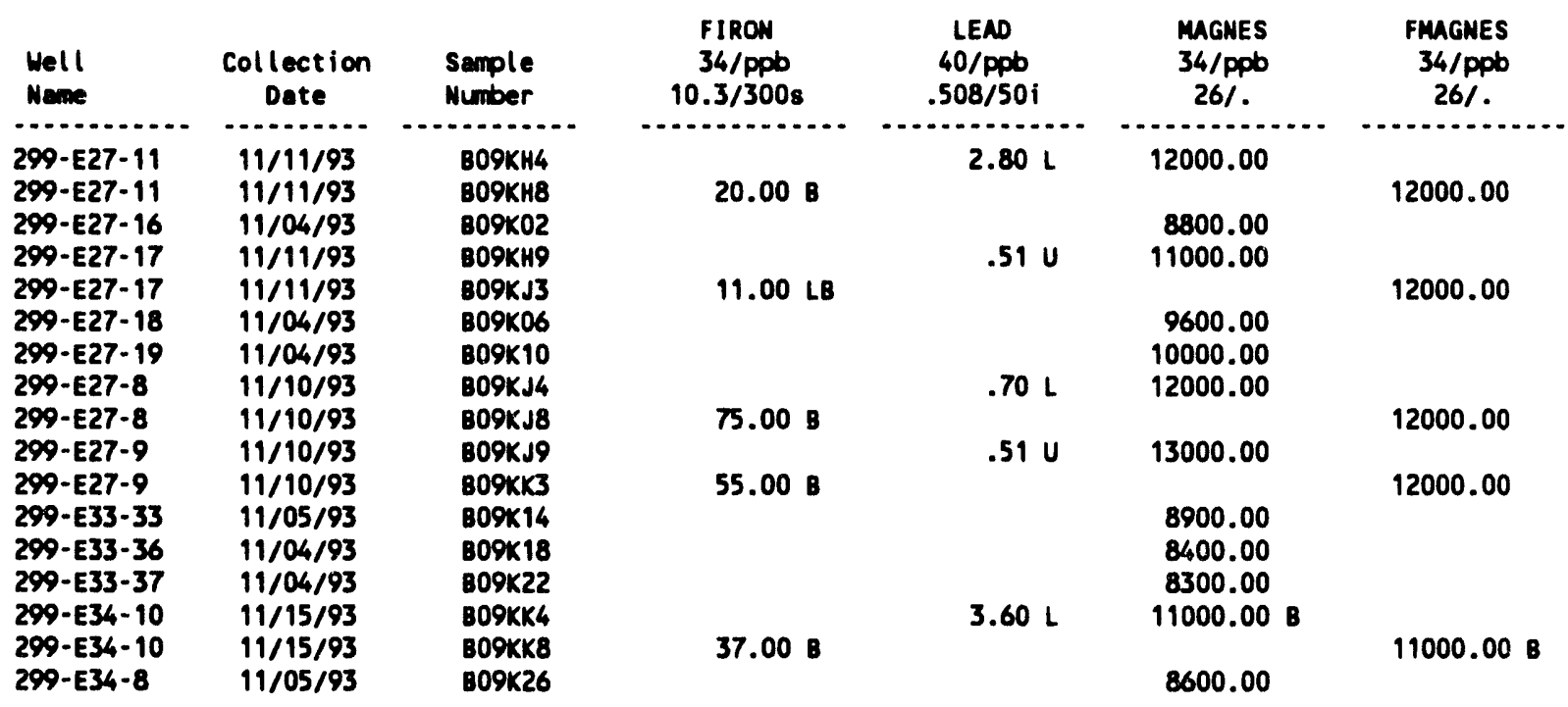

\begin{tabular}{|c|c|c|c|c|c|c|}
\hline $\begin{array}{l}\text { Well } \\
\text { Name }\end{array}$ & $\begin{array}{c}\text { Collection } \\
\text { Date }\end{array}$ & $\begin{array}{l}\text { Sample } \\
\text { Number }\end{array}$ & $\begin{array}{l}\text { MANGESE } \\
34 / \mathrm{ppb} \\
1.35 / 50 \mathrm{~s}\end{array}$ & $\begin{array}{l}\text { FMANGAN } \\
34 / \mathrm{ppb} \\
1.35 / 50 \mathrm{~s}\end{array}$ & $\begin{array}{l}\text { METHYCH } \\
25 / \mathrm{ppb} \\
.056 / .\end{array}$ & $\begin{array}{l}\text { NICKEL } \\
34 / \mathrm{ppb} \\
17.9 \%\end{array}$ \\
\hline $\begin{array}{l}299-E 27-11 \\
299-E 27-11\end{array}$ & $\begin{array}{l}11 / 11 / 93 \\
11 / 11 / 93\end{array}$ & $\begin{array}{l}\text { B09KH4 } \\
\text { B09KH8 }\end{array}$ & 16.00 & $2.40 \mathrm{~L}$ & $.06 \mathrm{U}$ & 79.00 \\
\hline $\begin{array}{l}299-\text { E27- } 16 \\
299-E 27-17\end{array}$ & $\begin{array}{l}11 / 04 / 93 \\
11 / 11 / 93\end{array}$ & B09K02 & $\begin{array}{l}6.00 \mathrm{~L} \\
9.00 \mathrm{~L}\end{array}$ & & $\begin{array}{l}.06 \mathrm{U} \\
.06 \mathrm{U}\end{array}$ & $\begin{array}{l}20.00 \mathrm{~L} \\
40.00\end{array}$ \\
\hline $\begin{array}{l}299-E 27-17 \\
299-E 27-18 \\
299-E 27-19\end{array}$ & $\begin{array}{l}11 / 11 / 93 \\
11 / 04 / 93 \\
11 / 04 / 93\end{array}$ & $\begin{array}{l}\text { B09KJ3 } \\
\text { B09K06 } \\
\text { B09K10 }\end{array}$ & $\begin{array}{l}2.70 \mathrm{~L} \\
3.80\end{array}$ & $1.90 \mathrm{~L}$ & $\begin{array}{l}.06 \mathrm{U} \\
.06 \mathrm{U}\end{array}$ & $\begin{array}{l}17.90 \mathrm{U} \\
17.90 \mathrm{U}\end{array}$ \\
\hline
\end{tabular}


Table 10-4. Constituents with at Least One Detected Value for the 216-B-63 Trench Data for Reporting Period October 1 through December 31, 1993. (sheet 4 of 6)

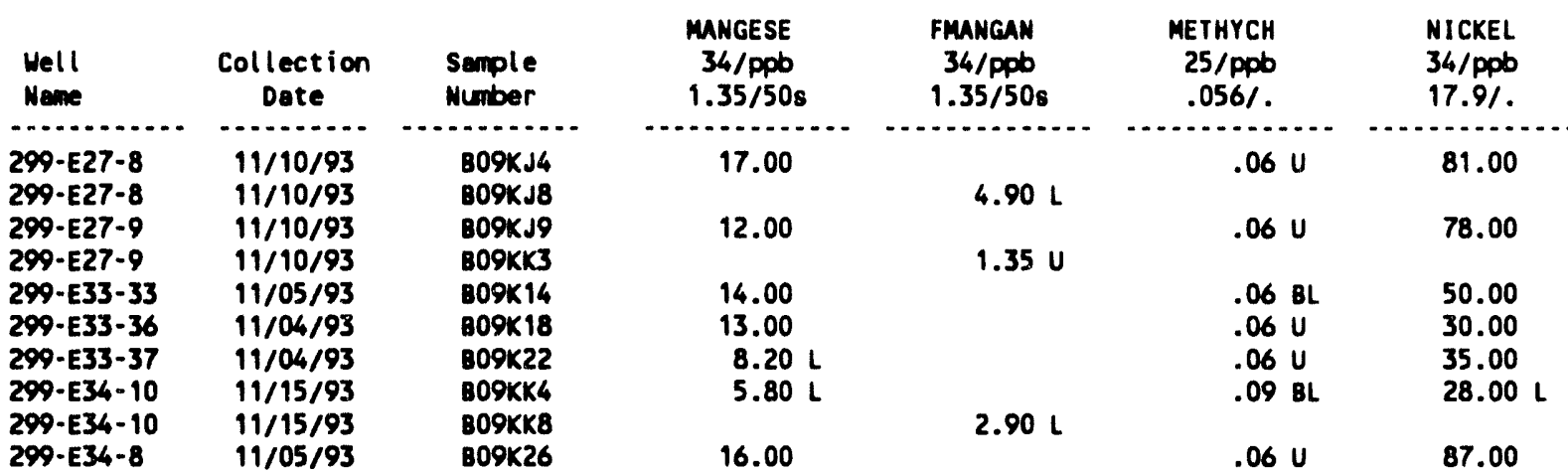

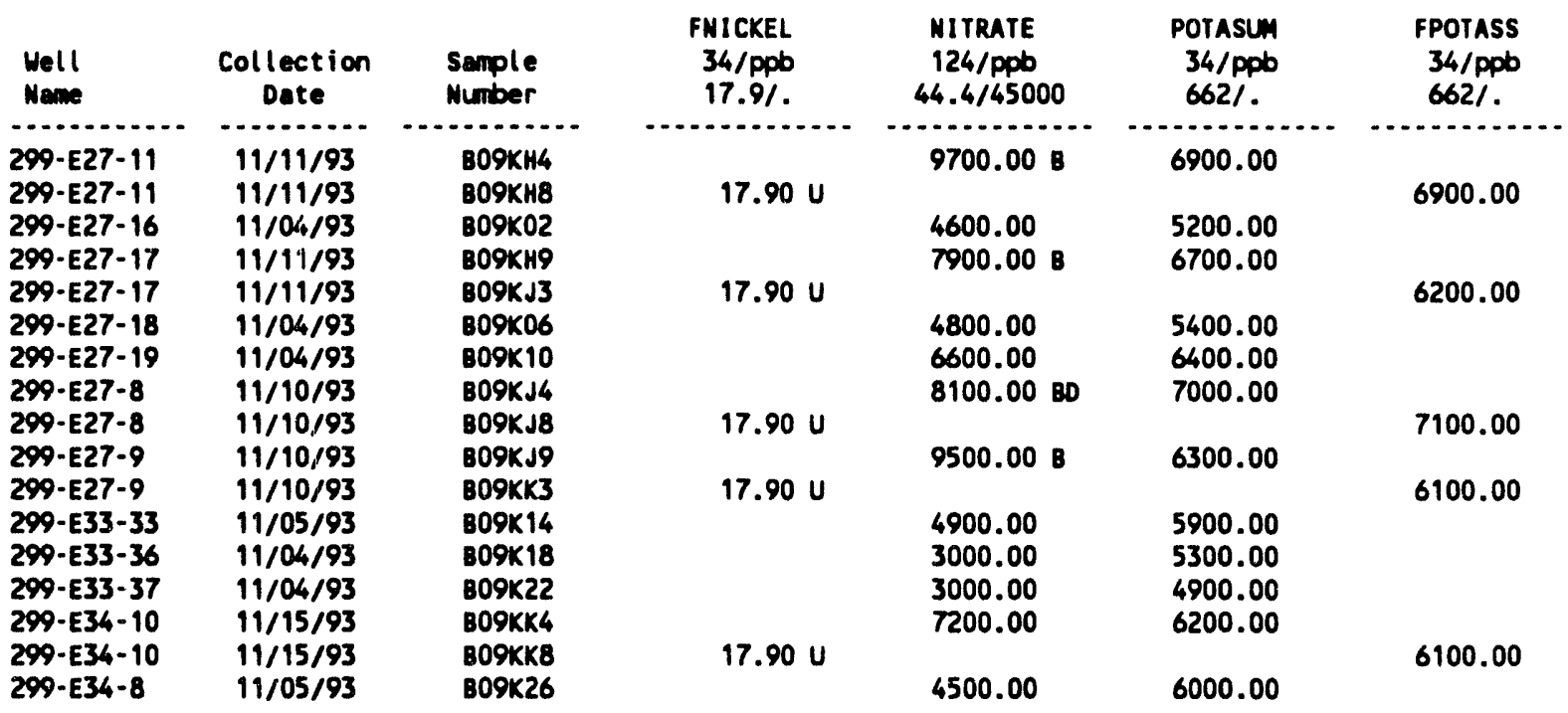

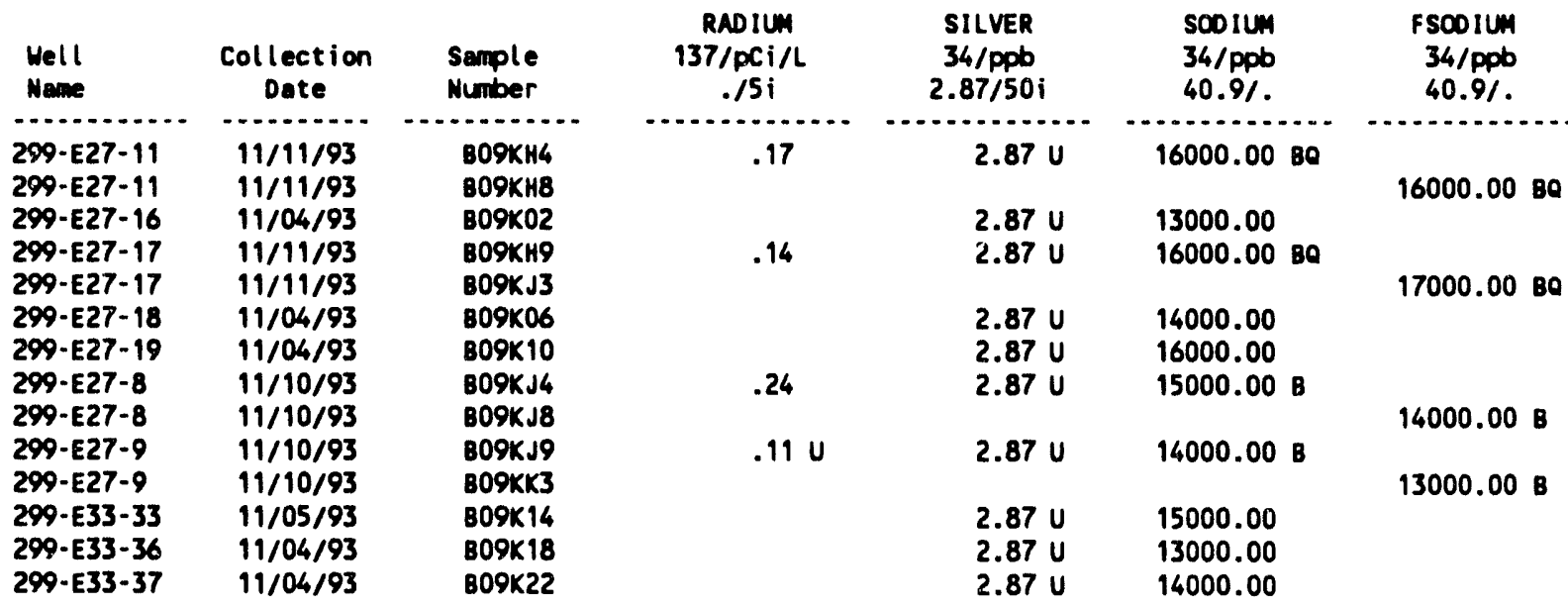


Table 10-4. Constituents with at Least One Detected Value for the 216-B-63 Trench Data for Reporting Period October 1 through December 31, 1993. (sheet 5 of 6)

\begin{tabular}{|c|c|c|c|c|c|c|}
\hline $\begin{array}{l}\text { Well } \\
\text { Nome }\end{array}$ & $\begin{array}{c}\text { Collection } \\
\text { Date }\end{array}$ & $\begin{array}{l}\text { Semple } \\
\text { Number }\end{array}$ & $\begin{array}{c}\text { RADIUM } \\
137 / \mathrm{PCI} / \mathrm{L} \\
.15 i\end{array}$ & $\begin{array}{l}\text { SILVER } \\
34 / \mathrm{ppb} \\
2.87 / 50 \mathrm{i}\end{array}$ & $\begin{array}{l}\text { soolum } \\
34 / \mathrm{ppb} \\
40.91\end{array}$ & $\begin{array}{c}\text { FSODIUN } \\
34 / \text { ppb } \\
40.9 /\end{array}$ \\
\hline $\begin{array}{l}299-E 34-10 \\
299-E 34-10 \\
299-E 34-8\end{array}$ & $\begin{array}{l}11 / 15 / 93 \\
11 / 15 / 93 \\
11 / 05 / 93\end{array}$ & $\begin{array}{l}\text { B09KK4 } \\
\text { B09KK8 } \\
\text { B09K26 }\end{array}$ & $.12 \mathrm{U}$ & $2.87 \mathrm{U}$ & $\begin{array}{l}16000.00 \mathrm{BO} \\
15000.00\end{array}$ & $16000.00 \mathrm{BO}$ \\
\hline $\begin{array}{l}\text { Hell } \\
\text { Nene }\end{array}$ & $\begin{array}{c}\text { Collection } \\
\text { Date }\end{array}$ & $\begin{array}{l}\text { Semple } \\
\text { Number }\end{array}$ & $\begin{array}{c}\text { SULFATE } \\
124 / \mathrm{ppb} \\
49.9 / 250000 \mathrm{~s}\end{array}$ & $\begin{array}{l}\text { TOLUENE } \\
25 / \mathrm{ppb} \\
.056 / 1000\end{array}$ & $\begin{array}{c}\text { TRITIUN } \\
\text { 142/pCi/L } \\
. / 20000\end{array}$ & $\begin{array}{c}\text { TURBID } \\
\text { 126/NTU } \\
.05 /\end{array}$ \\
\hline $\begin{array}{l}299-E 27-11 \\
299-E 27-16 \\
299-E 27-17 \\
299-E 27-18 \\
299-E 27-19 \\
299-E 27-8 \\
299-E 27-9 \\
299-E 33-33 \\
299-E 33-36 \\
299-E 33-37 \\
299-E 34-10 \\
299-E 34-8\end{array}$ & $\begin{array}{l}11 / 11 / 93 \\
11 / 04 / 93 \\
11 / 11 / 93 \\
11 / 04 / 93 \\
11 / 04 / 93 \\
11 / 10 / 93 \\
11 / 10 / 93 \\
11 / 05 / 93 \\
11 / 04 / 93 \\
11 / 04 / 93 \\
11 / 15 / 93 \\
11 / 05 / 93\end{array}$ & $\begin{array}{l}\text { B09KH4 } \\
\text { B09K02 } \\
809 K H 9 \\
809 K 06 \\
\text { B09K10 } \\
\text { B09KJ4 } \\
\text { B09KJ9 } \\
\text { B09K14 } \\
\text { B09K18 } \\
\text { B09K22 } \\
\text { B09KK4 } \\
\text { B09K26 }\end{array}$ & $\begin{array}{ll}67000.00 & 0 \\
35000.00 & 0 \\
64000.00 & D \\
39000.00 & D \\
52000.00 & D \\
69000.00 & D \\
75000.00 & D \\
30000.00 & D \\
24000.00 & D \\
25000.00 & D \\
56000.00 & D \\
37000.00 & D\end{array}$ & $\begin{array}{l}.06 \mathrm{U} \\
.06 \mathrm{U} \\
.06 \mathrm{U} \\
.06 \mathrm{U} \\
.06 \mathrm{U} \\
.06 \mathrm{BL} \\
.06 \mathrm{U} \\
.06 \mathrm{U} \\
.06 \mathrm{U} \\
.06 \mathrm{U} \\
.06 \mathrm{U} \\
.06 \mathrm{U}\end{array}$ & $\begin{array}{l}4360.00 \\
4790.00 \\
5080.00 \\
5160.00 \\
5220.00 \\
3090.00 \\
1640.00 \\
4760.00 \\
3050.00 \\
2950.00 \\
5010.00 \\
3090.00\end{array}$ & $\begin{array}{l}1.900 \\
1.000\end{array}$ \\
\hline
\end{tabular}

\begin{tabular}{|c|c|c|c|c|c|c|}
\hline $\begin{array}{l}\text { Holl } \\
\text { Meme }\end{array}$ & $\begin{array}{c}\text { Collection } \\
\text { Dote }\end{array}$ & $\begin{array}{l}\text { Semple } \\
\text { Number }\end{array}$ & $\begin{array}{l}\text { URANIUN } \\
\text { 145/ppb } \\
. / .\end{array}$ & $\begin{array}{c}U-234 \\
148 / P C i / L \\
.1\end{array}$ & $\begin{array}{c}U-235 \\
148 / p C i / L \\
. /\end{array}$ & $\begin{array}{c}U-238 \\
148 / P C i / L \\
.1\end{array}$ \\
\hline 299-E27-11 & $11 / 11 / 93$ & $809 \times 30$ & & 1.16 & $.01 \mathrm{U}$ & 1.00 \\
\hline $\begin{array}{l}299-E 27-11 \\
299-E 27-16\end{array}$ & $11 / 11 / 93$ & $\begin{array}{l}\text { B09KH4 } \\
\text { B09K0? }\end{array}$ & 2.56 & 112 & 04 & \\
\hline $299-E 27-17$ & $111 / 11 / 93$ & B09k34 & & $\begin{array}{l}1.16 \\
1.30\end{array}$ & .06 & $\begin{array}{r}.45 \\
1.13\end{array}$ \\
\hline 299-E27-17 & $11 / 11 / 93$ & BO9KHO & 2.87 & & & \\
\hline 299-E27-18 & $11 / 04 / 93$ & B09K06 & & 1.25 & .05 & 1.22 \\
\hline 299-E27-19 & $11 / 04 / 93$ & B09K10 & & 1.19 & .06 & 1.03 \\
\hline 299-E27-8 & $11 / 10 / 93$ & B09K38 & & 1.23 & .03 & .86 \\
\hline $299-\varepsilon 27-8$ & $11 / 10 / 93$ & B09KJ4 & 2.61 & & & \\
\hline 299-E27-9 & $11 / 10 / 93$ & B09K42 & & 1.32 & .04 & .98 \\
\hline 299-E27-9 & $11 / 10 / 93$ & B09KJ9 & 2.24 & & & \\
\hline 299-E33-33 & $11 / 05 / 93$ & B09K14 & & 1.20 & $.03 \mathrm{U}$ & .79 \\
\hline $299-E 33-36$ & $11 / 04 / 93$ & B09K18 & & 1.05 & .04 & .80 \\
\hline 299-E33-37 & $11 / 04 / 93$ & B09K22 & & .92 & $.02 u$ & .91 \\
\hline $299-E 34-10$ & $11 / 15 / 93$ & $809 \times 50$ & & 1.16 & .04 & .95 \\
\hline $299-E 34-10$ & $11 / 15 / 93$ & B09KK4 & 3.17 & & & \\
\hline $299-E 34-8$ & $11 / 05 / 93$ & B09K26 & & 1.16 & .05 & .87 \\
\hline
\end{tabular}


Table 10-4. Constituents with at Least One Detected Value for the 216-B-63 Trench Data for Reporting Period October 1 through December 31,1993 . (sheet 6 of 6 )

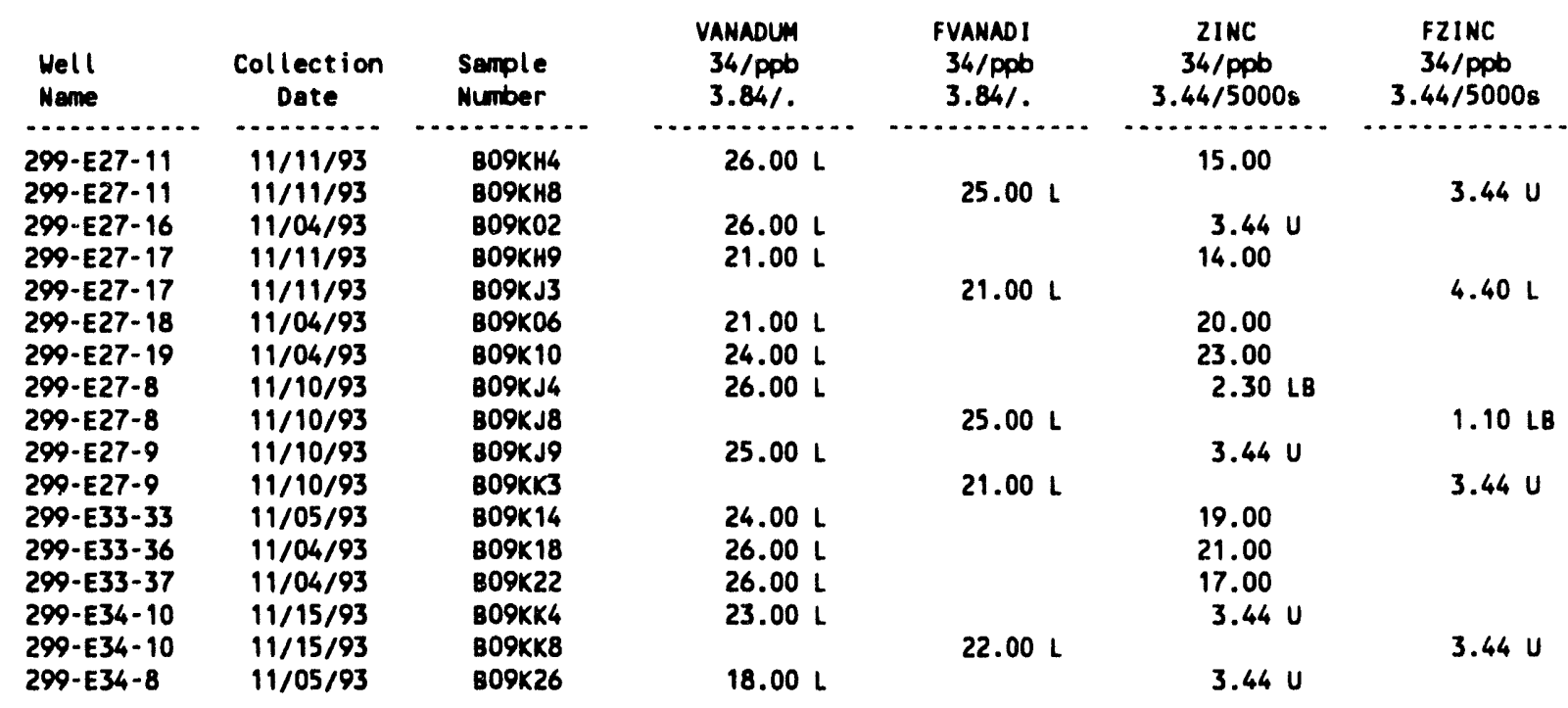

For explanation of this table, see Section 1.4 of report. 
Table 10-5. Contamination Indicator Parameters for the 216-B-63 Trench Data for Reporting Period October 1 through December 31, 1993. (sheet 1 of 2)

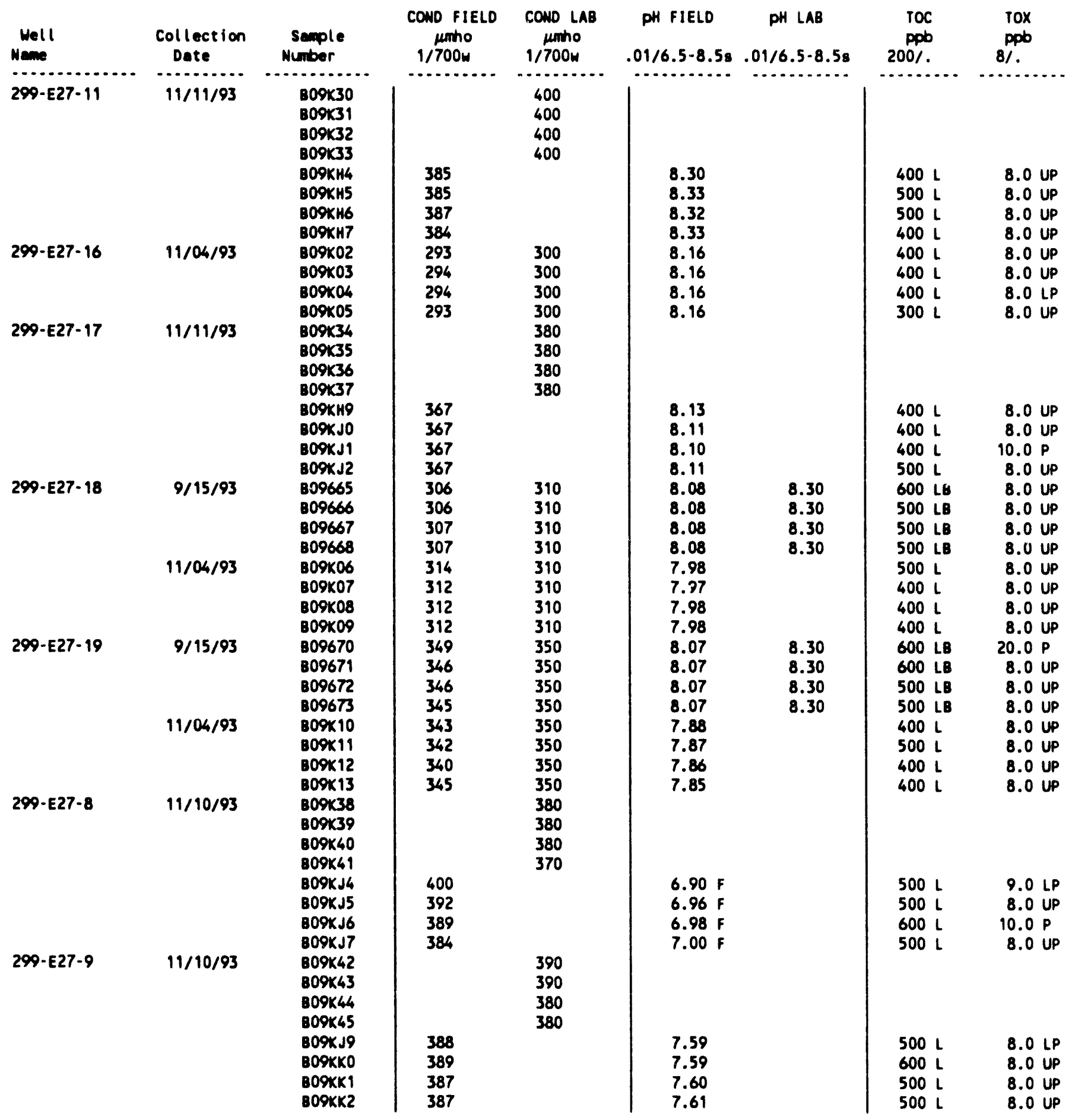


Table 10-5. Contamination Indicator Parameters for the 216-B-63 Trench Data for Reporting Period October 1 through December 31, 1993. (sheet 2 of 2)

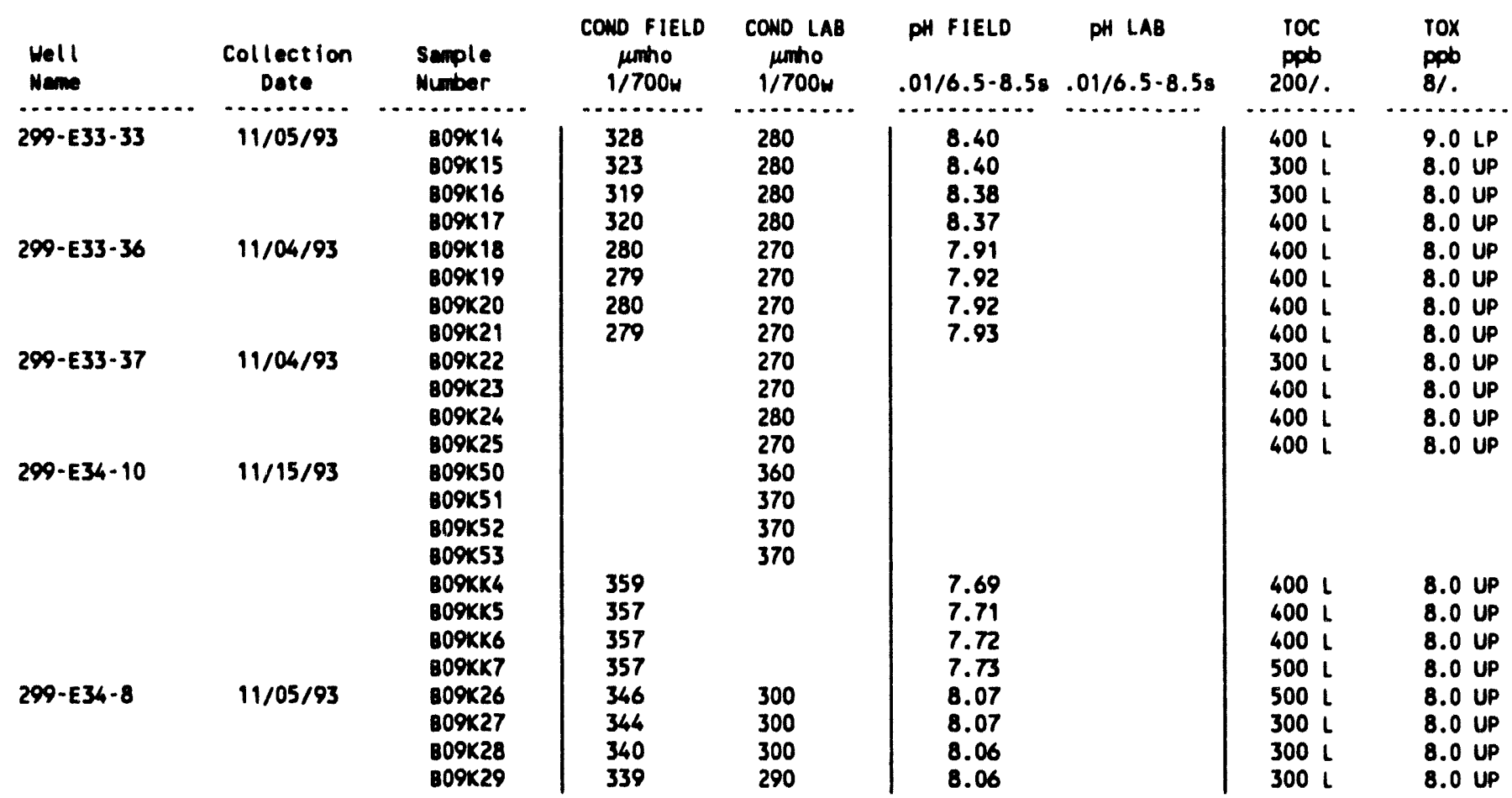

For explanation of this table, see Section 1.4 of report. 
DOE/RL-93-56-4

Table 10-6. Water Quality Standards Exceeded at the 216-B-63 Trench.

\begin{tabular}{|l|l|l|l|}
\hline Unfiltered chromium & $299-E 27-8$ & $299-E 27-16$ & $299-E 33-36$ \\
& $299-E 27-9$ & $299-E 27-17$ & $299-E 34-8$ \\
& $299-E 27-11$ & $299-E 33-33$ & $299-E 34-10$ \\
\hline Unfiltered iron & $299-E 27-8$ & $299-E 27-17$ & $299-E 34-8$ \\
& $299-E 27-9$ & $299-E 33-33$ & $299-E 34-10$ \\
& $299-E 27-11$ & $299-E 33-36$ & \\
\hline
\end{tabular}




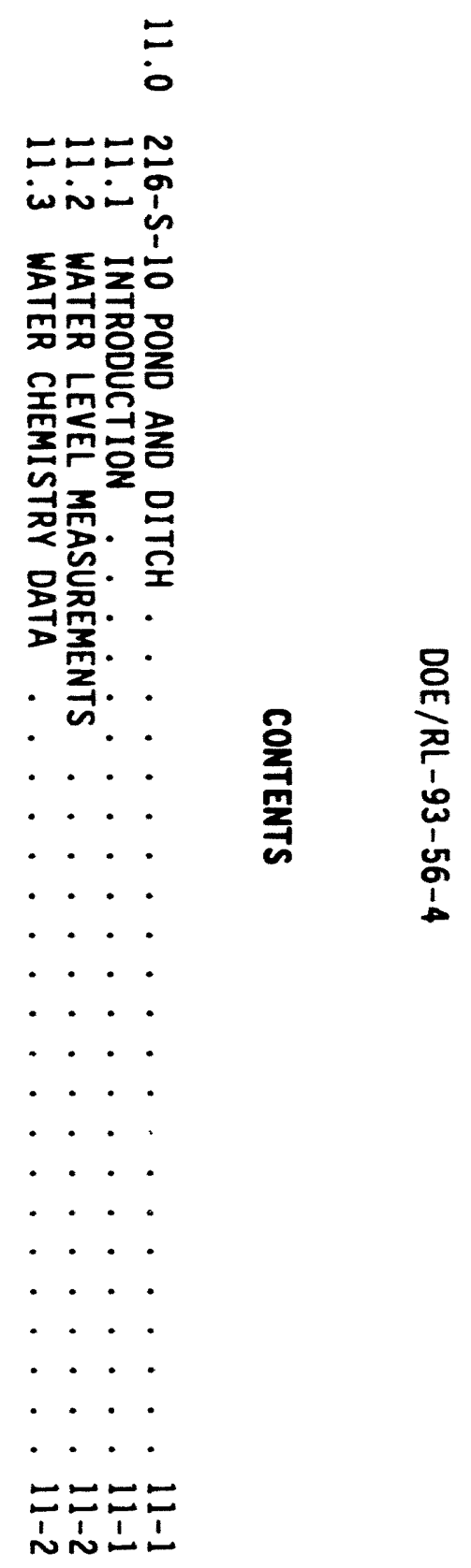


DOE/RL-93-56-4

LIST OF FIGURES

11-1 Monitoring Well Location for the 216-S-10 Facility . . . . . 11-3

\section{LIST OF TABLES}

11-1 Monitoring Well Purpose and Sampling Schedule for the 216-S-10 Facility . . . . . . . . . . . . . . . . . 11-4

11-2 RCRA Water Level Measurement Report for the 216-S-10 Facility, Fourth Quarter 1993 ........... 11-5

11-3 Constituent List and Summary of Results for the 216-S-10 Facility Data for Reporting Period October 1 through December $31,1993 \ldots$. . . . . . . . . . . . 11-6

11-4 Constituents with at Least One Detected Value for the 216-S-10 Facility Data for Reporting Period October 1 through December 31, $1993 \ldots$. . . . . . . . . . . . . 11-8

11-5 Contamination Indicator Parameters for the 216-S-10 Facility Data for Reporting Period October 1 through December 31, 1993 . . 11-9 


\title{
11.0 216-S-10 POND AND DITCH
}

\author{
J. W. Lindberg \\ Westinghouse Hanford Company
}

\subsection{INTRODUCTION}

The 216-S-10 Pond and Ditch (S-10 Facility) is located south-southwest of the 200 West Area, directly outside the perimeter fence. The S-10 Facility received wastewater from the Reduction-0xidation (REDOX) Plant (202-S Building) located in the 200 West Area. In the past, wastewater disposed at the S-10 Facility has contained hazardous waste and radioactive materials. Since 1985, physical controls and operating procedures have been modified to avoid inadvertent discharge of hazardous chemicals to the wastewater stream. The facility has been monitored under interim status since August 1991. In October 1991, the effluent waste stream, including the water source, to the 216-S-10 Ditch was deactivated. Because the S-10 Facility is not expected to receive additional hazardous substances, the facility will be closed under RCRA interim status.

The S-10 Facility initially comprised an open, unlined ditch $1.8 \mathrm{~m}(6 \mathrm{ft})$ deep, $1.2 \mathrm{~m}(4 \mathrm{ft})$ wide at the bottom, and $686 \mathrm{~m}(2,250 \mathrm{ft})$ long. In addition, an open, unlined percolation pond constructed at the southwest end of the ditch, which is approximately 2 hectares (5 acres) in size, was active during part of the time the ditch was receiving waste.

The 216-S-10 Ditch began receiving wastewater from the REDOX Plant in August 1951, and the 216-5-10 Pond was dug and placed in service in February 1954. In Dctober 1985, the 216-5-10 Pond and portions of the 216-S-10 Ditch were decommissioned, backfilled, and stabilized. The remaining portion of the 216-S-10 Ditch (approximately $594 \mathrm{~m}[1,950 \mathrm{ft}]$ ) received nonhazardous, nonregulated waste from the 202-S Building chemical sewer until deactivation in October 1991. This waste stream was composed of cooling water, steam condensate, water tower overflow, and drain effluent. These source streams had been routed so that they would not come into contact with hazardous materials. There has been no effluent discharge to the facility since October 1991.

Releases of hazardous materials and constituents to the S-10 Facility are poorly documented. Radioactive waste was reportedly disposed at the S-10 Facility as a result of contaminated floor and sewer drains at the REDOX Plant. Hazardous chemical releases were documented in 1954 and 1983 and included $\mathrm{Al}\left(\mathrm{NO}_{3}\right)_{3}, \mathrm{NaNO}_{3}, \mathrm{NaOH}, \mathrm{Na}_{3} \mathrm{PO}_{4}, \mathrm{NaF}, \mathrm{NaCl}$, and $\mathrm{K}_{2} \mathrm{Cr}_{2} \mathrm{O}_{7}$.

During 1991 the annual volume of effluent discharged to the 216-S-10 Ditch was between $1.9 \times 10^{8}$ and $5.7 \times 10^{8} \mathrm{~L}\left(5.0 \times 10^{7}\right.$ and $\left.1.5 \times 10^{8} \mathrm{gal}\right)$. This volume of discharge had created a localized recharge mound and an associated perched water table directly below the receiving (north) end of the ditch. This recharge mound has since dissipated, and the perched water table has been dry since shortly after the ditch was deactivated. 
DOE/RL-93-56-4

Groundwater beneath the 216-S-10 Ditch is sampled semiannually (except for well 299-W27-2, which is sampled quarterly) in accordance with the Interim-Status Groundwater Monitoring Plan for the 216-5-10 Pond and Ditch (WHC 1990b). The site is currently in the indicator parameters evaluation program.

The monitoring network currently consists of two upgradient wells, three downgradient wells, and one downgradient well that monitors the base of the unconfined aquifer (Figure 11-1). Table 11-1 1ists the wells and their sampling status. All of the wells now have at least four quarters of analytical data. The results of the background statistical calculations for the upgradient monitoring wells (based on the first four quarters of analytical data) were presented in the RCRA annual report (DOE-RL 1993a).

\subsection{MATER LEVEL MEASUREMENTS}

Water level measurements obtained during the fourth quarter of $\mathrm{CY} 1993$ are presented in Table 11-2. Water level measurements are obtained at all wells that are sampled. Also, quarterly water level measurements are made by WHC at four wells at the S-10 Facility, including 299-W26-8, 299-W26-9, 299-W26-10, 299-W26-11 (if the well is not dry), and 299-W26-12.

\subsection{MATER CHEMISTRY DATA}

Groundwater sampling during the fourth quarter of $\mathrm{Cr} 1993$ included all of the wells in the monitoring network. Table 11-1 lists the sampling dates for each of the wells. Available data for the reporting period October 1 through December 31, 1993, are shown in Tables 11-3 through 11-5. Table 11-3 includes a list of reported constituents and a summary of results. Constituents with at least one detected value are provided in Table 11-4. Table 11-5 1ists the results for the CIP analyses. No CIP replicate averages exceed the calculated critical means except for the case noted below.

The critical mean value for specific conductance was exceeded in well 299-W27-2 in both the field and laboratory analyses $(311 \mu \mathrm{mho} / \mathrm{cm}$ for the field and $350 \mu \mathrm{mho} / \mathrm{cm}$ for the laboratory). However, well 299-W27-2

(downgradient) monitors the base of the unconfined aquifer and is not used for statistical evaluation purposes. As more data become available from well 299-W27-2, time-versus-concentration plots will be developed to monitor the groundwater quality at the deeper horizon.

Tables 11-3 and 11-4 show that for all constituents analyzed, none of them exceeded DWSs. The $D$ flags for sulfate indicate that the samples were diluted for analysis. 

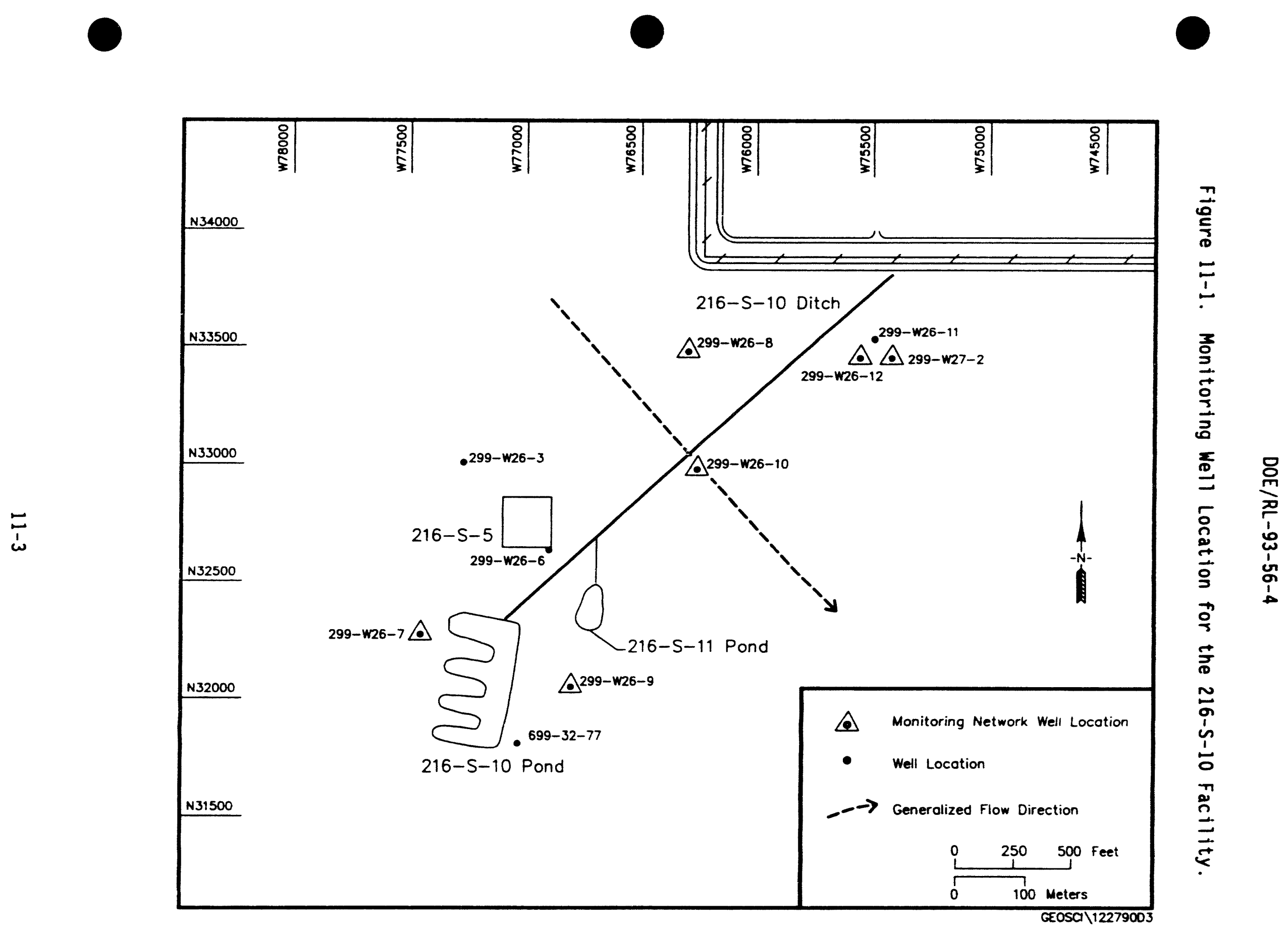
Table 11-1. Monitoring Well Purpose and Sampling Schedule for the 216-S-10 Facility.

\begin{tabular}{|c|c|c|c|c|}
\hline $\begin{array}{c}\text { Well no. } \\
(299-)\end{array}$ & $\begin{array}{c}\text { Relative } \\
\text { position }\end{array}$ & Hydrogeologic unit & $\begin{array}{c}\text { Sampling } \\
\text { frequency }\end{array}$ & $\begin{array}{c}\text { Sample date, } \\
\text { 4th Qtr 1993 }\end{array}$ \\
\hline W26-7 & Upgradient & Ringold: Water Table & Semiannually & $12 / 15 / 93$ \\
\hline W26-8 & Upgradient & Ringold: Water Table & Semiannually & $12 / 15 / 93$ \\
\hline W26-9 & Downgradient & Ringold: Water Table & Semiannually & $12 / 17 / 93$ \\
\hline W26-10 & Downgradient & Ringold: Water Table & Semiannually & $12 / 17 / 93$ \\
\hline W26-12 & Downgradient & $\begin{array}{l}\text { Ringold: Water Table } \\
\text { Wemiannually }\end{array}$ & $12 / 17 / 93$ \\
\hline W27-2 & Downgradient & $\begin{array}{c}\text { Ringold: Base of } \\
\text { Unconfined }\end{array}$ & Quarterly & $\begin{array}{l}09 / 27 / 93 \\
12 / 16 / 93\end{array}$ \\
\hline
\end{tabular}


Table 11-2. RCRA Water Level Measurement Report for the 216-S-10 Facility, Fourth Quarter 1993.

\begin{tabular}{cccc}
\hline We11 & Date & $\begin{array}{c}\text { Depth to } \\
\text { water }(\mathrm{ft})\end{array}$ & $\begin{array}{c}\text { Water level } \\
\text { elevation } \\
\text { above ms } 1 \mathrm{ft})\end{array}$ \\
\hline Wells Monitoring the Top of the Unconfined Aquifer \\
\hline $299-W 26-10$ & $12 / 08 / 93$ & 214.08 & 456.79 \\
$299-W 26-12$ & $12 / 08 / 93$ & 218.62 & 457.07 \\
$299-W 26-7$ & $12 / 08 / 93$ & 193.47 & 458.52 \\
$299-W 26-8$ & $12 / 08 / 93$ & 208.87 & 457.44 \\
$299-W 26-9$ & $12 / 08 / 93$ & 197.01 & 457.15 \\
\hline
\end{tabular}

Notes: 1. Water level elevations are calculated by subtracting the measured depth to water from the surveyed elevation for the well.

2. Depth-to-water values are transcribed from field records.

3. Depth-to-water measurements taken at the time of sampling were not available at the time of this report.

4. To convert feet to meters multiply by 0.3048 . 
Table 11-3. Constituent List and Summary of Results for the 216-S-10 Facility Data for Reporting Period October 1 through December 31, 1993.

(sheet 1 of 2)

CONTAMIMATION INDICATOR PARAMETERS

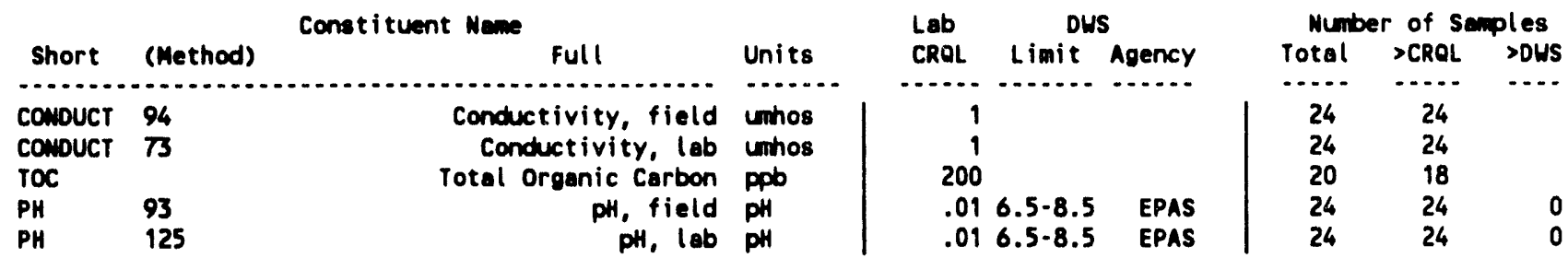

DRINKING WATER PARAMETERS

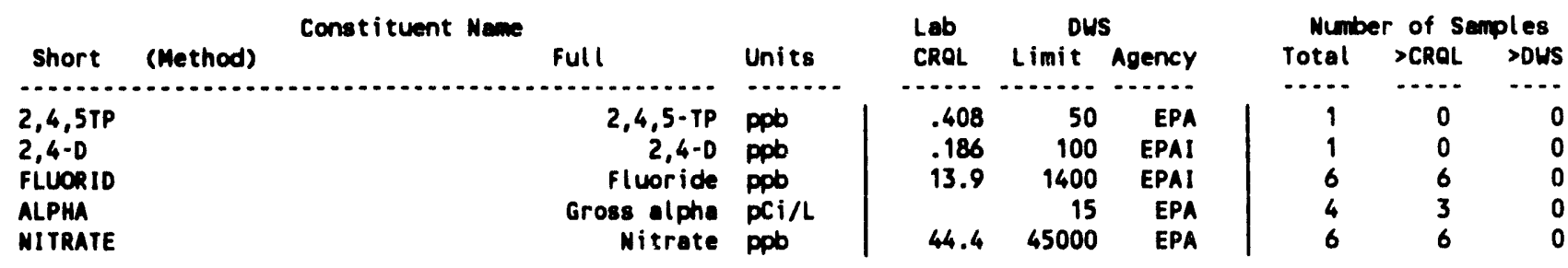

GROUNDWATER OUALITY PARAMETERS

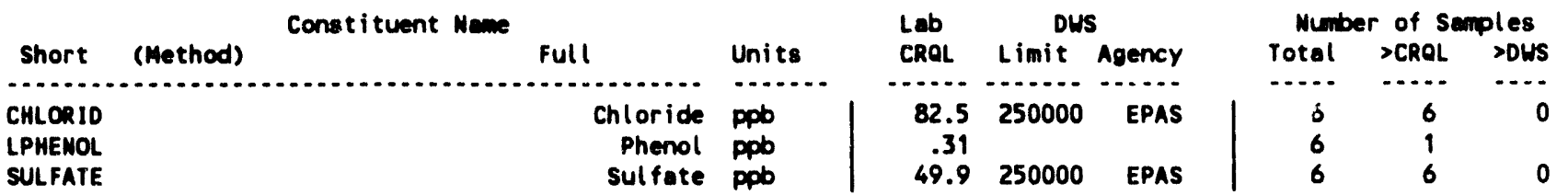

SITE SPECIFIC AND OTHER CONSTITUENTS

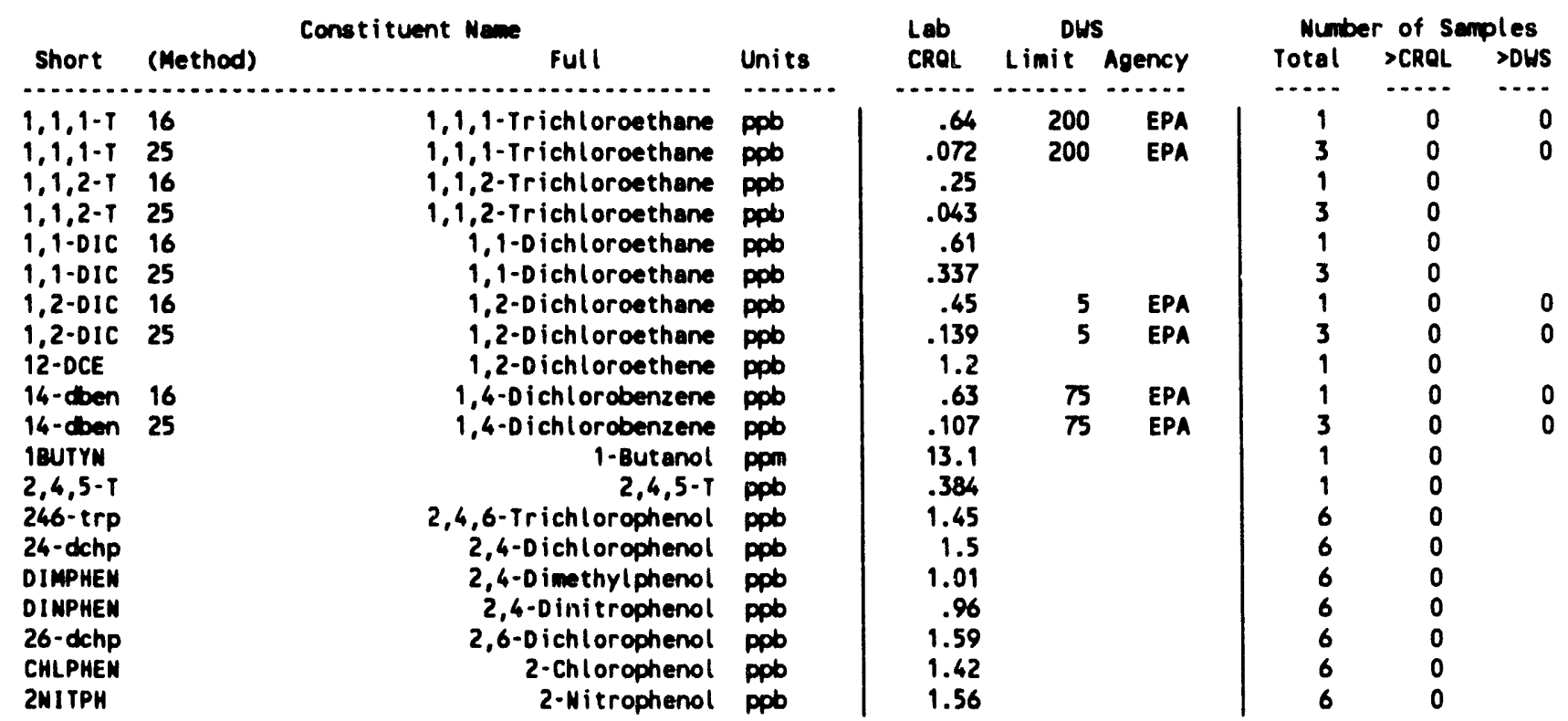


Table 11-3. Constituent List and Summary of Results for the 216-S-10 Facility Data for Reporting Period October 1 through December 31, 1993.

(sheet 2 of 2)

\begin{tabular}{|c|c|c|c|c|c|c|c|c|c|}
\hline & & Constituent Mane & & Lab & DWS & & Numit & of Se & ples \\
\hline Short & (Method) & Full & Units & CROL & Limit & Agency & Total & $>$ CROL & $>$ DWS \\
\hline SUTOIMP & 30 & 2-sec-Butyl-4,6-dinitrophenol (DN & mob & 1.35 & & & 6 & 0 & \\
\hline BUTDINP & 49 & 2-sec-Butyl-4,6-dini trophenol (DN & 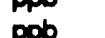 & 269 & & & 0 & 0 & \\
\hline 460N2NAP & & 4,6-0 initro-2-methylphenol & ppob & 1.18 & & & 6 & 0 & \\
\hline CHLCRES & & 4-Chloro-3-methylphenol & ppb & 1.12 & & & 6 & 0 & \\
\hline MIBK & & 4-Methyl-2-pentanone & ppb & .85 & & & 1 & 0 & \\
\hline MITPHEN & & 4-Nitrophenol & ppob & .65 & & & 6 & 0 & \\
\hline ACETONE & & Acetone & ppb & 13.4 & & & 1 & 0 & \\
\hline BENZENE & 16 & Benzene & ppb & .65 & 5 & EPA & 1 & 0 & 0 \\
\hline BENZENE & 25 & Benzene & ppo & .105 & 5 & EPA & 3 & 0 & 0 \\
\hline BROMIDE & & Bromide & ppb & 52.8 & & & 6 & 0 & \\
\hline CARBIDE & & Carbon disulfide & ppb & .95 & & & 1 & 0 & \\
\hline TETRAME & 16 & Carbon tetrachloride & ppob & .87 & 5 & EPA & 1 & 1 & 0 \\
\hline TETRANE & 25 & Carbon tetrachloride & ppb & .121 & 5 & EPA & 3 & 3 & $\mathbf{0}$ \\
\hline CHLFORM & 16 & Chtoroform & ppb & .4 & & & 1 & 1 & \\
\hline CHLFOAM & 25 & Chloroform & ppb & .043 & & & 3 & 3 & \\
\hline CRESOLS & & Cresols (wethylphenols) & ppb & 4.66 & & & 6 & 0 & \\
\hline ETMCYAN & & Ethyl cyanide & ppb & 4.34 & & & 1 & $\mathbf{0}$ & \\
\hline ETHBENZ & & Ethylbenzene & ppb & .046 & 700 & EPA & 3 & $\mathbf{0}$ & 0 \\
\hline BETA & & Gross bete & $\mathrm{PCi} / \mathrm{L}$ & & & & 4 & 4 & \\
\hline METHONE & & Methyl ethyl ketone & ppb & 4.25 & & & 1 & 0 & \\
\hline МЕTнYсн & 16 & Methylene chloride & ppb & .61 & & & 1 & 0 & \\
\hline МЕтнYсн & 25 & Methylene chloride & ppb & .056 & & & 3 & 0 & \\
\hline MITRITE & & Nitrite & ppb & 38.3 & 1000 & EPA & 6 & 0 & 0 \\
\hline PENTCHP & & Pentechlorophenol & ppb & .87 & 1 & EPA & 6 & 0 & 0 \\
\hline PHOSPHA & & Phosphate & ppb & 147 & & & 6 & 0 & \\
\hline TC- 99 & & Technet ium-99 & $\mathrm{PCi} / \mathrm{L}$ & & 900 & EPA & 6 & 3 & 0 \\
\hline PERCENE & 16 & Tetrachloroethene & ppb & 1.1 & 5 & EPA & 1 & 0 & 0 \\
\hline PERCENE & 25 & Tetrachloroethene & ppob & .049 & 5 & EPA & 3 & 0 & 0 \\
\hline $\begin{array}{l}\text { TETPHNL } \\
\text { TAF }\end{array}$ & & $\begin{array}{l}\text { Tetrachlorophenols } \\
\text { Tetrahydrofuran }\end{array}$ & $\begin{array}{l}\text { ppb } \\
\text { ppb }\end{array}$ & $\begin{array}{l}1.05 \\
3.67\end{array}$ & & & 6 & $\begin{array}{l}0 \\
0\end{array}$ & \\
\hline TOLUENE & 16 & Toluene & ppob & .73 & 1000 & EPA & 1 & 0 & 0 \\
\hline TOLUENE & 25 & Toluene & ppb & .056 & 1000 & EPA & 3 & 0 & 0 \\
\hline TRICENE & 16 & Trichloroethene & ppo & .77 & 5 & EPA & 1 & 0 & 0 \\
\hline TRICENE & 25 & Trichloroethene & ppb & .065 & 5 & EPA & 3 & 0 & 0 \\
\hline TRIPHNL & & Trichlorophenols & ppb & 1.11 & & & 6 & 0 & \\
\hline TRITIUM & & Tritiun & $\mathrm{PCi} / \mathrm{L}$ & & 20000 & EPA & 4 & 3 & 0 \\
\hline URANIUN & & Uraniva & ppb & & & & 6 & 6 & \\
\hline VIAYIOE & 16 & Vinyl chloride & ppb & .78 & 2 & EPA & 1 & 0 & 0 \\
\hline VINYIDE & 25 & Vinyl chloride & ppb & .266 & 2 & EPA & 3 & $\mathbf{0}$ & 0 \\
\hline XYLENE & 16 & Xylenes (total) & ppob & 1.7 & 10000 & EPA & 1 & 0 & 0 \\
\hline XYLENE & 25 & Xylenes (total) & ppob & .202 & 10000 & EPA & 3 & 0 & 0 \\
\hline $\begin{array}{l}\text { CIS1ZOE } \\
\text { TRANDCE }\end{array}$ & & $\begin{array}{l}\text { cis-1,2-Dichloroethylene } \\
\text { trans-1,2-Dichloroethylene }\end{array}$ & $\begin{array}{l}\text { ppb } \\
\text { ppb }\end{array}$ & $\begin{array}{l}.127 \\
.149\end{array}$ & $\begin{array}{r}70 \\
100\end{array}$ & $\begin{array}{l}\text { EPA } \\
\text { EPA }\end{array}$ & $\begin{array}{l}3 \\
3\end{array}$ & $\begin{array}{l}0 \\
0\end{array}$ & $\begin{array}{l}0 \\
0\end{array}$ \\
\hline innose & & & & & & & & 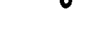 & \\
\hline
\end{tabular}

For explenation of this teble, see Section 1.4 of report. 
Table 11-4. Constituents with at Least One Detected Value for the 216-S-10 Facility Data for Reporting Period October 1 through December 31, 1993.

\begin{tabular}{|c|c|c|c|c|c|c|}
\hline $\begin{array}{l}\text { Well } \\
\text { Neme }\end{array}$ & $\begin{array}{c}\text { Collection } \\
\text { Dote }\end{array}$ & $\begin{array}{l}\text { Semple } \\
\text { Number }\end{array}$ & $\begin{array}{c}\text { TETRANE } \\
\text { 16/ppb } \\
.87 / 5\end{array}$ & $\begin{array}{l}\text { TE TRANE } \\
25 / \mathrm{ppb} \\
.121 / 5\end{array}$ & $\begin{array}{c}\text { CHLOR1D } \\
124 / \mathrm{ppb} \\
82.5 / 250000 \mathrm{~s}\end{array}$ & $\begin{array}{c}\text { CHLFORM } \\
16 / \mathrm{Ppb} \\
.4 / .\end{array}$ \\
\hline $\begin{array}{l}299-W 26-10 \\
299-W 26-12 \\
299-W 26-7 \\
299-W 26-8 \\
299-W 26-9 \\
299-W 27-2 \\
299-W 27-2\end{array}$ & $\begin{array}{r}12 / 17 / 93 \\
12 / 17 / 93 \\
12 / 15 / 93 \\
12 / 15 / 93 \\
12 / 17 / 93 \\
9 / 27 / 93 \\
12 / 16 / 93\end{array}$ & $\begin{array}{l}\text { B09N25 } \\
809 P 00 \\
809 P 05 \\
809 P 10 \\
809 P 15 \\
8096 Y 0 \\
809 P 20\end{array}$ & $4.90 \mathrm{~L}$ & $\begin{array}{l}.37 \mathrm{LO} \\
1.400 \\
3.700\end{array}$ & $\begin{array}{l}6200.00 \\
1500.00 \\
5500.00 \\
3100.00 \\
6800.00 \\
22000.00\end{array}$ & $.68 \mathrm{~L}$ \\
\hline $\begin{array}{l}\text { Mell } \\
\text { Mene }\end{array}$ & $\begin{array}{c}\text { Collection } \\
\text { Date }\end{array}$ & $\begin{array}{l}\text { Semple } \\
\text { Number }\end{array}$ & $\begin{array}{l}\text { CHLFORM } \\
25 / \text { PPb } \\
.043 / .\end{array}$ & $\begin{array}{l}\text { FLUORID } \\
124 / \mathrm{ppb} \\
13.9 / 1400 \mathrm{i}\end{array}$ & $\begin{array}{c}\text { ALPHA } \\
135 / \mathrm{PCI} / \mathrm{L} \\
. / 15\end{array}$ & $\begin{array}{c}\text { BETA } \\
136 / \mathrm{PCI} / \mathrm{L} \\
. /\end{array}$ \\
\hline $\begin{array}{l}299-\text { W26-10 } \\
299-\text { W26-12 } \\
299-\text { W26-7 } \\
299-\text { W26-8 } \\
299-W 26-9 \\
299-\text { W27-2 }\end{array}$ & $\begin{array}{l}12 / 17 / 93 \\
12 / 17 / 93 \\
12 / 15 / 93 \\
12 / 15 / 93 \\
12 / 17 / 93 \\
12 / 16 / 93\end{array}$ & $\begin{array}{l}\text { B09N25 } \\
\text { B09P00 } \\
\text { B09P05 } \\
\text { B09P10 } \\
\text { B09P15 } \\
\text { B09P20 }\end{array}$ & $\begin{array}{l}.14 \mathrm{~L} \\
.81 \\
.30 \mathrm{~L}\end{array}$ & $\begin{array}{r}800.00 \\
1000.00 \\
900.00 \\
800.00 \\
800.00 \\
900.00\end{array}$ & $\begin{array}{l}.98 \mathrm{U} \\
3.93 \\
1.36 \\
1.31\end{array}$ & $\begin{array}{r}5.97 \\
17.10\end{array}$ \\
\hline $\begin{array}{l}\text { Well } \\
\text { Memere }\end{array}$ & $\begin{array}{c}\text { Collection } \\
\text { Date }\end{array}$ & $\begin{array}{l}\text { Semple } \\
\text { Number }\end{array}$ & $\begin{array}{l}\text { NITRATE } \\
124 / \mathrm{ppb} \\
44.4 / 45000\end{array}$ & $\begin{array}{l}\text { LPHENOL } \\
30 / \mathrm{ppb} \\
.31 \%\end{array}$ & $\begin{array}{c}\text { SUL.FATE } \\
124 / \mathrm{ppb} \\
49.9 / 250000 \mathrm{~s}\end{array}$ & $\begin{array}{c}\text { TC- } 99 \\
143 / p C i / L \\
. / 900\end{array}$ \\
\hline $\begin{array}{l}299-W 26-10 \\
299-W 26-12 \\
299-W 26-7 \\
299-W 26-8 \\
299-W 26-9 \\
299-W 27-2\end{array}$ & $\begin{array}{l}12 / 17 / 93 \\
12 / 17 / 93 \\
12 / 15 / 93 \\
12 / 15 / 93 \\
12 / 17 / 93 \\
12 / 16 / 93\end{array}$ & $\begin{array}{l}\text { B09W25 } \\
\text { B09P00 } \\
\text { B09P05 } \\
\text { B09P10 } \\
\text { B09P15 } \\
\text { B09P20 }\end{array}$ & $\begin{array}{r}2600.00 \\
600.00 \\
7800.00 \\
400.00 \\
5800.00 \\
4600.00\end{array}$ & $\begin{array}{l}.31 \mathrm{U} \\
.40 \mathrm{~L} \\
.31 \mathrm{U} \\
.31 \mathrm{U} \\
.31 \mathrm{U} \\
.31 \mathrm{U}\end{array}$ & $\begin{array}{lll}13000.00 & \mathrm{D} \\
12000.00 & \mathrm{D} \\
18000.00 \mathrm{D} \\
10000.00 \mathrm{D} \\
18000.00 \mathrm{D} \\
18000.00 \mathrm{D}\end{array}$ & $\begin{array}{l}.69 \mathrm{U} \\
-.42 \mathrm{U} \\
2.32 \\
3.31 \\
1.07 \mathrm{U} \\
6.80\end{array}$ \\
\hline
\end{tabular}

\begin{tabular}{|c|c|c|c|c|}
\hline $\begin{array}{l}\text { Well } \\
\text { Mene }\end{array}$ & $\begin{array}{c}\text { Collection } \\
\text { Date }\end{array}$ & $\begin{array}{l}\text { Semple } \\
\text { Number }\end{array}$ & $\begin{array}{l}\text { TRITIUN } \\
\text { 142/pCi/L } \\
. / 20000\end{array}$ & $\begin{array}{c}\text { URANIUM } \\
\text { 145/ppb } \\
.1\end{array}$ \\
\hline $\begin{array}{l}299-\mathrm{W} 26-10 \\
299-\mathrm{W} 26-12 \\
299-\mathrm{W} 26-7 \\
299-\mathrm{W} 26-8 \\
299-\mathrm{W} 26-9 \\
299-\mathrm{W} 27-2\end{array}$ & $\begin{array}{l}12 / 17 / 93 \\
12 / 17 / 93 \\
12 / 15 / 93 \\
12 / 15 / 93 \\
12 / 17 / 93 \\
12 / 16 / 93\end{array}$ & $\begin{array}{l}\text { BO9NZ5 } \\
\text { B09P00 } \\
\text { BO9P05 } \\
\text { B09P10 } \\
\text { B09P15 } \\
\text { 809P20 }\end{array}$ & $\begin{array}{l}263.00 \\
157.00 \mathrm{U} \\
\\
359.00 \\
324.00\end{array}$ & $\begin{array}{r}1.58 \\
3.89 \\
1.64 \\
15.40 \\
1.61 \\
.63\end{array}$ \\
\hline
\end{tabular}

For explanation of this table, see Section 1.4 of report. 
Table 11-5. Contamination Indicator Parameters for the 216-S-10 Facility Data for Reporting Period October 1 through December 31, 1993.

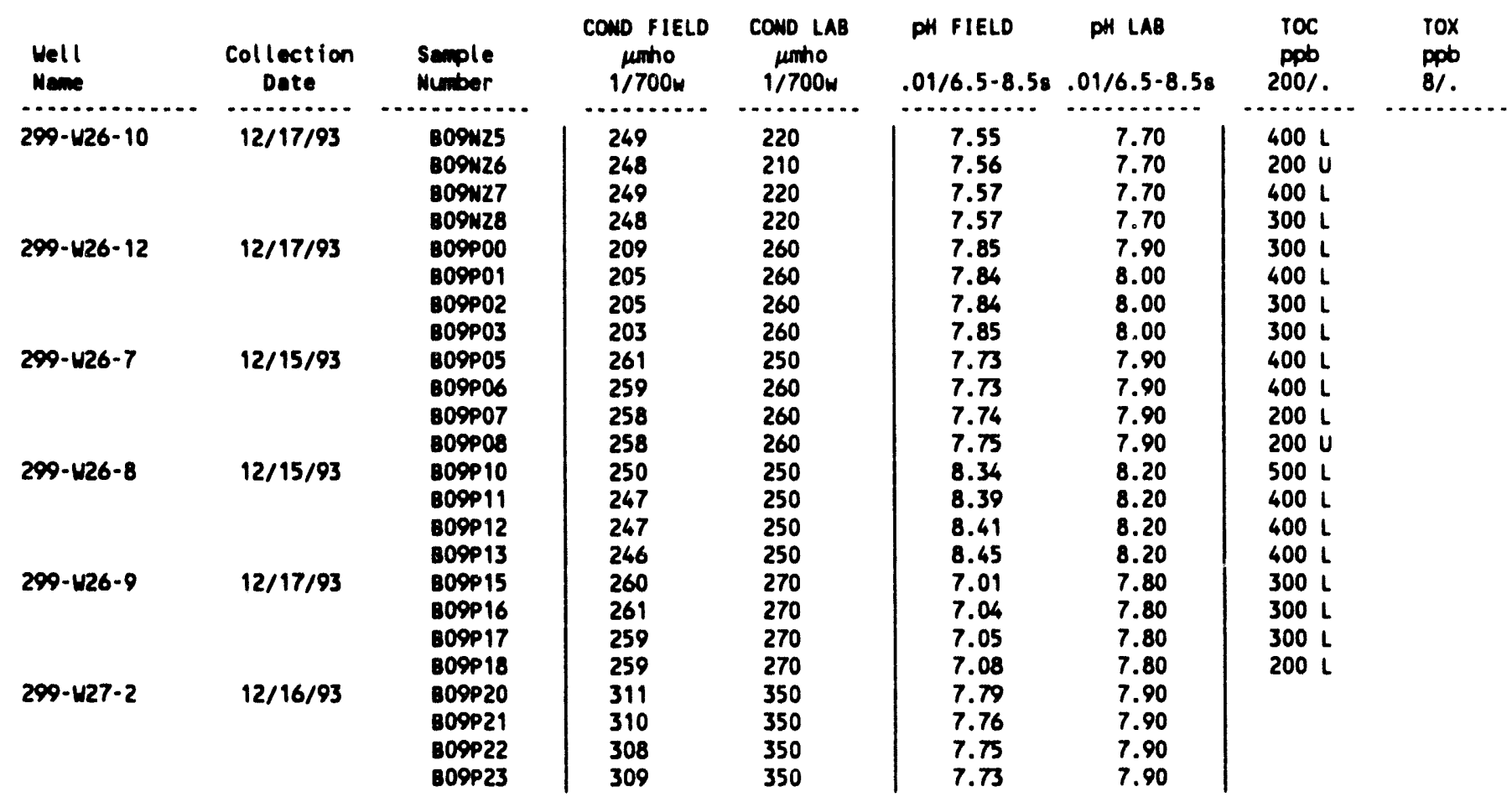

For explenation of tis teble, see Section 1.4 of report. 
DOE/RL-93-56-4

This page intentionally left blank. 
DOE/RL-93-56-4

\section{CONTENTS}

$12.0216-U-12$ CRIB $\ldots \ldots \ldots$. . . . . . . . . . . . 12-1

12.1 INTRODUCTION . . . . . . . . . . . . . . . 12-1

12.2 WATER LEVEL MEASUREMENTS . . . . . . . . . . . . . . 12-2

12.3 WATER CHEMISTRY DATA .............. . . 12-2 
DOE/RL-93-56-4

LIST OF FIGURES

12-1 Well Location Map for 216-U-12 Crib . . . . . . . . . . 12-3

\section{LIST OF TABLES}

12-1 Monitoring Well Purpose and Sampling Schedule for the 216-U-12 Crib................. 12-4

12-2 RCRA Water Level Measurement Report for the 216-U-12 Crib, Fourth Quarter 1993.............. 12-5

12-3 Constituent List and Summary of Results for the 216-U-12 Crib Data for Reporting Period October 1 through December 31, 1993 . . 12-6

12-4 Constituents with at Least One Detected Value for the 216-U-12 Crib Data for Reporting Period October 1 through December $31,1993 \ldots$. . . . . . . . . . . . .

12-5 Contamination Indicator Parameters for the 216-U-12 Crib Data for Reporting Period October 1 through December 31, 1993 . . . . 12-9 


\title{
$12.0216-\mathrm{U}-12$ CRIB
}

\author{
B. A. Williams \\ Hestinghouse Hanford Company
}

\subsection{INTRODUCTION}

A RCRA assessment-level groundwater monitoring network is established for the 216-U-12 $\mathrm{Crib}(\mathrm{U}-12 \mathrm{Crib})$. The $\mathrm{U}-12 \mathrm{Crib}$ received wastewater from the $U$ Plant in the 200 West Area from April 1960 unt 11 February 1988, when it was permanently retired and replaced by the 216-U-17 $\mathrm{Crib}$. Wastewater disposed in the U-12 Crib contained hazardous waste and radioactive materials. RL has proposed that the facility be closed under RCRA interim status ( 40 CFR 265).

The U-12 Crib is located approximately $609 \mathrm{~m}(1,998 \mathrm{ft})$ south of the $U$ Plant in the 200 West Area (see Figure 1-1). The crib is an unlined, gravel-bottom, percolation crib that is 3 by $30.5 \mathrm{~m}$ ( 10 by $100 \mathrm{ft}$ ) wide and $4 \mathrm{~m}(13 \mathrm{ft})$ deep. The crib has a plastic barrier cover and is backfilled with the original excavated soil. A vitrified clay distribution pipe, buried in the gravel, dispersed the effluent across the bottom of the crib.

The waste was composed of effluent from the $U$ Plant and included 291-U-1 Stack drainage and process condensate from the 224-U Building. The U-12 Crib received this waste stream from April 1960 until 1972, when it was deactivated. The U-12 Crib was reactivated in November 1981 and received waste until it was permanently retired in February 1988. An average of over $1,000,000 \mathrm{~L}(264,200 \mathrm{gal})$ of effluent each year were disposed of in the crib during its active 1 ife. The $U-12$ Crib has received low-level radioactive waste that is known to have included chemicals such as dilute nitric acid, as well as radioactive waste of plutonium, strontium, ruthenium, and uranium. Since 1985, physical controls and operating procedures have been modified to avoid inadvertent discharge of hazardous chemicals to the wastewater stream. Hydrogeologic evaluation (as of 1983) below the crib has indicated radioactive contamination to a depth of a least $43 \mathrm{~m}(140 \mathrm{ft})$. The depth to water below the crib is approximately $70 \mathrm{~m}(231 \mathrm{ft})$.

RCRA Interim-Status Groundwater Monitoring Plan for the 216-U-12 Crib (WHC 1990C) presents the program used to establish the background levels for the CIPs. The document summarizes the available data for the disposal facility. In accordance with the plan, four RCRA groundwater monitoring wells were installed in 1990. The monitoring network consisted of one upgradient and three downgradient wells (Figure 12-1).

The sample results for the fourth quarter of 1993 represent the tenth calendar quarter of data collected at the U-12 Crib. Data collected during the third quarter of 1992 from downgradient wells 299-W22-41 and 299-W22-42 showed elevated levels of specific conductivity. Verification sampling and analysis during that and subsequent quarters corroborated these findings. The U-12 Crib groundwater monitoring well network currently is sampled quarterly for assessment monitoring in accordance with the U-12 Crib groundwater 
assessment plan (WHC 1993a). Monthly sampling began in November 1993 for the field parameters $\mathrm{pH}$ and specific conductivity. The data from the monthly sampling will be reported when it is avallable.

Two existing wells, 299-W22-22 and 299-W22-23, have been remediated for inclusion in the monitoring network and were sampled for the first time during the second quarter of $\mathrm{CY} 1993$. Table 12-1 lists the network wells and their sampling status during the reporting period. Currently the network consists of six wells. Sample recovery in the two remediated wells has been poor due to low fluid flow into the wells. This problem is currently being evaluated to determine the continued useability of the wells.

\subsection{MATER LEVEL MEASUREMENTS}

Water levels in each network well are measured at least quarterly and when water samples are collected. Water level measurements obtained during the quarter are presented in Table 12-2.

\subsection{MATER CHEMISTRY DATA}

Groundwater samples were collected in December 1993 from all network wells except 299-W22-22, which is being evaluated for inadequate water sample volumes. Sampling results for well 299-W22-23 that were collected during September (representing the third quarter $C Y$ 1993) are also reported. All data currently available from this and the previous sampling period are shown in Tables 12-3 through 12-5. Table 12-3 includes the constituent 1 ist and a sumnary of results. Tables 12-4 and 12-5 show results for specific constituents. Analytical data unavailable at the time this report was prepared will be included in future quarterly reports.

Specific conductivity in downgradient wells 299-W22-41 and 299-W22-42 continue to exceed the critical mean of $437 \mu \mathrm{mho} / \mathrm{cm}$. Results for nitrate (DWS $=45,000 \mathrm{ppb}$ ), the principal constituent suspected of causing elevated conductivity, are also elevated in these wells; well 299-W22-41 had a value of $180,000 \mathrm{ppb}$ and well 299-W22-42 had a value of $240,000 \mathrm{ppb}$. Radiological gross beta and the related constituent technetium-99 al so have elevated results in these two wells, which are consistent with previous data. Carbon tetrachloride values were above the 5-ppb DWS in all network wells except 299-W22-23, which is awaiting the lab results. Tritium activity in well 299-W22-23 is $24,200 \mathrm{pCi} / \mathrm{L}$, which is above the $20,000 \mathrm{pCi} / \mathrm{L}$ DWS. This value is being investigated.

An explanation of any data flags can be found in Section 1.4.5. The D flags for chloride and sulfate indicated that the samples were diluted for analysis. 


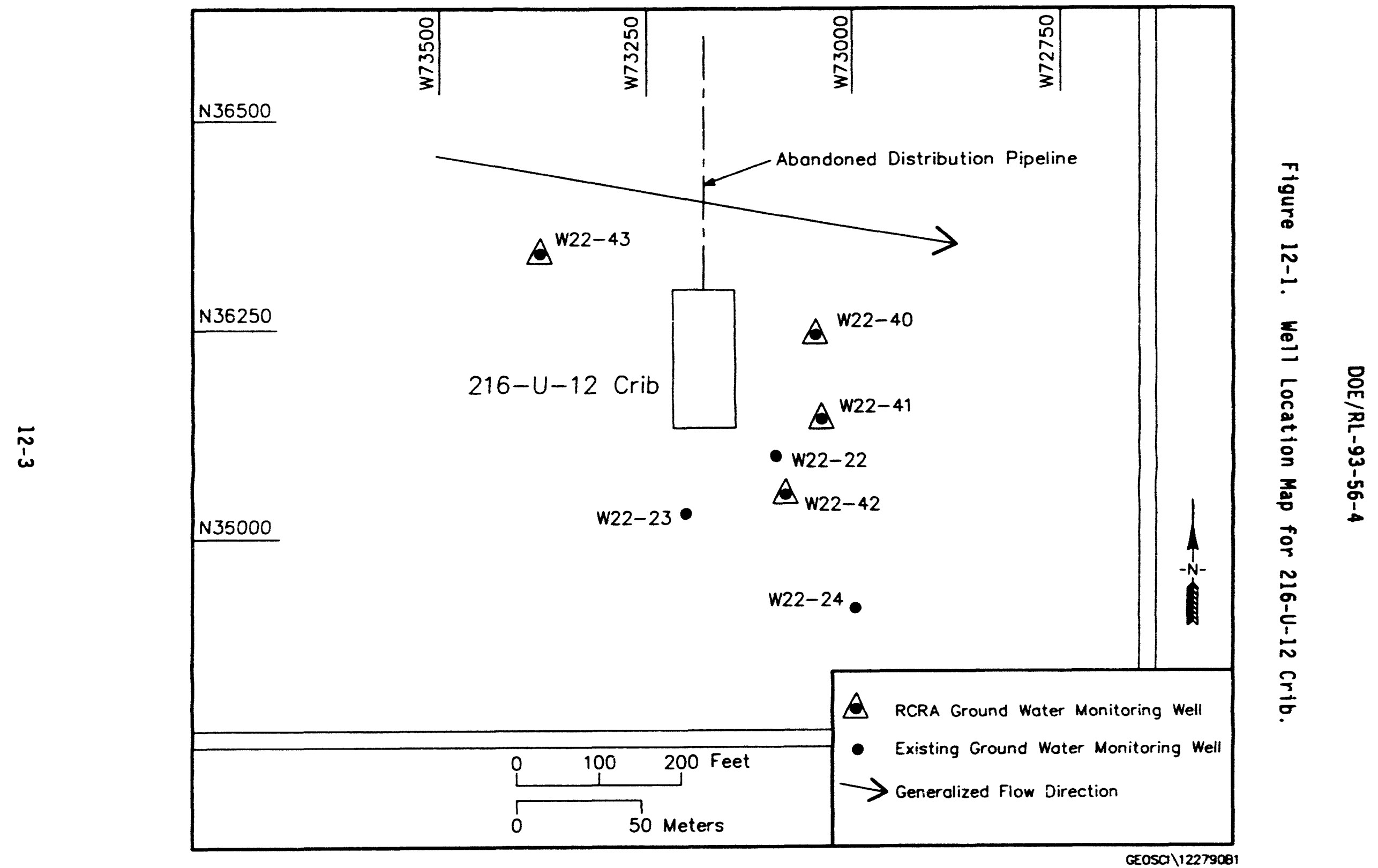


Table 12-1. Monitoring Well Purpose and Sampling Schedule for the 216-U-12 Crib.

\begin{tabular}{|c|c|c|c|c|}
\hline $\begin{array}{c}\text { Well no. } \\
(229-)\end{array}$ & $\begin{array}{c}\text { Relative } \\
\text { position }\end{array}$ & Hydrogeologic unit & $\begin{array}{c}\text { Sample } \\
\text { frequency }\end{array}$ & $\begin{array}{c}\text { Sample date, } \\
\text { 4th Qtr 1993 }\end{array}$ \\
\hline W22-43 & Upgradient & Ringold: Water Table & Quarterly & $12 / 20 / 93$ \\
\hline W22-40 & Downgradient & Ringold: Water Table & Quarterly & $12 / 21 / 93$ \\
\hline W22-41 & Downgradient & Ringold: Water Table & Quarterly & $12 / 20 / 93$ \\
\hline W22-42 & Downgradient & Ringold: Water Table & Quarterly & $12 / 21 / 93$ \\
\hline W22-22 & Downgradient & Ringold: Water Table & Quarterly & Not sampled \\
\hline W22-23 & Upgradient & Ringold: Water Table & Quarterly & $12 / 17 / 93$ \\
\hline
\end{tabular}

Note: Hydrogeologic units include the sandy gravels of the Hanford formation and silty sands of the Ringold Formation. 
Table 12-2. RCRA Water Level Measurement Report for the 216-U-12 Crib, Fourth Quarter 1993.

\begin{tabular}{rlll} 
Well & Date & $\begin{array}{c}\text { Depth to } \\
\text { water }(\mathrm{ft})\end{array}$ & $\begin{array}{c}\text { Water level } \\
\text { elevation } \\
\text { above ms }(\mathrm{ft})\end{array}$ \\
\hline Wells Monitoring the Top of the Unconfined Aquifer \\
\hline $299-W 22-40$ & $12 / 08 / 93$ & 236.42 & 455.81 \\
$299-W 22-41$ & $12 / 08 / 93$ & 235.97 & 455.77 \\
$299-W 22-42$ & $12 / 08 / 93$ & 235.45 & 455.71 \\
$299-W 22-43$ & $12 / 08 / 93$ & 234.73 & 456.62 \\
\hline
\end{tabular}

Notes: 1. Water level elevations are calculated by subtracting the measured depth to water from the surveyed elevation for the well.

2. Depth-to-water values are transcribed from field records.

3. To convert feet to meters multiply by 0.3048 . 
Table 12-3. Constituent List and Summary of Results for the 216-U-12 Crib Data for Reporting Period October 1 through December 31, 1993. (sheet 1 of 2)

CONTAMIMATION IMDICATOR PARAMETERS

\begin{tabular}{|c|c|c|c|c|c|c|c|c|c|}
\hline & & Const ituent Meme & & Lab & DH & & Mum & $r$ of $\mathrm{se}$ & mples \\
\hline Short & (Method) & Full & Units & CROL & Limit & Agency & Total & $>$ CROL & >DWS \\
\hline $\begin{array}{l}\text { CONDUCT } \\
\text { CONDUCT } \\
\text { TOC } \\
\text { PH }\end{array}$ & $\begin{array}{l}94 \\
73\end{array}$ & $\begin{array}{r}\text { Conductivity, field } \\
\text { Conductivity, lab } \\
\text { Total Organic Carbon } \\
\text { pH, field }\end{array}$ & 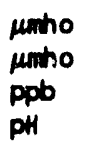 & $\begin{array}{r}1 \\
1 \\
200 \\
.01\end{array}$ & $6.5-8.5$ & EPAS & $\begin{array}{l}20 \\
20 \\
20 \\
20\end{array}$ & $\begin{array}{l}20 \\
20 \\
18 \\
20\end{array}$ & \\
\hline
\end{tabular}

DRINKING HATER PARAMETERS

\begin{tabular}{|c|c|c|c|c|c|c|c|c|c|}
\hline \multirow[b]{2}{*}{ Short } & \multicolumn{2}{|r|}{ Constituent Mane } & \multirow[b]{2}{*}{ Units } & \multirow{2}{*}{$\begin{array}{l}\text { Lab } \\
\text { CROL }\end{array}$} & \multicolumn{2}{|c|}{ DWS } & \multicolumn{3}{|c|}{ Number of Semples } \\
\hline & (Method) & Full & & & Limit & Agency & Total & $>$ CRQL & $>$ DWS \\
\hline $\begin{array}{l}\text { FLUORID } \\
\text { ALPHA } \\
\text { NITRATE }\end{array}$ & & $\begin{array}{r}\text { Fluoride } \\
\text { Gross alpha } \\
\text { Nitrate }\end{array}$ & $\begin{array}{l}p p b \\
p C i / L \\
p o b\end{array}$ & 13.9 & $\begin{array}{r}1400 \\
15 \\
45000\end{array}$ & $\begin{array}{r}\text { EPAI } \\
\text { EPA } \\
\text { EPA }\end{array}$ & $\begin{array}{l}6 \\
5 \\
6\end{array}$ & $\begin{array}{l}6 \\
4 \\
6\end{array}$ & $\begin{array}{l}0 \\
0 \\
2\end{array}$ \\
\hline
\end{tabular}

GROUNDHATER OUALITY PARAMETERS

\begin{tabular}{|c|c|c|c|c|c|c|c|c|c|}
\hline \multicolumn{4}{|c|}{ Constituent Nene } & \multicolumn{3}{|c|}{ DWS } & \multicolumn{3}{|c|}{ Number of Semples } \\
\hline Short & (Method) & Full & Units & CROL & Limit & Agency & Total & $>$ CROL & $>$ OWS \\
\hline $\begin{array}{l}\text { HLOR } \\
\text { ULFA }\end{array}$ & & $\begin{array}{l}\text { Chloride } \\
\text { Sulfate }\end{array}$ & $\begin{array}{l}\text { ppb } \\
\text { ppb }\end{array}$ & $\begin{array}{l}82.5 \\
49.9\end{array}$ & $\begin{array}{l}250000 \\
250000\end{array}$ & $\begin{array}{l}\text { EPAS } \\
\text { EPAS }\end{array}$ & $\begin{array}{l}6 \\
6\end{array}$ & $\begin{array}{l}6 \\
6\end{array}$ & \\
\hline
\end{tabular}

SITE SPECIFIC AND OTHER CONSTITUENTS

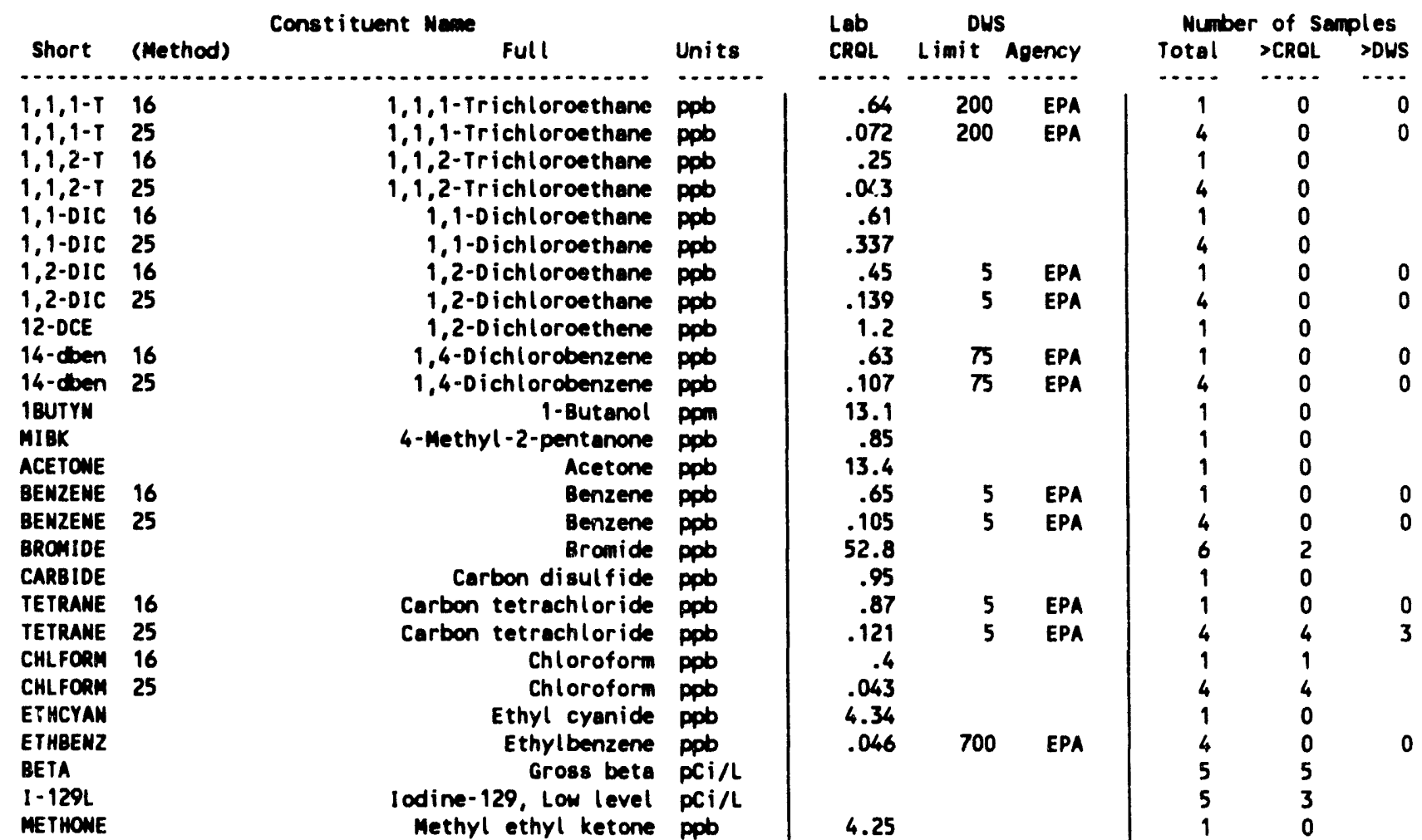


Table 12-3. Constituent List and Summary of Results for the 216-U-12 Crib Data for Reporting Period October 1 through December 31, 1993. (sheet 2 of 2)

\begin{tabular}{|c|c|c|c|c|c|c|c|c|c|}
\hline \multirow{3}{*}{ Short } & \multirow[b]{2}{*}{ (Method) } & \multirow{2}{*}{ Constituent Name Full } & \multirow[b]{2}{*}{ Units } & \multirow{2}{*}{$\begin{array}{l}\text { Lab } \\
\text { CRQL }\end{array}$} & \multicolumn{2}{|c|}{ DWS } & \multicolumn{3}{|c|}{ Number of Samples } \\
\hline & & & & & Limit & Agency & Total & $>$ CROL & $>$ DWS \\
\hline & 14 & (1) & $\cdots$ & . & $\cdots$ & $\cdots$ & $\cdots$ & $\cdots$ & $\cdots$ \\
\hline $\begin{array}{l}\text { METHYCH } \\
\text { METHYCH }\end{array}$ & $\begin{array}{l}16 \\
25\end{array}$ & Methylene chloride & ppb & .61 & & & 1 & $\begin{array}{l}0 \\
0\end{array}$ & \\
\hline $\begin{array}{l}\text { METHYCH } \\
\text { MITRITE }\end{array}$ & 25 & $\begin{array}{r}\text { Methylene chloride } \\
\text { Nitrite }\end{array}$ & $\begin{array}{l}\text { ppb } \\
\text { pppb }\end{array}$ & $\begin{array}{l}.056 \\
38.3\end{array}$ & 1000 & EPA & $\begin{array}{l}4 \\
6\end{array}$ & $\begin{array}{l}0 \\
0\end{array}$ & 0 \\
\hline PнOSPHA & & Phosphate & ppb & 147 & & & 6 & 0 & \\
\hline SR-90 & & strontium-90 & $\mathrm{PC} i / \mathrm{L}$ & & 8 & EPA & 5 & 0 & 0 \\
\hline TC- 99 & & Technet iun-99 & $\mathrm{PCi} / \mathrm{L}$ & & 900 & EPA & 5 & 5 & 0 \\
\hline PERCENE & 16 & Tet rachloroethene & ppob & 1.1 & 5 & EPA & 1 & 0 & 0 \\
\hline $\begin{array}{l}\text { PERCENE } \\
\text { TAF }\end{array}$ & 25 & $\begin{array}{l}\text { Tetrachloroethene } \\
\text { Tetrahydrofuran }\end{array}$ & $\begin{array}{l}\text { ppb } \\
\text { ppb }\end{array}$ & $\begin{array}{l}.049 \\
3.67\end{array}$ & 5 & EPA & $\begin{array}{l}4 \\
1\end{array}$ & $\begin{array}{l}0 \\
0\end{array}$ & 0 \\
\hline TOLUENE & 16 & Toluene & ppob & .73 & 1000 & EPA & 1 & 0 & 0 \\
\hline TOLUENE & 25 & Tol wene & ppob & .056 & 1000 & EPA & 4 & 0 & 0 \\
\hline TRICENE & 16 & Trichloroethene & ppob & .7 & $\mathbf{5}$ & EPA & 1 & 0 & 0 \\
\hline TRICENE & 25 & Trichloroethene & ppob & .055 & 5 & EPA & 4 & 0 & 0 \\
\hline TRITIU & & Tritiun & $\mathrm{PCi} / \mathrm{L}$ & & 20000 & EPA & 5 & 5 & 1 \\
\hline $\begin{array}{l}\text { TURB10 } \\
U-234\end{array}$ & & $\begin{array}{l}\text { Turbidity } \\
\text { Uraniun-236 }\end{array}$ & NTU & .03 & & & $\begin{array}{l}5 \\
5\end{array}$ & $\begin{array}{l}5 \\
5\end{array}$ & \\
\hline $\begin{array}{l}u-234 \\
u-235\end{array}$ & & $\begin{array}{l}\text { Uraniun-234 } \\
\text { Uranium-235 }\end{array}$ & $\mathrm{pCi} / \mathrm{L}$ & & & & $\begin{array}{l}5 \\
5\end{array}$ & $\begin{array}{l}3 \\
2\end{array}$ & \\
\hline$U-238$ & & Ureniu-238 & $\mathrm{PCi} / \mathrm{L}$ & & & & 5 & 5 & \\
\hline VINYIDE & 16 & Vinyl chloride & ppb & .78 & 2 & EPA & 1 & 0 & 0 \\
\hline VIAYIDE & 25 & Vinyl chloride & ppb & .266 & 2 & EPA & 4 & 0 & 0 \\
\hline XYLENE & 16 & Xylenes (total) & ppob & 1.7 & 10000 & EPA & 1 & 0 & 0 \\
\hline XYLENE & 25 & Xylenes (total) & ppo & .202 & 10000 & EPA & 4 & 0 & 0 \\
\hline $\begin{array}{l}\text { CISIZDE } \\
\text { TRAMDCE }\end{array}$ & & $\begin{array}{l}\text { cis-1,2-Dichloroethylene } \\
\text { trans-1,2-Dichloroethylene }\end{array}$ & $\begin{array}{l}p p b \\
p p b\end{array}$ & $\begin{array}{l}.127 \\
.149\end{array}$ & $\begin{array}{r}70 \\
100\end{array}$ & $\begin{array}{l}\text { EPA } \\
\text { EPA }\end{array}$ & $\begin{array}{l}4 \\
4\end{array}$ & $\begin{array}{l}0 \\
0\end{array}$ & $\begin{array}{l}0 \\
0\end{array}$ \\
\hline TR & & trans-1, e-urencoroetnylene & $\operatorname{mpr}$ & & & $\operatorname{con}$ & 4 & 0 & 0 \\
\hline
\end{tabular}

For explenation of this table, see Section 1.4 of report. 
Table 12-4. Constituents with at Least One Detected Value for the 216-U-12 $\mathrm{Crib}$ Data for Reporting Period October 1 through December 31, 1993.

\begin{tabular}{|c|c|c|c|c|c|c|}
\hline Well & $\begin{array}{c}\text { Collection } \\
\text { Dete }\end{array}$ & $\begin{array}{l}\text { Semple } \\
\text { Number }\end{array}$ & $\begin{array}{c}\text { BROAIDE } \\
124 / \mathrm{ppb} \\
52.8 /\end{array}$ & $\begin{array}{r}\text { TETRANE } \\
25 / \mathrm{ppb} \\
.121 / 5\end{array}$ & $\begin{array}{c}\text { CHLORID } \\
124 / \mathrm{ppb} \\
82.5 / 250000 \mathrm{~s}\end{array}$ & $\begin{array}{c}\text { CHLFORM } \\
16 / \mathrm{ppb} \\
.4 / .\end{array}$ \\
\hline 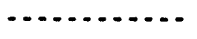 & $\cdots$ & $\ldots \ldots \ldots$ & $\ldots \ldots$ & 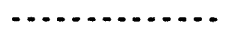 & 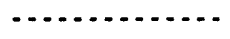 & \\
\hline $\begin{array}{l}299-122-23 \\
299-1122-23 \\
299-122-40 \\
299-W 22-41 \\
299-122-42 \\
299-w 22-43\end{array}$ & $\begin{array}{r}9 / 30 / 93 \\
12 / 17 / 93 \\
12 / 21 / 93 \\
12 / 20 / 93 \\
12 / 21 / 93 \\
12 / 20 / 93\end{array}$ & $\begin{array}{l}809622 \\
809 p 48 \\
809 p 53 \\
809 p 58 \\
809 P 63 \\
809 p 68\end{array}$ & $\begin{array}{l}52.80 \mathrm{U} \\
52.80 \mathrm{U} \\
70.00 \mathrm{~L} \\
52.80 \mathrm{U} \\
52.80 \mathrm{U} \\
60.00 \mathrm{~L}\end{array}$ & $\begin{array}{l}6.90 \\
6.70 \\
4.80 \\
7.00\end{array}$ & $\begin{array}{ll}18000.00 & D \\
15000.00 & D \\
17000.00 & D \\
13000.00 & D \\
11000.00 & D \\
17000.00 & D\end{array}$ & $.97 \mathrm{~L}$ \\
\hline
\end{tabular}

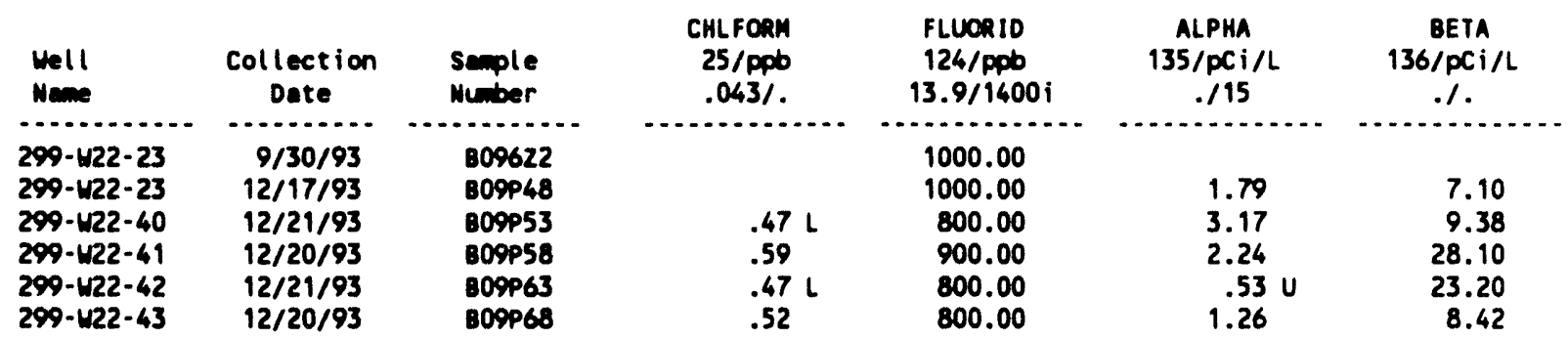

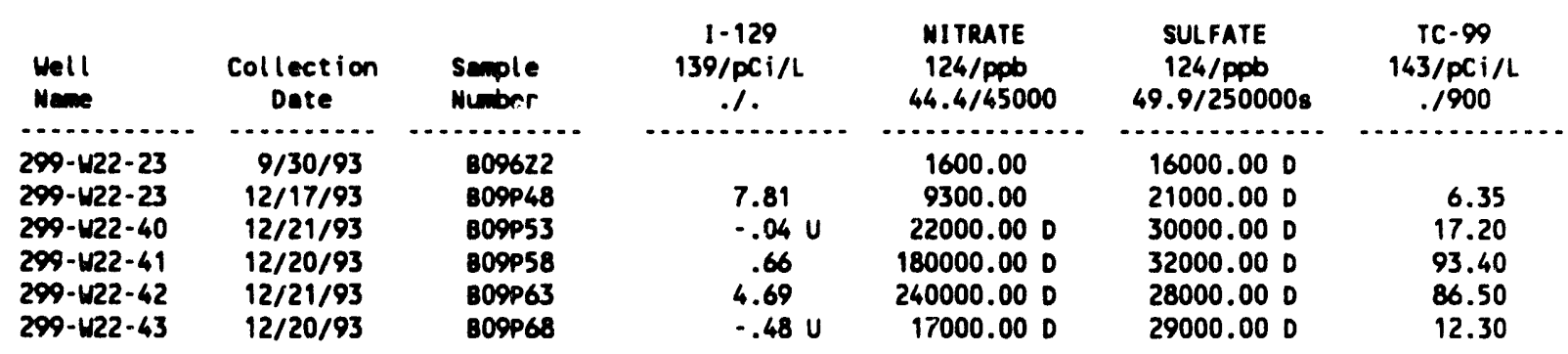

\begin{tabular}{|c|c|c|c|c|c|c|}
\hline $\begin{array}{l}\text { Well } \\
\text { Meme }\end{array}$ & $\begin{array}{c}\text { Collection } \\
\text { Date }\end{array}$ & $\begin{array}{l}\text { Semple } \\
\text { Number }\end{array}$ & $\begin{array}{c}\text { TRITIUA } \\
\text { 142/pCi/L } \\
. / 20000\end{array}$ & $\begin{array}{c}\text { TURBID } \\
\text { 126/NTU } \\
.05 /\end{array}$ & $\begin{array}{c}U-234 \\
148 / \mathrm{pCi} / \mathrm{L} \\
. /\end{array}$ & $\begin{array}{c}U-235 \\
148 / p C i / L \\
. /\end{array}$ \\
\hline$\ldots \ldots$ & $\ldots \ldots$ & $\ldots \ldots \ldots$ & 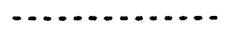 & $\cdots+\cdots$ & $\ldots \ldots$ & \\
\hline $\begin{array}{l}299-422-23 \\
299-122-40 \\
299-122-41 \\
299-422-42 \\
299-422-43\end{array}$ & $\begin{array}{l}12 / 17 / 93 \\
12 / 21 / 93 \\
12 / 20 / 93 \\
12 / 21 / 93 \\
12 / 20 / 93\end{array}$ & $\begin{array}{l}809 P 48 \\
809 P 53 \\
809 P 58 \\
809 P 63 \\
809 P 68\end{array}$ & $\begin{array}{r}24200.00 \\
2070.00 \\
1680.00 \\
11600.00 \\
1980.00\end{array}$ & $\begin{array}{r}37.00 \\
.53 \\
.85 \\
.93 \\
1.10\end{array}$ & $\begin{array}{l}1.16 \\
1.84 \\
.86 \\
1.45 \\
1.09\end{array}$ & $\begin{array}{l}.03 \mathrm{U} \\
.05 \\
.01 \mathrm{U} \\
.04 \\
.03 \mathrm{U}\end{array}$ \\
\hline
\end{tabular}

\begin{tabular}{lccc}
$\begin{array}{l}\text { Well } \\
\text { Nene }\end{array}$ & $\begin{array}{c}\text { Collection } \\
\text { Date }\end{array}$ & $\begin{array}{c}\text { Semple } \\
\text { Number }\end{array}$ & $\begin{array}{c}\text { U-238 } \\
148 / \mathrm{pCi} / \mathrm{L} \\
. / .\end{array}$ \\
\hdashline$\ldots 9-W 22-23$ & $12 / 17 / 93$ & $809 P 48$ & .58 \\
$299-W 22-40$ & $12 / 21 / 93$ & $809 P 53$ & 1.27 \\
$299-W 22-41$ & $12 / 20 / 93$ & $809 P 58$ & .62 \\
$299-W 22-42$ & $12 / 21 / 93$ & $809 P 63$ & 1.23 \\
$299-W 22-43$ & $12 / 20 / 93$ & $809 P 68$ & .91
\end{tabular}

For explenation of this table, see Section 1.4 of report. 
Table 12-5. Contamination Indicator Parameters for the 216-U-12 Crib Data for Reporting Period October 1 through December 31, 1993.

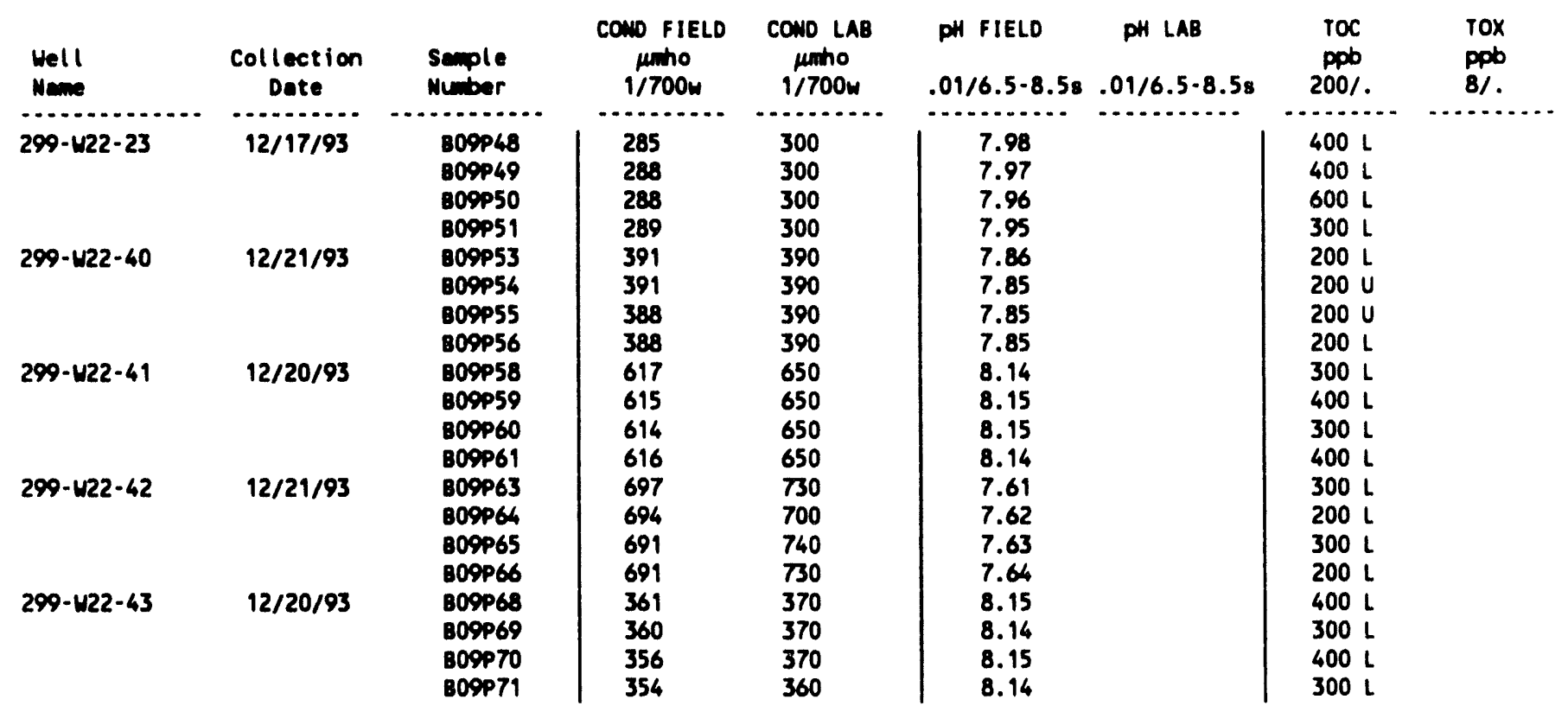

For explenation of this table, see section 1.4 of report. 
DOE/RL-93-56-4

This page intentionally left blank. 
DOE/RL-93-56-4

\section{CONTENTS}

13.0 LIQUID EFFLUENT RETENTION FACILITY . . . . . . . . . . . 13-1

13.1 INTRODUCTION . . . . . . . . . . . . . . . . 13-1

13.2 WATER LEVEL MEASUREMENTS . . . . . . . . . . . . . 13-1

13.3 WATER CHEMISTRY DATA ................. 13-1 
DOE/RL-93-56-4

\section{LIST OF FIGURES}

13-1 Monitoring Well Locations for the Liquid Effluent

Retention Facility .............. 13-3

\section{LIST OF TABLES}

13-1 Monitoring Well Purpose and Sampling Schedule for the Liquid Effluent Retention Facility Network . . . . . . . . . . . 13-4

13-2 RCRA Water Level Measurement Report for the Liquid Effluent Retention Facility, Fourth Quarter 1993 ......... 13-5

13-3 Constituent List and Summary of Results for the Liquid Effluent Retention Facility Data for Reporting Period October 1 through December 31, 1993 . . . . . . . . . . . 13-6

13-4 Constituents with at Least One Detected Value for the Liquid Effluent Retention Facility Data for Reporting Period October 1 through December 31, 1993 ......... . 13-9

13-5 Contamination Indicator Parameters for the Liquid Effluent Retention Facility Data for Reporting Period October 1 through December 31,1993 ............... 13-12 


\title{
13.0 LIQUID EFFLUENT RETENTION FACILITY
}

\author{
M. D. Sweeney \\ Westinghouse Hanford Company
}

\subsection{INTRODUCTION}

The Liquid Effluent Retention Facility (LERF) was constructed to provide interim storage of 242-A Evaporator process condensate effluent suspected of containing listed and dangerous (ammonia) waste constituents. Construction of the LERF began in May 1990 as a facility expansion under interim-status regulations. The LERF, classified as a surface impoundment for mixed waste storage, will be permitted in accordance with WAC 173-303. The evaporator was shut down in 1989 and is scheduled for restart. A treatment unit for the evaporator liquid waste is being designed and is scheduled for operation in 1994. The LERF basins will serve as temporary storage for the waste until that time.

The facility is classified as a surface impoundment and was originally planned to be four basins arranged side by side. Four excavations were made; only three of the four basins are lined and currently planned for use. The basins are located at the northeast corner of the 200 East Area. The liquid effluent may contain spent nonhalogenated solvents (F003 and F005, WAC 173-303-9004, "Dangerous Waste Source List"), i.e., acetone, methyl ethyl ketone, and methyl isobutyl ketone. The ammonia content in the liquid effluent may 31 so be cause for handling the effluent as a dangerous waste.

A groundwater monitoring network was installed, in accordance with the groundwater monitoring plan (WHC 1991), around the LERF in 1990. Four quarters of background groundwater sampling collection and analys is were completed. Currently the facility is in the detection-level indicator parameter evaluation program status until closure or other action. Three downgradient wells and one upgradient well constitute the monitoring network. Figure 13-1 shows the well locations. Table 13-1 provides the sampling event and last sampling date and interval, and defines the upgradient and downgradient wells.

\subsection{HATER LEVEL MEASUREMENTS}

Water levels are measured quarterly and when groundwater samples are collected at each of the four wells in the network. Water level measurements obtained during the quarter are presented in Table 13-2.

\subsection{MATER CHEMISTRY DATA}

The samples for the fourth quarter of 1993 were collected on October 10 , 1993. One of the downgradient wells, 299-E26-9, has been removed from the sampling schedule. This well has too little water to sample; less than $0.3 \mathrm{~m}$ $(1 \mathrm{ft})$ of water is left in the well casing. 
The data received between cutoff dates for this quarter are reported in Tables 13-2 through 13-5. Unfiltered chromium and iron continue to indicate fluctuating concentrations above the DWS for these constituents. Filtered analysis for these parameters are well below the DWS. A statistical evaluation has been completed for indicator parameters for the LERF. There were no exceedances for the current sampling period. No CIP replicate analysis exceeded the calculated critical means.

Data are flagged in Tables 13-4 and 13-5 according to the explanation found in Section 1.4.5. The D flags for sulfate indicate that the samples were diluted for analysis. 
Figure 13-1. Monitoring Well Locations for the Liquid Effluent Retention Facility.

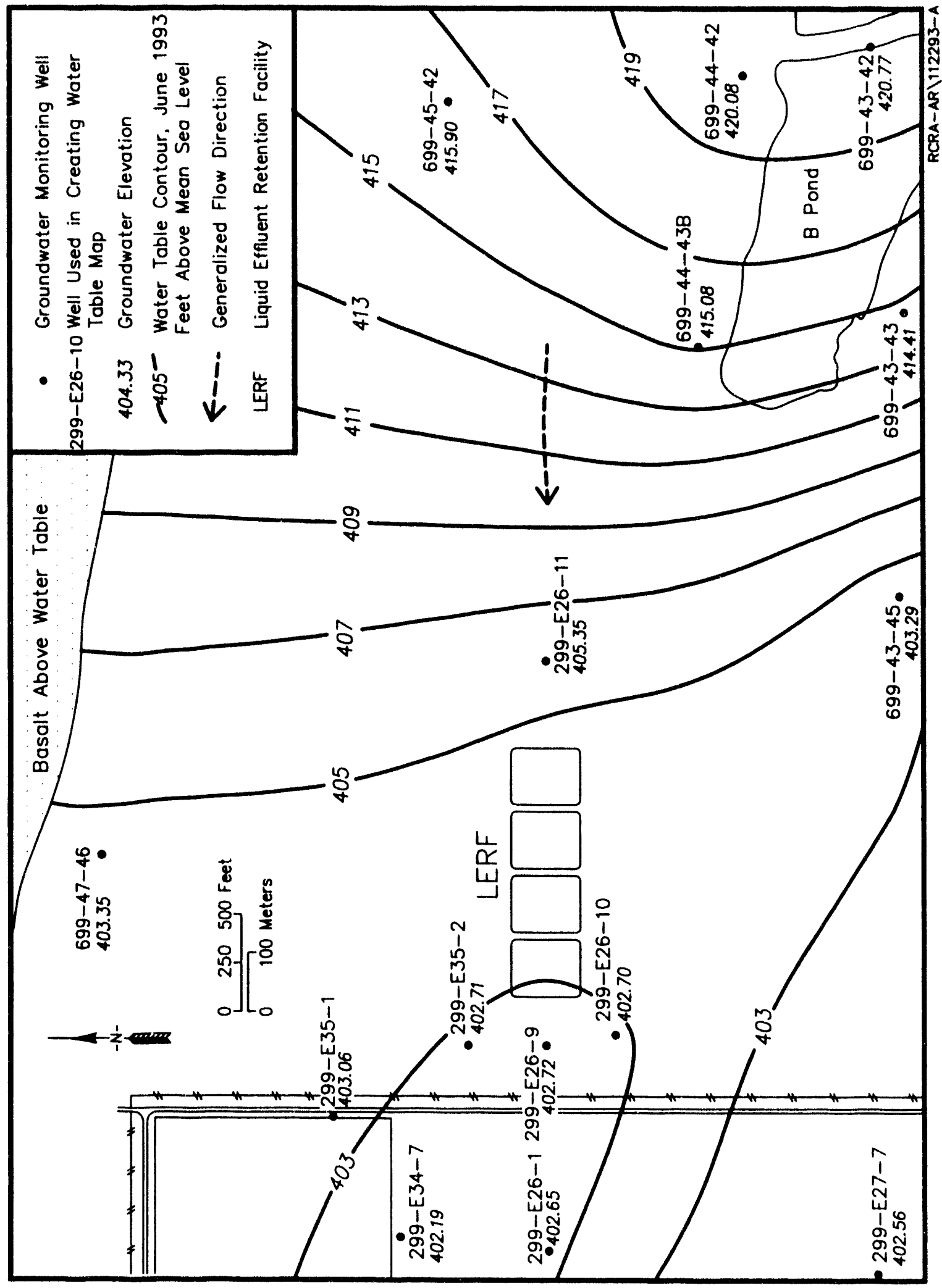


Table 13-1. Monitoring Well Purpose and Sampling Schedule for the Liquid Effluent Retention Facility Network.

\begin{tabular}{|c|c|c|c|c|}
\hline $\begin{array}{c}\text { Well no. } \\
(299-)\end{array}$ & $\begin{array}{c}\text { Relative } \\
\text { position }\end{array}$ & Hydrogeologic unit & $\begin{array}{c}\text { Sample } \\
\text { frequency }\end{array}$ & $\begin{array}{c}\text { Sample date, } \\
\text { 4th Qtr 1993 }\end{array}$ \\
\hline E26-9 & Downgradient & Hanford: Water Table & Semiannually & Not sampled \\
\hline E26-10 & Downgradient & Hanford: Water Table & Semiannually & $10 / 08 / 93$ \\
\hline E26-11 & Upgradient & Hanford: Water Table & Semiannually & $10 / 08 / 93$ \\
\hline E35-2 & Downgradient & Hanford: Water Table & Semiannually & $10 / 08 / 93$ \\
\hline
\end{tabular}


Table 13-2. RCRA Water Level Measurement Report for the Liquid Effiuent Retention Facility, Fourth Quarter 1993.

\begin{tabular}{lccc}
\hline Well & Date & $\begin{array}{c}\text { Depth to } \\
\text { water (ft) }\end{array}$ & $\begin{array}{c}\text { Water level } \\
\text { elevation } \\
\text { above ms (ft) }\end{array}$ \\
\hline $299-E 26-10$ & $10 / 08 / 93$ & $\begin{array}{l}198.85 \\
199.28\end{array}$ & $\begin{array}{l}402.62^{\star} \\
402.19\end{array}$ \\
$299-E 26-11$ & $12 / 10 / 93$ & 194.35 & $405.33^{\star}$ \\
$299-E 26-9$ & $10 / 08 / 93$ & 200.71 & 402.18 \\
$299-E 35-2$ & $12 / 10 / 93$ & 199.75 & $402.35^{\star}$ \\
& $10 / 08 / 93$ & 199.95 & 402.15 \\
\hline
\end{tabular}

Notes: 1. Water level elevations are calculated by subtracting the measured depth to water from the surveyed elevation for the well.

2. Depth-to-water values are transcribed from field records.

3. Elevations marked with an ' $*$ ' were measured at the time of sampling.

4. To convert feet to meters multiply by 0.3048 . 
Table 13-3. Constituent List and Summary of Results for the Liquid Effluent Retention Facility Data for Reporting Period October 1

through December 31, 1993. (sheet 1 of 3)

CONTAMIMATION INDICATOR PARAMETERS

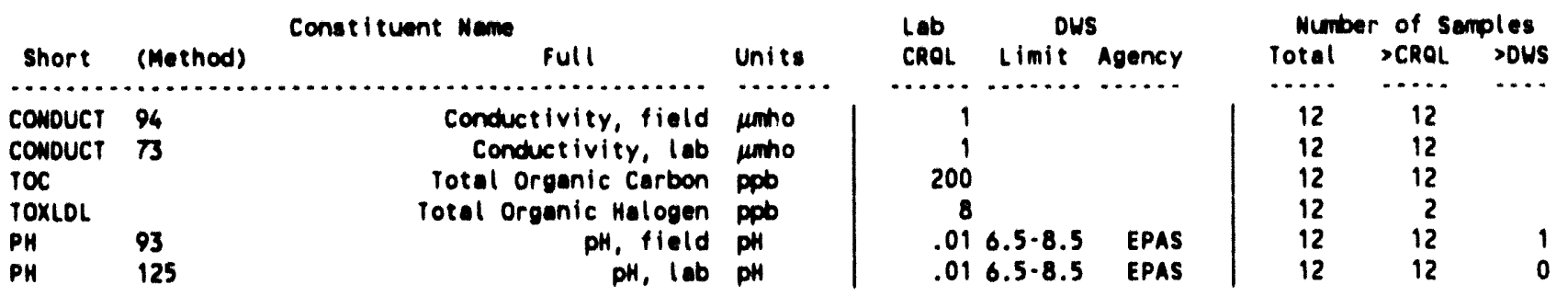

DRINKING MATER PARAMETERS

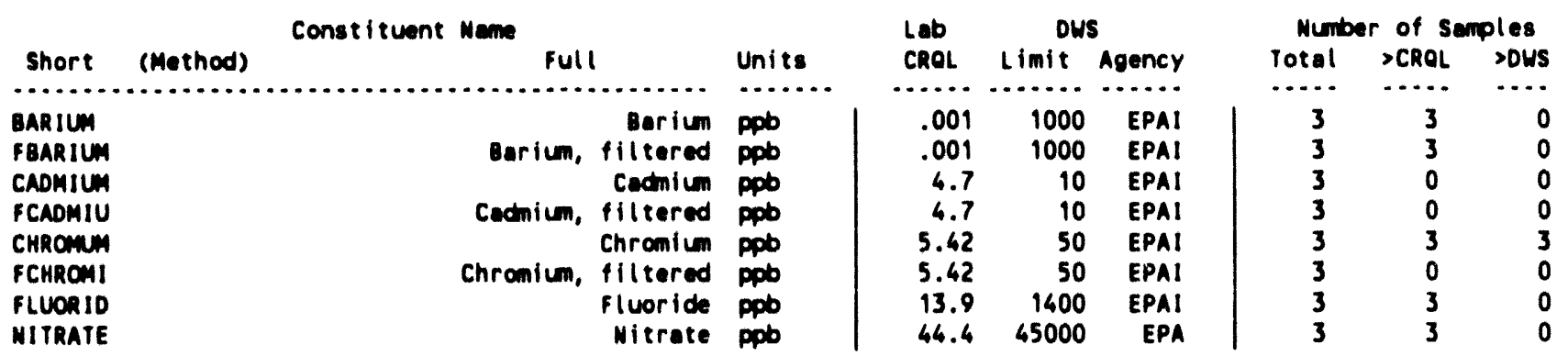

GROUNOWATER QUALITY PARAMETERS

\begin{tabular}{|c|c|c|c|c|c|c|c|c|c|}
\hline \multirow[b]{2}{*}{ Short } & \multirow[b]{2}{*}{ (Method) } & Constituent Meme & \multirow[b]{2}{*}{ Units } & \multirow{2}{*}{$\begin{array}{l}\text { Lab } \\
\text { CROL }\end{array}$} & \multicolumn{2}{|c|}{ Dus } & \multicolumn{3}{|c|}{ Number of Semples } \\
\hline & & Full & & & Limit & Agency & Total & $>$ CROL & $>$ DWS \\
\hline CHLORID & & chloride & ous & 82.5 & 250000 & EPAS & 3 & 3 & 0 \\
\hline IRON & & Iron & ppb & 10.3 & 300 & EPAS & 3 & 3 & 3 \\
\hline FIRON & & Iron, filtered & ppo & 10.3 & 300 & EPAS & 3 & 3 & 0 \\
\hline MANGESE & & Manganese & ppob & 1.35 & 50 & EPAS & 3 & 3 & 0 \\
\hline FMAMGAN & & Manganese, filtered & ppob & 1.35 & 50 & EPAS & 3 & 3 & 0 \\
\hline LPHENOL & & Phenol & ppob & .31 & & & 3 & 0 & \\
\hline Soolum & & Sodium & ppb & 40.9 & & & 3 & 3 & \\
\hline FSOOIUN & & Sodium, filtered & ppob & 40.9 & & & 3 & 3 & \\
\hline SULFATE & & Sulfate & popo & 49.9 & 250000 & EPAS & 3 & 3 & 0 \\
\hline
\end{tabular}

SITE SPECIFIC AND OTHER CONSTITUENTS

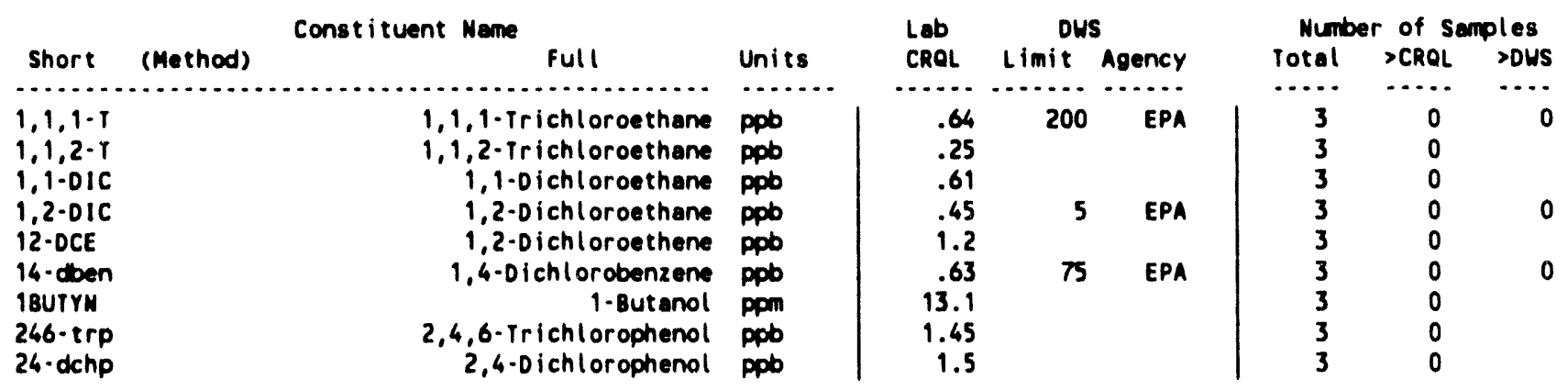


Table 13-3. Constituent List and Summary of Results for the Liquid Effluent Retention Facility Data for Reporting Period October 1

through December 31, 1993. (sheet 2 of 3 )

\begin{tabular}{|c|c|c|c|c|c|c|c|c|}
\hline short & Constituent Name full & Units & Lab & DWS & S Agency & Munt & $r$ of Se & $\begin{array}{l}\text { oles } \\
>\text { Dus }\end{array}$ \\
\hline$\ldots . .$. & ........... & …... & $\ldots \ldots$ & $\ldots . .$. & - is & $\ldots \ldots$ & $\cdots \cdots$ & $\ldots$ \\
\hline DIMPHEN & 2,4-D imethylphenol & ppob & 1.01 & & & 3 & 0 & \\
\hline DINPHEN & 2,4 -0 initrophenol & ppb & .96 & & & 3 & 0 & \\
\hline 26-dchp & 2,6-Dichlorophenol & ppb & 1.59 & & & 3 & 0 & \\
\hline CHLPHEN & 2-Chlorophenol & ppb & 1.42 & & & 3 & 0 & \\
\hline 2NITPH & 2-Nitrophenol & ppob & 1.56 & & & 3 & 0 & \\
\hline BUTDINP & 2-sec-Butyl-4,6-dinitrophenol (DN & ppob & 1.35 & & & 3 & 0 & \\
\hline 460N2MP & 4,6-0 initro-2-methylphenol & ppob & 1.18 & & & 3 & 0 & \\
\hline CHLCRES & 4-Chloro-3-methylphenol & ppb & 1.12 & & & 3 & 0 & \\
\hline MIBK & 4-Methyl-2-pentanone & ppob & .85 & & & 3 & 0 & \\
\hline MITPHEN & 4-Nitrophenol & ppob & .65 & & & 3 & 0 & \\
\hline ACETONE & Acetone & ppo & 13.4 & & & 3 & 0 & \\
\hline ALKALIN & Alkal inity & ppon & 1.96 & & & 3 & 3 & \\
\hline ALUMNUM & Aluninum & ppob & 32.5 & & & 3 & 2 & \\
\hline FALUNIN & Aluminum & ppo & 32.5 & & & 3 & 1 & \\
\hline AMHONIU & Amonium ion & ppob & 38.5 & & & 3 & 3 & \\
\hline ANTIONY - & Ant imony & ppob & 69.4 & & & 3 & 0 & \\
\hline FANTIMO & Antimony, filtered & ppob & 69.4 & & & 3 & 0 & \\
\hline BENZENE & Benzene & ppob & .65 & 5 & EPA & 3 & 0 & 0 \\
\hline BERYLUM & Beryllium & ppob & .814 & & & 3 & 0 & \\
\hline FBERYLL & Beryllium, filtered & ppb & .814 & & & 3 & 0 & \\
\hline BROMIDE & Bromide & ppb & 52.8 & & & 3 & 3 & \\
\hline CALCIUM & Calcium & ppb & 20.9 & & & 3 & 3 & \\
\hline FCALCIU & Calcium, filtered & ppob & 20.9 & & & 3 & 3 & \\
\hline CARBIDE & Carbon disulfide & ppb & .95 & & & 3 & 0 & \\
\hline TETRANE & Carbon tetrachloride & ppb & .87 & 5 & EPA & 3 & 0 & 0 \\
\hline CHLFORM & Chloroform & ppb & .4 & & & 3 & 0 & \\
\hline COBALt & Cobelt & ppb & 4.05 & & & 3 & 1 & \\
\hline FCכBALT & Cobalt, filtered & ppb & 4.05 & & & 3 & 0 & \\
\hline COPPER & Copper & ppb & 2.65 & 1000 & EPAS & 3 & 3 & 0 \\
\hline FCOPPER & Copper, filtered & ppob & 2.65 & 1000 & EPAS & 3 & 2 & 0 \\
\hline CRESOLS & Cresols (methylphenols) & ppb & 4.66 & & & 3 & 0 & \\
\hline ETHCYAN & Ethyl cyanide & ppb & 4.34 & & & 3 & 0 & \\
\hline MAGNES & Magnesium & ppob & 26 & & & 3 & 3 & \\
\hline FMAGNES & Magnesium, filtered & ppb & 26 & & & 3 & 3 & \\
\hline METHONE & Methyl ethyl ketone & ppob & 4.25 & & & 3 & 0 & \\
\hline METHYCH & Methylene chloride & ppob & .61 & & & 3 & 0 & \\
\hline NICKEL & Mickel & ppob & 17.9 & & & 3 & 3 & \\
\hline FNICKEL & Nickel, filtered & ppb & 17.9 & & & 3 & 0 & \\
\hline NITRITE & Nitrite & ppo & 38.3 & 1000 & EPA & 3 & 0 & 0 \\
\hline PENTCHP & Pentachlor ophenol & ppob & .87 & 1 & EPA & 3 & 0 & 0 \\
\hline PHOSPHA & Phosphate & ppb & 147 & & & 3 & 0 & \\
\hline POTASUA & Potassiun & ppb & 662 & & & 3 & 3 & \\
\hline FPOTASS & Potassium, filtered & ppob & 662 & & & 3 & 3 & \\
\hline SILVER & silver & ppob & 2.87 & 50 & EPAI & 3 & 0 & 0 \\
\hline FSILVER & silver, filtered & ppb & 2.87 & 511 & EPAI & 3 & 0 & 0 \\
\hline PERCENE & Tetrachloroethene & ppb & 1.1 & 5 & EPA & 3 & 0 & 0 \\
\hline TETPHNL & Tetrachlorophenols & ppb & 1.05 & & & 3 & 0 & \\
\hline TAF & Tetrahydrofuran & ppb & 3.67 & & & 3 & 0 & \\
\hline TIN & Tin & ppb & 51.1 & & & 3 & 0 & \\
\hline FTIN & Tin, filtered & ppob & 51.1 & & & 3 & 1 & \\
\hline TOLUENE & Tolvene & ppb & .73 & 1000 & EPA & 3 & 0 & 0 \\
\hline TOS & Total Dissolved Solids & ppm & 10 & 500 & EPAS & 3 & 3 & 0 \\
\hline TRICENE & Trichloroethene & ppb & .77 & 5 & EPA & 3 & 0 & 0 \\
\hline TRIPHNL & Trichlorophenols & ppb & 1.11 & & & 3 & 0 & \\
\hline TRITIUM & Tritium & $\mathrm{PCi} / \mathrm{L}$ & & 20000 & EPA & 3 & 3 & 0 \\
\hline VANADUM & Vanadiun & ppb & 3.84 & & & 3 & 3 & \\
\hline
\end{tabular}


Table 13-3. Constituent List and Summary of Results for the Liquid Effluent Retention Facility Data for Reporting Period October 1

through December 31,1993 . (sheet 3 of 3)

\begin{tabular}{|c|c|c|c|c|c|c|c|c|c|}
\hline \multirow[b]{2}{*}{ Short } & \multirow[b]{2}{*}{ (Method) } & \multirow{2}{*}{ Const ituent Name } & \multirow[b]{2}{*}{ Units } & \multirow{2}{*}{$\begin{array}{l}\text { Lab } \\
\text { CROL }\end{array}$} & \multicolumn{2}{|c|}{ DWS } & \multicolumn{3}{|c|}{ Number of Samples } \\
\hline & & & & & Limit & Agency & Total & $>C R Q L$ & $>$ DWS \\
\hline & & 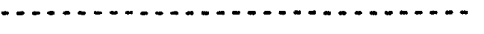 & & $\cdots$ & (n) & $\cdots$ & $\cdots$ & $\cdots \cdot$ & $\cdots$ \\
\hline FVANAD I & & Vanadium, filtered & ppb & 3.84 & & & 3 & 3 & \\
\hline VINYIDE & & Vinyl chloride & ppb & .78 & 2 & EPA & 3 & 0 & 0 \\
\hline XYLENE & & Xylenes (total) & ppb & 1.7 & 10000 & EPA & 3 & 0 & 0 \\
\hline ZINC & & 2 inc & ppb & 3.44 & 5000 & EPAS & 3 & 3 & 0 \\
\hline FZINC & & Zinc, filtered & ppb & 3.44 & 5000 & EPAS & 3 & 2 & 0 \\
\hline
\end{tabular}

For explanation of this table, see Section 1.4 of report. 
Table 13-4. Constituents with at Least One Detected Value for the Liquid Effluent Retention Facility Data for Reporting Period October 1 through December 31,1993 . (sheet 1 of 3 )

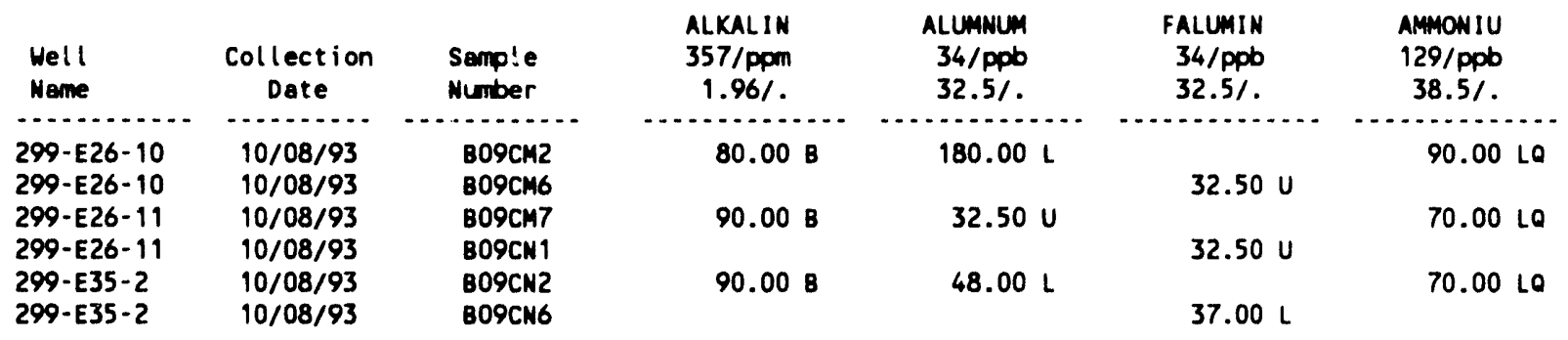

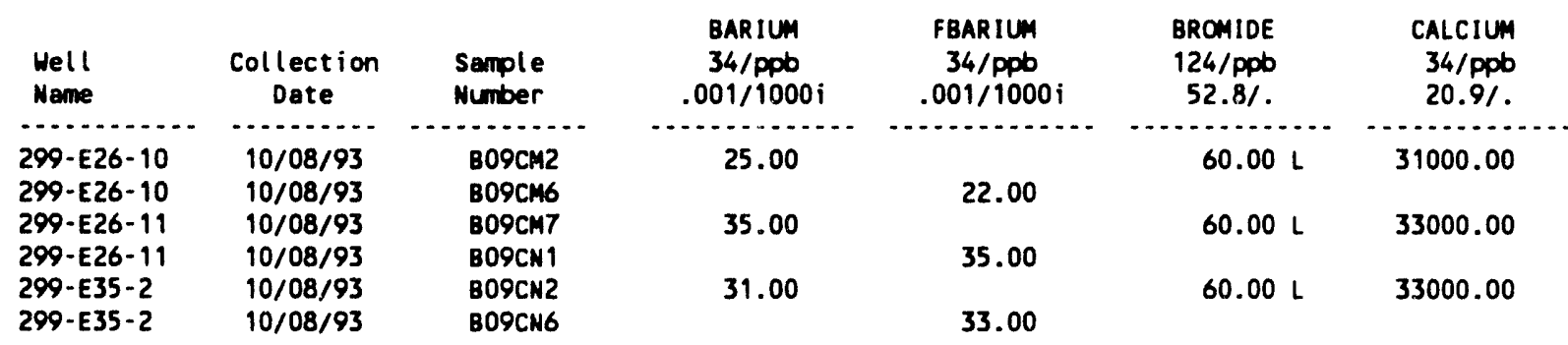

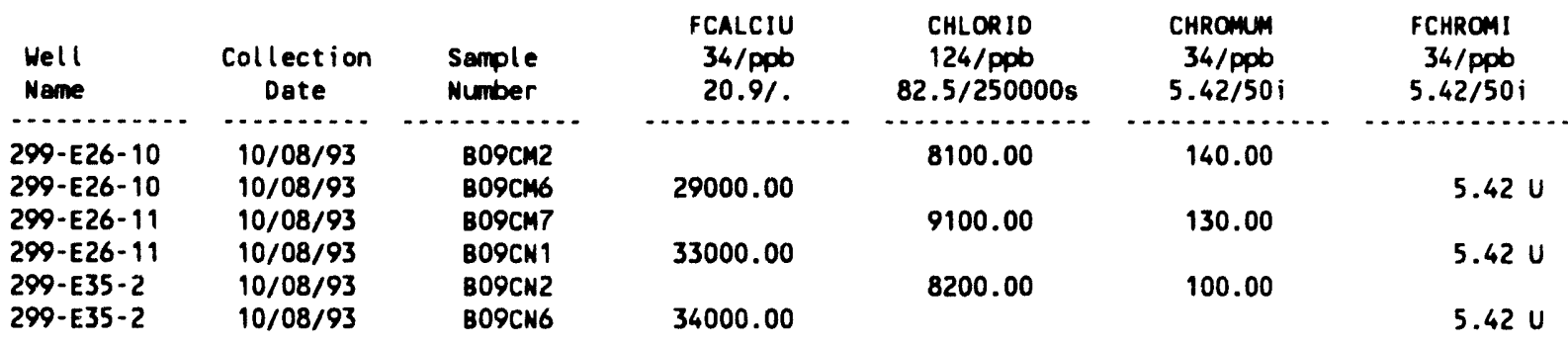

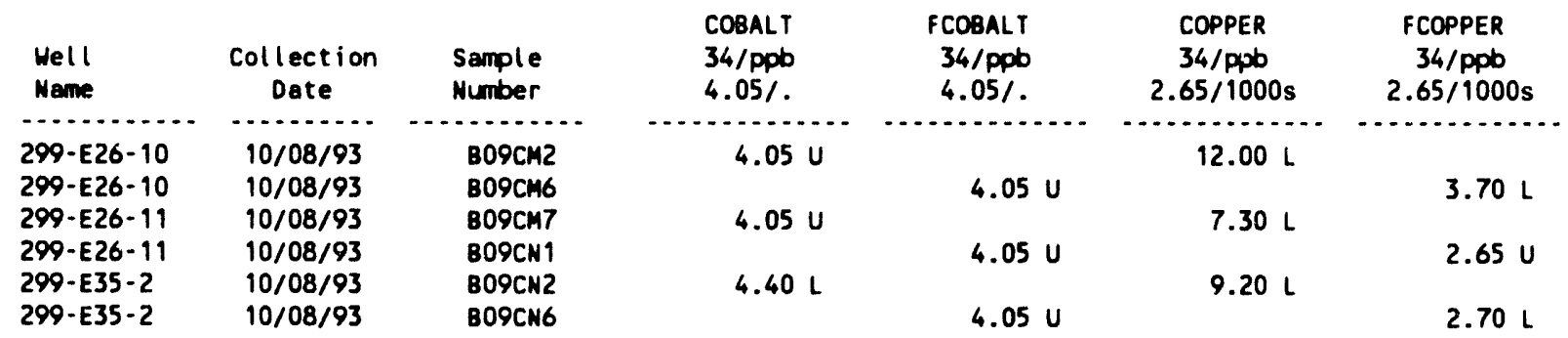


Table 13-4. Constituents with at Least One Detected Value for the Liquid Effluent Retention Facility Data for Reporting Period October 1 through December 31, 1993. (sheet 2 of 3)

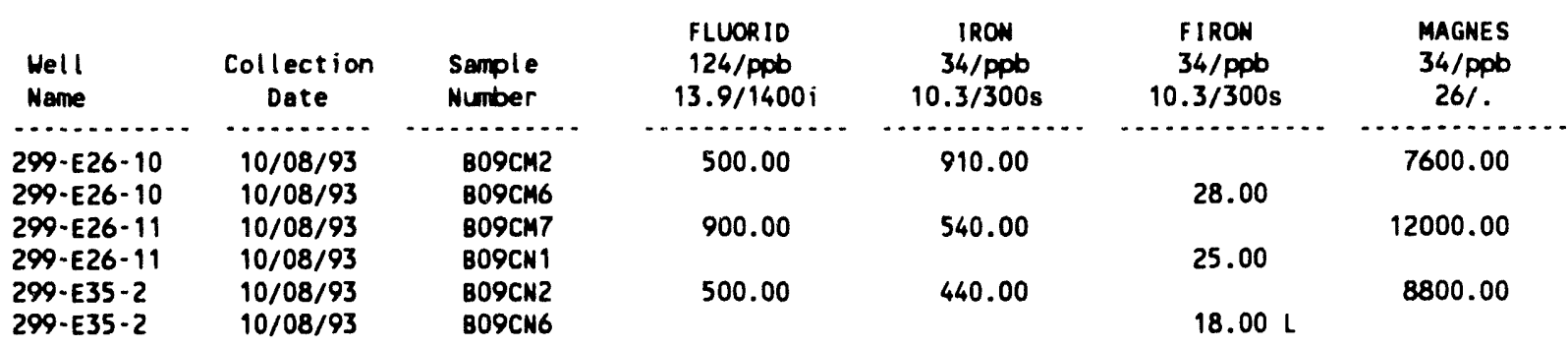

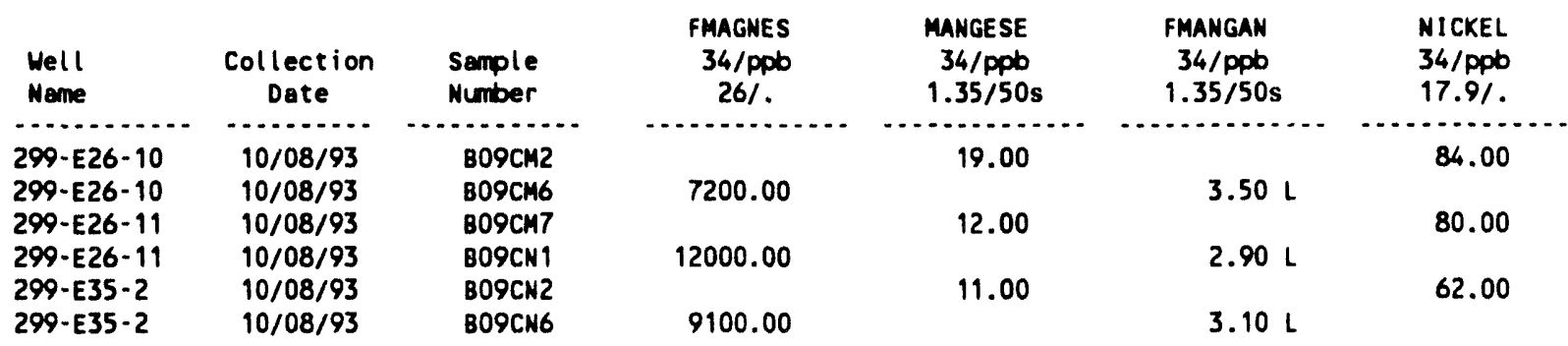

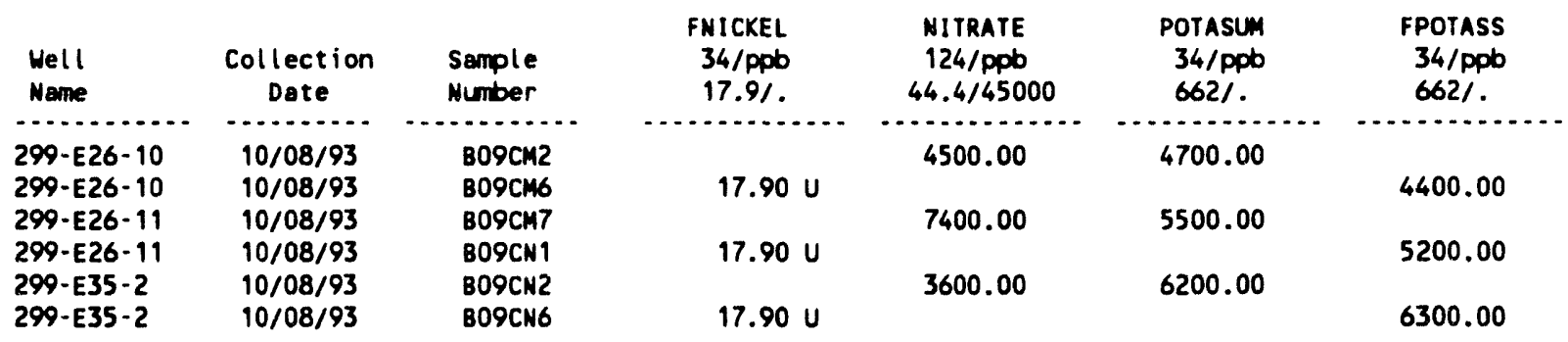

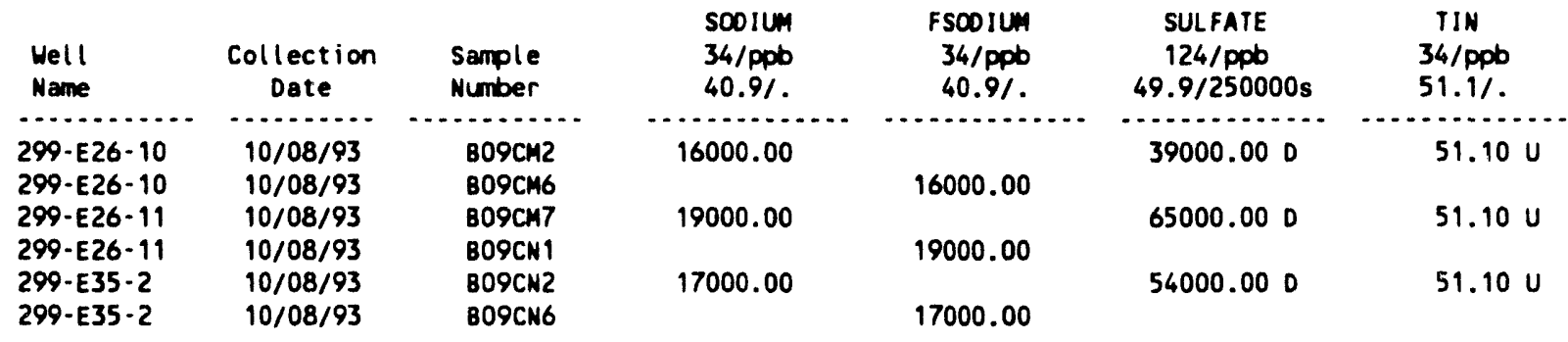

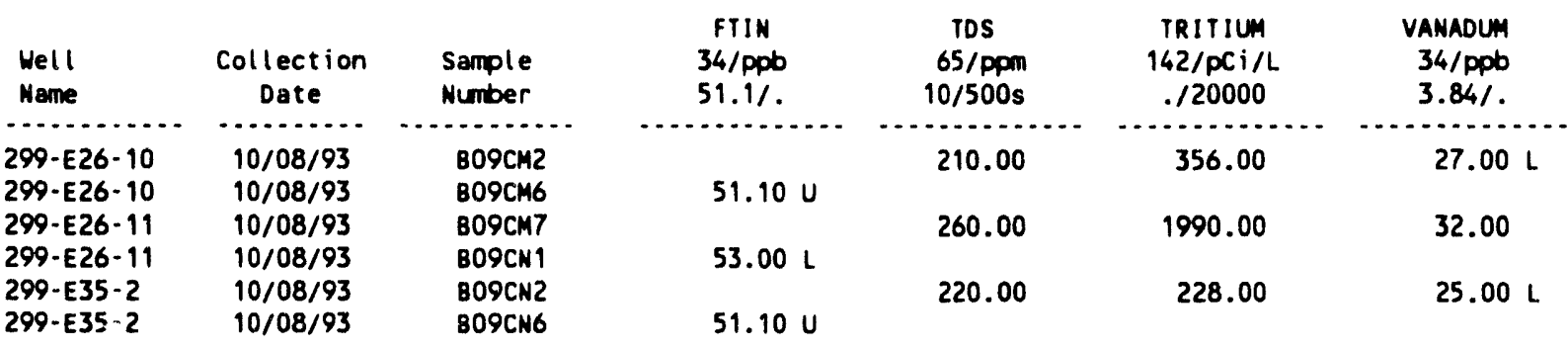


Table 13-4. Constituents with at Least One Detected Value for the Liquid Effluent Retention Facility Data for Reporting Period October 1

through December 31, 1993. (sheet 3 of 3)

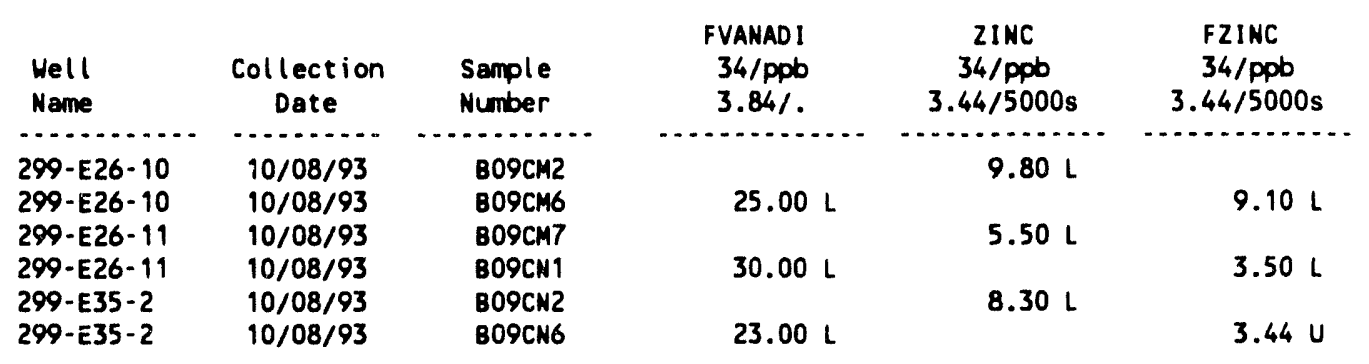

For explanation of this table, see Section 1.4 of report. 
Table 13-5. Contamination Indicator Parameters for the Liquid Effluent Retention Facility Data for Reporting Period October 1 through December 31, 1993.

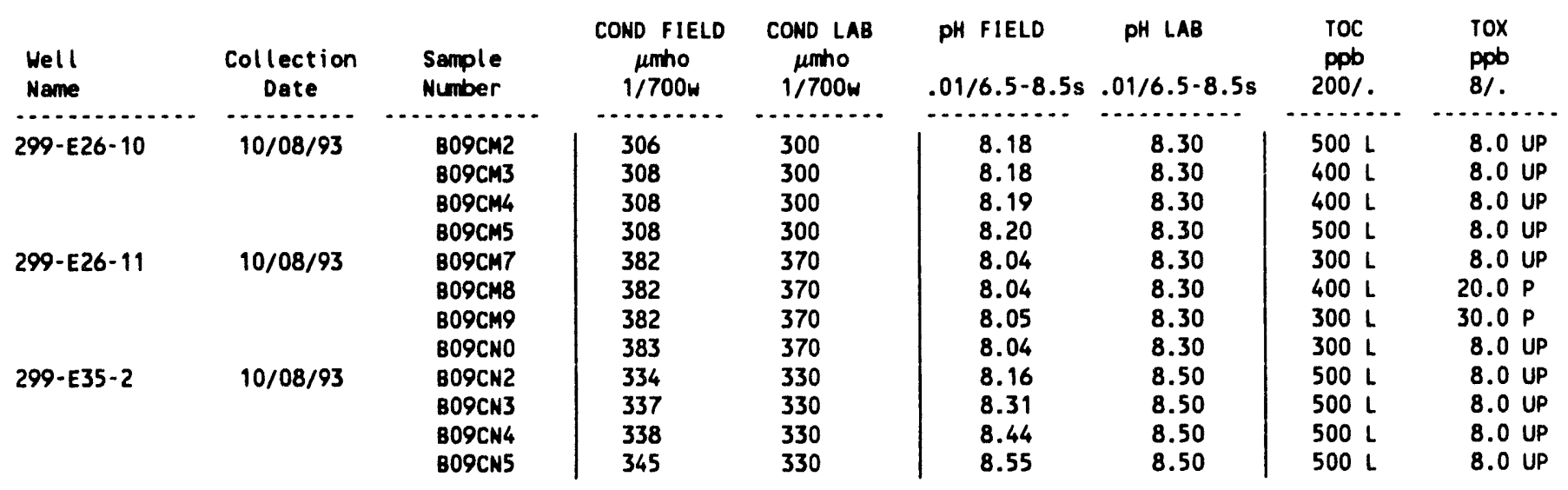

For explanation of this table, see Section 1.4 of report. 
DOE/RL-93-56-4

\section{CONTENTS}

$14.02101-M$ POND . . . . . . . . . . . . . . . . . 14-1

14.1 INTRODUCTION ................... . . . . . . . . . . . .

14.2 WATER LEVEL DATA . . . . . . . . . . . . 14-1

14.3 WATER CHEMISTRY DATA ................... 14-1 


\section{LIST OF FIGURES}

14-1 Monitoring Well Locations for the 2101-M Pond . . . . . . . . . 14-3

\section{LIST OF TABLES}

14-1 Monitoring Well Purpose and Sampling Schedule for the 2101-M Pond . . . . . . . . . . . . . . . . . . . . . 14-4

14-2 RCRA Water Level Measurement Report for the 2101-M Pond, Fourth Quarter 1993 . . . . . . . . . . . . . . 14-5

14-3 Constituent List and Summary of Results for the 2101-M Pond Data for Reporting Period October 1 through December 31, 1993 . . . 14-6

14-4 Constituents with at Least One Detected Value for the 2101-M Pond Data for Reporting Period October 1 through December 31, 1993 . . . . . . . . . . . . . . 14-8

14-5 Contamination Indicator Parameters for the 2101-M Pond Data for Reporting Period October 1 through December 31, 1993 . . . . . 14-9 


\title{
14.0 2101-M POND
}

\author{
E. C. Thornton \\ Westinghouse Hanford Company
}

\subsection{INTRODUCTION}

The 2101-M Pond is a U-shaped, unlined trench located in the 200 East Area. It has received wastewater from the 2101-M Building heating and air conditioning system since 1953. In 1981, Basalt Waste Isolation Project laboratories were plumbed into the discharge line from the 2101-M Building to the 2101-M Pond. From 1981 until mid-1985, these laboratories may have discharged dangerous waste into the 2101-M Pond. The most important chemicals used in the 2101-M laboratories were barium chloride and hydrochloric and nitric acids, and they are assumed to have been disposed in laboratory drains connected to the 2101-M Pond.

The groundwater monitoring network for 2101-M Pond consists of one upgradient (299-E18-1) and three downgradient (299-E18-2, 299-E18-3, and 299-E18-4) $1.11 \mathrm{~s}$ installed in the uppermost portion of the unconfined aquifer (Figure 14-1). Upgradient well 299-E18-1 is also used as a monitoring well for the 216-B-3 Pond System.

Groundwater sampling began in August 1988 for the 2101-M Pond and was conducted on a quarterly basis until August 1989 to establish background values. Semiannual indicator parameter evaluation monitoring was initiated in November 1989 under RCRA interim-status regulations. Table 14-1 provides information on the relative position of each well, the hydrogeologic unit monitored, sampling frequency, and the latest sampling date.

\subsection{WATER. LEVEL DATA}

Water level measurements were performed in the October through December 1993 period for all four wells. The data are presented in Table 14-2. Water levels have been corrected for vertical deviations for each well based on inclinometer measurements.

\subsection{HATER CHEMISTRY DATA}

Semiannual sampling was conducted for the fourth quarter of 1993 for the four wells associated with 2101-M Pond. Groundwater samples were collected on December 15, 1993, for wells 299-E18-1 and 299-E18-4, and on December 20, 1993, for wells 299-E18-2 and 299-E18-3. Those data received by March 1993 are reported in Tables 14-3 through 14-5. Critical means for TOC, $\mathrm{pH}$, and conductivity were not exceeded. TOX data have not yet been received.

A turbidity value of 8.70 NTUs was reported for well 299-E18-1, compared to a value of 47.3 NTUs of the last sample collected on June 11, 1993. Tha most recent sample was collected using a purging and sampling rate of less than $3.8 \mathrm{~L} / \mathrm{min}(1 \mathrm{gal} / \mathrm{min})$. This suggests that lowering the pumping rate 
reduces turbidity somewhat, but does not necessarily eliminate it. ICP metal analyses are not yet available for comparison with turbidity values. None of the reported constituent values were above the DWS.

Data are flagged in Tables 14-4 and 14-5 according to the explanation provided in Section 1.4.5. The $D$ flags for sulfate, chloride, and nitrate indicate that the samples were diluted for analysis. 
DOE/RL-93-56-4

Figure 14-1. Monitoring Well Locations for the 2101-M Pond.

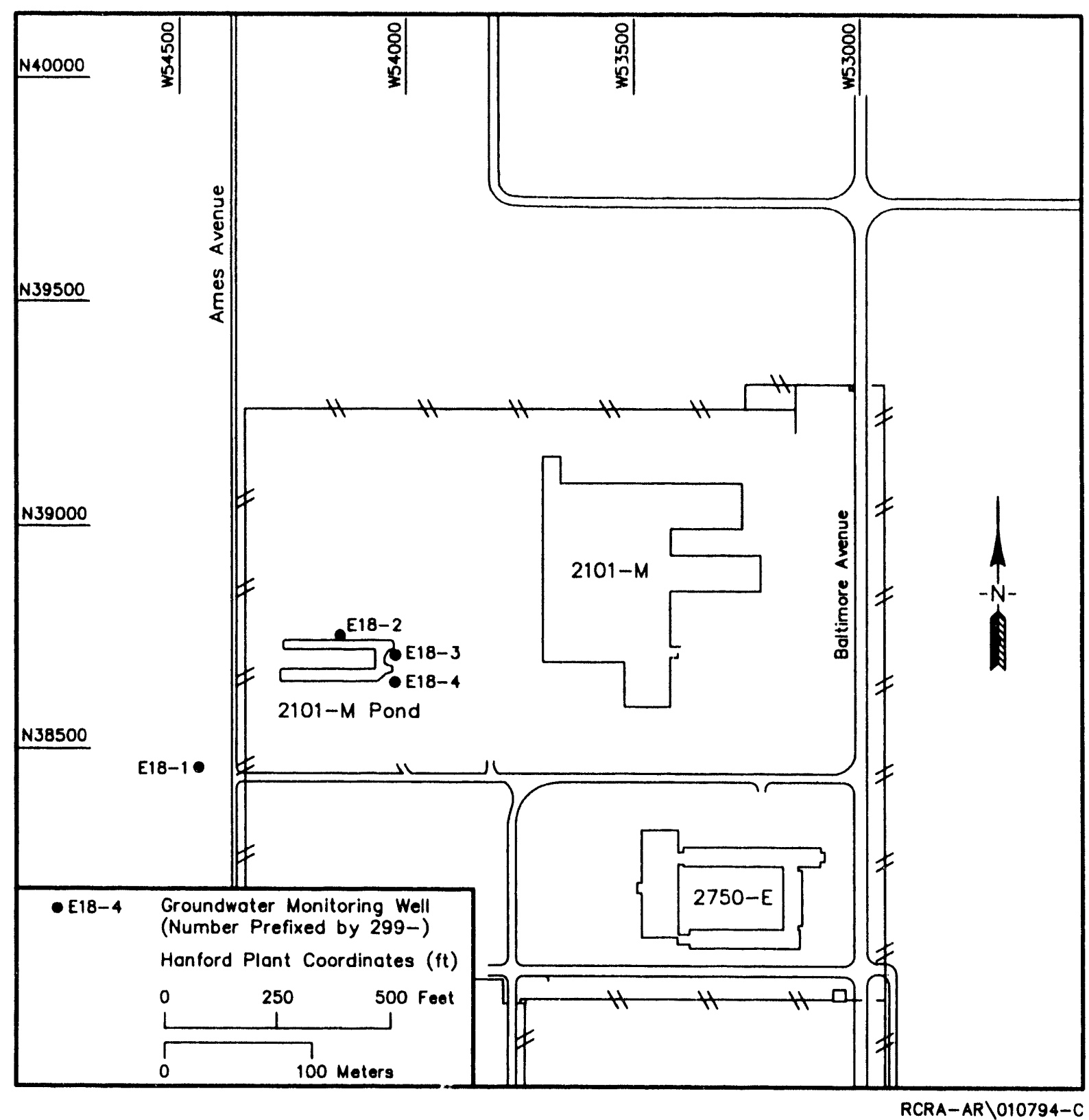


Table 14-1. Monitoring Well Purpose and Sampling Schedule for the 2101-M Pond.

\begin{tabular}{|c|c|c|c|c|}
\hline $\begin{array}{c}\text { Wel1 no. } \\
(299-)\end{array}$ & $\begin{array}{c}\text { Relative } \\
\text { position }\end{array}$ & \multicolumn{1}{|c|}{ Hydrogeologic unit } & $\begin{array}{c}\text { Sample } \\
\text { frequency }\end{array}$ & $\begin{array}{c}\text { Sample date, } \\
\text { 4th Qtr 1993 }\end{array}$ \\
\hline E18-1 & Upgradient & Ringold: Water Table & Semiannually & $12 / 15 / 93$ \\
\hline E18-2 & Downgradient & Ringold: Water Table & Semiannually & $12 / 20 / 93$ \\
\hline E18-3 & Downgradient & Ringold: Water Table & Semiannually & $12 / 20 / 93$ \\
\hline E18-4 & Downgradient & Ringold: Water Table & Semiannually & $12 / 15 / 93$ \\
\hline
\end{tabular}

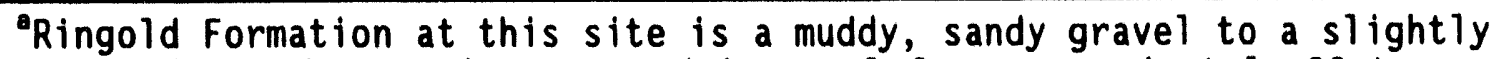
muddy gravelly sand over the screened interval from approximately 92 to $101 \mathrm{~m}$ (302 to $331 \mathrm{ft}$ ) below top of casing. 
Table 14-2. RCRA Water Level Measurement Report for the 2101-M Pond, Fourth Quarter 1993.

\begin{tabular}{lccc}
\hline We11 & Date & $\begin{array}{c}\text { Depth to } \\
\text { water }(\mathrm{ft})\end{array}$ & $\begin{array}{c}\text { Water leve1 } \\
\text { elevation } \\
\text { above ms }(\mathrm{ft})\end{array}$ \\
\hline $299-\mathrm{b} 18-1$ & $10 / 12 / 93$ & 318.37 & 401.93 \\
& $11 / 03 / 93$ & 318.25 & 402.05 \\
& $12 / 13 / 93$ & 318.35 & 401.95 \\
& $10 / 12 / 93$ & 319.70 & 401.73 \\
$299-E 18-2$ & $11 / 03 / 93$ & 319.63 & 401.80 \\
& $12 / 13 / 93$ & 319.62 & 401.81 \\
& $10 / 12 / 93$ & 321.97 & 400.10 \\
& $11 / 03 / 93$ & 320.31 & 401.76 \\
& $12 / 15 / 93$ & 320.72 & 401.35 \\
& $10 / 12 / 93$ & 319.67 & 401.90 \\
& $11 / 03 / 93$ & 319.63 & 401.94 \\
& $12 / 13 / 93$ & 319.72 & 401.85 \\
\hline
\end{tabular}

Notes: 1. Water level elevations are calculated by subtracting the measured depth to water from the surveyed elevation corrected for vertical deviations in the well. Vertical deviation correction factors based on inclinometer measurements are $+0.06 \mathrm{ft}$ for $299-E 18-1$, $+0.22 \mathrm{ft}$ for 299-E18-2, +0.03 ft for 299-E18-3, and +0.0 ft for 299-E18-4.

2. Depth-to-water values are transcribed from field records.

3. To convert feet to meters multiply by 0.3048 .

4. Elevations measured at the time of sampling were not available for inclusion in this report. 
Table 14-3. Constituent List and Summary of Results for the 2101-M Pond Data for Reporting Period October 1 through December 31, 1993. (sheet 1 of 2)

CONTAMINATION INDICATOR PARAMETERS

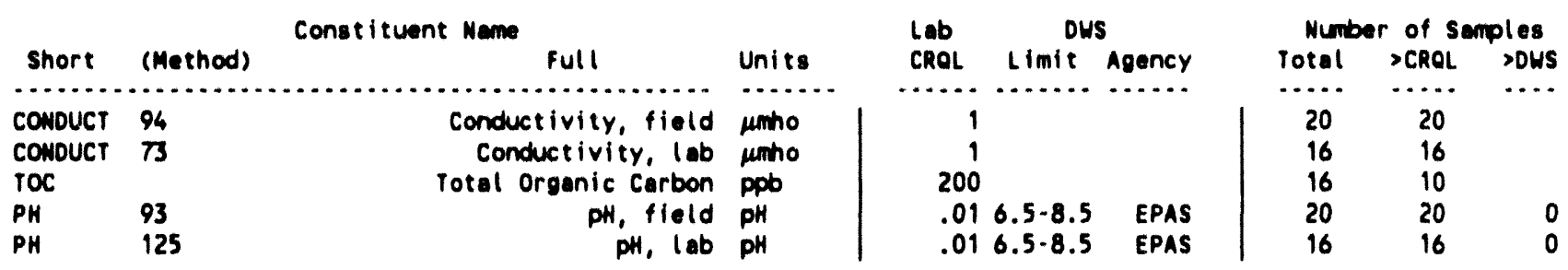

DRINKING WATER PARAMETERS

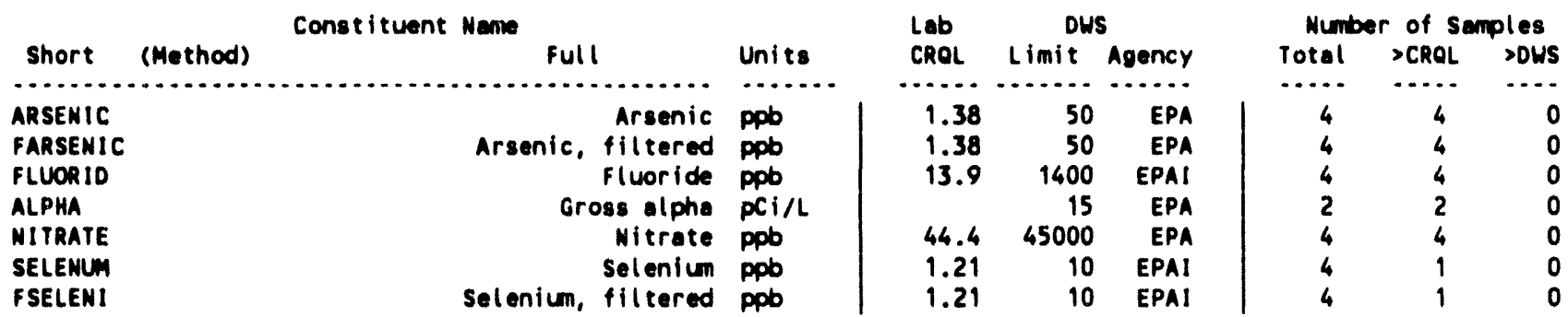

GROUNDWATER QUALITY PARAMETERS

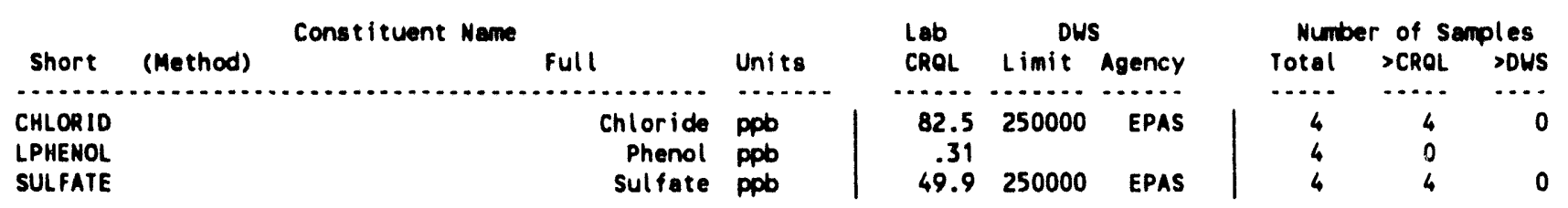

SITE SPECIFIC AND OTHER CONSTITUENTS

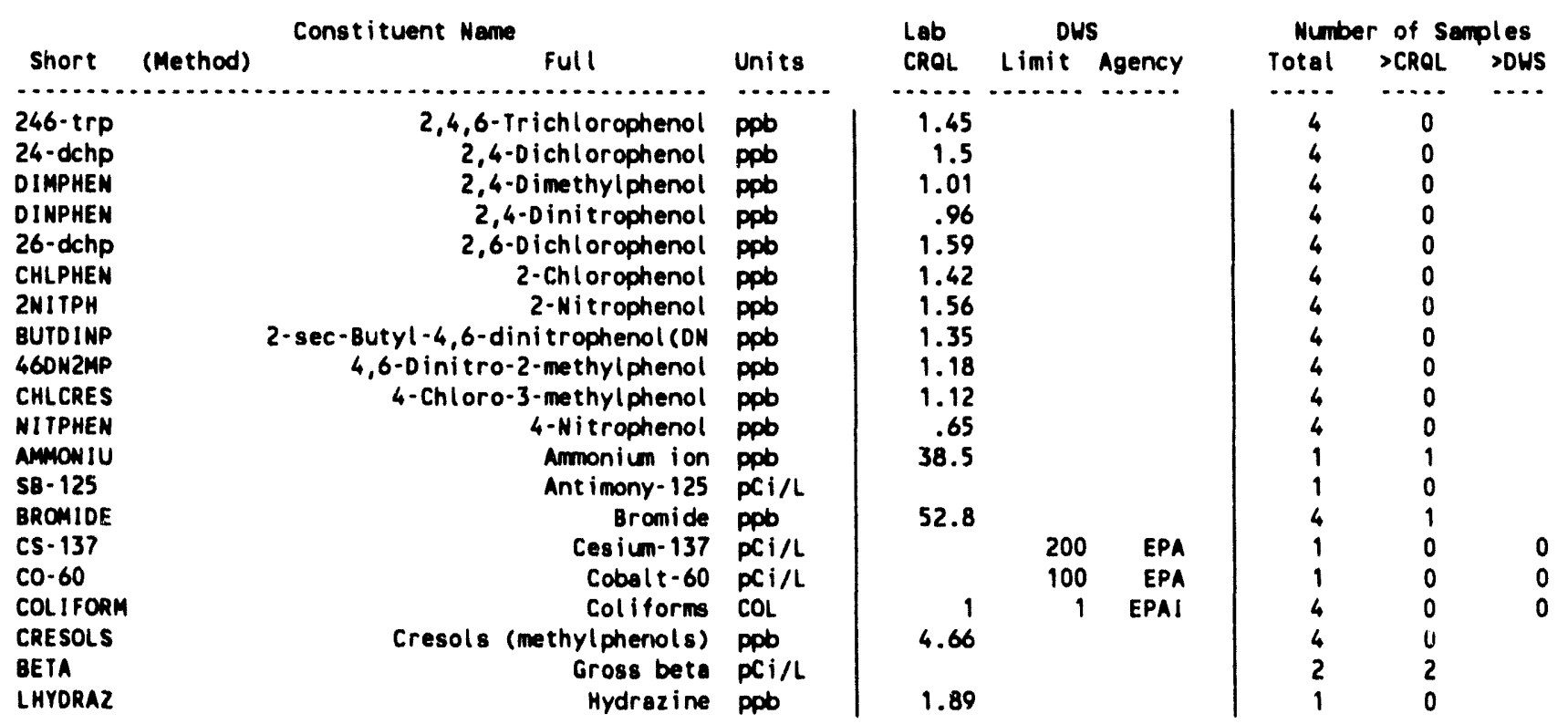


Table 14-3. Constituent List and Summary of Results for the 2101-M Pond Data for Reporting Period October 1 through December 31, 1993. (sheet 2 of 2)

\begin{tabular}{|c|c|c|c|c|c|c|c|c|c|}
\hline & & Const ituent Neme & & Lab & DWS & & Num & $r$ of $s$ & ples \\
\hline short & (Hethod) & Full & Units & CROL & Limit & Agency & Total & $>$ CRQL & $>$ DWS \\
\hline$\ldots . .$. & $\ldots \ldots \ldots$ & $\ldots \ldots \ldots$ & $\ldots \ldots$ & $\ldots \ldots$ & $\ldots \ldots$ & $\ldots \ldots$ & $\cdots \cdot$ & $\cdots \cdots$ & $\cdots$ \\
\hline NITRITE & & Nitrite & ppo & 38.3 & 1000 & EPA & 4 & 0 & 0 \\
\hline PENTCHP & & Pentachlorophenol & ppb & .87 & 1 & EPA & 4 & 0 & 0 \\
\hline PHOSPNA & & Phosphate & ppo & 147 & & & 4 & 0 & \\
\hline RU- 106 & & Ruthenium-106 & $\mathrm{PCi} / \mathrm{L}$ & & 30 & EPA & 1 & 0 & 0 \\
\hline TETPHNL & & Tetrachlor ophenols & ppo & 1.05 & & & 4 & 0 & \\
\hline TOS & & Total Dissolved Solids & ppm & 10 & 500 & EPAS & 1 & 1 & 0 \\
\hline TRIPHNL & & Trichlorophenols & ppb & 1.11 & & & 4 & 0 & \\
\hline TURBID & & Turbidity & NTU & .05 & & & 1 & 1 & \\
\hline URANIUA & & Uranium & ppb & & & & 4 & 4 & \\
\hline
\end{tabular}

For explanstion of this table, see Section 1.4 of report. 

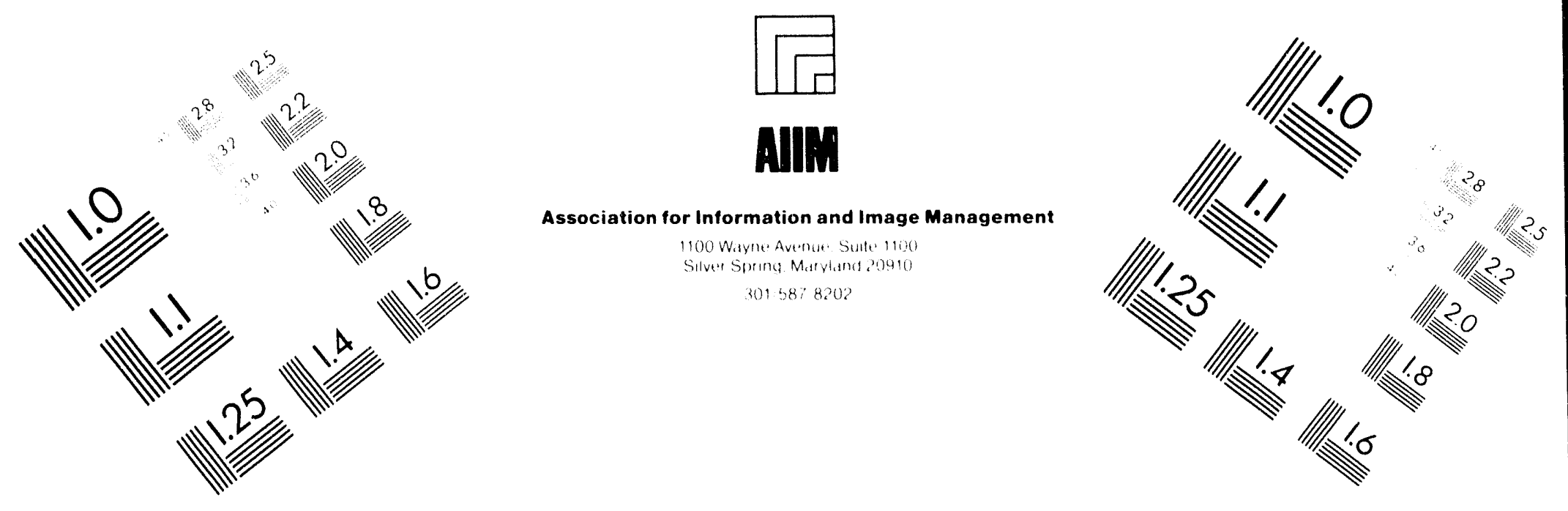

Centimeter

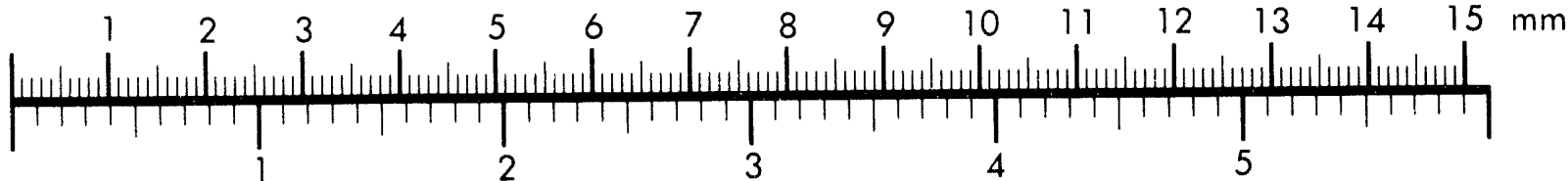

Inches
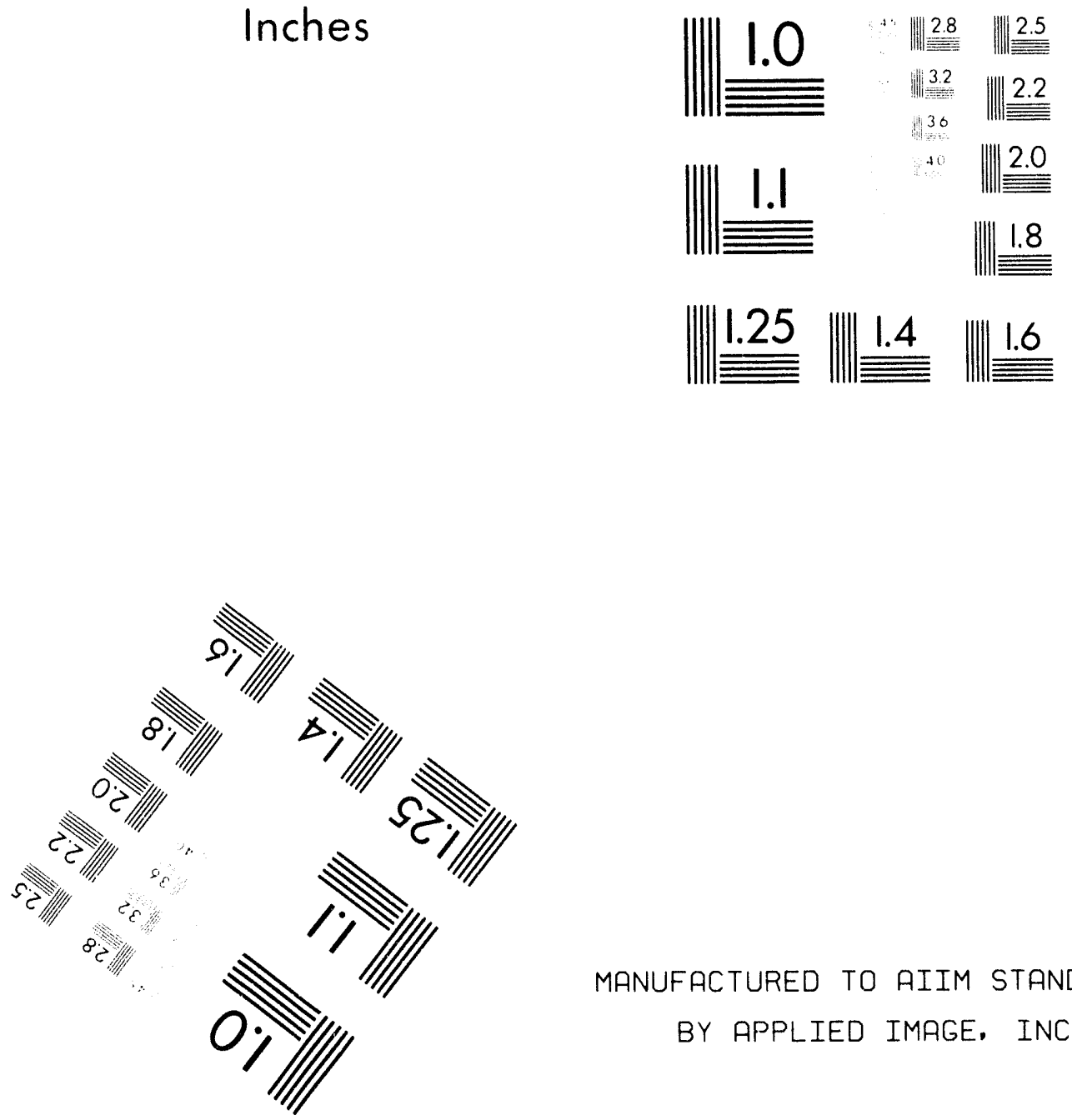

MANUFACTURED TO AIIM STANDARDS

BY APPLIED IMAGE, INC.

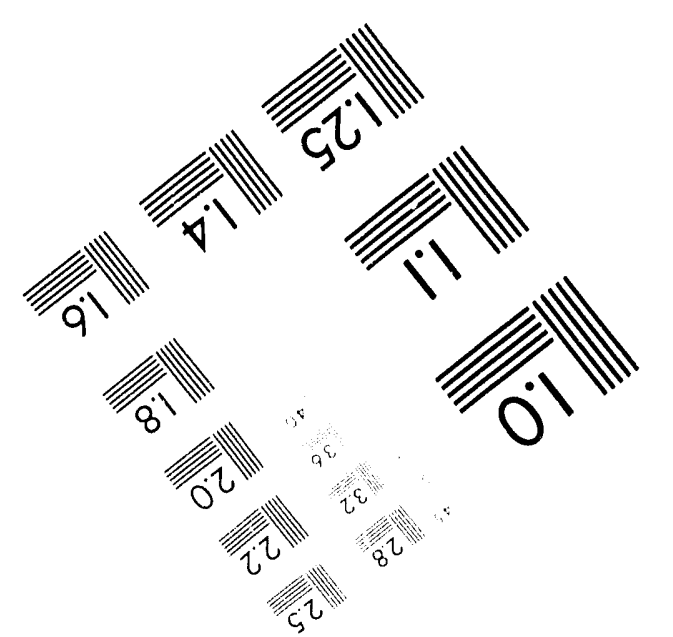



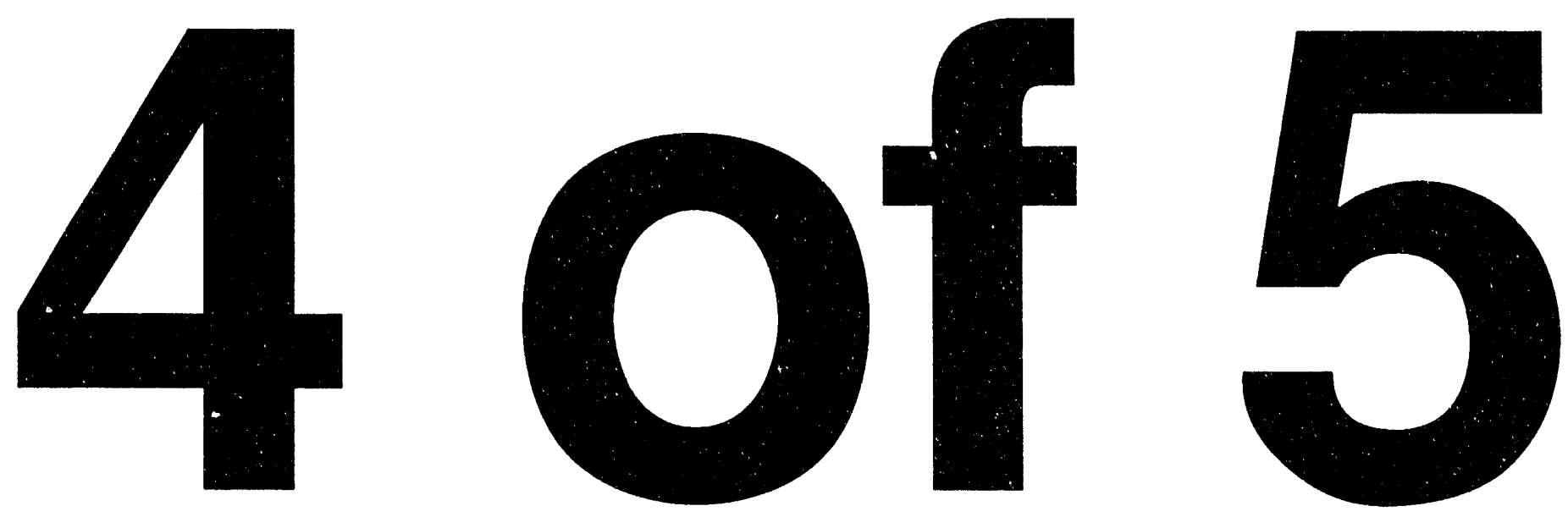
Table 14-4. Constituents with at Least One Detected Value for the 2101-M Pond Data for Reporting Period October 1 through December 31, 1993.

\begin{tabular}{|c|c|c|c|c|c|c|}
\hline $\begin{array}{l}\text { Hell } \\
\text { Name }\end{array}$ & $\begin{array}{c}\text { Collection } \\
\text { Date }\end{array}$ & $\begin{array}{l}\text { Sample } \\
\text { Number }\end{array}$ & $\begin{array}{c}\text { AMMONIU } \\
129 / \mathrm{ppD} \\
38.5 \%\end{array}$ & $\begin{array}{r}\text { ARSEN IC } \\
43 / \mathrm{ppb} \\
1.38 / 50\end{array}$ & $\begin{array}{c}\text { FARSENIC } \\
43 / \mathrm{ppb} \\
1.38 / 50\end{array}$ & $\begin{array}{c}\text { BROMIDE } \\
124 / \mathrm{ppb} \\
52.8 / .\end{array}$ \\
\hline$\ldots$. & - n. & . & 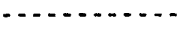 & $\cdots . .$. & 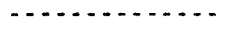 & \\
\hline 299-E18-1 & $12 / 15 / 93$ & B09NX1 & & $2.20 \mathrm{~L}$ & & $70.00 \mathrm{~L}$ \\
\hline 299-E 18-1 & $12 / 15 / 93$ & B09NX5 & & & $2.00 \mathrm{~L}$ & \\
\hline 299-E18-1 & $12 / 15 / 93$ & B09N21 & $100.00 Q$ & & & \\
\hline 299-E.18-2 & $12 / 20 / 93$ & BO9NX6 & & 6.00 & & $52.80 \mathrm{U}$ \\
\hline 299-E18-2 & $12 / 20 / 93$ & BOSNYO & & & 6.10 & \\
\hline $299-E 18-3$ & $12 / 20 / 93$ & BOSNY 1 & & 11.00 & & $52.80 \mathrm{U}$ \\
\hline 299-E18-3 & $12 / 20 / 93$ & B09NY5 & & & 11.00 & \\
\hline $299-E 18-4$ & $12 / 15 / 93$ & B09NY6 & & 13.00 & & $52.80 \mathrm{U}$ \\
\hline $299-E 18-4$ & $12 / 15 / 93$ & BO9N2O & & & 11.00 & \\
\hline
\end{tabular}

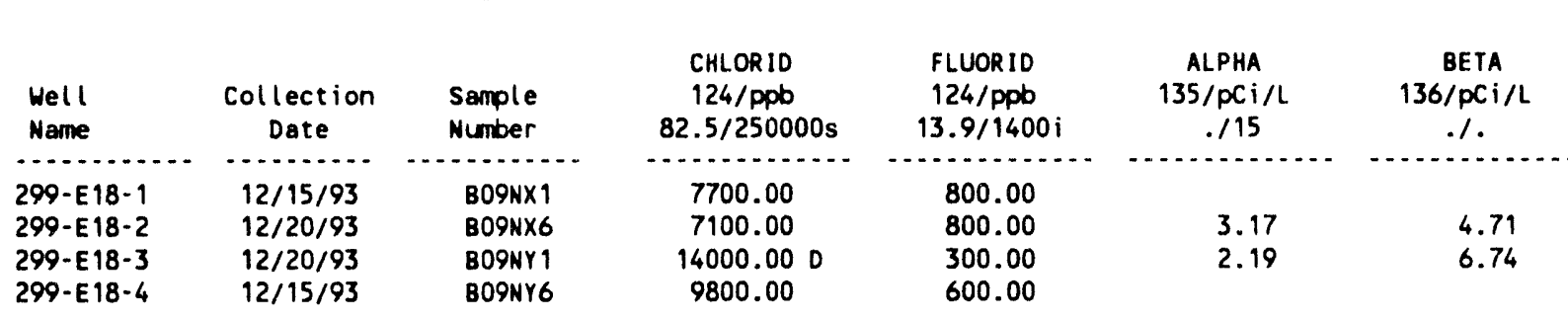

\begin{tabular}{|c|c|c|c|c|c|c|}
\hline $\begin{array}{l}\text { Hell } \\
\text { Name }\end{array}$ & $\begin{array}{c}\text { Collection } \\
\text { Date }\end{array}$ & $\begin{array}{l}\text { Sample } \\
\text { Number }\end{array}$ & $\begin{array}{l}\text { NITRATE } \\
124 / \mathrm{ppb} \\
44.4 / 45000\end{array}$ & $\begin{array}{l}\text { SELENUM } \\
48 / \mathrm{ppb} \\
1.21 / 10 \mathrm{i}\end{array}$ & $\begin{array}{l}\text { FSELENI } \\
48 / \mathrm{ppb} \\
1.21 / 10 \mathrm{i}\end{array}$ & $\begin{array}{c}\text { SULFATE } \\
124 / \mathrm{ppb} \\
49.9 / 250000 \mathrm{~s}\end{array}$ \\
\hline$\cdots$ & - & - . . . & 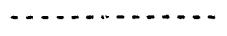 & $\cdots$ & 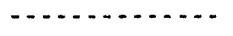 & \\
\hline $299-E 18-1$ & $12 / 15 / 93$ & B09NX1 & $13000.00 \mathrm{D}$ & $6.90 \mathrm{~L}$ & & $120000.00 \mathrm{D}$ \\
\hline $299-E 18-1$ & $12 / 15 / 93$ & 809NX5 & & & $5.50 \mathrm{~L}$ & \\
\hline 299-E18-2 & $12 / 20 / 93$ & $809 \mathrm{~N} \times 6$ & 500.00 & $1.21 \mathrm{U}$ & & $24000.00 \mathrm{D}$ \\
\hline 299-E 18-2 & $12 / 20 / 93$ & BO9NYO & & & $1.21 \mathrm{U}$ & \\
\hline $299-E 18-3$ & $12 / 20 / 93$ & BO9NY1 & 600.00 & $1.21 \mathrm{U}$ & & $11000.00 \mathrm{D}$ \\
\hline 299-E18-3 & $12 / 20 / 93$ & BO9NYS & & & $1.21 \mathrm{U}$ & \\
\hline $299-E 18-4$ & $12 / 15 / 93$ & BO9NYG & 400.00 & $1.21 \mathrm{U}$ & & $11000.00 \mathrm{D}$ \\
\hline 299-E18-4 & $12 / 15 / 93$ & BO9NZO & & & $1.21 \mathrm{U}$ & \\
\hline
\end{tabular}

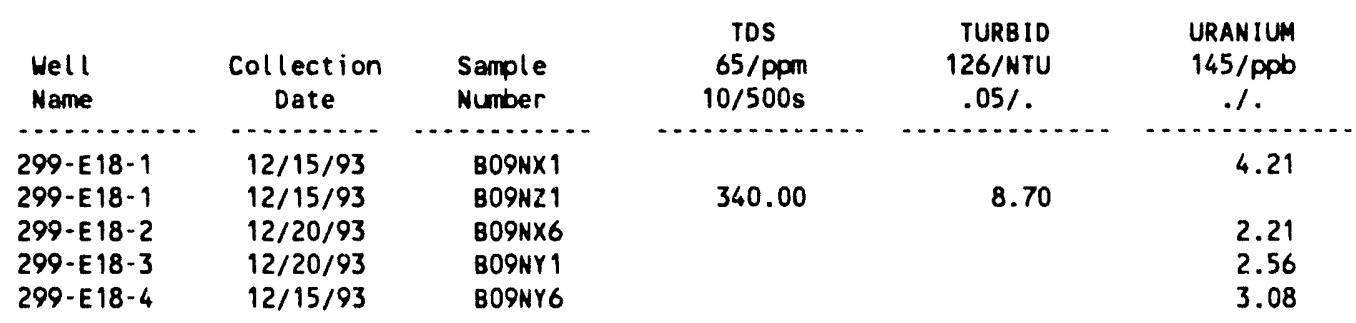

For explanation of this table, see Section 1.4 of report. 
Table 14-5. Contamination Indicator Parameters for the 2101-M Pond Data for Reporting Period October 1 through December 31, 1993.

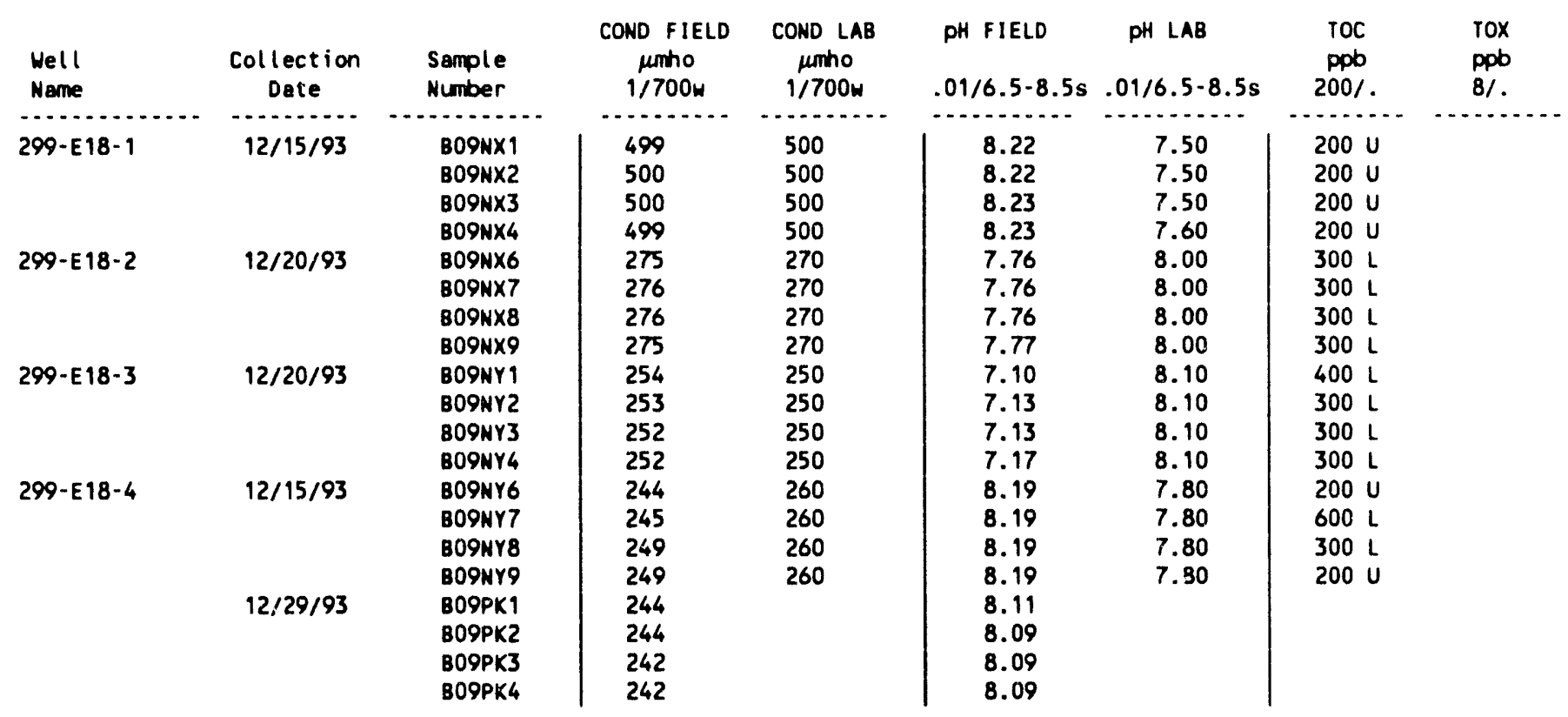

For explanation of this table, see Section 1.4 of report. 
$\mathrm{DOE} / \mathrm{RL}-93-56-4$

This page intentionally left blank. 
DOE/RL-93-56-4

\section{CONTENTS}

15.0 LOW-LEVEL BURIAL GROUNDS . . . . . . . . . . . . 15-1

15.1 INTRODUCTION . . . . . . . . . . . . . . 15-1

15.2 WATER LEVEL MEASUREMENTS . . . . . . . . . . . . . . 15-1

15.3 WATER CHEMISTRY DATA ..................... 15-1 


\section{LIST OF FIGURES}

15-1 Monitoring We11 Locations for Low-Level

Waste Management Area l. . . . . . . . . . . . . . 15-3

15-2 Monitoring Well Locations for Low-Level

Waste Management Area 2................ . . 15-4

15-3 Monitoring Well Locations for Low-Level

Waste Management Area 3... . . . . . . . . . . . 15-5

15-4 Monitoring Well Locations for Low-Level

Waste Management Area 4 . . . . . . . . . . . . . . 15-6

15-5 Monitoring Well Locations for Low-Level

Waste Management Area $5 \ldots 15-7$

\section{LIST OF TABLES}

15-1 Low-Level Burial Grounds . . . . . . . . . . . . . . . 15-8

15-2 Monitoring Well Purpose and Sampling Schedule

for Low-Level Waste Management Area 1 . . . . . . . . . . . 15-9

15-3 Monitoring Well Purpose and Sampling Schedule for Low-Level

Waste Management Area 2............... . . 15-10

15-4 Monitoring Well Purpose and Sampling Schedule for Low-Level

Waste Management Area 3................. 15-11

15-5 Monitoring Well Purpose and Sampling Schedule for Low-Level

Waste Management Area 4 .............. . . 15-12

15-6 Monitoring Well Purpose and Sampling Schedule for Low-Level

Waste Management Area 5................ . 15-13

15-7 RCRA Water Level Measurement Report for the LLWMA-1,

Fourth Quarter 1993 ................. 15-14

15-8 RCRA Water Level Measurement Report for the LLWMA-2,

Fourth Quarter 1993 ................. 15-17

15-9 RCRA Water Level Measurement Report for the LLWMA-3,

Fourth Quarter 1993 ........ . . . . . . . . 15-19

15-10 RCRA Water Level Measurement Report for the LLWMA-4,

Fourth Quarter 1993 ................. 15-22

15-11 RCRA Water Level Measurement Report for the LLWMA-5,

Fourth Quarter 1993 . . . . . . . . . . . . . . . . 15-24 
DOE/RL-93-56-4

\section{LIST OF TABLES (cont.)}

15-12 Constituent List and Summary of Results for the Low-Level

Waste Burial Grounds WMA 1 Data for Reporting Period

October 1 through December 31, 1993 . . . . . . . . . . . . 15-25

15-13 Constituents with at Least One Detected Value for the

Low-Level Waste Burial Grounds WMA 1 Data for Reporting

Period October 1 through December 31, 1993 . . . . . . . . . 15-28

15-14 Contamination Indicator Parameters for the Low-Level

Waste Burial Grounds WMA 1 Data for Reporting Period

October 1 through December 31, 1993 . . . . . . . . . . 15-39

15-15 Constituent List and Summary of Results for the Low-Level

Waste Burial Grounds WMA 2 Data for Reporting Period

October 1 through December 31, 1993 ............ 15-40

15-16 Constituents with at Least One Detected Value for the

Low-Level Waste Burial Grounds WMA 2 Data for Reporting

Period October 1 through December 31, 1993 . . . . . . . . 15-43

15-17 Contamination Indicator Parameters for the Low-Leve1

Waste Burial Grounds WMA 2 Data for Reporting Period

October 1 through December 31, 1993 ............ 15-51

15-18 Constituent List and Summary of Results for the

Low-Level Waste Burial Grounds WMA 3 Data for Reporting

Period October 1 through December 31, 1993 . . . . . . . . . 15-53

15-19 Constituents with at Least One Detected Value for the

Low-Level Waste Burial Grounds WMA 3 Data for Reporting

Period October 1 through December 31, 1993 . . . . . . . . . 15-56

15-20 Contamination Indicator Parameters for the Low-Level

Waste Burial Grounds WMA 3 Data for Reporting Period

October 1 through December 31, 1993 .. . . . . . . . . . 15-69

15-21 Constituent List and Summary of Results for the Low-Level

Waste Burial Grounds WMA 4 Data for Reporting Period

October 1 through December 31, 1993 .. . . . . . . . . . . 15-70

15-22 Constituents with at Least One Detected Value for the Low-Level Waste Burial Grounds WMA 4 Data for Reporting

Period October 1 through December 31, 1993 . . . . . . . . . 15-73

15-23 Contamination Indicator Parameters for the Low-Level

Waste Burial Grounds WMA 4 Data for Reporting Period

October 1 through December 31, 1993 . . . . . . . . . . . 15-83

15-24 Constituent List and Summary of Results for the Low-Leve1

Waste Burial Grounds WMA 5 Data for Reporting Period

October 1 through December 31, 1993 


\section{LIST OF TABLES (cont.)}

15-25 Constituents with at Least One Detected Value for the Low-Level Waste Burial Grounds WMA 5 Data for Reporting Period October 1 through December 31, 1993 ......... . 15-88

15-26 Contamination Indicator Parameters for the Low-Level Waste Burial Grounds WMA 5 Data for Reporting Period October 1 through December 31,1993 ............... 15-92

15-27 Water Quality Standards Exceeded at Low-Level Waste Management Area 1. . . . . . . . . . . . . . . 15-93

15-28 Water Quality Standards Exceeded at Low-Level Waste Management Area 2. . . . . . . . . . . . . . . . 15-93

15-29 Water Quality Standards Exceeded at Low-Level Waste Management Area 3.................... 15-94

15-30 Water Quality Standards Exceeded at Low-Level Waste Management Area 4. . . . . . . . . . . . . . . . 15-95

15-31 Water Quality Standards Exceeded at Low-Level Waste Management Area 5..................... 15-96 


\title{
15.0 LOW-LEVEL BURIAL GROUNDS
}

\author{
R. B. Mercer \\ Westinghouse Hanford Company
}

\subsection{INTRODUCTION}

The 200 Areas LLBGs consist of five Low-Level Waste Management Areas (LLWMA). LLWMA-1 and LLWMA-2 are located in the 200 East Area and LLWMAs-3, -4 , and -5 are located in the 200 West Area (Figures 15-1 through 15-5). A description of the waste contained in each burial ground is provided in Table 15-1.

The groundwater monitoring program for the LLBG began with the installation of 35 monitoring wells in 1987. Additional wells have been installed in subsequent years. The present monitoring networks are listed in Tables 15-2 through 15-6.

Currently, groundwater quality assessment programs are under way for LLWMA-1 and LLWMA-3. Groundwater sampling is conducted on a quarterly basis under the assessment programs. Semiannual detection-level sampling is being conducted at LLWMA-2 and LLWMA-4. The first four quarters of data have been collected at some of the LLWMA-5 monitoring wells, and background values have been established for the indicator parameters (except TOX). This site is now in a detection monitoring program with semiannual sampling.

\subsection{HATER LEVEL MEASUREMENTS}

In addition to measurements taken at the time of sampling, water levels are measured monthly at LLWMA-1 and LLWMA-3 (areas in assessment) and quarterly at LLWMAs-2, -4 , and -5 . Water level measurements are presented in Tables 15-7 through 15-11. Additional water level measurements are reported for some wells if they are monitored for other projects. Suspected errors in the water ' evel measurements occurred at several wells this quarter as indicated in the tables.

Water level measurements were not taken at wells 299-W6-5 or 299-W6-8 because of obstructed casings. The casing in well 299-W6-5 was crimped during installation. Remedial action enlarged the casing to permit the installation of the pump. There is not sufficient space in the well to measure water levels and additional well remediation may not be possible without damaging the casing. The obstruction has been removed from well 299-W6-8. Water levels cont inue to decline beneath LLWMA-2. Well 299-E34-4 has been dry since it was constructed. Water levels in wells 299-E34-6, 299-E34-7, and 299-E35-1 have fallen to the point that measurements may not be representative.

\subsection{WATER CHEMISTRY DATA}

Scheduled groundwater samples were collected as scheduled at all five LLWMAs. Five wells scheduled for semiannual sampling at LLWMA-2 were sampled this quarter for the 216-B-63 RCRA monitoring program. Samples were analyzed 
for CIPs, interim primary drinking water parameters, water quality parameters, and site-specific parameters. Results of analyses are listed in Tables 15-12 through 15-26. These tables also contain any previously unreported data. The replicate averages for the indicator parameters did not exceed the critical means at LLWMAs-2, -4 or -5 .

An NCR was issued during the construction of wel1 299-W18-32 because a portion of a broken casing shoe was left in the screened interval. A P flag is being assigned to the chemistry data for metals from this well. Results flagged with a ' $Q$ ' are associated with suspect $Q C$ data. All TOX results are flagged with a 'P,' indicating a problem with data. Data quality and data flags are discussed in Sections 1.2 and 1.4 , respectively. The $D$ flag on chloride, nitrate, and sulfate indicate that the samples were diluted for analysis. The cause of the $B$ flag on barium, calcium, iron, and sodium for LLWMA-2 and LLWMA-4 is under investigation. Tables 15-27 through 15-31 list the water quality standards that were exceeded for the data reported this quarter.

The $\mathrm{pH}$ values taken in November 1993 from wells 299-E27-8 and 299-E34-12 (LLWMA-2) were below the established critical range for this waste management area. Verification sampling was conducted on December 12, 1993, and confirmed that the November measurements were in error. An RADE has been submitted for this data. 
Figure 15-1. Monitoring Well Locations for Low-Level Waste Management Area 1.

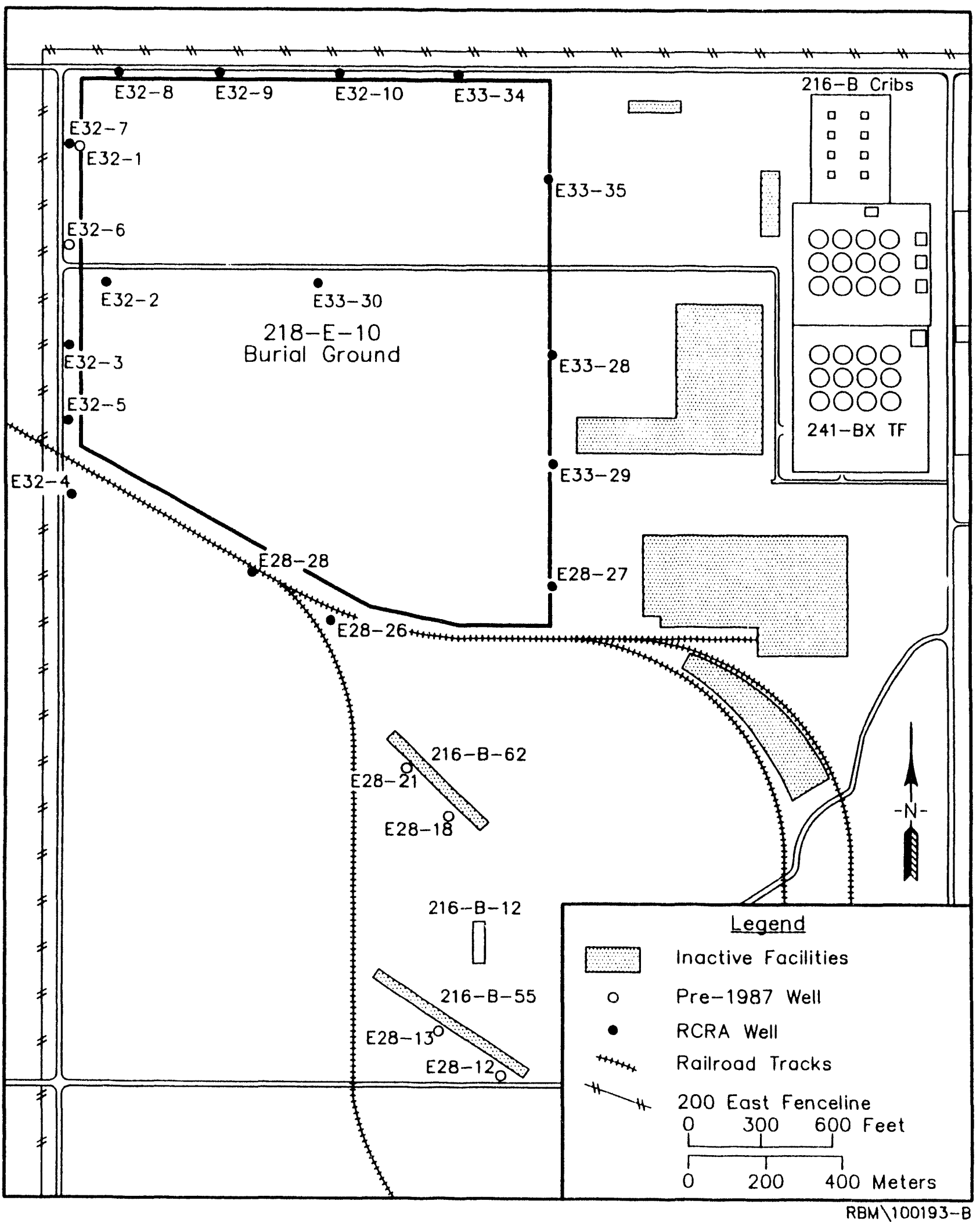




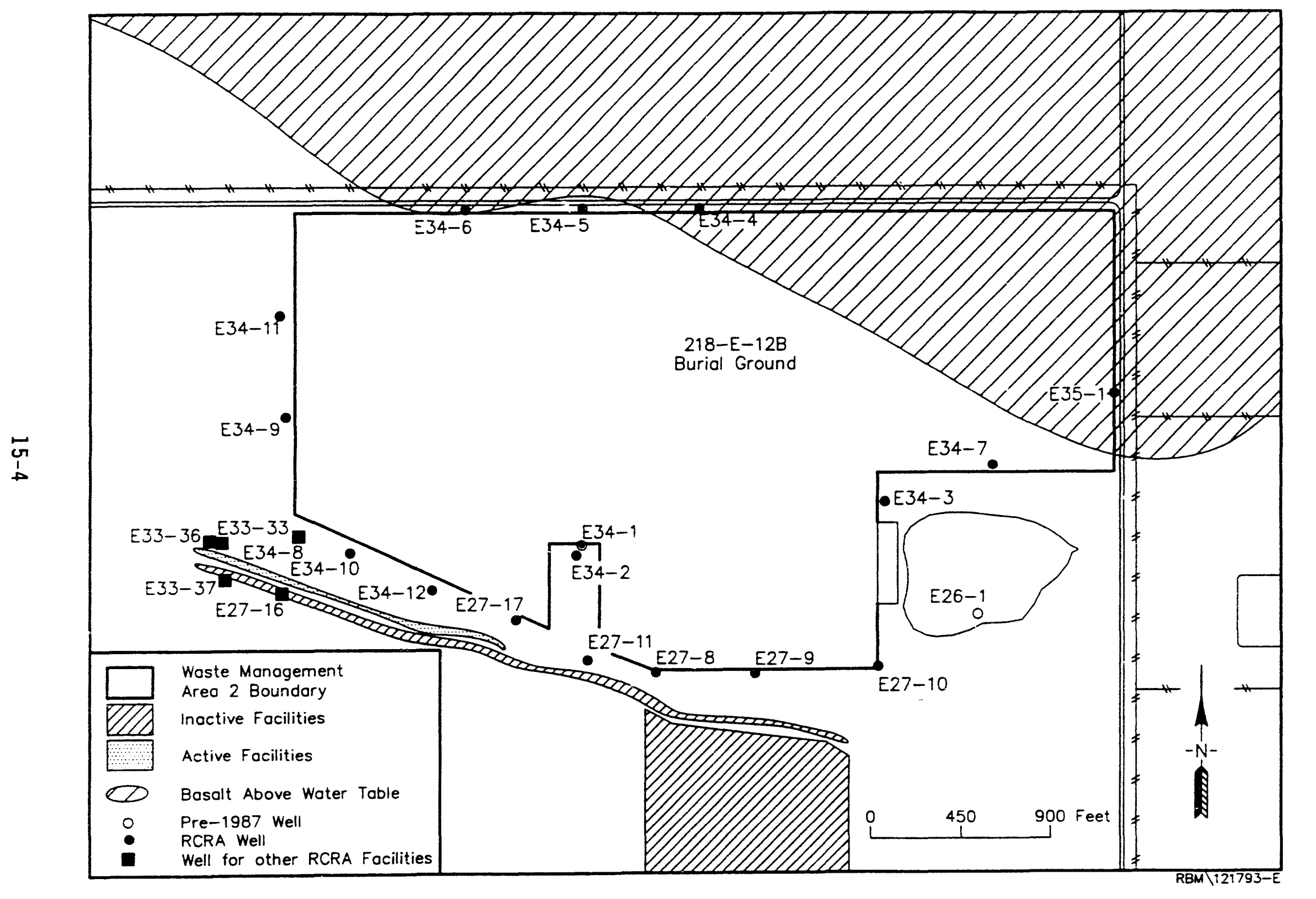

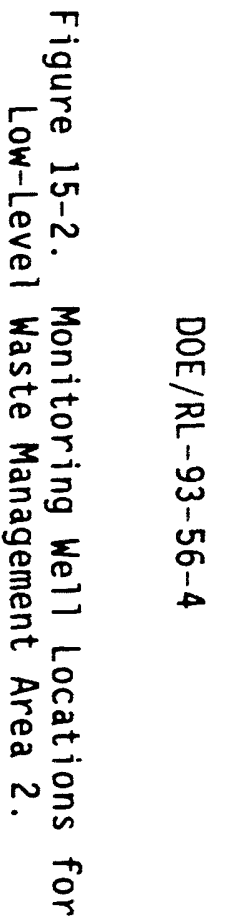




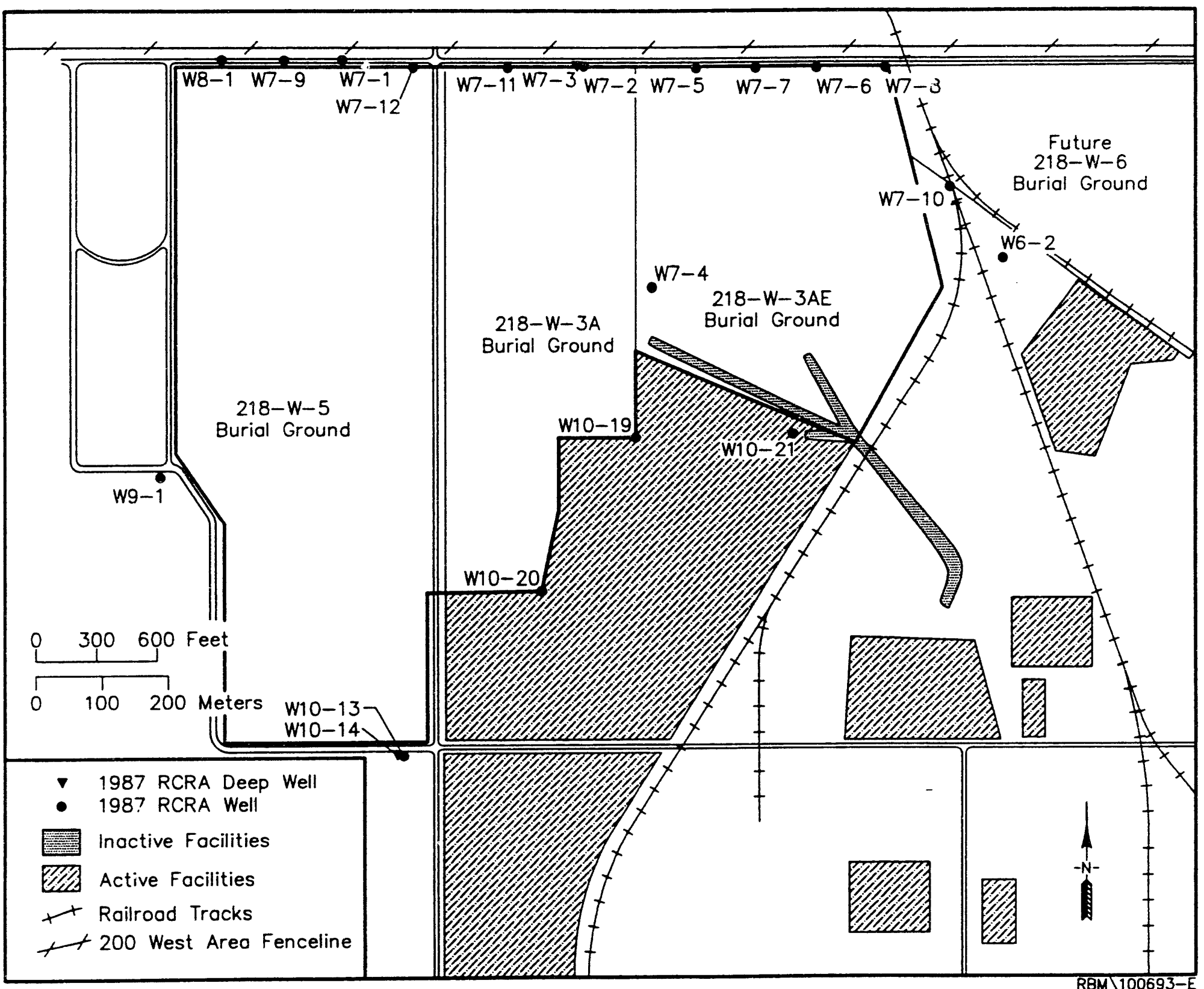

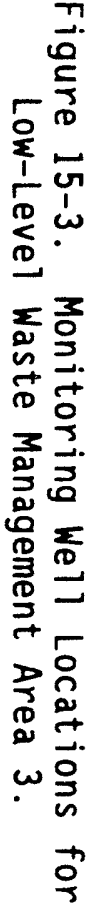


Figure 15-4. Monitoring Well Locations for Low-Level Waste Management Area 4.

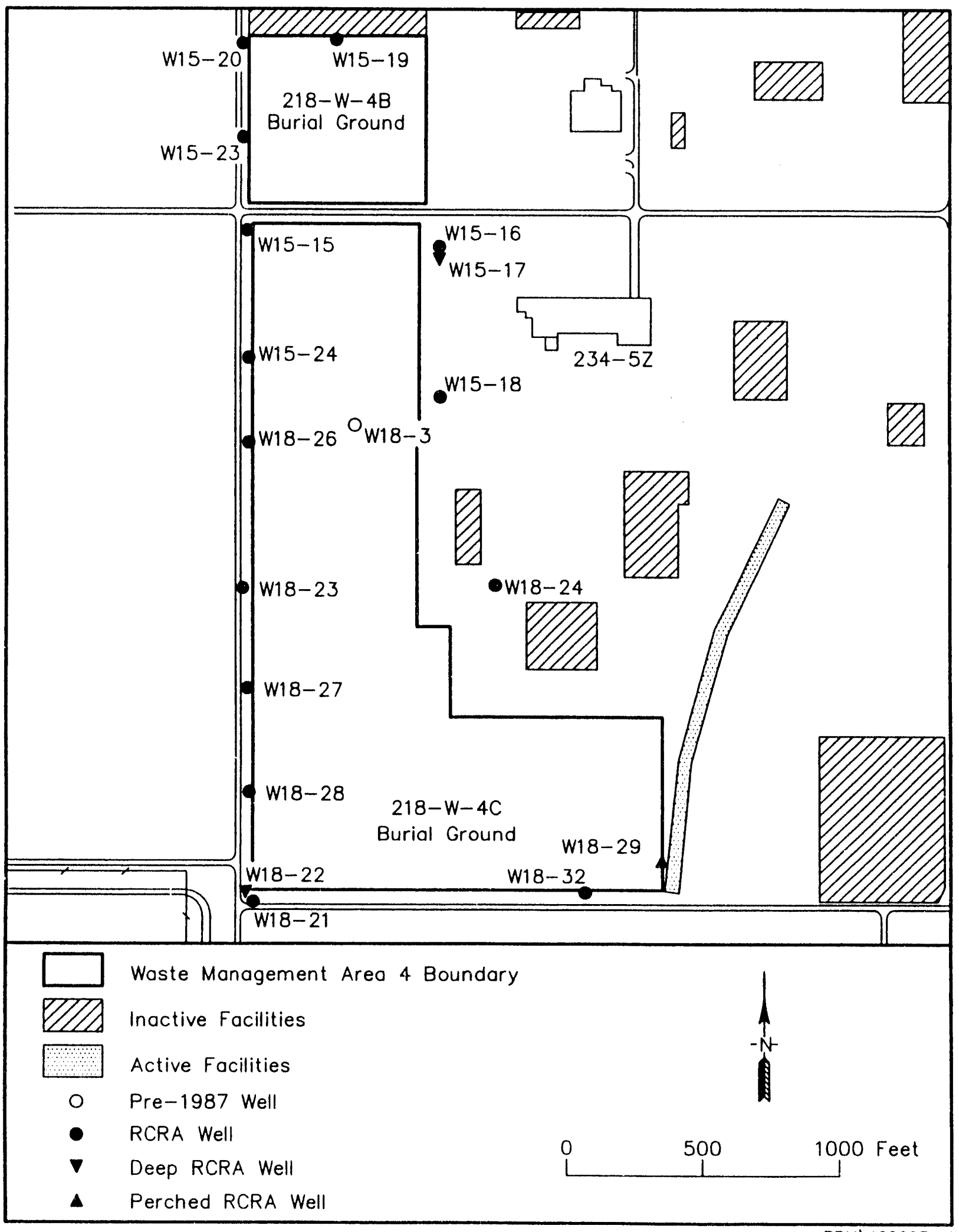



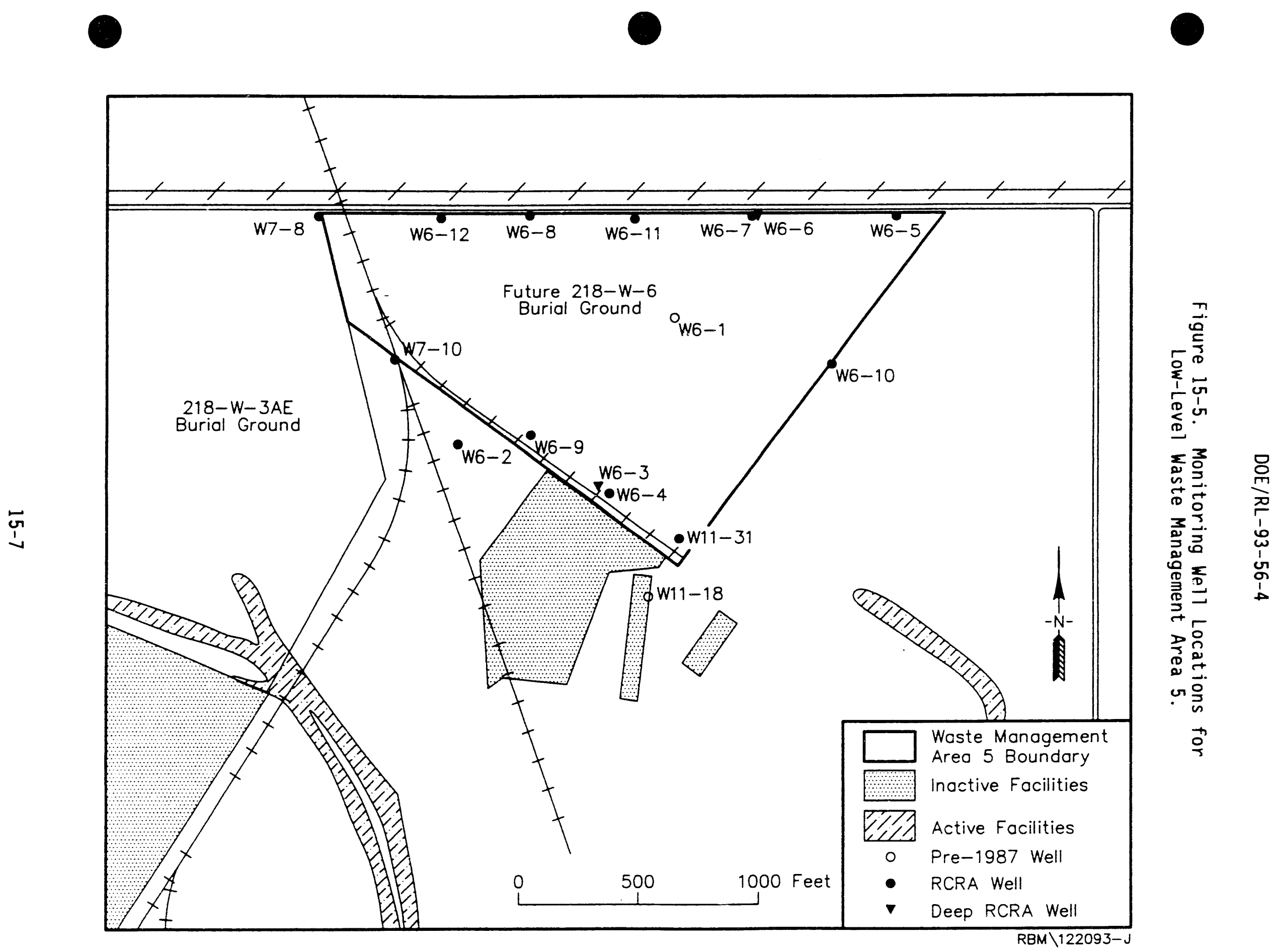
Table 15-1. Low-Level Burial Grounds.

\begin{tabular}{|c|c|c|c|}
\hline LLWMA- & Burial Ground & First use & Contents \\
\hline 1 & $218-E-10$ & 1960 & $\begin{array}{l}\text { Dragoff material } \\
\text { Failed equipment } \\
\text { Mixed waste }\end{array}$ \\
\hline 2 & $218-E-12 B$ & 1968 & $\begin{array}{l}\text { Miscellaneous dry waste } \\
\text { Transuranic waste } \\
\text { Submarine reactor compartments }\end{array}$ \\
\hline \multirow[t]{3}{*}{3} & $218-W-3 A$ & 1970 & $\begin{array}{l}\text { Ion exchange resins } \\
\text { Failed equipment }\end{array}$ \\
\hline & $218-W-3 A E$ & 1981 & $\begin{array}{l}\text { Low-level waste } \\
\text { Low-level mixed waste }\end{array}$ \\
\hline & $218-W-5$ & 1986 & $\begin{array}{l}\text { Low-level waste } \\
\text { Low-level mixed waste }\end{array}$ \\
\hline \multirow[t]{2}{*}{4} & $218-W-4 B$ & 1968 & $\begin{array}{l}\text { Mixed waste } \\
\text { Retrievable transuranic waste }\end{array}$ \\
\hline & $218-W-4 C$ & 1978 & $\begin{array}{l}\text { Transuranic waste } \\
\text { Mixed waste } \\
\text { Low-level waste }\end{array}$ \\
\hline 5 & $218-W-6$ & $N / A$ & None to date \\
\hline
\end{tabular}

$N / A=$ not applicable. 
Table 15-2. Monitoring Well Purpose and Sampling Schedule for Low-Level Waste Management Area 1.

\begin{tabular}{|c|c|c|c|c|}
\hline $\begin{array}{c}\text { Well no. } \\
(299-)\end{array}$ & $\begin{array}{l}\text { Relative } \\
\text { position }\end{array}$ & Hydrogeologic unit & $\begin{array}{c}\text { Sample } \\
\text { frequen } y\end{array}$ & $\begin{array}{l}\text { Sample date, } \\
\text { 4th Qtr } 1993\end{array}$ \\
\hline$E 28-26^{87}$ & Upgradient & $\begin{array}{l}\text { Hanford/Ringold: } \\
\text { Water Table }\end{array}$ & Quarterly & $10 / 01 / 93$ \\
\hline$E 28-27^{87}$ & Upgradient & Hanford: Water Table & Quarterly & $10 / 05 / 93$ \\
\hline$E 28-28^{90}$ & Upgradient & $\begin{array}{l}\text { Hanford/Ringold: } \\
\text { Water Table }\end{array}$ & Quarterly & $10 / 01 / 93$ \\
\hline$E 32-2^{87}$ & Downgradient & Hanford: Water Table & Quarterly & $10 / 01 / 93$ \\
\hline$E 32-3^{87}$ & Downgradient & $\begin{array}{l}\text { Hanford/Ringold: } \\
\text { Water Table }\end{array}$ & Quarterly & $10 / 01 / 93$ \\
\hline$E 32-4^{87}$ & Upgradient & $\begin{array}{l}\text { Hanford/Ringold: } \\
\text { Water Table }\end{array}$ & Quarterly & $10 / 15 / 93$ \\
\hline$\overline{E 32}-5^{89}$ & Downgradient & $\begin{array}{l}\text { Hanford/Ringold: } \\
\text { Water Table }\end{array}$ & Quarterly & $10 / 20 / 93$ \\
\hline$E 32-6^{91}$ & Downgradient & Hanford: Water Table & Quarterly & $10 / 04 / 93$ \\
\hline$E 32-7^{91}$ & Downgradient & Hanford: Water Table & Quarterly & $10 / 04 / 93$ \\
\hline$E 32-8^{91}$ & Downgradient & Hanford: Water Table & Quarterly & $10 / 05 / 93$ \\
\hline$E 32-9^{91}$ & Downgradient & Hanford: Water Table & Quarterly & $10-04 / 93$ \\
\hline$E 32-10^{92}$ & Downgradient & Hanford: Water Table & Quarterly & $10 / 01 / 93$ \\
\hline$E 33-28^{87}$ & Upgradient & Hanford: Water Table & Quarterly & $10 / 05 / 93$ \\
\hline$E 33-29^{87}$ & Upgradient & Hanford: Water Table & Quarterly & $10 / 05 / 93$ \\
\hline$E 33-30^{87}$ & Downgradient & Hanford: Water Table & Quarterly & $10 / 05 / 93$ \\
\hline$E 33-34^{90}$ & Downgradient & Hanford: Water Table & Quarterly & $10 / 06 / 93$ \\
\hline$E 33-35^{90}$ & Upgradient & Hanford: Water Table & Quarterly & $10 / 06 / 93$ \\
\hline
\end{tabular}

Note: Superscript following well number indicates year of installation. 
Table 15-3. Monitoring Well Purpose and Sampling Schedule for Low-Level Waste Management Area 2.

\begin{tabular}{|c|c|c|c|c|}
\hline $\begin{array}{l}\text { Wel1 no. } \\
(299-)\end{array}$ & $\begin{array}{l}\text { Relative } \\
\text { position }\end{array}$ & Hydrogeologic unit & $\begin{array}{c}\text { Sample } \\
\text { frequency }\end{array}$ & $\begin{array}{l}\text { Sample date, } \\
\text { 4th Qtr } 1993\end{array}$ \\
\hline$E 27-8^{87}$ & Downgradient & Hanford: Water Table & Semiannually & $11 / 10 / 93$ \\
\hline$E 27-9^{87}$ & Downgradient & Hanford: Water Table & Semiannually & $11 / 10 / 93$ \\
\hline$E 27-10^{87}$ & Upgradient & Hanford: Water Table & Semiannually & $11 / 11 / 93$ \\
\hline$E 27-11^{89}$ & Downgradient & Hanford: Water Table & Semi annually & $11 / 11 / 93$ \\
\hline $\mathrm{E} 27-17^{91}$ & Downgradient & Hanford: Water Table & Semiannually & $11 / 11 / 93$ \\
\hline$E 34-2^{87}$ & Downgradient & Hanford: Water Table & Semiannually & $11 / 10 / 93$ \\
\hline$E 34-3^{87}$ & Upgradient & Hanford: Water Table & Semiannually & $11 / 10 / 93$ \\
\hline t $34-4^{87}$ & Upgradient & Hanford: Water Table & $N / A$ & Dry \\
\hline$E 34-5^{87}$ & Upgradient & Hanford: Water Table & Semiannually & $11 / 10 / 93$ \\
\hline$E 34-6^{87}$ & Upgradient & Hanford: Water Table & $N / A$ & Dry \\
\hline$E 34-7^{89}$ & Upgradient & Hanford: Water Table & Semiannually & NS \\
\hline$E 34-9^{91}$ & Downgradient & Hanford: Water Table & Semiannually & $11 / 15 / 93$ \\
\hline$E 34-10^{91}$ & Downgradient & Hanford: Water Table & Semiannually & $11 / 15 / 93$ \\
\hline$E 34-11^{92}$ & Downgradient & Hanford: Water Table & Quarterly & $11 / 15 / 93$ \\
\hline$E 34-12^{92}$ & Downgradient & Hanford: Water Table & Quarterly & $11 / 15 / 93$ \\
\hline$E 35-1^{89}$ & Upgradient & Hanford: Water Table & $N / A$ & Dry \\
\hline
\end{tabular}

$N / A=$ not applicable.

NS = not sampled.

Note: Superscript following well number indicates year of installation. 
Table 15-4. Monitoring Well Purpose and Sampling Schedule for Low-Level Waste Management Area 3.

\begin{tabular}{|c|c|c|c|c|c|}
\hline $\begin{array}{c}\text { Wel1 no. } \\
(299-)\end{array}$ & $\begin{array}{l}\text { Relative } \\
\text { position }\end{array}$ & \multicolumn{2}{|c|}{ Hydrogeologic unit } & $\begin{array}{c}\text { Sample } \\
\text { frequency }\end{array}$ & $\begin{array}{l}\text { Sample date, } \\
\text { 4th Qtr } 1993\end{array}$ \\
\hline$W 6-2^{87 m}$ & Downgradient & Ringol & Water Table & Quarterly & $12 / 07 / 93$ \\
\hline$W 7-1^{87}$ & Downgradient & Ringold & Water Table & Quarterly & $12 / 06 / 93$ \\
\hline$W 7-2^{87}$ & Downgradient & Ringold & Water Table & Quarterly & $12 / 06 / 93$ \\
\hline$W 7-3^{87}$ & Downgradient & Ringold & Deep & Quarterly & $12 / 07 / 93$ \\
\hline$W 7-4^{87}$ & Downgradient & Ringold & Water Table & Quarterly & $12 / 03 / 93$ \\
\hline$W 7-5^{87}$ & Downgradient & Ringold & Water Table & Quarterly & $12 / 06 / 93$ \\
\hline$W 7-6^{87}$ & Downgradient & Ringold & Water Table & Quarterly & $12 / 03 / 93$ \\
\hline$W 7-7^{89}$ & Downgradient & Ringolc & Water Table & Quarterly & $12 / 03 / 93$ \\
\hline$W 7-8^{89}$ & Downgradient & Ringold & Water Table & Quarterly & $12 / 03 / 93$ \\
\hline$W 7-9^{90}$ & Downgradient & Ringold & Water Table & Quarterly & $12 / 03 / 93$ \\
\hline$W 7-10^{90 m}$ & Downgradient & Ringold & Water Table & Quarterly & $12 / 02 / 93$ \\
\hline$W 7-11^{91}$ & Downgradient & Ringold & Water Tabie & Quarterly & $12 / 06 / 93$ \\
\hline$W 7-12^{91}$ & Downgradient & Ringold & Water Table & Quarterly & $12 / 03 / 93$ \\
\hline$W 8-1^{87}$ & Downgradient & Ringold & Water Table & Quarterly & $12 / 03 / 93$ \\
\hline W9-1 $1^{87}$ & Upgradient & Ringold & Water Tabie & Quarterly & $12 / 02 / 93$ \\
\hline$W 10-13^{87}$ & Upgradient & Ringold & Water Table & Quarterly & $11 / 30 / 93$ \\
\hline$W 10-14^{87}$ & Upgradient & Ringold & Deep & Quarterly & $12 / 01 / 93$ \\
\hline$W 10-19^{92}$ & Upgradient & Ringold & Water Table & Quarterly & $12 / 02 / 93$ \\
\hline$W 10-21^{93}$ & Upgradient & Ringold & Water Table & Quarterly & $12 / 15 / 93$ \\
\hline
\end{tabular}

Notes: 1. Superscript following well number indicates year of installation.

2. Well monitors multiple Low-Level Waste Management Areas.

3. Deep indicates well monitors bottom of the unconfined aquifer. 
Table 15-5. Monitoring Well Purpose and Sampling Schedule for Low-Level Waste Management Area 4.

\begin{tabular}{|c|c|c|c|c|}
\hline $\begin{array}{c}\text { Well no. } \\
(299-)\end{array}$ & $\begin{array}{l}\text { Relative } \\
\text { position }\end{array}$ & Hydrogeologic unit & $\begin{array}{c}\text { Sample } \\
\text { frequency }\end{array}$ & $\begin{array}{l}\text { Sample date, } \\
4 \text { th Qtr } 1993\end{array}$ \\
\hline$W 15-15^{87}$ & Downgradient & Ringold: Water Table & Semiannually & $11 / 19 / 93$ \\
\hline$W 15-16^{87}$ & Upgradient & Ringold: Water Table & Semiannually & $11 / 18 / 93$ \\
\hline$W 15-17^{87}$ & Upgradient & Ringold: Deep & Semiannually & $11 / 18 / 93$ \\
\hline$W 15-18^{87}$ & Upgradient & Ringold: Water Table & Semiannually & $11 / 18 / 93$ \\
\hline$W 15-19^{89}$ & Downgradient & Ringold: Water Table & Semiannually & $12 / 03 / 93$ \\
\hline$W 15-20^{89}$ & Downgradient & Ringold: Water Table & Semiannually & $11 / 19 / 93$ \\
\hline$W 15-23^{90}$ & Downgradient & Ringold: Water Table & Semiannually & $11 / 19 / 93$ \\
\hline$W 15-24^{89}$ & Downgradient & Ringold: Water Table & Semiannually & $11 / 29 / 93$ \\
\hline$W 18-21^{87}$ & Downgradient & Ringold: Water Table & Semiannually & $11 / 19 / 93$ \\
\hline$W 18-22^{87}$ & Downgradient & Ringold: Deep & Semiannually & $11 / 30 / 93$ \\
\hline$W 18-23^{87}$ & Downgradient & Ringold: Water Table & Semiannually & $11 / 22 / 93$ \\
\hline$W 18-24^{87}$ & Upgradient & Ringold: Water Table & Semiannually & $11 / 29 / 93$ \\
\hline$W 18-26^{89}$ & Downgradient & Ringold: Water Table & Semiannually & $11 / 26 / 93$ \\
\hline$W 18-27^{91}$ & Downgradient & Ringold: Water Table & Quarterly & $11 / 30 / 93$ \\
\hline$W 18-28^{91}$ & Downgradient & Ringold: Water Table & Quarterly & $11 / 29 / 93$ \\
\hline$W 18-29^{91}$ & Upgradient & Hanford: Perched & Quarterly & $11 / 29 / 93$ \\
\hline$W 18-32^{92}$ & Upgradient & Ringold: Water Table & Quarterly & $11 / 29 / 93$ \\
\hline
\end{tabular}

Notes: 1. Superscript following well number indicates year of installation.

2. Deep indicates well monitors bottom of the unconfined aquifer. 
Table 15-6. Monitoring Well Purpose and Sampling Schedule for Low-Level Waste Management Area 5.

\begin{tabular}{|c|c|c|c|c|}
\hline $\begin{array}{c}\text { Wel1 no. } \\
(299-)\end{array}$ & $\begin{array}{l}\text { Relative } \\
\text { position }\end{array}$ & Hydrogeologic init & $\begin{array}{c}\text { Sample } \\
\text { frequency }\end{array}$ & $\begin{array}{l}\text { Sample date, } \\
\text { 4th Qtr } 1993\end{array}$ \\
\hline$W 6-2^{87 m}$ & Upgradient & Ringold: Water Table & Quarterly & $12 / 07 / 93$ \\
\hline$W 6-3^{91}$ & Upgradient & Ringold: Deep & Semiannually & NS \\
\hline$W 6-4^{91}$ & Upgradient & Ringold: Water Table & Semiannually & NS \\
\hline $16-5^{91}$ & Downgradient & Ringold: Water Table & Semiannually & NS \\
\hline W6- $6^{91}$ & Downgradient & Ringold: Deep & Semiannually & NS \\
\hline$W 6-7^{91}$ & Downgradient & Ringold: Water Table & Semiannually & NS \\
\hline$W 6-8^{91}$ & Downgradient & Ringold: Water Table & Semiannually & NS \\
\hline W6- $9^{92}$ & Upgradient & Ringold: Water Table & Semiannually & NS \\
\hline$w 6-10^{92}$ & Upgradient & Ringold: Water Table & Semiannually & NS \\
\hline W6-1192 & Downgradient & Ringold: Water Table & Semiannually & NS \\
\hline W6- $12^{92}$ & Downgradient & Ringold: Water Table & Semiannually & NS \\
\hline$W 7-10^{90 m}$ & Upgradient & Ringold: Water Table & Quarterly & $12 / 02 / 93$ \\
\hline$W 11-31^{92}$ & Upgradient & Ringold: Water Table & Semiannually & NS \\
\hline
\end{tabular}

NS = not sampled.

Notes: 1. Superscript following well number indicates year of installation.

2. WWe1l monitors multiple Low-Level Waste Management Areas.

3. Deep indicates well monitors bottom of the unconfined aquifer. 
Table 15-7. RCRA Water Level Measurement Report for the LLWMA-1, Fourth Quarter 1993. (sheet 1 of 3)

\begin{tabular}{|c|c|c|c|}
\hline Well & Date & $\begin{array}{l}\text { Depth to } \\
\text { water (ft) }\end{array}$ & $\begin{array}{l}\text { Water level } \\
\text { elevation } \\
\text { above msl ( } f t)\end{array}$ \\
\hline \multicolumn{4}{|c|}{ LLWMA-1 Wells M } \\
\hline 299-E28-26 & $\begin{array}{l}10 / 01 / 93 \\
10 / 14 / 93 \\
11 / 11 / 93 \\
12 / 16 / 93\end{array}$ & $\begin{array}{l}286.05 \\
285.68 \\
285.69 \\
285.81\end{array}$ & $\begin{array}{l}401.21 \star \\
401.58 \\
401.57 \\
401.45\end{array}$ \\
\hline 299-E28-27 & $\begin{array}{l}10 / 05 / 93 \\
10 / 14 / 93 \\
11 / 11 / 93 \\
12 / 16 / 93\end{array}$ & $\begin{array}{l}278.86 \\
278.85 \\
278.91 \\
279.01\end{array}$ & $\begin{array}{l}401.51^{\star} \\
401.52 \\
401.46 \\
401.36\end{array}$ \\
\hline $299-E 28-28$ & $\begin{array}{l}10 / 01 / 93 \\
10 / 14 / 93 \\
11 / 11 / 93 \\
12 / 16 / 93\end{array}$ & $\begin{array}{l}285.10 \\
285.09 \\
285.13 \\
285.28\end{array}$ & $\begin{array}{l}401.45^{\star} \\
401.46 \\
401.42 \\
401.27\end{array}$ \\
\hline 299-E32-10 & $\begin{array}{l}10 / 01 / 93 \\
10 / 14 / 93 \\
11 / 11 / 93 \\
12 / 16 / 93\end{array}$ & $\begin{array}{l}236.20 \\
236.21 \\
236.20 \\
236.37\end{array}$ & $\begin{array}{l}401.68 \star \\
401.67 \\
401.68 \\
401.51\end{array}$ \\
\hline 299-E32-2 & $\begin{array}{l}10 / 01 / 93 \\
10 / 14 / 93 \\
11 / 11 / 93 \\
12 / 16 / 93\end{array}$ & $\begin{array}{l}268.60 \\
268.68 \\
268.70 \\
268.86\end{array}$ & $\begin{array}{l}401.46^{\star} \\
401.38 \\
401.36 \\
401.20\end{array}$ \\
\hline 299-E32-3 & $\begin{array}{l}10 / 01 / 93 \\
10 / 14 / 93 \\
11 / 11 / 93 \\
12 / 16 / 93\end{array}$ & $\begin{array}{l}275.15 \\
275.98 \\
276.77 \\
275.15\end{array}$ & $\begin{array}{l}401.36 \star \\
400.53 \\
399.74+ \\
401.36\end{array}$ \\
\hline 299-E32-4 & $\begin{array}{l}10 / 14 / 93 \\
10 / 15 / 93 \\
11 / 11 / 93 \\
12 / 16 / 93\end{array}$ & $\begin{array}{l}284.52 \\
284.34 \\
284.52 \\
284.69\end{array}$ & $\begin{array}{l}401.36 \\
401.54^{\star} \\
401.36 \\
401.19\end{array}$ \\
\hline 299-E32-5 & $\begin{array}{l}10 / 14 / 93 \\
10 / 20 / 93 \\
11 / 11 / 93 \\
12 / 16 / 93\end{array}$ & $\begin{array}{l}280.68 \\
280.69 \\
280.79 \\
280.81\end{array}$ & $\begin{array}{l}401.46 \\
401.45^{\star} \\
401.35 \\
401.33\end{array}$ \\
\hline
\end{tabular}


Table 15-7. RCRA Water Level Measurement Report for the LLWMA-1, Fourth Quarter 1993. (sheet 2 of 3)

\begin{tabular}{|c|c|c|c|}
\hline Well & Dat 2 & $\begin{array}{l}\text { Depth to } \\
\text { water (ft) }\end{array}$ & $\begin{array}{l}\text { Water level } \\
\text { elevation } \\
\text { above msl (ft) }\end{array}$ \\
\hline \multicolumn{4}{|l|}{ LLWMA-1 } \\
\hline 299-E32-6 & $\begin{array}{l}10 / 04 / 93 \\
12 / 10 / 93\end{array}$ & $\begin{array}{l}265.69 \\
265.88\end{array}$ & $\begin{array}{l}401.76^{\star} \\
401.57\end{array}$ \\
\hline 299-E32-7 & $\begin{array}{l}10 / 04 / 93 \\
10 / 14 / 93 \\
11 / 11 / 93 \\
12 / 16 / 93\end{array}$ & $\begin{array}{l}256.67 \\
256.72 \\
256.75 \\
256.87\end{array}$ & $\begin{array}{l}401.75 \star \\
401.70 \\
401.67 \\
401.55\end{array}$ \\
\hline 299-E32-8 & $\begin{array}{l}10 / 05 / 93 \\
10 / 14 / 93 \\
11 / 11 / 93 \\
12 / 16 / 93\end{array}$ & $\begin{array}{l}243.89 \\
243.98 \\
244.01 \\
244.13\end{array}$ & $\begin{array}{l}401.70 \star \\
401.61 \\
401.58 \\
401.46\end{array}$ \\
\hline 299-E32-9 & $\begin{array}{l}10 / 04 / 93 \\
10 / 14 / 93 \\
11 / 11 / 93 \\
12 / 16 / 93\end{array}$ & $\begin{array}{l}241.56 \\
241.65 \\
241.70 \\
241.78\end{array}$ & $\begin{array}{l}401.77^{\star} \\
401.68 \\
401.63 \\
401.55\end{array}$ \\
\hline $299-E 33-28$ & $\begin{array}{l}10 / 05 / 93 \\
10 / 14 / 93 \\
11 / 11 / 93 \\
12 / 16 / 93\end{array}$ & $\begin{array}{l}262.62 \\
262.65 \\
262.71 \\
262.78\end{array}$ & $\begin{array}{l}401.61 * \\
401.58 \\
401.52 \\
401.45\end{array}$ \\
\hline 299-E33-29 & $\begin{array}{l}10 / 05 / 93 \\
10 / 14 / 93 \\
11 / 11 / 93 \\
12 / 16 / 93\end{array}$ & $\begin{array}{l}271.10 \\
272.19 \\
272.24 \\
272.32\end{array}$ & $\begin{array}{l}402.67{ }^{\star}+ \\
401.58 \\
401.53 \\
401.45\end{array}$ \\
\hline 299-E33-30 & $\begin{array}{l}10 / 05 / 93 \\
10 / 14 / 93 \\
11 / 11 / 93 \\
12 / 16 / 93\end{array}$ & $\begin{array}{l}261.90 \\
262.11 \\
262.17 \\
262.28\end{array}$ & $\begin{array}{l}401.80 \star \\
401.59 \\
401.53 \\
401.42\end{array}$ \\
\hline $299-E 33-34$ & $\begin{array}{l}10 / 06 / 93 \\
10 / 14 / 93 \\
11 / 11 / 93 \\
12 / 16 / 93\end{array}$ & $\begin{array}{l}231.81 \\
231.83 \\
231.88 \\
232.05\end{array}$ & $\begin{array}{l}401.52 \star \\
401.50 \\
401.45 \\
401.28\end{array}$ \\
\hline
\end{tabular}


Table 15-7. RCRA Water Level Measurement Report for the LLWMA-1, Fourth Quarter 1993. (sheet 3 of 3)

\begin{tabular}{cccc}
\hline Well & Date & $\begin{array}{c}\text { Depth to } \\
\text { water }(\mathrm{ft})\end{array}$ & $\begin{array}{c}\text { Water level } \\
\text { elevation } \\
\text { above msl (ft) }\end{array}$ \\
\hline LLWMA-1 Wells Monitoring the Top of the Unconfined Aquifer \\
\hline $299-$ E33-35 & $10 / 06 / 93$ & 241.53 & $401.48 *$ \\
$10 / 14 / 93$ & 241.54 & 401.47 \\
$11 / 11 / 93$ & 241.55 & 401.46 \\
$12 / 16 / 93$ & 241.72 & 401.29 \\
\hline
\end{tabular}

Notes: 1. Water level elevations are calculated by subtracting the measured depth to water from the surveyed elevation for the well.

2. Depth-to-water values are transcribed from field records.

3. Elevations marked with an ' $*$ ' were measured at the time of sampling.

4. Elevations marked with a ' + ' are outside of the expected range and are suspected of error.

5. To convert feet to meters multiply by 0.3048 . 
Table 15-8. RCRA Water Level Measurement Report for the LLWMA-2, Fourth Quarter 1993. (sheet 1 of 2)

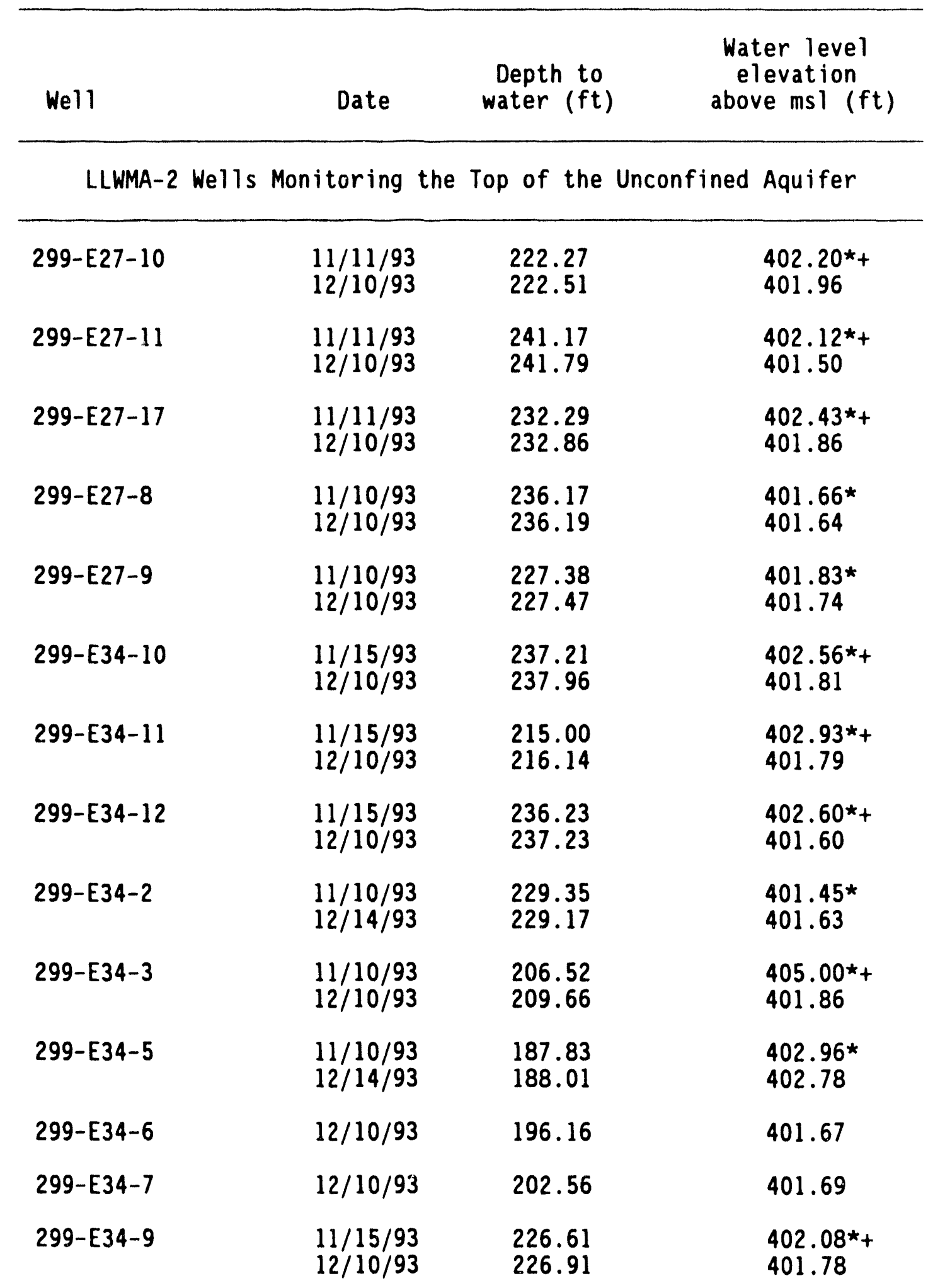


Table 15-8. RCRA Water Level Measurement Report for the LLWMA-2, Fourth Quarter 1993. (sheet 2 of 2)

We11 Date $\begin{gathered}\text { Depth to } \\ \text { water }(\mathrm{ft})\end{gathered} \begin{gathered}\text { Water level } \\ \text { elevation } \\ \text { above ms }(\mathrm{ft})\end{gathered}$

LLWMA-2 Wells Monitoring the Top of the Unconfined Aquifer

$\begin{array}{llll}299-E 35-1 & 12 / 10 / 93 & 195.31 & 402.99\end{array}$

Notes: 1. Water level elevations are calculated by subtracting the measured depth to water from the surveyed elevation for the well.

2. Depth-to-water values are transcribed from field records.

3. Elevations marked with an ' $*$ ' were measured at the time of sampling.

4. Elevations marked with a ' + ' are outside of the expected range and are suspected of error.

5. To convert feet to meters multipiy by 0.3048 . 
Table 15-9. RCRA Water Level Measurement Report for the LLWMA-3, Fourth Quarter 1993. (sheet 1 of 3)

\begin{tabular}{|c|c|c|c|}
\hline Well & Date & $\begin{array}{l}\text { Depth to } \\
\text { water (ft) }\end{array}$ & $\begin{array}{l}\text { Water level } \\
\text { elevation } \\
\text { above msl (ft) }\end{array}$ \\
\hline \multicolumn{4}{|c|}{ LLWMA-3 WE } \\
\hline 299-W10-13 & $\begin{array}{l}10 / 14 / 93 \\
11 / 11 / 93 \\
12 / 16 / 93\end{array}$ & $\begin{array}{l}237.05 \\
236.95 \\
237.62\end{array}$ & $\begin{array}{l}461.99 \\
462.09 \\
461.42\end{array}$ \\
\hline $299-W 10-19$ & $\begin{array}{l}10 / 15 / 93 \\
11 / 11 / 93 \\
12 / 16 / 93\end{array}$ & $\begin{array}{l}222.56 \\
222.50 \\
222.91\end{array}$ & $\begin{array}{l}460.43 \\
460.49 \\
460.08\end{array}$ \\
\hline $299-W 6-2$ & $\begin{array}{l}10 / 14 / 93 \\
11 / 11 / 93 \\
12 / 16 / 93\end{array}$ & $\begin{array}{l}234.98 \\
234.91 \\
235.39\end{array}$ & $\begin{array}{l}457.47 \\
457.54 \\
457.06\end{array}$ \\
\hline 299-W7-1 & $\begin{array}{l}10 / 14 / 93 \\
11 / 11 / 93 \\
12 / 16 / 93\end{array}$ & $\begin{array}{l}232.53 \\
232.47 \\
233.00\end{array}$ & $\begin{array}{l}458.18 \\
458.24 \\
457.71\end{array}$ \\
\hline 299-W7-10 & $\begin{array}{l}10 / 14 / 93 \\
11 / 11 / 93 \\
12 / 16 / 93\end{array}$ & $\begin{array}{l}233.84 \\
232.80 \\
233.28\end{array}$ & $\begin{array}{l}455.82+ \\
456.86 \\
456.38\end{array}$ \\
\hline 299-W7-11 & $\begin{array}{l}10 / 14 / 93 \\
11 / 11 / 93 \\
12 / 16 / 93\end{array}$ & $\begin{array}{l}223.61 \\
223.59 \\
224.01\end{array}$ & $\begin{array}{l}457.84 \\
457.86 \\
457.44\end{array}$ \\
\hline 299-W7-12 & $\begin{array}{l}10 / 14 / 93 \\
11 / 11 / 93 \\
12 / 16 / 93\end{array}$ & $\begin{array}{l}229.78 \\
229.77 \\
230.18\end{array}$ & $\begin{array}{l}458.15 \\
458.16 \\
457.75\end{array}$ \\
\hline 299-W7-2 & $\begin{array}{l}10 / 14 / 93 \\
11 / 11 / 93 \\
12 / 16 / 93\end{array}$ & $\begin{array}{l}218.73 \\
218.50 \\
218.94\end{array}$ & $\begin{array}{l}456.86 \\
457.09 \\
456.65\end{array}$ \\
\hline 299-W7-4 & $\begin{array}{l}10 / 15 / 93 \\
11 / 11 / 93 \\
12 / 16 / 93\end{array}$ & $\begin{array}{l}212.88 \\
212.85 \\
213.21\end{array}$ & $\begin{array}{l}458.81 \\
458.84 \\
458.48\end{array}$ \\
\hline $299-W 7-5$ & $\begin{array}{l}10 / 14 / 93 \\
11 / 11 / 93 \\
12 / 16 / 93\end{array}$ & $\begin{array}{l}216.47 \\
216.44 \\
216.87\end{array}$ & $\begin{array}{l}456.58 \\
456.61 \\
456.18\end{array}$ \\
\hline
\end{tabular}


Table 15-9. RCRA Water Level Measurement Report for the LLWMA-3, Fourth Quarter 1993. (sheet 2 of 3)

Well Date $\quad \begin{gathered}\text { Depth to } \\ \text { water }(\mathrm{ft})\end{gathered} \begin{gathered}\text { Water level } \\ \text { elevation } \\ \text { above ms }(\mathrm{ft})\end{gathered}$

LLWMA-3 Wells Monitoring the Top of the Unconfined Aquifer

\begin{tabular}{llll}
\hline 299-W7-6 & $10 / 14 / 93$ & 222.21 & 456.43 \\
& $11 / 11 / 93$ & 222.15 & 456.49 \\
& $12 / 16 / 93$ & 222.59 & 456.05 \\
$299-W 7-7$ & $10 / 14 / 93$ & 218.33 & 456.61 \\
& $11 / 11 / 93$ & 218.32 & 456.62 \\
& $12 / 16 / 93$ & 218.69 & 456.25 \\
$299-W 7-8$ & $10 / 14 / 93$ & 232.13 & 455.22 \\
& $11 / 11 / 93$ & 231.64 & 455.71 \\
& $12 / 16 / 93$ & 232.48 & 454.87 \\
$299-W 7-9$ & $10 / 14 / 93$ & 233.48 & 458.61 \\
& $11 / 11 / 93$ & 233.43 & 458.66 \\
& $12 / 16 / 93$ & 233.94 & 458.15 \\
$299-W 8-1$ & $10 / 14 / 93$ & 242.63 & 458.70 \\
& $11 / 11 / 93$ & 242.55 & 458.78 \\
& $12 / 16 / 93$ & 243.19 & 458.14 \\
$299-W 9-1$ & $10 / 14 / 93$ & 276.66 & 461.07 \\
& $11 / 11 / 93$ & 276.62 & 461.11 \\
& $12 / 16 / 93$ & 277.23 & 460.50 \\
\hline
\end{tabular}

LLWMA-3 Wells Monitoring the Bottom of the Unconfined Aquifer

\begin{tabular}{llll}
\hline $299-W 10-14$ & $10 / 14 / 93$ & 237.84 & 461.59 \\
& $11 / 11 / 93$ & 237.74 & 461.69 \\
& $12 / 16 / 93$ & 238.37 & 461.06 \\
$299-W 7-3$ & $10 / 14 / 93$ & 220.15 & 455.99 \\
& $11 / 11 / 93$ & 220.10 & 456.04 \\
& $12 / 16 / 93$ & 220.54 & 455.60
\end{tabular}


Table 15-9. RCRA Water Level Measurement Report for the LLWMA-3, Fourth Quarter 1993. (sheet 3 of 3)

LLWMA-3 Wells Monitoring the Top of the Unconfined Aquifer

$\begin{array}{llll}299-W 10-21 & 10 / 15 / 93 & 276.93 & 397.13+ \\ & 11 / 11 / 93 & 216.79 & 457.27\end{array}$

Notes: 1. Water level elevations are calculated by subtracting the measured depth to water from the surveyed elevation for the well.

2. Depth-to-water values are transcribed from field records.

3. Elevations marked with a ' + ' are outside of the expected range and are suspected of error.

4. To convert feet to meters multipiy by 0.3048 . 
Table 15-10. RCRA Water Level Measurement Report for the LLWMA-4, Fourth Quarter 1993. (sheet 1 of 2)

\begin{tabular}{cccc}
\hline We11 & Date & $\begin{array}{c}\text { Depth to } \\
\text { water (ft) }\end{array}$ & $\begin{array}{c}\text { Water level } \\
\text { elevation } \\
\text { above ms }\end{array}$ \\
\hline LLWMA-4 Wells
\end{tabular}


Table 15-10. RCRA Water Level Measurement Report for the LLWMA-4, Fourth Quarter 1993. (sheet 2 of 2)

Well Date $\begin{gathered}\text { Depth to } \\ \text { water }(\mathrm{ft})\end{gathered} \begin{gathered}\text { Water level } \\ \text { elevation } \\ \text { above msl (ft) }\end{gathered}$

LLWMA-4 Wells Monitoring the Bottom of the Unconfined Aquifer

\begin{tabular}{llll}
\hline $299-W 15-17$ & $11 / 18 / 93$ & 221.20 & $463.44 \star$ \\
& $12 / 10 / 93$ & 220.97 & 463.67 \\
$299-W 18-22$ & $12 / 10 / 93$ & 205.58 & 462.91 \\
\hline
\end{tabular}

LLWMA-4 Wells Monitoring the Perched Zone

\begin{tabular}{llll}
\hline $299-W 18-29$ & $12 / 10 / 93$ & 126.62 & 547.52 \\
\hline
\end{tabular}

Notes: 1. Water level elevations are calculated by subtracting the measured depth to water from the surveyed elevation for the well.

2. Depth-to-water values are transcribed from field records.

3. Elevations marked with an ' $*$ ' were measured at the time of sampling.

4. Elevations marked with a ' + ' are outside of the expected range and are suspected of error.

5. To convert feet to meters multipiy by 0.3048 . 
Table 15-11. RCRA Water Level Measurement Report for the LLWMA-5, Fourth Quarter 1993.

Well Date $\begin{gathered}\text { Depth to } \\ \text { water }(\mathrm{ft})\end{gathered} \begin{gathered}\text { Water level } \\ \text { elevation } \\ \text { above msl }(\mathrm{ft})\end{gathered}$

LLWMA-5 Wells Monitoring the Top of the Unconfined Aquifer

\begin{tabular}{llll}
\hline $299-W 6-9$ & $12 / 10 / 93$ & 238.59 & 457.51 \\
\hline
\end{tabular}

LLWMA-5 Wells Monitoring the Top of the Unconfined Aquifer

\begin{tabular}{llll}
\hline $299-W 6-10$ & $12 / 10 / 93$ & 254.95 & 457.53 \\
$299-W 6-11$ & $12 / 10 / 93$ & 248.03 & 454.83 \\
$299-W 6-12$ & $12 / 10 / 93$ & 236.60 & 455.91 \\
$299-W 6-2$ & $10 / 14 / 93$ & 234.98 & 457.47 \\
& $11 / 11 / 93$ & 234.91 & 457.54 \\
& $12 / 16 / 93$ & 235.39 & 457.06 \\
$299-W 6-4$ & $12 / 10 / 93$ & 243.73 & 457.52 \\
$299-W 6-7$ & $12 / 10 / 93$ & 256.27 & 454.01 \\
$299-W 7-10$ & $10 / 14 / 93$ & 233.84 & 455.82 \\
& $11 / 11 / 93$ & 232.80 & 456.86 \\
& $12 / 16 / 93$ & 233.28 & 456.38 \\
\hline
\end{tabular}

LLWMA-5 Wells Monitoring the Bottom of the Unconfined Aquifer

\begin{tabular}{llll}
\hline $299-W 6-3$ & $12 / 10 / 93$ & 242.91 & 456.92 \\
$299-W 6-6$ & $12 / 10 / 93$ & 256.03 & 453.97 \\
\hline
\end{tabular}

Notes: 1. Water level elevations are calculated by subtracting the measured depth to water from the surveyed elevation for the well.

2. Depth-to-water values are transcribed from field records.

3. To convert feet to meters multiply by 0.3048 . 
Table 15-12. Constituent List and Summary of Results for the Low-Level Waste Burial Grounds WMA 1 Data for Reporting Period October 1 through

December 31, 1993. (sheet 1 of 3)

CONTAMINATION INDICATOR PARAMETERS

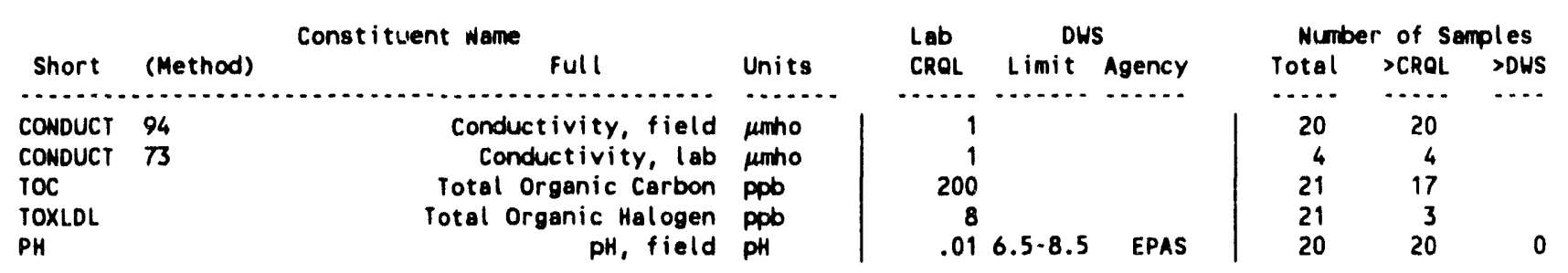

DRINKING WATER PARAMETERS

\begin{tabular}{|c|c|c|c|c|c|c|c|c|c|}
\hline \multirow[b]{2}{*}{ Short } & \multirow[b]{2}{*}{ (Method) } & \multirow[t]{2}{*}{ Constituent Name } & \multirow[b]{2}{*}{ Units } & \multirow{2}{*}{$\begin{array}{l}\text { Lab } \\
\text { CROL }\end{array}$} & \multicolumn{2}{|c|}{ DWS } & \multicolumn{3}{|c|}{ Number of Samples } \\
\hline & & & & & Limit & Agency & Total & $>$ CROL & $>$ DWS \\
\hline$\cdots$ & $\cdots$ & 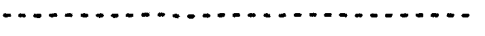 & $\cdots$ & $\cdots \ldots$ & 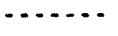 & $\cdots \ldots$ & ..... & $\cdots$ & $\cdots$ \\
\hline $2,4,5 T P$ & & $2,4,5-T P$ & ppb & .408 & 50 & EPA & 18 & 0 & 0 \\
\hline $2,4-0$ & & $2,4-0$ & ppb & .186 & 100 & EPAI & 18 & 0 & 0 \\
\hline ARSENIC & & Arsenic & ppob & 1.38 & 50 & EPA & 18 & 18 & 0 \\
\hline FARSENIC & & Arsenic, filtered & ppo & 1.38 & 50 & EPA & 18 & 18 & 0 \\
\hline BARIUM & & Barium & ppb & .001 & 1000 & EPAI & 18 & 18 & 0 \\
\hline FBARIUM & & Barium, filtered & ppb & .001 & 1000 & EPAI & 18 & 18 & 0 \\
\hline CADMIUM & & Cadmium & ppb & 4.7 & 10 & EPAI & 18 & 0 & 0 \\
\hline FCADMIU & & Cachnium, filtered & ppb & 4.7 & 10 & EPAI & 18 & 0 & 0 \\
\hline CHROMUM & & Chromium & ppb & 5.42 & 50 & EPAI & 18 & 18 & 17 \\
\hline FCHROM! & & Chromium, filtered & ppb & 5.42 & 50 & EPAI & 18 & 5 & 0 \\
\hline ENDRIN & & Endrin & ppb & .008 & .2 & EPA & 18 & 0 & 0 \\
\hline FLUORID & & Fluoride & ppb & 13.9 & 1400 & EPAI & 19 & 19 & 0 \\
\hline ALPHA & & Gross alpha & $\mathrm{pCi} / \mathrm{L}$ & & 15 & EPA & 18 & 16 & 0 \\
\hline MERCURY & & Mercury & ppb & .158 & 2 & EPA & 18 & 0 & 0 \\
\hline FMERCUR & & Mercury, filtered & ppb & .158 & 2 & EPA & 18 & 0 & 0 \\
\hline METHLOR & & Methoxychlor & ppb & .1 & 100 & EPAI & 18 & 0 & 0 \\
\hline NITRATE & & Nitrate & ppb & 44.4 & 45000 & EPA & 19 & 19 & 0 \\
\hline RADIUM & & Radiun & $\mathrm{PCi} / \mathrm{L}$ & & 5 & EPAI & 18 & 3 & 0 \\
\hline SELENUM & & Selenium & pob & 1.21 & 10 & EPAI & 18 & 13 & 0 \\
\hline FSELENI & & Selenium, filtered & ppb & 1.21 & 10 & EPAI & 18 & 14 & 0 \\
\hline TOXAENE & & Toxaphene & ppb & .89 & 5 & EPAI & 18 & 0 & 0 \\
\hline g-BHC & & gamma-BHC (Lindane) & ppb & .0021 & 4 & EPAI & 18 & 1 & 0 \\
\hline
\end{tabular}

GROUNDWATER QUALITY PARAMETERS

\begin{tabular}{|c|c|c|c|c|c|c|c|c|c|}
\hline \multirow[b]{2}{*}{ Short } & \multirow[b]{2}{*}{ (Method) } & \multirow{2}{*}{$\begin{array}{l}\text { Constituent Name full } \\
\text { fore }\end{array}$} & \multirow[b]{2}{*}{ Units } & \multirow{2}{*}{$\begin{array}{l}\text { Lob } \\
\text { CROL }\end{array}$} & \multicolumn{2}{|c|}{ DWS } & \multicolumn{3}{|c|}{ Number of Semples } \\
\hline & & & & & Limit & Agency & Total & $>C R Q L$ & $>$ OWS \\
\hline CHLORID & & chloride & mb & 825 & 250000 & EPAS & 10 & 10 & 0 \\
\hline IRON & & Iron & ppob & 10.3 & 300 & EPAS & $\begin{array}{l}18 \\
18\end{array}$ & $\begin{array}{l}19 \\
18\end{array}$ & 17 \\
\hline FIRON & & Iron, filtered & ppb & 10.3 & 300 & EPAS & 18 & 18 & 0 \\
\hline MANGESE & & Manganese & ppb & 1.35 & 50 & EPAS & 18 & 18 & 0 \\
\hline FMANGAN & & Manganese, filtered & ppb & 1.35 & 50 & EPAS & 18 & 16 & 0 \\
\hline LPHENOL & & Phenol & ppb & .31 & & & 18 & 1 & \\
\hline SOOIUM & & Sodium & ppb & 40.9 & & & 18 & 18 & \\
\hline FSODIUM & & Sodium, filtered & ppb & 40.9 & & & 18 & 18 & \\
\hline SULFATE & & Sulfate & ppb & 49.9 & 250000 & EPAS & 19 & 19 & 0 \\
\hline
\end{tabular}


Table 15-12. Constituent List and Summary of Results for the Low-Level Waste Burial Grounds WMA 1 Data for Reporting Period 0ctober 1 through

December 31,1993 . (sheet 2 of 3 )

SITE SPECIFIC ANO OTHER CONSTITUENTS

\begin{tabular}{|c|c|c|c|c|c|c|c|c|c|}
\hline \multirow[b]{2}{*}{ Short } & \multirow[b]{2}{*}{ (Method) } & \multirow{2}{*}{ Const ituent Mame } & \multirow[b]{2}{*}{ Units } & \multirow{2}{*}{$\begin{array}{l}\text { Lab } \\
\text { CRQL }\end{array}$} & \multicolumn{2}{|l|}{ DUS } & \multicolumn{3}{|c|}{ Number of Samples } \\
\hline & & & & & Limit & Agency & Total & $>$ CRQL & $>$ DWS \\
\hline $2,4,5-T$ & & $2,4,5-T$ & ppo & .384 & 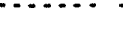 & $\cdots$ & 18 & $\begin{array}{c}\cdots \\
0\end{array}$ & $\cdots$ \\
\hline $246-t r p$ & & 2,4,6-Trichlorophenol & ppb & 9.45 & & & 18 & 0 & \\
\hline 24 -dchp & & 2,4-Dichlorophenol & ppob & 1.5 & & & 18 & 0 & \\
\hline DIMPHEN & & 2,4-D imethylphenol & ppob & 1.01 & & & 18 & 0 & \\
\hline DINPHEN & & 2,4-D ini trophenol & ppo & .96 & & & 18 & 0 & \\
\hline 26-dchp & & 2,6-Dichlorophenol & ppb & 1.59 & & & 18 & 0 & \\
\hline CHLPHEN & & 2-Chlorophenol & ppb & 1.42 & & & 18 & 1 & \\
\hline 2NITPH & & 2-Witrophenol & ppb & 1.56 & & & 18 & 0 & \\
\hline BUTDINP & 30 & 2-sec-8utyl-4,6-dini trophenol CON & ppb & 1.35 & & & 18 & 0 & \\
\hline BUTO INP & 49 & 2-sec-Butyl-4,6-dini trophenol (DN & ppob & .269 & & & 18 & 0 & \\
\hline & & $4,41-000$ & ppb & .0005 & & & 18 & 0 & \\
\hline DDE & & $4,41-D D E$ & ppob & .0009 & & & 18 & 1 & \\
\hline DDT & & $4,4^{\prime}-00 T$ & ppb & .011 & & & 18 & 0 & \\
\hline 460N2MP & & 4,6-0 initro-2-methylphenol & ppb & 1.18 & & & 18 & 0 & \\
\hline CHLCRES & & 4-Chloro-3-methylphenol & ppb & 1.12 & & & 18 & 1 & \\
\hline NITPHEN & & 4-Nitrophenol & pppb & .65 & & & 18 & 0 & \\
\hline ALDRIN & & Aldrin & ppb & .05 & & & 18 & 0 & \\
\hline Q-BHC & & Alpha-BHC & ppb & .012 & & & 18 & 0 & \\
\hline ALUMNUM & & Aluminum & ppob & 32.5 & & & 18 & 13 & \\
\hline FALUMIN & & Aluminum & ppo & 32.5 & & & 18 & 3 & \\
\hline AMMONIU & & Amnonium ion & ppb & 38.5 & & & 18 & 17 & \\
\hline ANTIONY & & Ant imony & ppb & 69.4 & & & 18 & 0 & \\
\hline FANTIMO & & Ant imony, filtered & ppo & 69.4 & & & 18 & 0 & \\
\hline SB-125 & & Ant imony-125 & $\mathrm{pCi} / \mathrm{L}$ & & & & 1 & 0 & \\
\hline BERYLUM & & Beryllium & ppo & .814 & & & 18 & 1 & \\
\hline FBERYLL & & Beryllium, filtered & ppb & .814 & & & 18 & 1 & \\
\hline b-BHC & & Beta-BHC & ppb & .0026 & & & 18 & 0 & \\
\hline BROMIDE & & Bromide & ppb & 52.8 & & & 19 & 5 & \\
\hline CALCIUM & & Calcium & ppb & 20.9 & & & 18 & 18 & \\
\hline FCALCIU & & Calcium, filtered & ppb & 20.9 & & & 18 & 18 & \\
\hline Cs-137 & & Cesiun- 137 & $\mathrm{pCi} / \mathrm{L}$ & & 200 & EPA & 1 & 0 & 0 \\
\hline CHLOANE & & Chlordane & ppb & .0057 & 2 & EPA & 18 & 0 & 0 \\
\hline COBALT & & Cobalt & ppb & 4.05 & & & 18 & 0 & \\
\hline FCOBALT & & Cobelt, filtered & ppb & 4.05 & & & 18 & 1 & \\
\hline $\mathrm{CO}-60$ & & Cobalt -60 & $\mathrm{PCi} / \mathrm{L}$ & & 100 & EPA & 1 & 0 & 0 \\
\hline COLIFORM & & Coliforms & $\mathrm{COL}$ & 1 & 1 & EPAI & 18 & 0 & 0 \\
\hline COPPER & & Copper & ppob & 2.65 & 1000 & EPAS & 18 & 16 & 0 \\
\hline FCOPPER & & Copper, filtered & ppb & 2.65 & 1000 & EPAS & 18 & 11 & 0 \\
\hline CRESOLS & & Cresols (methylphenols) & ppob & 4.66 & & & 18 & 0 & \\
\hline d-BHC & & Del ta-BHC & ppo & .0014 & & & 18 & 0 & \\
\hline DIELRIN & & Dieldrin & ppo & .019 & & & 18 & 0 & \\
\hline ENDO1 & & Endosulfan 1 & ppb & .0029 & & & 18 & 0 & \\
\hline ENDO2 & & Endosulf fan 11 & ppb & .004 & & & 18 & 0 & \\
\hline ENDSFAN & & Endosulfan sulfate & ppb & .0072 & & & 18 & 0 & \\
\hline ENDRALD & & Endrin Aldehyde & ppb & .011 & & & 18 & 0 & \\
\hline BETA & & Gross beta & $\mathrm{pCi} / \mathrm{L}$ & & & & 18 & 18 & \\
\hline HEPTLOR & & Heptachlor & ppo & .0019 & .4 & EPA & 18 & 1 & 0 \\
\hline HEPTIDE & & Heptachlor epoxide & ppb & .0008 & .2 & EPA & 18 & 0 & 0 \\
\hline LHYORAZ & & Hydrazine & ppo & 1.89 & & & 1 & 0 & \\
\hline LEAD & & Lead & ppb & .508 & 50 & EPAI & 18 & 11 & 0 \\
\hline FLEAD & & Lesd, filtered & ppb & .508 & 50 & EPAI & 18 & 5 & 0 \\
\hline MAGNES & & Magnesium & ppo & 26 & & & 18 & 18 & \\
\hline FMAGNES & & Magnesium, filtered & ppb & 26 & & & 18 & 18 & \\
\hline NICKEL & & Nickel & ppob & 17.9 & & & 18 & 18 & \\
\hline
\end{tabular}


Table 15-12. Constituent List and Summary of Results for the Low-Level Waste Burial Grounds WMA 1 Data for Reporting Period October 1 through

December 31,1993 . (sheet 3 of 3)

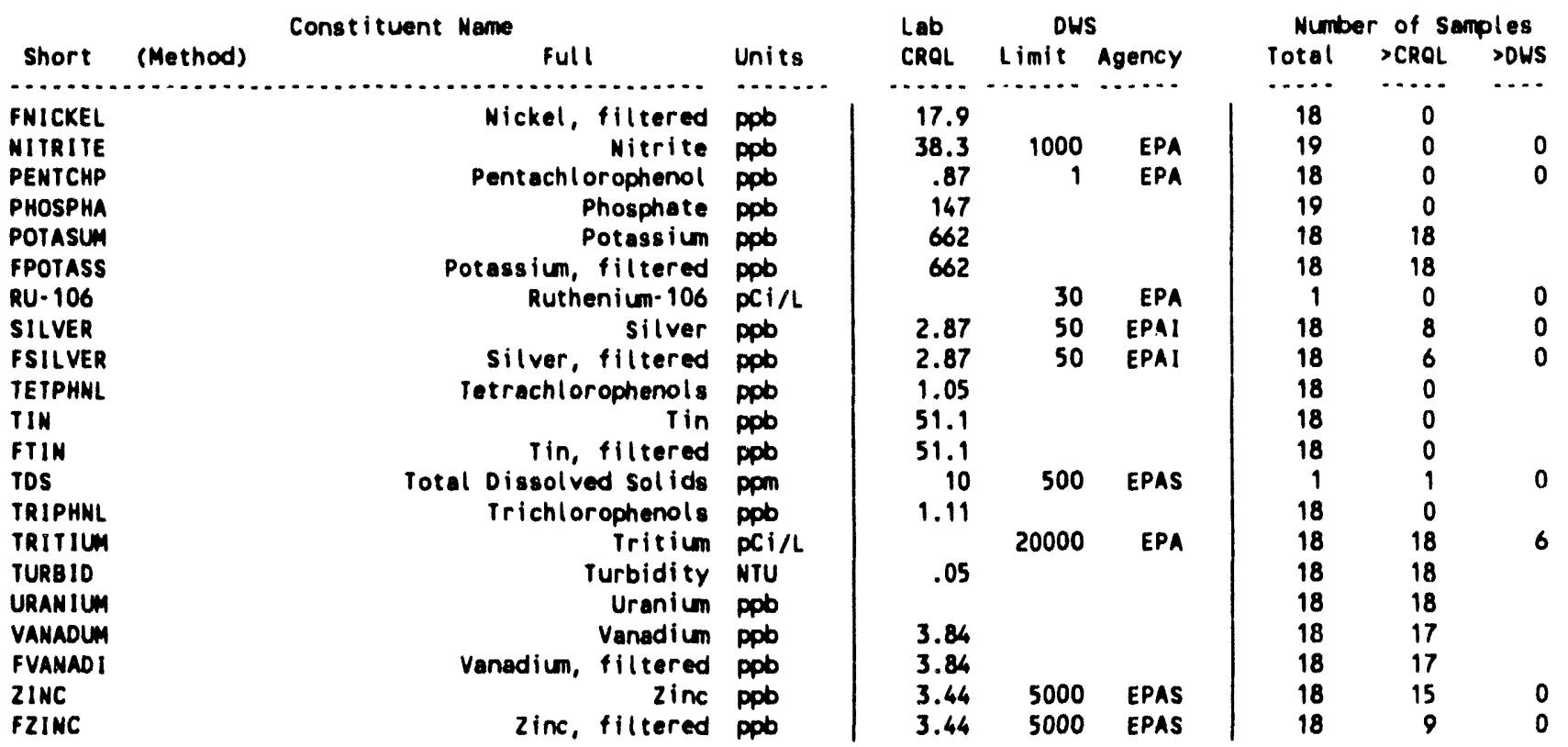

for explanation of this table, see section 1.4 of report. 
Table 15-13. Constituents with at Least One Detected Value for the Low-Level Waste Burial Grounds WMA 1 Data for Reporting Period October 1 through December 31, 1993. (sheet 1 of 11)

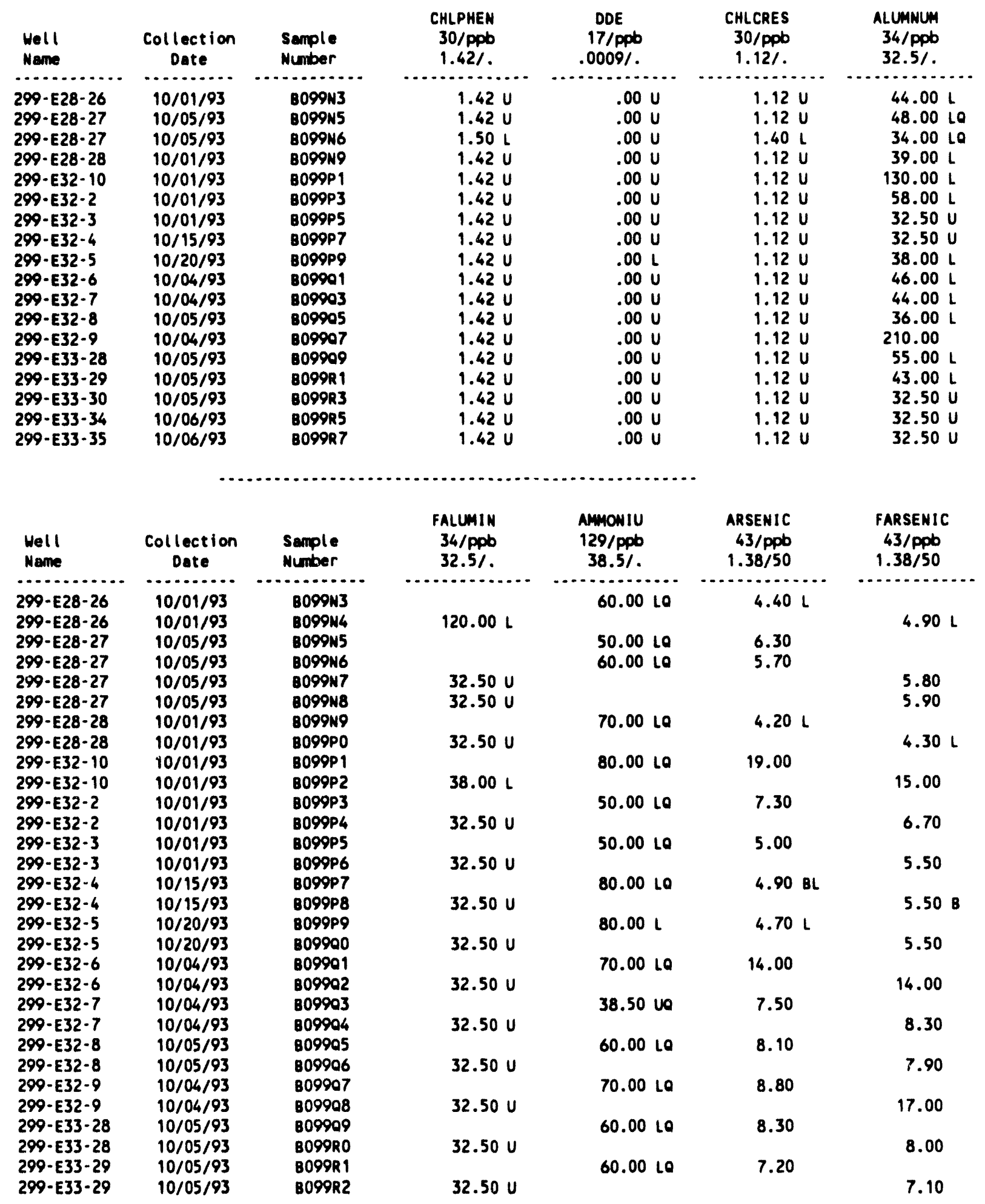


Table 15-13. Constituents with at Least One Detected Value for the Low-Level Waste Burial Grounds WMA 1 Data for Reporting Period October 1

through December 31, 1993. (sheet 2 of 11)

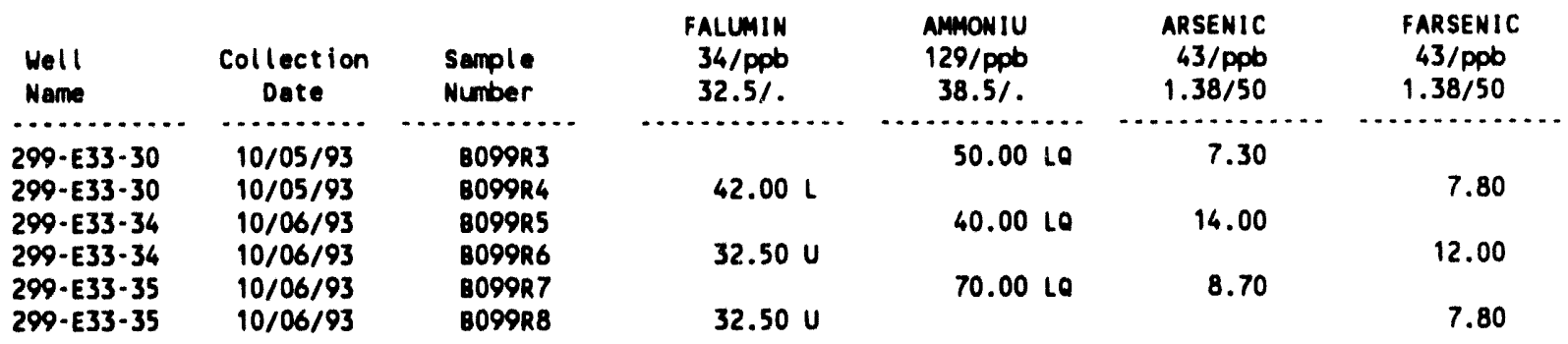

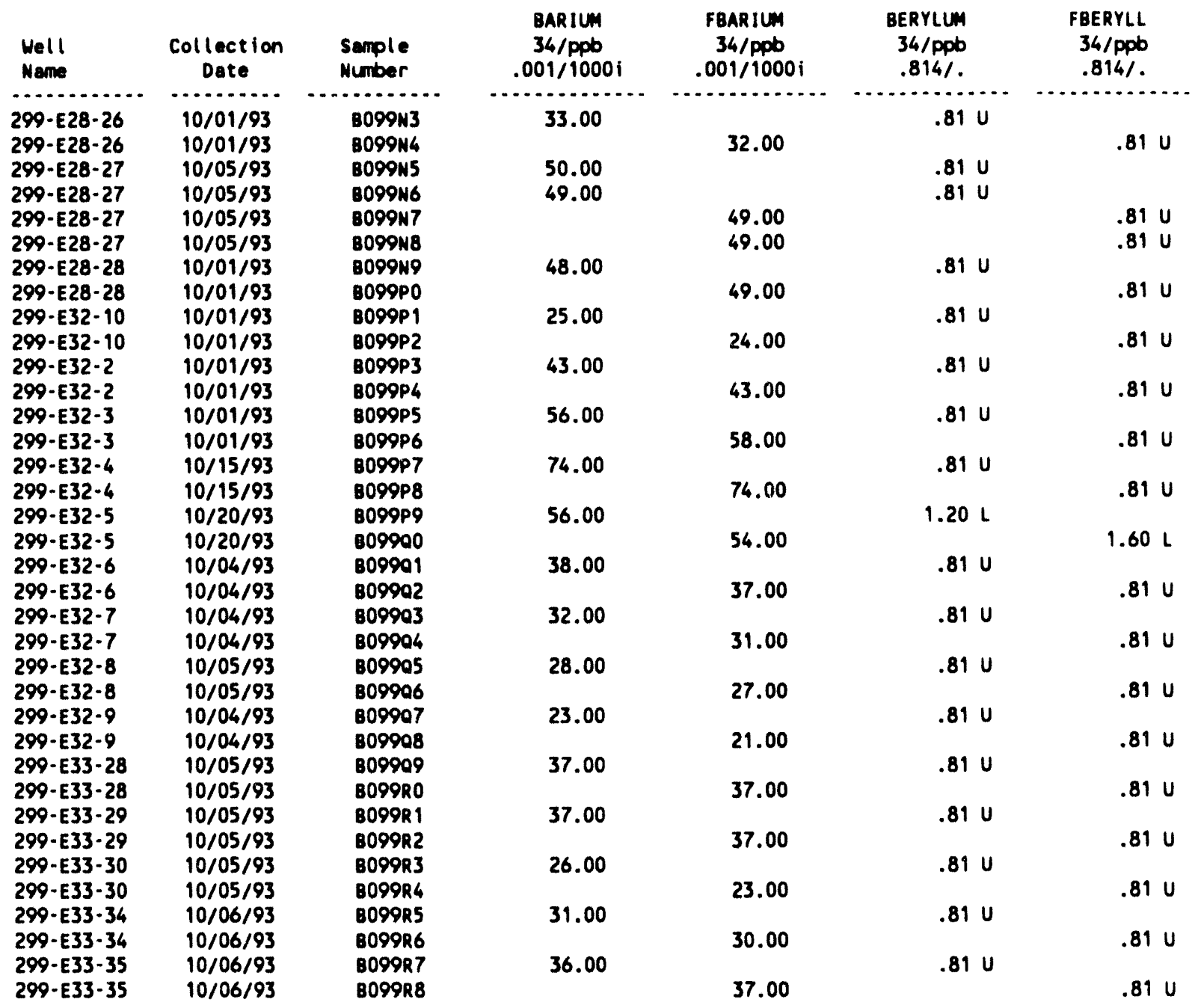


Table 15-13. Constituents with at Least One Detected Value for the Low-Level Waste Burial Grounds WMA 1 Data for Reporting Period October 1 through December 31, 1993. (sheet 3 of 11)

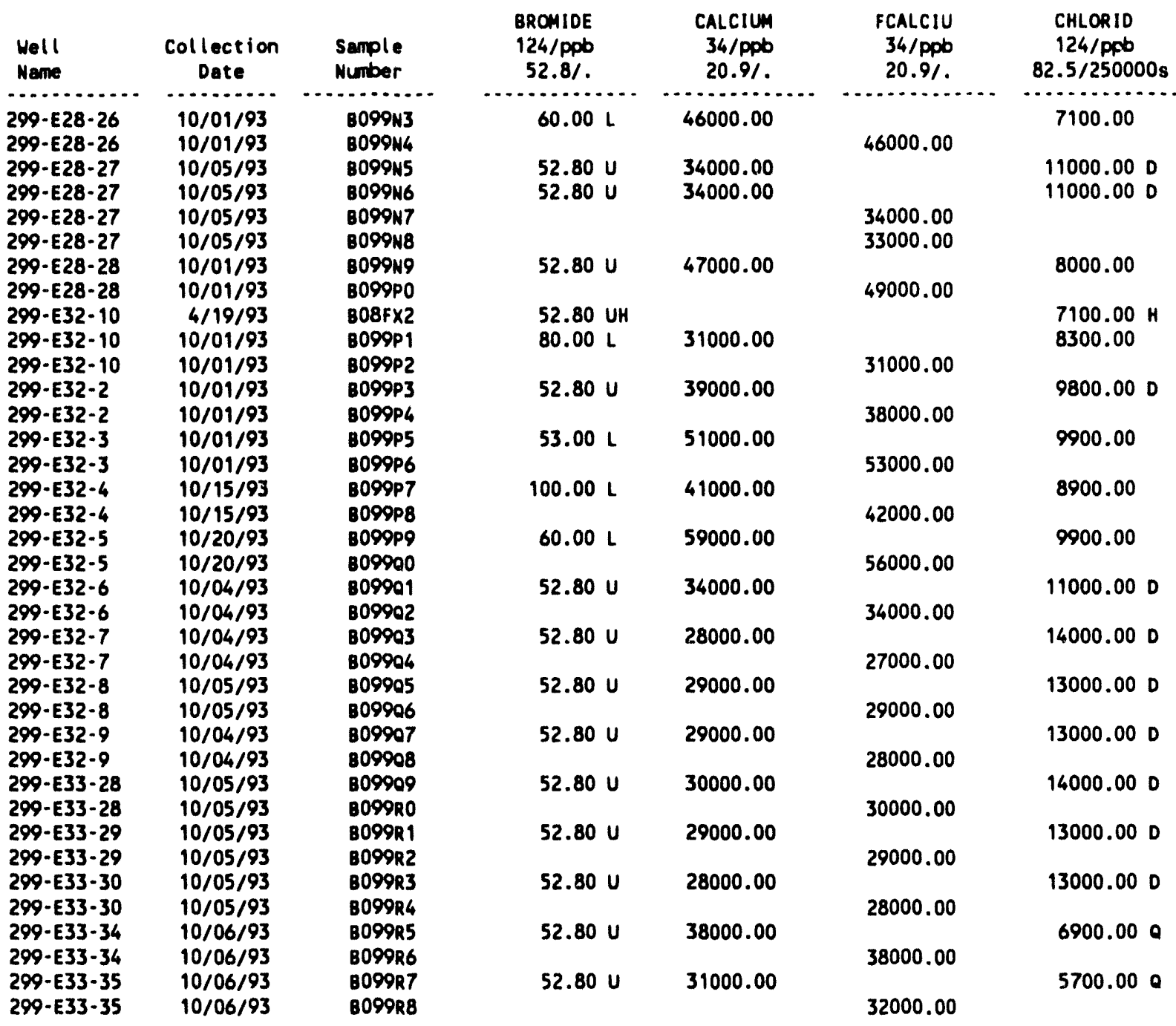

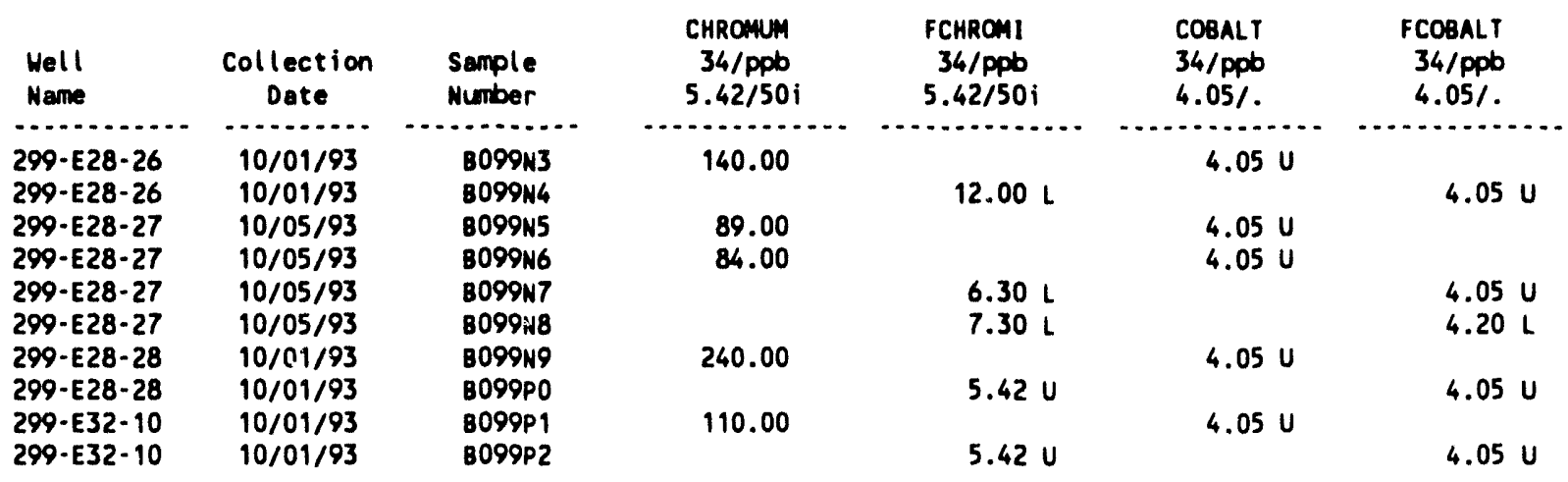


Table 15-13. Constituents with at Least One Detected Value for the Low-Level Waste Burial Grounds WMA 1 Data for Reporting Period October 1 through December 31, 1993. (sheet 4 of 11)

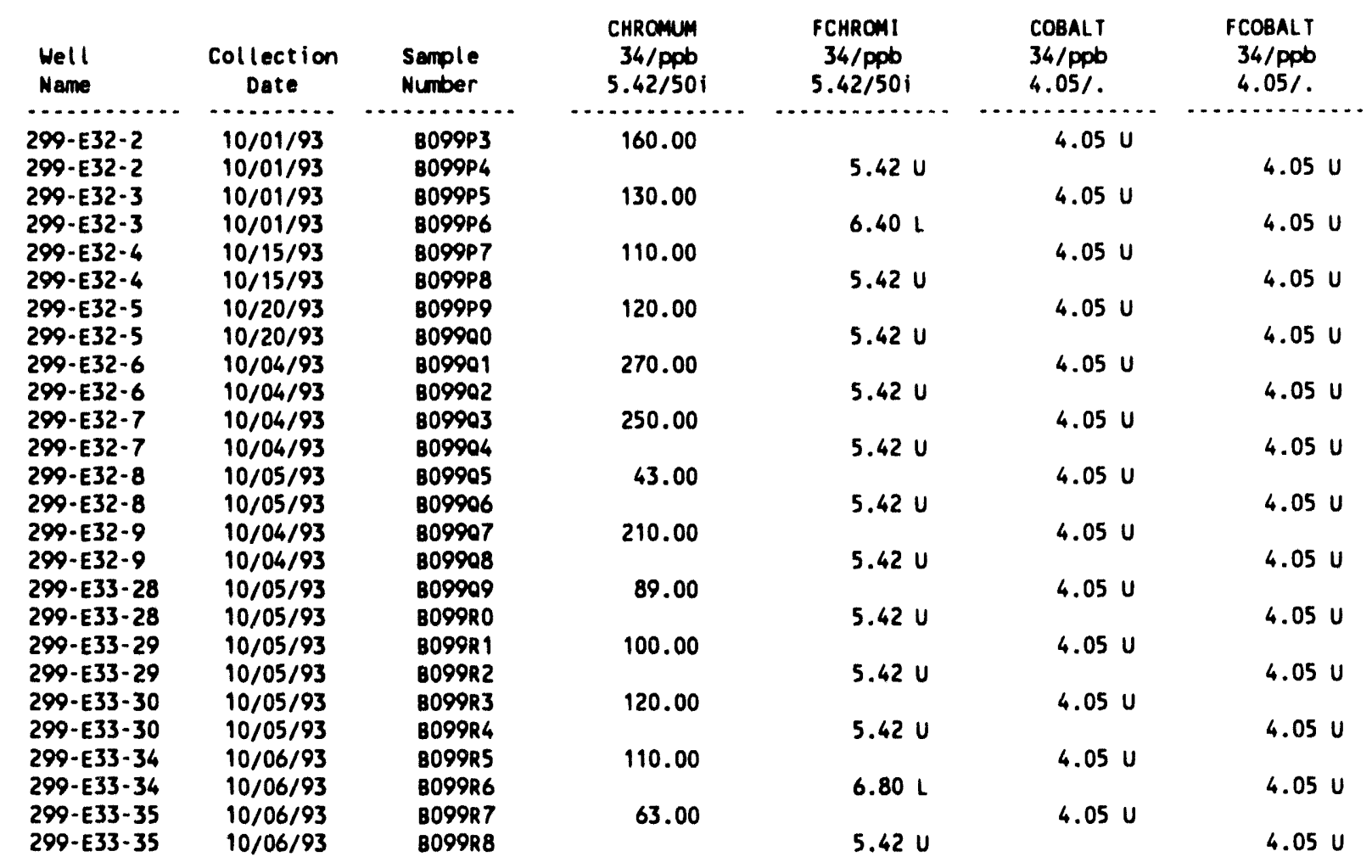

\begin{tabular}{|c|c|c|c|c|c|c|}
\hline $\begin{array}{l}\text { Well } \\
\text { Nane }\end{array}$ & $\begin{array}{c}\text { Collection } \\
\text { Date }\end{array}$ & $\begin{array}{l}\text { Sample } \\
\text { Number }\end{array}$ & $\begin{array}{c}\text { COPPER } \\
34 / \mathrm{ppb} \\
2.65 / 1000 \mathrm{~s}\end{array}$ & $\begin{array}{c}\text { FCOPPER } \\
34 / \mathrm{ppb} \\
2.65 / 1000 \mathrm{~s}\end{array}$ & $\begin{array}{l}\text { FLUORID } \\
124 / \mathrm{ppb} \\
13.9 / 1400 \mathrm{i}\end{array}$ & $\begin{array}{c}\text { ALPHA } \\
135 / \mathrm{PCi} / \mathrm{L} \\
. / 15\end{array}$ \\
\hline $299-E 28-26$ & $10 / 01 / 93$ & B099N3 & $9.70 \mathrm{~L}$ & 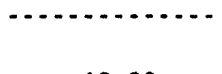 & 600.00 & 13.10 \\
\hline 299-E28-26 & $10 / 01 / 93$ & B099N4 & & $19.00 \mathrm{~L}$ & & \\
\hline $\begin{array}{l}299-E 28-27 \\
299-E 28-27\end{array}$ & & $\begin{array}{l}\text { B099N5 } \\
\text { B099N6 }\end{array}$ & $\begin{array}{l}3.20 \mathrm{LQ} \\
5.60 \mathrm{LQ}\end{array}$ & & $\begin{array}{l}600.00 \\
600.00\end{array}$ & $\begin{array}{l}8.510 \\
6.250\end{array}$ \\
\hline & & $\begin{array}{l}\text { B099N7 } \\
\text { BO99N8 }\end{array}$ & & $\begin{array}{l}2.65 \mathrm{U} \\
3.20 \mathrm{~L}\end{array}$ & & \\
\hline 299-E28-28 & $10 / 01 / 93$ & BO99N9 & $16.00 \mathrm{~L}$ & & 700.00 & 11.90 \\
\hline 299-E28-28 & $10 / 01 / 93$ & BO99PO & & $4.10 \mathrm{~L}$ & & \\
\hline $299-E 32-10$ & $4 / 19 / 93$ & B08FX2 & & & $300.00 \mathrm{H}$ & \\
\hline $\begin{array}{l}299-E 32-10 \\
299-E 32-10\end{array}$ & $\begin{array}{l}10 / 01 / 93 \\
10 / 01 / 93\end{array}$ & $\begin{array}{l}\text { B099P1 } \\
\text { B099P2 }\end{array}$ & $5.70 \mathrm{~L}$ & $2.65 \mathrm{U}$ & 700.00 & 1.81 \\
\hline $\begin{array}{l}299-E 32-2 \\
299-E 32-2\end{array}$ & $\begin{array}{l}10 / 01 / 93 \\
10 / 01 / 93\end{array}$ & $\begin{array}{l}8099 P 3 \\
8099 P 4\end{array}$ & $4.00 \mathrm{~L}$ & $2.65 \mathrm{U}$ & 700.00 & 8.46 \\
\hline $\begin{array}{l}299-E 32-3 \\
299-E 32 \cdot 3\end{array}$ & $\begin{array}{l}10 / 01 / 93 \\
10 / 01 / 93\end{array}$ & $\begin{array}{l}\text { B099P5 } \\
\text { B099P6 }\end{array}$ & $8.40 \mathrm{~L}$ & $3.20 \mathrm{~L}$ & 700.00 & 12.30 \\
\hline $\begin{array}{l}299-E 32-4 \\
299-E 32-4\end{array}$ & $\begin{array}{l}10 / 15 / 93 \\
10 / 15 / 93\end{array}$ & $\begin{array}{l}\text { B099P7 } \\
\text { B099P8 }\end{array}$ & $2.65 \mathrm{U}$ & $2.65 \mathrm{U}$ & 700.00 & 3.57 \\
\hline $\begin{array}{l}299-E 32-5 \\
299-E 32-5\end{array}$ & $\begin{array}{l}10 / 20 / 93 \\
10 / 20 / 93\end{array}$ & $\begin{array}{l}\text { B099P9 } \\
\text { B09900 }\end{array}$ & $9.90 \mathrm{~L}$ & $2.70 \mathrm{~L}$ & 1100.00 & 13.60 \\
\hline $\begin{array}{l}299-E 32-6 \\
299-E 32-6\end{array}$ & $\begin{array}{l}10 / 04 / 93 \\
10 / 04 / 93\end{array}$ & $\begin{array}{l}B 09901 \\
B 09902\end{array}$ & $15.00 \mathrm{~L}$ & $2.65 \mathrm{U}$ & 700.00 & 6.73 \\
\hline
\end{tabular}


Table 15-13. Constituents with at Least One Detected Value for the Low-Level Waste Burial Grounds WMA 1 Data for Reporting Period October 1 through December 31, 1993. (sheet 5 of 11)

\begin{tabular}{|c|c|c|c|c|c|c|}
\hline $\begin{array}{l}\text { Well } \\
\text { Name }\end{array}$ & $\begin{array}{c}\text { Collection } \\
\text { Date }\end{array}$ & $\begin{array}{l}\text { Sample } \\
\text { Number }\end{array}$ & $\begin{array}{c}\text { COPPER } \\
34 / \mathrm{ppb} \\
2.65 / 1000 \mathrm{~s}\end{array}$ & $\begin{array}{c}\text { FCOPPER } \\
34 / \text { PPb } \\
2.65 / 1000 \mathrm{~s}\end{array}$ & $\begin{array}{l}\text { FLUORIO } \\
124 / \mathrm{ppb} \\
13.9 / 1400 \mathrm{i}\end{array}$ & $\begin{array}{c}\text { ALPHA } \\
135 / \mathrm{pCi} / \mathrm{L} \\
. / 15\end{array}$ \\
\hline . & . & ……..... & (n........ & ...... & ........... & \\
\hline $\begin{array}{l}299-E 32-7 \\
299-E 32-7\end{array}$ & $\begin{array}{l}10 / 04 / 93 \\
10 / 04 / 93\end{array}$ & $\begin{array}{l}809903 \\
809904\end{array}$ & $12.00 \mathrm{~L}$ & $4.90 \mathrm{~L}$ & 600.00 & 4.05 \\
\hline $\begin{array}{l}299-E 32-8 \\
299-E 32-8\end{array}$ & $\begin{array}{l}10 / 05 / 93 \\
10 / 05 / 93\end{array}$ & $\begin{array}{l}809905 \\
809906\end{array}$ & $6.10 \mathrm{~L}$ & $2.80 \mathrm{~L}$ & 400.00 & 1.50 \\
\hline $\begin{array}{l}299-E 32-9 \\
299-E 32-9\end{array}$ & $\begin{array}{l}10 / 04 / 93 \\
10 / 04 / 93\end{array}$ & $\begin{array}{l}809907 \\
809908\end{array}$ & $12.00 \mathrm{~L}$ & $5.70 \mathrm{~L}$ & 500.00 & $.67 \mathrm{U}$ \\
\hline $\begin{array}{l}299-\text { E33-28 } \\
299-\text { E33-28 }\end{array}$ & $\begin{array}{l}10 / 05 / 93 \\
10 / 05 / 93\end{array}$ & $\begin{array}{l}\text { B09909 } \\
\text { B099RO }\end{array}$ & $2.80 \mathrm{~L}$ & & 400.00 & $.48 \mathrm{U}$ \\
\hline $\begin{array}{l}299-E 33-29 \\
299-E 33-29\end{array}$ & $\begin{array}{l}10 / 05 / 93 \\
10 / 05 / 93\end{array}$ & $\begin{array}{l}\text { BO99R1 } \\
\text { BO99R2 }\end{array}$ & $3.20 \mathrm{~L}$ & $2.65 \mathrm{U}$ & 500.00 & 1.54 \\
\hline $\begin{array}{l}299-\text { E33-30 } \\
299-\text { E33-30 }\end{array}$ & $\begin{array}{l}10 / 05 / 93 \\
10 / 05 / 93\end{array}$ & $\begin{array}{l}\text { B099R3 } \\
\text { B099R4 }\end{array}$ & $2.65 \mathrm{U}$ & $7.30 \mathrm{~L}$ & 600.00 & 2.43 \\
\hline $\begin{array}{l}299-E 33-34 \\
299-E 33-34\end{array}$ & $\begin{array}{l}10 / 06 / 93 \\
10 / 06 / 93\end{array}$ & $\begin{array}{l}\text { B099R5 } \\
\text { B099R6 }\end{array}$ & $8.10 \mathrm{~L}$ & $4.90 \mathrm{~L}$ & 500.00 & 5.30 \\
\hline $\begin{array}{l}299-\text { E33-35 } \\
299-\text { E33-35 }\end{array}$ & $\begin{array}{l}10 / 06 / 93 \\
10 / 06 / 93\end{array}$ & $\begin{array}{l}\text { B099R7 } \\
\text { B099R8 }\end{array}$ & $8.10 \mathrm{~L}$ & $2.80 \mathrm{~L}$ & 600.00 & 1.86 \\
\hline
\end{tabular}

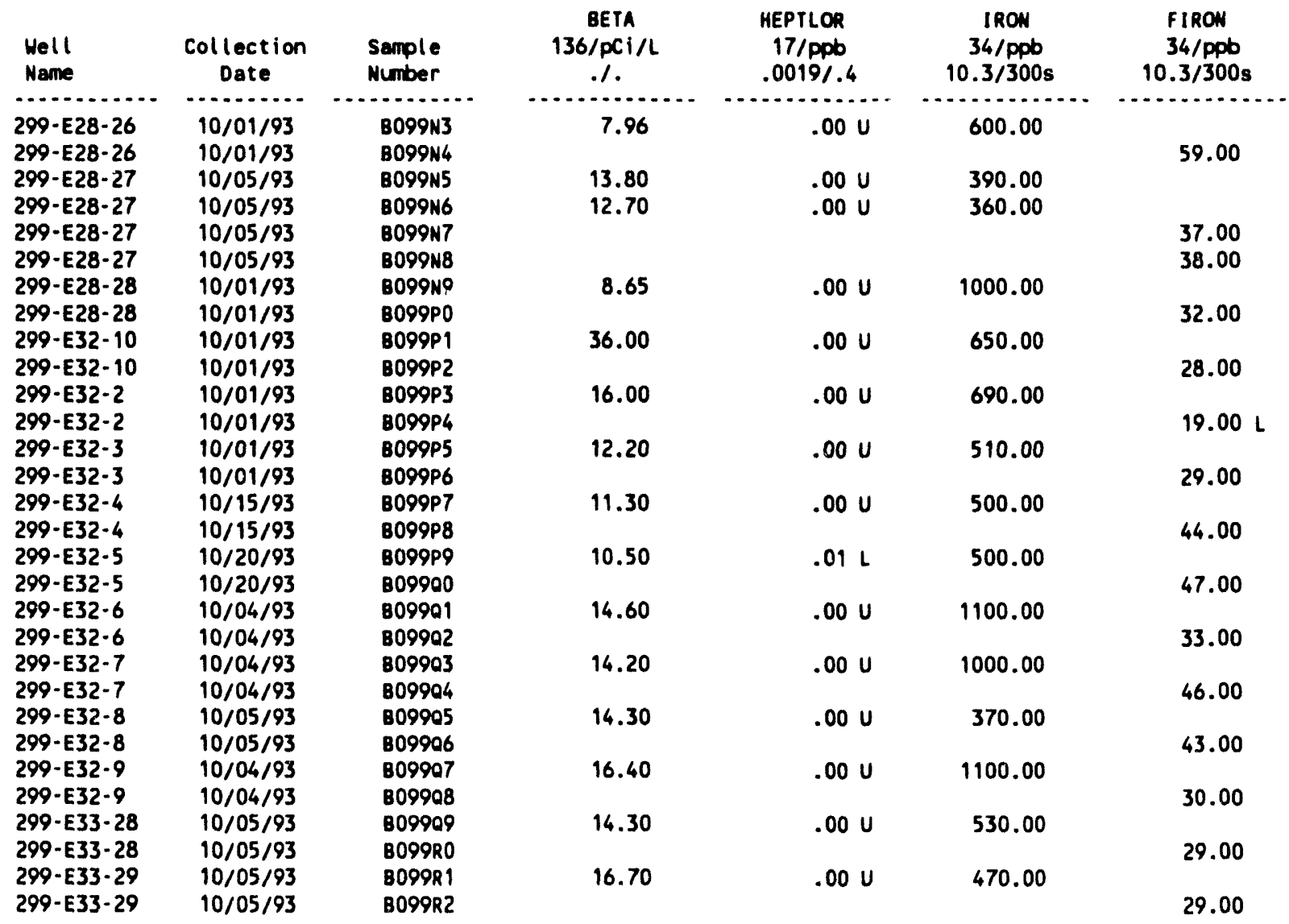


Table 15-13. Constituents with at Least One Detected Vaiue for the Low-Level Waste Burial Grounds WMA 1 Data for Reporting Period October 1 through December 31, 1993. (sheet 6 of 11)

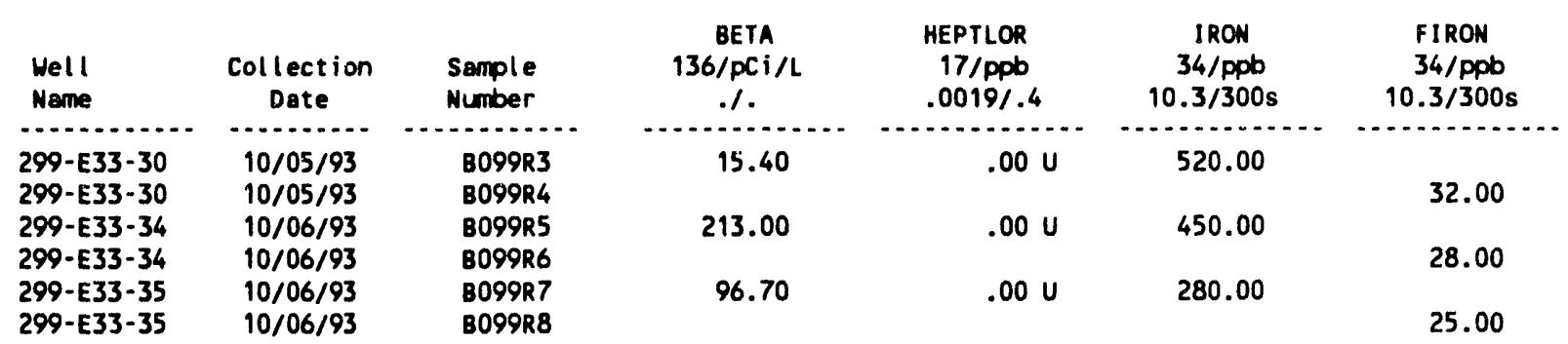

\begin{tabular}{|c|c|c|c|c|c|c|}
\hline $\begin{array}{l}\text { Well } \\
\text { Name }\end{array}$ & $\begin{array}{l}\text { Collection } \\
\text { Date }\end{array}$ & $\begin{array}{l}\text { Sample } \\
\text { Number }\end{array}$ & $\begin{array}{l}\text { LEAD } \\
40 / \text { ppb } \\
.508 / 50 i\end{array}$ & $\begin{array}{l}\text { MAGNES } \\
34 / \text { ppb } \\
26 / .\end{array}$ & $\begin{array}{c}\text { FMAGNES } \\
34 / \mathrm{ppb} \\
26 /\end{array}$ & $\begin{array}{c}\text { MANGESE } \\
34 / \mathrm{pPO} \\
1.35 / 50 \mathrm{~s}\end{array}$ \\
\hline (1) & (n) & (1) & $-\cdots$ & מרחמר & $\cdots$ & \\
\hline $\begin{array}{l}299-E 28-26 \\
299-E 28-26\end{array}$ & $\begin{array}{l}10 / 01 / 93 \\
10 / 01 / 93\end{array}$ & $\begin{array}{l}\text { B099N3 } \\
\text { B099N4 }\end{array}$ & $.66 \mathrm{BL}$ & 12000.00 & 12000.00 & 12.00 \\
\hline $\begin{array}{l}299-E 28-27 \\
299-E 28-27\end{array}$ & $\begin{array}{l}10 / 05 / 93 \\
10 / 05 / 93\end{array}$ & $\begin{array}{l}\text { B099N5 } \\
\text { B099N6 }\end{array}$ & $\begin{array}{l}.51 \text { UO } \\
1.30 \text { BLO }\end{array}$ & $\begin{array}{l}10000.00 \\
10000.00\end{array}$ & & $\begin{array}{l}8.30 \mathrm{~L} \\
7.40 \mathrm{~L}\end{array}$ \\
\hline 299-E28-27 & $10 / 05 / 93$ & B099N7 & & & 10000.00 & \\
\hline 299-E28-27 & $10 / 05 / 93$ & B099N8 & & & 10000.00 & \\
\hline 299-E28-28 & $10 / 01 / 93$ & B099N9 & $.67 \mathrm{BL}$ & 12000.00 & & 23.00 \\
\hline 299-E28-28 & $10 / 01 / 93$ & B099PO & & & 13000.00 & \\
\hline $299-E 32-10$ & $10 / 01 / 93$ & 8099P1 & $2.40 \mathrm{BL}$ & 9200.00 & & 14.00 \\
\hline 299-E32-10 & $10 / 01 / 93$ & B099P2 & & & 9200.00 & \\
\hline 299-E32-2 & $10 / 01 / 93$ & B099P3 & $.63 \mathrm{BL}$ & 12000.00 & & 14.00 \\
\hline 299-E32-2 & $10 / 01 / 93$ & B099P4 & & & 11000.00 & \\
\hline $299-E 32-3$ & $10 / 01 / 93$ & B099P5 & $.51 \mathrm{U}$ & 14000.00 & & 12.00 \\
\hline 299-E32-3 & $10 / 01 / 93$ & B099P6 & & & 15000.00 & \\
\hline $299-E 32-4$ & $10 / 15 / 93$ & B099P7 & .89 LB & 13000.00 & & 10.00 \\
\hline $299-E 32-4$ & $10 / 15 / 93$ & B099P8 & & & 14000.00 & \\
\hline $299-E 32-5$ & $10 / 20 / 93$ & B099P9 & $2.60 \mathrm{~L}$ & 15000.00 & & 11.00 \\
\hline 299-E32-5 & $10 / 20 / 93$ & B09900 & & & 14000.00 & \\
\hline $299-E 32-6$ & $10 / 04 / 93$ & B09901 & $.51 \mathrm{U}$ & 11000.00 & & 23.00 \\
\hline $299-E 32-6$ & $10 / 04 / 93$ & B09902 & & & 11000.00 & \\
\hline $299-E 32-7$ & $10 / 04 / 93$ & B09903 & $2.20 \mathrm{BL}$ & 8600.00 & & 20.00 \\
\hline 299-E32-7 & $10 / 04 / 93$ & B09994 & & & 8400.00 & \\
\hline $299-E 32 \cdot 8$ & $10 / 05 / 93$ & 809905 & $.51 \mathrm{U}$ & 8900.00 & & 5.701 \\
\hline $299-E 32-8$ & $10 / 05 / 93$ & B09906 & & & 8800.00 & \\
\hline 299-E32-9 & $10 / 04 / 93$ & B09907 & $.51 \mathrm{U}$ & 9100.00 & & 23.00 \\
\hline 299-E32-9 & $10 / 04 / 93$ & 809908 & & & 8900.00 & \\
\hline $299-E 33-28$ & $10 / 05 / 93$ & B09909 & $2.80 \mathrm{BL}$ & 8800.00 & & $8.80 \mathrm{~L}$ \\
\hline 299-E33-28 & $10 / 05 / 93$ & 8099RO & & & 8900.00 & \\
\hline 299-E33-29 & $10 / 05 / 93$ & B099R1 & $.95 \mathrm{BL}$ & 8800.00 & & $9.60 \mathrm{~L}$ \\
\hline 299-E33-29 & $10 / 05 / 93$ & B099R2 & & & 8900.00 & \\
\hline 299-E33-30 & $10 / 05 / 93$ & B099R3 & $1.30 \mathrm{BL}$ & 8700.00 & & $9.60 \mathrm{~L}$ \\
\hline 299-E33-30 & $10 / 05 / 93$ & B099R4 & & & 8700.00 & \\
\hline 299-E33-34 & $10 / 06 / 93$ & B099R5 & $.51 \mathrm{U}$ & 11000.00 & 110000 & $9.20 \mathrm{~L}$ \\
\hline $299-E 33-35$ & $10 / 06 / 93$ & BO99R7 & $.51 \mathrm{U}$ & 8900.00 & 17000.00 & $7.40 \mathrm{~L}$ \\
\hline $299-E 33-35$ & $10 / 06 / 93$ & B099R8 & & & 9000.00 & \\
\hline
\end{tabular}


Table 15-13. Constituents with at Least One Detected Value for the Low-Level Waste Burial Grounds WMA 1 Data for Reporting Period October 1 through December 31, 1993. (sheet 7 of 11)

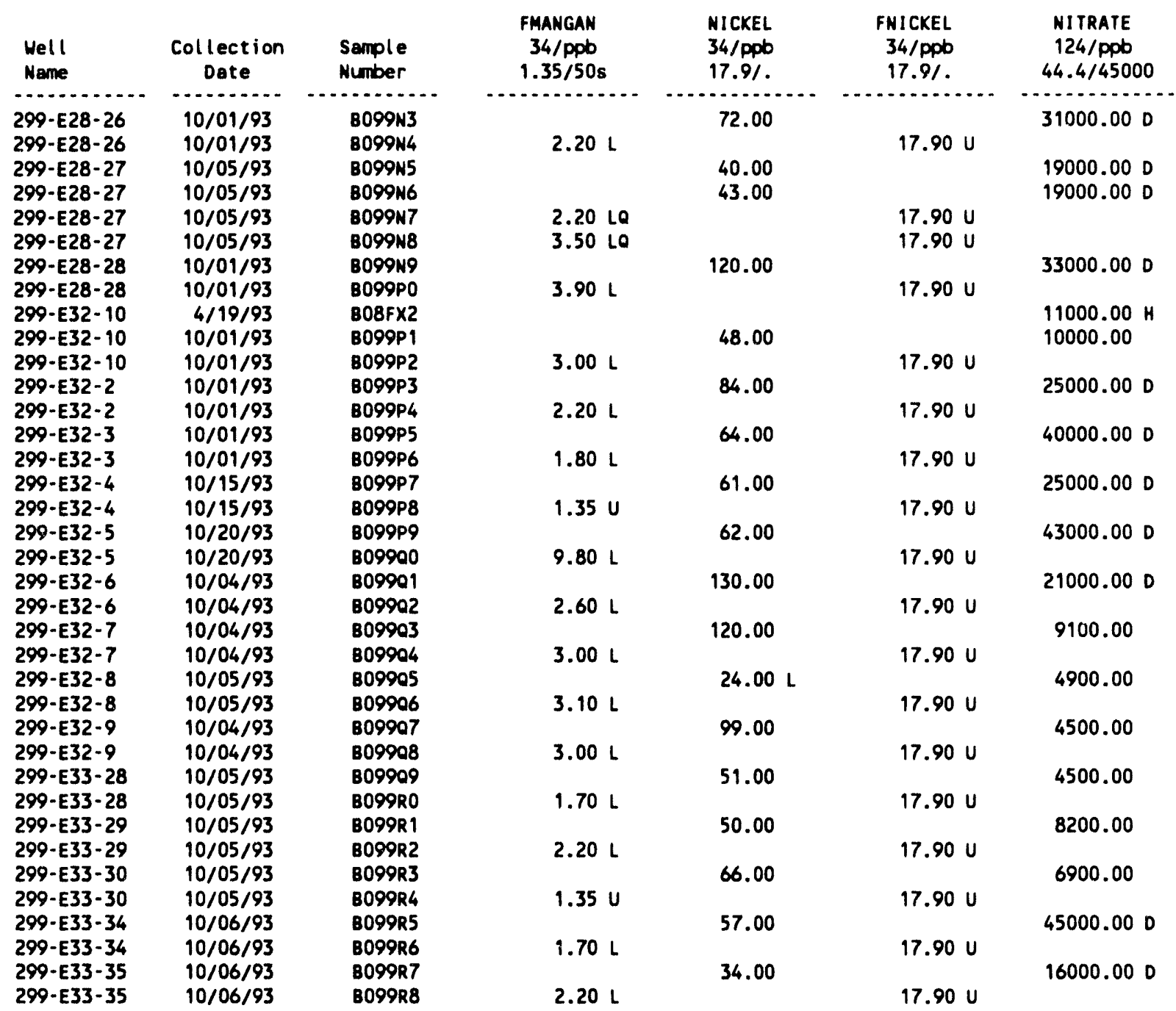

\begin{tabular}{|c|c|c|c|c|c|c|}
\hline $\begin{array}{l}\text { Hell } \\
\text { Name }\end{array}$ & $\begin{array}{c}\text { Collection } \\
\text { Date }\end{array}$ & $\begin{array}{l}\text { Sample } \\
\text { Number }\end{array}$ & $\begin{array}{l}\text { LPHENOL } \\
30 / \mathrm{ppb} \\
.31 \%\end{array}$ & $\begin{array}{l}\text { POTASUM } \\
34 / \mathrm{ppb} \\
662 \%\end{array}$ & $\begin{array}{l}\text { FPOTASS } \\
\text { 34/ppb } \\
662 / .\end{array}$ & $\begin{array}{c}\text { RADIUM } \\
137 / P C i / L \\
.15 i\end{array}$ \\
\hline 299-E28-26 & $10 / 01 / 93$ & B099N3 & $.31 \mathrm{U}$ & 5400.00 & & $.08 \mathrm{U}$ \\
\hline $\begin{array}{l}299-E 28-26 \\
299-E 28-27 \\
299-E 28-27\end{array}$ & $\begin{array}{l}10 / 01 / 93 \\
10 / 05 / 93 \\
10 / 05 / 93\end{array}$ & $\begin{array}{l}\text { B099N4 } \\
\text { B099N5 } \\
\text { B099N6 }\end{array}$ & $\begin{array}{l}.31 \text { UO } \\
.70 \text { LO }\end{array}$ & $\begin{array}{l}6000.00 \\
5700.00\end{array}$ & 5500.00 & $\begin{array}{l}.180 \\
.09 \text { vo }\end{array}$ \\
\hline $\begin{array}{l}299-E 28-27 \\
299-E 28-27\end{array}$ & $\begin{array}{l}10 / 05 / 93 \\
10 / 05 / 93\end{array}$ & $\begin{array}{l}\text { B099N7 } \\
\text { B099N8 }\end{array}$ & & & $\begin{array}{l}6400.00 \\
5900.00\end{array}$ & \\
\hline $\begin{array}{l}299-E 28-28 \\
299-E 28-28\end{array}$ & $\begin{array}{l}10 / 01 / 93 \\
10 / 01 / 93\end{array}$ & $\begin{array}{l}\text { B099N9 } \\
\text { B099PO }\end{array}$ & $.31 \mathrm{U}$ & 5900.00 & 5500.00 & .19 \\
\hline
\end{tabular}


Table 15-13. Constituents with at Least One Detected Value for the Low-Level Waste Burial Grounds WMA 1 Data for Reporting Period October 1

through December 31, 1993. (sheet 8 of 11)

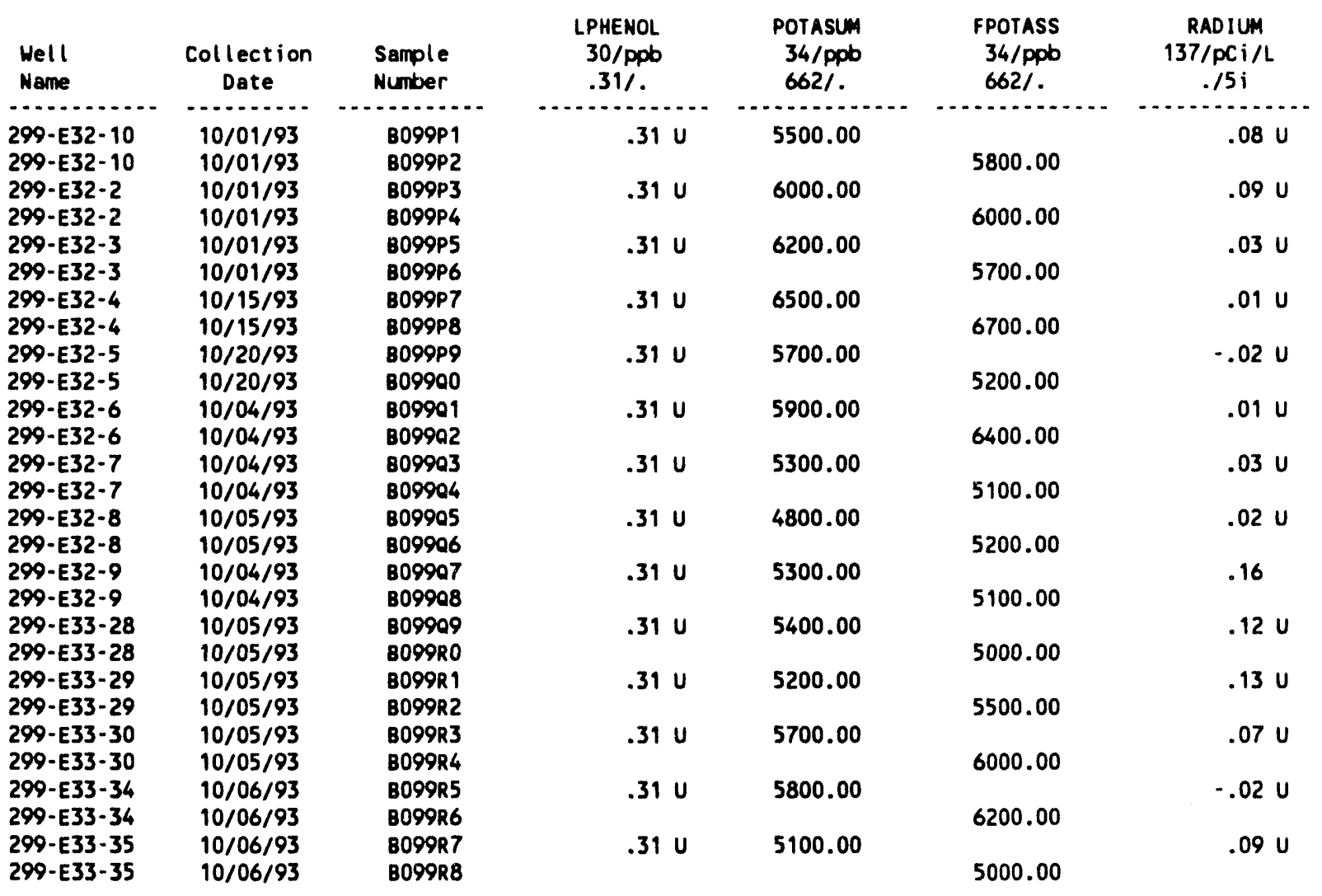

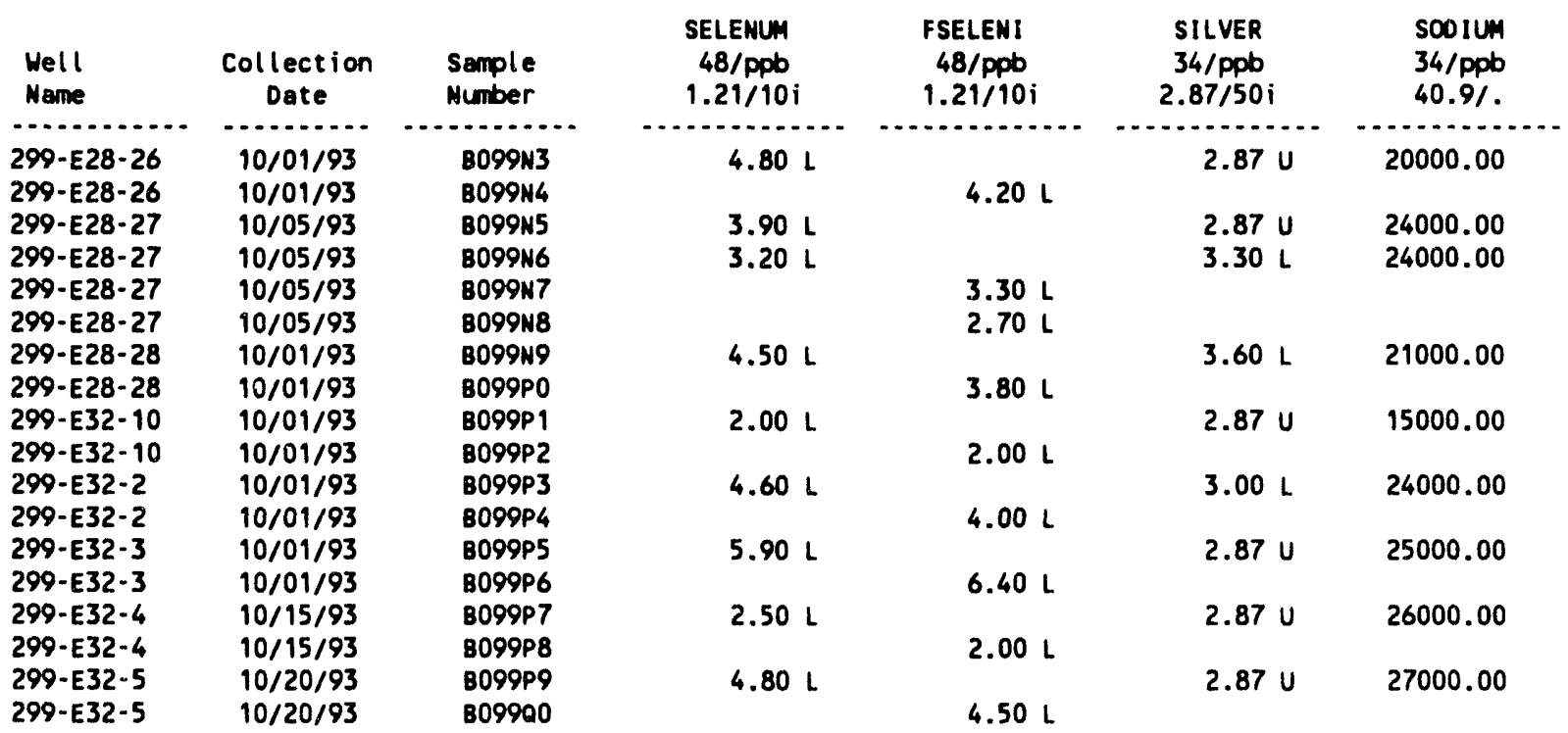


Table 15-13. Constituents with at Least One Detected Value for the Low-Level Waste Burial Grounds WMA 1 Data for Reporting Period October 1 through December 31, 1993. (sheet 9 of 11)

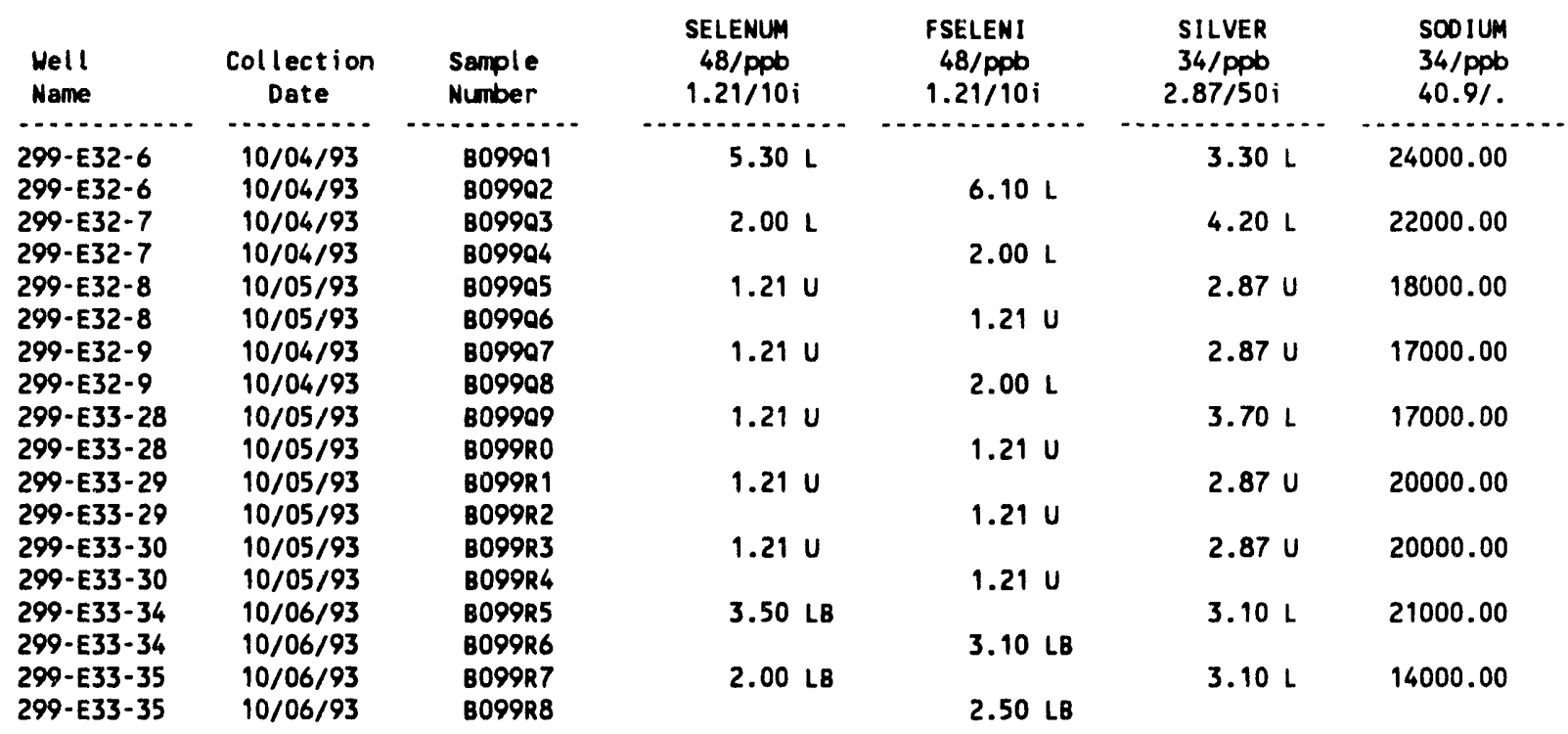

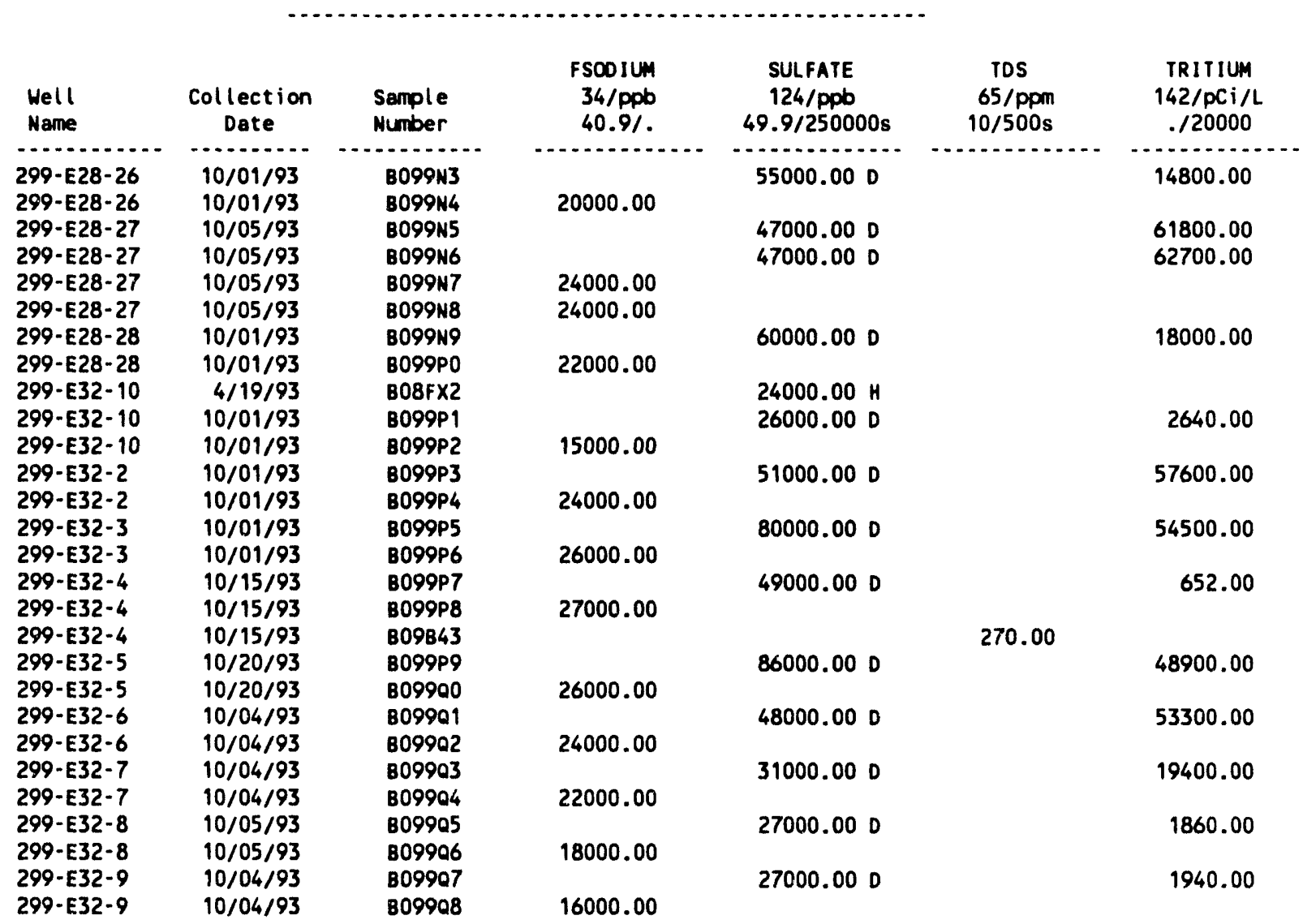


Table 15-13. Constituents with at Least One Detected Value for the Low-Level Waste Burial Grounds WMA 1 Data for Reporting Period October 1 through December 31, 1993. (sheet 10 of 11)

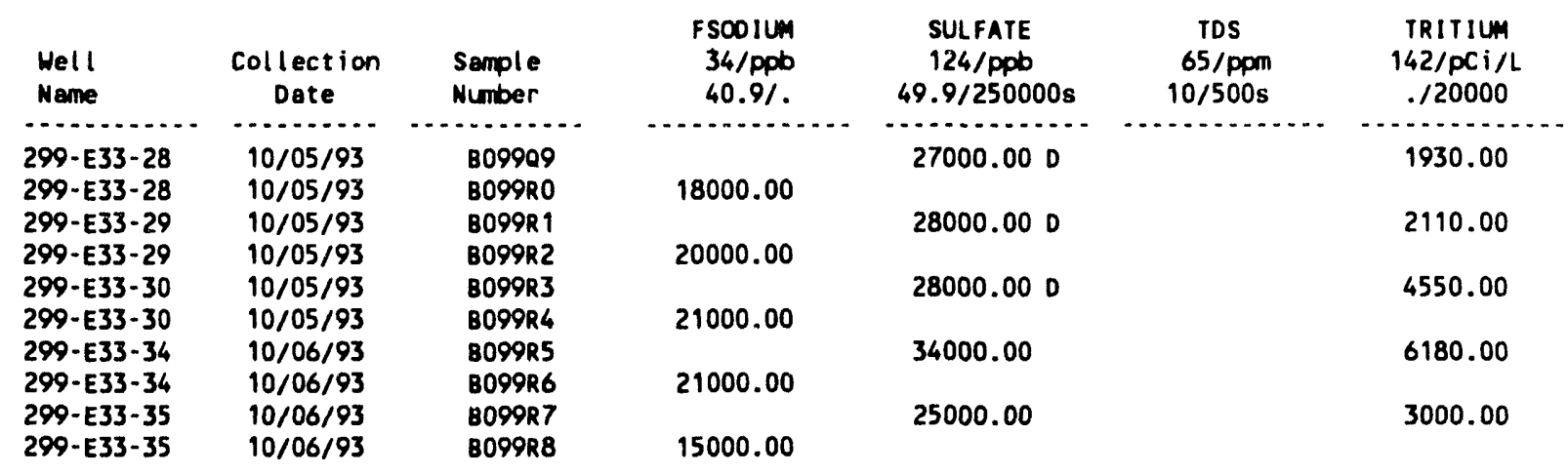

\begin{tabular}{|c|c|c|c|c|c|c|}
\hline $\begin{array}{l}\text { Well } \\
\text { Name }\end{array}$ & $\begin{array}{c}\text { Collection } \\
\text { Dote }\end{array}$ & $\begin{array}{l}\text { Sample } \\
\text { Number }\end{array}$ & $\begin{array}{c}\text { TURBID } \\
126 / \text { NTU } \\
.05 \%\end{array}$ & $\begin{array}{c}\text { URANIUM } \\
\text { 145/ppb } \\
. / .\end{array}$ & $\begin{array}{c}\text { VANADUM } \\
34 / \mathrm{ppb} \\
3.84 \%\end{array}$ & $\begin{array}{l}\text { FVANADI } \\
34 / \text { ppb } \\
3.84 / .\end{array}$ \\
\hline $\begin{array}{l}299-E 28-26 \\
299-E 28-26\end{array}$ & $\begin{array}{l}10 / 01 / 93 \\
10 / 01 / 93\end{array}$ & $\begin{array}{l}\text { B099N3 } \\
\text { B099N4 }\end{array}$ & 1.00 & 20.50 & $19.00 \mathrm{~L}$ & $16.00 \mathrm{~L}$ \\
\hline $299-E 28-27$ & $10 / 05 / 93$ & 8099 N5 & 1.400 & 8.19 & $23.00 \mathrm{~L}$ & $10.00 \mathrm{~L}$ \\
\hline 299-E28-27 & $10 / 05 / 93$ & B099N6 & 1.500 & 8.67 & $23.00 \mathrm{~L}$ & \\
\hline 299-E28-27 & 10/05/93 & B099N7 & & & & $26.00 \mathrm{~L}$ \\
\hline $\begin{array}{l}299-E 28-27 \\
299-E 28-28\end{array}$ & $\begin{array}{l}10 / 05 / 93 \\
10 / 01 / 93\end{array}$ & $\begin{array}{l}8099 \mathrm{N8} \\
8099 \mathrm{NO}\end{array}$ & 1.20 & 20.50 & 19.00 & $23.00 \mathrm{~L}$ \\
\hline $299-E 28-28$ & $10 / 01 / 93$ & B099PO & & & & $19.00 \mathrm{~L}$ \\
\hline 299-E32-10 & 10/01/93 & B099P1 & 2.40 & 2.03 & $24.00 \mathrm{~L}$ & \\
\hline 299-E32-10 & $10 / 01 / 93$ & B099P2 & & & & $23.00 \mathrm{~L}$ \\
\hline $299-E 32-2$ & $10 / 01 / 93$ & B099P3 & 1.20 & 11.90 & $23.00 \mathrm{~L}$ & \\
\hline 299-E32-2 & 10/01/93 & B099P4 & & & & $21.00 \mathrm{~L}$ \\
\hline $299-E 32-3$ & $10 / 01 / 93$ & B099P5 & .80 & 19.10 & $22.00 \mathrm{~L}$ & \\
\hline $299-E 32-3$ & $10 / 01 / 93$ & B099P6 & & & & $19.00 \mathrm{~L}$ \\
\hline $299-E 32-4$ & $10 / 15 / 93$ & B099P7 & 1.30 & 3.12 & $3.84 \mathrm{U}$ & \\
\hline $299-E 32-4$ & $10 / 15 / 93$ & B099P8 & & & & $3.84 \mathrm{U}$ \\
\hline 299-E32-5 & $10 / 20 / 93$ & B099P9 & 1.40 & 12.40 & $19.00 \mathrm{~L}$ & \\
\hline 299-E32-5 & $10 / 20 / 93$ & 809900 & & & & $12.00 \mathrm{~L}$ \\
\hline $299-E 32-6$ & $10 / 04 / 93$ & 809901 & 1.000 & 7.70 & $18.00 \mathrm{~L}$ & \\
\hline 299-E32-6 & $10 / 04 / 93$ & 809902 & & & & $19.00 \mathrm{~L}$ \\
\hline 299-E32-7 & $10 / 04 / 93$ & 809903 & 2.600 & 2.98 & $26.00 \mathrm{~L}$ & \\
\hline 299-E32-7 & $10 / 04 / 93$ & 809904 & & & & $23.00 \mathrm{~L}$ \\
\hline 299-E32-8 & $10 / 05 / 93$ & 809905 & 1.500 & 1.91 & $29.00 \mathrm{~L}$ & \\
\hline $299-E 32-8$ & $10 / 05 / 93$ & B09906 & & & & $24.00 \mathrm{~L}$ \\
\hline 299-E32-9 & $10 / 04 / 93$ & 809907 & $3.10 a$ & 1.89 & $25.00 \mathrm{~L}$ & \\
\hline 299-E32-9 & $10 / 04 / 93$ & 809908 & & & & $24.00 \mathrm{~L}$ \\
\hline $299-E 33-28$ & $10 / 05 / 93$ & 809909 & $1.50 \mathrm{a}$ & 1.72 & $28.00 \mathrm{~L}$ & \\
\hline $299-E 33-28$ & $10 / 05 / 93$ & BO99RO & & & & $23.00 \mathrm{~L}$ \\
\hline $299-E 33-29$ & $10 / 05 / 93$ & B099R1 & 1.500 & 1.87 & $25.00 \mathrm{~L}$ & \\
\hline $299-E 33-29$ & $10 / 05 / 93$ & B099R2 & & & & $24.00 \mathrm{~L}$ \\
\hline 299-E33-30 & $10 / 05 / 93$ & BO99R3 & 1.000 & 2.34 & $24.00 \mathrm{~L}$ & \\
\hline $299-E 33-30$ & $10 / 05 / 93$ & B099R4 & & & & $23.00 \mathrm{~L}$ \\
\hline $299-E 33-34$ & $10 / 06 / 93$ & B099R5 & .600 & 6.34 & $27.00 \mathrm{~L}$ & \\
\hline 299-E33-34 & $10 / 06 / 93$ & B099R6 & & & & $25.00 \mathrm{~L}$ \\
\hline 299-E33-35 & $10 / 06 / 93$ & B099R7 & .600 & 2.32 & $23.00 \mathrm{~L}$ & \\
\hline $299-E 33-35$ & $10 / 06 / 93$ & B099R8 & & & & $26.00 \mathrm{~L}$ \\
\hline
\end{tabular}


Table 15-13. Constituents with at Least One Detected Value for the Low-Level Waste Burial Grounds WMA 1 Data for Reporting Period October 1 through December 31, 1993. (sheet 11 of 11)

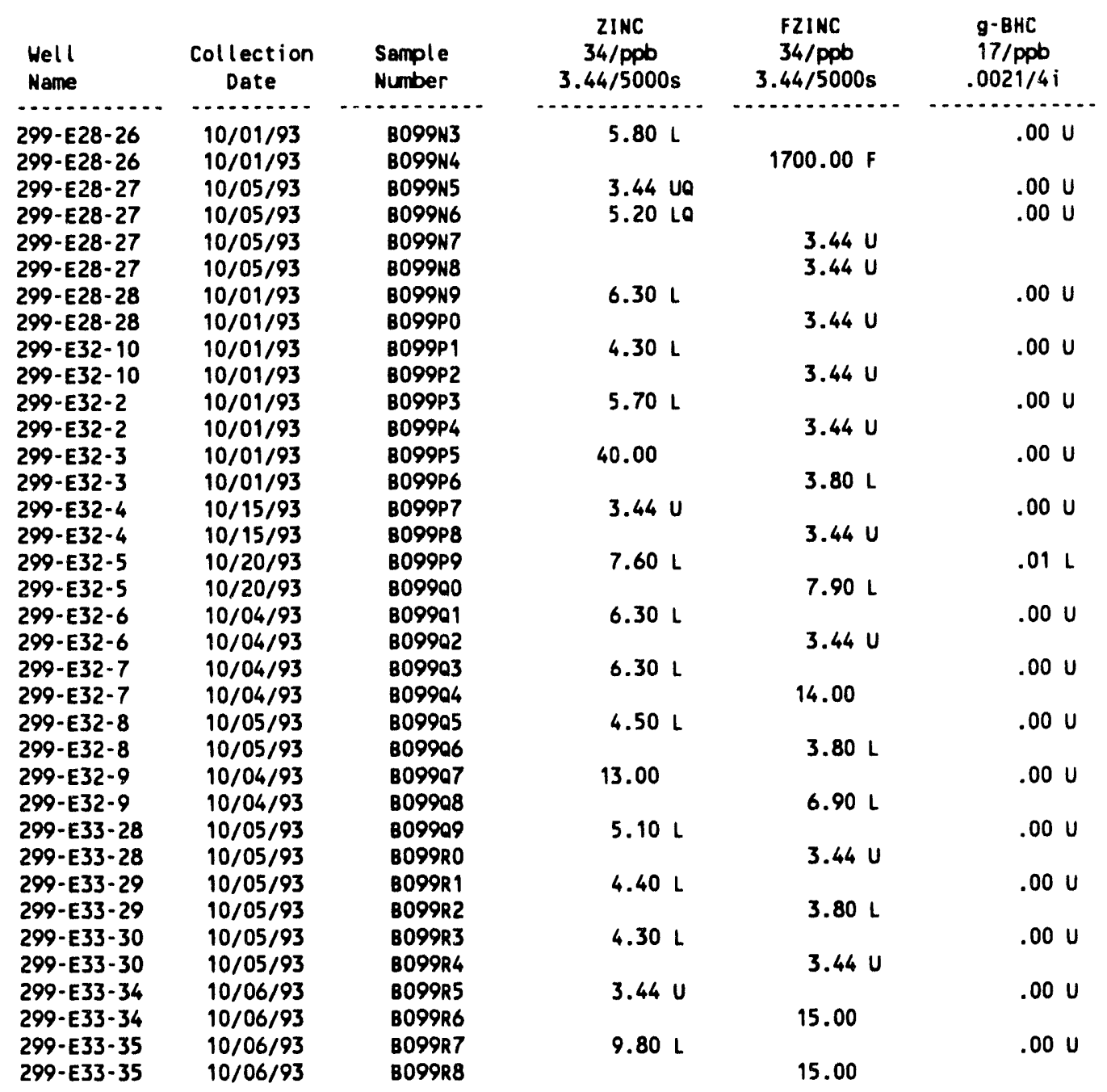

For explanation of this table, see Section 1.4 of report. 
Table 15-14. Contamination Indicator Parameters for the Low-Level Waste Burial Grounds WMA 1 Data for Reporting Period October 1 through December 31, 1993.

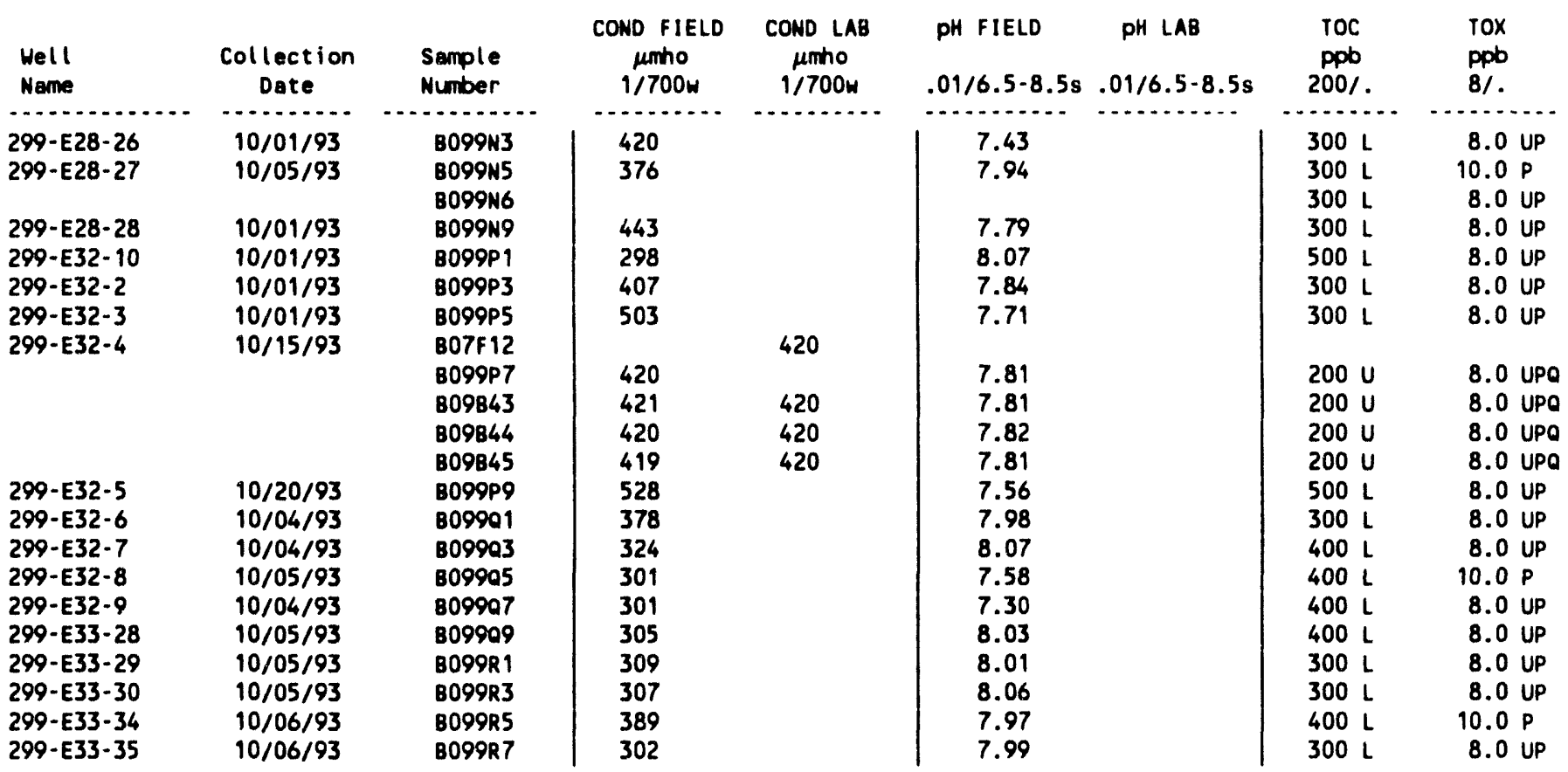

For explanation of this table, see Section 1.4 of report. 
Table 15-15. Constituent List and Summary of Results for the Low-Level Waste Burial Grounds WMA 2 Data for Reporting Period October 1 through December 31, 1993. (sheet 1 of 3)

CONTAMINATION INDICATOR PARAMETERS

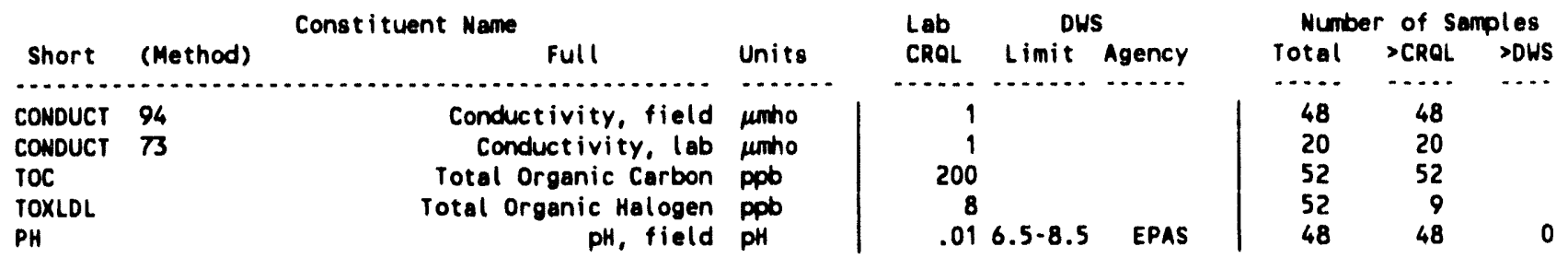

\section{DRINKING WATER PARAMETERS}

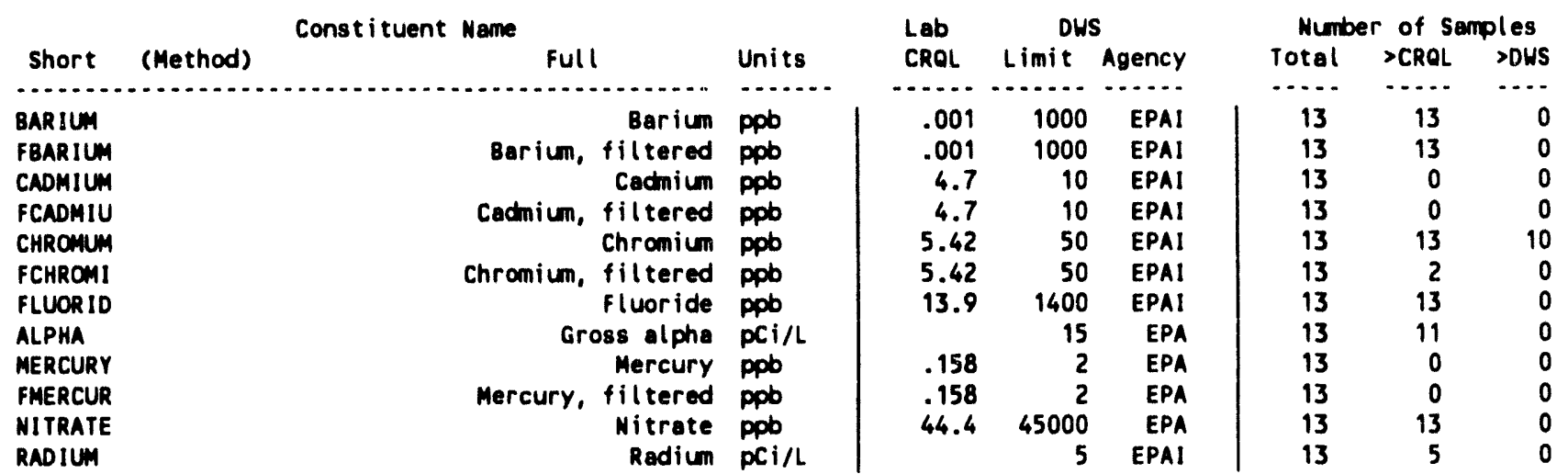

GROUNDWATER QUALITY PARAMETERS

\begin{tabular}{|c|c|c|c|c|c|c|c|c|c|}
\hline \multicolumn{4}{|c|}{ Constituent Name } & \multicolumn{3}{|c|}{ DHS } & \multicolumn{3}{|c|}{ Number of Semples } \\
\hline Short & (Method) & Full & Units & CRQL & Limit & Agency & Total & $>$ CRQL & $>$ DHS \\
\hline$\cdots \cdots$ & $\cdots \cdots$ & $\ldots \ldots \ldots$ & $\ldots . .$. & $\ldots \ldots$ & $\ldots \ldots$ & $\ldots \ldots$ & $\cdots$ & $\ldots \ldots$ & $\cdots$ \\
\hline $\begin{array}{l}\text { CHLOR ID } \\
\text { IRON }\end{array}$ & & $\begin{array}{r}\text { Chloride } \\
\text { Iron }\end{array}$ & $\begin{array}{l}p p b \\
p p b\end{array}$ & $\begin{array}{l}82.5 \\
10.3\end{array}$ & $\begin{array}{r}250000 \\
300\end{array}$ & $\begin{array}{l}\text { EPAS } \\
\text { EPAS }\end{array}$ & $\begin{array}{l}13 \\
13\end{array}$ & $\begin{array}{l}13 \\
13\end{array}$ & $\begin{array}{r}0 \\
10\end{array}$ \\
\hline FIRON & & Iron, filtered & ppb & 10.3 & 300 & EPAS & 13 & 12 & 0 \\
\hline MANGESE & & Manganese & ppb & 1.35 & 50 & EPAS & 13 & 13 & 0 \\
\hline FMANGAN & & Manganese, filtered & ppb & 1.35 & 50 & EPAS & 13 & 10 & 0 \\
\hline LPHENOL & 19 & Phenol & ppb & .833 & & & 13 & 0 & \\
\hline LPHENOL & 30 & Phenol & ppb & .31 & & & 13 & 0 & \\
\hline SOOIUM & & Sodium & ppo & 40.9 & & & 13 & 13 & \\
\hline FSOOIUN & & Sodiun, filtered & ppb & 40.9 & & & 13 & 13 & \\
\hline SULFATE & & Sulfate & ppb & 49.9 & 250000 & EPAS & 13 & 13 & 0 \\
\hline
\end{tabular}


Table 15-15. Constituent List and Summary of Results for the Low-Level Waste Burial Grounds WMA 2 Data for Reporting Period October 1

through December 31, 1993. (sheet 2 of 3 )

SITE SPECIFIC AND OTHER CONSTITUENTS

\begin{tabular}{|c|c|c|c|c|c|c|c|c|c|}
\hline \multirow{2}{*}{ Short } & \multirow[b]{2}{*}{ (Method) } & \multirow{2}{*}{$\begin{array}{l}\text { Const ituent Name Full } \\
\text { fons }\end{array}$} & \multirow[b]{2}{*}{ Units } & \multirow{2}{*}{$\begin{array}{l}\text { Lab } \\
\text { CRQL }\end{array}$} & \multicolumn{2}{|l|}{ DWS } & \multicolumn{3}{|c|}{ Number of Samples } \\
\hline & & & & & Limit & Agency & Total & $>$ CRQL & >DWS \\
\hline $1,1,1-1$ & $\cdots$ & 1,1,1-Trichloroethane & ppo & .072 & 200 & EPA & 13 & 4 & $\cdots$ \\
\hline $\begin{array}{l}1,1,2-1 \\
1,1-0,16\end{array}$ & & $1,1,2$-Trichlor oethane & ppo & .043 & 200 & ern & 13 & 0 & \\
\hline $\begin{array}{l}1,1-D 1 C \\
1,2-D I C\end{array}$ & & 1,1-Dichloroethene & ppb & .337 & & & 13 & 0 & \\
\hline $\begin{array}{l}1,2-01 C \\
14 \text {-cben }\end{array}$ & & 1,2-0 ichloroethane & ppb & .139 & 5 & EPA & 13 & 0 & 0 \\
\hline $\begin{array}{l}14 \text {-dben } \\
246-t r p\end{array}$ & & 1,4-0ichlorobenzene & ppb & .107 & 75 & EPA & 13 & 0 & 0 \\
\hline $\begin{array}{l}246-\operatorname{trp} \\
24-\text { dchp }\end{array}$ & & 2,4,6-Trichlorophenol & ppb & 1.45 & & & 13 & 0 & \\
\hline $\begin{array}{l}24-d c h p \\
24-d c h p\end{array}$ & 19 & 2,4-Dichlorophenol & ppb & 2.8 & & & 13 & 0 & \\
\hline $\begin{array}{l}\text { 24-dchp } \\
\text { DIMPHEN }\end{array}$ & 30 & 2,4-Dichlorophenol & ppb & 1.5 & & & 13 & 0 & \\
\hline $\begin{array}{l}\text { DIMPHEN } \\
\text { DINPHEN }\end{array}$ & & 2,4-D imethylphenol & ppo & 1.01 & & & 13 & 0 & \\
\hline $\begin{array}{l}\text { DINPHEN } \\
26 \text {-dchp }\end{array}$ & & 2,4-Dinitrophenol & ppb & .96 & & & 13 & 0 & \\
\hline $\begin{array}{l}\text { 26-dchp } \\
\text { CHLPHEN }\end{array}$ & & 2,6-0ichlorophenol & ppb & 1.59 & & & 13 & 0 & \\
\hline $\begin{array}{l}\text { CHLPHEN } \\
\text { 2METHPH }\end{array}$ & & 2-Chlorophenol & ppb & 1.42 & & & 13 & 0 & \\
\hline $\begin{array}{l}\text { 2METHPH } \\
\text { 2NITPH }\end{array}$ & & 2-Methyl phenol & ppb & 1.8 & & & 13 & 0 & \\
\hline $\begin{array}{l}\text { 2NITPH } \\
\text { 2NITPH }\end{array}$ & 19 & 2-Nitrophenol & ppob & 3.96 & & & 13 & 0 & \\
\hline $\begin{array}{l}\text { 2NITPH } \\
\text { BUTDINP }\end{array}$ & 30 & 2-Nitrophenol & ppb & 1.56 & & & 13 & 0 & \\
\hline $\begin{array}{l}\text { BUTD INP } \\
\text { 46DN2MP }\end{array}$ & & 2-sec-Butyl-4,6-dinitrophenol (ON & ppob & 1.35 & & & $\begin{array}{l}13 \\
13\end{array}$ & 0 & \\
\hline CHLCRES & & $\begin{array}{l}\text { 4,6-Dinitro-2-methyl phenol } \\
\text { 4-Chloro-3-methylphenol }\end{array}$ & ppob & $\begin{array}{l}1.18 \\
1.12\end{array}$ & & & $\begin{array}{l}13 \\
13\end{array}$ & $\begin{array}{l}0 \\
0\end{array}$ & \\
\hline 4METHPH & & & ppob & 3.54 & & & 13 & 0 & \\
\hline NITPHEN & & 4-Nitrophenol & ppb & .65 & & & 13 & 0 & \\
\hline ALUMNUM & & Aluminum & ppob & 32.5 & & & 13 & 2 & \\
\hline FALUMIN & & Aluminum & ppb & 32.5 & & & 13 & 0 & \\
\hline ANTIONY & & Ant imony & ppb & 69.4 & & & 13 & 0 & \\
\hline FANTIMO & & Antimony, filtered & ppb & 69.4 & & & 13 & 0 & \\
\hline AR1016 & & Aroclor-1016 & ppb & .19 & & & 13 & 0 & \\
\hline AR1221 & & Aroclor-1221 & ppb & .14 & & & 13 & 0 & \\
\hline AR1232 & & Aroclor-1232 & ppb & .11 & & & 13 & 0 & \\
\hline AR1242 & & Aroclor -1242 & ppb & .11 & & & 13 & 0 & \\
\hline AR1248 & & Aroclor -1248 & ppb & .04 & & & 13 & 0 & \\
\hline AR1254 & & Aroclor-1254 & ppb & .1 & & & 13 & 0 & \\
\hline AR1260 & & Aroclor -1260 & ppb & .11 & & & 13 & 0 & \\
\hline BENZENE & & Benzene & ppb & .105 & 5 & EPA & 13 & 1 & 0 \\
\hline BENZOTH & & Benzothiazole & ppb & 2.55 & & & 13 & 0 & \\
\hline BERYLUM & & Beryllium & ppb & .814 & & & 13 & 0 & \\
\hline FBERYLL & & Beryllium, filtered & ppb & .814 & & & 13 & 0 & \\
\hline BIS2EPH & & Bis(2-ethylhexyl) phthalate & ppb & 4.07 & & & 13 & 0 & \\
\hline BROMIDE & & Bromide & ppb & 52.8 & & & 13 & 8 & \\
\hline CALCIUM & & Calcium & ppb & 20.9 & & & 13 & 13 & \\
\hline FCALCIU & & Calcium, filtered & ppb & 20.9 & & & 13 & 13 & \\
\hline TETRANE & & Carbon tetrachloride & ppb & .121 & 5 & EPA & 13 & 1 & 0 \\
\hline CHLFORM & & Chloroform & ppb & .043 & & & 13 & 4 & \\
\hline COBALT & & Cobalt & ppb & 4.05 & & & 13 & 3 & \\
\hline FCOBALT & & Cobslt, filtered & ppb & 4.05 & & & 13 & 0 & \\
\hline COPPER & & Copper & ppob & 2.65 & 1000 & EPAS & 13 & 11 & 0 \\
\hline FCOPPER & & Copper, filtered & ppb & 2.65 & 1000 & EPAS & 13 & 7 & 0 \\
\hline CRESOLS & & Cresols (methylphenols) & ppb & 4.66 & & & 13 & 0 & \\
\hline CYANIDE & & Cyanide & $p p b$ & 1.24 & & & 13 & 0 & \\
\hline DECANE & & Decane & ppb & 4.03 & & & 13 & 0 & \\
\hline DODECAN & & Dodecane & ppb & 3.62 & & & 13 & 0 & \\
\hline ETHBENZ & & Ethylbenzene & ppb & .046 & 700 & EPA & 13 & 0 & 0 \\
\hline BETA & & Gross beta & $\mathrm{PCi} / \mathrm{L}$ & & & & 13 & 13 & \\
\hline $1-129 L$ & & lodine-129, Low level & $\mathrm{PCi} / \mathrm{L}$ & & & & 5 & 5 & \\
\hline LEAD & & Lead & ppb & .508 & 50 & EPAI & 13 & 7 & 0 \\
\hline FLEAD & & Lead, filtered & ppb & .508 & 50 & EPAI & 13 & 5 & 0 \\
\hline
\end{tabular}


Table 15-15. Constituent List and Summary of Results for the Low-Level Waste Burial Grounds WMA 2 Data for Reporting Period October 1 through December 31, 1993. (sheet 3 of 3)

\begin{tabular}{|c|c|c|c|c|c|c|c|c|c|}
\hline \multirow{2}{*}{$\begin{array}{l}\text { short } \\
\text { s... }\end{array}$} & \multirow[b]{2}{*}{ (Method) } & \multirow{2}{*}{ Constituent Neme Full } & \multirow[b]{2}{*}{ Units } & \multirow{2}{*}{$\begin{array}{l}\text { Lab } \\
\text { CROL }\end{array}$} & \multicolumn{2}{|l|}{ DUS } & \multicolumn{3}{|c|}{ Number of Semples } \\
\hline & & & & & Limit & Agency & Total & >CROL & $>$ OWS \\
\hline ….... & & . & $\cdots \cdot$ & $\cdots \cdot$ & ......... & $\cdots \cdot$ & $\cdots \cdot$ & $\cdots$ & $\cdots \cdot$ \\
\hline MAGNES & & Magnesium & ppob & 26 & & & 13 & 13 & \\
\hline FMAGNES & & Magnesium, filtered & ppob & 26 & & & 13 & 13 & \\
\hline METHYCH & & Methylene chloride & ppob & .056 & & & 13 & 3 & \\
\hline MAPHTHA & & Naphthalene & ppob & 6.5 & & & 13 & 0 & \\
\hline NICKEL & & Nickel & ppob & 17.9 & & & 13 & 10 & \\
\hline FWICKEL & & Nickel, filtered & ppo & 17.9 & & & 13 & 1 & \\
\hline NITRITE & & Nitrite & ppob & 38.3 & 1000 & EPA & 13 & 0 & 0 \\
\hline PENTCHP & 19 & Pentachlorophenol & ppob & 8.07 & 1 & EPA & 13 & 0 & $13 *$ \\
\hline PENTCHP & 30 & Pentachlorophenol & ppb & .87 & 1 & EPA & 13 & 0 & 0 \\
\hline PHOSPHA & & Phosphate & ppb & 147 & & & 13 & 0 & \\
\hline POTASUM & & Potassium & ppb & 662 & & & 13 & 13 & \\
\hline FPOTASS & & Potassium, filtered & Ppb & 662 & & & 13 & 13 & \\
\hline SILVER & & silver & ppob & 2.87 & 50 & EPAI & 13 & 0 & 0 \\
\hline FSILVER & & silver, filtered & ppb & 2.87 & 50 & EPAI & 13 & 1 & 0 \\
\hline PERCENE & & Tetrachloroethene & ppob & .049 & 5 & EPA & 13 & $\mathbf{0}$ & 0 \\
\hline TETPNNL & & Tetrachlorophenols & ppob & 1.05 & & & 13 & $\mathbf{0}$ & \\
\hline TETRADE & & Tetradecane & ppb & 2.43 & & & 13 & 0 & \\
\hline TIN & & $\operatorname{Tin}$ & ppo & 51.1 & & & 13 & $\mathbf{0}$ & \\
\hline FTIN & & Tin, filtered & ppb & 51.1 & & & 13 & 0 & \\
\hline TOLUENE & & Toluene & ppb & .056 & 1000 & EPA & 13 & 1 & 0 \\
\hline TRIBUTPH & & Tributyl Phosphate & ppb & 4.42 & & & 13 & 0 & \\
\hline TRICENE & & Trichloroethene & ppob & .065 & 5 & EPA & 13 & 0 & 0 \\
\hline TRIPHNL & & Irichlorophenol: & ppob & 1.11 & & & 13 & $\mathbf{0}$ & \\
\hline TOICHPH & & Tris-2-chloroethyl phosphate & ppob & 2.88 & & & 13 & 0 & \\
\hline TRITIUM & & Tritiun & $\mathrm{PCi} / \mathrm{L}$ & & 20000 & EPA & 13 & 11 & 0 \\
\hline TURBID & & Turbidity & NTU & .05 & & & 13 & 13 & \\
\hline URANIUM & & Uranium & ppb & & & & 13 & 13 & \\
\hline$U-234$ & & Uranium-234 & $\mathrm{PCi} / \mathrm{L}$ & & & & 5 & 5 & \\
\hline$U-235$ & & Uranium-235 & $\mathrm{PC} i / L$ & & & & 5 & 4 & \\
\hline$U-238$ & & Uranium-238 & $\mathrm{PCi} / \mathrm{L}$ & & & & 5 & 5 & \\
\hline VAMADUM & & Vanadium & ppob & 3.84 & & & 13 & 13 & \\
\hline FVANADI & & Vanadium, filtered & ppb & 3.84 & & & 13 & 13 & \\
\hline VINYIDE & & Vinyl chloride & ppob & .266 & 2 & EPA & 13 & 0 & 0 \\
\hline XYLENE & & Xylenes (total) & ppob & .202 & 10000 & EPA & 13 & 0 & 0 \\
\hline $21 N C$ & & Zinc & ppob & 3.44 & 5000 & EPAS & 13 & 5 & 0 \\
\hline FZINC & & zinc, filtered & ppb & 3.44 & 5000 & EPAS & 13 & 1 & 0 \\
\hline CIS120E & & cis-1,2-Dichloroethylene & ppb & .127 & 70 & EPA & 13 & 0 & 0 \\
\hline MCRESOL & & m-Cresol & ppb & 1.44 & & & 13 & 0 & \\
\hline TRANDCE & & trans-1,2-Dichloroethylene & Ppob & .149 & 100 & EPA & 13 & 0 & 0 \\
\hline
\end{tabular}


Table 15-16. Constituents with at Least One Detected Value for the Low-Level Waste Burial Grounds WMA 2 Data for Reporting Period October 1 through December 31, 1993. (sheet 1 of 8)

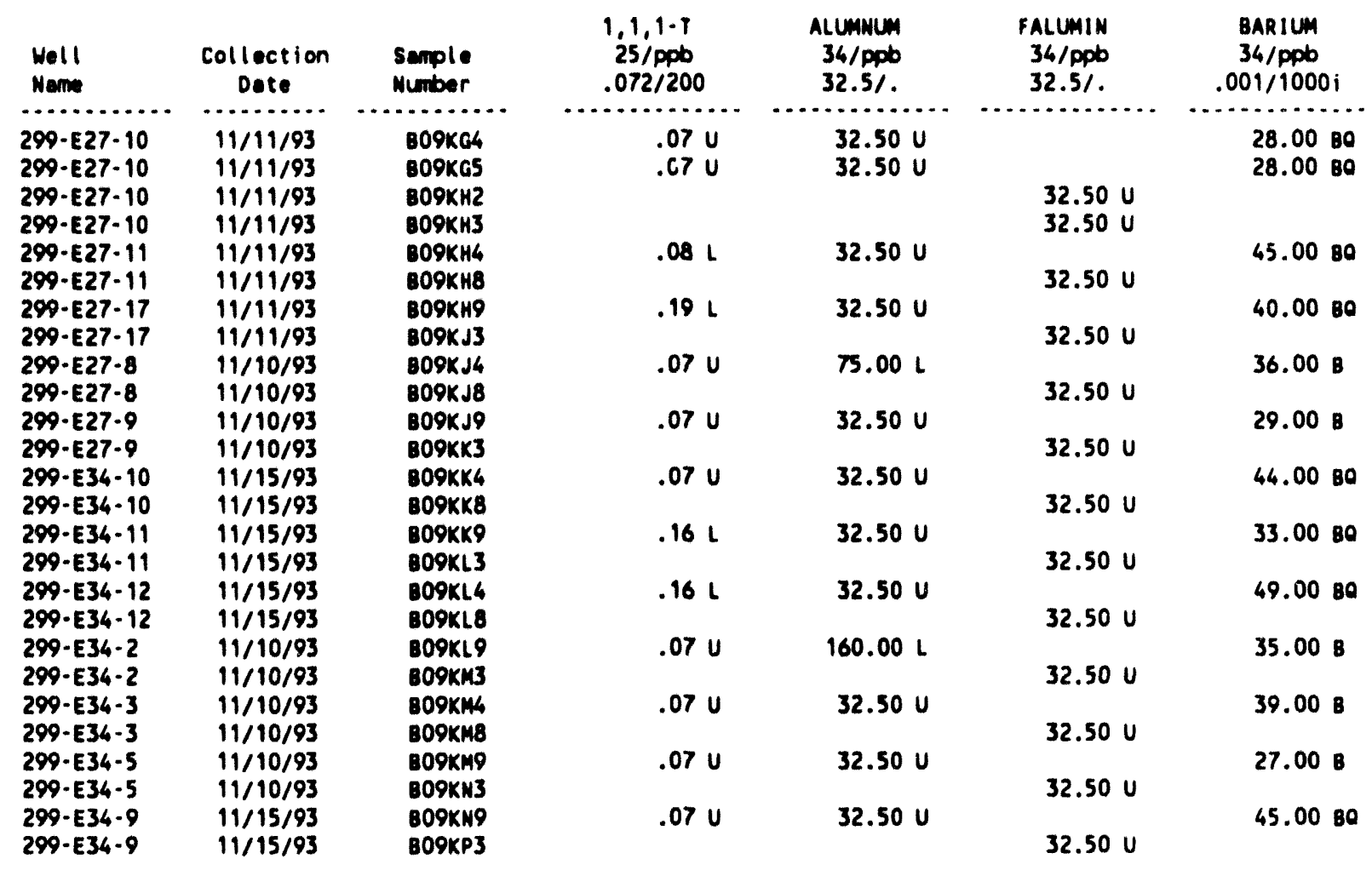

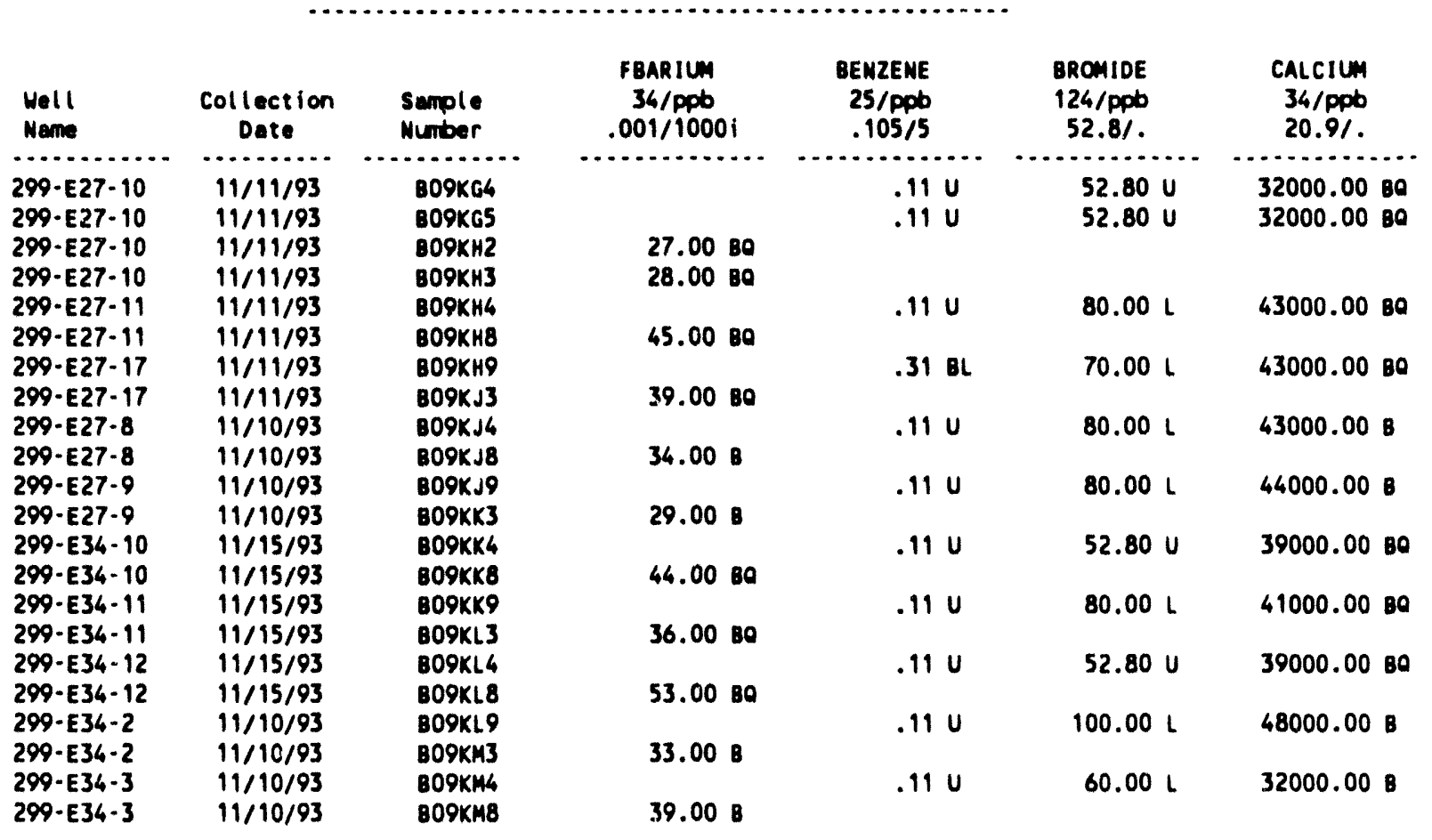


Table 15-16. Constituents with at Least One Detected Value for the Low-Level Waste Burial Grounds WMA 2 Data for Reporting Period October 1 through December 31, 1993. (sheet 2 of 8)

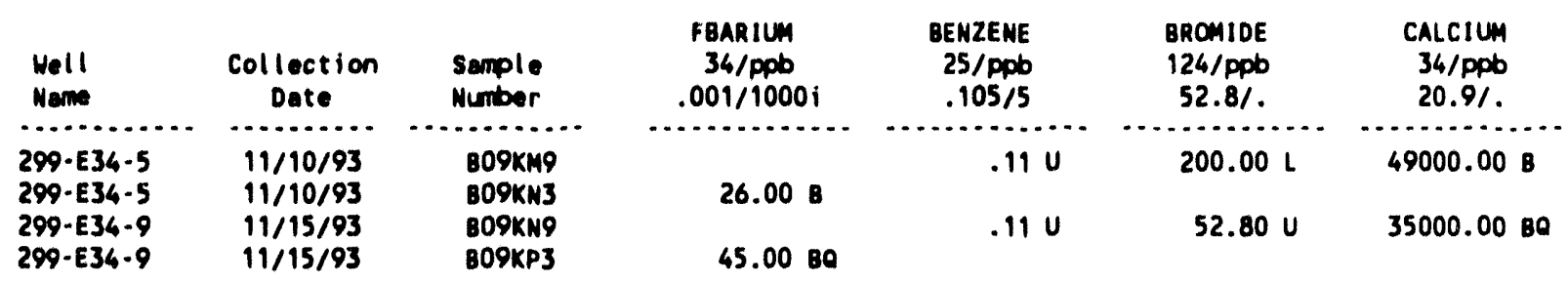

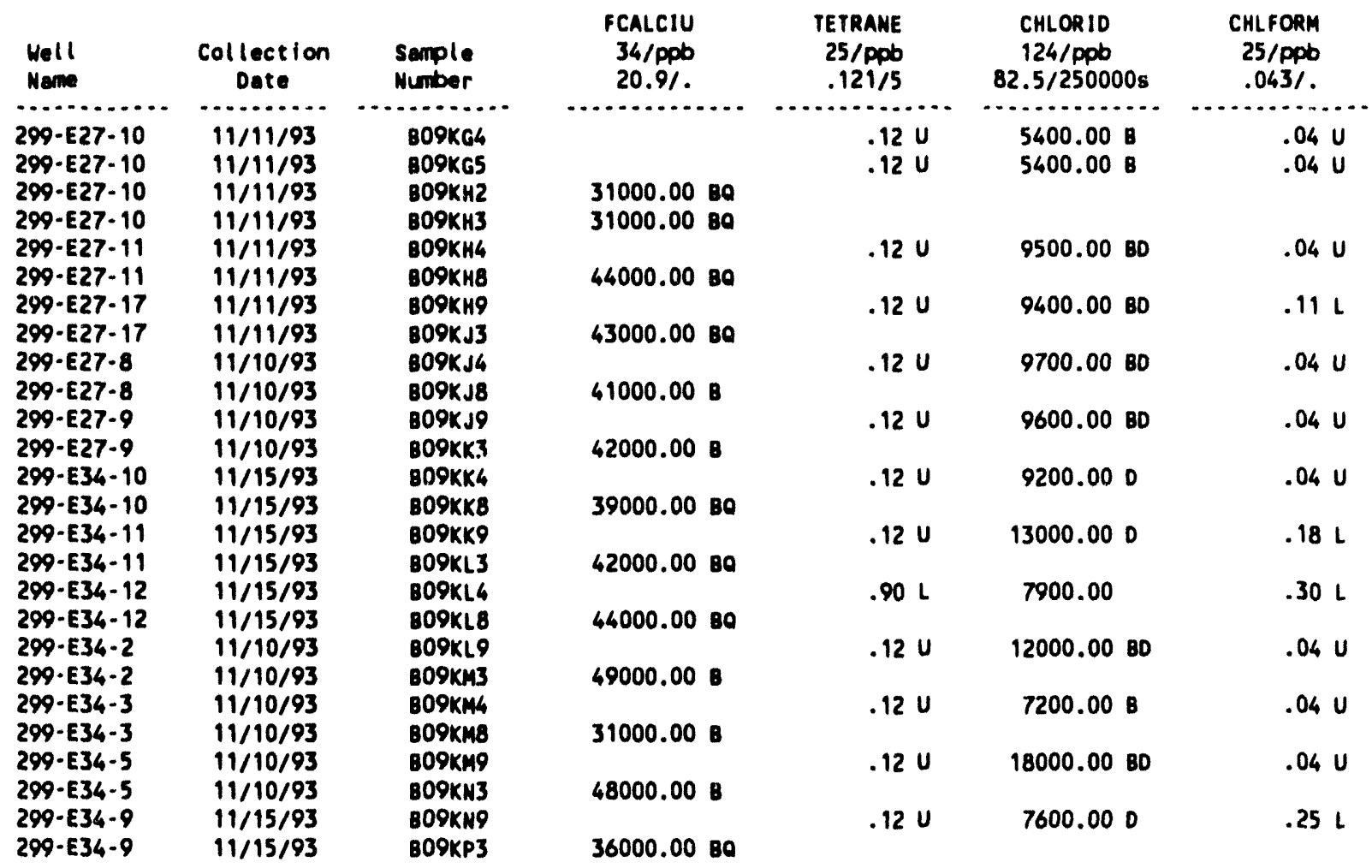

\begin{tabular}{|c|c|c|c|c|c|c|}
\hline $\begin{array}{l}\text { Hell } \\
\text { Name }\end{array}$ & $\begin{array}{c}\text { Collection } \\
\text { Date }\end{array}$ & $\begin{array}{l}\text { Semple } \\
\text { Number }\end{array}$ & $\begin{array}{l}\text { CHROMin } \\
34 / \text { ppb } \\
5.42 / 50 i\end{array}$ & $\begin{array}{l}\text { FCHROAI } \\
34 / \mathrm{ppb} \\
5.42 / 50 \mathrm{i}\end{array}$ & $\begin{array}{l}\text { COBALT } \\
34 / \mathrm{ppb} \\
4.05 /\end{array}$ & $\begin{array}{c}\text { FCOBALT } \\
34 / \mathrm{ppb} \\
4.05 / .\end{array}$ \\
\hline$\cdots \cdots \cdots+\cdots$ & $\ldots \ldots$ & $\ldots \ldots \ldots$ & 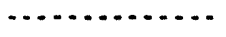 & 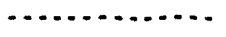 & $\ldots \ldots \ldots \ldots$ & $\ldots \ldots$ \\
\hline $\begin{array}{l}299 \cdot E 27-10 \\
299-E 27-10\end{array}$ & $\begin{array}{l}11 / 11 / 93 \\
11 / 11 / 93\end{array}$ & $\begin{array}{l}\text { 809KG4 } \\
\text { 809KG5 }\end{array}$ & $\begin{array}{l}63.00 \\
62.00\end{array}$ & & $\begin{array}{l}4.05 \text { U0 } \\
4.05 \text { v0 }\end{array}$ & \\
\hline $\begin{array}{l}299-E 27-10 \\
299-E 27-10\end{array}$ & $\begin{array}{l}11 / 11 / 93 \\
11 / 11 / 93\end{array}$ & $\begin{array}{l}\text { 809Kн2 } \\
809 \text { K } 3\end{array}$ & & $\begin{array}{l}5.42 \mathrm{U} \\
5.42 \mathrm{U}\end{array}$ & & $\begin{array}{l}4.05 \text { Ua } \\
4.05 \text { UQ }\end{array}$ \\
\hline 299-E27-11 & $11 / 11 / 93$ & 809KH4 & 160.00 & & 4.05 vo & \\
\hline $\begin{array}{l}299-E 27-11 \\
200-E 27-17\end{array}$ & $11 / 11 / 93$ & 809Кн8 & & $5.42 \mathrm{U}$ & & 4.0510 \\
\hline $299-E 27-17$ & $11 / 11 / 95$ & $\begin{array}{l}809 K \mathrm{H} 9 \\
809 \mathrm{KJ} 3\end{array}$ & .00 & $5.42 \mathrm{U}$ & Ua & 4.05 vo \\
\hline $\begin{array}{l}299-E 27-8 \\
299-E 27-8\end{array}$ & $\begin{array}{l}11 / 10 / 93 \\
11 / 10 / 93\end{array}$ & $\begin{array}{l}809 K J 4 \\
809 K J 8\end{array}$ & 150.00 & $8.70 \mathrm{~L}$ & $4.05 \mathrm{U}$ & $4.05 \mathrm{U}$ \\
\hline
\end{tabular}


Table 15-16. Constituents with at Least One Detected Value for the Low-Level Waste Burial Grounds WMA 2 Data for Reporting Period October 1

through December 31, 1993. (sheet 3 of 8)

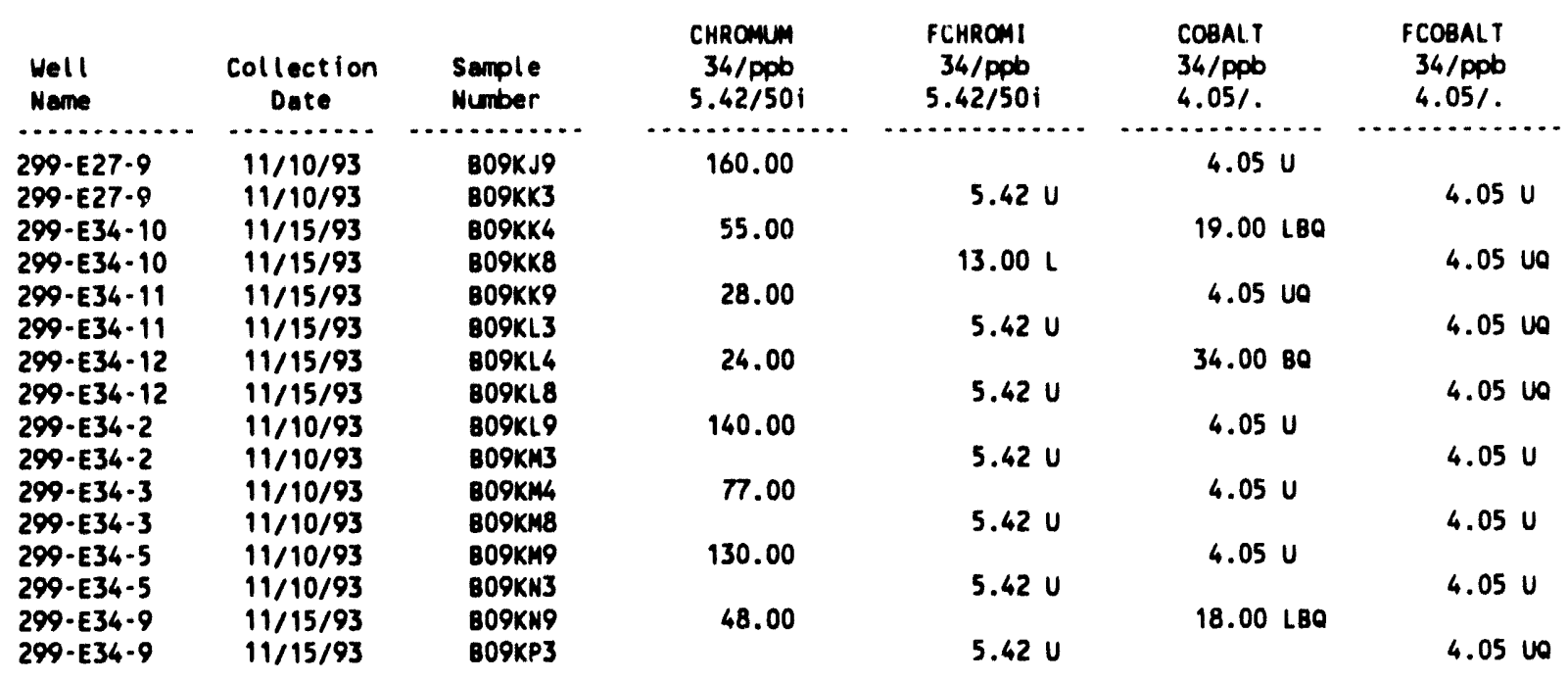

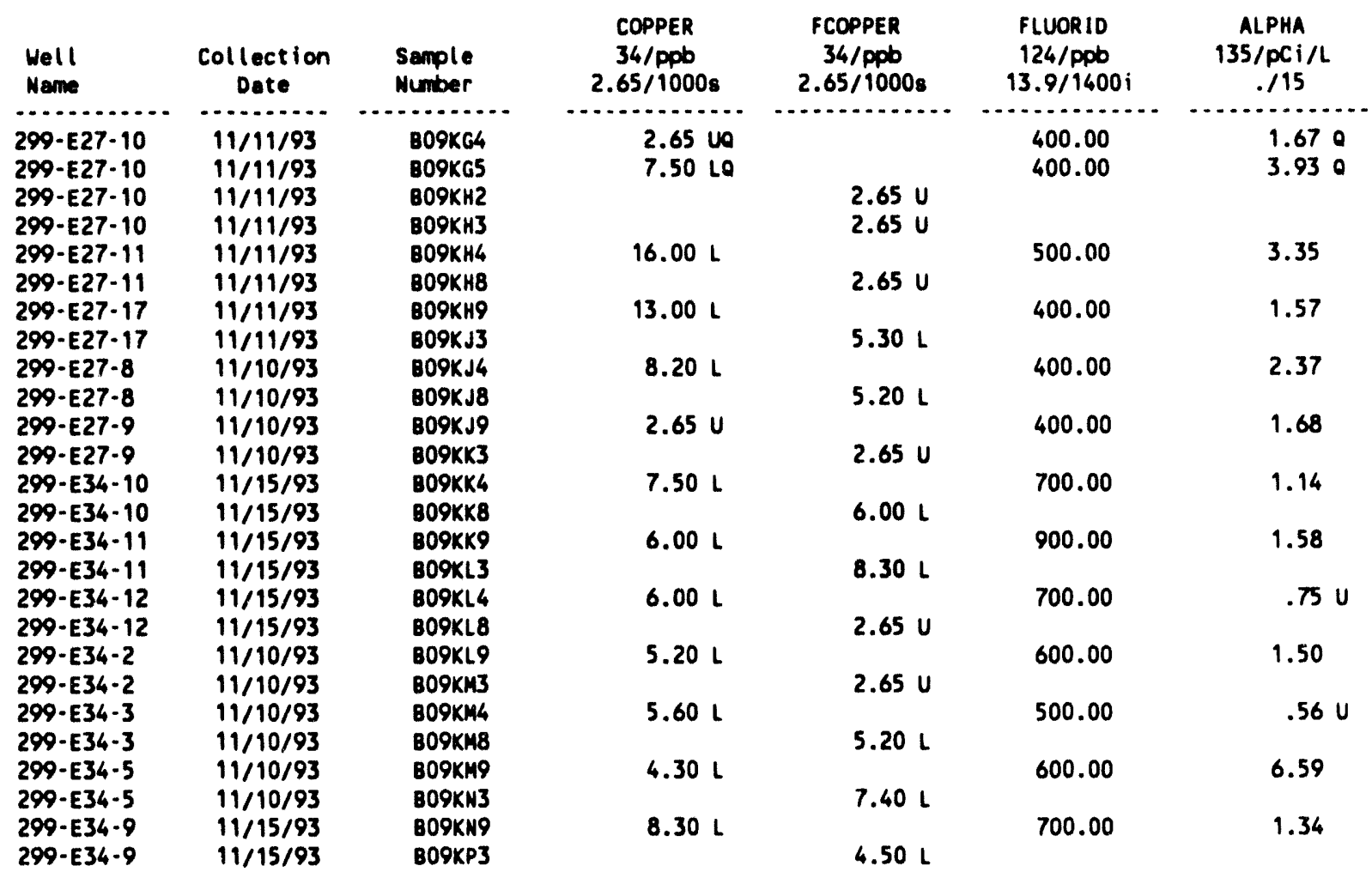


Table 15-16. Constituents with at Least One Detected Value for the Low-Leve1 Waste Burial Grounds WMA 2 Data for Reporting Period October 1

through December 31, 1993. (sheet 4 of 8)

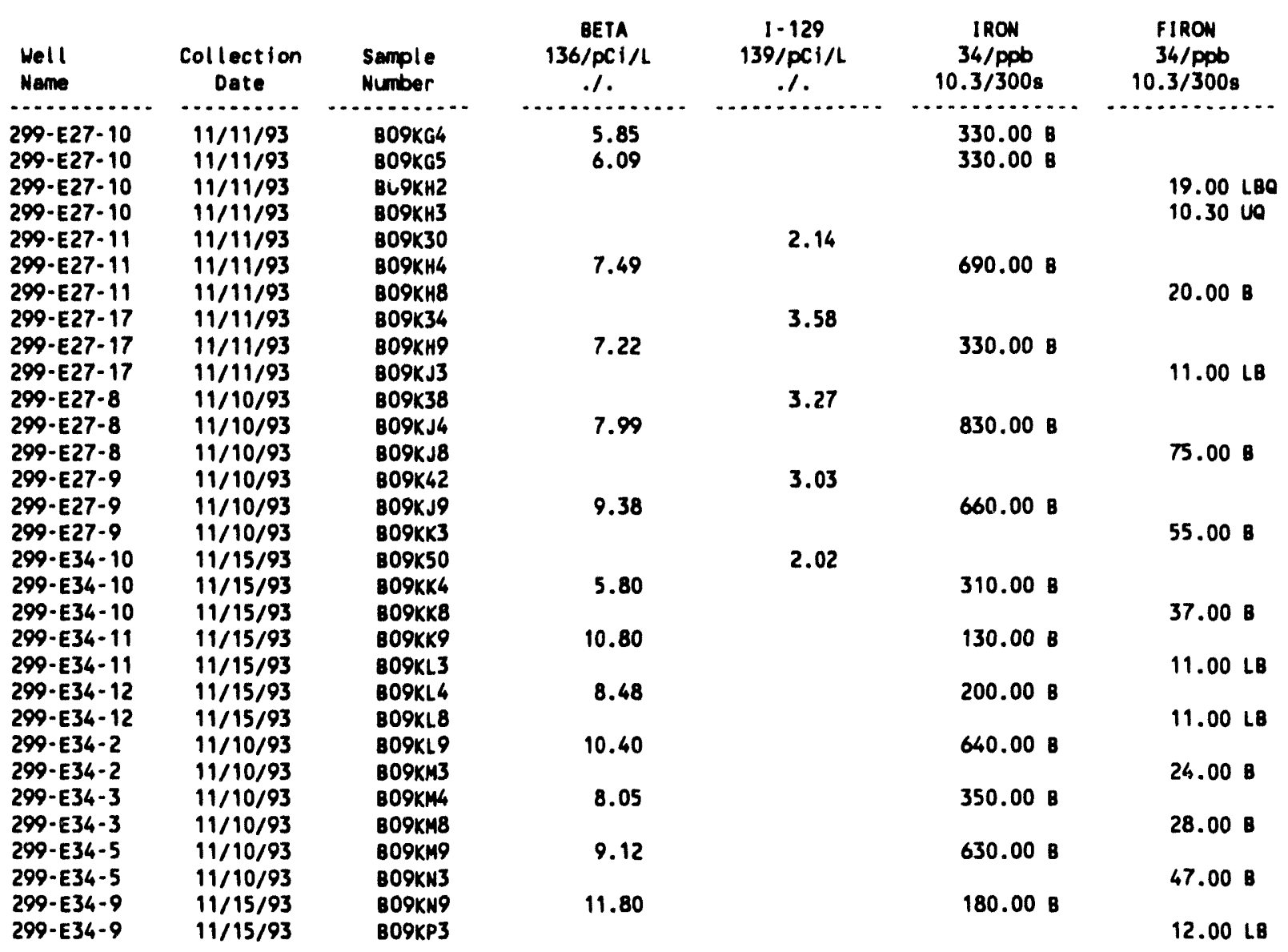

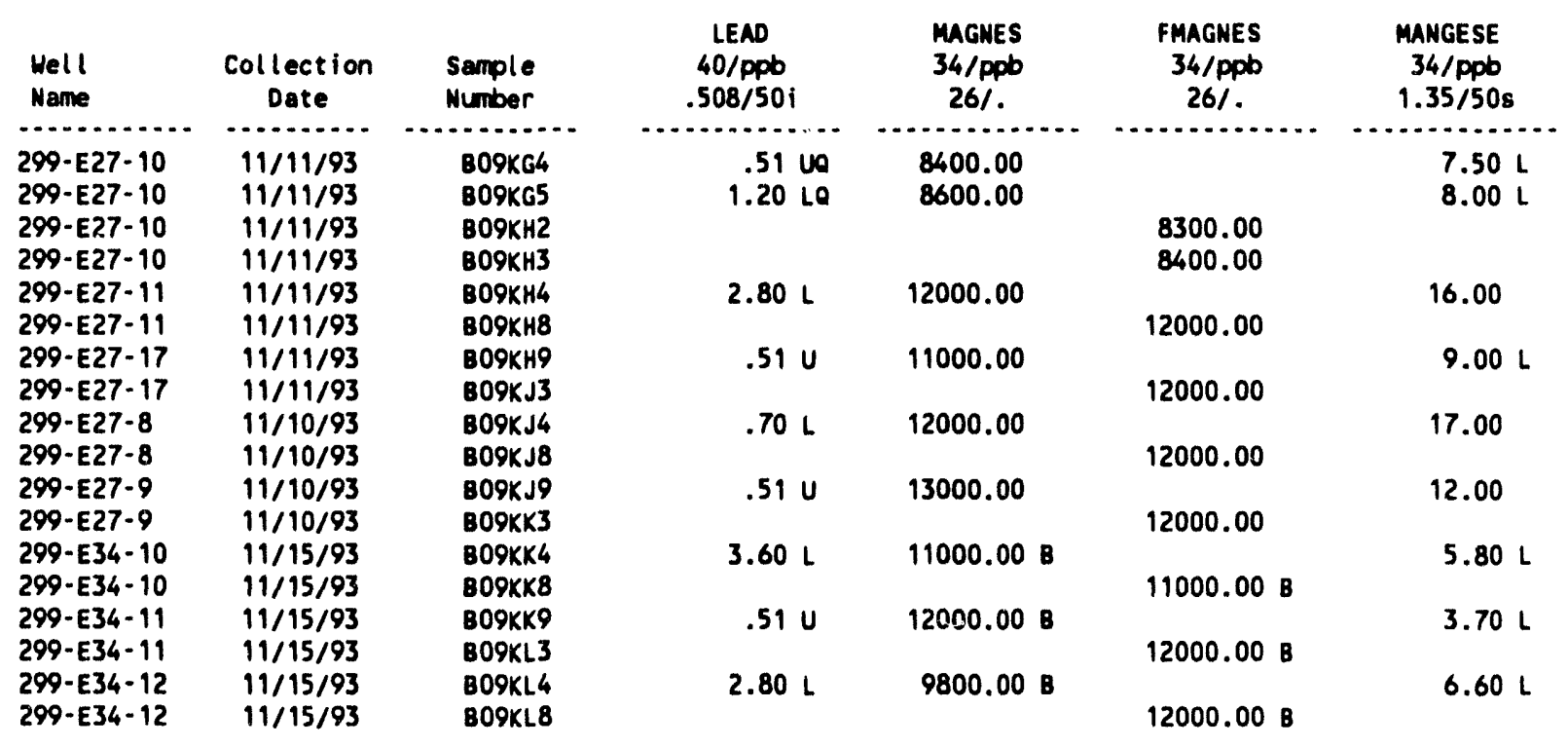


Table 15-16. Constituents with at Least One Detected Value for the Low-Level Waste Burial Grounds WMA 2 Data for Reporting Period October 1

through December 31, 1993. (sheet 5 of 8 )

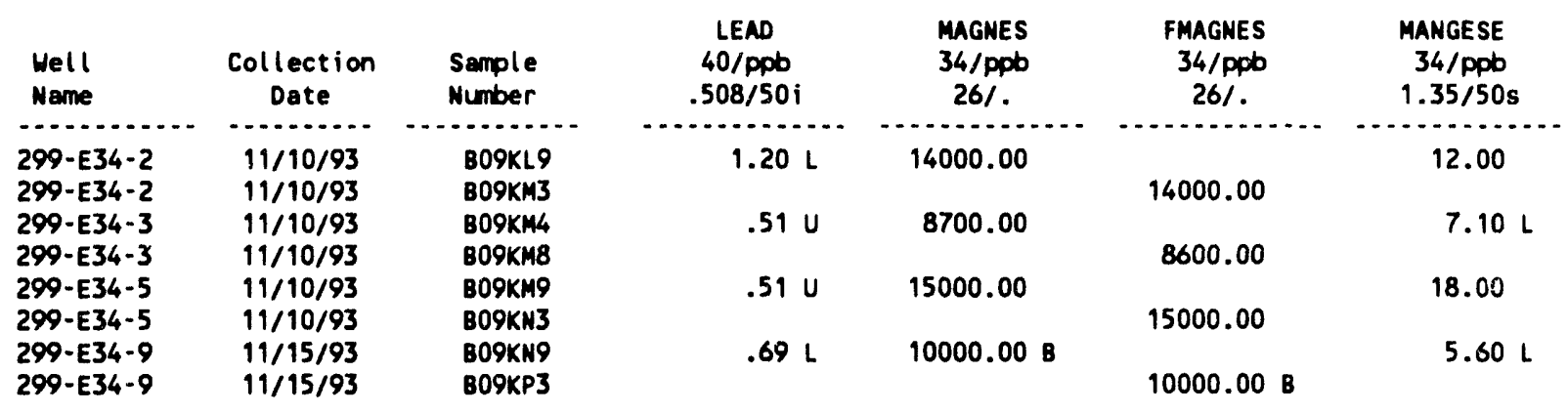

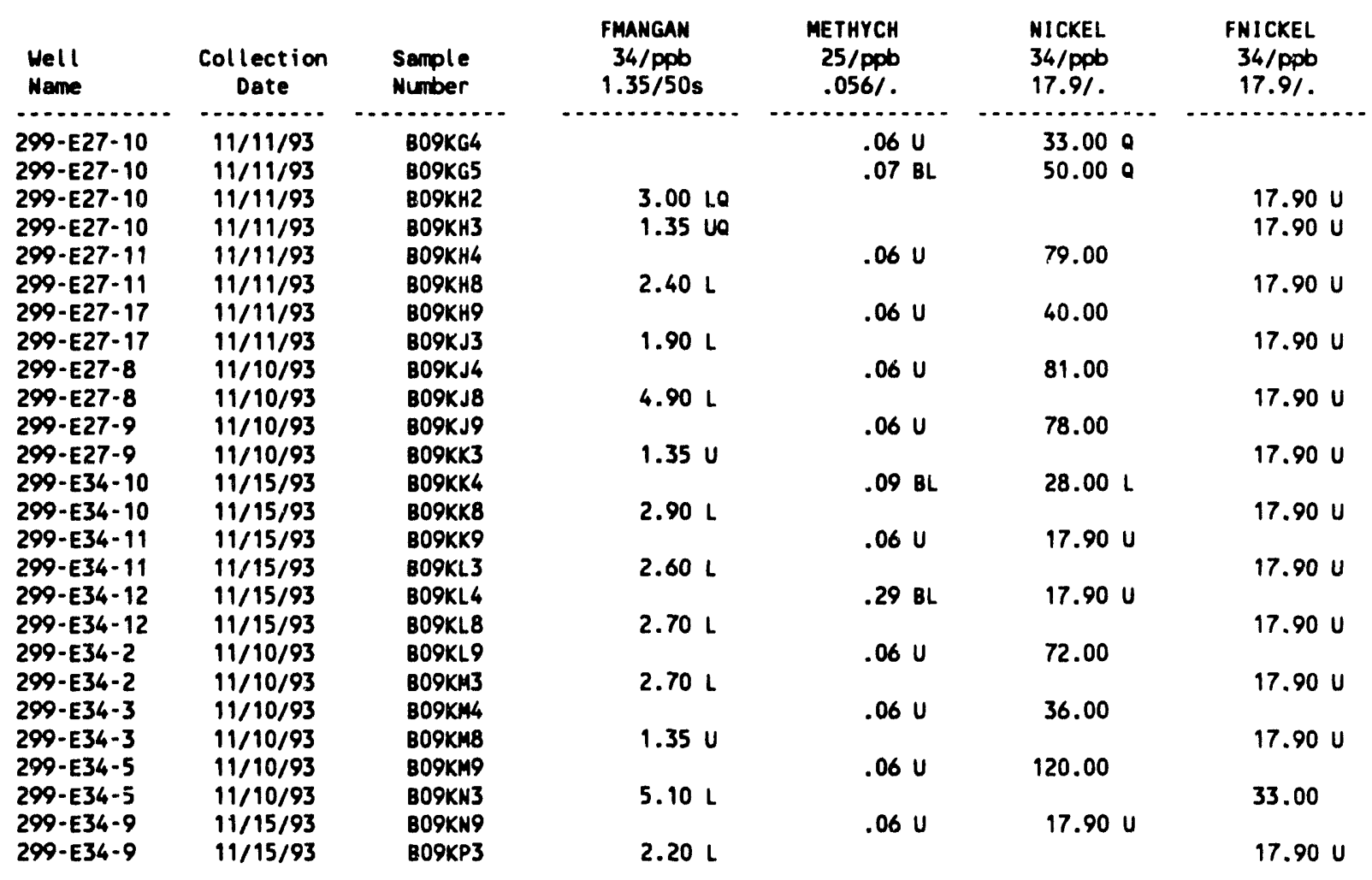

\begin{tabular}{|c|c|c|c|c|c|c|}
\hline $\begin{array}{l}\text { Well } \\
\text { Name }\end{array}$ & $\begin{array}{l}\text { Collection } \\
\text { Date }\end{array}$ & $\begin{array}{l}\text { Sample } \\
\text { Number }\end{array}$ & $\begin{array}{l}\text { NITRATE } \\
\text { 124/ppb } \\
44.4 / 45000\end{array}$ & $\begin{array}{c}\text { POTASUM } \\
34 / \mathrm{ppb} \\
662 \% .\end{array}$ & $\begin{array}{c}\text { FPOTASS } \\
34 / \mathrm{ppb} \\
662 \% .\end{array}$ & $\begin{array}{c}\text { RADIUM } \\
137 / \mathrm{pCi} / L \\
.15 \mathrm{i}\end{array}$ \\
\hline $\begin{array}{l}299-E 27-10 \\
299-E 27-10\end{array}$ & $\begin{array}{l}11 / 11 / 93 \\
11 / 11 / 93\end{array}$ & $\begin{array}{l}\text { B09KG4 } \\
\text { B09KG5 }\end{array}$ & $\begin{array}{l}3500.00 \mathrm{~B} \\
3600.00 \mathrm{~B}\end{array}$ & $\begin{array}{l}5800.00 \\
5200.00\end{array}$ & & $\begin{array}{l}.03 \mathrm{U} \\
.09 \mathrm{U}\end{array}$ \\
\hline $\begin{array}{l}299-E 27-10 \\
299-E 27-10\end{array}$ & $\begin{array}{l}11 / 11 / 93 \\
11 / 11 / 93\end{array}$ & $\begin{array}{l}\text { BO9KH2 } \\
\text { B09KH3 }\end{array}$ & & & $\begin{array}{l}5600.00 \\
5600.00\end{array}$ & \\
\hline 299-E27-11 & $11 / 11 / 93$ & B09KH4 & $9700.00 \mathrm{~B}$ & 6900.00 & & .17 \\
\hline 299-E27-11 & $11 / 11 / 93$ & 809KH8 & & & 6900.00 & \\
\hline
\end{tabular}


Table 15-16. Constituents with at Least One Detected Value for the Low-Level Waste Burial Grounds WMA 2 Data for Reporting Period October 1

through December 31, 1993. (sheet 6 of 8)

\begin{tabular}{|c|c|c|c|c|c|c|}
\hline $\begin{array}{l}\text { Well } \\
\text { Name }\end{array}$ & $\begin{array}{c}\text { Collection } \\
\text { Date }\end{array}$ & $\begin{array}{l}\text { Sample } \\
\text { Number }\end{array}$ & $\begin{array}{l}\text { NI TRATE } \\
\text { 124/ppb } \\
44.4 / 45000\end{array}$ & $\begin{array}{c}\text { POTASUM } \\
34 / \text { ppb } \\
662 /\end{array}$ & $\begin{array}{c}\text { FPOTASS } \\
34 / \mathrm{ppb} \\
662 / .\end{array}$ & $\begin{array}{c}\text { RADIUM } \\
137 / \mathrm{pCi} / \mathrm{L} \\
. / 5 \mathrm{i}\end{array}$ \\
\hline $299-\mathrm{E} 27-17$ & $11 / 11 / 93$ & В09КH9 & 7900.00 B & 6700.00 & & .14 \\
\hline $299-E 27-17$ & $11 / 11 / 93$ & B09KJ3 & & & 6200.00 & \\
\hline 299-E27-8 & $11 / 10 / 93$ & B09KJ4 & $8100.00 \mathrm{BD}$ & 7000.00 & & .24 \\
\hline $299-E 27-8$ & $11 / 10 / 93$ & B09KJ8 & & & 7100.00 & \\
\hline 299-E27-9 & $11 / 10 / 93$ & B09KJ9 & $9500.00 \mathrm{~B}$ & 6300.00 & & $.11 \mathrm{U}$ \\
\hline 299-E27-9 & $11 / 10 / 93$ & B09KK3 & & & 6100.00 & \\
\hline $299-E 34-10$ & $11 / 15 / 93$ & B09KK4 & 7200.00 & 6200.00 & & $.12 U$ \\
\hline $299-E 34-10$ & $11 / 15 / 93$ & B09KK8 & & & 6100.00 & \\
\hline $299-E 34-11$ & $11 / 15 / 93$ & B09KK9 & $12000.00 \mathrm{D}$ & 6300.00 & & $.11 \mathrm{U}$ \\
\hline $299-E 34-11$ & $11 / 15 / 93$ & B09KL3 & & & 6400.00 & \\
\hline $299-E 34-12$ & $11 / 15 / 93$ & B09KL4 & 6000.00 & 5400.00 & & $.10 \mathrm{U}$ \\
\hline $299-E 34-12$ & $11 / 15 / 93$ & B09KL8 & & & 5900.00 & \\
\hline 299-E34-2 & $11 / 10 / 93$ & B09KL9 & $11000.00 \mathrm{BD}$ & 7300.00 & & .22 \\
\hline $299-E 34-2$ & $11 / 10 / 93$ & 809kM3 & & & 7000.00 & \\
\hline $299-E 34-3$ & $11 / 10 / 93$ & B09KM4 & $5200.00 \mathrm{~B}$ & 6700.00 & & $.12 \mathrm{U}$ \\
\hline $299-E 34-3$ & $11 / 10 / 93$ & B09KM8 & & & 6600.00 & \\
\hline $299-E 34-5$ & $11 / 10 / 93$ & B09KM9 & $13000.00 \mathrm{BD}$ & 8300.00 & & .23 \\
\hline $299-E 34-5$ & $11 / 10 / 93$ & B09KN3 & & & 7800.00 & \\
\hline $299-E 34-9$ & $11 / 15 / 93$ & B09KN9 & $13000.00 \mathrm{D}$ & 6200.00 & & $.02 \mathrm{U}$ \\
\hline 299-E34-9 & $11 / 15 / 93$ & B09KP3 & & & .00 & \\
\hline
\end{tabular}

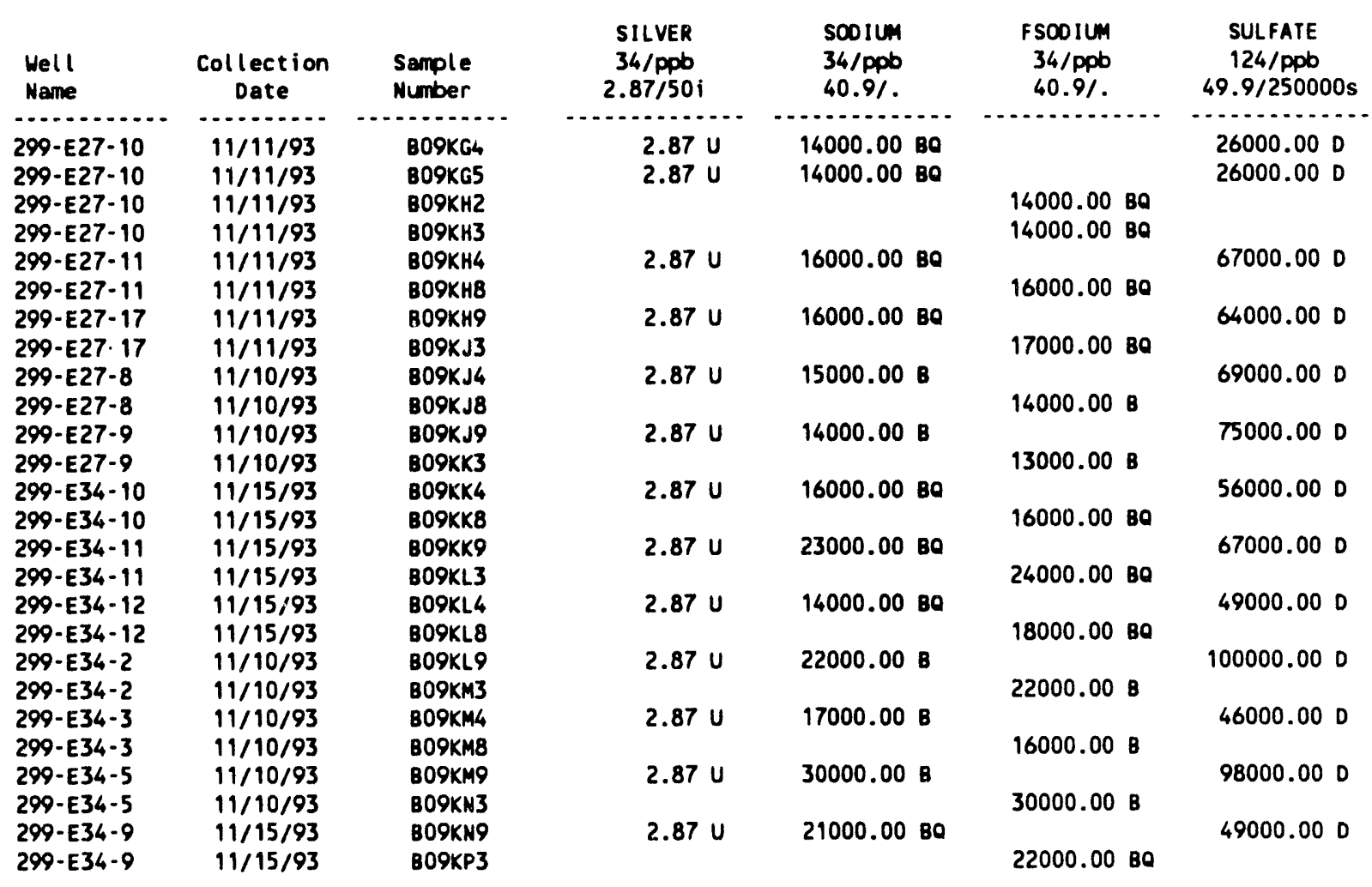


Table 15-16. Constituents with at Least One Detected Value for the Low-Level Waste Burial Grounds WMA 2 Data for Reporting Period October 1 through December 31, 1993. (sheet 7 of 8)

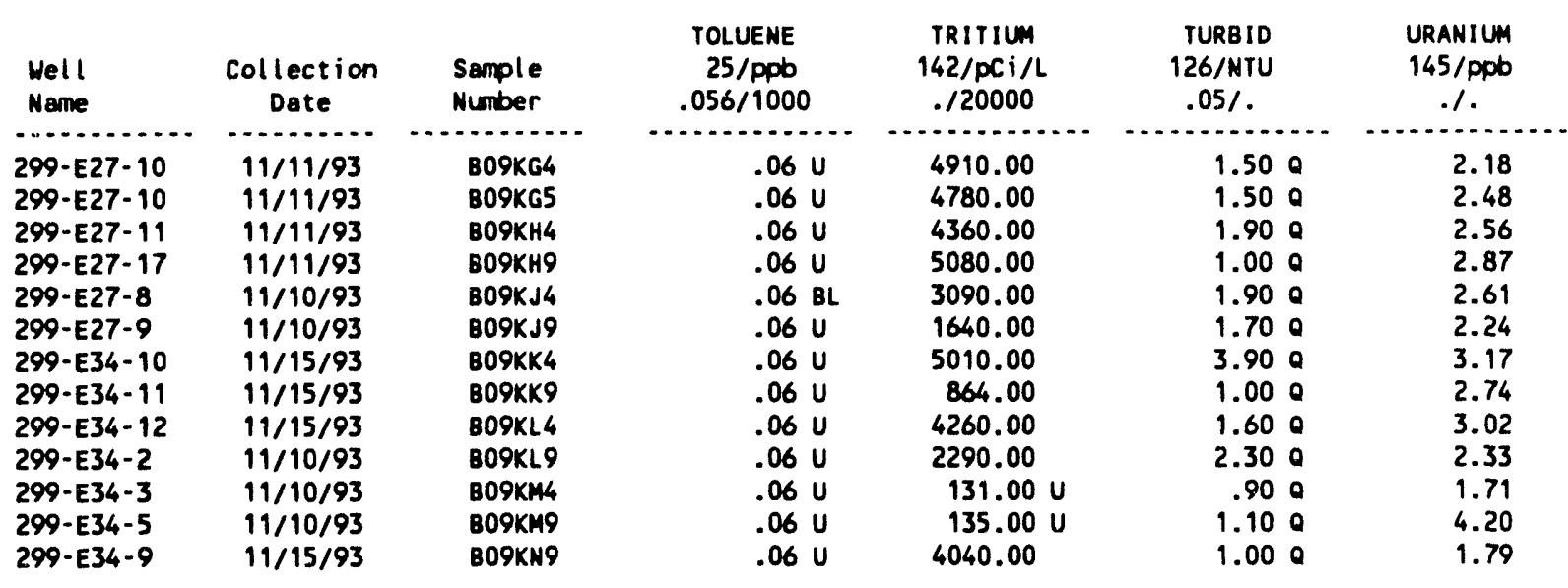

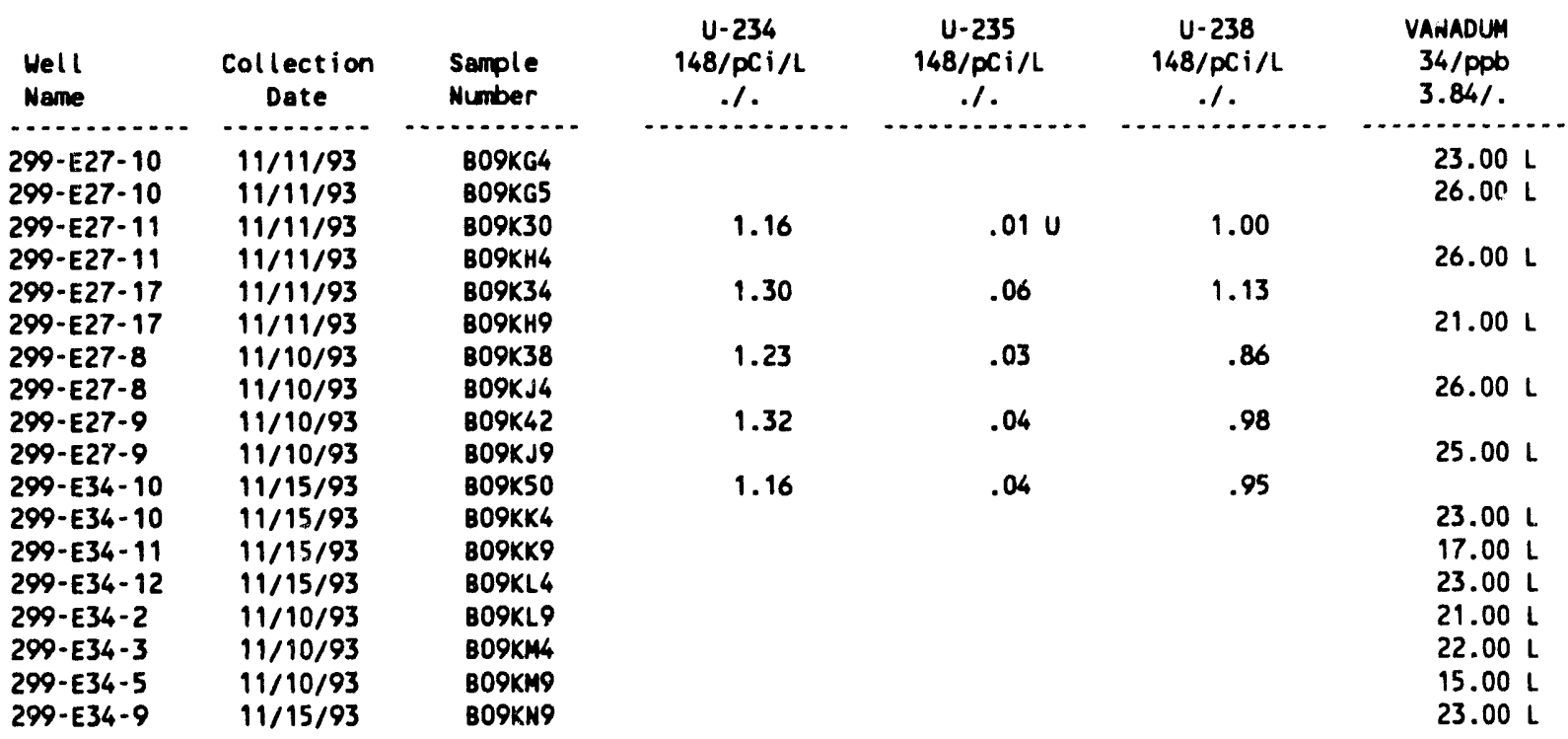

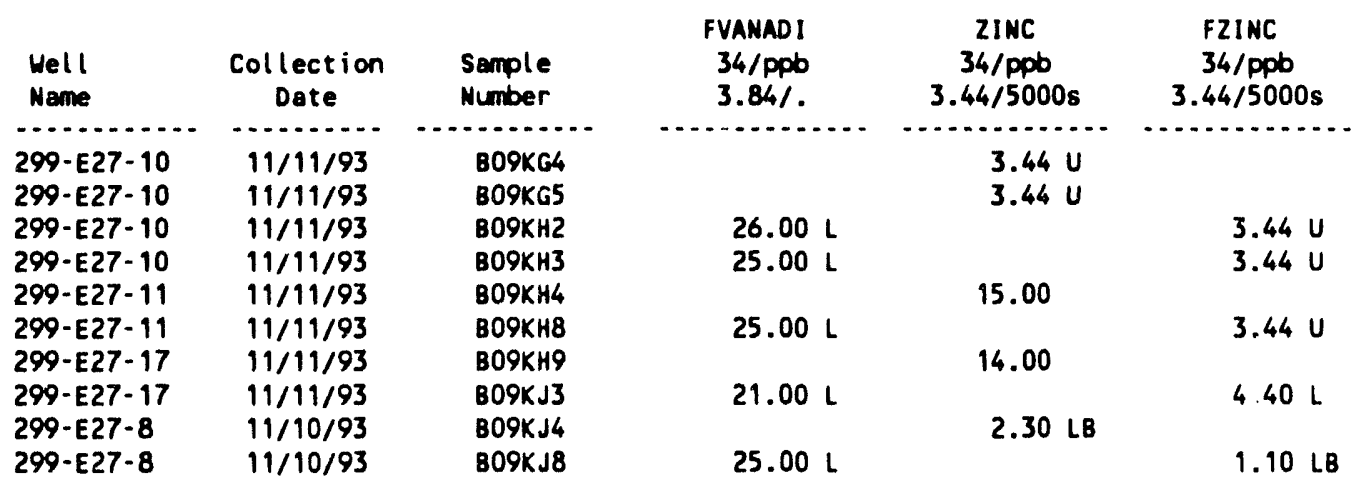


Table 15-16. Constituents with at Least One Detected Value for the Low-Level Waste Burial Grounds WMA 2 Data for Reporting Period October 1

through December 31, 1993. (sheet 8 of 8)

\begin{tabular}{|c|c|c|c|c|c|}
\hline $\begin{array}{l}\text { Well } \\
\text { Name }\end{array}$ & $\begin{array}{c}\text { Collection } \\
\text { Date }\end{array}$ & $\begin{array}{l}\text { Sample } \\
\text { Number }\end{array}$ & $\begin{array}{c}\text { FVANADI } \\
34 / \mathrm{ppb} \\
3.84 / .\end{array}$ & $\begin{array}{c}\text { ZINC } \\
34 / \mathrm{ppb} \\
3.44 / 5000 \mathrm{~s}\end{array}$ & $\begin{array}{c}\text { F21NC } \\
34 / \mathrm{ppb} \\
3.44 / 5000 \mathrm{~s}\end{array}$ \\
\hline $299-E 27-9$ & $11 / 10 / 93$ & 809kJ9 & & $3.44 \mathrm{U}$ & \\
\hline 299-E27-9 & $11 / 10 / 93$ & $809 \mathrm{KK} 3$ & $21.00 \mathrm{~L}$ & 3.448 & $3.44 \mathrm{U}$ \\
\hline 299-E34- 10 & $11 / 15 / 93$ & B09KK4 & & $3.44 \mathrm{U}$ & \\
\hline $299-E 34-10$ & $11 / 15 / 93$ & B09KK8 & $22.00 \mathrm{~L}$ & & $3.44 \mathrm{U}$ \\
\hline $299-E 34-11$ & $11 / 15 / 93$ & B09KK9 & & $3.44 \mathrm{U}$ & \\
\hline $299-E 34-11$ & $11 / 15 / 93$ & $809 \mathrm{KL} 3$ & $17.00 \mathrm{~L}$ & & $3.44 \mathrm{U}$ \\
\hline $299-E 34-12$ & $11 / 15 / 93$ & $809 K L 4$ & & 6.40 LB & \\
\hline $299-E 34-12$ & $11 / 15 / 93$ & 809KL8 & $23.00 \mathrm{~L}$ & & $2.40 \mathrm{LB}$ \\
\hline 299-E34-2 & $11 / 10 / 93$ & $809 \mathrm{KL} 9$ & & $4.40 \mathrm{LB}$ & \\
\hline $299-E 34-2$ & $11 / 10 / 93$ & B09KM3 & $18.00 \mathrm{~L}$ & & $3.44 \mathrm{U}$ \\
\hline 299-E34-3 & $11 / 10 / 93$ & B09KM4 & & $3.44 \mathrm{U}$ & \\
\hline $299-E 34-3$ & $11 / 10 / 93$ & 809kM8 & $21.00 \mathrm{~L}$ & & $3.44 \mathrm{U}$ \\
\hline $\begin{array}{l}299-E 34-5 \\
299-E 34-5\end{array}$ & $\begin{array}{l}11 / 10 / 93 \\
11 / 10 / 93\end{array}$ & $\begin{array}{l}\text { B09KM9 } \\
\text { B09KN3 }\end{array}$ & 178 & 5.20 LB & $3.44 \mathrm{U}$ \\
\hline 299-E34-9 & $11 / 15 / 93$ & BO9KN9 & 18.0 & $3.44 \mathrm{U}$ & 3.440 \\
\hline $299-E 34-9$ & $11 / 15 / 93$ & B09KP3 & $23.00 \mathrm{~L}$ & & $3.44 \mathrm{U}$ \\
\hline
\end{tabular}

for explanation of this table, see Section 1.4 of report. 
Table 15-17. Contamination Indicator Parameters for the Low-Level Waste Burial Grounds WMA 2 Data for Reporting Period October 1 through December 31, 1993. (sheet 1 of 2)

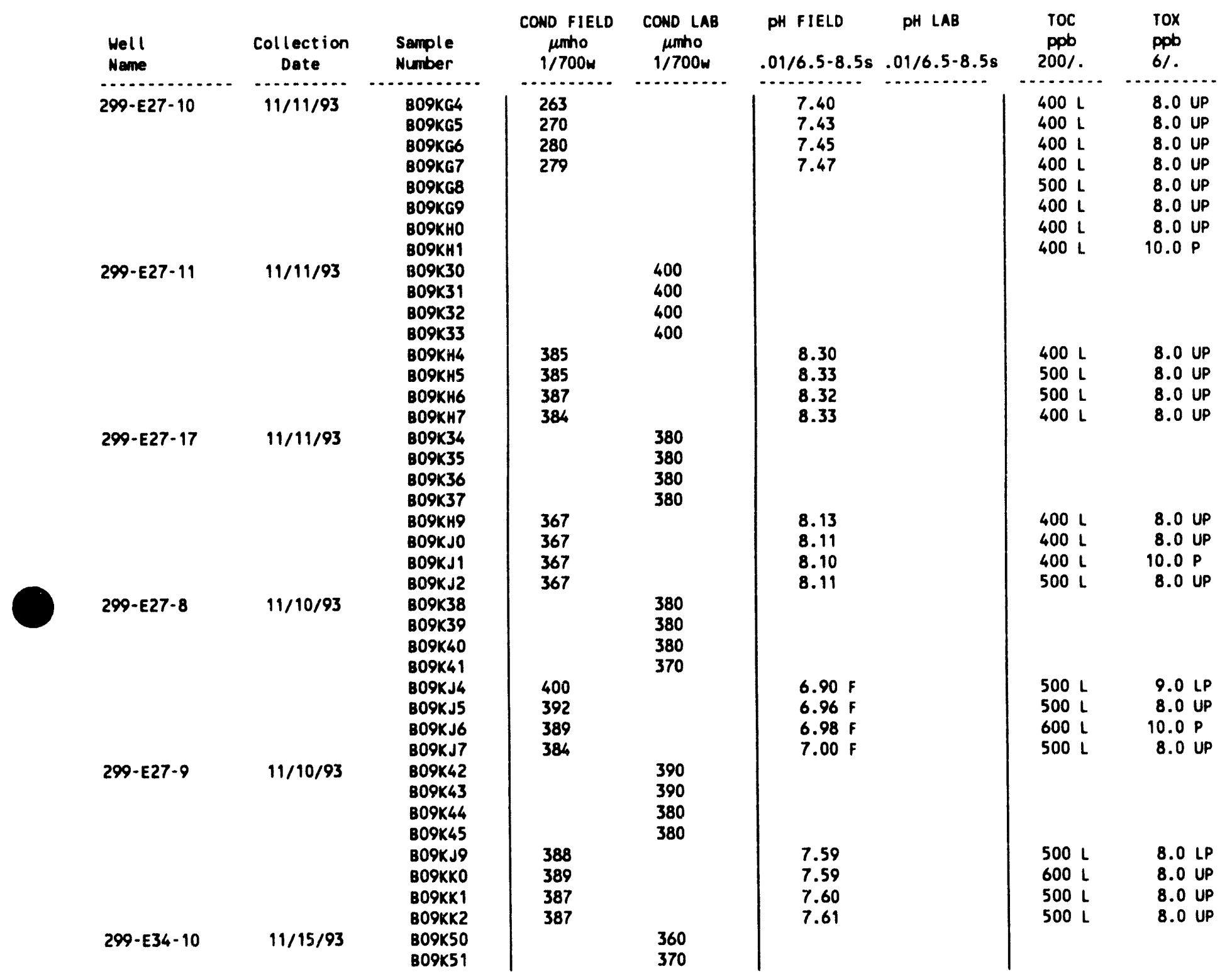


Table 15-17. Contamination Indicator Parameters for the Low-Level Waste Burial Grounds WMA 2 Data for Reporting Period October 1 through December 31, 1993.

(sheet 2 of 2)

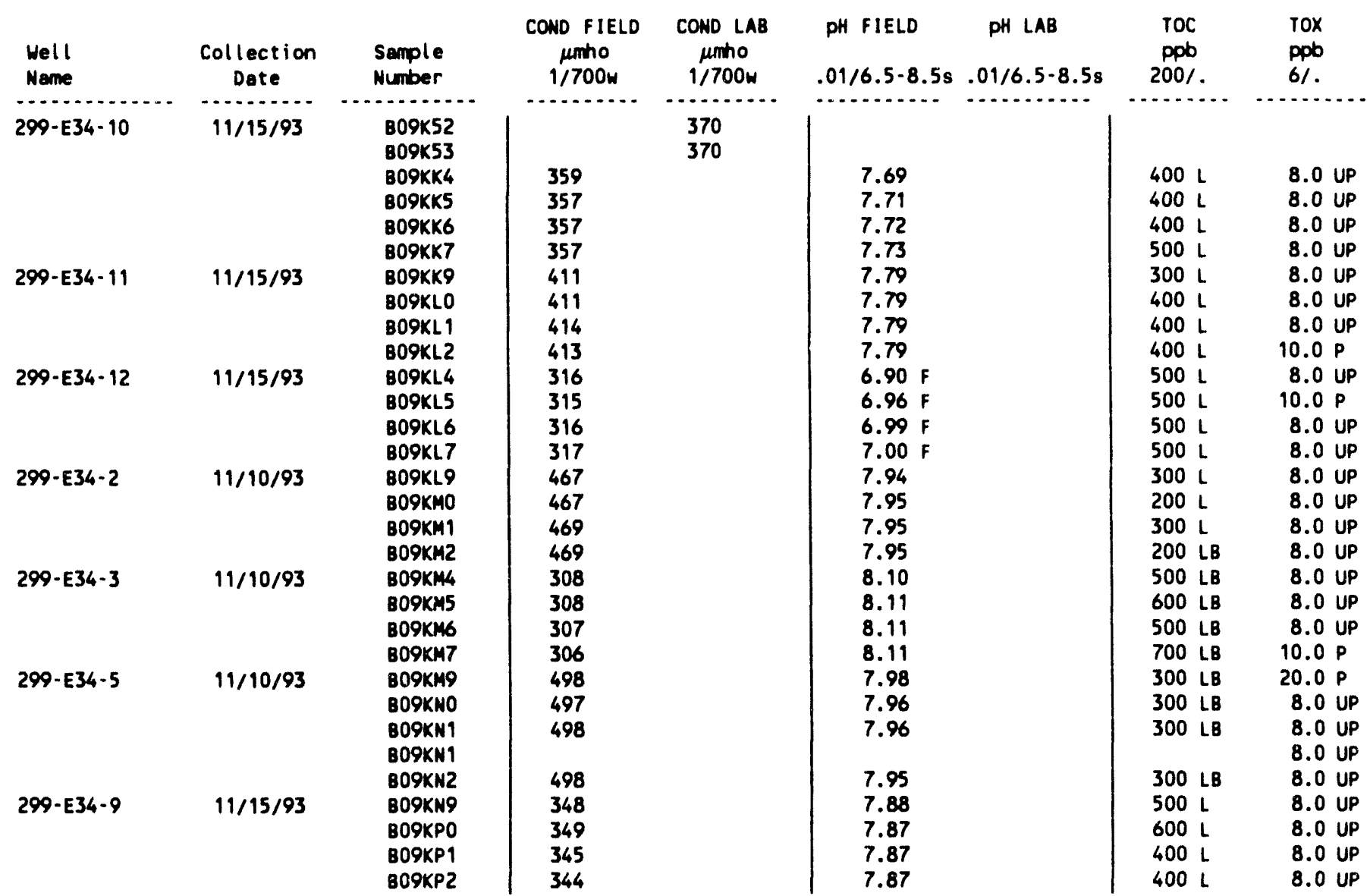


Table 15-18. Constituent List and Summary of Results for the Low-Level Waste Burial Grounds WMA 3 Data for Reporting Period October 1

through December 31, 1993. (sheet 1 of 3 )

CONTAMINATION INDICATOR PARAMETERS

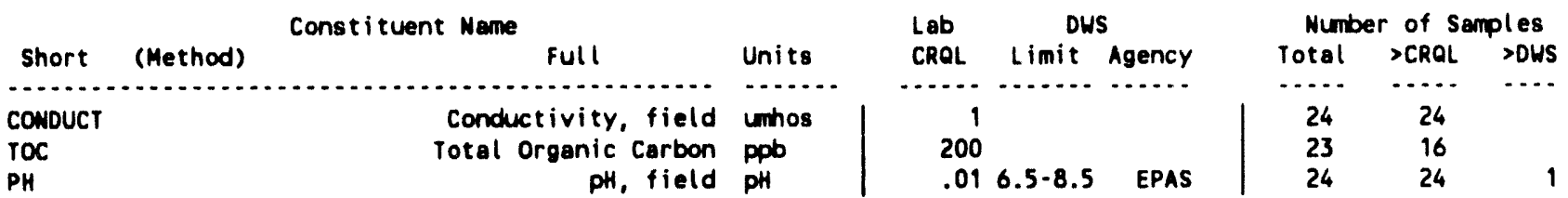

ORINKING WATER PARAMETERS

\begin{tabular}{|c|c|c|c|c|c|c|c|c|c|}
\hline \multirow[b]{2}{*}{ Short } & \multirow[b]{2}{*}{ (Method) } & \multirow{2}{*}{$\begin{array}{l}\text { Constituent Nane full } \\
\text { Ful }\end{array}$} & \multirow[b]{2}{*}{ Units } & \multirow{2}{*}{$\begin{array}{l}\text { Lab } \\
\text { CROL }\end{array}$} & \multicolumn{2}{|c|}{ DWS } & \multicolumn{3}{|c|}{ Number of Senples } \\
\hline & & & & & Limit & Agency & Total & $>$ CROL & $>$ OWS \\
\hline $2,4,5 T P$ & & $2,4,5-T P$ & ppb & .408 & 50 & EPA & 21 & 1 & 0 \\
\hline $2,4-0$ & & $2,4-0$ & ppb & .186 & 100 & EPAI & 21 & 2 & 0 \\
\hline ARSENIC & & Arsenic & ppb & 1.38 & 50 & EPA & 20 & 12 & 0 \\
\hline FARSENIC & & Arsenic, filtered & ppb & 1.38 & 50 & EPA & 20 & 16 & 0 \\
\hline BARIUM & & Barium & ppb & .001 & 1000 & EPAI & 21 & 21 & 0 \\
\hline FBARIUM & & Barium, filtered & ppb & .001 & 1000 & EPAI & 21 & 21 & 0 \\
\hline CADMIUM & & Cadmiun & ppb & 4.7 & 10 & EPAI & 21 & 0 & 0 \\
\hline FCADMIU & & Cadmium, filtered & ppo & 4.7 & 10 & EPAI & 21 & 0 & 0 \\
\hline CHROMUM & & Chromiun & ppb & 5.42 & 50 & EPAI & 21 & 21 & 17 \\
\hline FCHROMI & & Chromiun, filtered & ppb & 5.42 & 50 & EPAI & 21 & 19 & 0 \\
\hline ENDRIN & & Endr in & ppb & .008 & .2 & EPA & 20 & 0 & 0 \\
\hline FLUORID & & Fluoride & ppb & 13.9 & 1400 & EPAI & 20 & 20 & 0 \\
\hline ALPHA & & Gross alpha & $\mathrm{PC} i / \mathrm{L}$ & & 15 & EPA & 20 & 16 & 0 \\
\hline MERCURY & & Mercury & ppb & .158 & 2 & EPA & 20 & 0 & 0 \\
\hline FMERCUR & & Mercury, filtered & ppb & .158 & 2 & EPA & 20 & 0 & 0 \\
\hline METHLOR & & Methoxychlor & ppb & .1 & 100 & EPAI & 20 & 0 & 0 \\
\hline MITRATE & & Nitrate & ppb & 44.4 & 45000 & EPA & 20 & 20 & 5 \\
\hline RADIUM & & Radium & $\mathrm{PC} i / \mathrm{L}$ & & 5 & EPAI & 20 & 13 & 0 \\
\hline SELENUM & & Selenium & ppb & 1.21 & 10 & EPAI & 20 & 12 & 0 \\
\hline FSELENI & & Selenium, filtered & ppo & 1.21 & 10 & EPAI & 20 & 12 & 0 \\
\hline TOXAENE & & Toxaphene & ppb & .89 & 5 & EPAI & 20 & 0 & 0 \\
\hline g-BHC & & gamm-BHC (Lindane) & ppo & .0021 & 4 & EPAI & 20 & 0 & 0 \\
\hline
\end{tabular}

GROUNOWATER QUALITY PARAMETERS

\begin{tabular}{|c|c|c|c|c|c|c|c|c|c|}
\hline \multirow[b]{2}{*}{ Short } & \multicolumn{2}{|r|}{ Constituent Name } & \multirow[b]{2}{*}{ Units } & \multirow{2}{*}{$\begin{array}{l}\text { Lab } \\
\text { CRQL }\end{array}$} & \multicolumn{2}{|c|}{ DWS } & \multicolumn{3}{|c|}{ Number of Semples } \\
\hline & (Method) & Full & & & Limit & Agency & Total & $>C R Q L$ & $>$ DUS \\
\hline & & (n) & $\cdots$ & $\cdots .$. & -........ & a..... & $\cdots$ & $\cdots$ & $\cdots$ \\
\hline CHLORID & & $\begin{array}{c}\text { Chloride } \\
\text { Iron }\end{array}$ & ppb & $\begin{array}{l}82.5 \\
10.3\end{array}$ & $\begin{array}{r}250000 \\
300\end{array}$ & $\begin{array}{l}\text { EPAS } \\
\text { EPAS }\end{array}$ & $\begin{array}{l}20 \\
21\end{array}$ & $\begin{array}{l}20 \\
21\end{array}$ & $\begin{array}{r}0 \\
14\end{array}$ \\
\hline FIRON & & Iron, filtered & ppo & 10.3 & 300 & EPAS & 21 & 21 & 0 \\
\hline MANGESE & & Manganese & ppo & 1.35 & 50 & EPAS & 21 & 21 & 3 \\
\hline FMANGAN & & Manganese, filtered & ppb & 1.35 & 50 & EPAS & 21 & 17 & 0 \\
\hline LPHENOL & & Phenol & ppb & .31 & & & 20 & 0 & \\
\hline soolum & & Sodium & ppb & 40.9 & & & 21 & 21 & \\
\hline Fsoolum & & Sodium, filtered & ppb & 40.9 & & & 21 & 21 & \\
\hline SULFATE & & sulfate & $p p b$ & 49.9 & 250000 & EPAS & 20 & 20 & 0 \\
\hline
\end{tabular}


Table 15-18. Constituent List and Summary of Results for the Low-Level Waste Burial Grounds WMA 3 Data for Reporting Period October 1 through December 31,1993 . (sheet 2 of 3 )

SITE SPECIFIC AND OTHER CONSTITUENTS

\begin{tabular}{|c|c|c|c|c|c|c|c|c|c|}
\hline \multirow[b]{2}{*}{ Short } & \multirow[b]{2}{*}{ (Method) } & \multirow{2}{*}{$\begin{array}{l}\text { Constituent Name Full } \\
\text { for }\end{array}$} & \multirow[b]{2}{*}{ Units } & \multirow{2}{*}{$\begin{array}{l}\text { Lab } \\
\text { CROL }\end{array}$} & \multicolumn{2}{|c|}{ DWS } & \multicolumn{3}{|c|}{ Number of Samples } \\
\hline & & & & & Limit & Agency & Total & $>$ CRQL & $>$ DWS \\
\hline 1,1,1-T & 16 & $1,1,1-1$ richloroethane & ppb & .64 & 200 & EPA & 12 & 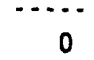 & 0 \\
\hline $1,1,1-1$ & 25 & $1,1,1$-Trichloroethane & ppb & .072 & 200 & EPA & 20 & 3 & 0 \\
\hline $1,1,2-T$ & 16 & $1,1,2-1$ richloroethane & ppb & .25 & & & 12 & 0 & \\
\hline $1,1,2-T$ & 25 & 1,1,2-Trichloroethane & ppob & .043 & & & 20 & 0 & \\
\hline $1,1-01 C$ & 16 & 1,1-Dichloroethane & ppb & .61 & & & 12 & 0 & \\
\hline $1,1 \cdot D 1 C$ & 25 & 1,1-Dichloroethane & ppb & .337 & & & 20 & 0 & \\
\hline $1,2-D 1 C$ & 16 & 1,2-Dichloroethane & ppob & .45 & 5 & EPA & 12 & 0 & 0 \\
\hline $1,2-01 C$ & 25 & 1.2-0ichloroethane & ppb & .139 & 5 & EPA & 20 & 0 & 0 \\
\hline $12-D C E$ & & 1,2-Dichloroethene & ppb & 1.2 & & & 12 & 0 & \\
\hline 14-cben & 16 & 1,4-0 ichlorobenzene & ppo & .63 & $\sqrt{3}$ & EPA & 12 & 0 & 0 \\
\hline 14-ctben & 25 & 1,4-Dichlordienzene & ppb & 107 & 75 & EPA & 20 & 0 & 0 \\
\hline 1BUTYN & & 1-Rutenol & ppm & 13.1 & & & 12. & 0 & \\
\hline $2,4,5-T$ & & $2,4,5-T$ & ppb & .384 & & & 21 & 2 & \\
\hline $\begin{array}{l}246-t r p \\
24-\text { dchp }\end{array}$ & & $\begin{array}{l}\text { 2,4,6-Trichlorophenol } \\
2,4-0 \text { ichlorophenol }\end{array}$ & $\begin{array}{l}\text { ppb } \\
\text { ppb }\end{array}$ & $\begin{array}{r}1.45 \\
1.5\end{array}$ & & & $\begin{array}{l}20 \\
20\end{array}$ & $\begin{array}{l}0 \\
0\end{array}$ & \\
\hline DIMPHEN & & 2,4-0 ime thyl phenol & ppb & 1.01 & & & 20 & 0 & \\
\hline DINPHEN & & 2,4-D initrophenol & ppb & .96 & & & 20 & 0 & \\
\hline 26-dchp & & 2,6-0ichlorophenol & ppb & 1.59 & & & 20 & 0 & \\
\hline CHLPHEN & & 2-Chlorophenol & ppb & 1.42 & & & 20 & 0 & \\
\hline 2NITPH & & 2-Nitrophenol & ppb & 1.56 & & & 20 & 0 & \\
\hline BUTDINP & 30 & 2-sec-Butyl-4,6-dinitrophenol (DN & ppb & 1.35 & & & 20 & 0 & \\
\hline BUTOINP & 49 & 2-sec-8utyl-4,6-dinitrophenol (DN & ppb & .269 & & & 21 & 0 & \\
\hline DDD & & $4,41-D D D$ & ppb & .0005 & & & 20 & 0 & \\
\hline DDE & & $4,4^{\prime}-D D E$ & ppo & .0009 & & & 20 & 0 & \\
\hline DDT & & $4,4^{\prime}-D D T$ & ppb & .011 & & & 20 & 0 & \\
\hline 460N2MP & & 4,6-0 initro-2-methylphenol & ppb & 1.18 & & & 20 & 0 & \\
\hline CHLCRES & & 4-Chloro-3-methylphenol & ppb & 1.12 & & & 20 & 0 & \\
\hline MIBK & & 4-Methyl-2-pentenone & ppob & .85 & & & 12 & 0 & \\
\hline NITPHEN & & 4-Nitrophenol & ppb & .65 & & & 20 & 0 & \\
\hline ACETONE & & Acetone & ppb & 13.4 & & & 12 & 0 & \\
\hline ALDRIN & & Aldrin & ppb & .05 & & & 20 & 0 & \\
\hline a-BHC & & Alpha-BHC & ppb & .012 & & & 20 & 0 & \\
\hline ALUMNUM & & Aluminum & ppb & 32.5 & & & 21 & 12 & \\
\hline FALUMIN & & Aluminum & ppb & 32.5 & & & 21 & 0 & \\
\hline ANTIONY & & Ant imony & ppb & 69.4 & & & 21 & 0 & \\
\hline FANTIMO & & Ant imony, filtered & ppob & 69.4 & & & 21 & 0 & \\
\hline BENZENE & 16 & Benzene & ppob & .65 & 5 & EPA & 12 & 0 & 0 \\
\hline BENZENE & 25 & Benzene & ppb & .105 & 5 & EPA & 20 & 6 & 0 \\
\hline BERYLUM & & Beryllium & ppb & .814 & & & 21 & 0 & \\
\hline FBERYLL & & Berylliun, filtered & ppb & .814 & & & 21 & 0 & \\
\hline b-BHC & & Beta-BHC & ppb & .0026 & & & 20 & 0 & \\
\hline BROMIDE & & Bromide & ppob & 52.8 & & & 20 & 12 & \\
\hline CALCIUN & & Colcium & ppb & 20.9 & & & 21 & 21 & \\
\hline FCALCIU & & Calcium, filtered & ppb & 20.9 & & & 21 & 21 & \\
\hline CARBIDE & & Carbon disulfide & ppb & .95 & & & 12 & 0 & \\
\hline TETRANE & 16 & Carbon tetrachloride & ppb & .87 & 5 & EPA & 12 & 6 & 5 \\
\hline TETRANE & 25 & Carbon tetrachloride & ppb & .121 & 5 & EPA & 20 & 19 & 9 \\
\hline CHLOANE & & Chlordene & ppb & .0057 & 2 & EPA & 20 & 0 & 0 \\
\hline CHLFORM & 16 & Chloroform & ppb & .4 & & & 12 & 0 & \\
\hline CHLFORM & 25 & Chloroform & ppb & .043 & & & 20 & 20 & \\
\hline COBALT & & Cobalt & ppb & 4.05 & & & 21 & 0 & \\
\hline FCOBALT & & Cobelt, filtered & ppb & 4.05 & & & 21 & 0 & \\
\hline COLIFORM & & Coliforms & $\mathrm{COL}$ & 1 & 1 & EPAI & 20 & 0 & 0 \\
\hline
\end{tabular}


Table 15-18. Constituent List and Summary of Results for the Low-Level Waste Burial Grounds WMA 3 Data for Reporting Period October 1 through December 31, 1993. (sheet 3 of 3)

\begin{tabular}{|c|c|c|c|c|c|c|c|c|c|}
\hline & & Const ituent Name & & Lab & DUS & & Numb & $r$ of 58 & ples \\
\hline Short & (Method) & Full & Units & CROL & Limit & Agency & Total & $>$ CROL & $>$ DUS \\
\hline COPPER & & Copper & pob & 2.65 & 1000 & EPAS & 21 & 9 & 0 \\
\hline FCOPPER & & Copper, filtered & ppb & 2.65 & 1000 & EPAS & 21 & 1 & 0 \\
\hline CRESOLS & & Cresols (methylphenols) & ppb & 4.66 & & & 20 & 0 & \\
\hline CYANIDE & & Cyanide & ppb & 1.24 & & & 1 & 1 & \\
\hline d-BHC & & Delta-BHC & ppb & .0014 & & & 20 & 1 & \\
\hline DIELRIN & & Dieldrin & ppb & .019 & & & 20 & 0 & \\
\hline ENDO1 & & Endosul fan I & ppb & .0029 & & & 20 & 0 & \\
\hline ENDO2 & & Endosul fan II & ppb & .004 & & & 20 & 0 & \\
\hline EMDSFAN & & Endosulfan sulfate & ppb & .0072 & & & 20 & 0 & \\
\hline ENORALD & & Endrin Aldehyde & ppb & .011 & & & 20 & 0 & \\
\hline ETHCYAN & & Ethyl cyanide & ppb & 4.34 & & & 12 & 0 & \\
\hline ETHBENZ & & Ethylbenzene & ppb & .046 & 700 & EPA & 20 & 1 & 0 \\
\hline BETA & & Gross beta & $\mathrm{PCI} / \mathrm{L}$ & & & & 20 & 20 & \\
\hline HEPTLOR & & Heptachlor & ppob & .0019 & .4 & EPA & 20 & 0 & 0 \\
\hline HEPTIDE & & Heptachlor epoxide & ppb & .0008 & .2 & EPA & 20 & 0 & 0 \\
\hline LEAD & & Lead & ppb & .508 & 50 & EPAI & 20 & 13 & 0 \\
\hline FLEAD & & Lead, filtered & ppob & .508 & 50 & EPAI & 20 & 13 & 0 \\
\hline MAGNES & & Magnesium & ppb & 26 & & & 21 & 21 & \\
\hline FMAGNES & & Magnesium, filtered & ppo & 26 & & & 21 & 21 & \\
\hline METHONE & & Methyl ethyl ketone & ppb & 4.25 & & & 12 & 0 & \\
\hline METHYCH & 16 & Methylene chloride & ppo & .61 & & & 12 & 0 & \\
\hline METHYCH & 25 & Methylene chloride & ppob & .056 & & & 20 & 15 & \\
\hline NICKEL & & Nickel & ppob & 17.9 & & & 21 & 17 & \\
\hline FNICKEL & & Nickel, filtered & ppb & 17.9 & & & 21 & 3 & \\
\hline NITRITE & & Nitrite & ppb & 38.3 & 1000 & EPA & 20 & 1 & 0 \\
\hline PENTCHP & & Pentachlorophenol & ppb & .87 & 1 & EPA & 20 & 0 & 0 \\
\hline PHOSPHA & & Phosphate & ppo & 147 & & & 20 & 0 & \\
\hline POTASUM & & Potassium & ppb & 662 & & & 21 & 21 & \\
\hline FPOTASS & & Potassium, filtered & ppb & 662 & & & 21 & 21 & \\
\hline SILVER & & Silver & ppo & 2.87 & 50 & EPAI & 21 & 1 & 0 \\
\hline FSILVER & & silver, filtered & ppb & 2.87 & 50 & EPAI & 21 & 2 & 0 \\
\hline PERCENE & 16 & Tetrachloroethene & ppb & 1.1 & 5 & EPA & 12 & 0 & 0 \\
\hline PERCENE & 25 & Tetrachloroethene & ppo & .049 & 5 & EPA & 20 & 6 & 0 \\
\hline TETPHNL & & Tetrachlorophenols & ppb & 1.05 & & & 20 & 0 & \\
\hline TAF & & Tetrahydrofuran & ppb & 3.67 & & & 12 & 0 & \\
\hline TIN & & Tin & ppb & 51.1 & & & 21 & 0 & \\
\hline FIIN & & Tin, filtered & ppb & 51.1 & & & 21 & 0 & \\
\hline TOLUENE & 16 & Tolvene & ppb & .73 & 1000 & EPA & 12 & 0 & 0 \\
\hline TOLUENE & 25 & Tolvene & $\mathrm{ppb}$ & .056 & 1000 & EPA & 20 & 1 & 0 \\
\hline TRICENE & 16 & Trichloroethene & ppob & .77 & 5 & EPA & 12 & 1 & 0 \\
\hline TRICENE & 25 & Irichloroethene & ppb & .065 & 5 & EPA & 20 & 8 & 2 \\
\hline TRIPHNL & & Trichlorophenols & ppb & 1.11 & & & 20 & 0 & \\
\hline TRITIUM & & Tritium & $\mathrm{pCi} / \mathrm{L}$ & & 20000 & EPA & 20 & 10 & 0 \\
\hline TURBID & & Turbidity & NTU & .05 & & & 20 & 20 & \\
\hline VANADUM & & Vanadium & ppob & 3.84 & & & 21 & 20 & \\
\hline FVANADI & & Vanadium, filtered & ppob & 3.84 & & & 21 & 20 & \\
\hline VINYIDE & 16 & Vinyl chloride & ppb & .78 & 2 & EPA & 12 & 0 & 0 \\
\hline VINYIDE & 25 & vinyl chloride & $p p b$ & .266 & 2 & EPA & 20 & 0 & 0 \\
\hline XYLENE & 16 & xylenes (total) & ppb & 1.7 & 10000 & EPA & 12 & 0 & 0 \\
\hline XYLENE & 25 & Xylenes (total) & ppb & .202 & 10000 & EPA & 20 & 0 & 0 \\
\hline ZINC & & 2 inc & ppob & 3.44 & 5000 & EPAS & 21 & 16 & 0 \\
\hline FZINC & & Zinc, filtered & ppo & 3.44 & 5000 & EPAS & 21 & 6 & 0 \\
\hline CIS12DE & & $\begin{array}{l}\text { cis-1,2-Dichloroethylene } \\
\text { trans-1,2-Dichloroethyl ene }\end{array}$ & ppob & $\begin{array}{l}.127 \\
.149\end{array}$ & $\begin{array}{r}70 \\
100\end{array}$ & $\begin{array}{l}\text { EPA } \\
\text { EPA }\end{array}$ & $\begin{array}{l}20 \\
20\end{array}$ & $\begin{array}{l}0 \\
0\end{array}$ & 0 \\
\hline TRANDCE & & & ppob & .149 & 100 & EPA & 20 & 0 & \\
\hline
\end{tabular}

For explanation of this table, see Section 1.4 of report. 
Table 15-19. Constituents with at Least One Detected Value for the Low-Level Waste Burial Grounds WMA 3 Data for Reporting Period October 1 through December 31, 1993. (sheet 1 of 13)

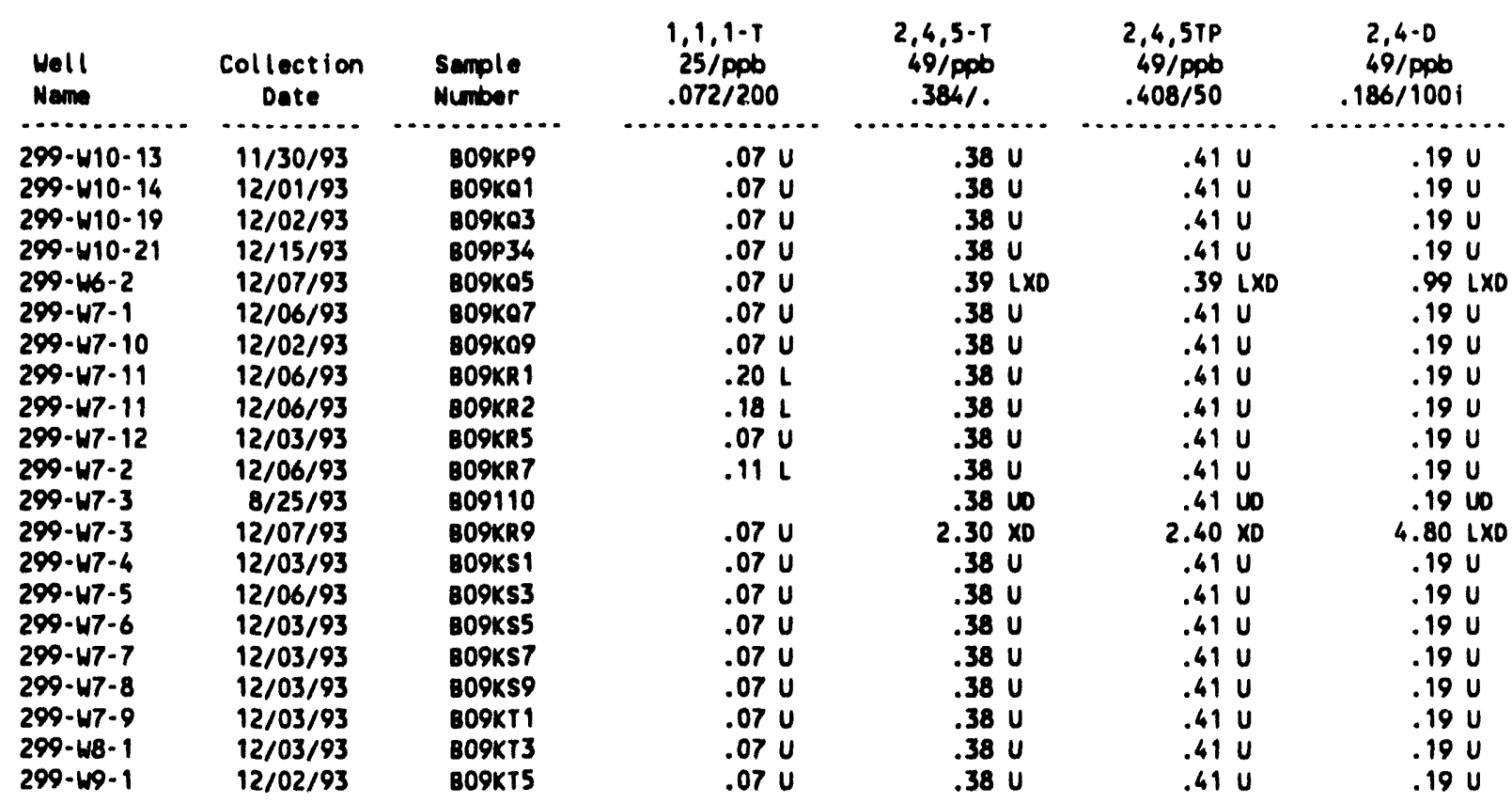

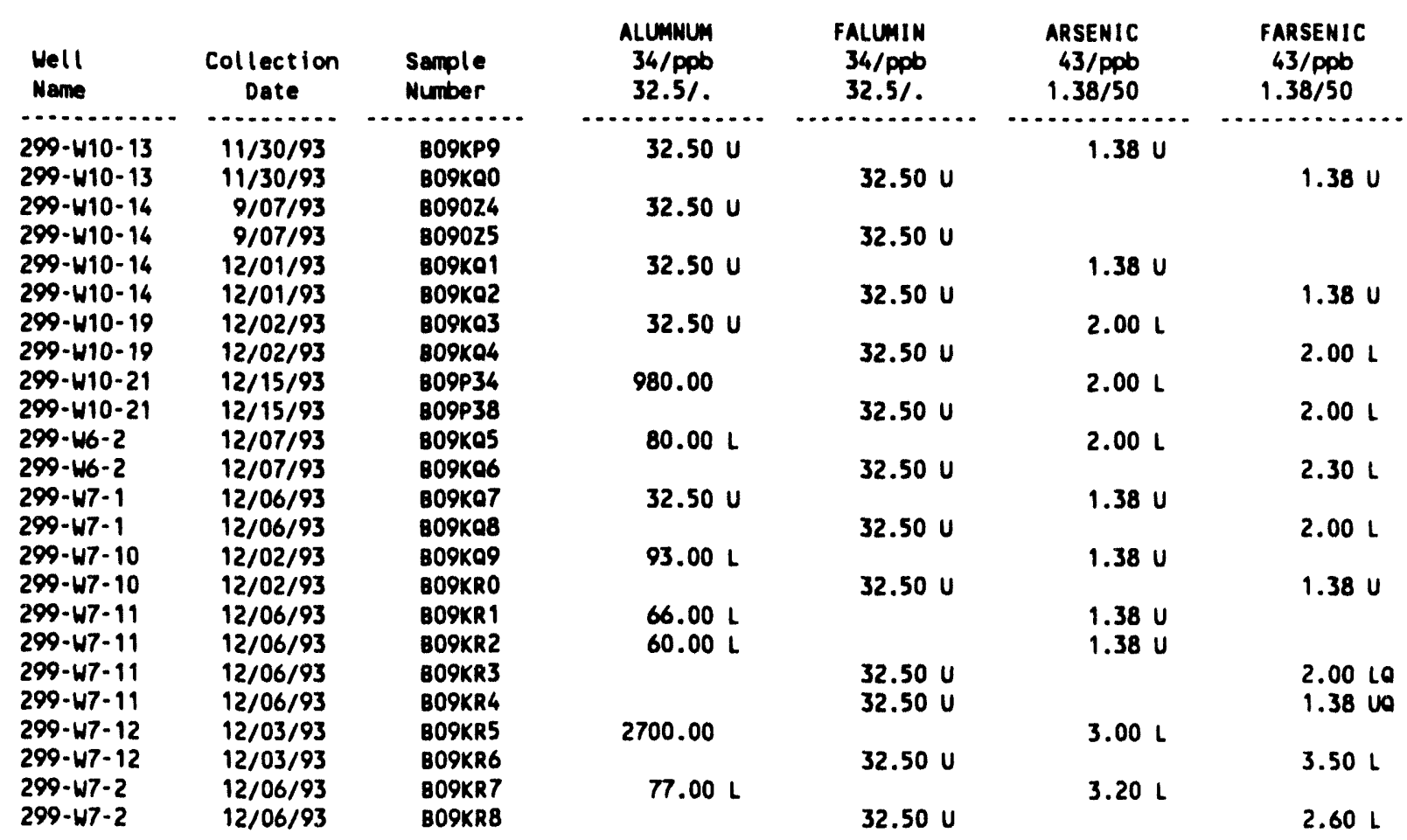


Table 15-19. Constituents with at Least One Detected Value for the Low-Level Waste Burial Grounds WMA 3 Data for Reporting Period October 1 through December 31, 1993. (sheet 2 of 13)

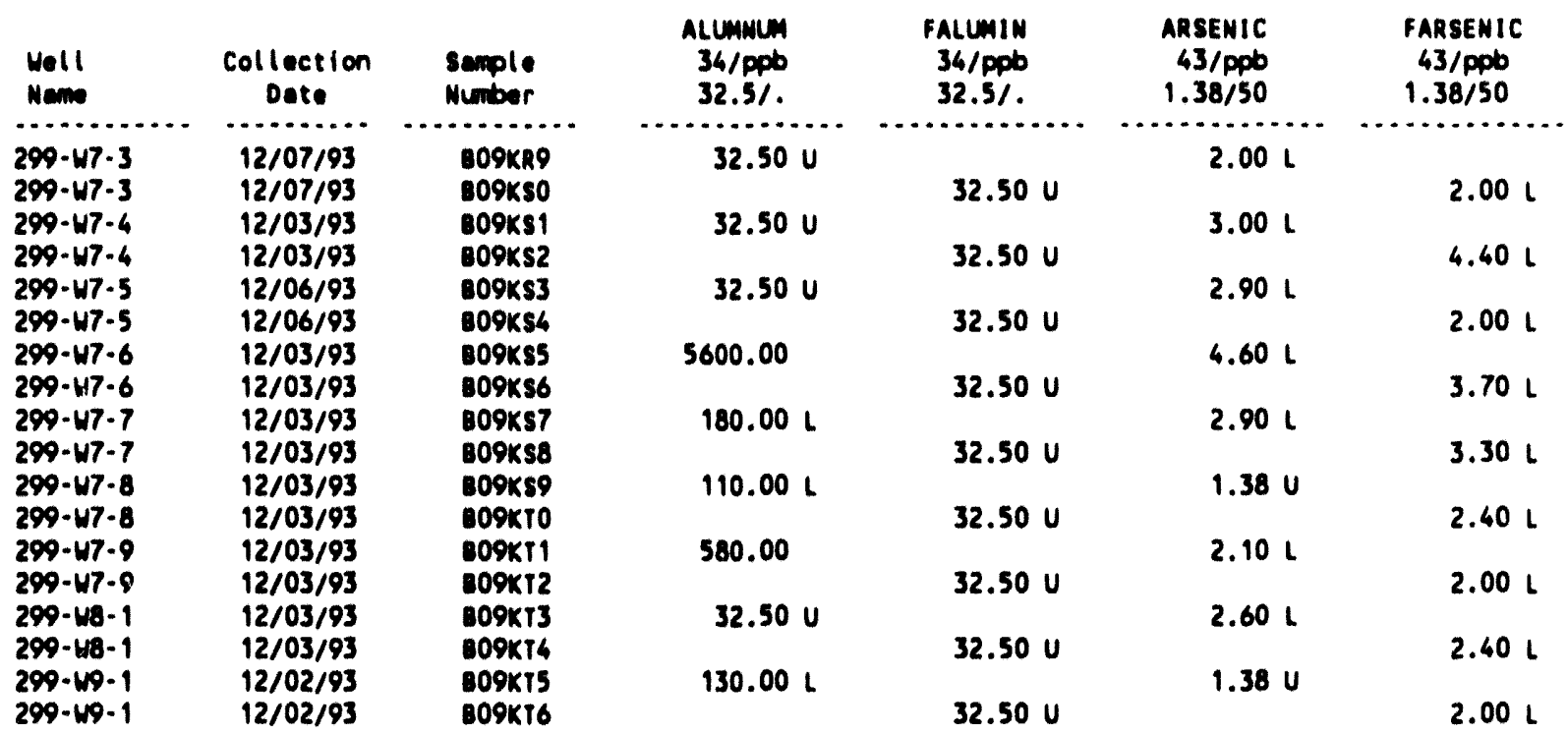

\begin{tabular}{|c|c|c|c|c|c|c|}
\hline $\begin{array}{l}\text { Well } \\
\text { Neme }\end{array}$ & $\begin{array}{c}\text { Collection } \\
\text { Date }\end{array}$ & $\begin{array}{l}\text { Sample } \\
\text { Number }\end{array}$ & $\begin{array}{c}\text { BARIUN } \\
34 / \text { ppb } \\
.001 / 1000 i\end{array}$ & $\begin{array}{l}\text { FBARIUN } \\
34 / \mathrm{ppb} \\
.001 / 10001\end{array}$ & $\begin{array}{r}\text { BENZENE } \\
25 / \mathrm{ppb} \\
.105 / 5\end{array}$ & $\begin{array}{c}\text { BAONIDE } \\
124 / \mathrm{ppb} \\
52.8 \%\end{array}$ \\
\hline $\begin{array}{l}299-W 10-13 \\
299-W 10-13 \\
299-W 10-14 \\
299-W 10-14\end{array}$ & $\begin{array}{l}11 / 30 / 93 \\
11 / 30 / 93 \\
9 / 07 / 93 \\
9 / 07 / 93\end{array}$ & $\begin{array}{l}809 K P 9 \\
809 K a 0 \\
809024 \\
809025\end{array}$ & $\begin{array}{l}44.00 \mathrm{BO} \\
68.00\end{array}$ & $\begin{array}{l}41.0080 \\
68.00\end{array}$ & $.11 \mathrm{u}$ & $200.00 \mathrm{~L}$ \\
\hline $\begin{array}{l}299-W 10-14 \\
299-W 10-14\end{array}$ & $\begin{array}{l}12 / 01 / 93 \\
12 / 01 / 93\end{array}$ & $\begin{array}{l}\text { 809Ka1 } \\
\text { 809Ka2 }\end{array}$ & 73.00 & 74.00 & $.11 \mathrm{U}$ & $100.00 \mathrm{~L}$ \\
\hline $\begin{array}{l}299-W 10-19 \\
299-W 10-19\end{array}$ & $\begin{array}{l}12 / 02 / 93 \\
12 / 02 / 93\end{array}$ & $\begin{array}{l}\text { 809K03 } \\
\text { 809K04 }\end{array}$ & 62.00 & 62.00 & $.17 \mathrm{BL}$ & $52.80 \mathrm{U}$ \\
\hline $\begin{array}{l}299-W 10-21 \\
299-W 10-21\end{array}$ & $\begin{array}{l}12 / 15 / 93 \\
12 / 15 / 93\end{array}$ & $\begin{array}{l}\text { 809P34 } \\
\text { B09P38 }\end{array}$ & $78.00 \mathrm{~B}$ & & $.11 \mathrm{U}$ & $52.80 \mathrm{U}$ \\
\hline $\begin{array}{l}299-116-2 \\
299-116-2\end{array}$ & $\begin{array}{l}12 / 07 / 93 \\
12 / 07 / 93\end{array}$ & $\begin{array}{l}\text { B09KQ5 } \\
\text { B09K06 }\end{array}$ & $39.00 \mathrm{~B}$ & $38.00 \mathrm{~B}$ & $.11 \mathrm{U}$ & $52.80 \mathrm{U}$ \\
\hline $\begin{array}{l}299-47-1 \\
299-47-1\end{array}$ & $\begin{array}{l}12 / 06 / 93 \\
12 / 06 / 93\end{array}$ & $\begin{array}{l}\text { 809Ka7 } \\
809 \mathrm{Ka8}\end{array}$ & 30.00 & 34.00 & $.24 \mathrm{BL}$ & $100.00 \mathrm{~L}$ \\
\hline $\begin{array}{l}299-47-10 \\
299-47-10\end{array}$ & $\begin{array}{l}12 / 02 / 93 \\
12 / 02 / 93\end{array}$ & $\begin{array}{l}\text { B09Ka9 } \\
\text { BO9KRO }\end{array}$ & 40.00 & 36.00 & $.45 \mathrm{BL}$ & $52.80 \mathrm{U}$ \\
\hline $\begin{array}{l}299-w 7-11 \\
299-47-11\end{array}$ & $\begin{array}{l}12 / 06 / 93 \\
12 / 06 / 93\end{array}$ & $\begin{array}{l}\text { 809KR1 } \\
\text { B09KR2 }\end{array}$ & $\begin{array}{l}37.00 \\
34.00\end{array}$ & & $\begin{array}{l}.11 \mathrm{U} \\
.11 \mathrm{U}\end{array}$ & $\begin{array}{l}200.00 \mathrm{~L} \\
200.00 \mathrm{~L}\end{array}$ \\
\hline $\begin{array}{l}299 \cdot-W 7-11 \\
299 \cdot-W 7-11\end{array}$ & $\begin{array}{l}12 / 06 / 93 \\
12 / 06 / 93\end{array}$ & $\begin{array}{l}\text { B09KR3 } \\
\text { B09KR4 }\end{array}$ & & $\begin{array}{l}35.00 \\
34.00\end{array}$ & & \\
\hline $\begin{array}{l}299-47-12 \\
299-47-12\end{array}$ & $\begin{array}{l}12 / 03 / 93 \\
12 / 03 / 93\end{array}$ & $\begin{array}{l}\text { 809KR5 } \\
809 \text { KR6 }\end{array}$ & 59.00 & 37.00 & $.11 \mathrm{U}$ & $80.00 \mathrm{~L}$ \\
\hline $\begin{array}{l}299-W 7-2 \\
299-w 7-2\end{array}$ & $\begin{array}{l}12 / 06 / 93 \\
12 / 06 / 93\end{array}$ & $\begin{array}{l}\text { 809KR7 } \\
\text { 809KR8 }\end{array}$ & 25.00 & 24.00 & $.11 \mathrm{U}$ & $80.00 \mathrm{~L}$ \\
\hline $\begin{array}{l}299-w 7-3 \\
299-w 7-3\end{array}$ & $\begin{array}{l}12 / 07 / 93 \\
12 / 07 / 93\end{array}$ & $\begin{array}{l}\text { 809KR9 } \\
\text { B09KSO }\end{array}$ & $31.00 \mathrm{~B}$ & $30.00 \mathrm{~B}$ & $.11 \mathrm{U}$ & $60.00 \mathrm{~L}$ \\
\hline $\begin{array}{l}299-47-4 \\
299-47-4\end{array}$ & $\begin{array}{l}12 / 03 / 93 \\
12 / 03 / 93\end{array}$ & $\begin{array}{l}\text { B09ks1 } \\
\text { B09ks2 }\end{array}$ & 40.00 & 39.00 & $.11 \mathrm{U}$ & $52.80 \mathrm{U}$ \\
\hline
\end{tabular}


Table 15-19. Constituents with at Least One Detected Value for the Low-Level Waste Burial Grounds WMA 3 Data for Reporting Period October 1 through December 31, 1993. (sheet 3 of 13)

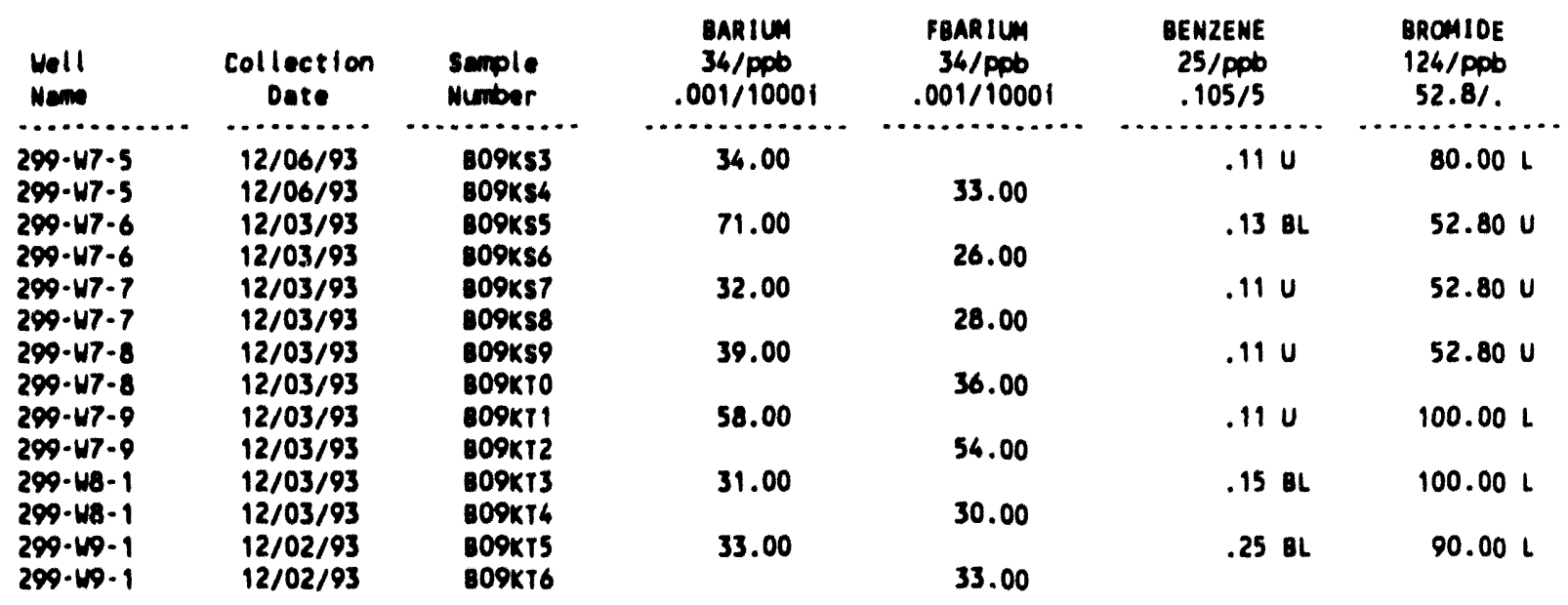

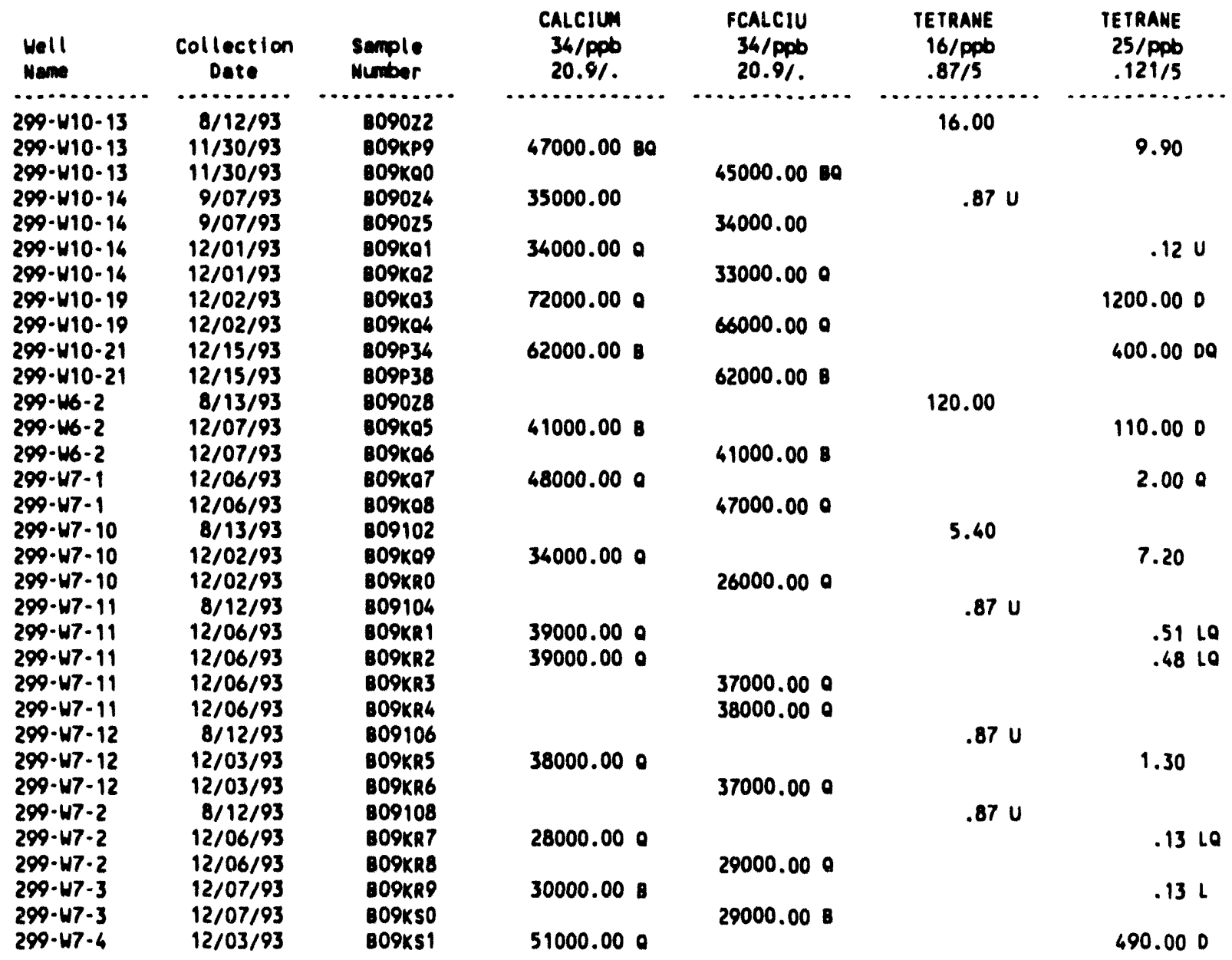


Table 15-19. Constituents with at Least One Detected Value for the Low-Level Waste Burial Grounds WMA 3 Data for Reporting Period October 1 through December 31, 1993. (sheet 4 of 13)

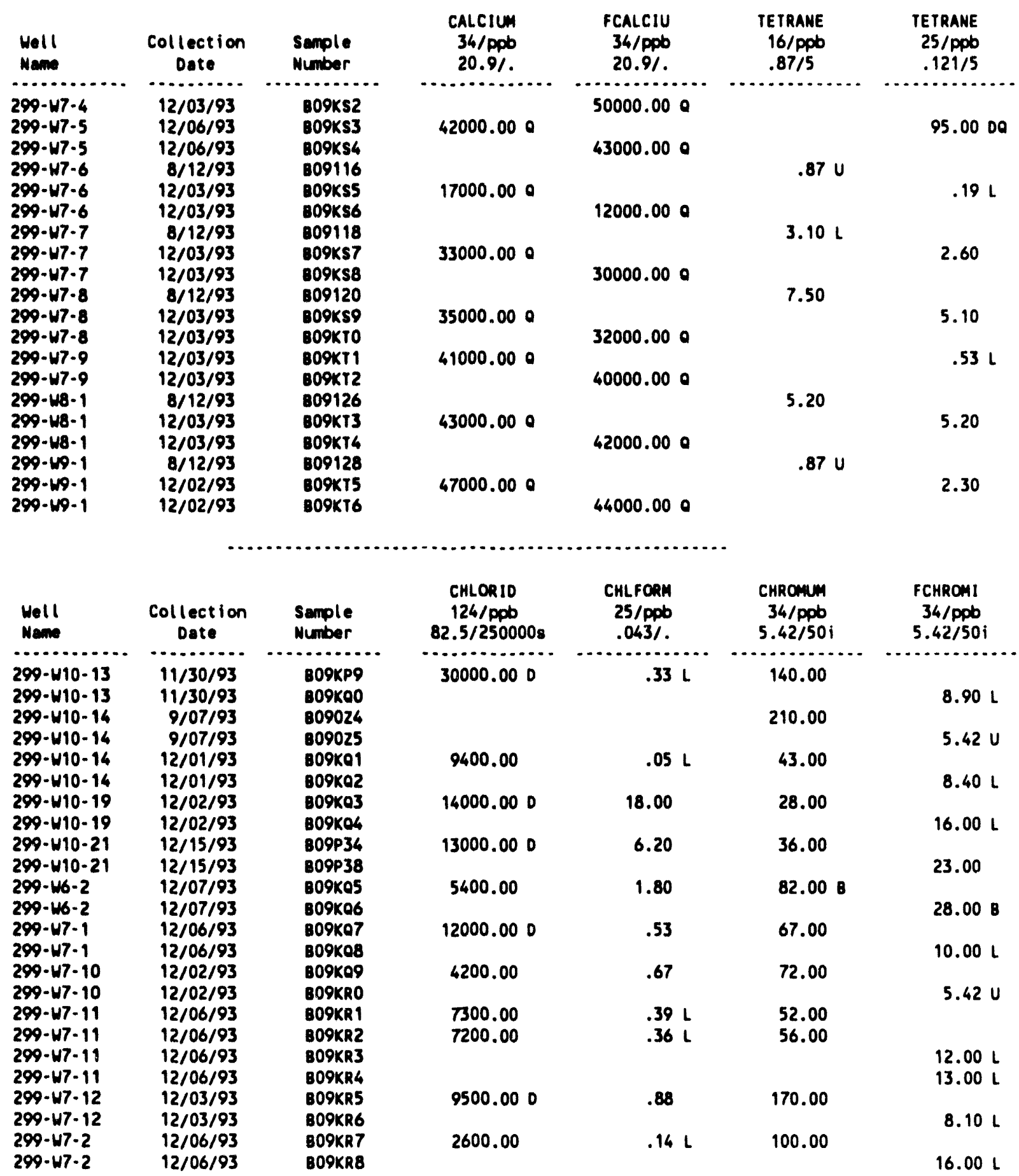


Table 15-19. Constituents with at Least One Detected Value for the Low-Level Waste Burial Grounds WMA 3 Data for Reporting Period October 1 through December 31, 1993. (sheet 5 of 13)

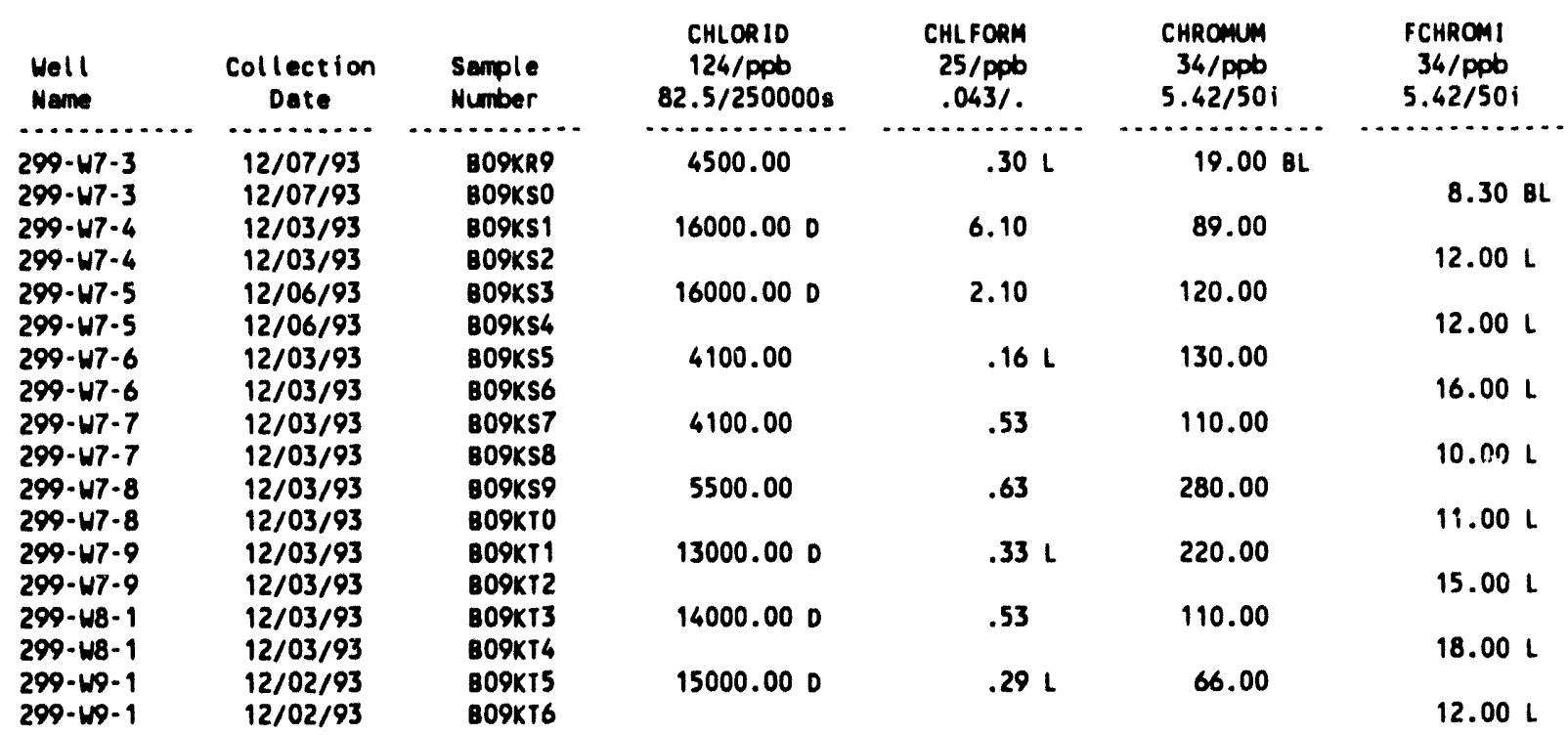

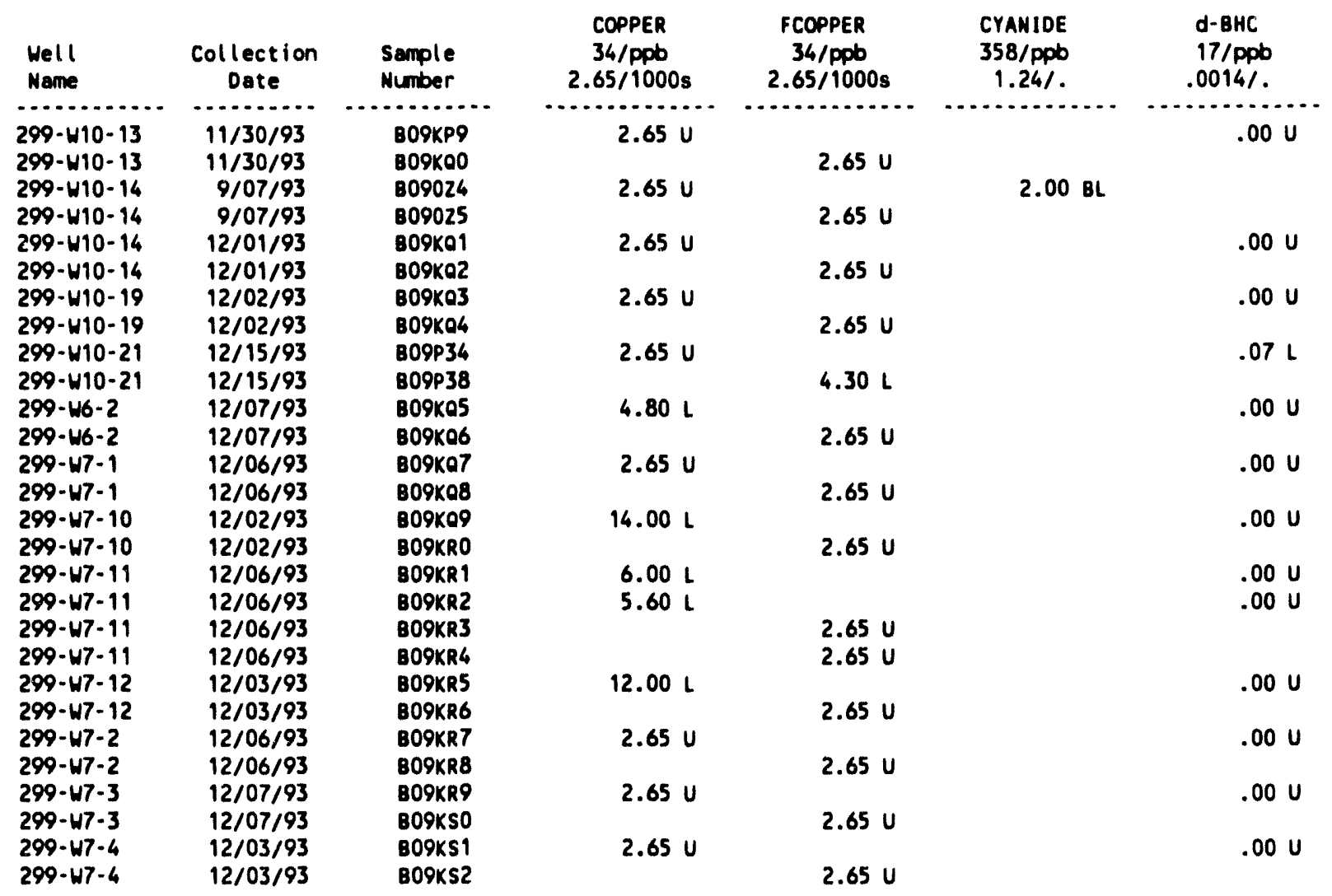


Table 15-19. Constituents with at Least One Detected Value for the Low-Level Waste Burial Grounds WMA 3 Data for Reporting Period October 1

through December 31, 1993. (sheet 6 of 13)

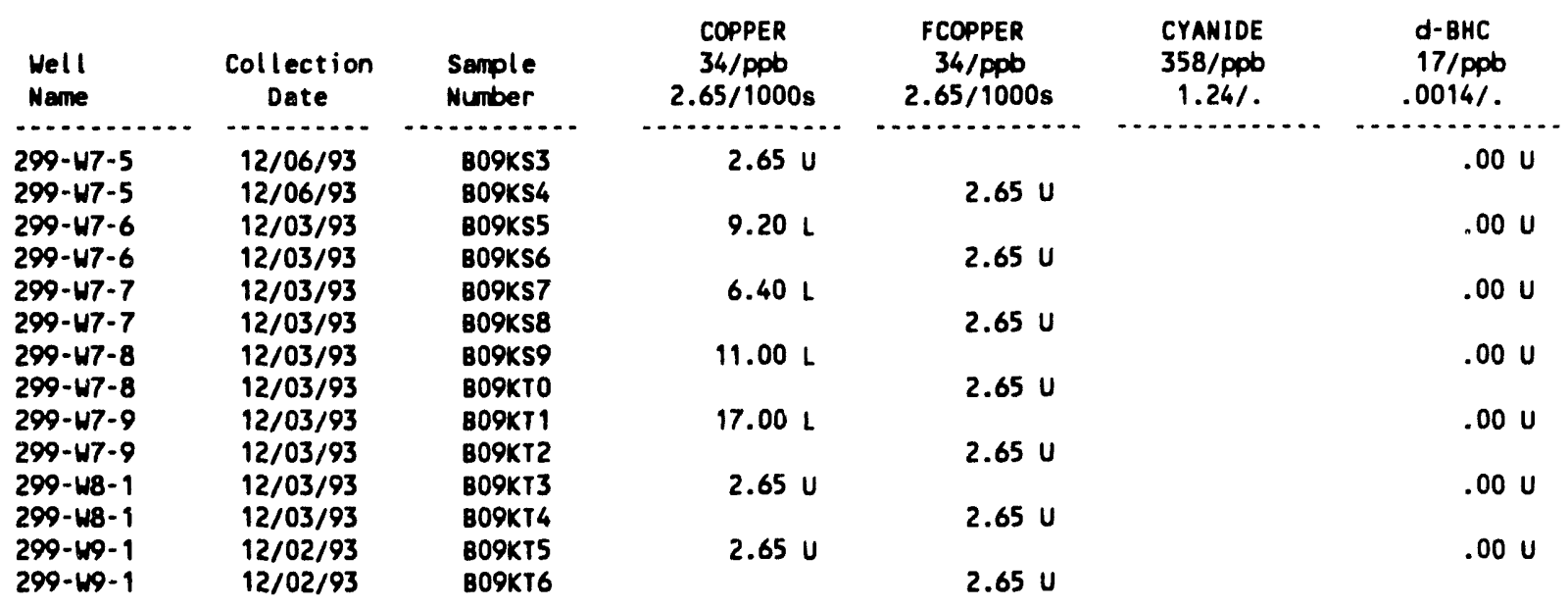

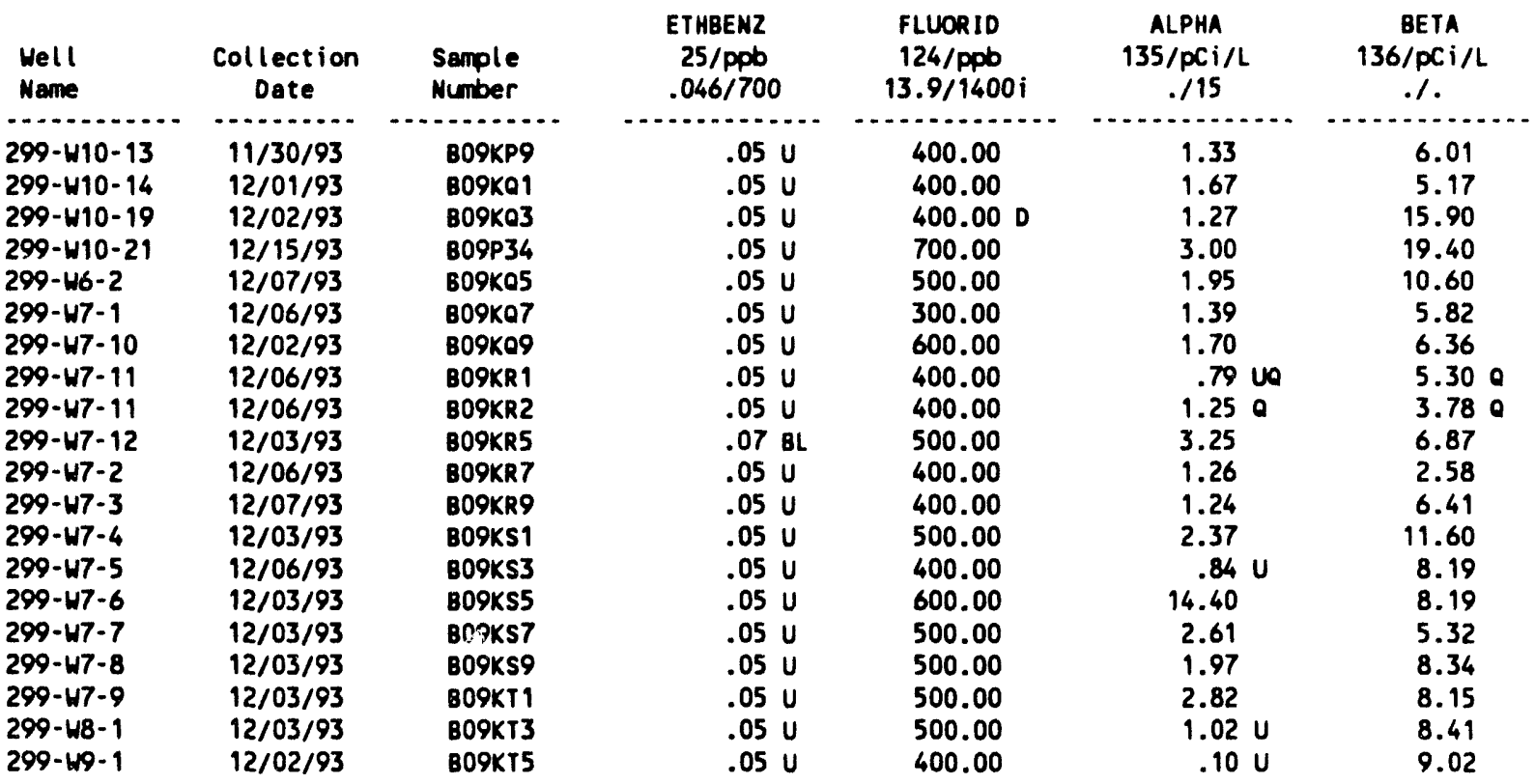

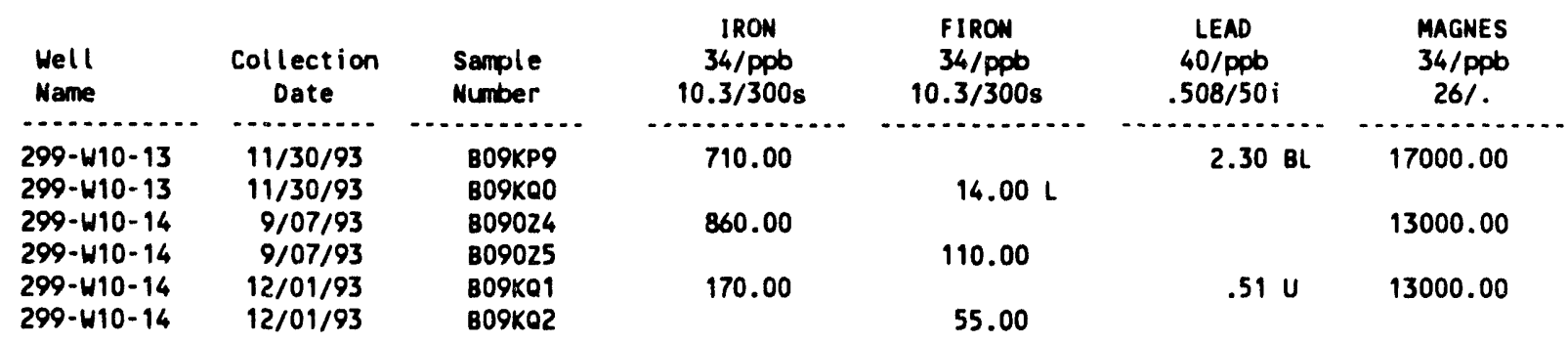


Table 15-19. Constituents with at Least One Detected Value for the Low-Level Waste Burial Grounds WMA 3 Data for Reporting Period October 1

through December 31, 1993. (sheet 7 of 13)

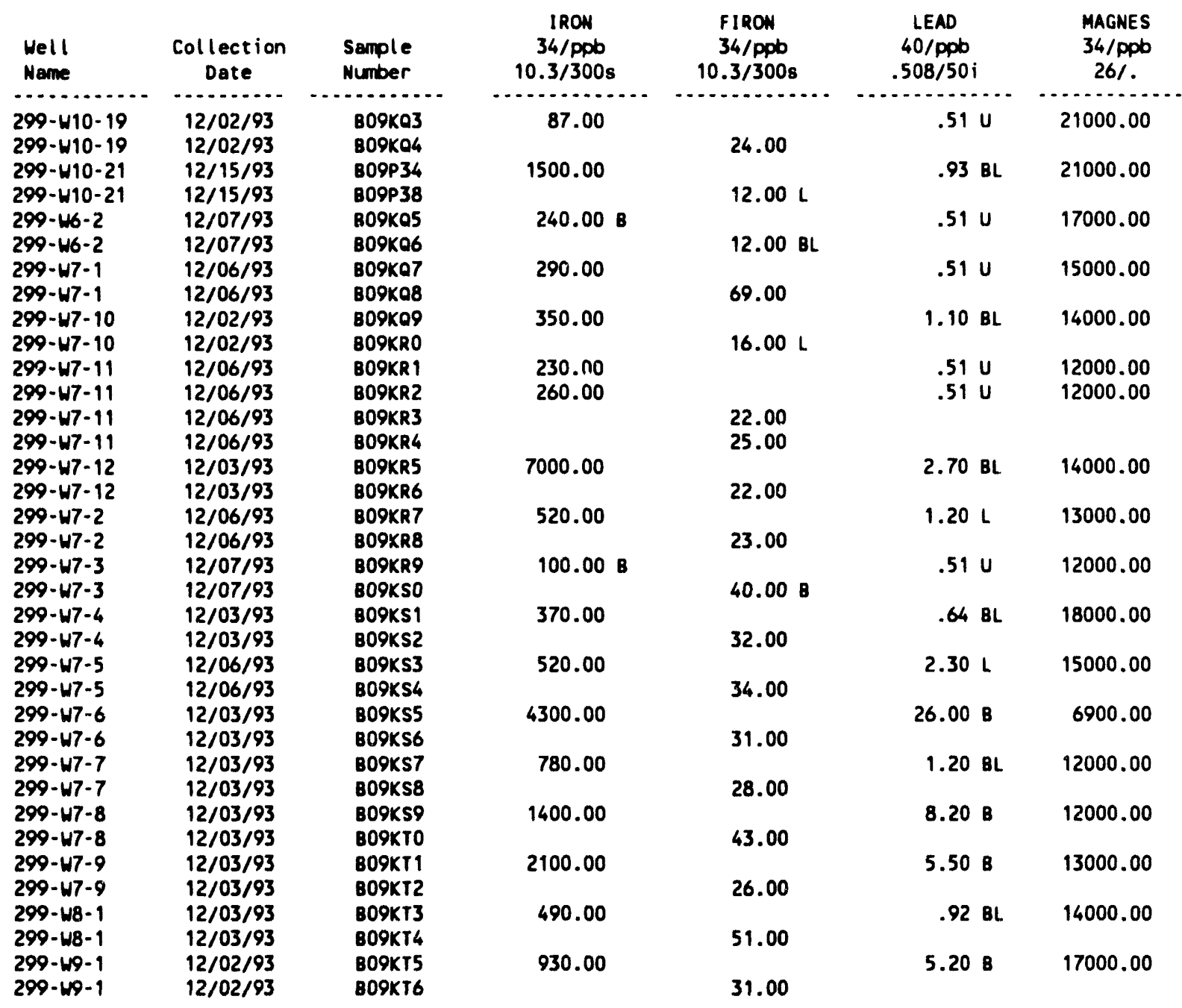

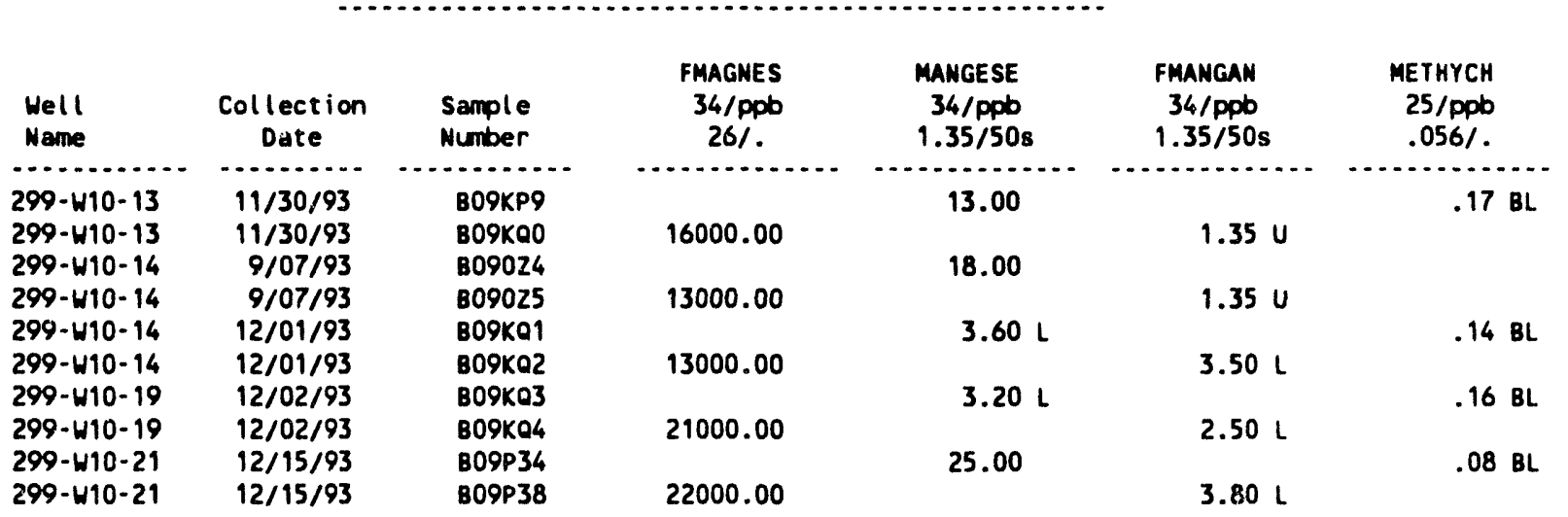


Table 15-19. Constituents with at Least One Detected Value for the Low-Level Waste Burial Grounds WMA 3 Data for Reporting Period October 1

through December 31, 1993. (sheet 8 of 13)

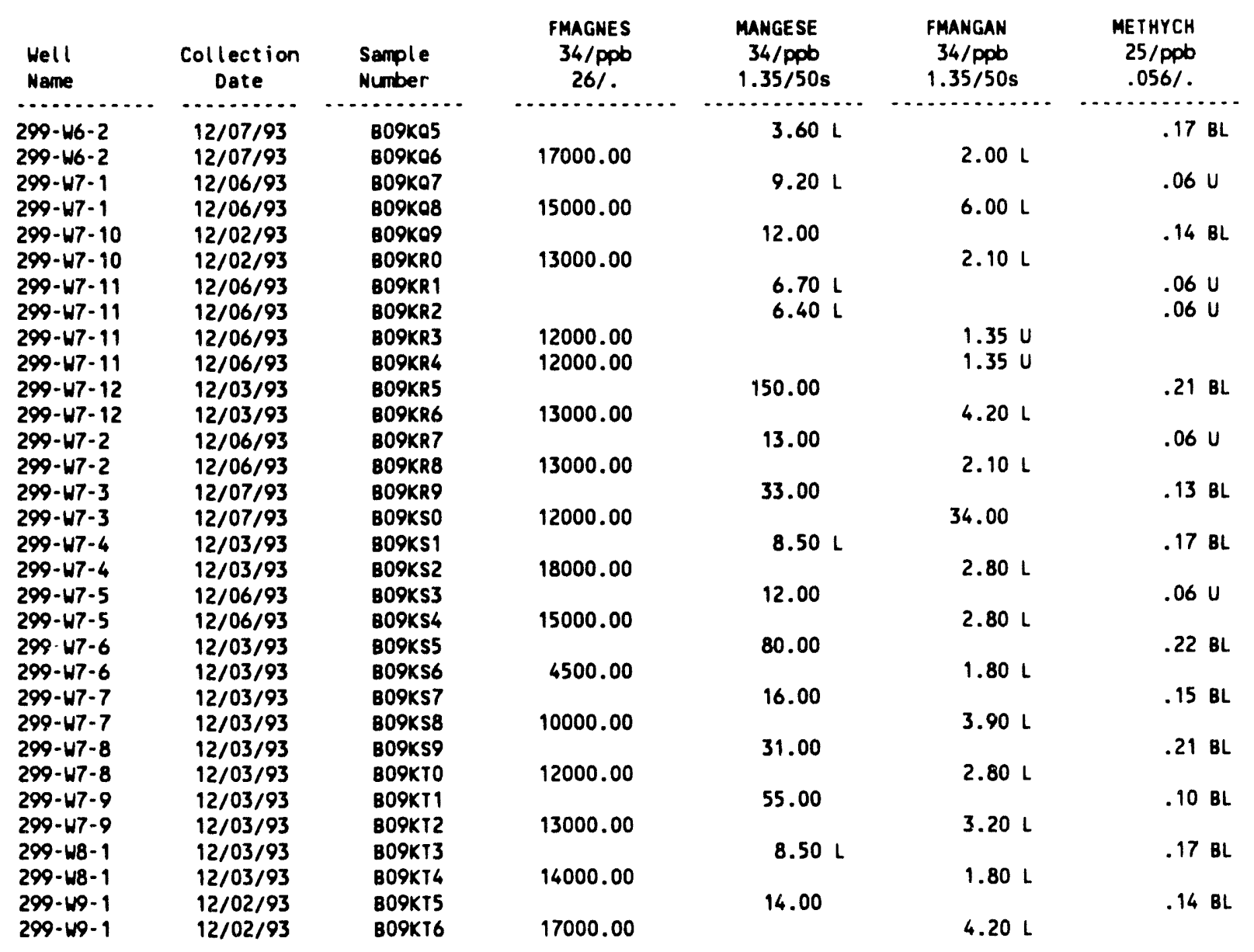

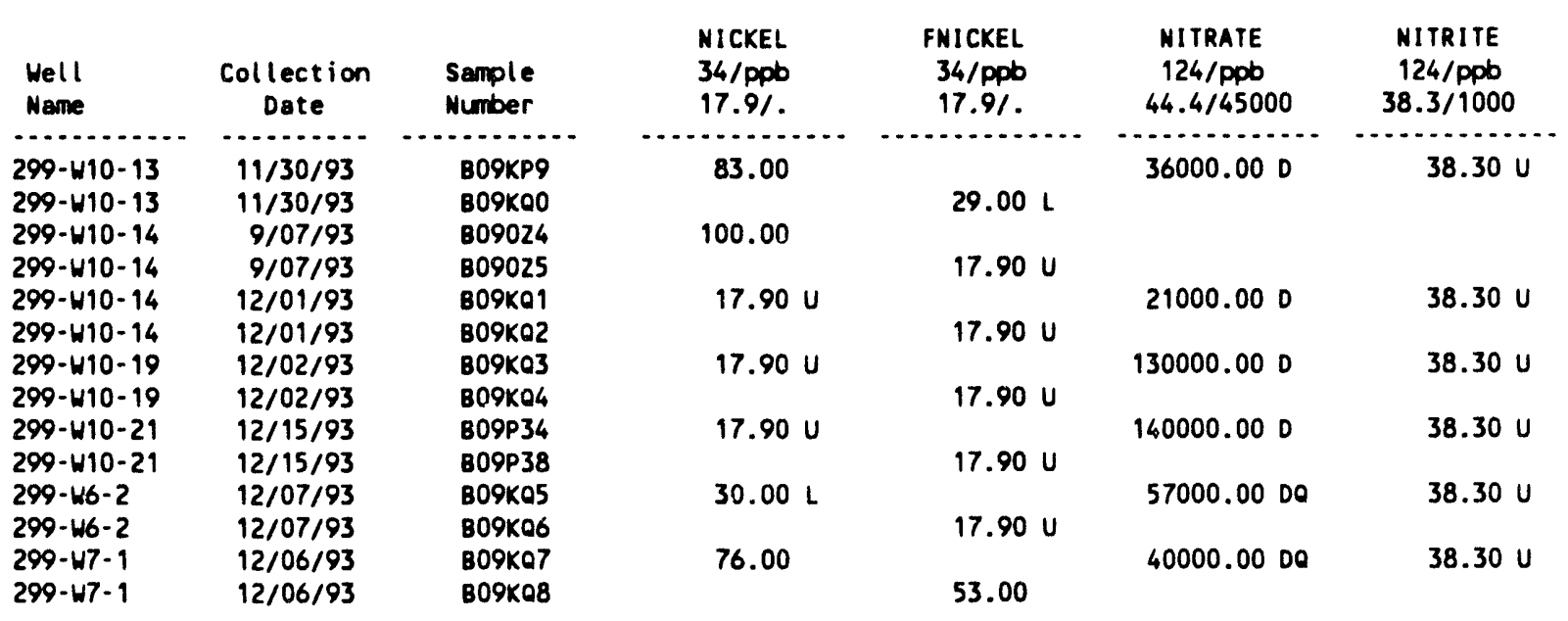


Table 15-19. Constituents with at Least One Detected Value for the Low-Level Waste Burial Grounds WMA 3 Data for Reporting Period October 1 through December 31, 1993. (sheet 9 of 13)

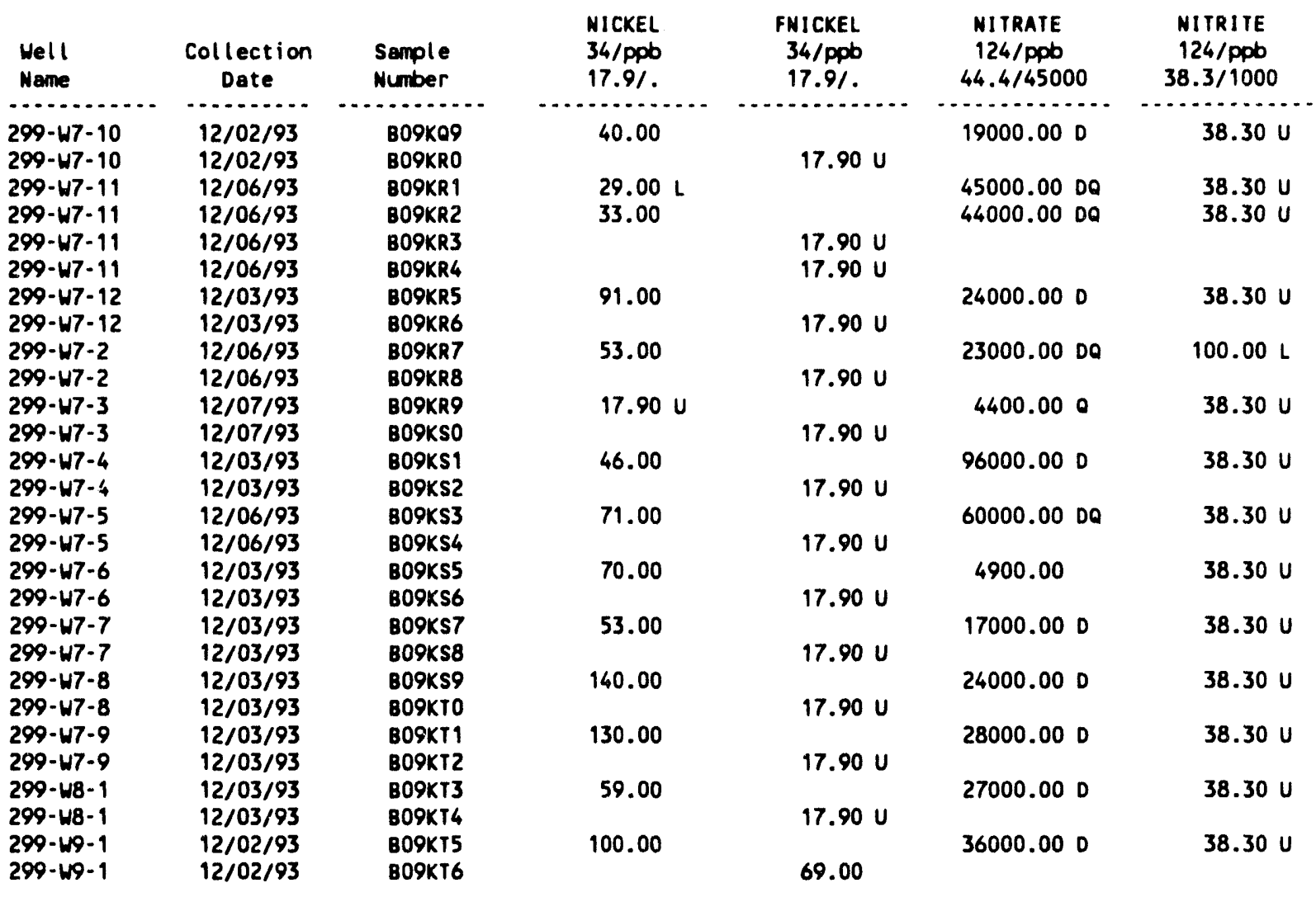

\begin{tabular}{|c|c|c|c|c|c|c|}
\hline $\begin{array}{l}\text { Hell } \\
\text { Name }\end{array}$ & $\begin{array}{c}\text { Collection } \\
\text { Date }\end{array}$ & $\begin{array}{l}\text { Sample } \\
\text { Number }\end{array}$ & $\begin{array}{l}\text { POTASUM } \\
\text { 34/ppb } \\
6621 .\end{array}$ & $\begin{array}{c}\text { FPOTASS } \\
34 / \mathrm{ppb} \\
6621 .\end{array}$ & $\begin{array}{c}\text { RADIUA } \\
137 / \mathrm{pCi} / \mathrm{L} \\
.15 \mathrm{i}\end{array}$ & $\begin{array}{l}\text { SELENUM } \\
48 / \mathrm{ppb} \\
1.21 / 10 \mathrm{i}\end{array}$ \\
\hline$\cdots \cdot$ & $\cdots$ & $\cdots$ & & & $x_{1}$ & \\
\hline $\begin{array}{l}299-w 10-13 \\
299-w 10-13\end{array}$ & $11 / 30 / 93$ & B09KP9 & 4200.00 & & $.06 \mathrm{U}$ & $4.60 \mathrm{~L}$ \\
\hline $\begin{array}{l}299-W 10-13 \\
299-W 10-14\end{array}$ & $\begin{array}{r}11 / 30 / 93 \\
9 / 07 / 93\end{array}$ & $\begin{array}{l}\text { B09KaO } \\
\text { B09074 }\end{array}$ & תח & 4000.00 & & \\
\hline $299-W 10-14$ & $9 / 07 / 93$ & & 4100.00 & 4000.00 & & \\
\hline $299-W 10-14$ & $12 / 01 / 93$ & B09Ka1 & 4500.00 & & $.05 \mathrm{U}$ & $1.21 \mathrm{U}$ \\
\hline $299-W 10-14$ & $12 / 01 / 93$ & B09Ka2 & & 4400.00 & & \\
\hline 299-W10-19 & $12 / 02 / 93$ & B09K03 & 4400.00 & & $.13 \mathrm{U}$ & $3.40 \mathrm{~L}$ \\
\hline 299-W10-19 & $12 / 02 / 93$ & B09K04 & & 4500.00 & & \\
\hline $299-W 10-21$ & $12 / 15 / 93$ & B09P34 & $3000.00 \mathrm{~B}$ & & .80 & $2.00 \mathrm{~L}$ \\
\hline $299-W 10-21$ & $12 / 15 / 93$ & B09P38 & & $4100.00 \mathrm{~B}$ & & \\
\hline 299-W6-2 & $12 / 07 / 93$ & B09Ka5 & $3500.00 \mathrm{~B}$ & & .16 & $1.21 \mathrm{U}$ \\
\hline $299-w 6-2$ & $12 / 07 / 93$ & B09Ka6 & & 3500.008 & & \\
\hline $299-47-1$ & $12 / 06 / 93$ & B09K07 & 3600.00 & & .17 & $2.70 \mathrm{~L}$ \\
\hline 299-W7-1 & $12 / 06 / 93$ & B09Ka8 & & 3400.00 & & \\
\hline $299-w 7-10$ & $12 / 02 / 93$ & B09Ka9 & 2900.00 & & .36 & $1.21 \mathrm{U}$ \\
\hline $299-w 7-10$ & $12 / 02 / 93$ & B09KRO & & 1900.00 & & \\
\hline $299-w 7-11$ & $12 / 06 / 93$ & B09KR1 & 3300.00 & & .130 & $2.00 \mathrm{~L}$ \\
\hline $299-w 7-11$ & $12 / 06 / 93$ & BO9KR2 & 3300.00 & & .270 & $2.00 \mathrm{~L}$ \\
\hline
\end{tabular}


Table 15-19. Constituents with at Least One Detected Value for the Low-Level Waste Burial Grounds WMA 3 Data for Reporting Period October 1

through December 31,1993 . (sheet 10 of 13)

\begin{tabular}{|c|c|c|c|c|c|c|}
\hline $\begin{array}{l}\text { Well } \\
\text { Name }\end{array}$ & $\begin{array}{c}\text { Collection } \\
\text { Date }\end{array}$ & $\begin{array}{l}\text { Sample } \\
\text { Number }\end{array}$ & $\begin{array}{l}\text { POTASUM } \\
34 / \mathrm{ppb} \\
6621 .\end{array}$ & $\begin{array}{l}\text { FPOTASS } \\
34 / \mathrm{ppb} \\
662 \%\end{array}$ & $\begin{array}{c}\text { RADIUM } \\
137 / \mathrm{PC} \mathrm{i} / \mathrm{L} \\
.15 \mathrm{i}\end{array}$ & $\begin{array}{l}\text { SELENUM } \\
48 / p p b \\
1.21 / 10 i\end{array}$ \\
\hline $\begin{array}{l}299-\omega 7-11 \\
299-\omega 7-11\end{array}$ & $\begin{array}{l}12 / 06 / 93 \\
12 / 06 / 93\end{array}$ & $\begin{array}{l}\text { B09KR3 } \\
\text { B09KR4 }\end{array}$ & $\cdots$ & $\begin{array}{l}3100.00 \\
3000.00\end{array}$ & & \\
\hline $\begin{array}{l}299-w 7-12 \\
299-\omega 7-12\end{array}$ & $\begin{array}{l}12 / 03 / 93 \\
12 / 03 / 93\end{array}$ & $\begin{array}{l}\text { B09KR5 } \\
\text { B09KR6 }\end{array}$ & 3700.00 & 3200.00 & .50 & $2.60 \mathrm{~L}$ \\
\hline $\begin{array}{l}299-w 7-2 \\
299-\omega 7-2\end{array}$ & $\begin{array}{l}12 / 06 / 93 \\
12 / 06 / 93\end{array}$ & $\begin{array}{l}\text { B09KR7 } \\
\text { B09KR8 }\end{array}$ & 1700.00 & 1800.00 & .48 & $1.21 \mathrm{U}$ \\
\hline $\begin{array}{l}299-w 7-3 \\
299-w 7-3\end{array}$ & $\begin{array}{l}12 / 07 / 93 \\
12 / 07 / 93\end{array}$ & $\begin{array}{l}\text { B09KR9 } \\
\text { B09KSO }\end{array}$ & $5200.00 \mathrm{~B}$ & $5300.00 \mathrm{~B}$ & .28 & $1.21 \mathrm{U}$ \\
\hline $\begin{array}{l}299-w 7-4 \\
299-w 7-4\end{array}$ & $\begin{array}{l}12 / 03 / 93 \\
12 / 03 / 93\end{array}$ & $\begin{array}{l}\text { B09KS1 } \\
\text { B09KS2 }\end{array}$ & 2600.00 & 2900.00 & $.04 \mathrm{U}$ & $2.80 \mathrm{~L}$ \\
\hline $\begin{array}{l}299-W 7-5 \\
299-W 7-5\end{array}$ & $\begin{array}{l}12 / 06 / 93 \\
12 / 06 / 93\end{array}$ & $\begin{array}{l}\text { 809KS3 } \\
\text { 809KS4 }\end{array}$ & 3400.00 & 3200.00 & .34 & $1.21 \mathrm{U}$ \\
\hline $\begin{array}{l}299-W 7-6 \\
299-W 7-6\end{array}$ & $\begin{array}{l}12 / 03 / 93 \\
12 / 03 / 93\end{array}$ & $\begin{array}{l}\text { B09KS5 } \\
\text { B09KS6 }\end{array}$ & 2900.00 & 2200.00 & 2.14 & $1.21 \mathrm{U}$ \\
\hline $\begin{array}{l}299-W 7-7 \\
299-w 7-7\end{array}$ & $\begin{array}{l}12 / 03 / 93 \\
12 / 03 / 93\end{array}$ & $\begin{array}{l}\text { B09KS7 } \\
\text { B09KS8 }\end{array}$ & 3100.00 & 2900.00 & $.12 \mathrm{U}$ & $2.00 \mathrm{~L}$ \\
\hline $\begin{array}{l}299-W 7-8 \\
299-W 7-8\end{array}$ & $\begin{array}{l}12 / 03 / 93 \\
12 / 03 / 93\end{array}$ & $\begin{array}{l}\text { B09K59 } \\
\text { BO9KT0 }\end{array}$ & 3100.00 & 2100.00 & .17 & $1.21 \mathrm{U}$ \\
\hline $\begin{array}{l}299-47-9 \\
299-47-9\end{array}$ & $\begin{array}{l}12 / 03 / 93 \\
12 / 03 / 93\end{array}$ & $\begin{array}{l}\text { 809KT1 } \\
\text { B09KT2 }\end{array}$ & 4500.00 & 4100.00 & .66 & $4.20 \mathrm{~L}$ \\
\hline $\begin{array}{l}299-48-1 \\
299-48-1\end{array}$ & $\begin{array}{l}12 / 03 / 93 \\
12 / 03 / 93\end{array}$ & $\begin{array}{l}\text { B09KT3 } \\
\text { B09KT4 }\end{array}$ & 3500.00 & 3600.00 & $.10 \mathrm{U}$ & $3.60 \mathrm{~L}$ \\
\hline $\begin{array}{l}299-w 9-1 \\
299-169-1\end{array}$ & $\begin{array}{l}12 / 02 / 93 \\
12 / 02 / 93\end{array}$ & $\begin{array}{l}\text { B09KT5 } \\
\text { B09KT6 }\end{array}$ & 5500.00 & 4800.00 & $-.01 \mathrm{U}$ & $4.50 \mathrm{~L}$ \\
\hline
\end{tabular}

\begin{tabular}{|c|c|c|c|c|c|c|}
\hline $\begin{array}{l}\text { Well } \\
\text { Name }\end{array}$ & $\begin{array}{c}\text { Collection } \\
\text { Date }\end{array}$ & $\begin{array}{l}\text { Sample } \\
\text { Number }\end{array}$ & $\begin{array}{c}\text { FSELENI } \\
48 / \mathrm{ppb} \\
1.21 / 10 \mathrm{i}\end{array}$ & $\begin{array}{l}\text { SILVER } \\
\text { 34/ppb } \\
2.87 / 50 i\end{array}$ & $\begin{array}{l}\text { soolum } \\
34 / \mathrm{ppb} \\
40.9 / .\end{array}$ & $\begin{array}{c}\text { FSOD IUM } \\
34 / \mathrm{ppb} \\
40.9 \%\end{array}$ \\
\hline $299-W 10-13$ & $11 / 30 / 93$ & B09KP9 & & $2.87 \mathrm{U}$ & $13000.00 \mathrm{BO}$ & \\
\hline $299-410-13$ & $11 / 30 / 93$ & BO9KaO & $5.00 \mathrm{~L}$ & (2) & 10000.00 & 13000.0080 \\
\hline $299-W 10-14$ & $9 / 07 / 93$ & 809024 & & $2.87 U$ & 15000.00 & \\
\hline $299-W 10-14$ & $9 / 07 / 93$ & 809025 & & & & 15000.00 \\
\hline $299-W 10-14$ & $12 / 01 / 93$ & 809K01 & & $2.87 \mathrm{U}$ & 17000.000 & \\
\hline $299-W 10-14$ & $12 / 01 / 93$ & B09K02 & $1.21 \mathrm{U}$ & & & 16000.000 \\
\hline $299-W 10-19$ & $12 / 02 / 93$ & B09K03 & & $2.87 \mathrm{U}$ & 13000.000 & \\
\hline 299-W10-19 & $12 / 02 / 93$ & B09KO4 & $3.90 \mathrm{~L}$ & & & 13000.000 \\
\hline $299-W 10-21$ & $12 / 15 / 93$ & BO9P34 & & $2.87 \mathrm{U}$ & $9100.00 \mathrm{~B}$ & \\
\hline $299-410-21$ & $12 / 15 / 93$ & B09P38 & $2.00 \mathrm{~L}$ & & & $9300.00 \mathrm{~B}$ \\
\hline 299-146-2 & $12 / 07 / 93$ & B09K05 & & $2.87 U$ & 11000.00 & \\
\hline 299-W6-2 & $12 / 07 / 93$ & B09K06 & $1.21 \mathrm{U}$ & & & 10000.00 \\
\hline $299-W 7-1$ & $12 / 06 / 93$ & B09K07 & & $2.87 \mathrm{U}$ & 9500.000 & \\
\hline $299-W 7-1$ & $12 / 06 / 93$ & B09K08 & $2.00 \mathrm{~L}$ & & & 9700.000 \\
\hline $299-w 7-10$ & $12 / 02 / 93$ & B09K09 & & $9.80 \mathrm{~L}$ & $8900.00 a$ & \\
\hline $299-w 7-10$ & $12 / 02 / 93$ & BO9KRO & $1.21 \mathrm{U}$ & & & 8800.000 \\
\hline $299-47-11$ & $12 / 06 / 93$ & B09KR 1 & & $2.87 \mathrm{U}$ & 11000.000 & \\
\hline $299-w 7-11$ & $12 / 06 / 93$ & B09KR2 & & $2.87 \mathrm{U}$ & $11000.00 a$ & \\
\hline $299-47-11$ & $12 / 06 / 93$ & B09KR3 & $2.00 \mathrm{~L}$ & & & 11000.000 \\
\hline $299-W 7-11$ & $12 / 06 / 93$ & 809KR4 & $2.00 \mathrm{~L}$ & & & $11000.00 a$ \\
\hline $\begin{aligned} 28 y-41-16 \\
200-47-90\end{aligned}$ & $12 / 03 / 93$ & BO9KR3 & & $2.81 \mathrm{U}$ & $10000.00 a$ & \\
\hline $299-47-12$ & $12 / 03 / 93$ & B09KR6 & $3.10 \mathrm{~L}$ & & & 9900.000 \\
\hline
\end{tabular}


Table 15-19. Constituents with at Least One Detected Value for the Low-Level Waste Burial Grounds WMA 3 Data for Reporting Period October 1

through Decemer 31, 1993. (sheet 11 of 13)

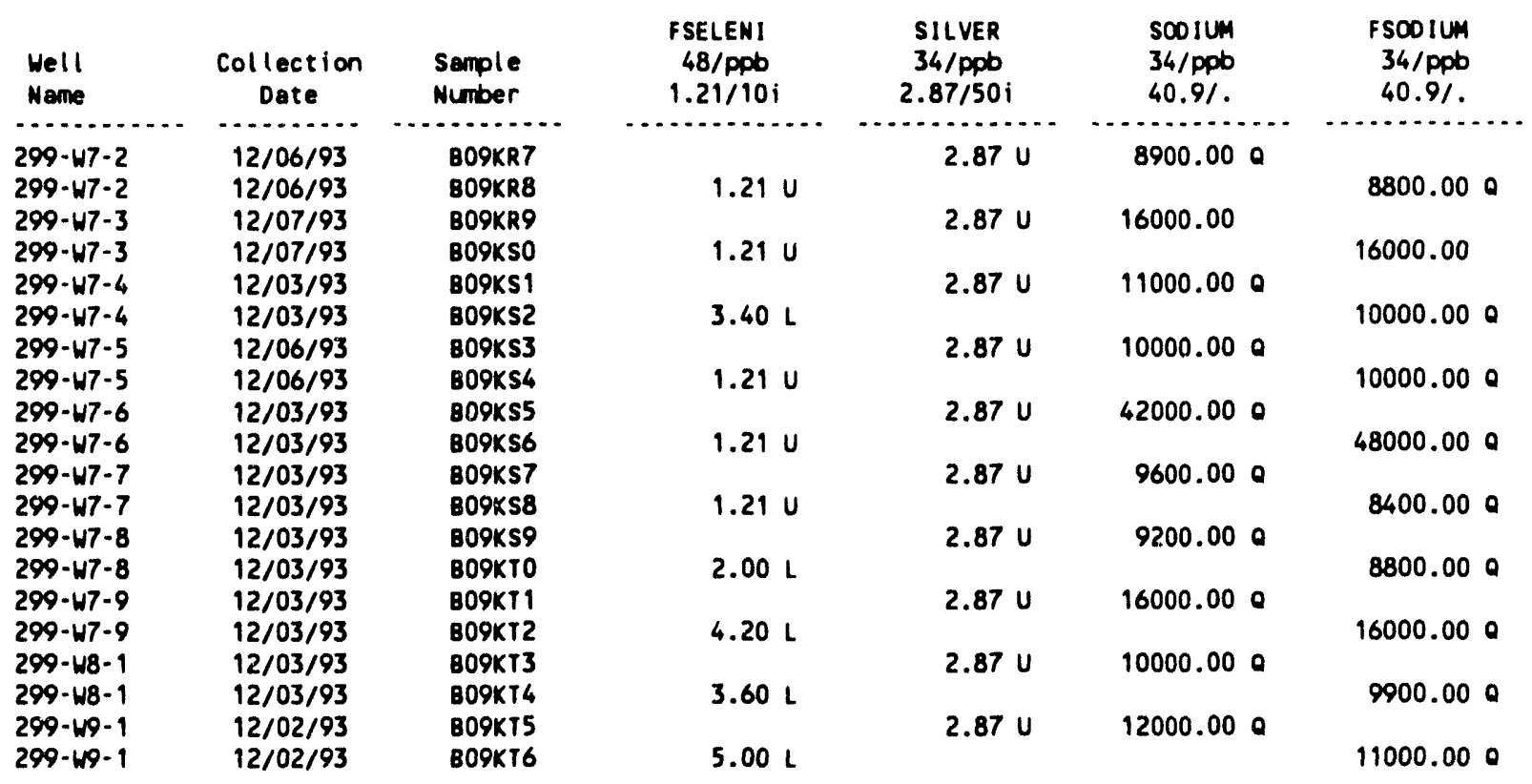

\begin{tabular}{|c|c|c|c|c|c|c|}
\hline $\begin{array}{l}\text { Well } \\
\text { Name }\end{array}$ & $\begin{array}{c}\text { Collection } \\
\text { Date }\end{array}$ & $\begin{array}{l}\text { Sample } \\
\text { Number }\end{array}$ & $\begin{array}{c}\text { SULFATE } \\
124 / \mathrm{ppb} \\
49.9 / 250000 \mathrm{~s}\end{array}$ & $\begin{array}{r}\text { PERCENE } \\
25 / \text { PpD } \\
.049 / 5\end{array}$ & $\begin{array}{l}\text { TOLUENE } \\
25 / \text { ppb } \\
.056 / 1000\end{array}$ & $\begin{array}{l}\text { TRICENE } \\
16 / \mathrm{Ppb} \\
.77 / 5\end{array}$ \\
\hline $299-410-13$ & $8 / 12 / 93$ & 809022 & & & & $.77 \mathrm{U}$ \\
\hline $\begin{array}{l}299-W 10-13 \\
299-W 10-14\end{array}$ & $\begin{array}{r}11 / 30 / 93 \\
9 / 07 / 93\end{array}$ & $\begin{array}{l}\text { 809KP9 } \\
809024\end{array}$ & $36000.00 \mathrm{D}$ & $.05 \mathrm{U}$ & $.06 \mathrm{U}$ & $.77 \mathrm{U}$ \\
\hline $299-W 10-14$ & $12 / 01 / 93$ & $809 \times 01$ & $25000.00 \mathrm{D}$ & $.05 \mathrm{U}$ & $.06 \mathrm{U}$ & \\
\hline $299-W 10-19$ & $12 / 02 / 93$ & B09K03 & $37000.00 \mathrm{D}$ & $.52 \mathrm{~B}$ & $.06 \mathrm{U}$ & \\
\hline $299-W 10-21$ & $12 / 15 / 93$ & B09P34 & $36000.00 \mathrm{D}$ & $.19 \mathrm{~L}$ & $.06 \mathrm{U}$ & \\
\hline $299-w 6-2$ & $8 / 13 / 93$ & 809028 & & & & $1.30 \mathrm{~L}$ \\
\hline $\begin{array}{l}299-W 6-2 \\
299-W 7-1\end{array}$ & $\begin{array}{l}12 / 07 / 93 \\
12 / 06 / 93\end{array}$ & $\begin{array}{l}\text { B09Ka5 } \\
\text { B09Ka7 }\end{array}$ & $\begin{array}{l}25000.00 \mathrm{D} \\
52000.00 \mathrm{D}\end{array}$ & $\begin{array}{l}.12 \mathrm{BL} \\
.05 \mathrm{U}\end{array}$ & $\begin{array}{l}.06 U \\
.06 U\end{array}$ & \\
\hline $299-47-10$ & $8 / 13 / 93$ & B09102 & & & & $.77 \mathrm{U}$ \\
\hline $299-W 7-10$ & $12 / 02 / 93$ & 809Ka9 & $21000.00 \mathrm{D}$ & $.05 \mathrm{U}$ & $.07 \mathrm{~L}$ & \\
\hline $299-W 7-11$ & $8 / 12 / 93$ & B09104 & & & & $.77 \mathrm{U}$ \\
\hline $299-47-11$ & $12 / 06 / 93$ & B09KR1 & $32000.00 \mathrm{D}$ & $.05 \mathrm{U}$ & $.06 \mathrm{U}$ & \\
\hline $299-47-11$ & $12 / 06 / 93$ & B09KR2 & $32000.00 \mathrm{D}$ & $.05 \mathrm{U}$ & $.06 \mathrm{U}$ & \\
\hline & $\begin{array}{r}8 / 12 / 93 \\
12 / 03 / 93\end{array}$ & $\begin{array}{l}\text { B09106 } \\
\text { B09KR5 }\end{array}$ & & $.05 \mathrm{U}$ & & .77 U \\
\hline $\begin{array}{l}299-W 7-12 \\
299-W 7-2\end{array}$ & $\begin{array}{r}12 / 03 / 93 \\
8 / 12 / 93\end{array}$ & $\begin{array}{l}\text { B09KR5 } \\
\text { B09108 }\end{array}$ & $19000.00 \mathrm{D}$ & .050 & $.06 \mathrm{U}$ & $.77 \mathrm{U}$ \\
\hline $299 \cdot w 7-2$ & $12 / 06 / 93$ & B09KR7 & $25000.00 \mathrm{D}$ & $.05 \mathrm{U}$ & $.06 \mathrm{U}$ & \\
\hline $299 \cdot w 7 \cdot 3$ & $12 / 07 / 93$ & B09KR9 & $25000.00 \mathrm{D}$ & $.05 \mathrm{U}$ & $.06 \mathrm{U}$ & \\
\hline $299-177-4$ & $12 / 03 / 93$ & B09KS1 & 33000.000 & $.24 \mathrm{BL}$ & $.06 \mathrm{U}$ & \\
\hline $299-47-5$ & $12 / 06 / 93$ & B09KS3 & 29000.000 & $.09 \mathrm{~L}$ & $.06 \mathrm{U}$ & \\
\hline $299-w 7-6$ & $8 / 12 / 93$ & B09116 & & & & .77 \\
\hline $299-47-6$ & $12 / 03 / 93$ & B09KS5 & $25000.00 \mathrm{D}$ & $.05 \mathrm{U}$ & $.06 \mathrm{U}$ & \\
\hline $299-177-7$ & $8 / 12 / 93$ & $B 09118$ & & & & .77 \\
\hline $99 \cdot-17 \cdot 7$ & $12 / 03 / 93$ & B09KS7 & 18000.00 & $.06 \mathrm{BL}$ & .06 & \\
\hline
\end{tabular}


Table 15-19. Constituents with at Least One Detected Value for the Low-Level Waste Burial Grounds WMA 3 Data for Reporting Period October 1

through December 31, 1993. (sheet 12 of 13)

$\begin{array}{lcccccc}\text { Well } & \begin{array}{c}\text { Collection } \\ \text { Date }\end{array} & \begin{array}{c}\text { Sample } \\ \text { Number }\end{array} & \begin{array}{c}\text { SULFATE } \\ 124 / \text { ppb } \\ 49.9 / 250000 \text { s }\end{array} & \begin{array}{c}\text { PERCENE } \\ 25 / \text { ppb } \\ .049 / 5\end{array} & \begin{array}{c}\text { TOLUENE } \\ 25 / \text { Ppb } \\ .056 / 1000\end{array} & \begin{array}{c}\text { TRICENE } \\ 16 / \text { PpD }\end{array} \\ \ldots .77 / 5\end{array}$

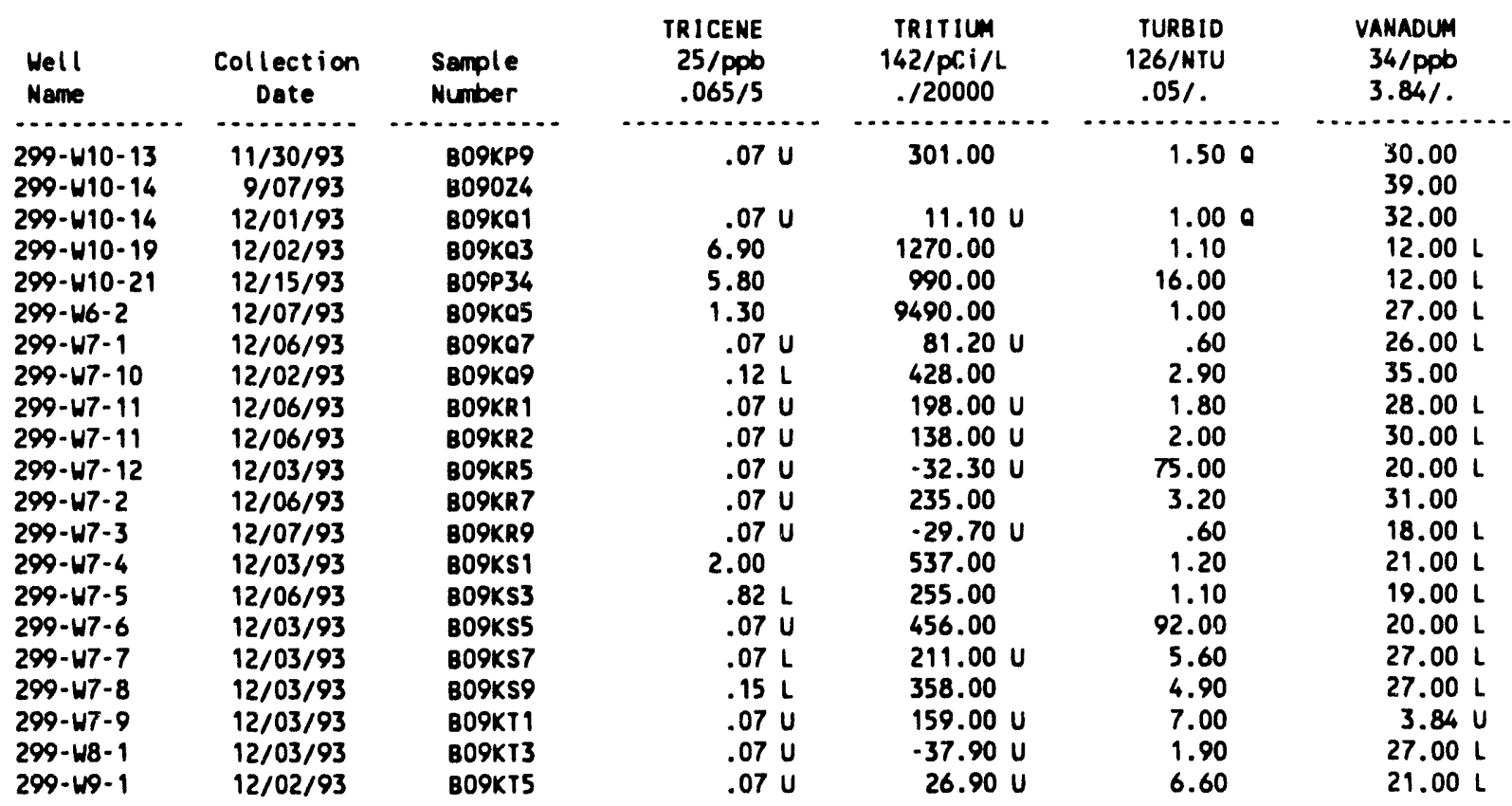

\begin{tabular}{|c|c|c|c|c|c|}
\hline $\begin{array}{l}\text { Well } \\
\text { Name }\end{array}$ & $\begin{array}{c}\text { Collection } \\
\text { Date }\end{array}$ & $\begin{array}{l}\text { Sample } \\
\text { Number }\end{array}$ & $\begin{array}{l}\text { FVANADI } \\
34 / p p b 6 \\
3.84 / .\end{array}$ & $\begin{array}{c}\text { 21NC } \\
34 / \mathrm{ppb} \\
3.44 / 5000 \mathrm{~s}\end{array}$ & $\begin{array}{c}\text { F21ACC } \\
34 / \mathrm{ppb} \\
3.44 / 5000 \mathrm{~s}\end{array}$ \\
\hline ....... & .............. & $\cdots . .$. & - & . & \\
\hline $\begin{array}{l}299-W 10-13 \\
299-W 10-13\end{array}$ & $\begin{array}{l}11 / 30 / 93 \\
11 / 30 / 93\end{array}$ & $\begin{array}{l}\text { B09KPQ } \\
\text { B09K00 }\end{array}$ & $26.00 \mathrm{~L}$ & $12.00 \mathrm{~B}$ & $3.44 \mathrm{U}$ \\
\hline $299-W 10-14$ & $9 / 07 / 93$ & B09024 & & $3.44 \mathrm{U}$ & \\
\hline $299-W 10-14$ & $9 / 07 / 93$ & 809025 & 32.00 & & $3.44 \mathrm{U}$ \\
\hline $299-W 10-14$ & $12 / 01 / 93$ & B09K01 & & $5.30<0$ & \\
\hline $299-W 10-14$ & $12 / 01 / 93$ & B09K02 & 35.00 & & $6.60 \mathrm{LQ}$ \\
\hline $299-W 10-19$ & $12 / 02 / 93$ & 809K03 & & 17.000 & \\
\hline $299-W 10-19$ & $12 / 02 / 93$ & 809Ka4 & $13.00 \mathrm{~L}$ & & $4.80 \mathrm{LO}$ \\
\hline $299-W 10-21$ & $12 / 15 / 93$ & B09P34 & & 64.008 & \\
\hline $299-\$ 10-21$ & $12 / 15 / 93$ & 809p38 & $14.00 \mathrm{~L}$ & & $28.00 \mathrm{~B}$ \\
\hline
\end{tabular}


Table 15-19. Constituents with at Least One Detected Value for the Low-Level Waste Burial Grounds WMA 3 Data for Reporting Period October 1 through December 31, 1993. (sheet 13 of 13)

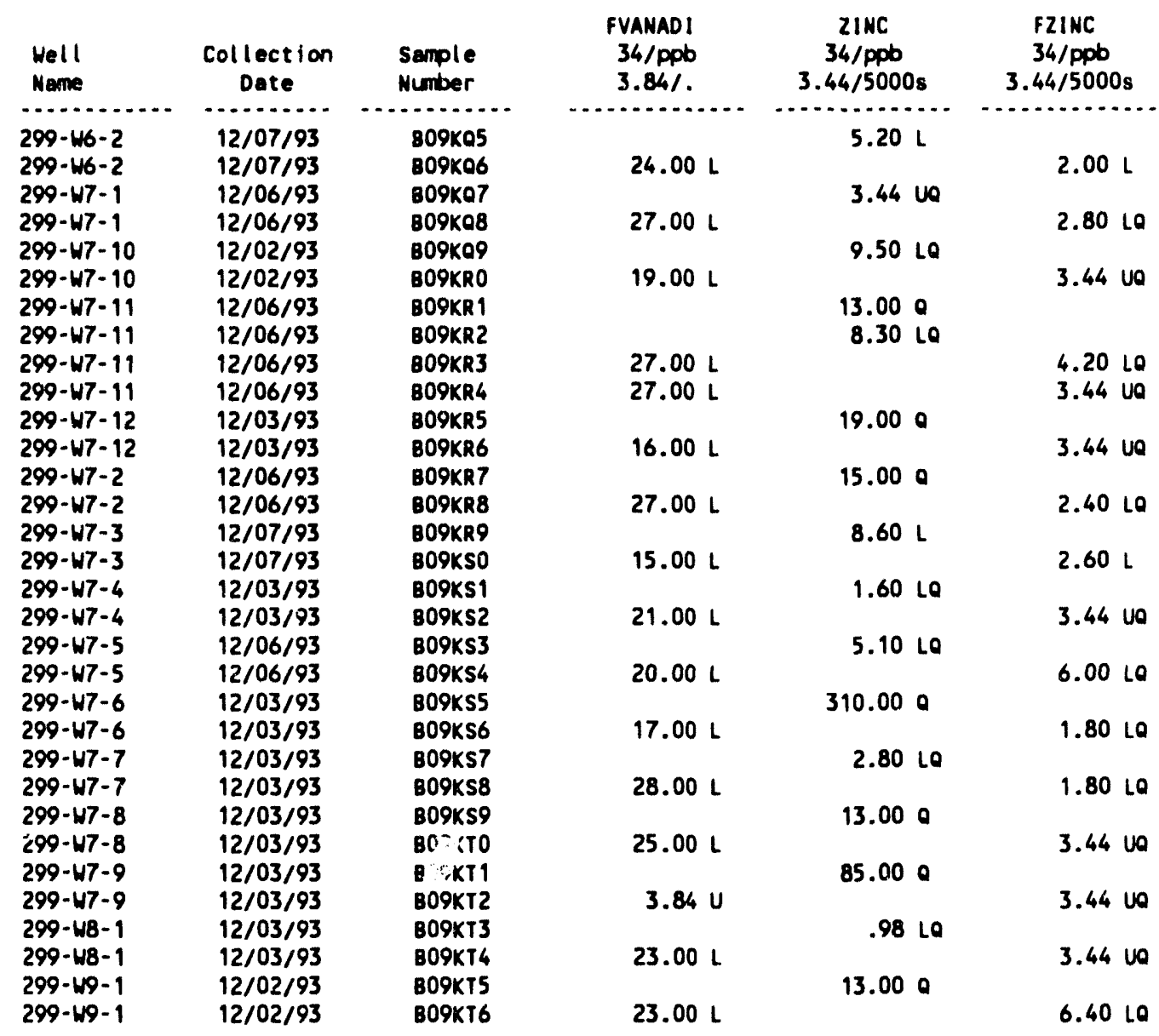


Table 15-20. Contamination Indicator Parameters for the Low-Level Waste Burial Grounds WMA 3 Data for Reporting Period October 1 through December 31, 1993.

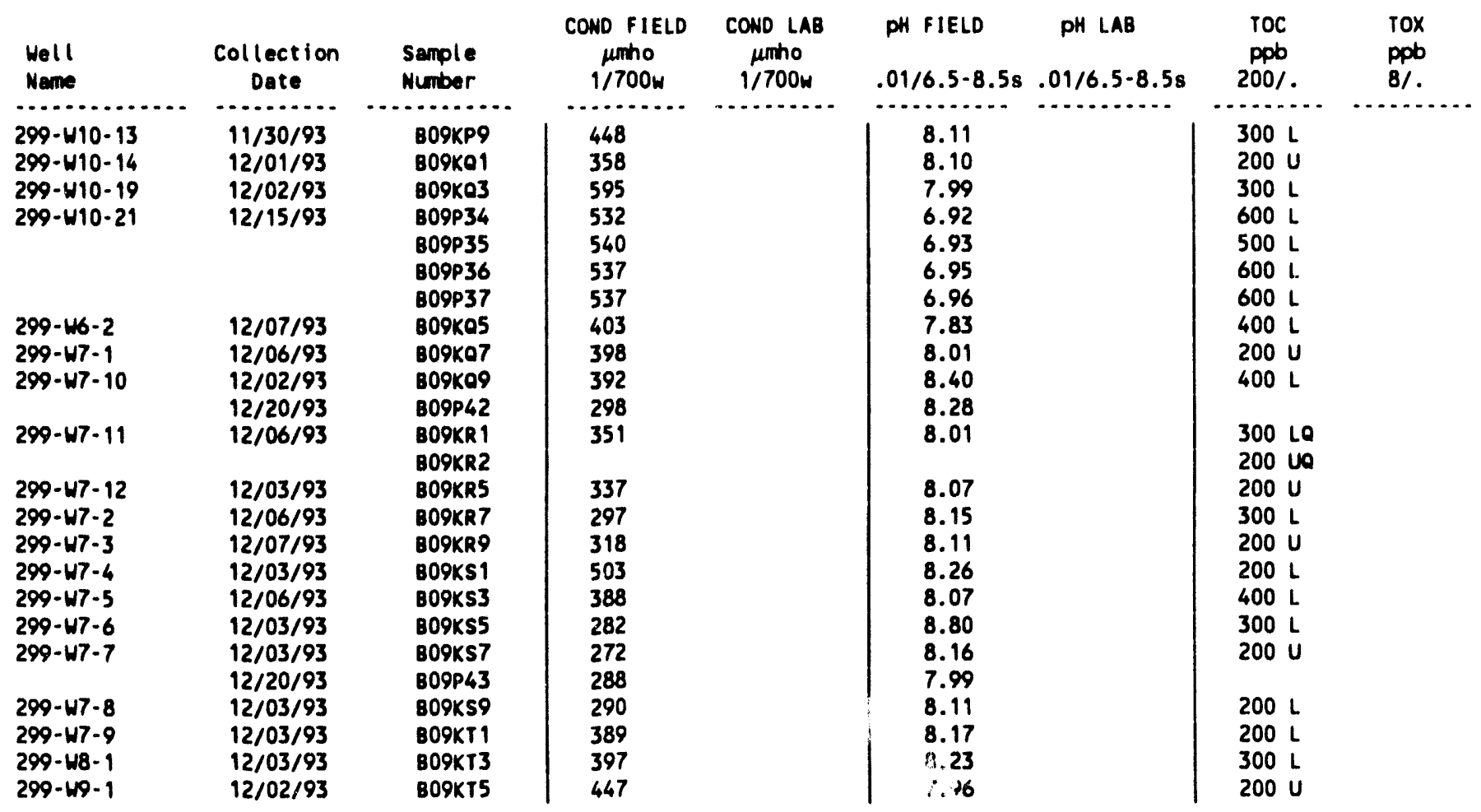

For explanation of this table, see section 1.4 of report. 
Table 15-21. Constituent List and Summary of Results for the Low-Level Waste Burial Grounds WMA 4 Data for Reporting Period October 1 through December 31, 1993. (sheet 1 of 3)

CONTAMINATION INDICATOR PARAMETERS

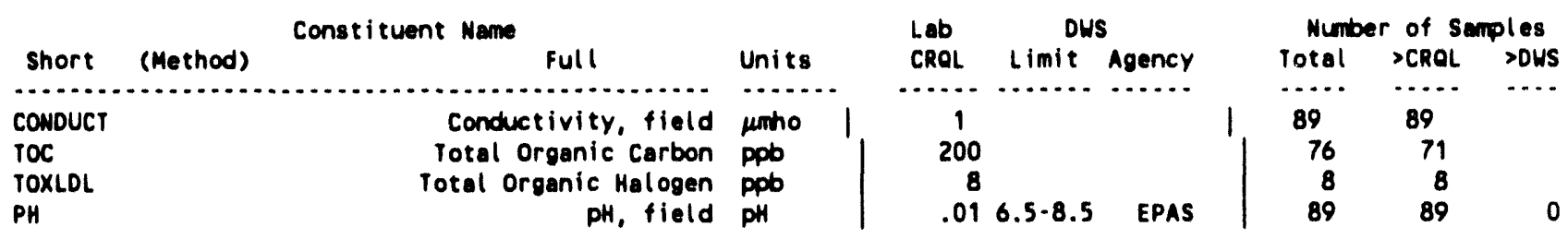

DRINKING HATER PARAMETERS

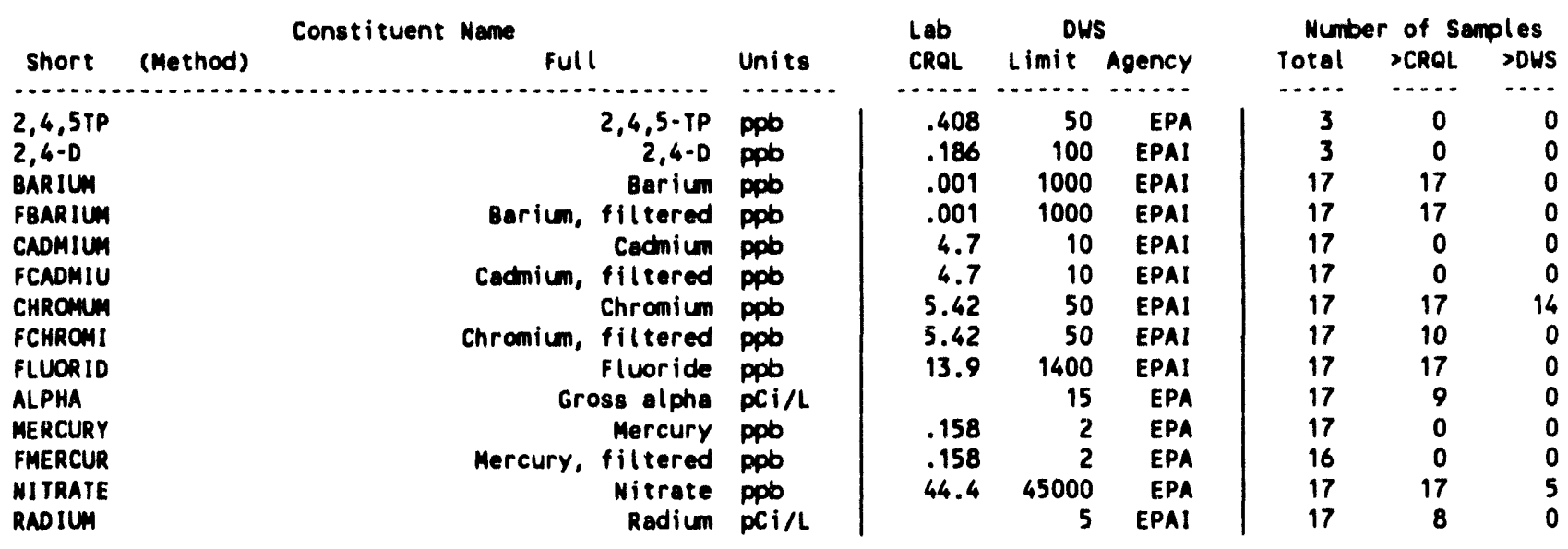

GROUNDWATER QUALITY PARAMETERS

\begin{tabular}{|c|c|c|c|c|c|c|c|c|c|}
\hline \multirow[b]{2}{*}{ Short } & \multirow[b]{2}{*}{ (Method) } & \multirow[t]{2}{*}{ Constituent Name } & \multirow[b]{2}{*}{ Units } & \multirow{2}{*}{$\begin{array}{l}\text { Lab } \\
\text { CROL }\end{array}$} & \multicolumn{2}{|c|}{ DWS } & \multicolumn{3}{|c|}{ Number of Semples } \\
\hline & & & & & Limit & Agency & Total & $>$ CROL & $>$ DWS \\
\hline & & $\cdots$ & & $\ldots \ldots$ & ....... & $\ldots . .$. & $\ldots$. & $\ldots$. & $\ldots$ \\
\hline CHLORID & & Chloride & $p p b$ & 82.5 & 250000 & EPAS & 17 & 17 & 0 \\
\hline IRON & & Iron & ppob & 10.3 & 300 & EPAS & 17 & 17 & 14 \\
\hline FIRON & & Iron, filtered & ppb & 10.3 & 300 & EPAS & 17 & 15 & 0 \\
\hline MANGESE & & Manganese & ppb & 1.35 & 50 & EPAS & 17 & 17 & 4 \\
\hline FMANGAN & & Manganese, filtered & ppob & 1.35 & 50 & EPAS & 17 & 17 & 0 \\
\hline LPHENOL & 19 & Phenol & ppb & .833 & & & 27 & 0 & \\
\hline LPHENOL & 30 & Phenol & $p p b$ & .31 & & & 17 & 0 & \\
\hline Soolum & & Sodium & pob & 40.9 & & & 17 & 17 & \\
\hline Fscolum & & Sodium, filtered & ppb & 40.9 & & & 17 & 17 & \\
\hline SULFATE & & sulfate & ppb & 49.9 & 250000 & EPAS & 17 & 17 & 0 \\
\hline
\end{tabular}


Table 15-21. Constituent List and Summary of Results for the Low-Level Waste Burial Grounds WMA 4 Data for Reporting Period October 1 through December 31, 1993. (sheet 2 of 3)

SITE SPECIFIC AMD OTHER CONSTITUEMTS

\begin{tabular}{|c|c|c|c|c|c|c|c|c|c|}
\hline \multirow{2}{*}{$\begin{array}{l}\text { short } \\
\text { s..... }\end{array}$} & \multirow[b]{2}{*}{ (Method) } & \multirow{2}{*}{ Constituent Mane full } & \multirow[b]{2}{*}{ Units } & \multirow{2}{*}{$\begin{array}{l}\text { Lab } \\
\text { CROL }\end{array}$} & \multicolumn{2}{|c|}{ DWS } & \multicolumn{3}{|c|}{ Number of semples } \\
\hline & & & & & Limit & Agency & Total & $>$ CROL & $>$ DWS \\
\hline $1,1,1 \cdot 1$ & 16 & $1,1,1$-Trichloroethene & pob & .64 & 200 & EPA & $\begin{array}{c}\cdots \\
5\end{array}$ & $\cdots$ & $\cdots_{0}$ \\
\hline $1,1,1-1$ & 25 & 1,1,1-Irichloroethane & ppob & .072 & 200 & EPA & 18 & 0 & $\begin{array}{l}0 \\
0\end{array}$ \\
\hline $1,1,2 \cdot 1$ & 16 & $1,1,2-$ irichloroethene & ppob & .25 & & & 5 & 0 & 0 \\
\hline $1,1,2-1$ & 25 & $1,1,2 \cdot$ Irichloroethane & ppo & .043 & & & 18 & 0 & \\
\hline $1,1-01 C$ & 16 & 1,1-0ichloroethane & ppob & .61 & & & 5 & $\mathbf{0}$ & \\
\hline $1,1-D I C$ & 25 & 1,1-Dichloroethane & ppo & .337 & & & 18 & 0 & \\
\hline 1,2-DIC & 16 & 1.2-Dichloroethene & ppob & .45 & 5 & EPA & 5 & 0 & 0 \\
\hline 1,2-01C & 25 & 1,2-Dichloroethene & ppb & .139 & 5 & EPA & 18 & 0 & 0 \\
\hline $\begin{array}{l}\text { 12-DCE } \\
14-\mathrm{cben}\end{array}$ & & 1,2-0ichloroethene & ppob & 1.2 & & & 5 & 0 & \\
\hline $\begin{array}{l}14-\mathrm{dben} \\
14-\mathrm{cben}\end{array}$ & 16 & 1.4-Dichlorobenzene & ppob & .63 & $\pi$ & EPA & 5 & 0 & 0 \\
\hline $\begin{array}{l}\text { 14-dben } \\
\text { 1BUTYN }\end{array}$ & 25 & 1.4-Dichlorobenzente & Ppb & 107 & $\pi$ & EPA & 18 & 0 & 0 \\
\hline $\begin{array}{l}\text { 1BUTYN } \\
2,4,5-1\end{array}$ & & $\begin{array}{r}\text { 1- But anol } \\
2,4,5-1\end{array}$ & ppm & 13.1 & & & 5 & 0 & \\
\hline $\begin{array}{l}2,4,5-1 \\
246-\operatorname{trp}\end{array}$ & & & ppo & .384 & & & 3 & 0 & \\
\hline $\begin{array}{l}246-\operatorname{trp} \\
26-\mathrm{dchp}\end{array}$ & 19 & $\begin{array}{l}\text { 2,4,6-Trichlorophenol } \\
\text { 2,4-0 ichlorophenol }\end{array}$ & Ppo & 1.45 & & & 17 & 0 & \\
\hline $\begin{array}{l}24-\text { dchp } \\
24-\text { dchp }\end{array}$ & 30 & & Ppo & $\begin{array}{l}2.8 \\
1.5\end{array}$ & & & 27 & 0 & \\
\hline DIMPHEN & & 2,4-0 imethylphenol & $\begin{array}{l}\text { Ppo } \\
\text { Ppb }\end{array}$ & 1.01 & & & $\begin{array}{l}17 \\
17\end{array}$ & $\begin{array}{l}0 \\
0\end{array}$ & \\
\hline DINPHEN & & 2,4-0 initrophenol & ppob & .96 & & & 17 & 0 & \\
\hline 26-dchp & & 2,6-0 ichlorophenol & ppb & 1.59 & & & 17 & 0 & \\
\hline CHLPHEN & & 2-Chlorophenol & ppo & 1.42 & & & 17 & 0 & \\
\hline 2METHPH & & 2-Methylphenol & ppob & 1.8 & & & 27 & 0 & \\
\hline 2NITPH & 19 & 2-Nitrophenol & ppo & 3.96 & & & 27 & 0 & \\
\hline 2NITPH & 30 & 2-Witrophenol & ppob & 1.56 & & & 17 & 0 & \\
\hline BUTOINP & 30 & 2-sec-Butyl-4,6-dinl trophenol (ON & ppb & 1.35 & & & 17 & 0 & \\
\hline BUTOINP & 49 & 2-sec-Butyl-4,6-dinitrophenol (ON & ppb & .269 & & & 3 & 0 & \\
\hline 460N2MP & & 4,6-0 initro-2-methylphenol & ppob & 1.18 & & & 17 & 0 & \\
\hline CHLCRES & & 4-Chloro-3-methylphenol & ppb & 1.12 & & & 17 & 0 & \\
\hline MIBK & & 4-Methyl-2-pentanone & Ppb & .85 & & & 5 & 0 & \\
\hline 4METHPH & & 4-Methylphenol & ppob & 3.54 & & & 27 & 0 & \\
\hline NITPHEN & & 4-Nitrophenol & ppb & .65 & & & 17 & 0 & \\
\hline ACETONE & & Acetone & ppb & 13.4 & & & 5 & 0 & \\
\hline ALUMNUM & & Aluminum & ppb & 32.5 & & & 17 & 7 & \\
\hline FALUUIN & & Aluminum & ppb & 32.5 & & & 17 & 0 & \\
\hline ANTIONY & & Ant imony & ppob & 69.4 & & & 17 & 0 & \\
\hline FANTIMO & & Antimony, filtered & ppb & 69.6 & & & 17 & 1 & \\
\hline BENZENE & 16 & Benzene & ppb & .65 & 5 & EPA & 5 & 0 & 0 \\
\hline BENZENE & 25 & Benzene & ppb & .105 & 5 & EPA & 18 & 3 & 0 \\
\hline BENZOTH & & Benzothiazole & ppb & 2.55 & & & 27 & 0 & \\
\hline BERYLUM & & Beryllium & ppb & .814 & & & 17 & 0 & \\
\hline FBERYLL & & Beryllium, filtered & ppb & .814 & & & 17 & 0 & \\
\hline BIS2EPH & & Bis(2-ethylhexyl) phthalate & ppb & 4.07 & & & 27 & 0 & \\
\hline BROMIDE & & Bromide & ppb & 52.8 & & & 17 & 3 & \\
\hline CALCIUN & & Calcium & ppb & 20.9 & & & 17 & 17 & \\
\hline FCALCIU & & Colcium, filtered & ppob & 20.9 & & & 17 & 17 & \\
\hline CARBIDE & & Carbon disulfide & ppb & .95 & & & 5 & 0 & \\
\hline TETRANE & 16 & Carbon tetrachloride & ppob & .87 & 5 & EPA & 5 & 4 & 3 \\
\hline TETRANE & 25 & Carbon tetrachloride & ppb & .121 & 5 & EPA & 18 & 17 & 16 \\
\hline CHLFORM & 16 & Chloroform & ppb & .4 & & & 5 & 3 & \\
\hline CHLFORM & 25 & Chloroform & ppb & .043 & & & 18 & 17 & \\
\hline COBALT & & Cobolt & ppb & 4.05 & & & 17 & 1 & \\
\hline FCOBALT & & Cobait, filtered & ppo & 4.05 & & & 17 & 0 & \\
\hline COPPER & & Copper & $p p b$ & 2.65 & 1000 & EPAS & 17 & 11 & 0 \\
\hline FCOPPER & & Copper, filtered & ppb & 2.65 & 1000 & EPAS & 17 & 1 & 0 \\
\hline CRESOLS & & Cresols (methylphenols) & ppob & 4.66 & & & 17 & 0 & \\
\hline
\end{tabular}


Table 15-21. Constituent List and Summary of Results for the Low-Level Waste Burial Grounds WMA 4 Data for Reporting Period October 1 through December 31, 1993. (sheet 3 of 3)

\begin{tabular}{|c|c|c|c|c|c|c|c|c|c|}
\hline Short & (Method) & Constl twent Mans & lin to & Lab & DUS & Spancr & Numb & $r$ of $\mathrm{se}$ & ples \\
\hline$\ldots . .$. & ........... & $\ldots \ldots \ldots \ldots$ & $\ldots . .$. & $\ldots .$. & ........ & ....... & $\ldots . .$. & ...... & $\ldots$ \\
\hline CrANIDE & & Cyenide & ppob & 1.26 & & & 18 & 2 & \\
\hline DECANE & & Decane & ppb & 4.03 & & & 27 & 0 & \\
\hline DODECAN & & Dodecene & ppob & 3.62 & & & 27 & 0 & \\
\hline ETHCYAN & & Ethyl cyenide & ppob & 4.34 & & & 5 & 0 & \\
\hline ETHBENZ & & Ethylbanzene & ppob & .046 & 700 & EPA & 18 & 2 & 0 \\
\hline BETA & & Grose bete & $\mathrm{pCi} / \mathrm{L}$ & & & & 17 & 17 & \\
\hline LEAD & & leed & ppos & .508 & 50 & EPAI & 17 & 16 & 0 \\
\hline PLEAD & & Leed, flltered & ppo & .508 & so & EPAI & 17 & 13 & 0 \\
\hline MAGNES & & Magnes ium & ppob & 26 & & & 17 & 17 & \\
\hline FMANES & & Magnesiun, filtered & pab & 26 & & & 17 & 17 & \\
\hline METHONE & & Methyl ethyl ketone & ppo & 4.25 & & & 5 & 0 & \\
\hline $\begin{array}{l}\text { METHYCH } \\
\text { METHYCH }\end{array}$ & 16 & & Ppo & .61 & & & 5 & $\begin{array}{r}0 \\
17\end{array}$ & \\
\hline $\begin{array}{l}\text { METHYCH } \\
\text { MAPHTHA }\end{array}$ & & $\begin{array}{l}\text { Methylene chloride } \\
\text { Naphthalene }\end{array}$ & $\begin{array}{l}\text { ppb } \\
\text { ppb }\end{array}$ & $\begin{array}{r}.056 \\
6.5\end{array}$ & & & 27 & 0 & \\
\hline MICKEL & & Nickel & Ppb & 17.9 & & & 17 & 15 & \\
\hline FNICKEL & & Nickel, filtered & ppo & 17.9 & & & 17 & 2 & \\
\hline NITRITE & & Nitrite & ppob & 38.3 & 1000 & EPA & 17 & $\mathbf{0}$ & 0 \\
\hline PENTCHP & 19 & Pentachlorophenol & ppob & 8.07 & 1 & EPA & 27 & 0 & $27 *$ \\
\hline PENTCHP & 30 & Pentechlorophenol & popo & .87 & 1 & EPA & 17 & 0 & 0 \\
\hline PHOSPHA & & Phosphate & ppob & 147 & & & 17 & 0 & \\
\hline $\begin{array}{l}\text { POTASUM } \\
\text { FPOTASS }\end{array}$ & & Potassium & ppb & 662 & & & $\begin{array}{l}17 \\
17\end{array}$ & 17 & \\
\hline $\begin{array}{l}\text { FPOTASS } \\
\text { SILVER }\end{array}$ & & $\begin{array}{l}\text { Potassium, filtered } \\
\text { silver }\end{array}$ & ppo & , 662 & & & $\begin{array}{l}17 \\
17\end{array}$ & 17 & \\
\hline $\begin{array}{l}\text { SILVER } \\
\text { FSILVER }\end{array}$ & & $\begin{array}{l}\text { sillver } \\
\text { silver, flitered }\end{array}$ & ppb & $\begin{array}{l}2.87 \\
2.87\end{array}$ & 50 & EPAI & $\begin{array}{l}17 \\
17\end{array}$ & 1 & 0 \\
\hline $\begin{array}{l}\text { FSILVER } \\
\text { PERCENE }\end{array}$ & 16 & $\begin{array}{l}\text { Silver, flltered } \\
\text { Tetrachioroethene }\end{array}$ & $\begin{array}{l}\text { ppb } \\
\text { ppb }\end{array}$ & $\begin{array}{r}2.87 \\
1.1\end{array}$ & $\begin{array}{r}50 \\
5\end{array}$ & $\begin{array}{r}\text { EPA! } \\
\text { EPA }\end{array}$ & $\begin{array}{r}17 \\
5\end{array}$ & $\begin{array}{l}0 \\
0\end{array}$ & $\begin{array}{l}0 \\
0\end{array}$ \\
\hline PERCENE & 25 & Tetrechloroethene & ppob & .049 & 5 & EPA & 18 & 10 & 0 \\
\hline TETPHNL & & Tetrachlorophenols & ppo & 1.05 & & & 17 & 0 & \\
\hline TETRADE & & Tetradecane & ppob & 2.43 & & & 27 & 0 & \\
\hline TAF & & Tetrahydrofuran & ppb & 3.67 & & & 5 & 0 & \\
\hline TIN & & Tin & ppb & 51.1 & & & 17 & 0 & \\
\hline FTIN & & Tin, filtered & ppb & 51.1 & & & 17 & 0 & \\
\hline TOLUENE & 16 & Toluene & ppob & .73 & 1000 & EPA & 5 & 0 & 0 \\
\hline TOLUENE & 25 & Tolvene & ppob & .056 & 1000 & EPI & 18 & 1 & 0 \\
\hline TRIBUTPH & & Tributyl Phosphate & ppo & 4.42 & & & 27 & 0 & \\
\hline TRICENE & 16 & Trichloroethene & ppob &.$\pi 7$ & 5 & EPA & 5 & 0 & 0 \\
\hline TRICENE & 25 & irichloroethene & ppob & .065 & 5 & EPA & 18 & 16 & 1 \\
\hline TRIPHNL & & Trichlorophenols & ppb & 1.11 & & & 17 & 0 & \\
\hline TOICHPH & & Tris-2-chloroethyl phosphate & ppob & 2.88 & & & 27 & 0 & \\
\hline TRITIUM & & Tritium & $\mathrm{PCI} / \mathrm{L}$ & & 20000 & EPA & 17 & 2 & 0 \\
\hline TURBID & & Turbidity & NTU & .05 & & & 17 & 17 & \\
\hline URANIUA & & Uranium & ppo & & & & 17 & 17 & \\
\hline VANADUM & & Vanadium & ppb & 3.84 & & & 17 & 16 & \\
\hline FVANADI & & Vanadium, filtered & ppb & 3.84 & & & 17 & 16 & \\
\hline VINYIOE & 16 & Vinyl chloride & ppb & .78 & 2 & EPA & 5 & 0 & 0 \\
\hline VINYIDE & 25 & Vinyl chloride & ppob & .266 & 2 & EPA & 18 & 0 & 0 \\
\hline XYLENE & 16 & Xylenes (total) & ppb & 1.7 & 10000 & EPA & 5 & 0 & 0 \\
\hline XYLENE & 25 & xylenes (total) & ppob & .202 & 10000 & EPA & 18 & 0 & 0 \\
\hline ZINC & & Zinc & ppb & 3.44 & 5000 & EPAS & 17 & 14 & 0 \\
\hline FZINC & & zinc, filtered & ppb & 3.44 & 5000 & EPAS & 17 & 8 & 0 \\
\hline CIS12DE & & cis-1,2-oichloroethylene & ppb & .127 & 70 & EPA & 18 & 0 & 0 \\
\hline MCRESOL & & $m$-Cresol & ppb & 1.44 & & & 27 & 0 & \\
\hline TRANDCE & & trans-1,2-Dichloroethylene & ppb & .149 & 100 & EPA & 18 & 0 & 0 \\
\hline
\end{tabular}

For explanation of this table, see section 1.4 of report. 
Table 15-22. Constituents with at Least One Detected Value for the Low-Level Waste Burial Grounds WMA 4 Data for Reporting Period October 1 through December 31, 1993. (sheet 1 of 10)

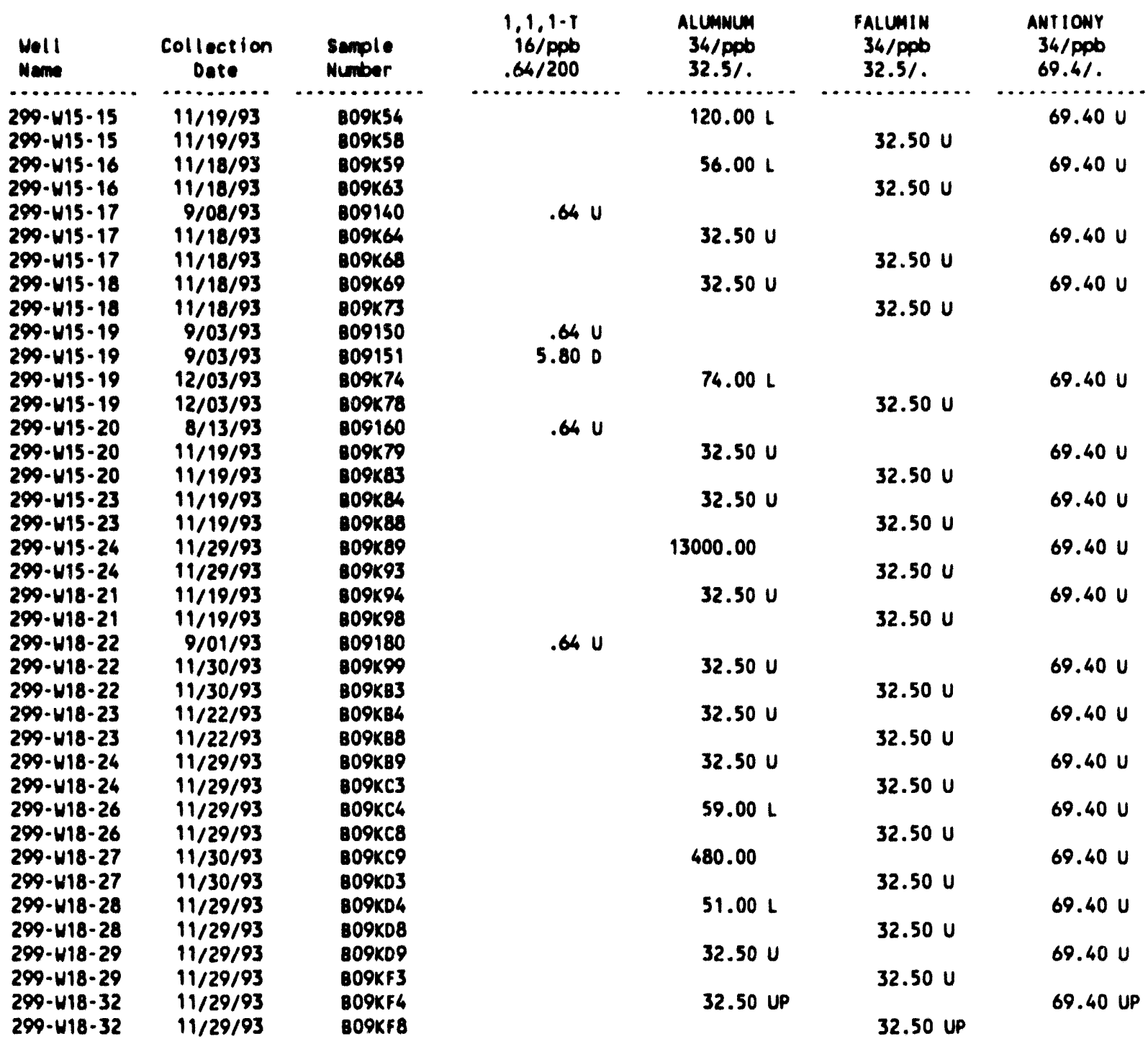

\begin{tabular}{|c|c|c|c|c|c|c|}
\hline $\begin{array}{l}\text { Well } \\
\text { Name }\end{array}$ & $\begin{array}{c}\text { Collection } \\
\text { Date }\end{array}$ & $\begin{array}{l}\text { Semple } \\
\text { Number }\end{array}$ & $\begin{array}{l}\text { FANTIMO } \\
34 / \mathrm{ppD} \\
69.4 / .\end{array}$ & $\begin{array}{c}\text { BARIUM } \\
\text { 34/ppb } \\
.001 / 1000 i\end{array}$ & $\begin{array}{l}\text { FBARIUM } \\
34 / \mathrm{ppb} \\
.001 / 1000 \mathrm{i}\end{array}$ & $\begin{array}{r}\text { BENZENE } \\
25 / \mathrm{pPD} \\
.105 / 5\end{array}$ \\
\hline … & $\cdots$ & a........ & $\cdots \cdots$ & . & . & $\cdots \cdots$ \\
\hline $\begin{array}{l}299-W 15-15 \\
299-W 15-15\end{array}$ & $\begin{array}{l}11 / 19 / 93 \\
11 / 19 / 93\end{array}$ & $\begin{array}{l}809 \times 54 \\
809 \times 58\end{array}$ & $69.40 \mathrm{U}$ & 44.0080 & 41.0080 & $.11 \mathrm{UH}$ \\
\hline $\begin{array}{l}299-W 15-16 \\
299-W 15-16\end{array}$ & $\begin{array}{l}11 / 18 / 93 \\
11 / 18 / 93\end{array}$ & $\begin{array}{l}809 \times 59 \\
809 \times 63\end{array}$ & $69.40 \mathrm{U}$ & 79.0080 & 76.0080 & $.11 \mathrm{U}$ \\
\hline $\begin{array}{l}299-\text { W15-16 } \\
299-\text { W15-17 }\end{array}$ & $\begin{array}{l}12 / 06 / 93 \\
11 / 18 / 93\end{array}$ & $\begin{array}{l}\text { B09N18 } \\
809 \times 64\end{array}$ & & $76.00 \mathrm{BO}$ & & $\begin{array}{l}.11 \mathrm{U} \\
.11 \mathrm{U}\end{array}$ \\
\hline $\begin{array}{l}299-W 15-17 \\
299-W 15-18\end{array}$ & $\begin{array}{l}11 / 18 / 93 \\
11 / 18 / 93\end{array}$ & $\begin{array}{l}809 \times 68 \\
809 \times 69\end{array}$ & $69.40 \mathrm{U}$ & $62.00 \mathrm{BO}$ & 75.0080 & $.11 \mathrm{U}$ \\
\hline
\end{tabular}


Table 15-22. Constituents with at Least One Detected Value for the Low-Level Waste Burial Grounds WMA 4 Data for Reporting Period October 1 through De cember 31, 1993. (sheet 2 of 10)

\begin{tabular}{|c|c|c|c|c|c|c|}
\hline $\begin{array}{l}\text { Well } \\
\text { Neme }\end{array}$ & $\begin{array}{c}\text { Collection } \\
\text { Date }\end{array}$ & $\begin{array}{l}\text { Sample } \\
\text { Number }\end{array}$ & $\begin{array}{l}\text { FANTIMO } \\
34 / \text { ppb } \\
69.4 / .\end{array}$ & $\begin{array}{c}\text { BARIUN } \\
34 / \text { ppb } \\
.001 / 10001\end{array}$ & $\begin{array}{l}\text { FBARIUA } \\
34 / \text { ppb } \\
.001 / 1000 i\end{array}$ & $\begin{array}{r}\text { BENZENE } \\
25 / \text { Pp6 } \\
.105 / 5\end{array}$ \\
\hline $\begin{array}{l}299-W 15-18 \\
299-W 15-19 \\
299-W 15-19 \\
299-W 15-20 \\
299-W 15-20 \\
299-W 15-23 \\
299-W 15-23 \\
299-W: 5-24 \\
299-W 15-24 \\
299-W 18-21 \\
299-W 18-21 \\
299-W 18-22 \\
299-W 18-22 \\
299-W 18-23 \\
299-W 18-23 \\
299-W 18-24 \\
299-W 18-24 \\
299-W 18-26 \\
299-W 18-26 \\
299-W 18-27 \\
299-W 18-27 \\
299-W 18-28 \\
299-W 18-28 \\
299-W 18-29 \\
299-W 18-29 \\
299-W 18-32 \\
299-W 18-32\end{array}$ & $\begin{array}{l}11 / 18 / 93 \\
12 / 03 / 93 \\
12 / 03 / 93 \\
11 / 19 / 93 \\
11 / 19 / 93 \\
11 / 19 / 93 \\
11 / 19 / 93 \\
11 / 29 / 93 \\
11 / 29 / 93 \\
11 / 19 / 93 \\
11 / 19 / 93 \\
11 / 30 / 93 \\
11 / 30 / 93 \\
11 / 22 / 93 \\
11 / 22 / 93 \\
11 / 29 / 93 \\
11 / 29 / 93 \\
11 / 29 / 93 \\
11 / 29 / 93 \\
11 / 30 / 93 \\
11 / 30 / 93 \\
11 / 29 / 93 \\
11 / 29 / 93 \\
11 / 29 / 93 \\
11 / 29 / 93 \\
11 / 29 / 93 \\
11 / 29 / 93\end{array}$ & 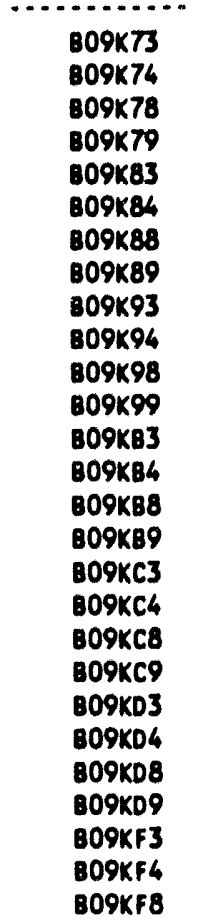 & $\begin{array}{l}69.40 \mathrm{U} \\
69.40 \mathrm{U} \\
69.40 \mathrm{U} \\
69.40 \mathrm{U} \\
69.40 \mathrm{U} \\
69.40 \mathrm{U} \\
69.40 \mathrm{U} \\
77.00 \mathrm{~L} \\
69.40 \mathrm{U} \\
69.40 \mathrm{U} \\
69.40 \mathrm{U} \\
69.40 \mathrm{U} \\
69.40 \mathrm{U} \\
69.40 \mathrm{UP}\end{array}$ & $\begin{array}{l}61.00 \\
44.0080 \\
30.0080 \\
220.0080 \\
30.0080 \\
56.0080 \\
28.0080 \\
24.0080 \\
44.0080 \\
40.0080 \\
63.0080 \\
28.0080\end{array}$ & $\begin{array}{l}66.00 \mathrm{BO} \\
58.00 \\
41.00 \mathrm{B0} \\
29.00 \mathrm{BO} \\
76.00 \mathrm{BO} \\
11.00 \mathrm{LBO} \\
57.00 \mathrm{BO} \\
28.00 \mathrm{BO} \\
24.00 \mathrm{BO} \\
42.00 \mathrm{BO} \\
32.00 \mathrm{BO} \\
59.00 \mathrm{BO} \\
29.00 \mathrm{BO}\end{array}$ & $\begin{array}{l}.11 \mathrm{U} \\
.16 \mathrm{LH} \\
.11 \mathrm{UH} \\
.24 \mathrm{BL} \\
.11 \mathrm{UH} \\
.11 \mathrm{U} \\
3.00 \\
.11 \mathrm{U} \\
.11 \mathrm{U} \\
.11 \mathrm{U} \\
.11 \mathrm{U} \\
.11 \mathrm{U} \\
.11 \mathrm{U}\end{array}$ \\
\hline
\end{tabular}

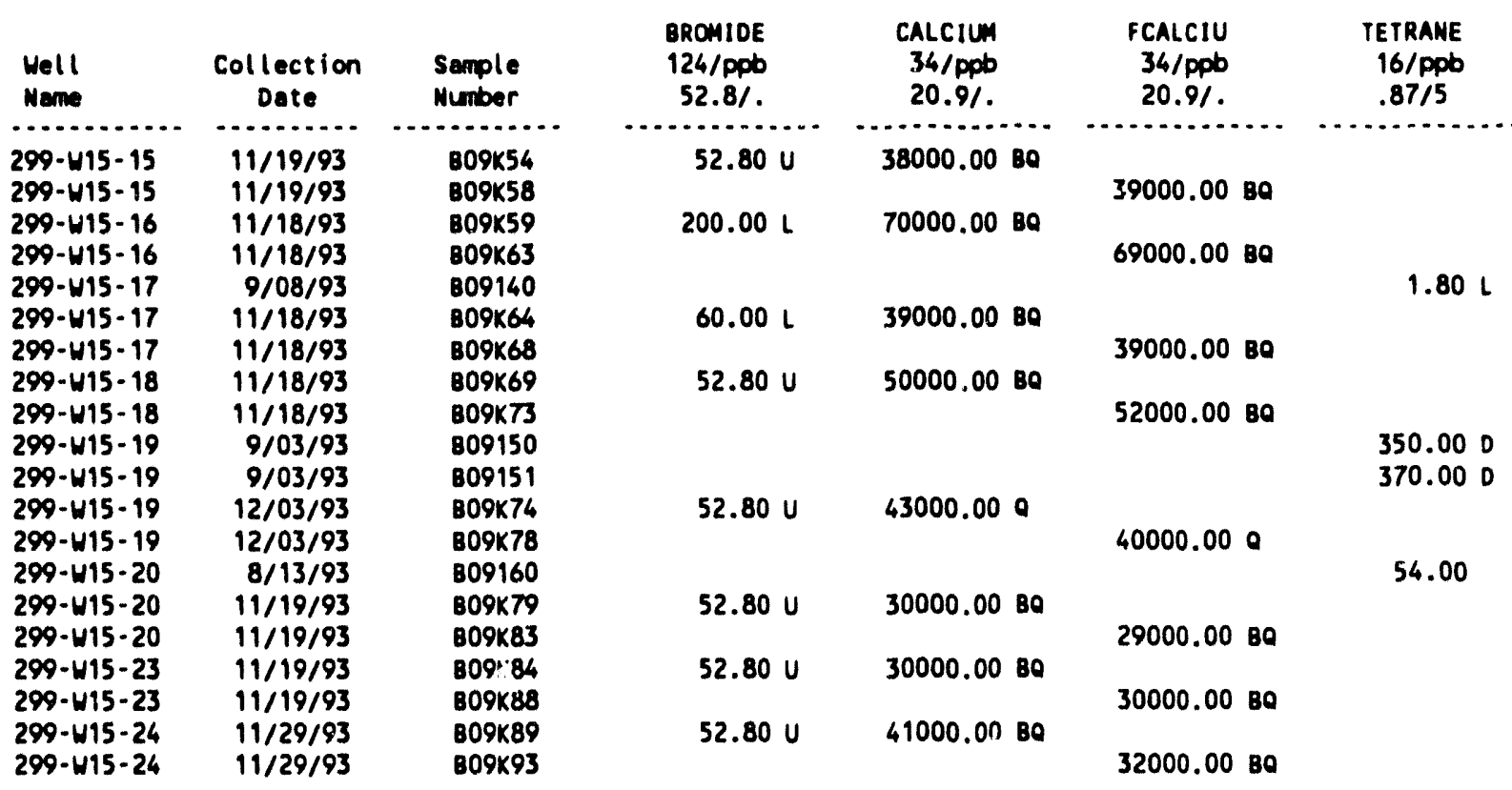


Table 15-22. Constituents with at Least One Detected Value for the Low-Level Waste Burial Grounds WMA 4 Data for Reporting Period October 1

through December 31, 1993. (sheet 3 of 10)

\begin{tabular}{|c|c|c|c|c|c|}
\hline $\begin{array}{l}\text { Well } \\
\text { Name }\end{array}$ & $\begin{array}{c}\text { Collection } \\
\text { Date }\end{array}$ & $\begin{array}{l}\text { Sample } \\
\text { Number }\end{array}$ & $\begin{array}{c}\text { BROMIDE } \\
124 / \mathrm{ppb} \\
52.8 \%\end{array}$ & $\begin{array}{c}\text { CALCIUM } \\
34 / \mathrm{ppb} \\
20.9 / .\end{array}$ & $\begin{array}{c}\text { FCALCIU } \\
34 / \mathrm{ppb} \\
20.91 .\end{array}$ \\
\hline $\begin{array}{l}299-W 18-21 \\
299-W 18-21 \\
299-W 18-22\end{array}$ & $\begin{array}{r}11 / 19 / 93 \\
11 / 19 / 93 \\
9 / 01 / 93\end{array}$ & $\begin{array}{l}\text { B09K94 } \\
809 K 98 \\
\text { B09180 }\end{array}$ & $52.80 \mathrm{U}$ & $26000.00 \mathrm{BQ}$ & $10000.00 \mathrm{BQ}$ \\
\hline $\begin{array}{l}299-W 18-22 \\
299-W 18-22\end{array}$ & $\begin{array}{l}11 / 30 / 93 \\
11 / 30 / 93\end{array}$ & $\begin{array}{l}\text { B09K99 } \\
\text { B09KB3 }\end{array}$ & $80.00 \mathrm{~L}$ & $37000.00 \mathrm{BO}$ & 37000.00 BO \\
\hline $\begin{array}{l}299-W 18-23 \\
290-W 18-23\end{array}$ & $\begin{array}{l}11 / 22 / 93 \\
11 / 22 / 93\end{array}$ & $\begin{array}{l}\text { B09KB4 } \\
\text { 809KB8 }\end{array}$ & $52.80 \mathrm{U}$ & $28000.00 B 0$ & $28000.00 \mathrm{BO}$ \\
\hline $\begin{array}{l}299-W 18-24 \\
299-W 18-24\end{array}$ & & $\begin{array}{l}\text { B09KB9 } \\
\text { B09KC3 }\end{array}$ & $52.80 \mathrm{U}$ & $22000.00 \mathrm{BO}$ & 22000.0080 \\
\hline $\begin{array}{l}299-W 18-26 \\
200-418-26\end{array}$ & $\begin{array}{l}11 / 29 / 93 \\
11 / 29 / 93\end{array}$ & $\begin{array}{l}809 \mathrm{KC4} \\
\text { B09KC8 }\end{array}$ & $52.80 \mathrm{U}$ & 31000.0080 & $30000.00 \mathrm{BO}$ \\
\hline $299-418-27$ & $11 / 30 / 93$ & B09KC9 & $52.80 \mathrm{U}$ & $25000.00 \mathrm{BO}$ & \\
\hline $299-N 18-27$ & $11 / 30 / 93$ & B09KD3 & & & $25000.00 \mathrm{BQ}$ \\
\hline $\begin{array}{l}299-w 18-28 \\
299-w 18-28\end{array}$ & $\begin{array}{l}11 / 29 / 93 \\
11 / 29 / 93\end{array}$ & $\begin{array}{l}\text { 809KD4 } \\
\text { B09KD8 }\end{array}$ & $52.80 \mathrm{U}$ & $29000.00 \mathrm{BO}$ & $28000.00 \mathrm{BO}$ \\
\hline $299-W 18-29$ & $11 / 29 / 93$ & B09KD9 & $52.80 \mathrm{U}$ & 25000.0080 & \\
\hline $299-418-29$ & $11 / 29 / 93$ & B09KF3 & & & $24000.00 \mathrm{BO}$ \\
\hline $\begin{array}{l}299--418-52 \\
299-W 18-32\end{array}$ & $\begin{array}{l}11 / 29 / 95 \\
11 / 29 / 93\end{array}$ & B09KF8 & 36 & $.00 \mathrm{BPO}$ & $20000.00 \mathrm{BPO}$ \\
\hline
\end{tabular}

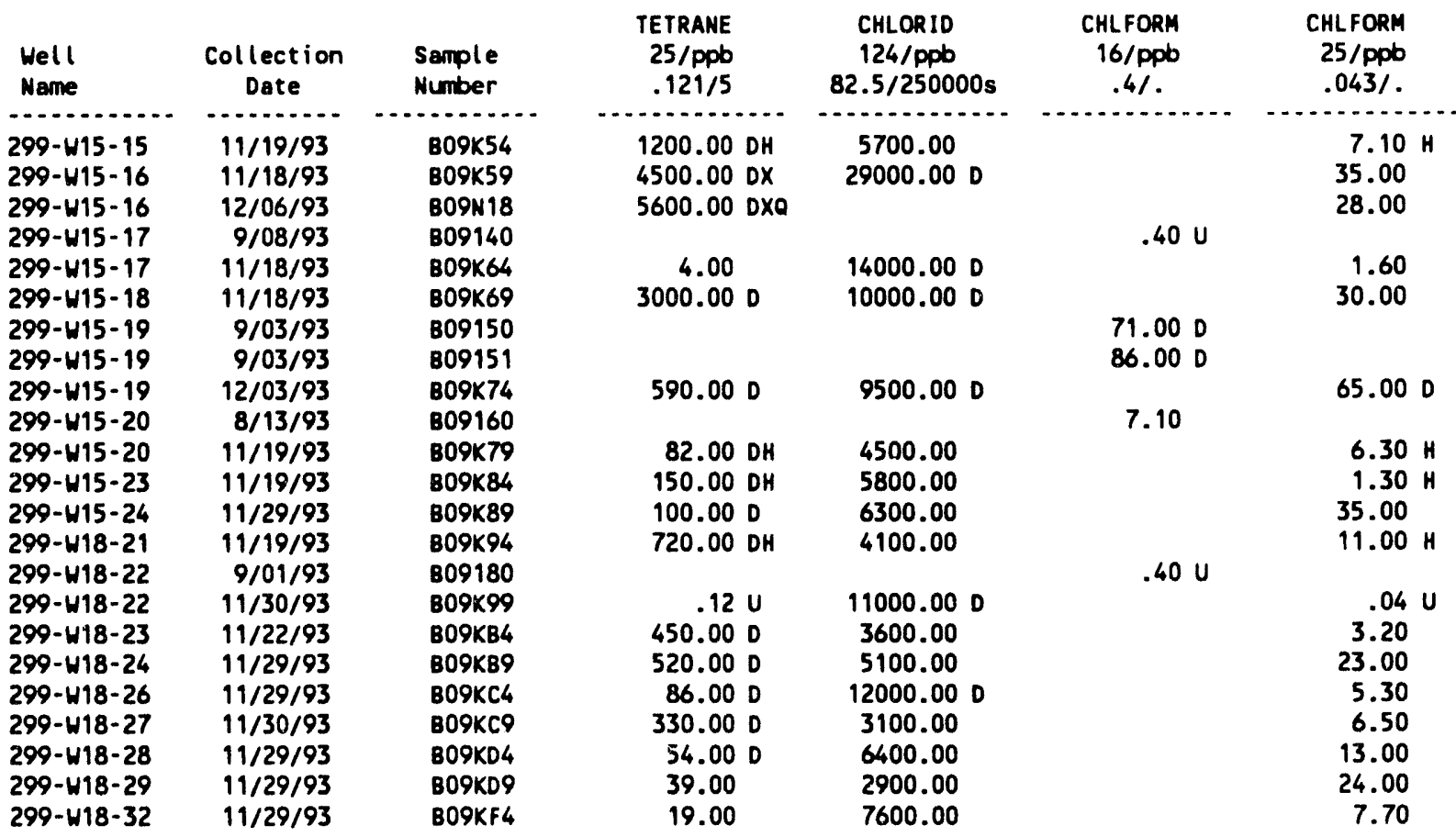


Table 15-22. Constituents with at Least One Detected Value for the Low-Level Waste Burial Grounds WMA 4 Data for Reporting Period October 1 through December 31, 1993. (sheet 4 of 10)

\begin{tabular}{|c|c|c|c|c|c|c|}
\hline $\begin{array}{l}\text { Well } \\
\text { Name }\end{array}$ & $\begin{array}{c}\text { Collection } \\
\text { Date }\end{array}$ & $\begin{array}{l}\text { Sample } \\
\text { Number }\end{array}$ & $\begin{array}{c}\text { CHROMUM } \\
34 / \mathrm{ppb} \\
5.42 / 50 \mathrm{i}\end{array}$ & $\begin{array}{c}\text { FCHROMI } \\
34 / \mathrm{ppb} \\
5.42 / 50 \mathrm{i}\end{array}$ & $\begin{array}{l}\text { COBALT } \\
34 / \mathrm{ppb} \\
4.05 /\end{array}$ & $\begin{array}{c}\text { FCOBALT } \\
34 / \mathrm{ppb} \\
4.05 / .\end{array}$ \\
\hline $299-415 \cdot 15$ & $11 / 19 / 93$ & $809 \times 54$ & 290.00 & $\cdots$ & 4.05 vo & \\
\hline $\begin{array}{l}299-W 15-15 \\
299-W 15-15\end{array}$ & $\begin{array}{l}11 / 19 / 45 \\
11 / 19 / 93\end{array}$ & B09K58 & 290.00 & $11.00 \mathrm{~L}$ & 4.0500 & 4.05 vo \\
\hline $299-W 15-16$ & $11 / 18 / 93$ & B09K59 & 110.00 & & 4.05 vo & \\
\hline $299-415-16$ & $11 / 18 / 93$ & 809K63 & & $12.00 \mathrm{~L}$ & & 4.05 vo \\
\hline $299-W 15-17$ & $11 / 18 / 93$ & $809 \times 64$ & 250.00 & & 4.0500 & \\
\hline $299-W 15-17$ & $11 / 18 / 93$ & B09K68 & & $12.00 \mathrm{~L}$ & & 4.05 vo \\
\hline $299-W 15-18$ & $11 / 18 / 93$ & B09K69 & 92.00 & & 4.05 vo & \\
\hline $299-415-18$ & $11 / 18 / 93$ & B09K73 & & 46.00 & & 4.05 va \\
\hline $299-W 15-19$ & $12 / 03 / 93$ & B09K74 & 640.00 & & $4.05 \mathrm{U}$ & \\
\hline $299-W 15-19$ & $12 / 03 / 93$ & $809 \times 78$ & & $13.00 \mathrm{~L}$ & & $4.05 \mathrm{U}$ \\
\hline $299-415-20$ & $11 / 19 / 93$ & B09K79 & 650.00 & & 4.05 vo & \\
\hline $299-W 15-20$ & $11 / 19 / 93$ & B09K83 & & $18.00 \mathrm{~L}$ & & 4.05 vo \\
\hline $299-W 15-23$ & $11 / 19 / 93$ & $809 \times 84$ & 170.00 & & 4.0500 & \\
\hline $299-415-23$ & $11 / 19 / 93$ & $809 \times 88$ & & $7.40 \mathrm{~L}$ & & 4.05 va \\
\hline $\begin{array}{l}299-W 15-24 \\
299-W 15-24\end{array}$ & $\begin{array}{l}11 / 29 / 93 \\
11 / 29 / 93\end{array}$ & $\begin{array}{l}\text { B09K89 } \\
\text { B09K93 }\end{array}$ & 1100.00 & $7.50 \mathrm{~L}$ & 4.05 vo & 4.05 va \\
\hline $299-418-21$ & $11 / 19 / 93$ & B09K94 & 130.00 & & 4.05 vo & \\
\hline $299-W 18-21$ & $11 / 19 / 93$ & $809 \times 98$ & & $5.42 U$ & & 4.05 vo \\
\hline $299-W 18-22$ & $11 / 30 / 93$ & $809 \times 99$ & 130.00 & & $16.00 \mathrm{LO}$ & \\
\hline $299-W 18-22$ & $91 / 30 / 93$ & $809 \times 83$ & & $9.80 \mathrm{~L}$ & & 4.05 vo \\
\hline $299-418-23$ & $11 / 22 / 93$ & B09KB4 & 30.00 & & 4.0500 & \\
\hline $\begin{array}{l}299-W 18-23 \\
299-W 18-24\end{array}$ & $\begin{array}{l}11 / 22 / 93 \\
11 / 29 / 93\end{array}$ & $\begin{array}{l}\text { 809KB8 } \\
\text { 809KB9 }\end{array}$ & 47.00 & $5.42 \mathrm{U}$ & 4.05 vo & 4.0500 \\
\hline $299-418-24$ & $11 / 29 / 93$ & B09KC3 & 81.00 & $5.42 \mathrm{U}$ & 4.0500 & 4.05 vo \\
\hline $299-418-26$ & $11 / 29 / 93$ & B09KC4 & 470.00 & & 4.0500 & \\
\hline $299-W 18-26$ & $11 / 29 / 93$ & B09KC8 & & $10.00 \mathrm{~L}$ & & 4.05 vo \\
\hline & $11 / 30 / 93$ & $\begin{array}{l}809 \times C 9 \\
800 \times 03\end{array}$ & 270.00 & & 4.0500 & \\
\hline $\begin{array}{l}299-W 18-27 \\
299-W 18-28\end{array}$ & $\begin{array}{l}11 / 30 / 93 \\
11 / 29 / 93\end{array}$ & $\begin{array}{l}\text { B09KD3 } \\
\text { B09KD4 }\end{array}$ & 84.00 & $5.42 \mathrm{U}$ & 40540 & 4.05 Ua \\
\hline $299-W 18-28$ & $11 / 29 / 93$ & B09KD8 & 84.00 & $5.42 U$ & 4.0500 & 4.05 vo \\
\hline $299-W 18-29$ & $11 / 29 / 93$ & 809KD9 & 72.00 & & 4.0500 & \\
\hline $299-418-29$ & $11 / 29 / 93$ & B09KF3 & & $5.42 \mathrm{U}$ & & 4.05 vo \\
\hline $299-418-32$ & $11 / 29 / 93$ & B09KF 4 & $24.00 \mathrm{P}$ & & 4.05 UPO & \\
\hline $299-W 18-32$ & $11 / 29 / 93$ & B09KF8 & & 5.42 UP & & 4.05 UPQ \\
\hline
\end{tabular}

\begin{tabular}{|c|c|c|c|c|c|c|}
\hline $\begin{array}{l}\text { Well } \\
\text { Name }\end{array}$ & $\begin{array}{c}\text { Collection } \\
\text { Date }\end{array}$ & $\begin{array}{l}\text { Sample } \\
\text { Number }\end{array}$ & $\begin{array}{c}\text { COPPER } \\
34 / \mathrm{ppb} \\
2.65 / 1000 \mathrm{~s}\end{array}$ & $\begin{array}{c}\text { FCOPPER } \\
34 / \mathrm{ppb} \\
2.65 / 1000 \mathrm{~s}\end{array}$ & $\begin{array}{c}\text { CYANIDE } \\
358 / \mathrm{ppb} \\
1.24 /\end{array}$ & $\begin{array}{l}\text { ETHBENZ } \\
25 / \text { ppb } \\
.046 / 700\end{array}$ \\
\hline $299-W 15-15$ & $11 / 19 / 93$ & B09k54 & $2.65 \mathrm{U}$ & - & $1.24 \mathrm{U}$ & $.05 \mathrm{BLH}$ \\
\hline $299-W 15-15$ & $11 / 19 / 93$ & B09K58 & & $2.65 \mathrm{U}$ & & \\
\hline $299-W 15-16$ & $11 / 18 / 93$ & 809K59 & $2.65 \mathrm{U}$ & & $1.24 \mathrm{U}$ & $.05 \mathrm{U}$ \\
\hline $299-w 15-16$ & $11 / 18 / 93$ & B09K63 & & $2.65 \mathrm{U}$ & & \\
\hline $299-W 15-16$ & $12 / 06 / 93$ & BO9N18 & & & & $.05 \mathrm{U}$ \\
\hline $299-W 15-17$ & $9 / 08 / 93$ & $B 09140$ & & & $2.00 \mathrm{BL}$ & \\
\hline $299-W 15-17$ & $11 / 18 / 93$ & $809 \times 64$ & $5.30 \mathrm{~L}$ & & $3.00 \mathrm{~L}$ & $.05 \mathrm{U}$ \\
\hline $299-W 15-17$ & $11 / 18 / 93$ & B09K68 & & $2.65 \mathrm{U}$ & & \\
\hline $299-W 15-18$ & $11 / 18 / 93$ & B09K69 & $2.65 \mathrm{U}$ & & $1.24 \mathrm{U}$ & $.05 \mathrm{U}$ \\
\hline $299-W 15-18$ & $11 / 18 / 93$ & B09K73 & & $8.30 \mathrm{~L}$ & & \\
\hline 299-W15-19 & $12 / 03 / 93$ & $809 \times 74$ & 20.00 & & $1.24 \mathrm{U}$ & $.05 \mathrm{U}$ \\
\hline $299-W 15-19$ & $12 / 03 / 93$ & B09K78 & & $2.65 \mathrm{U}$ & & \\
\hline $299-W 15-20$ & $11 / 19 / 93$ & $309 \times 79$ & 26.00 & & $1.24 \mathrm{U}$ & $.06 \mathrm{BLH}$ \\
\hline
\end{tabular}


Table 15-22. Constituents with at Least One Detected Value for the Low-Level Waste Burial Grounds WMA 4 Data for Reporting Period October 1 through December 31, 1993. (sheet 5 of 10)

\begin{tabular}{|c|c|c|c|c|c|c|}
\hline $\begin{array}{l}\text { Well } \\
\text { Name }\end{array}$ & $\begin{array}{c}\text { Collection } \\
\text { Date }\end{array}$ & $\begin{array}{l}\text { Sample } \\
\text { Number }\end{array}$ & $\begin{array}{c}\text { COPPER } \\
34 / P p b \\
2.65 / 1000 \mathrm{~s}\end{array}$ & $\begin{array}{c}\text { FCOPPER } \\
34 / \text { ppb } \\
2.65 / 1000 \mathrm{~s}\end{array}$ & $\begin{array}{c}\text { CYANIDE } \\
\text { 358/ppb } \\
1.24 / .\end{array}$ & $\begin{array}{l}\text { ETHBENZ } \\
25 / \mathrm{ppb} \\
.046 / 700\end{array}$ \\
\hline $299-\$ 15-20$ & $11 / 19 / 93$ & $809 \times 83$ & - & $2.65 \mathrm{U}$ & & \\
\hline $299-W 15-23$ & $11 / 19 / 93$ & 809K84 & $16.00 \mathrm{~L}$ & & $1.24 \mathrm{U}$ & .05 UH \\
\hline $299-415-23$ & $11 / 19 / 93$ & $809 \times 88$ & & $2.65 \mathrm{U}$ & & \\
\hline $299-W 15-24$ & $11 / 29 / 93$ & B09K89 & 67.00 & & $1.24 \mathrm{U}$ & $.05 \mathrm{U}$ \\
\hline $299-\$ 15-24$ & $11 / 29 / 93$ & $809 \times 93$ & & $2.65 \mathrm{U}$ & & \\
\hline $299-W 18-21$ & $11 / 19 / 93$ & B09K94 & $2.65 \mathrm{U}$ & & $1.24 \mathrm{U}$ & $.05 \mathrm{UH}$ \\
\hline $299-418-21$ & $11 / 19 / 93$ & B09K98 & & $2.65 \mathrm{U}$ & & \\
\hline $299-W 18-22$ & $11 / 30 / 93$ & B09K99 & $5.30 \mathrm{~L}$ & & $1.24 \mathrm{U}$ & $.05 \mathrm{U}$ \\
\hline $299-418-22$ & $11 / 30 / 93$ & B09KB3 & & $2.65 \mathrm{U}$ & & \\
\hline $299-418-23$ & $11 / 22 / 93$ & B09KB4 & $6.80 \mathrm{~L}$ & & $1.24 \mathrm{U}$ & $.05 \mathrm{U}$ \\
\hline $299-H 18-23$ & $11 / 22 / 93$ & B09KB8 & & $2.65 \mathrm{U}$ & & \\
\hline $299-W 18-24$ & $11 / 29 / 93$ & B09KB9 & $2.65 \mathrm{U}$ & & $1.24 \mathrm{U}$ & $.05 U$ \\
\hline 299-W18-24 & $11 / 29 / 93$ & B09KC3 & & $2.65 \mathrm{U}$ & & \\
\hline $299-418-26$ & $11 / 29 / 93$ & B09KC4 & 25.00 & & $1.2+U$ & $.05 U$ \\
\hline $299-W 18-26$ & $11 / 29 / 93$ & B09KC8 & & $2.65 \mathrm{U}$ & & \\
\hline $299-H 18-27$ & $11 / 30 / 93$ & B09KC9 & $16.00 \mathrm{~L}$ & & $1.24 \mathrm{U}$ & $.05 \mathrm{U}$ \\
\hline $299-W 18-27$ & $11 / 30 / 93$ & B09KD3 & & $2.65 \mathrm{U}$ & & \\
\hline $299-W 18-28$ & $11 / 29 / 93$ & B09KD4 & $11.00 \mathrm{~L}$ & & $1.24 \mathrm{U}$ & $.05 \mathrm{U}$ \\
\hline $299-W 18-28$ & $11 / 29 / 93$ & B09KD8 & & $2.65 \mathrm{U}$ & & \\
\hline $299-W 18-29$ & $11 / 29 / 93$ & B09KD9 & $6.00 \mathrm{~L}$ & & $1.24 \mathrm{U}$ & $.05 \mathrm{U}$ \\
\hline $299-W 18-29$ & $11 / 29 / 93$ & B09KF3 & & $2.65 \mathrm{U}$ & & \\
\hline $299-W 18-32$ & $11 / 29 / 93$ & B09KF4 & 2.65 UP & & $1.24 \mathrm{U}$ & $.05 \mathrm{U}$ \\
\hline $299-W 18-32$ & $11 / 29 / 93$ & B09KF8 & & 2.65 UP & & \\
\hline
\end{tabular}

\begin{tabular}{|c|c|c|c|c|c|c|}
\hline $\begin{array}{l}\text { Well } \\
\text { Name }\end{array}$ & $\begin{array}{c}\text { Collection } \\
\text { Date }\end{array}$ & $\begin{array}{l}\text { Sample } \\
\text { Number }\end{array}$ & $\begin{array}{l}\text { FLUORID } \\
124 / \mathrm{ppD} \\
13.9 / 1400 \mathrm{i}\end{array}$ & $\begin{array}{c}\text { ALPHA } \\
135 / \mathrm{pCi} / \mathrm{L} \\
. / 15\end{array}$ & $\begin{array}{c}\text { BETA } \\
136 / \mathrm{pCi} / \mathrm{L} \\
. \%\end{array}$ & $\begin{array}{c}\text { IRON } \\
\text { 34/ppb } \\
10.3 / 300 \mathrm{~s}\end{array}$ \\
\hline $299-W 15-15$ & $11 / 19 / 93$ & B09K54 & 500.00 & 5.18 & 10.80 & 1500.00 \\
\hline $299-W 15-16$ & $11 / 18 / 93$ & B09K59 & 1100.00 & 2.09 & 6.85 & 630.00 \\
\hline $299-W 15-17$ & $11 / 18 / 93$ & $809 \times 64$ & 700.00 & $.67 \mathrm{U}$ & 8.11 & 1100.00 \\
\hline $299-W 15-18$ & $11 / 18 / 93$ & B09K69 & 900.00 & $.04 \mathrm{U}$ & 6.28 & 390.00 \\
\hline 299-W15-19 & $12 / 03 / 93$ & $809 \times 74$ & 600.00 & 2.82 & 9.81 & 2800.00 \\
\hline $299-415-20$ & $11 / 19 / 93$ & B09K79 & 500.00 & 1.35 & 3.74 & 2700.00 \\
\hline $299-W 15-23$ & $11 / 19 / 93$ & B09K84 & 400.00 & $.52 \mathrm{U}$ & 4.04 & 700.00 \\
\hline $299-W 15-24$ & $11 / 29 / 93$ & B09K89 & 600.00 & 1.53 & 9.46 & 24000.00 \\
\hline $299-W 18-21$ & $11 / 19 / 93$ & B09K94 & 600.00 & 9.39 & 6.04 & 640.00 \\
\hline $299-w 18-22$ & $11 / 30 / 93$ & $809 \times 99$ & 400.00 & $.75 \mathrm{U}$ & 5.35 & 510.00 \\
\hline $299-W 18-23$ & $11 / 22 / 93$ & B09KB4 & 1000.00 & $.95 \mathrm{U}$ & 3.73 & 120.00 \\
\hline $299-418-24$ & $11 / 29 / 93$ & B09KB9 & 800.00 & $.17 \mathrm{U}$ & 3.96 & 170.00 \\
\hline $299-418-26$ & $11 / 29 / 93$ & B09KC4 & 600.00 & $.77 \mathrm{u}$ & 4.42 & 2000.00 \\
\hline $299-418-27$ & $11 / 30 / 93$ & B09KC9 & 500.00 & 2.03 & 3.13 & 2000.00 \\
\hline $299-H 18-28$ & $11 / 29 / 93$ & B09KD4 & 600.00 & $1.05 \mathrm{U}$ & 5.21 & 540.00 \\
\hline $299-W 18-29$ & $11 / 29 / 93$ & B09KD9 & 300.00 & 1.76 & 3.07 & 370.00 \\
\hline $299-W 18-32$ & $11 / 29 / 93$ & B09KF4 & 600.00 & 1.74 & 2.78 & $270.00 F$ \\
\hline
\end{tabular}


Table 15-22. Constituents with at Least One Detected Value for the Low-Level Waste Burial Grounds WMA 4 Data for Reporting Period October 1 through December 31, 1993. (sheet 6 of 10)

\begin{tabular}{|c|c|c|c|c|c|c|}
\hline $\begin{array}{l}\text { Well } \\
\text { Name }\end{array}$ & $\begin{array}{c}\text { Collection } \\
\text { Date }\end{array}$ & $\begin{array}{l}\text { Sample } \\
\text { Number }\end{array}$ & $\begin{array}{c}\text { FIRON } \\
\text { 34/ppb } \\
10.3 / 300 \mathrm{~s}\end{array}$ & $\begin{array}{l}\text { LEAD } \\
\text { 40/ppb } \\
.508 / 50 \mathrm{i}\end{array}$ & $\begin{array}{c}\text { MAGNES } \\
34 / p p b \\
26 /\end{array}$ & $\begin{array}{c}\text { FMAGNES } \\
34 / \text { ppb } \\
26 /\end{array}$ \\
\hline $299-W 15-15$ & $11 / 19 / 93$ & 809K54 & - & $1.30 \mathrm{BL}$ & 12000.00 & \\
\hline $299-W 15-15$ & $11 / 19 / 93$ & B09K58 & 46.00 & & & 12000.00 \\
\hline $299-W 15-16$ & $11 / 18 / 93$ & 809K59 & & $.60 \mathrm{~L}$ & 20000.00 & \\
\hline $299-W 15-16$ & $11 / 18 / 93$ & $809 K 63$ & $18.00 \mathrm{~L}$ & & & 19000.00 \\
\hline $299-W 15-17$ & $11 / 18 / 93$ & $809 \times 64$ & & $2.20 \mathrm{~L}$ & 14000.00 & \\
\hline $299-415-17$ & $11 / 18 / 93$ & B09K68 & 40.00 & & & 14000.00 \\
\hline $299-W 15-18$ & $11 / 18 / 93$ & B09K69 & & $.51 \mathrm{U}$ & 15000.00 & \\
\hline $299-W 15-18$ & $11 / 18 / 93$ & B09k73 & 140.00 & & & 16000.00 \\
\hline $299-415-19$ & $12 / 03 / 93$ & $809 \times 74$ & & $3.80 \mathrm{BL}$ & 13000.00 & \\
\hline $299-W 15-19$ & $12 / 03 / 93$ & B09K78 & 48.00 & & & 13000.00 \\
\hline $299-W 15-20$ & $11 / 19 / 93$ & $809 \times 79$ & & $5.20 \mathrm{~B}$ & 8600.00 & \\
\hline $299-W 15-20$ & $11 / 19 / 93$ & $809 k 83$ & 60.00 & & & 8400,00 \\
\hline $299-415-23$ & $11 / 19 / 93$ & B09K84 & & $1.10 \mathrm{BL}$ & 8300.00 & \\
\hline $299-W 15-23$ & $11 / 19 / 93$ & $809 \times 88$ & $10.30 \mathrm{U}$ & & & 8300.00 \\
\hline $299-W 15 \cdot 24$ & $11 / 29 / 93$ & 809K89 & & $12.00 \mathrm{~B}$ & 14000.00 & \\
\hline $299-W 15-24$ & $11 / 29 / 93$ & B09K93 & $15.00 \mathrm{~L}$ & & & 9800.00 \\
\hline $299-418-21$ & $11 / 19 / 93$ & B09K94 & & $3.50 \mathrm{BL}$ & 8300.00 & \\
\hline $299-W 18-21$ & $11 / 19 / 93$ & $809 \times 98$ & $10.30 \mathrm{U}$ & & & 3200.00 \\
\hline $299-W 18-22$ & $11 / 30 / 93$ & 809K99 & & $.85 \mathrm{BL}$ & 13000.00 & \\
\hline $299-418-22$ & $11 / 30 / 93$ & B09KB3 & 39.00 & & & 13000.00 \\
\hline $299-418-23$ & $11 / 22 / 93$ & B09KB4 & & $5.30 \mathrm{~B}$ & 7900.00 & \\
\hline $299-W 18-23$ & $11 / 22 / 93$ & B09KB8 & $17.00 \mathrm{~L}$ & & & 8000.00 \\
\hline $299-W 18-24$ & $11 / 29 / 93$ & BO9KB9 & & $1.30 \mathrm{BL}$ & 7000.00 & \\
\hline $299-W 18-24$ & $11 / 29 / 93$ & B09KC3 & $11.00 \mathrm{~L}$ & & & 7100.00 \\
\hline $299-W 18-26$ & $11 / 29 / 93$ & B09KC4 & & $2.90 \mathrm{BL}$ & 8400.00 & \\
\hline $299-W 18-26$ & $11 / 29 / 93$ & B09KC8 & 20.00 & & & 8200.00 \\
\hline $299-418-27$ & $11 / 30 / 93$ & B09KC9 & & $6.90 \mathrm{~B}$ & 7700.00 & \\
\hline $299-W 18-27$ & $11 / 30 / 93$ & B09KD3 & 25.00 & & & 7700.00 \\
\hline $299-418-28$ & $11 / 29 / 93$ & B09KD 4 & & $.81 \mathrm{BL}$ & 8900.00 & \\
\hline $299-W 18-28$ & $11 / 29 / 93$ & B09KD8 & 23.00 & & & 8700.00 \\
\hline $299-418-29$ & $11 / 29 / 93$ & $809 \mathrm{KD} 9$ & & 5.00 & 5300.00 & \\
\hline $299-W 18-29$ & $11 / 29 / 93$ & B09KF3 & 24.00 & & & 5100.00 \\
\hline $299-W 18-32$ & $11 / 29 / 93$ & B09KF4 & & $4.10 \mathrm{BL}$ & $7000.00 P$ & \\
\hline $299-w 18-32$ & $11 / 29 / 93$ & B09KF8 & $21.00 P$ & & & 6600.00 \\
\hline
\end{tabular}

\begin{tabular}{|c|c|c|c|c|c|c|}
\hline $\begin{array}{l}\text { Well } \\
\text { Name }\end{array}$ & $\begin{array}{c}\text { Collection } \\
\text { Date }\end{array}$ & $\begin{array}{l}\text { Sample } \\
\text { Number }\end{array}$ & $\begin{array}{c}\text { MANGESE } \\
34 / \mathrm{ppb} \\
1.35 / 50 \mathrm{~s}\end{array}$ & $\begin{array}{l}\text { FMANGAN } \\
34 / \text { PpD } \\
1.35 / 50 \mathrm{~s}\end{array}$ & $\begin{array}{l}\text { METHYCH } \\
25 / \mathrm{ppb} \\
.056 / .\end{array}$ & $\begin{array}{l}\text { NICKEL } \\
34 / \mathrm{ppb} \\
17.9 \%\end{array}$ \\
\hline $299-W 15-15$ & $11 / 19 / 93$ & B09K54 & 35.00 & & .15 BLH & 140.00 \\
\hline $299-H 15-15$ & $11 / 19 / 93$ & $809 \times 58$ & & $2.90 \mathrm{~L}$ & & \\
\hline $299-\$ 15-16$ & $11 / 18 / 93$ & $809 \times 59$ & 13.00 & & $.47 \mathrm{BL}$ & 52.00 \\
\hline $299-\$ 15-16$ & $11 / 18 / 93$ & B09K63 & & $2.00 \mathrm{~L}$ & & \\
\hline $299-W 15-16$ & $12 / 06 / 93$ & BO9N18 & & & $.06 \mathrm{U}$ & \\
\hline $299-W 15-17$ & $11 / 18 / 93$ & $809 \times 64$ & 27.00 & & $.55 \mathrm{BL}$ & 140.00 \\
\hline $299-\$ 15-17$ & $11 / 18 / 93$ & B09K68 & & $5.00 \mathrm{~L}$ & & \\
\hline $299-W 15 \cdot 18$ & $11 / 18 / 93$ & $809 \times 69$ & 12.00 & & $.27 \mathrm{BL}$ & 52.00 \\
\hline $299-W 15-18$ & $11 / 18 / 93$ & $809 \times 73$ & & $3.10 \mathrm{~L}$ & & \\
\hline $299-W 15-19$ & $12 / 03 / 93$ & $809 \times 74$ & 57.00 & & $.14 \mathrm{BL}$ & 320.00 \\
\hline $299-W 15-19$ & $12 / 03 / 93$ & $809 \times 78$ & & $4.20 \mathrm{~L}$ & & \\
\hline $299-W 15-20$ & $11 / 19 / 93$ & B09K79 & 60.00 & & $.30 \mathrm{BLH}$ & 330.00 \\
\hline $299-W 15-20$ & $11 / 19 / 93$ & B09K83 & & $5.60 \mathrm{~L}$ & & \\
\hline $299-W 15-23$ & $11 / 19 / 93$ & 809K84 & 20.00 & & $.33 \mathrm{BLH}$ & 88.00 \\
\hline $299-W 15-23$ & $11 / 19 / 93$ & B09K88 & & $5.10 \mathrm{~L}$ & & \\
\hline
\end{tabular}


Table 15-22. Constituents with at Least One Detected Value for the Low-Level Waste Burial Grounds WMA 4 Data for Reporting Period October 1

through December 31, 1993. (sheet 7 of 10)

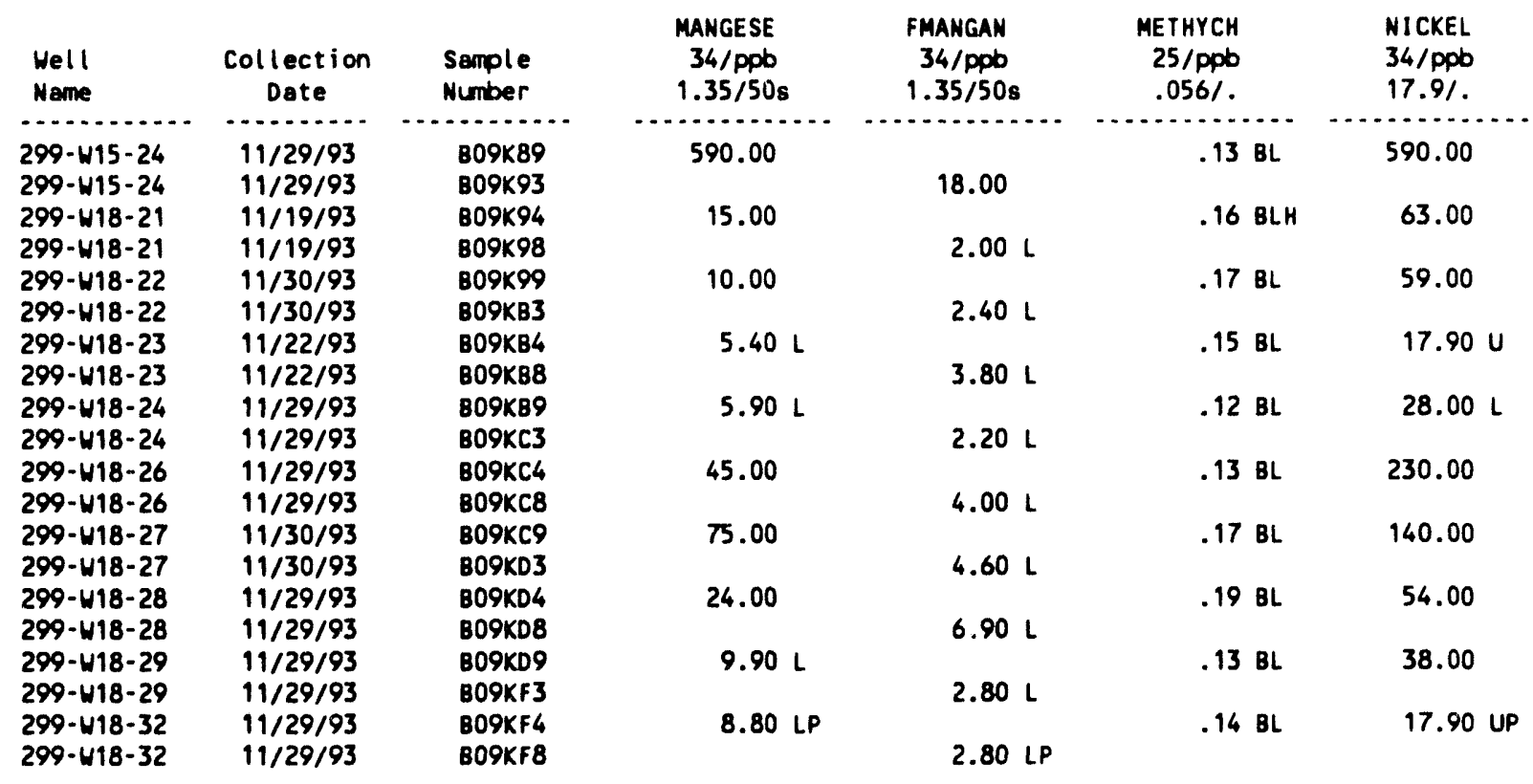

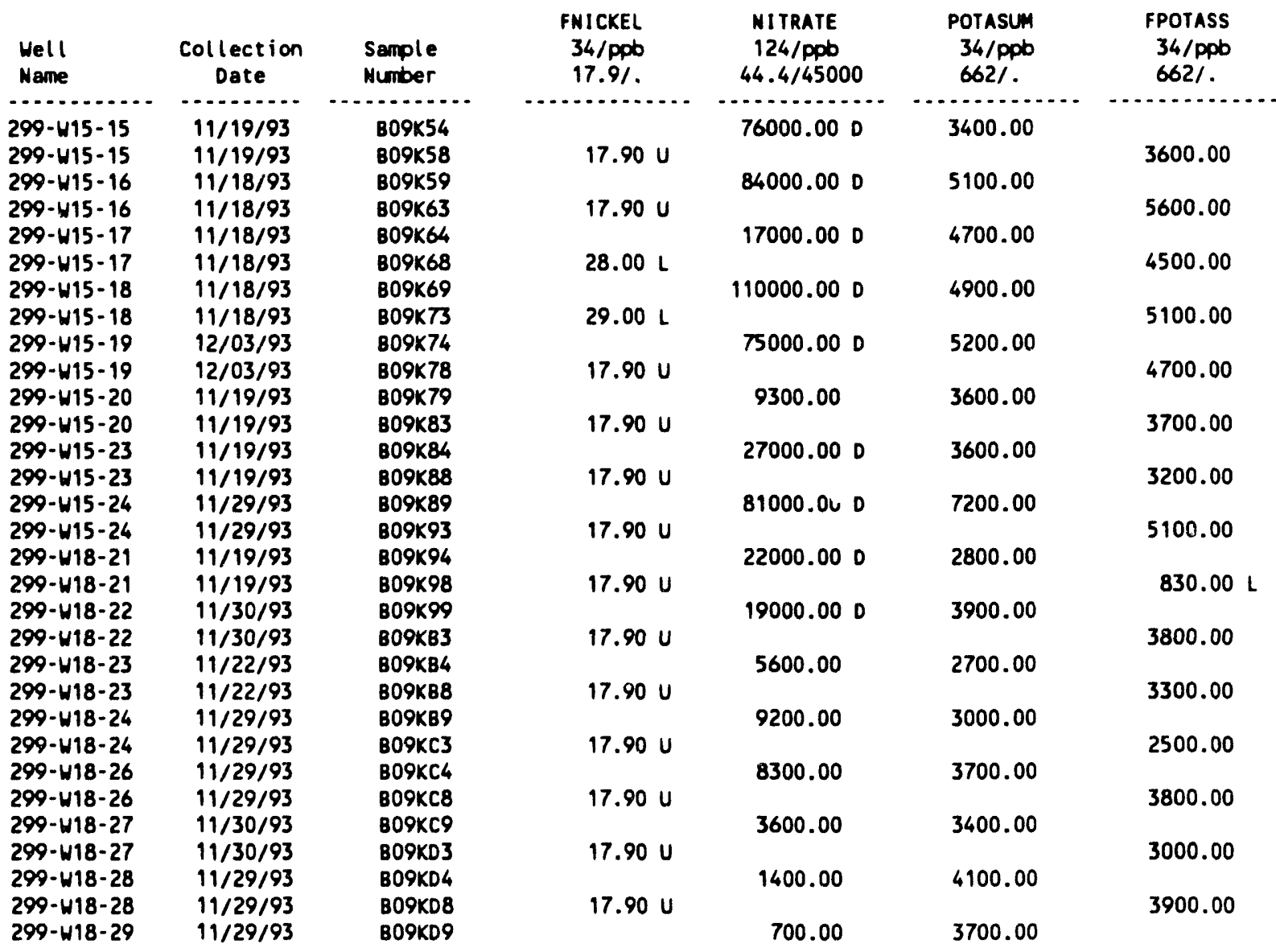


Table 15-22. Constituents with at Least One Detected Value for the Low-Level Waste Burial Grounds WMA 4 Data for Reporting Period October 1 through December 31,1993 . (sheet 8 of 10)

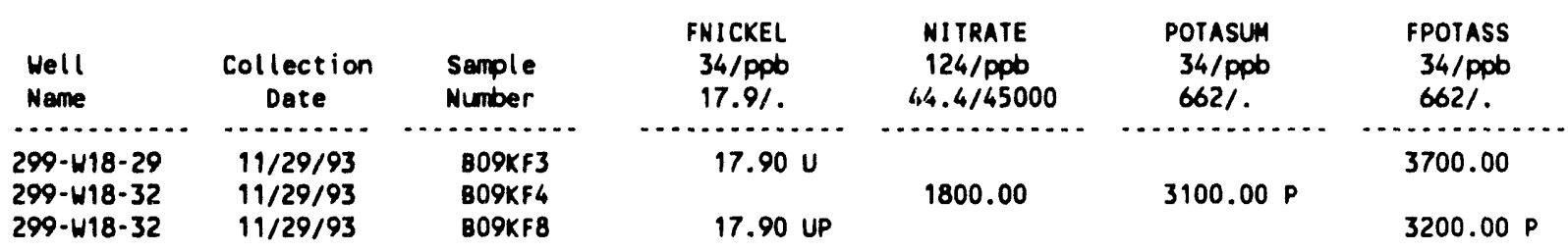

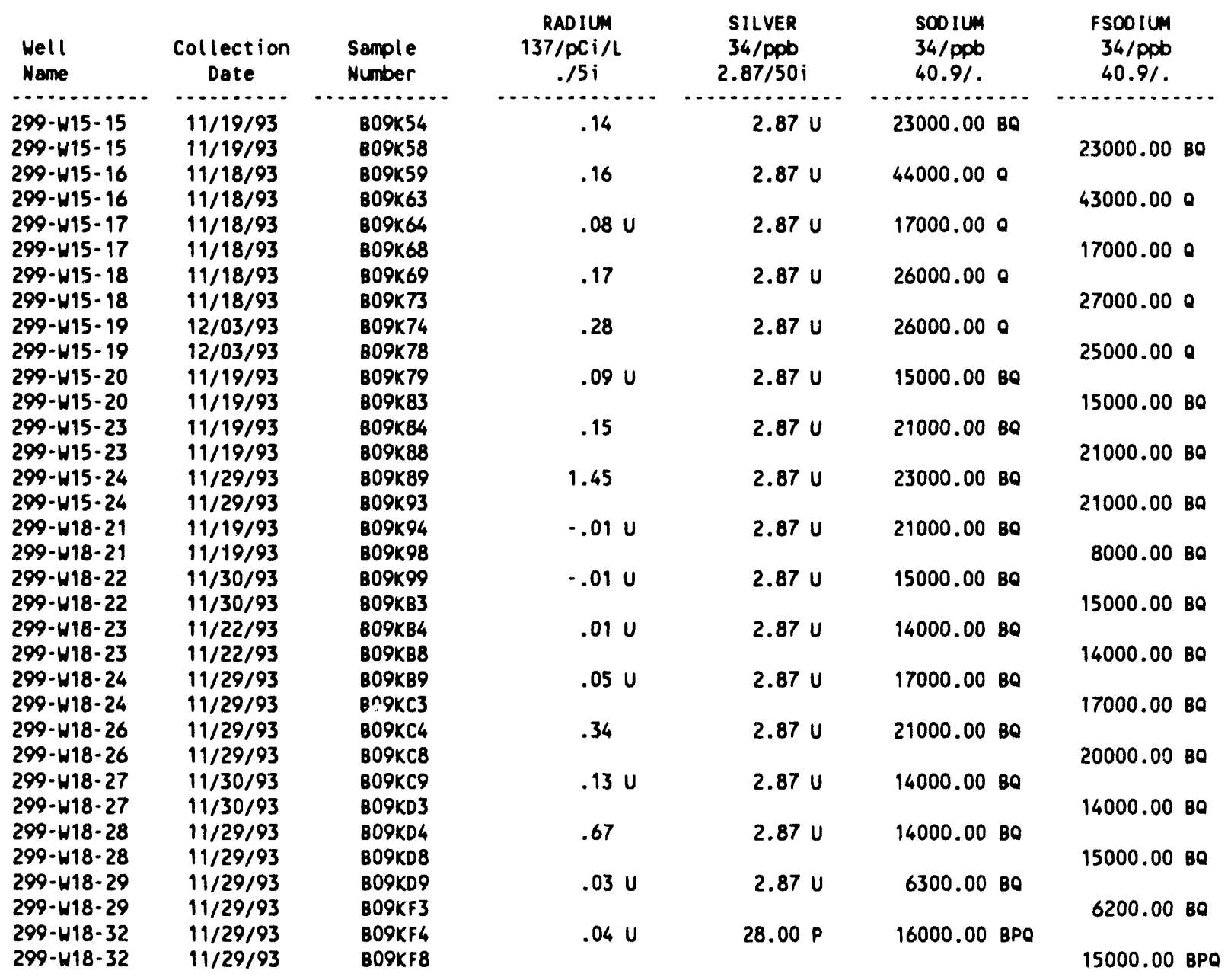

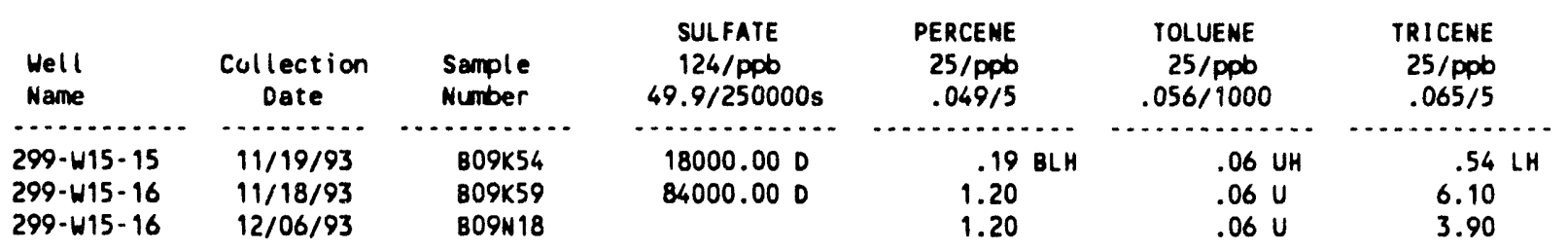


Table 15-22. Constituents with at Least One Detected Value for the Low-Level Waste Burial Grounds WMA 4 Data for Reporting Period October 1

through December 31, 1993. (sheet 9 of 10)

\begin{tabular}{|c|c|c|c|c|c|c|}
\hline $\begin{array}{l}\text { Well } \\
\text { Name }\end{array}$ & $\begin{array}{c}\text { Collection } \\
\text { Date }\end{array}$ & $\begin{array}{l}\text { Sample } \\
\text { Number }\end{array}$ & $\begin{array}{c}\text { SULFATE } \\
124 / \mathrm{ppb} \\
49.9 / 250000 \mathrm{~s}\end{array}$ & $\begin{array}{r}\text { PERCENE } \\
25 / \mathrm{ppb} \\
.049 / 5\end{array}$ & $\begin{array}{c}\text { TOLUENE } \\
25 / \mathrm{ppb} \\
.056 / 1000\end{array}$ & $\begin{array}{r}\text { TRI CENE } \\
25 / \mathrm{ppb} \\
.065 / 5\end{array}$ \\
\hline $\begin{array}{l}299-W 15-17 \\
299-W 15-18 \\
299-W 15-19 \\
299-W 15-20 \\
299-W 15-23 \\
299-W 15-24 \\
299-W 18-21 \\
299-W 18-22 \\
299-W 18-23 \\
299-W 18-24 \\
299-W 18-26 \\
299-W 18-27 \\
299-W 18-28 \\
299-W 18-29 \\
299-W 18-32\end{array}$ & $\begin{array}{l}11 / 18 / 93 \\
11 / 18 / 93 \\
12 / 03 / 93 \\
11 / 19 / 93 \\
11 / 19 / 93 \\
11 / 29 / 93 \\
11 / 19 / 93 \\
11 / 30 / 93 \\
11 / 22 / 93 \\
11 / 29 / 93 \\
11 / 29 / 93 \\
11 / 30 / 93 \\
11 / 29 / 93 \\
11 / 29 / 93 \\
11 / 29 / 93\end{array}$ & $\begin{array}{l}809 K 64 \\
809 K 69 \\
809 K 74 \\
809 K 79 \\
809 K 84 \\
809 K 89 \\
809 K 94 \\
809 K 99 \\
809 K B 4 \\
809 K B 9 \\
809 K C 4 \\
809 K C 9 \\
B 09 K D 4 \\
809 K D 9 \\
809 K F 4\end{array}$ & $\begin{array}{l}29000.00 \mathrm{D} \\
31000.00 \mathrm{D} \\
26000.00 \mathrm{D} \\
15000.00 \mathrm{D} \\
16000.00 \mathrm{D} \\
19000.00 \mathrm{D} \\
17000.00 \mathrm{D} \\
19000.00 \mathrm{D} \\
16000.00 \mathrm{D} \\
19000.00 \mathrm{D} \\
20000.00 \mathrm{D} \\
16000.00 \mathrm{D} \\
16000.00 \mathrm{D} \\
17000.00 \mathrm{D} \\
19000.00 \mathrm{D}\end{array}$ & $\begin{array}{l}.05 \mathrm{U} \\
.77 \\
.11 \mathrm{~L} \\
.06 \mathrm{BLH} \\
.05 \mathrm{UH} \\
.05 \mathrm{U} \\
.19 \mathrm{BLH} \\
.05 \mathrm{U} \\
.08 \mathrm{BL} \\
.19 \mathrm{BL} \\
.05 \mathrm{U} \\
.05 \mathrm{U} \\
.05 \mathrm{U} \\
.06 \mathrm{BL} \\
.05 \mathrm{U}\end{array}$ & $\begin{array}{l}.06 \mathrm{U} \\
.06 \mathrm{U} \\
.06 \mathrm{U} \\
.07 \mathrm{LH} \\
.06 \mathrm{UH} \\
.06 \mathrm{U} \\
.06 \mathrm{UH} \\
.06 \mathrm{U} \\
.06 \mathrm{U} \\
.06 \mathrm{U} \\
.06 \mathrm{U} \\
.06 \mathrm{U} \\
.06 \mathrm{U} \\
.06 \mathrm{U} \\
.06 \mathrm{U}\end{array}$ & $\begin{array}{l}.35 \mathrm{~L} \\
3 \times 1 \\
.11 \mathrm{~L} \\
.10 \mathrm{LH} \\
.07 \mathrm{U} \\
.79 \mathrm{LH} \\
.07 \mathrm{U} \\
.29 \mathrm{~L} \\
.78 \mathrm{~L} \\
.11 \mathrm{~L} \\
.16 \mathrm{~L} \\
.36 \mathrm{~L} \\
.32 \mathrm{~L} \\
.09 \mathrm{~L}\end{array}$ \\
\hline
\end{tabular}

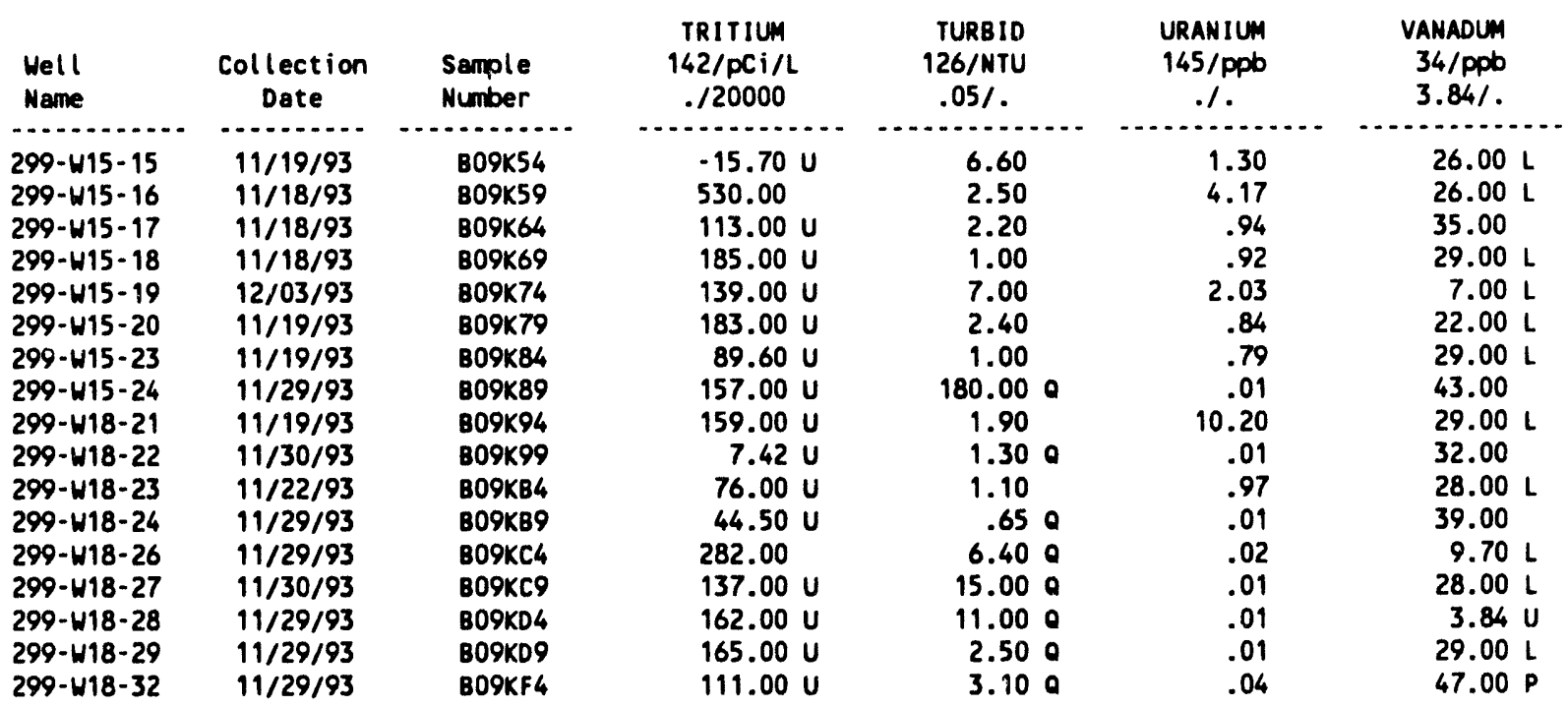

\begin{tabular}{|c|c|c|c|c|c|}
\hline $\begin{array}{l}\text { Well } \\
\text { Name }\end{array}$ & $\begin{array}{c}\text { Collection } \\
\text { Date }\end{array}$ & $\begin{array}{l}\text { Sample } \\
\text { Number }\end{array}$ & $\begin{array}{l}\text { FVANADI } \\
34 / \text { ppb } \\
3.84 / .\end{array}$ & $\begin{array}{c}\text { ZINC } \\
34 / \mathrm{ppb} \\
3.44 / 5000 \mathrm{~s}\end{array}$ & $\begin{array}{c}\text { FZINC } \\
34 / \mathrm{ppb} \\
3.44 / 5000 \mathrm{~s}\end{array}$ \\
\hline $200-415-15$ & $11 / 10 / 03$ & B00K54 & . & 070 & \\
\hline $\begin{array}{l}299-W 15-15 \\
299-W 15-15 \\
299-\psi 15-16\end{array}$ & $\begin{array}{l}11 / 19 / 93 \\
11 / 19 / 93 \\
11 / 18 / 93\end{array}$ & $\begin{array}{l}\text { 809K54 } \\
\text { 809K58 } \\
809 \times 59\end{array}$ & $26.00 \mathrm{~L}$ & $\begin{array}{l}9.70 \mathrm{LB} \\
13.00 \mathrm{~B}\end{array}$ & $5.30 \mathrm{LB}$ \\
\hline $299-415-16$ & $11 / 18 / 93$ & B09K63 & $27.00 \mathrm{~L}$ & & $7.00 \mathrm{LB}$ \\
\hline $299-W 15-17$ & $11 / 18 / 93$ & $809 \times 64$ & & $18.00 \mathrm{~B}$ & \\
\hline 299-W15-17 & $11 / 18 / 93$ & B09K68 & 31.00 & & $8.00 \mathrm{LB}$ \\
\hline $299-W 15-18$ & $11 / 18 / 93$ & $809 K 69$ & & $11.00 \mathrm{~B}$ & \\
\hline $299-W 15-18$ & $11 / 18 / 93$ & $809 \times 73$ & $28.00 \mathrm{~L}$ & & $11.00 \mathrm{~B}$ \\
\hline
\end{tabular}


Table 15-22. Constituents with at Least One Detected Value for the Low-Level Waste Burial Grounds WMA 4 Data for Reporting Period October 1 through December 31, 1993. (sheet 10 of 10)

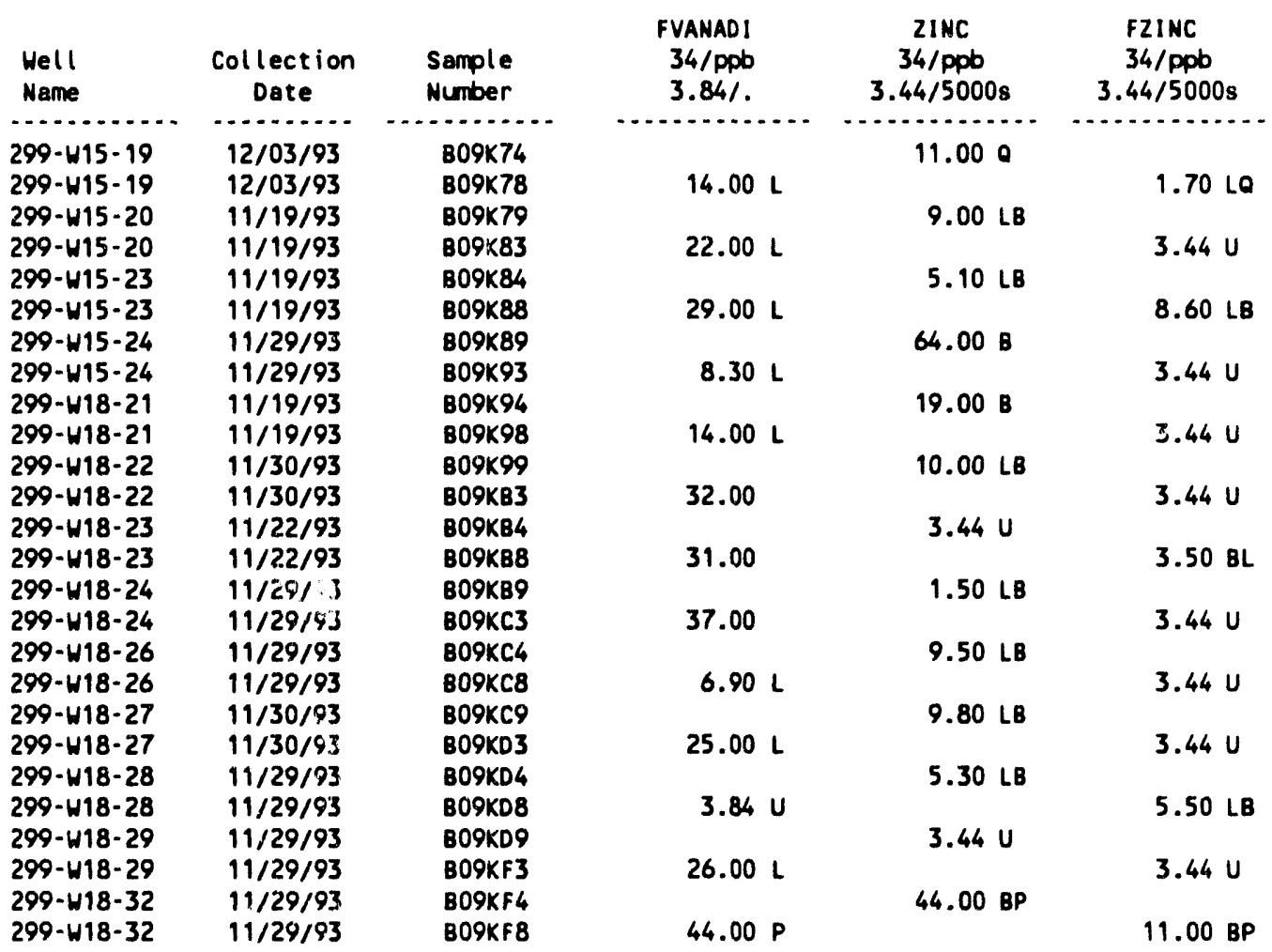

For explanation of this table, see Section 1.4 of report. 
Table 15-23. Contamination Indicator Parameters for the Low-Level Waste Burial Grounds WMA 4 Data for Reporting Period October 1 through

December 31, 1993. (sheet 1 of 2)

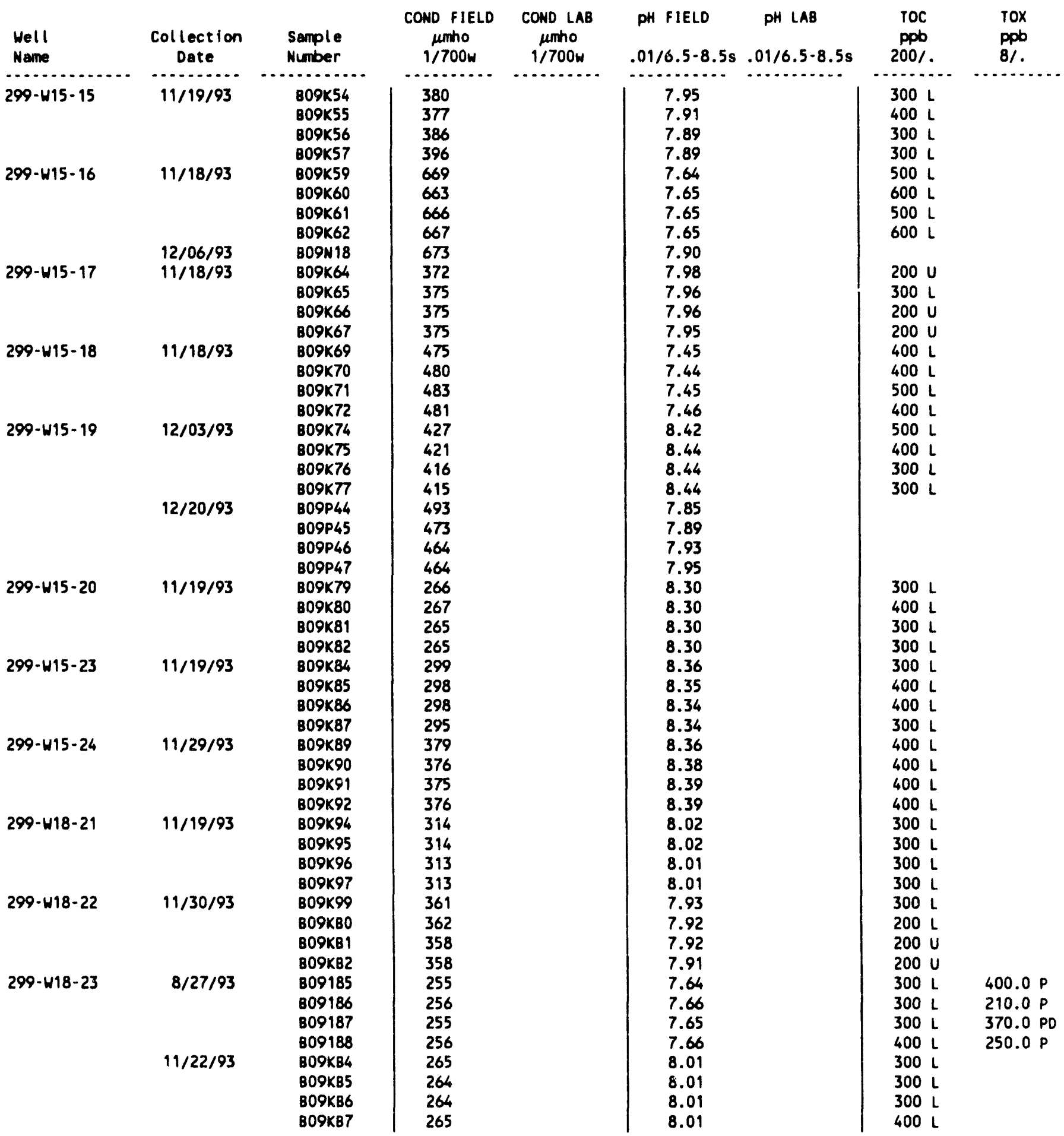


Table 15-23. Contamination Indicator Parameters for the Low-Level Waste Burial Grounds WMA 4 Data for Reporting Period October 1 through

December 31, 1993. (sheet 2 of 2)

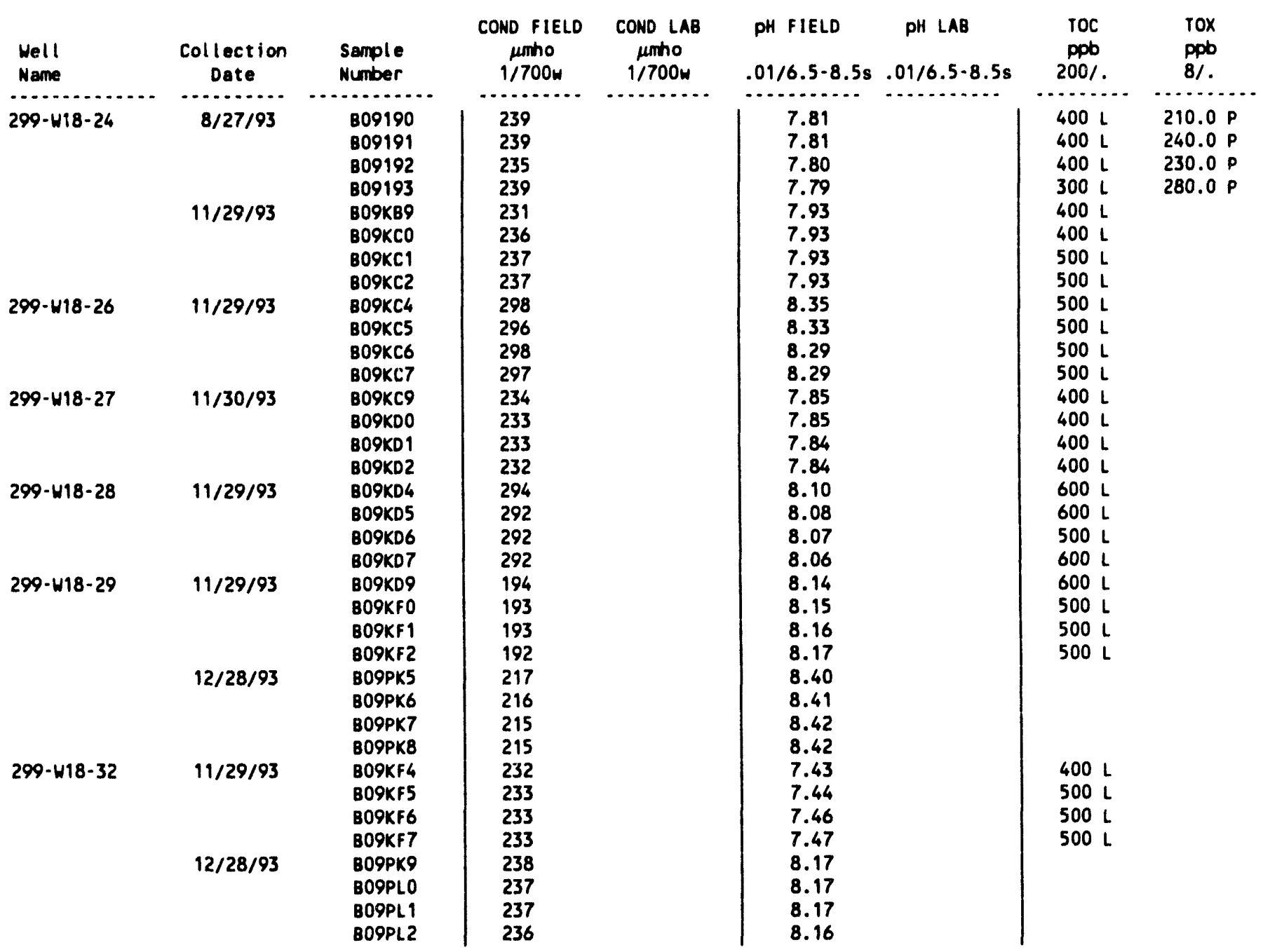

$\overline{\text { For explanation of this table, see Section } 1.4 \text { of report. }}$ 
Table 15-24. Constituent List and Summary of Results for the Low-Level Waste Burial Grounds WMA 5 Data for Reporting Period October 1

through December 31, 1993. (sheet 1 of 3)

CONTAMINATION IMDICATOR PARAMETERS

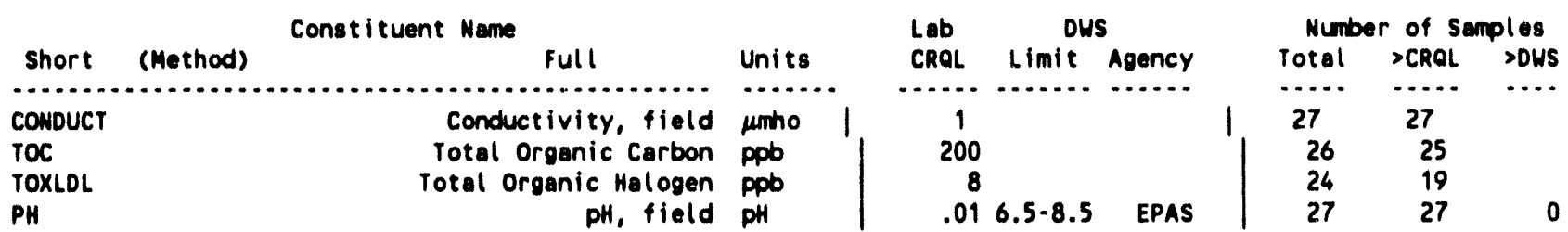

DRINKING WATER PARAMETERS

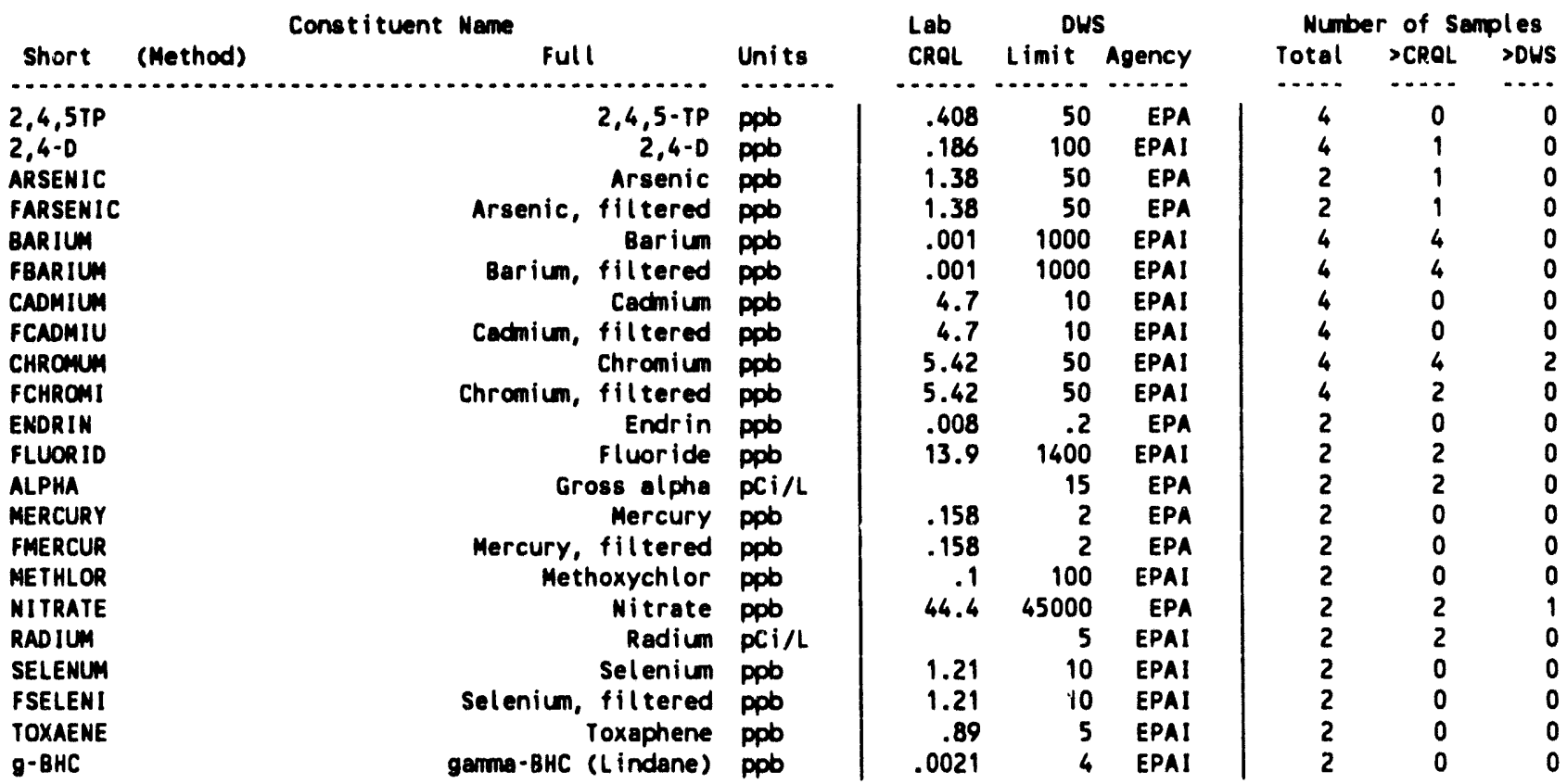

GROUNDWATER QUALITY PARAMETERS

\begin{tabular}{|c|c|c|c|c|c|c|c|c|c|}
\hline \multirow[b]{2}{*}{ Short } & \multirow[b]{2}{*}{ (Method) } & \multirow{2}{*}{ Constituent Name } & \multirow[b]{2}{*}{ Units } & \multirow{2}{*}{$\begin{array}{l}\text { Lab } \\
\text { CROL }\end{array}$} & \multicolumn{2}{|c|}{ Dus } & \multicolumn{3}{|c|}{ Number of Semples } \\
\hline & & & & & Limit & Agency & Total & $>$ CROL & >DWS \\
\hline$\cdots$ & 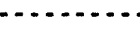 & $\cdots>$ & $\cdots$ & ...... & ........ & …... & $\cdots$ & $\ldots$. & $\cdots$ \\
\hline CHLORIO & & Chloride & ppb & 82.5 & 250000 & EPAS & 2 & 2 & 0 \\
\hline IRON & & Iron & ppb & 10.3 & 300 & EPAS & 4 & 4 & 1 \\
\hline FIRON & & Iron, filtered & ppb & 10.3 & 300 & EPAS & 4 & 4 & 0 \\
\hline MANGESE & & Manganese & ppb & 1.35 & 50 & EPAS & 4 & 3 & 1 \\
\hline FMANGAN & & Manganese, filtered & ppb & 1.35 & 50 & EPAS & 4 & 3 & 1 \\
\hline LPHENOL & & Phenol & ppb & .31 & & & 4 & 0 & \\
\hline SOOIUM & & Sodium & ppb & 40.9 & & & 4 & 4 & \\
\hline FSOOIUN & & Sodium, filtered & ppb & 40.9 & & & 4 & 4 & \\
\hline SULFATE & & Sulfate & ppb & 49.9 & 250000 & EPAS & 2 & 2 & 0 \\
\hline
\end{tabular}


Table 15-24. Constituent List and Summary of Results for the Low-Level Waste Burial Grounds WMA 5 Data for Reporting Period October 1 through December 31, 1993. (sheet 2 of 3)

SITE SPECIFIC AND OTHER CONSTITUENTS

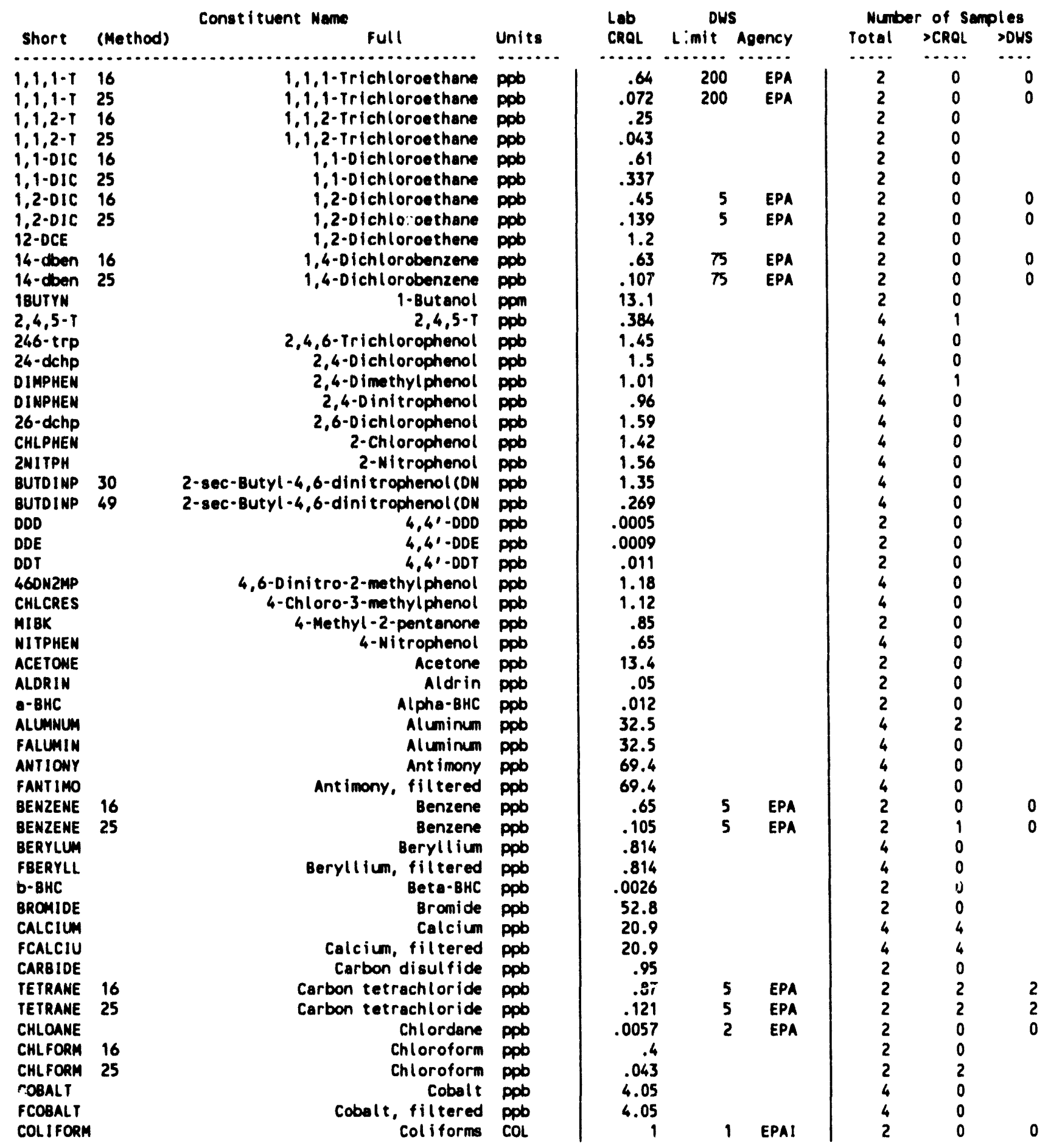


Table 15-25. Constituents with at Least One Detected Value for the Low-Level Waste Burial Grounds WMA 5 Data for Reporting Period October 1

through December 31, 1993. (sheet 1 of 4)

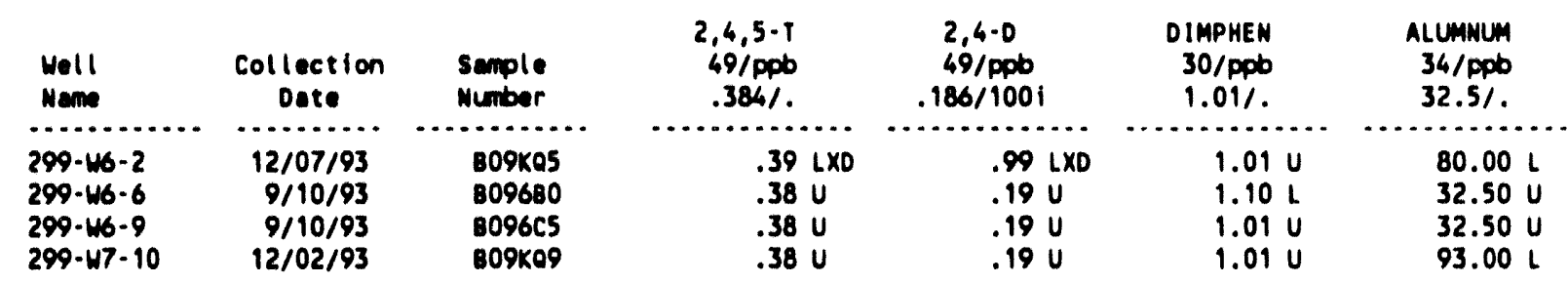

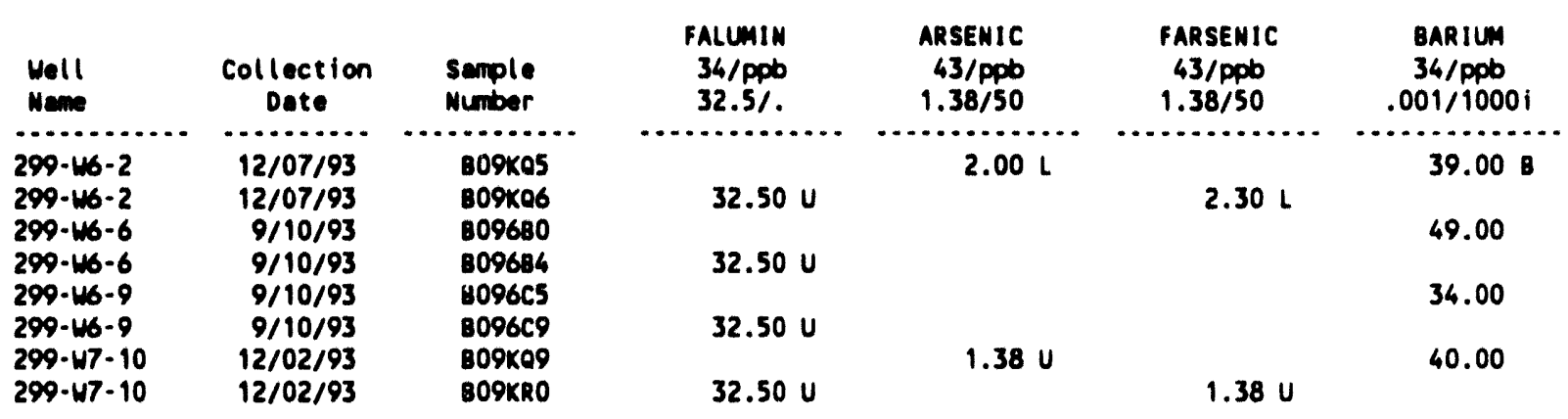

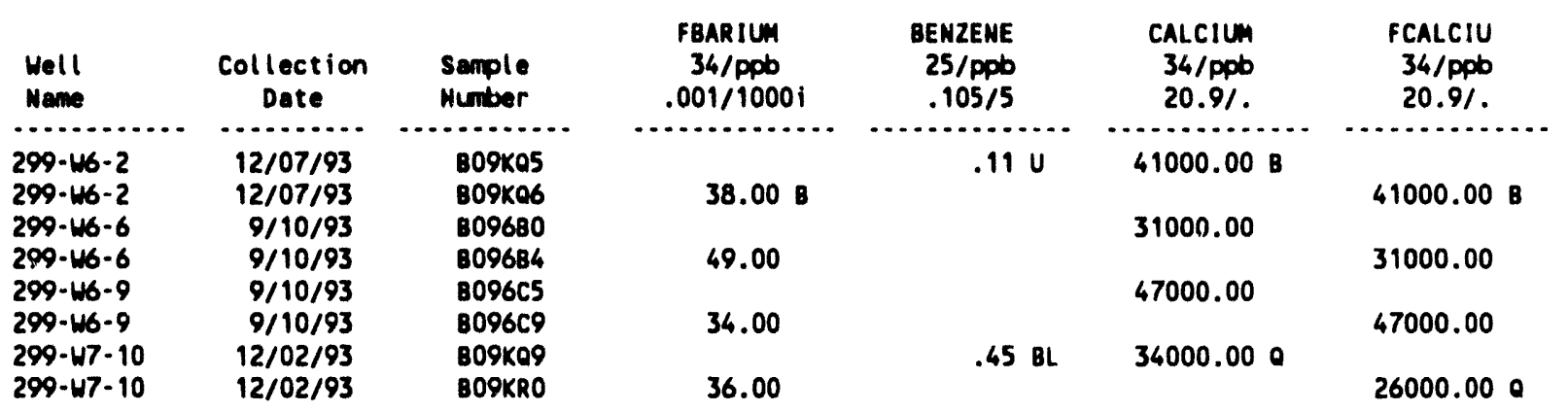

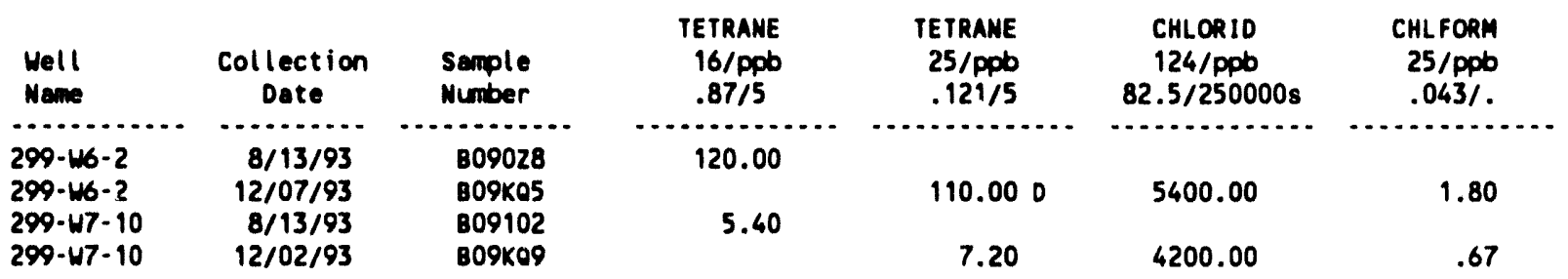


Table 15-24. Constituent List and Summary of Results for the Low-Level Waste Burial Grounds WMA 5 Data for Reporting Period October 1

through December 31, 1993. (sheet 3 of 3)

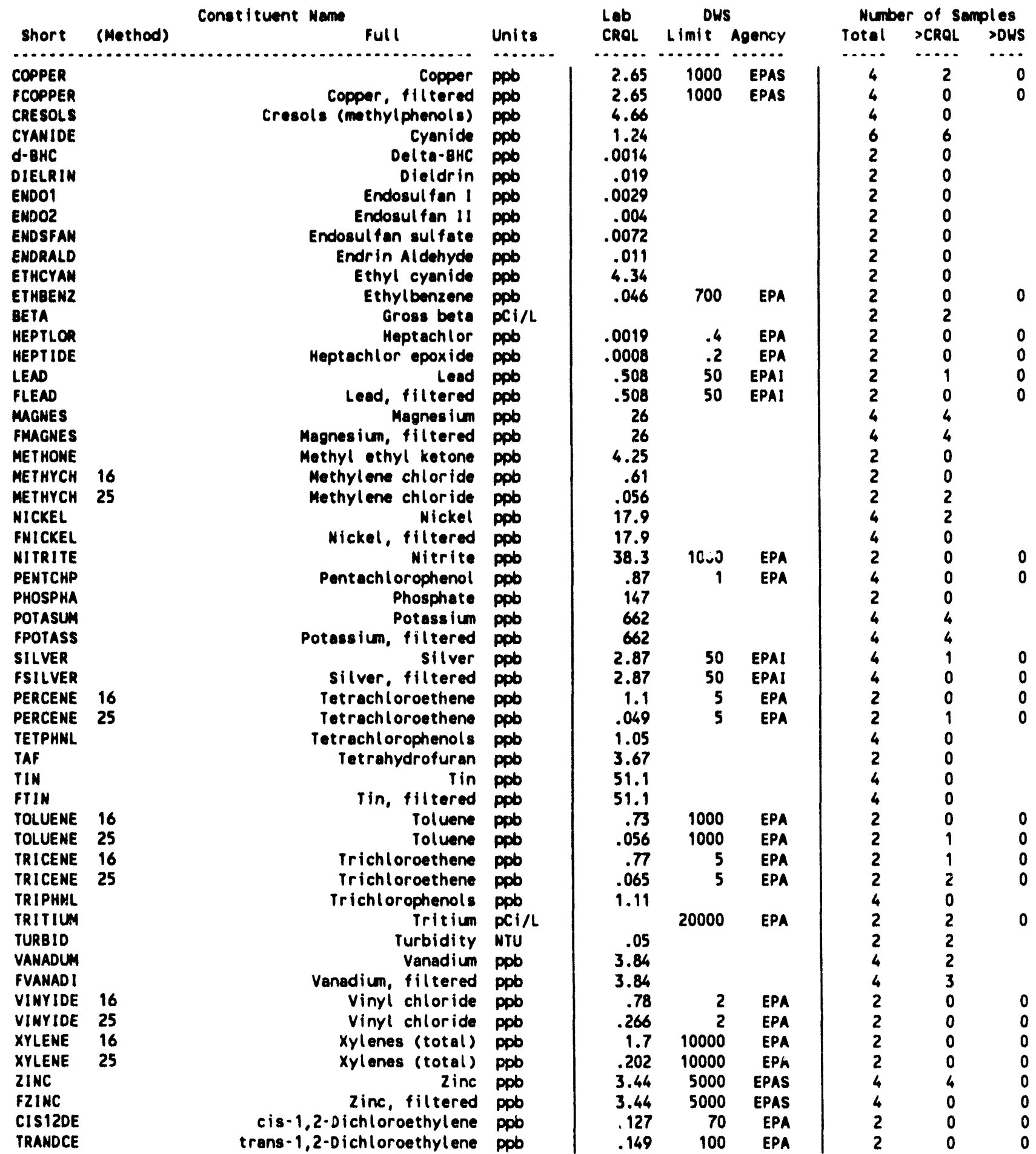

For explanation of this table, see Section 1.4 of report. 

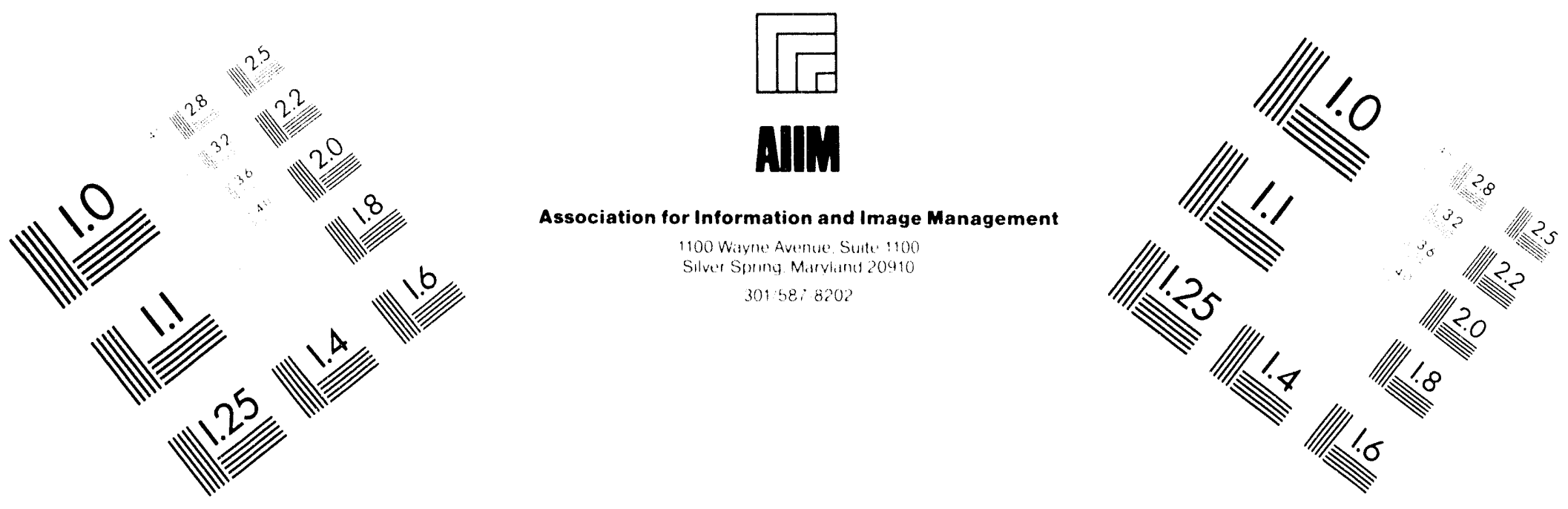

\section{Centimeter}

C)

Inches
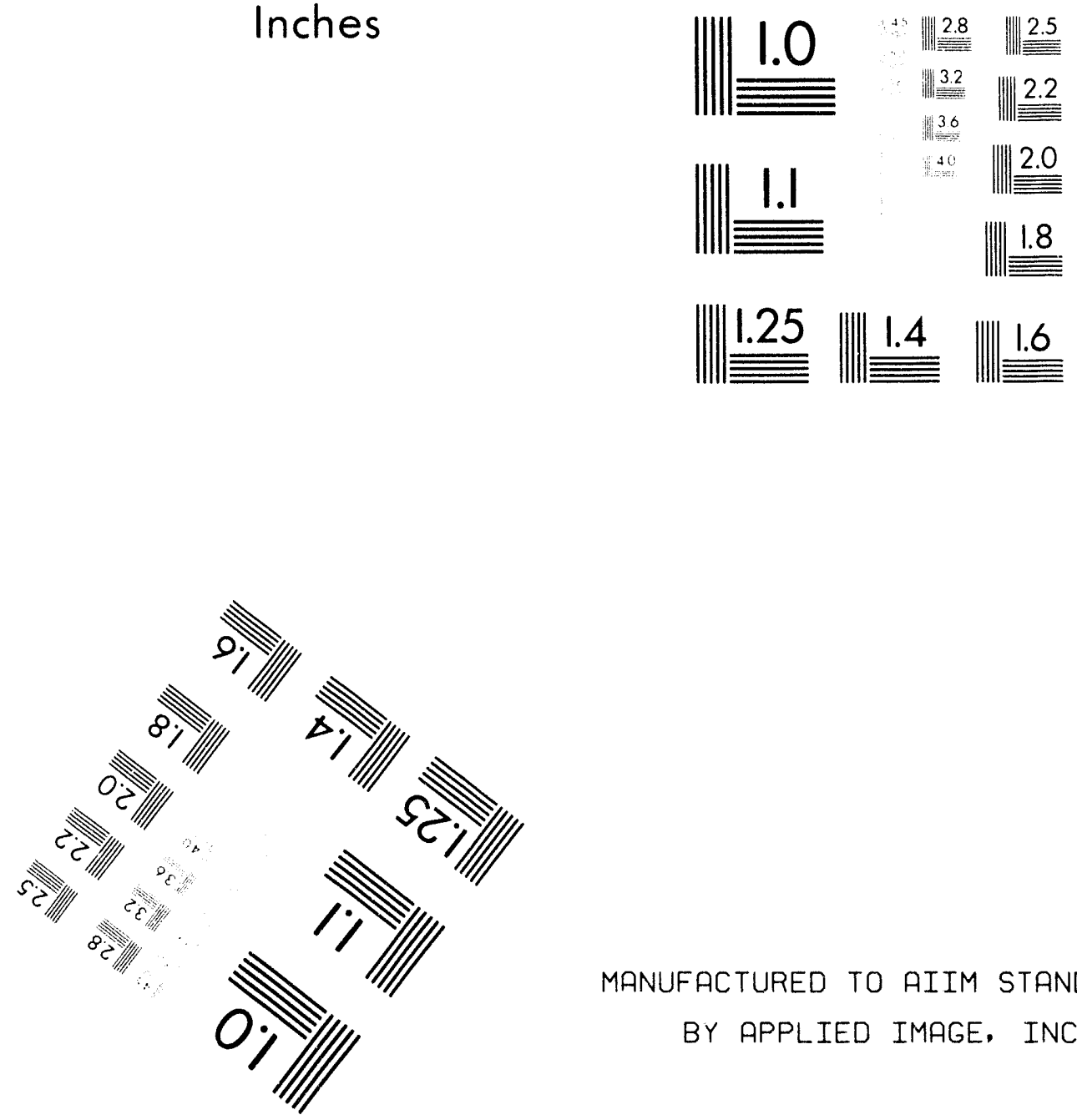

MANUFACTURED TO AIIM STANDARDS

BY APPLIED IMAGE, INC.

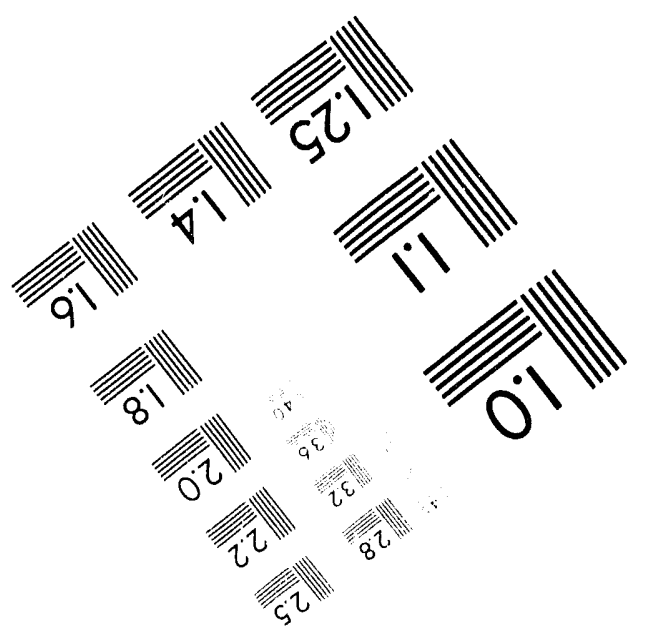



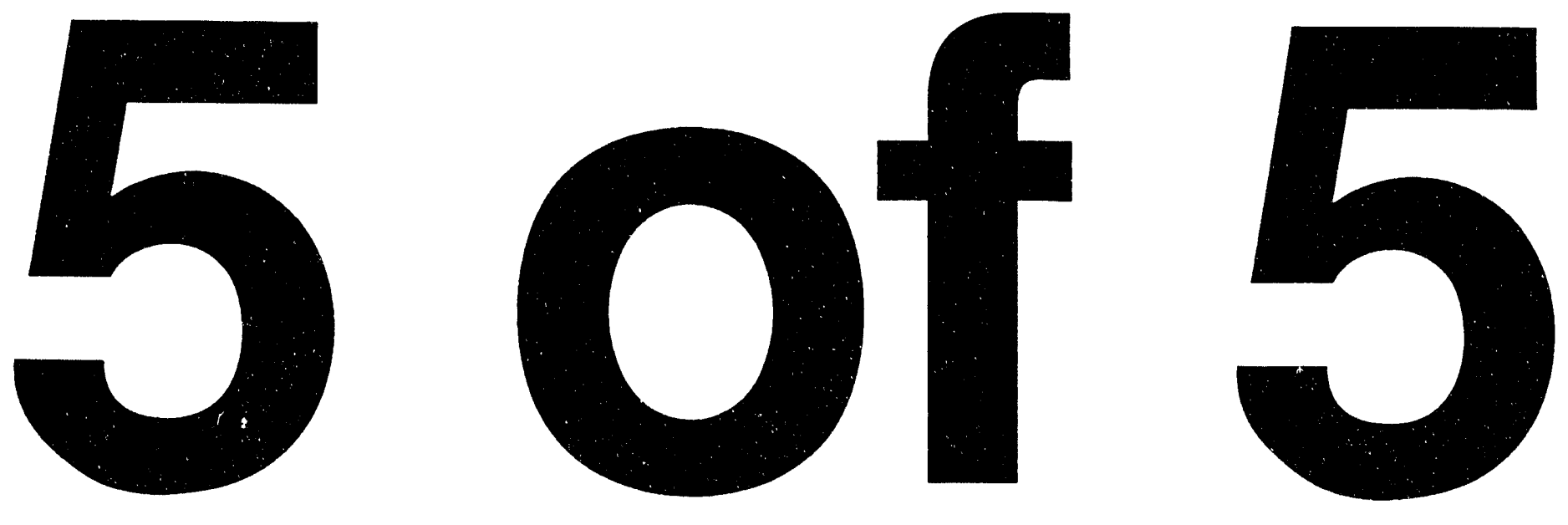
Table 15-25. Constituents with at Least One Detected Value for the Low-Level Waste Burial Grounds WMA 5 Data for Reporting Period October 1 through December 31, 1993. (sheet 1 of 4)

\begin{tabular}{|c|c|c|c|c|c|c|}
\hline $\begin{array}{l}\text { Well } \\
\text { Name }\end{array}$ & $\begin{array}{c}\text { Collection } \\
\text { Date }\end{array}$ & $\begin{array}{l}\text { Sample } \\
\text { Number }\end{array}$ & $\begin{array}{c}2,4,5-1 \\
49 / 0 \mathrm{pb} \\
.3 \mathrm{~cm} / .\end{array}$ & $\begin{array}{c}2,4-D \\
49 / p p b \\
.186 / 100 i\end{array}$ & $\begin{array}{c}\text { DIMPHEN } \\
30 / \text { ppb } \\
1.01 / .\end{array}$ & $\begin{array}{c}\text { ALUMNUM } \\
34 / \mathrm{ppb} \\
32.5 /\end{array}$ \\
\hline $\begin{array}{l}299-\omega 6-2 \\
299-w 6-6 \\
299-\omega 6-9 \\
299-W 7-10\end{array}$ & $\begin{array}{r}12 / 07 / 93 \\
9 / 10 / 93 \\
9 / 10 / 93 \\
12 / 02 / 93\end{array}$ & $\begin{array}{l}\text { B09KO5 } \\
\text { B096BO } \\
\text { B096C5 } \\
\text { B09Ka9 }\end{array}$ & $\begin{array}{l}.39 \mathrm{LXD} \\
.38 \mathrm{U} \\
.38 \mathrm{U} \\
.38 \mathrm{U}\end{array}$ & $\begin{array}{l}.99 \mathrm{LXD} \\
.19 \mathrm{U} \\
.19 \mathrm{U} \\
.19 \mathrm{U}\end{array}$ & $\begin{array}{l}1.01 \mathrm{U} \\
1.10 \mathrm{~L} \\
1.01 \mathrm{U} \\
1.01 \mathrm{U}\end{array}$ & $\begin{array}{l}80.00 \\
32.50 \\
32.50 \\
93.00\end{array}$ \\
\hline
\end{tabular}

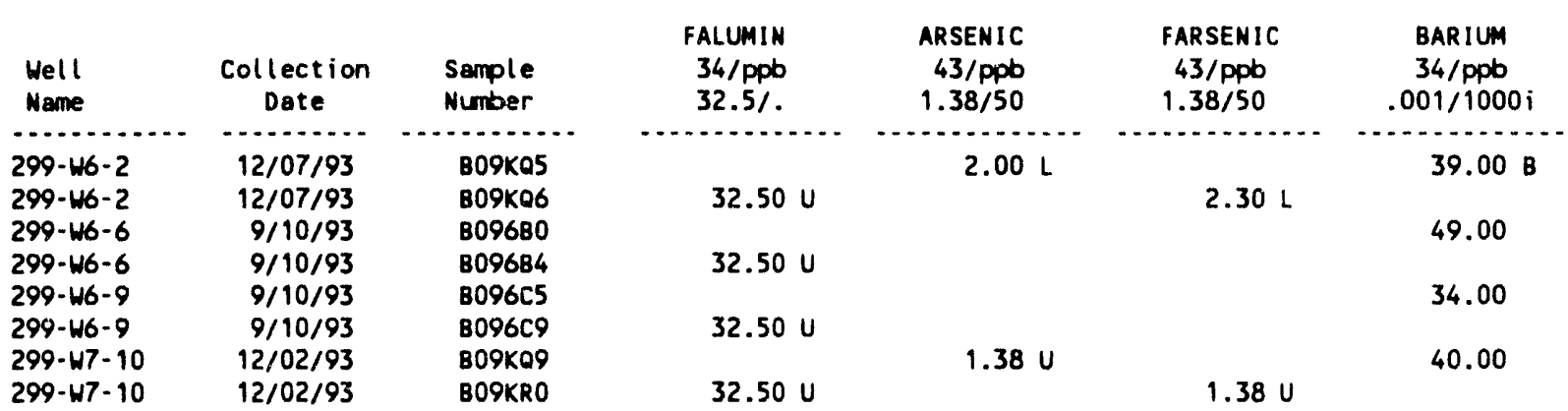

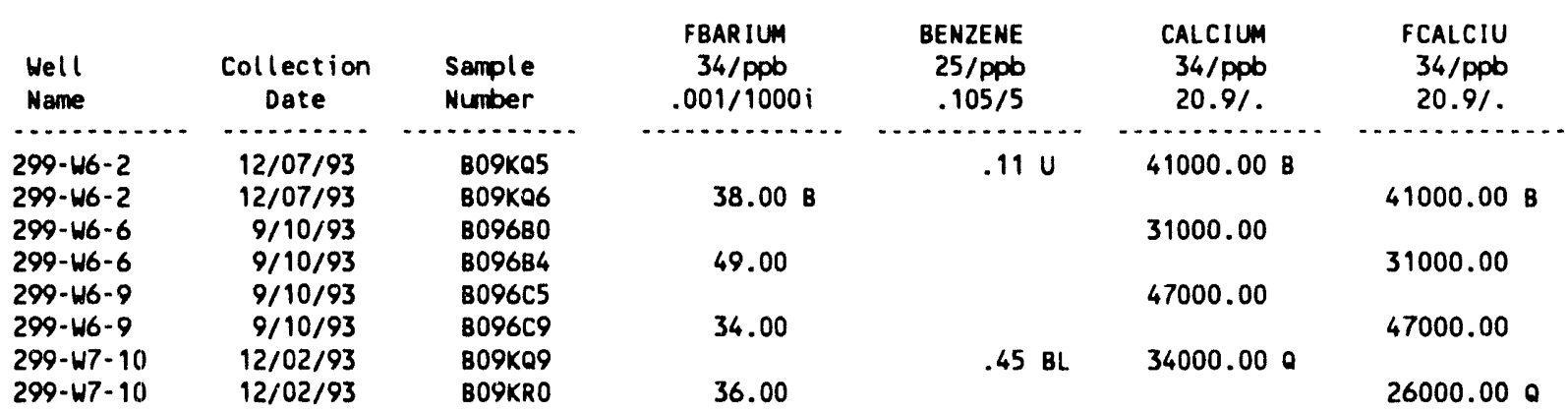

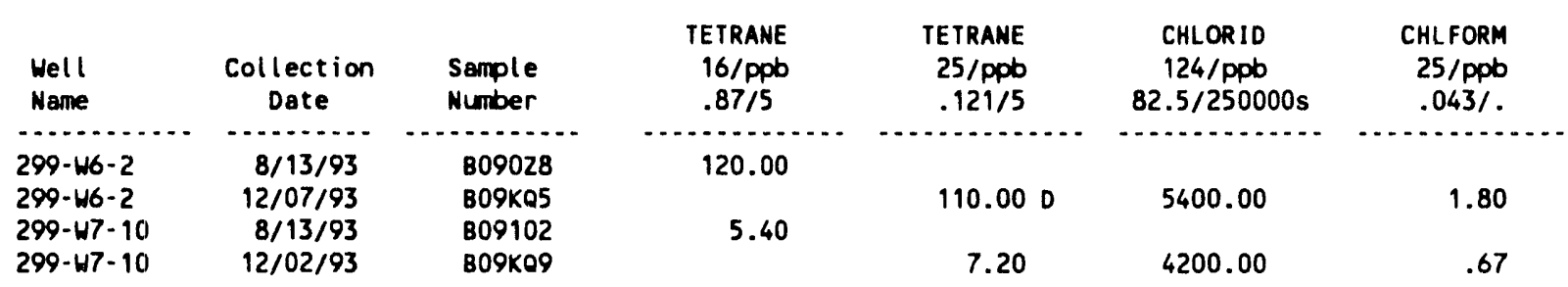


Table 15-25. Constituents with at Least One Detected Value for the Low-Level Waste Burial Grounds WMA 5 Data for Reporting Period October 1

through December 31, 1993. (sheet 2 of 4)

\begin{tabular}{|c|c|c|c|c|c|c|}
\hline $\begin{array}{l}\text { Well } \\
\text { Name }\end{array}$ & $\begin{array}{c}\text { Collection } \\
\text { Date }\end{array}$ & $\begin{array}{l}\text { Sample } \\
\text { Number }\end{array}$ & $\begin{array}{l}\text { CHROMUM } \\
34 / \mathrm{ppb} \\
5.42 / 50 ;\end{array}$ & $\begin{array}{l}\text { FCHROMI } \\
34 / \text { ppb } \\
5.42 / 50 \mathrm{i}\end{array}$ & $\begin{array}{c}\text { COPPER } \\
34 / \mathrm{ppb} \\
2.65 / 1000 \mathrm{~s}\end{array}$ & $\begin{array}{c}\text { FCOPPER } \\
34 / \text { ppb } \\
2.65 / 1000 \mathrm{~s}\end{array}$ \\
\hline ….... & ................. & $\ldots \ldots \ldots$ & (....... & & & \\
\hline $\begin{array}{l}299-W 6-2 \\
299-W 6-2\end{array}$ & $\begin{array}{l}12 / 07 / 93 \\
12 / 07 / 93\end{array}$ & $\begin{array}{l}\text { B09K05 } \\
\text { B09K06 }\end{array}$ & $82.00 \mathrm{~B}$ & $28.00 \mathrm{~B}$ & $4.80 \mathrm{~L}$ & $2.65 \mathrm{U}$ \\
\hline $\begin{array}{l}299-w 6-6 \\
299-166-6\end{array}$ & $\begin{array}{l}9 / 10 / 93 \\
9 / 10 / 93\end{array}$ & $\begin{array}{l}\text { B09680 } \\
\text { B09684 }\end{array}$ & 24.00 & $5.42 \mathrm{U}$ & $2.65 \mathrm{U}$ & $2.65 \mathrm{U}$ \\
\hline $299-116-9$ & $9 / 10 / 93$ & $8096 \mathrm{CS}$ & 36.00 & & $2.65 \mathrm{U}$ & \\
\hline 299-W6-9 & $9 / 10 / 93$ & B096C9 & & 28.00 & & $2.65 \mathrm{U}$ \\
\hline $\begin{array}{l}299-w 7-10 \\
299-\omega 7-10\end{array}$ & $\begin{array}{l}12 / 02 / 93 \\
12 / 02 / 93\end{array}$ & $\begin{array}{l}\text { 809K09 } \\
\text { BO9KRO }\end{array}$ & 72.00 & & $14.00 \mathrm{~L}$ & $2.65 \mathrm{U}$ \\
\hline
\end{tabular}

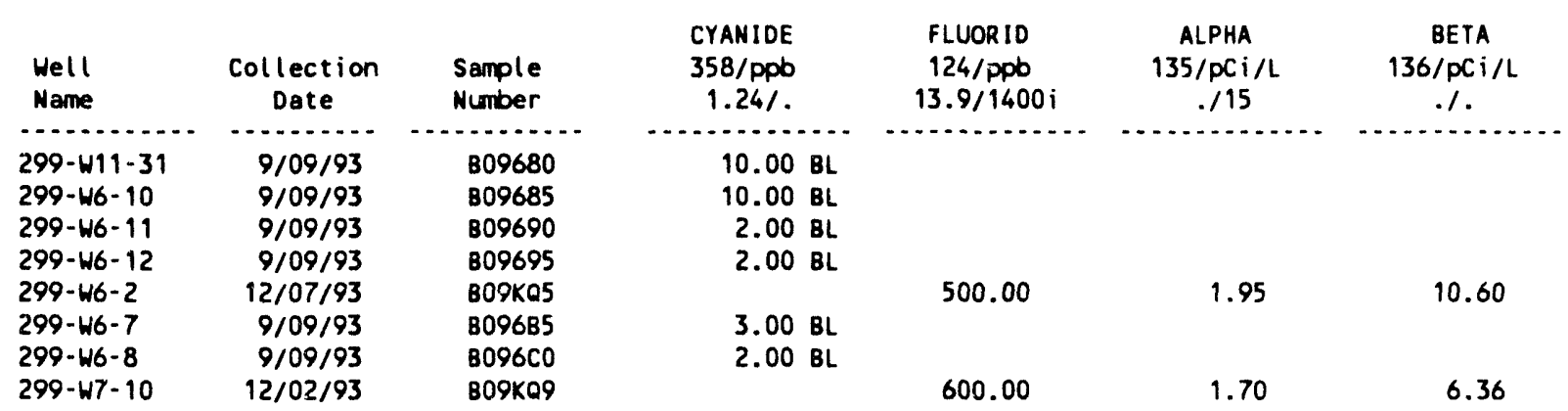

\begin{tabular}{|c|c|c|c|c|c|c|}
\hline $\begin{array}{l}\text { Well } \\
\text { Name }\end{array}$ & $\begin{array}{c}\text { Collection } \\
\text { Date }\end{array}$ & $\begin{array}{l}\text { Sample } \\
\text { Number }\end{array}$ & $\begin{array}{c}\text { IRON } \\
34 / \mathrm{ppb} \\
10.3 / 300 \mathrm{~s}\end{array}$ & $\begin{array}{c}\text { FIRON } \\
\text { 34/ppt } \\
10.3 / 300 \mathrm{~s}\end{array}$ & $\begin{array}{l}\text { LEAD } \\
40 / \mathrm{ppb} \\
.508 / 50 \mathrm{i}\end{array}$ & $\begin{array}{c}\text { MAGNES } \\
34 / \mathrm{ppb} \\
26 / .\end{array}$ \\
\hline $299-166-2$ & $12 / 07 / 93$ & B09Ka5 & $240.00 \mathrm{~B}$ & $\cdots$ & $.51 \mathrm{U}$ & 17000.00 \\
\hline $\begin{array}{l}299-w 6-2 \\
299-w 6-6 \\
299-w 6-6\end{array}$ & $\begin{array}{r}12 / 07 / 93 \\
9 / 10 / 93 \\
9 / 10 / 93\end{array}$ & $\begin{array}{l}\text { B09K06 } \\
\text { B096B0 } \\
\text { B096B4 }\end{array}$ & & $\begin{array}{l}12.00 \mathrm{BL} \\
76.00\end{array}$ & & 13000.00 \\
\hline $\begin{array}{l}299-w 6-9 \\
299-w 6-9\end{array}$ & $\begin{array}{l}9 / 10 / 93 \\
9 / 10 / 93\end{array}$ & $\begin{array}{l}8096 C 5 \\
8096 C 9\end{array}$ & 140.00 & 34.00 & & 18000.00 \\
\hline $\begin{array}{l}299-\omega 7-10 \\
299-\omega 7-10\end{array}$ & $\begin{array}{l}12 / 02 / 93 \\
12 / 02 / 93\end{array}$ & $\begin{array}{l}\text { BO9KO9 } \\
\text { BO9KRO }\end{array}$ & 350.00 & $16.00 \mathrm{~L}$ & $1.10 \mathrm{BL}$ & 14000.00 \\
\hline
\end{tabular}

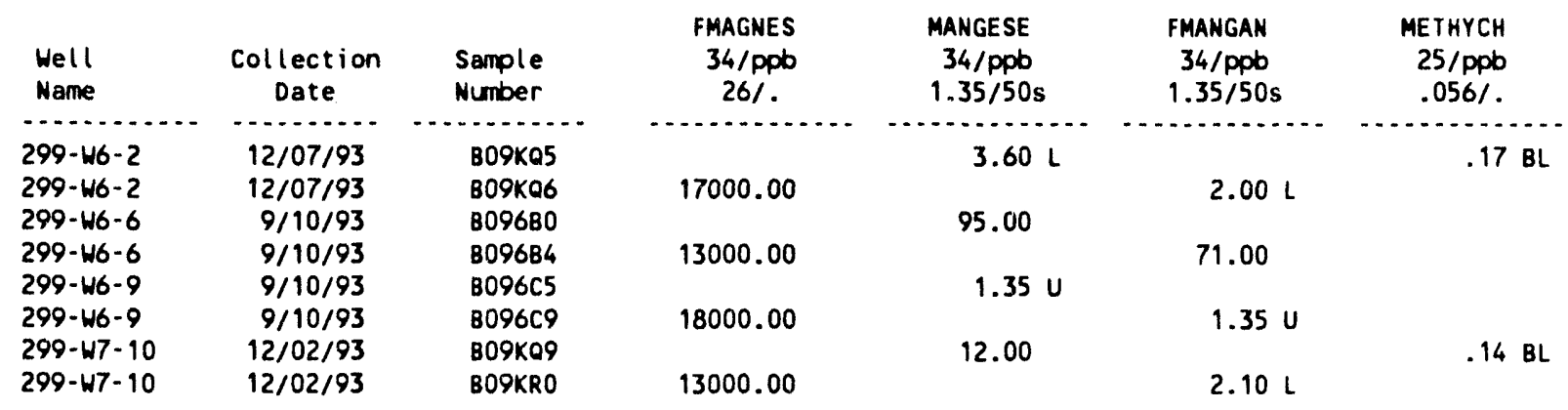


Table 15-25. Constituents with at Least One Detected Value for the Low-Level Waste Burial Grounds WMA 5 Data for Reporting Period October 1 through December 31, 1993. (sheet 3 of 4)

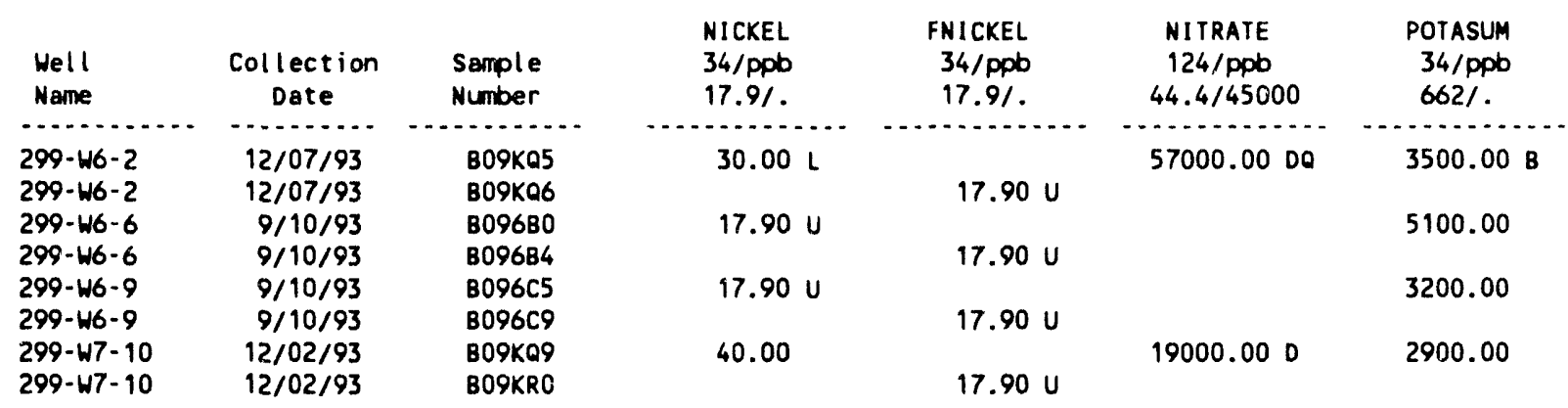

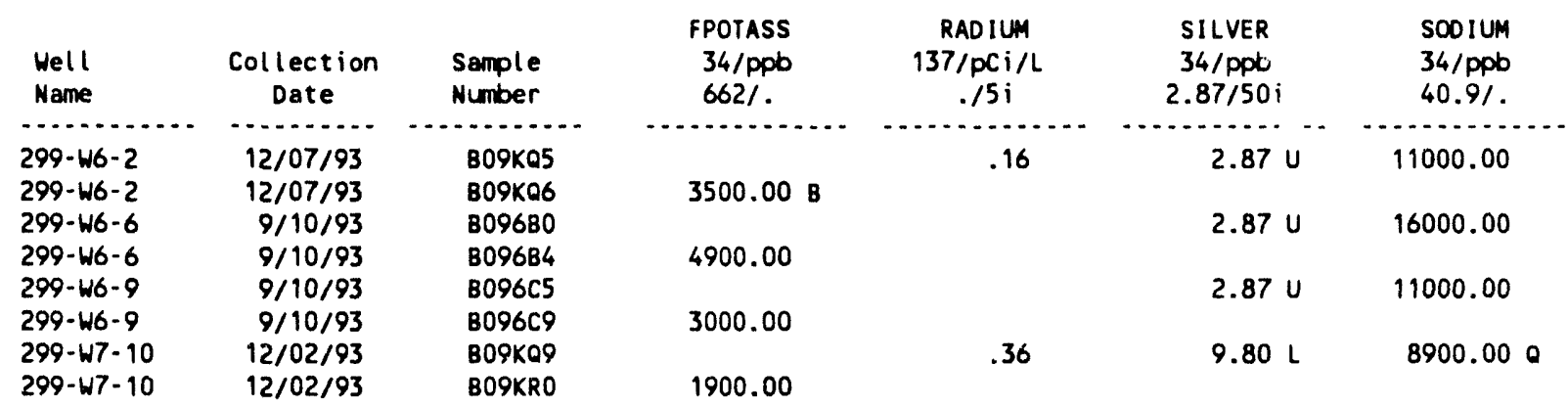

\begin{tabular}{|c|c|c|c|c|c|c|}
\hline $\begin{array}{l}\text { Well } \\
\text { Name }\end{array}$ & $\begin{array}{c}\text { Collection } \\
\text { Date }\end{array}$ & $\begin{array}{l}\text { Sample } \\
\text { Number }\end{array}$ & $\begin{array}{l}\text { FSOD IUM } \\
34 / \mathrm{ppb} \\
40.91\end{array}$ & $\begin{array}{c}\text { SULFATE } \\
124 / \text { ppb } \\
49.9 / 250000 \mathrm{~s}\end{array}$ & $\begin{array}{r}\text { PERCENE } \\
25 / \mathrm{ppb} \\
.049 / 5\end{array}$ & $\begin{array}{l}\text { TOLUENE } \\
25 / \mathrm{ppb} \\
.056 / 1000\end{array}$ \\
\hline$\ldots . . . . . . . . . . .$. & $\ldots . . . .$. & $\ldots . . . . . .$. & $\ldots$ & - .............. & $\ldots . . .6 . . . .$. & \\
\hline $\begin{array}{l}299-W 6-2 \\
299-46-2\end{array}$ & $\begin{array}{l}12 / 07 / 93 \\
12 / 07 / 93\end{array}$ & $\begin{array}{l}\text { B09K05 } \\
\text { B09K06 }\end{array}$ & 10000.00 & 25000.000 & $.12 \mathrm{BL}$ & $.06 \mathrm{U}$ \\
\hline $\begin{array}{l}299-W 6-6 \\
299-W 6-9\end{array}$ & $\begin{array}{l}9 / 10 / 93 \\
9 / 10 / 93\end{array}$ & $\begin{array}{l}\text { B09684 } \\
\text { B096C9 }\end{array}$ & $\begin{array}{l}16000.00 \\
11000.00\end{array}$ & & & \\
\hline $\begin{array}{l}299-W 7-10 \\
299-w 7-10\end{array}$ & $\begin{array}{l}12 / 02 / 93 \\
12 / 02 / 93\end{array}$ & $\begin{array}{l}\text { BO9KQ9 } \\
\text { BO9KRO }\end{array}$ & 8800.00 & $21000.00 \mathrm{D}$ & $.05 \mathrm{U}$ & .07 \\
\hline
\end{tabular}

\begin{tabular}{|c|c|c|c|c|c|c|}
\hline $\begin{array}{l}\text { Hell } \\
\text { Name }\end{array}$ & $\begin{array}{c}\text { Collection } \\
\text { Date }\end{array}$ & $\begin{array}{l}\text { Sample } \\
\text { Number }\end{array}$ & $\begin{array}{c}\text { TRICENE } \\
16 / \text { ppb } \\
.77 / 5\end{array}$ & $\begin{array}{r}\text { TRICENE } \\
25 / \mathrm{ppb} \\
.065 / 5\end{array}$ & $\begin{array}{c}\text { TRITIUM } \\
\text { 142/pCi/L } \\
. / 20000\end{array}$ & $\begin{array}{c}\text { TURBID } \\
126 / N T U \\
.05 / .\end{array}$ \\
\hline$\cdots+\cdots$ & $\cdots \ldots$ & 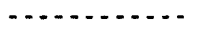 & 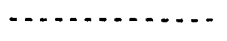 & 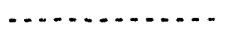 & 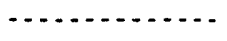 & \\
\hline 299-W6-2 & $8 / 13 / 93$ & 809028 & $1.30 \mathrm{~L}$ & & & \\
\hline $\begin{array}{l}299-w 6-2 \\
299-w 7-10\end{array}$ & $\begin{array}{r}12 / 07 / 93 \\
8 / 13 / 93\end{array}$ & $\begin{array}{l}\text { B09K05 } \\
\text { B09102 }\end{array}$ & $.77 \mathrm{U}$ & 1.30 & 9490.00 & 1.00 \\
\hline $299-w 7-10$ & $12 / 02 / 93$ & BO9K09 & & $.12 \mathrm{~L}$ & 428.00 & 2.90 \\
\hline
\end{tabular}


Table 15-25. Constituents with at Least One Detected Value for the Low-Level Waste Burial Grounds WMA 5 Data for Reporting Period October 1 through December 31, 1993. (sheet 4 of 4)

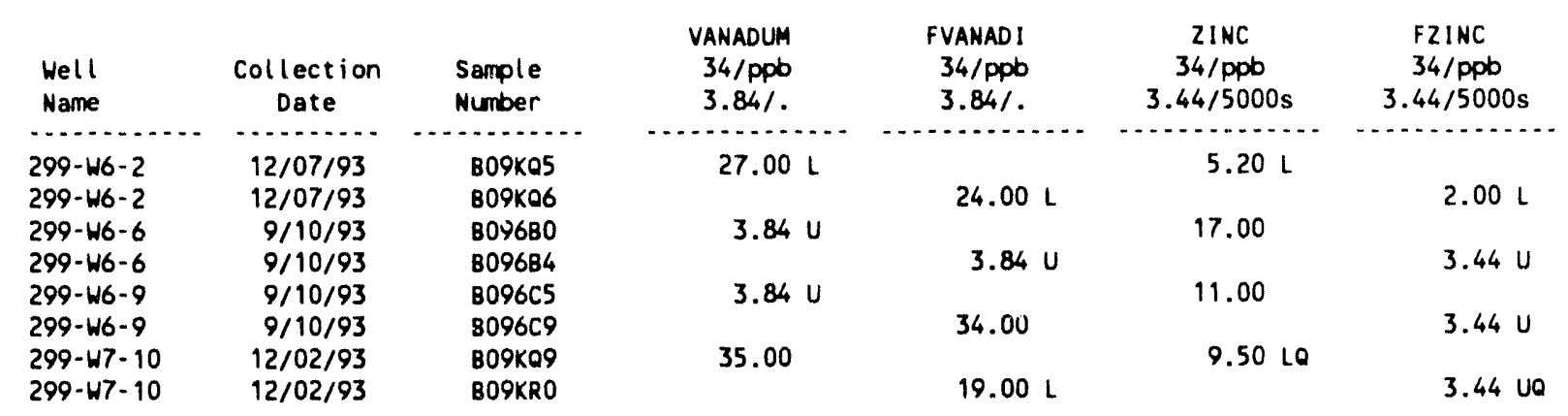

For explanation of this table, see Section 1.4 of report. 
Table 15-26. Contamination Indicator Parameters for the Low-Level Waste Burial Grounds WMA 5 Data for Reporting Period October 1 through December 31, 1993.

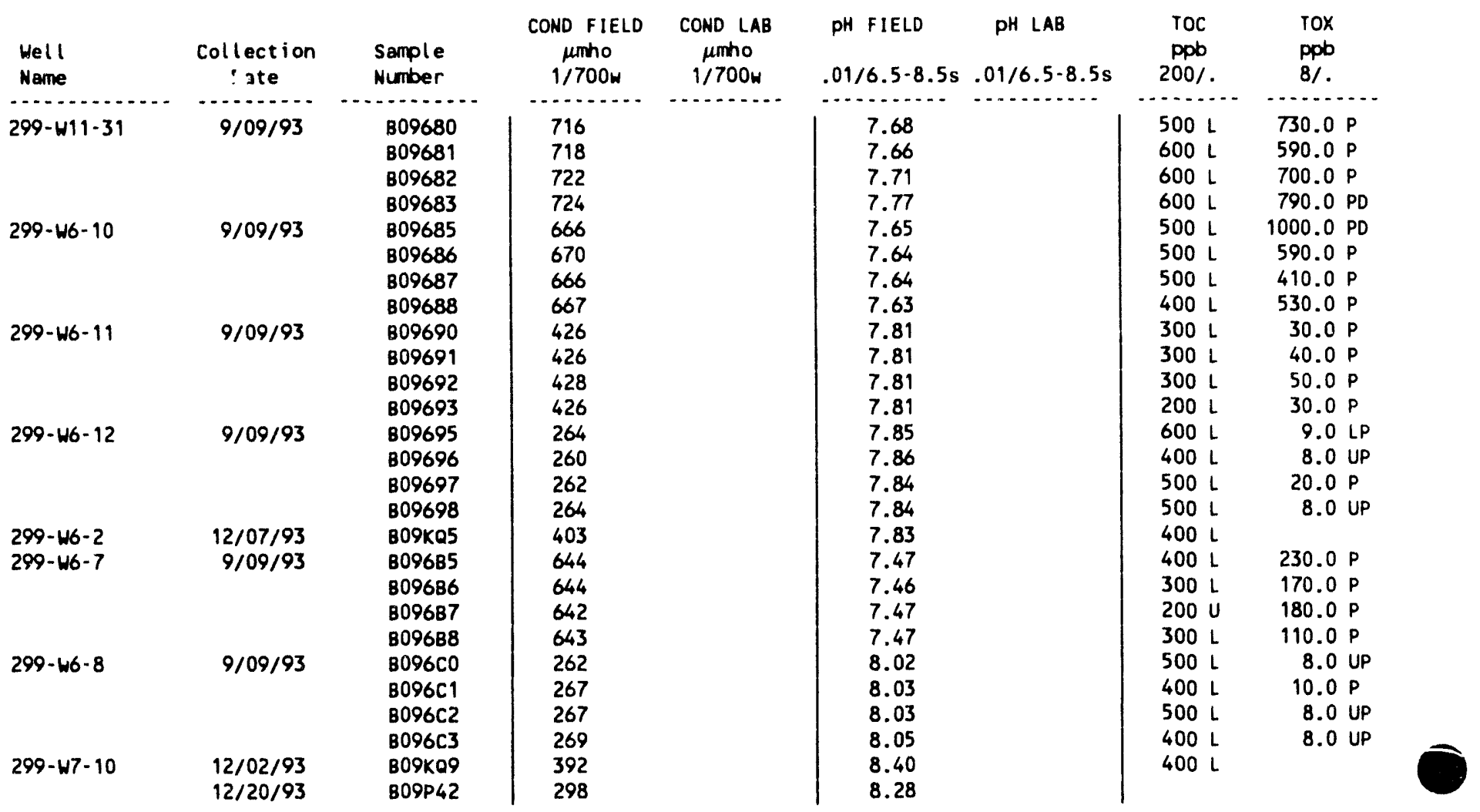

For explanation of this table, see section 1.4 of report. 


\begin{tabular}{|c|c|c|c|}
\hline $\begin{array}{l}\text { Chromium } \\
\text { (50 pṕb) }\end{array}$ & $\begin{array}{l}299-E 28-26 \\
299-E 32-2 \\
299-E 32-5 \\
299-E 32-9 \\
299-E 33-29 \\
299-E 33-35\end{array}$ & $\begin{array}{l}299-E 28-27 \\
299-E 32-3 \\
299-E 32-6 \\
299-E 32-10 \\
299-E 33-30\end{array}$ & $\begin{array}{l}299-E 28-28 \\
299-E 32-4 \\
299-E 32-7 \\
299-E 33-28 \\
299-E 33-34\end{array}$ \\
\hline $\begin{array}{l}\text { Iron } \\
\text { (300 ppb) }\end{array}$ & $\begin{array}{l}299-E 28-26 \\
299-E 32-2 \\
299-E 32-5 \\
299-E 32-8 \\
299-E 33-28 \\
299-E 33-34\end{array}$ & $\begin{array}{l}299-E 28-27 \\
299-E 32-3 \\
299-E 32-6 \\
299-E 32-9 \\
299-E 33-29\end{array}$ & $\begin{array}{l}299-E 28-28 \\
299-E 32-4 \\
299-E 32-7 \\
299-E 32-10 \\
299-E 33-30\end{array}$ \\
\hline $\begin{array}{l}\text { Tritium } \\
(20,000 \mathrm{pC} i / L)\end{array}$ & $\begin{array}{l}299-E 28-27 \\
299-E 32-5\end{array}$ & $\begin{array}{l}299-E 32-2 \\
299-E 32-6\end{array}$ & $299-E 32-3$ \\
\hline
\end{tabular}

Table 15-28. Water Quality Standards Exceeded at Low-Level Waste Management Area 2.

\begin{tabular}{|llll|}
\hline Chromium & $299-E 27-8$ & $299-E 27-9$ & $299-E 27-10$ \\
$(50 \mathrm{ppb})$ & $299-\mathrm{E} 27-11$ & $299-\mathrm{E} 27-17$ & $299-\mathrm{E} 34-2$ \\
& $299-\mathrm{E} 32-3$ & $299-\mathrm{E} 32-5$ & $299-\mathrm{E} 34-10$ \\
\hline Iron & $299-\mathrm{E} 27-8^{\mathrm{a}}$ & $299-\mathrm{E} 27-9^{\mathrm{a}}$ & $299-\mathrm{E} 27-10^{\mathrm{a}}$ \\
(300 ppb) & $299-\mathrm{E} 27-11^{\mathrm{a}}$ & $299-\mathrm{E} 27-17^{\mathrm{a}}$ & $299-\mathrm{E} 34-2^{\mathrm{a}}$ \\
& $299-\mathrm{E} 32-3^{\mathrm{a}}$ & $299-\mathrm{E} 32-5^{\mathrm{a}}$ & $299-\mathrm{E} 34-10^{\mathrm{a}}$ \\
\hline
\end{tabular}

${ }^{8}$ Field QC samples associated with this data exceeded the reliable detection limits. 
Table 15-29. Water Quality Standards Exceeded at Low-Level Waste Management Area 3.

\begin{tabular}{|llll|}
\hline $\begin{array}{l}\text { Field pH } \\
(>8.5)\end{array}$ & $299-W 7-6$ & & \\
\hline Chromium & & & \\
$(50 \mathrm{ppb})$ & $299-W 6-2^{2}$ & $299-W 7-1$ & $299-W 7-2$ \\
& $299-W 7-4$ & $299-W 7-5$ & $299-W 7-6$ \\
& $299-W 7-7$ & $299-W 7-8$ & $299-W 7-9$ \\
& $299-W 7-10$ & $299-W 7-11$ & $299-W 7-12$ \\
& $299-W 8-1$ & $299-W 9-1$ & $299-W 10-13$ \\
& $299-W 10-14$ & & \\
\hline Nitrate & $299-W 6-2$ & $299-W 7-4$ & $299-W 7-5$ \\
$(45,000$ ppb) & $299-W 10-19$ & $299-W 10-21$ & \\
\hline Iron & $299-W 7-2$ & $299-W 7-4$ & $299-W 7-5$ \\
$(300 \mathrm{ppb})$ & $299-W 7-6$ & $299-W 7-7$ & $299-W 7-8$ \\
& $299-W 7-9$ & $299-W 7-10$ & $299-W 7-12$ \\
& $299-W 8-1$ & $299-W 9-1$ & $299-W 10-13$ \\
& $299-W 10-14$ & $299-W 10-21$ & \\
\hline $\begin{array}{l}\text { Manganese } \\
\text { (50 ppb) }\end{array}$ & $299-W 7-6$ & $299-W 7-9$ & $299-W 7-12$ \\
\hline Carbon tetrachloride & $299-W 6-2$ & $299-W 7-4$ & $299-W 7-5$ \\
(5 ppb) & $299-W 7-8$ & $299-W 7-10$ & $299-W 8-1$ \\
& $299-W 10-13$ & $299-W 10-19$ & $299-W 10-21$ \\
\hline Trichloroethene & $299-W 10-19$ & $299-W 10-21$ & \\
(5 ppb) & & & \\
\hline
\end{tabular}

${ }^{\text {a } F i e l d ~ Q C ~ s a m p l e s ~ a s s o c i a t e d ~ w i t h ~ t h i s ~ d a t a ~ e x c e e d e d ~ t h e ~ r e l i a b l e ~}$ detection limits. 
Table 15-30. Water Quality Standards Exceeded at Low-Level Waste Management Area 4.

\begin{tabular}{|llll|}
\hline Chromium & $299-W 15-15$ & $299-W 15-16$ & $299-W 15-17$ \\
(50 ppb) & $299-W 15-18$ & $299-W 15-19$ & $299-W 15-20$ \\
& $299-W 15-23$ & $299-W 15-24$ & $299-W 18-21$ \\
& $299-W 18-22$ & $299-W 18-26$ & $299-W 18-27$ \\
& $299-W 18-28$ & $299-W 18-29$ & \\
\hline Nitrate & $299-W 15-15$ & $299-W 15-16$ & $299-W 15-18$ \\
$(45,000 \mathrm{ppb})$ & $299-W 15-19$ & $299-W 15-24$ & \\
\hline Iron & $299-W 15-15$ & $299-W 15-16$ & $299-W 15-17$ \\
(300 ppb) & $299-W 15-18$ & $299-W 15-19$ & $299-W 15-20$ \\
& $299-W 15-23$ & $299-W 15-24$ & $299-W 18-21$ \\
& $299-W 18-22$ & $299-W 18-26$ & $299-W 18-27$ \\
& $299-W 18-28$ & $299-W 18-29$ & \\
\hline Manganese & $299-W 15-19$ & $299-W 15-20$ & $299-W 15-24$ \\
(50 ppb) & $299-W 18-27$ & & \\
\hline Carbon tetrachloride & $299-W 15-15^{\circ}$ & $299-W 15-16^{b}$ & $299-W 15-18$ \\
(5 ppb) & $299-W 15-19$ & $299-W 15-20^{\circ}$ & $299-W 15-23^{\circ}$ \\
& $299-W 15-24$ & $299-W 18-21^{\circ}$ & $299-W 18-23$ \\
& $299-W 18-24$ & $299-W 18-26$ & $299-W 18-27$ \\
& $299-W 18-28$ & $299-W 18-29$ & $299-W 18-32$ \\
\hline Trichloroethene & $299-W 15-16$ & & \\
(5 ppb) & & & \\
\hline
\end{tabular}

Holding time exceeded. limits.

${ }^{\circ} \mathrm{QC}$ samples associated with this data exceeded the reliable detection 
Table 15-31. Water Quality Standards Exceeded at Low-Level Waste Management Area 5.

\begin{tabular}{|c|c|c|}
\hline $\begin{array}{l}\text { Chromium } \\
\text { (50 ppb) }\end{array}$ & $299-W 6-2^{a}$ & $299-W 7-10$ \\
\hline $\begin{array}{l}\text { Nitrate } \\
(45,000 \mathrm{ppb})\end{array}$ & $299-W 6-2$ & \\
\hline $\begin{array}{l}\text { Iron } \\
(300 \mathrm{ppb})\end{array}$ & $299-W 7-10$ & \\
\hline $\begin{array}{l}\text { Manganese } \\
(50 \mathrm{ppb})\end{array}$ & 299-W6-6 & \\
\hline $\begin{array}{l}\text { Filtered manganese } \\
(50)\end{array}$ & 299-W6-6 & \\
\hline $\begin{array}{l}\text { Carbon tetrachloride } \\
\text { (5 ppb) }\end{array}$ & 299-W6-2 & $299-W 7-10$ \\
\hline
\end{tabular}

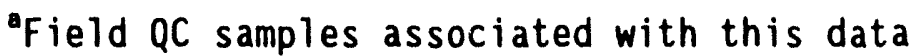
exceeded the reliable detection limits. 


$$
\text { DOE/RL-93-56-4 }
$$

\section{CONTENTS}

16.0 SINGLE-SHELL TANKS . . . . . . . . . . . . . 16-1

16.1 INTRODUCTION . . . . . . . . . . . . . . . . 16-1

16.2 WATER LEVEL MEASUREMENTS . . . . . . . . . . . . . 16-2

16.3 WATER CHEMISTRY DATA .............. . . 16-3

16.3.1 Waste Management Area A-AX . . . . . . . . 16-4

16.3.2 Waste Management Area B-BX-BY . . . . . . . . . . 16-4

16.3.3 Waste Management Area $C$. . . . . . . . . . . . . 16-4

16.3.4 Waste Management Area S-SX . . . . . . . . . . . 16-4

16.3.5 Waste Management Area $T \ldots . . . . . . .16-4$

16.3.6 Waste Management Area TX-TY . . . . . . . . . . 16-5

16.3.7 Waste Management Area U . . . . . . . . . 16-6 


\section{LIST OF FIGURES}

16-1 Single-She11 Tank Waste Management Areas in the $16-7$

16-2 Single-She11 Tank Waste Management Areas in the 200 West Area . . . . . . . . . . . . . . . . 16-8

16-3 Monitoring Well Locations for the Single-Shell Tank Waste Management Areas in the 200 East Area . . . . . . . . . . . 16-9

16-4 Monitoring Well Locations for the Single-Shell Tank Waste

Management Areas in the 200 West Area . . . . . . . . . . 16-10

\section{LIST OF TABLES}

16-1 Groundwater Monitoring Welis for the Single-She11 Tanks . . . 16-11

16-2 RCRA Water Level Measurement Report for the Single-Shell Tanks, Fourth Quarter 1993 ................ 16-13

16-3 Constituent List and Summary of Results for the Single-Shell Tank Waste Management Area A-AX Data for Reporting Period October 1 through December 31, 1993 . . . . . . . . . . . 16-20

16-4 Constituents with at Least One Detected Value for the Single-Shell Tank Waste Management Area A-AX Data for Reporting Period October 1 through December 31, 1993.

16-5 Contamination Indicator Parameters for the Single-Shell Tank Waste Management Area A-AX Data for Reporting Period October 1 through December 31, 1993 .. . . . . . . . . . 16-22

16-6 Constituent List and Summary of Results for the Single-She 11 Tank Waste Management Area B-BY-BX Data for Reporting Period October 1 through December 31, 1993

16-7 Constituents with at Least One Detected Value for ine Single-Shell Tank Waste Management Area B-BY-BX Data for Reporting Period October 1 through December 31, 1993 . . . . . 16-25

16-8 Contamination Indicator Parameters for the Single-Shell Tank Waste Management Area B-BY-BX Data for Reporting Period October 1 through December 31, 1993

16-9 Constituent List and Summary of Results for the Single-Shell Tank Waste Management Area C Data for Reporting Period October 1 through December 31, 1993 


\section{LIST OF TABLES (cont.)}

16-10 Constituents with at Least One Detected Value for the Single-She11 Tank Waste Management Area C Data for Reporting Period October 1 through December 31, 1993 . . . . . 16-29

16-11 Contamination Indicator Parameters for the Single-Shell Tank Waste Management Area C Data for Reporting Period October 1 through December 31, 1993 ................ 16-30

16-12 Constituent List and Summary of Results for the Single-Shell Tank Waste Management Area S-SX Data for Reporting Period October 1 through December 31, 1993 . . . . . . . . . . . 16-31

16-13 Constituents with at Least One Detected Value for the Single-Shell Tank Waste Management Area S-SX Data for Reporting Period October 1 through December 31, 1993 . . . . . 16-32

16-14 Contamination Indicator Parameters for the Single-Shell Tank Waste Management Area S-SX Data for Reporting Period October 1 through December 31, 1993.............. 16-33

16-15 Constituent List and Summary of Results for the Single-Shell Tank Waste Management Area T Data for Reporting Period October 1 through December 31, 1993 . . . . . . . . . . . 16-34

16-16 Constituents with at Least One Detected Value for the Single-Shell Tank Waste Management Area T Data for Reporting Period October 1 through December 31, 1993......... . 16-36

16-17 Contamination Indicator Parameters for the Single-Shell Tank Waste Management Area T Data for Reporting Period October 1 through December 31, 1993 ................ 16-38

16-18 Constituent List and Summary of Results for the Single-Shell Tank Waste Management Area TX-TY Data for Reporting Period October 1 through December 31, 1993 ............ 16-39

16-19 Constituents with at Least One Detected Value for the Single-Shell Tank Waste Management Area TX-TY Data for Reporting Period October 1 through December 31, 1993 .. . . . . 16-41

16-20 Contamination Indicator Parameters for the Single-Shell Tank Waste Management Area TX-TY Data for Reporting Period October 1 through December 1993 .............. 16-43

16-21 Constituent List and Summary of Results for the Single-Shell Tank Waste Management Area U Data for Reporting Period October 1 through December 31, 1993. 
DOE/RL-93-56-4

\section{LIST OF TABLES (cont.)}

16-22 Constituents with at Least One Detected Value for the Single-Shell Tank Waste Management Area U Data for Reporting Period October 1 through December 31, 1993 . . . . . . . . 16-45

16-23 Contamination Indicator Parameters for the Single-Shell Tank Waste Management Area U Data for Reporting Period October 1 through December $31,1993 \ldots$. . . . . . . . . . . . 16-46 
DOE/RL-93-56-4

\title{
16.0 SINGLE-SHELL TANKS
}

\author{
J. A. Caggiano \\ Westinghouse Hanford Company
}

\subsection{INTRODUCTION}

Although decommissioned in 1980, the SSTs are considered to be actively storing hazardous and radioactive waste and have been designated as RCRA facilities. The SSTs received metal and first-cycle waste from the chemical processing of uranium-bearing spent fuel rods. Spent fuel rods from reactors in the 100 Areas were processed using the bismuth phosphate, REDOX, or PUREX process. Metal waste is that which was produced in the first step of the process of dissolving spent fuels, and contains about $90 \%$ of fission product radionuclides as well as uranium and some plutonium. Other waste streams (e.g., coating, noncomplexed, and deionizing waste) also were sent to SSTs at some time in their history (see Anderson 1990 for a discussion of types of waste sent to various SSTs from startup until the tank was no longer used). Chemical and radiological contents of the various SSTs were summarized in July 1993 (WHC 1993C).

The 149 SSTs are located in seven waste management areas (WMA) containing one or more tank farms (Figures 16-1 and 16-2). Three WMAs are in the 200 East Area (A-AX, B-BX-BY, and C) and four are in the 200 West Area (S-SX, $T, T X-T Y$, and $U$ ). Each tank farm contains from 4 (AX Tank Farm) to 18 (TX Tank Farm) tanks, each of which is an underground, reinforced-concrete tank with a single liner of carbon steel. The larger tanks have a diameter of $23 \mathrm{~m}(75 \mathrm{ft})$, varying height, and are buried at least $2 \mathrm{~m}(6 \mathrm{ft})$ below the ground surface. The capacity of these tanks varies from 2,006,000 to $3,785,000 \mathrm{~L}(530,000$ to $1,000,000 \mathrm{gal})$. There are sixteen 200 -series tanks, each of which hold $208,175 \mathrm{~L}(5 \mathrm{~s}, 000 \mathrm{gal})$. Four 200 -series tanks are located in each of the original four tank farms: 241-B, 241-C, 241-T, and 241- $U$. The original four tank farms were built in 1944; SSTs were built from 1944 to 1964 .

Interim-status, detection-level groundwater monitoring was initiated at the SSTs in 1989 with the construction of 12 RCRA standard wells. Sampling was initiated in February 1990 at WMAs A-AX, B-BX-BY, C, and T, but was suspended until July 1991 because of the absence of analytical laboratory support. Sampling resumed in July 1991 at WMAs A-AX, B-BX-BY, and T, and continued quarterly through early 1993. By October 1991, all existing RCRA standard wells in all WMAs were being sampled quarterly. Most wells have been sampled quarterly more than four times. All WMAs, except for T and TX-TY, are now in detection-level, semiannual indicator parameter evaluation status. WMAs $T$ and TX-TY continue to be sampled quarterly, as these sites are now in assessment-level monitoring status because of elevated specific conductance. A groundwater quality assessment monitoring plan (Caggiano and Chou 1993) for WMAs T and TX-TY was submitted to Ecology in July 1993. The SST WMA well locations are shown in Figures 16-3 and 16-4. Wells in the SST monitoring networks are listed in Table 16-1. Groundwater monitoring at the SSTs follows the groundwater monitoring plan (Jensen et al. 1989) as revised (Caggiano and Goodwin 1991). 
Numerous older wells in the vicinity of the tank farms are constructed of carbon steel casing that is perforated and open to different levels in the unconfined aquifer. These wells were part of an operational groundwater monitoring system at some point in their history and currently are used only for water level measurements or to collect samples for operational monitoring. Some are used for environmental surveillance monitoring to check for conformance to DOE orders (e.g., Schmidt et al. 1992). These older wells do not comply with the construction specifications of WAC 173-160 and are not used for collection of RCRA samples. One exception is well 2-E27-7, a well constructed in 1982 that has a carbon steel casing, an annular seal, and a stainless steel screen below the carbon steel casing. There is no carbon steel in contact with groundwater in well 2-E27-7. Samples are collected from this well, which is upgradient of WMA $C$.

\subsection{MATER LEVEL MEASUREMENTS}

Water levels measured in 61 wells for this quarter are given in Table 16-2. These data include rout ine water level measurements taken when sampling, as well as nonroutine monthly measurements made by Environmental Field Services personnel. Geodetic survey elevations used to calculate water levels this quarter were changed to National Geodetic Vertical Datum 1929 Standard (NGVD'29). Many previous surveys used locally established benchmarks as the datum, but there has been a mix of elevation data obtained from surveying at different times using a different datum.

The water table in the 200 East Area is nearly flat because of declining water levels and high transmissivity of the Hanford formation. The water table map for June 1993 (DOE-RL 1994a, Fig 4.13-5) illustrates the very low slope to the water table. The maximum difference in elevation for all monitoring wells in the 200 East Area SST networks (A-AX, B-BX-BY, and C) for December 1993 is $16 \mathrm{~cm}(0.55 \mathrm{ft})$ between wells 2-E25-2 and 2-E33-39, we11 s that are nearly $2.4 \mathrm{~km}(1.5 \mathrm{mi}$ ) apart (a slope of approximately $6.3 \mathrm{~cm} / \mathrm{km}$ [4 in./mi ]).

Water levels in upgradient wells 2-W18-25 and 2-W18-31 continue to be declining faster than in downgradient well 2-W19-32 at WMA U. A possible change in flow direction is suggested and this trend is being watched. Hydrographs of wells in WMA $U$ were included in the recently issued annual report (DOE-RL 1994a, Figure 4.13-10). Five wells have been added to the water level monitoring network in the vicinity of the 241-U Tank Farm to determine the extent and possible source for this change (see Table 16-2). Quarterly sampling for anions has also been instituted, as chloride is the best tracer for water recharging the unconfined aquifer from the 284Powerhouse Pond. Background values will be modified if this is a permanent change in groundwater flow direction.

Water levels in a number of older carbon steel wells located inside the perimeter fences of the tank farms have not been measured since July 1993 and are no longer found in Table 16-2. Access to the tank farms was restricted to essential safety-related activities in August 1993 because of several incidents, and these wells were eliminated from the SST water level monitoring network. 
DOE/RL-93-56-4

Well 2-E33-24, located adjacent to the 241-BY Tank Farm (WMA B-BX-BY), was decommissioned in September 1993 so that a cover could be constructed over the adjacent 216-B-57 Crib as part of remedial measures for operable unit 200-BP-1. This well no longer appears in Table 16-2.

\subsection{WATER CHEM̈ISTRY DATA}

Data received to date from analyses of samples collected during the fourth quarter of 1993 are provided in Tables 16-3 through 16-23. The D flag following data for sulfate, nitrate, and chloride indicates that the samples were diluted for analysis. Most SST wells were not sampled this quarter. Quarterly sampling is presently conducted in WMAs T and TX-TY because these sites are in groundwater assessment monitoring under interim status

(40 CFR 265). Anions are also sampled quarterly at WMA U. Some samples from SST wells were collected in late September 1993, and the data were not received in time to be included in that quarterly report (DOE-RL 1994b). Missing data appear in this quarterly report.

Several standards for constituent concentration have been added to the data tables, resulting in some constituents exceeding limits that had not previously been ident ified in quarterly reports. For example, carbon tetrachloride exceeds the 5-ppb standard in a number of wells in the 200 West Area because there is a widespread plume of this constituent in the groundwater (DOE-RL 1994a, Fig 4.1-19). Similarly, trichloroethene has exceeded the limit in some 200 West Area wells.

Inconsistency exists in published regulatory limits for fluoride. The primary DWS for fluoride is 4,000 ppb ( 40 CFR 141); the secondary standard (40 CFR 143.3) is 2,000 ppb. Washington State standards in Revised Code of Washington (RCW) 248 and WAC 173-200 are 4,000 ppb. However, Appendix III of 40 CFR 265 (EPA Interim Primary DWS) lists a range of 1.4 to $2.4 \mathrm{ppm}(1,400$ to $2,400 \mathrm{ppb})$ for fluoride. The preponderance of promulgated standards is 4,000 ppb and this will be used in comparing values to a regulatory limit. Groundwater quality beneath WMA $T$ is degraded because of fluoride, which has exceeded the 4,000-ppb standard in the past and continues to do so in some wells. Fluorine-bearing compounds were used in the bismuth phosphate process in 221-T (T P1ant) and were discharged to SSTs as well as liquid effluent facilities in the northern part of 200 West Area. Tables 16-3 through 16-23 use the 1,400-ppb standard for fluoride.

Remediation of well 2-W11-28 at WMA T was completed and it was scheduled to be sampled in December 1993, but was delayed. The well has not been sampled since May 1992 because of problems with the well and the sampling pump.

Four quarters of background data have been obtained from wells in all WMAs and the data have been statistically analyzed to calculate a background for TOC, specific conductance, and critical range (for $\mathrm{pH}$ ) for each site. Values for TOX were not used to calculate a critical mean for each WMA because of $Q C$ problems in the laboratory. Replicate average values from downgradient wells are compared to the critical means for indicator parameters to determine whether the facilities in any SST WMAs may be affecting groundwater quality. 


$$
\mathrm{DOE} / \mathrm{RL}-93-56-4
$$

Additional wells to supplement the groundwater quality assessment program are being identified and checked before having any remedial work performed and adding them to the SST network for WMAs T and TX-TY. These two WMAs are in groundwater quality assessment status because of elevated specific

conductance. These wells are being added to these networks to provide data for determining the possible extent of the high conductivity plume in groundwater beneath these two WMAs, and whether the SSTs are the source responsible for this elevated specific conductance.

\subsubsection{Waste Management Area A-AX}

Well 2-E25-46 was sampled late during the third quarter of 1993. Data not reported for the September 29, 1993 sampling are reported in Tables 16-3 and 16-4.

\subsubsection{Waste Management Area B-BX-BY}

Unfiltered analyses of chromium and iron exceed the DWS and secondary maximum contaminant level (SMCL) in wells 2-E33-33 and 2-E33-36. Results of filtered samples were not reported this quarter.

Values for $\mathrm{pH}$, specific conductance, and TOC did not exceed the critical mean or critical range for wells for this quarter. All values for TOC were above the MDL but below the CRQL; values for TOX were at or below the MDL except for a single value for we1l 2-E33-33.

\subsubsection{Waste Management Area C}

The only data reported for WMA C were September 1993 sampling results for total dissolved solids (TDS) and sulfide, which were not included in the previous quarterly report.

\subsubsection{Waste Management Area S-SX}

Data from the September sampling for a few constituents that were not reported in the previous quarterly report are included in Tables 16-12 and 16-13.

\subsubsection{Waste Management Area T}

Nitrate exceeds the DWS in upgradient wel1 2-W10-16 and downgradient well 2-W10-15 for the September and December 1993 sampling. Fluoride in downgradient well 2-W10-15 exceeds the 4,000-ppb DWS for both September and December sampling. TDS exceed the SMCL in both wells 2-W10-15 and 2-W10-16. Values for wells 2-W10-15 and 2-W10-16 are 810 and $520 \mathrm{ppm}$, respectively. Tritium is elevated above the 20,000-pCi/L standard in well 2-W10-16. Carbon tetrachloride exceeds the DWS in wells 2-W10-15 and 2-W11-27. Trichloroethene exceeds the DWS in downgradient well 2-W10-15. 
Remediation of well 2-W11-28 to repair the casing damaged during completion of the well in December 1991 was completed. However, the inside diameter of the well in the damaged interval (between 52 and $53 \mathrm{~m} \mathrm{[171.8}$ and $175.2 \mathrm{ft}$ ] deep) has been narrowed to less than $6 \mathrm{~cm}(2.5 \mathrm{in.})$; consequently, a steel tape used to measure water levels will not pass between the riser pipes and the narrowed casing. A dedicated $E$ tape has been installed in a pump riser that will be used to measure water levels in this well. Carbon steel casing rollers used in the remediation of this well broke from the drill string and now rest at the bottom of the screen. Data are being flagged to evaluate any effects of the steel on groundwater quality. After remediation, an NCR was prepiared because the well departs from conditions in the generic specification for well construction. Because of previous problems with turbidity in this well (the single sample taken from this well in May 1992 had a turbidity of 170 NTUs), turbidity will be checked to determine whether the remediation has corrected this condition.

Values for TOC samples for the fourth quarter of 1993 are incomplete. Those reported are below the CRQL but above the MDL. All reported values for $\mathrm{pH}$ are within the critical range for all wells. No TOX data were reported.

The average of quadruplicate measurements of field-specific conductance continues to exceed the critical mean for specific conductance in downgradient well 2-W10-15, following a pattern that triggered this site into assessment monitoring. Measurements of field-and laboratory-specific conductance in all other wells are below the critical mean for conductivity; however, specific conductance in well 2-W10-16 exceeds a 700-mmho/cm limit in RCW 248.

\subsubsection{Waste Management Area TX-TY}

Tritium is elevated above the DWS in all downgradient wells (2-W10-17, 2-W14-12, and 2-W10-18) at WMA TX-TY. Turbidity is elevated in wells 2-W10-18 and 2-W15-22 at WMA TX-TY. Gross beta of $834 \mathrm{pCi} / \mathrm{L}$ in well 2-W14-12 is apparently due to elevated technetium-99 at $7,040 \mathrm{pCi} / \mathrm{L}$. Fluoride exceeded the 4,000-ppb DWS in September 1993 in well 2-W14-12, but not in December 1993. Fluoride is elevated in other wells at WMA TX-TY, compared with usual values in Hanford Site groundwater (Johnson 1993, Table A-1-2), but does not exceed the 4,000-ppb DWS. Nitrate exceeded the DWS in all wells for samples taken in September as well as December. Trichloroethene exceeds the DWS in upgradient we11 2-W15-22 and downgradient well 2-W10-17. TDS is at or above the SMCL in downgradient wells 2-W10-17 and 2-W14-12 at 500 and 760 ppm, respectively. Turbidity is elevated in upgradient well 2-W15-22 and downgradient well 2-W10-18.

Field conductivities measured at downgradient wells 2-W10-17 and 2-W14-12 continue to exceed the SMCL and the critical mean for two measurements in December 1993 in each well. Elevated specific conductance has triggered this site into assessment monitoring. Measurements of $\mathrm{pH}$ are within the critical range. Data for TOC for the December 1993 sample from well 2-W15-22 exceeds the MDL, but TOC and TOX data are not reported for other wells. 


\subsubsection{Waste Management Area $U$}

Carbon tetrachloride exceeds the DWS for all wells reporting this constituent this quarter.

Chloride is significantly higher in downgradient well 2-W19-32 $(28,000 \mathrm{ppb}$ ) than in all other wells (range $=2,900$ to $10,000 \mathrm{ppb})$ this quarter, although it is well below the SMCL of 250,000 ppb.

Values for specific conductance are consistent with previous quarters and do not exceed the SMCL for conductivity. No laboratory data for $\mathrm{pH}$ and specific conductance were reported for any wells this quarter.

TOC and TOX results were not reported this quarter. 
Figure 16-1. Single-Shell Tank Waste Management Areas in the 200 East Area.

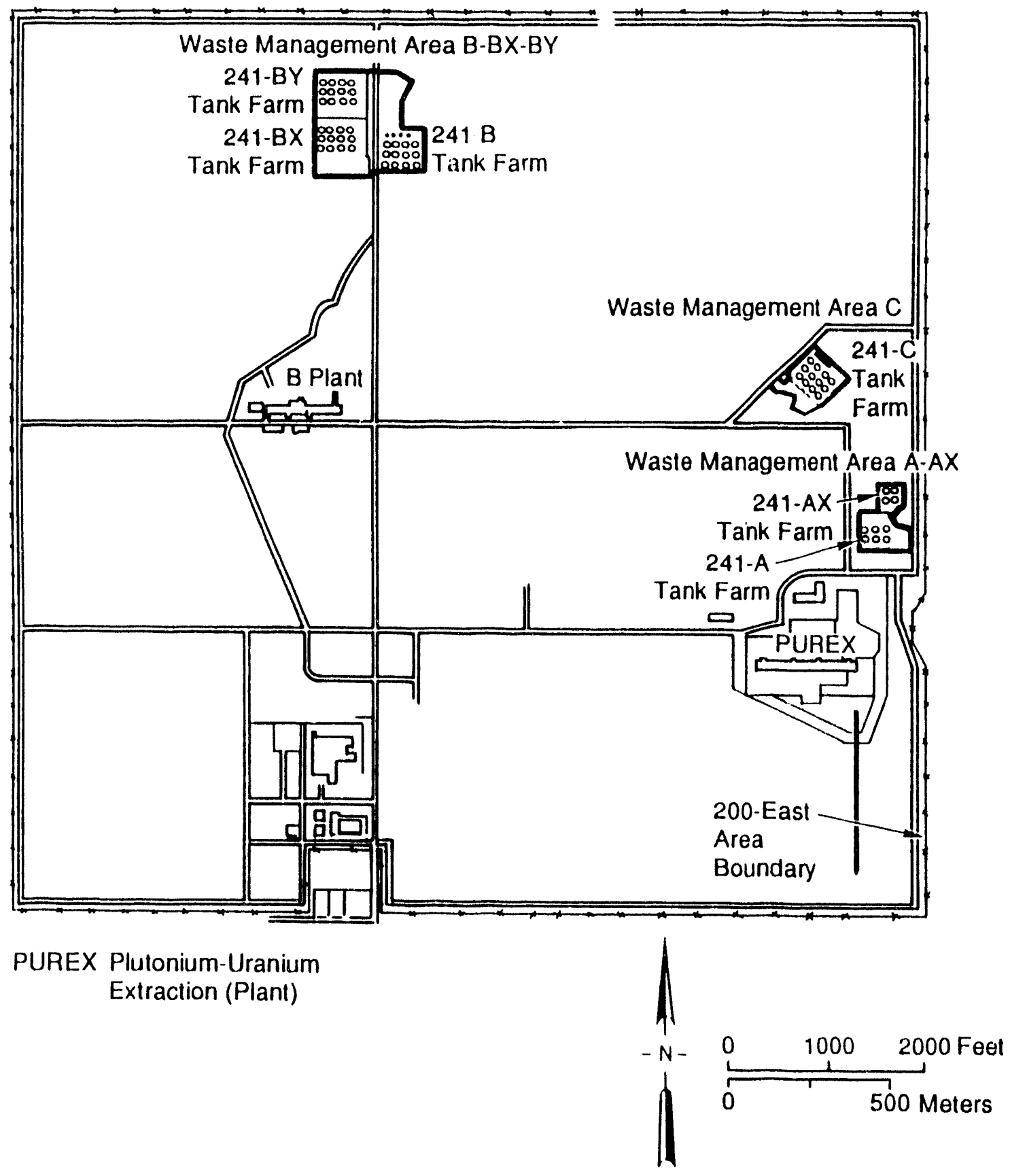

PS $90 \cdot 71$ 
Figure 16-2. Single-Shell Tank Waste Management Areas in the 200 West Area.

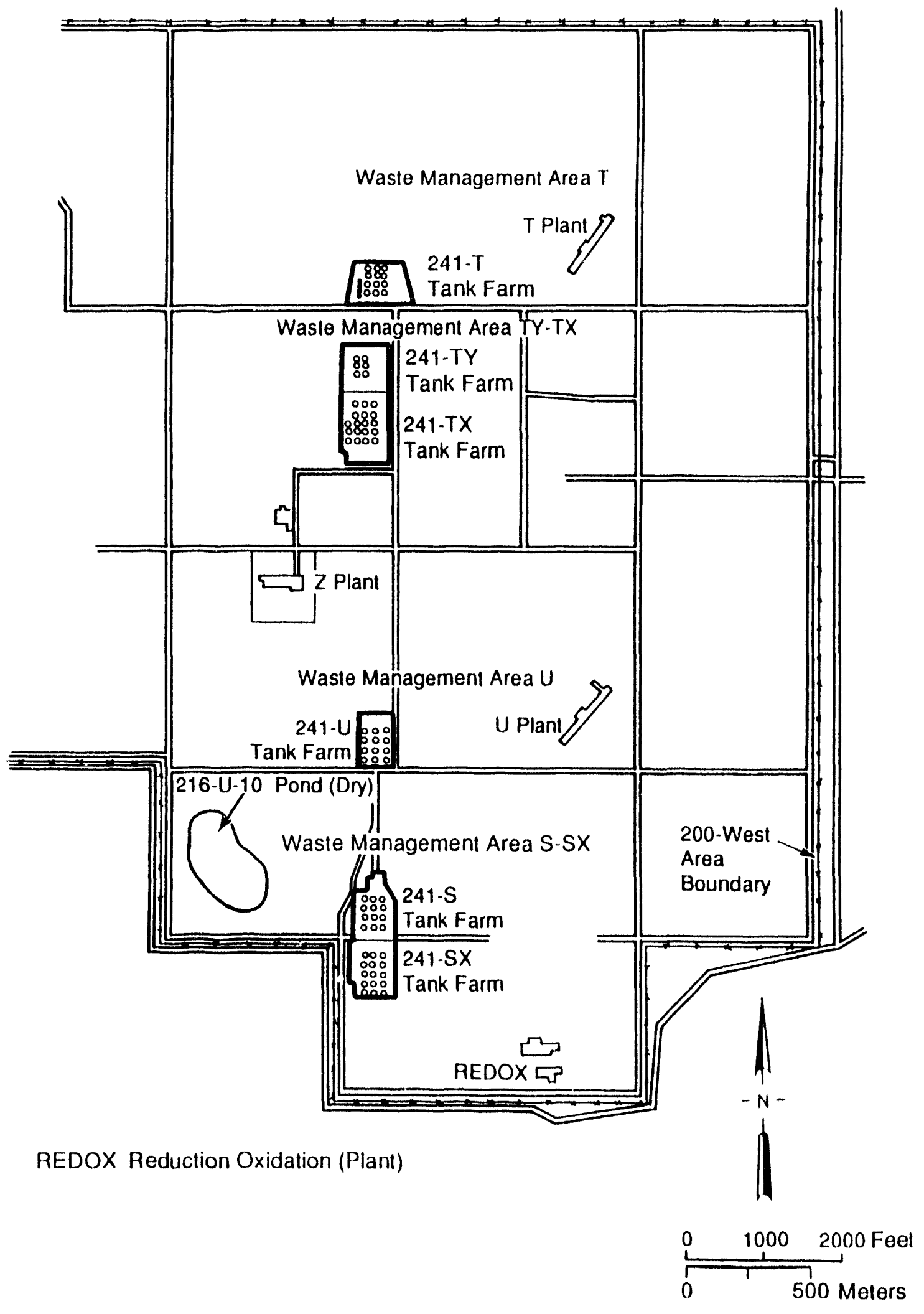

PS.90 69 


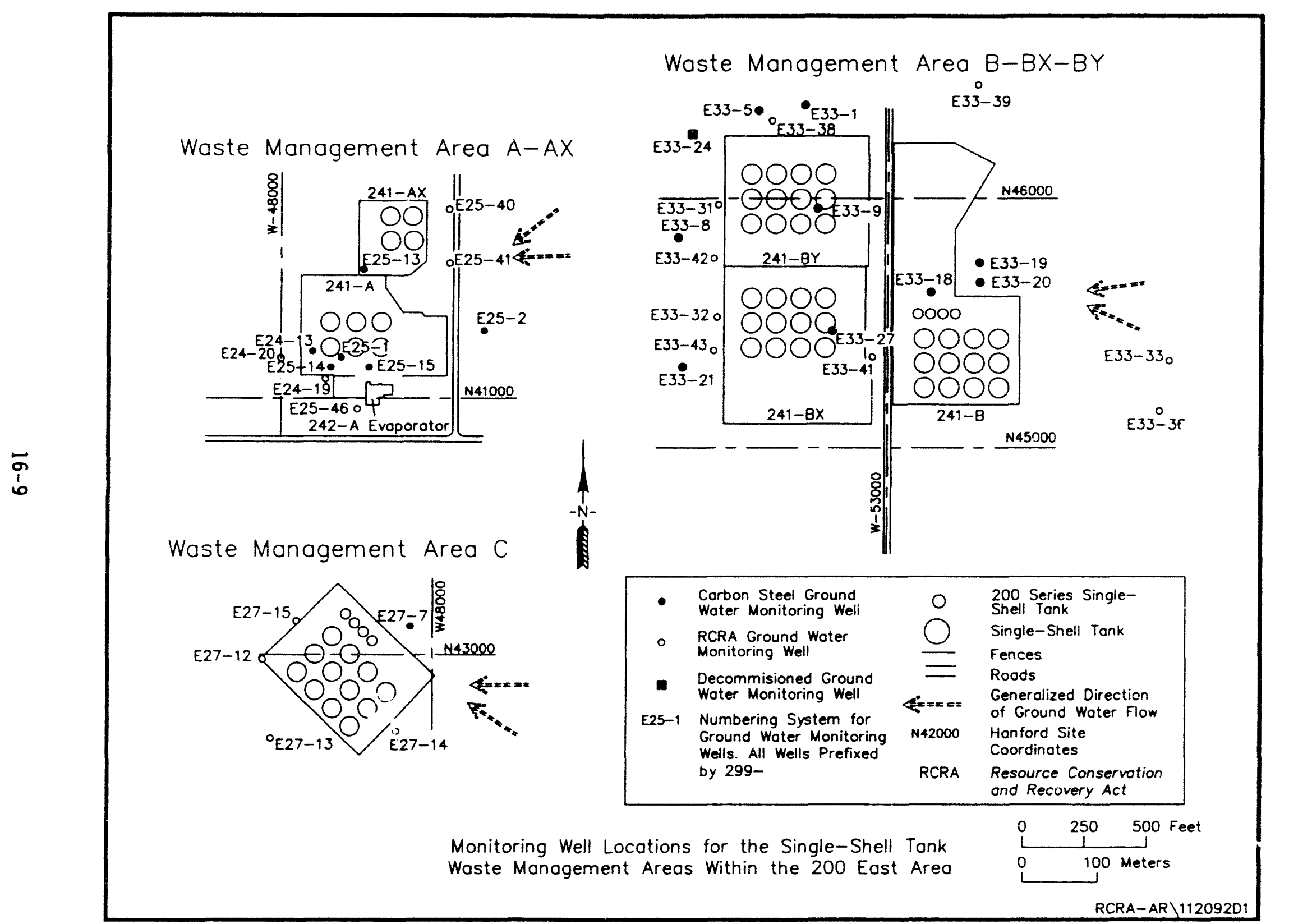

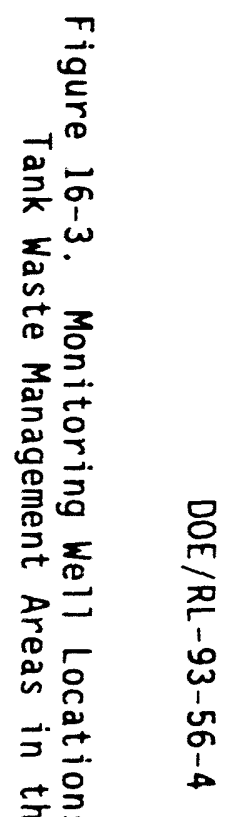

ํํㅇ

可

$\rightarrow$

交合

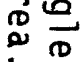


Woste Management Areo S-SX

Waste Management Areo $T$

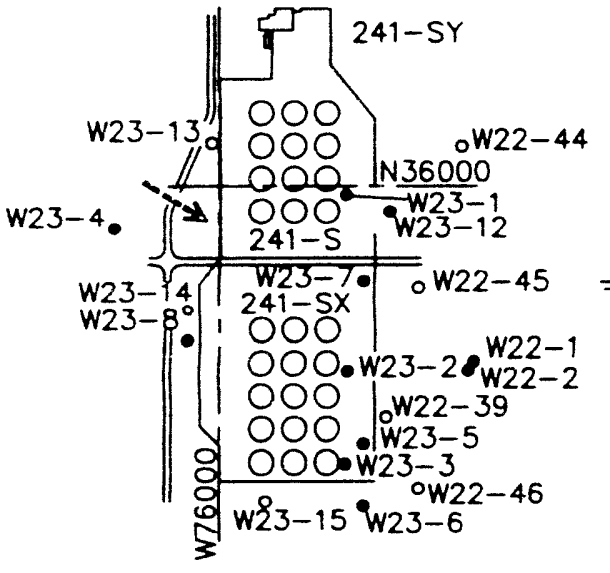

Waste Management Area TX-TY

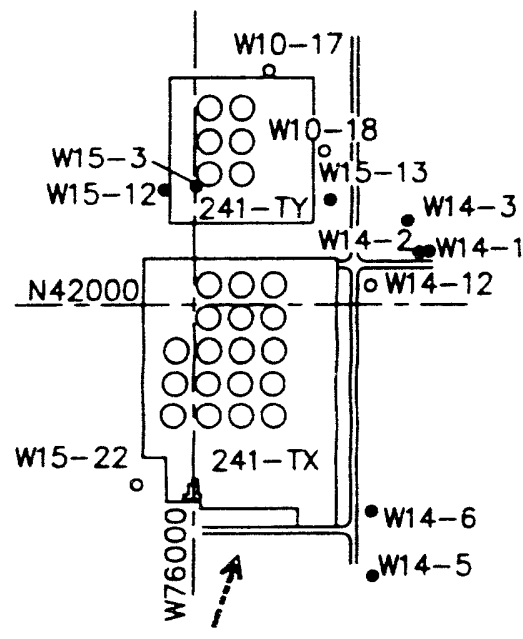

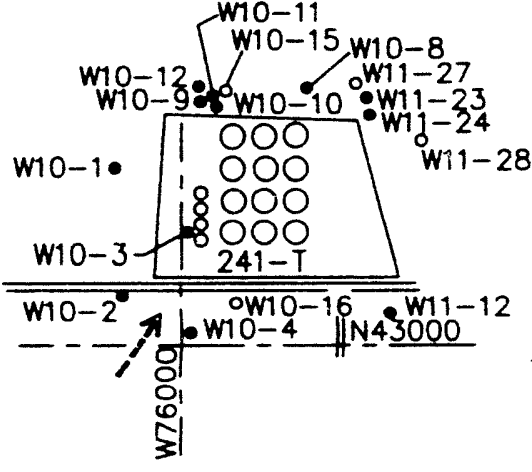
2 N38000

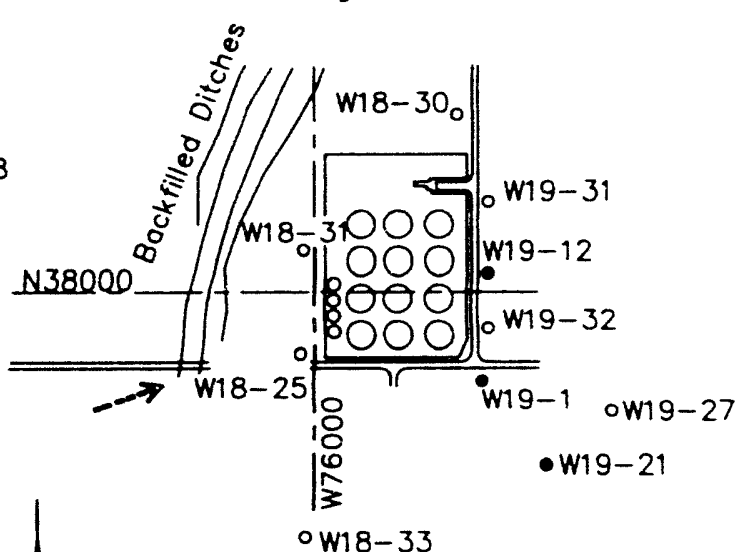

w19-6•

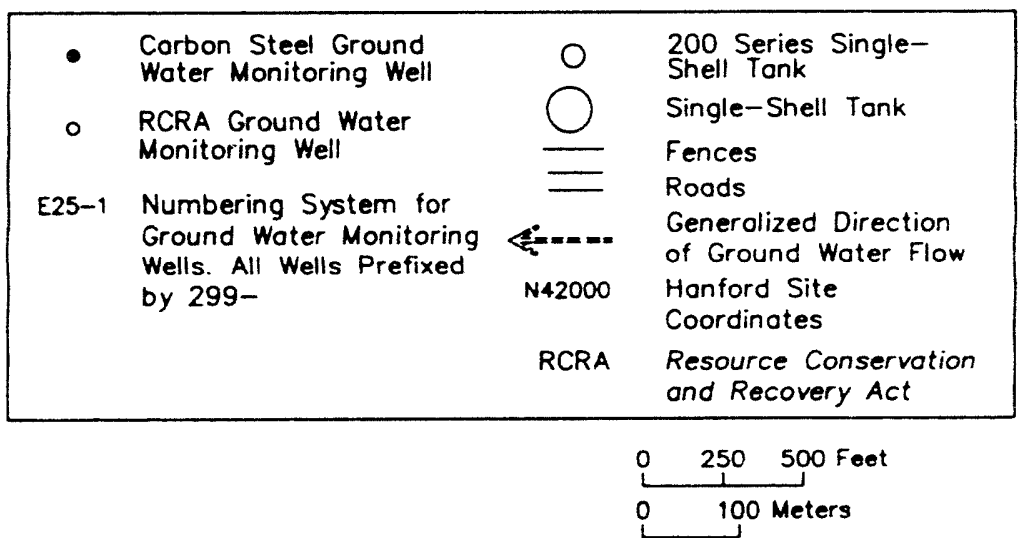

Monitoring Well Locations for the Single-Shell Tank Waste Management Areas Within the 200 West Area.

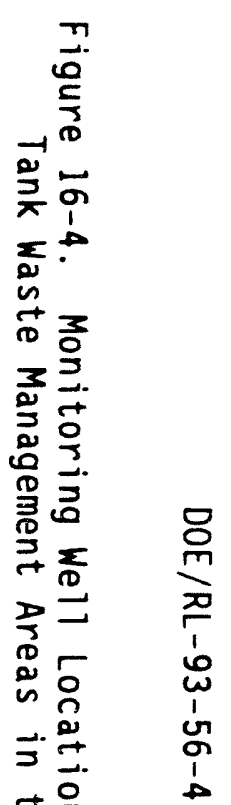

俩的

용학

要古

皮

굴

象

告 
Table 16-1. Groundwater Monitoring Wells for the Single-Shell Tanks. (sheet 1 of 2)

\begin{tabular}{|c|c|c|c|}
\hline $\begin{array}{c}\text { Well no. } \\
(299-)\end{array}$ & Aquifer & $\begin{array}{c}\text { Sample } \\
\text { frequency }\end{array}$ & $\begin{array}{l}\text { Sample date, } \\
4 \text { th Qtr } 1993\end{array}$ \\
\hline \multicolumn{4}{|c|}{ Single-Shell Tanks Waste Management Area A-AX } \\
\hline$E 24-19^{89}$ & Ringold: Water Table & Semiannually & NS \\
\hline $\mathrm{E} 24-20^{90}$ & Ringold: Water Table & Semiannually & NS \\
\hline$E 25-40^{89}$ & Ringold: Water Table & Semiannually & NS \\
\hline$E 25-41^{89}$ & Ringold: Water Table & Semiannually & NS \\
\hline$E 25-46^{92}$ & Ringold: Water Table & Semiannually & $9 / 29 / 93$ \\
\hline \multicolumn{4}{|c|}{ Single-Shell Tanks Waste Management Area B-BX-BY } \\
\hline$E 33-31^{89}$ & Hanford: Water Table & Semiannually & NS \\
\hline$E 33-32^{89}$ & Hanford: Water Table & Semiannually & NS \\
\hline$E 33-33^{89}$ & Hanford: Water Table & Semiannually & $11 / 05 / 93$ \\
\hline$E 33-36^{90}$ & Hanford: Water Table & Semiannually & $11 / 04 / 93$ \\
\hline$E 33-41^{90 b}$ & Hanford: Water Table & Semiannually & NS \\
\hline$E 33-38^{90 c}$ & Hanford: Water Table & Semiannually & NS \\
\hline$E 33-39^{90 c}$ & Hanford: Water Table & Semiannually & NS \\
\hline$E 33-42^{91}$ & Hanford: Water Table & Semiannually & NS \\
\hline$E 33-43^{91}$ & Hanford: Water Table & Semiannually & NS \\
\hline \multicolumn{4}{|c|}{ Single-Shell Tank Waste Management Area C } \\
\hline$E 27-12^{89}$ & Ringold: Water Table & Semiannually & NS \\
\hline$E 27-13^{89}$ & Ringold: Water Table & Semiannually & NS \\
\hline$E 27-14^{89}$ & Ringold: Water Table & Semiannually & NS \\
\hline $\mathrm{E} 27-15^{89}$ & Ringold: Water Table & Semiannually & NS \\
\hline$E 27-7^{82}$ & Ringold: Water Table & Semiannually & NS \\
\hline \multicolumn{4}{|c|}{ Single-Shell Tanks Waste Management Area S-SX } \\
\hline$W 22-39^{90}$ & Ringold: Water Table & Semiannually & NS \\
\hline$W 22-44^{91}$ & Ringold: Water Table & Semiannually & NS \\
\hline$W 22-45^{92}$ & Ringold: Water Table & Semiannually & NS \\
\hline$W 22-46^{91}$ & Ringold: Water Table & Semiannually & NS \\
\hline$W 23-13^{91}$ & Ringold: Water Table & Semiannually & NS \\
\hline
\end{tabular}


Table 16-1. Groundwater Monitoring Wells for the Single-Shell Tanks. (sheet 2 of 2)

\begin{tabular}{|c|c|c|c|}
\hline $\begin{array}{c}\text { Well no. } \\
(299-)\end{array}$ & Aquifer & $\begin{array}{c}\text { Sample } \\
\text { frequency }\end{array}$ & $\begin{array}{l}\text { Sample date, } \\
\text { 4th Qtr } 1993\end{array}$ \\
\hline $123-14^{90}$ & Ringold: Water Table & Semiannually & NS \\
\hline$W 23-15^{91}$ & Ringold: Water Table & Semiannually & NS \\
\hline \multicolumn{4}{|c|}{ Single-She 11 Tanks Waste Management Area $\mathrm{T}$} \\
\hline$W 10-15^{89}$ & Ringold: Water Table & Quarterly & $12 / 10 / 93$ \\
\hline$W 10-16^{89}$ & Ringold: Water Table & Quarterly & $12 / 16 / 93$ \\
\hline$W 11-27^{91}$ & Ringold: Water Table & Quarterly & $12 / 13 / 93$ \\
\hline$W 11-28^{91}$ & Ringold: Water Table & Quarterly & NS \\
\hline \multicolumn{4}{|c|}{ Single-Shell Tanks Waste Management Area TX-TY } \\
\hline $\mathrm{W} 10-17^{90}$ & Ringold: Water Table & Quarterly & $\begin{array}{l}12 / 16 / 93 \\
12 / 28 / 93 \\
\end{array}$ \\
\hline$W 10-18^{90}$ & Ringold: Water Table & Quarterly & $\begin{array}{l}12 / 16 / 93 \\
12 / 28 / 93\end{array}$ \\
\hline$W 14-12^{91}$ & Ringold: Water Table & Quarterly & $\begin{array}{l}12 / 16 / 93 \\
12 / 28 / 93\end{array}$ \\
\hline$W 15-22^{90}$ & Ringold: Water Table & Quarterly & $12 / 10 / 93$ \\
\hline \multicolumn{4}{|c|}{ Single-Shell Tanks Waste Management Area U } \\
\hline$W 18-25^{90}$ & Ringold: Water Table & Semiannually & $12 / 09 / 93$ \\
\hline$W 18-30^{91}$ & Ringold: Water Table & Semiannual1y & $12 / 09 / 93$ \\
\hline$W 18-31^{91}$ & Ringold: Water Table & Semiannual1y & $12 / 09 / 93$ \\
\hline$W 19-31^{90}$ & Ringold: Water Table & Semiannually & $12 / 09 / 93$ \\
\hline$W 19-32^{90}$ & Ringold: Water Table & Semiannually & $12 / 09 / 93$ \\
\hline$W 19-12^{83}$ & Ringold: Water Table & Semiannually & NS \\
\hline
\end{tabular}

NS = not sampled.

Notes: Shading denotes upgradient wells. Superscript following well number denotes the year of installation.

Wells are screened either in sand and gravel of the Hanford formation or in matrix-supported silty sandy gravel of the Ringold Formation.

Well in middle of WMA B-BX-BY and not clearly upgradient or downgradient. Therefore, data from sampling/analyses in this well are not used in statistical evaluations.

'Part of 200-BP-1 network. Not sampled regularly as RCRA wells. 
Table 16-2. RCRA Water Level Measurement Report for the Single-Shell Tanks, Fourth Quarter 1993. (sheet 1 of 7)

\begin{tabular}{|c|c|c|c|}
\hline Well & Date & $\begin{array}{l}\text { Depth to } \\
\text { water }(f t)\end{array}$ & $\begin{array}{c}\text { Water level } \\
\text { elevation } \\
\text { above msl (ft) }\end{array}$ \\
\hline \multicolumn{4}{|c|}{ 241-A/AX Tank Farm Wells } \\
\hline 299-E24-19 & $\begin{array}{l}10 / 27 / 93 \\
11 / 24 / 93 \\
12 / 15 / 93\end{array}$ & $\begin{array}{l}291.91 \\
292.20 \\
292.12\end{array}$ & $\begin{array}{l}401.94 \\
401.65 \\
401.73\end{array}$ \\
\hline 299-E24-20 & $\begin{array}{l}10 / 27 / 93 \\
11 / 24 / 93 \\
12 / 15 / 93\end{array}$ & $\begin{array}{l}287.22 \\
287.68 \\
287.46\end{array}$ & $\begin{array}{l}402.06 \\
401.60 \\
401.82\end{array}$ \\
\hline 299-E25-2 & $\begin{array}{l}10 / 27 / 93 \\
11 / 24 / 93 \\
12 / 15 / 93\end{array}$ & $\begin{array}{l}273.52 \\
273.81 \\
273.71\end{array}$ & $\begin{array}{l}402.13 \\
401.84 \\
401.94\end{array}$ \\
\hline 299-E25-40 & $\begin{array}{l}10 / 27 / 93 \\
11 / 24 / 93 \\
12 / 15 / 93\end{array}$ & $\begin{array}{l}263.82 \\
264.09 \\
264.03\end{array}$ & $\begin{array}{l}402.09 \\
401.82 \\
401.88\end{array}$ \\
\hline $299-E 25-41$ & $\begin{array}{l}10 / 27 / 93 \\
11 / 24 / 93 \\
12 / 15 / 93\end{array}$ & $\begin{array}{l}269.29 \\
269.59 \\
269.53\end{array}$ & $\begin{array}{l}402.17 \\
401.87 \\
401.93\end{array}$ \\
\hline 299-E25-46 & $\begin{array}{l}10 / 27 / 93 \\
11 / 24 / 93 \\
12 / 15 / 93\end{array}$ & $\begin{array}{l}292.78 \\
292.90 \\
292.92\end{array}$ & $\begin{array}{l}402.03 \\
401.91 \\
401.89\end{array}$ \\
\hline \multicolumn{4}{|c|}{ 241-B/BX/BY Tank Farm Wells } \\
\hline 299-E33-1 & $\begin{array}{l}10 / 27 / 93 \\
11 / 24 / 93 \\
12 / 15 / 93\end{array}$ & $\begin{array}{l}230.16 \\
230.40 \\
230.42\end{array}$ & $\begin{array}{l}401.95 \\
401.71 \\
401.69\end{array}$ \\
\hline $299-E 33-18$ & $\begin{array}{l}10 / 27 / 93 \\
11 / 24 / 93 \\
12 / 15 / 93\end{array}$ & $\begin{array}{l}249.84 \\
250.10 \\
250.13\end{array}$ & $\begin{array}{l}402.02 \\
401.76 \\
401.73\end{array}$ \\
\hline 299-E33-21 & $\begin{array}{l}10 / 27 / 93 \\
11 / 24 / 93 \\
12 / 15 / 93\end{array}$ & $\begin{array}{l}266.34 \\
266.95 \\
266.64\end{array}$ & $\begin{array}{l}401.99 \\
401.38 \\
401.69\end{array}$ \\
\hline
\end{tabular}


DOE/RL-93-56-4

Table 16-2. RCRA Water Level Measurement Report for the Single-Shell Tanks, Fourth Quarter 1993. (sheet 2 of 7)

\begin{tabular}{|c|c|c|c|}
\hline Well & Date & $\begin{array}{l}\text { Depth to } \\
\text { water }(f t)\end{array}$ & $\begin{array}{l}\text { Water level } \\
\text { elevation } \\
\text { above msl (ft) }\end{array}$ \\
\hline \multicolumn{4}{|c|}{ 241-B/BX/BY Tank Farm Wells } \\
\hline $299-E 33-31$ & $\begin{array}{l}10 / 27 / 93 \\
11 / 24 / 93 \\
12 / 15 / 93\end{array}$ & $\begin{array}{l}245.63 \\
245.82 \\
245.82\end{array}$ & $\begin{array}{l}401.85 \\
401.66 \\
401.66\end{array}$ \\
\hline 299-E33-32 & $\begin{array}{l}10 / 27 / 93 \\
11 / 24 / 93 \\
12 / 15 / 93\end{array}$ & $\begin{array}{l}258.53 \\
258.98 \\
258.56\end{array}$ & $\begin{array}{l}401.50 \\
401.05 \\
401.47\end{array}$ \\
\hline 299-E33-33 & $\begin{array}{l}10 / 27 / 93 \\
11 / 05 / 93 \\
11 / 24 / 93 \\
12 / 15 / 93\end{array}$ & $\begin{array}{l}238.48 \\
237.39 \\
238.73 \\
238.45\end{array}$ & $\begin{array}{l}401.89 \\
402.98^{\star}+ \\
401.64 \\
401.92\end{array}$ \\
\hline 299-E33-36 & $\begin{array}{l}11 / 04 / 93 \\
12 / 08 / 93\end{array}$ & $\begin{array}{l}246.35 \\
246.13\end{array}$ & $\begin{array}{l}400.52^{\star} \\
400.74\end{array}$ \\
\hline 299-E33-38 & $12 / 14 / 93$ & 230.42 & 401.53 \\
\hline 299-E33-39 & $12 / 14 / 93$ & 221.93 & 401.39 \\
\hline $299-E 33-41$ & $\begin{array}{l}10 / 27 / 93 \\
11 / 24 / 93 \\
12 / 15 / 93\end{array}$ & $\begin{array}{l}253.04 \\
253.26 \\
253.28\end{array}$ & $\begin{array}{l}401.91 \\
401.69 \\
401.67\end{array}$ \\
\hline 299-E33-42 & $\begin{array}{l}10 / 27 / 93 \\
11 / 24 / 93 \\
12 / 15 / 93\end{array}$ & $\begin{array}{l}252.49 \\
252.57 \\
252.58\end{array}$ & $\begin{array}{l}401.81 \\
401.73 \\
401.72\end{array}$ \\
\hline $299-E 33-43$ & $\begin{array}{l}10 / 27 / 93 \\
11 / 24 / 93 \\
12 / 15 / 93\end{array}$ & $\begin{array}{l}260.97 \\
261.43 \\
261.08\end{array}$ & $\begin{array}{l}401.71 \\
401.25 \\
401.60\end{array}$ \\
\hline 299-E33-5 & $\begin{array}{l}10 / 27 / 93 \\
11 / 24 / 93 \\
12 / 15 / 93\end{array}$ & $\begin{array}{l}232.72 \\
232.94 \\
232.96\end{array}$ & $\begin{array}{l}402.00 \\
401.78 \\
401.76\end{array}$ \\
\hline 299-E33-8 & $\begin{array}{l}10 / 27 / 93 \\
11 / 24 / 93 \\
12 / 15 / 93\end{array}$ & $\begin{array}{l}248.94 \\
249.19 \\
249.20\end{array}$ & $\begin{array}{l}401.99 \\
401.74 \\
401.73\end{array}$ \\
\hline
\end{tabular}


Table 16-2. RCRA Water Level Measurement Report for the Single-Shell Tanks, Fourth Quarter 1993. (sheet 3 of 7)

\begin{tabular}{|c|c|c|c|}
\hline Well & Date & $\begin{array}{l}\text { Depth to } \\
\text { water (ft) }\end{array}$ & $\begin{array}{l}\text { Water level } \\
\text { elevation } \\
\text { above msl (ft) }\end{array}$ \\
\hline \multicolumn{4}{|c|}{ 241-C Tank Farm Wells } \\
\hline $299-E 27-12$ & $\begin{array}{l}10 / 27 / 93 \\
11 / 24 / 93 \\
12 / 15 / 93\end{array}$ & $\begin{array}{l}259.18 \\
259.44 \\
259.38\end{array}$ & $\begin{array}{l}401.97 \\
401.71 \\
401.77\end{array}$ \\
\hline $299-E 27-13$ & $\begin{array}{l}10 / 27 / 93 \\
11 / 24 / 93 \\
12 / 15 / 93\end{array}$ & $\begin{array}{l}267.04 \\
267.29 \\
267.24\end{array}$ & $\begin{array}{l}402.15 \\
401.90 \\
401.95\end{array}$ \\
\hline 299-E27-14 & $\begin{array}{l}10 / 27 / 93 \\
11 / 24 / 93 \\
12 / 15 / 93\end{array}$ & $\begin{array}{l}256.55 \\
256.77 \\
256.74\end{array}$ & $\begin{array}{l}401.99 \\
401.77 \\
401.80\end{array}$ \\
\hline $299-E 27-15$ & $\begin{array}{l}10 / 27 / 93 \\
11 / 24 / 93 \\
12 / 15 / 93\end{array}$ & $\begin{array}{l}251.25 \\
251.46 \\
251.97\end{array}$ & $\begin{array}{l}401.62 \\
401.41 \\
400.90\end{array}$ \\
\hline 299-E27-7 & $\begin{array}{l}10 / 27 / 93 \\
11 / 24 / 93 \\
12 / 15 / 93\end{array}$ & $\begin{array}{l}232.76 \\
232.95 \\
232.94\end{array}$ & $\begin{array}{l}402.11 \\
401.92 \\
401.93\end{array}$ \\
\hline \multicolumn{4}{|c|}{ 241-S/SX Tank Farm Wells } \\
\hline $299-W 22-39$ & $\begin{array}{l}10 / 26 / 93 \\
11 / 23 / 93 \\
12 / 15 / 93\end{array}$ & $\begin{array}{l}210.17 \\
210.43 \\
210.35\end{array}$ & $\begin{array}{l}458.09 \\
457.83 \\
457.91\end{array}$ \\
\hline $299-W 22-44$ & $10 / 26 / 93$ & 217.79 & 460.34 \\
\hline 299-W22-45 & $\begin{array}{l}10 / 26 / 93 \\
11 / 23 / 93 \\
12 / 15 / 93\end{array}$ & $\begin{array}{l}207.35 \\
207.63 \\
207.58\end{array}$ & $\begin{array}{l}458.86 \\
458.58 \\
458.63\end{array}$ \\
\hline 299-W22-46 & $\begin{array}{l}10 / 26 / 93 \\
11 / 23 / 93 \\
12 / 15 / 93\end{array}$ & $\begin{array}{l}213.31 \\
213.97 \\
213.51\end{array}$ & $\begin{array}{l}457.87 \\
457.21 \\
457.67\end{array}$ \\
\hline $299-W 23-13$ & $\begin{array}{l}10 / 26 / 93 \\
11 / 23 / 93 \\
12 / 15 / 93\end{array}$ & $\begin{array}{l}205.46 \\
205.77 \\
205.72\end{array}$ & $\begin{array}{l}460.87 \\
460.56 \\
460.61\end{array}$ \\
\hline
\end{tabular}


Table 16-2. RCRA Water Level Measurement Report for the Single-Shell Tanks, Fourth Quarter 1993. (sheet 4 of 7)

\begin{tabular}{|c|c|c|c|}
\hline Well & Date & $\begin{array}{l}\text { Depth to } \\
\text { water }(f t)\end{array}$ & $\begin{array}{l}\text { Water level } \\
\text { elevation } \\
\text { above msl (ft) }\end{array}$ \\
\hline \multicolumn{4}{|c|}{ 241-S/SX Tank Farm Wells } \\
\hline 299-W23-14 & $\begin{array}{l}10 / 26 / 93 \\
11 / 23 / 93 \\
12 / 15 / 93\end{array}$ & $\begin{array}{l}203.92 \\
204.26 \\
204.16\end{array}$ & $\begin{array}{l}460.08 \\
459.74 \\
459.84\end{array}$ \\
\hline $299-W 23-15$ & $\begin{array}{l}10 / 26 / 93 \\
11 / 23 / 93 \\
12 / 15 / 93\end{array}$ & $\begin{array}{l}196.60 \\
196.85 \\
196.82\end{array}$ & $\begin{array}{l}458.84 \\
458.59 \\
458.62\end{array}$ \\
\hline 299-W23-6 & $\begin{array}{l}10 / 26 / 93 \\
11 / 23 / 93 \\
12 / 15 / 93\end{array}$ & $\begin{array}{l}208.80 \\
209.04 \\
208.97\end{array}$ & $\begin{array}{l}458.22 \\
457.98 \\
458.05\end{array}$ \\
\hline 299-W23-8 & $\begin{array}{l}10 / 26 / 93 \\
11 / 23 / 93 \\
12 / 15 / 93\end{array}$ & $\begin{array}{l}203.72 \\
204.02 \\
203.93\end{array}$ & $\begin{array}{l}460.23 \\
459.93 \\
460.02\end{array}$ \\
\hline \multicolumn{4}{|c|}{ 241-T Tank Farm Wells } \\
\hline 299-W10-10 & $\begin{array}{l}10 / 26 / 93 \\
11 / 23 / 93 \\
12 / 15 / 93\end{array}$ & $\begin{array}{l}214.24 \\
214.39 \\
214.19\end{array}$ & $\begin{array}{l}461.11 \\
460.96 \\
461.16\end{array}$ \\
\hline 299-W10-11 & $\begin{array}{l}10 / 26 / 93 \\
11 / 23 / 93 \\
12 / 15 / 93\end{array}$ & $\begin{array}{l}214.10 \\
214.26 \\
214.08\end{array}$ & $\begin{array}{l}461.42 \\
461.26 \\
461.44\end{array}$ \\
\hline 299-W10-12 & $\begin{array}{l}10 / 26 / 93 \\
11 / 23 / 93 \\
12 / 15 / 93\end{array}$ & $\begin{array}{l}213.84 \\
213.95 \\
213.79\end{array}$ & $\begin{array}{l}461.17 \\
461.06 \\
461.22\end{array}$ \\
\hline 299-W10-15 & $\begin{array}{l}10 / 26 / 93 \\
11 / 23 / 93 \\
12 / 15 / 93\end{array}$ & $\begin{array}{l}214.82 \\
214.94 \\
214.77\end{array}$ & $\begin{array}{l}461.08 \\
460.96 \\
461.13\end{array}$ \\
\hline $299-W 10-16$ & $\begin{array}{l}10 / 26 / 93 \\
11 / 23 / 93 \\
12 / 15 / 93\end{array}$ & $\begin{array}{l}211.54 \\
211.60 \\
211.48\end{array}$ & $\begin{array}{l}461.48 \\
461.42 \\
4 J 1.54\end{array}$ \\
\hline
\end{tabular}


Table 16-2. RCRA Water Level Measurement Report for the Single-Shell Tanks, Fourth Quarter 1993. (sheet 5 of 7)

\begin{tabular}{|c|c|c|c|}
\hline Well & Date & $\begin{array}{l}\text { Depth to } \\
\text { water (ft) }\end{array}$ & $\begin{array}{l}\text { Water level } \\
\text { elevation } \\
\text { above msl (ft) }\end{array}$ \\
\hline \multicolumn{4}{|c|}{ 241-T Tank Farm Wells } \\
\hline $299-W 10-8$ & $\begin{array}{l}10 / 26 / 93 \\
11 / 23 / 93 \\
12 / 15 / 93\end{array}$ & $\begin{array}{l}219.44 \\
219.57 \\
219.48\end{array}$ & $\begin{array}{l}460.87 \\
460.74 \\
460.83\end{array}$ \\
\hline $299-W 10-9$ & $\begin{array}{l}10 / 26 / 93 \\
11 / 23 / 93 \\
12 / 15 / 93\end{array}$ & $\begin{array}{l}213.64 \\
213.77 \\
213.58\end{array}$ & $\begin{array}{l}461.29 \\
461.16 \\
461.35\end{array}$ \\
\hline $299-W 11-23$ & $\begin{array}{l}10 / 26 / 93 \\
11 / 23 / 93 \\
12 / 15 / 93\end{array}$ & $\begin{array}{l}227.98 \\
227.79 \\
227.58\end{array}$ & $\begin{array}{l}460.16 \\
460.35 \\
460.56\end{array}$ \\
\hline $299-W 11-24$ & $\begin{array}{l}10 / 26 / 93 \\
11 / 23 / 93 \\
12 / 15 / 93\end{array}$ & $\begin{array}{l}226.49 \\
226.64 \\
226.39\end{array}$ & $\begin{array}{l}460.94 \\
460.79 \\
461.04\end{array}$ \\
\hline $299-W 11-27$ & $\begin{array}{l}10 / 26 / 93 \\
11 / 23 / 93 \\
12 / 15 / 93\end{array}$ & $\begin{array}{l}224.53 \\
224.66 \\
224.49\end{array}$ & $\begin{array}{l}460.74 \\
460.61 \\
460.78\end{array}$ \\
\hline \multicolumn{4}{|c|}{ 241-TX/TY Tank Farm Wells } \\
\hline 299-W10-17 & $\begin{array}{l}10 / 26 / 93 \\
11 / 23 / 93 \\
12 / 15 / 93\end{array}$ & $\begin{array}{l}209.23 \\
209.41 \\
209.22\end{array}$ & $\begin{array}{l}461.61 \\
461.43 \\
461.62\end{array}$ \\
\hline 299-W10-18 & $\begin{array}{l}10 / 26 / 93 \\
11 / 23 / 93 \\
12 / 15 / 93\end{array}$ & $\begin{array}{l}208.52 \\
208.60 \\
208.41\end{array}$ & $\begin{array}{l}462.41 \\
462.33 \\
462.52\end{array}$ \\
\hline $299-W 14-12$ & $\begin{array}{l}10 / 26 / 93 \\
11 / 23 / 93 \\
12 / 15 / 93\end{array}$ & $\begin{array}{l}207.28 \\
207.38 \\
207.16\end{array}$ & $\begin{array}{l}463.24 \\
463.14 \\
463.36\end{array}$ \\
\hline $299-W 15-12$ & $\begin{array}{l}10 / 26 / 93 \\
11 / 23 / 93 \\
12 / 15 / 93\end{array}$ & $\begin{array}{l}207.49 \\
207.64 \\
207.44\end{array}$ & $\begin{array}{l}462.58 \\
462.43 \\
462.63\end{array}$ \\
\hline
\end{tabular}


Table 16-2. RCRA Water Level Measurement Report for the Single-Shell Tanks, Fourth Quarter 1993. (sheet 6 of 7)

\begin{tabular}{|c|c|c|c|}
\hline Well & Date & $\begin{array}{l}\text { Depth to } \\
\text { water }(f t)\end{array}$ & $\begin{array}{l}\text { Water level } \\
\text { elevation } \\
\text { above msl ( } \mathrm{ft} \text { ) }\end{array}$ \\
\hline \multicolumn{4}{|c|}{ 241-TX/TY Tank Farm Wells } \\
\hline $299-W 15-13$ & $\begin{array}{l}10 / 26 / 93 \\
11 / 23 / 93 \\
12 / 15 / 93\end{array}$ & $\begin{array}{l}207.52 \\
207.64 \\
207.45\end{array}$ & $\begin{array}{l}462.60 \\
462.48 \\
462.67\end{array}$ \\
\hline $299-W 15-22$ & $\begin{array}{l}10 / 26 / 93 \\
11 / 23 / 93 \\
12 / 15 / 93\end{array}$ & $\begin{array}{l}206.28 \\
206.45 \\
206.26\end{array}$ & $\begin{array}{l}464.49 \\
464.32 \\
464.51\end{array}$ \\
\hline \multicolumn{4}{|c|}{ 241-U Tank Farm Wells } \\
\hline $299-W 18-25$ & $\begin{array}{l}10 / 26 / 93 \\
11 / 23 / 93 \\
12 / 15 / 93\end{array}$ & $\begin{array}{l}201.55 \\
201.93 \\
201.87\end{array}$ & $\begin{array}{l}464.49 \\
464.11 \\
464.17\end{array}$ \\
\hline $299-W 18-30$ & $\begin{array}{l}10 / 26 / 93 \\
11 / 23 / 93 \\
12 / 15 / 93\end{array}$ & $\begin{array}{l}208.33 \\
208.59 \\
208.43\end{array}$ & $\begin{array}{l}464.51 \\
464.25 \\
464.41\end{array}$ \\
\hline 299-W18-31 & $\begin{array}{l}10 / 26 / 93 \\
11 / 23 / 93 \\
12 / 15 / 93\end{array}$ & $\begin{array}{l}199.66 \\
199.92 \\
199.88\end{array}$ & $\begin{array}{l}464.50 \\
464.24 \\
464.28\end{array}$ \\
\hline $299-W 18-33$ & $\begin{array}{l}10 / 26 / 93 \\
11 / 23 / 93 \\
12 / 15 / 93\end{array}$ & $\begin{array}{l}204.90 \\
205.40 \\
205.23\end{array}$ & $\begin{array}{l}464.01 \\
463.51 \\
463.68\end{array}$ \\
\hline $299-W 19-1$ & $12 / 02 / 93$ & 208.34 & 465.43 \\
\hline $299-W 19-12$ & $\begin{array}{l}10 / 26 / 93 \\
11 / 23 / 93 \\
12 / 15 / 93\end{array}$ & $\begin{array}{l}208.72 \\
208.95 \\
208.81\end{array}$ & $\begin{array}{l}464.53 \\
464.30 \\
464.44\end{array}$ \\
\hline $299-W 19-21$ & $\begin{array}{l}10 / 26 / 93 \\
12 / 15 / 93\end{array}$ & $\begin{array}{l}213.51 \\
213.88\end{array}$ & $\begin{array}{l}465.28 \\
464.91\end{array}$ \\
\hline $299-W 19-27$ & $\begin{array}{l}10 / 26 / 93 \\
11 / 23 / 93 \\
12 / 15 / 93\end{array}$ & $\begin{array}{l}219.19 \\
219.51 \\
219.42\end{array}$ & $\begin{array}{l}464.72 \\
464.40 \\
464.49\end{array}$ \\
\hline
\end{tabular}


Table 16-2. RCRA Water Level Measurement Report for the Single-She11 Tanks, Fourth Quarter 1993. (sheet 7 of 7)

\begin{tabular}{|c|c|c|c|}
\hline Well & Date & $\begin{array}{l}\text { Depth to } \\
\text { water (ft) }\end{array}$ & $\begin{array}{l}\text { Water level } \\
\text { elevation } \\
\text { above msl (ft) }\end{array}$ \\
\hline \multicolumn{4}{|c|}{ 241-U Tank Farm Wells } \\
\hline $299-W 19-31$ & $\begin{array}{l}10 / 26 / 93 \\
11 / 23 / 93 \\
12 / 15 / 93\end{array}$ & $\begin{array}{l}209.65 \\
209.83 \\
209.77\end{array}$ & $\begin{array}{l}464.54 \\
464.36 \\
464.42\end{array}$ \\
\hline 299-W19-32 & $\begin{array}{l}10 / 26 / 93 \\
11 / 23 / 93 \\
12 / 15 / 93\end{array}$ & $\begin{array}{l}210.13 \\
210.44 \\
210.33\end{array}$ & $\begin{array}{l}464.77 \\
464.46 \\
464.57\end{array}$ \\
\hline 299-W19-6 & $\begin{array}{l}10 / 26 / 93 \\
11 / 23 / 93 \\
12 / 15 / 93\end{array}$ & $\begin{array}{l}226.62 \\
226.90 \\
226.81\end{array}$ & $\begin{array}{l}459.98 \\
459.70 \\
459.79\end{array}$ \\
\hline
\end{tabular}

Notes: 1. Water level elevations are calculated by subtracting the measured depth to water from the surveyed elevation for the well.

2. Depth-to-water values are transcribed from field records.

3. Elevations marked with an $1 * 1$ were measured at the time of sampling.

4. Elevations marked with a ' + ' are outside of the expected range and are suspected of error.

5. To convert feet to meters multiply by 0.3048 . 
Table 16-3. Constituent List and Summary of Results for the Single-Shell Tank Waste Management Area A-AX Data for Reporting Period October 1 through December $31,1993$.

DRINKING WATER PARAMETERS

\begin{tabular}{|c|c|c|c|c|c|c|c|c|c|c|}
\hline \multirow[b]{2}{*}{ Short } & \multirow[b]{2}{*}{ (Method) } & \multirow[t]{2}{*}{ Constituent Name } & \multirow[b]{2}{*}{ Full } & \multirow[b]{2}{*}{ Units } & \multirow{2}{*}{$\begin{array}{l}\text { Lab } \\
\text { CROL }\end{array}$} & \multicolumn{2}{|c|}{ DWS } & \multicolumn{3}{|c|}{ Number of Samples } \\
\hline & & & & & & Limit & Agency & Total & $>\mathrm{CRQL}$ & $>$ DWS \\
\hline$\ldots \ldots$ & $\ldots . . .$. & & $\ldots \ldots \ldots$ & $\ldots$. & $\ldots \ldots$ & $\ldots \ldots$ & $\ldots .$. & $\ldots$. & $\ldots$. & $\ldots$ \\
\hline ARSENIC & & & Arsenic & ppb & 1.38 & 50 & EPA & 1 & 1 & 0 \\
\hline FARSENIC & & Arsenic, & filtered & ppb & 1.38 & 50 & EPA & 1 & 1 & 0 \\
\hline FLUOR10 & & & Fluoride & ppb & 13.9 & 1400 & EPAI & 1 & 1 & 0 \\
\hline NITRATE & & & Nitrate & ppb & 44.4 & 45000 & EPA & 1 & 1 & 0 \\
\hline SELENUM & & & Selenium & ppb & 1.21 & 10 & EPAI & 1 & 0 & 0 \\
\hline FSELENI & & Selenium, & filtered & ppb & 1.21 & 10 & EPAI & 1 & 0 & 0 \\
\hline
\end{tabular}

GROUNDWATER QUALITY PARAMETERS

\begin{tabular}{|c|c|c|c|c|c|c|c|c|c|}
\hline \multirow[b]{2}{*}{ Short } & \multirow[b]{2}{*}{ (Method) } & \multirow{2}{*}{ Constituent Name } & \multirow[b]{2}{*}{ Units } & \multirow{2}{*}{$\begin{array}{l}\text { Lab } \\
\text { CROL }\end{array}$} & \multicolumn{2}{|c|}{ DWS } & \multicolumn{3}{|c|}{ Number of Samples } \\
\hline & & & & & Limit & Agency & Total & $>$ CROL & $>$ DWS \\
\hline$\cdots \ldots$ & & $\ldots \ldots$ & $\ldots \ldots$ & $\cdots \cdots$ & $\ldots \ldots$ & $\ldots \ldots$ & $\cdots \cdots$ & $\cdots \cdot$ & $\cdots$ \\
\hline $\begin{array}{l}\text { CHLORID } \\
\text { SULFATE }\end{array}$ & & $\begin{array}{l}\text { Chloride } \\
\text { sulfate }\end{array}$ & $\begin{array}{l}\text { ppb } \\
\text { ppb }\end{array}$ & $\begin{array}{l}82.5 \\
49.9\end{array}$ & $\begin{array}{l}250000 \\
250000\end{array}$ & $\begin{array}{l}\text { EPAS } \\
\text { EPAS }\end{array}$ & $\begin{array}{l}1 \\
1\end{array}$ & $\begin{array}{l}1 \\
1\end{array}$ & $\begin{array}{l}0 \\
0\end{array}$ \\
\hline
\end{tabular}

SITE SPECIFIC AND OTHER CONSTITUENTS

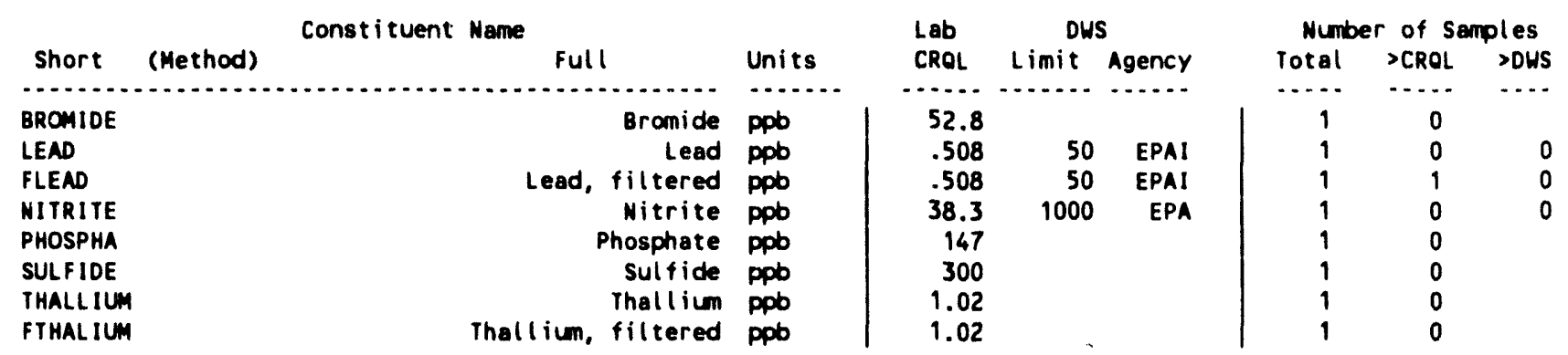

For explanation of this table, see Section 1.4 of report. 
Table 16-4. Constituents with at Least One Detected Value for the Single-Shell Tank Waste Management Area A-AX Data for Reporting Period October 1 through December 31, 1993.

\begin{tabular}{|c|c|c|c|c|c|c|}
\hline $\begin{array}{l}\text { Well } \\
\text { Name }\end{array}$ & $\begin{array}{c}\text { Collection } \\
\text { Date }\end{array}$ & $\begin{array}{l}\text { Sample } \\
\text { Number }\end{array}$ & $\begin{array}{r}\text { ARSENIC } \\
43 / \mathrm{ppb} \\
1.38 / 50\end{array}$ & $\begin{array}{c}\text { FARSENIC } \\
43 / \mathrm{pob} \\
1.38 / 50\end{array}$ & $\begin{array}{c}\text { CHLORID } \\
124 / \mathrm{ppb} \\
82.5 / 250000 \mathrm{~s}\end{array}$ & $\begin{array}{l}\text { FLUORID } \\
124 / \mathrm{ppb} \\
13.9 / 1400 \mathrm{i}\end{array}$ \\
\hline$\ldots \ldots \ldots$ & $\ldots \ldots \ldots$ & $\ldots \ldots \ldots$ & $\ldots \ldots \ldots$ & $\ldots \ldots . . . .$. & ............. & $\ldots \ldots . . . . .$. \\
\hline $\begin{array}{l}299-E 25-46 \\
299-E 25-46\end{array}$ & $\begin{array}{l}9 / 29 / 93 \\
9 / 29 / 93\end{array}$ & $\begin{array}{l}8096 \times 5 \\
8096 \times 9\end{array}$ & 13.00 & 15.00 & 4700.00 & 200.00 \\
\hline $\begin{array}{l}\text { Nell } \\
\text { Name }\end{array}$ & $\begin{array}{c}\text { Collection } \\
\text { Date }\end{array}$ & $\begin{array}{l}\text { Semple } \\
\text { Number }\end{array}$ & $\begin{array}{l}\text { LEAD } \\
\text { 40/ppb } \\
.508 / 50 \mathrm{i}\end{array}$ & $\begin{array}{l}\text { NITRATE } \\
124 / \text { ppb } \\
44.4 / 45000\end{array}$ & $\begin{array}{c}\text { SULFATE } \\
124 / \mathrm{ppb} \\
49.9 / 250000 \mathrm{~s}\end{array}$ & \\
\hline $299-E 25-46$ & $9 / 29 / 93$ & $\begin{array}{c}8096 k 5 \\
0.3 .\end{array}$ & $.51 \mathrm{u}$ & 6200.00 & $23000.00 \mathrm{D}$ & \\
\hline
\end{tabular}

For explanation of this table, see section 1.4 of report. 
Table 16-5. Contamination Indicator Parameters for the Single-Shell Tank Waste Management Area A-AX Data for Reporting Period October 1 through December 31, 1993.

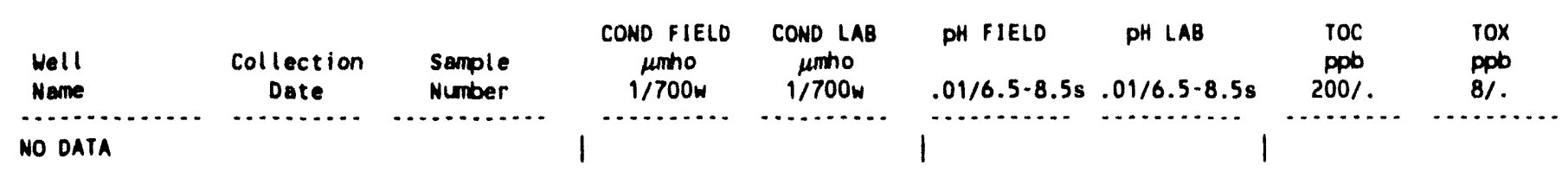

For explanation of this table, see section 1.4 of report. 
Table 16-6. Constituent List and Summary of Results for the Single-Shell Tank Waste Management Area B-BY-BX Data for Reporting Period October 1

through December 31, 1993. (sheet 1 of 2)

CONTAMINATION INDICATOR PARAMETERS

\begin{tabular}{|c|c|c|c|c|c|c|c|c|}
\hline & & Constituent Name & & Lab & & Numt & $r$ of $s$ & ples \\
\hline Short & (Method) & Full & Units & Limit & Agency & Total & $>$ CRQL & $>$ DWS \\
\hline $\begin{array}{l}\text { CONDUCT } \\
\text { CONDUCT } \\
\text { TOC } \\
\text { TOXLDL } \\
\text { PH }\end{array}$ & $\begin{array}{l}94 \\
73\end{array}$ & $\begin{array}{l}\text { Conductivity, field } \\
\text { Conductivity, lab } \\
\text { Total Organic Carbon } \\
\text { Total Organic Halogen } \\
\text { pH, field }\end{array}$ & $\begin{array}{l}\mu m h o \\
\mu m h o \\
\text { ppb } \\
\text { Ppb } \\
\text { pH }\end{array}$ & $\begin{array}{r}1 \\
1 \\
200 \\
8 \\
.016 .5 \cdot 8.5\end{array}$ & EPAS & $\begin{array}{l}8 \\
8 \\
8 \\
8 \\
8\end{array}$ & $\begin{array}{l}8 \\
8 \\
8 \\
1 \\
8\end{array}$ & 0 \\
\hline
\end{tabular}

DRINKING WATER PARAMETERS

\begin{tabular}{|c|c|c|c|c|c|c|c|c|c|c|}
\hline \multirow[b]{2}{*}{ Short } & \multirow[b]{2}{*}{ (Method) } & \multirow{2}{*}{ Constituent Name } & \multirow[b]{2}{*}{ Full } & \multirow[b]{2}{*}{ Units } & \multirow{2}{*}{$\begin{array}{l}\text { Lab } \\
\text { CROL }\end{array}$} & \multicolumn{2}{|c|}{ DWS } & \multicolumn{3}{|c|}{ Number of Samples } \\
\hline & & & & & & Limit & Agency & Total & $>C R O L$ & $>$ DWS \\
\hline & & & Doriim & & (n) & 1000 & FPA & $\cdots$ & 3 & \\
\hline $\begin{array}{l}\text { BARIUM } \\
\text { CADMIUM }\end{array}$ & & & $\begin{array}{l}\text { Barium } \\
\text { Cadnium }\end{array}$ & $\begin{array}{l}\text { ppb } \\
\text { ppb }\end{array}$ & $\begin{array}{r}.001 \\
4.7\end{array}$ & $\begin{array}{r}1000 \\
10\end{array}$ & $\begin{array}{l}\text { EPAI } \\
\text { EPAI }\end{array}$ & $\begin{array}{l}2 \\
2\end{array}$ & $\begin{array}{l}2 \\
0\end{array}$ & 0 \\
\hline CHROMUN & & & Chromium & ppb & 5.42 & 50 & EPAI & 2 & 2 & 2 \\
\hline FLUORID & & & Fluoride & ppb & 13.9 & 1400 & EPAI & 2 & 2 & 0 \\
\hline NITRATE & & & Nitrate & ppb & 44.4 & 45000 & EPA & 2 & 2 & 0 \\
\hline
\end{tabular}

\section{GROUNDWATER QUALITY PARAMETERS}

\begin{tabular}{|c|c|c|c|c|c|c|c|c|c|c|}
\hline \multirow[b]{2}{*}{ Short } & \multirow[b]{2}{*}{ (Method) } & \multirow{2}{*}{ Constituent Name } & \multirow[b]{2}{*}{ Full } & \multirow[b]{2}{*}{ Units } & \multirow{2}{*}{$\begin{array}{l}\text { Lab } \\
\text { CRQL }\end{array}$} & \multicolumn{2}{|c|}{ DUS } & \multicolumn{3}{|c|}{ Number of Samples } \\
\hline & & & & & & Limit & Agency & Total & $>$ CRQL & $>$ OWS \\
\hline & & & & ........ & ..... & 250000 & anse & $\cdots$ & $\overline{3}^{2}$ & \\
\hline $\begin{array}{l}\text { CHLORID } \\
\text { IRON }\end{array}$ & & & $\begin{array}{r}\text { Chloride } \\
\text { Iron }\end{array}$ & $\begin{array}{l}\text { ppb } \\
\text { ppb }\end{array}$ & $\begin{array}{l}82.5 \\
10.3\end{array}$ & $\begin{array}{r}250000 \\
300\end{array}$ & $\begin{array}{l}\text { EPAS } \\
\text { EPAS }\end{array}$ & $\begin{array}{l}2 \\
2\end{array}$ & $\begin{array}{l}2 \\
2\end{array}$ & 2 \\
\hline MANGESE & & & Manganese & ppo & 1.35 & 50 & EPAS & 2 & 2 & 0 \\
\hline LPHENOL & & & Phenol & ppb & .31 & & & 2 & 0 & \\
\hline SODIun & & & Sodium & ppb & 40.9 & & & 2 & 2 & \\
\hline SULFATE & & & Sulfate & ppb & 49.9 & 250000 & EPAS & 2 & 2 & 0 \\
\hline
\end{tabular}

SITE SPECIFIC AND OTHER CONSTITUENTS

\begin{tabular}{|c|c|c|c|c|c|c|c|c|c|}
\hline \multirow[b]{2}{*}{ Short } & \multirow[b]{2}{*}{ (Method) } & \multirow[t]{2}{*}{ Constituent Name } & \multirow[b]{2}{*}{ Units } & \multirow{2}{*}{$\begin{array}{l}\text { Lab } \\
\text { CROL }\end{array}$} & \multicolumn{2}{|c|}{ DWS } & \multicolumn{3}{|c|}{ Number of Semples } \\
\hline & & & & & Limit & Agency & Total & $>$ CRQL & $>$ DUS \\
\hline & & , & $\cdots$ & (n) & ……. & ...... & $\cdots$ & $\cdots$ & $\cdots$ \\
\hline $1,1,1 \cdot T$ & & 1,1,1-irichloroethane & ppob & .072 & 200 & EPA & $\begin{array}{l}2 \\
2\end{array}$ & $\begin{array}{l}2 \\
0\end{array}$ & 0 \\
\hline $\begin{array}{l}1,1,2 \cdot T \\
1,1-D / C\end{array}$ & & $\begin{array}{r}\text { 1,1,2-irichloroethane } \\
\text { 1,1-Dichloroethane }\end{array}$ & $\begin{array}{l}\text { ppb } \\
\text { ppb }\end{array}$ & $\begin{array}{l}.043 \\
.337\end{array}$ & & & $\begin{array}{l}2 \\
2\end{array}$ & $\begin{array}{l}0 \\
0\end{array}$ & \\
\hline $\begin{array}{l}1,1-D I C \\
1,2-0 I C\end{array}$ & & & $\begin{array}{l}p p o \\
p p b\end{array}$ & (139 & 5 & EPA & 2 & 0 & \\
\hline 14 -dben & & $\begin{array}{l}1,2-0 \text { ichlor oethane } \\
1,4-0 \text { ichlorobenzene }\end{array}$ & ppb & .107 & 75 & EPA & 2 & 0 & \\
\hline $246-t r p$ & & $2,4,6$ - Trichlorophenol & ppo & 1.45 & & & 2 & 0 & \\
\hline $24-d c h p$ & & 2,4-Dichlorophenol & ppb & 1.5 & & & 2 & 0 & \\
\hline DIMPHEN & & 2,4-D imethylphenol & ppo & 1.01 & & & 2 & 0 & \\
\hline DINPHEN & & 2,4 -D init rophenol & ppb & .96 & & & 2 & 0 & \\
\hline 26-dchp & & 2,6-0 ichlorophenol & ppb & 1.59 & & & 2 & 0 & \\
\hline CHLPHEN & & 2.-Chlorophenol & ppob & 1.42 & & & 2 & 0 & \\
\hline 2NITPH & & 2-Nitrophenol & ppb & 1.56 & & & 2 & 0 & \\
\hline BUTDINP & & 2-sec-Butyl-4,6-dinitrophenol (DN & ppb & 1.35 & & & 2 & 0 & \\
\hline $460 N 2 M P$ & & 4,6-Dinitro-2-methylphenol & ppob & 1.18 & & & 2 & 0 & \\
\hline CHLCRES & & 4-Chloro-3-methylphenol & ppob & 1.12 & & & 2 & 0 & \\
\hline MITPHEN & & 4-Nitrophenol & طب̣ & .65 & & & 2 & 0 & \\
\hline ALUMNUM & & Aluminizn & Fin & 32.5 & & & 2 & 1 & \\
\hline
\end{tabular}


Table 16-6. Constituent List and Summary of Results for the Single-Shell Tank Waste Management Area B-BY-BX Data for Reporting Period October 1

through December 31, 1993. (sheet 2 of 2)

\begin{tabular}{|c|c|c|c|c|c|c|c|c|c|}
\hline \multirow[b]{2}{*}{ short } & \multirow[b]{2}{*}{ (Method) } & \multirow{2}{*}{ 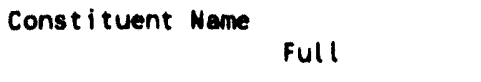 } & \multirow[b]{2}{*}{ Units } & \multirow{2}{*}{$\begin{array}{l}\text { Lab } \\
\text { CROL }\end{array}$} & \multicolumn{2}{|l|}{ DUS } & \multicolumn{3}{|c|}{ Number of Semples } \\
\hline & & & & & Limit & Agency & Total & $>$ CRQL & $>$ DWS \\
\hline ANTIONY & & Ant imony & pob & 69.4 & $\ldots$ & ….... & 2 & 0 & $\cdots$ \\
\hline BENZENE & & $\begin{array}{l}\text { Antimony } \\
\text { Benzene }\end{array}$ & ppob & .105 & 5 & EPA & 2 & 0 & 0 \\
\hline BERYLUM & & Beryllium & ppob & .814 & & & 2 & 0 & \\
\hline BROMIDE & & Bromide & ppob & 52.8 & & & 2 & 0 & \\
\hline CALCIUM & & Calcium & ppb & 20.9 & & & 2 & 2 & \\
\hline TETRANE & & Carbon tetrachloride & ppb & .121 & 5 & EPA & 2 & 0 & 0 \\
\hline CHLFORM & & Chloroform & ppob & .043 & & & 2 & 2 & \\
\hline COBALT & & Cobalt & ppb & 4.05 & & & 2 & 2 & \\
\hline COPPER & & Copper & ppob & 2.65 & 1600 & EPAS & 2 & 2 & 0 \\
\hline CRESOLS & & Cresols (methylphenols) & ppb & 4.66 & & & 2 & 0 & \\
\hline ETHBENZ & & Ethylbenzene & ppob & .046 & 700 & EPA & 2 & 0 & 0 \\
\hline $1-129 L$ & & lodine-129, Low level & $\mathrm{PCi} / \mathrm{L}$ & & & & 2 & 2 & \\
\hline MAGNES & & Magnesium & ppb & 26 & & & 2 & 2 & \\
\hline METHYCH & & Methylene chloride & ppb & .056 & & & 2 & 1 & \\
\hline NICKEL & & Nickel & ppb & 17.9 & & & 2 & 2 & \\
\hline NITRITE & & Nitrite & ppb & 38.3 & 1000 & EPA & 2 & 0 & 0 \\
\hline PENTCHP & & Pentachlorophenol & ppb & .87 & 1 & EPA & 2 & 0 & 0 \\
\hline PHOSPHA & & Phosphate & ppb & 147 & & & 2 & 0 & \\
\hline POTASUM & & Potassium & ppb & 662 & & & 2 & 2 & \\
\hline SILVER & & silver & ppob & 2.87 & 50 & EPAI & 2 & 0 & 0 \\
\hline SULFIDE & & Sulfide & ppob & 300 & & & 2 & 1 & \\
\hline PERCENE & & Tetrachloroethene & ppb & .049 & 5 & EPA & 2 & 0 & 0 \\
\hline TETPHNL & & Tetrachlorophenols & ppb & 1.05 & & & 2 & 0 & \\
\hline TIN & & $\operatorname{Tin}$ & ppo & 51.1 & & & 2 & 0 & \\
\hline TOLUENE & & Tolvene & ppo & .056 & 1000 & EPA & 2 & 0 & 0 \\
\hline TDS & & Total Dissolved Solids & ppm & 10 & 500 & EPAS & 2 & 2 & 0 \\
\hline TRICENE & & Trichloroethene & ppb & .065 & 5 & EPA & 2 & 0 & 0 \\
\hline TRIPHNL & & Trichlorophenols & ppo & 1.11 & & & 2 & 0 & \\
\hline TRITIUM & & Tritium & $\mathrm{pCi/L}$ & & 20000 & EPA & 2 & 2 & 0 \\
\hline$U-234$ & & Uranium-234 & $\mathrm{pCi} / \mathrm{L}$ & & & & 2 & 2 & \\
\hline$u-235$ & & Uranium-235 & $\mathrm{pCi} / \mathrm{L}$ & & & & 2 & 1 & \\
\hline$U-238$ & & Uranium-238 & $\mathrm{PCi} / \mathrm{L}$ & & & & 2 & 2 & \\
\hline VAKADUM & & Vanadium & ppb & 3.84 & & & 2 & 2 & \\
\hline VINYIDE & & Vinyl chloride & ppb & .266 & 2 & EPA & 2 & 0 & 0 \\
\hline XYLENE & & xylenes (total) & ppb & .202 & 10000 & EPA & 2 & 0 & 0 \\
\hline ZINC & & Zinc & ppb & 3.44 & 5000 & EPAS & 2 & 2 & 0 \\
\hline CIS120E & & cis-1,2-Dichloroethylene & ppb & .127 & 70 & EPA & 2 & 0 & 0 \\
\hline TRANDCE & & trans-1,2-Dichloroethylene & ppb & .149 & 100 & EPA & 2 & 0 & 0 \\
\hline
\end{tabular}

For explanation of this table, see Section 1.4 of report. 
Table 16-7. Constituents with at Least One Detected Value for the Single-Shell Tank Waste Management Area B-BY-BX Data for Reporting Period October 1 through December 31, 1993. (sheet 1 of 2)

\begin{tabular}{|c|c|c|c|c|c|c|}
\hline $\begin{array}{l}\text { Well } \\
\text { Name }\end{array}$ & $\begin{array}{c}\text { Collection } \\
\text { Date }\end{array}$ & $\begin{array}{l}\text { Sample } \\
\text { Number }\end{array}$ & $\begin{array}{l}1,1,1-1 \\
25 / \mathrm{ppb} \\
.072 / 200\end{array}$ & $\begin{array}{l}\text { ALUANUM } \\
34 / \mathrm{ppb} \\
32.5 /\end{array}$ & $\begin{array}{l}\text { BARIUM } \\
34 / \text { ppb } \\
.001 / 1000 \mathrm{i}\end{array}$ & $\begin{array}{c}\text { CALCIUM } \\
34 / \mathrm{ppb} \\
20.9 \%\end{array}$ \\
\hline $\begin{array}{l}299-E 33-33 \\
299-E 33-36\end{array}$ & $\begin{array}{l}11 / 05 / 93 \\
11 / 04 / 93\end{array}$ & $\begin{array}{l}\text { 809K14 } \\
\text { 809K18 }\end{array}$ & $\begin{array}{l}.12 \mathrm{~L} \\
.09 \mathrm{~L}\end{array}$ & $\begin{array}{l}94.00 \mathrm{~L} \\
32.50 \mathrm{U}\end{array}$ & $\begin{array}{l}34.00 \\
35.000\end{array}$ & $\begin{array}{l}30000.00 \\
30000.00\end{array}$ \\
\hline
\end{tabular}

\begin{tabular}{|c|c|c|c|c|c|c|}
\hline $\begin{array}{l}\text { Well } \\
\text { Name }\end{array}$ & $\begin{array}{c}\text { Collection } \\
\text { Date }\end{array}$ & $\begin{array}{l}\text { Sample } \\
\text { Number }\end{array}$ & $\begin{array}{c}\text { CHLORID } \\
124 / \mathrm{ppb} \\
82.5 / 250000 \mathrm{~s}\end{array}$ & $\begin{array}{l}\text { CHLFORM } \\
25 / \text { PpD } \\
.043 / .\end{array}$ & $\begin{array}{c}\text { CHROMUM } \\
34 / \mathrm{ppb} \\
5.42 / 50 \mathrm{i}\end{array}$ & $\begin{array}{l}\text { COBALT } \\
34 / \text { ppb } \\
4.05 \%\end{array}$ \\
\hline $\begin{array}{l}299-E 33-33 \\
299-E 33-36\end{array}$ & $\begin{array}{l}11 / 05 / 93 \\
11 / 04 / 93\end{array}$ & $\begin{array}{l}\text { 809K14 } \\
\text { B09K18 }\end{array}$ & $\begin{array}{l}5300.00 \\
4800.00\end{array}$ & $\begin{array}{l}.23 \mathrm{~L} \\
.10 \mathrm{~L}\end{array}$ & $\begin{array}{l}140.00 \\
120.00\end{array}$ & $\begin{array}{l}6.70 \mathrm{~L} \\
4.10 \mathrm{~L}\end{array}$ \\
\hline
\end{tabular}

\begin{tabular}{|c|c|c|c|c|c|c|}
\hline $\begin{array}{l}\text { Well } \\
\text { Name }\end{array}$ & $\begin{array}{c}\text { Collection } \\
\text { Date }\end{array}$ & $\begin{array}{l}\text { Sample } \\
\text { Number }\end{array}$ & $\begin{array}{c}\text { COPPER } \\
34 / \mathrm{ppb} \\
2.65 / 1000 \mathrm{~s}\end{array}$ & $\begin{array}{l}\text { FLUORID } \\
124 / \text { ppb } \\
13.9 / 1400 \mathrm{i}\end{array}$ & $\begin{array}{c}1-129 \\
139 / \mathrm{pCi} / \mathrm{L} \\
. / .\end{array}$ & $\begin{array}{c}\text { IRON } \\
34 / \mathrm{ppb} \\
10.3 / 300 \mathrm{~s}\end{array}$ \\
\hline $299-E 33-33$ & $\begin{array}{l}11 / 05 / 93 \\
11 / 04 / 93\end{array}$ & $\begin{array}{l}\text { 809K14 } \\
809 \times 18\end{array}$ & $\begin{array}{r}14.00 \mathrm{~L} \\
7.00 \mathrm{~L}\end{array}$ & 600.00 & $\begin{array}{l}3.11 \\
5.53\end{array}$ & $\begin{array}{l}630.00 \\
510.00\end{array}$ \\
\hline
\end{tabular}

\begin{tabular}{|c|c|c|c|c|c|c|}
\hline $\begin{array}{l}\text { Well } \\
\text { Name }\end{array}$ & $\begin{array}{c}\text { Collection } \\
\text { Date }\end{array}$ & $\begin{array}{l}\text { Sample } \\
\text { Number }\end{array}$ & $\begin{array}{c}\text { MAGNES } \\
34 / \mathrm{ppb} \\
26 / .\end{array}$ & $\begin{array}{c}\text { MANGESE } \\
34 / \mathrm{ppb} \\
1.35 / 50 \mathrm{~s}\end{array}$ & $\begin{array}{c}\text { METHYCH } \\
25 / \mathrm{ppb} \\
.056 / .\end{array}$ & $\begin{array}{l}\text { NICKEL } \\
34 / \mathrm{ppb} \\
17.9 \%\end{array}$ \\
\hline - & .............. & $\cdots$ & . & $\cdots$ & & \\
\hline $\begin{array}{l}299-E 33-33 \\
299-E 33-36\end{array}$ & $\begin{array}{l}11 / 05 / 93 \\
11 / 04 / 93\end{array}$ & $\begin{array}{l}\text { 809K } 14 \\
\text { B09K } 18\end{array}$ & $\begin{array}{l}8900.00 \\
8400.00\end{array}$ & $\begin{array}{l}14.00 \\
13.00\end{array}$ & $\begin{array}{l}.06 \mathrm{BL} \\
.06 \mathrm{U}\end{array}$ & $\begin{array}{l}50.00 \\
30.00\end{array}$ \\
\hline
\end{tabular}

\begin{tabular}{|c|c|c|c|c|c|c|}
\hline $\begin{array}{l}\text { Nell } \\
\text { Name }\end{array}$ & $\begin{array}{c}\text { Collection } \\
\text { Date }\end{array}$ & $\begin{array}{l}\text { Sample } \\
\text { Number }\end{array}$ & $\begin{array}{c}\text { NITRATE } \\
124 / \mathrm{ppb} \\
44.4 / 45000\end{array}$ & $\begin{array}{l}\text { POTASUM } \\
\text { 34/ppb } \\
6621 .\end{array}$ & $\begin{array}{l}\text { so0lum } \\
34 / p p b \\
40.9 \%\end{array}$ & $\begin{array}{c}\text { SULFATE } \\
124 / \text { ppb } \\
49.9 / 250000 \mathrm{~s}\end{array}$ \\
\hline $\begin{array}{l}299-E 33-33 \\
299-E 33-36\end{array}$ & $\begin{array}{l}11 / 05 / 93 \\
11 / 04 / 93\end{array}$ & $\begin{array}{l}\text { 809K } 14 \\
\text { 809K } 18\end{array}$ & $\begin{array}{l}4900.00 \\
3000.00\end{array}$ & $\begin{array}{l}5900.00 \\
5300.00\end{array}$ & $\begin{array}{l}15000.00 \\
13000.00\end{array}$ & $\begin{array}{l}30000.000 \\
24000.000\end{array}$ \\
\hline
\end{tabular}

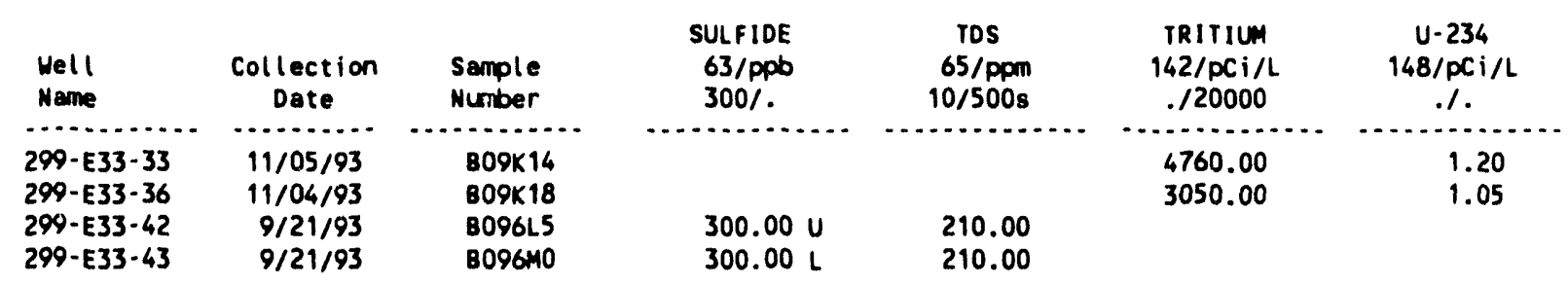


Table 16-7. Constituents with at Least One Detected Value for the Single-Shell Tank Waste Management Area B-BY-BX Data for Reporting Period October 1 through December 31,1993 . (sheet 2 of 2)

\begin{tabular}{|c|c|c|c|c|c|c|}
\hline $\begin{array}{l}\text { Well } \\
\text { Hame }\end{array}$ & $\begin{array}{c}\text { Collection } \\
\text { Date }\end{array}$ & $\begin{array}{l}\text { Sample } \\
\text { Number }\end{array}$ & $\begin{array}{c}U \cdot 235 \\
148 / p C i / L \\
.1 .\end{array}$ & $\begin{array}{c}U-238 \\
148 / p C i / L \\
. / .\end{array}$ & $\begin{array}{l}\text { VANADUM } \\
34 / \mathrm{ppD} \\
3.84 / .\end{array}$ & $\begin{array}{c}21 \mathrm{NC} \\
34 / \mathrm{ppb} \\
3.44 / 5000 \mathrm{~s}\end{array}$ \\
\hline $\begin{array}{l}299-E 33-33 \\
299-E 33-36\end{array}$ & $\begin{array}{l}11 / 05 / 93 \\
11 / 04 / 93\end{array}$ & $\begin{array}{l}809 \times 14 \\
809 \times 18\end{array}$ & $\begin{array}{l}.03 U \\
.04\end{array}$ & $\begin{array}{l}.79 \\
.80\end{array}$ & $\begin{array}{l}24.00 \mathrm{~L} \\
26.00 \mathrm{~L}\end{array}$ & $\begin{array}{l}19.00 \\
21.00\end{array}$ \\
\hline
\end{tabular}

For explanation of this table, see Section 1.4 of report. 
Table 16-8. Contamination Indicator Parameters for the Single-Shell Tank Waste Management Area B-BY-BX Data for Reporting Period October 1

through December 31, 1993.

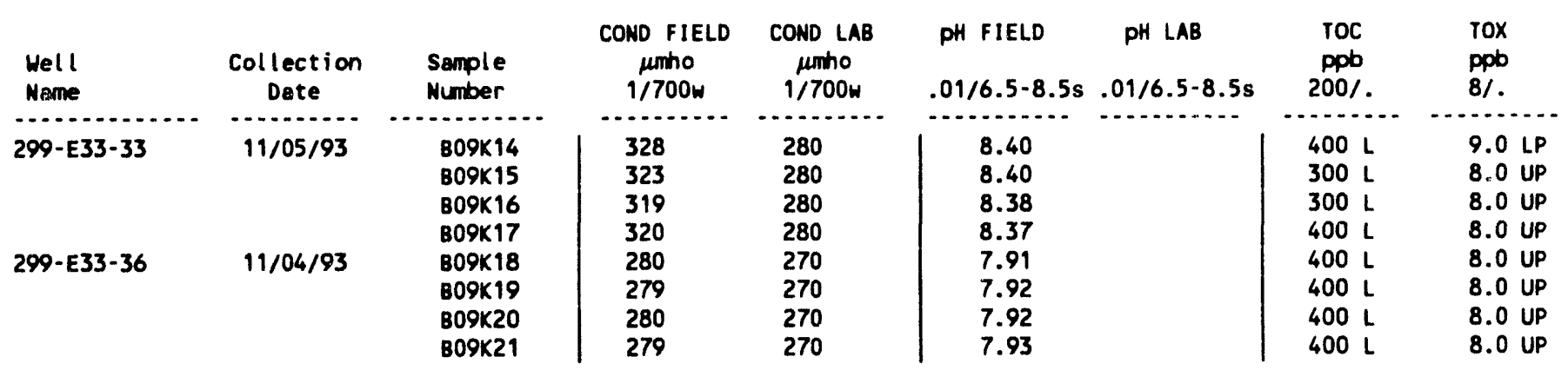

For explanation of this table, see Section 1.4 of report. 
Table 16-9. Constituent List and Summary of Results for the Single-Shell Tank Waste Management Area C Data for Reporting Period October 1 through December 31, 1993.

SITE SPECIFIC AND OTHER CONSTITUENTS

\begin{tabular}{|c|c|c|c|c|c|c|c|c|c|}
\hline \multirow[b]{2}{*}{ Short } & \multirow{2}{*}{\multicolumn{2}{|c|}{ Const ituent Name }} & \multirow[b]{2}{*}{ Units } & \multirow{2}{*}{$\begin{array}{l}\text { Lab } \\
\text { CRQL }\end{array}$} & \multicolumn{2}{|c|}{ DWS } & \multicolumn{3}{|c|}{ Number of Samples } \\
\hline & & Full & & & Limit & Agency & Total & $>C R Q L$ & $>$ DWS \\
\hline SULFIDE & & Sulfide & oob & 300 & & & 1 & ${ }_{0}$ & \\
\hline TDS & & Total Dissolved Solids & pom & 10 & 500 & EPAS & 1 & 1 & 0 \\
\hline
\end{tabular}

For explanation of this table, see Section 1.4 of report. 
Table 16-10. Constituents with at Least One Detected Value for the Single-Shell Tank Waste Management Area C Data for Reporting Period October 1 through December 31, 1993.

\begin{tabular}{|c|c|c|c|}
\hline $\begin{array}{l}\text { Well } \\
\text { Name }\end{array}$ & $\begin{array}{l}\text { Collection } \\
\text { Date }\end{array}$ & $\begin{array}{l}\text { Sample } \\
\text { Number }\end{array}$ & $\begin{array}{c}\text { TDS } \\
65 / \mathrm{ppm} \\
10 / 500 \mathrm{~s}\end{array}$ \\
\hline 299-E27-7 & $9 / 21 / 93$ & BO96LO & 180.00 \\
\hline
\end{tabular}

For explanation of this table, see Section 1.4 of report. 
Table 16-11. Contamination Indicator Parameters for the Single-Shell Tank Waste Management Area C Data for Reporting Period October 1 through December 31, 1993.

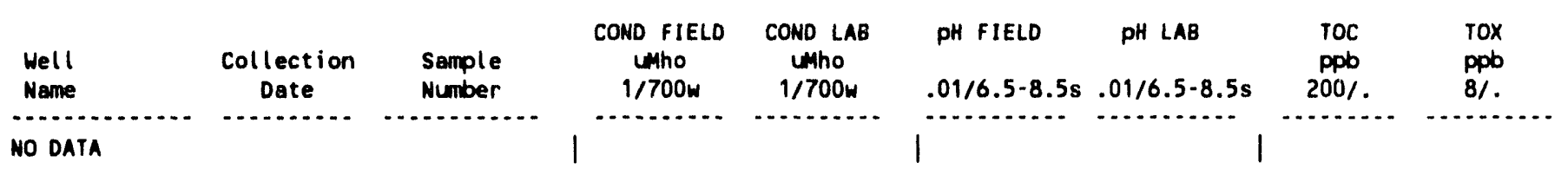

For explanation of this table, see Section 1.4 of report. 
Table 16-12. Constituent List and Summary of Results for the Single-Shell Tank Waste Management Area S-SX Data for Reporting

Period October 1 through December 31, 1993.

DRINKING WATER PARAMETERS

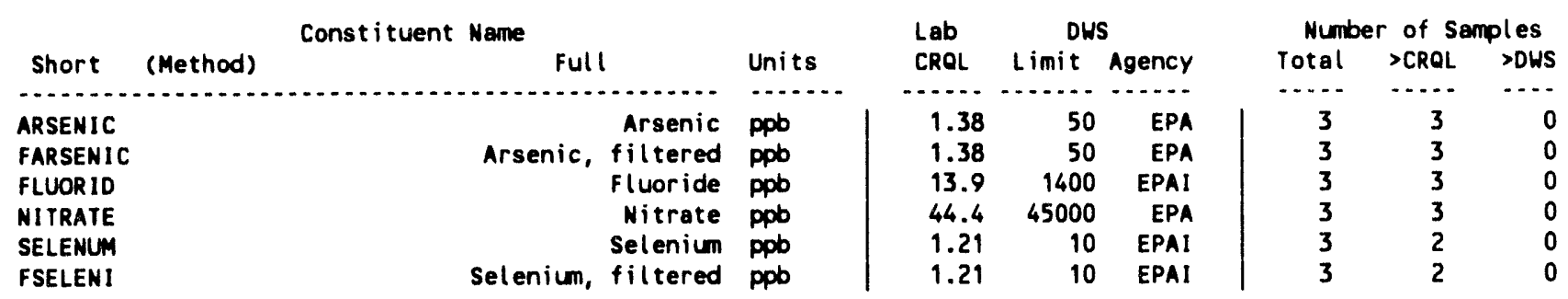

GROUNDWATER QUALITY PARAMETERS

\begin{tabular}{|c|c|c|c|c|c|c|c|c|c|}
\hline \multirow[b]{2}{*}{ Short } & \multirow{2}{*}{\multicolumn{2}{|c|}{ Constituent Name }} & \multirow[b]{2}{*}{ Units } & \multirow{2}{*}{$\begin{array}{l}\text { Lab } \\
\text { CROL }\end{array}$} & \multicolumn{2}{|c|}{ DWS } & \multicolumn{3}{|c|}{ Number of Samples } \\
\hline & & & & & Limit & Agency & Total & $>C R Q L$ & $>$ DWS \\
\hline $\begin{array}{l}\text { CHLORID } \\
\text { SULFATE }\end{array}$ & & $\begin{array}{l}\text { Chloride } \\
\text { Sulfate }\end{array}$ & $\begin{array}{l}\text { ppb } \\
\text { ppb }\end{array}$ & $\begin{array}{l}82.5 \\
49.9\end{array}$ & $\begin{array}{l}250000 \\
250000\end{array}$ & $\begin{array}{l}\text { EPAS } \\
\text { EPAS }\end{array}$ & $\begin{array}{l}3 \\
3\end{array}$ & $\begin{array}{l}3 \\
3\end{array}$ & $\begin{array}{l}0 \\
0\end{array}$ \\
\hline
\end{tabular}

SITE SPECIFIC AND OTHER CONSTITUENTS

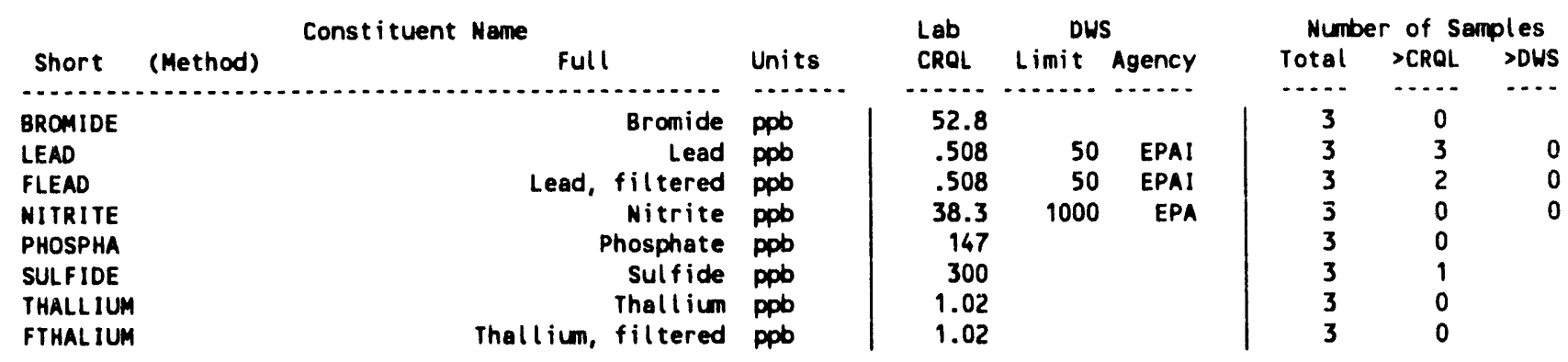

$\overline{\text { For explanation of this table, see Section } 1.4 \text { of report. }}$ 
Table 16-13. Constituents with at Least One Detected Value for the Single-Shell Tank Waste Management Area S-SX Data for Reporting Period October 1 through December 31, 1993.

\begin{tabular}{|c|c|c|c|c|c|c|}
\hline $\begin{array}{l}\text { Well } \\
\text { Name }\end{array}$ & $\begin{array}{c}\text { Collection } \\
\text { Date }\end{array}$ & $\begin{array}{l}\text { Sample } \\
\text { Number }\end{array}$ & $\begin{array}{r}\text { ARSENIC } \\
43 / \mathrm{ppb} \\
1.38 / 50\end{array}$ & $\begin{array}{l}\text { FARSENIC } \\
43 / \mathrm{ppb} \\
1.38 / 50\end{array}$ & $\begin{array}{c}\text { CHLORID } \\
124 / \mathrm{ppb} \\
82.5 / 250000 \mathrm{~s}\end{array}$ & $\begin{array}{l}\text { FLUORID } \\
124 / \mathrm{ppb} \\
13.9 / 1400 \mathrm{i}\end{array}$ \\
\hline $\begin{array}{l}299-W 22-44 \\
299-W 22-44\end{array}$ & $\begin{array}{l}9 / 29 / 93 \\
9 / 29 / 93\end{array}$ & $\begin{array}{l}\text { B09650 } \\
\text { B09654 }\end{array}$ & 6.60 & 6.40 & 1300.00 & 400.00 \\
\hline $\begin{array}{l}299-W 22-45 \\
299-W 22-45 \\
299-W 22-45 \\
299-W 22-45\end{array}$ & $\begin{array}{l}9 / 29 / 93 \\
9 / 29 / 93 \\
9 / 29 / 93 \\
9 / 29 / 93\end{array}$ & $\begin{array}{l}\text { B096S5 } \\
\text { B096S6 } \\
8096 T 3 \\
\text { B096T4 }\end{array}$ & $\begin{array}{l}6.50 \\
6.80\end{array}$ & $\begin{array}{l}6.70 \\
6.00\end{array}$ & $\begin{array}{l}3400.00 \\
3300.00\end{array}$ & $\begin{array}{l}500.00 \\
500.00\end{array}$ \\
\hline
\end{tabular}

\begin{tabular}{|c|c|c|c|c|c|c|}
\hline $\begin{array}{l}\text { Well } \\
\text { Name }\end{array}$ & $\begin{array}{c}\text { Collection } \\
\text { Date }\end{array}$ & $\begin{array}{l}\text { Sample } \\
\text { Number }\end{array}$ & $\begin{array}{l}\text { LEAD } \\
40 / p p b \\
.508 / 50 \mathrm{i}\end{array}$ & $\begin{array}{l}\text { NITRATE } \\
124 / \mathrm{ppb} \\
44.4 / 45000\end{array}$ & $\begin{array}{l}\text { SELENUM } \\
48 / \mathrm{ppb} \\
1.21 / 10 \mathrm{i}\end{array}$ & $\begin{array}{l}\text { FSELENI } \\
48 / \mathrm{ppb} \\
1.21 / 10 i\end{array}$ \\
\hline $\begin{array}{l}299-W 22-44 \\
299-W 22-44 \\
299-W 22-45 \\
299-W 22-45 \\
299-W 22-45 \\
299-W 22-45\end{array}$ & $\begin{array}{l}9 / 29 / 93 \\
9 / 29 / 93 \\
9 / 29 / 93 \\
9 / 29 / 93 \\
9 / 29 / 93 \\
9 / 29 / 93\end{array}$ & $\begin{array}{l}\text { B096SO } \\
\text { B096S4 } \\
\text { B096S5 } \\
\text { B096S6 } \\
\text { B096T3 } \\
\text { B096T4 }\end{array}$ & $\begin{array}{r}1.70 \mathrm{BL} \\
.80 \mathrm{BL} \\
2.00 \mathrm{BL}\end{array}$ & $\begin{array}{l}900.00 \\
9400.00 \\
9000.00\end{array}$ & $\begin{array}{l}1.21 \mathrm{U} \\
2.40 \mathrm{~L} \\
2.40 \mathrm{~L}\end{array}$ & $\begin{array}{l}1.21 \mathrm{U} \\
2.10 \mathrm{~L} \\
3.50 \mathrm{~L}\end{array}$ \\
\hline $\begin{array}{l}\text { Well } \\
\text { Name }\end{array}$ & $\begin{array}{c}\text { Collection } \\
\text { Date }\end{array}$ & $\begin{array}{l}\text { Sample } \\
\text { Number }\end{array}$ & $\begin{array}{c}\text { SULFATE } \\
124 / \mathrm{ppb} \\
49.9 / 250000 \mathrm{~s}\end{array}$ & $\begin{array}{l}\text { SULFIDE } \\
63 / p p b \\
300 \%\end{array}$ & & \\
\hline $\begin{array}{l}299-W 22-44 \\
299-W 22-45 \\
299-W 22-45\end{array}$ & $\begin{array}{l}9 / 29 / 93 \\
9 / 29 / 93 \\
9 / 29 / 93\end{array}$ & $\begin{array}{l}\text { B096SO } \\
\text { B096S5 } \\
\text { B096S6 }\end{array}$ & $\begin{array}{l}10000.00 \mathrm{D} \\
16000.00 \mathrm{D} \\
16000.00 \mathrm{D}\end{array}$ & $\begin{array}{l}300.00 \mathrm{~L} \\
300.00 \mathrm{U} \\
300.00 \mathrm{U}\end{array}$ & & \\
\hline
\end{tabular}

For explanation of this table, see Section 1.4 of report. 


\section{Table 16-14. Contamination Indicator Parameters for the Single-Shell Tank Waste Management Area S-SX Data for Reporting Period October 1 through December 31, 1993.}

\begin{tabular}{|c|c|c|c|c|c|c|c|c|}
\hline $\begin{array}{l}\text { Well } \\
\text { Name }\end{array}$ & $\begin{array}{c}\text { Collection } \\
\text { Date }\end{array}$ & $\begin{array}{l}\text { Sample } \\
\text { Number }\end{array}$ & $\begin{array}{c}\text { COND FIELD } \\
\mu \text { mho } \\
1 / 700 \mathrm{~W}\end{array}$ & $\begin{array}{c}\text { COND LAB } \\
\mu \text { mho } \\
1 / 700 \mathrm{~W}\end{array}$ & $\begin{array}{l}\text { PH FIELD } \\
.01 / 6.5-8.5 \mathrm{~s}\end{array}$ & $\begin{array}{c}\text { PH LAB } \\
.01 / 6.5-8.5 \mathrm{~s}\end{array}$ & $\begin{array}{c}10 C \\
p p b \\
200 \%\end{array}$ & $\begin{array}{l}\text { TOX } \\
\text { ppb } \\
8 \% .\end{array}$ \\
\hline NO DATA & - & & & & & .... & . . & \\
\hline
\end{tabular}

For explanation of this table, see Section 1.4 of report. 
Table 16-15. Constituent List and Summary of Results for the Single-Shell Tank Waste Management Area T Data for Reporting Period October 1

through December 31, 1993. (sheet 1 of 2)

CONTAMINATION INDICATOR PARAMETERS

\begin{tabular}{|c|c|c|c|c|c|c|c|c|c|}
\hline \multirow[b]{2}{*}{ Short } & \multirow[b]{2}{*}{ (Method) } & \multirow{2}{*}{ Constituent Name Full } & \multirow[b]{2}{*}{ Units } & \multirow{2}{*}{$\begin{array}{l}\text { Lab } \\
\text { CROL }\end{array}$} & \multicolumn{2}{|c|}{ DWS } & \multicolumn{3}{|c|}{ Number of Samples } \\
\hline & & & & & Limit & Agency & Total & $>\mathrm{CRQL}$ & $>$ DWS \\
\hline (......... & & (n......... & & $\cdots$ & -...... & $\cdots$ & $\cdots$ & $\cdots \cdot$ & $\cdots$ \\
\hline CONDUCT & 94 & Conductivity, field & $\mu$ mho & 1 & & & 12 & 12 & \\
\hline CONDUCT & 73 & Conductivity, lab & umho & 1 & & & 12 & 12 & \\
\hline TOC & & Total Organic Carbon & ppob & 200 & & & 8 & 8 & \\
\hline PH & 93 & pH, field & PH & .01 & $6.5-8.5$ & EPAS & 12 & 12 & 0 \\
\hline PH & 125 & $\mathrm{pH}, \mathrm{lab}$ & pH & .01 & $6.5-8.5$ & EPAS & 12 & 12 & 0 \\
\hline
\end{tabular}

DRINKING WATER PARAMETERS

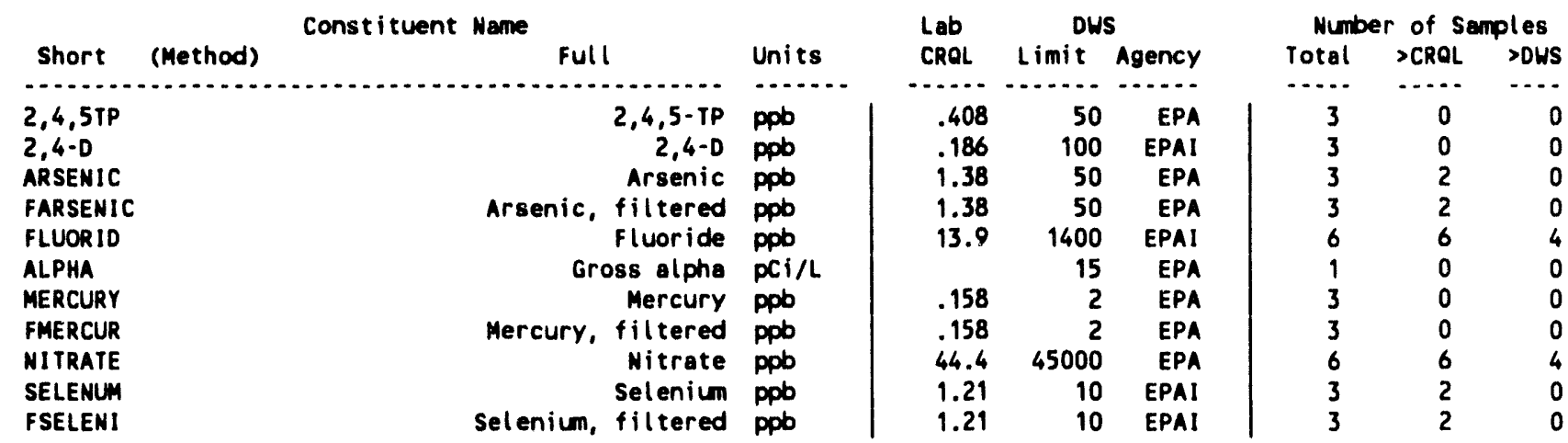

GROUNDWATER QUALITY PARAMETERS

\begin{tabular}{|c|c|c|c|c|c|c|c|c|c|}
\hline \multirow[b]{2}{*}{ Short } & \multirow[b]{2}{*}{ (Method) } & Constituent Name & \multirow[b]{2}{*}{ Units } & \multirow{2}{*}{$\begin{array}{l}\text { Lab } \\
\text { CRQL }\end{array}$} & \multicolumn{2}{|c|}{ DWS } & \multicolumn{3}{|c|}{ Number of Semples } \\
\hline & & Full & & & Limit & Agency & Total & $>C R Q L$ & $>$ DWS \\
\hline $\mathrm{CH} O \mathrm{O}$ & & & & ... & (n... & (n) & $\cdots$ & $\cdots$ & $\cdots$ \\
\hline $\begin{array}{l}\text { CHLORID } \\
\text { SULFATE }\end{array}$ & & $\begin{array}{l}\text { Chloride } \\
\text { Sulfate }\end{array}$ & $\begin{array}{l}\text { ppb } \\
\text { ppb }\end{array}$ & $\begin{array}{l}82.5 \\
49.9\end{array}$ & $\begin{array}{l}250000 \\
250000\end{array}$ & $\begin{array}{l}\text { EPAS } \\
\text { EPAS }\end{array}$ & $\begin{array}{l}6 \\
6\end{array}$ & $\begin{array}{l}6 \\
6\end{array}$ & $\begin{array}{l}0 \\
0\end{array}$ \\
\hline
\end{tabular}

SITE SPECIFIC AND OTHER CONSTITUENTS

\begin{tabular}{|c|c|c|c|c|c|c|c|c|c|}
\hline \multicolumn{4}{|c|}{ Constituent Name } & \multicolumn{3}{|c|}{ DUS } & \multicolumn{3}{|c|}{ Number of Semples } \\
\hline Short & (Method) & Full & Units & CROL & Limit & Agency & Total & $>$ CRQL & $>$ DHS \\
\hline$\ldots \ldots$ & $\cdots$ & 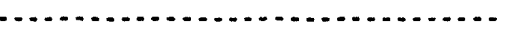 & $\ldots \ldots$ & $\ldots \ldots$ & $\ldots \ldots$ & $\ldots \ldots$ & $\ldots .$. & $\ldots$. & $\ldots$ \\
\hline $\begin{array}{l}1,1,1-T \\
1,1,2-T \\
1,1-D I C\end{array}$ & & $\begin{array}{r}1,1,1-\text { Trichloroethane } \\
1,1,2-\text { Trichloroethane } \\
\text { 1,1-Dichloroethane }\end{array}$ & $\begin{array}{l}\text { ppb } \\
\text { ppb } \\
\text { ppb }\end{array}$ & $\begin{array}{l}.072 \\
.043 \\
.337\end{array}$ & 200 & EPA & $\begin{array}{l}2 \\
2 \\
2\end{array}$ & $\begin{array}{l}0 \\
0 \\
0\end{array}$ & 0 \\
\hline $1,2-D I C$ & & 1,2-Dichloroethane & Pplo & .139 & 5 & EPA & 2 & 0 & 0 \\
\hline 14-cben & & 1,4-Dichlorobenzene & ppb & .107 & 75 & EPA & 2 & 0 & 0 \\
\hline $\begin{array}{l}2,4,5-1 \\
\text { BUTDINP }\end{array}$ & & 2-sec-Butyl-4,6-dinitromengl $2,4,3-1$ & Ppb & .384 & & & 3 & 0 & \\
\hline ALKALIN & & Alkal inity & ppm & 1.96 & & & 3 & 3 & \\
\hline BENZENE & & Benzene & ppb & .105 & 5 & EPA & 2 & 0 & 0 \\
\hline BROMIDE & & Bromide & ppb & 52.8 & & & 6 & 0 & \\
\hline TETRANE & & Carbon tetrachloride & ppo & .121 & 5 & EPA & 2 & 2 & 2 \\
\hline CHLFORM & & Chloroform & ppb & .043 & & & 2 & 2 & \\
\hline ETHBENZ & & Ethylbenzene & ppb & .046 & 700 & EPA & 2 & 0 & 0 \\
\hline BETA & & Gross beta & $\mathrm{pCi} / \mathrm{L}$ & & & & 1 & 1 & \\
\hline $1-129 L$ & & lodine-129, Low level & $\mathrm{pCi} / \mathrm{L}$ & & & & 3 & 3 & \\
\hline
\end{tabular}


Table 16-15. Constituent List and Summary of Results for the Single-Shell Tank Waste Management Area T Data for Reporting Period October 1

through December 31, 1993. (sheet 2 of 2)

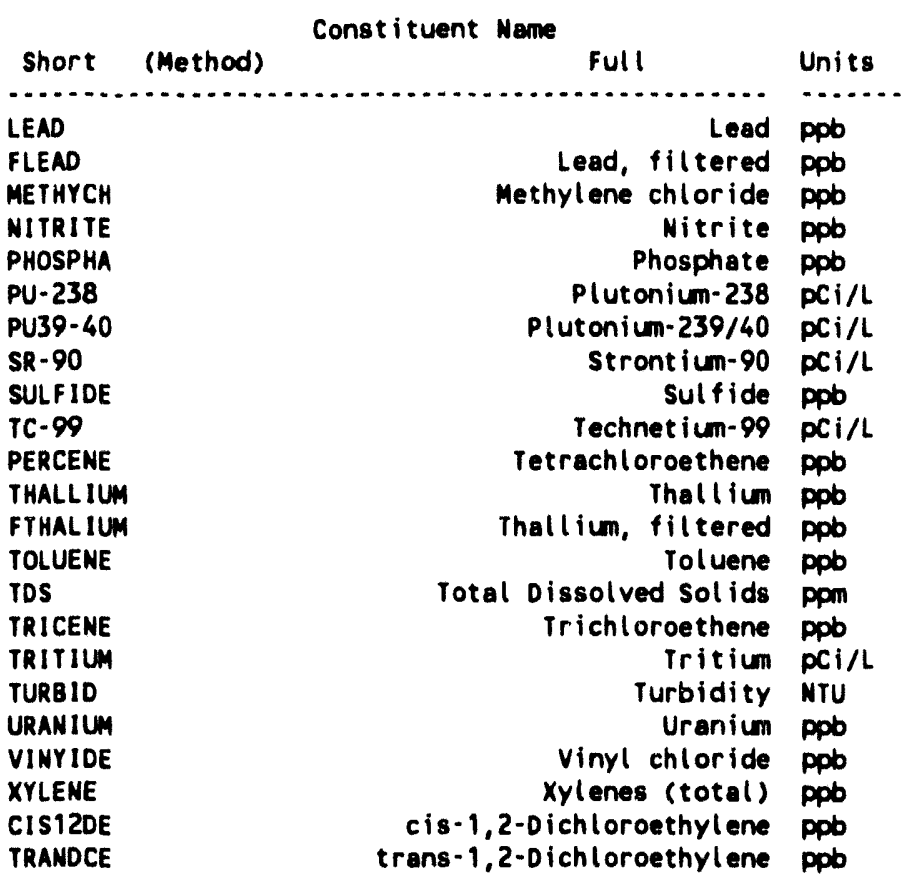

\begin{tabular}{rrr} 
Lab & \multicolumn{2}{c}{ DWS } \\
CRQL & Limit & Agency \\
$\cdots . .5$ & $\ldots .$. & $\ldots .$. \\
.508 & 50 & EPAI \\
.508 & 50 & EPAI \\
.056 & & \\
38.3 & 1000 & EPA \\
147 & & \\
& & \\
& & \\
300 & 8 & EPA \\
& 900 & EPA \\
.049 & 5 & EPA \\
1.02 & & \\
1.02 & & \\
.056 & 1000 & EPA \\
10 & 500 & EPAS \\
.065 & 5 & EPA \\
& 20000 & EPA \\
.05 & & \\
& & \\
.266 & 2 & EPA \\
.202 & 10000 & EPA \\
.127 & 70 & EPA \\
.149 & 100 & EPA
\end{tabular}

Number of Samples Total >CRQL >DWS -....

$\begin{array}{lll}6 & 6 & 0 \\ 6 & 3 & 0 \\ 2 & 0 & \\ 6 & 0 & 0 \\ 6 & 0 & \\ 3 & 0 & \\ 3 & 0 & \\ 3 & 0 & 0 \\ 3 & 0 & \\ 3 & 2 & 0 \\ 2 & 1 & 0 \\ 6 & 0 & \\ 6 & 0 & \\ 2 & 0 & 0 \\ 3 & 3 & 2 \\ 2 & 1 & 1 \\ 1 & 1 & 1 \\ 3 & 3 & \\ 3 & 3 & \\ 2 & 0 & 0 \\ 2 & 0 & 0 \\ 2 & 0 & 0 \\ 2 & 0 & 0\end{array}$

for explanation of this table, see Section 1.4 of report. 
Table 16-16. Constituents with at Least One Detected Value for the Single-Shell Tank Waste Management Area T Data for Reporting Period October 1

through December 31, 1993. (sheet 1 of 2)

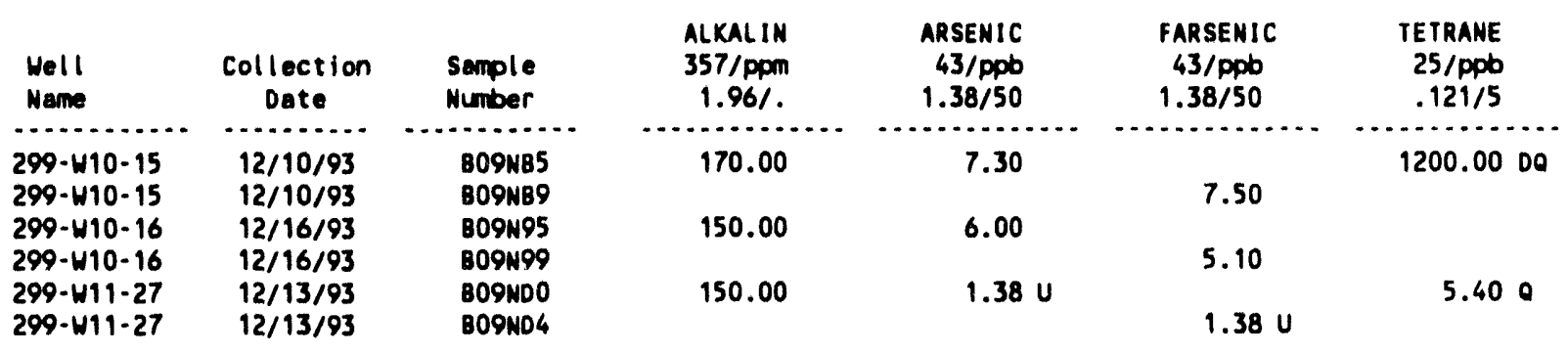

\begin{tabular}{|c|c|c|c|c|c|c|}
\hline $\begin{array}{l}\text { Well } \\
\text { Name }\end{array}$ & $\begin{array}{c}\text { Collection } \\
\text { Date }\end{array}$ & $\begin{array}{l}\text { Sample } \\
\text { Number }\end{array}$ & $\begin{array}{c}\text { CHLORID } \\
124 / \mathrm{ppb} \\
82.5 / 250000 \mathrm{~s}\end{array}$ & $\begin{array}{l}\text { CHLFORM } \\
25 / \mathrm{ppb} \\
.043 / .\end{array}$ & $\begin{array}{l}\text { FLUORID } \\
124 / \mathrm{ppO} \\
13.9 / 1400 \mathrm{i}\end{array}$ & $\begin{array}{c}\text { BETA } \\
\text { 136/PCi/L } \\
. / .\end{array}$ \\
\hline $\begin{array}{l}299-W 10-15 \\
299-W 10-15 \\
299-W 10-16 \\
299-W 10-16 \\
299-W 11-27 \\
299-W 11-27\end{array}$ & $\begin{array}{r}9 / 28 / 93 \\
12 / 10 / 93 \\
9 / 28 / 93 \\
12 / 16 / 93 \\
9 / 28 / 93 \\
12 / 13 / 93\end{array}$ & $\begin{array}{l}\text { B096M5 } \\
\text { B09NB5 } \\
\text { B096NO } \\
\text { B09N95 } \\
\text { 8096P5 } \\
\text { B09NDO }\end{array}$ & $\begin{array}{c}28000.00 \mathrm{D} \\
26000.00 \mathrm{DQ} \\
30000.00 \mathrm{D} \\
31000.00 \mathrm{D} \\
2100.00 \\
2000.00\end{array}$ & 9.80 & $\begin{array}{l}4700.00 \\
4800.00 \\
2500.00 \\
2600.00 \\
900.00 \\
1300.00\end{array}$ & 30.60 \\
\hline
\end{tabular}

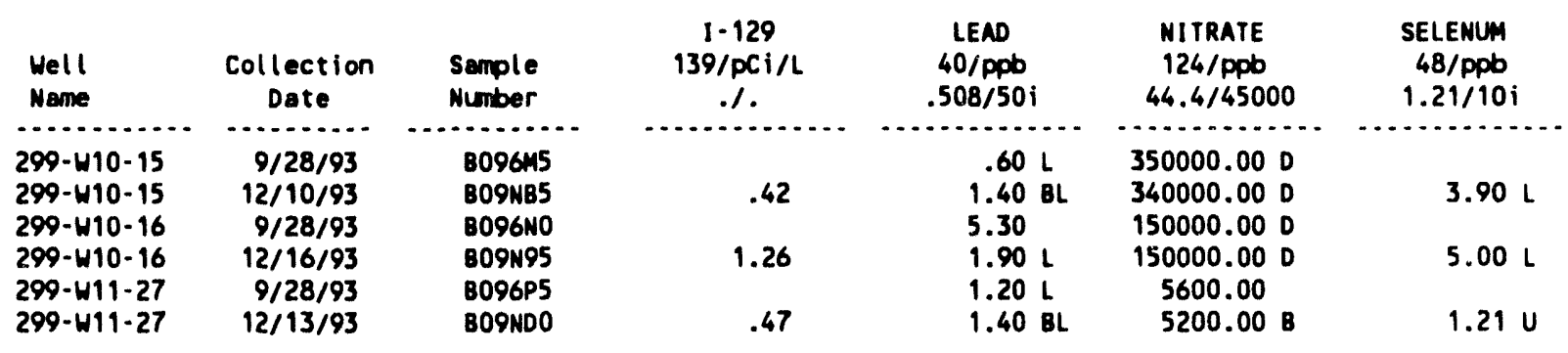

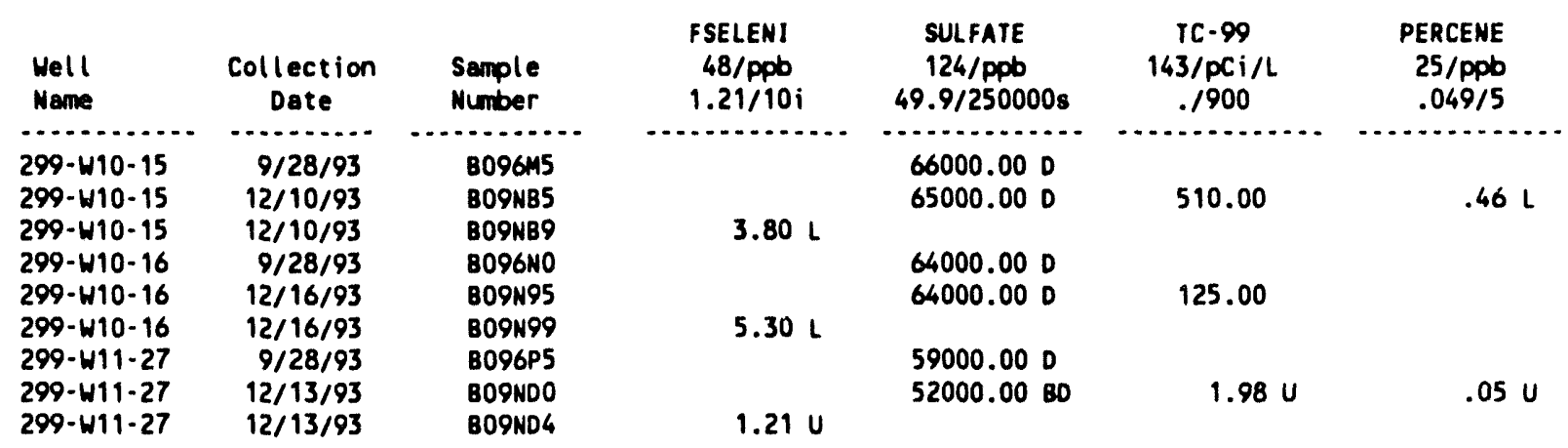


Table 16-16. Constituents with at Least One Detected Value for the Single-Shell Tank Waste Management Area T Data for Reporting Period October 1 through December 31, 1993. (sheet 2 of 2)

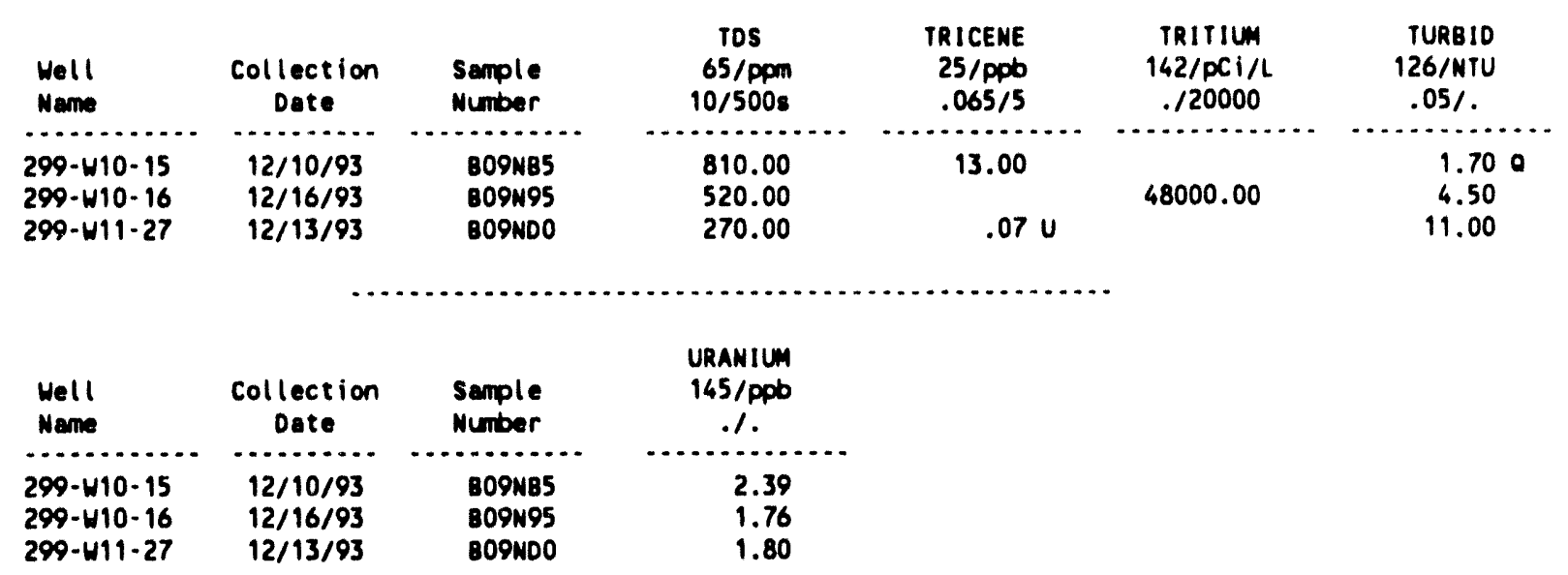

For explanation of this table, see Section 1.4 of report. 
Table 16-17. Contamination Indicator Parameters for the Single-Shell Tank Waste Management Area $T$ Data for Reporting Period October 1 through December 31, 1993.

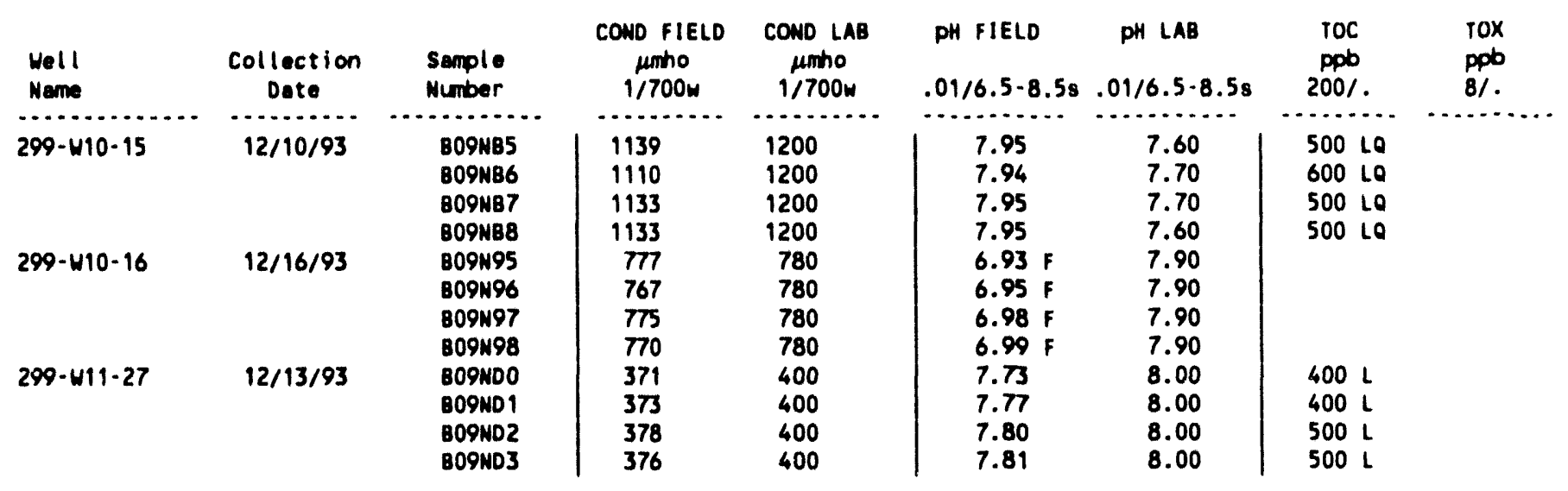

For explanation of this table, see section 1.4 of report. 
Table 16-18. Constituent List and Summary of Results for the Single-Shell Tank Waste Management Area TX-TY Data for Reporting Period October 1 through

December 31, 1993. (sheet 1 of 2)

CONTAMINATION INDICATOR PARAMETERS

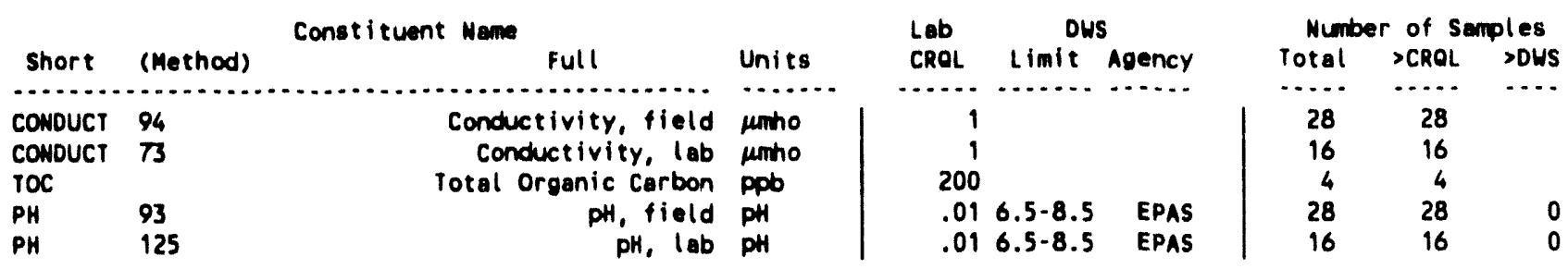

DRINKING WATER PARAMETERS

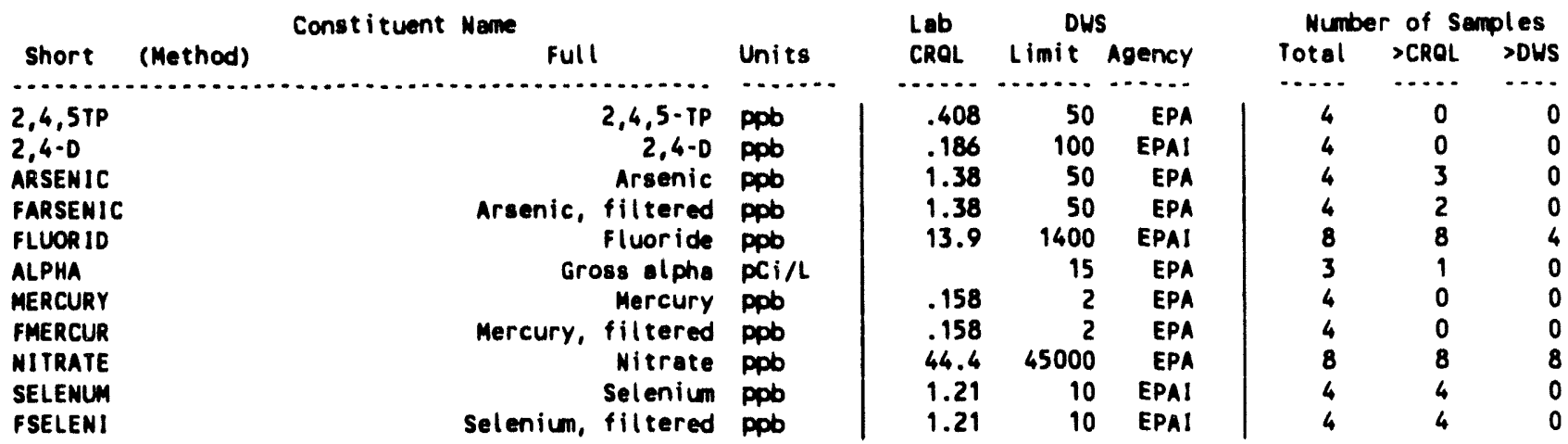

\section{GROUNDWATER OUALITY PARAMETERS}

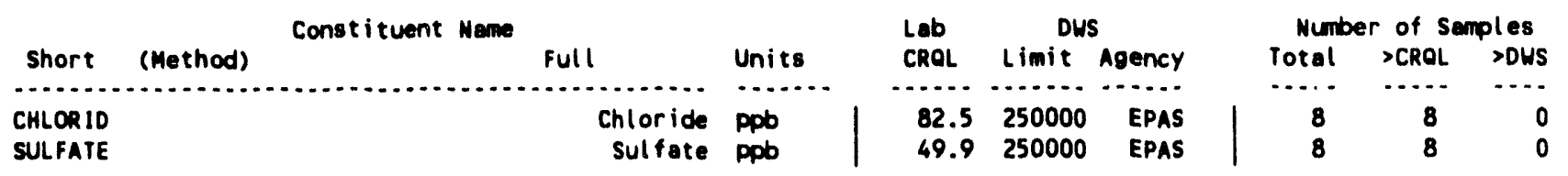

SITE SPECIFIC AND OTHER CONSTITUENTS

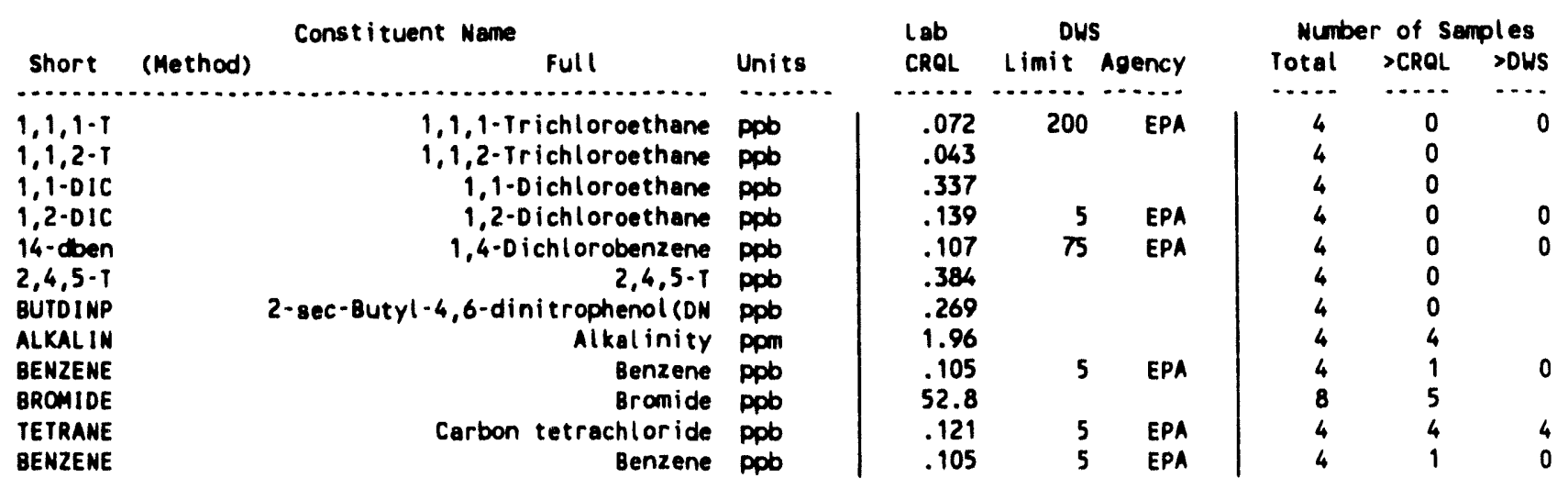


Table 16-18. Constituent List and Summary of Results for the Single-Shell Tank Waste Management Area TX-TY Data for Reporting Period October 1 through December 31, 1993. (sheet 2 of 2)

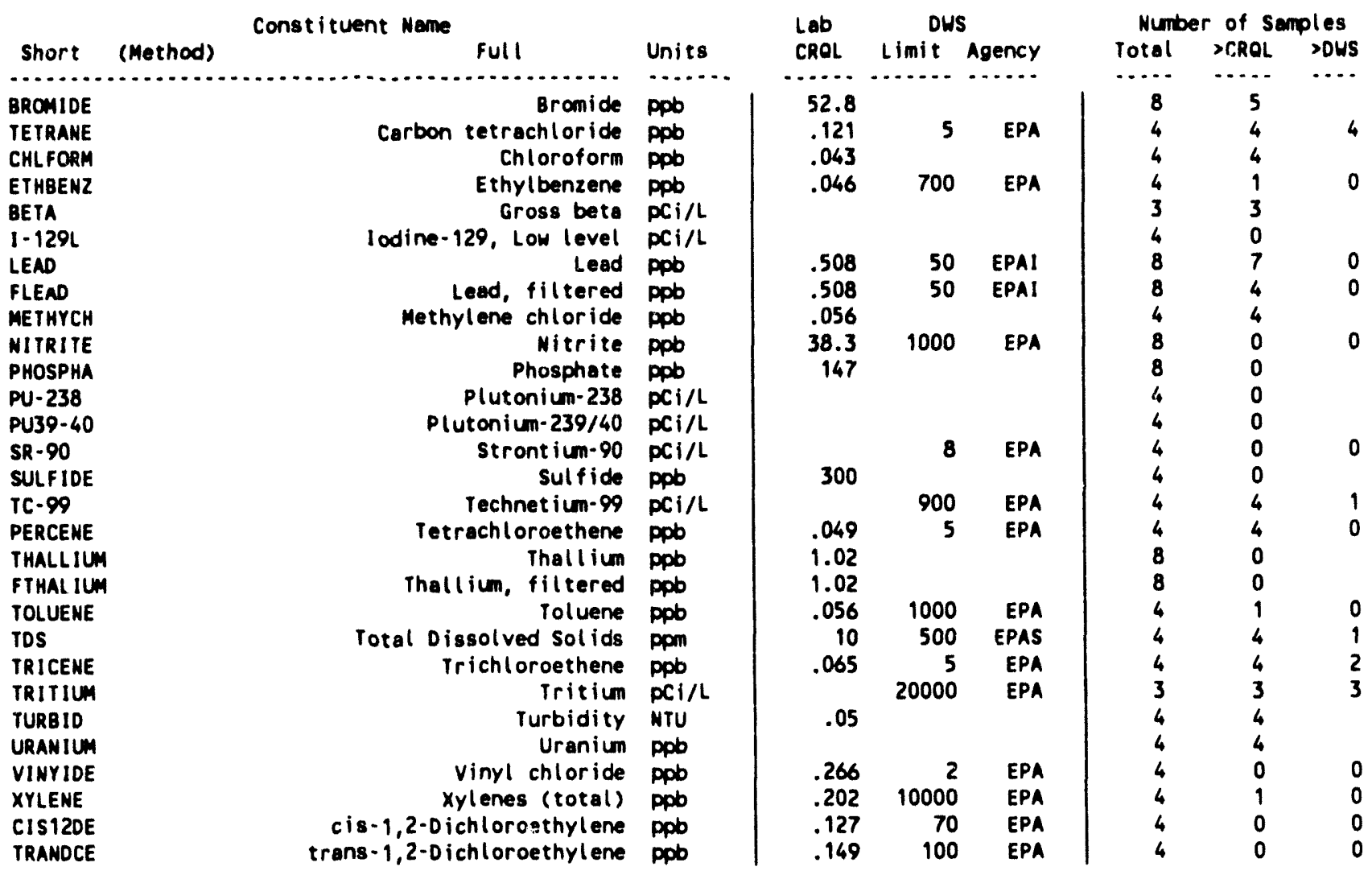

For explanation of this table, see section 1.4 of report. 
Table 16-19. Constituents with at Least One Detected Value for the Single-Shell Tank Waste Management Area TX-TY Data for Reporting Period October 1 through December 31, 1993. (sheet 1 of 2)

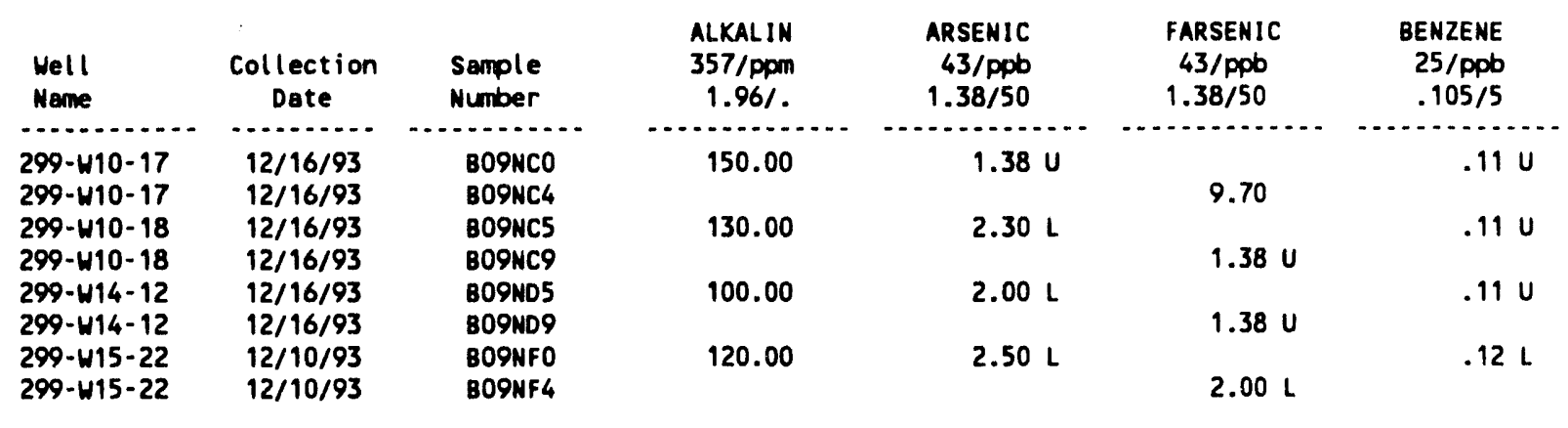

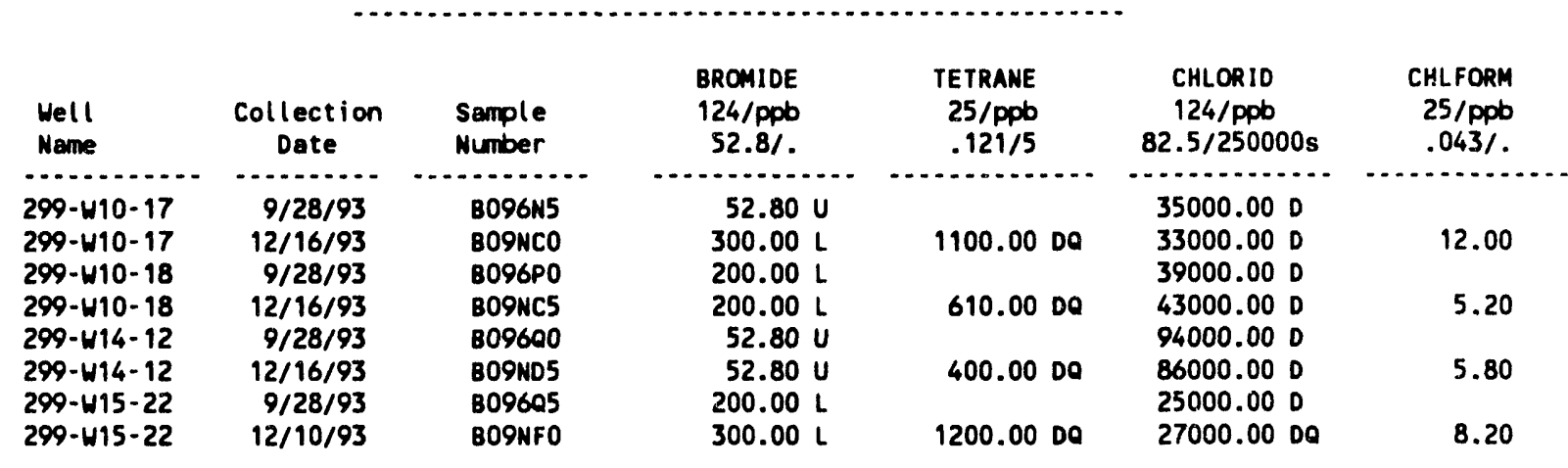

\begin{tabular}{|c|c|c|c|c|c|c|}
\hline $\begin{array}{l}\text { Hell } \\
\text { Mame }\end{array}$ & $\begin{array}{c}\text { Collection } \\
\text { Date }\end{array}$ & $\begin{array}{l}\text { Sample } \\
\text { Number }\end{array}$ & $\begin{array}{l}\text { ETHBENZ } \\
25 / \mathrm{ppb} \\
.046 / 700\end{array}$ & $\begin{array}{l}\text { FLUORID } \\
124 / \mathrm{ppb} \\
13.9 / 1400 \mathrm{i}\end{array}$ & $\begin{array}{c}\text { ALPHA } \\
135 / P C i / L \\
. / 15\end{array}$ & $\begin{array}{c}\text { BETA } \\
136 / \mathrm{pCi} / \mathrm{L} \\
. / .\end{array}$ \\
\hline $\begin{array}{l}299-w 10-17 \\
299-w 10-17 \\
299-w 10-18 \\
299-w 10-18 \\
299-w 14-12 \\
299-w 14-12 \\
299-W 15-22 \\
299-w 15-22\end{array}$ & $\begin{array}{r}9 / 28 / 93 \\
12 / 16 / 93 \\
9 / 28 / 93 \\
12 / 16 / 93 \\
9 / 28 / 93 \\
12 / 16 / 93 \\
9 / 28 / 93 \\
12 / 10 / 93\end{array}$ & $\begin{array}{l}\text { B096N5 } \\
\text { B09NCO } \\
\text { B096PO } \\
\text { B09NC5 } \\
\text { B09600 } \\
\text { B09ND5 } \\
\text { B09605 } \\
\text { B09NFO }\end{array}$ & $\begin{array}{l}.05 \mathrm{U} \\
.05 \mathrm{U} \\
.06 \mathrm{~L} \\
.05 \mathrm{U}\end{array}$ & $\begin{array}{c}2200.00 \\
2200.00 \\
1400.00 \\
1500.00 \\
5300.00 \\
900.00 \\
500.00 \\
500.00\end{array}$ & $\begin{array}{l}1.99 \\
.84 \mathrm{U} \\
2.22 \mathrm{U}\end{array}$ & $\begin{array}{r}37.50 \\
17.60 \\
834.00\end{array}$ \\
\hline
\end{tabular}

\begin{tabular}{|c|c|c|c|c|c|c|}
\hline $\begin{array}{l}\text { Well } \\
\text { Name }\end{array}$ & $\begin{array}{l}\text { Collection } \\
\text { Date }\end{array}$ & $\begin{array}{l}\text { Sample } \\
\text { Number }\end{array}$ & $\begin{array}{l}\text { LEAD } \\
40 / \mathrm{ppb} \\
.508 / 50 \mathrm{i}\end{array}$ & $\begin{array}{l}\text { METHYCH } \\
25 / \mathrm{ppb} \\
.056 / .\end{array}$ & $\begin{array}{l}\text { NITRATE } \\
124 / \mathrm{ppb} \\
44.4 / 45000\end{array}$ & $\begin{array}{l}\text { SELENUM } \\
48 / \mathrm{ppb} \\
1.21 / 10 \mathrm{i}\end{array}$ \\
\hline $299-410-17$ & $9 / 28 / 93$ & 809645 & $51 \mathrm{u}$ & . . . & 000 & \\
\hline $\begin{array}{l}299-W 10-17 \\
299-W 10-17 \\
299-W 10-18\end{array}$ & $\begin{array}{r}9 / 28 / 93 \\
12 / 16 / 93 \\
9 / 28 / 93\end{array}$ & $\begin{array}{l}\text { BO96NS } \\
\text { BO9NCO } \\
\text { B096PO }\end{array}$ & $\begin{array}{r}.21 \mathrm{~L} \\
.90 \mathrm{~L} \\
4.90 \mathrm{~L}\end{array}$ & $.19 \mathrm{BL}$ & $\begin{array}{r}120000.00 \mathrm{D} \\
57000.00 \mathrm{D}\end{array}$ & $3.80 \mathrm{~L}$ \\
\hline & & $\begin{array}{l}\text { B09NC5 } \\
\text { B09600 }\end{array}$ & $\begin{array}{l}5.00 \\
1.00 \mathrm{~L}\end{array}$ & $.10 \mathrm{BL}$ & $\begin{array}{rl}59000.00 & D \\
520000.00 & D\end{array}$ & $2.00 \mathrm{~L}$ \\
\hline $\begin{array}{l}299-W 14-12 \\
299-W 15-22 \\
299-W 15-22\end{array}$ & $\begin{array}{r}12 / 96 / 93 \\
9 / 28 / 93 \\
12 / 10 / 93\end{array}$ & $\begin{array}{l}\text { B09ND5 } \\
\text { BO9605 } \\
\text { BO9NFO }\end{array}$ & $\begin{array}{l}3.30 \mathrm{~L} \\
3.00 \mathrm{~L} \\
3.70 \mathrm{BL}\end{array}$ & $.28 \mathrm{BL}$ & $\begin{array}{r}390000.00 \mathrm{D} \\
95000.00 \mathrm{D} \\
110000.00 \mathrm{D}\end{array}$ & $2.40 \mathrm{~L}$ \\
\hline
\end{tabular}


Table 16-19. Constituents with at Least One Detected Value for the Single-Shell Tank Waste Management Area TX-TY Data for Reporting Period October 1 through December 31, 1993. (sheet 2 of 2)

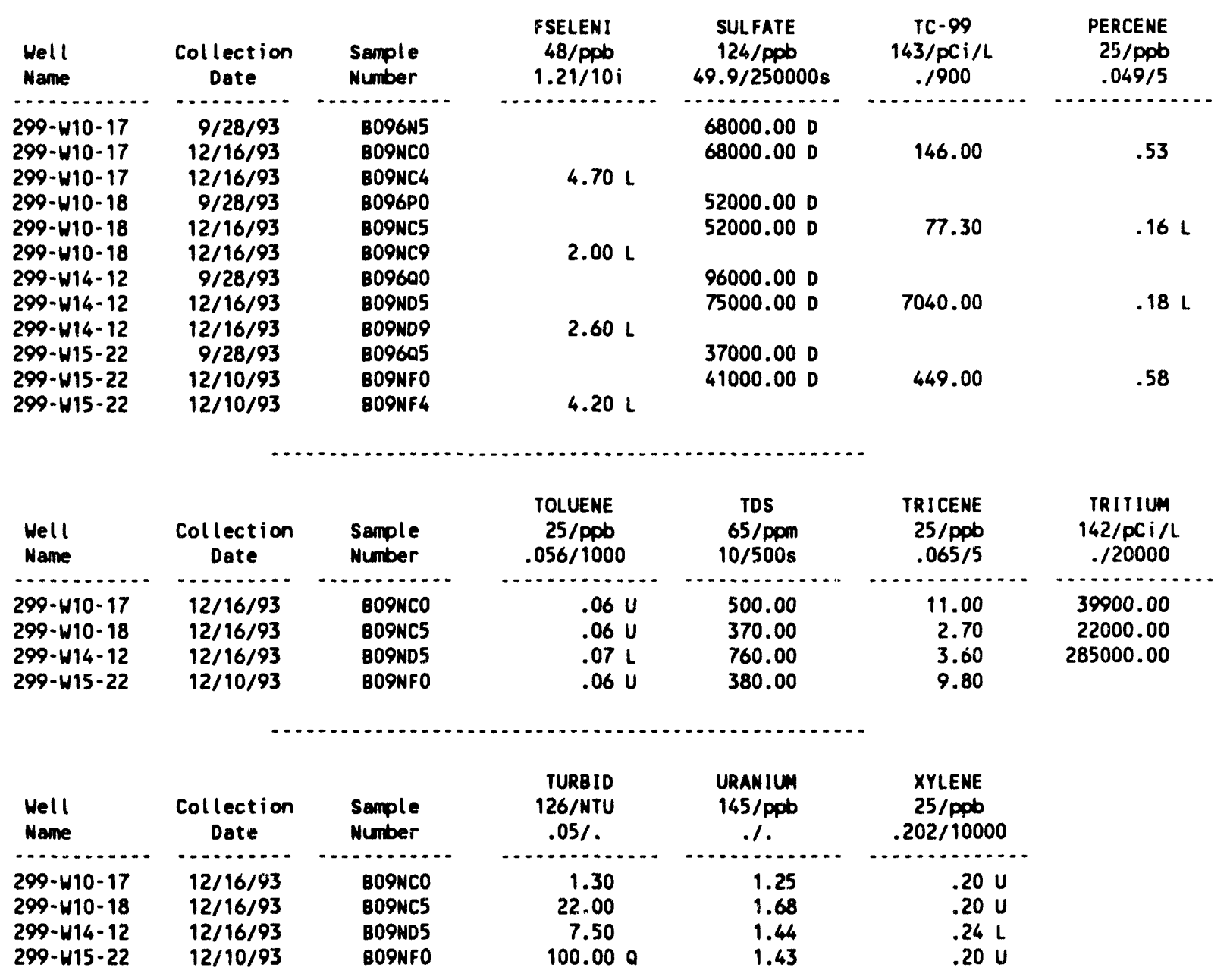

For explanation of this table, see Section 1.4 of report. 
Table 16-20. Contamination Indicator Parameters for the Single-Shell Tank Waste Management Area TX-TY Data for Reporting Period October 1 through December 1993.

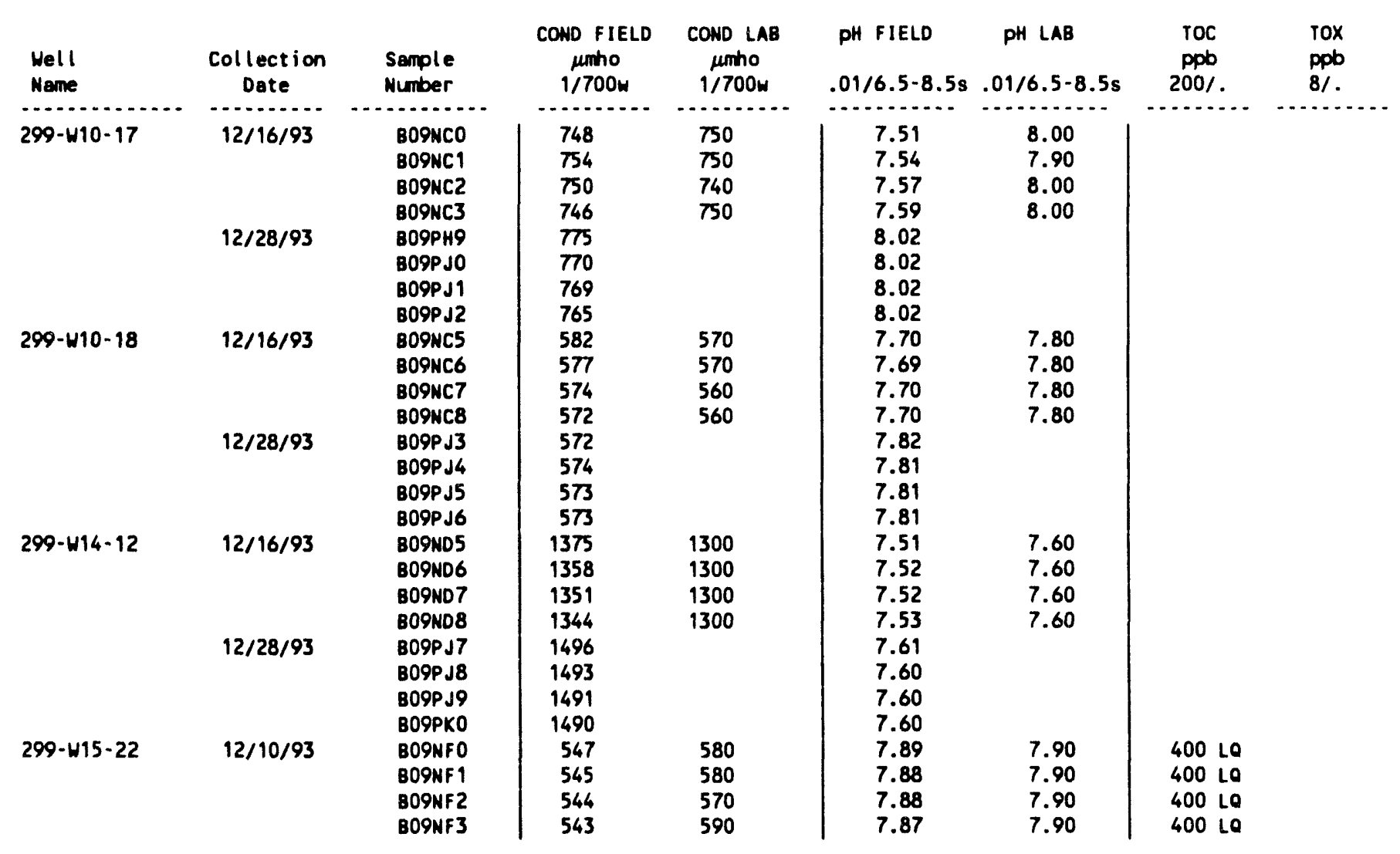

For explanation of this table, see Section 1.4 of report. 
Table 16-21. Constituent List and Summary of Results for the Single-Shell Tank Waste Management Area U Data for Reporting Period October 1

through December 31, 1993.

CONTAMINATION INDICATOR PARAMETERS

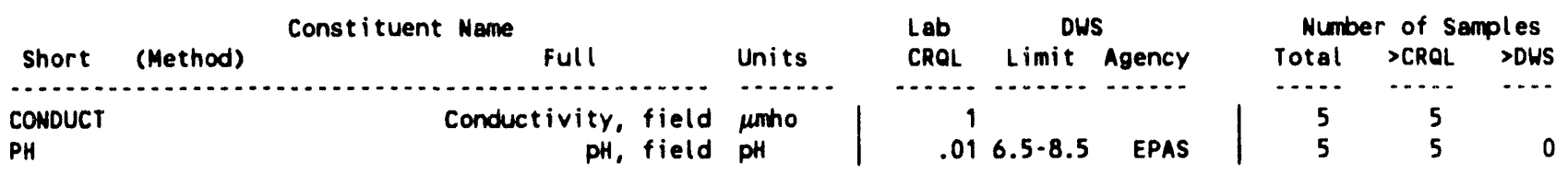

DRINKING WATER PARAMETERS

\begin{tabular}{|c|c|c|c|c|c|c|c|c|c|}
\hline \multirow[b]{2}{*}{ Short } & \multirow{2}{*}{\multicolumn{2}{|c|}{ Const ituent Name }} & \multirow[b]{2}{*}{ Units } & \multirow{2}{*}{$\begin{array}{l}\text { Lab } \\
\text { CROL }\end{array}$} & \multicolumn{2}{|c|}{ DWS } & \multicolumn{3}{|c|}{ Number of Samples } \\
\hline & & full & & & Limit & Agency & Total & $>C R Q L$ & $>$ DWS \\
\hline & & Fluoride & ppb & 13.9 & 1400 & EPA! & 6 & 6 & 0 \\
\hline
\end{tabular}

GROUNDWATER QUALITY PARAMETERS

\begin{tabular}{|c|c|c|c|c|c|c|c|c|c|}
\hline \multirow[b]{2}{*}{ Short } & \multirow[b]{2}{*}{ (Method) } & \multirow[t]{2}{*}{ Constituent Nane } & \multirow[b]{2}{*}{ Units } & \multirow{2}{*}{$\begin{array}{l}\text { Lab } \\
\text { CROL }\end{array}$} & \multicolumn{2}{|c|}{ DUS } & \multicolumn{3}{|c|}{ Number of Semples } \\
\hline & & & & & Limit & Agency & Total & $>$ CROL & $>$ DWS \\
\hline 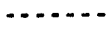 & & $\ldots .$. & $\cdots$ & $\ldots .$. & ........ & $\cdots . .$. & $\cdots \cdot$ & $\cdots$ & $\cdots$ \\
\hline $\begin{array}{l}\text { CHLORID } \\
\text { SULFATE }\end{array}$ & & $\begin{array}{l}\text { Chloride } \\
\text { Sulfate }\end{array}$ & $\begin{array}{l}p p b \\
p p b\end{array}$ & $\begin{array}{l}82.5 \\
49.9\end{array}$ & $\begin{array}{l}250000 \\
250000\end{array}$ & $\begin{array}{l}\text { EPAS } \\
\text { EPAS }\end{array}$ & $\begin{array}{l}6 \\
6\end{array}$ & $\begin{array}{l}6 \\
6\end{array}$ & $\begin{array}{l}0 \\
0\end{array}$ \\
\hline
\end{tabular}

SITE SPECIFIC AND OTHER CONSTITUENTS

\begin{tabular}{|c|c|c|c|c|c|c|c|c|c|}
\hline \multirow[b]{2}{*}{ Short } & & \multirow{2}{*}{ Constituent Name } & \multirow[b]{2}{*}{ Units } & \multicolumn{3}{|c|}{ DWS } & \multicolumn{3}{|c|}{ Number of Samples } \\
\hline & (Method) & & & CROL & Limit & Agency & Total & $>$ CRQL & $>$ DWS \\
\hline $1,1,1-T$ & & 1,1,1-Irichloroethane & pob & 072 & 200 & EPA & 4 & 0 & $\cdots$ \\
\hline $1,1,2-1$ & & 1,1,2-Trichloroethane & Ppo & .043 & 200 & EPA & 4 & 0 & 0 \\
\hline $1,1-D I C$ & & 1,1-Dichloroethane & ppb & .337 & & & 4 & 0 & \\
\hline $1,2-01 C$ & & 1,2-Dichloroethane & ppb & .139 & 5 & EPA & 4 & 0 & 0 \\
\hline 14 -dben & & 1,4-Dichlor obenzene & ppb & .107 & 75 & EPA & 4 & 0 & 0 \\
\hline BENZENE & & Benzene & ppb & .105 & 5 & EPA & 4 & 0 & 0 \\
\hline BROMIDE & & Bromide & ppb & 52.8 & & & 6 & 2 & \\
\hline TETRANE & & Carbon tetrachloride & ppb & .121 & 5 & EPA & 4 & 4 & 4 \\
\hline CHLFORM & & Chloroform & ppo & .043 & & & 4 & 4 & \\
\hline ETHBENZ & & Ethylbenzene & ppb & .046 & 700 & EPA & 4 & 0 & 0 \\
\hline METHYCH & & Methylene chloride & ppb & .056 & & & 4 & 4 & \\
\hline NITRITE & & Nitrite & ppb & 38.3 & 1000 & EPA & 6 & 1 & 0 \\
\hline PHOSPHA & & Phosphate & ppob & 147 & & & 6 & 0 & \\
\hline SULFIDE & & Sulfide & ppb & 300 & & & 2 & 0 & \\
\hline PERCENE & & Tetrachloroethene & ppob & .049 & 5 & EPA & 4 & 2 & 0 \\
\hline TOLUENE & & Tolvene & ppb & .056 & 1000 & EPA & 4 & 0 & 0 \\
\hline TDS & & Total Dissolved Solids & ppm & 10 & 500 & EPAS & 2 & 2 & 0 \\
\hline TRICEAE & & Trichloroethene & ppb & .065 & $\mathbf{5}$ & EPA & 4 & 3 & 0 \\
\hline VINYIDE & & Vinyl chloride & ppo & .266 & 2 & EPA & 4 & 0 & 0 \\
\hline XYLENE & & Xylenes (total) & ppb & .202 & 10000 & EPA & 4 & 0 & 0 \\
\hline CISIZDE & & 2-Dichloroethylene & ppb & .127 & 70 & EPA & 4 & 0 & 0 \\
\hline TRANDCE & & trans-1,2-Dichloroethylene & ppo & .149 & 100 & EPA & 4 & 0 & 0 \\
\hline
\end{tabular}

For explanation of this table, see Section 1.4 of report. 
Table 16-22. Constituents with at Least One Detected Value for the Single-Shell Tank Waste Management Area U Data for Reporting Period October 1 through December 31, 1993.

\begin{tabular}{|c|c|c|c|c|c|c|}
\hline $\begin{array}{l}\text { Nell } \\
\text { Name }\end{array}$ & $\begin{array}{c}\text { Collection } \\
\text { Date }\end{array}$ & $\begin{array}{l}\text { Sample } \\
\text { Number }\end{array}$ & $\begin{array}{c}\text { BROMIDE } \\
124 / \text { Ppb } \\
52.8 \%\end{array}$ & $\begin{array}{c}\text { TETRANE } \\
25 / \text { ppb } \\
.121 / 5\end{array}$ & $\begin{array}{c}\text { CHLORID } \\
124 / \mathrm{ppb} \\
82.5 / 250000 \mathrm{~s}\end{array}$ & $\begin{array}{c}\text { CHLFORM } \\
25 / \text { Pp6 } \\
.043 \%\end{array}$ \\
\hline $\begin{array}{l}299-W 18-25 \\
299-W 18-25 \\
299-W 18-30 \\
299-W 18-31 \\
299-W 19-31 \\
299-W 19-32\end{array}$ & $\begin{array}{l}12 / 09 / 93 \\
12 / 09 / 93 \\
12 / 09 / 93 \\
12 / 09 / 93 \\
12 / 09 / 93 \\
12 / 09 / 93\end{array}$ & $\begin{array}{l}\text { B09N35 } \\
\text { B09N36 } \\
B 09 N 37 \\
809 N 33 \\
\text { B09N34 } \\
\text { B09N38 }\end{array}$ & $\begin{array}{l}52.80 \mathrm{UO} \\
70.00 \mathrm{LO} \\
52.80 \mathrm{U} \\
52.80 \mathrm{U} \\
52.80 \mathrm{U} \\
54.00 \mathrm{~L}\end{array}$ & $\begin{array}{l}38.000 \\
37.000 \\
71.000 \\
16.00\end{array}$ & $\begin{array}{c}10000.00 \\
9500.00 \\
2900.00 \\
5300.00 \\
4900.00 \\
28000.00\end{array}$ & $\begin{array}{l}2.00 \\
1.90 \\
1.10\end{array}$ \\
\hline
\end{tabular}

\begin{tabular}{|c|c|c|c|c|c|c|}
\hline $\begin{array}{l}\text { Well } \\
\text { Name }\end{array}$ & $\begin{array}{c}\text { Collection } \\
\text { Date }\end{array}$ & $\begin{array}{l}\text { Sample } \\
\text { Number }\end{array}$ & $\begin{array}{l}\text { FLUORID } \\
124 / \mathrm{pPb} \\
13.9 / 1400 \mathrm{i}\end{array}$ & $\begin{array}{l}\text { METHYCH } \\
25 / \mathrm{ppb} \\
.056 / .\end{array}$ & $\begin{array}{l}\text { NITRATE } \\
124 / \mathrm{ppb} \\
44.4 / 45000\end{array}$ & $\begin{array}{c}\text { NITRITE } \\
124 / \mathrm{ppb} \\
38.3 / 1000\end{array}$ \\
\hline $\begin{array}{l}299-W 18-25 \\
299-W 18-25 \\
299-W 18-30 \\
299-W 18-31 \\
299-W 19-31 \\
299-W 19-32\end{array}$ & $\begin{array}{l}12 / 09 / 93 \\
12 / 09 / 93 \\
12 / 09 / 93 \\
12 / 09 / 93 \\
12 / 09 / 93 \\
12 / 09 / 93\end{array}$ & $\begin{array}{l}\text { B09N35 } \\
\text { B09N36 } \\
B 09 N 37 \\
B 09 N 33 \\
B 09 N 34 \\
B 09 N 38\end{array}$ & $\begin{array}{l}800.000 \\
500.00 a \\
100.00 \\
500.00 \\
900.00 \\
900.00\end{array}$ & $\begin{array}{ll}.14 \mathrm{BL} \\
.12 \mathrm{BL} \\
.13 \mathrm{BL}\end{array}$ & $\begin{array}{c}11000.00 \mathrm{D} \\
11000.00 \mathrm{D} \\
4000.00 \\
9200.00 \\
5300.00 \\
18000.00 \mathrm{D}\end{array}$ & $\begin{array}{l}38.30 \mathrm{U} \\
38.30 \mathrm{U} \\
38.30 \mathrm{U} \\
400.00 \\
38.30 \mathrm{U} \\
38.30 \mathrm{U}\end{array}$ \\
\hline
\end{tabular}

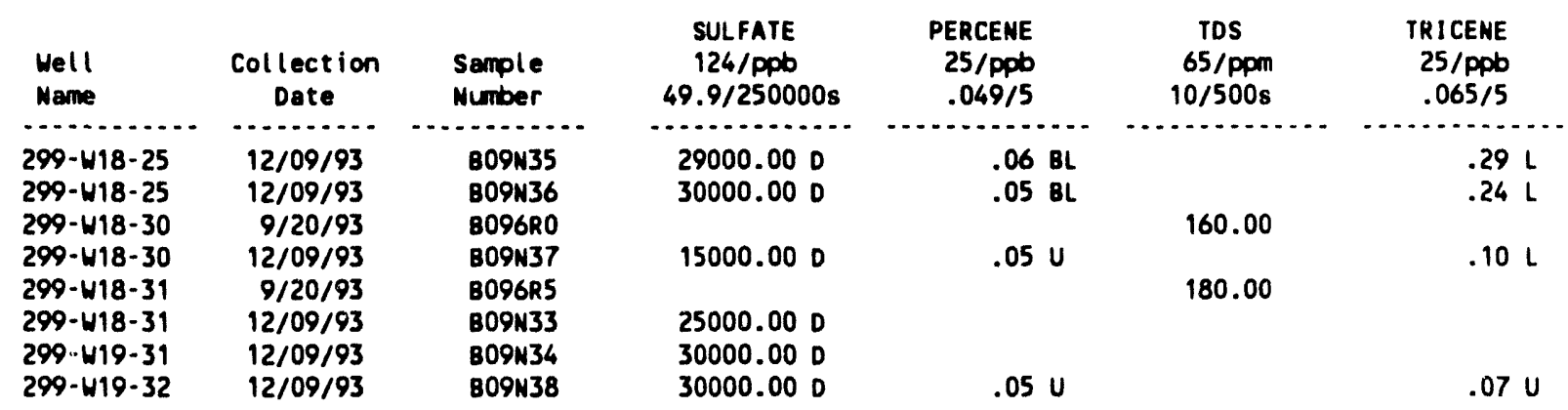

For explanation of this table, see Section 1.4 of report. 
Table 16-23. Contamination Indicator Parameters for the Single-Shell Tank Waste Management Area U Data for Reporting Period October 1 through December 31, 1993.

\begin{tabular}{|c|c|c|c|c|c|c|c|c|}
\hline $\begin{array}{l}\text { Well } \\
\text { Name }\end{array}$ & $\begin{array}{c}\text { Collection } \\
\text { Date }\end{array}$ & $\begin{array}{l}\text { Sample } \\
\text { Number }\end{array}$ & $\begin{array}{c}\text { COND FIELD } \\
\mu m h o \\
1 / 700 \mathrm{~W}\end{array}$ & $\begin{array}{c}\text { COND LAB } \\
\substack{\mu \mathrm{mho} \\
1 / 700 \mathrm{~W}}\end{array}$ & $\begin{array}{l}\text { PH FIELD } \\
.01 / 6.5-8.5 \mathrm{~s}\end{array}$ & $\begin{array}{c}\text { PH LAB } \\
.01 / 6.5-8.5 \mathrm{~s}\end{array}$ & $\begin{array}{c}\text { TOC } \\
\text { Ppb } \\
200 \%\end{array}$ & $\begin{array}{l}\text { TOX } \\
\text { ppb } \\
8 / .\end{array}$ \\
\hline $\begin{array}{l}299-W 18-25 \\
299-W 18-30 \\
299-W 18-31 \\
299-W 19-31 \\
299-W 19-32\end{array}$ & $\begin{array}{l}12 / 09 / 93 \\
12 / 09 / 93 \\
12 / 09 / 93 \\
12 / 09 / 93 \\
12 / 09 / 93\end{array}$ & $\begin{array}{l}\text { B09N35 } \\
809 N 37 \\
B 09 N 33 \\
809 N 34 \\
809 N 38\end{array}$ & $\begin{array}{l}306 \\
221 \\
293 \\
344 \\
416\end{array}$ & & $\begin{array}{l}7.87 \\
8.02 \\
7.90 \\
8.04 \\
8.19\end{array}$ & & & \\
\hline
\end{tabular}

For explanation of this table, see Section 1.4 of report. 
DOE/RL-93-56-4

\section{CONTENTS}

17.0300 AREA PROCESS TRENCHES . . . . . . . . . . . . 17-1

17.1 INTRODUCTION . . . . . . . . . . . . . . . . . 17-1

17.2 WATER LEVEL MEASUREMENTS . . . . . . . . . . . . 17-1

17.3 WATER CHEMISTRY DATA ...................... 


\section{LIST OF FIGURES}

17-1 Monitoring Well Locations for the 300 Area Process Trenches . . 17-2

\section{LIST OF TABLES}

17-1 Monitoring Well Purpose and Sampling Schedule for the 300 Area Process Trenches Network . . . . . . . . . . . . 17-3

17-2 RCRA Water Level Measurement Report for the 300 Area Process Trenches, Fourth Quarter 1993 . . . . . . . . . . . 17-4

17-3 Constituent List and Summary of Results for the 300 Area Process Trenches Data for Keporting Period October 1 through December 31, 1993 .................. . . 17-9

17-4 Constituents with at Least One Detected Value for the 300 Area Process Trenches Data for Reporting Period October 1 through December 31, 1993 ................. 17-12

17-5 Contamination Indicator Parameters for the 300 Area Process Trenches Data for Reporting Period October 1 through December 31, 1993 
DOE/RL-93-56-4

\subsection{AREA PROCESS TRENCHES \\ J. V. Borghese \\ Pacific Northwest Laboratory}

\subsection{INTRODUCTION}

The groundwater near the 300 Area Process Trenches (316-5) has been monitored by a RCRA well network since June 1985. The groundwater monitoring is being conducted under interim-status regulations (40 CFR 265) and is an assessment level program (Schalla 1988). The process trenches are located in the northern portion of the 300 Area and are used for the disposal of wastewater from various 300 Area operations. The wastewater primarily consists of cooling water, with small quantities of nonhazardous maintenance and process waste.

Monitoring wells were constructed in response to a Consent Agreement and Compliance Order (Ecology and EPA 1986). Groundwater wells currently used to monitor the facility are shown in Figure 17-1 and are 1isted in Table 17-1; they consist of both pre-RCRA and RCRA standard wells.

\subsection{MATER LEVEL MEASUREMENTS}

Water levels are measured monthly and at the time of sampling in selected 300 Area wells. Water level measurements obtained during the fourth quarter of 1993 are presented in Table 17-2.

\subsection{MATER CHEMISTRY DATA}

Water chemistry samples are collected semiannually from the 300 Area process trenches monitoring wells. In addition to semiannual samples, samples are collected quarterly from well 399-1-17A to provide near-trench monitoring of contaminants. Sampling dates are listed in Table 17-1. Analytical results received for the sampling events are presented in Tables 17-3 through 17-5. These tables include previously unreported data. An explanation of the data flags is given in Section 1.4. The D flags shown in Table 17-3 indicate that the sample was diluted for analysis. A discussion of the data quality can be found in Section 1.2 .

None of the results received for this quarter had constituents that were above the DWS. However, previously unreported data for the September 1993 sampling event having the constituents of iron, chromium, and manganese exceeded the DWS. 
Figure 17-1. Monitoring Well Locations for the 300 Area Process Trenches.

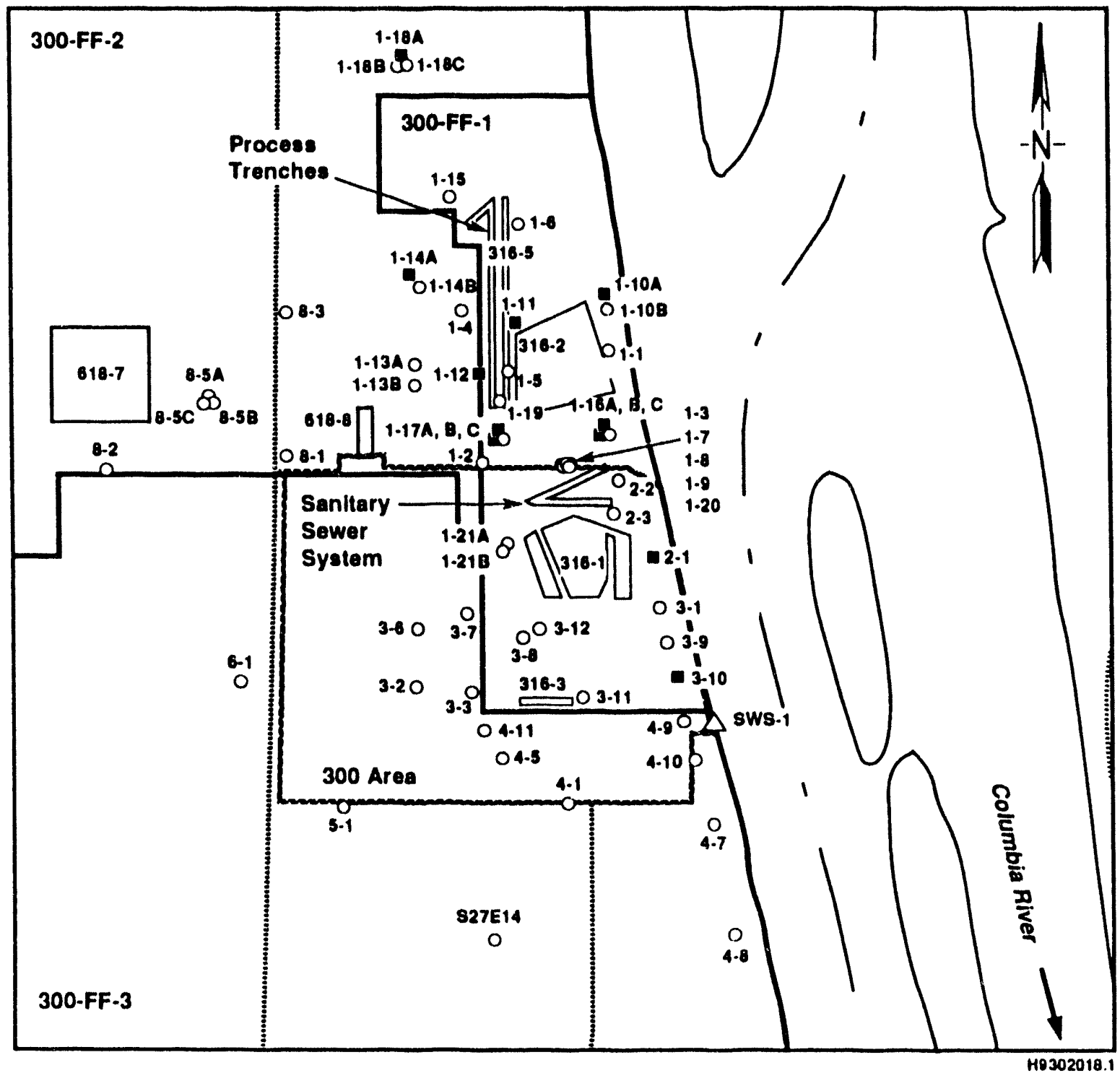
O $\quad 1.12$
- 4.7 Monitoring Network Well
$\triangle$ sws-1 Surface-Water Monitoring Station
Roads

Well Location and Number (Wells Preflxed by 399-,

Except Those Beginning with S are Prefixed with 699-) 
Table 17-1. Monitoring Well Purpose and Sampling Schedule for the 300 Area Process Trenches Network.

\begin{tabular}{|c|c|l|c|c|}
\hline $\begin{array}{c}\text { Well no. } \\
(399-)\end{array}$ & $\begin{array}{c}\text { Relative } \\
\text { position }\end{array}$ & \multicolumn{1}{|c|}{ Hydrogeologic unit } & $\begin{array}{c}\text { Sample } \\
\text { frequency }\end{array}$ & $\begin{array}{c}\text { Sample date, } \\
\text { 4th Qtr 1993 }\end{array}$ \\
\hline $1-10 \mathrm{~A}$ & Downgradient & Hanford/Ringold: Water Table & Semiannually & -- \\
\hline $1-11$ & Adjacent & Hanford/Ringold: Water Table & Semiannually & -- \\
\hline $1-12$ & Downgradient & Hanford/Ringold: Water Table & Semiannually & -- \\
\hline $1-14 \mathrm{~A}$ & Adjacent & Hanford: Water Table & Semiannually & -- \\
\hline $1-16 \mathrm{~A}$ & Downgradient & Ringold: Water Table & Semiannually & -- \\
\hline $1-16 \mathrm{~B}$ & Downgradient & $\begin{array}{l}\text { Ringold: Bottom of } \\
\text { Unconfined Aquifer }\end{array}$ & Semiannually & -- \\
\hline $1-17 \mathrm{~A}$ & Downgradient & Ringold: Water Table & Quarterly & $12 / 09 / 93$ \\
\hline $1-17 \mathrm{~B}$ & Downgradient & $\begin{array}{l}\text { Ringold: Bottom of } \\
\text { Unconfined Aquifer }\end{array}$ & Semiannually & -- \\
\hline $1-18 \mathrm{~A}$ & Upgradient & Ringold: Water Table & Semiannually & -- \\
\hline $2-1$ & Downgradient & Hanford/Ringold: Water Table & Semiannually & -- \\
\hline $3-10$ & Downgradient & Hanford: Water Table & Semiannually & -- \\
\hline
\end{tabular}

Note: Hydrogeologic units include the sandy gravels of the Hanford formation and silty sands of the Ringold Formation. Water levels are measured in all wells monthly. 
Table 17-2. RCRA Water Level Measurement Report for the 300 Area Process Trenches, Fourth Quarter 1993. (sheet 1 of 5)

Wel1 Date $\begin{gathered}\text { Depth to } \\ \text { water }(\mathrm{ft})\end{gathered} \begin{gathered}\text { Water level } \\ \text { elevation } \\ \text { above ms }(\mathrm{ft})\end{gathered}$

Wells Monitoring the Top of the Unconfined Aquifer

\begin{tabular}{|c|c|c|c|}
\hline $399-1-1$ & $\begin{array}{l}10 / 20 / 93 \\
11 / 22 / 93 \\
12 / 17 / 93\end{array}$ & $\begin{array}{l}36.50 \\
35.41 \\
34.27\end{array}$ & $\begin{array}{l}340.16 \\
341.25 \\
342.39\end{array}$ \\
\hline $399-1-10 A$ & $\begin{array}{l}10 / 20 / 93 \\
11 / 22 / 93 \\
12 / 17 / 93\end{array}$ & $\begin{array}{l}33.40 \\
32.42 \\
31.15\end{array}$ & $\begin{array}{l}340.17 \\
341.15 \\
342.42\end{array}$ \\
\hline $399-1-11$ & $\begin{array}{l}10 / 20 / 93 \\
11 / 22 / 93 \\
12 / 17 / 93\end{array}$ & $\begin{array}{l}37.40 \\
36.05 \\
35.41\end{array}$ & $\begin{array}{l}340.32 \\
341.67 \\
342.31\end{array}$ \\
\hline $399-1-12$ & $\begin{array}{l}10 / 20 / 93 \\
11 / 22 / 93 \\
12 / 17 / 93\end{array}$ & $\begin{array}{l}44.44 \\
42.94 \\
42.33\end{array}$ & $\begin{array}{l}339.97 \\
341.47 \\
342.08\end{array}$ \\
\hline $399-1-14 A$ & $\begin{array}{l}10 / 20 / 93 \\
11 / 22 / 93 \\
12 / 17 / 93\end{array}$ & $\begin{array}{l}42.54 \\
41.32 \\
40.87\end{array}$ & $\begin{array}{l}340.64 \\
341.86 \\
342.31\end{array}$ \\
\hline $399-1-15$ & $\begin{array}{l}10 / 20 / 93 \\
11 / 22 / 93 \\
12 / 17 / 93\end{array}$ & $\begin{array}{l}38.80 \\
37.61 \\
37.10\end{array}$ & $\begin{array}{l}340.73 \\
341.92 \\
342.43\end{array}$ \\
\hline $399-1-16 A$ & $\begin{array}{l}10 / 20 / 93 \\
11 / 22 / 93 \\
12 / 17 / 93\end{array}$ & $\begin{array}{l}41.68 \\
40.25 \\
39.38\end{array}$ & $\begin{array}{l}339.79 \\
341.22 \\
342.09\end{array}$ \\
\hline $399-1-17 A$ & $\begin{array}{l}10 / 20 / 93 \\
11 / 17 / 93 \\
11 / 22 / 93 \\
12 / 17 / 93\end{array}$ & $\begin{array}{l}37.60 \\
35.92 \\
36.10 \\
35.45\end{array}$ & $\begin{array}{l}339.84 \\
341.52 \\
341.34 \\
341.99\end{array}$ \\
\hline $399-1-18 A$ & $\begin{array}{l}10 / 20 / 93 \\
11 / 22 / 93 \\
12 / 17 / 93\end{array}$ & $\begin{array}{l}49.48 \\
48.45 \\
47.93\end{array}$ & $\begin{array}{l}341.30 \\
342.33 \\
342.85\end{array}$ \\
\hline $399-1-19$ & $\begin{array}{l}10 / 20 / 93 \\
11 / 22 / 93 \\
12 / 17 / 93\end{array}$ & $\begin{array}{l}34.65 \\
33.15 \\
32.52\end{array}$ & $\begin{array}{l}339.99 \\
341.49 \\
342.12\end{array}$ \\
\hline
\end{tabular}


Table 17-2. RCRA Water Level Measurement Report for the 300 Area Process Trenches, Fourth Quarter 1993. (sheet 2 of 5)

We11 Date $\begin{gathered}\text { Depth to } \\ \text { water }(\mathrm{ft})\end{gathered} \begin{gathered}\text { Water level } \\ \text { elevation } \\ \text { above ms } 1 \text { (ft) }\end{gathered}$

Wells Monitoring the Top of the Unconfined Aquifer

\begin{tabular}{|c|c|c|c|}
\hline $399-1-3$ & $\begin{array}{l}10 / 20 / 93 \\
11 / 22 / 93 \\
12 / 17 / 93\end{array}$ & $\begin{array}{l}44.90 \\
43.49 \\
42.94\end{array}$ & $\begin{array}{l}339.81 \\
341.22 \\
341.77\end{array}$ \\
\hline $399-1-4$ & $\begin{array}{l}10 / 20 / 93 \\
11 / 22 / 93 \\
12 / 17 / 93\end{array}$ & $\begin{array}{l}40.05 \\
38.78 \\
38.27\end{array}$ & $\begin{array}{l}340.53 \\
341.80 \\
342.31\end{array}$ \\
\hline $399-1-5$ & $\begin{array}{l}10 / 20 / 93 \\
11 / 22 / 93 \\
12 / 17 / 93\end{array}$ & $\begin{array}{l}39.83 \\
38.36 \\
37.68\end{array}$ & $\begin{array}{l}339.94 \\
341.41 \\
342.09\end{array}$ \\
\hline $399-1-7$ & $\begin{array}{l}10 / 20 / 93 \\
11 / 22 / 93 \\
12 / 17 / 93\end{array}$ & $\begin{array}{l}45.80 \\
44.45 \\
43.52\end{array}$ & $\begin{array}{l}339.80 \\
341.15 \\
342.08\end{array}$ \\
\hline $399-1-8$ & $\begin{array}{l}10 / 20 / 93 \\
11 / 22 / 93 \\
12 / 17 / 93\end{array}$ & $\begin{array}{l}45.06 \\
43.69 \\
42.81\end{array}$ & $\begin{array}{l}339.82 \\
341.19 \\
342.07\end{array}$ \\
\hline $399-2-1$ & $\begin{array}{l}10 / 20 / 93 \\
11 / 22 / 93 \\
12 / 17 / 93\end{array}$ & $\begin{array}{l}35.44 \\
34.36 \\
33.15\end{array}$ & $\begin{array}{l}339.79 \\
340.87 \\
342.08\end{array}$ \\
\hline $399-2-2$ & $\begin{array}{l}10 / 07 / 93 \\
10 / 20 / 93 \\
11 / 22 / 93 \\
12 / 17 / 93\end{array}$ & $\begin{array}{l}37.32 \\
37.72 \\
36.52 \\
35.43\end{array}$ & $\begin{array}{l}340.20 * \\
339.80 \\
341.00 \\
342.09\end{array}$ \\
\hline $399-2-3$ & $\begin{array}{l}10 / 20 / 93 \\
11 / 22 / 93 \\
12 / 17 / 93\end{array}$ & $\begin{array}{l}35.63 \\
34.98 \\
33.34\end{array}$ & $\begin{array}{l}339.79 \\
340.44 \\
342.08\end{array}$ \\
\hline $399-3-1$ & $\begin{array}{l}10 / 20 / 93 \\
11 / 22 / 93 \\
12 / 17 / 93\end{array}$ & $\begin{array}{l}44.69 \\
43.46 \\
42.39\end{array}$ & $\begin{array}{l}339.70 \\
340.93 \\
342.00\end{array}$ \\
\hline
\end{tabular}


Table 17-2. RCRA Water Level Measurement Report for the 300 Area Process Trenches, Fourth Quarter 1993. (sheet 3 of 5)

Well Date $\begin{gathered}\text { Depth to } \\ \text { water }(\mathrm{ft})\end{gathered} \begin{gathered}\text { Water level } \\ \text { elevation } \\ \text { above ms } 1 \text { ( } \mathrm{ft})\end{gathered}$

Wells Monitoring the Top of the Unconfined Aquifer

\begin{tabular}{|c|c|c|c|}
\hline $399-3-10$ & $\begin{array}{l}10 / 20 / 93 \\
11 / 22 / 93 \\
12 / 17 / 93\end{array}$ & $\begin{array}{l}45.84 \\
44.43 \\
43.39\end{array}$ & $\begin{array}{l}339.51 \\
340.92 \\
341.96\end{array}$ \\
\hline $399-3-12$ & $\begin{array}{l}11 / 22 / 93 \\
12 / 17 / 93\end{array}$ & $\begin{array}{l}46.97 \\
46.34\end{array}$ & $\begin{array}{l}341.07 \\
341.70\end{array}$ \\
\hline $399-3-6$ & $\begin{array}{l}10 / 20 / 93 \\
12 / 17 / 93\end{array}$ & $\begin{array}{l}53.05 \\
50.97\end{array}$ & $\begin{array}{l}339.75 \\
341.83\end{array}$ \\
\hline $399-3-9$ & $\begin{array}{l}10 / 20 / 93 \\
11 / 22 / 93 \\
12 / 17 / 93\end{array}$ & $\begin{array}{l}48.60 \\
47.09 \\
46.25\end{array}$ & $\begin{array}{l}339.48 \\
340.99 \\
341.83\end{array}$ \\
\hline $399-4-1$ & $\begin{array}{l}10 / 20 / 93 \\
11 / 22 / 93 \\
12 / 17 / 93\end{array}$ & $\begin{array}{l}56.50 \\
54.71 \\
53.79\end{array}$ & $\begin{array}{l}339.10 \\
340.89 \\
341.81\end{array}$ \\
\hline $399-4-10$ & $\begin{array}{l}10 / 20 / 93 \\
11 / 22 / 93 \\
12 / 17 / 93\end{array}$ & $\begin{array}{l}38.99 \\
37.70 \\
36.56\end{array}$ & $\begin{array}{l}339.55 \\
340.84 \\
341.98\end{array}$ \\
\hline $399-4-11$ & $\begin{array}{l}10 / 20 / 93 \\
11 / 22 / 93 \\
12 / 17 / 93\end{array}$ & $\begin{array}{l}64.99 \\
63.39 \\
62.61\end{array}$ & $\begin{array}{l}339.46 \\
341.06 \\
341.84\end{array}$ \\
\hline $399-4-7$ & $\begin{array}{l}10 / 20 / 93 \\
11 / 22 / 93 \\
12 / 17 / 93\end{array}$ & $\begin{array}{l}39.14 \\
37.76 \\
36.64\end{array}$ & $\begin{array}{l}339.42 \\
340.80 \\
341.92\end{array}$ \\
\hline $399-4-9$ & $\begin{array}{l}11 / 22 / 93 \\
12 / 17 / 93\end{array}$ & $\begin{array}{l}41.23 \\
40.16\end{array}$ & $\begin{array}{l}340.93 \\
342.00\end{array}$ \\
\hline $399-5-1$ & $\begin{array}{l}10 / 20 / 93 \\
11 / 22 / 93 \\
12 / 17 / 93\end{array}$ & $\begin{array}{l}55.36 \\
54.01 \\
53.63\end{array}$ & $\begin{array}{l}340.17 \\
341.52 \\
341.90\end{array}$ \\
\hline $399-6-1$ & $\begin{array}{l}10 / 20 / 93 \\
11 / 22 / 93 \\
12 / 17 / 93\end{array}$ & $\begin{array}{l}48.28 \\
47.07 \\
46.81\end{array}$ & $\begin{array}{l}340.51 \\
341.72 \\
341.98\end{array}$ \\
\hline
\end{tabular}




$$
\mathrm{DOE} / \mathrm{RL}-93-56-4
$$

Table 17-2. RCRA Water Level Measurement Report for the 300 Area Process Trenches, Fourth Quarter 1993. (sheet 4 of 5)

\begin{tabular}{lccc}
\hline Well & Date & $\begin{array}{c}\text { Depth to } \\
\text { water }(\mathrm{ft})\end{array}$ & $\begin{array}{c}\text { Water level } \\
\text { elevation } \\
\text { above ms }\end{array}$ \\
\hline & Wells $(\mathrm{ft})$ \\
\hline $399-8-1$ & $10 / 20 / 93$ & 55.81 & 340.32 \\
& $11 / 22 / 93$ & 54.47 & 341.66 \\
$399-8-2$ & $12 / 17 / 93$ & 54.14 & 341.99 \\
& $10 / 20 / 93$ & 56.60 & 341.42 \\
& $11 / 22 / 93$ & 55.82 & 342.20 \\
$399-8-3$ & $12 / 17 / 93$ & 55.61 & 342.41 \\
& $10 / 20 / 93$ & 54.04 & 340.71 \\
& $11 / 22 / 93$ & 52.84 & 341.91 \\
& $12 / 17 / 93$ & 52.58 & 342.17 \\
$699-527-E 14$ & $10 / 20 / 93$ & 60.38 & 339.30 \\
& $11 / 17 / 93$ & 58.99 & $340.69 *$ \\
& $11 / 22 / 93$ & 58.78 & 340.90 \\
& $12 / 17 / 93$ & 57.92 & 341.76 \\
\hline
\end{tabular}

Wells Monitoring the Bottom of the Unconfined Aquifer

\begin{tabular}{llll}
\hline $399-1-16 B$ & $10 / 20 / 93$ & 41.85 & 339.23 \\
& $11 / 22 / 93$ & 39.95 & 341.13 \\
$399-1-17 B$ & $12 / 17 / 93$ & 38.90 & 342.18 \\
& $10 / 20 / 93$ & 37.89 & 339.91 \\
$399-1-18 B$ & $11 / 22 / 93$ & 36.44 & 341.36 \\
& $12 / 17 / 93$ & 35.72 & 342.08 \\
& $10 / 20 / 93$ & 48.25 & 341.64 \\
& $11 / 22 / 93$ & 47.34 & 342.55 \\
\hline
\end{tabular}

Wells Monitoring the Confined Aquifer

\begin{tabular}{llll}
\hline $399-1-17 C$ & $10 / 20 / 93$ & 2.50 & 375.56 \\
& $11 / 22 / 93$ & 2.76 & 375.30 \\
& $12 / 17 / 93$ & 2.18 & 375.88
\end{tabular}


Table 17-2. RCRA Water Level Measurement Report for the 300 Area Process Trenches, Fourth Quarter 1993. (sheet 5 of 5)

\begin{tabular}{llll}
\hline Wel1 & Date & $\begin{array}{c}\text { Depth to } \\
\text { water }(\mathrm{ft})\end{array}$ & $\begin{array}{c}\text { Water level } \\
\text { elevation } \\
\text { above ms }(\mathrm{ft})\end{array}$ \\
\hline $399-1-18 \mathrm{C}$ & Wells Monitoring the Confined Aquifer \\
\hline & $10 / 20 / 93$ & 45.75 & 342.26 \\
$11 / 22 / 93$ & 44.87 & 343.14 \\
$12 / 17 / 93$ & 44.36 & 343.65 \\
\hline
\end{tabular}

Notes: 1. Water level elevations are calculated by subtracting the measured depth to water from the surveyed elevation for the well.

2. Depth-to-water values are transcribed from field records.

3. Elevations marked with an $' * 1$ were measured at the time of sampling.

4. To convert feet to meters multiply by 0.3048 . 
Table 17-3. Constituent List and Summary of Results for the 300 Area Process Trenches Data for Reporting Period October 1 through

December 31, 1993. (sheet 1 of 3)

CONTAMINATION INDICATOR PARAMETERS

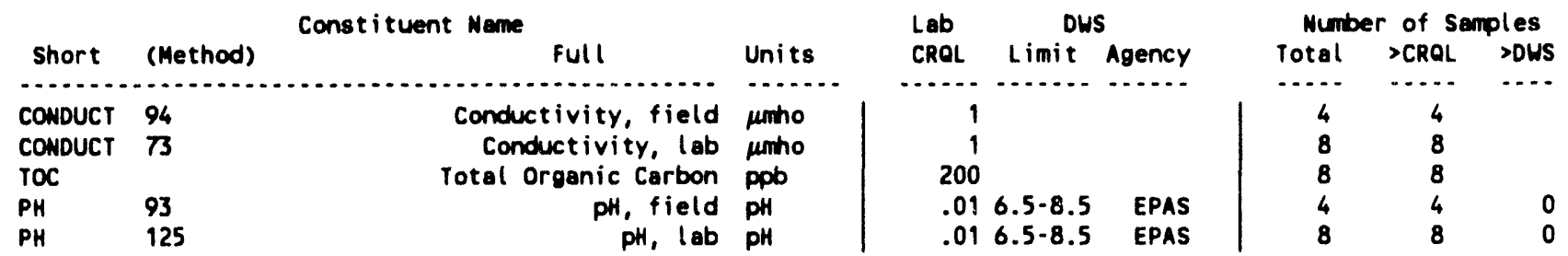

DRINKING HATER PARAMETERS

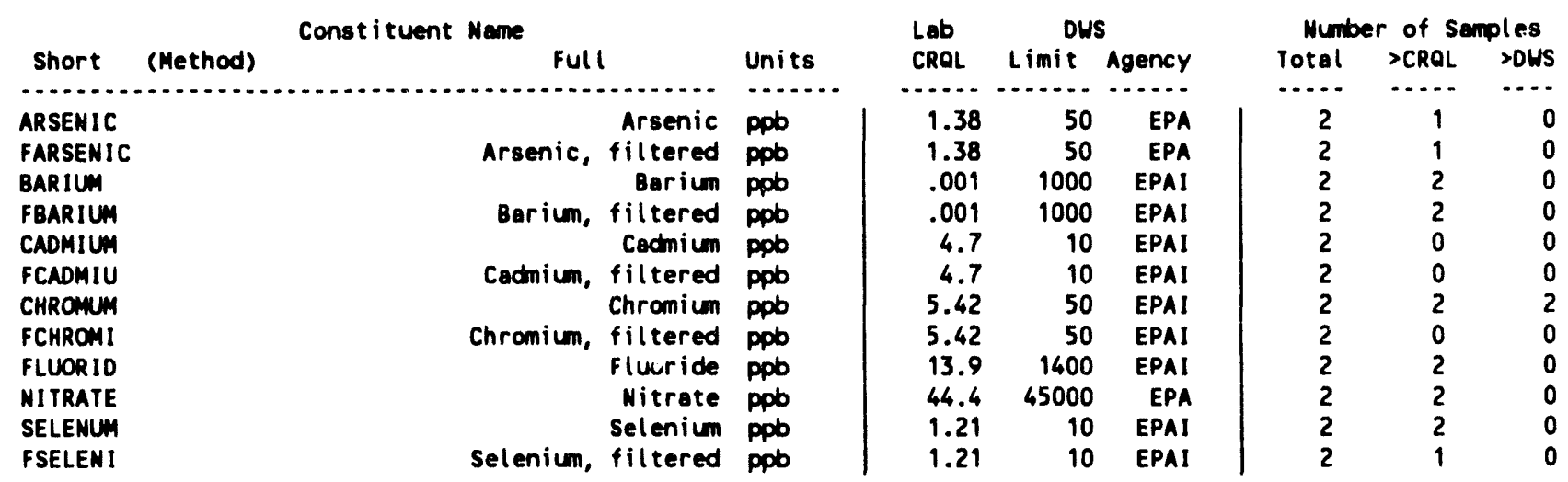

GROUNDWATER QUALITY PARAMETERS

\begin{tabular}{|c|c|c|c|c|c|c|c|c|c|}
\hline \multirow[b]{2}{*}{ Short } & \multirow[b]{2}{*}{ (Method) } & \multirow[t]{2}{*}{ Constituent Neme } & \multirow[b]{2}{*}{ Units } & \multirow{2}{*}{$\begin{array}{l}\text { Lab } \\
\text { CRQL }\end{array}$} & \multicolumn{2}{|c|}{ DWS } & \multicolumn{3}{|c|}{ Number of Samples } \\
\hline & & & & & Limit & Agency & Total & $>$ CRQL & $>0$ WS \\
\hline CHLORIO & & Chloride & ppb & 82.5 & 250000 & EPAS & 2 & 2 & 0 \\
\hline IRON & & Iron & ppo & 10.3 & 300 & EPAS & 2 & 2 & 2 \\
\hline FIRON & & Iron, filtered & ppb & 10.3 & 300 & EPAS & 2 & 1 & 1 \\
\hline MANGESE & & Manganese & ppo & 1.35 & 50 & EPAS & 2 & 2 & 1 \\
\hline FMANGAN & & Mangenese, filtered & ppob & 1.35 & 50 & EPAS & 2 & 1 & 1 \\
\hline soolun & & Sodium & ppob & 40.9 & & & 2 & 2 & \\
\hline FSOOIUM & & Sodium, filtered & ppb & 40.9 & & & 2 & 2 & \\
\hline SULFATE & & Sulfate & ppb & 49.9 & 250000 & EPAS & 2 & 2 & 0 \\
\hline
\end{tabular}

SITE SPECIFIC AND OTHER CONSTITUENTS

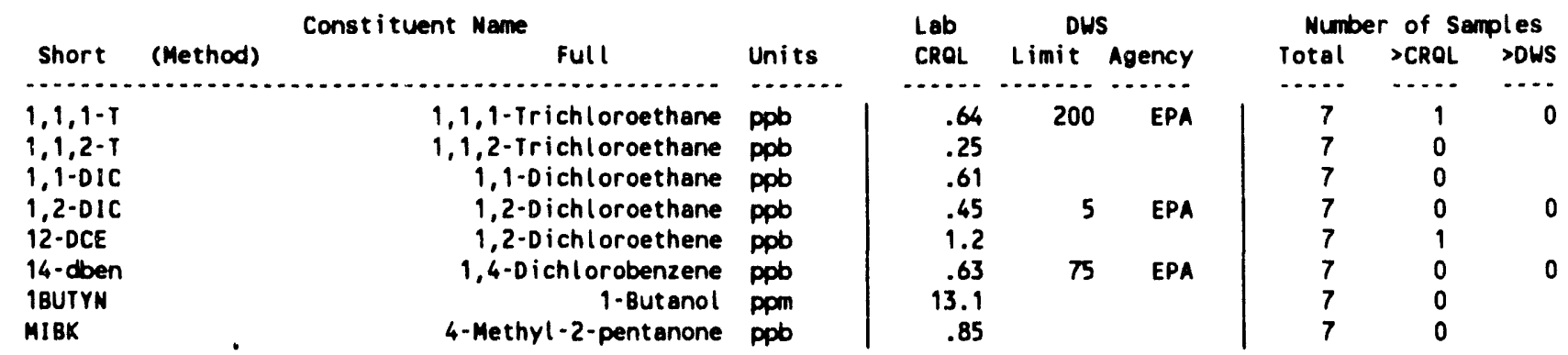


Table 17-3. Constituent List and Summary of Results for the 300 Area Process Trenches Data for Reporting Period October 1 through

December 31, 1993. (sheet 2 of 3 )

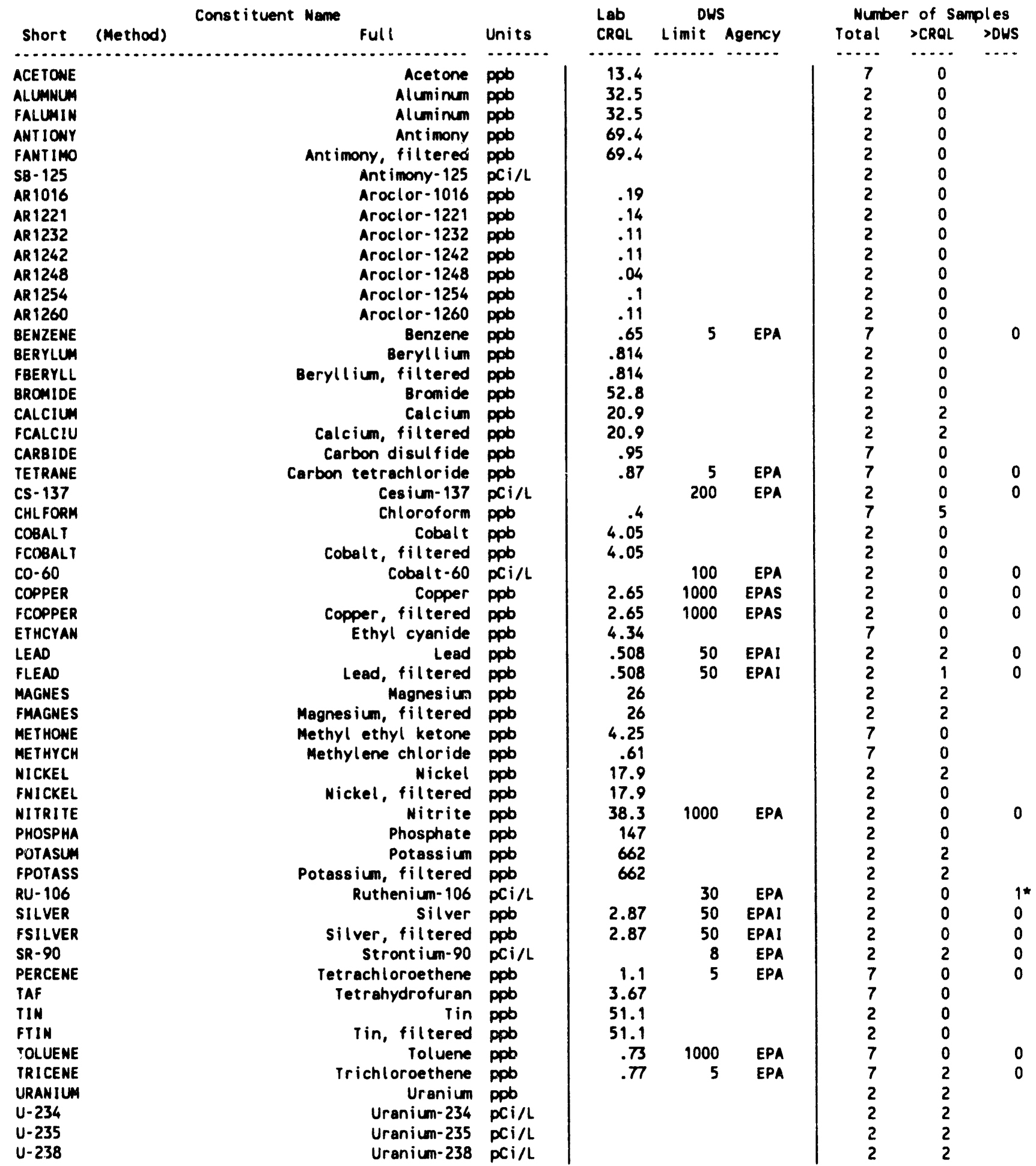


Table 17-3. Constituent List and Summary of Results for the 300 Area Process Trenches Data for Reporting Period October 1 through

December 31, 1993. (sheet 3 of 3)

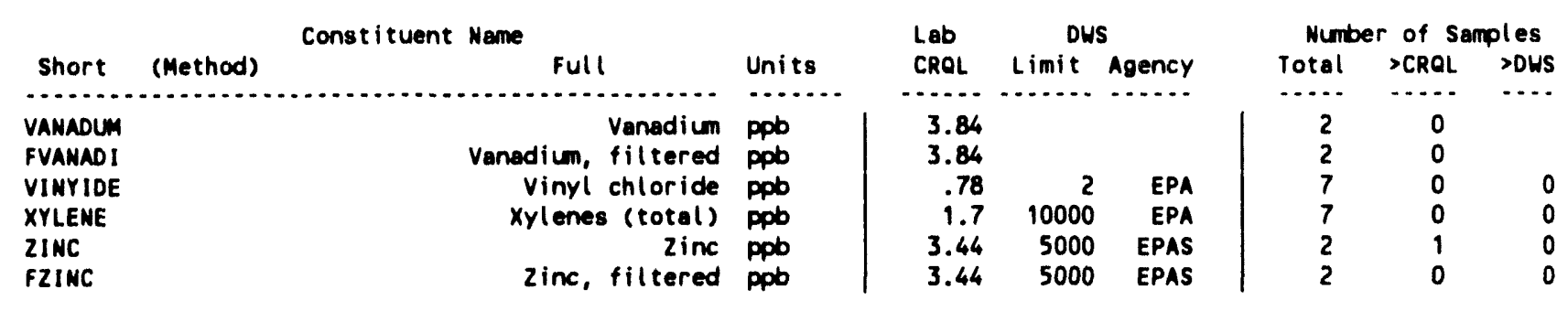

For explanation of this table, see Section 1.4 of report. 
Table 17-4. Constituents with at Least One Detected Value for the 300 Area Process Trenches Data for Reporting Period October 1

through December 31, 1993. (sheet 1 of 3)

\begin{tabular}{|c|c|c|c|c|c|c|}
\hline $\begin{array}{l}\text { Well } \\
\text { Name }\end{array}$ & $\begin{array}{c}\text { Collection } \\
\text { Date }\end{array}$ & $\begin{array}{l}\text { Sample } \\
\text { Number }\end{array}$ & $\begin{array}{r}1,1,1-1 \\
16 / \mathrm{ppb} \\
.64 / 200\end{array}$ & $\begin{array}{l}\text { 12-DCE } \\
16 / \mathrm{ppb} \\
1.2 \%\end{array}$ & $\begin{array}{r}\text { ARSENIC } \\
43 / \mathrm{ppb} \\
1.38 / 50\end{array}$ & $\begin{array}{l}\text { FARSENIC } \\
43 / \mathrm{ppO} \\
1.38 / 50\end{array}$ \\
\hline $\begin{array}{l}399-1-16 A \\
399-1-16 A \\
399-1-16 B \\
399-1-16 B \\
399-1-17 A \\
399-1-17 A \\
399-1-17 A \\
399-1-17 B \\
399-1-18 A \\
399-3-10 \\
399-3-10\end{array}$ & $\begin{array}{c}9 / 29 / 93 \\
9 / 29 / 93 \\
9 / 29 / 93 \\
9 / 29 / 93 \\
9 / 03 / 93 \\
12 / 09 / 93 \\
12 / 09 / 93 \\
9 / 07 / 93 \\
9 / 07 / 93 \\
9 / 03 / 93 \\
9 / 03 / 93\end{array}$ & $\begin{array}{l}B 09649 \\
B 09650 \\
B 09651 \\
809652 \\
B 09653 \\
B 094 \times 4 \\
B 094 \times 5 \\
B 09655 \\
B 09657 \\
B 09659 \\
B 09660\end{array}$ & $\begin{array}{l}.64 \mathrm{U} \\
.64 \mathrm{UO} \\
.64 \mathrm{UO} \\
2.30 \mathrm{~L} \\
.64 \mathrm{U} \\
.64 \mathrm{U} \\
.64 \mathrm{U}\end{array}$ & $\begin{array}{l}1.20 \mathrm{U} \\
1.20 \mathrm{U} \\
1.20 \mathrm{U} \\
5.10 \\
1.20 \mathrm{U} \\
1.20 \mathrm{U} \\
1.20 \mathrm{U}\end{array}$ & $\begin{array}{l}3.10 \mathrm{~L} \\
1.38 \mathrm{U}\end{array}$ & $\begin{array}{l}2.70 \mathrm{~L} \\
1.38 \mathrm{U}\end{array}$ \\
\hline $\begin{array}{l}\text { Hell } \\
\text { Nane }\end{array}$ & $\begin{array}{c}\text { Collection } \\
\text { Date }\end{array}$ & $\begin{array}{l}\text { Sample } \\
\text { Number }\end{array}$ & $\begin{array}{c}\text { BARIUM } \\
34 / \mathrm{ppb} \\
.001 / 1000 \mathrm{i}\end{array}$ & $\begin{array}{l}\text { FBARIUM } \\
34 / \mathrm{ppb} \\
.001 / 1000 \mathrm{i}\end{array}$ & $\begin{array}{c}\text { CALCIUM } \\
34 / \text { ppb } \\
20.9 / .\end{array}$ & $\begin{array}{l}\text { FCALCIU } \\
34 / \mathrm{ppb} \\
20.9 / .\end{array}$ \\
\hline $\begin{array}{l}399-1-178 \\
399-1-178 \\
399-1-184 \\
399-1-184\end{array}$ & $\begin{array}{l}9 / 07 / 93 \\
9 / 07 / 93 \\
9 / 07 / 93 \\
9 / 07 / 93\end{array}$ & $\begin{array}{l}809655 \\
809656 \\
B 09657 \\
B 09658\end{array}$ & $\begin{array}{l}66.00 \\
47.00\end{array}$ & $\begin{array}{l}67.00 \\
46.00\end{array}$ & $\begin{array}{l}19000.00 \\
46000.00\end{array}$ & $\begin{array}{l}19000.00 \\
46000.00\end{array}$ \\
\hline $\begin{array}{l}\text { Well } \\
\text { Name }\end{array}$ & $\begin{array}{c}\text { Collection } \\
\text { Date }\end{array}$ & $\begin{array}{l}\text { Sample } \\
\text { Number }\end{array}$ & $\begin{array}{c}\text { CHLORID } \\
124 / \mathrm{ppb} \\
82.5 / 250000 \mathrm{~s}\end{array}$ & $\begin{array}{c}\text { CHLFORM } \\
16 / \text { ppb } \\
.4 / .\end{array}$ & $\begin{array}{l}\text { CHROMUM } \\
34 / \mathrm{ppb} \\
5.42 / 50 \mathrm{i}\end{array}$ & $\begin{array}{l}\text { FCHROMI } \\
34 / \mathrm{ppb} \\
5.42 / 50 \mathrm{i}\end{array}$ \\
\hline $\begin{array}{l}399-1-17 A \\
399-1-17 A \\
399-1-17 A \\
399-1-17 B \\
399-1-17 B \\
399-1-18 A \\
399-1-18 A \\
399-3-10 \\
399-3-10\end{array}$ & $\begin{array}{r}9 / 03 / 93 \\
12 / 09 / 93 \\
12 / 09 / 93 \\
9 / 07 / 93 \\
9 / 07 / 93 \\
9 / 07 / 93 \\
9 / 07 / 93 \\
9 / 03 / 93 \\
9 / 03 / 93\end{array}$ & $\begin{array}{l}B 09653 \\
B 094 \times 4 \\
B 094 \times 5 \\
809655 \\
B 09656 \\
809657 \\
B 09658 \\
B 09659 \\
B 09660\end{array}$ & $\begin{array}{l}140000.00 \mathrm{D} \\
140000.00 \mathrm{D}\end{array}$ & $\begin{array}{l}22.00 \\
7.20 \mathrm{~B} \\
7.20 \mathrm{~B} \\
.40 \mathrm{U} \\
.40 \mathrm{U} \\
3.00 \mathrm{~L} \\
3.10 \mathrm{~L}\end{array}$ & $\begin{array}{l}76.00 \\
81.00\end{array}$ & $\begin{array}{l}5.42 \mathrm{U} \\
5.42 \mathrm{U}\end{array}$ \\
\hline
\end{tabular}

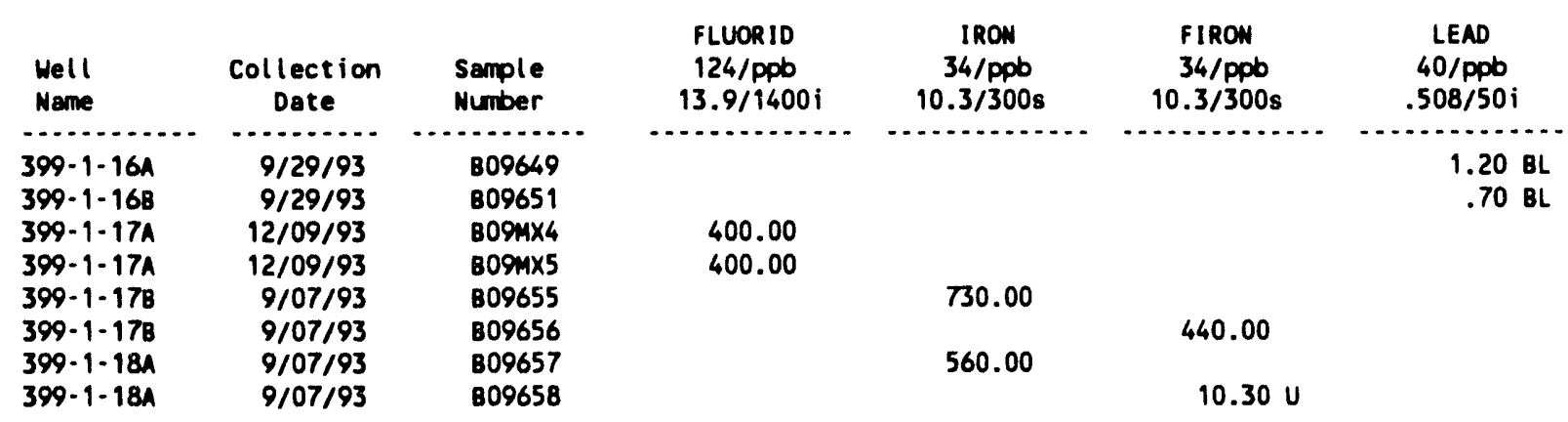


Table 17-4. Constituents with at Least One Detected Value for the 300 Area Process Trenches Data for Reporting Period October 1

through December 31, 1993. (sheet 2 of 3)

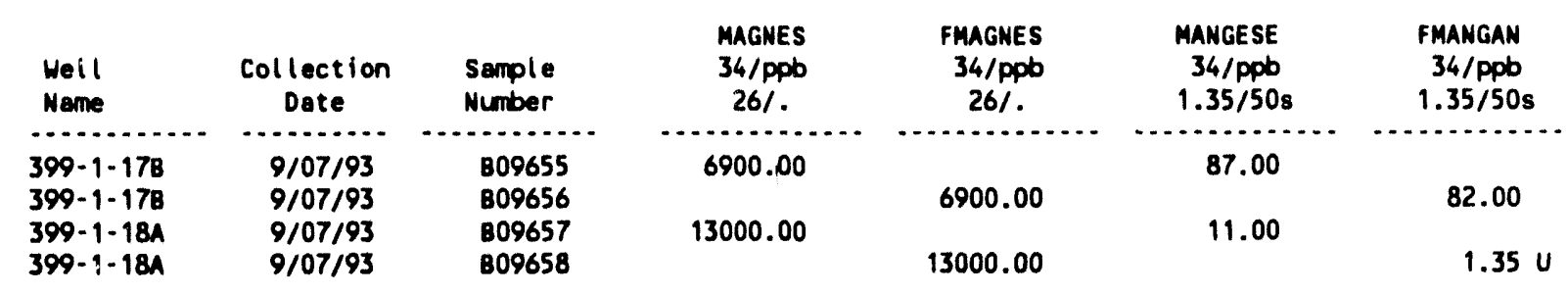

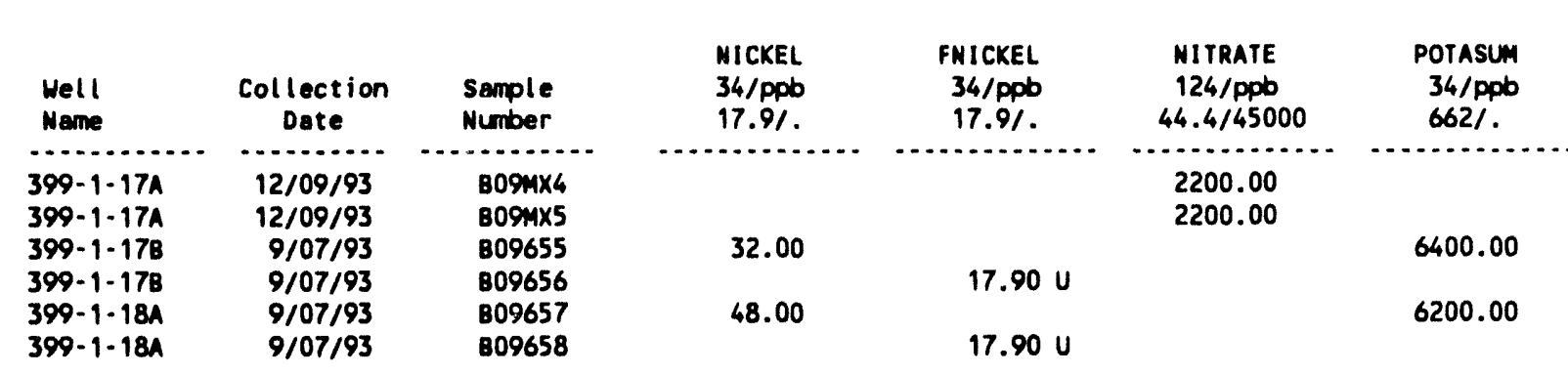

\begin{tabular}{|c|c|c|c|c|c|c|}
\hline $\begin{array}{l}\text { Well } \\
\text { Name }\end{array}$ & $\begin{array}{c}\text { Collection } \\
\text { Date }\end{array}$ & $\begin{array}{l}\text { Semple } \\
\text { Number }\end{array}$ & $\begin{array}{l}\text { FPOTASS } \\
34 / \mathrm{ppb} \\
662 / .\end{array}$ & $\begin{array}{l}\text { SELENUM } \\
48 / \mathrm{ppb} \\
1.21 / 10 \mathrm{i}\end{array}$ & $\begin{array}{c}\text { FSELENI } \\
48 / \mathrm{ppb} \\
1.21 / 10 \mathrm{i}\end{array}$ & $\begin{array}{l}\text { soolum } \\
34 / \mathrm{ppb} \\
40.91 .\end{array}$ \\
\hline $\begin{array}{l}399-1-16 A \\
399-1-16 A \\
399-1-168 \\
399-1-168 \\
399-1-178 \\
399-1-17 B \\
399-1-18 A \\
399-1-18 A\end{array}$ & $\begin{array}{l}9 / 29 / 93 \\
9 / 29 / 93 \\
9 / 29 / 93 \\
9 / 29 / 93 \\
9 / 07 / 93 \\
9 / 07 / 93 \\
9 / 07 / 93 \\
9 / 07 / 93\end{array}$ & $\begin{array}{l}B 09649 \\
B 09650 \\
B 09651 \\
809652 \\
B 09655 \\
809656 \\
809657 \\
809658\end{array}$ & $\begin{array}{l}5800.00 \\
6400.00\end{array}$ & $\begin{array}{l}3.40 \mathrm{~L} \\
2.00 \mathrm{~L}\end{array}$ & $\begin{array}{l}2.20 \mathrm{~L} \\
1.2111\end{array}$ & $\begin{array}{r}49000.00 \\
23000.00\end{array}$ \\
\hline
\end{tabular}

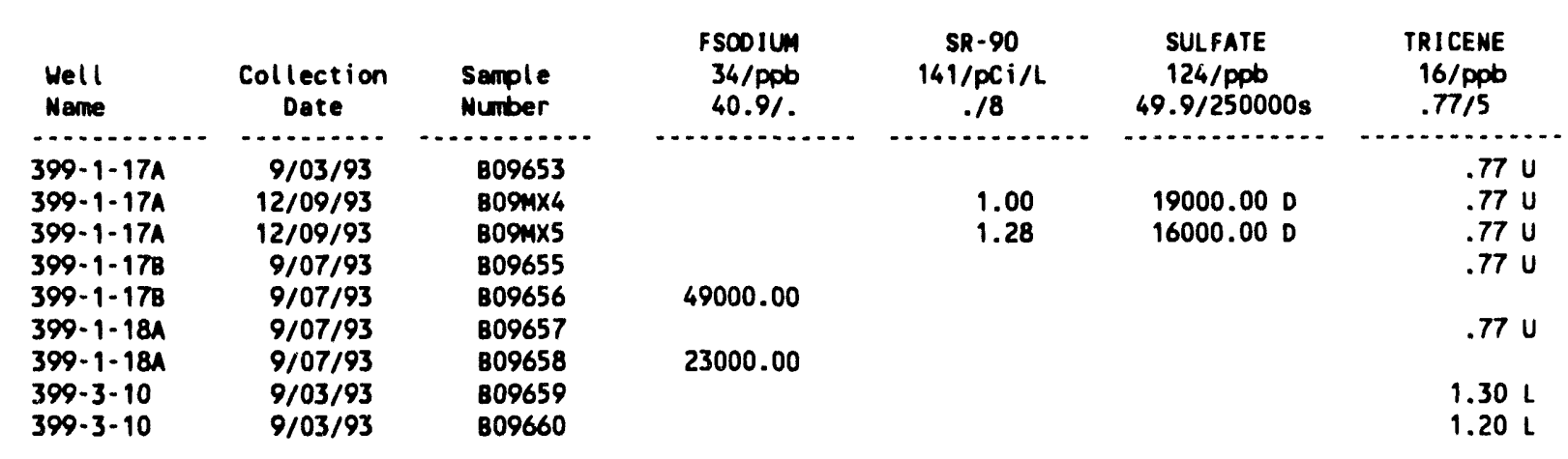

\begin{tabular}{|c|c|c|c|c|c|c|}
\hline $\begin{array}{l}\text { Well } \\
\text { Name }\end{array}$ & $\begin{array}{c}\text { Collection } \\
\text { Date }\end{array}$ & $\begin{array}{l}\text { Sample } \\
\text { Number }\end{array}$ & $\begin{array}{c}\text { URANIUM } \\
\text { 145/PpD } \\
. / .\end{array}$ & $\begin{array}{c}U-234 \\
148 / p C i / L \\
. / .\end{array}$ & $\begin{array}{c}U-235 \\
148 / p C i / L \\
. / .\end{array}$ & $\begin{array}{c}U-238 \\
148 / \mathrm{pCi} / \mathrm{L} \\
. /\end{array}$ \\
\hline $\begin{array}{l}399-1-17 A \\
399-1-17 A\end{array}$ & $\begin{array}{l}12 / 09 / 93 \\
12 / 09 / 93\end{array}$ & $\begin{array}{l}\text { Bo9MX4 } \\
\text { B09MX5 }\end{array}$ & $\begin{array}{l}19.80 \\
24.40\end{array}$ & $\begin{array}{l}11.80 \\
11.30\end{array}$ & $\begin{array}{l}.570 \\
.77 a\end{array}$ & $\begin{array}{l}8.40 \\
7.97\end{array}$ \\
\hline
\end{tabular}


Table 17-4. Constituents with at Least One Detected Value for the 300 Area Process Trenches Data for Reporting Period October 1 through December 31, 1993. (sheet 3 of 3)

\begin{tabular}{|c|c|c|c|c|}
\hline $\begin{array}{l}\text { Hell } \\
\text { Name }\end{array}$ & $\begin{array}{c}\text { Collection } \\
\text { Date }\end{array}$ & $\begin{array}{l}\text { Sample } \\
\text { Number }\end{array}$ & $\begin{array}{c}\text { ZINC } \\
34 / \mathrm{ppb} \\
3.44 / 5000 \mathrm{~s}\end{array}$ & $\begin{array}{l}\text { F21NC } \\
34 / \mathrm{ppb} \\
3.44 / 5000 \mathrm{~s}\end{array}$ \\
\hline $\begin{array}{l}399-1-178 \\
399-1-178 \\
399-1-18 A \\
399-1-184\end{array}$ & $\begin{array}{l}9 / 07 / 93 \\
9 / 07 / 93 \\
9 / 07 / 93 \\
9 / 07 / 93\end{array}$ & $\begin{array}{l}809655 \\
809656 \\
809657 \\
809658\end{array}$ & $\begin{array}{l}3.44 \mathrm{U} \\
100.00\end{array}$ & $\begin{array}{l}3.46 \mathrm{U} \\
3.44 \mathrm{U}\end{array}$ \\
\hline
\end{tabular}

For explanation of this table, see Section 1.4 of report. 
Table 17-5. Contamination Indicator Parameters for the 300 Area Process Trenches Data for Reporting Period October 1 through December 31, 1993.

\begin{tabular}{|c|c|c|c|c|c|c|c|c|}
\hline $\begin{array}{l}\text { Well } \\
\text { Name }\end{array}$ & $\begin{array}{c}\text { Collection } \\
\text { Date }\end{array}$ & $\begin{array}{l}\text { Semple } \\
\text { Number }\end{array}$ & $\begin{array}{c}\text { COND FIELO } \\
\mu \mathrm{mho} \\
1 / 700 \mathrm{~W}\end{array}$ & $\begin{array}{c}\text { COND LAB } \\
\text { mmino } \\
1 / 700 \mathrm{H}\end{array}$ & $\begin{array}{l}\text { PH FIELD } \\
.01 / 6.5-8.58\end{array}$ & $\begin{array}{c}\text { PH LAB } \\
.01 / 6.5-8.58\end{array}$ & $\begin{array}{c}\text { TOC } \\
\text { ppb } \\
200 / .\end{array}$ & $\begin{array}{l}\text { Tox } \\
\text { ppb } \\
8 /\end{array}$ \\
\hline$\ldots \ldots \ldots \ldots$ & $\ldots \ldots \ldots$ & $\ldots \ldots \ldots$ & $\ldots \ldots$ & $\ldots \ldots$ & $\ldots \ldots$ & $\ldots$ & $\cdots \ldots$ & $\ldots \ldots$ \\
\hline $399-1-17 A$ & $12 / 09 / 93$ & 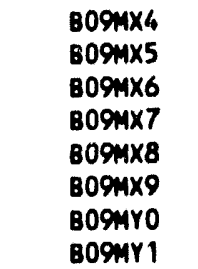 & $\begin{array}{l}609 \\
609 \\
609 \\
612\end{array}$ & $\begin{array}{l}630 \\
610 \\
630 \\
630 \\
630 \\
630 \\
630 \\
630\end{array}$ & $\begin{array}{l}7.32 \\
7.31 \\
7.31 \\
7.30\end{array}$ & $\begin{array}{l}6.90 \\
6.90 \\
6.90 \\
6.80 \\
6.90 \\
6.80 \\
6.90 \\
6.90\end{array}$ & $\begin{array}{l}700 \mathrm{~L} \\
600 \mathrm{~L} \\
700 \mathrm{~L} \\
600 \mathrm{~L} \\
700 \mathrm{~L} \\
700 \mathrm{~L} \\
700 \mathrm{~L} \\
600 \mathrm{~L}\end{array}$ & \\
\hline
\end{tabular}

For explanation of this table, see Section 1.4 of report. 
DOE/RL-93-56-4

This page intentionally left blank. 
DOE/RL-93-56-4

\section{CONTENTS}

18.0 NONRADIOACTIVE DANGEROUS WASTE LANDFILL . . . . . . . . . 18-1

18.1 INTRODUCTION . . . . . . . . . . . . . . . . . . . 18-1

18.2 WATER LEVEL DATA . . . . . . . . . . . . . . . 18-1

18.3 WATER CHEMISTRY DATA ............... 18-1 
18-1 Monitoring Well Locations for the Nonradioactive Dangerous Waste Landfill and Solid Waste Landfill ............ 18-2

\section{LIST OF TABLES}

18-1 Monitoring Well Purpose and Sampling Schedule for the Nonradioactive Dangerous Waste Landfill Network. . . . . . . 18-3

18-2 RCRA Water Level Measurement Report for the NRDWL, Fourth Quarter 1993.................. 18-4

18-3 Constituent List and Summary of Results for the NRDWL Data for Reporting Period October 1 through December $31,1993 \ldots \ldots$. . . . . . . . . . . . . .

18-4 Constituents with at Least One Detected Value for the NRDWL Data for Reporting Period October 1 through December 31, 1993 . . . . . . . . . . . . . . 18-8

18-5 Contamination Indicator Parameters for the NRDWL Data for Reporting Period October 1 through December 31, 1993 ..... . 18-10 
$D O E / R L-93-56-4$

\title{
18.0 NONRADIOACTIVE DANGEROUS WASTE LANDFILL
}

\author{
F. N. Hodges \\ Westinghouse Hanford Company
}

\subsection{INTRODUCTION}

The Nonradioactive Dangerous Waste Landfill (NRDWL) is part of the Hanford Central Landfill located approximately $5.6 \mathrm{~km}$ (3.5 mi) southeast of the 200 East Area. The NRDWL, covered under the Consent Agreement and Compliance Order (Ecology and EPA 1986), is currently under an interim-status, detection-level groundwater monitoring program (WHC 1993b). The groundwater monitoring network consists of three upgradient and six downgradient wells (Table 18-1). Two upgradient and five downgradient wells are screened at the top of the water table. One upgradient and one downgradient well are screened at the top of a low-permeability unit approximately $21.3 \mathrm{~m}(70 \mathrm{ft})$ below the top of the water table. Figure 18-1 shows well locations. It should be noted that well 699-26-34A was previously named 699-26-34.

\subsection{WATER LEVEL DATA}

Nonroutine water level measurements were carried out on a monthly schedule for nine NRDWL monitoring wells, the six monitoring wells of the adjacent Solid Waste Landfill, and in several nearby non-RCRA wells. Results of the water level measurements are reported in Table 18-2.

\subsection{HATER CHEMISTRY DATA}

The monitoring network for the NRDWL is on a semiannual sampling schedule; the two new downgradient monitoring wells (699-25-340 and 699-26-34B) completed their quarterly sampling schedule in September 1993. All wells in the monitoring network were sampled in September 1993. The next scheduled sampling for the NRDWL network is in February 1994. Previously unreported data for the September 1993 samplings are included in this report (Tables 18-3 through 18-5).

Values of specific conductance, field $\mathrm{pH}$, and TOC reported in this quarter's report do not exceed their critical means for this site. Tox values were not evaluated because the data are suspect.

A followup audit of the Datachem Laboratory, as indicated in the second quarterly report of 1993, indicates that problems with TOX analyses found in the first audit have not been corrected. The potential usefuiness of the TOX data is under evaluation (see Section 1.2.4). Until this problem is resolved, all TOX data will be flagged with ' $P$. ' An indication of this continuing problem is provided by the large variation within replicate data sets in Table 18-5. 
Figure 18-1. Monitoring Well Locations for the Nonradioactive Dangerous Waste Landf 111 and Solid Waste Landfill.

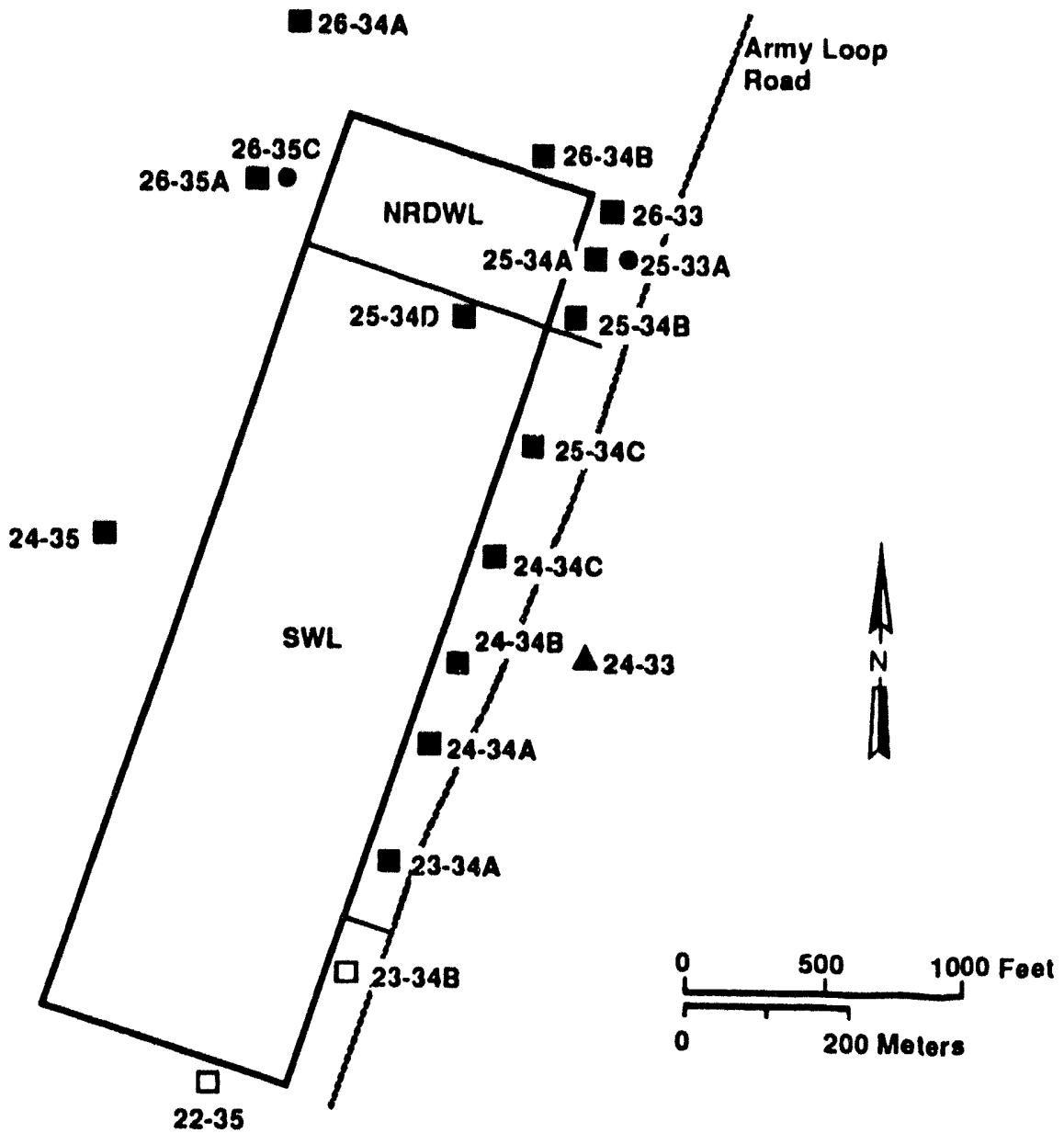

Well completed at the top of the unconfined aquifer

- Well completed in the upper Ringold Formation

- Well not constructed to RCRA specifications

D Now RCRA wells comploted in 1993.

NRDWL Nonradioactive Dangerous Waste Landfill

SWL Solid Waste Landfill

All wells prefixed by 699 - 
Table 18-1. Monitoring Well Purpose and Sampling Schedule for the Nonradioactive Dangerous Waste Landfill Network.

\begin{tabular}{|c|c|l|c|c|}
\hline $\begin{array}{c}\text { Well no. } \\
(699-)\end{array}$ & $\begin{array}{c}\text { Relative } \\
\text { position }\end{array}$ & \multicolumn{1}{|c|}{ Hydrogeologic unit } & $\begin{array}{c}\text { Sample } \\
\text { frequency }\end{array}$ & $\begin{array}{c}\text { Sample date, } \\
\text { 4th Qtr 1993 }\end{array}$ \\
\hline $26-34 A$ & Upgradient & Hanford: Water Table & Semiannually & -- \\
\hline $26-35 A^{\circ}$ & Upgradient & Hanford: Water Table & Semiannually & -- \\
\hline $26-35 C$ & Upgradient & Top of Ringold & Semiannually & -- \\
\hline $25-33 A$ & Downgradient & Top of Ringold & Semiannually & -- \\
\hline $25-34 A$ & Downgradient & Hanford: Water Table & Semiannually & -- \\
\hline $25-34 B$ & Downgradient & Hanford: Water Table & Semiannually & -- \\
\hline $25-340$ & Downgradient & Hanford: Water Table & Semiannually & -- \\
\hline $26-33$ & Downgradient & Hanford: Water Table & Semiannually & -- \\
\hline $26-34 B$ & Downgradient & Hanford: Water Table & Semiannually & -- \\
\hline
\end{tabular}

Note: Hydrogeologic units include the sandy gravels of the Hanford formation and silty sands of the Ringold Formation. Water levels are measured monthly in all wells.

${ }^{a}$ Well also sampled as part of Solid Waste Landfill network. 
Table 18-2. RCRA Water Level Measurement Report for the NRDWL, Fourth Quarter 1993. (sheet 1 of 3)

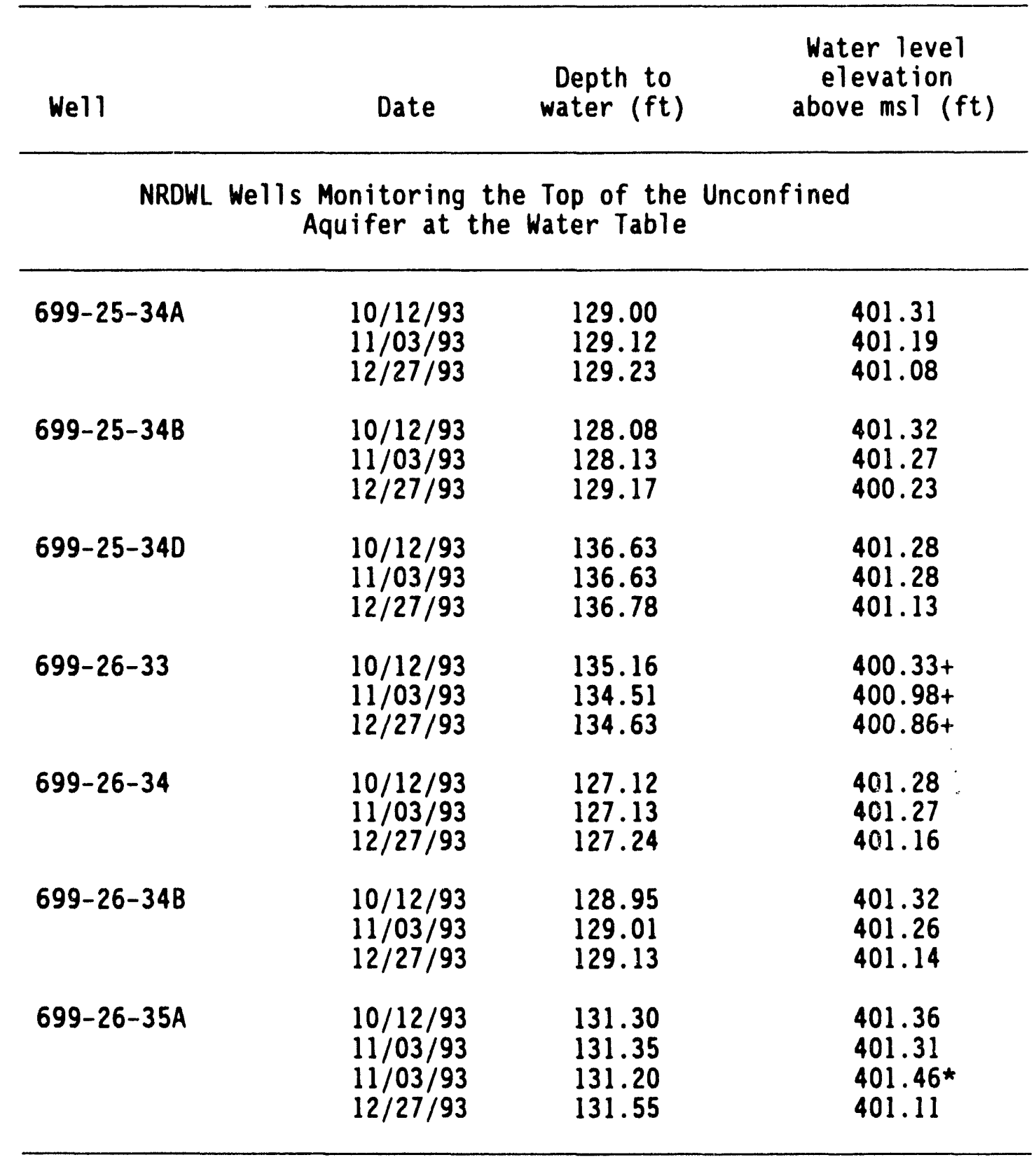

NRDWL Wells Monitoring the Upper Ringold

\begin{tabular}{llll}
\hline $699-25-33 A$ & $10 / 12 / 93$ & 127.75 & 401.22 \\
& $11 / 03 / 93$ & 127.76 & 401.21 \\
& $12 / 27 / 93$ & 128.00 & 400.97 \\
$699-26-35 C$ & $10 / 12 / 93$ & 131.31 & 401.37 \\
& $11 / 03 / 93$ & 131.41 & 401.27 \\
& $12 / 27 / 93$ & 131.51 & 401.17
\end{tabular}


Table 18-2. RCRA Water Level Measurement Report for the NRDWL, Fourth Quarter 1993. (sheet 2 of 3 )

Well Date $\begin{gathered}\text { Depth to } \\ \text { water }(\mathrm{ft})\end{gathered} \begin{gathered}\text { Water level } \\ \text { elevation } \\ \text { above msl (ft) }\end{gathered}$

NRDWL Wells Monitoring the Upper Ringold

Other Wells Associated with NRDWL

\begin{tabular}{|c|c|c|c|}
\hline $699-20-20$ & $\begin{array}{l}10 / 12 / 93 \\
10 / 15 / 93 \\
11 / 03 / 93 \\
11 / 17 / 93 \\
12 / 27 / 93\end{array}$ & $\begin{array}{l}105.75 \\
105.66 \\
105.78 \\
105.76 \\
105.92\end{array}$ & $\begin{array}{l}399.83 \\
399.92^{\star} \\
399.80 \\
399.82^{\star} \\
399.66\end{array}$ \\
\hline $699-20-39$ & $\begin{array}{l}10 / 12 / 93 \\
11 / 03 / 93 \\
12 / 27 / 93\end{array}$ & $\begin{array}{l}138.42 \\
138.38 \\
138.58\end{array}$ & $\begin{array}{l}401.56 \\
401.60 \\
401.40\end{array}$ \\
\hline $699-23-34$ & $\begin{array}{l}10 / 12 / 93 \\
11 / 03 / 93 \\
12 / 27 / 93\end{array}$ & $\begin{array}{l}131.59 \\
131.61 \\
131.75\end{array}$ & $\begin{array}{l}401.27 \\
401.25 \\
401.11\end{array}$ \\
\hline $699-24-33$ & $\begin{array}{l}10 / 12 / 93 \\
11 / 03 / 93 \\
11 / 03 / 93 \\
12 / 27 / 93\end{array}$ & $\begin{array}{l}123.03 \\
123.05 \\
122.98 \\
123.20\end{array}$ & $\begin{array}{l}401.18 \\
401.16 \\
401.23 * \\
401.01\end{array}$ \\
\hline $699-24-34 A$ & $\begin{array}{l}10 / 12 / 93 \\
11 / 03 / 93 \\
11 / 03 / 93 \\
12 / 27 / 93\end{array}$ & $\begin{array}{l}132.63 \\
132.64 \\
132.61 \\
132.77\end{array}$ & $\begin{array}{l}401.26 \\
401.25 \\
401.28 * \\
401.12\end{array}$ \\
\hline $699-24-34 B$ & $\begin{array}{l}10 / 12 / 93 \\
11 / 03 / 93 \\
11 / 03 / 93 \\
12 / 27 / 93\end{array}$ & $\begin{array}{l}132.21 \\
132.24 \\
132.22 \\
132.36\end{array}$ & $\begin{array}{l}401.29 \\
401.26 \star \\
401.28 \\
401.14\end{array}$ \\
\hline $699-24-34 C$ & $\begin{array}{l}10 / 12 / 93 \\
11 / 03 / 93 \\
11 / 03 / 93 \\
12 / 27 / 93\end{array}$ & $\begin{array}{l}131.32 \\
131.34 \\
131.29 \\
131.47\end{array}$ & $\begin{array}{l}401.26 \\
401.24 \\
401.29 \star \\
401.11\end{array}$ \\
\hline
\end{tabular}


Table 18-2. RCRA Water Level Measurement Report for the NRDWL, Fourth Quarter 1993. (sheet 3 of 3)

\begin{tabular}{|c|c|c|c|}
\hline Well & Date & $\begin{array}{l}\text { Depth to } \\
\text { water (ft) }\end{array}$ & $\begin{array}{l}\text { Water level } \\
\text { elevation } \\
\text { above msl }(\mathrm{ft})\end{array}$ \\
\hline \multicolumn{4}{|c|}{ Other Wells Associated with NRDWL } \\
\hline $699-24-35$ & $\begin{array}{l}10 / 12 / 93 \\
11 / 03 / 93 \\
11 / 03 / 93 \\
12 / 27 / 93\end{array}$ & $\begin{array}{l}137.53 \\
137.49 \\
137.47 \\
137.66\end{array}$ & $\begin{array}{l}401.28 \\
401.32^{*} \\
401.34 \\
401.15\end{array}$ \\
\hline $699-25-34 C$ & $\begin{array}{l}10 / 12 / 93 \\
11 / 03 / 93 \\
11 / 03 / 93 \\
12 / 27 / 93\end{array}$ & $\begin{array}{l}134.19 \\
134.31 \\
134.20 \\
134.35\end{array}$ & $\begin{array}{l}401.27 \\
401.15 \\
401.26^{\star} \\
401.11\end{array}$ \\
\hline $699-28-40$ & $\begin{array}{l}10 / 12 / 93 \\
11 / 03 / 93 \\
12 / 27 / 93\end{array}$ & $\begin{array}{l}157.90 \\
158.02 \\
158.05\end{array}$ & $\begin{array}{l}401.54 \\
401.42 \\
401.39\end{array}$ \\
\hline $699-31-31$ & $\begin{array}{l}10 / 12 / 93 \\
11 / 03 / 93 \\
12 / 27 / 93\end{array}$ & $\begin{array}{l}128.17 \\
127.97 \\
128.27\end{array}$ & $\begin{array}{l}401.15 \\
401.35 \\
401.05\end{array}$ \\
\hline $699-34-39 A$ & $\begin{array}{l}10 / 12 / 93 \\
11 / 03 / 93 \\
12 / 27 / 93\end{array}$ & $\begin{array}{l}135.44 \\
135.45 \\
135.60\end{array}$ & $\begin{array}{l}401.63 \\
401.62 \\
401.47\end{array}$ \\
\hline
\end{tabular}

Notes: 1. Water level elevations are calculated by subtracting the measured depth to water from the surveyed elevation for the well.

2. Depth-to-water values are transcribed from field records.

3. Elevations marked with an ' $*$ ' were measured at the time of sampling.

4. Elevations marked with a ' + ' are outside of the expected range and are suspected of error.

5. To convert feet to meters multiply by 0.3048 . 
Table 18-3. Constituent List and Summary of Results for the NRDWL Data for Reporting Period October 1 through December 31, 1993.

CONTAMIMATION INDICATOR PARAMETERS

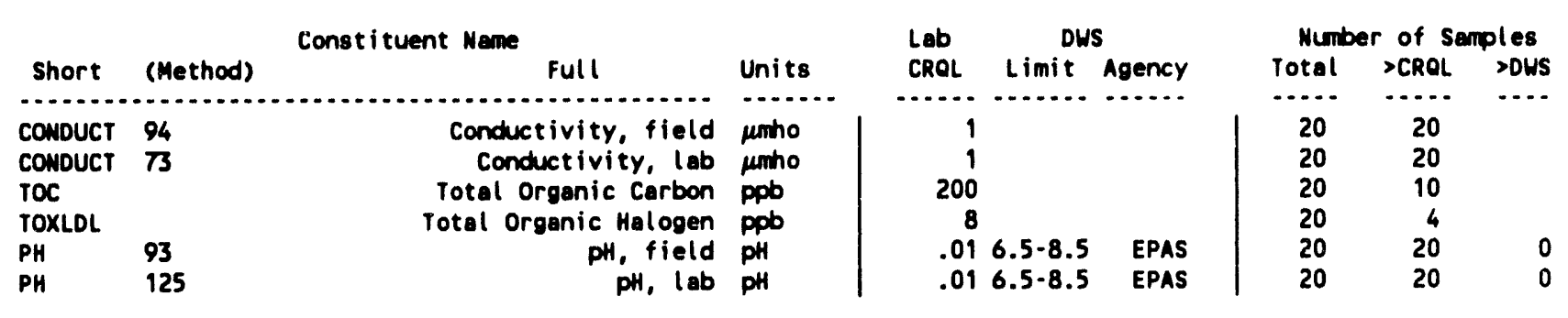

SITE SPECIFIC AND OTHER CONSTITUENTS

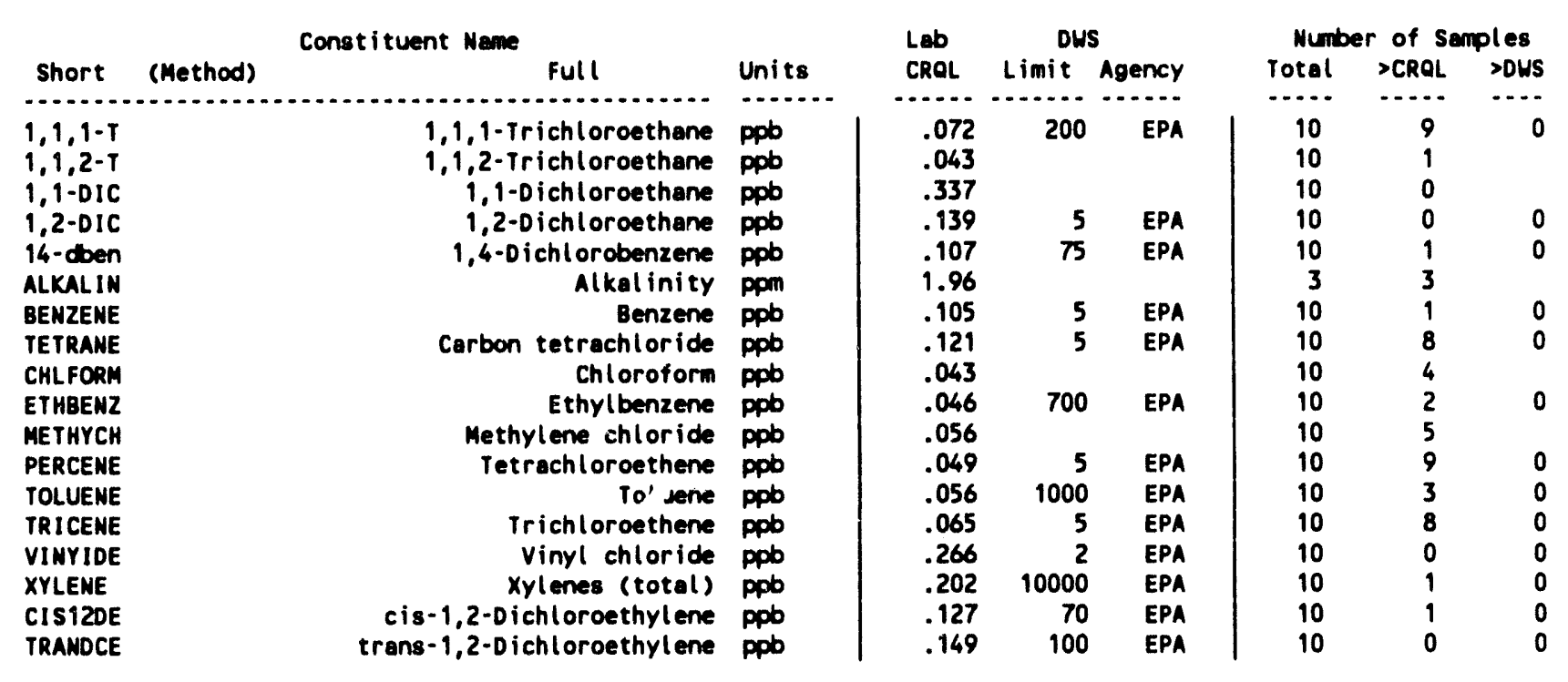

For explanation of this table, see Section 1.4 of report. 
Table 18-4. Constituents with at Least One Detected Value for the NRDWL Data for Reporting Period October 1 through December 31, 1993. (sheet 1 of 2)

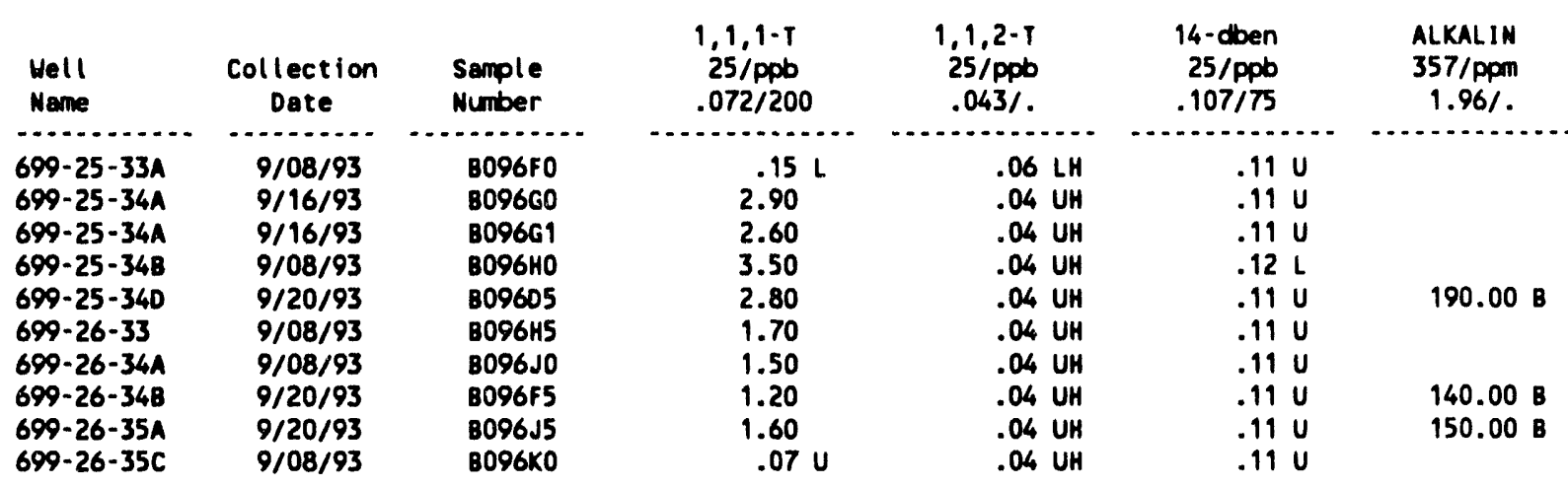

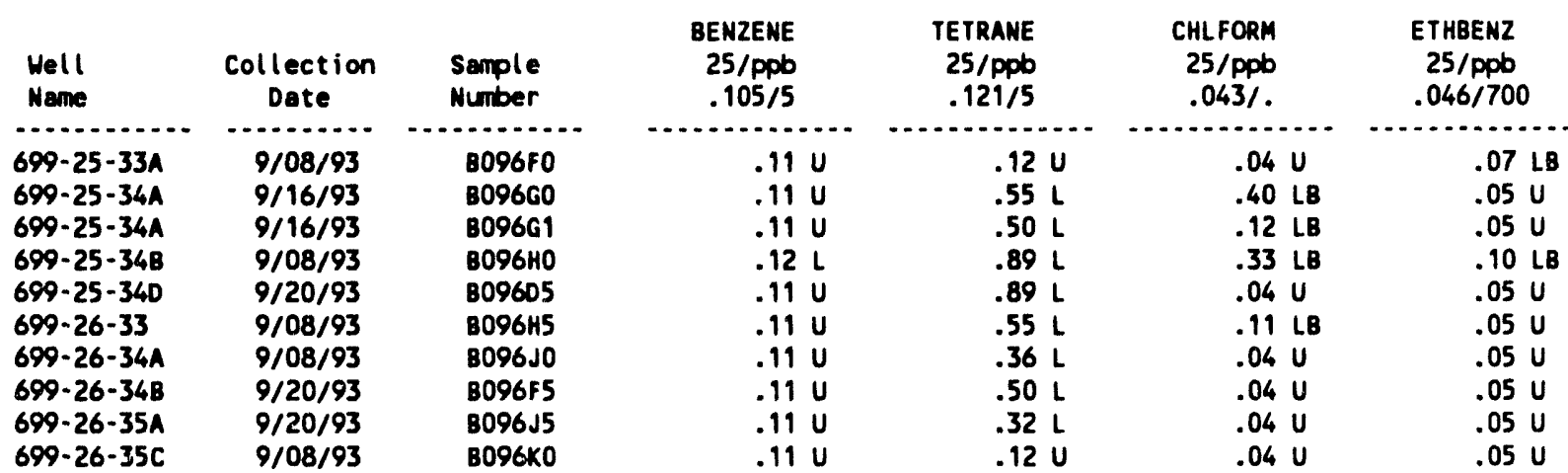

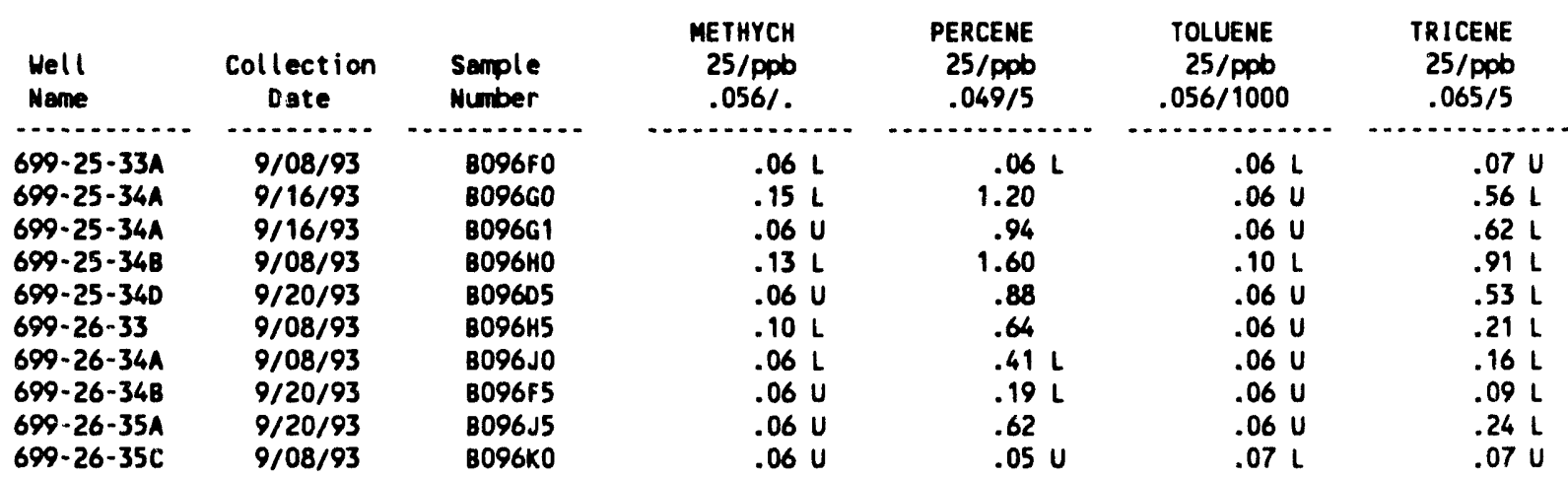

\begin{tabular}{|c|c|c|c|c|}
\hline $\begin{array}{l}\text { Mell } \\
\text { Name }\end{array}$ & $\begin{array}{c}\text { Collection } \\
\text { Date }\end{array}$ & $\begin{array}{l}\text { Sample } \\
\text { Number }\end{array}$ & $\begin{array}{c}\text { XYLENE } \\
25 / \text { ppb } \\
.202 / 10000\end{array}$ & $\begin{array}{r}\text { CIS12DE } \\
25 / p p b \\
.127 / 70\end{array}$ \\
\hline 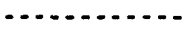 & $\ldots$ & $\ldots \ldots$ & . & $\ldots \ldots$ \\
\hline $\begin{array}{l}699-25-33 A \\
699-25-34 A \\
699-25-34 A \\
699-25-34 B\end{array}$ & $\begin{array}{l}9 / 08 / 93 \\
9 / 16 / 93 \\
9 / 16 / 93 \\
9 / 08 / 93\end{array}$ & $\begin{array}{l}\text { B096FO } \\
\text { B096GO } \\
\text { B096G1 } \\
\text { B096HO }\end{array}$ & $\begin{array}{l}.20 \mathrm{U} \\
.20 \mathrm{U} \\
.20 \mathrm{U} \\
.24 \mathrm{LB}\end{array}$ & $\begin{array}{l}.13 \mathrm{U} \\
.13 \mathrm{U} \\
.15 \mathrm{~L} \\
.13 \mathrm{U}\end{array}$ \\
\hline
\end{tabular}


Table 18-4. Constituents with at Least One Detected Value for the NRDWL Data for Reporting Period October 1 through December 31, 1993. (sheet 2 of 2 )

\begin{tabular}{|c|c|c|c|c|}
\hline $\begin{array}{l}\text { Hell } \\
\text { Name }\end{array}$ & $\begin{array}{c}\text { Collection } \\
\text { Dote }\end{array}$ & $\begin{array}{l}\text { Sample } \\
\text { Number }\end{array}$ & $\begin{array}{c}\text { XYLENE } \\
25 / p p b \\
.202 / 10000\end{array}$ & $\begin{array}{r}\text { CIS12DE } \\
25 / p p b \\
.127 / 70\end{array}$ \\
\hline $\begin{array}{l}699-25-340 \\
699-26-33 \\
699-26-34 A \\
699-26-34 B \\
699-26-35 A \\
699-26-35 C\end{array}$ & $\begin{array}{l}9 / 20 / 93 \\
9 / 08 / 93 \\
9 / 08 / 93 \\
9 / 20 / 93 \\
9 / 20 / 93 \\
9 / 08 / 93\end{array}$ & $\begin{array}{l}\text { B09605 } \\
\text { B096H5 } \\
\text { B096J0 } \\
\text { B096F5 } \\
\text { B096J5 } \\
\text { B096K0 }\end{array}$ & $\begin{array}{l}.20 \mathrm{U} \\
.20 \mathrm{U} \\
.20 \mathrm{U} \\
.20 \mathrm{U} \\
.20 \mathrm{U} \\
.20 \mathrm{U}\end{array}$ & $\begin{array}{l}.13 U \\
.13 U \\
.13 U \\
.13 U \\
.13 U \\
.13 U\end{array}$ \\
\hline
\end{tabular}

For explanation of this table, see Section 1.4 of report. 
Table 18-5. Contamination Indicator Parameters for the NRDWL Data for Reporting Period October 1 through December 31, 1993.

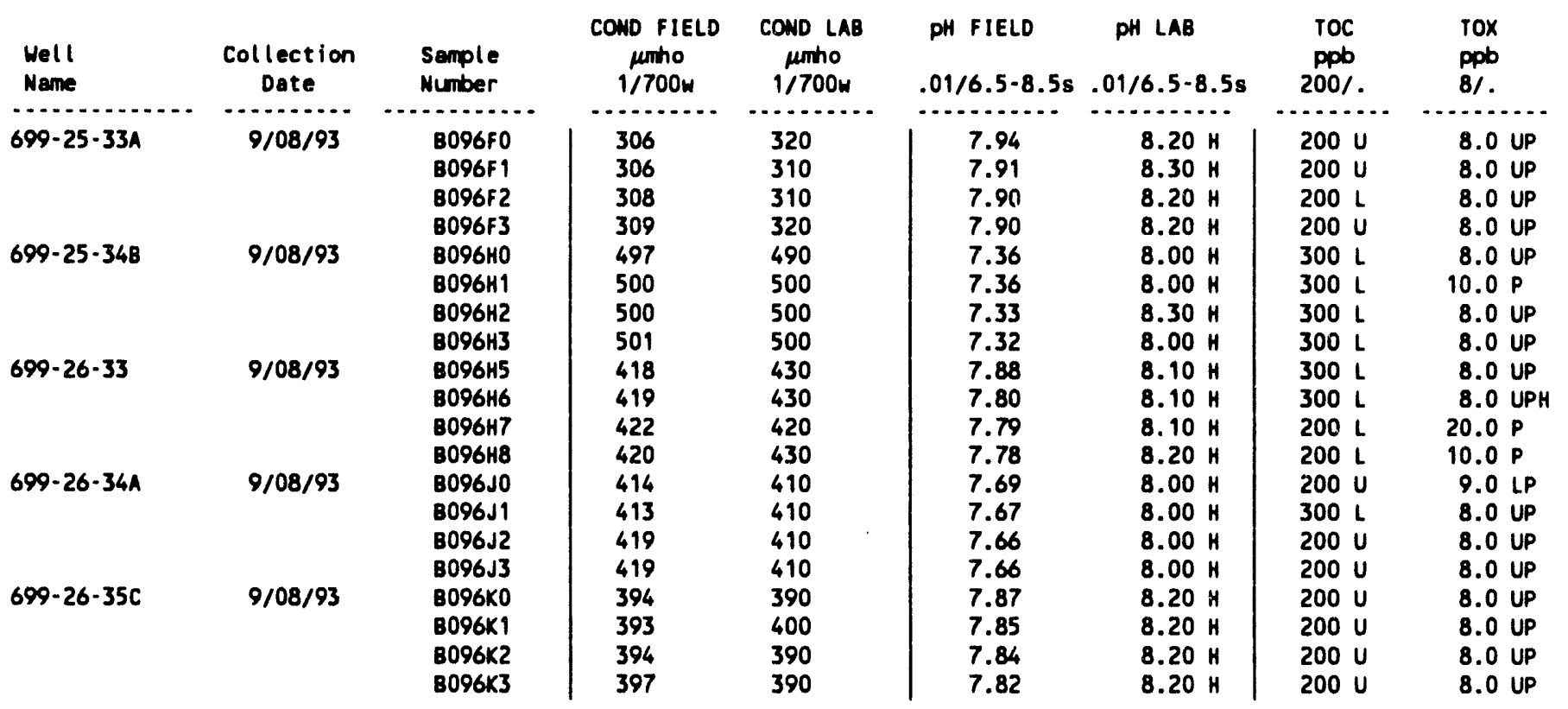

For explanation of this table, see Section 1.4 of report. 


\subsection{REFERENCES}

40 CFR 141, "National Primary Drinking Water Regulations," Code of Federal Regulations, as amended.

40 CFR 143, "National Secondary Drinking Water Regulations," Code of Federal Regulations, as amended.

40 CFR 265, "Interim Status Standards for Owners and Operators of Hazardous Waste Treatment, Storage, and Disposal Facilities," Code of Federal Regulations, as amended.

Anderson, J. D., 1990, A History of the 200 Area Tank Farms, WHC-MR-0132, Westinghouse Hanford Company, Richland, Washington.

APHA, 1989, Standard Methods for Evaluation of Water and Wastewater, 17th ed., American Public Health Association, Washington, D.C.

ASTM, 1991, Annual Book of ASTM Standards, Vo1. 11.01, Water and Environmental Technology, Philadelphia, Pennsylvania.

Caggiano, J. A., and C. J. Chou, 1993, Interim-Status Groundwater Quality Assessment Plan for the Single Shell Tank Waste Management Areas $T$ and TX-TY: Richland, Washington,. Westinghouse Hanford Company, WHC-SD-EN-AP-132, Rev. 0, Westinghouse Hanford Company, Richland, Washington.

Chou, C. J., G. L. Kasza, and R. B. Mercer, 1990, Interim-Status Groundwater Quality Assessment Plan for the 216-A-29 Ditch, WHC-SD-EN-AP-031, Rev. 0 , Westinghouse Hanford Company, Richland, Washington.

DOE, 1987, 216-A-29 Ditch Preliminary Closure/Post Closure Plan, TRAC-0095, U.S. Department of Energy, Richland Operations Office, Richland, Washington.

DOE-RL, 1993a, Annual Report for RCRA Groundwater Monitoring Projects at Hanford Site Facilities for 1992, D0E/RL-93-09, U.S. Department of Energy, Richland Operations Office, Richland, Washington.

DOE-RL, 1993b, Quarterly Report of RCRA Groundwater Monitoring Data for Period April 1, 1993 through June 30, 1993, DOE/RL-93-56-2, U.S. Department of Energy, Richland Operations Office, Richiand, Washington.

DOE-RL, 1994a, Annual Report for RCRA Groundwater Monitoring Projects at Hanford Site Facilities for 1993, DOE/RL-93-88, U.S. Department of Energy, Richland Operations Office, Richland, Washington.

DOE-RL, 1994b, Quarterly Report of RCRA Groundwater Monitoring Data for Period July 1, 1993 through September 30, 1993, DOE/RL-93-56-3, U.S. Department of Energy, Richland Operations Office, Richland, Washington. 
Ecology and EPA, 1986, Consent Agreement and Compliance Order, Ecology No. DE 86-133, Washington State Department of Ecology and the U.S. Environmental Protection Agency, Olympia, Washington.

Ecology, EPA, and DOE, 1992, Hanford Federal Facility Agreement and Consent Order, 2 vols, Washington State Department of Ecology, U.S. Environmental Protection Agency, and U.S. Department of Energy, Olympla, Washington.

EPA, 1979, Methods for Chemical Analysis of Water and Waste, EPA-600/4-79-020, U.S. Environmerital Protection Agency, Environmental Monitoring and Support Laboratory, Cincinnati, Ohio.

EPA, 1986a, RCRA Groundwater Monitoring Technical Enforcement Guidance Document (TEGD), OSWER-9950.1, U.S. Environmental Protection Agency, Washington, D.C.

EPA, 1986b, Test Methods for Evaluating Solid Waste, SW-846, Third"Edition, U.S. Environmental Protection Agency, Washington, D.C.

Harris, S. F., 1990, Groundwater Quality Assessment Plan for the 216-8-3 Pond System, WHC-SD-EN-AP-030, Rev. 0, Westinghouse Hanford Company, Richland, Washington.

Hartman, M. J., 1991, Groundwater Monitoring Plan for the 100-D Ponds, WHC-SD-EN-AP-048, Rev. 0, Westinghouse Hanford Company, Richland, Washington.

Hartman, M. J., 1993, Groundwater Quality Assessment Plan for the 1324-N/NA Site, WHC-SD-EN-AP-005, Rev. 1, Westinghouse Hanford Company, Richland, Washington.

Jensen, E. J., S. P. Airhart, M. A. Chamness, T. J. Gilmore, D. R. Newcomer, and K. R. Oster, 1989, 40 CFR 265 Interim-Status Ground-Water Monitoring Plan for the Single-Shell Tanks, WHC-SD-EN-AP-012, Westinghouse Hanford Company, Richland, Washington

Johnson, V. G., 1993, Westinghouse Hanford Company Operational Groundwater Status Report, 1990-1992, WHC-EP-0595, Westinghouse Hanford Company, Richland, Washington.

Kasza, G. L., and S. M. Goodwin, 1991, Groundwater Monitoring Plan for the 216-A-29 Ditch, WHC-SD-EN-AP-045, Rev. 0, Westinghouse Hanford Company, Richland, Washington.

PNL, 1989, 40 CFR 265 Interim-Status Indicator-Evaluation Groundwater Monitoring Plan for the 216-B-63 Trench, PNL-6862, Pacific Northwest Laboratory, Richland, Washington.

RCW 248, "Public Water Supplies," Revised Code of Washington, as amended.

Resource Conservation and Recovery Act of 1976, 42 USC 6901, et seq. 
Schalla, R., 1988, Revised Ground-Water Monitoring Compliance Plan for the 300 Area Process Trenches, PNL-6671, Pacific Northwest Laboratory, Richland, Washington.

Schmidt, J. W., A. R. Johnson, S. M. McKinney, C. J. Perkins, and C. R. Webb, 1992, West inghouse Hanford Company Environmental Surveillance Annual Report, WHC-EP-0573, West inghouse Hanford Company, Richland, Washington.

Teel, S. S., B. N. Bjornstad, and D. R. Newcomer, 1989, Interim-Status Ground Water Monitoring Plan for the Grout Treatment Facility, WHC-SD-EN-AP-006, Rev. 0, Westinghouse Hanford Company, Richland, Washington.

WAC 173-160, "Minimum Standards for Construction and Maintenance of Wells," Washington Administrative Code, as amended.

WAC 173-200, "Water Quality Standards of the State of Washington, "Washington Administrative Code, as amended.

WAC 173-303, "Dangerous Waste Regulations," Washington Administrative Code, as amended.

WHC, 1990a, 183-D Filter Backwash Wastewater Stream-Specific Report, WHC-EP-0342, Addendum 33, Westinghouse Hanford Company, Richland, Washington.

WHC, 1990b, Interim-Status Groundwater Monitoring Plan for the 216-S-10 Pond and Ditch, WHC-SD-EN-AP-018, Rev. 0, Westinghouse Hanford Company, Richland, Washington.

WHC, 1990C, Interim-Status Groundwater Monitoring Plan for the 216-U-12 Crib, WHC-SD-EN-AP-019, Rev. 0, Westinghouse Hanford Company, Richland, Washington.

WHC, 1991, Interim Status Groundwater Monitoring Plan for the 200 East Area Liquid Effluent Retention Facility, WHC-SD-EN-AP-024, Rev. 1, Westinghouse Hanford Company, Richland, Washington.

WHC, 1992, Quality Assurance Project Plan for RCRA Groundwater Monitoring Activities, WHC-SD-EN-QAPP-001, Rev. 1, Westinghouse Hanford Company, Richland, Washington.

WHC, 1993a, Interim-Status Groundwater Quality Assessment Plan for the 216-U-12 Crib, WHC-SD-EN-AP-108, Rev. 0, Westinghouse Hanford Company, Richland, Washington.

WHC, 1993b, Interim Status Groundwater Monitoring Plan for the Nonradioactive Dangerous Waste Landfill, Hanford, Washington, WHC-SD-EN-AP-026, Rev. 0 , Westinghouse Hanford Company, Richland, Washington.

WHC, 1993c, Radionuclide and Chemical Inventories for the Single-Shell Tanks: Report Prepared for Westinghouse Hanford Company by WASTREN, Inc., WHC-SD-WM-TI-565, Rev. 1, Westinghouse Hanford Company, Richland, Washington. 
DOE/RL-93-56-4

This page intentionally left blank. 
DOE/RL-93-56-4

\section{DISTRIBUTION}

\section{Number of coples}

OFESITE

2

Washington State Department of

Ecology - Kennewick Branch

7601 W. Clearwater, Sulte 102

Kennewick, Washington 99336

J. Wallace

1

Westinghouse Sayannah River Company

P. 0. Box A

Aiken, South Carolina 29801

R. C. Tuckfield

ONSITE

10

\section{U.S. Department of Energy,} Richland Operations office
R. D. Freeberg
A5-19
M. J. Furnan (4)
R3-80
J. M. Hennig
R3-80
R. G. Mcleod
A5-19
R. P. Saget
A5-54
RL Public Reading Room (2)
H2-53

4

Pacific Northwest Laboratory

R. W. Bryce

K6-96

B. E. Opitz

$K 6-79$

D. L. Stewart

K6-96

Technical Files

$K 1-11$

69

Westinghouse Hanford Company

M. R. Adams

H6-01

L. S. Angerman

L6-61

D. B. Barnett

H6-06

D. J. Brown

B4-63

R. W. Brown

L6-79

W. R. Brown

H6-30

J. A. Caggiano

H6-06

C. J. Chou

J. J. Dorian

H6-06

D. G. Farwick

H6-30

H4-16

K. R. Fecht

H6-06 
DOE/RL-93-56-4

DISTRIBUTION (cont.)

Number of copies

ONSITE

Westinghouse Hanford Company (cont.)

B. H. Ford

H6-06

M. J. Galgoul

H6-03

M. G. Gardner

N3-06

R. D. Gustavson

R1-51

W. M. Hayward

T7-05

F. N. Hodges

D. J. Hoff

D. G. Horton

H6-06

M. C. Hughes

H6-23

L. C. Hulstrom

H6-06

R. L. Jackson

V. G. Johnson

J. C. Johnston

$D$. K. Jungers (10)

$\times 5-55$

H6-03

J. R. Kasper

G. L. Kasza

A. J. Knepp

A. G. Law

L. D. Lockard

H6-06

H6-06

H6-06

H6-06

R2-54

H6-06

H6-06

H6-06

M. M. McCarthy

S3-90

A. K. McDowe 11

N3-13

R. B. Mercer

R1-51

M. A. Mihalic

H6-06

D. L. Parker

$\times 5-55$

R. D. Pierce

D. A. Pratt

H6-03

S. M. Price

H5-11

T4-03

W. A. Retterer

H6-23

J. T. Sams

J. S. Schmid

J. A. Serkowski

S2-37

H5-14

H6-06

K. R. Tanasse

H6-06

$R$. R. Thompson

N3-05

E. C. Thornton

H6-32

D. J. Watson

H6-06

$6 / 27194$

B. A. Williams

$\times 0-41$

C. D. Wittreich

H6-06

M. T. York

Central Files

H6-03

H6-32

EPIC (7)

Information Release Administration

L8-04

ENd

DATE

Document Processing and Distribution (2)

H4-17

L8-15 